\title{
$\beta$-Hematin Crystal Formation: New Insights from Molecular Dynamics Simulations of Small Clusters in Condensed Phase
}

\section{Supporting Information}

Jean-Paul Becker, Fan Wang, Pascal Sonnet and François-Yves Dupradeau* UMR CNRS 7378, UFR de Pharmacie, Université de Picardie Jules Verne, 1, rue des Louvels, F-80037 Amiens, France

*corresponding author: Email: fyd@q4md-forcefieldtools.org

Phone: $+33(0) 3-2282-7498$

Fax: $\quad+33(0) 3-2282-7469$ 


\section{Contents}

1 Becker et al. force field 2

1.1 Force field parameter files . . . . . . . . . . . . . . . . . . 2

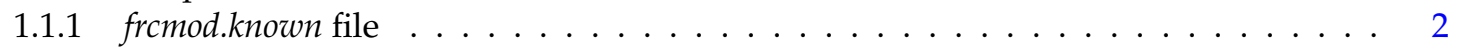

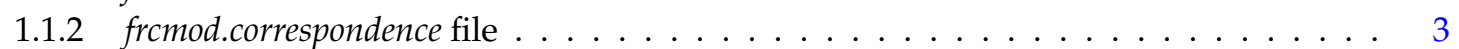

1.2 Force field library files . . . . . . . . . . . . . . . . . . . . . . . . . . 4

1.2.1 m1-c1_f1.mol2 file ......................... 4

$1.2 .2 m 2-c 1$ f1.mol2 file ........................ 6

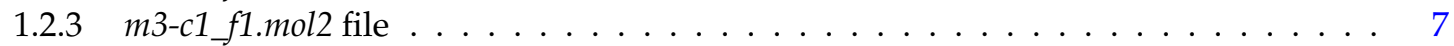

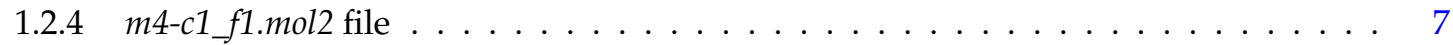

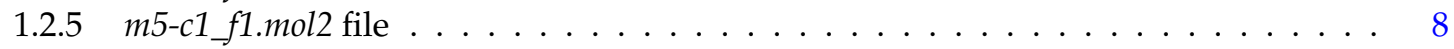

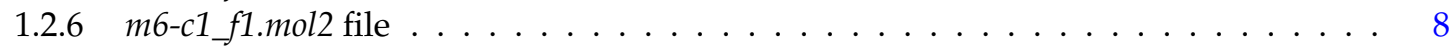

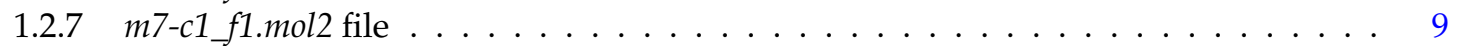

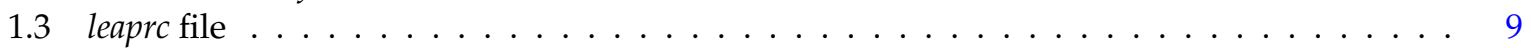

2 Cartesian coordinates of input structures of molecular dynamics in the PDB file format 31

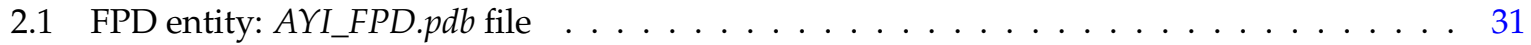

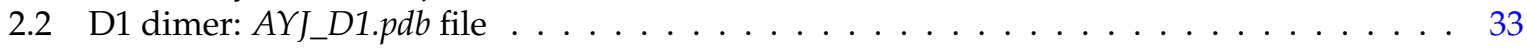

2.3 D2 dimer: A01_D2.pdb file . . . . . . . . . . . . . . . . . . . . . . 38

2.4 D3 dimer: D04_D3.pdb file . . . . . . . . . . . . . . . . . . . . . . . 42

2.5 D4 dimer: D05_D4.pdb file . . . . . . . . . . . . . . . . . . . . . . . . . . . . . . 46

2.6 D5 dimer: AYK_D5.pdb file . . . . . . . . . . . . . . . . . . . . . . . . . . . . 50

2.7 T1 dimer: $A Y Y_{-} T 1 . p d b$ file . . . . . . . . . . . . . . . . . . . . . . . . 54

2.8 T2 dimer: A02_T2.pdb file . . . . . . . . . . . . . . . . . . . . 61

2.9 T3 dimer: A03_T3.pdb file . . . . . . . . . . . . . . . . . . . . . 67

2.10 T4 dimer: AYR_T4.pdb file . . . . . . . . . . . . . . . . . . . . . 73

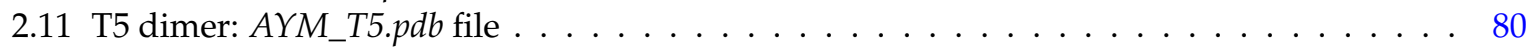

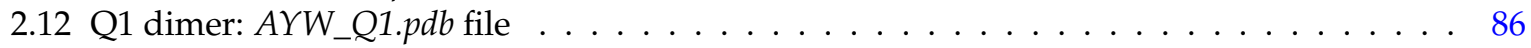

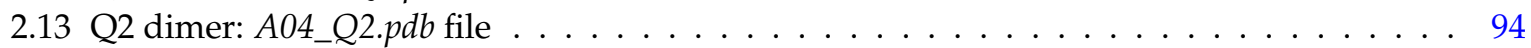

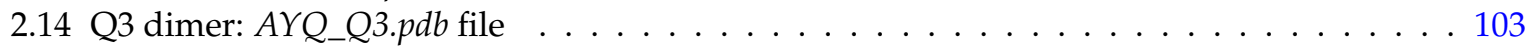

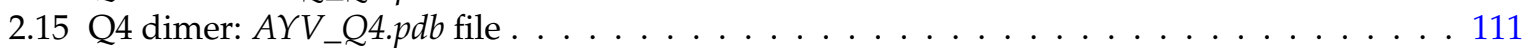

2.16 Q5 dimer: Q05_Q5.pdb file . . . . . . . . . . . . . . . . . . . . . . . . . . . . . . . . . . . . . . . . . . . . . . . . . . . . . . . . . . .

2.17 Crystal model: Q05_Q5.pdb file . . . . . . . . . . . . . . . . . . . . 128

3 Introduction to supporting figures $\quad 184$

4 List of abbreviations used in the Supporting Information $\quad 185$

$\begin{array}{lr}\text { List of figures } & 186\end{array}$ 


\section{Becker et al. force field}

\subsection{Force field parameter files}

\subsection{1 frcmod.known file}

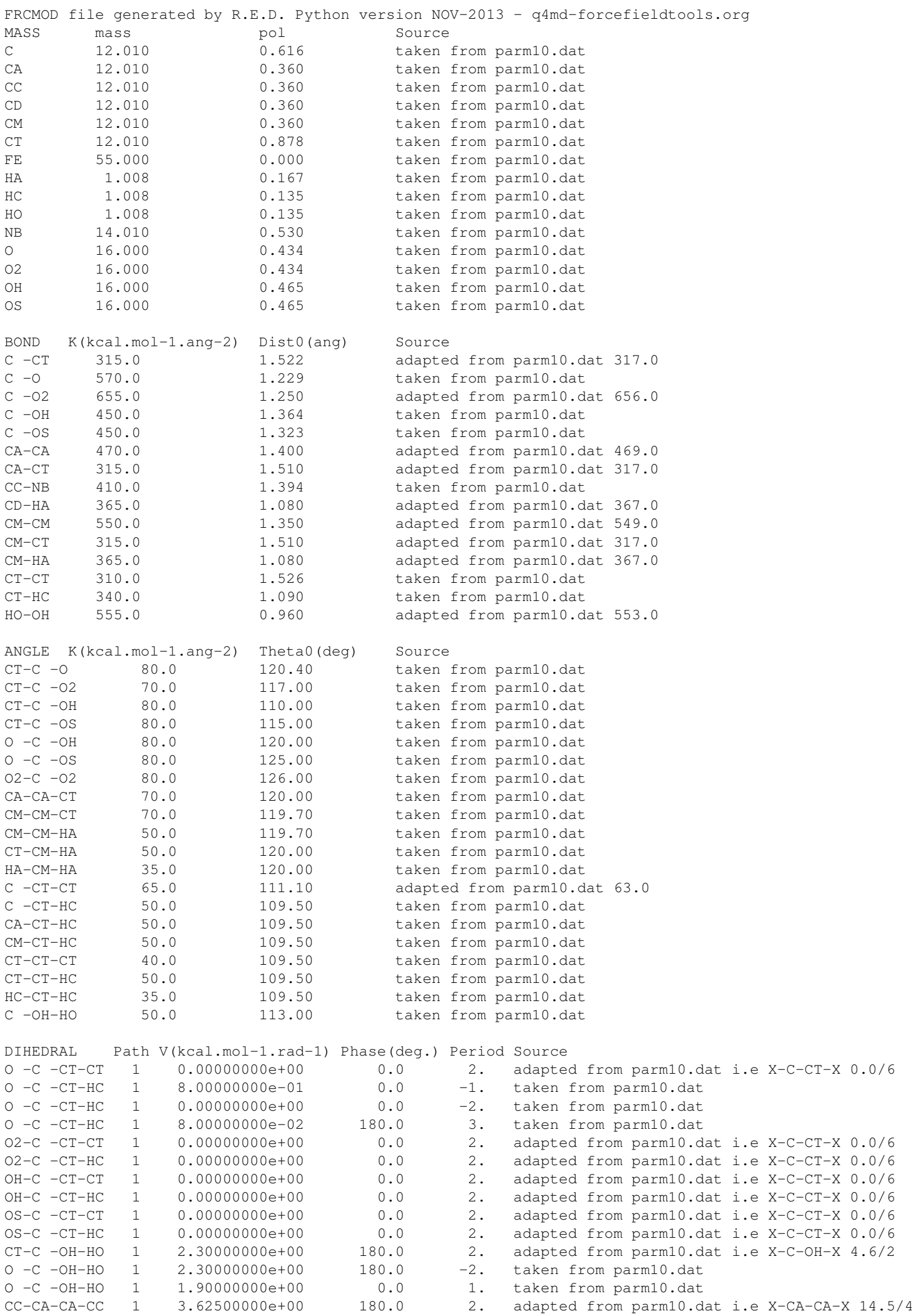




\begin{tabular}{|c|c|c|c|c|c|c|}
\hline $\mathrm{CC}-\mathrm{CA}-\mathrm{CA}-\mathrm{CT}$ & 1 & $3.62500000 e+00$ & 180.0 & 2 . & adapted from parm10. dat i.e $\mathrm{X}-\mathrm{CA}-\mathrm{CA}-\mathrm{X}$ & $14.5 / 4$ \\
\hline $\mathrm{CT}-\mathrm{CA}-\mathrm{CA}-\mathrm{CT}$ & 1 & $3.62500000 e+00$ & 180.0 & 2 . & adapted from parm10.dat i.e X-CA-CA-X & $14.5 / 4$ \\
\hline $\mathrm{CA}-\mathrm{CA}-\mathrm{CT}-\mathrm{HC}$ & 1 & $0.00000000 e+00$ & 0.0 & 2 . & adapted from parm10.dat i.e X-CA-CT-X & $0.0 / 6$ \\
\hline $\mathrm{CC}-\mathrm{CA}-\mathrm{CT}-\mathrm{HC}$ & 1 & $0.00000000 e+00$ & 0.0 & 2 . & adapted from parm10.dat i.e X-CA-CT-X & $0.0 / 6$ \\
\hline $\mathrm{CA}-\mathrm{CC}-\mathrm{NB}-\mathrm{CC}$ & 1 & $2.40000000 e+00$ & 180.0 & 2 . & adapted from parm10.dat i.e X-CC-NB-X & $4.8 / 2$ \\
\hline $\mathrm{CD}-\mathrm{CC}-\mathrm{NB}-\mathrm{CC}$ & 1 & $2.40000000 e+00$ & 180.0 & 2 . & adapted from parm10.dat i.e X-CC-NB-X & $4.8 / 2$ \\
\hline $\mathrm{CT}-\mathrm{CM}-\mathrm{CM}-\mathrm{HA}$ & 1 & $6.65000000 e+00$ & 180.0 & 2 . & adapted from parm10.dat i.e X-CM-CM-X & $26.6 / 4$ \\
\hline $\mathrm{HA}-\mathrm{CM}-\mathrm{CM}-\mathrm{HA}$ & 1 & $6.65000000 e+00$ & 180.0 & 2 . & adapted from parm10.dat i.e X-CM-CM-X & $26.6 / 4$ \\
\hline $\mathrm{CM}-\mathrm{CM}-\mathrm{CT}-\mathrm{HC}$ & 1 & $3.80000000 e-01$ & 180.0 & -3 . & taken from parm10.dat & \\
\hline $\mathrm{CM}-\mathrm{CM}-\mathrm{CT}-\mathrm{HC}$ & 1 & $1.15000000 e+00$ & 0.0 & 1. & taken from parm10.dat & \\
\hline $\mathrm{HA}-\mathrm{CM}-\mathrm{CT}-\mathrm{HC}$ & 1 & $0.00000000 e+00$ & 0.0 & 3. & adapted from parm10.dat i.e X-CM-CT-X & $0.0 / 6$ \\
\hline $\mathrm{C}-\mathrm{CT}-\mathrm{CT}-\mathrm{CT}$ & 1 & $1.55555556 \mathrm{e}-01$ & 0.0 & 3. & adapted from parm10.dat i.e X-CT-CT-X & $1.4 / 9$ \\
\hline $\mathrm{C}-\mathrm{CT}-\mathrm{CT}-\mathrm{HC}$ & 1 & $1.55555556 \mathrm{e}-01$ & 0.0 & 3 . & adapted from parm10.dat i.e X-CT-CT-X & $1.4 / 9$ \\
\hline $\mathrm{CT}-\mathrm{CT}-\mathrm{CT}-\mathrm{CT}$ & 1 & $1.80000000 e-01$ & 0.0 & -3 & taken from parm10.dat & \\
\hline $\mathrm{CT}-\mathrm{CT}-\mathrm{CT}-\mathrm{CT}$ & 1 & $2.50000000 e-01$ & 180.0 & -2 . & taken from parm10.dat & \\
\hline $\mathrm{CT}-\mathrm{CT}-\mathrm{CT}-\mathrm{CT}$ & 1 & $2.00000000 e-01$ & 180.0 & 1. & taken from parm10.dat & \\
\hline $\mathrm{CT}-\mathrm{CT}-\mathrm{CT}-\mathrm{HC}$ & 1 & $1.60000000 e-01$ & 0.0 & 3 . & taken from parm10.dat & \\
\hline $\mathrm{HC}-\mathrm{CT}-\mathrm{CT}-\mathrm{HC}$ & 1 & $1.50000000 e-01$ & 0.0 & 3 . & taken from parm10.dat & \\
\hline $\mathrm{FE}-\mathrm{N} 4-\mathrm{CC}-\mathrm{CA}$ & 1 & $0.00000000 e+00$ & 0.0 & 1 . & taken from q4md-forcefileldtools & \\
\hline $\mathrm{FE}-\mathrm{N} 4-\mathrm{CC}-\mathrm{CD}$ & 1 & $0.00000000 e+00$ & 0.0 & 1. & taken from q4md-forcefileldtools & \\
\hline $\mathrm{CC}-\mathrm{N} 4-\mathrm{FE}-\mathrm{N} 4$ & 1 & $0.00000000 e+00$ & 0.0 & 1. & taken from q4md-forcefileldtools & \\
\hline $\mathrm{CC}-\mathrm{N} 4-\mathrm{FE}-\mathrm{N} 5$ & 1 & $0.00000000 e+00$ & 0.0 & 1. & taken from q4md-forcefileldtools & \\
\hline $\mathrm{CC}-\mathrm{N} 4-\mathrm{EE}-\mathrm{OS}$ & 1 & $0.00000000 e+00$ & 0.0 & 1. & taken from q4md-forcefileldtools & \\
\hline $\mathrm{FE}-\mathrm{N} 5-\mathrm{CC}-\mathrm{CA}$ & 1 & $0.00000000 e+00$ & 0.0 & 1 . & taken from q4md-forcefileldtools & \\
\hline $\mathrm{FE}-\mathrm{N} 5-\mathrm{CC}-\mathrm{CD}$ & 1 & $0.00000000 e+00$ & 0.0 & 1 . & taken from q4md-forcefileldtools & \\
\hline $\mathrm{CC}-\mathrm{N} 5-\mathrm{EE}-\mathrm{N} 4$ & 1 & $0.00000000 e+00$ & 0.0 & 1. & taken from q4md-forcefileldtools & \\
\hline $\mathrm{CC}-\mathrm{N} 5-\mathrm{EE}-\mathrm{N} 5$ & 1 & $0.00000000 e+00$ & 0.0 & 1 . & taken from q4md-forcefileldtools & \\
\hline $\mathrm{CC}-\mathrm{N} 5-\mathrm{EE}-\mathrm{OS}$ & 1 & $0.00000000 e+00$ & 0.0 & 1. & taken from q4md-forcefileldtools & \\
\hline $\mathrm{FE}-\mathrm{OS}-\mathrm{C}-\mathrm{CT}$ & 1 & $0.00000000 e+00$ & 0.0 & 1 . & taken from q4md-forcefileldtools & \\
\hline $\mathrm{FE}-\mathrm{OS}-\mathrm{C}-\mathrm{O}$ & 1 & $0.00000000 e+00$ & 0.0 & 1. & taken from q4md-forcefileldtools & \\
\hline $\mathrm{C}-\mathrm{OS}-\mathrm{EE}-\mathrm{N} 4$ & 1 & $0.00000000 e+00$ & 0.0 & 1. & taken from q4md-forcefileldtools & \\
\hline $\mathrm{C}-\mathrm{OS}-\mathrm{FE}-\mathrm{N} 5$ & 1 & $0.00000000 e+00$ & 0.0 & 1 . & taken from q4md-forcefileldtools & \\
\hline IMPROPER & & $\mathrm{V}(\mathrm{kcal} \cdot \mathrm{mol}-1 \cdot \mathrm{rad}-1)$ & Phase (deg.) & Period & Source & \\
\hline $\mathrm{CT}-\mathrm{O}-\mathrm{C}-\mathrm{OH}$ & & $1.05000000 e+01$ & 180.0 & 2 . & taken from parm10. dat & \\
\hline $\mathrm{CT}-\mathrm{O}-\mathrm{C}-\mathrm{OS}$ & & $1.05000000 e+01$ & 180.0 & 2 . & adapted from parm10.dat i.e $\mathrm{X}-\mathrm{X}-\mathrm{C}-\mathrm{O}$ & \\
\hline $\mathrm{CT}-\mathrm{O} 2-\mathrm{C}-\mathrm{O} 2$ & & $1.05000000 e+01$ & 180.0 & 2 . & adapted from parm10.dat i.e $\mathrm{X}-\mathrm{O} 2-\mathrm{C}-\mathrm{O} 2$ & \\
\hline $\mathrm{CM}-\mathrm{CT}-\mathrm{CM}-\mathrm{HA}$ & & $1.10000000 e+00$ & 180.0 & 2 . & taken from parm10.dat & \\
\hline $\mathrm{CM}-\mathrm{HA}-\mathrm{CM}-\mathrm{HA}$ & & $1.10000000 e+00$ & 180.0 & 2 . & adapted from parm10.dat i.e X-X-CM-HA & \\
\hline NONBON & $\mathrm{R} \star($ ang $)$ & $\operatorname{Eps}(\mathrm{kcal} \cdot \mathrm{mol}-1)$ & Source & & & \\
\hline $\mathrm{C}$ & 1.9080 & 0.08600000 & taken & from pa & arm10. dat & \\
\hline $\mathrm{CA}$ & 1.9080 & 0.08600000 & taken & from pa & arm10. dat & \\
\hline $\mathrm{CC}$ & 1.9080 & 0.08600000 & taken & from pa & barm10.dat & \\
\hline$C D$ & 1.9080 & 0.08600000 & taken & from pa & barm10. dat & \\
\hline $\mathrm{CM}$ & 1.9080 & 0.08600000 & taken & from pa & arm10. dat & \\
\hline $\mathrm{CT}$ & 1.9080 & 0.10940000 & taken & from $p$ & arm10.dat & \\
\hline $\mathrm{HA}$ & 1.4590 & 0.01500000 & taken & from $p$ & arm10.dat & \\
\hline $\mathrm{HC}$ & 1.4870 & 0.01570000 & taken & from pa & barm10.dat & \\
\hline $\mathrm{HO}$ & 0.0000 & 0.00000000 & taken & from pa & arm10.dat & \\
\hline NB & 1.8240 & 0.17000000 & taken & from pa & barm10.dat & \\
\hline 0 & 1.6612 & 0.21000000 & taken & from $p$ & barm10. dat & \\
\hline 02 & 1.6612 & 0.21000000 & taken & from pa & darm10.dat & \\
\hline $\mathrm{OH}$ & 1.7210 & 0.21040000 & taken & from pa & barm10.dat & \\
\hline OS & 1.6837 & 0.17000000 & taken & from pa & darm10.dat & \\
\hline $\mathrm{FE}$ & 1.2000 & 0.05000000 & Amber & contrik & b. . . & \\
\hline
\end{tabular}

\subsection{2 frcmod.correspondence file}

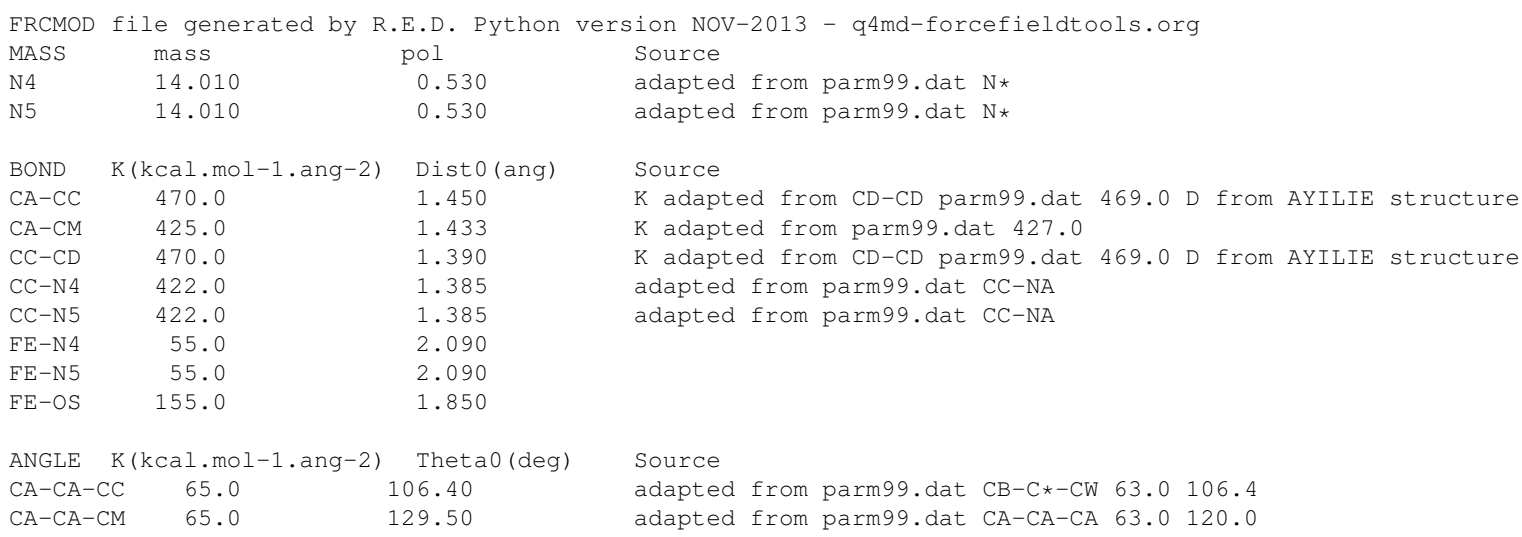




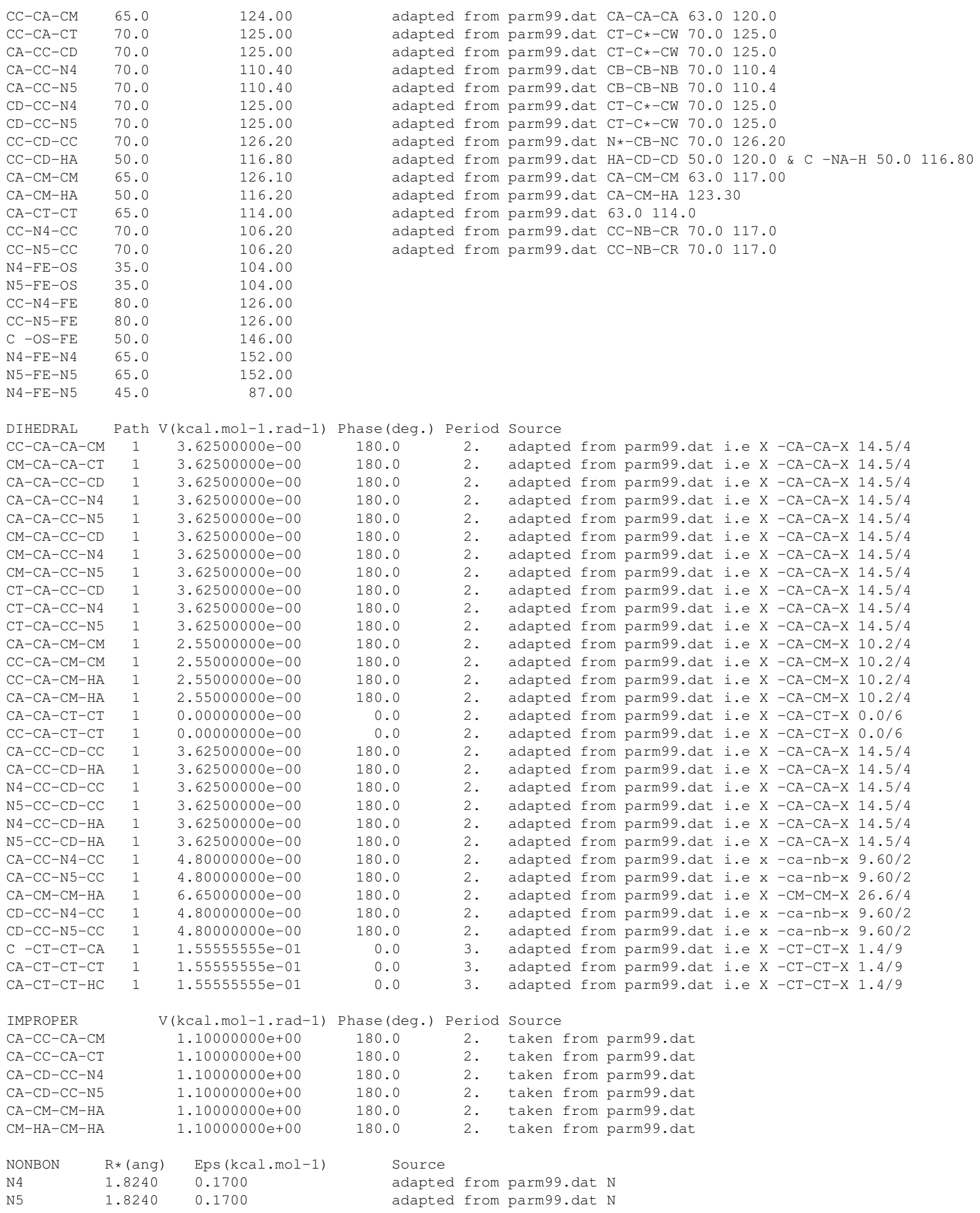

\subsection{Force field library files}

\subsection{1 m1-c1_f1.mol2 file}

$\#$

\# Generated by PyRED version APR-2014

\# http://q4md-forcefieldtools.org

adapted from parm99. dat $N$ 
(a $<$ TRIPOS $>$ MOLECULE

F00

\begin{tabular}{|c|c|c|c|c|c|c|c|c|c|c|}
\hline \multicolumn{11}{|c|}{1} \\
\hline \multicolumn{11}{|c|}{ SMALL } \\
\hline \multicolumn{11}{|c|}{ USER_CHARGES } \\
\hline \multicolumn{11}{|c|}{ d $<$ TRIPOS $>$ ATOM } \\
\hline 1 & $\mathrm{FE}$ & 0.461466 & 0.004161 & -0.103993 & $\mathrm{FE}$ & 1 & FOO & 0.2837 & 0.0000 & $\star \star \star \star *$ \\
\hline 2 & $\mathrm{C} 1$ & 2.293058 & -2.492593 & -0.144246 & $\mathrm{CC}$ & 1 & FOO & -0.0319 & 0.3600 & 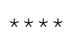 \\
\hline 3 & $\mathrm{C} 2$ & 3.644698 & -2.923582 & 0.163959 & $\mathrm{CA}$ & 1 & FOO & 0.0456 & 0.3600 & 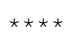 \\
\hline 4 & C3 & 4.388238 & -1.783121 & 0.330439 & $\mathrm{CA}$ & 1 & FOO & 0.0456 & 0.3600 & $\star \star \star \star *$ \\
\hline 5 & $\mathrm{C} 4$ & 3.482753 & -0.666558 & 0.126646 & $\mathrm{CC}$ & - & F00 & -0.0319 & 0.3600 & $\star \star \star \star *$ \\
\hline 6 & C5 & 3.846270 & 0.674589 & 0.207378 & $C D$ & - & FOO & -0.0627 & 0.3600 & 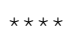 \\
\hline 7 & H5 & 4.882514 & 0.886919 & 0.448343 & $\mathrm{HA}$ & & FOO & 0.1241 & 0.1670 & 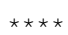 \\
\hline 8 & $\mathrm{C} 6$ & 3.011255 & 1.770070 & 0.007570 & $\mathrm{CC}$ & & FOO & -0.0319 & 0.3600 & 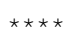 \\
\hline 9 & $\mathrm{C} 7$ & 3.433250 & 3.157005 & 0.084164 & $\mathrm{CA}$ & & FOO & 0.0456 & 0.3600 & $\star \star \star \star *$ \\
\hline 10 & $\mathrm{C} 8$ & 2.322490 & 3.918046 & -0.178103 & $\mathrm{CA}$ & & FOO & 0.0456 & 0.3600 & $\star \star \star \star *$ \\
\hline 11 & C9 & 1.233358 & 2.988629 & -0.416590 & $\mathrm{CC}$ & & FOO & -0.0319 & 0.3600 & $\star \star \star \star *$ \\
\hline 12 & $\mathrm{C} 10$ & -0.067500 & 3.356225 & -0.748466 & $C D$ & 1 & FOO & -0.0627 & 0.3600 & 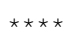 \\
\hline 13 & $\mathrm{H} 10$ & -0.273696 & 4.419449 & -0.812222 & $\mathrm{HA}$ & 1 & F00 & 0.1241 & 0.1670 & 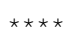 \\
\hline 14 & $\mathrm{C} 11$ & -1.119634 & 2.495760 & -1.049527 & $\mathrm{CC}$ & 1 & FOO & -0.0319 & 0.3600 & 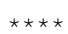 \\
\hline 15 & $\mathrm{C} 12$ & -2.430226 & 2.928345 & -1.496215 & $\mathrm{CA}$ & 1 & F00 & 0.0456 & 0.3600 & 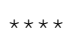 \\
\hline 16 & $\mathrm{C} 13$ & -3.151877 & 1.789313 & -1.751454 & $\mathrm{CA}$ & 1 & FOO & 0.0456 & 0.3600 & 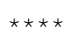 \\
\hline 17 & $\mathrm{C} 14$ & -2.279148 & 0.671266 & -1.449695 & $\mathrm{CC}$ & 1 & FOO & -0.0319 & 0.3600 & 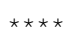 \\
\hline 18 & C15 & -2.632090 & -0.667468 & -1.585234 & $\mathrm{CD}$ & 1 & F00 & -0.0627 & 0.3600 & 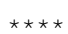 \\
\hline 19 & H15 & -3.635550 & -0.878743 & -1.939190 & $\mathrm{HA}$ & 1 & FOO & 0.1241 & 0.1670 & 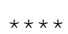 \\
\hline 20 & $\mathrm{C} 16$ & -1.812757 & -1.760373 & -1.319789 & $\mathrm{CC}$ & 1 & FOO & -0.0319 & 0.3600 & $\star \star \star \star *$ \\
\hline 21 & $\mathrm{C} 17$ & -2.201298 & -3.146738 & -1.494838 & $\mathrm{CA}$ & 1 & F00 & 0.0456 & 0.3600 & 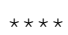 \\
\hline 22 & $\mathrm{C} 18$ & -1.111797 & -3.904464 & -1.146696 & $\mathrm{CA}$ & 1 & FOO & 0.0456 & 0.3600 & 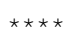 \\
\hline 23 & C19 & -0.064297 & -2.973964 & -0.770439 & $\mathrm{CC}$ & 1 & FOO & -0.0319 & 0.3600 & $*$ \\
\hline 24 & $\mathrm{C} 20$ & 1.227733 & -3.347590 & -0.411534 & $C D$ & 1 & F00 & -0.0627 & 600 & \\
\hline 25 & $\mathrm{H} 2 \mathrm{O}$ & 1.432680 & -4.4 & -0.3 & $\mathrm{HA}$ & 1 & FOO & 241 & & \\
\hline 26 & N21 & 2.2 & -1.1 & -0.1 & N4 & 1 & F00 & -0 & & $*$ \\
\hline 27 & $\mathrm{~N} 22$ & 1.6 & 1.6 & -0.300079 & N5 & 1 & FOO & -0.0954 & 0.0000 & 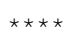 \\
\hline 28 & N23 & -1.053555 & 1.1 & -1.013964 & N4 & 1 & FOO & -0.0954 & 0.0000 & 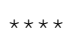 \\
\hline 29 & N24 & -0.515378 & -1.676274 & -0.869216 & N5 & 1 & F00 & -0.0954 & 0.0000 & 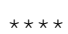 \\
\hline 30 & C99 & -0.888044 & -0.040561 & 2.620889 & $\mathrm{C}$ & 1 & F00 & 0.4992 & 0.6160 & $\star \star \star \star *$ \\
\hline 31 & 091 & 0.075807 & 0.007826 & 1.700798 & OS & 1 & FOO & -0.3125 & 0.4650 & $\star \star \star \star *$ \\
\hline 32 & 092 & -0.650061 & -0.141334 & 3.808499 & 0 & 1 & F00 & -0.5085 & 0.4340 & 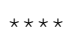 \\
\hline 33 & СT91 & -2.304506 & 0.048344 & 2.057984 & $\mathrm{CT}$ & 1 & FOO & 0.0565 & 0.8780 & \\
\hline 34 & H911 & -2.387466 & 0.989419 & 1.498548 & $\mathrm{HC}$ & 1 & F00 & 0.0050 & 0.1350 & \\
\hline 35 & H912 & -2.426841 & -0.748462 & 1.313535 & $\mathrm{HC}$ & 1 & F00 & 0.0050 & 0.1350 & \\
\hline 36 & СT92 & -3.392300 & -0.040588 & 3.130515 & $\mathrm{CT}$ & $\begin{array}{l}1 \\
1\end{array}$ & F00 & -0.0632 & 0.8780 & 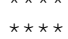 \\
\hline 37 & H921 & -3.236044 & 0.756124 & 3.868663 & $\mathrm{HC}$ & 1 & FOO & 0.0306 & 0.1350 & 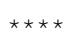 \\
\hline 38 & H922 & -3.277431 & -0.981872 & 3.683039 & $\mathrm{HC}$ & 1 & $\mathrm{~F} 00$ & 0.0306 & 0.1350 & $\star \star \star * *$ \\
\hline
\end{tabular}
a $<$ TRIPOS $>$ BOND

$\begin{array}{rrrr}1 & 1 & 26 & 1 \\ 2 & 1 & 27 & 1 \\ 3 & 1 & 28 & 1 \\ 4 & 1 & 29 & 1 \\ 5 & 1 & 31 & 1 \\ 6 & 2 & 3 & 1 \\ 7 & 2 & 24 & 1 \\ 8 & 2 & 26 & 1 \\ 9 & 3 & 4 & 1 \\ 10 & 4 & 5 & 1 \\ 11 & 5 & 6 & 1 \\ 12 & 5 & 26 & 1 \\ 13 & 6 & 7 & 1 \\ 14 & 6 & 8 & 1 \\ 15 & 8 & 9 & 1 \\ 16 & 8 & 27 & 1 \\ 17 & 9 & 10 & 1 \\ 18 & 10 & 11 & 1 \\ 19 & 11 & 12 & 1 \\ 20 & 11 & 27 & 1 \\ 21 & 12 & 13 & 1 \\ 22 & 12 & 14 & 1 \\ 23 & 14 & 15 & 1 \\ 24 & 14 & 28 & 1 \\ 25 & 15 & 16 & 1 \\ 26 & 16 & 17 & 1 \\ 27 & 17 & 18 & 1 \\ 28 & 17 & 28 & 1 \\ 29 & 18 & 19 & 1 \\ 30 & 18 & 20 & 1 \\ 31 & 20 & 21 & 1 \\ 32 & 20 & 29 & 1 \\ 33 & 21 & 22 & 1 \\ 34 & 22 & 23 & 1 \\ & & & \end{array}$




$\begin{array}{llll}35 & 23 & 24 & 1 \\ 36 & 23 & 29 & 1 \\ 37 & 24 & 25 & 1 \\ 38 & 30 & 31 & 1 \\ 39 & 30 & 32 & 1 \\ 40 & 30 & 33 & 1 \\ 41 & 33 & 34 & 1 \\ 42 & 33 & 35 & 1 \\ 43 & 33 & 36 & 1 \\ 44 & 36 & 37 & 1 \\ 45 & 36 & 38 & 1\end{array}$

(a< $<$ TRIPOS $>$ SUBSTRUCTURE

$$
39 \mathrm{~F} 00
$$

a $<$ TRIPOS $>$ HEADTAIL

C2 1

$0 \quad 0$

a $<$ TRIPOS $>$ RES IDUECONNECT

$\begin{array}{lllllll}1 & \mathrm{C} 2 & 0 & \mathrm{C} 3 & 0 & 0 & 0\end{array}$

\subsection{2 m2-c1_f1.mol2 file}

$\#$

\# Generated by PyRED version APR-2014

\# http://q4md-forcefieldtools.org

a $<$ TRIPOS $>$ MOLECULE

F01

38
SMALL

USER_CHARGES

a $<$ TRIPOS $>$ ATOM

\begin{tabular}{|c|c|c|c|c|c|}
\hline 1 & $\mathrm{FE}$ & 0.461466 & 0.004161 & -0.103993 & $\mathrm{FE}$ \\
\hline 2 & $\mathrm{C} 1$ & 2.293058 & -2.492593 & -0.144246 & $\mathrm{CC}$ \\
\hline 3 & $\mathrm{C} 2$ & 3.644698 & -2.923582 & 0.163959 & $\mathrm{CA}$ \\
\hline 4 & C3 & 4.388238 & -1.783121 & 0.330439 & $\mathrm{CA}$ \\
\hline 5 & $\mathrm{C} 4$ & 3.482753 & -0.666558 & 0.126646 & $\mathrm{CC}$ \\
\hline 6 & C5 & 3.846270 & 0.674589 & 0.207378 & $C D$ \\
\hline 7 & H5 & 4.882514 & 0.886919 & 0.448343 & $\mathrm{HA}$ \\
\hline 8 & $\mathrm{C} 6$ & 3.011255 & 1.770070 & 0.007570 & $\mathrm{CC}$ \\
\hline 9 & C7 & 3.433250 & 3.157005 & 0.084164 & $\mathrm{CA}$ \\
\hline 10 & $\mathrm{C} 8$ & 2.322490 & 3.918046 & -0.178103 & $\mathrm{CA}$ \\
\hline 11 & $\mathrm{C} 9$ & 1.233358 & 2.988629 & -0.416590 & $\mathrm{CC}$ \\
\hline 12 & $\mathrm{C} 10$ & -0.067500 & 3.356225 & -0.748466 & $\mathrm{CD}$ \\
\hline 13 & $\mathrm{H} 10$ & -0.273696 & 4.419449 & -0.812222 & $\mathrm{HA}$ \\
\hline 14 & $\mathrm{C} 11$ & -1.119634 & 2.495760 & -1.049527 & $\mathrm{CC}$ \\
\hline 15 & $\mathrm{C} 12$ & -2.430226 & 2.928345 & -1.496215 & $\mathrm{CA}$ \\
\hline 16 & $\mathrm{C} 13$ & -3.151877 & 1.789313 & -1.751454 & $\mathrm{CA}$ \\
\hline 17 & C14 & -2.279148 & 0.671266 & -1.449695 & $\mathrm{CC}$ \\
\hline 18 & C15 & -2.632090 & -0.667468 & -1.585234 & $\mathrm{CD}$ \\
\hline 19 & H15 & -3.635550 & -0.878743 & -1.939190 & $\mathrm{HA}$ \\
\hline 20 & $\mathrm{C} 16$ & -1.812757 & -1.760373 & -1.319789 & $\mathrm{CC}$ \\
\hline 21 & $\mathrm{C} 17$ & -2.201298 & -3.146738 & -1.494838 & $\mathrm{CA}$ \\
\hline 22 & $\mathrm{C} 18$ & -1.111797 & -3.904464 & -1.146696 & $\mathrm{CA}$ \\
\hline 23 & C19 & -0.064297 & -2.973964 & -0.770439 & $\mathrm{CC}$ \\
\hline 24 & $\mathrm{C} 20$ & 1.227733 & -3.347590 & -0.411534 & $C D$ \\
\hline 25 & $\mathrm{H} 2 \mathrm{O}$ & 1.432680 & -4.411997 & -0.367022 & $\mathrm{HA}$ \\
\hline 26 & N21 & 2.219824 & -1.121708 & -0.164444 & NB \\
\hline 27 & N22 & 1.674270 & 1.693155 & -0.300079 & NB \\
\hline 28 & N23 & -1.053555 & 1.119828 & -1.013964 & $\mathrm{NB}$ \\
\hline 29 & N24 & -0.515378 & -1.676274 & -0.869216 & $\mathrm{NB}$ \\
\hline 30 & C99 & -0.888044 & -0.040561 & 2.620889 & $\mathrm{C}$ \\
\hline 31 & 091 & 0.075807 & 0.007826 & 1.700798 & 02 \\
\hline 32 & 092 & -0.650061 & -0.141334 & 3.808499 & 02 \\
\hline 33 & CT91 & -2.304506 & 0.048344 & 2.057984 & $\mathrm{CT}$ \\
\hline 34 & H911 & -2.387466 & 0.989419 & 1.498548 & $\mathrm{HC}$ \\
\hline 35 & H912 & -2.426841 & -0.748462 & 1.313535 & $\mathrm{HC}$ \\
\hline 36 & CT92 & -3.392300 & -0.040588 & 3.130515 & $\mathrm{CT}$ \\
\hline 37 & H921 & -3.236044 & 0.756124 & 3.868663 & $\mathrm{HC}$ \\
\hline & H922 & -3.277431 & -0.981872 & 3.683039 & $\mathrm{HC}$ \\
\hline
\end{tabular}

F01 $3.0000 \quad 0.0000 * * * *$

F01 $0.42520 .3600 * * * *$

F01 $-0.0037 \quad 0.3600 * * * *$

F01 $-0.0037 \quad 0.3600 * * * *$

F01 $0.42520 .3600 * * * *$

F01 $-0.3364 \quad 0.3600 * \star * *$

$\begin{array}{lll}\mathrm{F} 01 & 0.1312 & 0.1670 * * * *\end{array}$

$\mathrm{F} 010.42520 .3600 * * * *$

F01 $-0.0037 \quad 0.3600 * * * *$

F01 - $0.0037-0.3600 * * * *$

$\begin{array}{lll}\text { F01 } & 0.4252 & 0.3600 * * * *\end{array}$

F01 - $0.33640 .3600 * \star * *$

F01 $0.13120 .1670 * \star \star * *$

F01 $0.42520 .3600 * \star \star * *$

$\begin{array}{llll}F 01 & -0.0037 & 0.3600 * * * *\end{array}$

$\mathrm{F} 01-0.00370 .3600 * * * *$

$\begin{array}{lllll}\text { F01 } & 0.4252 & 0.3600 * \star * *\end{array}$

F01 $-0.33640 .3600 * * * *$

$\begin{array}{lll}\text { F01 } & 0.1312 & 0.1670 * * * *\end{array}$

F01 $0.42520 .3600 * * * *$

F01-0.0037 $0.3600 * * * *$

$\begin{array}{lll}\mathrm{F} 01 & -0.0037 & 0.3600 * * * *\end{array}$

F01 $0.42520 .3600 * * * *$

F01 $-0.33640 .3600 * \star * \star *$

F01 $0.13120 .1670 * \star * \star *$

F01 - $1.22140 .5300 * * * *$

$\mathrm{F} 01-1.22140 .5300 * * * *$

F01 - $1.22140 .5300 * \star \star * *$

F01 - $1.22140 .5300 * * * *$

$\begin{array}{llll}\mathrm{F} 01 & 0.0644 & 0.6160 * * *\end{array}$

F01 $-0.5198 \quad 0.4340 * * *$

F01 $-0.5198 \quad 0.4340 * \star * * *$

$\mathrm{F} 010.8504 \quad 0.8780 * \star \star * *$

F01 - $0.30610 .1350 * \star \star * *$

F01 $-0.30610 .1350 * * * *$

F01 $0.0040 \quad 0.8780 * * * *$

$\begin{array}{llll}\text { F01 } & 0.0337 & 0.1350 * * * *\end{array}$

F01 $0.03370 .1350 * * * *$ 


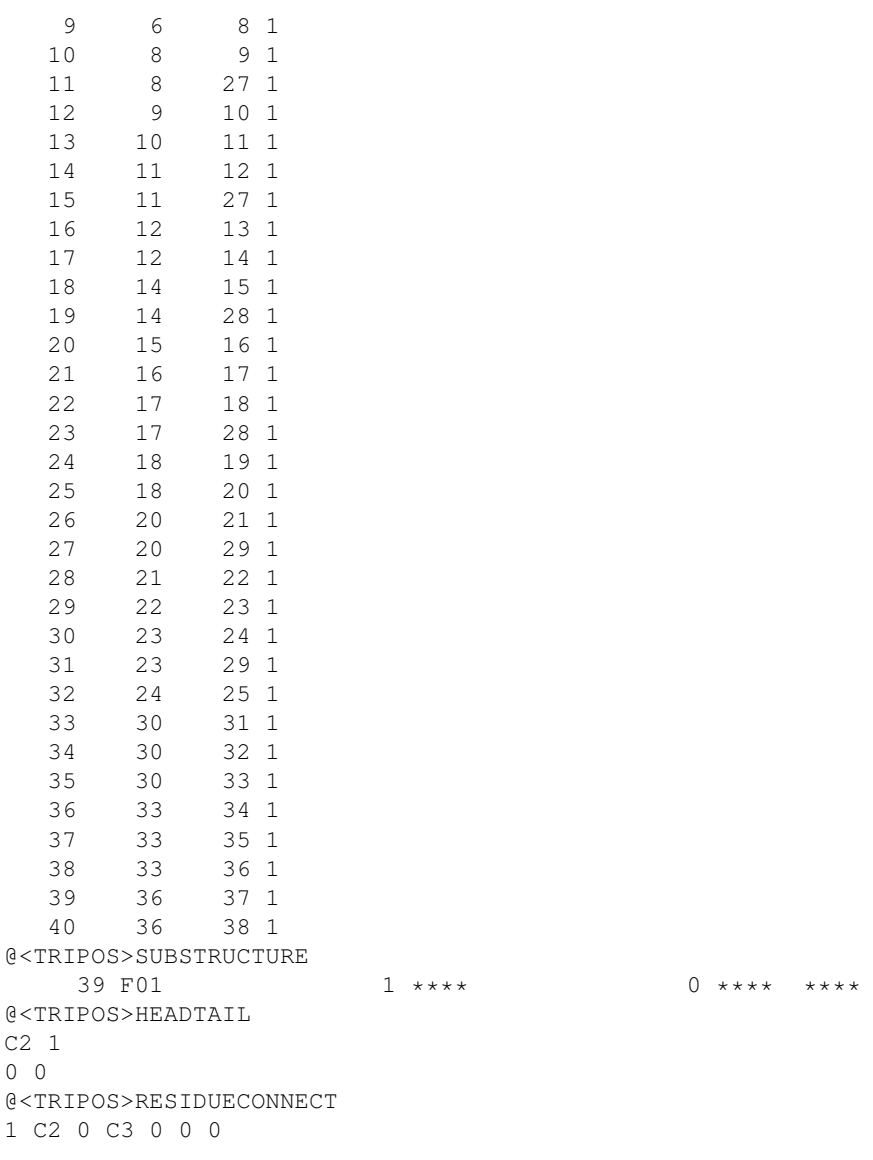

\subsection{3 m3-c1_f1.mol2 file}

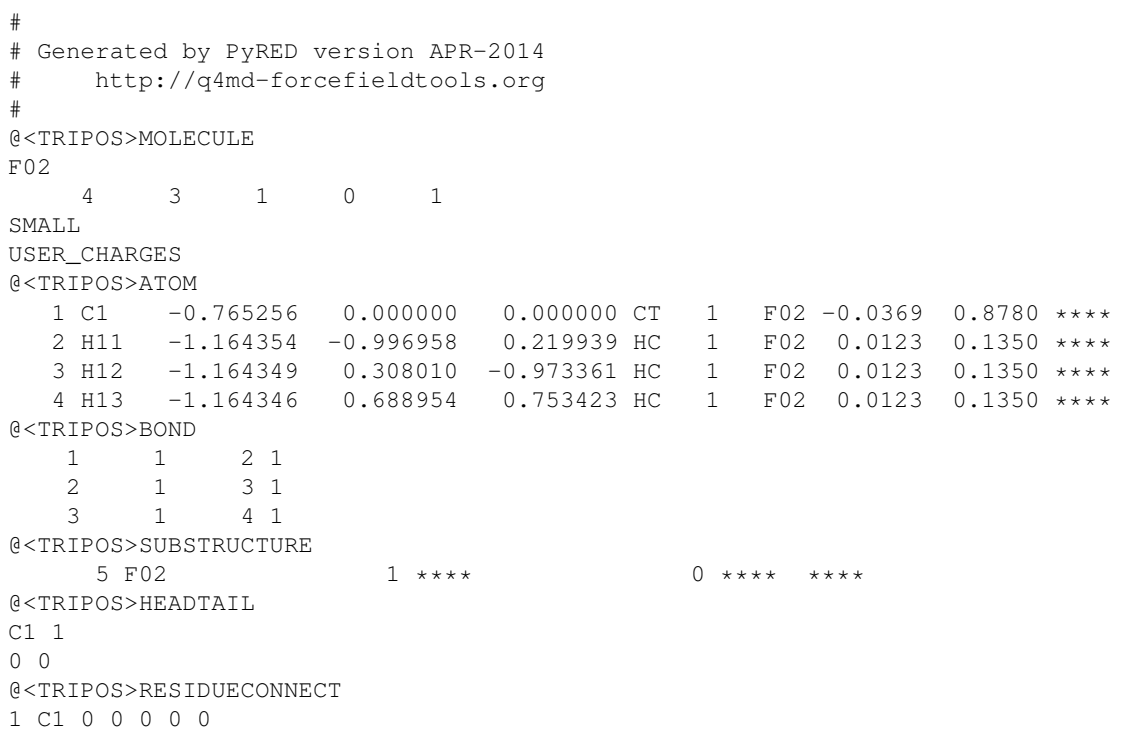

\subsection{4 m4-c1_f1.mol2 file}

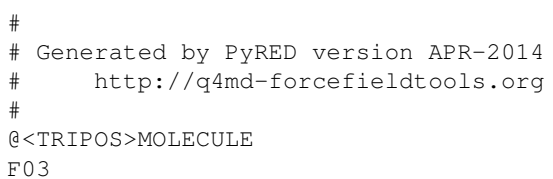


SMALL

USER_CHARGES

Q $<<$ TRIPOS $>$ ATOM

\begin{tabular}{|c|c|c|c|c|c|c|c|c|c|}
\hline $1 \mathrm{C} 1$ & 1.283100 & -0.220395 & 0.000003 & $\mathrm{CM}$ & 1 & $\mathrm{~F} 03$ & -0.5188 & 0.3600 & 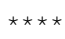 \\
\hline H11 & 1.305701 & -1.308635 & -0.000011 & $\mathrm{HA}$ & 1 & F03 & 0.1850 & 0.1670 & 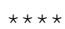 \\
\hline $\mathrm{H} 12$ & 2.245282 & 0.284820 & -0.000003 & $\mathrm{HA}$ & 1 & $\mathrm{~F} 03$ & 0.1850 & 0.1670 & 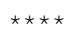 \\
\hline $\mathrm{C} 2$ & 0.133801 & 0.455499 & -0.000001 & $\mathrm{CM}$ & 1 & F03 & 0.0790 & 0.3600 & 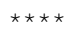 \\
\hline H 21 & 0.164044 & 1.546252 & -0.000005 & $\mathrm{HA}$ & 1 & F03 & 0.0698 & 0.1670 & 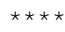 \\
\hline
\end{tabular}
a $<$ TRIPOS $>$ BOND

$\begin{array}{llll}1 & 1 & 2 & 1 \\ 2 & 1 & 3 & 1 \\ 3 & 1 & 4 & 1 \\ 4 & 4 & 5 & 1\end{array}$

$a<$ TRIPOS $>$ SUBSTRUCTURE

$$
6 \mathrm{~F} 03
$$

@ $<$ TRIPOS $>$ HEADTAII

C2

$0 \quad 0$

a $<$ TRIPOS $>$ RES IDUECONNECT

$\begin{array}{llllllll}1 & C 2 & 0 & 0 & 0 & 0 & 0\end{array}$

\subsection{5 m5-c1_f1.mol2 file}

\# Generated by PyRED version APR-2014

\# http://q4md-forcefieldtools.org

Q $<$ TRIPOS $>$ MOLECULE

F04

SMALL
USER_CHARGES
(d<TRIPOS $>$ ATOM

$\begin{array}{llrrrl}1 & 03 & -2.005597 & -1.216125 & 0.212440 & \mathrm{OH} \\ 2 & \mathrm{H} 3 & -2.873788 & -1.399224 & -0.193499 & \mathrm{HO} \\ 3 & \mathrm{C} 1 & -1.772509 & 0.108539 & 0.008720 & \mathrm{C} \\ 4 & \mathrm{O} 2 & -2.564091 & 0.821005 & -0.567771 & \mathrm{O} \\ 5 & \mathrm{C} 4 & -0.432120 & 0.531774 & 0.565285 & \mathrm{CT} \\ 6 & \mathrm{H} 41 & -0.386036 & 1.622560 & 0.503471 & \mathrm{HC} \\ 7 & \mathrm{H} 42 & -0.388596 & 0.245126 & 1.623720 & \mathrm{HC} \\ 8 & \mathrm{C} 5 & 0.752186 & -0.108142 & -0.186399 & \mathrm{CT} \\ 9 & \mathrm{H} 51 & 0.670091 & -1.200174 & -0.124138 & \mathrm{HC} \\ 0 & \mathrm{H} 52 & 0.686068 & 0.151910 & -1.251812 & \mathrm{HC}\end{array}$

$\mathrm{F} 04-0.5435 \quad 0.4650 * \star \star * *$

F04 $0.40890 .1350 * * * *$

F04 $0.53450 .6160 * \star * *$

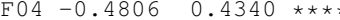

F04 $-0.0673 \quad 0.8780 * * * *$

F04 $0.0378 \quad 0.1350 * * * *$

$\begin{array}{lll}\mathrm{F} 04 & 0.0378 & 0.1350 * * * *\end{array}$

F04 $0.0444-0.8780 * * * *$

F04 $0.0140 \quad 0.1350 * * * *$

$\mathrm{F} 040.0140 \quad 0.1350 * * * *$ d $<$ TRIPOS $>$ BOND

$\begin{array}{rrrr}1 & 1 & 2 & 1 \\ 2 & 1 & 3 & 1 \\ 3 & 3 & 4 & 1 \\ 4 & 3 & 5 & 1 \\ 5 & 5 & 6 & 1 \\ 6 & 5 & 7 & 1 \\ 7 & 5 & 8 & 1 \\ 8 & 8 & 9 & 1 \\ 9 & 8 & 10 & 1\end{array}$

a $<$ TRIPOS $>$ SUBSTRUCTURE

$$
11 \mathrm{~F} 04
$$

31

5

61

81

TRIPOS $>$ HEADTAIL

C5 1

$0 \quad 0$

a $<$ TRIPOS $>$ RES IDUECONNECT

1 C5 0000000

\subsection{6 m6-c1_f1.mol2 file}

\# Generated by PyRED version APR-2014 \# http://q4md-forcefieldtools.org

\#

d $<$ TRIPOS $>$ MOLECULE

F05

\section{SMALL}

USER_CHARGES

(d) $<$ TRIPOS $>$ ATOM

$\begin{array}{lllrrl}1 & \mathrm{C} 1 & -1.876088 & 0.091069 & -0.006643 & \mathrm{C} \\ 2 & 02 & -2.876954 & -0.624915 & 0.239136 & 02 \\ 3 & 03 & -1.799540 & 1.336200 & -0.176800 & 02 \\ 4 & \mathrm{C} 4 & -0.515765 & -0.698035 & -0.167398 & \mathrm{CT}\end{array}$

F05 $0.64710 .6160 * * * *$

F05 $-0.72120 .4340 * * * *$

F05 - $0.72120 .4340 * \star * *$

F05 $-0.01440 .8780 * \star \star *$ 


$\begin{array}{rlrrrrrrr}5 & \mathrm{H} 41 & -0.563416 & -1.614377 & 0.437388 \mathrm{HC} & 1 & \mathrm{~F} 05 & -0.0427 & 0.1350 * * * * \\ 6 \mathrm{H} 42 & -0.450456 & -1.029057 & -1.218661 \mathrm{HC} & 1 & \mathrm{~F} 05 & -0.0427 & 0.1350 * * * * \\ 7 & \mathrm{C} 5 & 0.724544 & 0.131032 & 0.170622 \mathrm{CT} & 1 & \mathrm{~F} 05 & -0.1413 & 0.8780 * * * * \\ 8 & \mathrm{H} 51 & 0.611883 & 1.097553 & -0.335993 \mathrm{HC} & 1 & \mathrm{~F} 05 & 0.0182 & 0.1350 * * * * \\ 9 & \mathrm{H} 52 & 0.727039 & 0.361941 & 1.248026 \mathrm{HC} & 1 & \mathrm{~F} 05 & 0.0182 & 0.1350 * * * *\end{array}$

$\begin{array}{cccc}\text { a }<\text { TRIPOS }>\text { BOND } & & \\ 1 & 1 & 2 & 1 \\ 2 & 1 & 3 & 1 \\ 3 & 1 & 4 & 1 \\ 4 & 4 & 5 & 1 \\ 5 & 4 & 6 & 1 \\ 6 & 4 & 7 & 1 \\ 7 & 7 & 8 & 1 \\ 8 & 7 & 9 & 1\end{array}$

(a) $<$ TRIPOS $>$ SUBSTRUCTURE

10 F05

a $<$ TRIPOS $>$ HEADTAII

C5 1

$0 \quad 0$

e $<$ TRIPOS $>$ RES IDUECONNECT

1 C5 0000000

\subsection{7 m7-c1_f1.mol2 file}

\# Generated by PyRED version APR-2014

\# http://q4md-forcefieldtools.org

(d) $<$ TRIPOS $>$ MOLECULE

F06

10

USER_CHARGES

a $<$ TRIPOS $>$ ATOM

$\begin{array}{rlrrrr}1 & \mathrm{C} 1 & -2.560908 & -0.323627 & 0.000000 & \mathrm{CT} \\ 2 & \mathrm{H} 11 & -2.607386 & -0.970852 & -0.884600 & \mathrm{HC} \\ 3 & \mathrm{H} 12 & -2.607385 & -0.970851 & 0.884601 & \mathrm{HC} \\ 4 & \mathrm{H} 13 & -3.458968 & 0.304763 & 0.000001 & \mathrm{HC} \\ 5 & \mathrm{C} 2 & -1.283937 & 0.522983 & 0.000000 & \mathrm{CT} \\ 6 & \mathrm{H} 21 & -1.283423 & 1.184647 & -0.878037 & \mathrm{HC} \\ 7 & \mathrm{H} 22 & -1.283423 & 1.184648 & 0.878036 & \mathrm{HC} \\ 8 & \mathrm{C} 3 & 0.000000 & -0.316406 & 0.000000 & \mathrm{CT} \\ 9 & \mathrm{H} 31 & 0.000000 & -0.979273 & 0.878508 & \mathrm{HC} \\ 10 & \mathrm{H} 32 & 0.000000 & -0.979272 & -0.878509 & \mathrm{HC}\end{array}$

$10 \mathrm{H} 32 \quad 0.000000$

a $<$ TRIPOS $>$ BOND

$\begin{array}{rrrr}1 & 1 & 2 & 1 \\ 2 & 1 & 3 & 1 \\ 3 & 1 & 4 & 1 \\ 4 & 1 & 5 & 1 \\ 5 & 5 & 6 & 1 \\ 6 & 5 & 7 & 1 \\ 7 & 5 & 8 & 1 \\ 8 & 8 & 9 & 1 \\ 9 & 8 & 10 & 1\end{array}$

(d) $<$ TRIPOS $>$ SUBSTRUCTURE

$$
\begin{aligned}
& 11 \text { F0 } 6 \\
& \text { POS }>\text { HEADTAIL }
\end{aligned}
$$

a $<$ TRIPOS $>$ HEADTA

C3 1

$0 \quad 0$

a $<$ TRIPOS $>$ RES IDUECONNECT

$\begin{array}{lllllll}1 & \mathrm{C} 3 & 0 & 0 & 0 & 0 & 0\end{array}$

\section{3 leaprc file}

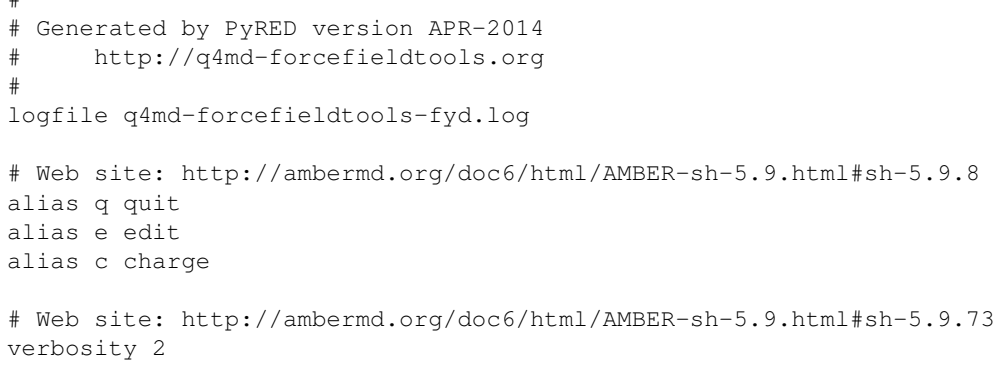




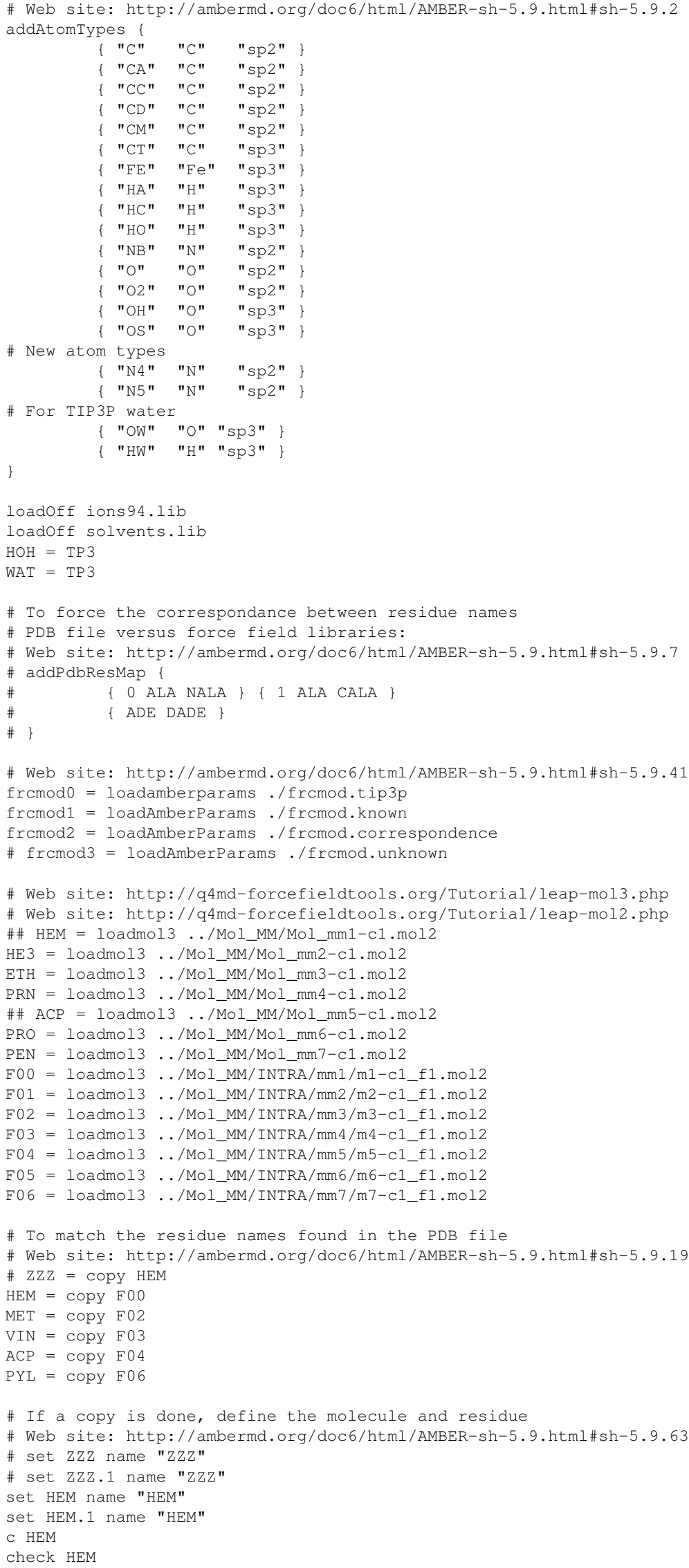

\# Web site: http://ambermd.org/doc6/html/AMBER-sh-5.9.html\#sh-5.9.41

frcmod0 = loadamberparams ./frcmod.tip $3 p$

frcmod1 = loadAmberParams . /frcmod.known

frcmod2 = loadAmberParams./frcmod.correspondence

\# frcmod3 = loadAmberParams ./frcmod.unknown

\# Web site: http://q4md-forcefieldtools.org/Tutorial/leap-mol3.php \# Web site: http://q4md-forcefieldtools.org/Tutorial/leap-mol2.php \#\# HEM = loadmol3 $\ldots /$ Mol_MM/Mol_mm1-c1.mol2 
set MET name "MET"

set MET.1 name "MET"

C MET

check MET

set VIN name "VIN"

set VIN.I name "VIN"

c VIN

check VIN

set $A C P$ name "ACP"

set ACP.1 name "ACP"

C $\mathrm{ACP}$

check ACP

set PYL name "PYL"

set PYL.1 name "PYL"

C PYL

check PYL

\# Let's load the PDB file

\# Web site: http://ambermd.org/doc6/html/AMBER-sh-5.9.html\#sh-5.9.44

\# $\mathrm{VAR}=$ loadPdb Your-PDB-file.ent

\#AYI = loadpdb AYILIE_nom.pdb

AYI $=$ loadpdb AYI_FPD.pdb

set AYI name "AYI"

\#AYJ = loadpdb AYILIE 1 4 rd.pdb

AYJ = loadpdb AYJ_D1.pdb

set AYJ name "AYJ"

\#AYK = loadpdb AYILIE_1_14_rd.pdb

$A Y K=10 a d p d b$ AYK_D5.pdb

set AYK name "AYK"

\#AYM = loadpdb AYILIE_1_14_21_rd.pdb

$\mathrm{AYM}=$ loadpdb AYM_T5.pdb

set AYM name "AYM"

\#AYO = loadpdb AYILIE_27_rd.pdb

$\mathrm{AYO}=$ loadpdb AYO_27_mer.pdb

set AYO name "AYO"

$\# A Y Q=10 a d p d b$ AYILIE_1_7_11_14_rd.pdb

$\mathrm{AYQ}=10 \mathrm{adpdb}$ AYQ_Q3.pdb

set AYQ name "AYQ"

\#AYR = loadpdb AYILIE_1_7_14_rd.pdb

$\mathrm{AYR}=$ loadpdb AYR_T4.pdb

set AYR name "AYR"

\#AYV = loadpdb AYILIE_1_4_11_14_rd.pdb

$\mathrm{AYV}=$ loadpdb AYV_Q4.pdb

set AYV name "AYV"

\#AYW = 1oadpdb AYILIE_1_51_100_150_rd.pdb

$A Y W=10 a d p d b$ AYW_Q1.pdb AYW_Q1.pdb

set AYW name "AYW"

\#AYY = loadpdb Ligne_1_2_4_rd.pdb

$\mathrm{AYY}=$ loadpdb AYY_T1.pdb

set AYY name "AYY"

\#A01 = loadpdb AYILIE_1_9_rd.pdb

$\mathrm{A} 01=$ loadpdb A01_D2.pdb

set A01 name "A01"

\#A02 = loadpdb AYILIE_1_2_4_rd.pdb

$\mathrm{A} 02=10 a d p d b$ A02_T2.pdb

set A02 name "A02"

\#A03 = loadpdb AYILIE_1 4 9 rd.pdb

$\mathrm{A03}=10 \mathrm{adpdb}$ A03_T3.pdb

set A03 name "A03"

\#A04 = loadpdb AYILIE_1_2_4_9_rd.pdb

$\mathrm{A} 04$ = loadpdb A04 $02 \cdot \mathrm{pdb}$

set A04 name "A04"

\#D04 = loadpdb D4_rd.pdb

D04 = loadpdb D04_D3.pdb

set D04 name "D04"

\#D05 = loadpdb D5 rd.pdb

D05 = loadpdb D05_D4.pdb

set D05 name "D05"

\#005 = loadpdb Q5_rd.pdb

$Q 05=10 a d p d b$ Q05_Q5.pdb

set Q05 name "Q05"

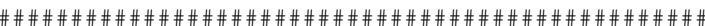

bond AYI.1.C2 AYI.3.C2

bond AYI.1.C3 AYI.4.C1

bond AYI.1.C7 AYI.5.C2

bond AYI.1.C8 AYI.6.C1

bond AYI. 1.C17 AYI. 7.C5 
bond AYI.1.C12 AYI.8.C1 bond AYI.1.C18 AYI.9.C1

bond AYI.1.C13 AYI.2.CT92 bond AYI.2.C13 AYI.1.CT92

bond AYI.2.C2 AYI.10.C2 bond AYI.2.C3 AYI.11.C1 bond AYI.2.C7 AYI.12.C2 bond AYI.2.C8 AYI.13.C1 bond AYI.2.C17 AYI.14.C5 bond AYI.2.C12 AYI.15.C1 bond AYI.2.C18 AYI.16.C1

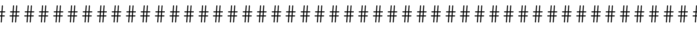

\# The 1 FPD.

bond AYJ.1.C2 AYJ.3.C2

bond AYJ.1.C3 AYJ.4.C1 bond AYJ.1.C7 AYJ.5.C2

bond AYJ.1.C8 AYJ.6.C1 bond AYJ.1.C17 AYJ.7.C5 bond AYJ.1.C12 AYJ.8.C1 bond AYJ.1.C18 AYJ.9.C1

bond AYJ.1.C13 AYJ.2.CT92 bond AYJ.2.C13 AYJ.1.CT92

bond AYJ.2.C2 AYJ.10.C2 bond AYJ.2.C3 AYJ.11.C1 bond AYJ.2.C7 AYJ.12.C2 bond AYJ.2.C8 AYJ.13.C1 bond AYJ.2.C17 AYJ.14.C5 bond AYJ.2.C12 AYJ.15.C1 bond AYJ.2.C18 AYJ.16.C1

\# The 2 FPD.

bond AYJ.17.C2 AYJ.19.C2 bond AYJ.17.C3 AYJ.20.C1 bond AYJ.17.C7 AYJ.21.C2 bond AYJ.17.C8 AYJ.22.C1 bond AYJ.17.C17 AYJ.23.C5 bond AYJ.17.C12 AYJ.24.C1 bond AYJ.17.C18 AYJ.25.C1

bond AYJ.17.C13 AYJ.18.CT92 bond AYJ.18.C13 AYJ.17.CT92

bond AYJ.18.C2 AYJ.26.C2 bond AYJ.18.C3 AYJ.27.C1 bond AYJ.18.C7 AYJ.28.C2 bond AYJ.18.C8 AYJ.29.C1 bond AYJ.18.C17 AYJ.30.C5 bond AYJ.18.C12 AYJ.31.C1 bond AYJ.18.C18 AYJ.32.C1

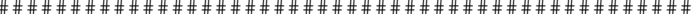

\# The 1 FPD.

bond AYK.1.C2 AYK.3.C2 bond AYK.1.C3 AYK.4.C1 bond AYK.1.C7 AYK.5.C2 bond AYK.1.C8 AYK.6.C1 bond AYK.1.C17 AYK.7.C5 bond AYK.1.C12 AYK. 8.C1 bond AYK.1.C18 AYK.9.C1

bond AYK.1.C13 AYK.2.CT92 bond AYK.2.C13 AYK.1.CT92

bond AYK.2.C2 AYK.10.C2 bond AYK.2.C3 AYK.11.C1 bond AYK.2.C7 AYK.12.C2 bond AYK.2.C8 AYK.13.C1 bond AYK.2.C17 AYK.14.C5 bond AYK.2.C12 AYK.15.C1 bond AYK.2.C18 AYK.16.C1

\# The 2 FPD. 
bond AYK.17.C2 AYK.19.C2 bond AYK.17.C3 AYK.20.C1 bond AYK.17.C7 AYK.21.C2 bond AYK.17.C8 AYK.22.C1 bond AYK.17.C17 AYK.23.C5 bond AYK.17.C12 AYK.24.C1 bond AYK.17.C18 AYK.25.C1

bond AYK.17.C13 AYK.18.CT92 bond AYK.18.C13 AYK.17.CT92

bond AYK.18.C2 AYK.26.C2 bond AYK.18.C3 AYK.27.C1 bond AYK.18.C7 AYK.28.C2 bond AYK.18.C8 AYK.29.C1 bond AYK.18.C17 AYK.30.C5 bond AYK.18.C12 AYK.31.C1 bond AYK.18.C18 AYK.32.C1

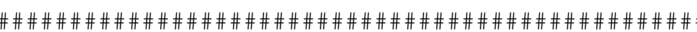

\# The 1 FPD.

bond AYM.1.C2 AYM.3.C2 bond AYM.1.C3 AYM.4.C1

bond AYM.1.C7 AYM. 5.C2

bond AYM.1.C8 AYM. 6.C1 bond AYM.1.C17 AYM.7.C5 bond AYM.1.C12 AYM.8.C1 bond AYM.1.C18 AYM.9.C1

bond AYM.1.C13 AYM.2.CT92 bond AYM.2.C13 AYM.1.CT92

bond AYM.2.C2 AYM.10.C2 bond AYM. 2.C3 AYM.11.C1 bond AYM.2.C7 AYM.12.C2 bond AYM.2.C8 AYM.13.C1 bond AYM.2.C17 AYM.14.C5 bond AYM. 2.C12 AYM.15.C1 bond AYM. 2.C18 AYM.16.C1

\# The 2 FPD.

bond AYM.17.C2 AYM.19.C2 bond AYM.17.C3 AYM.20.C1 bond AYM.17.C7 AYM.21.C2 bond AYM.17.C8 AYM.22.C1

bond AYM.17.C17 AYM.23.C5 bond AYM.17.C12 AYM.24.C1 bond AYM.17.C18 AYM.25.C1

bond AYM.17.C13 AYM.18.CT92 bond AYM.18.C13 AYM.17.CT92

bond AYM.18.C2 AYM.26.C2 bond AYM.18.C3 AYM. 27.C1 bond AYM.18.C7 AYM.28.C2 bond AYM.18.C8 AYM.29.C1

bond AYM.18.C17 AYM.30.C5 bond AYM.18.C12 AYM.31.C1 bond AYM.18.C18 AYM.32.C1

\# The 3 FPD.

bond AYM.33.C2 AYM.35.C2 bond AYM.33.C3 AYM.36.C1 bond AYM.33.C7 AYM. 37.C2 bond AYM. 33.C8 AYM. 38.C1 bond AYM.33.C17 AYM.39.C5 bond AYM. 33.C12 AYM.40.C1 bond AYM.33.C18 AYM.41.C1

bond AYM.33.C13 AYM.34.CT92 bond AYM.34.C13 AYM.33.CT92

bond AYM.34.C2 AYM.42.C2 bond AYM.34.C3 AYM.43.C bond AYM.34.C7 AYM.44.C2 bond AYM. 34.C8 AYM. 45.C1 bond AYM. 34.C17 AYM.46.C5 bond AYM.34.C12 AYM. 47.C1 
\# The 1 FPD.

bond AYO.1.C2 AYO.3.C2

bond AYO.1.C3 AYO.4.C1

bond AYO.1.C7 AYO.5.C2

bond AYO.1.C8 AYO.6.C1

bond AYO.1.C17 AYO.7.C5

bond AYO.1.C12 AYO.8.C1

bond AYO.1.C18 AYO.9.C1

bond AYO.1.C13 AYO.2.CT92

bond AYO.2.C13 AYO.1.CT92

bond AYO.2.C2 AYO.10.C2

bond AYO.2.C3 AYO.11.C1

bond AYO.2.C7 AYO.12.C2

bond AYO.2.C8 AYO.13.C1

bond AYO.2.C17 AYO.14.C5

bond AYO.2.C12 AYO.15.C1

bond AYO.2.C18 AYO.16.C1

\# The 2 FPD.

bond AYO.17.C2 AYO.19.C2 bond AYO.17.C3 AYO.20.C1 bond AYO.17.C7 AYO.21.C2 bond AYO.17.C8 AYO.22.C1 bond AYO.17.C17 AYO.23.C5 bond AYO.17.C12 AYO.24.C1 bond AYO.17.C18 AYO.25.C1

bond AYO.17.C13 AYO.18.СT92 bond AYO.18.C13 AYO.17.CT92

bond AYO.18.C2 AYO.26.C2 bond AYO.18.C3 AYO.27.C1 bond AYO.18.C7 AYO.28.C2 bond AYO.18.C8 AYO.29.C1 bond AYO.18.C17 AYO.30.C5 bond AYO.18.C12 AYO.31.C1 bond AYO.18.C18 AYO.32.C1

\section{\# The 3 FPD.}

bond AYO.33.C2 AYO.35.C2 bond AYO.33.C3 AYO.36.C1 bond AYO.33.C7 AYO.37.C2 bond AYO.33.C8 AYO.38.C1 bond AYO.33.C17 AYO.39.C5 bond AYO.33.C12 AYO.40.C1 bond AYO.33.C18 AYO.41.C1

bond AYO.33.C13 AYO.34.CT92 bond AYO.34.C13 AYO.33.CT92

bond AYO.34.C2 AYO.42.C2 bond AYO.34.C3 AYO.43.C1 bond AYO.34.C7 AYO.44.C2 bond AYO.34.C8 AYO.45.C1 bond AYO.34.C17 AYO.46.C5 bond AYO.34.C12 AYO.47.C1 bond AYO.34.C18 AYO.48.C1

\section{\# The 4 FPD.}

bond AYO.49.C2 AYO.51.C2 bond AYO.49.C3 AYO.52.C1 bond AYO.49.C7 AYO.53.C2 bond AYO.49.C8 AYO.54.C1 bond AYO.49.C17 AYO.55.C5 bond AYO.49.C12 AYO.56.C1 bond AYO.49.C18 AYO.57.C1

bond AYO.49.C13 AYO.50.СT92 bond AYO.50.C13 AYO.49.CT92

bond AYO.50.C2 AYO.58.C2 bond AYO.50.C3 AYO.59.C1 bond AYO.50.C7 AYO.60.C2 
bond AYO.50.C8 AYO.61.C1 bond AYO.50.C17 AYO.62.C5 bond AYO.50.C12 AYO.63.C1 bond AYO.50.C18 AYO.64.C1

\# The 5 FPD.

bond AYO.65.C2 AYO.67.C2

bond AYO.65.C3 AYO.68.C1 bond AYO.65.C7 AYO.69.C2

bond AYO.65.C8 AYO.70.C1 bond AYO.65.C17 AYO.71.C5 bond AYO.65.C12 AYO.72.C1 bond AYO.65.C18 AYO.73.C1

bond AYO.65.C13 AYO.66.СT92 bond AYO.66.C13 AYO.65.CT92

bond AYO.66.C2 AYO.74.C2 bond AYO.66.C3 AYO.75.C1 bond AYO.66.C7 AYO.76.C2 bond AYO.66.C8 AYO.77.C1 bond AYO.66.C17 AYO.78.C5 bond AYO.66.C12 AYO.79.C1 bond AYO.66.C18 AYO.80.C1

\# The 6 FPD.

bond AYO.81.C2 AYO.83.C2 bond AYO.81.C3 AYO.84.C1 bond AYO.81.C7 AYO.85.C2 bond AYO.81.C8 AYO.86.C1 bond AYO.81.C17 AYO.87.C5 bond AYO.81.C12 AYO.88.C1 bond AYO.81.C18 AYO.89.C1

bond AYO.81.C13 AYO.82.CT92 bond AYO.82.C13 AYO.81.CT92

bond AYO.82.C2 AYO.90.C2 bond AYO.82.C3 AYO.91.C1 bond AYO.82.C7 AYO.92.C2 bond AYO.82.C8 AYO.93.C1 bond AYO.82.C17 AYO.94.C5 bond AYO.82.C12 AYO.95.C1 bond AYO.82.C18 AYO.96.C1

\# The 7 FPD.

bond AYO.97.C2 AYO.99.C2 bond AYO.97.C3 AYO.100.C1 bond AYO.97.C7 AYO.101.C2 bond AYO.97.C8 AYO.102.C1 bond AYO.97.C17 AYO.103.C5 bond AYO.97.C12 AYO.104.C1 bond AYO.97.C18 AYO.105.C1

bond AYO.97.C13 AYO.98.CT92 bond AYO.98.C13 AYO.97.CT92

bond AYO.98.C2 AYO.106.C2 bond AYO.98.C3 AYO.107.C1 bond AYO.98.C7 AYO.108.C2 bond AYO.98.C8 AYO.109.C1 bond AYO.98.C17 AYO.110.C5 bond AYO.98.C12 AYO.111.C1 bond AYO.98.C18 AYO.112.C1

\# The 8 FPD.

bond AYO.113.C2 AYO.115.C2 bond AYO.113.C3 AYO.116.C1 bond AYO.113.C7 AYO.117.C2 bond AYO.113.C8 AYO.118.C1 bond AYO.113.C17 AYO.119.C5 bond AYO.113.C12 AYO.120.C bond AYO.113.C18 AYO.121.C1

bond AYO.113.C13 AYO.114.CT92 bond AYO.114.C13 AYO.113.CT92

bond AYO.114.C2 AYO.122.C2 bond AYO.114.C3 AYO.123.C1 
bond AYO.114.C7 AYO.124.C2 bond AYO.114.C8 AYO.125.C1 bond AYO.114.C17 AYO.126.C5 bond AYO.114.C12 AYO.127.C1 bond AYO.114.C18 AYO.128.C1

\# The 9 FPD.

bond AYO.129.C2 AYO.131.C2 bond AYO.129.C3 AYO.132.C1 bond AYO.129.C7 AYO.133.C2 bond AYO.129.C8 AYO.134.C1 bond AYO.129.C17 AYO.135.C5 bond AYO.129.C12 AYO.136.C bond AYO.129.C18 AYO.137.C1

bond AYO.129.C13 AYO.130.CT92 bond AYO.130.C13 AYO.129.CT92

bond AYO.130.C2 AYO.138.C2 bond AYO.130.C3 AYO.139.C1 bond AYO.130.C7 AYO.140.C2 bond AYO.130.C8 AYO.141.C1 bond AYO.130.C17 AYO.142.C5 bond AYO.130.C12 AYO.143.C1 bond AYO.130.C18 AYO.144.C1

\# The 10 FPD.

bond AYO.145.C2 AYO.147.C2 bond AYO.145.C3 AYO.148.C1 bond AYO.145.C7 AYO.149.C2 bond AYO.145.C8 AYO.150.C1 bond AYO.145.C17 AYO.151.C5 bond AYO.145.C12 AYO.152.C1 bond AYO.145.C18 AYO.153.C1

bond AYO.145.C13 AYO.146.CT92 bond AYO.146.C13 AYO.145.CT92

bond AYO.146.C2 AYO.154.C2 bond AYO.146.C3 AYO.155.C1 bond AYO.146.C7 AYO.156.C2 bond AYO.146.C8 AYO.157.C1 bond AYO.146.C17 AYO.158.C5 bond AYO.146.C12 AYO.159.C1 bond AYO.146.C18 AYO.160.C1

\# The 11 FPD.

bond AYO.161.C2 AYO.163.C2 bond AYO.161.C3 AYO.164.C1 bond AYO.161.C7 AYO.165.C2 bond AYO.161.C8 AYO.166.C1 bond AYO.161.C17 AYO.167.C5 bond AYO.161.C12 AYO.168.C1 bond AYO.161.C18 AYO.169.C1

bond AYO.161.C13 AYO.162.CT92 bond AYO.162.C13 AYO.161.CT92

bond AYO.162.C2 AYO.170.C2 bond AYO.162.C3 AYO.171.C1 bond AYO.162.C7 AYO.172.C2 bond AYO.162.C8 AYO.173.C1 bond AYO.162.C17 AYO.174.C5 bond AYO.162.C12 AYO.175.C1 bond AYO.162.C18 AYO.176.C1

\# The 12 FPD.

bond AYO.177.C2 AYO.179.C2

bond AYO.177.C3 AYO.180.C1 bond AYO.177.C7 AYO.181.C2 bond AYO.177.C8 AYO.182.C1 bond AYO.177.C17 AYO.183.C5 bond AYO.177.C12 AYO.184.C1 bond AYO.177.C18 AYO.185.C1

bond AYO.177.C13 AYO.178.CT92 bond AYO.178.C13 AYO.177.CT92

bond AYO.178.C2 AYO.186.C2 
bond AYO.178.C3 AYO.187.C1 bond AYO.178.C7 AYO.188.C2 bond AYO.178.C8 AYO.189.C1 bond AYO.178.C17 AYO.190.C5 bond AYO.178.C12 AYO.191.C1 bond AYO.178.C18 AYO.192.C1

\# The 13 FPD.

bond AYO.193.C2 AYO.195.C2

bond AYO.193.C3 AYO.196.C1 bond AYO.193.C7 AYO.197.C2 bond AYO.193.C8 AYO.198.C1 bond AYO.193.C17 AYO.199.C5 bond AYO.193.C12 AYO.200.C1 bond AYO.193.C18 AYO.201.C1

bond AYO.193.C13 AYO.194.CT92 bond AYO.194.C13 AYO.193.CT92

bond AYO.194.C2 AYO.202.C2 bond AYO.194.C3 AYO.203.C1 bond AYO.194.C7 AYO.204.C2 bond AYO.194.C8 AYO.205.C1 bond AYO.194.C17 AYO.206.C5 bond AYO.194.C12 AYO.207.C1 bond AYO.194.C18 AYO.208.C1

\# The 14 FPD.

bond AYO.209.C2 AYO.211.C2 bond AYO.209.C3 AYO.212.C1 bond AYO.209.C7 AYO.213.C2 bond AYO.209.C8 AYO.214.C1 bond AYO.209.C17 AYO.215.C5 bond AYO.209.C12 AYO.216.C1 bond AYO.209.C18 AYO.217.C1

bond AYO.209.C13 AYO.210.CT92 bond AYO.210.C13 AYO.209.CT92

bond AYO.210.C2 AYO.218.C2 bond AYO.210.C3 AYO.219.C1 bond AYO.210.C7 AYO.220.C2 bond AYO.210.C8 AYO.221.C1 bond AYO.210.C17 AYO.222.C5 bond AYO.210.C12 AYO.223.C1 bond AYO.210.C18 AYO.224.C1

\# The 15 FPD.

bond AYO.225.C2 AYO.227.C2 bond AYO.225.C3 AYO.228.C1 bond AYO.225.C7 AYO.229.C2 bond AYO.225.C8 AYO.230.C1 bond AYO.225.C17 AYO.231.C5 bond AYO.225.C12 AYO.232.C1 bond AYO.225.C18 AYO.233.C1

bond AYO.225.C13 AYO.226.CT92 bond AYO.226.C13 AYO.225.CT92

bond AYO.226.C2 AYO.234.C2 bond AYO.226.C3 AYO.235.C1 bond AYO.226.C7 AYO.236.C2 bond AYO.226.C8 AYO.237.C1 bond AYO.226.C17 AYO.238.C5 bond AYO.226.C12 AYO.239.C1 bond AYO.226.C18 AYO.240.C1

\# The 16 FPD.

bond AYO.241.C2 AYO.243.C2

bond AYO.241.C3 AYO.244.C1 bond AYO.241.C7 AYO.245.C2 bond AYO.241.C8 AYO.246.C1 bond AYO.241.C17 AYO.247.C5 bond AYO.241.C12 AYO.248.C1 bond AYO.241.C18 AYO.249.C1

bond AYO.241.C13 AYO.242.CT92 bond AYO.242.C13 AYO.241.CT92 
bond AYO.242.C2 AYO.250.C2 bond AYO.242.C3 AYO.251.C1 bond AYO.242.C7 AYO.252.C2 bond AYO.242.C8 AYO.253.C1 bond AYO.242.C17 AYO.254.C5 bond AYO.242.C12 AYO.255.C1 bond AYO.242.C18 AYO.256.C1

\# The 17 FPD.

bond AYO.257.C2 AYO.259.C2 bond AYO.257.C3 AYO.260.C1 bond AYO.257.C7 AYO.261.C2 bond AYO.257.C8 AYO.262.C1 bond AYO.257.C17 AYO.263.C5 bond AYO.257.C12 AYO.264.C1 bond AYO.257.C18 AYO.265.C1

bond AYO.257.C13 AYO.258.CT92 bond AYO.258.C13 AYO.257.CT92

bond AYO.258.C2 AYO.266.C2 bond AYO.258.C3 AYO.267.C1 bond AYO.258.C7 AYO.268.C2 bond AYO.258.C8 AYO.269.C1 bond AYO.258.C17 AYO.270.C5 bond AYO.258.C12 AYO.271.C1 bond AYO.258.C18 AYO.272.C1

\# The 18 FPD.

bond AYO.273.C2 AYO.275.C2 bond AYO.273.C3 AYO.276.C1 bond AYO.273.C7 AYO.277.C2 bond AYO.273.C8 AYO.278.C1 bond AYO.273.C17 AYO.279.C5 bond AYO.273.C12 AYO.280.C1 bond AYO.273.C18 AYO.281.C1

bond AYO.273.C13 AYO.274.CT92 bond AYO.274.C13 AYO.273.CT92

bond AYO.274.C2 AYO.282.C2 bond AYO.274.C3 AYO.283.C1 bond AYO.274.C7 AYO.284.C2 bond AYO.274.C8 AYO.285.C1 bond AYO.274.C17 AYO.286.C5 bond AYO.274.C12 AYO.287.C1 bond AYO.274.C18 AYO.288.C1

\# The 19 FPD.

bond AYO.289.C2 AYO.291.C2 bond AYO.289.C3 AYO.292.C1 bond AYO.289.C7 AYO.293.C2 bond AYO.289.C8 AYO.294.C1 bond AYO.289.C17 AYO.295.C5 bond AYO.289.C12 AYO.296.C1 bond AYO.289.C18 AYO.297.C1

bond AYO.289.C13 AYO.290.CT92 bond AYO.290.C13 AYO.289.CT92

bond AYO.290.C2 AYO.298.C2 bond AYO.290.C3 AYO.299.C1 bond AYO.290.C7 AYO.300.C2 bond AYO.290.C8 AYO.301.C1 bond AYO.290.C17 AYO.302.C5 bond AYO.290.C12 AYO.303.C1 bond AYO.290.C18 AYO.304.C1

\# The 20 FPD.

bond AYO.305.C2 AYO.307.C2 bond AYO.305.C3 AYO.308.C1 bond AYO.305.C7 AYO.309.C2 bond AYO.305.C8 AYO.310.C1 bond AYO.305.C17 AYO.311.C5 bond AYO.305.C12 AYO.312.C1 bond AYO.305.C18 AYO.313.C1

bond AYO.305.C13 AYO.306.CT92 bond AYO.306.C13 AYO.305.CT92 
bond AYO.306.C2 AYO.314.C2 bond AYO.306.C3 AYO.315.C1 bond AYO.306.C7 AYO.316.C2 bond AYO.306.C8 AYO.317.C1 bond AYO.306.C17 AYO.318.C5 bond AYO.306.C12 AYO.319.C1 bond AYO.306.C18 AYO.320.C1

\# The 21 FPD.

bond AYO.321.C2 AYO.323.C2 bond AYO.321.C3 AYO.324.C1 bond AYO.321.C7 AYO.325.C2 bond AYO.321.C8 AYO.326.C1 bond AYO.321.C17 AYO.327.C5 bond AYO.321.C12 AYO.328.C1 bond AYO.321.C18 AYO.329.C1

bond AYO.321.C13 AYO.322.CT92 bond AYO.322.C13 AYO.321.CT92

bond AYO.322.C2 AYO.330.C2 bond AYO.322.C3 AYO.331.C1 bond AYO.322.C7 AYO.332.C2 bond AYO.322.C8 AYO.333.C1 bond AYO.322.C17 AYO.334.C5 bond AYO.322.C12 AYO.335.C1 bond AYO.322.C18 AYO.336.C1

\# The 22 FPD.

bond AYO.337.C2 AYO.339.C2 bond AYO.337.C3 AYO.340.C1 bond AYO.337.C7 AYO.341.C2 bond AYO.337.C8 AYO.342.C1 bond AYO.337.C17 AYO.343.C5 bond AYO.337.C12 AYO.344.C1 bond AYO.337.C18 AYO.345.C1

bond AYO.337.C13 AYO.338.CT92 bond AYO.338.C13 AYO.337.CT92

bond AYO.338.C2 AYO.346.C2 bond AYO.338.C3 AYO.347.C1 bond AYO.338.C7 AYO.348.C2 bond AYO.338.C8 AYO.349.C1 bond AYO.338.C17 AYO.350.C5 bond AYO.338.C12 AYO.351.C1 bond AYO.338.C18 AYO.352.C1

\# The 23 FPD.

bond AYO.353.C2 AYO.355.C2 bond AYO.353.C3 AYO.356.C1 bond AYO.353.C7 AYO.357.C2 bond AYO.353.C8 AYO.358.C1 bond AYO.353.C17 AYO.359.C5 bond AYO.353.C12 AYO.360.C1 bond AYO.353.C18 AYO.361.CI

bond AYO.353.C13 AYO.354.CT92 bond AYO.354.C13 AYO.353.CT92

bond AYO.354.C2 AYO.362.C2 bond AYO.354.C3 AYO.363.C1 bond AYO.354.C7 AYO.364.C2 bond AYO.354.C8 AYO.365.C1 bond AYO.354.C17 AYO.366.C5 bond AYO.354.C12 AYO.367.C1 bond AYO.354.C18 AYO.368.C1

\# The 24 FPD.

bond AYO.369.C2 AYO.371.C2 bond AYO.369.C3 AYO.372.C1 bond AYO.369.C7 AYO.373.C2 bond AYO.369.C8 AYO.374.C1 bond AYO.369.C17 AYO.375.C5 bond AYO.369.C12 AYO.376.C1 bond AYO.369.C18 AYO.377.C1

bond AYO.369.C13 AYO.370.CT92 
bond AYO.370.C13 AYO.369.CT92

bond AYO.370.C2 AYO.378.C2 bond AYO.370.C3 AYO.379.C1 bond AYO.370.C7 AYO.380.C2 bond AYO.370.C8 AYO.381.C1 bond AYO.370.C17 AYO.382.C5 bond AYO.370.C12 AYO.383.C1 bond AYO.370.C18 AYO.384.C1

\# The 25 FPD.

bond AYO.385.C2 AYO.387.C2 bond AYO.385.C3 AYO.388.C1 bond AYO.385.C7 AYO.389.C2 bond AYO.385.C8 AYO.390.C1 bond AYO.385.C17 AYO.391.C5 bond AYO.385.C12 AYO.392.C1 bond AYO.385.C18 AYO.393.C1

bond AYO.385.C13 AYO.386.CT92 bond AYO.386.C13 AYO.385.CT92

bond AYO.386.C2 AYO.394.C2 bond AYO.386.C3 AYO.395.C bond AYO.386.C7 AYO.396.C2 bond AYO.386.C8 AYO.397.C1 bond AYO.386.C17 AYO.398.C5 bond AYO.386.C12 AYO.399.C1 bond AYO.386.C18 AYO.400.C1

\# The 26 FPD.

bond AYO.401.C2 AYO.403.C2 bond AYO.401.C3 AYO.404.C1 bond AYO.401.C7 AYO.405.C2 bond AYO.401.C8 AYO.406.C1 bond AYO.401.C17 AYO.407.C5 bond AYO.401.C12 AYO.408.C1 bond AYO.401.C18 AYO.409.C1

bond AYO.401.C13 AYO.402.CT92 bond AYO.402.C13 AYO.401.CT92

bond AYO.402.C2 AYO.410.C2 bond AYO. 402.C3 AYO .411.C1 bond AYO.402.C7 AYO.412.C2 bond AYO.402.C8 AYO.413.C1 bond AYO.402.C17 AYO.414.C5 bond AYO.402.C12 AYO.415.C1 bond AYO.402.C18 AYO.416.C1

\# The 27 FPD.

bond AYO.417.C2 AYO.419.C2 bond AYO.417.C3 AYO.420.C1 bond AYO.417.C7 AYO.421.C2 bond AYO.417.C8 AYO.422.C1 bond AYO.417.C17 AYO.423.C5 bond AYO.417.C12 AYO.424.C1 bond AYO.417.C18 AYO.425.C1

bond AYO.417.C13 AYO.418.CT92 bond AYO.418.C13 AYO.417.CT92

bond AYO.418.C2 AYO.426.C2 bond AYO.418.C3 AYO.427.C1 bond AYO. 418.C7 AYO.428.C2 bond AYO. 418.C8 AYO.429.C1 bond AYO.418.C17 AYO.430.C5 bond AYO.418.C12 AYO.431.CI bond AYO.418.C18 AYO.432.C1

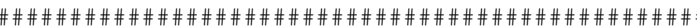

\# The 1 FPD.

bond AYQ.1.C2 AYQ.3.C2 bond AYQ.1.C3 AYQ.4.C1 bond AYQ.1.C7 AYQ.5.C2 bond AYO.1.C8 AYO.6.C1 bond AYQ.1.C17 AYQ.7.C5 bond AYQ.1.C12 AYQ.8.C1 
bond AYQ.1.C18 AYQ.9.C1

bond AYQ.1.C13 AYQ.2.CT92 bond AYQ.2.C13 AYQ.1.CT92

bond AYQ.2.C2 AYQ.10.C2 bond AYQ.2.C3 AYQ.11.C1 bond AYQ.2.C7 AYQ.12.C2 bond AYQ.2.C8 AYQ.13.C1 bond AYQ.2.C17 AYQ.14.C5 bond AYQ.2.C12 AYQ.15.C1 bond AYQ.2.C18 AYQ.16.C1

\# The 2 FPD.

bond AYQ.17.C2 AYQ.19.C2 bond AYQ.17.C3 AYQ.20.C1 bond AYQ.17.C7 AYQ.21.C2 bond AYQ.17.C8 AYQ.22.C1 bond AYQ.17.C17 AYQ.23.C5 bond AYQ.17.C12 AYQ.24.C1 bond AYQ.17.C18 AYQ.25.C1

bond AYQ.17.C13 AYQ.18.CT92 bond AYQ.18.C13 AYQ.17.CT92

bond AYQ.18.C2 AYQ.26.C2 bond AYQ.18.C3 AYQ.27.C1 bond AYQ.18.C7 AYQ.28.C2 bond AYQ.18.C8 AYQ.29.C1 bond AYQ.18.C17 AYQ.30.C5 bond AYQ.18.C12 AYQ.31.C1 bond AYQ.18.C18 AYQ.32.C1

\section{\# The 3 FPD.}

bond AYQ.33.C2 AYQ.35.C2 bond AYQ.33.C3 AYQ.36.C1 bond AYQ.33.C7 AYQ.37.C2 bond AYQ.33.C8 AYQ.38.C1 bond AYQ.33.C17 AYQ.39.C5 bond AYQ.33.C12 AYQ.40.C1 bond AYQ.33.C18 AYQ.41.C1

bond AYQ.33.C13 AYQ.34.CT92 bond AYQ.34.C13 AYQ.33.CT92

bond AYQ.34.C2 AYQ.42.C2 bond AYQ.34.C3 AYQ.43.C1 bond AYQ.34.C7 AYQ.44.C2 bond AYQ.34.C8 AYQ.45.C1 bond AYQ.34.C17 AYQ.46.C5 bond AYQ.34.C12 AYQ.47.C1 bond AYQ.34.C18 AYQ.48.C1

\section{\# The 4 FPD.}

bond AYQ.49.C2 AYQ.51.C2 bond AYQ.49.C3 AYQ.52.C1 bond AYQ.49.C7 AYQ.53.C2 bond AYQ.49.C8 AYQ.54.C1 bond AYQ.49.C17 AYQ.55.C5 bond AYQ.49.C12 AYQ.56.C1 bond AYQ.49.C18 AYQ.57.C1

bond AYQ.49.C13 AYQ.50.CT92 bond AYQ.50.C13 AYQ.49.CT92

bond AYQ.50.C2 AYQ.58.C2 bond AYQ.50.C3 AYQ.59.C1 bond AYQ.50.C7 AYQ.60.C2 bond AYQ.50.C8 AYQ.61.C1 bond AYQ.50.C17 AYQ.62.C5 bond AYQ.50.C12 AYQ.63.C1 bond AYQ.50.C18 AYQ.64.C1

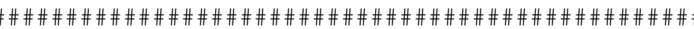

\# The 1 FPD.

bond AYR.1.C2 AYR.3.C2

bond AYR.1.C3 AYR.4.C1

bond AYR. 1.C7 AYR , 5.C2 
bond AYR.1.C8 AYR.6.C1 bond AYR.1.C17 AYR.7.C5 bond AYR.1.C12 AYR.8.C1 bond AYR.1.C18 AYR.9.C1

bond AYR.1.C13 AYR.2.CT92 bond AYR.2.C13 AYR.1.CT92

bond AYR.2.C2 AYR.10.C2 bond AYR.2.C3 AYR.11.C1 bond AYR.2.C7 AYR.12.C2 bond AYR.2.C8 AYR.13.C1 bond AYR.2.C17 AYR.14.C5 bond AYR.2.C12 AYR.15.C1 bond AYR.2.C18 AYR.16.C1

\# The 2 FPD.

bond AYR.17.C2 AYR.19.C2 bond AYR.17.C3 AYR.20.C1 bond AYR.17.C7 AYR.21.C2 bond AYR. 17.C8 AYR.22.C1 bond AYR.17.C17 AYR.23.C5 bond AYR.17.C12 AYR.24.C1 bond AYR.17.C18 AYR. 25.C1

bond AYR.17.C13 AYR.18.CT92 bond AYR.18.C13 AYR.17.CT92

bond AYR.18.C2 AYR.26.C2 bond AYR.18.C3 AYR.27.C1 bond AYR.18.C7 AYR.28.C2 bond AYR.18.C8 AYR.29.C1 bond AYR.18.C17 AYR.30.C5 bond AYR.18.C12 AYR.31.C1 bond AYR.18.C18 AYR.32.C1

\# The 3 FPD.

bond AYR.33.C2 AYR.35.C2 bond AYR.33.C3 AYR. 36.C1 bond AYR.33.C7 AYR.37.C2 bond AYR.33.C8 AYR.38.C1 bond AYR. 33.C17 AYR.39.C5 bond AYR.33.C12 AYR.40.C1 bond AYR. 33.C18 AYR.41.C1

bond AYR.33.C13 AYR.34.CT92 bond AYR. 34.C13 AYR.33.CT92

bond AYR.34.C2 AYR.42.C2 bond AYR.34.C3 AYR.43.C1 bond AYR.34.C7 AYR. $44 . C 2$ bond AYR.34.C8 AYR.45.C1 bond AYR. 34.C17 AYR.46.C5 bond AYR. 34.C12 AYR. 47.C1 bond AYR. 34.C18 AYR.48.C1

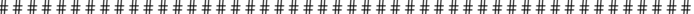

\# The 1 FPD.

bond AYV.1.C2 AYV.3.C2 bond AYV.1.C3 AYV.4.C1 bond AYV.1.C7 AYV.5.C2 bond AYV.1.C8 AYV.6.C1 bond AYV.1.C17 AYV.7.C5 bond AYV.1.C12 AYV.8.C1 bond AYV.1.C18 AYV.9.C1

bond AYV.1.C13 AYV.2.CT92 bond AYV.2.C13 AYV.1.CT92

bond AYV.2.C2 AYV.10.C2 bond AYV.2.C3 AYV.11.C1 bond AYV.2.C7 AYV.12.C2 bond AYV.2.C8 AYV.13.C1 bond AYV.2.C17 AYV.14.C5 bond AYV.2.C12 AYV.15.C1 bond AYV.2.C18 AYV.16.C1

\# The 2 FPD. 
bond AYV.17.C2 AYV.19.C2 bond AYV.17.C3 AYV.20.C1 bond AYV.17.C7 AYV.21.C2 bond AYV.17.C8 AYV.22.C1 bond AYV.17.C17 AYV.23.C5 bond AYV.17.C12 AYV.24.C1 bond AYV.17.C18 AYV.25.C1

bond AYV.17.C13 AYV.18.CT92 bond AYV.18.C13 AYV.17.CT92

bond AYV.18.C2 AYV.26.C2 bond AYV.18.C3 AYV.27.C1 bond AYV.18.C7 AYV.28.C2 bond AYV.18.C8 AYV.29.C1 bond AYV.18.C17 AYV.30.C5 bond AYV.18.C12 AYV.31.C1 bond AYV.18.C18 AYV. 32.C1

\# The 3 FPD

bond AYV.33.C2 AYV.35.C2 bond AYV.33.C3 AYV.36.C1 bond AYV.33.C7 AYV.37.C2 bond AYV.33.C8 AYV. 38.C1

bond AYV. 33.C17 AYV. 39.C5 bond AYV. 33.C12 AYV.40.C1 bond AYV.33.C18 AYV.41.C1

bond AYV.33.C13 AYV.34.CT92 bond AYV. 34.C13 AYV.33.CT92

bond AYV.34.C2 AYV.42.C2 bond AYV. 34.C3 AYV. 43.C1 bond AYV.34.C7 AYV.44.C2 bond AYV.34.C8 AYV. 45.C1

bond AYV. 34.C17 AYV. 46.C5 bond AYV.34.C12 AYV.47.C1 bond AYV.34.C18 AYV.48.C1

\# The 4 FPD.

bond AYV.49.C2 AYV.51.C2 bond AYV.49.C3 AYV.52.C1 bond AYV.49.C7 AYV.53.C2 bond AYV.49.C8 AYV.54.C1 bond AYV. 49.C17 AYV.55.C5 bond AYV.49.C12 AYV. 56.C1 bond AYV. 49.C18 AYV.57.C1

bond AYV.49.C13 AYV.50.CT92 bond AYV.50.C13 AYV.49.CT92

bond AYV.50.C2 AYV.58.C2 bond AYV.50.C3 AYV.59.C1 bond AYV.50.C7 AYV.60.C2 bond AYV.50.C8 AYV.61.C1 bond AYV.50.C17 AYV.62.C5 bond AYV.50.C12 AYV.63.C1 bond AYV.50.C18 AYV.64.C1

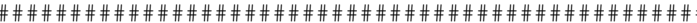

\# The 1 FPD.

bond AYW.1.C2 AYW.3.C2 bond AYW.1.C3 AYW.4.C1 bond AYW.1.C7 AYW. 5.C2 bond AYW. 1.C8 AYW , 6.C1 bond AYW.1.C17 AYW. 7.C5 bond AYW.1.C12 AYW. 8.C1 bond AYW.1.C18 AYW. 9.C1

bond AYW.1.C13 AYW.2.CT92 bond AYW.2.C13 AYW.1.CT92

bond AYW.2.C2 AYW.10.C2 bond AYW.2.C3 AYW.11.C1 bond AYW.2.C7 AYW.12.C2 bond AYW.2.C8 AYW.13.C1 bond AYW.2.C17 AYW.14.C5 bond AYW.2.C12 AYW.15.C1 
\# The 2 FPD.

bond AYW.17.C2 AYW.19.C2 bond AYW.17.C3 AYW.20.C1 bond AYW.17.C7 AYW.21.C2 bond AYW.17.C8 AYW.22.C1 bond AYW.17.C17 AYW.23.C5 bond AYW.17.C12 AYW.24.C1 bond AYW.17.C18 AYW.25.C1

bond AYW.17.C13 AYW.18.CT92 bond AYW.18.C13 AYW.17.CT92

bond AYW.18.C2 AYW.26.C2 bond AYW.18.C3 AYW.27.C1 bond AYW.18.C7 AYW.28.C2 bond AYW.18.C8 AYW.29.C1 bond AYW.18.C17 AYW.30.C5 bond AYW.18.C12 AYW.31.C1 bond AYW.18.C18 AYW.32.C1

\# The 3 FPD.

bond AYW. 33.C2 AYW.35.C2

bond AYW.33.C3 AYW.36.C1 bond AYW.33.C7 AYW.37.C2 bond AYW.33.C8 AYW.38.C1 bond AYW.33.C17 AYW.39.C5 bond AYW.33.C12 AYW.40.C1 bond AYW.33.C18 AYW.41.C1

bond AYW.33.C13 AYW.34.CT92 bond AYW. 34.C13 AYW.33.CT92

bond AYW.34.C2 AYW.42.C2 bond AYW.34.C3 AYW.43.C1 bond AYW.34.C7 AYW. 44.C2 bond AYW.34.C8 AYW.45.C1 bond AYW.34.C17 AYW.46.C5 bond AYW.34.C12 AYW.47.C1 bond AYW.34.C18 AYW.48.C1

\# The 4 FPD.

bond AYW.49.C2 AYW.51.C2 bond AYW.49.C3 AYW.52.C1 bond AYW.49.C7 AYW.53.C2 bond AYW.49.C8 AYW.54.C1 bond AYW. 49.C17 AYW.55.C5 bond AYW. 49.C12 AYW.56.C1 bond AYW.49.C18 AYW.57.C1

bond AYW.49.C13 AYW.50.CT92 bond AYW.50.C13 AYW.49.CT92

bond AYW.50.C2 AYW.58.C2 bond AYW.50.C3 AYW.59.C1 bond AYW.50.C7 AYW.60.C2 bond AYW.50.C8 AYW.61.C1 bond AYW.50.C17 AYW.62.C5 bond AYW.50.C12 AYW.63.C1 bond AYW. 50.C18 AYW.64.C1

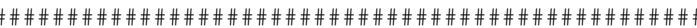

\# The 1 FPD.

bond AYY.1.C2 AYY.3.C2 bond AYY.1.C3 AYY.4.C1 bond AYY.1.C7 AYY.5.C2 bond AYY.1.C8 AYY.6.C1 bond AYY.1.C17 AYY.7.C5 bond AYY.1.C12 AYY.8.C1 bond AYY.1.C18 AYY.9.C1

bond AYY.1.C13 AYY.2.CT92 bond AYY.2.C13 AYY.1.CT92

bond AYY.2.C2 AYY.10.C2 bond AYY.2.C3 AYY.11.C1 bond AYY.2.C7 AYY.12.C2 
bond AYY.2.C8 AYY.13.C1 bond AYY.2.C17 AYY.14.C5 bond AYY.2.C12 AYY.15.C1 bond AYY.2.C18 AYY.16.C1

\# The 2 FPD.

bond AYY.17.C2 AYY.19.C2 bond AYY.17.C3 AYY.20.C1 bond AYY.17.C7 AYY.21.C2 bond AYY.17.C8 AYY.22.C1 bond AYY.17.C17 AYY.23.C5 bond AYY.17.C12 AYY.24.C1 bond AYY.17.C18 AYY.25.C1

bond AYY.17.C13 AYY.18.CT92 bond AYY.18.C13 AYY.17.CT92

bond AYY.18.C2 AYY.26.C2 bond AYY.18.C3 AYY.27.C1 bond AYY.18.C7 AYY.28.C2 bond AYY.18.C8 AYY.29.C1 bond AYY.18.C17 AYY.30.C5 bond AYY.18.C12 AYY.31.C1 bond AYY.18.C18 AYY.32.C1

\# The 3 FPD.

bond AYY.33.C2 AYY.35.C2 bond AYY.33.C3 AYY.36.C1 bond AYY.33.C7 AYY.37.C2 bond AYY.33.C8 AYY.38.C1 bond AYY.33.C17 AYY.39.C5 bond AYY.33.C12 AYY.40.C1 bond AYY.33.C18 AYY.41.C1

bond AYY.33.C13 AYY.34.CT92 bond AYY.34.C13 AYY.33.CT92

bond AYY.34.C2 AYY.42.C2 bond AYY.34.C3 AYY.43.C1 bond AYY.34.C7 AYY.44.C2 bond AYY.34.C8 AYY.45.C1 bond AYY.34.C17 AYY.46.C5 bond AYY. 34.C12 AYY. 47.C1 bond AYY.34.C18 AYY.48.C1

\section{\#\#\#\#\#\#\#\#\#\#\#\#\#\#\#\#\#\#\#\#\#\#\#\#\#\#\#\#\#}

\# The 1 FPD.

bond A04.1.C2 A04.3.C2 bond A04.1.C3 A04.4.C1 bond A04.1.C7 A04.5.C2 bond A04.1.C8 A04.6.C1 bond A04.1.C17 A04.7.C5 bond A04.1.C12 A04.8.C1 bond A04.1.C18 A04.9.C1

bond A04.1.C13 A04.2.CT92 bond A04.2.C13 A04.1.CT92

bond A04.2.C2 A04.10.C2 bond A04.2.C3 A04.11.C1 bond A04.2.C7 A04.12.C2 bond A04.2.C8 A04.13.C1 bond A04.2.C17 A04.14.C5 bond A04.2.C12 A04.15.C1 bond A04.2.C18 A04.16.C1

\# The 2 FPD.

bond A04.17.C2 A04.19.C2 bond A04.17.C3 A04.20.C1 bond A04.17.C7 A04.21.C2 bond $\mathrm{A0} 4.17 . \mathrm{C} 8 \mathrm{A0} 4.22 . \mathrm{Cl}$ bond $\mathrm{A} 04.17 . \mathrm{C} 17 \mathrm{~A} 04.23 . \mathrm{C} 5$ bond A04.17.C12 A04.24.C1 bond A04.17.C18 A04.25.C1

bond A04.17.C13 A04.18.CT92 bond A04.18.C13 A04.17.СT92 
bond A04.18.C2 A04.26.C2 bond A04.18.C3 A04.27.C1 bond A04.18.C7 A04.28.C2 bond A04.18.C8 A04.29.C1 bond A04.18.C17 A04.30.C5 bond A04.18.C12 A04.31.C1 bond A04.18.C18 A04.32.C1

\# The 3 FPD.

bond A04.33.C2 A04.35.C2 bond $\mathrm{A} 04.33 . \mathrm{C} 3 \mathrm{A04} \cdot 36 . \mathrm{C} 1$ bond A04.33.C7 A04.37.C2 bond A04.33.C8 A04.38.C1 bond A04.33.C17 A04.39.C5 bond A04.33.C12 A04.40.C1 bond A04.33.C18 A04.41.C1

bond A04.33.C13 A04.34.CT92 bond A04.34.C13 A04.33.CT92

bond A04.34.C2 A04.42.C2 bond $\mathrm{A0} 4.34 . \mathrm{C} 3 \mathrm{~A} 04.43 . \mathrm{Cl}$ bond A04.34.C7 A04.44.C2 bond A04.34.C8 A04.45.C1

bond A04.34.C17 A04.46.C5 bond A04.34.C12 A04.47.C1 bond A04.34.C18 A04.48.C1

\# The 4 FPD.

bond A04.49.C2 A04.51.C2 bond A04.49.C3 A04.52.C1 bond A04.49.C7 A04.53.C2 bond A04.49.C8 A04.54.C1 bond A04.49.C17 A04.55.C5 bond A04.49.C12 A04.56.C1 bond A04.49.C18 A04.57.C1

bond A04.49.C13 A04.50.CT92 bond A04.50.C13 A04.49.CT92

bond A04.50.C2 A04.58.C2 bond A04.50.C3 A04.59.C1 bond A04.50.C7 A04.60.C2 bond A04.50.C8 A04.61.C1 bond A04.50.C17 A04.62.C5 bond $\mathrm{A} 04.50, \mathrm{C} 12 \mathrm{~A} 04.63 . \mathrm{C} 1$ bond A04.50.C18 A04.64.C1

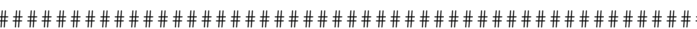

\# The 1 FPD.

bond A01.1.C2 A01.3.C2 bond A01.1.C3 A01.4.C1 bond A01.1.C7 A01.5.C2 bond A01.1.C8 A01.6.C1 bond A01.1.C17 A01.7.C5 bond A01.1.C12 A01.8.C1 bond A01.1.C18 A01.9.C1

bond A01.1.C13 A01.2.CT92 bond A01.2.C13 A01.1.CT92

bond A01.2.C2 A01.10.C2 bond A01.2.C3 A01.11.C1 bond A01.2.C7 A01.12.C2 bond A01.2.C8 A01.13.C1 bond A01.2.C17 A01.14.C5 bond A01.2.C12 A01.15.C1 bond A01.2.C18 A01.16.C1

\# The 2 FPD.

bond A01.17.C2 A01.19.C2 bond A01.17.C3 A01.20.C1 bond A01.17.C7 A01.21.C2 bond A01.17.C8 A01.22.C1 bond A01.17.C17 A01.23.C5 bond A01.17.C12 A01.24.C1 bond A01.17.C18 A01.25.C1 
bond A01.17.C13 A01.18.CT92 bond A01.18.C13 A01.17.CT92

bond A01.18.C2 A01.26.C2 bond A01.18.C3 A01.27.C1 bond $\mathrm{A} 01.18 . \mathrm{C} 7 \mathrm{~A} 01.28 . \mathrm{C} 2$ bond A01.18.C8 A01.29.C1 bond A01.18.C17 A01.30.C5 bond A01.18.C12 A01.31.C1 bond A01.18.C18 A01.32.C1

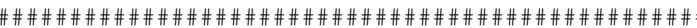

\# The 1 FPD.

bond A02.1.C2 A02.3.C2 bond A02.1.C3 A02.4.C1 bond $\mathrm{A} 02.1 . \mathrm{C} 7 \mathrm{~A} 02.5 . \mathrm{C} 2$ bond $\mathrm{A} 02.1 . \mathrm{C} 8 \mathrm{~A} 02.6 . \mathrm{C} 1$ bond A02.1.C17 A02.7.C5 bond A02.1.C12 A02.8.C1 bond $\mathrm{A} 02.1 . \mathrm{C} 18 \mathrm{~A} 02.9 . \mathrm{C} 1$

bond A02.1.C13 A02.2.СT92 bond A02.2.C13 A02.1.CT92

bond A02.2.C2 A02.10.C2 bond A02.2.C3 A02.11.C1 bond $\mathrm{A} 02.2 . \mathrm{C} 7 \mathrm{A02} .12 . \mathrm{C} 2$ bond A02.2.C8 A02.13.C1 bond A02.2.C17 A02.14.C5 bond A02.2.C12 A02.15.C1 bond A02.2.C18 A02.16.C1

\# The 2 FPD.

bond A02.17.C2 A02.19.C2 bond A02.17.C3 A02.20.C1 bond A02.17.C7 A02.21.C2 bond A02.17.C8 A02.22.C1 bond A02.17.C17 A02.23.C5 bond $\mathrm{A} 02.17 . \mathrm{C} 12 \mathrm{~A} 02.24 . \mathrm{C} 1$ bond A02.17.C18 A02.25.C1

bond A02.17.C13 A02.18.CT92 bond A02.18.C13 A02.17.CT92

bond A02.18.C2 A02.26.C2 bond A02.18.C3 A02.27.C1 bond A02.18.C7 A02.28.C2 bond A02.18.C8 A02.29.C1 bond A02.18.C17 A02.30.C5 bond A02.18.C12 A02.31.C1 bond A02.18.C18 A02.32.C1

\# The 3 FPD.

bond A02.33.C2 A02.35.C2 bond $\mathrm{A02} .33 . \mathrm{C3} \mathrm{A02} \cdot 36 . \mathrm{Cl}$ bond A02.33.C7 A02.37.C2 bond $\mathrm{A} 02.33 . \mathrm{C} 8 \mathrm{A02} .38 . \mathrm{Cl}$ bond A02.33.C17 A02.39.C5 bond A02.33.C12 A02.40.C1 bond A02.33.C18 A02.41.C1

bond A02.33.C13 A02.34.CT92 bond A02.34.C13 A02.33.СT92

bond $\mathrm{A} 02.34 . \mathrm{C} 2 \mathrm{~A} 02.42 . \mathrm{C} 2$ bond $\mathrm{A} 02.34 . \mathrm{C} 3 \mathrm{~A} 02.43 . \mathrm{Cl}$ bond A02.34.C7 A02.44.C2 bond $\mathrm{A} 02.34 . \mathrm{C} 8 \mathrm{A02} .45 . \mathrm{Cl}$ bond A02.34.C17 A02.46.C5 bond $\mathrm{A} 02.34 . \mathrm{C} 12 \mathrm{~A} 02.47 . \mathrm{C} 1$ bond A02.34.C18 A02.48.C1

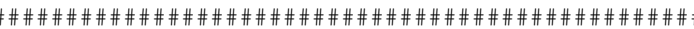

\# The 1 FPD.

bond A03.1.C2 A03.3.C2

bond A03.1.C3 A03.4.C1

bond $\mathrm{A} 03.1 . \mathrm{C} 7 \mathrm{A03}, 5 . \mathrm{C} 2$ 
bond A03.1.C8 A03.6.C1 bond A03.1.C17 A03.7.C5 bond A03.1.C12 A03.8.C1 bond A03.1.C18 A03.9.C1

bond A03.1.C13 A03.2.CT92 bond A03.2.C13 A03.1.CT92

bond A03.2.C2 A03.10.C2 bond A03.2.C3 A03.11.C1 bond A03.2.C7 A03.12.C2 bond A03.2.C8 A03.13.C1 bond A03.2.C17 A03.14.C5 bond $\mathrm{A} 03.2 . \mathrm{C} 12 \mathrm{~A} 03.15 . \mathrm{Cl}$ bond A03.2.C18 A03.16.C1

\# The 2 FPD.

bond A03.17.C2 A03.19.C2 bond A03.17.C3 A03.20.C1 bond $\mathrm{A} 03.17 . \mathrm{C} 7 \mathrm{~A} 03.21 . \mathrm{C} 2$ bond A03.17.C8 A03.22.C1 bond A03.17.C17 A03.23.C5 bond A03.17.C12 A03.24.C1 bond A03.17.C18 A03.25.C1

bond A03.17.C13 A03.18.СT92 bond A03.18.C13 A03.17.CT92

bond $\mathrm{A} 03.18 . \mathrm{C} 2 \mathrm{~A} 03.26 . \mathrm{C} 2$ bond A03.18.C3 A03.27.C1 bond $\mathrm{A} 03.18 . \mathrm{C} 7 \mathrm{~A} 03.28 . \mathrm{C} 2$ bond A03.18.C8 A03.29.C1 bond A03.18.C17 A03.30.C5 bond $\mathrm{A} 03.18 . \mathrm{C} 12 \mathrm{A03} .31 . \mathrm{C} 1$ bond $\mathrm{A} 03.18 . \mathrm{C} 18 \mathrm{A03}, 32 . \mathrm{C} 1$

\# The $3 \mathrm{FPD}$.

bond A03.33.C2 A03.35.C2 bond $\mathrm{A} 03.33 . \mathrm{C} 3 \mathrm{~A} 03.36 . \mathrm{C} 1$ bond $\mathrm{A03} .33 . \mathrm{C} 7 \mathrm{A03} .37 . \mathrm{C} 2$ bond A03.33.C8 A03.38.C1 bond A03.33.C17 A03.39.C5 bond $\mathrm{A} 03.33 . \mathrm{C} 12 \mathrm{A03.40.C1}$ bond A03.33.C18 A03.41.C1

bond A03.33.C13 A03.34.СT92 bond $\mathrm{A} 03.34 . \mathrm{C} 13 \mathrm{A03} .33 . \mathrm{CT} 92$

bond A03.34.C2 A03.42.C2 bond $\mathrm{A} 03.34 . \mathrm{C} 3 \mathrm{~A} 03.43 . \mathrm{C} 1$ bond $\mathrm{A03} .34 . \mathrm{C} 7 \mathrm{A03} .44 . \mathrm{C} 2$ bond A03.34.C8 A03.45.C1 bond A03.34.C17 A03.46.C5 bond $\mathrm{A} 03.34 . \mathrm{C} 12 \mathrm{~A} 03.47 . \mathrm{C} 1$ bond $\mathrm{A} 03.34 . \mathrm{C} 18 \mathrm{A03.48. \textrm {C } 1}$

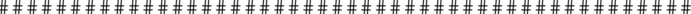

\# The 1 FPD.

bond D04.1.C2 D04.3.C2 bond D04.1.C3 D04.4.C1 bond D04.1.C7 D04.5.C2 bond D04.1.C8 D04.6.C1 bond D04.1.C17 D04.7.C5 bond D04.1.C12 D04.8.C1 bond D04.1.C18 D04.9.C1

bond D04.1.C13 D04.2.CT92 bond D04.2.C13 D04.1.CT92

bond D04.2.C2 D04.10.C2 bond D04.2.C3 D04.11.C1 bond D04.2.C7 D04.12.C2 bond D04.2.C8 D04.13.C1 bond D04.2.C17 D04.14.C5 bond D04.2.C12 D04.15.C1 bond D04.2.C18 D04.16.C1

\# The 2 FPD. 
bond D04.17.C2 D04.19.C2 bond D04.17.C3 D04.20.C1 bond D04.17.C7 D04.21.C2 bond D04.17.C8 D04.22.C1 bond D04.17.C17 D04.23.C5 bond D04.17.C12 D04.24.C1 bond D04.17.C18 D04.25.C1

bond D04.17.C13 D04.18.CT92 bond D04.18.C13 D04.17.CT92

bond D04.18.C2 D04.26.C2 bond D04.18.C3 D04.27.C1 bond D04.18.C7 D04.28.C2 bond D04.18.C8 D04.29.C1 bond D04.18.C17 D04.30.C5 bond D04.18.C12 D04.31.C1 bond D04.18.C18 D04.32.C1

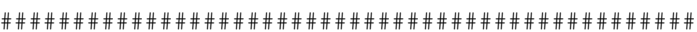

\# The 1 FPD.

bond D05.1.C2 D05.3.C2 bond D05.1.C3 D05.4.C1

bond D05.1.C7 D05.5.C2

bond D05.1.C8 D05.6.C1 bond D05.1.C17 D05.7.C5 bond D05.1.C12 D05.8.C1 bond D05.1.C18 D05.9.C1

bond D05.1.C13 D05.2.CT92 bond D05.2.C13 D05.1.CT92

bond D05.2.C2 D05.10.C2 bond D05.2.C3 D05.11.C1 bond D05.2.C7 D05.12.C2 bond D05.2.C8 D05.13.C1 bond D05.2.C17 D05.14.C5 bond D05.2.C12 D05.15.C1 bond D05.2.C18 D05.16.C1

\# The 2 FPD.

bond D05.17.C2 D05.19.C2 bond D05.17.C3 D05.20.C1 bond D05.17.C7 D05.21.C2 bond D05.17.C8 D05.22.C1

bond D05.17.C17 D05.23.C5 bond D05.17.C12 D05.24.C1 bond D05.17.C18 D05.25.C1

bond D05.17.C13 D05.18.CT92 bond D05.18.C13 D05.17.CT92

bond D05.18.C2 D05.26.C2 bond D05.18.C3 D05.27.C1 bond D05.18.C7 D05.28.C2 bond D05.18.C8 D05.29.C1

bond D05.18.C17 D05.30.C5 bond D05.18.C12 D05.31.C1 bond D05.18.C18 D05.32.C1

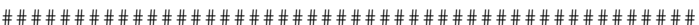

\# The 1 FPD.

bond Q05.1.C2 Q05.3.C2 bond $005.1 . \mathrm{C} 3005.4 . \mathrm{Cl}$ bond Q05.1.C7 Q05.5.C2 bond Q05.1.C8 Q05.6.C1

bond 005.1.C17 005.7.C5 bond Q05.1.C12 Q05.8.C bond Q05.1.C18 Q05.9.C1

bond 005.1.C13 005.2.CT92 bond Q05.2.C13 Q05.1.CT92

bond Q05.2.C2 Q05.10.C2 bond Q05.2.C3 Q05.11.C1 bond Q05.2.C7 Q05.12.C2 bond $005.2 . \mathrm{C} 8 \mathrm{0} 05.13 . \mathrm{Cl}$ 
bond Q05.2.C17 Q05.14.C5 bond Q05.2.C12 Q05.15.C1 bond Q05.2.C18 Q05.16.C1

\# The 2 FPD.

bond Q05.17.C2 Q05.19.C2 bond Q05.17.C3 Q05.20.C1 bond Q05.17.C7 Q05.21.C2 bond Q05.17.C8 Q05.22.C1 bond Q05.17.C17 Q05.23.C5 bond Q05.17.C12 Q05.24.C1 bond Q05.17.C18 Q05.25.C1

bond Q05.17.C13 Q05.18.CT92 bond Q05.18.C13 Q05.17.CT92

bond Q05.18.C2 Q05.26.C2 bond $005.18 . \mathrm{C} 3 \mathrm{Q} 05.27 . \mathrm{C} 1$ bond Q05.18.C7 Q05.28.C2 bond Q05.18.C8 Q05.29.C1 bond $005.18 . C 17005.30 . C 5$ bond Q05.18.C12 Q05.31.C1 bond Q05.18.C18 Q05.32.C1

\# The 3 FPD.

bond Q05.33.C2 Q05.35.C2 bond Q05.33.C3 Q05.36.C1 bond $005.33 . \mathrm{C} 7 \mathrm{Q} 05.37 . \mathrm{C} 2$ bond Q05.33.C8 Q05.38.C1 bond Q05.33.C17 Q05.39.C5 bond Q05.33.C12 Q05.40.C1 bond Q05.33.C18 Q05.41.C1

bond Q05.33.C13 Q05.34.CT92 bond Q05.34.C13 Q05.33.CT92

bond Q05.34.C2 Q05.42.C2 bond Q05.34.C3 Q05.43.C1 bond Q05.34.C7 Q05.44.C2 bond Q05.34.C8 Q05.45.C1 bond Q05.34.C17 Q05.46.C5 bond $205.34 . C 12$ Q05.47.C1 bond Q05.34.C18 Q05.48.C1

\section{\# The 4 FPD.}

bond Q05.49.C2 Q05.51.C2

bond $005.49 . \mathrm{C} 3005.52 . \mathrm{Cl}$

bond Q05.49.C7 Q05.53.C2 bond Q05.49.C8 Q05.54.C1 bond $005.49 . \mathrm{C} 17 \mathrm{Q} 05.55 . \mathrm{C5}$ bond Q05.49.C12 Q05.56.C1 bond Q05.49.C18 Q05.57.C1

bond Q05.49.C13 Q05.50.CT92 bond Q05.50.C13 Q05.49.CT92

bond Q05.50.C2 Q05.58.C2 bond $005.50 . \mathrm{C} 3 \mathrm{Q} 05.59 . \mathrm{C} 1$ bond $Q 05.50 . C 7 \mathrm{Q} 05.60 . \mathrm{C} 2$ bond Q05.50.C8 Q05.61.C1 bond $005.50 . C 17005.62 . C 5$ bond $\mathrm{Q} 05.50 . \mathrm{C} 12 \mathrm{Q} 05.63 . \mathrm{Cl}$ bond $\mathrm{Q} 05.50 . \mathrm{C} 18 \mathrm{Q} 05.64 . \mathrm{C} 1$

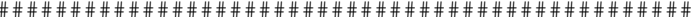

\# Let's save the prmtop and prmcrd file with specific file extensions

(to be automatically recognized by VMD http://www.ks.uiuc.edu/Research/vmd/)

\# Web site: http://ambermd.org/doc6/html/AMBER-sh-5.9.html\#sh-5.9.54

\# saveAmberParm HEM HEM.parm7 HEM.rst7

solvateoct AYI TIP3PBOX 10.0

solvateoct AYJ TIP3PBOX 10.0

solvateoct AYK TIP3PBOX 10.0 solvateoct AYM TIP3PBOX 10.0

solvateoct AYO TIP3PBOX 10.0 solvateoct $\mathrm{AYQ}$ TIP3PBOX 10.0 solvateoct AYR TIP3PBOX 10.0 solvateoct AYV TIP3PBOX 10.0 
solvateoct AYW TIP 3PBOX 10.0 solvateoct AYY TIP3PBOX 10.0 solvateoct $\mathrm{A} 01$ TIP 3PBOX 10.0 solvateoct A02 TIP3PBOX 10.0 solvateoct A03 TIP 3PBOX 10.0 solvateoct A04 TIP3PBOX 10.0 solvateoct D04 TIP3PBOX 10.0 solvateoct D05 TIP3PBOX 10.0 solvateoct Q05 TIP3PBOX 10.0

savepdb AYI AYI.pdb savepdb AYJ AYJ.pdb savepdb AYK AYK.pdb savepdb AYM AYM.pdb savepdb AYO AYO.pdb savepdb AYQ AYQ.pdb savepdb AYR AYR.pdb savepdb AYV AYV.pdb savepdb AYW AYW.pdb savepdb AYY AYY.pdb savepdb A01 A01.pdb savepdb A02 A02.pdb savepdb A03 A03.pdb savepdb A04 A04.pdb savepdb D04 D04.pdb savepdb D05 D05.pdb savepdb Q05 Q05.pdb

saveAmberParm AYI AYI.parm7 AYI.rst7 saveAmberParm AYJ AYJ.parm7 AYJ.rst7 saveAmberParm AYK AYK.parm7 AYK.rst7 saveAmberParm AYM AYM.parm7 AYM.rst7 saveAmberParm AYO AYO.parm7 AYO.rst7 saveAmberParm AYQ AYQ.parm7 AYQ.rst7 saveAmberParm AYR AYR.parm7 AYR.rst7 saveAmberParm AYV AYV.parm7 AYV.rst7 saveAmberParm AYW AYW.parm7 AYW.rst7 saveAmberParm AYY AYY.parm7 AYY.rst7 saveAmberParm A01 A01.parm7 A01.rst7 saveAmberParm A02 A02.parm7 A02.rst7 saveAmberParm A03 A03.parm7 A03.rst7 saveAmberParm A04 A04.parm7 A04.rst7 saveAmberParm D04 D04.parm7 D04.rst7 saveAmberParm D05 D05.parm7 D05.rst7 saveAmberParm Q05 Q05.parm7 Q05.rst7

\section{Cartesian coordinates of input structures of molecular dynamics in the PDB file format}

\subsection{FPD entity: $A Y I \_F P D . p d b$ file}

\begin{tabular}{|c|c|c|c|c|c|c|c|c|c|}
\hline HETATM & 1 & $\mathrm{FE}$ & HEM & 1 & 4.263 & 10.757 & 1.491 & 1.00 & 0.00 \\
\hline HETATM & 2 & $\mathrm{C} 1$ & HEM & 1 & 2.035 & 12.629 & 0.429 & 1.00 & 0.00 \\
\hline HETATM & 3 & $\mathrm{C} 2$ & HEM & 1 & 1.676 & 13.420 & -0.742 & 1.00 & 0.00 \\
\hline HETATM & 4 & C3 & HEM & 1 & 2.822 & 13.517 & -1.517 & 1.00 & 0.00 \\
\hline HETATM & 5 & C4 & HEM & 1 & 3.851 & 12.800 & -0.809 & 1.00 & 0.00 \\
\hline HETATM & 6 & C5 & HEM & 1 & 5.167 & 12.681 & -1.247 & 1.00 & 0.00 \\
\hline HETATM & 7 & H5 & HEM & 1 & 5.420 & 13.177 & -2.182 & 1.00 & 0.00 \\
\hline HETATM & 8 & C6 & HEM & 1 & 6.186 & 11.982 & -0.611 & 1.00 & 0.00 \\
\hline HETATM & 9 & C7 & HEM & 1 & 7.555 & 11.910 & -1.081 & 1.00 & 0.00 \\
\hline HETATM & 10 & $\mathrm{C} 8$ & HEM & 1 & 8.260 & 11.190 & -0.134 & 1.00 & 0.00 \\
\hline HETATM & 11 & C9 & HEM & 1 & 7.309 & 10.825 & 0.891 & 1.00 & 0.00 \\
\hline HETATM & 12 & $\mathrm{C} 10$ & HEM & 1 & 7.614 & 10.090 & 2.034 & 1.00 & 0.00 \\
\hline HETATM & 13 & $\mathrm{H} 10$ & HEM & 1 & 8.641 & 9.746 & 2.140 & 1.00 & 0.00 \\
\hline HETATM & 14 & $\mathrm{C} 11$ & HEM & 1 & 6.739 & 9.750 & 3.057 & 1.00 & 0.00 \\
\hline HETATM & 15 & $\mathrm{C} 12$ & HEM & 1 & 7.120 & 9.025 & 4.247 & 1.00 & 0.00 \\
\hline HETATM & 16 & $\mathrm{C} 13$ & HEM & 1 & 5.981 & 8.878 & 5.004 & 1.00 & 0.00 \\
\hline HETATM & 17 & $\mathrm{C} 14$ & HEM & 1 & 4.908 & 9.519 & 4.273 & 1.00 & 0.00 \\
\hline HETATM & 18 & C15 & HEM & 1 & 3.580 & 9.574 & 4.694 & 1.00 & 0.00 \\
\hline HETATM & 19 & H15 & HEM & 1 & 3.340 & 9.089 & 5.642 & 1.00 & 0.00 \\
\hline HETATM & 20 & C16 & HEM & 1 & 2.550 & 10.255 & 4.051 & 1.00 & 0.00 \\
\hline HETATM & 21 & $\mathrm{C} 17$ & HEM & 1 & 1.184 & 10.369 & 4.533 & 1.00 & 0.00 \\
\hline HETATM & 22 & $\mathrm{C} 18$ & HEM & 1 & 0.508 & 11.137 & 3.609 & 1.00 & 0.00 \\
\hline
\end{tabular}




\begin{tabular}{|c|c|c|c|c|c|c|c|c|c|}
\hline HETATM & 23 & C19 & HEM & 1 & 1.458 & 11.489 & 2.573 & 1.00 & 0.00 \\
\hline HETATM & 24 & $\mathrm{C} 20$ & HEM & 1 & 1.167 & 12.280 & 1.462 & 1.00 & 0.00 \\
\hline HETATM & 25 & H2O & HEM & 1 & 0.152 & 12.669 & 1.402 & 1.00 & 0.00 \\
\hline HETATM & 26 & N21 & HEM & 1 & 3.357 & 12.266 & 0.367 & 1.00 & 0.00 \\
\hline HETATM & 27 & N22 & HEM & 1 & 6.062 & 11.319 & 0.590 & 1.00 & 0.00 \\
\hline HETATM & 28 & N23 & HEM & 1 & 5.391 & 10.056 & 3.090 & 1.00 & 0.00 \\
\hline HETATM & 29 & N24 & HEM & 1 & 2.691 & 10.941 & 2.854 & 1.00 & 0.00 \\
\hline HETATM & 30 & C99 & HEM & 1 & 3.598 & 8.076 & 0.352 & 1.00 & 0.00 \\
\hline HETATM & 31 & 091 & HEM & 1 & 3.757 & 9.389 & 0.365 & 1.00 & 0.00 \\
\hline HETATM & 32 & 092 & HEM & 1 & 3.443 & 7.458 & -0.703 & 1.00 & 0.00 \\
\hline HETATM & 33 & CT91 & HEM & 1 & 3.614 & 7.394 & 1.709 & 1.00 & 0.00 \\
\hline HETATM & 34 & H911 & HEM & 1 & 4.593 & 7.590 & 2.171 & 1.00 & 0.00 \\
\hline HETATM & 35 & H912 & HEM & 1 & 2.866 & 7.884 & 2.352 & 1.00 & 0.00 \\
\hline HETATM & 36 & CT92 & HEM & 1 & 3.354 & 5.890 & 1.597 & 1.00 & 0.00 \\
\hline HETATM & 37 & H921 & HEM & 1 & 4.151 & 5.429 & 1.000 & 1.00 & 0.00 \\
\hline HETATM & 38 & H922 & HEM & 1 & 2.429 & 5.734 & 1.027 & 1.00 & 0.00 \\
\hline \multicolumn{10}{|l|}{ TER } \\
\hline HETATM & 39 & $\mathrm{FE}$ & HEM & 2 & 4.955 & 3.343 & 6.440 & 1.00 & 0.00 \\
\hline HETATM & 40 & $\mathrm{C} 1$ & HEM & 2 & 7.183 & 1.471 & 7.502 & 1.00 & 0.00 \\
\hline HETATM & 41 & $\mathrm{C} 2$ & HEM & 2 & 7.542 & 0.680 & 8.674 & 1.00 & 0.00 \\
\hline HETATM & 42 & C3 & HEM & 2 & 6.397 & 0.583 & 9.448 & 1.00 & 0.00 \\
\hline HETATM & 43 & C4 & HEM & 2 & 5.367 & 1.301 & 8.740 & 1.00 & 0.00 \\
\hline HETATM & 44 & C5 & HEM & 2 & 4.052 & 1.420 & 9.178 & 1.00 & 0.00 \\
\hline HETATM & 45 & H5 & HEM & 2 & 3.798 & 0.923 & 10.113 & 1.00 & 0.00 \\
\hline HETATM & 46 & $\mathrm{C} 6$ & HEM & 2 & 3.033 & 2.118 & 8.542 & 1.00 & 0.00 \\
\hline HETATM & 47 & C7 & HEM & 2 & 1.664 & 2.191 & 9.012 & 1.00 & 0.00 \\
\hline HETATM & 48 & $\mathrm{C} 8$ & HEM & 2 & 0.958 & 2.910 & 8.065 & 1.00 & 0.00 \\
\hline HETATM & 49 & C9 & HEM & 2 & 1.909 & 3.275 & 7.041 & 1.00 & 0.00 \\
\hline HETATM & 50 & $\mathrm{C} 10$ & HEM & 2 & 1.605 & 4.010 & 5.897 & 1.00 & 0.00 \\
\hline HETATM & 51 & $\mathrm{H} 10$ & HEM & 2 & 0.578 & 4.354 & 5.791 & 1.00 & 0.00 \\
\hline HETATM & 52 & $\mathrm{C} 11$ & HEM & 2 & 2.479 & 4.350 & 4.874 & 1.00 & 0.00 \\
\hline HETATM & 53 & $\mathrm{C} 12$ & HEM & 2 & 2.098 & 5.076 & 3.684 & 1.00 & 0.00 \\
\hline HETATM & 54 & C13 & HEM & 2 & 3.238 & 5.223 & 2.927 & 1.00 & 0.00 \\
\hline HETATM & 55 & C14 & HEM & 2 & 4.311 & 4.581 & 3.659 & 1.00 & 0.00 \\
\hline HETATM & 56 & C15 & HEM & 2 & 5.639 & 4.527 & 3.237 & 1.00 & 0.00 \\
\hline HETATM & 57 & H15 & HEM & 2 & 5.879 & 5.011 & 2.289 & 1.00 & 0.00 \\
\hline HETATM & 58 & $\mathrm{C} 16$ & HEM & 2 & 6.669 & 3.845 & 3.880 & 1.00 & 0.00 \\
\hline HETATM & 59 & $\mathrm{C} 17$ & HEM & 2 & 8.035 & 3.732 & 3.398 & 1.00 & 0.00 \\
\hline HETATM & 60 & $\mathrm{C} 18$ & HEM & 2 & 8.710 & 2.964 & 4.322 & 1.00 & 0.00 \\
\hline HETATM & 61 & C19 & HEM & 2 & 7.761 & 2.612 & 5.358 & 1.00 & 0.00 \\
\hline HETATM & 62 & $\mathrm{C} 20$ & HEM & 2 & 8.052 & 1.821 & 6.469 & 1.00 & 0.00 \\
\hline HETATM & 63 & H2O & HEM & 2 & 9.066 & 1.432 & 6.530 & 1.00 & 0.00 \\
\hline HETATM & 64 & N21 & HEM & 2 & 5.862 & 1.834 & 7.564 & 1.00 & 0.00 \\
\hline HETATM & 65 & N22 & HEM & 2 & 3.157 & 2.781 & 7.342 & 1.00 & 0.00 \\
\hline HETATM & 66 & N23 & HEM & 2 & 3.828 & 4.044 & 4.841 & 1.00 & 0.00 \\
\hline HETATM & 67 & N24 & HEM & 2 & 6.527 & 3.160 & 5.077 & 1.00 & 0.00 \\
\hline HETATM & 68 & C99 & HEM & 2 & 5.621 & 6.025 & 7.579 & 1.00 & 0.00 \\
\hline HETATM & 69 & 091 & HEM & 2 & 5.461 & 4.711 & 7.567 & 1.00 & 0.00 \\
\hline HETATM & 70 & 092 & HEM & 2 & 5.776 & 6.642 & 8.635 & 1.00 & 0.00 \\
\hline HETATM & 71 & CT91 & HEM & 2 & 5.605 & 6.706 & 6.223 & 1.00 & 0.00 \\
\hline HETATM & 72 & H911 & HEM & 2 & 4.626 & 6.511 & 5.760 & 1.00 & 0.00 \\
\hline HETATM & 73 & H912 & HEM & 2 & 6.353 & 6.216 & 5.580 & 1.00 & 0.00 \\
\hline HETATM & 74 & СТ92 & HEM & 2 & 5.864 & 8.211 & 6.334 & 1.00 & 0.00 \\
\hline HETATM & 75 & H921 & HEM & 2 & 6.789 & 8.367 & 6.905 & 1.00 & 0.00 \\
\hline HETATM & 76 & H922 & HEM & 2 & 5.068 & 8.671 & 6.931 & 1.00 & 0.00 \\
\hline \multicolumn{10}{|l|}{ TER } \\
\hline HETATM & 77 & $\mathrm{C} 2$ & VIN & 3 & 0.345 & 13.923 & -1.002 & 1.00 & 0.00 \\
\hline HETATM & 78 & H2 1 & VIN & 3 & -0.471 & 13.224 & -0.837 & 1.00 & 0.00 \\
\hline HETATM & 79 & $\mathrm{C} 1$ & VIN & 3 & 0.012 & 15.147 & -1.403 & 1.00 & 0.00 \\
\hline HETATM & 80 & $\mathrm{H} 11$ & VIN & 3 & 0.743 & 15.926 & -1.511 & 1.00 & 0.00 \\
\hline HETATM & 81 & $\mathrm{H} 12$ & VIN & 3 & -1.030 & 15.417 & -1.570 & 1.00 & 0.00 \\
\hline \multicolumn{10}{|l|}{ TER } \\
\hline HETATM & 82 & $\mathrm{C} 1$ & MET & 4 & 3.008 & 14.177 & -2.840 & 1.00 & 0.00 \\
\hline HETATM & 83 & H11 & MET & 4 & 3.243 & 15.248 & -2.720 & 1.00 & 0.00 \\
\hline HETATM & 84 & $\mathrm{H} 12$ & $\mathrm{MET}$ & 4 & 3.821 & 13.713 & -3.410 & 1.00 & 0.00 \\
\hline HETATM & 85 & $\mathrm{H} 13$ & MET & 4 & 2.101 & 14.100 & -3.450 & 1.00 & 0.00 \\
\hline \multicolumn{10}{|l|}{ TER } \\
\hline HETATM & 86 & $\mathrm{C} 2$ & VIN & 5 & 8.018 & 12.499 & -2.325 & 1.00 & 0.00 \\
\hline HETATM & 87 & H 21 & VIN & 5 & 7.352 & 12.399 & -3.184 & 1.00 & 0.00 \\
\hline HETATM & 88 & $\mathrm{C} 1$ & VIN & 5 & 9.185 & 13.144 & -2.484 & 1.00 & 0.00 \\
\hline HETATM & 89 & H11 & VIN & 5 & 9.865 & 13.300 & -1.654 & 1.00 & 0.00 \\
\hline HETATM & 90 & $\mathrm{H} 12$ & VIN & 5 & 9.490 & 13.542 & -3.450 & 1.00 & 0.00 \\
\hline \multicolumn{10}{|l|}{ TER } \\
\hline HETATM & 91 & $\mathrm{C} 1$ & MET & 6 & 9.703 & 10.816 & -0.121 & 1.00 & 0.00 \\
\hline HETATM & 92 & $\mathrm{H} 11$ & MET & 6 & 10.294 & 11.480 & 0.528 & 1.00 & 0.00 \\
\hline HETATM & 93 & $\mathrm{H} 12$ & $\mathrm{MET}$ & 6 & 9.859 & 9.794 & 0.246 & 1.00 & 0.00 \\
\hline HETATM & 94 & $\mathrm{H} 13$ & MET & 6 & 10.135 & 10.882 & -1.126 & 1.00 & 0.00 \\
\hline TER & & & & & & & & & \\
\hline HETATM & 95 & C5 & $\mathrm{ACP}$ & 7 & 0.615 & 9.735 & 5.770 & 1.00 & 0.00 \\
\hline
\end{tabular}




\begin{tabular}{|c|c|c|c|c|c|c|c|c|c|}
\hline HETATM & 96 & H5 1 & $\mathrm{ACP}$ & 7 & 1.354 & 9.723 & 6.582 & 1.00 & 0.00 \\
\hline HETATM & 97 & H 52 & $\mathrm{ACP}$ & 7 & -0.226 & 10.343 & 6.134 & 1.00 & 0.00 \\
\hline HETATM & 98 & $\mathrm{C} 4$ & $\mathrm{ACP}$ & 7 & 0.136 & 8.297 & 5.497 & 1.00 & 0.00 \\
\hline HETATM & 99 & H 41 & $\mathrm{ACP}$ & 7 & -0.422 & 8.236 & 4.549 & 1.00 & 0.00 \\
\hline HETATM & 100 & $\mathrm{H} 42$ & $\mathrm{ACP}$ & 7 & 1.007 & 7.630 & 5.390 & 1.00 & 0.00 \\
\hline HETATM & 101 & $\mathrm{C} 1$ & $\mathrm{ACP}$ & 7 & -0.728 & 7.685 & 6.573 & 1.00 & 0.00 \\
\hline HETATM & 102 & $\mathrm{O} 2$ & $\mathrm{ACP}$ & 7 & -0.956 & 8.208 & 7.671 & 1.00 & 0.00 \\
\hline HETATM & 103 & 03 & $\mathrm{ACP}$ & 7 & -1.244 & 6.505 & 6.209 & 1.00 & 0.00 \\
\hline HETATM & 104 & H3 & $\mathrm{ACP}$ & 7 & -1.853 & 6.151 & 6.945 & 1.00 & 0.00 \\
\hline \multicolumn{10}{|l|}{ TER } \\
\hline HETATM & 105 & $\mathrm{C} 1$ & MET & 8 & 8.511 & 8.608 & 4.597 & 1.00 & 0.00 \\
\hline HETATM & 106 & H11 & $\mathrm{MET}$ & 8 & 9.063 & 9.448 & 5.047 & 1.00 & 0.00 \\
\hline HETATM & 107 & H12 & MET & 8 & 8.517 & 7.782 & 5.319 & 1.00 & 0.00 \\
\hline HETATM & 108 & H13 & MET & 8 & 9.082 & 8.289 & 3.717 & 1.00 & 0.00 \\
\hline \multicolumn{10}{|l|}{ TER } \\
\hline HETATM & 109 & $\mathrm{C} 1$ & $\mathrm{MET}$ & 9 & -0.923 & 11.557 & 3.646 & 1.00 & 0.00 \\
\hline HETATM & 110 & H11 & MET & 9 & -1.368 & 11.348 & 4.626 & 1.00 & 0.00 \\
\hline HETATM & 111 & H12 & $\mathrm{MET}$ & 9 & -1.033 & 12.637 & 3.471 & 1.00 & 0.00 \\
\hline HETATM & 112 & H13 & $\mathrm{MET}$ & 9 & -1.523 & 11.031 & 2.889 & 1.00 & 0.00 \\
\hline \multicolumn{10}{|l|}{ TER } \\
\hline HETATM & 113 & $\mathrm{C} 2$ & VIN & 10 & 8.874 & 0.178 & 8.933 & 1.00 & 0.00 \\
\hline HETATM & 114 & $\mathrm{H} 21$ & VIN & 10 & 9.690 & 0.876 & 8.768 & 1.00 & 0.00 \\
\hline HETATM & 115 & $\mathrm{C} 1$ & VIN & 10 & 9.207 & -1.047 & 9.335 & 1.00 & 0.00 \\
\hline HETATM & 116 & $\mathrm{H} 11$ & VIN & 10 & 8.475 & -1.826 & 9.442 & 1.00 & 0.00 \\
\hline HETATM & 117 & $\mathrm{H} 12$ & VIN & 10 & 10.249 & -1.316 & 9.501 & 1.00 & 0.00 \\
\hline \multicolumn{10}{|l|}{ TER } \\
\hline HETATM & 118 & $\mathrm{C} 1$ & $\mathrm{MET}$ & 11 & 6.210 & -0.077 & 10.771 & 1.00 & 0.00 \\
\hline HETATM & 119 & H11 & MET & 11 & 5.975 & -1.148 & 10.652 & 1.00 & 0.00 \\
\hline HETATM & 120 & H12 & MET & 11 & 5.397 & 0.387 & 11.341 & 1.00 & 0.00 \\
\hline HETATM & 121 & $\mathrm{H} 13$ & MET & 11 & 7.118 & 0.001 & 11.382 & 1.00 & 0.00 \\
\hline \multicolumn{10}{|l|}{ TER } \\
\hline HETATM & 122 & C2 & VIN & 12 & 1.200 & 1.601 & 10.256 & 1.00 & 0.00 \\
\hline HETATM & 123 & $\mathrm{H} 21$ & VIN & 12 & 1.866 & 1.702 & 11.115 & 1.00 & 0.00 \\
\hline HETATM & 124 & $\mathrm{C} 1$ & VIN & 12 & 0.033 & 0.957 & 10.415 & 1.00 & 0.00 \\
\hline HETATM & 125 & H11 & VIN & 12 & -0.647 & 0.800 & 9.585 & 1.00 & 0.00 \\
\hline HETATM & 126 & $\mathrm{H} 12$ & VIN & 12 & -0.272 & 0.559 & 11.381 & 1.00 & 0.00 \\
\hline \multicolumn{10}{|l|}{ TER } \\
\hline HETATM & 127 & $\mathrm{C} 1$ & MET & 13 & -0.484 & 3.284 & 8.052 & 1.00 & 0.00 \\
\hline HETATM & 128 & $\mathrm{H} 11$ & $\mathrm{MET}$ & 13 & -0.641 & 4.306 & 7.685 & 1.00 & 0.00 \\
\hline HETATM & 129 & H12 & MET & 13 & -0.917 & 3.219 & 9.057 & 1.00 & 0.00 \\
\hline HETATM & 130 & $\mathrm{H} 13$ & MET & 13 & -1.076 & 2.620 & 7.403 & 1.00 & 0.00 \\
\hline \multicolumn{10}{|l|}{ TER } \\
\hline HETATM & 131 & C5 & $\mathrm{ACP}$ & 14 & 8.604 & 4.366 & 2.161 & 1.00 & 0.00 \\
\hline HETATM & 132 & H51 & $\mathrm{ACP}$ & 14 & 7.865 & 4.377 & 1.349 & 1.00 & 0.00 \\
\hline HETATM & 133 & H5 2 & $\mathrm{ACP}$ & 14 & 9.445 & 3.758 & 1.798 & 1.00 & 0.00 \\
\hline HETATM & 134 & $\mathrm{C} 4$ & $\mathrm{ACP}$ & 14 & 9.082 & 5.803 & 2.434 & 1.00 & 0.00 \\
\hline HETATM & 135 & H4 1 & $\mathrm{ACP}$ & 14 & 9.640 & 5.865 & 3.382 & 1.00 & 0.00 \\
\hline HETATM & 136 & $\mathrm{H} 42$ & $\mathrm{ACP}$ & 14 & 8.212 & 6.470 & 2.541 & 1.00 & 0.00 \\
\hline HETATM & 137 & $\mathrm{C} 1$ & $\mathrm{ACP}$ & 14 & 9.946 & 6.416 & 1.358 & 1.00 & 0.00 \\
\hline HETATM & 138 & 02 & $\mathrm{ACP}$ & 14 & 10.174 & 5.893 & 0.260 & 1.00 & 0.00 \\
\hline HETATM & 139 & 03 & $\mathrm{ACP}$ & 14 & 10.462 & 7.595 & 1.722 & 1.00 & 0.00 \\
\hline HETATM & 140 & H3 & $\mathrm{ACP}$ & 14 & 11.071 & 7.949 & 0.986 & 1.00 & 0.00 \\
\hline \multicolumn{10}{|l|}{ TER } \\
\hline HETATM & 141 & $\mathrm{C} 1$ & $\mathrm{MET}$ & 15 & 0.708 & 5.492 & 3.334 & 1.00 & 0.00 \\
\hline HETATM & 142 & H11 & $\mathrm{MET}$ & 15 & 0.155 & 4.653 & 2.884 & 1.00 & 0.00 \\
\hline HETATM & 143 & $\mathrm{H} 12$ & MET & 15 & 0.701 & 6.318 & 2.613 & 1.00 & 0.00 \\
\hline HETATM & 144 & $\mathrm{H} 13$ & MET & 15 & 0.137 & 5.812 & 4.214 & 1.00 & 0.00 \\
\hline \multicolumn{10}{|l|}{ TER } \\
\hline HETATM & 145 & $\mathrm{C} 1$ & MET & 16 & 10.141 & 2.543 & 4.285 & 1.00 & 0.00 \\
\hline HETATM & 146 & $\mathrm{H} 11$ & MET & 16 & 10.586 & 2.752 & 3.305 & 1.00 & 0.00 \\
\hline HETATM & 147 & $\mathrm{H} 12$ & $\mathrm{MET}$ & 16 & 10.252 & 1.463 & 4.460 & 1.00 & 0.00 \\
\hline HETATM & 148 & $\mathrm{H} 13$ & $\mathrm{MET}$ & 16 & 10.742 & 3.069 & 5.042 & 1.00 & 0.00 \\
\hline \multicolumn{10}{|l|}{ TER } \\
\hline END & & & & & & & & & \\
\hline
\end{tabular}

\subsection{D1 dimer: $A Y J \_D 1 . p d b$ file}

\begin{tabular}{|c|c|c|c|c|c|c|c|c|c|c|}
\hline HETATM & 1 & $\mathrm{FE}$ & HEM & 1 & 4.263 & 10.757 & 1.491 & 1.00 & 0.00 & $\mathrm{FE}$ \\
\hline HETATM & 2 & $\mathrm{C} 1$ & HEM & 1 & 2.035 & 12.629 & 0.429 & 1.00 & 0.00 & C \\
\hline HETATM & 3 & $\mathrm{C} 2$ & HEM & 1 & 1.676 & 13.420 & -0.742 & 1.00 & 0.00 & C \\
\hline HETATM & 4 & C3 & HEM & 1 & 2.822 & 13.517 & -1.517 & 1.00 & 0.00 & C \\
\hline HETATM & 5 & C4 & HEM & 1 & 3.851 & 12.800 & -0.809 & 1.00 & 0.00 & C \\
\hline HETATM & 6 & C5 & HEM & 1 & 5.167 & 12.681 & -1.247 & 1.00 & 0.00 & C \\
\hline HETATM & 7 & H5 & HEM & 1 & 5.420 & 13.177 & -2.182 & 1.00 & 0.00 & $\mathrm{H}$ \\
\hline HETATM & 8 & $\mathrm{C} 6$ & HEM & 1 & 6.186 & 11.982 & -0.611 & 1.00 & 0.00 & C \\
\hline HETATM & 9 & C7 & HEM & 1 & 7.555 & 11.910 & -1.081 & 1.00 & 0.00 & C \\
\hline HETATM & 10 & $\mathrm{C} 8$ & HEM & 1 & 8.260 & 11.190 & -0.134 & 1.00 & 0.00 & C \\
\hline HETATM & 11 & $\mathrm{C} 9$ & HEM & 1 & 7.309 & 10.825 & 0.891 & 1.00 & 0.00 & C \\
\hline
\end{tabular}




\begin{tabular}{|c|c|c|c|c|c|c|c|c|c|c|}
\hline HETATM & 12 & $\mathrm{C} 10$ & HEM & 1 & 7.614 & 10.090 & 2.034 & 1.00 & 0.00 & C \\
\hline HETATM & 13 & $\mathrm{H} 10$ & HEM & 1 & 8.641 & 9.746 & 2.140 & 1.00 & 0.00 & $\mathrm{H}$ \\
\hline HETATM & 14 & $\mathrm{C} 11$ & HEM & 1 & 6.739 & 9.750 & 3.057 & 1.00 & 0.00 & C \\
\hline HETATM & 15 & $\mathrm{C} 12$ & HEM & 1 & 7.120 & 9.025 & 4.247 & 1.00 & 0.00 & C \\
\hline HETATM & 16 & C13 & HEM & 1 & 5.981 & 8.878 & 5.004 & 1.00 & 0.00 & C \\
\hline HETATM & 17 & $\mathrm{C} 14$ & HEM & 1 & 4.908 & 9.519 & 4.273 & 1.00 & 0.00 & C \\
\hline HETATM & 18 & C15 & HEM & 1 & 3.580 & 9.574 & 4.694 & 1.00 & 0.00 & C \\
\hline HETATM & 19 & H15 & HEM & 1 & 3.340 & 9.089 & 5.642 & 1.00 & 0.00 & $\mathrm{H}$ \\
\hline HETATM & 20 & $\mathrm{C} 16$ & HEM & 1 & 2.550 & 10.255 & 4.051 & 1.00 & 0.00 & C \\
\hline HETATM & 21 & $\mathrm{C} 17$ & HEM & 1 & 1.184 & 10.369 & 4.533 & 1.00 & 0.00 & C \\
\hline HETATM & 22 & $\mathrm{C} 18$ & HEM & 1 & 0.508 & 11.137 & 3.609 & 1.00 & 0.00 & C \\
\hline HETATM & 23 & C19 & HEM & 1 & 1.458 & 11.489 & 2.573 & 1.00 & 0.00 & C \\
\hline HETATM & 24 & $\mathrm{C} 20$ & HEM & 1 & 1.167 & 12.280 & 1.462 & 1.00 & 0.00 & C \\
\hline HETATM & 25 & $\mathrm{H} 2 \mathrm{O}$ & HEM & 1 & 0.152 & 12.669 & 1.402 & 1.00 & 0.00 & $\mathrm{H}$ \\
\hline HETATM & 26 & N21 & HEM & 1 & 3.357 & 12.266 & 0.367 & 1.00 & 0.00 & $\mathrm{~N}$ \\
\hline HETATM & 27 & N22 & HEM & 1 & 6.062 & 11.319 & 0.590 & 1.00 & 0.00 & $\mathrm{~N}$ \\
\hline HETATM & 28 & N23 & HEM & 1 & 5.391 & 10.056 & 3.090 & 1.00 & 0.00 & $\mathrm{~N}$ \\
\hline HETATM & 29 & N24 & HEM & 1 & 2.691 & 10.941 & 2.854 & 1.00 & 0.00 & $\mathrm{~N}$ \\
\hline HETATM & 30 & C99 & HEM & 1 & 3.598 & 8.076 & 0.352 & 1.00 & 0.00 & C \\
\hline HETATM & 31 & 091 & HEM & 1 & 3.757 & 9.389 & 0.365 & 1.00 & 0.00 & o \\
\hline HETATM & 32 & 092 & HEM & 1 & 3.443 & 7.458 & -0.703 & 1.00 & 0.00 & O \\
\hline HETATM & 33 & СТ91 & HEM & 1 & 3.614 & 7.394 & 1.709 & 1.00 & 0.00 & C \\
\hline HETATM & 34 & H911 & HEM & 1 & 4.593 & 7.590 & 2.171 & 1.00 & 0.00 & $\mathrm{H}$ \\
\hline HETATM & 35 & H912 & HEM & 1 & 2.866 & 7.884 & 2.352 & 1.00 & 0.00 & $\mathrm{H}$ \\
\hline HETATM & 36 & СТ92 & HEM & 1 & 3.354 & 5.890 & 1.597 & 1.00 & 0.00 & C \\
\hline HETATM & 37 & H921 & HEM & 1 & 4.151 & 5.429 & 1.000 & 1.00 & 0.00 & $\mathrm{H}$ \\
\hline HETATM & 38 & H922 & HEM & 1 & 2.429 & 5.734 & 1.027 & 1.00 & 0.00 & $\mathrm{H}$ \\
\hline \multicolumn{11}{|l|}{ TER } \\
\hline HETATM & 39 & $\mathrm{FE}$ & HEM & 2 & 4.955 & 3.343 & 6.440 & 1.00 & 0.00 & $\mathrm{FE}$ \\
\hline HETATM & 40 & $\mathrm{C} 1$ & HEM & 2 & 7.183 & 1.471 & 7.502 & 1.00 & 0.00 & C \\
\hline HETATM & 41 & $\mathrm{C} 2$ & HEM & 2 & 7.542 & 0.680 & 8.674 & 1.00 & 0.00 & C \\
\hline HETATM & 42 & C3 & HEM & 2 & 6.397 & 0.583 & 9.448 & 1.00 & 0.00 & C \\
\hline HETATM & 43 & $\mathrm{C} 4$ & HEM & 2 & 5.367 & 1.301 & 8.740 & 1.00 & 0.00 & C \\
\hline HETATM & 44 & C5 & HEM & 2 & 4.052 & 1.420 & 9.178 & 1.00 & 0.00 & C \\
\hline HETATM & 45 & H5 & HEM & 2 & 3.798 & 0.923 & 10.113 & 1.00 & 0.00 & $\mathrm{H}$ \\
\hline HETATM & 46 & C6 & HEM & 2 & 3.033 & 2.118 & 8.542 & 1.00 & 0.00 & C \\
\hline HETATM & 47 & C7 & HEM & 2 & 1.664 & 2.191 & 9.012 & 1.00 & 0.00 & C \\
\hline HETATM & 48 & $\mathrm{C} 8$ & HEM & 2 & 0.958 & 2.910 & 8.065 & 1.00 & 0.00 & C \\
\hline HETATM & 49 & $\mathrm{C} 9$ & HEM & 2 & 1.909 & 3.275 & 7.041 & 1.00 & 0.00 & C \\
\hline HETATM & 50 & $\mathrm{C} 10$ & HEM & 2 & 1.605 & 4.010 & 5.897 & 1.00 & 0.00 & C \\
\hline HETATM & 51 & $\mathrm{H} 10$ & HEM & 2 & 0.578 & 4.354 & 5.791 & 1.00 & 0.00 & $\mathrm{H}$ \\
\hline HETATM & 52 & $\mathrm{C} 11$ & HEM & 2 & 2.479 & 4.350 & 4.874 & 1.00 & 0.00 & C \\
\hline HETATM & 53 & $\mathrm{C} 12$ & HEM & 2 & 2.098 & 5.076 & 3.684 & 1.00 & 0.00 & C \\
\hline HETATM & 54 & $\mathrm{C} 13$ & HEM & 2 & 3.238 & 5.223 & 2.927 & 1.00 & 0.00 & C \\
\hline HETATM & 55 & $\mathrm{C} 14$ & HEM & 2 & 4.311 & 4.581 & 3.659 & 1.00 & 0.00 & $\mathrm{C}$ \\
\hline HETATM & 56 & C15 & HEM & 2 & 5.639 & 4.527 & 3.237 & 1.00 & 0.00 & C \\
\hline HETATM & 57 & H15 & HEM & 2 & 5.879 & 5.011 & 2.289 & 1.00 & 0.00 & $\mathrm{H}$ \\
\hline HETATM & 58 & $\mathrm{C} 16$ & HEM & 2 & 6.669 & 3.845 & 3.880 & 1.00 & 0.00 & C \\
\hline HETATM & 59 & $\mathrm{C} 17$ & HEM & 2 & 8.035 & 3.732 & 3.398 & 1.00 & 0.00 & $\mathrm{C}$ \\
\hline HETATM & 60 & $\mathrm{C} 18$ & HEM & 2 & 8.710 & 2.964 & 4.322 & 1.00 & 0.00 & C \\
\hline HETATM & 61 & C19 & HEM & 2 & 7.761 & 2.612 & 5.358 & 1.00 & 0.00 & $\mathrm{C}$ \\
\hline HETATM & 62 & $\mathrm{C} 20$ & HEM & 2 & 8.052 & 1.821 & 6.469 & 1.00 & 0.00 & C \\
\hline HETATM & 63 & $\mathrm{H} 2 \mathrm{O}$ & HEM & 2 & 9.066 & 1.432 & 6.530 & 1.00 & 0.00 & $\mathrm{H}$ \\
\hline HETATM & 64 & N21 & HEM & 2 & 5.862 & 1.834 & 7.564 & 1.00 & 0.00 & $\mathrm{~N}$ \\
\hline HETATM & 65 & N22 & HEM & 2 & 3.157 & 2.781 & 7.342 & 1.00 & 0.00 & $\mathrm{~N}$ \\
\hline HETATM & 66 & N23 & HEM & 2 & 3.828 & 4.044 & 4.841 & 1.00 & 0.00 & $\mathrm{~N}$ \\
\hline HETATM & 67 & N24 & HEM & 2 & 6.527 & 3.160 & 5.077 & 1.00 & 0.00 & $\mathrm{~N}$ \\
\hline HETATM & 68 & C99 & HEM & 2 & 5.621 & 6.025 & 7.579 & 1.00 & 0.00 & C \\
\hline HETATM & 69 & 091 & HEM & 2 & 5.461 & 4.711 & 7.567 & 1.00 & 0.00 & O \\
\hline HETATM & 70 & 092 & HEM & 2 & 5.776 & 6.642 & 8.635 & 1.00 & 0.00 & 0 \\
\hline HETATM & 71 & СТ91 & HEM & 2 & 5.605 & 6.706 & 6.223 & 1.00 & 0.00 & C \\
\hline HETATM & 72 & H911 & HEM & 2 & 4.626 & 6.511 & 5.760 & 1.00 & 0.00 & $\mathrm{H}$ \\
\hline HETATM & 73 & H912 & HEM & 2 & 6.353 & 6.216 & 5.580 & 1.00 & 0.00 & $\mathrm{H}$ \\
\hline HETATM & 74 & СТ92 & HEM & 2 & 5.864 & 8.211 & 6.334 & 1.00 & 0.00 & C \\
\hline HETATM & 75 & H921 & HEM & 2 & 6.789 & 8.367 & 6.905 & 1.00 & 0.00 & $\mathrm{H}$ \\
\hline HETATM & 76 & H922 & HEM & 2 & 5.068 & 8.671 & 6.931 & 1.00 & 0.00 & $\mathrm{H}$ \\
\hline \multicolumn{11}{|l|}{ TER } \\
\hline HETATM & 77 & $\mathrm{C} 2$ & VIN & 3 & 0.345 & 13.923 & -1.002 & 1.00 & 0.00 & C \\
\hline HETATM & 78 & H 21 & VIN & 3 & -0.471 & 13.224 & -0.837 & 1.00 & 0.00 & $\mathrm{H}$ \\
\hline HETATM & 79 & $\mathrm{C} 1$ & VIN & 3 & 0.012 & 15.147 & -1.403 & 1.00 & 0.00 & $\mathrm{C}$ \\
\hline HETATM & 80 & $\mathrm{H} 11$ & VIN & 3 & 0.743 & 15.926 & -1.511 & 1.00 & 0.00 & $\mathrm{H}$ \\
\hline HETATM & 81 & $\mathrm{H} 12$ & VIN & 3 & -1.030 & 15.417 & -1.570 & 1.00 & 0.00 & $\mathrm{H}$ \\
\hline \multicolumn{11}{|l|}{ TER } \\
\hline HETATM & 82 & $\mathrm{C} 1$ & MET & 4 & 3.008 & 14.177 & -2.840 & 1.00 & 0.00 & C \\
\hline HETATM & 83 & $\mathrm{H} 11$ & MET & 4 & 3.243 & 15.248 & -2.720 & 1.00 & 0.00 & $\mathrm{H}$ \\
\hline HETATM & 84 & $\mathrm{H} 12$ & MET & 4 & 3.821 & 13.713 & -3.410 & 1.00 & 0.00 & $\mathrm{H}$ \\
\hline HETATM & 85 & H13 & $\mathrm{MET}$ & 4 & 2.101 & 14.100 & -3.450 & 1.00 & 0.00 & $\mathrm{H}$ \\
\hline \multicolumn{11}{|l|}{ TER } \\
\hline HETATM & 86 & $\mathrm{C} 2$ & VIN & 5 & 8.018 & 12.499 & -2.325 & 1.00 & 0.00 & C \\
\hline
\end{tabular}




\begin{tabular}{|c|c|c|c|}
\hline HETATM & 87 & H 21 & VIN \\
\hline HETATM & 88 & $\mathrm{C} 1$ & VIN \\
\hline HETATM & 89 & $\mathrm{H} 11$ & VIN \\
\hline HETATM & 90 & $\mathrm{H} 12$ & VIN \\
\hline \multicolumn{4}{|l|}{ TER } \\
\hline HETATM & 91 & $\mathrm{C} 1$ & MET \\
\hline HETATM & 92 & H11 & MET \\
\hline HETATM & 93 & $\mathrm{H} 12$ & MET \\
\hline HETATM & 94 & H13 & MET \\
\hline \multicolumn{4}{|l|}{ TER } \\
\hline HETATM & 95 & C5 & $\mathrm{ACP}$ \\
\hline HETATM & 96 & H51 & $\mathrm{ACP}$ \\
\hline HETATM & 97 & H52 & $\mathrm{ACP}$ \\
\hline HETATM & 98 & C4 & $\mathrm{ACP}$ \\
\hline HETATM & 99 & $\mathrm{H} 41$ & $\mathrm{ACP}$ \\
\hline HETATM & 100 & $\mathrm{H} 42$ & $\mathrm{ACP}$ \\
\hline HETATM & 101 & $\mathrm{C} 1$ & $\mathrm{ACP}$ \\
\hline HETATM & 102 & $\mathrm{O} 2$ & $\mathrm{ACP}$ \\
\hline HETATM & 103 & 03 & $\mathrm{ACP}$ \\
\hline HETATM & 104 & H3 & $\mathrm{ACP}$ \\
\hline \multicolumn{4}{|l|}{ TER } \\
\hline HETATM & 105 & $\mathrm{C} 1$ & MET \\
\hline HETATM & 106 & H11 & MET \\
\hline HETATM & 107 & $\mathrm{H} 12$ & MET \\
\hline HETATM & 108 & $\mathrm{H} 13$ & MET \\
\hline \multicolumn{4}{|l|}{ TER } \\
\hline HETATM & 109 & $\mathrm{C} 1$ & MET \\
\hline HETATM & 110 & $\mathrm{H} 11$ & MET \\
\hline HETATM & 111 & $\mathrm{H} 12$ & MET \\
\hline HETATM & 112 & H13 & MET \\
\hline \multicolumn{4}{|l|}{ TER } \\
\hline HETATM & 113 & $\mathrm{C} 2$ & VIN \\
\hline HETATM & 114 & H 21 & VIN \\
\hline HETATM & 115 & $\mathrm{C} 1$ & VIN \\
\hline HETATM & 116 & $\mathrm{H} 11$ & VIN \\
\hline HETATM & 117 & $\mathrm{H} 12$ & VIN \\
\hline \multicolumn{4}{|l|}{ TER } \\
\hline HETATM & 118 & $\mathrm{C} 1$ & MET \\
\hline HETATM & 119 & $\mathrm{H} 11$ & MET \\
\hline HETATM & 120 & $\mathrm{H} 12$ & MET \\
\hline HETATM & 121 & H13 & MET \\
\hline \multicolumn{4}{|l|}{ TER } \\
\hline HETATM & 122 & $\mathrm{C} 2$ & VIN \\
\hline HETATM & 123 & $\mathrm{H} 21$ & VIN \\
\hline HETATM & 124 & $\mathrm{C} 1$ & VIN \\
\hline HETATM & 125 & H11 & VIN \\
\hline HETATM & 126 & $\mathrm{H} 12$ & VIN \\
\hline \multicolumn{4}{|l|}{ TER } \\
\hline HETATM & 127 & $\mathrm{C} 1$ & MET \\
\hline HETATM & 128 & H11 & MET \\
\hline HETATM & 129 & $\mathrm{H} 12$ & MET \\
\hline HETATM & 130 & $\mathrm{H} 13$ & MET \\
\hline TER & & & \\
\hline HETATM & 131 & C5 & $\mathrm{ACP}$ \\
\hline HETATM & 132 & H51 & $\mathrm{ACP}$ \\
\hline HETATM & 133 & H52 & $\mathrm{ACP}$ \\
\hline HETATM & 134 & C4 & $\mathrm{ACP}$ \\
\hline HETATM & 135 & $\mathrm{H} 41$ & $\mathrm{ACP}$ \\
\hline HETATM & 136 & $\mathrm{H} 42$ & $\mathrm{ACP}$ \\
\hline HETATM & 137 & $\mathrm{C} 1$ & $\mathrm{ACP}$ \\
\hline HETATM & 138 & $\mathrm{O} 2$ & $\mathrm{ACP}$ \\
\hline HETATM & 139 & 03 & $\mathrm{ACP}$ \\
\hline HETATM & 140 & H3 & $\mathrm{ACP}$ \\
\hline TER & & & \\
\hline HETATM & 141 & $\mathrm{C} 1$ & MET \\
\hline HETATM & 142 & $\mathrm{H} 11$ & MET \\
\hline HETATM & 143 & $\mathrm{H} 12$ & MET \\
\hline HETATM & 144 & H13 & MET \\
\hline TER & & & \\
\hline HETATM & 145 & $\mathrm{C} 1$ & MET \\
\hline HETATM & 146 & H11 & MET \\
\hline HETATM & 147 & $\mathrm{H} 12$ & MET \\
\hline HETATM & 148 & $\mathrm{H} 13$ & MET \\
\hline TER & & & \\
\hline HETATM & 149 & $\mathrm{FE}$ & HEM \\
\hline HETATM & 150 & $\mathrm{C} 1$ & HEM \\
\hline HETATM & 151 & $\mathrm{C} 2$ & HEM \\
\hline HETATM & 152 & $\mathrm{C} 3$ & HEM \\
\hline HETATM & 153 & $\mathrm{C} 4$ & HEM \\
\hline
\end{tabular}

\begin{tabular}{|c|c|c|c|c|c|}
\hline 7.352 & 12.399 & -3.184 & 1.00 & 0.00 & $\mathrm{H}$ \\
\hline 9.185 & 13.144 & -2.484 & 1.00 & 0.00 & C \\
\hline 9.865 & 13.300 & -1.654 & 1.00 & 0.00 & $\mathrm{H}$ \\
\hline 9.490 & 13.542 & -3.450 & 1.00 & 0.00 & $\mathrm{H}$ \\
\hline 9.703 & 10.816 & -0.121 & 1.00 & 0.00 & C \\
\hline 10.294 & 11.480 & 0.528 & 1.00 & 0.00 & $\mathrm{H}$ \\
\hline 9.859 & 9.794 & 0.246 & 1.00 & 0.00 & $\mathrm{H}$ \\
\hline 10.135 & 10.882 & -1.126 & 1.00 & 0.00 & $\mathrm{H}$ \\
\hline 0.615 & 9.735 & 5.770 & 1.00 & 0.00 & C \\
\hline 1.354 & 9.723 & 6.582 & 1.00 & 0.00 & $\mathrm{H}$ \\
\hline-0.226 & 10.343 & 6.134 & 1.00 & 0.00 & $\mathrm{H}$ \\
\hline 0.136 & 8.297 & 5.497 & 1.00 & 0.00 & C \\
\hline-0.422 & 8.236 & 4.549 & 1.00 & 0.00 & $\mathrm{H}$ \\
\hline 1.007 & 7.630 & 5.390 & 1.00 & 0.00 & $\mathrm{H}$ \\
\hline-0.728 & 7.685 & 6.573 & 1.00 & 0.00 & C \\
\hline-0.956 & 8.208 & 7.671 & 1.00 & 0.00 & O \\
\hline-1.244 & 6.505 & 6.209 & 1.00 & 0.00 & O \\
\hline-1.853 & 6.151 & 6.945 & 1.00 & 0.00 & $\mathrm{H}$ \\
\hline 8.511 & 8.608 & 4.597 & 1.00 & 0.00 & C \\
\hline 9.063 & 9.448 & 5.047 & 1.00 & 0.00 & $\mathrm{H}$ \\
\hline 8.517 & 7.782 & 5.319 & 1.00 & 0.00 & $\mathrm{H}$ \\
\hline 9.082 & 8.289 & 3.717 & 1.00 & 0.00 & $\mathrm{H}$ \\
\hline-0.923 & 11.557 & 3.646 & 1.00 & 0.00 & C \\
\hline-1.368 & 11.348 & 4.626 & 1.00 & 0.00 & $\mathrm{H}$ \\
\hline-1.033 & 12.637 & 3.471 & 1.00 & 0.00 & $\mathrm{H}$ \\
\hline-1.523 & 11.031 & 2.889 & 1.00 & 0.00 & $\mathrm{H}$ \\
\hline 8.874 & 0.178 & 8.933 & 1.00 & 0.00 & C \\
\hline 9.690 & 0.876 & 8.768 & 1.00 & 0.00 & $\mathrm{H}$ \\
\hline 9.207 & -1.047 & 9.335 & 1.00 & 0.00 & C \\
\hline 8.475 & -1.826 & 9.442 & 1.00 & 0.00 & $\mathrm{H}$ \\
\hline 10.249 & -1.316 & 9.501 & 1.00 & 0.00 & $\mathrm{H}$ \\
\hline 6.210 & -0.077 & 10.771 & 1.00 & 0.00 & C \\
\hline 5.975 & -1.148 & 10.652 & 1.00 & 0.00 & $\mathrm{H}$ \\
\hline 5.397 & 0.387 & 11.341 & 1.00 & 0.00 & $\mathrm{H}$ \\
\hline 7.118 & 0.001 & 11.382 & 1.00 & 0.00 & $\mathrm{H}$ \\
\hline 1.200 & 1.601 & 10.256 & 1.00 & 0.00 & C \\
\hline 1.866 & 1.702 & 11.115 & 1.00 & 0.00 & $\mathrm{H}$ \\
\hline 0.033 & 0.957 & 10.415 & 1.00 & 0.00 & C \\
\hline-0.647 & 0.800 & 9.585 & 1.00 & 0.00 & $\mathrm{H}$ \\
\hline-0.272 & 0.559 & 11.381 & 1.00 & 0.00 & $\mathrm{H}$ \\
\hline-0.484 & 3.284 & 8.052 & 1.00 & 0.00 & C \\
\hline-0.641 & 4.306 & 7.685 & 1.00 & 0.00 & $\mathrm{H}$ \\
\hline-0.917 & 3.219 & 9.057 & 1.00 & 0.00 & $\mathrm{H}$ \\
\hline-1.076 & 2.620 & 7.403 & 1.00 & 0.00 & $\mathrm{H}$ \\
\hline 8.604 & 4.366 & 2.161 & 1.00 & 0.00 & C \\
\hline 7.865 & 4.377 & 1.349 & 1.00 & 0.00 & $\mathrm{H}$ \\
\hline 9.445 & 3.758 & 1.798 & 1.00 & 0.00 & $\mathrm{H}$ \\
\hline 9.082 & 5.803 & 2.434 & 1.00 & 0.00 & C \\
\hline 9.640 & 5.865 & 3.382 & 1.00 & 0.00 & $\mathrm{H}$ \\
\hline 8.212 & 6.470 & 2.541 & 1.00 & 0.00 & $\mathrm{H}$ \\
\hline 9.946 & 6.416 & 1.358 & 1.00 & 0.00 & C \\
\hline 10.174 & 5.893 & 0.260 & 1.00 & 0.00 & 0 \\
\hline 10.462 & 7.595 & 1.722 & 1.00 & 0.00 & O \\
\hline 11.071 & 7.949 & 0.986 & 1.00 & 0.00 & $\mathrm{H}$ \\
\hline 0.708 & 5.492 & 3.334 & 1.00 & 0.00 & C \\
\hline 0.155 & 4.653 & 2.884 & 1.00 & 0.00 & $\mathrm{H}$ \\
\hline 0.701 & 6.318 & 2.613 & 1.00 & 0.00 & $\mathrm{H}$ \\
\hline 0.137 & 5.812 & 4.214 & 1.00 & 0.00 & $\mathrm{H}$ \\
\hline 10.141 & 2.543 & 4.285 & 1.00 & 0.00 & $\mathrm{C}$ \\
\hline 10.586 & 2.752 & 3.305 & 1.00 & 0.00 & $\mathrm{H}$ \\
\hline 10.252 & 1.463 & 4.460 & 1.00 & 0.00 & $\mathrm{H}$ \\
\hline 10.742 & 3.069 & 5.042 & 1.00 & 0.00 & $\mathrm{H}$ \\
\hline 3.316 & 10.511 & 9.423 & 1.00 & 0.00 & $\mathrm{FE}$ \\
\hline 1.088 & 12.383 & 8.361 & 1.00 & 0.00 & C \\
\hline 0.729 & 13.174 & 7.189 & 1.00 & 0.00 & C \\
\hline 1.874 & 13.271 & 6.414 & 1.00 & 0.00 & C \\
\hline 2.904 & 12.553 & 7.122 & 1.00 & 0.00 & C \\
\hline
\end{tabular}




\begin{tabular}{|c|c|c|c|c|c|c|c|c|c|c|}
\hline HETATM & 154 & C5 & HEM & 17 & 4.219 & 12.435 & 6.684 & 1.00 & 0.00 & $\mathrm{C}$ \\
\hline HETATM & 155 & H5 & HEM & 17 & 4.472 & 12.931 & 5.749 & 1.00 & 0.00 & $\mathrm{H}$ \\
\hline HETATM & 156 & $\mathrm{C} 6$ & HEM & 17 & 5.238 & 11.736 & 7.320 & 1.00 & 0.00 & C \\
\hline HETATM & 157 & C7 & HEM & 17 & 6.607 & 11.664 & 6.851 & 1.00 & 0.00 & C \\
\hline HETATM & 158 & $\mathrm{C} 8$ & HEM & 17 & 7.313 & 10.944 & 7.798 & 1.00 & 0.00 & C \\
\hline HETATM & 159 & $\mathrm{C} 9$ & HEM & 17 & 6.362 & 10.579 & 8.822 & 1.00 & 0.00 & C \\
\hline HETATM & 160 & $\mathrm{C} 10$ & HEM & 17 & 6.666 & 9.844 & 9.965 & 1.00 & 0.00 & C \\
\hline HETATM & 161 & $\mathrm{H} 10$ & HEM & 17 & 7.693 & 9.500 & 10.071 & 1.00 & 0.00 & $\mathrm{H}$ \\
\hline HETATM & 162 & C11 & HEM & 17 & 5.792 & 9.504 & 10.988 & 1.00 & 0.00 & $\mathrm{C}$ \\
\hline HETATM & 163 & $\mathrm{C} 12$ & HEM & 17 & 6.173 & 8.779 & 12.178 & 1.00 & 0.00 & $\mathrm{C}$ \\
\hline HETATM & 164 & $\mathrm{C} 13$ & HEM & 17 & 5.033 & 8.631 & 12.935 & 1.00 & 0.00 & $\mathrm{C}$ \\
\hline HETATM & 165 & C14 & HEM & 17 & 3.960 & 9.273 & 12.204 & 1.00 & 0.00 & $\mathrm{C}$ \\
\hline HETATM & 166 & $\mathrm{C} 15$ & HEM & 17 & 2.632 & 9.328 & 12.625 & 1.00 & 0.00 & C \\
\hline HETATM & 167 & H15 & HEM & 17 & 2.392 & 8.843 & 13.573 & 1.00 & 0.00 & $\mathrm{H}$ \\
\hline HETATM & 168 & $\mathrm{C} 16$ & HEM & 17 & 1.602 & 10.009 & 11.982 & 1.00 & 0.00 & $\mathrm{C}$ \\
\hline HETATM & 169 & C17 & HEM & 17 & 0.236 & 10.123 & 12.464 & 1.00 & 0.00 & C \\
\hline HETATM & 170 & $\mathrm{C} 18$ & HEM & 17 & -0.439 & 10.890 & 11.541 & 1.00 & 0.00 & C \\
\hline HETATM & 171 & C19 & HEM & 17 & 0.510 & 11.242 & 10.504 & 1.00 & 0.00 & C \\
\hline HETATM & 172 & $\mathrm{C} 20$ & HEM & 17 & 0.219 & 12.033 & 9.393 & 1.00 & 0.00 & C \\
\hline HETATM & 173 & $\mathrm{H} 20$ & HEM & 17 & -0.795 & 12.423 & 9.333 & 1.00 & 0.00 & $\mathrm{H}$ \\
\hline HETATM & 174 & $\mathrm{~N} 21$ & HEM & 17 & 2.409 & 12.020 & 8.298 & 1.00 & 0.00 & $\mathrm{~N}$ \\
\hline HETATM & 175 & N22 & HEM & 17 & 5.114 & 11.073 & 8.521 & 1.00 & 0.00 & $\mathrm{~N}$ \\
\hline HETATM & 176 & $\mathrm{~N} 23$ & HEM & 17 & 4.443 & 9.810 & 11.021 & 1.00 & 0.00 & $\mathrm{~N}$ \\
\hline HETATM & 177 & $\mathrm{~N} 24$ & HEM & 17 & 1.743 & 10.694 & 10.786 & 1.00 & 0.00 & $\mathrm{~N}$ \\
\hline HETATM & 178 & C99 & HEM & 17 & 2.650 & 7.829 & 8.283 & 1.00 & 0.00 & C \\
\hline HETATM & 179 & 091 & HEM & 17 & 2.810 & 9.143 & 8.296 & 1.00 & 0.00 & 0 \\
\hline HETATM & 180 & 092 & HEM & 17 & 2.495 & 7.212 & 7.228 & 1.00 & 0.00 & 0 \\
\hline HETATM & 181 & СТ 91 & HEM & 17 & 2.666 & 7.148 & 9.640 & 1.00 & 0.00 & $\mathrm{C}$ \\
\hline HETATM & 182 & H911 & HEM & 17 & 3.645 & 7.343 & 10.103 & 1.00 & 0.00 & $\mathrm{H}$ \\
\hline HETATM & 183 & H912 & HEM & 17 & 1.918 & 7.638 & 10.283 & 1.00 & 0.00 & $\mathrm{H}$ \\
\hline HETATM & 184 & СТ92 & HEM & 17 & 2.407 & 5.644 & 9.528 & 1.00 & 0.00 & C \\
\hline HETATM & 185 & H921 & HEM & 17 & 3.203 & 5.183 & 8.931 & 1.00 & 0.00 & $\mathrm{H}$ \\
\hline HETATM & 186 & H922 & HEM & 17 & 1.482 & 5.488 & 8.958 & 1.00 & 0.00 & $\mathrm{H}$ \\
\hline \multicolumn{11}{|l|}{ TER } \\
\hline HETATM & 187 & $\mathrm{FE}$ & HEM & 18 & 4.008 & 3.097 & 14.371 & 1.00 & 0.00 & $\mathrm{FE}$ \\
\hline HETATM & 188 & $\mathrm{C} 1$ & HEM & 18 & 6.236 & 1.225 & 15.433 & 1.00 & 0.00 & C \\
\hline HETATM & 189 & $\mathrm{C} 2$ & HEM & 18 & 6.595 & 0.434 & 16.605 & 1.00 & 0.00 & C \\
\hline HETATM & 190 & $\mathrm{C} 3$ & HEM & 18 & 5.449 & 0.337 & 17.380 & 1.00 & 0.00 & C \\
\hline HETATM & 191 & $\mathrm{C} 4$ & HEM & 18 & 4.419 & 1.055 & 16.672 & 1.00 & 0.00 & C \\
\hline HETATM & 192 & C5 & HEM & 18 & 3.104 & 1.173 & 17.109 & 1.00 & 0.00 & C \\
\hline HETATM & 193 & H5 & HEM & 18 & 2.851 & 0.677 & 18.045 & 1.00 & 0.00 & $\mathrm{H}$ \\
\hline HETATM & 194 & $\mathrm{C} 6$ & HEM & 18 & 2.085 & 1.872 & 16.473 & 1.00 & 0.00 & C \\
\hline HETATM & 195 & $\mathrm{C} 7$ & HEM & 18 & 0.716 & 1.944 & 16.943 & 1.00 & 0.00 & C \\
\hline HETATM & 196 & $\mathrm{C} 8$ & HEM & 18 & 0.011 & 2.664 & 15.996 & 1.00 & 0.00 & C \\
\hline HETATM & 197 & $\mathrm{C} 9$ & HEM & 18 & 0.962 & 3.029 & 14.972 & 1.00 & 0.00 & C \\
\hline HETATM & 198 & $\mathrm{C} 10$ & HEM & 18 & 0.657 & 3.764 & 13.828 & 1.00 & 0.00 & C \\
\hline HETATM & 199 & $\mathrm{H} 10$ & HEM & 18 & -0.370 & 4.108 & 13.722 & 1.00 & 0.00 & $\mathrm{H}$ \\
\hline HETATM & 200 & C11 & HEM & 18 & 1.532 & 4.104 & 12.806 & 1.00 & 0.00 & $\mathrm{C}$ \\
\hline HETATM & 201 & $\mathrm{C} 12$ & HEM & 18 & 1.151 & 4.829 & 11.615 & 1.00 & 0.00 & $\mathrm{C}$ \\
\hline HETATM & 202 & $\mathrm{C} 13$ & HEM & 18 & 2.290 & 4.977 & 10.858 & 1.00 & 0.00 & C \\
\hline HETATM & 203 & C14 & HEM & 18 & 3.363 & 4.335 & 11.590 & 1.00 & 0.00 & C \\
\hline HETATM & 204 & $\mathrm{C} 15$ & HEM & 18 & 4.691 & 4.280 & 11.168 & 1.00 & 0.00 & C \\
\hline HETATM & 205 & $\mathrm{H} 15$ & HEM & 18 & 4.931 & 4.765 & 10.221 & 1.00 & 0.00 & $\mathrm{H}$ \\
\hline HETATM & 206 & $\mathrm{C} 16$ & HEM & 18 & 5.721 & 3.599 & 11.812 & 1.00 & 0.00 & $\mathrm{C}$ \\
\hline HETATM & 207 & C17 & HEM & 18 & 7.087 & 3.485 & 11.329 & 1.00 & 0.00 & C \\
\hline HETATM & 208 & $\mathrm{C} 18$ & HEM & 18 & 7.763 & 2.718 & 12.253 & 1.00 & 0.00 & C \\
\hline HETATM & 209 & C19 & HEM & 18 & 6.813 & 2.366 & 13.289 & 1.00 & 0.00 & C \\
\hline HETATM & 210 & $\mathrm{C} 20$ & HEM & 18 & 7.104 & 1.575 & 14.401 & 1.00 & 0.00 & C \\
\hline HETATM & 211 & $\mathrm{H} 20$ & HEM & 18 & 8.118 & 1.185 & 14.461 & 1.00 & 0.00 & $\mathrm{H}$ \\
\hline HETATM & 212 & N21 & HEM & 18 & 4.914 & 1.588 & 15.495 & 1.00 & 0.00 & $\mathrm{~N}$ \\
\hline HETATM & 213 & $\mathrm{~N} 22$ & HEM & 18 & 2.209 & 2.535 & 15.273 & 1.00 & 0.00 & $\mathrm{~N}$ \\
\hline HETATM & 214 & $\mathrm{~N} 23$ & HEM & 18 & 2.880 & 3.798 & 12.772 & 1.00 & 0.00 & $\mathrm{~N}$ \\
\hline HETATM & 215 & $\mathrm{~N} 24$ & HEM & 18 & 5.580 & 2.914 & 13.008 & 1.00 & 0.00 & $\mathrm{~N}$ \\
\hline HETATM & 216 & C99 & HEM & 18 & 4.673 & 5.779 & 15.510 & 1.00 & 0.00 & C \\
\hline HETATM & 217 & 091 & HEM & 18 & 4.514 & 4.465 & 15.498 & 1.00 & 0.00 & 0 \\
\hline HETATM & 218 & 092 & HEM & 18 & 4.828 & 6.396 & 16.566 & 1.00 & 0.00 & 0 \\
\hline HETATM & 219 & СТ 91 & HEM & 18 & 4.657 & 6.460 & 14.154 & 1.00 & 0.00 & C \\
\hline HETATM & 220 & H911 & HEM & 18 & 3.678 & 6.265 & 13.691 & 1.00 & 0.00 & $\mathrm{H}$ \\
\hline HETATM & 221 & H912 & HEM & 18 & 5.405 & 5.970 & 13.511 & 1.00 & 0.00 & $\mathrm{H}$ \\
\hline HETATM & 222 & СТ92 & HEM & 18 & 4.917 & 7.964 & 14.265 & 1.00 & 0.00 & C \\
\hline HETATM & 223 & H921 & HEM & 18 & 5.842 & 8.120 & 14.836 & 1.00 & 0.00 & $\mathrm{H}$ \\
\hline HETATM & 224 & H922 & HEM & 18 & 4.120 & 8.425 & 14.862 & 1.00 & 0.00 & $\mathrm{H}$ \\
\hline \multicolumn{11}{|l|}{ TER } \\
\hline HETATM & 225 & $\mathrm{C} 2$ & VIN & 19 & -0.603 & 13.676 & 6.929 & 1.00 & 0.00 & C \\
\hline HETATM & 226 & H2 1 & VIN & 19 & -1.419 & 12.978 & 7.094 & 1.00 & 0.00 & $\mathrm{H}$ \\
\hline HETATM & 227 & $\mathrm{C} 1$ & VIN & 19 & -0.936 & 14.901 & 6.528 & 1.00 & 0.00 & C \\
\hline HETATM & 228 & H11 & VIN & 19 & -0.205 & 15.680 & 6.421 & 1.00 & 0.00 & $\mathrm{H}$ \\
\hline HETATM & 229 & $\mathrm{H} 12$ & VIN & 19 & -1.978 & 15.170 & 6.361 & 1.00 & 0.00 & $\mathrm{H}$ \\
\hline
\end{tabular}




\begin{tabular}{|c|c|c|c|c|c|c|c|c|c|c|}
\hline HETATM & 230 & $\mathrm{C} 1$ & MET & 20 & 2.061 & 13.931 & 5.091 & 1.00 & 0.00 & C \\
\hline HETATM & 231 & $\mathrm{H} 11$ & MET & 20 & 2.295 & 15.002 & 5.211 & 1.00 & 0.00 & $\mathrm{H}$ \\
\hline HETATM & 232 & $\mathrm{H} 12$ & MET & 20 & 2.874 & 13.467 & 4.522 & 1.00 & 0.00 & $\mathrm{H}$ \\
\hline HETATM & 233 & $\mathrm{H} 13$ & MET & 20 & 1.153 & 13.854 & 4.481 & 1.00 & 0.00 & $\mathrm{H}$ \\
\hline \multicolumn{11}{|l|}{ TER } \\
\hline HETATM & 234 & $\mathrm{C} 2$ & VIN & 21 & 7.071 & 12.253 & 5.606 & 1.00 & 0.00 & C \\
\hline HETATM & 235 & H 21 & VIN & 21 & 6.405 & 12.152 & 4.747 & 1.00 & 0.00 & $\mathrm{H}$ \\
\hline HETATM & 236 & $\mathrm{C} 1$ & VIN & 21 & 8.238 & 12.898 & 5.447 & 1.00 & 0.00 & C \\
\hline HETATM & 237 & $\mathrm{H} 11$ & VIN & 21 & 8.917 & 13.054 & 6.277 & 1.00 & 0.00 & $\mathrm{H}$ \\
\hline HETATM & 238 & $\mathrm{H} 12$ & VIN & 21 & 8.543 & 13.296 & 4.482 & 1.00 & 0.00 & $\mathrm{H}$ \\
\hline \multicolumn{11}{|l|}{ TER } \\
\hline HETATM & 239 & $\mathrm{C} 1$ & MET & 22 & 8.755 & 10.570 & 7.810 & 1.00 & 0.00 & C \\
\hline HETATM & 240 & $\mathrm{H} 11$ & MET & 22 & 9.347 & 11.234 & 8.459 & 1.00 & 0.00 & $\mathrm{H}$ \\
\hline HETATM & 241 & $\mathrm{H} 12$ & MET & 22 & 8.912 & 9.548 & 8.177 & 1.00 & 0.00 & $\mathrm{H}$ \\
\hline HETATM & 242 & $\mathrm{H} 13$ & MET & 22 & 9.188 & 10.636 & 6.805 & 1.00 & 0.00 & $\mathrm{H}$ \\
\hline \multicolumn{11}{|c|}{ TER } \\
\hline HETATM & 243 & C5 & $\mathrm{ACP}$ & 23 & -0.333 & 9.488 & 13.701 & 1.00 & 0.00 & C \\
\hline HETATM & 244 & H51 & $\mathrm{ACP}$ & 23 & 0.406 & 9.477 & 14.514 & 1.00 & 0.00 & $\mathrm{H}$ \\
\hline HETATM & 245 & H52 & $\mathrm{ACP}$ & 23 & -1.174 & 10.097 & 14.065 & 1.00 & 0.00 & $\mathrm{H}$ \\
\hline HETATM & 246 & $\mathrm{C} 4$ & $\mathrm{ACP}$ & 23 & -0.812 & 8.051 & 13.428 & 1.00 & 0.00 & C \\
\hline HETATM & 247 & H4 1 & $\mathrm{ACP}$ & 23 & -1.369 & 7.990 & 12.481 & 1.00 & 0.00 & $\mathrm{H}$ \\
\hline HETATM & 248 & $\mathrm{H} 42$ & $\mathrm{ACP}$ & 23 & 0.059 & 7.384 & 13.321 & 1.00 & 0.00 & $\mathrm{H}$ \\
\hline HETATM & 249 & $\mathrm{C} 1$ & $\mathrm{ACP}$ & 23 & -1.676 & 7.439 & 14.504 & 1.00 & 0.00 & C \\
\hline HETATM & 250 & $\mathrm{O} 2$ & $\mathrm{ACP}$ & 23 & -1.903 & 7.961 & 15.603 & 1.00 & 0.00 & 0 \\
\hline HETATM & 251 & 03 & $\mathrm{ACP}$ & 23 & -2.191 & 6.259 & 14.140 & 1.00 & 0.00 & 0 \\
\hline HETATM & 252 & H3 & $\mathrm{ACP}$ & 23 & -2.800 & 5.905 & 14.876 & 1.00 & 0.00 & $\mathrm{H}$ \\
\hline \multicolumn{11}{|c|}{ TER } \\
\hline HETATM & 253 & $\mathrm{C} 1$ & MET & 24 & 7.563 & 8.362 & 12.528 & 1.00 & 0.00 & $\mathrm{C}$ \\
\hline HETATM & 254 & $\mathrm{H} 11$ & MET & 24 & 8.115 & 9.201 & 12.978 & 1.00 & 0.00 & $\mathrm{H}$ \\
\hline HETATM & 255 & $\mathrm{H} 12$ & MET & 24 & 7.570 & 7.536 & 13.250 & 1.00 & 0.00 & $\mathrm{H}$ \\
\hline HETATM & 256 & $\mathrm{H} 13$ & MET & 24 & 8.134 & 8.042 & 11.649 & 1.00 & 0.00 & $\mathrm{H}$ \\
\hline TER & & & & & & & & & & \\
\hline HETATM & 257 & $\mathrm{C} 1$ & MET & 25 & -1.870 & 11.311 & 11.577 & 1.00 & 0.00 & C \\
\hline HETATM & 258 & $\mathrm{H} 11$ & MET & 25 & -2.316 & 11.102 & 12.557 & 1.00 & 0.00 & $\mathrm{H}$ \\
\hline HETATM & 259 & $\mathrm{H} 12$ & MET & 25 & -1.981 & 12.391 & 11.402 & 1.00 & 0.00 & $\mathrm{H}$ \\
\hline HETATM & 260 & $\mathrm{H} 13$ & MET & 25 & -2.471 & 10.785 & 10.821 & 1.00 & 0.00 & $\mathrm{H}$ \\
\hline TER & & & & & & & & & & \\
\hline HETATM & 261 & $\mathrm{C} 2$ & VIN & 26 & 7.926 & -0.068 & 16.864 & 1.00 & 0.00 & C \\
\hline HETATM & 262 & H 21 & VIN & 26 & 8.742 & 0.630 & 16.699 & 1.00 & 0.00 & $\mathrm{H}$ \\
\hline HETATM & 263 & $\mathrm{C} 1$ & VIN & 26 & 8.259 & -1.293 & 17.266 & 1.00 & 0.00 & C \\
\hline HETATM & 264 & H11 & VIN & 26 & 7.528 & -2.072 & 17.373 & 1.00 & 0.00 & $\mathrm{H}$ \\
\hline HETATM & 265 & $\mathrm{H} 12$ & VIN & 26 & 9.301 & -1.562 & 17.432 & 1.00 & 0.00 & $\mathrm{H}$ \\
\hline TER & & & & & & & & & & \\
\hline HETATM & 266 & $\mathrm{C} 1$ & MET & 27 & 5.263 & -0.323 & 18.702 & 1.00 & 0.00 & C \\
\hline HETATM & 267 & $\mathrm{H} 11$ & MET & 27 & 5.028 & -1.394 & 18.583 & 1.00 & 0.00 & $\mathrm{H}$ \\
\hline HETATM & 268 & $\mathrm{H} 12$ & MET & 27 & 4.450 & 0.141 & 19.272 & 1.00 & 0.00 & $\mathrm{H}$ \\
\hline HETATM & 269 & $\mathrm{H} 13$ & MET & 27 & 6.170 & -0.246 & 19.313 & 1.00 & 0.00 & $\mathrm{H}$ \\
\hline TER & & & & & & & & & & \\
\hline HETATM & 270 & $\mathrm{C} 2$ & VIN & 28 & 0.252 & 1.355 & 18.188 & 1.00 & 0.00 & $\mathrm{C}$ \\
\hline HETATM & 271 & H 21 & VIN & 28 & 0.918 & 1.456 & 19.046 & 1.00 & 0.00 & $\mathrm{H}$ \\
\hline HETATM & 272 & $\mathrm{C} 1$ & VIN & 28 & -0.914 & 0.710 & 18.346 & 1.00 & 0.00 & C \\
\hline HETATM & 273 & $\mathrm{H} 11$ & VIN & 28 & -1.594 & 0.554 & 17.516 & 1.00 & 0.00 & $\mathrm{H}$ \\
\hline HETATM & 274 & $\mathrm{H} 12$ & VIN & 28 & -1.219 & 0.312 & 19.312 & 1.00 & 0.00 & $\mathrm{H}$ \\
\hline TER & & & & & & & & & & \\
\hline HETATM & 275 & $\mathrm{C} 1$ & MET & 29 & -1.432 & 3.038 & 15.984 & 1.00 & 0.00 & C \\
\hline HETATM & 276 & H11 & MET & 29 & -1.588 & 4.060 & 15.617 & 1.00 & 0.00 & $\mathrm{H}$ \\
\hline HETATM & 277 & $\mathrm{H} 12$ & MET & 29 & -1.864 & 2.972 & 16.989 & 1.00 & 0.00 & $\mathrm{H}$ \\
\hline HETATM & 278 & $\mathrm{H} 13$ & MET & 29 & -2.023 & 2.374 & 15.335 & 1.00 & 0.00 & $\mathrm{H}$ \\
\hline TER & & & & & & & & & & \\
\hline HETATM & 279 & C5 & $\mathrm{ACP}$ & 30 & 7.656 & 4.120 & 10.092 & 1.00 & 0.00 & C \\
\hline HETATM & 280 & H51 & $\mathrm{ACP}$ & 30 & 6.917 & 4.131 & 9.280 & 1.00 & 0.00 & $\mathrm{H}$ \\
\hline HETATM & 281 & H52 & $\mathrm{ACP}$ & 30 & 8.497 & 3.511 & 9.729 & 1.00 & 0.00 & $\mathrm{H}$ \\
\hline HETATM & 282 & $\mathrm{C} 4$ & $\mathrm{ACP}$ & 30 & 8.135 & 5.557 & 10.365 & 1.00 & 0.00 & C \\
\hline HETATM & 283 & H 41 & $\mathrm{ACP}$ & 30 & 8.693 & 5.618 & 11.313 & 1.00 & 0.00 & $\mathrm{H}$ \\
\hline HETATM & 284 & $\mathrm{H} 42$ & $\mathrm{ACP}$ & 30 & 7.264 & 6.224 & 10.472 & 1.00 & 0.00 & $\mathrm{H}$ \\
\hline HETATM & 285 & $\mathrm{C} 1$ & $\mathrm{ACP}$ & 30 & 8.999 & 6.169 & 9.289 & 1.00 & 0.00 & C \\
\hline HETATM & 286 & $\mathrm{O} 2$ & $\mathrm{ACP}$ & 30 & 9.227 & 5.647 & 8.191 & 1.00 & 0.00 & 0 \\
\hline HETATM & 287 & 03 & $\mathrm{ACP}$ & 30 & 9.515 & 7.349 & 9.653 & 1.00 & 0.00 & 0 \\
\hline HETATM & 288 & H3 & $\mathrm{ACP}$ & 30 & 10.124 & 7.703 & 8.917 & 1.00 & 0.00 & $\mathrm{H}$ \\
\hline TER & & & & & & & & & & \\
\hline HETATM & 289 & $\mathrm{C} 1$ & MET & 31 & -0.240 & 5.246 & 11.266 & 1.00 & 0.00 & $\mathrm{C}$ \\
\hline HETATM & 290 & $\mathrm{H} 11$ & MET & 31 & -0.792 & 4.407 & 10.815 & 1.00 & 0.00 & $\mathrm{H}$ \\
\hline HETATM & 291 & $\mathrm{H} 12$ & MET & 31 & -0.246 & 6.072 & 10.544 & 1.00 & 0.00 & $\mathrm{H}$ \\
\hline HETATM & 292 & $\mathrm{H} 13$ & MET & 31 & -0.811 & 5.565 & 12.145 & 1.00 & 0.00 & $\mathrm{H}$ \\
\hline TER & & & & & & & & & & \\
\hline HETATM & 293 & $\mathrm{C} 1$ & MET & 32 & 9.194 & 2.297 & 12.216 & 1.00 & 0.00 & $\mathrm{C}$ \\
\hline HETATM & 294 & $\mathrm{H} 11$ & MET & 32 & 9.639 & 2.506 & 11.236 & 1.00 & 0.00 & $\mathrm{H}$ \\
\hline HETATM & 295 & $\mathrm{H} 12$ & MET & 32 & 9.304 & 1.217 & 12.391 & 1.00 & 0.00 & $\mathrm{H}$ \\
\hline HETATM & 296 & H13 & MET & 32 & 9.794 & 2.823 & 12.973 & 1.00 & 0.00 & $\mathrm{H}$ \\
\hline
\end{tabular}




\subsection{D2 dimer: A01_D2.pdb file}

\begin{tabular}{|c|c|c|c|c|c|c|c|c|c|c|}
\hline ATOM & 1 & $\mathrm{FE}$ & HEM & 1 & 4.263 & 10.757 & 1.491 & 1.00 & 0.00 & $\mathrm{FE}$ \\
\hline ATOM & 2 & $\mathrm{C} 1$ & HEM & 1 & 2.035 & 12.629 & 0.429 & 1.00 & 0.00 & C \\
\hline ATOM & 3 & $\mathrm{C} 2$ & HEM & 1 & 1.676 & 13.420 & -0.742 & 1.00 & 0.00 & C \\
\hline ATOM & 4 & C3 & HEM & 1 & 2.822 & 13.517 & -1.517 & 1.00 & 0.00 & C \\
\hline ATOM & 5 & C4 & HEM & 1 & 3.851 & 12.800 & -0.809 & 1.00 & 0.00 & C \\
\hline ATOM & 6 & C5 & HEM & 1 & 5.167 & 12.681 & -1.247 & 1.00 & 0.00 & C \\
\hline ATOM & 7 & H5 & HEM & 1 & 5.420 & 13.177 & -2.182 & 1.00 & 0.00 & $\mathrm{H}$ \\
\hline ATOM & 8 & C6 & HEM & 1 & 6.186 & 11.982 & -0.611 & 1.00 & 0.00 & C \\
\hline ATOM & 9 & $\mathrm{C} 7$ & HEM & 1 & 7.555 & 11.910 & -1.081 & 1.00 & 0.00 & C \\
\hline ATOM & 10 & $\mathrm{C} 8$ & HEM & 1 & 8.260 & 11.190 & -0.134 & 1.00 & 0.00 & C \\
\hline ATOM & 11 & C9 & HEM & 1 & 7.309 & 10.825 & 0.891 & 1.00 & 0.00 & C \\
\hline ATOM & 12 & $\mathrm{C} 10$ & HEM & 1 & 7.614 & 10.090 & 2.034 & 1.00 & 0.00 & C \\
\hline AтOM & 13 & H1O & HEM & 1 & 8.641 & 9.746 & 2.140 & 1.00 & 0.00 & $\mathrm{H}$ \\
\hline ATOM & 14 & $\mathrm{C} 11$ & HEM & 1 & 6.739 & 9.750 & 3.057 & 1.00 & 0.00 & C \\
\hline ATOM & 15 & $\mathrm{C} 12$ & HEM & 1 & 7.120 & 9.025 & 4.247 & 1.00 & 0.00 & C \\
\hline ATOM & 16 & C13 & HEM & 1 & 5.981 & 8.878 & 5.004 & 1.00 & 0.00 & C \\
\hline ATOM & 17 & C14 & HEM & 1 & 4.908 & 9.519 & 4.273 & 1.00 & 0.00 & C \\
\hline ATOM & 18 & C15 & HEM & 1 & 3.580 & 9.574 & 4.694 & 1.00 & 0.00 & C \\
\hline ATOM & 19 & H1 5 & HEM & 1 & 3.340 & 9.089 & 5.642 & 1.00 & 0.00 & $\mathrm{H}$ \\
\hline ATOM & 20 & $\mathrm{C} 16$ & HEM & 1 & 2.550 & 10.255 & 4.051 & 1.00 & 0.00 & C \\
\hline ATOM & 21 & $\mathrm{C} 17$ & HEM & 1 & 1.184 & 10.369 & 4.533 & 1.00 & 0.00 & C \\
\hline ATOM & 22 & $\mathrm{C} 18$ & HEM & 1 & 0.508 & 11.137 & 3.609 & 1.00 & 0.00 & C \\
\hline ATOM & 23 & C19 & HEM & 1 & 1.458 & 11.489 & 2.573 & 1.00 & 0.00 & C \\
\hline ATOM & 24 & $\mathrm{C} 20$ & HEM & 1 & 1.167 & 12.280 & 1.462 & 1.00 & 0.00 & C \\
\hline ATOM & 25 & $\mathrm{H} 2 \mathrm{O}$ & HEM & 1 & 0.152 & 12.669 & 1.402 & 1.00 & 0.00 & $\mathrm{H}$ \\
\hline ATOM & 26 & N21 & HEM & 1 & 3.357 & 12.266 & 0.367 & 1.00 & 0.00 & $\mathrm{~N}$ \\
\hline ATOM & 27 & N22 & HEM & 1 & 6.062 & 11.319 & 0.590 & 1.00 & 0.00 & $\mathrm{~N}$ \\
\hline ATOM & 28 & N23 & HEM & 1 & 5.391 & 10.056 & 3.090 & 1.00 & 0.00 & $\mathrm{~N}$ \\
\hline ATOM & 29 & N24 & HEM & 1 & 2.691 & 10.941 & 2.854 & 1.00 & 0.00 & $\mathrm{~N}$ \\
\hline ATOM & 30 & C99 & HEM & 1 & 3.598 & 8.076 & 0.352 & 1.00 & 0.00 & C \\
\hline ATOM & 31 & 091 & HEM & 1 & 3.757 & 9.389 & 0.365 & 1.00 & 0.00 & 0 \\
\hline ATOM & 32 & 092 & HEM & 1 & 3.443 & 7.458 & -0.703 & 1.00 & 0.00 & o \\
\hline ATOM & 33 & СТ91 & HEM & 1 & 3.614 & 7.394 & 1.709 & 1.00 & 0.00 & C \\
\hline ATOM & 34 & H911 & HEM & 1 & 4.593 & 7.590 & 2.171 & 1.00 & 0.00 & $\mathrm{H}$ \\
\hline ATOM & 35 & H912 & HEM & 1 & 2.866 & 7.884 & 2.352 & 1.00 & 0.00 & $\mathrm{H}$ \\
\hline ATOM & 36 & CT92 & HEM & 1 & 3.354 & 5.890 & 1.597 & 1.00 & 0.00 & C \\
\hline ATOM & 37 & H921 & HEM & 1 & 4.151 & 5.429 & 1.000 & 1.00 & 0.00 & $\mathrm{H}$ \\
\hline ATOM & 38 & H922 & HEM & 1 & 2.429 & 5.734 & 1.027 & 1.00 & 0.00 & $\mathrm{H}$ \\
\hline \multicolumn{11}{|l|}{ TER } \\
\hline ATOM & 39 & $\mathrm{FE}$ & HEM & 2 & 4.955 & 3.343 & 6.440 & 1.00 & 0.00 & $\mathrm{FE}$ \\
\hline ATOM & 40 & $\mathrm{C} 1$ & HEM & 2 & 7.183 & 1.471 & 7.502 & 1.00 & 0.00 & C \\
\hline ATOM & 41 & $\mathrm{C} 2$ & HEM & 2 & 7.542 & 0.680 & 8.674 & 1.00 & 0.00 & C \\
\hline ATOM & 42 & $\mathrm{C} 3$ & HEM & 2 & 6.397 & 0.583 & 9.448 & 1.00 & 0.00 & $\mathrm{C}$ \\
\hline ATOM & 43 & $\mathrm{C} 4$ & HEM & 2 & 5.367 & 1.301 & 8.740 & 1.00 & 0.00 & C \\
\hline ATOM & 44 & C5 & HEM & 2 & 4.052 & 1.420 & 9.178 & 1.00 & 0.00 & C \\
\hline ATOM & 45 & H5 & HEM & 2 & 3.798 & 0.923 & 10.113 & 1.00 & 0.00 & $\mathrm{H}$ \\
\hline ATOM & 46 & C 6 & HEM & 2 & 3.033 & 2.118 & 8.542 & 1.00 & 0.00 & C \\
\hline ATOM & 47 & $\mathrm{C} 7$ & HEM & 2 & 1.664 & 2.191 & 9.012 & 1.00 & 0.00 & C \\
\hline ATOM & 48 & $\mathrm{C} 8$ & HEM & 2 & 0.958 & 2.910 & 8.065 & 1.00 & 0.00 & C \\
\hline ATOM & 49 & C9 & HEM & 2 & 1.909 & 3.275 & 7.041 & 1.00 & 0.00 & C \\
\hline ATOM & 50 & $\mathrm{C} 10$ & HEM & 2 & 1.605 & 4.010 & 5.897 & 1.00 & 0.00 & C \\
\hline ATOM & 51 & H1O & HEM & 2 & 0.578 & 4.354 & 5.791 & 1.00 & 0.00 & $\mathrm{H}$ \\
\hline ATOM & 52 & $\mathrm{C} 11$ & HEM & 2 & 2.479 & 4.350 & 4.874 & 1.00 & 0.00 & C \\
\hline ATOM & 53 & $\mathrm{C} 12$ & HEM & 2 & 2.098 & 5.076 & 3.684 & 1.00 & 0.00 & C \\
\hline ATOM & 54 & $\mathrm{C} 13$ & HEM & 2 & 3.238 & 5.223 & 2.927 & 1.00 & 0.00 & C \\
\hline ATOM & 55 & $\mathrm{C} 14$ & HEM & 2 & 4.311 & 4.581 & 3.659 & 1.00 & 0.00 & C \\
\hline ATOM & 56 & C15 & HEM & 2 & 5.639 & 4.527 & 3.237 & 1.00 & 0.00 & C \\
\hline ATOM & 57 & H15 & HEM & 2 & 5.879 & 5.011 & 2.289 & 1.00 & 0.00 & $\mathrm{H}$ \\
\hline AтOM & 58 & $\mathrm{C} 16$ & HEM & 2 & 6.669 & 3.845 & 3.880 & 1.00 & 0.00 & C \\
\hline АтOM & 59 & $\mathrm{C} 17$ & HEM & 2 & 8.035 & 3.732 & 3.398 & 1.00 & 0.00 & C \\
\hline ATOM & 60 & $\mathrm{C} 18$ & HEM & 2 & 8.710 & 2.964 & 4.322 & 1.00 & 0.00 & C \\
\hline ATOM & 61 & C19 & HEM & 2 & 7.761 & 2.612 & 5.358 & 1.00 & 0.00 & C \\
\hline ATOM & 62 & $\mathrm{C} 20$ & HEM & 2 & 8.052 & 1.821 & 6.469 & 1.00 & 0.00 & C \\
\hline ATOM & 63 & $\mathrm{H} 2 \mathrm{O}$ & HEM & 2 & 9.066 & 1.432 & 6.530 & 1.00 & 0.00 & $\mathrm{H}$ \\
\hline ATOM & 64 & N21 & HEM & 2 & 5.862 & 1.834 & 7.564 & 1.00 & 0.00 & $\mathrm{~N}$ \\
\hline ATOM & 65 & N22 & HEM & 2 & 3.157 & 2.781 & 7.342 & 1.00 & 0.00 & $\mathrm{~N}$ \\
\hline ATOM & 66 & N23 & HEM & 2 & 3.828 & 4.044 & 4.841 & 1.00 & 0.00 & $\mathrm{~N}$ \\
\hline АTOM & 67 & N24 & HEM & 2 & 6.527 & 3.160 & 5.077 & 1.00 & 0.00 & $\mathrm{~N}$ \\
\hline ATOM & 68 & C99 & HEM & 2 & 5.621 & 6.025 & 7.579 & 1.00 & 0.00 & C \\
\hline ATOM & 69 & 091 & HEM & 2 & 5.461 & 4.711 & 7.567 & 1.00 & 0.00 & 0 \\
\hline ATOM & 70 & 092 & HEM & 2 & 5.776 & 6.642 & 8.635 & 1.00 & 0.00 & 0 \\
\hline ATOM & 71 & СТ 91 & HEM & 2 & 5.605 & 6.706 & 6.223 & 1.00 & 0.00 & C \\
\hline ATOM & 72 & H911 & HEM & 2 & 4.626 & 6.511 & 5.760 & 1.00 & 0.00 & $\mathrm{H}$ \\
\hline
\end{tabular}




\begin{tabular}{|c|c|c|c|c|c|c|c|c|c|c|}
\hline ATOM & 73 & H912 & HEM & 2 & 6.353 & 6.216 & 5.580 & 1.00 & 0.00 & $\mathrm{H}$ \\
\hline ATOM & 74 & СТ92 & HEM & 2 & 5.864 & 8.211 & 6.334 & 1.00 & 0.00 & C \\
\hline ATOM & 75 & H921 & HEM & 2 & 6.789 & 8.367 & 6.905 & 1.00 & 0.00 & $\mathrm{H}$ \\
\hline ATOM & 76 & H922 & HEM & 2 & 5.068 & 8.671 & 6.931 & 1.00 & 0.00 & $\mathrm{H}$ \\
\hline \multicolumn{10}{|l|}{ TER } & \\
\hline ATOM & 77 & $\mathrm{C} 2$ & VIN & 3 & 0.345 & 13.923 & -1.002 & 1.00 & 0.00 & C \\
\hline ATOM & 78 & $\mathrm{H} 21$ & VIN & 3 & -0.471 & 13.224 & -0.837 & 1.00 & 0.00 & $\mathrm{H}$ \\
\hline ATOM & 79 & $\mathrm{C} 1$ & VIN & 3 & 0.012 & 15.147 & -1.403 & 1.00 & 0.00 & C \\
\hline ATOM & 80 & $\mathrm{H} 11$ & VIN & 3 & 0.743 & 15.926 & -1.511 & 1.00 & 0.00 & $\mathrm{H}$ \\
\hline ATOM & 81 & $\mathrm{H} 12$ & VIN & 3 & -1.030 & 15.417 & -1.570 & 1.00 & 0.00 & $\mathrm{H}$ \\
\hline \multicolumn{10}{|l|}{ TER } & \\
\hline ATOM & 82 & $\mathrm{C} 1$ & MET & 4 & 3.008 & 14.177 & -2.840 & 1.00 & 0.00 & C \\
\hline ATOM & 83 & H11 & MET & 4 & 3.243 & 15.248 & -2.720 & 1.00 & 0.00 & H \\
\hline ATOM & 84 & $\mathrm{H} 12$ & $\mathrm{MET}$ & 4 & 3.821 & 13.713 & -3.410 & 1.00 & 0.00 & 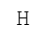 \\
\hline ATOM & 85 & $\mathrm{H} 13$ & $\mathrm{MET}$ & 4 & 2.101 & 14.100 & -3.450 & 1.00 & 0.00 & . \\
\hline \multicolumn{10}{|l|}{ TER } & \\
\hline ATOM & 86 & $\mathrm{C} 2$ & VIN & 5 & 8.018 & 12.499 & -2.325 & 1.00 & 0.00 & C \\
\hline ATOM & 87 & H 21 & VIN & 5 & 7.352 & 12.399 & -3.184 & 1.00 & 0.00 & 1 \\
\hline ATOM & 88 & $\mathrm{C} 1$ & VIN & 5 & 9.185 & 13.144 & -2.484 & 1.00 & 0.00 & C \\
\hline ATOM & 89 & $\mathrm{H} 11$ & VIN & 5 & 9.865 & 13.300 & -1.654 & 1.00 & 0.00 & $\mathrm{H}$ \\
\hline ATOM & 90 & $\mathrm{H} 12$ & VIN & 5 & 9.490 & 13.542 & -3.450 & 1.00 & 0.00 & $\mathrm{H}$ \\
\hline \multicolumn{10}{|l|}{ TER } & \\
\hline ATOM & 91 & $\mathrm{C} 1$ & $\mathrm{MET}$ & 6 & 9.703 & 10.816 & -0.121 & 1.00 & 0.00 & C \\
\hline ATOM & 92 & $\mathrm{H} 11$ & $\mathrm{MET}$ & 6 & 10.294 & 11.480 & 0.528 & 1.00 & 0.00 & $\mathrm{H}$ \\
\hline ATOM & 93 & $\mathrm{H} 12$ & MET & 6 & 9.859 & 9.794 & 0.246 & 1.00 & 0.00 & $\mathrm{H}$ \\
\hline ATOM & 94 & $\mathrm{H} 13$ & $\mathrm{MET}$ & 6 & 10.135 & 10.882 & -1.126 & 1.00 & 0.00 & $\mathrm{H}$ \\
\hline TER & & & & & & & & & & \\
\hline ATOM & 95 & C5 & $\mathrm{ACP}$ & 7 & 0.615 & 9.735 & 5.770 & 1.00 & 0.00 & C \\
\hline ATOM & 96 & H51 & $\mathrm{ACP}$ & 7 & 1.354 & 9.723 & 6.582 & 1.00 & 0.00 & $\mathrm{H}$ \\
\hline ATOM & 97 & H52 & $\mathrm{ACP}$ & 7 & -0.226 & 10.343 & 6.134 & 1.00 & 0.00 & $\mathrm{H}$ \\
\hline ATOM & 98 & $\mathrm{C} 4$ & $\mathrm{ACP}$ & 7 & 0.136 & 8.297 & 5.497 & 1.00 & 0.00 & C \\
\hline ATOM & 99 & $\mathrm{H} 41$ & $\mathrm{ACP}$ & 7 & -0.422 & 8.236 & 4.549 & 1.00 & 0.00 & $\mathrm{H}$ \\
\hline ATOM & 100 & $\mathrm{H} 42$ & $\mathrm{ACP}$ & 7 & 1.007 & 7.630 & 5.390 & 1.00 & 0.00 & $\mathrm{H}$ \\
\hline ATOM & 101 & $\mathrm{C} 1$ & $\mathrm{ACP}$ & 7 & -0.728 & 7.685 & 6.573 & 1.00 & 0.00 & C \\
\hline ATOM & 102 & $\mathrm{O} 2$ & $\mathrm{ACP}$ & 7 & -0.956 & 8.208 & 7.671 & 1.00 & 0.00 & 0 \\
\hline ATOM & 103 & 03 & $\mathrm{ACP}$ & 7 & -1.244 & 6.505 & 6.209 & 1.00 & 0.00 & o \\
\hline ATOM & 104 & H3 & $\mathrm{ACP}$ & 7 & -1.853 & 6.151 & 6.945 & 1.00 & 0.00 & $\mathrm{H}$ \\
\hline TER & & & & & & & & & & \\
\hline ATOM & 105 & $\mathrm{C} 1$ & $\mathrm{MET}$ & 8 & 8.511 & 8.608 & 4.597 & 1.00 & 0.00 & C \\
\hline ATOM & 106 & $\mathrm{H} 11$ & $\mathrm{MET}$ & 8 & 9.063 & 9.448 & 5.047 & 1.00 & 0.00 & $\mathrm{H}$ \\
\hline ATOM & 107 & $\mathrm{H} 12$ & $\mathrm{MET}$ & 8 & 8.517 & 7.782 & 5.319 & 1.00 & 0.00 & $\mathrm{H}$ \\
\hline ATOM & 108 & $\mathrm{H} 13$ & $\mathrm{MET}$ & 8 & 9.082 & 8.289 & 3.717 & 1.00 & 0.00 & $\mathrm{H}$ \\
\hline TER & & & & & & & & & & \\
\hline ATOM & 109 & $\mathrm{C} 1$ & $\mathrm{MET}$ & 9 & -0.923 & 11.557 & 3.646 & 1.00 & 0.00 & C \\
\hline ATOM & 110 & $\mathrm{H} 11$ & $\mathrm{MET}$ & 9 & -1.368 & 11.348 & 4.626 & 1.00 & 0.00 & $\mathrm{H}$ \\
\hline ATOM & 111 & $\mathrm{H} 12$ & $\mathrm{MET}$ & 9 & -1.033 & 12.637 & 3.471 & 1.00 & 0.00 & $\mathrm{H}$ \\
\hline ATOM & 112 & $\mathrm{H} 13$ & $\mathrm{MET}$ & 9 & -1.523 & 11.031 & 2.889 & 1.00 & 0.00 & $\mathrm{H}$ \\
\hline TER & & & & & & & & & & \\
\hline ATOM & 113 & $\mathrm{C} 2$ & VIN & 10 & 8.874 & 0.178 & 8.933 & 1.00 & 0.00 & C \\
\hline ATOM & 114 & $\mathrm{H} 21$ & VIN & 10 & 9.690 & 0.876 & 8.768 & 1.00 & 0.00 & $\mathrm{H}$ \\
\hline ATOM & 115 & $\mathrm{C} 1$ & VIN & 10 & 9.207 & -1.047 & 9.335 & 1.00 & 0.00 & C \\
\hline ATOM & 116 & $\mathrm{H} 11$ & VIN & 10 & 8.475 & -1.826 & 9.442 & 1.00 & 0.00 & $\mathrm{H}$ \\
\hline ATOM & 117 & $\mathrm{H} 12$ & VIN & 10 & 10.249 & -1.316 & 9.501 & 1.00 & 0.00 & $\mathrm{H}$ \\
\hline TER & & & & & & & & & & \\
\hline ATOM & 118 & $\mathrm{C} 1$ & $\mathrm{MET}$ & 11 & 6.210 & -0.077 & 10.771 & 1.00 & 0.00 & C \\
\hline ATOM & 119 & $\mathrm{H} 11$ & MET & 11 & 5.975 & -1.148 & 10.652 & 1.00 & 0.00 & $\mathrm{H}$ \\
\hline ATOM & 120 & $\mathrm{H} 12$ & $\mathrm{MET}$ & 11 & 5.397 & 0.387 & 11.341 & 1.00 & 0.00 & $\mathrm{H}$ \\
\hline ATOM & 121 & $\mathrm{H} 13$ & $\mathrm{MET}$ & 11 & 7.118 & 0.001 & 11.382 & 1.00 & 0.00 & $\mathrm{H}$ \\
\hline TER & & & & & & & & & & \\
\hline ATOM & 122 & $\mathrm{C} 2$ & VIN & 12 & 1.200 & 1.601 & 10.256 & 1.00 & 0.00 & C \\
\hline ATOM & 123 & $\mathrm{H} 21$ & VIN & 12 & 1.866 & 1.702 & 11.115 & 1.00 & 0.00 & $\mathrm{H}$ \\
\hline ATOM & 124 & $\mathrm{C} 1$ & VIN & 12 & 0.033 & 0.957 & 10.415 & 1.00 & 0.00 & C \\
\hline ATOM & 125 & $\mathrm{H} 11$ & VIN & 12 & -0.647 & 0.800 & 9.585 & 1.00 & 0.00 & $\mathrm{H}$ \\
\hline ATOM & 126 & $\mathrm{H} 12$ & VIN & 12 & -0.272 & 0.559 & 11.381 & 1.00 & 0.00 & $\mathrm{H}$ \\
\hline TER & & & & & & & & & & \\
\hline ATOM & 127 & $\mathrm{C} 1$ & $\mathrm{MET}$ & 13 & -0.484 & 3.284 & 8.052 & 1.00 & 0.00 & a \\
\hline ATOM & 128 & $\mathrm{H} 11$ & $\mathrm{MET}$ & 13 & -0.641 & 4.306 & 7.685 & 1.00 & 0.00 & $\mathrm{H}$ \\
\hline ATOM & 129 & $\mathrm{H} 12$ & $\mathrm{MET}$ & 13 & -0.917 & 3.219 & 9.057 & 1.00 & 0.00 & $\mathrm{H}$ \\
\hline ATOM & 130 & $\mathrm{H} 13$ & MET & 13 & -1.076 & 2.620 & 7.403 & 1.00 & 0.00 & $\mathrm{H}$ \\
\hline TER & & & & & & & & & & \\
\hline ATOM & 131 & $\mathrm{C} 5$ & $\mathrm{ACP}$ & 14 & 8.604 & 4.366 & 2.161 & 1.00 & 0.00 & C \\
\hline ATOM & 132 & H51 & $\mathrm{ACP}$ & 14 & 7.865 & 4.377 & 1.349 & 1.00 & 0.00 & $\mathrm{H}$ \\
\hline ATOM & 133 & H52 & $\mathrm{ACP}$ & 14 & 9.445 & 3.758 & 1.798 & 1.00 & 0.00 & $H$ \\
\hline ATOM & 134 & $\mathrm{C} 4$ & $\mathrm{ACP}$ & 14 & 9.082 & 5.803 & 2.434 & 1.00 & 0.00 & $\mathrm{C}$ \\
\hline ATOM & 135 & $\mathrm{H} 41$ & $\mathrm{ACP}$ & 14 & 9.640 & 5.865 & 3.382 & 1.00 & 0.00 & \\
\hline ATOM & 136 & $\mathrm{H} 42$ & $\mathrm{ACP}$ & 14 & 8.212 & 6.470 & 2.541 & 1.00 & 0.00 & \\
\hline ATOM & 137 & $\mathrm{C} 1$ & $\mathrm{ACP}$ & 14 & 9.946 & 6.416 & 1.358 & 1.00 & 0.00 & \\
\hline ATOM & 138 & $\mathrm{O} 2$ & $\mathrm{ACP}$ & 14 & 10.174 & 5.893 & 0.260 & 1.00 & 0.00 & \\
\hline ATOM & 139 & 03 & $\mathrm{ACP}$ & 14 & 10.462 & 7.595 & 1.722 & 1.00 & 0.00 & \\
\hline
\end{tabular}




\begin{tabular}{|c|c|c|c|c|c|c|c|c|c|c|}
\hline $\begin{array}{l}\text { ATOM } \\
\text { TER }\end{array}$ & 140 & H3 & $\mathrm{ACP}$ & 14 & 11.071 & 7.949 & 0.986 & 1.00 & 0.00 & $\mathrm{H}$ \\
\hline ATOM & 141 & $\mathrm{C} 1$ & MET & 15 & 0.708 & 5.492 & 3.334 & 1.00 & 0.00 & C \\
\hline $\mathrm{ATOM}$ & 142 & $\mathrm{H} 11$ & $\mathrm{MET}$ & 15 & 0.155 & 4.653 & 2.884 & 1.00 & 0.00 & $\mathrm{H}$ \\
\hline ATOM & 143 & $\mathrm{H} 12$ & MET & 15 & 0.701 & 6.318 & 2.613 & 1.00 & 0.00 & $\mathrm{H}$ \\
\hline ATOM & 144 & H13 & MET & 15 & 0.137 & 5.812 & 4.214 & 1.00 & 0.00 & H \\
\hline \multicolumn{10}{|l|}{ TER } & \\
\hline ATOM & 145 & $\mathrm{C} 1$ & MET & 16 & 10.141 & 2.543 & 4.285 & 1.00 & 0.00 & C \\
\hline ATOM & 146 & H11 & MET & 16 & 10.586 & 2.752 & 3.305 & 1.00 & 0.00 & $H$ \\
\hline ATOM & 147 & $\mathrm{H} 12$ & MET & 16 & 10.252 & 1.463 & 4.460 & 1.00 & 0.00 & 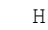 \\
\hline ATOM & 148 & H13 & MET & 16 & 10.742 & 3.069 & 5.042 & 1.00 & 0.00 & 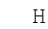 \\
\hline \multicolumn{11}{|l|}{ TER } \\
\hline ATOM & 149 & $\mathrm{FE}$ & HEM & 17 & 3.294 & 25.350 & -6.440 & 1.00 & 0.00 & $\mathrm{FE}$ \\
\hline ATOM & 150 & $\mathrm{C} 1$ & HEM & 17 & 1.066 & 27.222 & -7.502 & 1.00 & 0.00 & C \\
\hline ATOM & 151 & $\mathrm{C} 2$ & HEM & 17 & 0.707 & 28.013 & -8.674 & 1.00 & 0.00 & C \\
\hline ATOM & 152 & C3 & HEM & 17 & 1.853 & 28.110 & -9.448 & 1.00 & 0.00 & C \\
\hline ATOM & 153 & $\mathrm{C} 4$ & HEM & 17 & 2.882 & 27.392 & -8.740 & 1.00 & 0.00 & C \\
\hline ATOM & 154 & C5 & HEM & 17 & 4.197 & 27.274 & -9.178 & 1.00 & 0.00 & C \\
\hline ATOM & 155 & H5 & HEM & 17 & 4.451 & 27.770 & -10.113 & 1.00 & 0.00 & $\mathrm{H}$ \\
\hline ATOM & 156 & $\mathrm{C} 6$ & HEM & 17 & 5.217 & 26.575 & -8.542 & 1.00 & 0.00 & C \\
\hline ATOM & 157 & C7 & HEM & 17 & 6.586 & 26.503 & -9.012 & 1.00 & 0.00 & C \\
\hline ATOM & 158 & $\mathrm{C} 8$ & HEM & 17 & 7.291 & 25.783 & -8.065 & 1.00 & 0.00 & C \\
\hline ATOM & 159 & $\mathrm{C} 9$ & HEM & 17 & 6.340 & 25.418 & -7.041 & 1.00 & 0.00 & C \\
\hline ATOM & 160 & $\mathrm{C} 10$ & HEM & 17 & 6.645 & 24.683 & -5.897 & 1.00 & 0.00 & C \\
\hline ATOM & 161 & $\mathrm{H} 1 \mathrm{O}$ & HEM & 17 & 7.671 & 24.339 & -5.791 & 1.00 & 0.00 & $\mathrm{H}$ \\
\hline ATOM & 162 & C11 & HEM & 17 & 5.770 & 24.343 & -4.874 & 1.00 & 0.00 & C \\
\hline ATOM & 163 & C12 & HEM & 17 & 6.151 & 23.618 & -3.684 & 1.00 & 0.00 & C \\
\hline ATOM & 164 & $\mathrm{C} 13$ & HEM & 17 & 5.012 & 23.470 & -2.927 & 1.00 & 0.00 & C \\
\hline ATOM & 165 & $\mathrm{C} 14$ & HEM & 17 & 3.939 & 24.112 & -3.659 & 1.00 & 0.00 & C \\
\hline ATOM & 166 & C15 & HEM & 17 & 2.611 & 24.167 & -3.237 & 1.00 & 0.00 & $\mathrm{C}$ \\
\hline ATOM & 167 & $\mathrm{H} 15$ & HEM & 17 & 2.370 & 23.682 & -2.289 & 1.00 & 0.00 & $\mathrm{H}$ \\
\hline ATOM & 168 & C16 & HEM & 17 & 1.580 & 24.848 & -3.880 & 1.00 & 0.00 & C \\
\hline ATOM & 169 & C17 & HEM & 17 & 0.215 & 24.962 & -3.398 & 1.00 & 0.00 & C \\
\hline ATOM & 170 & C18 & HEM & 17 & -0.461 & 25.729 & -4.322 & 1.00 & 0.00 & $\mathrm{C}$ \\
\hline ATOM & 171 & C19 & HEM & 17 & 0.489 & 26.081 & -5.358 & 1.00 & 0.00 & C \\
\hline ATOM & 172 & $\mathrm{C} 20$ & HEM & 17 & 0.197 & 26.872 & -6.469 & 1.00 & 0.00 & C \\
\hline ATOM & 173 & $\mathrm{H} 2 \mathrm{O}$ & HEM & 17 & -0.817 & 27.262 & -6.530 & 1.00 & 0.00 & $\mathrm{H}$ \\
\hline ATOM & 174 & N21 & HEM & 17 & 2.388 & 26.859 & -7.564 & 1.00 & 0.00 & $\mathrm{~N}$ \\
\hline ATOM & 175 & N22 & HEM & 17 & 5.092 & 25.912 & -7.342 & 1.00 & 0.00 & $\mathrm{~N}$ \\
\hline ATOM & 176 & N23 & HEM & 17 & 4.422 & 24.649 & -4.841 & 1.00 & 0.00 & $\mathrm{~N}$ \\
\hline ATOM & 177 & N24 & HEM & 17 & 1.722 & 25.533 & -5.077 & 1.00 & 0.00 & $\mathrm{~N}$ \\
\hline ATOM & 178 & C99 & HEM & 17 & 2.629 & 22.668 & -7.579 & 1.00 & 0.00 & C \\
\hline ATOM & 179 & 091 & HEM & 17 & 2.788 & 23.982 & -7.567 & 1.00 & 0.00 & O \\
\hline ATOM & 180 & 092 & HEM & 17 & 2.474 & 22.051 & -8.635 & 1.00 & 0.00 & 0 \\
\hline ATOM & 181 & СТ 91 & HEM & 17 & 2.645 & 21.987 & -6.223 & 1.00 & 0.00 & C \\
\hline ATOM & 182 & H911 & HEM & 17 & 3.623 & 22.182 & -5.760 & 1.00 & 0.00 & $\mathrm{H}$ \\
\hline ATOM & 183 & H912 & HEM & 17 & 1.897 & 22.477 & -5.580 & 1.00 & 0.00 & $\mathrm{H}$ \\
\hline ATOM & 184 & СТ 92 & HEM & 17 & 2.385 & 20.483 & -6.334 & 1.00 & 0.00 & C \\
\hline ATOM & 185 & H921 & HEM & 17 & 3.181 & 20.022 & -6.931 & 1.00 & 0.00 & $\mathrm{H}$ \\
\hline ATOM & 186 & H922 & HEM & 17 & 1.460 & 20.327 & -6.905 & 1.00 & 0.00 & $\mathrm{H}$ \\
\hline \multicolumn{11}{|l|}{ TER } \\
\hline ATOM & 187 & $\mathrm{FE}$ & HEM & 18 & 3.986 & 17.936 & -1.491 & 1.00 & 0.00 & $\mathrm{FE}$ \\
\hline ATOM & 188 & $\mathrm{C} 1$ & HEM & 18 & 6.214 & 16.064 & -0.429 & 1.00 & 0.00 & $\mathrm{C}$ \\
\hline ATOM & 189 & $\mathrm{C} 2$ & HEM & 18 & 6.573 & 15.273 & 0.742 & 1.00 & 0.00 & C \\
\hline ATOM & 190 & $\mathrm{C} 3$ & HEM & 18 & 5.427 & 15.176 & 1.517 & 1.00 & 0.00 & C \\
\hline ATOM & 191 & $\mathrm{C} 4$ & HEM & 18 & 4.398 & 15.894 & 0.809 & 1.00 & 0.00 & C \\
\hline ATOM & 192 & C5 & HEM & 18 & 3.083 & 16.012 & 1.247 & 1.00 & 0.00 & $\mathrm{C}$ \\
\hline ATOM & 193 & H5 & HEM & 18 & 2.829 & 15.516 & 2.182 & 1.00 & 0.00 & $\mathrm{H}$ \\
\hline ATOM & 194 & $\mathrm{C} 6$ & HEM & 18 & 2.063 & 16.711 & 0.611 & 1.00 & 0.00 & $\mathrm{C}$ \\
\hline ATOM & 195 & $\mathrm{C} 7$ & HEM & 18 & 0.694 & 16.783 & 1.081 & 1.00 & 0.00 & C \\
\hline $\mathrm{ATOM}$ & 196 & $\mathrm{C} 8$ & HEM & 18 & -0.011 & 17.503 & 0.134 & 1.00 & 0.00 & C \\
\hline ATOM & 197 & $\mathrm{C} 9$ & HEM & 18 & 0.940 & 17.868 & -0.891 & 1.00 & 0.00 & $\mathrm{C}$ \\
\hline ATOM & 198 & $\mathrm{C} 10$ & HEM & 18 & 0.636 & 18.603 & -2.034 & 1.00 & 0.00 & C \\
\hline ATOM & 199 & $\mathrm{H} 10$ & HEM & 18 & -0.391 & 18.947 & -2.140 & 1.00 & 0.00 & $\mathrm{H}$ \\
\hline ATOM & 200 & C11 & HEM & 18 & 1.510 & 18.943 & -3.057 & 1.00 & 0.00 & C \\
\hline ATOM & 201 & C12 & HEM & 18 & 1.129 & 19.668 & -4.247 & 1.00 & 0.00 & C \\
\hline ATOM & 202 & $\mathrm{C} 13$ & HEM & 18 & 2.269 & 19.816 & -5.004 & 1.00 & 0.00 & C \\
\hline ATOM & 203 & C14 & HEM & 18 & 3.341 & 19.174 & -4.273 & 1.00 & 0.00 & $\mathrm{C}$ \\
\hline ATOM & 204 & C15 & HEM & 18 & 4.670 & 19.119 & -4.694 & 1.00 & 0.00 & C \\
\hline ATOM & 205 & H15 & HEM & 18 & 4.910 & 19.604 & -5.642 & 1.00 & 0.00 & $\mathrm{H}$ \\
\hline ATOM & 206 & $\mathrm{C} 16$ & HEM & 18 & 5.700 & 18.438 & -4.051 & 1.00 & 0.00 & C \\
\hline ATOM & 207 & $\mathrm{C} 17$ & HEM & 18 & 7.066 & 18.324 & -4.533 & 1.00 & 0.00 & C \\
\hline ATOM & 208 & $\mathrm{C} 18$ & HEM & 18 & 7.741 & 17.557 & -3.609 & 1.00 & 0.00 & C \\
\hline ATOM & 209 & C19 & HEM & 18 & 6.791 & 17.205 & -2.573 & 1.00 & 0.00 & C \\
\hline ATOM & 210 & $\mathrm{C} 20$ & HEM & 18 & 7.083 & 16.414 & -1.462 & 1.00 & 0.00 & C \\
\hline ATOM & 211 & $\mathrm{H} 2 \mathrm{O}$ & HEM & 18 & 8.097 & 16.024 & -1.402 & 1.00 & 0.00 & $\mathrm{H}$ \\
\hline ATOM & 212 & N21 & HEM & 18 & 4.892 & 16.427 & -0.367 & 1.00 & 0.00 & $\mathrm{~N}$ \\
\hline ATOM & 213 & N22 & HEM & 18 & 2.188 & 17.374 & -0.590 & 1.00 & 0.00 & $\mathrm{~N}$ \\
\hline ATOM & 214 & N23 & HEM & 18 & 2.859 & 18.637 & -3.090 & 1.00 & 0.00 & $\mathrm{~N}$ \\
\hline
\end{tabular}




\begin{tabular}{|c|c|c|c|}
\hline ATOM & 215 & N24 & HEM \\
\hline ATOM & 216 & C99 & HEM \\
\hline AтOM & 217 & 091 & HEM \\
\hline ATOM & 218 & 092 & HEM \\
\hline ATOM & 219 & СТ91 & HEM \\
\hline ATOM & 220 & H911 & HEM \\
\hline ATOM & 221 & H912 & HEM \\
\hline ATOM & 222 & CT92 & HEM \\
\hline ATOM & 223 & H921 & HEM \\
\hline ATOM & 224 & H922 & HEM \\
\hline \multicolumn{4}{|l|}{ TER } \\
\hline ATOM & 225 & $\mathrm{C} 2$ & VIN \\
\hline ATOM & 226 & H 21 & VIN \\
\hline ATOM & 227 & $\mathrm{C} 1$ & VIN \\
\hline ATOM & 228 & H11 & VIN \\
\hline ATOM & 229 & $\mathrm{H} 12$ & VIN \\
\hline \multicolumn{4}{|l|}{ TER } \\
\hline ATOM & 230 & $\mathrm{C} 1$ & MET \\
\hline ATOM & 231 & $\mathrm{H} 11$ & MET \\
\hline ATOM & 232 & $\mathrm{H} 12$ & MET \\
\hline ATOM & 233 & H13 & $\mathrm{MET}$ \\
\hline \multicolumn{4}{|l|}{ TER } \\
\hline AтOM & 234 & $\mathrm{C} 2$ & VIN \\
\hline ATOM & 235 & H21 & VIN \\
\hline ATOM & 236 & $\mathrm{C} 1$ & VIN \\
\hline ATOM & 237 & H11 & VIN \\
\hline ATOM & 238 & $\mathrm{H} 12$ & VIN \\
\hline \multicolumn{4}{|l|}{ TER } \\
\hline ATOM & 239 & $\mathrm{C} 1$ & MET \\
\hline ATOM & 240 & H11 & MET \\
\hline ATOM & 241 & H12 & MET \\
\hline ATOM & 242 & H13 & MET \\
\hline \multicolumn{4}{|l|}{ TER } \\
\hline ATOM & 243 & C5 & $\mathrm{ACP}$ \\
\hline ATOM & 244 & H51 & $\mathrm{ACP}$ \\
\hline ATOM & 245 & H52 & $\mathrm{ACP}$ \\
\hline ATOM & 246 & $\mathrm{C} 4$ & $\mathrm{ACP}$ \\
\hline ATOM & 247 & $\mathrm{H} 41$ & $\mathrm{ACP}$ \\
\hline ATOM & 248 & $\mathrm{H} 42$ & $\mathrm{ACP}$ \\
\hline ATOM & 249 & $\mathrm{C} 1$ & $\mathrm{ACP}$ \\
\hline ATOM & 250 & $\mathrm{O} 2$ & $\mathrm{ACP}$ \\
\hline AтOM & 251 & 03 & $\mathrm{ACP}$ \\
\hline ATOM & 252 & Н3 & $\mathrm{ACP}$ \\
\hline \multicolumn{4}{|l|}{ TER } \\
\hline ATOM & 253 & $\mathrm{C} 1$ & $\mathrm{MET}$ \\
\hline ATOM & 254 & H11 & $\mathrm{MET}$ \\
\hline ATOM & 255 & H12 & MET \\
\hline ATOM & 256 & H13 & MET \\
\hline \multicolumn{4}{|l|}{ TER } \\
\hline ATOM & 257 & $\mathrm{C} 1$ & MET \\
\hline ATOM & 258 & H11 & MET \\
\hline ATOM & 259 & H12 & MET \\
\hline ATOM & 260 & $\mathrm{H} 13$ & MET \\
\hline \multicolumn{4}{|l|}{ TER } \\
\hline ATOM & 261 & $\mathrm{C} 2$ & VIN \\
\hline ATOM & 262 & H 21 & VIN \\
\hline ATOM & 263 & $\mathrm{C} 1$ & VIN \\
\hline ATOM & 264 & $\mathrm{H} 11$ & VIN \\
\hline ATOM & 265 & $\mathrm{H} 12$ & VIN \\
\hline TER & & & \\
\hline ATOM & 266 & $\mathrm{C} 1$ & MET \\
\hline ATOM & 267 & H11 & MET \\
\hline ATOM & 268 & H12 & $\mathrm{MET}$ \\
\hline ATOM & 269 & H13 & $\mathrm{MET}$ \\
\hline TER & & & \\
\hline ATOM & 270 & $\mathrm{C} 2$ & VIN \\
\hline ATOM & 271 & H21 & VIN \\
\hline ATOM & 272 & $\mathrm{C} 1$ & VIN \\
\hline ATOM & 273 & H11 & VIN \\
\hline ATOM & 274 & H12 & VIN \\
\hline TER & & & \\
\hline ATOM & 275 & C1 & MET \\
\hline ATOM & 276 & $\mathrm{H} 11$ & MET \\
\hline ATOM & 277 & H12 & MET \\
\hline ATOM & 278 & H13 & $\mathrm{MET}$ \\
\hline TER & & & \\
\hline ATOM & 279 & $\mathrm{C} 5$ & $\mathrm{ACP}$ \\
\hline ATOM & 280 & H51 & $\mathrm{ACP}$ \\
\hline ATOM & 281 & H52 & $\mathrm{ACP}$ \\
\hline
\end{tabular}

\begin{tabular}{|c|c|c|c|c|c|}
\hline 5.558 & 17.753 & -2.854 & 1.00 & 0.00 & $\mathrm{~N}$ \\
\hline 4.652 & 20.618 & -0.352 & 1.00 & 0.00 & C \\
\hline 4.492 & 19.304 & -0.365 & 1.00 & 0.00 & 0 \\
\hline 4.806 & 21.235 & 0.703 & 1.00 & 0.00 & O \\
\hline 4.635 & 21.299 & -1.709 & 1.00 & 0.00 & C \\
\hline 3.657 & 21.104 & -2.171 & 1.00 & 0.00 & $\mathrm{H}$ \\
\hline 5.383 & 20.809 & -2.352 & 1.00 & 0.00 & $\mathrm{H}$ \\
\hline 4.895 & 22.803 & -1.597 & 1.00 & 0.00 & $\mathrm{C}$ \\
\hline 5.820 & 22.959 & -1.027 & 1.00 & 0.00 & $\mathrm{H}$ \\
\hline 4.099 & 23.264 & -1.000 & 1.00 & 0.00 & $\mathrm{H}$ \\
\hline-0.625 & 28.515 & -8.933 & 1.00 & 0.00 & C \\
\hline-1.440 & 27.817 & -8.768 & 1.00 & 0.00 & $\mathrm{H}$ \\
\hline-0.958 & 29.740 & -9.335 & 1.00 & 0.00 & $\mathrm{C}$ \\
\hline-0.226 & 30.519 & -9.442 & 1.00 & 0.00 & $\mathrm{H}$ \\
\hline-1.999 & 30.010 & -9.501 & 1.00 & 0.00 & $\mathrm{H}$ \\
\hline 2.039 & 28.770 & -10.771 & 1.00 & 0.00 & C \\
\hline 2.274 & 29.841 & -10.652 & 1.00 & 0.00 & $\mathrm{H}$ \\
\hline 2.852 & 28.306 & -11.341 & 1.00 & 0.00 & $\mathrm{H}$ \\
\hline 1.131 & 28.693 & -11.382 & 1.00 & 0.00 & $\mathrm{H}$ \\
\hline 7.049 & 27.092 & -10.256 & 1.00 & 0.00 & C \\
\hline 6.383 & 26.991 & -11.115 & 1.00 & 0.00 & $\mathrm{H}$ \\
\hline 8.216 & 27.737 & -10.415 & 1.00 & 0.00 & C \\
\hline 8.896 & 27.893 & -9.585 & 1.00 & 0.00 & $\mathrm{H}$ \\
\hline 8.521 & 28.135 & -11.381 & 1.00 & 0.00 & $\mathrm{H}$ \\
\hline 8.734 & 25.409 & -8.052 & 1.00 & 0.00 & C \\
\hline 9.325 & 26.073 & -7.403 & 1.00 & 0.00 & $\mathrm{H}$ \\
\hline 8.890 & 24.387 & -7.685 & 1.00 & 0.00 & $\mathrm{H}$ \\
\hline 9.166 & 25.475 & -9.057 & 1.00 & 0.00 & $\mathrm{H}$ \\
\hline-0.355 & 24.327 & -2.161 & 1.00 & 0.00 & C \\
\hline 0.384 & 24.316 & -1.349 & 1.00 & 0.00 & $\mathrm{H}$ \\
\hline-1.195 & 24.936 & -1.798 & 1.00 & 0.00 & $\mathrm{H}$ \\
\hline-0.833 & 22.890 & -2.434 & 1.00 & 0.00 & C \\
\hline-1.391 & 22.829 & -3.382 & 1.00 & 0.00 & $\mathrm{H}$ \\
\hline 0.037 & 22.223 & -2.541 & 1.00 & 0.00 & $\mathrm{H}$ \\
\hline-1.697 & 22.278 & -1.358 & 1.00 & 0.00 & C \\
\hline-1.925 & 22.800 & -0.260 & 1.00 & 0.00 & 0 \\
\hline-2.213 & 21.098 & -1.722 & 1.00 & 0.00 & O \\
\hline-2.822 & 20.744 & -0.986 & 1.00 & 0.00 & $\mathrm{H}$ \\
\hline 7.541 & 23.201 & -3.334 & 1.00 & 0.00 & C \\
\hline 8.094 & 24.040 & -2.884 & 1.00 & 0.00 & $\mathrm{H}$ \\
\hline 7.548 & 22.375 & -2.613 & 1.00 & 0.00 & $\mathrm{H}$ \\
\hline 8.112 & 22.882 & -4.214 & 1.00 & 0.00 & $\mathrm{H}$ \\
\hline-1.892 & 26.150 & -4.285 & 1.00 & 0.00 & C \\
\hline-2.337 & 25.941 & -3.305 & 1.00 & 0.00 & $\mathrm{H}$ \\
\hline-2.002 & 27.230 & -4.460 & 1.00 & 0.00 & $\mathrm{H}$ \\
\hline-2.493 & 25.624 & -5.042 & 1.00 & 0.00 & $\mathrm{H}$ \\
\hline 7.905 & 14.771 & 1.002 & 1.00 & 0.00 & C \\
\hline 8.721 & 15.469 & 0.837 & 1.00 & 0.00 & $\mathrm{H}$ \\
\hline 8.238 & 13.546 & 1.403 & 1.00 & 0.00 & C \\
\hline 7.506 & 12.767 & 1.511 & 1.00 & 0.00 & $\mathrm{H}$ \\
\hline 9.279 & 13.277 & 1.570 & 1.00 & 0.00 & $\mathrm{H}$ \\
\hline 5.241 & 14.516 & 2.840 & 1.00 & 0.00 & C \\
\hline 5.006 & 13.445 & 2.720 & 1.00 & 0.00 & $\mathrm{H}$ \\
\hline 4.428 & 14.980 & 3.410 & 1.00 & 0.00 & $\mathrm{H}$ \\
\hline 6.149 & 14.593 & 3.450 & 1.00 & 0.00 & $\mathrm{H}$ \\
\hline 0.231 & 16.194 & 2.325 & 1.00 & 0.00 & C \\
\hline 0.897 & 16.295 & 3.184 & 1.00 & 0.00 & $\mathrm{H}$ \\
\hline-0.936 & 15.550 & 2.484 & 1.00 & 0.00 & C \\
\hline-1.616 & 15.393 & 1.654 & 1.00 & 0.00 & $\mathrm{H}$ \\
\hline-1.241 & 15.151 & 3.450 & 1.00 & 0.00 & $\mathrm{H}$ \\
\hline-1.454 & 17.877 & 0.121 & 1.00 & 0.00 & $\mathrm{C}$ \\
\hline-1.610 & 18.899 & -0.246 & 1.00 & 0.00 & $\mathrm{H}$ \\
\hline-1.886 & 17.811 & 1.126 & 1.00 & 0.00 & $\mathrm{H}$ \\
\hline-2.045 & 17.213 & -0.528 & 1.00 & 0.00 & $\mathrm{H}$ \\
\hline 7.635 & 18.959 & -5.770 & 1.00 & 0.00 & C \\
\hline 6.896 & 18.970 & -6.582 & 1.00 & 0.00 & $\mathrm{H}$ \\
\hline 8.475 & 18.350 & -6.134 & 1.00 & 0.00 & $\mathrm{H}$ \\
\hline
\end{tabular}




$\begin{array}{llrll}\text { ATOM } & 282 & \text { C4 } & \text { ACP } & 30 \\ \text { ATOM } & 283 & \text { H41 } & \text { ACP } & 30 \\ \text { ATOM } & 284 & \text { H42 } & \text { ACP } & 30 \\ \text { ATOM } & 285 & \text { C1 } & \text { ACP } & 30 \\ \text { ATOM } & 286 & \text { O2 } & \text { ACP } & 30 \\ \text { ATOM } & 287 & \text { O3 } & \text { ACP } & 30 \\ \text { ATOM } & 288 & \text { H3 } & \text { ACP } & 30 \\ \text { TER } & & & & \\ \text { ATOM } & 289 & \text { C1 } & \text { MET } & 31 \\ \text { ATOM } & 290 & \text { H11 } & \text { MET } & 31 \\ \text { ATOM } & 291 & \text { H12 } & \text { MET } & 31 \\ \text { ATOM } & 292 & \text { H13 } & \text { MET } & 31 \\ \text { TER } & & & & \\ \text { ATOM } & 293 & \text { C1 } & \text { MET } & 32 \\ \text { ATOM } & 294 & \text { H11 } & \text { MET } & 32 \\ \text { ATOM } & 295 & \text { H12 } & \text { MET } & 32 \\ \text { ATOM } & 296 & \text { H13 } & \text { MET } & 32 \\ \text { TER }\end{array}$

ATOM 296 H13 MET 32

TER

$\begin{array}{rrrrrl}8.113 & 20.396 & -5.497 & 1.00 & 0.00 & \mathrm{C} \\ 8.671 & 20.457 & -4.549 & 1.00 & 0.00 & \mathrm{H} \\ 7.243 & 21.063 & -5.390 & 1.00 & 0.00 & \mathrm{H} \\ 8.977 & 21.008 & -6.573 & 1.00 & 0.00 & \mathrm{C} \\ 9.205 & 20.486 & -7.671 & 1.00 & 0.00 & \mathrm{O} \\ 9.493 & 22.188 & -6.209 & 1.00 & 0.00 & \mathrm{O} \\ 10.102 & 22.542 & -6.945 & 1.00 & 0.00 & \mathrm{H} \\ -0.261 & 20.085 & -4.597 & 1.00 & 0.00 & \mathrm{C} \\ -0.814 & 19.246 & -5.047 & 1.00 & 0.00 & \mathrm{H} \\ -0.268 & 20.911 & -5.319 & 1.00 & 0.00 & \mathrm{H} \\ -0.832 & 20.405 & -3.717 & 1.00 & 0.00 & \mathrm{H} \\ 9.172 & 17.136 & -3.646 & 1.00 & 0.00 & \mathrm{C} \\ 9.617 & 17.345 & -4.626 & 1.00 & 0.00 & \mathrm{H} \\ 9.283 & 16.056 & -3.471 & 1.00 & 0.00 & \mathrm{H} \\ 9.773 & 17.662 & -2.889 & 1.00 & 0.00 & \mathrm{H}\end{array}$

\subsection{D3 dimer: D04_D3.pdb file}

\begin{tabular}{|c|c|c|c|c|c|c|c|c|c|c|}
\hline HETATM & 1 & $\mathrm{FE}$ & HEM & 1 & 4.263 & 10.757 & 1.491 & 1.00 & 0.00 & $\mathrm{Fe}$ \\
\hline HETATM & 2 & $\mathrm{C} 1$ & HEM & 1 & 2.035 & 12.629 & 0.429 & 1.00 & 0.00 & C \\
\hline HETATM & 3 & $\mathrm{C} 2$ & HEM & 1 & 1.676 & 13.420 & -0.742 & 1.00 & 0.00 & C \\
\hline HETATM & 4 & $\mathrm{C} 3$ & HEM & 1 & 2.822 & 13.517 & -1.517 & 1.00 & 0.00 & $\mathrm{C}$ \\
\hline HETATM & 5 & C4 & HEM & 1 & 3.851 & 12.800 & -0.809 & 1.00 & 0.00 & C \\
\hline HETATM & 6 & C5 & HEM & 1 & 5.167 & 12.681 & -1.247 & 1.00 & 0.00 & C \\
\hline HETATM & 7 & H5 & HEM & 1 & 5.420 & 13.177 & -2.182 & 1.00 & 0.00 & $\mathrm{H}$ \\
\hline HETATM & 8 & C6 & HEM & 1 & 6.186 & 11.982 & -0.611 & 1.00 & 0.00 & C \\
\hline HETATM & 9 & C7 & HEM & 1 & 7.555 & 11.910 & -1.081 & 1.00 & 0.00 & C \\
\hline HETATM & 10 & $\mathrm{C} 8$ & HEM & 1 & 8.260 & 11.190 & -0.134 & 1.00 & 0.00 & C \\
\hline HETATM & 11 & C9 & HEM & 1 & 7.309 & 10.825 & 0.891 & 1.00 & 0.00 & C \\
\hline HETATM & 12 & $\mathrm{C} 10$ & HEM & 1 & 7.614 & 10.090 & 2.034 & 1.00 & 0.00 & C \\
\hline HETATM & 13 & $\mathrm{H} 10$ & HEM & 1 & 8.641 & 9.746 & 2.140 & 1.00 & 0.00 & $\mathrm{H}$ \\
\hline HETATM & 14 & $\mathrm{C} 11$ & HEM & 1 & 6.739 & 9.750 & 3.057 & 1.00 & 0.00 & C \\
\hline HETATM & 15 & $\mathrm{C} 12$ & HEM & 1 & 7.120 & 9.025 & 4.247 & 1.00 & 0.00 & $\mathrm{C}$ \\
\hline HETATM & 16 & $\mathrm{C} 13$ & HEM & 1 & 5.981 & 8.878 & 5.004 & 1.00 & 0.00 & C \\
\hline HETATM & 17 & $\mathrm{C} 14$ & HEM & 1 & 4.908 & 9.519 & 4.273 & 1.00 & 0.00 & C \\
\hline HETATM & 18 & $\mathrm{C} 15$ & HEM & 1 & 3.580 & 9.574 & 4.694 & 1.00 & 0.00 & C \\
\hline HETATM & 19 & H15 & HEM & 1 & 3.340 & 9.089 & 5.642 & 1.00 & 0.00 & $\mathrm{H}$ \\
\hline HETATM & 20 & $\mathrm{C} 16$ & HEM & 1 & 2.550 & 10.255 & 4.051 & 1.00 & 0.00 & C \\
\hline HETATM & 21 & $\mathrm{C} 17$ & HEM & 1 & 1.184 & 10.369 & 4.533 & 1.00 & 0.00 & C \\
\hline HETATM & 22 & $\mathrm{C} 18$ & HEM & 1 & 0.508 & 11.137 & 3.609 & 1.00 & 0.00 & C \\
\hline HETATM & 23 & C19 & HEM & 1 & 1.458 & 11.489 & 2.573 & 1.00 & 0.00 & C \\
\hline HETATM & 24 & $\mathrm{C} 20$ & HEM & 1 & 1.167 & 12.280 & 1.462 & 1.00 & 0.00 & C \\
\hline HETATM & 25 & $\mathrm{H} 20$ & HEM & 1 & 0.152 & 12.669 & 1.402 & 1.00 & 0.00 & $\mathrm{H}$ \\
\hline HETATM & 26 & $\mathrm{~N} 21$ & HEM & 1 & 3.357 & 12.266 & 0.367 & 1.00 & 0.00 & $\mathrm{~N}$ \\
\hline HETATM & 27 & N22 & HEM & 1 & 6.062 & 11.319 & 0.590 & 1.00 & 0.00 & $\mathrm{~N}$ \\
\hline HETATM & 28 & N23 & HEM & 1 & 5.391 & 10.056 & 3.090 & 1.00 & 0.00 & $\mathrm{~N}$ \\
\hline HETATM & 29 & N24 & HEM & 1 & 2.691 & 10.941 & 2.854 & 1.00 & 0.00 & $\mathrm{~N}$ \\
\hline HETATM & 30 & C99 & HEM & 1 & 3.598 & 8.076 & 0.352 & 1.00 & 0.00 & C \\
\hline HETATM & 31 & 091 & HEM & 1 & 3.757 & 9.389 & 0.365 & 1.00 & 0.00 & 0 \\
\hline HETATM & 32 & 092 & HEM & 1 & 3.443 & 7.458 & -0.703 & 1.00 & 0.00 & O \\
\hline HETATM & 33 & СТ91 & HEM & 1 & 3.614 & 7.394 & 1.709 & 1.00 & 0.00 & $\mathrm{C}$ \\
\hline HETATM & 34 & H911 & HEM & 1 & 4.593 & 7.590 & 2.171 & 1.00 & 0.00 & $\mathrm{H}$ \\
\hline HETATM & 35 & H912 & HEM & 1 & 2.866 & 7.884 & 2.352 & 1.00 & 0.00 & $\mathrm{H}$ \\
\hline HETATM & 36 & СТ92 & HEM & 1 & 3.354 & 5.890 & 1.597 & 1.00 & 0.00 & C \\
\hline HETATM & 37 & H921 & HEM & 1 & 4.151 & 5.429 & 1.000 & 1.00 & 0.00 & $\mathrm{H}$ \\
\hline HETATM & 38 & H922 & HEM & 1 & 2.429 & 5.734 & 1.027 & 1.00 & 0.00 & $\mathrm{H}$ \\
\hline \multicolumn{11}{|l|}{ TER } \\
\hline HETATM & 39 & $\mathrm{FE}$ & HEM & 2 & 4.955 & 3.343 & 6.440 & 1.00 & 0.00 & $\mathrm{Fe}$ \\
\hline HETATM & 40 & $\mathrm{C} 1$ & HEM & 2 & 7.183 & 1.471 & 7.502 & 1.00 & 0.00 & C \\
\hline HETATM & 41 & $\mathrm{C} 2$ & HEM & 2 & 7.542 & 0.680 & 8.674 & 1.00 & 0.00 & C \\
\hline HETATM & 42 & C3 & HEM & 2 & 6.397 & 0.583 & 9.448 & 1.00 & 0.00 & C \\
\hline HETATM & 43 & $\mathrm{C} 4$ & HEM & 2 & 5.367 & 1.301 & 8.740 & 1.00 & 0.00 & C \\
\hline HETATM & 44 & C5 & HEM & 2 & 4.052 & 1.420 & 9.178 & 1.00 & 0.00 & C \\
\hline HETATM & 45 & H5 & HEM & 2 & 3.798 & 0.923 & 10.113 & 1.00 & 0.00 & $\mathrm{H}$ \\
\hline HETATM & 46 & $\mathrm{C} 6$ & HEM & 2 & 3.033 & 2.118 & 8.542 & 1.00 & 0.00 & C \\
\hline HETATM & 47 & C7 & HEM & 2 & 1.664 & 2.191 & 9.012 & 1.00 & 0.00 & C \\
\hline HETATM & 48 & $\mathrm{C} 8$ & HEM & 2 & 0.958 & 2.910 & 8.065 & 1.00 & 0.00 & C \\
\hline HETATM & 49 & $\mathrm{C} 9$ & HEM & 2 & 1.909 & 3.275 & 7.041 & 1.00 & 0.00 & C \\
\hline HETATM & 50 & $\mathrm{C} 10$ & HEM & 2 & 1.605 & 4.010 & 5.897 & 1.00 & 0.00 & C \\
\hline HETATM & 51 & $\mathrm{H} 10$ & HEM & 2 & 0.578 & 4.354 & 5.791 & 1.00 & 0.00 & $\mathrm{H}$ \\
\hline HETATM & 52 & $\mathrm{C} 11$ & HEM & 2 & 2.479 & 4.350 & 4.874 & 1.00 & 0.00 & $\mathrm{C}$ \\
\hline HETATM & 53 & $\mathrm{C} 12$ & HEM & 2 & 2.098 & 5.076 & 3.684 & 1.00 & 0.00 & C \\
\hline HETATM & 54 & $\mathrm{C} 13$ & HEM & 2 & 3.238 & 5.223 & 2.927 & 1.00 & 0.00 & C \\
\hline HETATM & 55 & C14 & HEM & 2 & 4.311 & 4.581 & 3.659 & 1.00 & 0.00 & C \\
\hline
\end{tabular}




\begin{tabular}{|c|c|c|c|}
\hline HETATM & 56 & C15 & HEM \\
\hline HETATM & 57 & H15 & HEM \\
\hline HETATM & 58 & C16 & HEM \\
\hline HETATM & 59 & C17 & HEM \\
\hline HETATM & 60 & C18 & HEM \\
\hline HETATM & 61 & C19 & HEM \\
\hline HETATM & 62 & C20 & HEM \\
\hline HETATM & 63 & $\mathrm{H} 2 \mathrm{O}$ & HEM \\
\hline HETATM & 64 & N21 & HEM \\
\hline HETATM & 65 & N22 & HEM \\
\hline HETATM & 66 & N23 & HEM \\
\hline HETATM & 67 & N24 & HEM \\
\hline HETATM & 68 & C99 & HEM \\
\hline HETATM & 69 & 091 & HEM \\
\hline HETATM & 70 & 092 & HEM \\
\hline HETATM & 71 & CT91 & HEM \\
\hline HETATM & 72 & H911 & HEM \\
\hline HETATM & 73 & H912 & HEM \\
\hline HETATM & 74 & CT92 & HEM \\
\hline HETATM & 75 & H921 & HEM \\
\hline HETATM & 76 & H922 & HEM \\
\hline \multicolumn{4}{|l|}{ TER } \\
\hline HETATM & 77 & $\mathrm{C} 2$ & VIN \\
\hline HETATM & 78 & H 21 & VIN \\
\hline HETATM & 79 & $\mathrm{C} 1$ & VIN \\
\hline HETATM & 80 & $\mathrm{H} 11$ & VIN \\
\hline HETATM & 81 & $\mathrm{H} 12$ & VIN \\
\hline \multicolumn{4}{|l|}{ TER } \\
\hline HETATM & 82 & $\mathrm{C} 1$ & MET \\
\hline HETATM & 83 & $\mathrm{H} 11$ & MET \\
\hline HETATM & 84 & $\mathrm{H} 12$ & MET \\
\hline HETATM & 85 & H13 & MET \\
\hline \multicolumn{4}{|l|}{ TER } \\
\hline HETATM & 86 & $\mathrm{C} 2$ & VIN \\
\hline HETATM & 87 & H 21 & VIN \\
\hline HETATM & 88 & $\mathrm{C} 1$ & VIN \\
\hline HETATM & 89 & H11 & VIN \\
\hline HETATM & 90 & $\mathrm{H} 12$ & VIN \\
\hline \multicolumn{4}{|l|}{ TER } \\
\hline HETATM & 91 & $\mathrm{C} 1$ & MET \\
\hline HETATM & 92 & $\mathrm{H} 11$ & MET \\
\hline HETATM & 93 & H12 & MET \\
\hline HETATM & 94 & H13 & $\mathrm{MET}$ \\
\hline \multicolumn{4}{|l|}{ TER } \\
\hline HETATM & 95 & C5 & $\mathrm{ACP}$ \\
\hline HETATM & 96 & H51 & $\mathrm{ACP}$ \\
\hline HETATM & 97 & H52 & $\mathrm{ACP}$ \\
\hline HETATM & 98 & C4 & $\mathrm{ACP}$ \\
\hline HETATM & 99 & $\mathrm{H} 41$ & $\mathrm{ACP}$ \\
\hline HETATM & 100 & $\mathrm{H} 42$ & $\mathrm{ACP}$ \\
\hline HETATM & 101 & $\mathrm{C} 1$ & $\mathrm{ACP}$ \\
\hline HETATM & 102 & $\mathrm{O} 2$ & $\mathrm{ACP}$ \\
\hline HETATM & 103 & 03 & $\mathrm{ACP}$ \\
\hline HETATM & 104 & H3 & $\mathrm{ACP}$ \\
\hline \multicolumn{4}{|l|}{ TER } \\
\hline HETATM & 105 & C1 & MET \\
\hline HETATM & 106 & $\mathrm{H} 11$ & MET \\
\hline HETATM & 107 & $\mathrm{H} 12$ & MET \\
\hline HETATM & 108 & H13 & $\mathrm{MET}$ \\
\hline \multicolumn{4}{|l|}{ TER } \\
\hline HETATM & 109 & $\mathrm{C} 1$ & MET \\
\hline HETATM & 110 & H11 & MET \\
\hline HETATM & 111 & $\mathrm{H} 12$ & MET \\
\hline HETATM & 112 & $\mathrm{H} 13$ & MET \\
\hline \multicolumn{4}{|l|}{ TER } \\
\hline HETATM & 113 & C2 & VIN \\
\hline HETATM & 114 & H 21 & VIN \\
\hline HETATM & 115 & $\mathrm{C} 1$ & VIN \\
\hline HETATM & 116 & $\mathrm{H} 11$ & VIN \\
\hline HETATM & 117 & $\mathrm{H} 12$ & VIN \\
\hline TER & & & \\
\hline HETATM & 118 & $\mathrm{C} 1$ & MET \\
\hline HETATM & 119 & H11 & MET \\
\hline HETATM & 120 & $\mathrm{H} 12$ & MET \\
\hline HETATM & 121 & $\mathrm{H} 13$ & MET \\
\hline TER & & & \\
\hline HETATM & 122 & $\mathrm{C} 2$ & VIN \\
\hline HETATM & 123 & H 21 & VIN \\
\hline HETATM & 124 & $\mathrm{C} 1$ & VIN \\
\hline
\end{tabular}

\begin{tabular}{|c|c|c|c|c|c|}
\hline 5.639 & 4.527 & 3.237 & 1.00 & 0.00 & C \\
\hline 5.879 & 5.011 & 2.289 & 1.00 & 0.00 & $\mathrm{H}$ \\
\hline 6.669 & 3.845 & 3.880 & 1.00 & 0.00 & C \\
\hline 8.035 & 3.732 & 3.398 & 1.00 & 0.00 & C \\
\hline 8.710 & 2.964 & 4.322 & 1.00 & 0.00 & C \\
\hline 7.761 & 2.612 & 5.358 & 1.00 & 0.00 & C \\
\hline 8.052 & 1.821 & 6.469 & 1.00 & 0.00 & C \\
\hline 9.066 & 1.432 & 6.530 & 1.00 & 0.00 & $\mathrm{H}$ \\
\hline 5.862 & 1.834 & 7.564 & 1.00 & 0.00 & $\mathrm{~N}$ \\
\hline 3.157 & 2.781 & 7.342 & 1.00 & 0.00 & $\mathrm{~N}$ \\
\hline 3.828 & 4.044 & 4.841 & 1.00 & 0.00 & $\mathrm{~N}$ \\
\hline 6.527 & 3.160 & 5.077 & 1.00 & 0.00 & $\mathrm{~N}$ \\
\hline 5.621 & 6.025 & 7.579 & 1.00 & 0.00 & C \\
\hline 5.461 & 4.711 & 7.567 & 1.00 & 0.00 & o \\
\hline 5.776 & 6.642 & 8.635 & 1.00 & 0.00 & 0 \\
\hline 5.605 & 6.706 & 6.223 & 1.00 & 0.00 & C \\
\hline 4.626 & 6.511 & 5.760 & 1.00 & 0.00 & $\mathrm{H}$ \\
\hline 6.353 & 6.216 & 5.580 & 1.00 & 0.00 & $\mathrm{H}$ \\
\hline 5.864 & 8.211 & 6.334 & 1.00 & 0.00 & C \\
\hline 6.789 & 8.367 & 6.905 & 1.00 & 0.00 & $\mathrm{H}$ \\
\hline 5.068 & 8.671 & 6.931 & 1.00 & 0.00 & $\mathrm{H}$ \\
\hline 0.345 & 13.923 & -1.002 & 1.00 & 0.00 & C \\
\hline-0.471 & 13.224 & -0.837 & 1.00 & 0.00 & $\mathrm{H}$ \\
\hline 0.012 & 15.147 & -1.403 & 1.00 & 0.00 & C \\
\hline 0.743 & 15.926 & -1.511 & 1.00 & 0.00 & $\mathrm{H}$ \\
\hline-1.030 & 15.417 & -1.570 & 1.00 & 0.00 & $\mathrm{H}$ \\
\hline 3.008 & 14.177 & -2.840 & 1.00 & 0.00 & C \\
\hline 3.243 & 15.248 & -2.720 & 1.00 & 0.00 & $\mathrm{H}$ \\
\hline 3.821 & 13.713 & -3.410 & 1.00 & 0.00 & $\mathrm{H}$ \\
\hline 2.101 & 14.100 & -3.450 & 1.00 & 0.00 & $\mathrm{H}$ \\
\hline 8.018 & 12.499 & -2.325 & 1.00 & 0.00 & C \\
\hline 7.352 & 12.399 & -3.184 & 1.00 & 0.00 & $\mathrm{H}$ \\
\hline 9.185 & 13.144 & -2.484 & 1.00 & 0.00 & C \\
\hline 9.865 & 13.300 & -1.654 & 1.00 & 0.00 & $\mathrm{H}$ \\
\hline 9.490 & 13.542 & -3.450 & 1.00 & 0.00 & $\mathrm{H}$ \\
\hline 9.703 & 10.816 & -0.121 & 1.00 & 0.00 & C \\
\hline 10.294 & 11.480 & 0.528 & 1.00 & 0.00 & $\mathrm{H}$ \\
\hline 9.859 & 9.794 & 0.246 & 1.00 & 0.00 & $\mathrm{H}$ \\
\hline 10.135 & 10.882 & -1.126 & 1.00 & 0.00 & $\mathrm{H}$ \\
\hline 0.615 & 9.735 & 5.770 & 1.00 & 0.00 & C \\
\hline 1.354 & 9.723 & 6.582 & 1.00 & 0.00 & $\mathrm{H}$ \\
\hline-0.226 & 10.343 & 6.134 & 1.00 & 0.00 & $\mathrm{H}$ \\
\hline 0.136 & 8.297 & 5.497 & 1.00 & 0.00 & C \\
\hline-0.422 & 8.236 & 4.549 & 1.00 & 0.00 & $\mathrm{H}$ \\
\hline 1.007 & 7.630 & 5.390 & 1.00 & 0.00 & $\mathrm{H}$ \\
\hline-0.728 & 7.685 & 6.573 & 1.00 & 0.00 & C \\
\hline-0.956 & 8.208 & 7.671 & 1.00 & 0.00 & 0 \\
\hline-1.244 & 6.505 & 6.209 & 1.00 & 0.00 & 0 \\
\hline-1.853 & 6.151 & 6.945 & 1.00 & 0.00 & $\mathrm{H}$ \\
\hline 8.511 & 8.608 & 4.597 & 1.00 & 0.00 & C \\
\hline 9.063 & 9.448 & 5.047 & 1.00 & 0.00 & $\mathrm{H}$ \\
\hline 8.517 & 7.782 & 5.319 & 1.00 & 0.00 & $\mathrm{H}$ \\
\hline 9.082 & 8.289 & 3.717 & 1.00 & 0.00 & $\mathrm{H}$ \\
\hline-0.923 & 11.557 & 3.646 & 1.00 & 0.00 & C \\
\hline-1.368 & 11.348 & 4.626 & 1.00 & 0.00 & $\mathrm{H}$ \\
\hline-1.033 & 12.637 & 3.471 & 1.00 & 0.00 & $\mathrm{H}$ \\
\hline-1.523 & 11.031 & 2.889 & 1.00 & 0.00 & $\mathrm{H}$ \\
\hline 8.874 & 0.178 & 8.933 & 1.00 & 0.00 & C \\
\hline 9.690 & 0.876 & 8.768 & 1.00 & 0.00 & $\mathrm{H}$ \\
\hline 9.207 & -1.047 & 9.335 & 1.00 & 0.00 & C \\
\hline 8.475 & -1.826 & 9.442 & 1.00 & 0.00 & $\mathrm{H}$ \\
\hline 10.249 & -1.316 & 9.501 & 1.00 & 0.00 & $\mathrm{H}$ \\
\hline 6.210 & -0.077 & 10.771 & 1.00 & 0.00 & C \\
\hline 5.975 & -1.148 & 10.652 & 1.00 & 0.00 & $\mathrm{H}$ \\
\hline 5.397 & 0.387 & 11.341 & 1.00 & 0.00 & $\mathrm{H}$ \\
\hline 7.118 & 0.001 & 11.382 & 1.00 & 0.00 & $\mathrm{H}$ \\
\hline 1.200 & 1.601 & 10.256 & 1.00 & 0.00 & C \\
\hline 1.866 & 1.702 & 11.115 & 1.00 & 0.00 & $\mathrm{H}$ \\
\hline 0.033 & 0.957 & 10.415 & 1.00 & 0.00 & C \\
\hline
\end{tabular}




\begin{tabular}{|c|c|c|c|c|c|c|c|c|c|c|}
\hline HETATM & 125 & H11 & VIN & 12 & -0.647 & 0.800 & 9.585 & 1.00 & 0.00 & $\mathrm{H}$ \\
\hline HETATM & 126 & $\mathrm{H} 12$ & VIN & 12 & -0.272 & 0.559 & 11.381 & 1.00 & 0.00 & $\mathrm{H}$ \\
\hline \multicolumn{11}{|l|}{ TER } \\
\hline HETATM & 127 & $\mathrm{C} 1$ & MET & 13 & -0.484 & 3.284 & 8.052 & 1.00 & 0.00 & C \\
\hline HETATM & 128 & H11 & $\mathrm{MET}$ & 13 & -0.641 & 4.306 & 7.685 & 1.00 & 0.00 & $\mathrm{H}$ \\
\hline HETATM & 129 & $\mathrm{H} 12$ & MET & 13 & -0.917 & 3.219 & 9.057 & 1.00 & 0.00 & $\mathrm{H}$ \\
\hline HETATM & 130 & $\mathrm{H} 13$ & MET & 13 & -1.076 & 2.620 & 7.403 & 1.00 & 0.00 & $\mathrm{H}$ \\
\hline \multicolumn{11}{|l|}{ TER } \\
\hline HETATM & 131 & C5 & $\mathrm{ACP}$ & 14 & 8.604 & 4.366 & 2.161 & 1.00 & 0.00 & $\mathrm{C}$ \\
\hline HETATM & 132 & H51 & $\mathrm{ACP}$ & 14 & 7.865 & 4.377 & 1.349 & 1.00 & 0.00 & $\mathrm{H}$ \\
\hline HETATM & 133 & H52 & $\mathrm{ACP}$ & 14 & 9.445 & 3.758 & 1.798 & 1.00 & 0.00 & $\mathrm{H}$ \\
\hline HETATM & 134 & $\mathrm{C} 4$ & $\mathrm{ACP}$ & 14 & 9.082 & 5.803 & 2.434 & 1.00 & 0.00 & C \\
\hline HETATM & 135 & $\mathrm{H} 41$ & $\mathrm{ACP}$ & 14 & 9.640 & 5.865 & 3.382 & 1.00 & 0.00 & $\mathrm{H}$ \\
\hline HETATM & 136 & $\mathrm{H} 42$ & $\mathrm{ACP}$ & 14 & 8.212 & 6.470 & 2.541 & 1.00 & 0.00 & $\mathrm{H}$ \\
\hline HETATM & 137 & $\mathrm{C} 1$ & $\mathrm{ACP}$ & 14 & 9.946 & 6.416 & 1.358 & 1.00 & 0.00 & C \\
\hline HETATM & 138 & $\mathrm{O} 2$ & $\mathrm{ACP}$ & 14 & 10.174 & 5.893 & 0.260 & 1.00 & 0.00 & o \\
\hline HETATM & 139 & 03 & $\mathrm{ACP}$ & 14 & 10.462 & 7.595 & 1.722 & 1.00 & 0.00 & 0 \\
\hline HETATM & 140 & $\mathrm{H} 3$ & $\mathrm{ACP}$ & 14 & 11.071 & 7.949 & 0.986 & 1.00 & 0.00 & $\mathrm{H}$ \\
\hline \multicolumn{11}{|l|}{ TER } \\
\hline HETATM & 141 & $\mathrm{C} 1$ & MET & 15 & 0.708 & 5.492 & 3.334 & 1.00 & 0.00 & C \\
\hline HETATM & 142 & H11 & MET & 15 & 0.155 & 4.653 & 2.884 & 1.00 & 0.00 & $\mathrm{H}$ \\
\hline HETATM & 143 & $\mathrm{H} 12$ & MET & 15 & 0.701 & 6.318 & 2.613 & 1.00 & 0.00 & $\mathrm{H}$ \\
\hline HETATM & 144 & H13 & MET & 15 & 0.137 & 5.812 & 4.214 & 1.00 & 0.00 & $\mathrm{H}$ \\
\hline \multicolumn{11}{|c|}{ TER } \\
\hline HETATM & 145 & $\mathrm{C} 1$ & MET & 16 & 10.141 & 2.543 & 4.285 & 1.00 & 0.00 & C \\
\hline HETATM & 146 & H11 & MET & 16 & 10.586 & 2.752 & 3.305 & 1.00 & 0.00 & $\mathrm{H}$ \\
\hline HETATM & 147 & H12 & MET & 16 & 10.252 & 1.463 & 4.460 & 1.00 & 0.00 & $\mathrm{H}$ \\
\hline HETATM & 148 & $\mathrm{H} 13$ & MET & 16 & 10.742 & 3.069 & 5.042 & 1.00 & 0.00 & $\mathrm{H}$ \\
\hline TER & & & & & & & & & & \\
\hline HETATM & 149 & $\mathrm{FE}$ & HEM & 17 & 16.346 & 10.757 & 1.491 & 1.00 & 0.00 & $\mathrm{Fe}$ \\
\hline HETATM & 150 & $\mathrm{C} 1$ & HEM & 17 & 14.118 & 12.629 & 0.429 & 1.00 & 0.00 & C \\
\hline HETATM & 151 & $\mathrm{C} 2$ & HEM & 17 & 13.759 & 13.420 & -0.742 & 1.00 & 0.00 & C \\
\hline HETATM & 152 & $\mathrm{C} 3$ & HEM & 17 & 14.905 & 13.517 & -1.517 & 1.00 & 0.00 & C \\
\hline HETATM & 153 & $\mathrm{C} 4$ & HEM & 17 & 15.934 & 12.800 & -0.809 & 1.00 & 0.00 & C \\
\hline HETATM & 154 & $\mathrm{C} 5$ & HEM & 17 & 17.249 & 12.681 & -1.247 & 1.00 & 0.00 & C \\
\hline HETATM & 155 & H5 & HEM & 17 & 17.503 & 13.177 & -2.182 & 1.00 & 0.00 & $\mathrm{H}$ \\
\hline HETATM & 156 & $\mathrm{C} 6$ & HEM & 17 & 18.269 & 11.982 & -0.611 & 1.00 & 0.00 & C \\
\hline HETATM & 157 & C7 & HEM & 17 & 19.638 & 11.910 & -1.081 & 1.00 & 0.00 & C \\
\hline HETATM & 158 & $\mathrm{C} 8$ & HEM & 17 & 20.343 & 11.190 & -0.134 & 1.00 & 0.00 & C \\
\hline HETATM & 159 & $\mathrm{C} 9$ & HEM & 17 & 19.392 & 10.825 & 0.891 & 1.00 & 0.00 & C \\
\hline HETATM & 160 & $\mathrm{C} 10$ & HEM & 17 & 19.697 & 10.090 & 2.034 & 1.00 & 0.00 & C \\
\hline HETATM & 161 & $\mathrm{H} 10$ & HEM & 17 & 20.723 & 9.746 & 2.140 & 1.00 & 0.00 & $\mathrm{H}$ \\
\hline HETATM & 162 & $\mathrm{C} 11$ & HEM & 17 & 18.822 & 9.750 & 3.057 & 1.00 & 0.00 & C \\
\hline HETATM & 163 & $\mathrm{C} 12$ & HEM & 17 & 19.203 & 9.025 & 4.247 & 1.00 & 0.00 & C \\
\hline HETATM & 164 & $\mathrm{C} 13$ & HEM & 17 & 18.064 & 8.878 & 5.004 & 1.00 & 0.00 & $\mathrm{C}$ \\
\hline HETATM & 165 & C14 & HEM & 17 & 16.991 & 9.519 & 4.273 & 1.00 & 0.00 & $\mathrm{C}$ \\
\hline HETATM & 166 & C15 & HEM & 17 & 15.663 & 9.574 & 4.694 & 1.00 & 0.00 & C \\
\hline HETATM & 167 & H15 & HEM & 17 & 15.423 & 9.089 & 5.642 & 1.00 & 0.00 & $\mathrm{H}$ \\
\hline HETATM & 168 & C16 & HEM & 17 & 14.632 & 10.255 & 4.051 & 1.00 & 0.00 & C \\
\hline HETATM & 169 & $\mathrm{C} 17$ & HEM & 17 & 13.267 & 10.369 & 4.533 & 1.00 & 0.00 & C \\
\hline HETATM & 170 & $\mathrm{C} 18$ & HEM & 17 & 12.591 & 11.137 & 3.609 & 1.00 & 0.00 & C \\
\hline HETATM & 171 & C19 & HEM & 17 & 13.541 & 11.489 & 2.573 & 1.00 & 0.00 & C \\
\hline HETATM & 172 & $\mathrm{C} 20$ & HEM & 17 & 13.250 & 12.280 & 1.462 & 1.00 & 0.00 & C \\
\hline HETATM & 173 & $\mathrm{H} 2 \mathrm{O}$ & HEM & 17 & 12.235 & 12.669 & 1.402 & 1.00 & 0.00 & $\mathrm{H}$ \\
\hline HETATM & 174 & N21 & HEM & 17 & 15.440 & 12.266 & 0.367 & 1.00 & 0.00 & $\mathrm{~N}$ \\
\hline HETATM & 175 & $\mathrm{~N} 22$ & HEM & 17 & 18.144 & 11.319 & 0.590 & 1.00 & 0.00 & $\mathrm{~N}$ \\
\hline HETATM & 176 & N23 & HEM & 17 & 17.474 & 10.056 & 3.090 & 1.00 & 0.00 & $\mathrm{~N}$ \\
\hline HETATM & 177 & N2 4 & HEM & 17 & 14.774 & 10.941 & 2.854 & 1.00 & 0.00 & $\mathrm{~N}$ \\
\hline HETATM & 178 & C99 & HEM & 17 & 15.681 & 8.076 & 0.352 & 1.00 & 0.00 & C \\
\hline HETATM & 179 & 091 & HEM & 17 & 15.840 & 9.389 & 0.365 & 1.00 & 0.00 & o \\
\hline HETATM & 180 & 092 & HEM & 17 & 15.526 & 7.458 & -0.703 & 1.00 & 0.00 & 0 \\
\hline HETATM & 181 & СТ 91 & HEM & 17 & 15.697 & 7.394 & 1.709 & 1.00 & 0.00 & C \\
\hline HETATM & 182 & H911 & HEM & 17 & 16.676 & 7.590 & 2.171 & 1.00 & 0.00 & $\mathrm{H}$ \\
\hline HETATM & 183 & H912 & HEM & 17 & 14.949 & 7.884 & 2.352 & 1.00 & 0.00 & $\mathrm{H}$ \\
\hline HETATM & 184 & СТ 92 & HEM & 17 & 15.437 & 5.890 & 1.597 & 1.00 & 0.00 & C \\
\hline HETATM & 185 & H921 & HEM & 17 & 16.234 & 5.429 & 1.000 & 1.00 & 0.00 & $\mathrm{H}$ \\
\hline HETATM & 186 & H922 & HEM & 17 & 14.512 & 5.734 & 1.027 & 1.00 & 0.00 & $\mathrm{H}$ \\
\hline TER & & & & & & & & & & \\
\hline HETATM & 187 & $\mathrm{FE}$ & HEM & 18 & 17.038 & 3.343 & 6.440 & 1.00 & 0.00 & $\mathrm{Fe}$ \\
\hline HETATM & 188 & $\mathrm{C} 1$ & HEM & 18 & 19.266 & 1.471 & 7.502 & 1.00 & 0.00 & C \\
\hline HETATM & 189 & $\mathrm{C} 2$ & HEM & 18 & 19.625 & 0.680 & 8.674 & 1.00 & 0.00 & C \\
\hline HETATM & 190 & $\mathrm{C} 3$ & HEM & 18 & 18.479 & 0.583 & 9.448 & 1.00 & 0.00 & C \\
\hline HETATM & 191 & $\mathrm{C} 4$ & HEM & 18 & 17.450 & 1.301 & 8.740 & 1.00 & 0.00 & C \\
\hline HETATM & 192 & $\mathrm{C} 5$ & HEM & 18 & 16.135 & 1.420 & 9.178 & 1.00 & 0.00 & C \\
\hline HETATM & 193 & H5 & HEM & 18 & 15.881 & 0.923 & 10.113 & 1.00 & 0.00 & $\mathrm{H}$ \\
\hline HETATM & 194 & $\mathrm{C} 6$ & HEM & 18 & 15.115 & 2.118 & 8.542 & 1.00 & 0.00 & C \\
\hline HETATM & 195 & C7 & HEM & 18 & 13.747 & 2.191 & 9.012 & 1.00 & 0.00 & C \\
\hline HETATM & 196 & C8 & HEM & 18 & 13.041 & 2.910 & 8.065 & 1.00 & 0.00 & C \\
\hline HETATM & 197 & C9 & HEM & 18 & 13.992 & 3.275 & 7.041 & 1.00 & 0.00 & C \\
\hline
\end{tabular}




\begin{tabular}{|c|c|c|c|c|c|c|c|c|c|c|}
\hline HETATM & 198 & $\mathrm{C} 10$ & HEM & 18 & 13.688 & 4.010 & 5.897 & 1.00 & 0.00 & C \\
\hline HETATM & 199 & H1O & HEM & 18 & 12.661 & 4.354 & 5.791 & 1.00 & 0.00 & $\mathrm{H}$ \\
\hline HETATM & 200 & C11 & HEM & 18 & 14.562 & 4.350 & 4.874 & 1.00 & 0.00 & $\mathrm{C}$ \\
\hline HETATM & 201 & $\mathrm{C} 12$ & HEM & 18 & 14.181 & 5.076 & 3.684 & 1.00 & 0.00 & C \\
\hline HETATM & 202 & C13 & HEM & 18 & 15.321 & 5.223 & 2.927 & 1.00 & 0.00 & C \\
\hline HETATM & 203 & C14 & HEM & 18 & 16.394 & 4.581 & 3.659 & 1.00 & 0.00 & C \\
\hline HETATM & 204 & C15 & HEM & 18 & 17.722 & 4.527 & 3.237 & 1.00 & 0.00 & C \\
\hline HETATM & 205 & H15 & HEM & 18 & 17.962 & 5.011 & 2.289 & 1.00 & 0.00 & $\mathrm{H}$ \\
\hline HETATM & 206 & C16 & HEM & 18 & 18.752 & 3.845 & 3.880 & 1.00 & 0.00 & $\mathrm{C}$ \\
\hline HETATM & 207 & C17 & HEM & 18 & 20.118 & 3.732 & 3.398 & 1.00 & 0.00 & $\mathrm{C}$ \\
\hline HETATM & 208 & $\mathrm{C} 18$ & HEM & 18 & 20.793 & 2.964 & 4.322 & 1.00 & 0.00 & C \\
\hline HETATM & 209 & C19 & HEM & 18 & 19.843 & 2.612 & 5.358 & 1.00 & 0.00 & C \\
\hline HETATM & 210 & $\mathrm{C} 20$ & HEM & 18 & 20.135 & 1.821 & 6.469 & 1.00 & 0.00 & C \\
\hline HETATM & 211 & $\mathrm{H} 2 \mathrm{O}$ & HEM & 18 & 21.149 & 1.432 & 6.530 & 1.00 & 0.00 & $\mathrm{H}$ \\
\hline HETATM & 212 & $\mathrm{~N} 21$ & HEM & 18 & 17.944 & 1.834 & 7.564 & 1.00 & 0.00 & $\mathrm{~N}$ \\
\hline HETATM & 213 & N22 & HEM & 18 & 15.240 & 2.781 & 7.342 & 1.00 & 0.00 & $\mathrm{~N}$ \\
\hline HETATM & 214 & N23 & HEM & 18 & 15.911 & 4.044 & 4.841 & 1.00 & 0.00 & $\mathrm{~N}$ \\
\hline HETATM & 215 & $\mathrm{~N} 24$ & HEM & 18 & 18.610 & 3.160 & 5.077 & 1.00 & 0.00 & $\mathrm{~N}$ \\
\hline HETATM & 216 & C99 & HEM & 18 & 17.704 & 6.025 & 7.579 & 1.00 & 0.00 & C \\
\hline HETATM & 217 & 091 & HEM & 18 & 17.544 & 4.711 & 7.567 & 1.00 & 0.00 & 0 \\
\hline HETATM & 218 & 092 & HEM & 18 & 17.858 & 6.642 & 8.635 & 1.00 & 0.00 & 0 \\
\hline HETATM & 219 & СТ 91 & HEM & 18 & 17.688 & 6.706 & 6.223 & 1.00 & 0.00 & C \\
\hline HETATM & 220 & H911 & HEM & 18 & 16.709 & 6.511 & 5.760 & 1.00 & 0.00 & $\mathrm{H}$ \\
\hline HETATM & 221 & H912 & HEM & 18 & 18.436 & 6.216 & 5.580 & 1.00 & 0.00 & $\mathrm{H}$ \\
\hline HETATM & 222 & СТ 92 & HEM & 18 & 17.947 & 8.211 & 6.334 & 1.00 & 0.00 & C \\
\hline HETATM & 223 & H921 & HEM & 18 & 18.872 & 8.367 & 6.905 & 1.00 & 0.00 & $\mathrm{H}$ \\
\hline HETATM & 224 & H922 & HEM & 18 & 17.151 & 8.671 & 6.931 & 1.00 & 0.00 & $\mathrm{H}$ \\
\hline \multicolumn{11}{|l|}{ TER } \\
\hline HETATM & 225 & $\mathrm{C} 2$ & VIN & 19 & 12.427 & 13.923 & -1.002 & 1.00 & 0.00 & $\mathrm{C}$ \\
\hline HETATM & 226 & H2 1 & VIN & 19 & 11.612 & 13.224 & -0.837 & 1.00 & 0.00 & $\mathrm{H}$ \\
\hline HETATM & 227 & $\mathrm{C} 1$ & VIN & 19 & 12.095 & 15.147 & -1.403 & 1.00 & 0.00 & C \\
\hline HETATM & 228 & H11 & VIN & 19 & 12.826 & 15.926 & -1.511 & 1.00 & 0.00 & $\mathrm{H}$ \\
\hline HETATM & 229 & $\mathrm{H} 12$ & VIN & 19 & 11.053 & 15.417 & -1.570 & 1.00 & 0.00 & $\mathrm{H}$ \\
\hline \multicolumn{11}{|l|}{ TER } \\
\hline HETATM & 230 & $\mathrm{C} 1$ & MET & 20 & 15.091 & 14.177 & -2.840 & 1.00 & 0.00 & C \\
\hline HETATM & 231 & H11 & MET & 20 & 15.326 & 15.248 & -2.720 & 1.00 & 0.00 & $\mathrm{H}$ \\
\hline HETATM & 232 & $\mathrm{H} 12$ & MET & 20 & 15.904 & 13.713 & -3.410 & 1.00 & 0.00 & $\mathrm{H}$ \\
\hline HETATM & 233 & H13 & MET & 20 & 14.184 & 14.100 & -3.450 & 1.00 & 0.00 & $\mathrm{H}$ \\
\hline \multicolumn{11}{|l|}{ TER } \\
\hline HETATM & 234 & $\mathrm{C} 2$ & VIN & 21 & 20.101 & 12.499 & -2.325 & 1.00 & 0.00 & C \\
\hline HETATM & 235 & H 21 & VIN & 21 & 19.435 & 12.399 & -3.184 & 1.00 & 0.00 & $\mathrm{H}$ \\
\hline HETATM & 236 & $\mathrm{C} 1$ & VIN & 21 & 21.268 & 13.144 & -2.484 & 1.00 & 0.00 & C \\
\hline HETATM & 237 & H11 & VIN & 21 & 21.948 & 13.300 & -1.654 & 1.00 & 0.00 & $\mathrm{H}$ \\
\hline HETATM & 238 & $\mathrm{H} 12$ & VIN & 21 & 21.573 & 13.542 & -3.450 & 1.00 & 0.00 & $\mathrm{H}$ \\
\hline \multicolumn{11}{|l|}{ TER } \\
\hline HETATM & 239 & $\mathrm{C} 1$ & MET & 22 & 21.786 & 10.816 & -0.121 & 1.00 & 0.00 & $\mathrm{C}$ \\
\hline HETATM & 240 & H11 & MET & 22 & 22.377 & 11.480 & 0.528 & 1.00 & 0.00 & $\mathrm{H}$ \\
\hline HETATM & 241 & $\mathrm{H} 12$ & MET & 22 & 21.942 & 9.794 & 0.246 & 1.00 & 0.00 & $\mathrm{H}$ \\
\hline HETATM & 242 & H13 & MET & 22 & 22.218 & 10.882 & -1.126 & 1.00 & 0.00 & $\mathrm{H}$ \\
\hline TER & & & & & & & & & & \\
\hline HETATM & 243 & C5 & $\mathrm{ACP}$ & 23 & 12.697 & 9.735 & 5.770 & 1.00 & 0.00 & C \\
\hline HETATM & 244 & H51 & $\mathrm{ACP}$ & 23 & 13.436 & 9.723 & 6.582 & 1.00 & 0.00 & $\mathrm{H}$ \\
\hline HETATM & 245 & H52 & $\mathrm{ACP}$ & 23 & 11.857 & 10.343 & 6.134 & 1.00 & 0.00 & $\mathrm{H}$ \\
\hline HETATM & 246 & $\mathrm{C} 4$ & $\mathrm{ACP}$ & 23 & 12.219 & 8.297 & 5.497 & 1.00 & 0.00 & C \\
\hline HETATM & 247 & $\mathrm{H} 4 \mathrm{I}$ & $\mathrm{ACP}$ & 23 & 11.661 & 8.236 & 4.549 & 1.00 & 0.00 & $\mathrm{H}$ \\
\hline HETATM & 248 & $\mathrm{H} 42$ & $\mathrm{ACP}$ & 23 & 13.089 & 7.630 & 5.390 & 1.00 & 0.00 & $\mathrm{H}$ \\
\hline HETATM & 249 & $\mathrm{C} 1$ & $\mathrm{ACP}$ & 23 & 11.355 & 7.685 & 6.573 & 1.00 & 0.00 & C \\
\hline HETATM & 250 & $\mathrm{O} 2$ & $\mathrm{ACP}$ & 23 & 11.127 & 8.208 & 7.671 & 1.00 & 0.00 & 0 \\
\hline HETATM & 251 & 03 & $\mathrm{ACP}$ & 23 & 10.839 & 6.505 & 6.209 & 1.00 & 0.00 & 0 \\
\hline HETATM & 252 & H3 & $\mathrm{ACP}$ & 23 & 10.230 & 6.151 & 6.945 & 1.00 & 0.00 & $\mathrm{H}$ \\
\hline TER & & & & & & & & & & \\
\hline HETATM & 253 & $\mathrm{C} 1$ & MET & 24 & 20.594 & 8.608 & 4.597 & 1.00 & 0.00 & C \\
\hline HETATM & 254 & H11 & MET & 24 & 21.146 & 9.448 & 5.047 & 1.00 & 0.00 & $\mathrm{H}$ \\
\hline HETATM & 255 & $\mathrm{H} 12$ & MET & 24 & 20.600 & 7.782 & 5.319 & 1.00 & 0.00 & $\mathrm{H}$ \\
\hline HETATM & 256 & H13 & MET & 24 & 21.165 & 8.289 & 3.717 & 1.00 & 0.00 & $\mathrm{H}$ \\
\hline TER & & & & & & & & & & \\
\hline HETATM & 257 & $\mathrm{C} 1$ & MET & 25 & 11.160 & 11.557 & 3.646 & 1.00 & 0.00 & C \\
\hline HETATM & 258 & H11 & MET & 25 & 10.715 & 11.348 & 4.626 & 1.00 & 0.00 & $\mathrm{H}$ \\
\hline HETATM & 259 & $\mathrm{H} 12$ & MET & 25 & 11.050 & 12.637 & 3.471 & 1.00 & 0.00 & $\mathrm{H}$ \\
\hline HETATM & 260 & H13 & MET & 25 & 10.559 & 11.031 & 2.889 & 1.00 & 0.00 & $\mathrm{H}$ \\
\hline TER & & & & & & & & & & \\
\hline HETATM & 261 & $\mathrm{C} 2$ & VIN & 26 & 20.957 & 0.178 & 8.933 & 1.00 & 0.00 & C \\
\hline HETATM & 262 & H 21 & VIN & 26 & 21.773 & 0.876 & 8.768 & 1.00 & 0.00 & $\mathrm{H}$ \\
\hline HETATM & 263 & $\mathrm{C} 1$ & VIN & 26 & 21.290 & -1.047 & 9.335 & 1.00 & 0.00 & C \\
\hline HETATM & 264 & H11 & VIN & 26 & 20.558 & -1.826 & 9.442 & 1.00 & 0.00 & $\mathrm{H}$ \\
\hline HETATM & 265 & $\mathrm{H} 12$ & VIN & 26 & 22.331 & -1.316 & 9.501 & 1.00 & 0.00 & $\mathrm{H}$ \\
\hline TER & & & & & & & & & & \\
\hline HETATM & 266 & $\mathrm{C} 1$ & MET & 27 & 18.293 & -0.077 & 10.771 & 1.00 & 0.00 & C \\
\hline HETATM & 267 & H11 & MET & 27 & 18.058 & -1.148 & 10.652 & 1.00 & 0.00 & \\
\hline
\end{tabular}




\begin{tabular}{|c|c|c|c|c|c|c|c|c|c|c|}
\hline HETATM & 268 & $\mathrm{H} 12$ & MET & 27 & 17.480 & 0.387 & 11.341 & 1.00 & 0.00 & $\mathrm{H}$ \\
\hline HETATM & 269 & $\mathrm{H} 13$ & MET & 27 & 19.201 & 0.001 & 11.382 & 1.00 & 0.00 & $\mathrm{H}$ \\
\hline \multicolumn{11}{|l|}{ TER } \\
\hline HETATM & 270 & $\mathrm{C} 2$ & VIN & 28 & 13.283 & 1.601 & 10.256 & 1.00 & 0.00 & C \\
\hline HETATM & 271 & H21 & VIN & 28 & 13.949 & 1.702 & 11.115 & 1.00 & 0.00 & $\mathrm{H}$ \\
\hline HETATM & 272 & $\mathrm{C} 1$ & VIN & 28 & 12.116 & 0.957 & 10.415 & 1.00 & 0.00 & C \\
\hline HETATM & 273 & $\mathrm{H} 11$ & VIN & 28 & 11.436 & 0.800 & 9.585 & 1.00 & 0.00 & $\mathrm{H}$ \\
\hline HETATM & 274 & $\mathrm{H} 12$ & VIN & 28 & 11.811 & 0.559 & 11.381 & 1.00 & 0.00 & $\mathrm{H}$ \\
\hline \multicolumn{11}{|l|}{ TER } \\
\hline HETATM & 275 & $\mathrm{C} 1$ & $\mathrm{MET}$ & 29 & 11.598 & 3.284 & 8.052 & 1.00 & 0.00 & C \\
\hline HETATM & 276 & $\mathrm{H} 11$ & $\mathrm{MET}$ & 29 & 11.442 & 4.306 & 7.685 & 1.00 & 0.00 & $\mathrm{H}$ \\
\hline HETATM & 277 & $\mathrm{H} 12$ & $\mathrm{MET}$ & 29 & 11.166 & 3.219 & 9.057 & 1.00 & 0.00 & $\mathrm{H}$ \\
\hline HETATM & 278 & $\mathrm{H} 13$ & $\mathrm{MET}$ & 29 & 11.007 & 2.620 & 7.403 & 1.00 & 0.00 & $\mathrm{H}$ \\
\hline \multicolumn{11}{|l|}{ TER } \\
\hline HETATM & 279 & C5 & $\mathrm{ACP}$ & 30 & 20.687 & 4.366 & 2.161 & 1.00 & 0.00 & C \\
\hline HETATM & 280 & H51 & $\mathrm{ACP}$ & 30 & 19.948 & 4.377 & 1.349 & 1.00 & 0.00 & $\mathrm{H}$ \\
\hline HETATM & 281 & H52 & $\mathrm{ACP}$ & 30 & 21.527 & 3.758 & 1.798 & 1.00 & 0.00 & $\mathrm{H}$ \\
\hline HETATM & 282 & $\mathrm{C} 4$ & $\mathrm{ACP}$ & 30 & 21.165 & 5.803 & 2.434 & 1.00 & 0.00 & C \\
\hline HETATM & 283 & H4 1 & $\mathrm{ACP}$ & 30 & 21.723 & 5.865 & 3.382 & 1.00 & 0.00 & $\mathrm{H}$ \\
\hline HETATM & 284 & $\mathrm{H} 42$ & $\mathrm{ACP}$ & 30 & 20.295 & 6.470 & 2.541 & 1.00 & 0.00 & $\mathrm{H}$ \\
\hline HETATM & 285 & $\mathrm{C} 1$ & $\mathrm{ACP}$ & 30 & 22.029 & 6.416 & 1.358 & 1.00 & 0.00 & C \\
\hline HETATM & 286 & $\mathrm{O} 2$ & $\mathrm{ACP}$ & 30 & 22.257 & 5.893 & 0.260 & 1.00 & 0.00 & 0 \\
\hline HETATM & 287 & 03 & $\mathrm{ACP}$ & 30 & 22.545 & 7.595 & 1.722 & 1.00 & 0.00 & O \\
\hline HETATM & 288 & H3 & $\mathrm{ACP}$ & 30 & 23.154 & 7.949 & 0.986 & 1.00 & 0.00 & $\mathrm{H}$ \\
\hline \multicolumn{11}{|l|}{ TER } \\
\hline HETATM & 289 & $\mathrm{C} 1$ & $\mathrm{MET}$ & 31 & 12.791 & 5.492 & 3.334 & 1.00 & 0.00 & C \\
\hline HETATM & 290 & $\mathrm{H} 11$ & MET & 31 & 12.238 & 4.653 & 2.884 & 1.00 & 0.00 & $\mathrm{H}$ \\
\hline HETATM & 291 & $\mathrm{H} 12$ & $\mathrm{MET}$ & 31 & 12.784 & 6.318 & 2.613 & 1.00 & 0.00 & $\mathrm{H}$ \\
\hline HETATM & 292 & H13 & $\mathrm{MET}$ & 31 & 12.220 & 5.812 & 4.214 & 1.00 & 0.00 & $\mathrm{H}$ \\
\hline \multicolumn{11}{|l|}{ TER } \\
\hline HETATM & 293 & $\mathrm{C} 1$ & $\mathrm{MET}$ & 32 & 22.224 & 2.543 & 4.285 & 1.00 & 0.00 & C \\
\hline HETATM & 294 & H11 & MET & 32 & 22.669 & 2.752 & 3.305 & 1.00 & 0.00 & $\mathrm{H}$ \\
\hline HETATM & 295 & $\mathrm{H} 12$ & MET & 32 & 22.335 & 1.463 & 4.460 & 1.00 & 0.00 & $\mathrm{H}$ \\
\hline HETATM & 296 & H13 & $\mathrm{MET}$ & 32 & 22.825 & 3.069 & 5.042 & 1.00 & 0.00 & $\mathrm{H}$ \\
\hline
\end{tabular}

TER

END

\subsection{D4 dimer: D05 D4.pdb file}

\begin{tabular}{|c|c|c|c|c|c|c|c|c|c|c|}
\hline HETATM & 1 & $\mathrm{FE}$ & HEM & 1 & 4.263 & 10.757 & 1.491 & 1.00 & 0.00 & $\mathrm{Fe}$ \\
\hline HETATM & 2 & $\mathrm{C} 1$ & HEM & 1 & 2.035 & 12.629 & 0.429 & 1.00 & 0.00 & C \\
\hline HETATM & 3 & $\mathrm{C} 2$ & HEM & 1 & 1.676 & 13.420 & -0.742 & 1.00 & 0.00 & C \\
\hline HETATM & 4 & C3 & HEM & 1 & 2.822 & 13.517 & -1.517 & 1.00 & 0.00 & $\mathrm{C}$ \\
\hline HETATM & 5 & $\mathrm{C} 4$ & HEM & 1 & 3.851 & 12.800 & -0.809 & 1.00 & 0.00 & $\mathrm{C}$ \\
\hline HETATM & 6 & $\mathrm{C} 5$ & HEM & 1 & 5.167 & 12.681 & -1.247 & 1.00 & 0.00 & $\mathrm{C}$ \\
\hline HETATM & 7 & H5 & HEM & 1 & 5.420 & 13.177 & -2.182 & 1.00 & 0.00 & $\mathrm{H}$ \\
\hline HETATM & 8 & $\mathrm{C} 6$ & HEM & 1 & 6.186 & 11.982 & -0.611 & 1.00 & 0.00 & C \\
\hline HETATM & 9 & $\mathrm{C} 7$ & HEM & 1 & 7.555 & 11.910 & -1.081 & 1.00 & 0.00 & $\mathrm{C}$ \\
\hline HETATM & 10 & $\mathrm{C} 8$ & HEM & 1 & 8.260 & 11.190 & -0.134 & 1.00 & 0.00 & C \\
\hline HETATM & 11 & $\mathrm{C} 9$ & HEM & 1 & 7.309 & 10.825 & 0.891 & 1.00 & 0.00 & C \\
\hline HETATM & 12 & $\mathrm{C} 10$ & HEM & 1 & 7.614 & 10.090 & 2.034 & 1.00 & 0.00 & C \\
\hline HETATM & 13 & $\mathrm{H} 10$ & HEM & 1 & 8.641 & 9.746 & 2.140 & 1.00 & 0.00 & $\mathrm{H}$ \\
\hline HETATM & 14 & $\mathrm{C} 11$ & HEM & 1 & 6.739 & 9.750 & 3.057 & 1.00 & 0.00 & C \\
\hline HETATM & 15 & $\mathrm{C} 12$ & HEM & 1 & 7.120 & 9.025 & 4.247 & 1.00 & 0.00 & C \\
\hline HETATM & 16 & $\mathrm{C} 13$ & HEM & 1 & 5.981 & 8.878 & 5.004 & 1.00 & 0.00 & $\mathrm{C}$ \\
\hline HETATM & 17 & C14 & HEM & 1 & 4.908 & 9.519 & 4.273 & 1.00 & 0.00 & C \\
\hline HETATM & 18 & C15 & HEM & 1 & 3.580 & 9.574 & 4.694 & 1.00 & 0.00 & $\mathrm{C}$ \\
\hline HETATM & 19 & H15 & HEM & 1 & 3.340 & 9.089 & 5.642 & 1.00 & 0.00 & $\mathrm{H}$ \\
\hline HETATM & 20 & $\mathrm{C} 16$ & HEM & 1 & 2.550 & 10.255 & 4.051 & 1.00 & 0.00 & C \\
\hline HETATM & 21 & $\mathrm{C} 17$ & HEM & 1 & 1.184 & 10.369 & 4.533 & 1.00 & 0.00 & C \\
\hline HETATM & 22 & $\mathrm{C} 18$ & HEM & 1 & 0.508 & 11.137 & 3.609 & 1.00 & 0.00 & C \\
\hline HETATM & 23 & C19 & HEM & 1 & 1.458 & 11.489 & 2.573 & 1.00 & 0.00 & $\mathrm{C}$ \\
\hline HETATM & 24 & $\mathrm{C} 20$ & HEM & 1 & 1.167 & 12.280 & 1.462 & 1.00 & 0.00 & $\mathrm{C}$ \\
\hline HETATM & 25 & $\mathrm{H} 20$ & HEM & 1 & 0.152 & 12.669 & 1.402 & 1.00 & 0.00 & $\mathrm{H}$ \\
\hline HETATM & 26 & $\mathrm{~N} 21$ & HEM & 1 & 3.357 & 12.266 & 0.367 & 1.00 & 0.00 & $\mathrm{~N}$ \\
\hline HETATM & 27 & $\mathrm{~N} 22$ & HEM & 1 & 6.062 & 11.319 & 0.590 & 1.00 & 0.00 & $\mathrm{~N}$ \\
\hline HETATM & 28 & $\mathrm{~N} 23$ & HEM & 1 & 5.391 & 10.056 & 3.090 & 1.00 & 0.00 & $\mathrm{~N}$ \\
\hline HETATM & 29 & $\mathrm{~N} 24$ & HEM & 1 & 2.691 & 10.941 & 2.854 & 1.00 & 0.00 & $\mathrm{~N}$ \\
\hline HETATM & 30 & C99 & HEM & 1 & 3.598 & 8.076 & 0.352 & 1.00 & 0.00 & C \\
\hline HETATM & 31 & 091 & HEM & 1 & 3.757 & 9.389 & 0.365 & 1.00 & 0.00 & o \\
\hline HETATM & 32 & 092 & HEM & 1 & 3.443 & 7.458 & -0.703 & 1.00 & 0.00 & 0 \\
\hline HETATM & 33 & СТ 91 & HEM & 1 & 3.614 & 7.394 & 1.709 & 1.00 & 0.00 & C \\
\hline HETATM & 34 & H911 & HEM & 1 & 4.593 & 7.590 & 2.171 & 1.00 & 0.00 & $\mathrm{H}$ \\
\hline HETATM & 35 & H912 & HEM & 1 & 2.866 & 7.884 & 2.352 & 1.00 & 0.00 & $\mathrm{H}$ \\
\hline HETATM & 36 & CT92 & HEM & 1 & 3.354 & 5.890 & 1.597 & 1.00 & 0.00 & C \\
\hline HETATM & 37 & H921 & HEM & 1 & 4.151 & 5.429 & 1.000 & 1.00 & 0.00 & $\mathrm{H}$ \\
\hline HETATM & 38 & H922 & HEM & 1 & 2.429 & 5.734 & 1.027 & 1.00 & 0.00 & $\mathrm{H}$ \\
\hline
\end{tabular}




\begin{tabular}{|c|c|c|c|c|c|c|c|c|c|c|}
\hline HETATM & 39 & $\mathrm{FE}$ & HEM & 2 & 4.955 & 3.343 & 6.440 & 1.00 & 0.00 & $\mathrm{Fe}$ \\
\hline HETATM & 40 & $\mathrm{C} 1$ & HEM & 2 & 7.183 & 1.471 & 7.502 & 1.00 & 0.00 & C \\
\hline HETATM & 41 & $\mathrm{C} 2$ & HEM & 2 & 7.542 & 0.680 & 8.674 & 1.00 & 0.00 & C \\
\hline HETATM & 42 & $\mathrm{C} 3$ & HEM & 2 & 6.397 & 0.583 & 9.448 & 1.00 & 0.00 & C \\
\hline HETATM & 43 & $\mathrm{C} 4$ & HEM & 2 & 5.367 & 1.301 & 8.740 & 1.00 & 0.00 & C \\
\hline HETATM & 44 & C5 & HEM & 2 & 4.052 & 1.420 & 9.178 & 1.00 & 0.00 & C \\
\hline HETATM & 45 & H5 & HEM & 2 & 3.798 & 0.923 & 10.113 & 1.00 & 0.00 & $\mathrm{H}$ \\
\hline HETATM & 46 & $\mathrm{C} 6$ & HEM & 2 & 3.033 & 2.118 & 8.542 & 1.00 & 0.00 & C \\
\hline HETATM & 47 & C7 & HEM & 2 & 1.664 & 2.191 & 9.012 & 1.00 & 0.00 & $\mathrm{C}$ \\
\hline HETATM & 48 & C8 & HEM & 2 & 0.958 & 2.910 & 8.065 & 1.00 & 0.00 & $\mathrm{C}$ \\
\hline HETATM & 49 & $\mathrm{C} 9$ & HEM & 2 & 1.909 & 3.275 & 7.041 & 1.00 & 0.00 & C \\
\hline HETATM & 50 & $\mathrm{C} 10$ & HEM & 2 & 1.605 & 4.010 & 5.897 & 1.00 & 0.00 & C \\
\hline HETATM & 51 & $\mathrm{H} 10$ & HEM & 2 & 0.578 & 4.354 & 5.791 & 1.00 & 0.00 & $\mathrm{H}$ \\
\hline HETATM & 52 & $\mathrm{C} 11$ & HEM & 2 & 2.479 & 4.350 & 4.874 & 1.00 & 0.00 & C \\
\hline HETATM & 53 & $\mathrm{C} 12$ & HEM & 2 & 2.098 & 5.076 & 3.684 & 1.00 & 0.00 & C \\
\hline HETATM & 54 & $\mathrm{C} 13$ & HEM & 2 & 3.238 & 5.223 & 2.927 & 1.00 & 0.00 & C \\
\hline HETATM & 55 & C14 & HEM & 2 & 4.311 & 4.581 & 3.659 & 1.00 & 0.00 & C \\
\hline HETATM & 56 & $\mathrm{C} 15$ & HEM & 2 & 5.639 & 4.527 & 3.237 & 1.00 & 0.00 & C \\
\hline HETATM & 57 & H15 & HEM & 2 & 5.879 & 5.011 & 2.289 & 1.00 & 0.00 & $\mathrm{H}$ \\
\hline HETATM & 58 & C16 & HEM & 2 & 6.669 & 3.845 & 3.880 & 1.00 & 0.00 & C \\
\hline HETATM & 59 & C17 & HEM & 2 & 8.035 & 3.732 & 3.398 & 1.00 & 0.00 & C \\
\hline HETATM & 60 & $\mathrm{C} 18$ & HEM & 2 & 8.710 & 2.964 & 4.322 & 1.00 & 0.00 & C \\
\hline HETATM & 61 & C19 & HEM & 2 & 7.761 & 2.612 & 5.358 & 1.00 & 0.00 & C \\
\hline HETATM & 62 & $\mathrm{C} 20$ & HEM & 2 & 8.052 & 1.821 & 6.469 & 1.00 & 0.00 & C \\
\hline HETATM & 63 & $\mathrm{H} 20$ & HEM & 2 & 9.066 & 1.432 & 6.530 & 1.00 & 0.00 & $\mathrm{H}$ \\
\hline HETATM & 64 & $\mathrm{~N} 21$ & HEM & 2 & 5.862 & 1.834 & 7.564 & 1.00 & 0.00 & $\mathrm{~N}$ \\
\hline HETATM & 65 & N22 & HEM & 2 & 3.157 & 2.781 & 7.342 & 1.00 & 0.00 & $\mathrm{~N}$ \\
\hline HETATM & 66 & $\mathrm{~N} 23$ & HEM & 2 & 3.828 & 4.044 & 4.841 & 1.00 & 0.00 & $\mathrm{~N}$ \\
\hline HETATM & 67 & N2 4 & HEM & 2 & 6.527 & 3.160 & 5.077 & 1.00 & 0.00 & $\mathrm{~N}$ \\
\hline HETATM & 68 & C99 & HEM & 2 & 5.621 & 6.025 & 7.579 & 1.00 & 0.00 & $\mathrm{C}$ \\
\hline HETATM & 69 & 091 & HEM & 2 & 5.461 & 4.711 & 7.567 & 1.00 & 0.00 & 0 \\
\hline HETATM & 70 & 092 & HEM & 2 & 5.776 & 6.642 & 8.635 & 1.00 & 0.00 & 0 \\
\hline HETATM & 71 & СТ91 & HEM & 2 & 5.605 & 6.706 & 6.223 & 1.00 & 0.00 & C \\
\hline HETATM & 72 & H911 & HEM & 2 & 4.626 & 6.511 & 5.760 & 1.00 & 0.00 & $\mathrm{H}$ \\
\hline HETATM & 73 & H912 & HEM & 2 & 6.353 & 6.216 & 5.580 & 1.00 & 0.00 & $\mathrm{H}$ \\
\hline HETATM & 74 & СТ92 & HEM & 2 & 5.864 & 8.211 & 6.334 & 1.00 & 0.00 & C \\
\hline HETATM & 75 & H921 & HEM & 2 & 6.789 & 8.367 & 6.905 & 1.00 & 0.00 & $\mathrm{H}$ \\
\hline HETATM & 76 & H922 & HEM & 2 & 5.068 & 8.671 & 6.931 & 1.00 & 0.00 & $\mathrm{H}$ \\
\hline \multicolumn{11}{|l|}{ TER } \\
\hline HETATM & 77 & $\mathrm{C} 2$ & VIN & 3 & 0.345 & 13.923 & -1.002 & 1.00 & 0.00 & C \\
\hline HETATM & 78 & $\mathrm{H} 21$ & VIN & 3 & -0.471 & 13.224 & -0.837 & 1.00 & 0.00 & $\mathrm{H}$ \\
\hline HETATM & 79 & $\mathrm{C} 1$ & VIN & 3 & 0.012 & 15.147 & -1.403 & 1.00 & 0.00 & C \\
\hline HETATM & 80 & H11 & VIN & 3 & 0.743 & 15.926 & -1.511 & 1.00 & 0.00 & $\mathrm{H}$ \\
\hline HETATM & 81 & $\mathrm{H} 12$ & VIN & 3 & -1.030 & 15.417 & -1.570 & 1.00 & 0.00 & $\mathrm{H}$ \\
\hline \multicolumn{11}{|l|}{ TER } \\
\hline HETATM & 82 & $\mathrm{C} 1$ & $\mathrm{MET}$ & 4 & 3.008 & 14.177 & -2.840 & 1.00 & 0.00 & C \\
\hline HETATM & 83 & H11 & $\mathrm{MET}$ & 4 & 3.243 & 15.248 & -2.720 & 1.00 & 0.00 & $\mathrm{H}$ \\
\hline HETATM & 84 & $\mathrm{H} 12$ & $\mathrm{MET}$ & 4 & 3.821 & 13.713 & -3.410 & 1.00 & 0.00 & $\mathrm{H}$ \\
\hline HETATM & 85 & H13 & MET & 4 & 2.101 & 14.100 & -3.450 & 1.00 & 0.00 & $\mathrm{H}$ \\
\hline \multicolumn{11}{|l|}{ TER } \\
\hline HETATM & 86 & $\mathrm{C} 2$ & VIN & 5 & 8.018 & 12.499 & -2.325 & 1.00 & 0.00 & C \\
\hline HETATM & 87 & H2 1 & VIN & 5 & 7.352 & 12.399 & -3.184 & 1.00 & 0.00 & $\mathrm{H}$ \\
\hline HETATM & 88 & $\mathrm{C} 1$ & VIN & 5 & 9.185 & 13.144 & -2.484 & 1.00 & 0.00 & $\mathrm{C}$ \\
\hline HETATM & 89 & $\mathrm{H} 11$ & VIN & 5 & 9.865 & 13.300 & -1.654 & 1.00 & 0.00 & $\mathrm{H}$ \\
\hline HETATM & 90 & $\mathrm{H} 12$ & VIN & 5 & 9.490 & 13.542 & -3.450 & 1.00 & 0.00 & $\mathrm{H}$ \\
\hline \multicolumn{11}{|l|}{ TER } \\
\hline HETATM & 91 & $\mathrm{C} 1$ & $\mathrm{MET}$ & 6 & 9.703 & 10.816 & -0.121 & 1.00 & 0.00 & C \\
\hline HETATM & 92 & H11 & $\mathrm{MET}$ & 6 & 10.294 & 11.480 & 0.528 & 1.00 & 0.00 & $\mathrm{H}$ \\
\hline HETATM & 93 & $\mathrm{H} 12$ & $\mathrm{MET}$ & 6 & 9.859 & 9.794 & 0.246 & 1.00 & 0.00 & $\mathrm{H}$ \\
\hline HETATM & 94 & $\mathrm{H} 13$ & MET & 6 & 10.135 & 10.882 & -1.126 & 1.00 & 0.00 & $\mathrm{H}$ \\
\hline TER & & & & & & & & & & \\
\hline HETATM & 95 & C5 & $\mathrm{ACP}$ & 7 & 0.615 & 9.735 & 5.770 & 1.00 & 0.00 & C \\
\hline HETATM & 96 & H51 & $\mathrm{ACP}$ & 7 & 1.354 & 9.723 & 6.582 & 1.00 & 0.00 & $\mathrm{H}$ \\
\hline HETATM & 97 & H52 & $\mathrm{ACP}$ & 7 & -0.226 & 10.343 & 6.134 & 1.00 & 0.00 & $\mathrm{H}$ \\
\hline HETATM & 98 & $\mathrm{C} 4$ & $\mathrm{ACP}$ & 7 & 0.136 & 8.297 & 5.497 & 1.00 & 0.00 & C \\
\hline HETATM & 99 & $\mathrm{H} 4 \mathrm{I}$ & $\mathrm{ACP}$ & 7 & -0.422 & 8.236 & 4.549 & 1.00 & 0.00 & $\mathrm{H}$ \\
\hline HETATM & 100 & $\mathrm{H} 42$ & $\mathrm{ACP}$ & 7 & 1.007 & 7.630 & 5.390 & 1.00 & 0.00 & $\mathrm{H}$ \\
\hline HETATM & 101 & $\mathrm{C} 1$ & $\mathrm{ACP}$ & 7 & -0.728 & 7.685 & 6.573 & 1.00 & 0.00 & C \\
\hline HETATM & 102 & $\mathrm{O} 2$ & $\mathrm{ACP}$ & 7 & -0.956 & 8.208 & 7.671 & 1.00 & 0.00 & 0 \\
\hline HETATM & 103 & 03 & $\mathrm{ACP}$ & 7 & -1.244 & 6.505 & 6.209 & 1.00 & 0.00 & 0 \\
\hline HETATM & 104 & H3 & $\mathrm{ACP}$ & 7 & -1.853 & 6.151 & 6.945 & 1.00 & 0.00 & $\mathrm{H}$ \\
\hline TER & & & & & & & & & & \\
\hline HETATM & 105 & $\mathrm{C} 1$ & $\mathrm{MET}$ & 8 & 8.511 & 8.608 & 4.597 & 1.00 & 0.00 & C \\
\hline HETATM & 106 & H11 & MET & 8 & 9.063 & 9.448 & 5.047 & 1.00 & 0.00 & $\mathrm{H}$ \\
\hline HETATM & 107 & $\mathrm{H} 12$ & MET & 8 & 8.517 & 7.782 & 5.319 & 1.00 & 0.00 & $\mathrm{H}$ \\
\hline HETATM & 108 & $\mathrm{H} 13$ & MET & 8 & 9.082 & 8.289 & 3.717 & 1.00 & 0.00 & $\mathrm{H}$ \\
\hline TER & & & & & & & & & & \\
\hline HETATM & 109 & $\mathrm{C} 1$ & $\mathrm{MET}$ & 9 & -0.923 & 11.557 & 3.646 & 1.00 & 0.00 & C \\
\hline HETATM & 110 & H11 & MET & 9 & -1.368 & 11.348 & 4.626 & 1.00 & 0.00 & $\mathrm{H}$ \\
\hline
\end{tabular}




\begin{tabular}{|c|c|c|c|}
\hline HETATM & 111 & $\mathrm{H} 12$ & MET \\
\hline HETATM & 112 & H13 & MET \\
\hline \multicolumn{4}{|l|}{ TER } \\
\hline HETATM & 113 & $\mathrm{C} 2$ & VIN \\
\hline HETATM & 114 & H 21 & VIN \\
\hline HETATM & 115 & $\mathrm{C} 1$ & VIN \\
\hline HETATM & 116 & H11 & VIN \\
\hline HETATM & 117 & $\mathrm{H} 12$ & VIN \\
\hline \multicolumn{4}{|l|}{ TER } \\
\hline HETATM & 118 & $\mathrm{C} 1$ & $\mathrm{MET}$ \\
\hline HETATM & 119 & H11 & MET \\
\hline HETATM & 120 & $\mathrm{H} 12$ & MET \\
\hline HETATM & 121 & H13 & MET \\
\hline \multicolumn{4}{|l|}{ TER } \\
\hline HETATM & 122 & $\mathrm{C} 2$ & VIN \\
\hline HETATM & 123 & H21 & VIN \\
\hline HETATM & 124 & $\mathrm{C} 1$ & VIN \\
\hline HETATM & 125 & H11 & VIN \\
\hline HETATM & 126 & $\mathrm{H} 12$ & VIN \\
\hline \multicolumn{4}{|l|}{ TER } \\
\hline HETATM & 127 & $\mathrm{C} 1$ & MET \\
\hline HETATM & 128 & H11 & MET \\
\hline HETATM & 129 & H12 & MET \\
\hline HETATM & 130 & H13 & MET \\
\hline \multicolumn{4}{|l|}{ TER } \\
\hline HETATM & 131 & C5 & $\mathrm{ACP}$ \\
\hline HETATM & 132 & H51 & $\mathrm{ACP}$ \\
\hline HETATM & 133 & H52 & $\mathrm{ACP}$ \\
\hline HETATM & 134 & $\mathrm{C} 4$ & $\mathrm{ACP}$ \\
\hline HETATM & 135 & H 41 & $\mathrm{ACP}$ \\
\hline HETATM & 136 & $\mathrm{H} 42$ & $\mathrm{ACP}$ \\
\hline HETATM & 137 & $\mathrm{C} 1$ & $\mathrm{ACP}$ \\
\hline HETATM & 138 & $\mathrm{O} 2$ & $\mathrm{ACP}$ \\
\hline HETATM & 139 & 03 & $\mathrm{ACP}$ \\
\hline HETATM & 140 & H3 & $\mathrm{ACP}$ \\
\hline \multicolumn{4}{|l|}{ TER } \\
\hline HETATM & 141 & $\mathrm{C} 1$ & MET \\
\hline HETATM & 142 & $\mathrm{H} 11$ & MET \\
\hline HETATM & 143 & $\mathrm{H} 12$ & MET \\
\hline HETATM & 144 & H13 & MET \\
\hline \multicolumn{4}{|l|}{ TER } \\
\hline HETATM & 145 & $\mathrm{C} 1$ & MET \\
\hline HETATM & 146 & $\mathrm{H} 11$ & MET \\
\hline HETATM & 147 & $\mathrm{H} 12$ & MET \\
\hline HETATM & 148 & $\mathrm{H} 13$ & MET \\
\hline \multicolumn{4}{|l|}{ TER } \\
\hline HETATM & 149 & $\mathrm{FE}$ & HEM \\
\hline HETATM & 150 & $\mathrm{C} 1$ & HEM \\
\hline HETATM & 151 & $\mathrm{C} 2$ & HEM \\
\hline HETATM & 152 & $\mathrm{C} 3$ & HEM \\
\hline HETATM & 153 & $\mathrm{C} 4$ & HEM \\
\hline HETATM & 154 & C5 & HEM \\
\hline HETATM & 155 & H5 & HEM \\
\hline HETATM & 156 & C6 & HEM \\
\hline HETATM & 157 & $\mathrm{C} 7$ & HEM \\
\hline HETATM & 158 & $\mathrm{C} 8$ & HEM \\
\hline HETATM & 159 & $\mathrm{C} 9$ & HEM \\
\hline HETATM & 160 & $\mathrm{C} 10$ & HEM \\
\hline HETATM & 161 & $\mathrm{H} 10$ & HEM \\
\hline HETATM & 162 & $\mathrm{C} 11$ & HEM \\
\hline HETATM & 163 & $\mathrm{C} 12$ & HEM \\
\hline HETATM & 164 & C13 & HEM \\
\hline HETATM & 165 & C14 & HEM \\
\hline HETATM & 166 & C15 & HEM \\
\hline HETATM & 167 & H15 & HEM \\
\hline HETATM & 168 & $\mathrm{C} 16$ & HEM \\
\hline HETATM & 169 & $\mathrm{C} 17$ & HEM \\
\hline HETATM & 170 & $\mathrm{C} 18$ & HEM \\
\hline HETATM & 171 & C19 & HEM \\
\hline HETATM & 172 & $\mathrm{C} 20$ & HEM \\
\hline HETATM & 173 & H 20 & HEM \\
\hline HETATM & 174 & N21 & HEM \\
\hline HETATM & 175 & N22 & HEM \\
\hline HETATM & 176 & N23 & HEM \\
\hline HETATM & 177 & $\mathrm{~N} 24$ & HEM \\
\hline HETATM & 178 & C99 & HEM \\
\hline HETATM & 179 & 091 & HEM \\
\hline HETATM & 180 & 092 & HEM \\
\hline HETATM & 181 & CT91 & HEM \\
\hline
\end{tabular}

\begin{tabular}{|c|c|c|c|c|c|}
\hline-1.033 & 12.637 & 3.471 & 1.00 & 0.00 & $\mathrm{H}$ \\
\hline-1.523 & 11.031 & 2.889 & 1.00 & 0.00 & $\mathrm{H}$ \\
\hline 8.874 & 0.178 & 8.933 & 1.00 & 0.00 & 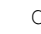 \\
\hline 9.690 & 0.876 & 8.768 & 1.00 & 0.00 & \\
\hline 9.207 & -1.047 & 9.335 & 1.00 & 0.00 & \\
\hline 8.475 & -1.826 & 9.442 & 1.00 & 0.00 & \\
\hline 10.249 & -1.316 & 9.501 & 1.00 & 0.00 & \\
\hline 6.210 & -0.077 & 10.771 & 1.00 & 0.00 & c \\
\hline 5.975 & -1.148 & 10.652 & 1.00 & 0.00 & \\
\hline 5.397 & 0.387 & 11.341 & 1.00 & 0.00 & \\
\hline 7.118 & 0.001 & 11.382 & 1.00 & 0.00 & \\
\hline 1.200 & 1.601 & 10.256 & 1.00 & 0.00 & 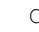 \\
\hline 1.866 & 1.702 & 11.115 & 1.00 & 0.00 & \\
\hline 0.033 & 0.957 & 10.415 & 1.00 & 0.00 & \\
\hline-0.647 & 0.800 & 9.585 & 1.00 & 0.00 & \\
\hline-0.272 & 0.559 & 11.381 & 1.00 & 0.00 & \\
\hline-0.484 & 3.284 & 8.052 & 1.00 & 0.00 & \\
\hline-0.641 & 4.306 & 7.685 & 1.00 & 0.00 & \\
\hline-0.917 & 3.219 & 9.057 & 1.00 & 0.00 & \\
\hline-1.076 & 2.620 & 7.403 & 1.00 & 0.00 & $\mathrm{H}$ \\
\hline 8.604 & 4.366 & 2.161 & 1.00 & 0.00 & 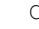 \\
\hline 7.865 & 4.377 & 1.349 & 1.00 & 0.00 & $\mathrm{H}$ \\
\hline 9.445 & 3.758 & 1.798 & 1.00 & 0.00 & H \\
\hline 9.082 & 5.803 & 2.434 & 1.00 & 0.00 & c \\
\hline 9.640 & 5.865 & 3.382 & 1.00 & 0.00 & H \\
\hline 8.212 & 6.470 & 2.541 & 1.00 & 0.00 & $\mathrm{H}$ \\
\hline 9.946 & 6.416 & 1.358 & 1.00 & 0.00 & c \\
\hline 10.174 & 5.893 & 0.260 & 1.00 & 0.00 & 0 \\
\hline 10.462 & 7.595 & 1.722 & 1.00 & 0.00 & 0 \\
\hline 11.071 & 7.949 & 0.986 & 1.00 & 0.00 & 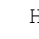 \\
\hline 0.708 & 5.492 & 3.334 & 1.00 & 0.00 & 0 \\
\hline 0.155 & 4.653 & 2.884 & 1.00 & 0.00 & $\mathrm{H}$ \\
\hline 0.701 & 6.318 & 2.613 & 1.00 & 0.00 & $\mathrm{H}$ \\
\hline 0.137 & 5.812 & 4.214 & 1.00 & 0.00 & $\mathrm{H}$ \\
\hline 10.141 & 2.543 & 4.285 & 1.00 & 0.00 & 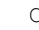 \\
\hline 10.586 & 2.752 & 3.305 & 1.00 & 0.00 & $\mathrm{H}$ \\
\hline 10.252 & 1.463 & 4.460 & 1.00 & 0.00 & $\mathrm{H}$ \\
\hline 10.742 & 3.069 & 5.042 & 1.00 & 0.00 & $\mathrm{H}$ \\
\hline 4.285 & -4.082 & 17.354 & 1.00 & 0.00 & $\mathrm{Fe}$ \\
\hline 2.057 & -2.210 & 16.292 & 1.00 & 0.00 & c \\
\hline 1.698 & -1.419 & 15.120 & 1.00 & 0.00 & c \\
\hline 2.844 & -1.322 & 14.345 & 1.00 & 0.00 & c \\
\hline 3.873 & -2.039 & 15.053 & 1.00 & 0.00 & $c$ \\
\hline 5.188 & -2.158 & 14.615 & 1.00 & 0.00 & c \\
\hline 5.442 & -1.662 & 13.680 & 1.00 & 0.00 & H \\
\hline 6.208 & -2.857 & 15.251 & 1.00 & 0.00 & c \\
\hline 7.576 & -2.929 & 14.782 & 1.00 & 0.00 & c \\
\hline 8.282 & -3.649 & 15.729 & 1.00 & 0.00 & c \\
\hline 7.331 & -4.014 & 16.753 & 1.00 & 0.00 & c \\
\hline 7.635 & -4.749 & 17.897 & 1.00 & 0.00 & c \\
\hline 8.662 & -5.093 & 18.002 & 1.00 & 0.00 & H \\
\hline 6.761 & -5.089 & 18.919 & 1.00 & 0.00 & c \\
\hline 7.142 & -5.814 & 20.109 & 1.00 & 0.00 & $c$ \\
\hline 6.002 & -5.961 & 20.866 & 1.00 & 0.00 & c \\
\hline 4.929 & -5.320 & 20.135 & 1.00 & 0.00 & c \\
\hline 3.601 & -5.265 & 20.557 & 1.00 & 0.00 & $c$ \\
\hline 3.361 & -5.750 & 21.504 & 1.00 & 0.00 & $\mathrm{H}$ \\
\hline 2.571 & -4.584 & 19.913 & 1.00 & 0.00 & $c$ \\
\hline 1.205 & -4.470 & 20.396 & 1.00 & 0.00 & $c$ \\
\hline 0.530 & -3.702 & 19.472 & 1.00 & 0.00 & $c$ \\
\hline 1.480 & -3.351 & 18.435 & 1.00 & 0.00 & c \\
\hline 1.188 & -2.560 & 17.324 & 1.00 & 0.00 & c \\
\hline 0.174 & -2.170 & 17.264 & 1.00 & 0.00 & H \\
\hline 3.379 & -2.573 & 16.230 & 1.00 & 0.00 & $\mathbb{N}$ \\
\hline 6.083 & -3.520 & 16.452 & 1.00 & 0.00 & N \\
\hline 5.412 & -4.783 & 18.952 & 1.00 & 0.00 & 1 \\
\hline 2.713 & -3.898 & 18.717 & 1.00 & 0.00 & \\
\hline 3.619 & -6.763 & 16.214 & 1.00 & 0.00 & c \\
\hline 3.779 & -5.450 & 16.227 & 1.00 & 0.00 & 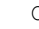 \\
\hline 3.465 & -7.381 & 15.159 & 1.00 & 0.00 & 0 \\
\hline 3.635 & -7.445 & 17.571 & 1.00 & 0.00 & \\
\hline
\end{tabular}



HETATM HETATM 185 H921 HEM HETATM 186 H922 HEM TER

HETATM 187 FE HEM HETATM $188 \quad \mathrm{C} 1 \mathrm{HE}$ HETATM 189 C2 HEM HETATM 190 C3 HFM HETATM 191 C4 HEM HETATM 192 C5 HEM HETATM 193 H5 HEM HETATM 194 C6 HEM HETATM 195 C7 HEM HETATM 196 C8 HEM HETATM 197 C9 HEM HETATM 198 C10 HEM HETATM 199 H1O HEM HETATM 200 C11 HEM HETATM 201 C12 HEM HETATM 202 C13 HEM HETATM 203 C14 HEM HETATM 204 C15 HEM HETATM 205 H15 HEM HETATM 206 C16 HEM HETATM $207 \quad$ C17 HEM HETATM 208 C18 HEM HETATM 209 C19 HEM HETATM 210 C20 HEM HETATM 211 H20 HEM HETATM 212 N21 HEM HETATM 213 N22 HEM HETATM 214 N23 HEM HETATM 215 N24 HEM HETATM 216 C99 HEM HETATM $217 \quad 091$ HEM HETATM 218092 HEM HETATM 219 CT91 HEM HETATM 220 H911 HEM HETATM 221 H912 HEM HETATM 222 CT92 HEM HETATM 223 H921 HEM HETATM 224 H922 HEM TER

HETATM 225 C2 VIN HETATM 226 H21 VIN HETATM 227 C1 VIN HETATM 228 H11 VIN HETATM 229 H12 VIN $T E R$

HETATM $230 \quad$ C1 ME HETATM 231 H11 MET HETATM 232 H12 MET HETATM 233 H13 MET TER

HETATM 234 C2 VIN $\begin{array}{lll}\text { HETATM } & 235 & \text { H21 VIN }\end{array}$ HETATM 236 C1 VIN HETATM 237 H11 VIN HETATM 238 H12 VIN $T E R$

HETATM 239 C1 MET HETATM 240 H11 MET HETATM 241 H12 MET HETATM 242 H13 MET TER

HETATM 243 C5 ACP HETATM 244 H51 ACP HETATM 245 H52 ACP HETATM 246 C4 ACP HETATM 247 H41 ACP HETATM 248 H42 ACP HETATM 249 C1 ACP HETATM 250 O2 ACP HETATM 251 O3 ACP HETATM 252 H3 ACP TER

HETATM 253 C1 MET $\begin{array}{lllll}4.614 & -7.250 & 18.034 & 1.00 & 0.00\end{array}$ $\begin{array}{lllll}2.887 & -6.955 & 18.214 & 1.00 & 0.00\end{array}$ $\begin{array}{lllll}3.376 & -8.949 & 17.459 & 1.00 & 0.00\end{array}$ $\begin{array}{lllll}4.172 & -9.410 & 16.863 & 1.00 & 0.00\end{array}$ $\begin{array}{lllll}2.451 & -9.105 & 16.889 & 1.00 & 0.00\end{array}$

$\begin{array}{lllll}4.977 & -11.496 & 22.302 & 1.00 & 0.00\end{array}$ $\begin{array}{llllll}7.205 & -13.368 & 23.364 & 1.00 & 0.00\end{array}$ $\begin{array}{lllll}7.564 & -14.159 & 24.536 & 1.00 & 0.00\end{array}$ $\begin{array}{lllll}6.418 & -14.256 & 25.311 & 1.00 & 0.00\end{array}$ $\begin{array}{lllll}5.389 & -13.538 & 24.603 & 1.00 & 0.00\end{array}$ $\begin{array}{lllll}4.074 & -13.419 & 25.041 & 1.00 & 0.00\end{array}$ $\begin{array}{lllll}3.820 & -13.916 & 25.976 & 1.00 & 0.00\end{array}$ $\begin{array}{lllll}3.054 & -12.721 & 24.405 & 1.00 & 0.00\end{array}$ $\begin{array}{lllll}1.685 & -12.648 & 24.874 & 1.00 & 0.00\end{array}$ $\begin{array}{lllll}0.980 & -11.929 & 23.927 & 1.00 & 0.00\end{array}$ $\begin{array}{lllll}1.931 & -11.564 & 22.903 & 1.00 & 0.00\end{array}$ $\begin{array}{lllll}1.626 & -10.829 & 21.759 & 1.00 & 0.00\end{array}$ $\begin{array}{llllll}0.600 & -10.485 & 21.654 & 1.00 & 0.00\end{array}$ $\begin{array}{lllll}2.501 & -10.489 & 20.737 & 1.00 & 0.00\end{array}$ $\begin{array}{lllll}2.120 & -9.763 & 19.547 & 1.00 & 0.00\end{array}$ $\begin{array}{lllll}3.259 & -9.616 & 18.790 & 1.00 & 0.00\end{array}$ $\begin{array}{lllll}4.332 & -10.258 & 19.521 & 1.00 & 0.00\end{array}$ $\begin{array}{lllll}5.660 & -10.312 & 19.099 & 1.00 & 0.00\end{array}$ $\begin{array}{lllll}5.900 & -9.828 & 18.152 & 1.00 & 0.00\end{array}$ $\begin{array}{lllll}6.691 & -10.994 & 19.743 & 1.00 & 0.00\end{array}$ $\begin{array}{lllll}8.056 & -11.108 & 19.260 & 1.00 & 0.00\end{array}$ $\begin{array}{lllll}8.732 & -11.875 & 20.184 & 1.00 & 0.00\end{array}$ $\begin{array}{lllll}7.782 & -12.227 & 21.221 & 1.00 & 0.00\end{array}$ $\begin{array}{lllll}8.073 & -13.018 & 22.332 & 1.00 & 0.00\end{array}$ $\begin{array}{lllll}9.088 & -13.407 & 22.392 & 1.00 & 0.00\end{array}$ $\begin{array}{lllll}5.883 & -13.005 & 23.426 & 1.00 & 0.00\end{array}$ $\begin{array}{lllll}3.179 & -12.058 & 23.204 & 1.00 & 0.00\end{array}$ $\begin{array}{lllll}3.849 & -10.795 & 20.704 & 1.00 & 0.00\end{array}$ $\begin{array}{lllll}6.549 & -11.679 & 20.939 & 1.00 & 0.00\end{array}$ $\begin{array}{lllll}5.642 & -8.814 & 23.442 & 1.00 & 0.00\end{array}$ $\begin{array}{lllll}5.483 & -10.128 & 23.429 & 1.00 & 0.00\end{array}$ $\begin{array}{lllll}5.797 & -8.197 & 24.497 & 1.00 & 0.00\end{array}$ $\begin{array}{lllll}5.626 & -8.133 & 22.085 & 1.00 & 0.00\end{array}$ $\begin{array}{lllll}4.647 & -8.328 & 21.622 & 1.00 & 0.00\end{array}$ $\begin{array}{lllll}6.374 & -8.623 & 21.442 & 1.00 & 0.00\end{array}$ $\begin{array}{lllll}5.886 & -6.628 & 22.197 & 1.00 & 0.00\end{array}$ $\begin{array}{lllll}6.811 & -6.473 & 22.767 & 1.00 & 0.00\end{array}$ $\begin{array}{llllll}5.089 & -6.168 & 22.793 & 1.00 & 0.00\end{array}$

$\begin{array}{lllll}0.366 & -0.917 & 14.860 & 1.00 & 0.00\end{array}$ $\begin{array}{lllll}-0.450 & -1.615 & 15.025 & 1.00 & 0.00\end{array}$ $\begin{array}{lllll}0.033 & 0.308 & 14.459 & 1.00 & 0.00\end{array}$ $\begin{array}{lllll}0.765 & 1.087 & 14.352 & 1.00 & 0.00\end{array}$

$\begin{array}{lllll}-1.008 & 0.578 & 14.292 & 1.00 & 0.00\end{array}$

$\begin{array}{lllll}3.030 & -0.662 & 13.022 & 1.00 & 0.00\end{array}$ $\begin{array}{lllll}3.265 & 0.409 & 13.142 & 1.00 & 0.00\end{array}$ $\begin{array}{lllll}3.843 & -1.126 & 12.453 & 1.00 & 0.00\end{array}$ $\begin{array}{lllll}2.122 & -0.739 & 12.412 & 1.00 & 0.00\end{array}$

$\begin{array}{llllll}8.040 & -2.340 & 13.537 & 1.00 & 0.00\end{array}$ $\begin{array}{llllll}7.374 & -2.440 & 12.679 & 1.00 & 0.00\end{array}$ $\begin{array}{lllll}9.207 & -1.695 & 13.379 & 1.00 & 0.00\end{array}$ $\begin{array}{lllll}9.887 & -1.539 & 14.209 & 1.00 & 0.00\end{array}$ $\begin{array}{lllll}9.512 & -1.297 & 12.413 & 1.00 & 0.00\end{array}$

$\begin{array}{lllll}9.725 & -4.023 & 15.741 & 1.00 & 0.00\end{array}$ $\begin{array}{lllll}10.316 & -3.359 & 16.390 & 1.00 & 0.00\end{array}$ $\begin{array}{lllll}9.881 & -5.045 & 16.108 & 1.00 & 0.00\end{array}$ $\begin{array}{lllll}10.157 & -3.957 & 14.736 & 1.00 & 0.00\end{array}$

$\begin{array}{lllll}0.636 & -5.105 & 21.633 & 1.00 & 0.00\end{array}$ $\begin{array}{lllll}1.375 & -5.116 & 22.445 & 1.00 & 0.00\end{array}$ $\begin{array}{lllll}-0.204 & -4.496 & 21.996 & 1.00 & 0.00\end{array}$ $\begin{array}{lllll}0.158 & -6.542 & 21.360 & 1.00 & 0.00\end{array}$ $\begin{array}{lllll}-0.400 & -6.603 & 20.412 & 1.00 & 0.00\end{array}$

$\begin{array}{lllll}1.028 & -7.209 & 21.253 & 1.00 & 0.00\end{array}$

$\begin{array}{llllll}-0.706 & -7.154 & 22.436 & 1.00 & 0.00\end{array}$

$\begin{array}{llllll}-0.934 & -6.631 & 23.534 & 1.00 & 0.00\end{array}$

$\begin{array}{lllll}-1.222 & -8.334 & 22.071 & 1.00 & 0.00\end{array}$

$\begin{array}{lllll}-1.831 & -8.688 & 22.808 & 1.00 & 0.00\end{array}$

$\begin{array}{llllll}8.532 & -6.231 & 20.459 & 1.00 & 0.00\end{array}$ 


\begin{tabular}{|c|c|c|c|}
\hline HETATM & 254 & $\mathrm{H} 11$ & MET \\
\hline HETATM & 255 & $\mathrm{H} 12$ & MET \\
\hline HETATM & 256 & $\mathrm{H} 13$ & MET \\
\hline \multicolumn{4}{|l|}{ TER } \\
\hline HETATM & 257 & $\mathrm{C} 1$ & MET \\
\hline HETATM & 258 & $\mathrm{H} 11$ & MET \\
\hline HETATM & 259 & $\mathrm{H} 12$ & MET \\
\hline HETATM & 260 & $\mathrm{H} 13$ & MET \\
\hline \multicolumn{4}{|l|}{ TER } \\
\hline HETATM & 261 & C2 & VIN \\
\hline HETATM & 262 & H 21 & VIN \\
\hline HETATM & 263 & $\mathrm{C} 1$ & VIN \\
\hline HETATM & 264 & H11 & VIN \\
\hline HETATM & 265 & $\mathrm{H} 12$ & VIN \\
\hline \multicolumn{4}{|l|}{ TER } \\
\hline HETATM & 266 & $\mathrm{C} 1$ & MET \\
\hline HETATM & 267 & $\mathrm{H} 11$ & MET \\
\hline HETATM & 268 & $\mathrm{H} 12$ & MET \\
\hline HETATM & 269 & $\mathrm{H} 13$ & MET \\
\hline \multicolumn{4}{|l|}{ TER } \\
\hline HETATM & 270 & $\mathrm{C} 2$ & VIN \\
\hline HETATM & 271 & $\mathrm{H} 21$ & VIN \\
\hline HETATM & 272 & $\mathrm{C} 1$ & VIN \\
\hline HETATM & 273 & $\mathrm{H} 11$ & VIN \\
\hline HETATM & 274 & $\mathrm{H} 12$ & VIN \\
\hline \multicolumn{4}{|l|}{ TER } \\
\hline HETATM & 275 & $\mathrm{C} 1$ & $\mathrm{MET}$ \\
\hline HETATM & 276 & $\mathrm{H} 11$ & MET \\
\hline HETATM & 277 & $\mathrm{H} 12$ & MET \\
\hline HETATM & 278 & $\mathrm{H} 13$ & MET \\
\hline \multicolumn{4}{|l|}{ TER } \\
\hline HETATM & 279 & C5 & $\mathrm{ACP}$ \\
\hline HETATM & 280 & H51 & $\mathrm{ACP}$ \\
\hline HETATM & 281 & H52 & $\mathrm{ACP}$ \\
\hline HETATM & 282 & C4 & $\mathrm{ACP}$ \\
\hline HETATM & 283 & H 41 & $\mathrm{ACP}$ \\
\hline HETATM & 284 & $\mathrm{H} 42$ & $\mathrm{ACP}$ \\
\hline HETATM & 285 & $\mathrm{C} 1$ & $\mathrm{ACP}$ \\
\hline HETATM & 286 & 02 & $\mathrm{ACP}$ \\
\hline HETATM & 287 & $\mathrm{O} 3$ & $\mathrm{ACP}$ \\
\hline HETATM & 288 & H3 & $\mathrm{ACP}$ \\
\hline \multicolumn{4}{|l|}{ TER } \\
\hline HETATM & 289 & $\mathrm{C} 1$ & MET \\
\hline HETATM & 290 & $\mathrm{H} 11$ & MET \\
\hline HETATM & 291 & $\mathrm{H} 12$ & MET \\
\hline HETATM & 292 & $\mathrm{H} 13$ & $\mathrm{MET}$ \\
\hline \multicolumn{4}{|l|}{ TER } \\
\hline HETATM & 293 & $\mathrm{C} 1$ & MET \\
\hline HETATM & 294 & $\mathrm{H} 11$ & MET \\
\hline HETATM & 295 & $\mathrm{H} 12$ & MET \\
\hline HETATM & 296 & $\mathrm{H} 13$ & MET \\
\hline
\end{tabular}

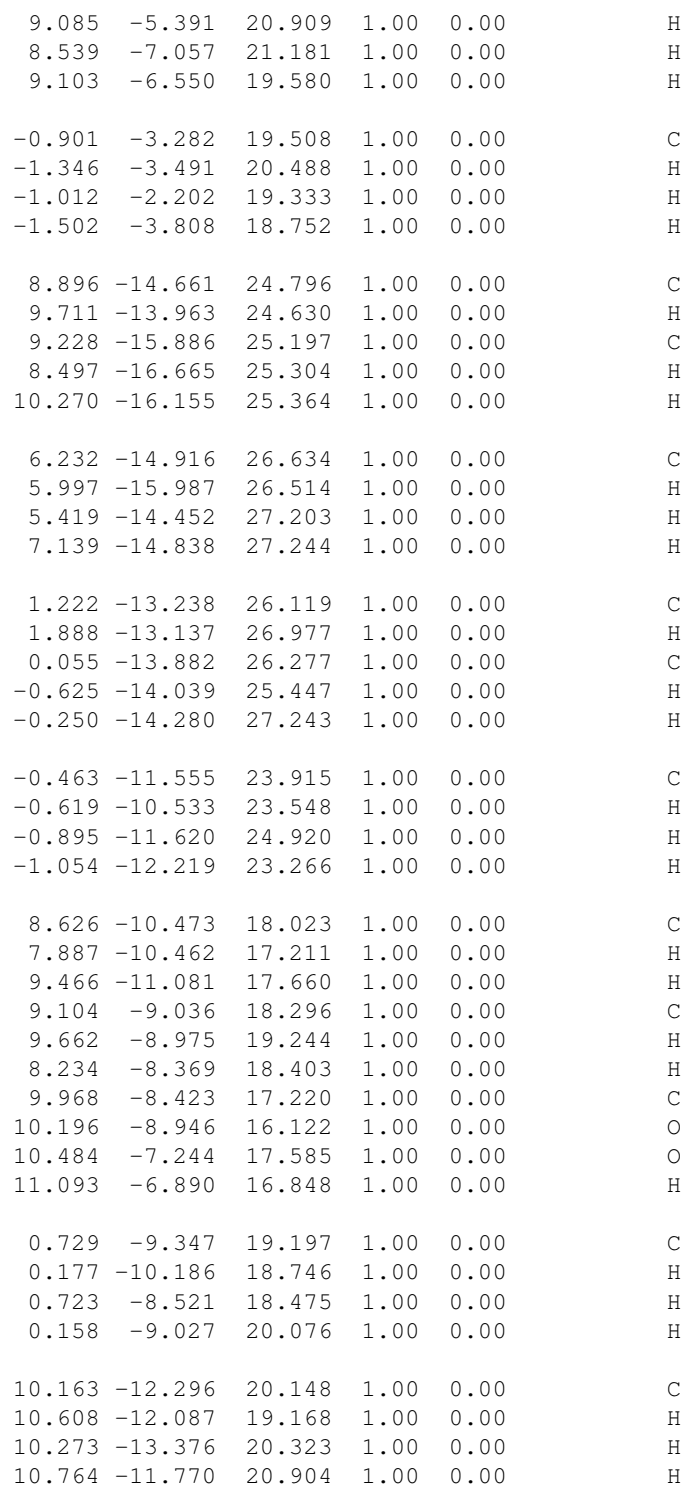
TER

END

\subsection{D5 dimer: AYK_D5.pdb file}

$\begin{array}{lrlll}\text { HETATM } & 1 & \text { FE } & \text { HEM } & 1 \\ \text { HETATM } & 2 & \text { C1 } & \text { HEM } & 1 \\ \text { HETATM } & 3 & \text { C2 } & \text { HEM } & 1 \\ \text { HETATM } & 4 & \text { C3 } & \text { HEM } & 1 \\ \text { HETATM } & 5 & \text { C4 } & \text { HEM } & 1 \\ \text { HETATM } & 6 & \text { C5 } & \text { HEM } & 1 \\ \text { HETATM } & 7 & \text { H5 } & \text { HEM } & 1 \\ \text { HETATM } & 8 & \text { C6 } & \text { HEM } & 1 \\ \text { HETATM } & 9 & \text { C7 } & \text { HEM } & 1 \\ \text { HETATM } & 10 & \text { C8 } & \text { HEM } & 1 \\ \text { HETATM } & 11 & \text { C9 } & \text { HEM } & 1 \\ \text { HETATM } & 12 & \text { C10 } & \text { HEM } & 1 \\ \text { HETATM } & 13 & \text { H10 } & \text { HEM } & 1 \\ \text { HETATM } & 14 & \text { C11 } & \text { HEM } & 1 \\ \text { HETATM } & 15 & \text { C12 } & \text { HEM } & 1 \\ \text { HETATM } & 16 & \text { C13 } & \text { HEM } & 1 \\ \text { HETATM } & 17 & \text { C14 } & \text { HEM } & 1 \\ \text { HETATM } & 18 & \text { C15 } & \text { HEM } & 1 \\ \text { HETATM } & 19 & \text { H15 } & \text { HEM } & 1 \\ \text { HETATM } & 20 & \text { C16 } & \text { HEM } & 1 \\ \text { HETATM } & 21 & \text { C17 } & \text { HEM } & 1 \\ \text { HETATM } & 22 & \text { C18 } & \text { HEM } & 1\end{array}$

$\begin{array}{rrrrr}4.263 & 10.757 & 1.491 & 1.00 & 0.00 \\ 2.035 & 12.629 & 0.429 & 1.00 & 0.00 \\ 1.676 & 13.420 & -0.742 & 1.00 & 0.00 \\ 2.822 & 13.517 & -1.517 & 1.00 & 0.00 \\ 3.851 & 12.800 & -0.809 & 1.00 & 0.00 \\ 5.167 & 12.681 & -1.247 & 1.00 & 0.00 \\ 5.420 & 13.177 & -2.182 & 1.00 & 0.00 \\ 6.186 & 11.982 & -0.611 & 1.00 & 0.00 \\ 7.555 & 11.910 & -1.081 & 1.00 & 0.00 \\ 8.260 & 11.190 & -0.134 & 1.00 & 0.00 \\ 7.309 & 10.825 & 0.891 & 1.00 & 0.00 \\ 7.614 & 10.090 & 2.034 & 1.00 & 0.00 \\ 8.641 & 9.746 & 2.140 & 1.00 & 0.00 \\ 6.739 & 9.750 & 3.057 & 1.00 & 0.00 \\ 7.120 & 9.025 & 4.247 & 1.00 & 0.00 \\ 5.981 & 8.878 & 5.004 & 1.00 & 0.00 \\ 4.908 & 9.519 & 4.273 & 1.00 & 0.00 \\ 3.580 & 9.574 & 4.694 & 1.00 & 0.00 \\ 3.340 & 9.089 & 5.642 & 1.00 & 0.00 \\ 2.550 & 10.255 & 4.051 & 1.00 & 0.00 \\ 1.184 & 10.369 & 4.533 & 1.00 & 0.00 \\ 0.508 & 11.137 & 3.609 & 1.00 & 0.00\end{array}$




\begin{tabular}{|c|c|c|c|c|c|c|c|c|c|c|}
\hline HETATM & 23 & C19 & HEM & 1 & 1.458 & 11.489 & 2.573 & 1.00 & 0.00 & C \\
\hline HETATM & 24 & $\mathrm{C} 20$ & HEM & 1 & 1.167 & 12.280 & 1.462 & 1.00 & 0.00 & C \\
\hline HETATM & 25 & $\mathrm{H} 2 \mathrm{O}$ & HEM & 1 & 0.152 & 12.669 & 1.402 & 1.00 & 0.00 & $\mathrm{H}$ \\
\hline HETATM & 26 & N21 & HEM & 1 & 3.357 & 12.266 & 0.367 & 1.00 & 0.00 & $\mathrm{~N}$ \\
\hline HETATM & 27 & N22 & HEM & 1 & 6.062 & 11.319 & 0.590 & 1.00 & 0.00 & $\mathrm{~N}$ \\
\hline HETATM & 28 & N23 & HEM & 1 & 5.391 & 10.056 & 3.090 & 1.00 & 0.00 & $\mathrm{~N}$ \\
\hline HETATM & 29 & N24 & HEM & 1 & 2.691 & 10.941 & 2.854 & 1.00 & 0.00 & $\mathrm{~N}$ \\
\hline HETATM & 30 & C99 & HEM & 1 & 3.598 & 8.076 & 0.352 & 1.00 & 0.00 & C \\
\hline HETATM & 31 & 091 & HEM & 1 & 3.757 & 9.389 & 0.365 & 1.00 & 0.00 & o \\
\hline HETATM & 32 & 092 & HEM & 1 & 3.443 & 7.458 & -0.703 & 1.00 & 0.00 & 0 \\
\hline HETATM & 33 & СТ91 & HEM & 1 & 3.614 & 7.394 & 1.709 & 1.00 & 0.00 & $\mathrm{C}$ \\
\hline HETATM & 34 & H911 & HEM & 1 & 4.593 & 7.590 & 2.171 & 1.00 & 0.00 & $\mathrm{H}$ \\
\hline HETATM & 35 & H912 & HEM & 1 & 2.866 & 7.884 & 2.352 & 1.00 & 0.00 & $\mathrm{H}$ \\
\hline HETATM & 36 & СТ92 & HEM & 1 & 3.354 & 5.890 & 1.597 & 1.00 & 0.00 & C \\
\hline HETATM & 37 & H921 & HEM & 1 & 4.151 & 5.429 & 1.000 & 1.00 & 0.00 & $\mathrm{H}$ \\
\hline HETATM & 38 & H922 & HEM & 1 & 2.429 & 5.734 & 1.027 & 1.00 & 0.00 & $\mathrm{H}$ \\
\hline \multicolumn{11}{|l|}{ TER } \\
\hline HETATM & 39 & $\mathrm{FE}$ & HEM & 2 & 4.955 & 3.343 & 6.440 & 1.00 & 0.00 & $\mathrm{FE}$ \\
\hline HETATM & 40 & $\mathrm{C} 1$ & HEM & 2 & 7.183 & 1.471 & 7.502 & 1.00 & 0.00 & C \\
\hline HETATM & 41 & $\mathrm{C} 2$ & HEM & 2 & 7.542 & 0.680 & 8.674 & 1.00 & 0.00 & C \\
\hline HETATM & 42 & C3 & HEM & 2 & 6.397 & 0.583 & 9.448 & 1.00 & 0.00 & C \\
\hline HETATM & 43 & $\mathrm{C} 4$ & HEM & 2 & 5.367 & 1.301 & 8.740 & 1.00 & 0.00 & C \\
\hline HETATM & 44 & C5 & HEM & 2 & 4.052 & 1.420 & 9.178 & 1.00 & 0.00 & C \\
\hline HETATM & 45 & H5 & HEM & 2 & 3.798 & 0.923 & 10.113 & 1.00 & 0.00 & $\mathrm{H}$ \\
\hline HETATM & 46 & $\mathrm{C} 6$ & HEM & 2 & 3.033 & 2.118 & 8.542 & 1.00 & 0.00 & C \\
\hline HETATM & 47 & $\mathrm{C} 7$ & HEM & 2 & 1.664 & 2.191 & 9.012 & 1.00 & 0.00 & C \\
\hline HETATM & 48 & $\mathrm{C} 8$ & HEM & 2 & 0.958 & 2.910 & 8.065 & 1.00 & 0.00 & C \\
\hline HETATM & 49 & $\mathrm{C} 9$ & HEM & 2 & 1.909 & 3.275 & 7.041 & 1.00 & 0.00 & C \\
\hline HETATM & 50 & $\mathrm{C} 10$ & HEM & 2 & 1.605 & 4.010 & 5.897 & 1.00 & 0.00 & C \\
\hline HETATM & 51 & $\mathrm{H} 10$ & HEM & 2 & 0.578 & 4.354 & 5.791 & 1.00 & 0.00 & $\mathrm{H}$ \\
\hline HETATM & 52 & $\mathrm{C} 11$ & HEM & 2 & 2.479 & 4.350 & 4.874 & 1.00 & 0.00 & C \\
\hline HETATM & 53 & $\mathrm{C} 12$ & HEM & 2 & 2.098 & 5.076 & 3.684 & 1.00 & 0.00 & C \\
\hline HETATM & 54 & $\mathrm{C} 13$ & HEM & 2 & 3.238 & 5.223 & 2.927 & 1.00 & 0.00 & C \\
\hline HETATM & 55 & $\mathrm{C} 14$ & HEM & 2 & 4.311 & 4.581 & 3.659 & 1.00 & 0.00 & C \\
\hline HETATM & 56 & $\mathrm{C} 15$ & HEM & 2 & 5.639 & 4.527 & 3.237 & 1.00 & 0.00 & C \\
\hline HETATM & 57 & H15 & HEM & 2 & 5.879 & 5.011 & 2.289 & 1.00 & 0.00 & $\mathrm{H}$ \\
\hline HETATM & 58 & $\mathrm{C} 16$ & HEM & 2 & 6.669 & 3.845 & 3.880 & 1.00 & 0.00 & C \\
\hline HETATM & 59 & $\mathrm{C} 17$ & HEM & 2 & 8.035 & 3.732 & 3.398 & 1.00 & 0.00 & C \\
\hline HETATM & 60 & $\mathrm{C} 18$ & HEM & 2 & 8.710 & 2.964 & 4.322 & 1.00 & 0.00 & C \\
\hline HETATM & 61 & $\mathrm{C} 19$ & HEM & 2 & 7.761 & 2.612 & 5.358 & 1.00 & 0.00 & C \\
\hline HETATM & 62 & $\mathrm{C} 20$ & HEM & 2 & 8.052 & 1.821 & 6.469 & 1.00 & 0.00 & C \\
\hline HETATM & 63 & $\mathrm{H} 20$ & HEM & 2 & 9.066 & 1.432 & 6.530 & 1.00 & 0.00 & $\mathrm{H}$ \\
\hline HETATM & 64 & N21 & HEM & 2 & 5.862 & 1.834 & 7.564 & 1.00 & 0.00 & $\mathrm{~N}$ \\
\hline HETATM & 65 & N22 & HEM & 2 & 3.157 & 2.781 & 7.342 & 1.00 & 0.00 & $\mathrm{~N}$ \\
\hline HETATM & 66 & N23 & HEM & 2 & 3.828 & 4.044 & 4.841 & 1.00 & 0.00 & $\mathrm{~N}$ \\
\hline HETATM & 67 & N24 & HEM & 2 & 6.527 & 3.160 & 5.077 & 1.00 & 0.00 & $\mathrm{~N}$ \\
\hline HETATM & 68 & C99 & HEM & 2 & 5.621 & 6.025 & 7.579 & 1.00 & 0.00 & C \\
\hline HETATM & 69 & 091 & HEM & 2 & 5.461 & 4.711 & 7.567 & 1.00 & 0.00 & O \\
\hline HETATM & 70 & 092 & HEM & 2 & 5.776 & 6.642 & 8.635 & 1.00 & 0.00 & O \\
\hline HETATM & 71 & СТ91 & HEM & 2 & 5.605 & 6.706 & 6.223 & 1.00 & 0.00 & C \\
\hline HETATM & 72 & H911 & HEM & 2 & 4.626 & 6.511 & 5.760 & 1.00 & 0.00 & $\mathrm{H}$ \\
\hline HETATM & 73 & H912 & HEM & 2 & 6.353 & 6.216 & 5.580 & 1.00 & 0.00 & $\mathrm{H}$ \\
\hline HETATM & 74 & СТ92 & HEM & 2 & 5.864 & 8.211 & 6.334 & 1.00 & 0.00 & C \\
\hline HETATM & 75 & H921 & HEM & 2 & 6.789 & 8.367 & 6.905 & 1.00 & 0.00 & $\mathrm{H}$ \\
\hline HETATM & 76 & H922 & HEM & 2 & 5.068 & 8.671 & 6.931 & 1.00 & 0.00 & $\mathrm{H}$ \\
\hline \multicolumn{11}{|l|}{ TER } \\
\hline HETATM & 77 & $\mathrm{C} 2$ & VIN & 3 & 0.345 & 13.923 & -1.002 & 1.00 & 0.00 & C \\
\hline HETATM & 78 & H 21 & VIN & 3 & -0.471 & 13.224 & -0.837 & 1.00 & 0.00 & $\mathrm{H}$ \\
\hline HETATM & 79 & $\mathrm{C} 1$ & VIN & 3 & 0.012 & 15.147 & -1.403 & 1.00 & 0.00 & C \\
\hline HETATM & 80 & $\mathrm{H} 11$ & VIN & 3 & 0.743 & 15.926 & -1.511 & 1.00 & 0.00 & $\mathrm{H}$ \\
\hline HETATM & 81 & $\mathrm{H} 12$ & VIN & 3 & -1.030 & 15.417 & -1.570 & 1.00 & 0.00 & $\mathrm{H}$ \\
\hline \multicolumn{11}{|l|}{ TER } \\
\hline HETATM & 82 & $\mathrm{C} 1$ & $\mathrm{MET}$ & 4 & 3.008 & 14.177 & -2.840 & 1.00 & 0.00 & C \\
\hline HETATM & 83 & H11 & MET & 4 & 3.243 & 15.248 & -2.720 & 1.00 & 0.00 & $\mathrm{H}$ \\
\hline HETATM & 84 & $\mathrm{H} 12$ & $\mathrm{MET}$ & 4 & 3.821 & 13.713 & -3.410 & 1.00 & 0.00 & $\mathrm{H}$ \\
\hline HETATM & 85 & $\mathrm{H} 13$ & $\mathrm{MET}$ & 4 & 2.101 & 14.100 & -3.450 & 1.00 & 0.00 & $\mathrm{H}$ \\
\hline \multicolumn{11}{|l|}{ TER } \\
\hline HETATM & 86 & $\mathrm{C} 2$ & VIN & 5 & 8.018 & 12.499 & -2.325 & 1.00 & 0.00 & C \\
\hline HETATM & 87 & H 21 & VIN & 5 & 7.352 & 12.399 & -3.184 & 1.00 & 0.00 & $\mathrm{H}$ \\
\hline HETATM & 88 & $\mathrm{C} 1$ & VIN & 5 & 9.185 & 13.144 & -2.484 & 1.00 & 0.00 & C \\
\hline HETATM & 89 & $\mathrm{H} 11$ & VIN & 5 & 9.865 & 13.300 & -1.654 & 1.00 & 0.00 & $\mathrm{H}$ \\
\hline HETATM & 90 & $\mathrm{H} 12$ & VIN & 5 & 9.490 & 13.542 & -3.450 & 1.00 & 0.00 & $\mathrm{H}$ \\
\hline TER & & & & & & & & & & \\
\hline HETATM & 91 & $\mathrm{C} 1$ & MET & 6 & 9.703 & 10.816 & -0.121 & 1.00 & 0.00 & C \\
\hline HETATM & 92 & $\mathrm{H} 11$ & MET & 6 & 10.294 & 11.480 & 0.528 & 1.00 & 0.00 & $\mathrm{H}$ \\
\hline HETATM & 93 & $\mathrm{H} 12$ & MET & 6 & 9.859 & 9.794 & 0.246 & 1.00 & 0.00 & $\mathrm{H}$ \\
\hline HETATM & 94 & $\mathrm{H} 13$ & $\mathrm{MET}$ & 6 & 10.135 & 10.882 & -1.126 & 1.00 & 0.00 & $\mathrm{H}$ \\
\hline TER & & & & & & & & & & \\
\hline HETATM & 95 & C5 & $\mathrm{ACP}$ & 7 & 0.615 & 9.735 & 5.770 & 1.00 & 0.00 & C \\
\hline
\end{tabular}




\begin{tabular}{|c|c|c|c|}
\hline HETATM & 96 & H51 & $\mathrm{ACP}$ \\
\hline HETATM & 97 & H52 & $\mathrm{ACP}$ \\
\hline HETATM & 98 & C4 & $\mathrm{ACP}$ \\
\hline HETATM & 99 & $\mathrm{H} 41$ & $\mathrm{ACP}$ \\
\hline HETATM & 100 & $\mathrm{H} 42$ & $\mathrm{ACP}$ \\
\hline HETATM & 101 & $\mathrm{C} 1$ & $\mathrm{ACP}$ \\
\hline HETATM & 102 & $\mathrm{O} 2$ & $\mathrm{ACP}$ \\
\hline HETATM & 103 & $\mathrm{O} 3$ & $\mathrm{ACP}$ \\
\hline HETATM & 104 & H3 & $\mathrm{ACP}$ \\
\hline \multicolumn{4}{|l|}{ TER } \\
\hline HETATM & 105 & C1 & MET \\
\hline HETATM & 106 & H11 & MET \\
\hline HETATM & 107 & $\mathrm{H} 12$ & MET \\
\hline HETATM & 108 & H13 & $\mathrm{MET}$ \\
\hline \multicolumn{4}{|l|}{ TER } \\
\hline HETATM & 109 & C1 & MET \\
\hline HETATM & 110 & H11 & MET \\
\hline HETATM & 111 & $\mathrm{H} 12$ & MET \\
\hline HETATM & 112 & H13 & MET \\
\hline \multicolumn{4}{|l|}{ TER } \\
\hline HETATM & 113 & $\mathrm{C} 2$ & VIN \\
\hline HETATM & 114 & $\mathrm{H} 21$ & VIN \\
\hline HETATM & 115 & $\mathrm{C} 1$ & VIN \\
\hline HETATM & 116 & H11 & VIN \\
\hline HETATM & 117 & H12 & VIN \\
\hline \multicolumn{4}{|l|}{$\mathrm{TER}$} \\
\hline HETATM & 118 & C1 & MET \\
\hline HETATM & 119 & H11 & MET \\
\hline HETATM & 120 & $\mathrm{H} 12$ & MET \\
\hline HETATM & 121 & H13 & MET \\
\hline \multicolumn{4}{|l|}{ TER } \\
\hline HETATM & 122 & $\mathrm{C} 2$ & VIN \\
\hline HETATM & 123 & H21 & VIN \\
\hline HETATM & 124 & $\mathrm{C} 1$ & VIN \\
\hline HETATM & 125 & $\mathrm{H} 11$ & VIN \\
\hline HETATM & 126 & $\mathrm{H} 12$ & VIN \\
\hline \multicolumn{4}{|l|}{ TER } \\
\hline HETATM & 127 & C1 & MET \\
\hline HETATM & 128 & H11 & MET \\
\hline HETATM & 129 & $\mathrm{H} 12$ & $\mathrm{MET}$ \\
\hline HETATM & 130 & $\mathrm{H} 13$ & MET \\
\hline \multicolumn{4}{|l|}{ TER } \\
\hline HETATM & 131 & C5 & $\mathrm{ACP}$ \\
\hline HETATM & 132 & H51 & $\mathrm{ACP}$ \\
\hline HETATM & 133 & H52 & $\mathrm{ACP}$ \\
\hline HETATM & 134 & $\mathrm{C} 4$ & $\mathrm{ACP}$ \\
\hline HETATM & 135 & H4 1 & $\mathrm{ACP}$ \\
\hline HETATM & 136 & $\mathrm{H} 42$ & $\mathrm{ACP}$ \\
\hline HETATM & 137 & C1 & $\mathrm{ACP}$ \\
\hline HETATM & 138 & $\mathrm{O} 2$ & $\mathrm{ACP}$ \\
\hline HETATM & 139 & 03 & $\mathrm{ACP}$ \\
\hline HETATM & 140 & H3 & $\mathrm{ACP}$ \\
\hline \multicolumn{4}{|l|}{ TER } \\
\hline HETATM & 141 & $\mathrm{C} 1$ & $\mathrm{MET}$ \\
\hline HETATM & 142 & H11 & MET \\
\hline HETATM & 143 & $\mathrm{H} 12$ & MET \\
\hline HETATM & 144 & $\mathrm{H} 13$ & MET \\
\hline TER & & & \\
\hline HETATM & 145 & $\mathrm{C} 1$ & $\mathrm{MET}$ \\
\hline HETATM & 146 & H11 & $\mathrm{MET}$ \\
\hline HETATM & 147 & $\mathrm{H} 12$ & $\mathrm{MET}$ \\
\hline HETATM & 148 & H13 & $\mathrm{MET}$ \\
\hline TER & & & \\
\hline HETATM & 149 & $\mathrm{FE}$ & HEM \\
\hline HETATM & 150 & $\mathrm{C} 1$ & HEM \\
\hline HETATM & 151 & $\mathrm{C} 2$ & HEM \\
\hline HETATM & 152 & C3 & HEM \\
\hline HETATM & 153 & C4 & HEM \\
\hline HETATM & 154 & C5 & HEM \\
\hline HETATM & 155 & H5 & HEM \\
\hline HETATM & 156 & C6 & HEM \\
\hline HETATM & 157 & C7 & HEM \\
\hline HETATM & 158 & $\mathrm{C} 8$ & HEM \\
\hline HETATM & 159 & C9 & HEM \\
\hline HETATM & 160 & $\mathrm{C} 10$ & HEM \\
\hline HETATM & 161 & H1O & HEM \\
\hline HETATM & 162 & $\mathrm{C} 11$ & HEM \\
\hline HETATM & 163 & $\mathrm{C} 12$ & HEM \\
\hline HETATM & 164 & $\mathrm{C} 13$ & HEM \\
\hline
\end{tabular}

\begin{tabular}{|c|c|c|c|c|c|}
\hline 1.354 & 9.723 & 6.582 & 1.00 & 0.00 & $\mathrm{H}$ \\
\hline-0.226 & 10.343 & 6.134 & 1.00 & 0.00 & $\mathrm{H}$ \\
\hline 0.136 & 8.297 & 5.497 & 1.00 & 0.00 & C \\
\hline-0.422 & 8.236 & 4.549 & 1.00 & 0.00 & $\mathrm{H}$ \\
\hline 1.007 & 7.630 & 5.390 & 1.00 & 0.00 & $\mathrm{H}$ \\
\hline-0.728 & 7.685 & 6.573 & 1.00 & 0.00 & C \\
\hline-0.956 & 8.208 & 7.671 & 1.00 & 0.00 & 0 \\
\hline-1.244 & 6.505 & 6.209 & 1.00 & 0.00 & 0 \\
\hline-1.853 & 6.151 & 6.945 & 1.00 & 0.00 & $\mathrm{H}$ \\
\hline 8.511 & 8.608 & 4.597 & 1.00 & 0.00 & C \\
\hline 9.063 & 9.448 & 5.047 & 1.00 & 0.00 & $\mathrm{H}$ \\
\hline 8.517 & 7.782 & 5.319 & 1.00 & 0.00 & $\mathrm{H}$ \\
\hline 9.082 & 8.289 & 3.717 & 1.00 & 0.00 & $\mathrm{H}$ \\
\hline-0.923 & 11.557 & 3.646 & 1.00 & 0.00 & $\mathrm{C}$ \\
\hline-1.368 & 11.348 & 4.626 & 1.00 & 0.00 & $\mathrm{H}$ \\
\hline-1.033 & 12.637 & 3.471 & 1.00 & 0.00 & $\mathrm{H}$ \\
\hline-1.523 & 11.031 & 2.889 & 1.00 & 0.00 & $\mathrm{H}$ \\
\hline 8.874 & 0.178 & 8.933 & 1.00 & 0.00 & $\mathrm{C}$ \\
\hline 9.690 & 0.876 & 8.768 & 1.00 & 0.00 & $\mathrm{H}$ \\
\hline 9.207 & -1.047 & 9.335 & 1.00 & 0.00 & C \\
\hline 8.475 & -1.826 & 9.442 & 1.00 & 0.00 & $\mathrm{H}$ \\
\hline 10.249 & -1.316 & 9.501 & 1.00 & 0.00 & $\mathrm{H}$ \\
\hline 6.210 & -0.077 & 10.771 & 1.00 & 0.00 & C \\
\hline 5.975 & -1.148 & 10.652 & 1.00 & 0.00 & $\mathrm{H}$ \\
\hline 5.397 & 0.387 & 11.341 & 1.00 & 0.00 & $\mathrm{H}$ \\
\hline 7.118 & 0.001 & 11.382 & 1.00 & 0.00 & $\mathrm{H}$ \\
\hline 1.200 & 1.601 & 10.256 & 1.00 & 0.00 & C \\
\hline 1.866 & 1.702 & 11.115 & 1.00 & 0.00 & $\mathrm{H}$ \\
\hline 0.033 & 0.957 & 10.415 & 1.00 & 0.00 & C \\
\hline-0.647 & 0.800 & 9.585 & 1.00 & 0.00 & $\mathrm{H}$ \\
\hline-0.272 & 0.559 & 11.381 & 1.00 & 0.00 & $\mathrm{H}$ \\
\hline-0.484 & 3.284 & 8.052 & 1.00 & 0.00 & C \\
\hline-0.641 & 4.306 & 7.685 & 1.00 & 0.00 & $\mathrm{H}$ \\
\hline-0.917 & 3.219 & 9.057 & 1.00 & 0.00 & $\mathrm{H}$ \\
\hline-1.076 & 2.620 & 7.403 & 1.00 & 0.00 & $\mathrm{H}$ \\
\hline 8.604 & 4.366 & 2.161 & 1.00 & 0.00 & C \\
\hline 7.865 & 4.377 & 1.349 & 1.00 & 0.00 & $\mathrm{H}$ \\
\hline 9.445 & 3.758 & 1.798 & 1.00 & 0.00 & $\mathrm{H}$ \\
\hline 9.082 & 5.803 & 2.434 & 1.00 & 0.00 & C \\
\hline 9.640 & 5.865 & 3.382 & 1.00 & 0.00 & $\mathrm{H}$ \\
\hline 8.212 & 6.470 & 2.541 & 1.00 & 0.00 & $\mathrm{H}$ \\
\hline 9.946 & 6.416 & 1.358 & 1.00 & 0.00 & C \\
\hline 10.174 & 5.893 & 0.260 & 1.00 & 0.00 & 0 \\
\hline 10.462 & 7.595 & 1.722 & 1.00 & 0.00 & 0 \\
\hline 11.071 & 7.949 & 0.986 & 1.00 & 0.00 & $\mathrm{H}$ \\
\hline 0.708 & 5.492 & 3.334 & 1.00 & 0.00 & C \\
\hline 0.155 & 4.653 & 2.884 & 1.00 & 0.00 & $\mathrm{H}$ \\
\hline 0.701 & 6.318 & 2.613 & 1.00 & 0.00 & $\mathrm{H}$ \\
\hline 0.137 & 5.812 & 4.214 & 1.00 & 0.00 & $\mathrm{H}$ \\
\hline 10.141 & 2.543 & 4.285 & 1.00 & 0.00 & $\mathrm{C}$ \\
\hline 10.586 & 2.752 & 3.305 & 1.00 & 0.00 & $\mathrm{H}$ \\
\hline 10.252 & 1.463 & 4.460 & 1.00 & 0.00 & $\mathrm{H}$ \\
\hline 10.742 & 3.069 & 5.042 & 1.00 & 0.00 & $\mathrm{H}$ \\
\hline 17.294 & 11.003 & -6.440 & 1.00 & 0.00 & $\mathrm{FE}$ \\
\hline 15.066 & 12.876 & -7.502 & 1.00 & 0.00 & C \\
\hline 14.707 & 13.666 & -8.674 & 1.00 & 0.00 & C \\
\hline 15.852 & 13.763 & -9.448 & 1.00 & 0.00 & C \\
\hline 16.882 & 13.046 & -8.740 & 1.00 & 0.00 & C \\
\hline 18.197 & 12.927 & -9.178 & 1.00 & 0.00 & C \\
\hline 18.451 & 13.423 & -10.113 & 1.00 & 0.00 & $\mathrm{H}$ \\
\hline 19.216 & 12.229 & -8.542 & 1.00 & 0.00 & $\mathrm{C}$ \\
\hline 20.585 & 12.156 & -9.012 & 1.00 & 0.00 & C \\
\hline 21.291 & 11.436 & -8.065 & 1.00 & 0.00 & C \\
\hline 20.340 & 11.071 & -7.041 & 1.00 & 0.00 & C \\
\hline 20.644 & 10.336 & -5.897 & 1.00 & 0.00 & C \\
\hline 21.671 & 9.993 & -5.791 & 1.00 & 0.00 & $\mathrm{H}$ \\
\hline 19.770 & 9.996 & -4.874 & 1.00 & 0.00 & C \\
\hline 20.151 & 9.271 & -3.684 & 1.00 & 0.00 & C \\
\hline 19.011 & 9.124 & -2.927 & 1.00 & 0.00 & C \\
\hline
\end{tabular}




\begin{tabular}{|c|c|c|c|c|c|c|c|c|c|c|}
\hline HETATM & 165 & $\mathrm{C} 14$ & HEM & 17 & 17.938 & 9.765 & -3.659 & 1.00 & 0.00 & C \\
\hline HETATM & 166 & C15 & HEM & 17 & 16.610 & 9.820 & -3.237 & 1.00 & 0.00 & C \\
\hline HETATM & 167 & H15 & HEM & 17 & 16.370 & 9.335 & -2.289 & 1.00 & 0.00 & $\mathrm{H}$ \\
\hline HETATM & 168 & C16 & HEM & 17 & 15.580 & 10.502 & -3.880 & 1.00 & 0.00 & C \\
\hline HETATM & 169 & C17 & HEM & 17 & 14.214 & 10.615 & -3.398 & 1.00 & 0.00 & C \\
\hline HETATM & 170 & C18 & HEM & 17 & 13.539 & 11.383 & -4.322 & 1.00 & 0.00 & C \\
\hline HETATM & 171 & C19 & HEM & 17 & 14.488 & 11.735 & -5.358 & 1.00 & 0.00 & C \\
\hline HETATM & 172 & $\mathrm{C} 20$ & HEM & 17 & 14.197 & 12.526 & -6.469 & 1.00 & 0.00 & $\mathrm{C}$ \\
\hline HETATM & 173 & H2O & HEM & 17 & 13.183 & 12.915 & -6.530 & 1.00 & 0.00 & $\mathrm{H}$ \\
\hline HETATM & 174 & N21 & HEM & 17 & 16.387 & 12.513 & -7.564 & 1.00 & 0.00 & $\mathrm{~N}$ \\
\hline HETATM & 175 & N22 & HEM & 17 & 19.092 & 11.566 & -7.342 & 1.00 & 0.00 & $\mathrm{~N}$ \\
\hline HETATM & 176 & N23 & HEM & 17 & 18.421 & 10.303 & -4.841 & 1.00 & 0.00 & $\mathrm{~N}$ \\
\hline HETATM & 177 & N24 & HEM & 17 & 15.722 & 11.187 & -5.077 & 1.00 & 0.00 & $\mathrm{~N}$ \\
\hline HETATM & 178 & C99 & HEM & 17 & 16.628 & 8.322 & -7.579 & 1.00 & 0.00 & $\mathrm{C}$ \\
\hline HETATM & 179 & 091 & HEM & 17 & 16.788 & 9.635 & -7.567 & 1.00 & 0.00 & O \\
\hline HETATM & 180 & 092 & HEM & 17 & 16.473 & 7.705 & -8.635 & 1.00 & 0.00 & O \\
\hline HETATM & 181 & CT91 & HEM & 17 & 16.644 & 7.640 & -6.223 & 1.00 & 0.00 & C \\
\hline HETATM & 182 & H911 & HEM & 17 & 17.623 & 7.836 & -5.760 & 1.00 & 0.00 & $\mathrm{H}$ \\
\hline HETATM & 183 & H912 & HEM & 17 & 15.896 & 8.130 & -5.580 & 1.00 & 0.00 & $\mathrm{H}$ \\
\hline HETATM & 184 & CT92 & HEM & 17 & 16.385 & 6.136 & -6.334 & 1.00 & 0.00 & C \\
\hline HETATM & 185 & H921 & HEM & 17 & 17.181 & 5.676 & -6.931 & 1.00 & 0.00 & $\mathrm{H}$ \\
\hline HETATM & 186 & H922 & HEM & 17 & 15.460 & 5.980 & -6.905 & 1.00 & 0.00 & $\mathrm{H}$ \\
\hline \multicolumn{11}{|l|}{ TER } \\
\hline HETATM & 187 & $\mathrm{FE}$ & HEM & 18 & 17.986 & 3.589 & -1.491 & 1.00 & 0.00 & $\mathrm{FE}$ \\
\hline HETATM & 188 & $\mathrm{C} 1$ & HEM & 18 & 20.214 & 1.717 & -0.429 & 1.00 & 0.00 & $\mathrm{C}$ \\
\hline HETATM & 189 & $\mathrm{C} 2$ & HEM & 18 & 20.573 & 0.927 & 0.742 & 1.00 & 0.00 & $\mathrm{C}$ \\
\hline HETATM & 190 & C3 & HEM & 18 & 19.427 & 0.830 & 1.517 & 1.00 & 0.00 & C \\
\hline HETATM & 191 & $\mathrm{C} 4$ & HEM & 18 & 18.398 & 1.547 & 0.809 & 1.00 & 0.00 & C \\
\hline HETATM & 192 & C5 & HEM & 18 & 17.082 & 1.666 & 1.247 & 1.00 & 0.00 & C \\
\hline HETATM & 193 & H5 & HEM & 18 & 16.829 & 1.170 & 2.182 & 1.00 & 0.00 & $\mathrm{H}$ \\
\hline HETATM & 194 & $\mathrm{C} 6$ & HEM & 18 & 16.063 & 2.364 & 0.611 & 1.00 & 0.00 & C \\
\hline HETATM & 195 & C7 & HEM & 18 & 14.694 & 2.437 & 1.081 & 1.00 & 0.00 & C \\
\hline HETATM & 196 & $\mathrm{C} 8$ & HEM & 18 & 13.989 & 3.157 & 0.134 & 1.00 & 0.00 & $\mathrm{C}$ \\
\hline HETATM & 197 & $\mathrm{C} 9$ & HEM & 18 & 14.940 & 3.522 & -0.891 & 1.00 & 0.00 & $\mathrm{C}$ \\
\hline HETATM & 198 & $\mathrm{C} 10$ & HEM & 18 & 14.635 & 4.256 & -2.034 & 1.00 & 0.00 & C \\
\hline HETATM & 199 & $\mathrm{H} 10$ & HEM & 18 & 13.608 & 4.600 & -2.140 & 1.00 & 0.00 & $\mathrm{H}$ \\
\hline HETATM & 200 & $\mathrm{C} 11$ & HEM & 18 & 15.510 & 4.596 & -3.057 & 1.00 & 0.00 & C \\
\hline HETATM & 201 & $\mathrm{C} 12$ & HEM & 18 & 15.129 & 5.322 & -4.247 & 1.00 & 0.00 & C \\
\hline HETATM & 202 & $\mathrm{C} 13$ & HEM & 18 & 16.268 & 5.469 & -5.004 & 1.00 & 0.00 & C \\
\hline HETATM & 203 & C14 & HEM & 18 & 17.341 & 4.828 & -4.273 & 1.00 & 0.00 & C \\
\hline HETATM & 204 & C15 & HEM & 18 & 18.669 & 4.773 & -4.694 & 1.00 & 0.00 & C \\
\hline HETATM & 205 & H15 & HEM & 18 & 18.909 & 5.258 & -5.642 & 1.00 & 0.00 & $\mathrm{H}$ \\
\hline HETATM & 206 & C16 & HEM & 18 & 19.699 & 4.091 & -4.051 & 1.00 & 0.00 & C \\
\hline HETATM & 207 & $\mathrm{C} 17$ & HEM & 18 & 21.065 & 3.978 & -4.533 & 1.00 & 0.00 & $\mathrm{C}$ \\
\hline HETATM & 208 & $\mathrm{C} 18$ & HEM & 18 & 21.741 & 3.210 & -3.609 & 1.00 & 0.00 & C \\
\hline HETATM & 209 & C19 & HEM & 18 & 20.791 & 2.858 & -2.573 & 1.00 & 0.00 & C \\
\hline HETATM & 210 & $\mathrm{C} 20$ & HEM & 18 & 21.082 & 2.067 & -1.462 & 1.00 & 0.00 & C \\
\hline HETATM & 211 & $\mathrm{H} 2 \mathrm{O}$ & HEM & 18 & 22.097 & 1.678 & -1.402 & 1.00 & 0.00 & $\mathrm{H}$ \\
\hline HETATM & 212 & N21 & HEM & 18 & 18.892 & 2.080 & -0.367 & 1.00 & 0.00 & $\mathrm{~N}$ \\
\hline HETATM & 213 & N22 & HEM & 18 & 16.187 & 3.027 & -0.590 & 1.00 & 0.00 & $\mathrm{~N}$ \\
\hline HETATM & 214 & N23 & HEM & 18 & 16.858 & 4.290 & -3.090 & 1.00 & 0.00 & $\mathrm{~N}$ \\
\hline HETATM & 215 & N24 & HEM & 18 & 19.558 & 3.406 & -2.854 & 1.00 & 0.00 & $\mathrm{~N}$ \\
\hline HETATM & 216 & C99 & HEM & 18 & 18.651 & 6.271 & -0.352 & 1.00 & 0.00 & $\mathrm{C}$ \\
\hline HETATM & 217 & 091 & HEM & 18 & 18.492 & 4.958 & -0.365 & 1.00 & 0.00 & O \\
\hline HETATM & 218 & 092 & HEM & 18 & 18.806 & 6.888 & 0.703 & 1.00 & 0.00 & o \\
\hline HETATM & 219 & СТ91 & HEM & 18 & 18.635 & 6.952 & -1.709 & 1.00 & 0.00 & C \\
\hline HETATM & 220 & H911 & HEM & 18 & 17.656 & 6.757 & -2.171 & 1.00 & 0.00 & $\mathrm{H}$ \\
\hline HETATM & 221 & H912 & HEM & 18 & 19.383 & 6.463 & -2.352 & 1.00 & 0.00 & $\mathrm{H}$ \\
\hline HETATM & 222 & СТ92 & HEM & 18 & 18.895 & 8.457 & -1.597 & 1.00 & 0.00 & C \\
\hline HETATM & 223 & H921 & HEM & 18 & 19.820 & 8.613 & -1.027 & 1.00 & 0.00 & $\mathrm{H}$ \\
\hline HETATM & 224 & H922 & HEM & 18 & 18.098 & 8.917 & -1.000 & 1.00 & 0.00 & $\mathrm{H}$ \\
\hline \multicolumn{11}{|l|}{ TER } \\
\hline HETATM & 225 & $\mathrm{C} 2$ & VIN & 19 & 13.375 & 14.169 & -8.933 & 1.00 & 0.00 & $\mathrm{C}$ \\
\hline HETATM & 226 & Н 21 & VIN & 19 & 12.559 & 13.470 & -8.768 & 1.00 & 0.00 & $\mathrm{H}$ \\
\hline HETATM & 227 & $\mathrm{C} 1$ & VIN & 19 & 13.042 & 15.394 & -9.335 & 1.00 & 0.00 & C \\
\hline HETATM & 228 & H11 & VIN & 19 & 13.774 & 16.172 & -9.442 & 1.00 & 0.00 & $\mathrm{H}$ \\
\hline HETATM & 229 & $\mathrm{H} 12$ & VIN & 19 & 12.000 & 15.663 & -9.501 & 1.00 & 0.00 & $\mathrm{H}$ \\
\hline \multicolumn{11}{|l|}{ TER } \\
\hline HETATM & 230 & $\mathrm{C} 1$ & $\mathrm{MET}$ & 20 & 16.039 & 14.424 & -10.771 & 1.00 & 0.00 & C \\
\hline HETATM & 231 & H11 & $\mathrm{MET}$ & 20 & 16.274 & 15.494 & -10.652 & 1.00 & 0.00 & $\mathrm{H}$ \\
\hline HETATM & 232 & H12 & $\mathrm{MET}$ & 20 & 16.852 & 13.959 & -11.341 & 1.00 & 0.00 & $\mathrm{H}$ \\
\hline HETATM & 233 & $\mathrm{H} 13$ & $\mathrm{MET}$ & 20 & 15.131 & 14.346 & $-11 \cdot 382$ & 1.00 & 0.00 & $\mathrm{H}$ \\
\hline \multicolumn{11}{|l|}{ TER } \\
\hline HETATM & 234 & $\mathrm{C} 2$ & VIN & 21 & 21.049 & 12.746 & -10.256 & 1.00 & 0.00 & C \\
\hline HETATM & 235 & H 21 & VIN & 21 & 20.383 & 12.645 & -11.115 & 1.00 & 0.00 & $\mathrm{H}$ \\
\hline HETATM & 236 & $\mathrm{C} 1$ & VIN & 21 & 22.216 & 13.390 & -10.415 & 1.00 & 0.00 & C \\
\hline HETATM & 237 & H11 & VIN & 21 & 22.896 & 13.546 & -9.585 & 1.00 & 0.00 & $\mathrm{H}$ \\
\hline HETATM & 238 & $\mathrm{H} 12$ & VIN & 21 & 22.521 & 13.788 & -11.381 & 1.00 & 0.00 & $\mathrm{H}$ \\
\hline
\end{tabular}




\begin{tabular}{|c|c|c|c|}
\hline HETATM & 239 & $\mathrm{C} 1$ & MET \\
\hline HETATM & 240 & H11 & MET \\
\hline HETATM & 241 & $\mathrm{H} 12$ & MET \\
\hline HETATM & 242 & H13 & MET \\
\hline \multicolumn{4}{|l|}{ TER } \\
\hline HETATM & 243 & C5 & $\mathrm{ACP}$ \\
\hline HETATM & 244 & H51 & $\mathrm{ACP}$ \\
\hline HETATM & 245 & H52 & $\mathrm{ACP}$ \\
\hline HETATM & 246 & C4 & $\mathrm{ACP}$ \\
\hline HETATM & 247 & H4 1 & $\mathrm{ACP}$ \\
\hline HETATM & 248 & $\mathrm{H} 42$ & $\mathrm{ACP}$ \\
\hline HETATM & 249 & $\mathrm{C} 1$ & $\mathrm{ACP}$ \\
\hline HETATM & 250 & 02 & $\mathrm{ACP}$ \\
\hline HETATM & 251 & 03 & $\mathrm{ACP}$ \\
\hline HETATM & 252 & H3 & $\mathrm{ACP}$ \\
\hline \multicolumn{4}{|l|}{ TER } \\
\hline HETATM & 253 & $\mathrm{C} 1$ & $\mathrm{MET}$ \\
\hline HETATM & 254 & H11 & MET \\
\hline HETATM & 255 & $\mathrm{H} 12$ & MET \\
\hline HETATM & 256 & H13 & MET \\
\hline \multicolumn{4}{|l|}{ TER } \\
\hline HETATM & 257 & $\mathrm{C} 1$ & MET \\
\hline HETATM & 258 & H11 & MET \\
\hline HETATM & 259 & $\mathrm{H} 12$ & MET \\
\hline HETATM & 260 & H13 & MET \\
\hline \multicolumn{4}{|l|}{ TER } \\
\hline HETATM & 261 & C2 & VIN \\
\hline HETATM & 262 & H 21 & VIN \\
\hline HETATM & 263 & $\mathrm{C} 1$ & VIN \\
\hline HETATM & 264 & H11 & VIN \\
\hline HETATM & 265 & $\mathrm{H} 12$ & VIN \\
\hline \multicolumn{4}{|l|}{ TER } \\
\hline HETATM & 266 & $\mathrm{C} 1$ & MET \\
\hline HETATM & 267 & H11 & $\mathrm{MET}$ \\
\hline HETATM & 268 & $\mathrm{H} 12$ & MET \\
\hline HETATM & 269 & $\mathrm{H} 13$ & MET \\
\hline \multicolumn{4}{|l|}{ TER } \\
\hline HETATM & 270 & $\mathrm{C} 2$ & VIN \\
\hline HETATM & 271 & H21 & VIN \\
\hline HETATM & 272 & C1 & VIN \\
\hline HETATM & 273 & H11 & VIN \\
\hline HETATM & 274 & $\mathrm{H} 12$ & VIN \\
\hline \multicolumn{4}{|l|}{ TER } \\
\hline HETATM & 275 & $\mathrm{C} 1$ & MET \\
\hline HETATM & 276 & H11 & $\mathrm{MET}$ \\
\hline HETATM & 277 & $\mathrm{H} 12$ & $\mathrm{MET}$ \\
\hline HETATM & 278 & H13 & MET \\
\hline \multicolumn{4}{|l|}{ TER } \\
\hline HETATM & 279 & C5 & $\mathrm{ACP}$ \\
\hline HETATM & 280 & H51 & $\mathrm{ACP}$ \\
\hline HETATM & 281 & H52 & $\mathrm{ACP}$ \\
\hline HETATM & 282 & $\mathrm{C} 4$ & $\mathrm{ACP}$ \\
\hline HETATM & 283 & H 41 & $\mathrm{ACP}$ \\
\hline HETATM & 284 & $\mathrm{H} 42$ & $\mathrm{ACP}$ \\
\hline HETATM & 285 & $\mathrm{C} 1$ & $\mathrm{ACP}$ \\
\hline HETATM & 286 & 02 & $\mathrm{ACP}$ \\
\hline HETATM & 287 & 03 & $\mathrm{ACP}$ \\
\hline HETATM & 288 & H3 & $\mathrm{ACP}$ \\
\hline \multicolumn{4}{|l|}{ TER } \\
\hline HETATM & 289 & $\mathrm{C} 1$ & MET \\
\hline HETATM & 290 & H11 & MET \\
\hline HETATM & 291 & $\mathrm{H} 12$ & MET \\
\hline HETATM & 292 & $\mathrm{H} 13$ & $\mathrm{MET}$ \\
\hline \multicolumn{4}{|l|}{ TER } \\
\hline HETATM & 293 & $\mathrm{C} 1$ & MET \\
\hline HETATM & 294 & H11 & MET \\
\hline HETATM & 295 & $\mathrm{H} 12$ & MET \\
\hline HETATM & 296 & $\mathrm{H} 13$ & MET \\
\hline TER & & & \\
\hline
\end{tabular}

\begin{tabular}{|c|c|c|c|c|c|}
\hline 22.733 & 11.062 & -8.052 & 1.00 & 0.00 & C \\
\hline 23.325 & 11.727 & -7.403 & 1.00 & 0.00 & $\mathrm{H}$ \\
\hline 22.890 & 10.040 & -7.685 & 1.00 & 0.00 & $\mathrm{H}$ \\
\hline 23.166 & 11.128 & -9.057 & 1.00 & 0.00 & $\mathrm{H}$ \\
\hline 13.645 & 9.981 & -2.161 & 1.00 & 0.00 & C \\
\hline 14.384 & 9.969 & -1.349 & 1.00 & 0.00 & $\mathrm{H}$ \\
\hline 12.804 & 10.589 & -1.798 & 1.00 & 0.00 & $\mathrm{H}$ \\
\hline 13.167 & 8.543 & -2.434 & 1.00 & 0.00 & $\mathrm{C}$ \\
\hline 12.609 & 8.482 & -3.382 & 1.00 & 0.00 & $\mathrm{H}$ \\
\hline 14.037 & 7.877 & -2.541 & 1.00 & 0.00 & $\mathrm{H}$ \\
\hline 12.303 & 7.931 & -1.358 & 1.00 & 0.00 & $\mathrm{C}$ \\
\hline 12.075 & 8.454 & -0.260 & 1.00 & 0.00 & 0 \\
\hline 11.787 & 6.751 & -1.722 & 1.00 & 0.00 & O \\
\hline 11.178 & 6.397 & -0.986 & 1.00 & 0.00 & $\mathrm{H}$ \\
\hline 21.541 & 8.855 & -3.334 & 1.00 & 0.00 & C \\
\hline 22.094 & 9.694 & -2.884 & 1.00 & 0.00 & $\mathrm{H}$ \\
\hline 21.548 & 8.029 & -2.613 & 1.00 & 0.00 & $\mathrm{H}$ \\
\hline 22.112 & 8.535 & -4.214 & 1.00 & 0.00 & $\mathrm{H}$ \\
\hline 12.108 & 11.803 & -4.285 & 1.00 & 0.00 & C \\
\hline 11.663 & 11.594 & -3.305 & 1.00 & 0.00 & $\mathrm{H}$ \\
\hline 11.997 & 12.883 & -4.460 & 1.00 & 0.00 & $\mathrm{H}$ \\
\hline 11.507 & 11.278 & -5.042 & 1.00 & 0.00 & $\mathrm{H}$ \\
\hline 21.904 & 0.424 & 1.002 & 1.00 & 0.00 & C \\
\hline 22.720 & 1.123 & 0.837 & 1.00 & 0.00 & $\mathrm{H}$ \\
\hline 22.237 & -0.801 & 1.403 & 1.00 & 0.00 & C \\
\hline 21.506 & -1.579 & 1.511 & 1.00 & 0.00 & $\mathrm{H}$ \\
\hline 23.279 & -1.070 & 1.570 & 1.00 & 0.00 & $\mathrm{H}$ \\
\hline 19.241 & 0.169 & 2.840 & 1.00 & 0.00 & C \\
\hline 19.006 & -0.902 & 2.720 & 1.00 & 0.00 & $\mathrm{H}$ \\
\hline 18.428 & 0.633 & 3.410 & 1.00 & 0.00 & $\mathrm{H}$ \\
\hline 20.148 & 0.247 & 3.450 & 1.00 & 0.00 & $\mathrm{H}$ \\
\hline 14.231 & 1.847 & 2.325 & 1.00 & 0.00 & C \\
\hline 14.897 & 1.948 & 3.184 & 1.00 & 0.00 & $\mathrm{H}$ \\
\hline 13.064 & 1.203 & 2.484 & 1.00 & 0.00 & C \\
\hline 12.384 & 1.047 & 1.654 & 1.00 & 0.00 & $\mathrm{H}$ \\
\hline 12.759 & 0.805 & 3.450 & 1.00 & 0.00 & $\mathrm{H}$ \\
\hline 12.546 & 3.530 & 0.121 & 1.00 & 0.00 & C \\
\hline 12.390 & 4.553 & -0.246 & 1.00 & 0.00 & $\mathrm{H}$ \\
\hline 12.114 & 3.465 & 1.126 & 1.00 & 0.00 & $\mathrm{H}$ \\
\hline 11.955 & 2.866 & -0.528 & 1.00 & 0.00 & $\mathrm{H}$ \\
\hline 21.634 & 4.612 & -5.770 & 1.00 & 0.00 & C \\
\hline 20.895 & 4.623 & -6.582 & 1.00 & 0.00 & $\mathrm{H}$ \\
\hline 22.475 & 4.004 & -6.134 & 1.00 & 0.00 & $\mathrm{H}$ \\
\hline 22.113 & 6.050 & -5.497 & 1.00 & 0.00 & C \\
\hline 22.671 & 6.111 & -4.549 & 1.00 & 0.00 & $\mathrm{H}$ \\
\hline 21.242 & 6.716 & -5.390 & 1.00 & 0.00 & $\mathrm{H}$ \\
\hline 22.977 & 6.662 & -6.573 & 1.00 & 0.00 & C \\
\hline 23.205 & 6.139 & -7.671 & 1.00 & 0.00 & O \\
\hline 23.493 & 7.841 & -6.209 & 1.00 & 0.00 & O \\
\hline 24.102 & 8.196 & -6.945 & 1.00 & 0.00 & $\mathrm{H}$ \\
\hline 13.738 & 5.738 & -4.597 & 1.00 & 0.00 & C \\
\hline 13.186 & 4.899 & -5.047 & 1.00 & 0.00 & $\mathrm{H}$ \\
\hline 13.732 & 6.564 & -5.319 & 1.00 & 0.00 & $\mathrm{H}$ \\
\hline 13.167 & 6.058 & -3.717 & 1.00 & 0.00 & $\mathrm{H}$ \\
\hline 23.172 & 2.790 & -3.646 & 1.00 & 0.00 & C \\
\hline 23.617 & 2.998 & -4.626 & 1.00 & 0.00 & $\mathrm{H}$ \\
\hline 23.282 & 1.709 & -3.471 & 1.00 & 0.00 & $\mathrm{H}$ \\
\hline 23.772 & 3.315 & -2.889 & 1.00 & 0.00 & $\mathrm{H}$ \\
\hline
\end{tabular}

END

\subsection{T1 dimer: AYY_T1.pdb file}

$\begin{array}{lllllllll}\text { HETATM } & 1 & \text { FE HEM } & 1 & 4.263 & 10.757 & 1.491 & 1.00 & 0.00 \\ \text { HETATM } & 2 & \text { C1 HEM } & 1 & 2.035 & 12.629 & 0.429 & 1.00 & 0.00 \\ \text { HETATM } & 3 & \text { C2 HEM } & 1 & 1.676 & 13.420 & -0.742 & 1.00 & 0.00 \\ \text { HETATM } & 4 & \text { C3 HEM } & 1 & 2.822 & 13.517 & -1.517 & 1.00 & 0.00 \\ \text { HETATM } & 5 & \text { C4 HEM } & 1 & 3.851 & 12.800 & -0.809 & 1.00 & 0.00\end{array}$




\begin{tabular}{|c|c|c|c|}
\hline HETATM & 6 & C5 & HEM \\
\hline HETATM & 7 & H5 & HEM \\
\hline HETATM & 8 & $\mathrm{C} 6$ & HEM \\
\hline HETATM & 9 & C7 & HEM \\
\hline HETATM & 10 & $\mathrm{C} 8$ & HEM \\
\hline HETATM & 11 & $\mathrm{C} 9$ & HEM \\
\hline HETATM & 12 & $\mathrm{C} 10$ & HEM \\
\hline HETATM & 13 & $\mathrm{H} 10$ & HEM \\
\hline HETATM & 14 & C11 & HEM \\
\hline HETATM & 15 & $\mathrm{C} 12$ & HEM \\
\hline HETATM & 16 & $\mathrm{C} 13$ & HEM \\
\hline HETATM & 17 & C14 & HEM \\
\hline HETATM & 18 & C15 & HEM \\
\hline HETATM & 19 & H15 & HEM \\
\hline HETATM & 20 & $\mathrm{C} 16$ & HEM \\
\hline HETATM & 21 & C17 & HEM \\
\hline HETATM & 22 & C18 & HEM \\
\hline HETATM & 23 & C19 & HEM \\
\hline HETATM & 24 & $\mathrm{C} 20$ & HEM \\
\hline HETATM & 25 & $\mathrm{H} 2 \mathrm{O}$ & HEM \\
\hline HETATM & 26 & N21 & HEM \\
\hline HETATM & 27 & N22 & HEM \\
\hline HETATM & 28 & N23 & HEM \\
\hline HETATM & 29 & N24 & HEM \\
\hline HETATM & 30 & C99 & HEM \\
\hline HETATM & 31 & 091 & HEM \\
\hline HETATM & 32 & 092 & HEM \\
\hline HETATM & 33 & СТ91 & HEM \\
\hline HETATM & 34 & H911 & HEM \\
\hline HETATM & 35 & H912 & HEM \\
\hline HETATM & 36 & CT 92 & HEM \\
\hline HETATM & 37 & H921 & HEM \\
\hline HETATM & 38 & H922 & HEM \\
\hline \multicolumn{4}{|l|}{ TER } \\
\hline HETATM & 39 & $\mathrm{FE}$ & HEM \\
\hline HETATM & 40 & $\mathrm{C} 1$ & HEM \\
\hline HETATM & 41 & $\mathrm{C} 2$ & HEM \\
\hline HETATM & 42 & $\mathrm{C} 3$ & HEM \\
\hline HETATM & 43 & C4 & HEM \\
\hline HETATM & 44 & C5 & HEM \\
\hline HETATM & 45 & H5 & HEM \\
\hline HETATM & 46 & $\mathrm{C} 6$ & HEM \\
\hline HETATM & 47 & C7 & HEM \\
\hline HETATM & 48 & $\mathrm{C} 8$ & HEM \\
\hline HETATM & 49 & $\mathrm{C} 9$ & HEM \\
\hline HETATM & 50 & $\mathrm{C} 10$ & HEM \\
\hline HETATM & 51 & $\mathrm{H} 10$ & HEM \\
\hline HETATM & 52 & $\mathrm{C} 11$ & HEM \\
\hline HETATM & 53 & C12 & HEM \\
\hline HETATM & 54 & $\mathrm{C} 13$ & HEM \\
\hline HETATM & 55 & C14 & HEM \\
\hline HETATM & 56 & C15 & HEM \\
\hline HETATM & 57 & H15 & HEM \\
\hline HETATM & 58 & C16 & HEM \\
\hline HETATM & 59 & C17 & HEM \\
\hline HETATM & 60 & C18 & HEM \\
\hline HETATM & 61 & C19 & HEM \\
\hline HETATM & 62 & $\mathrm{C} 20$ & HEM \\
\hline HETATM & 63 & $\mathrm{H} 2 \mathrm{O}$ & HEM \\
\hline HETATM & 64 & N21 & HEM \\
\hline HETATM & 65 & N22 & HEM \\
\hline HETATM & 66 & N23 & HEM \\
\hline HETATM & 67 & $\mathrm{~N} 24$ & HEM \\
\hline HETATM & 68 & C99 & HEM \\
\hline HETATM & 69 & 091 & HEM \\
\hline HETATM & 70 & 092 & HEM \\
\hline HETATM & 71 & СТ 91 & HEM \\
\hline HETATM & 72 & H911 & HEM \\
\hline HETATM & 73 & H912 & HEM \\
\hline HETATM & 74 & СТ92 & HEM \\
\hline HETATM & 75 & H921 & HEM \\
\hline HETATM & 76 & H922 & HEM \\
\hline \multicolumn{4}{|l|}{ TER } \\
\hline HETATM & 77 & $\mathrm{C} 2$ & VIN \\
\hline HETATM & 78 & H 21 & VIN \\
\hline HETATM & 79 & $\mathrm{C} 1$ & VIN \\
\hline HETATM & 80 & $\mathrm{H} 11$ & VIN \\
\hline HETATM & 81 & $\mathrm{H} 12$ & VIN \\
\hline
\end{tabular}

\begin{tabular}{|c|c|c|c|c|c|}
\hline 5.167 & 12.681 & -1.247 & 1.00 & 0.00 & C \\
\hline 5.420 & 13.177 & -2.182 & 1.00 & 0.00 & $\mathrm{H}$ \\
\hline 6.186 & 11.982 & -0.611 & 1.00 & 0.00 & C \\
\hline 7.555 & 11.910 & -1.081 & 1.00 & 0.00 & $\mathrm{C}$ \\
\hline 8.260 & 11.190 & -0.134 & 1.00 & 0.00 & C \\
\hline 7.309 & 10.825 & 0.891 & 1.00 & 0.00 & C \\
\hline 7.614 & 10.090 & 2.034 & 1.00 & 0.00 & C \\
\hline 8.641 & 9.746 & 2.140 & 1.00 & 0.00 & $\mathrm{H}$ \\
\hline 6.739 & 9.750 & 3.057 & 1.00 & 0.00 & C \\
\hline 7.120 & 9.025 & 4.247 & 1.00 & 0.00 & C \\
\hline 5.981 & 8.878 & 5.004 & 1.00 & 0.00 & C \\
\hline 4.908 & 9.519 & 4.273 & 1.00 & 0.00 & C \\
\hline 3.580 & 9.574 & 4.694 & 1.00 & 0.00 & C \\
\hline 3.340 & 9.089 & 5.642 & 1.00 & 0.00 & $\mathrm{H}$ \\
\hline 2.550 & 10.255 & 4.051 & 1.00 & 0.00 & C \\
\hline 1.184 & 10.369 & 4.533 & 1.00 & 0.00 & C \\
\hline 0.508 & 11.137 & 3.609 & 1.00 & 0.00 & C \\
\hline 1.458 & 11.489 & 2.573 & 1.00 & 0.00 & $\mathrm{C}$ \\
\hline 1.167 & 12.280 & 1.462 & 1.00 & 0.00 & C \\
\hline 0.152 & 12.669 & 1.402 & 1.00 & 0.00 & $\mathrm{H}$ \\
\hline 3.357 & 12.266 & 0.367 & 1.00 & 0.00 & $\mathrm{~N}$ \\
\hline 6.062 & 11.319 & 0.590 & 1.00 & 0.00 & $\mathrm{~N}$ \\
\hline 5.391 & 10.056 & 3.090 & 1.00 & 0.00 & $\mathrm{~N}$ \\
\hline 2.691 & 10.941 & 2.854 & 1.00 & 0.00 & $\mathrm{~N}$ \\
\hline 3.598 & 8.076 & 0.352 & 1.00 & 0.00 & $\mathrm{C}$ \\
\hline 3.757 & 9.389 & 0.365 & 1.00 & 0.00 & O \\
\hline 3.443 & 7.458 & -0.703 & 1.00 & 0.00 & 0 \\
\hline 3.614 & 7.394 & 1.709 & 1.00 & 0.00 & C \\
\hline 4.593 & 7.590 & 2.171 & 1.00 & 0.00 & $\mathrm{H}$ \\
\hline 2.866 & 7.884 & 2.352 & 1.00 & 0.00 & $\mathrm{H}$ \\
\hline 3.354 & 5.890 & 1.597 & 1.00 & 0.00 & C \\
\hline 4.151 & 5.429 & 1.000 & 1.00 & 0.00 & $\mathrm{H}$ \\
\hline 2.429 & 5.734 & 1.027 & 1.00 & 0.00 & $\mathrm{H}$ \\
\hline 4.955 & 3.343 & 6.440 & 1.00 & 0.00 & $\mathrm{FE}$ \\
\hline 7.183 & 1.471 & 7.502 & 1.00 & 0.00 & C \\
\hline 7.542 & 0.680 & 8.674 & 1.00 & 0.00 & $\mathrm{C}$ \\
\hline 6.397 & 0.583 & 9.448 & 1.00 & 0.00 & $\mathrm{C}$ \\
\hline 5.367 & 1.301 & 8.740 & 1.00 & 0.00 & C \\
\hline 4.052 & 1.420 & 9.178 & 1.00 & 0.00 & C \\
\hline 3.798 & 0.923 & 10.113 & 1.00 & 0.00 & $\mathrm{H}$ \\
\hline 3.033 & 2.118 & 8.542 & 1.00 & 0.00 & $\mathrm{C}$ \\
\hline 1.664 & 2.191 & 9.012 & 1.00 & 0.00 & C \\
\hline 0.958 & 2.910 & 8.065 & 1.00 & 0.00 & C \\
\hline 1.909 & 3.275 & 7.041 & 1.00 & 0.00 & $\mathrm{C}$ \\
\hline 1.605 & 4.010 & 5.897 & 1.00 & 0.00 & C \\
\hline 0.578 & 4.354 & 5.791 & 1.00 & 0.00 & $\mathrm{H}$ \\
\hline 2.479 & 4.350 & 4.874 & 1.00 & 0.00 & C \\
\hline 2.098 & 5.076 & 3.684 & 1.00 & 0.00 & C \\
\hline 3.238 & 5.223 & 2.927 & 1.00 & 0.00 & C \\
\hline 4.311 & 4.581 & 3.659 & 1.00 & 0.00 & C \\
\hline 5.639 & 4.527 & 3.237 & 1.00 & 0.00 & C \\
\hline 5.879 & 5.011 & 2.289 & 1.00 & 0.00 & $\mathrm{H}$ \\
\hline 6.669 & 3.845 & 3.880 & 1.00 & 0.00 & C \\
\hline 8.035 & 3.732 & 3.398 & 1.00 & 0.00 & $\mathrm{C}$ \\
\hline 8.710 & 2.964 & 4.322 & 1.00 & 0.00 & $\mathrm{C}$ \\
\hline 7.761 & 2.612 & 5.358 & 1.00 & 0.00 & $\mathrm{C}$ \\
\hline 8.052 & 1.821 & 6.469 & 1.00 & 0.00 & $\mathrm{C}$ \\
\hline 9.066 & 1.432 & 6.530 & 1.00 & 0.00 & $\mathrm{H}$ \\
\hline 5.862 & 1.834 & 7.564 & 1.00 & 0.00 & $\mathrm{~N}$ \\
\hline 3.157 & 2.781 & 7.342 & 1.00 & 0.00 & $\mathrm{~N}$ \\
\hline 3.828 & 4.044 & 4.841 & 1.00 & 0.00 & $\mathrm{~N}$ \\
\hline 6.527 & 3.160 & 5.077 & 1.00 & 0.00 & $\mathrm{~N}$ \\
\hline 5.621 & 6.025 & 7.579 & 1.00 & 0.00 & C \\
\hline 5.461 & 4.711 & 7.567 & 1.00 & 0.00 & O \\
\hline 5.776 & 6.642 & 8.635 & 1.00 & 0.00 & o \\
\hline 5.605 & 6.706 & 6.223 & 1.00 & 0.00 & C \\
\hline 4.626 & 6.511 & 5.760 & 1.00 & 0.00 & $\mathrm{H}$ \\
\hline 6.353 & 6.216 & 5.580 & 1.00 & 0.00 & $\mathrm{H}$ \\
\hline 5.864 & 8.211 & 6.334 & 1.00 & 0.00 & C \\
\hline 6.789 & 8.367 & 6.905 & 1.00 & 0.00 & $\mathrm{H}$ \\
\hline 5.068 & 8.671 & 6.931 & 1.00 & 0.00 & $\mathrm{H}$ \\
\hline 0.345 & 13.923 & -1.002 & 1.00 & 0.00 & C \\
\hline-0.471 & 13.224 & -0.837 & 1.00 & 0.00 & $\mathrm{H}$ \\
\hline 0.012 & 15.147 & -1.403 & 1.00 & 0.00 & $\mathrm{C}$ \\
\hline 0.743 & 15.926 & -1.511 & 1.00 & 0.00 & $\mathrm{H}$ \\
\hline-1.030 & 15.417 & -1.570 & 1.00 & 0.00 & 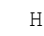 \\
\hline
\end{tabular}




\begin{tabular}{|c|c|c|c|}
\hline HETATM & 82 & $\mathrm{C} 1$ & MET \\
\hline HETATM & 83 & H11 & MET \\
\hline HETATM & 84 & H12 & MET \\
\hline HETATM & 85 & H13 & MET \\
\hline \multicolumn{4}{|l|}{ TER } \\
\hline HETATM & 86 & $\mathrm{C} 2$ & VIN \\
\hline HETATM & 87 & H21 & VIN \\
\hline HETATM & 88 & $\mathrm{C} 1$ & VIN \\
\hline HETATM & 89 & $\mathrm{H} 11$ & VIN \\
\hline HETATM & 90 & $\mathrm{H} 12$ & VIN \\
\hline \multicolumn{4}{|l|}{ TER } \\
\hline HETATM & 91 & $\mathrm{C} 1$ & MET \\
\hline HETATM & 92 & H11 & MET \\
\hline HETATM & 93 & H12 & MET \\
\hline HETATM & 94 & H13 & MET \\
\hline \multicolumn{4}{|l|}{ TER } \\
\hline HETATM & 95 & C5 & $\mathrm{ACP}$ \\
\hline HETATM & 96 & H51 & $\mathrm{ACP}$ \\
\hline HETATM & 97 & H52 & $\mathrm{ACP}$ \\
\hline HETATM & 98 & $\mathrm{C} 4$ & $\mathrm{ACP}$ \\
\hline HETATM & 99 & H 41 & $\mathrm{ACP}$ \\
\hline HETATM & 100 & $\mathrm{H} 42$ & $\mathrm{ACP}$ \\
\hline HETATM & 101 & $\mathrm{C} 1$ & $\mathrm{ACP}$ \\
\hline HETATM & 102 & $\mathrm{O} 2$ & $\mathrm{ACP}$ \\
\hline HETATM & 103 & 03 & $\mathrm{ACP}$ \\
\hline HETATM & 104 & H3 & $\mathrm{ACP}$ \\
\hline \multicolumn{4}{|l|}{ TER } \\
\hline HETATM & 105 & $\mathrm{C} 1$ & MET \\
\hline HETATM & 106 & $\mathrm{H} 11$ & MET \\
\hline HETATM & 107 & $\mathrm{H} 12$ & MET \\
\hline HETATM & 108 & H13 & MET \\
\hline \multicolumn{4}{|l|}{ TER } \\
\hline HETATM & 109 & $\mathrm{C} 1$ & MET \\
\hline HETATM & 110 & H11 & MET \\
\hline HETATM & 111 & $\mathrm{H} 12$ & MET \\
\hline HETATM & 112 & H13 & MET \\
\hline \multicolumn{4}{|l|}{ TER } \\
\hline HETATM & 113 & $\mathrm{C} 2$ & VIN \\
\hline HETATM & 114 & H 21 & VIN \\
\hline HETATM & 115 & $\mathrm{C} 1$ & VIN \\
\hline HETATM & 116 & H11 & VIN \\
\hline HETATM & 117 & $\mathrm{H} 12$ & VIN \\
\hline \multicolumn{4}{|l|}{ TER } \\
\hline HETATM & 118 & $\mathrm{C} 1$ & MET \\
\hline HETATM & 119 & $\mathrm{H} 11$ & MET \\
\hline HETATM & 120 & $\mathrm{H} 12$ & MET \\
\hline HETATM & 121 & $\mathrm{H} 13$ & MET \\
\hline \multicolumn{4}{|l|}{ TER } \\
\hline HETATM & 122 & $\mathrm{C} 2$ & VIN \\
\hline HETATM & 123 & H 21 & VIN \\
\hline HETATM & 124 & $\mathrm{C} 1$ & VIN \\
\hline HETATM & 125 & H11 & VIN \\
\hline HETATM & 126 & H12 & VIN \\
\hline TER & & & \\
\hline HETATM & 127 & $\mathrm{C} 1$ & MET \\
\hline HETATM & 128 & $\mathrm{H} 11$ & MET \\
\hline HETATM & 129 & $\mathrm{H} 12$ & MET \\
\hline HETATM & 130 & $\mathrm{H} 13$ & MET \\
\hline TER & & & \\
\hline HETATM & 131 & $\mathrm{C} 5$ & $\mathrm{ACP}$ \\
\hline HETATM & 132 & H51 & $\mathrm{ACP}$ \\
\hline HETATM & 133 & H52 & $\mathrm{ACP}$ \\
\hline HETATM & 134 & $\mathrm{C} 4$ & $\mathrm{ACP}$ \\
\hline HETATM & 135 & H 41 & $\mathrm{ACP}$ \\
\hline HETATM & 136 & H 42 & $\mathrm{ACP}$ \\
\hline HETATM & 137 & $\mathrm{C} 1$ & $\mathrm{ACP}$ \\
\hline HETATM & 138 & $\mathrm{O} 2$ & $\mathrm{ACP}$ \\
\hline HETATM & 139 & 03 & $\mathrm{ACP}$ \\
\hline HETATM & 140 & H3 & $\mathrm{ACP}$ \\
\hline TER & & & \\
\hline HETATM & 141 & $\mathrm{C} 1$ & MET \\
\hline HETATM & 142 & H11 & MET \\
\hline HETATM & 143 & H12 & MET \\
\hline HETATM & 144 & H13 & MET \\
\hline TER & & & \\
\hline HETATM & 145 & $\mathrm{C} 1$ & MET \\
\hline HETATM & 146 & $\mathrm{H} 11$ & MET \\
\hline HETATM & 147 & H12 & MET \\
\hline HETATM & 148 & H13 & MET \\
\hline
\end{tabular}

\begin{tabular}{|c|c|c|c|c|}
\hline 3.008 & 14.177 & -2.840 & 1.00 & 0.00 \\
\hline 3.243 & 15.248 & -2.720 & 1.00 & 0.00 \\
\hline 3.821 & 13.713 & -3.410 & 1.00 & 0.00 \\
\hline 2.101 & 14.100 & -3.450 & 1.00 & 0.00 \\
\hline 8.018 & 12.499 & -2.325 & 1.00 & 0.00 \\
\hline 7.352 & 12.399 & -3.184 & 1.00 & 0.00 \\
\hline 9.185 & 13.144 & -2.484 & 1.00 & 0.00 \\
\hline 9.865 & 13.300 & -1.654 & 1.00 & 0.00 \\
\hline 9.490 & 13.542 & -3.450 & 1.00 & 0.00 \\
\hline 9.703 & 10.816 & -0.121 & 1.00 & 0.00 \\
\hline 10.294 & 11.480 & 0.528 & 1.00 & 0.00 \\
\hline 9.859 & 9.794 & 0.246 & 1.00 & 0.00 \\
\hline 10.135 & 10.882 & -1.126 & 1.00 & 0.00 \\
\hline 0.615 & 9.735 & 5.770 & 1.00 & 0.00 \\
\hline 1.354 & 9.723 & 6.582 & 1.00 & 0.00 \\
\hline-0.226 & 10.343 & 6.134 & 1.00 & 0.00 \\
\hline 0.136 & 8.297 & 5.497 & 1.00 & 0.00 \\
\hline-0.422 & 8.236 & 4.549 & 1.00 & 0.00 \\
\hline 1.007 & 7.630 & 5.390 & 1.00 & 0.00 \\
\hline-0.728 & 7.685 & 6.573 & 1.00 & 0.00 \\
\hline-0.956 & 8.208 & 7.671 & 1.00 & 0.00 \\
\hline-1.244 & 6.505 & 6.209 & 1.00 & 0.00 \\
\hline-1.853 & 6.151 & 6.945 & 1.00 & 0.00 \\
\hline 8.511 & 8.608 & 4.597 & 1.00 & 0.00 \\
\hline 9.063 & 9.448 & 5.047 & 1.00 & 0.00 \\
\hline 8.517 & 7.782 & 5.319 & 1.00 & 0.00 \\
\hline 9.082 & 8.289 & 3.717 & 1.00 & 0.00 \\
\hline-0.923 & 11.557 & 3.646 & 1.00 & 0.00 \\
\hline-1.368 & 11.348 & 4.626 & 1.00 & 0.00 \\
\hline-1.033 & 12.637 & 3.471 & 1.00 & 0.00 \\
\hline-1.523 & 11.031 & 2.889 & 1.00 & 0.00 \\
\hline 8.874 & 0.178 & 8.933 & 1.00 & 0.00 \\
\hline 9.690 & 0.876 & 8.768 & 1.00 & 0.00 \\
\hline 9.207 & -1.047 & 9.335 & 1.00 & 0.00 \\
\hline 8.475 & -1.826 & 9.442 & 1.00 & 0.00 \\
\hline 10.249 & -1.316 & 9.501 & 1.00 & 0.00 \\
\hline 6.210 & -0.077 & 10.771 & 1.00 & 0.00 \\
\hline 5.975 & -1.148 & 10.652 & 1.00 & 0.00 \\
\hline 5.397 & 0.387 & 11.341 & 1.00 & 0.00 \\
\hline 7.118 & 0.001 & 11.382 & 1.00 & 0.00 \\
\hline 1.200 & 1.601 & 10.256 & 1.00 & 0.00 \\
\hline 1.866 & 1.702 & 11.115 & 1.00 & 0.00 \\
\hline 0.033 & 0.957 & 10.415 & 1.00 & 0.00 \\
\hline-0.647 & 0.800 & 9.585 & 1.00 & 0.00 \\
\hline-0.272 & 0.559 & 11.381 & 1.00 & 0.00 \\
\hline-0.484 & 3.284 & 8.052 & 1.00 & 0.00 \\
\hline-0.641 & 4.306 & 7.685 & 1.00 & 0.00 \\
\hline-0.917 & 3.219 & 9.057 & 1.00 & 0.00 \\
\hline-1.076 & 2.620 & 7.403 & 1.00 & 0.00 \\
\hline 8.604 & 4.366 & 2.161 & 1.00 & 0.00 \\
\hline 7.865 & 4.377 & 1.349 & 1.00 & 0.00 \\
\hline 9.445 & 3.758 & 1.798 & 1.00 & 0.00 \\
\hline 9.082 & 5.803 & 2.434 & 1.00 & 0.00 \\
\hline 9.640 & 5.865 & 3.382 & 1.00 & 0.00 \\
\hline 8.212 & 6.470 & 2.541 & 1.00 & 0.00 \\
\hline 9.946 & 6.416 & 1.358 & 1.00 & 0.00 \\
\hline 10.174 & 5.893 & 0.260 & 1.00 & 0.00 \\
\hline 10.462 & 7.595 & 1.722 & 1.00 & 0.00 \\
\hline 11.071 & 7.949 & 0.986 & 1.00 & 0.00 \\
\hline 0.708 & 5.492 & 3.334 & 1.00 & 0.00 \\
\hline 0.155 & 4.653 & 2.884 & 1.00 & 0.00 \\
\hline 0.701 & 6.318 & 2.613 & 1.00 & 0.00 \\
\hline 0.137 & 5.812 & 4.214 & 1.00 & 0.00 \\
\hline 10.141 & 2.543 & 4.285 & 1.00 & 0.00 \\
\hline 10.586 & 2.752 & 3.305 & 1.00 & 0.00 \\
\hline 10.252 & 1.463 & 4.460 & 1.00 & 0.00 \\
\hline 10.742 & 3.069 & 5.042 & 1.00 & 0.00 \\
\hline
\end{tabular}


TER

\begin{tabular}{|c|c|c|c|c|c|c|c|c|c|c|}
\hline HETATM & 149 & $\mathrm{FE}$ & HEM & 17 & 5.211 & 11.003 & -6.440 & 1.00 & 0.00 & $\mathrm{FE}$ \\
\hline HETATM & 150 & $\mathrm{C} 1$ & HEM & 17 & 2.983 & 12.876 & -7.502 & 1.00 & 0.00 & C \\
\hline HETATM & 151 & $\mathrm{C} 2$ & HEM & 17 & 2.624 & 13.666 & -8.674 & 1.00 & 0.00 & C \\
\hline HETATM & 152 & $\mathrm{C} 3$ & HEM & 17 & 3.770 & 13.763 & -9.448 & 1.00 & 0.00 & C \\
\hline HETATM & 153 & $\mathrm{C} 4$ & HEM & 17 & 4.799 & 13.046 & -8.740 & 1.00 & 0.00 & C \\
\hline HETATM & 154 & C5 & HEM & 17 & 6.114 & 12.927 & -9.178 & 1.00 & 0.00 & C \\
\hline HETATM & 155 & H5 & HEM & 17 & 6.368 & 13.423 & -10.113 & 1.00 & 0.00 & $\mathrm{H}$ \\
\hline HETATM & 156 & $\mathrm{C} 6$ & HEM & 17 & 7.134 & 12.229 & -8.542 & 1.00 & 0.00 & C \\
\hline HETATM & 157 & C7 & HEM & 17 & 8.502 & 12.156 & -9.012 & 1.00 & 0.00 & C \\
\hline HETATM & 158 & $\mathrm{C} 8$ & HEM & 17 & 9.208 & 11.436 & -8.065 & 1.00 & 0.00 & C \\
\hline HETATM & 159 & $\mathrm{C} 9$ & HEM & 17 & 8.257 & 11.071 & -7.041 & 1.00 & 0.00 & C \\
\hline HETATM & 160 & $\mathrm{C} 10$ & HEM & 17 & 8.561 & 10.336 & -5.897 & 1.00 & 0.00 & C \\
\hline HETATM & 161 & $\mathrm{H} 10$ & HEM & 17 & 9.588 & 9.993 & -5.791 & 1.00 & 0.00 & $\mathrm{H}$ \\
\hline HETATM & 162 & $\mathrm{C} 11$ & HEM & 17 & 7.687 & 9.996 & -4.874 & 1.00 & 0.00 & C \\
\hline HETATM & 163 & $\mathrm{C} 12$ & HEM & 17 & 8.068 & 9.271 & -3.684 & 1.00 & 0.00 & C \\
\hline HETATM & 164 & C13 & HEM & 17 & 6.928 & 9.124 & -2.927 & 1.00 & 0.00 & C \\
\hline HETATM & 165 & C14 & HEM & 17 & 5.855 & 9.765 & -3.659 & 1.00 & 0.00 & C \\
\hline HETATM & 166 & C15 & HEM & 17 & 4.527 & 9.820 & -3.237 & 1.00 & 0.00 & C \\
\hline HETATM & 167 & H15 & HEM & 17 & 4.287 & 9.335 & -2.289 & 1.00 & 0.00 & $\mathrm{H}$ \\
\hline HETATM & 168 & C16 & HEM & 17 & 3.497 & 10.502 & -3.880 & 1.00 & 0.00 & C \\
\hline HETATM & 169 & $\mathrm{C} 17$ & HEM & 17 & 2.131 & 10.615 & -3.398 & 1.00 & 0.00 & C \\
\hline HETATM & 170 & C18 & HEM & 17 & 1.456 & 11.383 & -4.322 & 1.00 & 0.00 & C \\
\hline HETATM & 171 & C19 & HEM & 17 & 2.406 & 11.735 & -5.358 & 1.00 & 0.00 & C \\
\hline HETATM & 172 & $\mathrm{C} 20$ & HEM & 17 & 2.114 & 12.526 & -6.469 & 1.00 & 0.00 & C \\
\hline HETATM & 173 & $\mathrm{H} 2 \mathrm{O}$ & HEM & 17 & 1.100 & 12.915 & -6.530 & 1.00 & 0.00 & $\mathrm{H}$ \\
\hline HETATM & 174 & N21 & HEM & 17 & 4.305 & 12.513 & -7.564 & 1.00 & 0.00 & $\mathrm{~N}$ \\
\hline HETATM & 175 & $\mathrm{~N} 22$ & HEM & 17 & 7.009 & 11.566 & -7.342 & 1.00 & 0.00 & $\mathrm{~N}$ \\
\hline HETATM & 176 & N23 & HEM & 17 & 6.338 & 10.303 & -4.841 & 1.00 & 0.00 & $\mathrm{~N}$ \\
\hline HETATM & 177 & N24 & HEM & 17 & 3.639 & 11.187 & -5.077 & 1.00 & 0.00 & $\mathrm{~N}$ \\
\hline HETATM & 178 & C99 & HEM & 17 & 4.545 & 8.322 & -7.579 & 1.00 & 0.00 & C \\
\hline HETATM & 179 & 091 & HEM & 17 & 4.705 & 9.635 & -7.567 & 1.00 & 0.00 & O \\
\hline HETATM & 180 & 092 & HEM & 17 & 4.391 & 7.705 & -8.635 & 1.00 & 0.00 & O \\
\hline HETATM & 181 & СТ91 & HEM & 17 & 4.561 & 7.640 & -6.223 & 1.00 & 0.00 & C \\
\hline HETATM & 182 & H911 & HEM & 17 & 5.540 & 7.836 & -5.760 & 1.00 & 0.00 & $\mathrm{H}$ \\
\hline HETATM & 183 & H912 & HEM & 17 & 3.813 & 8.130 & -5.580 & 1.00 & 0.00 & $\mathrm{H}$ \\
\hline HETATM & 184 & СТ92 & HEM & 17 & 4.302 & 6.136 & -6.334 & 1.00 & 0.00 & C \\
\hline HETATM & 185 & H921 & HEM & 17 & 5.098 & 5.676 & -6.931 & 1.00 & 0.00 & $\mathrm{H}$ \\
\hline HETATM & 186 & H922 & HEM & 17 & 3.377 & 5.980 & -6.905 & 1.00 & 0.00 & $\mathrm{H}$ \\
\hline TER & & & & & & & & & & \\
\hline HETATM & 187 & $\mathrm{FE}$ & HEM & 18 & 5.903 & 3.589 & -1.491 & 1.00 & 0.00 & $\mathrm{FE}$ \\
\hline HETATM & 188 & $\mathrm{C} 1$ & HEM & 18 & 8.131 & 1.717 & -0.429 & 1.00 & 0.00 & C \\
\hline HETATM & 189 & $\mathrm{C} 2$ & HEM & 18 & 8.490 & 0.927 & 0.742 & 1.00 & 0.00 & C \\
\hline HETATM & 190 & $\mathrm{C} 3$ & HEM & 18 & 7.344 & 0.830 & 1.517 & 1.00 & 0.00 & C \\
\hline HETATM & 191 & $\mathrm{C} 4$ & HEM & 18 & 6.315 & 1.547 & 0.809 & 1.00 & 0.00 & C \\
\hline HETATM & 192 & C5 & HEM & 18 & 5.000 & 1.666 & 1.247 & 1.00 & 0.00 & C \\
\hline HETATM & 193 & H5 & HEM & 18 & 4.746 & 1.170 & 2.182 & 1.00 & 0.00 & $\mathrm{H}$ \\
\hline HETATM & 194 & $\mathrm{C} 6$ & HEM & 18 & 3.980 & 2.364 & 0.611 & 1.00 & 0.00 & C \\
\hline HETATM & 195 & C7 & HEM & 18 & 2.611 & 2.437 & 1.081 & 1.00 & 0.00 & C \\
\hline HETATM & 196 & $\mathrm{C} 8$ & HEM & 18 & 1.906 & 3.157 & 0.134 & 1.00 & 0.00 & C \\
\hline HETATM & 197 & $\mathrm{C} 9$ & HEM & 18 & 2.857 & 3.522 & -0.891 & 1.00 & 0.00 & C \\
\hline HETATM & 198 & $\mathrm{C} 10$ & HEM & 18 & 2.552 & 4.256 & -2.034 & 1.00 & 0.00 & C \\
\hline HETATM & 199 & $\mathrm{H} 10$ & HEM & 18 & 1.526 & 4.600 & -2.140 & 1.00 & 0.00 & $\mathrm{H}$ \\
\hline HETATM & 200 & $\mathrm{C} 11$ & HEM & 18 & 3.427 & 4.596 & -3.057 & 1.00 & 0.00 & C \\
\hline HETATM & 201 & $\mathrm{C} 12$ & HEM & 18 & 3.046 & 5.322 & -4.247 & 1.00 & 0.00 & C \\
\hline HETATM & 202 & C13 & HEM & 18 & 4.185 & 5.469 & -5.004 & 1.00 & 0.00 & C \\
\hline HETATM & 203 & C14 & HEM & 18 & 5.258 & 4.828 & -4.273 & 1.00 & 0.00 & C \\
\hline HETATM & 204 & C15 & HEM & 18 & 6.586 & 4.773 & -4.694 & 1.00 & 0.00 & C \\
\hline HETATM & 205 & H15 & HEM & 18 & 6.826 & 5.258 & -5.642 & 1.00 & 0.00 & $\mathrm{H}$ \\
\hline HETATM & 206 & C16 & HEM & 18 & 7.617 & 4.091 & -4.051 & 1.00 & 0.00 & C \\
\hline HETATM & 207 & C17 & HEM & 18 & 8.982 & 3.978 & -4.533 & 1.00 & 0.00 & C \\
\hline HETATM & 208 & $\mathrm{C} 18$ & HEM & 18 & 9.658 & 3.210 & -3.609 & 1.00 & 0.00 & C \\
\hline HETATM & 209 & C19 & HEM & 18 & 8.708 & 2.858 & -2.573 & 1.00 & 0.00 & C \\
\hline HETATM & 210 & $\mathrm{C} 20$ & HEM & 18 & 8.999 & 2.067 & -1.462 & 1.00 & 0.00 & $\mathrm{C}$ \\
\hline HETATM & 211 & $\mathrm{H} 20$ & HEM & 18 & 10.014 & 1.678 & -1.402 & 1.00 & 0.00 & $\mathrm{H}$ \\
\hline HETATM & 212 & N21 & HEM & 18 & 6.809 & 2.080 & -0.367 & 1.00 & 0.00 & $\mathrm{~N}$ \\
\hline HETATM & 213 & N22 & HEM & 18 & 4.105 & 3.027 & -0.590 & 1.00 & 0.00 & $\mathrm{~N}$ \\
\hline HETATM & 214 & N23 & HEM & 18 & 4.775 & 4.290 & -3.090 & 1.00 & 0.00 & $\mathrm{~N}$ \\
\hline HETATM & 215 & N24 & HEM & 18 & 7.475 & 3.406 & -2.854 & 1.00 & 0.00 & $\mathrm{~N}$ \\
\hline HETATM & 216 & C99 & HEM & 18 & 6.568 & 6.271 & -0.352 & 1.00 & 0.00 & C \\
\hline HETATM & 217 & 091 & HEM & 18 & 6.409 & 4.958 & -0.365 & 1.00 & 0.00 & $\mathrm{O}$ \\
\hline HETATM & 218 & 092 & HEM & 18 & 6.723 & 6.888 & 0.703 & 1.00 & 0.00 & $\mathrm{O}$ \\
\hline HETATM & 219 & СТ91 & HEM & 18 & 6.552 & 6.952 & -1.709 & 1.00 & 0.00 & C \\
\hline HETATM & 220 & H911 & HEM & 18 & 5.573 & 6.757 & -2.171 & 1.00 & 0.00 & $\mathrm{H}$ \\
\hline HETATM & 221 & H912 & HEM & 18 & 7.300 & 6.463 & -2.352 & 1.00 & 0.00 & $\mathrm{H}$ \\
\hline HETATM & 222 & СT92 & HEM & 18 & 6.812 & 8.457 & -1.597 & 1.00 & 0.00 & C \\
\hline HETATM & 223 & H921 & HEM & 18 & 7.737 & 8.613 & -1.027 & 1.00 & 0.00 & $\mathrm{H}$ \\
\hline HETATM & 224 & H922 & HEM & 18 & 6.015 & 8.917 & -1.000 & 1.00 & 0.00 & $\mathrm{H}$ \\
\hline
\end{tabular}




\begin{tabular}{|c|c|c|c|}
\hline HETATM & 225 & $\mathrm{C} 2$ & VIN \\
\hline HETATM & 226 & H 21 & VIN \\
\hline HETATM & 227 & $\mathrm{C} 1$ & VIN \\
\hline HETATM & 228 & H11 & VIN \\
\hline HETATM & 229 & H12 & VIN \\
\hline \multicolumn{4}{|l|}{ TER } \\
\hline HETATM & 230 & $\mathrm{C} 1$ & MET \\
\hline HETATM & 231 & H11 & MET \\
\hline HETATM & 232 & H12 & MET \\
\hline HETATM & 233 & H13 & MET \\
\hline \multicolumn{4}{|l|}{ TER } \\
\hline HETATM & 234 & $\mathrm{C} 2$ & VIN \\
\hline HETATM & 235 & H 21 & VIN \\
\hline HETATM & 236 & $\mathrm{C} 1$ & VIN \\
\hline HETATM & 237 & H11 & VIN \\
\hline HETATM & 238 & H12 & VIN \\
\hline \multicolumn{4}{|l|}{ TER } \\
\hline HETATM & 239 & $\mathrm{C} 1$ & MET \\
\hline HETATM & 240 & H11 & MET \\
\hline HETATM & 241 & $\mathrm{H} 12$ & MET \\
\hline HETATM & 242 & $\mathrm{H} 13$ & MET \\
\hline \multicolumn{4}{|l|}{ TER } \\
\hline HETATM & 243 & C5 & $\mathrm{ACP}$ \\
\hline HETATM & 244 & H51 & $\mathrm{ACP}$ \\
\hline HETATM & 245 & H52 & $\mathrm{ACP}$ \\
\hline HETATM & 246 & $\mathrm{C} 4$ & $\mathrm{ACP}$ \\
\hline HETATM & 247 & H4 1 & $\mathrm{ACP}$ \\
\hline HETATM & 248 & H4 2 & $\mathrm{ACP}$ \\
\hline HETATM & 249 & $\mathrm{C} 1$ & $\mathrm{ACP}$ \\
\hline HETATM & 250 & $\mathrm{O} 2$ & $\mathrm{ACP}$ \\
\hline HETATM & 251 & 03 & $\mathrm{ACP}$ \\
\hline HETATM & 252 & H3 & $\mathrm{ACP}$ \\
\hline \multicolumn{4}{|l|}{ TER } \\
\hline HETATM & 253 & $\mathrm{C} 1$ & MET \\
\hline HETATM & 254 & H11 & MET \\
\hline HETATM & 255 & $\mathrm{H} 12$ & MET \\
\hline HETATM & 256 & H13 & MET \\
\hline \multicolumn{4}{|l|}{ TER } \\
\hline HETATM & 257 & $\mathrm{C} 1$ & MET \\
\hline HETATM & 258 & $\mathrm{H} 11$ & MET \\
\hline HETATM & 259 & $\mathrm{H} 12$ & MET \\
\hline HETATM & 260 & H13 & $\mathrm{MET}$ \\
\hline \multicolumn{4}{|l|}{ TER } \\
\hline HETATM & 261 & C2 & VIN \\
\hline HETATM & 262 & H 21 & VIN \\
\hline HETATM & 263 & $\mathrm{C} 1$ & VIN \\
\hline HETATM & 264 & H11 & VIN \\
\hline HETATM & 265 & $\mathrm{H} 12$ & VIN \\
\hline \multicolumn{4}{|l|}{ TER } \\
\hline HETATM & 266 & $\mathrm{C} 1$ & MET \\
\hline HETATM & 267 & H11 & MET \\
\hline HETATM & 268 & $\mathrm{H} 12$ & MET \\
\hline HETATM & 269 & $\mathrm{H} 13$ & MET \\
\hline TER & & & \\
\hline HETATM & 270 & $\mathrm{C} 2$ & VIN \\
\hline HETATM & 271 & H 21 & VIN \\
\hline HETATM & 272 & $\mathrm{C} 1$ & VIN \\
\hline HETATM & 273 & H11 & VIN \\
\hline HETATM & 274 & $\mathrm{H} 12$ & VIN \\
\hline TER & & & \\
\hline HETATM & 275 & $\mathrm{C} 1$ & MET \\
\hline HETATM & 276 & $\mathrm{H} 11$ & MET \\
\hline HETATM & 277 & $\mathrm{H} 12$ & MET \\
\hline HETATM & 278 & $\mathrm{H} 13$ & MET \\
\hline TER & & & \\
\hline HETATM & 279 & C5 & $\mathrm{ACP}$ \\
\hline HETATM & 280 & H51 & $\mathrm{ACP}$ \\
\hline HETATM & 281 & H52 & $\mathrm{ACP}$ \\
\hline HETATM & 282 & C4 & $\mathrm{ACP}$ \\
\hline HETATM & 283 & H 41 & $\mathrm{ACP}$ \\
\hline HETATM & 284 & H4 2 & $\mathrm{ACP}$ \\
\hline HETATM & 285 & $\mathrm{C} 1$ & $\mathrm{ACP}$ \\
\hline HETATM & 286 & $\mathrm{O} 2$ & $\mathrm{ACP}$ \\
\hline HETATM & 287 & 03 & $\mathrm{ACP}$ \\
\hline HETATM & 288 & H3 & $\mathrm{ACP}$ \\
\hline TER & & & \\
\hline HETATM & 289 & $\mathrm{C} 1$ & MET \\
\hline HETATM & 290 & H11 & MET \\
\hline HETATM & 291 & $\mathrm{H} 12$ & MET \\
\hline
\end{tabular}

\begin{tabular}{|c|c|c|c|c|}
\hline 1.292 & 14.169 & -8.933 & 1.00 & 0.00 \\
\hline 0.476 & 13.470 & -8.768 & 1.00 & 0.00 \\
\hline 0.959 & 15.394 & -9.335 & 1.00 & 0.00 \\
\hline 1.691 & 16.172 & -9.442 & 1.00 & 0.00 \\
\hline-0.082 & 15.663 & -9.501 & 1.00 & 0.00 \\
\hline 3.956 & 14.424 & -10.771 & 1.00 & 0.00 \\
\hline 4.191 & 15.494 & -10.652 & 1.00 & 0.00 \\
\hline 4.769 & 13.959 & $-11 \cdot 341$ & 1.00 & 0.00 \\
\hline 3.048 & 14.346 & -11.382 & 1.00 & 0.00 \\
\hline 8.966 & 12.746 & -10.256 & 1.00 & 0.00 \\
\hline 8.300 & 12.645 & -11.115 & 1.00 & 0.00 \\
\hline 10.133 & 13.390 & -10.415 & 1.00 & 0.00 \\
\hline 10.813 & 13.546 & -9.585 & 1.00 & 0.00 \\
\hline 10.438 & 13.788 & -11.381 & 1.00 & 0.00 \\
\hline 10.651 & 11.062 & -8.052 & 1.00 & 0.00 \\
\hline 11.242 & 11.727 & -7.403 & 1.00 & 0.00 \\
\hline 10.807 & 10.040 & -7.685 & 1.00 & 0.00 \\
\hline 11.083 & 11.128 & -9.057 & 1.00 & 0.00 \\
\hline 1.562 & 9.981 & -2.161 & 1.00 & 0.00 \\
\hline 2.301 & 9.969 & -1.349 & 1.00 & 0.00 \\
\hline 0.722 & 10.589 & -1.798 & 1.00 & 0.00 \\
\hline 1.084 & 8.543 & -2.434 & 1.00 & 0.00 \\
\hline 0.526 & 8.482 & -3.382 & 1.00 & 0.00 \\
\hline 1.954 & 7.877 & -2.541 & 1.00 & 0.00 \\
\hline 0.220 & 7.931 & -1.358 & 1.00 & 0.00 \\
\hline-0.008 & 8.454 & -0.260 & 1.00 & 0.00 \\
\hline-0.296 & 6.751 & -1.722 & 1.00 & 0.00 \\
\hline-0.905 & 6.397 & -0.986 & 1.00 & 0.00 \\
\hline 9.458 & 8.855 & -3.334 & 1.00 & 0.00 \\
\hline 10.011 & 9.694 & -2.884 & 1.00 & 0.00 \\
\hline 9.465 & 8.029 & -2.613 & 1.00 & 0.00 \\
\hline 10.029 & 8.535 & -4.214 & 1.00 & 0.00 \\
\hline 0.025 & 11.803 & -4.285 & 1.00 & 0.00 \\
\hline-0.420 & 11.594 & -3.305 & 1.00 & 0.00 \\
\hline-0.086 & 12.883 & -4.460 & 1.00 & 0.00 \\
\hline-0.576 & 11.278 & -5.042 & 1.00 & 0.00 \\
\hline 9.822 & 0.424 & 1.002 & 1.00 & 0.00 \\
\hline 10.637 & 1.123 & 0.837 & 1.00 & 0.00 \\
\hline 10.154 & -0.801 & 1.403 & 1.00 & 0.00 \\
\hline 9.423 & -1.579 & 1.511 & 1.00 & 0.00 \\
\hline 11.196 & -1.070 & 1.570 & 1.00 & 0.00 \\
\hline 7.158 & 0.169 & 2.840 & 1.00 & 0.00 \\
\hline 6.923 & -0.902 & 2.720 & 1.00 & 0.00 \\
\hline 6.345 & 0.633 & 3.410 & 1.00 & 0.00 \\
\hline 8.065 & 0.247 & 3.450 & 1.00 & 0.00 \\
\hline 2.148 & 1.847 & 2.325 & 1.00 & 0.00 \\
\hline 2.814 & 1.948 & 3.184 & 1.00 & 0.00 \\
\hline 0.981 & 1.203 & 2.484 & 1.00 & 0.00 \\
\hline 0.301 & 1.047 & 1.654 & 1.00 & 0.00 \\
\hline 0.676 & 0.805 & 3.450 & 1.00 & 0.00 \\
\hline 0.463 & 3.530 & 0.121 & 1.00 & 0.00 \\
\hline 0.307 & 4.553 & -0.246 & 1.00 & 0.00 \\
\hline 0.031 & 3.465 & 1.126 & 1.00 & 0.00 \\
\hline-0.128 & 2.866 & -0.528 & 1.00 & 0.00 \\
\hline 9.552 & 4.612 & -5.770 & 1.00 & 0.00 \\
\hline 8.813 & 4.623 & -6.582 & 1.00 & 0.00 \\
\hline 10.392 & 4.004 & -6.134 & 1.00 & 0.00 \\
\hline 10.030 & 6.050 & -5.497 & 1.00 & 0.00 \\
\hline 10.588 & 6.111 & -4.549 & 1.00 & 0.00 \\
\hline 9.160 & 6.716 & -5.390 & 1.00 & 0.00 \\
\hline 10.894 & 6.662 & -6.573 & 1.00 & 0.00 \\
\hline 11.122 & 6.139 & -7.671 & 1.00 & 0.00 \\
\hline 11.410 & 7.841 & -6.209 & 1.00 & 0.00 \\
\hline 12.019 & 8.196 & -6.945 & 1.00 & 0.00 \\
\hline 1.655 & 5.738 & -4.597 & 1.00 & 0.00 \\
\hline 1.103 & 4.899 & -5.047 & 1.00 & 0.00 \\
\hline 1.649 & 6.564 & -5.319 & 1.00 & 0.00 \\
\hline
\end{tabular}




\begin{tabular}{|c|c|c|c|c|c|c|c|c|c|c|}
\hline $\begin{array}{l}\text { HETATM } \\
\text { TER }\end{array}$ & 292 & H13 & MET & 31 & 1.084 & 6.058 & -3.717 & 1.00 & 0.00 & $\mathrm{H}$ \\
\hline HETATM & 293 & $\mathrm{C} 1$ & $\mathrm{MET}$ & 32 & 11.089 & 2.790 & -3.646 & 1.00 & 0.00 & C \\
\hline HETATM & 294 & H11 & $\mathrm{MET}$ & 32 & 11.534 & 2.998 & -4.626 & 1.00 & 0.00 & $\mathrm{H}$ \\
\hline HETATM & 295 & $\mathrm{H} 12$ & $\mathrm{MET}$ & 32 & 11.199 & 1.709 & -3.471 & 1.00 & 0.00 & $\mathrm{H}$ \\
\hline HETATM & 296 & H13 & $\mathrm{MET}$ & 32 & 11.690 & 3.315 & -2.889 & 1.00 & 0.00 & 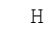 \\
\hline \multicolumn{11}{|l|}{ TER } \\
\hline HETATM & 297 & $\mathrm{FE}$ & HEM & 33 & 3.316 & 10.511 & 9.423 & 1.00 & 0.00 & $\mathrm{FE}$ \\
\hline HETATM & 298 & $\mathrm{C} 1$ & HEM & 33 & 1.088 & 12.383 & 8.361 & 1.00 & 0.00 & C \\
\hline HETATM & 299 & $\mathrm{C} 2$ & HEM & 33 & 0.729 & 13.174 & 7.189 & 1.00 & 0.00 & C \\
\hline HETATM & 300 & C3 & HEM & 33 & 1.874 & 13.271 & 6.414 & 1.00 & 0.00 & C \\
\hline HETATM & 301 & C4 & HEM & 33 & 2.904 & 12.553 & 7.122 & 1.00 & 0.00 & C \\
\hline HETATM & 302 & C5 & HEM & 33 & 4.219 & 12.435 & 6.684 & 1.00 & 0.00 & $\mathrm{C}$ \\
\hline HETATM & 303 & H5 & HEM & 33 & 4.472 & 12.931 & 5.749 & 1.00 & 0.00 & $\mathrm{H}$ \\
\hline HETATM & 304 & C6 & HEM & 33 & 5.238 & 11.736 & 7.320 & 1.00 & 0.00 & C \\
\hline HETATM & 305 & C7 & HEM & 33 & 6.607 & 11.664 & 6.851 & 1.00 & 0.00 & C \\
\hline HETATM & 306 & $\mathrm{C} 8$ & HEM & 33 & 7.313 & 10.944 & 7.798 & 1.00 & 0.00 & C \\
\hline HETATM & 307 & C9 & HEM & 33 & 6.362 & 10.579 & 8.822 & 1.00 & 0.00 & C \\
\hline HETATM & 308 & $\mathrm{C} 10$ & HEM & 33 & 6.666 & 9.844 & 9.965 & 1.00 & 0.00 & C \\
\hline HETATM & 309 & H1O & HEM & 33 & 7.693 & 9.500 & 10.071 & 1.00 & 0.00 & $\mathrm{H}$ \\
\hline HETATM & 310 & C11 & HEM & 33 & 5.792 & 9.504 & 10.988 & 1.00 & 0.00 & C \\
\hline HETATM & 311 & $\mathrm{C} 12$ & HEM & 33 & 6.173 & 8.779 & 12.178 & 1.00 & 0.00 & C \\
\hline HETATM & 312 & $\mathrm{C} 13$ & HEM & 33 & 5.033 & 8.631 & 12.935 & 1.00 & 0.00 & C \\
\hline HETATM & 313 & C14 & HEM & 33 & 3.960 & 9.273 & 12.204 & 1.00 & 0.00 & C \\
\hline HETATM & 314 & C15 & HEM & 33 & 2.632 & 9.328 & 12.625 & 1.00 & 0.00 & C \\
\hline HETATM & 315 & H15 & HEM & 33 & 2.392 & 8.843 & 13.573 & 1.00 & 0.00 & $\mathrm{H}$ \\
\hline HETATM & 316 & $\mathrm{C} 16$ & HEM & 33 & 1.602 & 10.009 & 11.982 & 1.00 & 0.00 & C \\
\hline HETATM & 317 & C17 & HEM & 33 & 0.236 & 10.123 & 12.464 & 1.00 & 0.00 & C \\
\hline HETATM & 318 & $\mathrm{C} 18$ & HEM & 33 & -0.439 & 10.890 & 11.541 & 1.00 & 0.00 & $\mathrm{C}$ \\
\hline HETATM & 319 & C19 & HEM & 33 & 0.510 & 11.242 & 10.504 & 1.00 & 0.00 & $\mathrm{C}$ \\
\hline HETATM & 320 & $\mathrm{C} 20$ & HEM & 33 & 0.219 & 12.033 & 9.393 & 1.00 & 0.00 & C \\
\hline HETATM & 321 & H 20 & HEM & 33 & -0.795 & 12.423 & 9.333 & 1.00 & 0.00 & $\mathrm{H}$ \\
\hline HETATM & 322 & N21 & HEM & 33 & 2.409 & 12.020 & 8.298 & 1.00 & 0.00 & $\mathrm{~N}$ \\
\hline HETATM & 323 & N22 & HEM & 33 & 5.114 & 11.073 & 8.521 & 1.00 & 0.00 & $\mathrm{~N}$ \\
\hline HETATM & 324 & N23 & HEM & 33 & 4.443 & 9.810 & 11.021 & 1.00 & 0.00 & $\mathrm{~N}$ \\
\hline HETATM & 325 & N2 4 & HEM & 33 & 1.743 & 10.694 & 10.786 & 1.00 & 0.00 & $\mathrm{~N}$ \\
\hline HETATM & 326 & C99 & HEM & 33 & 2.650 & 7.829 & 8.283 & 1.00 & 0.00 & C \\
\hline HETATM & 327 & 091 & HEM & 33 & 2.810 & 9.143 & 8.296 & 1.00 & 0.00 & O \\
\hline HETATM & 328 & 092 & HEM & 33 & 2.495 & 7.212 & 7.228 & 1.00 & 0.00 & O \\
\hline HETATM & 329 & СТ91 & HEM & 33 & 2.666 & 7.148 & 9.640 & 1.00 & 0.00 & C \\
\hline HETATM & 330 & H911 & HEM & 33 & 3.645 & 7.343 & 10.103 & 1.00 & 0.00 & $\mathrm{H}$ \\
\hline HETATM & 331 & H912 & HEM & 33 & 1.918 & 7.638 & 10.283 & 1.00 & 0.00 & $\mathrm{H}$ \\
\hline HETATM & 332 & СТ 92 & HEM & 33 & 2.407 & 5.644 & 9.528 & 1.00 & 0.00 & C \\
\hline HETATM & 333 & H921 & HEM & 33 & 3.203 & 5.183 & 8.931 & 1.00 & 0.00 & $\mathrm{H}$ \\
\hline HETATM & 334 & H922 & HEM & 33 & 1.482 & 5.488 & 8.958 & 1.00 & 0.00 & $\mathrm{H}$ \\
\hline \multicolumn{11}{|l|}{ TER } \\
\hline HETATM & 335 & $\mathrm{FE}$ & HEM & 34 & 4.008 & 3.097 & 14.371 & 1.00 & 0.00 & $\mathrm{FE}$ \\
\hline HETATM & 336 & $\mathrm{C} 1$ & HEM & 34 & 6.236 & 1.225 & 15.433 & 1.00 & 0.00 & $\mathrm{C}$ \\
\hline HETATM & 337 & $\mathrm{C} 2$ & HEM & 34 & 6.595 & 0.434 & 16.605 & 1.00 & 0.00 & $\mathrm{C}$ \\
\hline HETATM & 338 & $\mathrm{C} 3$ & HEM & 34 & 5.449 & 0.337 & 17.380 & 1.00 & 0.00 & C \\
\hline HETATM & 339 & $\mathrm{C} 4$ & HEM & 34 & 4.419 & 1.055 & 16.672 & 1.00 & 0.00 & C \\
\hline HETATM & 340 & C5 & HEM & 34 & 3.104 & 1.173 & 17.109 & 1.00 & 0.00 & C \\
\hline HETATM & 341 & H5 & HEM & 34 & 2.851 & 0.677 & 18.045 & 1.00 & 0.00 & $\mathrm{H}$ \\
\hline HETATM & 342 & $\mathrm{C} 6$ & HEM & 34 & 2.085 & 1.872 & 16.473 & 1.00 & 0.00 & C \\
\hline HETATM & 343 & C7 & HEM & 34 & 0.716 & 1.944 & 16.943 & 1.00 & 0.00 & C \\
\hline HETATM & 344 & $\mathrm{C} 8$ & HEM & 34 & 0.011 & 2.664 & 15.996 & 1.00 & 0.00 & C \\
\hline HETATM & 345 & $\mathrm{C} 9$ & HEM & 34 & 0.962 & 3.029 & 14.972 & 1.00 & 0.00 & C \\
\hline HETATM & 346 & C10 & HEM & 34 & 0.657 & 3.764 & 13.828 & 1.00 & 0.00 & C \\
\hline HETATM & 347 & H1O & HEM & 34 & -0.370 & 4.108 & 13.722 & 1.00 & 0.00 & $\mathrm{H}$ \\
\hline HETATM & 348 & $\mathrm{C} 11$ & HEM & 34 & 1.532 & 4.104 & 12.806 & 1.00 & 0.00 & C \\
\hline HETATM & 349 & $\mathrm{C} 12$ & HEM & 34 & 1.151 & 4.829 & 11.615 & 1.00 & 0.00 & C \\
\hline HETATM & 350 & $\mathrm{C} 13$ & HEM & 34 & 2.290 & 4.977 & 10.858 & 1.00 & 0.00 & C \\
\hline HETATM & 351 & C14 & HEM & 34 & 3.363 & 4.335 & 11.590 & 1.00 & 0.00 & C \\
\hline HETATM & 352 & C15 & HEM & 34 & 4.691 & 4.280 & 11.168 & 1.00 & 0.00 & C \\
\hline HETATM & 353 & H15 & HEM & 34 & 4.931 & 4.765 & 10.221 & 1.00 & 0.00 & $\mathrm{H}$ \\
\hline HETATM & 354 & C16 & HEM & 34 & 5.721 & 3.599 & 11.812 & 1.00 & 0.00 & C \\
\hline HETATM & 355 & C17 & HEM & 34 & 7.087 & 3.485 & 11.329 & 1.00 & 0.00 & C \\
\hline HETATM & 356 & $\mathrm{C} 18$ & HEM & 34 & 7.763 & 2.718 & 12.253 & 1.00 & 0.00 & C \\
\hline HETATM & 357 & C19 & HEM & 34 & 6.813 & 2.366 & 13.289 & 1.00 & 0.00 & C \\
\hline HETATM & 358 & $\mathrm{C} 20$ & HEM & 34 & 7.104 & 1.575 & 14.401 & 1.00 & 0.00 & C \\
\hline HETATM & 359 & $\mathrm{H} 20$ & HEM & 34 & 8.118 & 1.185 & 14.461 & 1.00 & 0.00 & $\mathrm{H}$ \\
\hline HETATM & 360 & N21 & HEM & 34 & 4.914 & 1.588 & 15.495 & 1.00 & 0.00 & $\mathrm{~N}$ \\
\hline HETATM & 361 & N22 & HEM & 34 & 2.209 & 2.535 & 15.273 & 1.00 & 0.00 & $\mathrm{~N}$ \\
\hline HETATM & 362 & N23 & HEM & 34 & 2.880 & 3.798 & 12.772 & 1.00 & 0.00 & $\mathrm{~N}$ \\
\hline HETATM & 363 & $\mathrm{~N} 24$ & HEM & 34 & 5.580 & 2.914 & 13.008 & 1.00 & 0.00 & $\mathrm{~N}$ \\
\hline HETATM & 364 & C99 & HEM & 34 & 4.673 & 5.779 & 15.510 & 1.00 & 0.00 & C \\
\hline HETATM & 365 & 091 & HEM & 34 & 4.514 & 4.465 & 15.498 & 1.00 & 0.00 & 0 \\
\hline HETATM & 366 & 092 & HEM & 34 & 4.828 & 6.396 & 16.566 & 1.00 & 0.00 & O \\
\hline HETATM & 367 & СТ91 & HEM & 34 & 4.657 & 6.460 & 14.154 & 1.00 & 0.00 & C \\
\hline
\end{tabular}




\begin{tabular}{|c|c|c|c|}
\hline HETATM & 368 & H911 & HEM \\
\hline HETATM & 369 & H912 & HEM \\
\hline HETATM & 370 & CT92 & HEM \\
\hline HETATM & 371 & H921 & HEM \\
\hline HETATM & 372 & H922 & HEM \\
\hline \multicolumn{4}{|l|}{ TER } \\
\hline HETATM & 373 & $\mathrm{C} 2$ & VIN \\
\hline HETATM & 374 & $\mathrm{H} 21$ & VIN \\
\hline HETATM & 375 & $\mathrm{C} 1$ & VIN \\
\hline HETATM & 376 & H11 & VIN \\
\hline HETATM & 377 & H12 & VIN \\
\hline \multicolumn{4}{|l|}{ TER } \\
\hline HETATM & 378 & C1 & MET \\
\hline HETATM & 379 & H11 & MET \\
\hline HETATM & 380 & $\mathrm{H} 12$ & MET \\
\hline HETATM & 381 & H13 & MET \\
\hline \multicolumn{4}{|l|}{ TER } \\
\hline HETATM & 382 & C2 & VIN \\
\hline HETATM & 383 & H 21 & VIN \\
\hline HETATM & 384 & $\mathrm{C} 1$ & VIN \\
\hline HETATM & 385 & $\mathrm{H} 11$ & VIN \\
\hline HETATM & 386 & $\mathrm{H} 12$ & VIN \\
\hline \multicolumn{4}{|l|}{ TER } \\
\hline HETATM & 387 & $\mathrm{C} 1$ & $\mathrm{MET}$ \\
\hline HETATM & 388 & H11 & MET \\
\hline HETATM & 389 & $\mathrm{H} 12$ & MET \\
\hline HETATM & 390 & $\mathrm{H} 13$ & $\mathrm{MET}$ \\
\hline \multicolumn{4}{|l|}{ TER } \\
\hline HETATM & 391 & C5 & $\mathrm{ACP}$ \\
\hline HETATM & 392 & H51 & $\mathrm{ACP}$ \\
\hline HETATM & 393 & H52 & $\mathrm{ACP}$ \\
\hline HETATM & 394 & C4 & $\mathrm{ACP}$ \\
\hline HETATM & 395 & H4 1 & $\mathrm{ACP}$ \\
\hline HETATM & 396 & $\mathrm{H} 42$ & $\mathrm{ACP}$ \\
\hline HETATM & 397 & $\mathrm{C} 1$ & $\mathrm{ACP}$ \\
\hline HETATM & 398 & 02 & $\mathrm{ACP}$ \\
\hline HETATM & 399 & 03 & $\mathrm{ACP}$ \\
\hline HETATM & 400 & H3 & $\mathrm{ACP}$ \\
\hline \multicolumn{4}{|l|}{ TER } \\
\hline HETATM & 401 & $\mathrm{C} 1$ & MET \\
\hline HETATM & 402 & H11 & MET \\
\hline HETATM & 403 & $\mathrm{H} 12$ & $\mathrm{MET}$ \\
\hline HETATM & 404 & H13 & MET \\
\hline \multicolumn{4}{|l|}{ TER } \\
\hline HETATM & 405 & $\mathrm{C} 1$ & MET \\
\hline HETATM & 406 & H11 & MET \\
\hline HETATM & 407 & $\mathrm{H} 12$ & $\mathrm{MET}$ \\
\hline HETATM & 408 & H13 & MET \\
\hline \multicolumn{4}{|l|}{ TER } \\
\hline HETATM & 409 & $\mathrm{C} 2$ & VIN \\
\hline HETATM & 410 & H 21 & VIN \\
\hline HETATM & 411 & $\mathrm{C} 1$ & VIN \\
\hline HETATM & 412 & H11 & VIN \\
\hline HETATM & 413 & $\mathrm{H} 12$ & VIN \\
\hline TER & & & \\
\hline HETATM & 414 & $\mathrm{C} 1$ & MET \\
\hline HETATM & 415 & H11 & MET \\
\hline HETATM & 416 & $\mathrm{H} 12$ & MET \\
\hline HETATM & 417 & H13 & MET \\
\hline TER & & & \\
\hline HETATM & 418 & $\mathrm{C} 2$ & VIN \\
\hline HETATM & 419 & H 21 & VIN \\
\hline HETATM & 420 & $\mathrm{C} 1$ & VIN \\
\hline HETATM & 421 & $\mathrm{H} 11$ & VIN \\
\hline HETATM & 422 & H12 & VIN \\
\hline TER & & & \\
\hline HETATM & 423 & $\mathrm{C} 1$ & MET \\
\hline HETATM & 424 & H11 & MET \\
\hline HETATM & 425 & H12 & MET \\
\hline HETATM & 426 & H13 & MET \\
\hline TER & & & \\
\hline HETATM & 427 & C5 & $\mathrm{ACP}$ \\
\hline HETATM & 428 & H51 & $\mathrm{ACP}$ \\
\hline HETATM & 429 & H52 & $\mathrm{ACP}$ \\
\hline HETATM & 430 & C4 & $\mathrm{ACP}$ \\
\hline HETATM & 431 & H 41 & $\mathrm{ACP}$ \\
\hline HETATM & 432 & $\mathrm{H} 42$ & $\mathrm{ACP}$ \\
\hline HETATM & 433 & $\mathrm{C} 1$ & $\mathrm{ACP}$ \\
\hline HETATN & 434 & 02 & Da \\
\hline
\end{tabular}

\begin{tabular}{|c|c|c|c|c|}
\hline 3.678 & 6.265 & 13.691 & 1.00 & 0.00 \\
\hline 5.405 & 5.970 & 13.511 & 1.00 & 0.00 \\
\hline 4.917 & 7.964 & 14.265 & 1.00 & 0.00 \\
\hline 5.842 & 8.120 & 14.836 & 1.00 & 0.00 \\
\hline 4.120 & 8.425 & 14.862 & 1.00 & 0.00 \\
\hline-0.603 & 13.676 & 6.929 & 1.00 & 0.00 \\
\hline-1.419 & 12.978 & 7.094 & 1.00 & 0.00 \\
\hline-0.936 & 14.901 & 6.528 & 1.00 & 0.00 \\
\hline-0.205 & 15.680 & 6.421 & 1.00 & 0.00 \\
\hline-1.978 & 15.170 & 6.361 & 1.00 & 0.00 \\
\hline 2.061 & 13.931 & 5.091 & 1.00 & 0.00 \\
\hline 2.295 & 15.002 & 5.211 & 1.00 & 0.00 \\
\hline 2.874 & 13.467 & 4.522 & 1.00 & 0.00 \\
\hline 1.153 & 13.854 & 4.481 & 1.00 & 0.00 \\
\hline 7.071 & 12.253 & 5.606 & 1.00 & 0.00 \\
\hline 6.405 & 12.152 & 4.747 & 1.00 & 0.00 \\
\hline 8.238 & 12.898 & 5.447 & 1.00 & 0.00 \\
\hline 8.917 & 13.054 & 6.277 & 1.00 & 0.00 \\
\hline 8.543 & 13.296 & 4.482 & 1.00 & 0.00 \\
\hline 8.755 & 10.570 & 7.810 & 1.00 & 0.00 \\
\hline 9.347 & 11.234 & 8.459 & 1.00 & 0.00 \\
\hline 8.912 & 9.548 & 8.177 & 1.00 & 0.00 \\
\hline 9.188 & 10.636 & 6.805 & 1.00 & 0.00 \\
\hline-0.333 & 9.488 & 13.701 & 1.00 & 0.00 \\
\hline 0.406 & 9.477 & 14.514 & 1.00 & 0.00 \\
\hline-1.174 & 10.097 & 14.065 & 1.00 & 0.00 \\
\hline-0.812 & 8.051 & 13.428 & 1.00 & 0.00 \\
\hline-1.369 & 7.990 & 12.481 & 1.00 & 0.00 \\
\hline 0.059 & 7.384 & 13.321 & 1.00 & 0.00 \\
\hline-1.676 & 7.439 & 14.504 & 1.00 & 0.00 \\
\hline-1.903 & 7.961 & 15.603 & 1.00 & 0.00 \\
\hline-2.191 & 6.259 & 14.140 & 1.00 & 0.00 \\
\hline-2.800 & 5.905 & 14.876 & 1.00 & 0.00 \\
\hline 7.563 & 8.362 & 12.528 & 1.00 & 0.00 \\
\hline 8.115 & 9.201 & 12.978 & 1.00 & 0.00 \\
\hline 7.570 & 7.536 & 13.250 & 1.00 & 0.00 \\
\hline 8.134 & 8.042 & 11.649 & 1.00 & 0.00 \\
\hline-1.870 & 11.311 & 11.577 & 1.00 & 0.00 \\
\hline-2.316 & 11.102 & 12.557 & 1.00 & 0.00 \\
\hline-1.981 & 12.391 & 11.402 & 1.00 & 0.00 \\
\hline-2.471 & 10.785 & 10.821 & 1.00 & 0.00 \\
\hline 7.926 & -0.068 & 16.864 & 1.00 & 0.00 \\
\hline 8.742 & 0.630 & 16.699 & 1.00 & 0.00 \\
\hline 8.259 & -1.293 & 17.266 & 1.00 & 0.00 \\
\hline 7.528 & -2.072 & 17.373 & 1.00 & 0.00 \\
\hline 9.301 & -1.562 & 17.432 & 1.00 & 0.00 \\
\hline 5.263 & -0.323 & 18.702 & 1.00 & 0.00 \\
\hline 5.028 & -1.394 & 18.583 & 1.00 & 0.00 \\
\hline 4.450 & 0.141 & 19.272 & 1.00 & 0.00 \\
\hline 6.170 & -0.246 & 19.313 & 1.00 & 0.00 \\
\hline 0.252 & 1.355 & 18.188 & 1.00 & 0.00 \\
\hline 0.918 & 1.456 & 19.046 & 1.00 & 0.00 \\
\hline-0.914 & 0.710 & 18.346 & 1.00 & 0.00 \\
\hline-1.594 & 0.554 & 17.516 & 1.00 & 0.00 \\
\hline-1.219 & 0.312 & 19.312 & 1.00 & 0.00 \\
\hline-1.432 & 3.038 & 15.984 & 1.00 & 0.00 \\
\hline-1.588 & 4.060 & 15.617 & 1.00 & 0.00 \\
\hline-1.864 & 2.972 & 16.989 & 1.00 & 0.00 \\
\hline-2.023 & 2.374 & 15.335 & 1.00 & 0.00 \\
\hline 7.656 & 4.120 & 10.092 & 1.00 & 0.00 \\
\hline 6.917 & 4.131 & 9.280 & 1.00 & 0.00 \\
\hline 8.497 & 3.511 & 9.729 & 1.00 & 0.00 \\
\hline 8.135 & 5.557 & 10.365 & 1.00 & 0.00 \\
\hline 8.693 & 5.618 & 11.313 & 1.00 & 0.00 \\
\hline 7.264 & 6.224 & 10.472 & 1.00 & 0.00 \\
\hline 8.999 & 6.169 & 9.289 & 1.00 & 0.00 \\
\hline 9.227 & 5.647 & 8.191 & 1.00 & 0.00 \\
\hline
\end{tabular}




\begin{tabular}{|c|c|c|c|c|c|c|c|c|c|c|}
\hline HETATM & 435 & 03 & $\mathrm{ACP}$ & 46 & 9.515 & 7.349 & 9.653 & 1.00 & 0.00 & 0 \\
\hline HETATM & 436 & H3 & $\mathrm{ACP}$ & 46 & 10.124 & 7.703 & 8.917 & 1.00 & 0.00 & $\mathrm{H}$ \\
\hline \multicolumn{11}{|l|}{ TER } \\
\hline HETATM & 437 & $\mathrm{C} 1$ & MET & 47 & -0.240 & 5.246 & 11.266 & 1.00 & 0.00 & C \\
\hline HETATM & 438 & $\mathrm{H} 11$ & MET & 47 & -0.792 & 4.407 & 10.815 & 1.00 & 0.00 & \\
\hline HETATM & 439 & $\mathrm{H} 12$ & MET & 47 & -0.246 & 6.072 & 10.544 & 1.00 & 0.00 & \\
\hline HETATM & 440 & $\mathrm{H} 13$ & MET & 47 & -0.811 & 5.565 & 12.145 & 1.00 & 0.00 & \\
\hline \multicolumn{10}{|l|}{ TER } & \\
\hline HETATM & 441 & $\mathrm{C} 1$ & MET & 48 & 9.194 & 2.297 & 12.216 & 1.00 & 0.00 & \\
\hline HETATM & 442 & H11 & MET & 48 & 9.639 & 2.506 & 11.236 & 1.00 & 0.00 & \\
\hline HETATM & 443 & $\mathrm{H} 12$ & MET & 48 & 9.304 & 1.217 & 12.391 & 1.00 & 0.00 & \\
\hline HETATM & 444 & $\mathrm{H} 13$ & MET & 48 & 9.794 & 2.823 & 12.973 & 1.00 & 0.00 & \\
\hline
\end{tabular}

TER

END

\subsection{T2 dimer: A02_T2.pdb file}

\begin{tabular}{|c|c|c|c|c|c|c|c|c|c|c|}
\hline ATOM & 1 & $\mathrm{FE}$ & HEM & 1 & 4.263 & 10.757 & 1.491 & 1.00 & 0.00 & $\mathrm{FE}$ \\
\hline ATOM & 2 & $\mathrm{C} 1$ & HEM & 1 & 2.035 & 12.629 & 0.429 & 1.00 & 0.00 & C \\
\hline ATOM & 3 & $\mathrm{C} 2$ & HEM & 1 & 1.676 & 13.420 & -0.742 & 1.00 & 0.00 & C \\
\hline ATOM & 4 & C3 & HEM & 1 & 2.822 & 13.517 & -1.517 & 1.00 & 0.00 & C \\
\hline ATOM & 5 & $\mathrm{C} 4$ & HEM & 1 & 3.851 & 12.800 & -0.809 & 1.00 & 0.00 & C \\
\hline ATOM & 6 & $\mathrm{C} 5$ & HEM & 1 & 5.167 & 12.681 & -1.247 & 1.00 & 0.00 & C \\
\hline ATOM & 7 & H5 & HEM & 1 & 5.420 & 13.177 & -2.182 & 1.00 & 0.00 & $\mathrm{H}$ \\
\hline ATOM & 8 & $\mathrm{C} 6$ & HEM & 1 & 6.186 & 11.982 & -0.611 & 1.00 & 0.00 & C \\
\hline ATOM & 9 & $\mathrm{C} 7$ & HEM & 1 & 7.555 & 11.910 & -1.081 & 1.00 & 0.00 & C \\
\hline ATOM & 10 & $\mathrm{C} 8$ & HEM & 1 & 8.260 & 11.190 & -0.134 & 1.00 & 0.00 & C \\
\hline ATOM & 11 & C9 & HEM & 1 & 7.309 & 10.825 & 0.891 & 1.00 & 0.00 & C \\
\hline ATOM & 12 & $\mathrm{C} 10$ & HEM & 1 & 7.614 & 10.090 & 2.034 & 1.00 & 0.00 & C \\
\hline ATOM & 13 & $\mathrm{H} 10$ & HEM & 1 & 8.641 & 9.746 & 2.140 & 1.00 & 0.00 & $\mathrm{H}$ \\
\hline ATOM & 14 & $\mathrm{C} 11$ & HEM & 1 & 6.739 & 9.750 & 3.057 & 1.00 & 0.00 & C \\
\hline ATOM & 15 & $\mathrm{C} 12$ & HEM & 1 & 7.120 & 9.025 & 4.247 & 1.00 & 0.00 & C \\
\hline ATOM & 16 & $\mathrm{C} 13$ & HEM & 1 & 5.981 & 8.878 & 5.004 & 1.00 & 0.00 & C \\
\hline ATOM & 17 & C14 & HEM & 1 & 4.908 & 9.519 & 4.273 & 1.00 & 0.00 & C \\
\hline ATOM & 18 & C15 & HEM & 1 & 3.580 & 9.574 & 4.694 & 1.00 & 0.00 & C \\
\hline ATOM & 19 & $\mathrm{H} 15$ & HEM & 1 & 3.340 & 9.089 & 5.642 & 1.00 & 0.00 & $\mathrm{H}$ \\
\hline ATOM & 20 & C16 & HEM & 1 & 2.550 & 10.255 & 4.051 & 1.00 & 0.00 & $\mathrm{C}$ \\
\hline ATOM & 21 & C17 & HEM & 1 & 1.184 & 10.369 & 4.533 & 1.00 & 0.00 & $\mathrm{C}$ \\
\hline ATOM & 22 & C18 & HEM & 1 & 0.508 & 11.137 & 3.609 & 1.00 & 0.00 & C \\
\hline ATOM & 23 & C19 & HEM & 1 & 1.458 & 11.489 & 2.573 & 1.00 & 0.00 & C \\
\hline ATOM & 24 & C20 & HEM & 1 & 1.167 & 12.280 & 1.462 & 1.00 & 0.00 & C \\
\hline ATOM & 25 & $\mathrm{H} 2 \mathrm{O}$ & HEM & 1 & 0.152 & 12.669 & 1.402 & 1.00 & 0.00 & $\mathrm{H}$ \\
\hline ATOM & 26 & $\mathrm{~N} 21$ & HEM & 1 & 3.357 & 12.266 & 0.367 & 1.00 & 0.00 & $\mathrm{~N}$ \\
\hline ATOM & 27 & N22 & HEM & 1 & 6.062 & 11.319 & 0.590 & 1.00 & 0.00 & $\mathrm{~N}$ \\
\hline ATOM & 28 & N23 & HEM & 1 & 5.391 & 10.056 & 3.090 & 1.00 & 0.00 & $\mathrm{~N}$ \\
\hline ATOM & 29 & N24 & HEM & 1 & 2.691 & 10.941 & 2.854 & 1.00 & 0.00 & $\mathrm{~N}$ \\
\hline ATOM & 30 & C99 & HEM & 1 & 3.598 & 8.076 & 0.352 & 1.00 & 0.00 & C \\
\hline ATOM & 31 & 091 & HEM & 1 & 3.757 & 9.389 & 0.365 & 1.00 & 0.00 & o \\
\hline ATOM & 32 & 092 & HEM & 1 & 3.443 & 7.458 & -0.703 & 1.00 & 0.00 & 0 \\
\hline ATOM & 33 & CT91 & HEM & 1 & 3.614 & 7.394 & 1.709 & 1.00 & 0.00 & C \\
\hline ATOM & 34 & H911 & HEM & 1 & 4.593 & 7.590 & 2.171 & 1.00 & 0.00 & $\mathrm{H}$ \\
\hline ATOM & 35 & H912 & HEM & 1 & 2.866 & 7.884 & 2.352 & 1.00 & 0.00 & $\mathrm{H}$ \\
\hline ATOM & 36 & CT92 & HEM & 1 & 3.354 & 5.890 & 1.597 & 1.00 & 0.00 & C \\
\hline ATOM & 37 & H921 & HEM & 1 & 4.151 & 5.429 & 1.000 & 1.00 & 0.00 & $\mathrm{H}$ \\
\hline ATOM & 38 & H922 & HEM & 1 & 2.429 & 5.734 & 1.027 & 1.00 & 0.00 & $\mathrm{H}$ \\
\hline \multicolumn{11}{|l|}{ TER } \\
\hline ATOM & 39 & $\mathrm{FE}$ & HEM & 2 & 4.955 & 3.343 & 6.440 & 1.00 & 0.00 & $\mathrm{FE}$ \\
\hline ATOM & 40 & $\mathrm{C} 1$ & HEM & 2 & 7.183 & 1.471 & 7.502 & 1.00 & 0.00 & C \\
\hline ATOM & 41 & $\mathrm{C} 2$ & HEM & 2 & 7.542 & 0.680 & 8.674 & 1.00 & 0.00 & C \\
\hline ATOM & 42 & C3 & HEM & 2 & 6.397 & 0.583 & 9.448 & 1.00 & 0.00 & C \\
\hline ATOM & 43 & C4 & HEM & 2 & 5.367 & 1.301 & 8.740 & 1.00 & 0.00 & C \\
\hline ATOM & 44 & C5 & HEM & 2 & 4.052 & 1.420 & 9.178 & 1.00 & 0.00 & C \\
\hline ATOM & 45 & $\mathrm{H} 5$ & HEM & 2 & 3.798 & 0.923 & 10.113 & 1.00 & 0.00 & $\mathrm{H}$ \\
\hline ATOM & 46 & $\mathrm{C} 6$ & HEM & 2 & 3.033 & 2.118 & 8.542 & 1.00 & 0.00 & C \\
\hline ATOM & 47 & $\mathrm{C} 7$ & HEM & 2 & 1.664 & 2.191 & 9.012 & 1.00 & 0.00 & C \\
\hline ATOM & 48 & $\mathrm{C} 8$ & HEM & 2 & 0.958 & 2.910 & 8.065 & 1.00 & 0.00 & C \\
\hline ATOM & 49 & $\mathrm{C} 9$ & HEM & 2 & 1.909 & 3.275 & 7.041 & 1.00 & 0.00 & C \\
\hline ATOM & 50 & $\mathrm{C} 10$ & HEM & 2 & 1.605 & 4.010 & 5.897 & 1.00 & 0.00 & C \\
\hline ATOM & 51 & H1O & HEM & 2 & 0.578 & 4.354 & 5.791 & 1.00 & 0.00 & $\mathrm{H}$ \\
\hline ATOM & 52 & C11 & HEM & 2 & 2.479 & 4.350 & 4.874 & 1.00 & 0.00 & 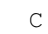 \\
\hline ATOM & 53 & $\mathrm{C} 12$ & HEM & 2 & 2.098 & 5.076 & 3.684 & 1.00 & 0.00 & 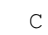 \\
\hline ATOM & 54 & $\mathrm{C} 13$ & HEM & 2 & 3.238 & 5.223 & 2.927 & 1.00 & 0.00 & \\
\hline ATOM & 55 & C14 & HEM & 2 & 4.311 & 4.581 & 3.659 & 1.00 & 0.00 & \\
\hline ATOM & 56 & C15 & HEM & 2 & 5.639 & 4.527 & 3.237 & 1.00 & 0.00 & \\
\hline ATOM & 57 & H15 & HEM & 2 & 5.879 & 5.011 & 2.289 & 1.00 & 0.00 & \\
\hline ATOM & 58 & C16 & HEM & 2 & 6.669 & 3.845 & 3.880 & 1.00 & 0.00 & \\
\hline ATOM & 59 & $\mathrm{C} 17$ & HEM & 2 & 8.035 & 3.732 & 3.398 & 1.00 & 0.00 & c \\
\hline ATOM & 60 & C18 & HEM & 2 & 8.710 & 2.964 & 4.322 & 1.00 & 0.00 & \\
\hline
\end{tabular}




\begin{tabular}{|c|c|c|c|c|c|c|c|c|c|c|}
\hline ATOM & 61 & $\mathrm{C} 19$ & HEM & 2 & 7.761 & 2.612 & 5.358 & 1.00 & 0.00 & C \\
\hline ATOM & 62 & $\mathrm{C} 20$ & HEM & 2 & 8.052 & 1.821 & 6.469 & 1.00 & 0.00 & $\mathrm{C}$ \\
\hline ATOM & 63 & $\mathrm{H} 2 \mathrm{O}$ & HEM & 2 & 9.066 & 1.432 & 6.530 & 1.00 & 0.00 & $\mathrm{H}$ \\
\hline ATOM & 64 & N21 & HEM & 2 & 5.862 & 1.834 & 7.564 & 1.00 & 0.00 & $\mathrm{~N}$ \\
\hline ATOM & 65 & N22 & HEM & 2 & 3.157 & 2.781 & 7.342 & 1.00 & 0.00 & $\mathrm{~N}$ \\
\hline ATOM & 66 & N23 & HEM & 2 & 3.828 & 4.044 & 4.841 & 1.00 & 0.00 & $\mathrm{~N}$ \\
\hline ATOM & 67 & N24 & HEM & 2 & 6.527 & 3.160 & 5.077 & 1.00 & 0.00 & $\mathrm{~N}$ \\
\hline ATOM & 68 & C99 & HEM & 2 & 5.621 & 6.025 & 7.579 & 1.00 & 0.00 & $\mathrm{C}$ \\
\hline ATOM & 69 & 091 & HEM & 2 & 5.461 & 4.711 & 7.567 & 1.00 & 0.00 & 0 \\
\hline ATOM & 70 & 092 & HEM & 2 & 5.776 & 6.642 & 8.635 & 1.00 & 0.00 & 0 \\
\hline ATOM & 71 & Ст91 & HEM & 2 & 5.605 & 6.706 & 6.223 & 1.00 & 0.00 & $\mathrm{C}$ \\
\hline ATOM & 72 & H911 & HEM & 2 & 4.626 & 6.511 & 5.760 & 1.00 & 0.00 & $\mathrm{H}$ \\
\hline ATOM & 73 & H912 & HEM & 2 & 6.353 & 6.216 & 5.580 & 1.00 & 0.00 & $\mathrm{H}$ \\
\hline ATOM & 74 & СТ92 & HEM & 2 & 5.864 & 8.211 & 6.334 & 1.00 & 0.00 & $\mathrm{C}$ \\
\hline ATOM & 75 & H921 & HEM & 2 & 6.789 & 8.367 & 6.905 & 1.00 & 0.00 & $\mathrm{H}$ \\
\hline ATOM & 76 & H922 & HEM & 2 & 5.068 & 8.671 & 6.931 & 1.00 & 0.00 & 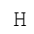 \\
\hline \multicolumn{11}{|l|}{ TER } \\
\hline ATOM & 77 & $\mathrm{C} 2$ & VIN & 3 & 0.345 & 13.923 & -1.002 & 1.00 & 0.00 & $\mathrm{C}$ \\
\hline ATOM & 78 & $\mathrm{H} 21$ & VIN & 3 & -0.471 & 13.224 & -0.837 & 1.00 & 0.00 & $\mathrm{H}$ \\
\hline ATOM & 79 & $\mathrm{C} 1$ & VIN & 3 & 0.012 & 15.147 & -1.403 & 1.00 & 0.00 & $\mathrm{C}$ \\
\hline ATOM & 80 & $\mathrm{H} 11$ & VIN & 3 & 0.743 & 15.926 & -1.511 & 1.00 & 0.00 & $\mathrm{H}$ \\
\hline ATOM & 81 & $\mathrm{H} 12$ & VIN & 3 & -1.030 & 15.417 & -1.570 & 1.00 & 0.00 & $\mathrm{H}$ \\
\hline \multicolumn{11}{|l|}{ TER } \\
\hline ATOM & 82 & $\mathrm{C} 1$ & MET & 4 & 3.008 & 14.177 & -2.840 & 1.00 & 0.00 & $\tau$ \\
\hline ATOM & 83 & $\mathrm{H} 11$ & MET & 4 & 3.243 & 15.248 & -2.720 & 1.00 & 0.00 & $\mathrm{H}$ \\
\hline ATOM & 84 & $\mathrm{H} 12$ & MET & 4 & 3.821 & 13.713 & -3.410 & 1.00 & 0.00 & $\mathrm{H}$ \\
\hline ATOM & 85 & $\mathrm{H} 13$ & $\mathrm{MET}$ & 4 & 2.101 & 14.100 & -3.450 & 1.00 & 0.00 & $\mathrm{H}$ \\
\hline \multicolumn{11}{|l|}{ TER } \\
\hline ATOM & 86 & $\mathrm{C} 2$ & VIN & 5 & 8.018 & 12.499 & -2.325 & 1.00 & 0.00 & $\mathrm{C}$ \\
\hline ATOM & 87 & $\mathrm{H} 21$ & VIN & 5 & 7.352 & 12.399 & -3.184 & 1.00 & 0.00 & $\mathrm{H}$ \\
\hline ATOM & 88 & $\mathrm{C} 1$ & VIN & 5 & 9.185 & 13.144 & -2.484 & 1.00 & 0.00 & $\mathrm{C}$ \\
\hline ATOM & 89 & $\mathrm{H} 11$ & VIN & 5 & 9.865 & 13.300 & -1.654 & 1.00 & 0.00 & $\mathrm{H}$ \\
\hline ATOM & 90 & $\mathrm{H} 12$ & VIN & 5 & 9.490 & 13.542 & -3.450 & 1.00 & 0.00 & $\mathrm{H}$ \\
\hline \multicolumn{11}{|l|}{ TER } \\
\hline ATOM & 91 & $\mathrm{C} 1$ & $\mathrm{MET}$ & 6 & 9.703 & 10.816 & -0.121 & 1.00 & 0.00 & C \\
\hline ATOM & 92 & $\mathrm{H} 11$ & MET & 6 & 10.294 & 11.480 & 0.528 & 1.00 & 0.00 & $\mathrm{H}$ \\
\hline ATOM & 93 & $\mathrm{H} 12$ & MET & 6 & 9.859 & 9.794 & 0.246 & 1.00 & 0.00 & $\mathrm{H}$ \\
\hline ATOM & 94 & $\mathrm{H} 13$ & MET & 6 & 10.135 & 10.882 & -1.126 & 1.00 & 0.00 & $\mathrm{H}$ \\
\hline TER & & & & & & & & & & \\
\hline ATOM & 95 & C5 & $\mathrm{ACP}$ & 7 & 0.615 & 9.735 & 5.770 & 1.00 & 0.00 & $\mathrm{C}$ \\
\hline ATOM & 96 & H51 & $\mathrm{ACP}$ & 7 & 1.354 & 9.723 & 6.582 & 1.00 & 0.00 & $\mathrm{H}$ \\
\hline ATOM & 97 & H52 & $\mathrm{ACP}$ & 7 & -0.226 & 10.343 & 6.134 & 1.00 & 0.00 & $\mathrm{H}$ \\
\hline ATOM & 98 & $\mathrm{C} 4$ & $\mathrm{ACP}$ & 7 & 0.136 & 8.297 & 5.497 & 1.00 & 0.00 & $\mathrm{C}$ \\
\hline ATOM & 99 & $\mathrm{H} 41$ & $\mathrm{ACP}$ & 7 & -0.422 & 8.236 & 4.549 & 1.00 & 0.00 & $\mathrm{H}$ \\
\hline ATOM & 100 & $\mathrm{H} 42$ & $\mathrm{ACP}$ & 7 & 1.007 & 7.630 & 5.390 & 1.00 & 0.00 & $\mathrm{H}$ \\
\hline ATOM & 101 & $\mathrm{C} 1$ & $\mathrm{ACP}$ & 7 & -0.728 & 7.685 & 6.573 & 1.00 & 0.00 & C \\
\hline ATOM & 102 & 02 & $\mathrm{ACP}$ & 7 & -0.956 & 8.208 & 7.671 & 1.00 & 0.00 & 0 \\
\hline ATOM & 103 & 03 & $\mathrm{ACP}$ & 7 & -1.244 & 6.505 & 6.209 & 1.00 & 0.00 & 0 \\
\hline ATOM & 104 & H3 & $\mathrm{ACP}$ & 7 & -1.853 & 6.151 & 6.945 & 1.00 & 0.00 & $\mathrm{H}$ \\
\hline TER & & & & & & & & & & \\
\hline ATOM & 105 & $\mathrm{C} 1$ & MET & 8 & 8.511 & 8.608 & 4.597 & 1.00 & 0.00 & C \\
\hline ATOM & 106 & $\mathrm{H} 11$ & MET & 8 & 9.063 & 9.448 & 5.047 & 1.00 & 0.00 & $\mathrm{H}$ \\
\hline ATOM & 107 & $\mathrm{H} 12$ & MET & 8 & 8.517 & 7.782 & 5.319 & 1.00 & 0.00 & $\mathrm{H}$ \\
\hline ATOM & 108 & $\mathrm{H} 13$ & $\mathrm{MET}$ & 8 & 9.082 & 8.289 & 3.717 & 1.00 & 0.00 & $\mathrm{H}$ \\
\hline TER & & & & & & & & & & \\
\hline ATOM & 109 & $\mathrm{C} 1$ & MET & 9 & -0.923 & 11.557 & 3.646 & 1.00 & 0.00 & C \\
\hline ATOM & 110 & $\mathrm{H} 11$ & $\mathrm{MET}$ & 9 & -1.368 & 11.348 & 4.626 & 1.00 & 0.00 & $\mathrm{H}$ \\
\hline ATOM & 111 & $\mathrm{H} 12$ & MET & 9 & -1.033 & 12.637 & 3.471 & 1.00 & 0.00 & $\mathrm{H}$ \\
\hline ATOM & 112 & $\mathrm{H} 13$ & $\mathrm{MET}$ & 9 & -1.523 & 11.031 & 2.889 & 1.00 & 0.00 & $\mathrm{H}$ \\
\hline TER & & & & & & & & & & \\
\hline ATOM & 113 & $\mathrm{C} 2$ & VIN & 10 & 8.874 & 0.178 & 8.933 & 1.00 & 0.00 & c \\
\hline ATOM & 114 & H21 & VIN & 10 & 9.690 & 0.876 & 8.768 & 1.00 & 0.00 & $\mathrm{H}$ \\
\hline ATOM & 115 & $\mathrm{C} 1$ & VIN & 10 & 9.207 & -1.047 & 9.335 & 1.00 & 0.00 & c \\
\hline ATOM & 116 & $\mathrm{H} 11$ & VIN & 10 & 8.475 & -1.826 & 9.442 & 1.00 & 0.00 & $\mathrm{H}$ \\
\hline ATOM & 117 & $\mathrm{H} 12$ & VIN & 10 & 10.249 & -1.316 & 9.501 & 1.00 & 0.00 & $\mathrm{H}$ \\
\hline TER & & & & & & & & & & \\
\hline ATOM & 118 & $\mathrm{C} 1$ & MET & 11 & 6.210 & -0.077 & 10.771 & 1.00 & 0.00 & $\mathrm{C}$ \\
\hline ATOM & 119 & $\mathrm{H} 11$ & $\mathrm{MET}$ & 11 & 5.975 & -1.148 & 10.652 & 1.00 & 0.00 & $\mathrm{H}$ \\
\hline ATOM & 120 & $\mathrm{H} 12$ & MET & 11 & 5.397 & 0.387 & 11.341 & 1.00 & 0.00 & $\mathrm{H}$ \\
\hline ATOM & 121 & $\mathrm{H} 13$ & MET & 11 & 7.118 & 0.001 & 11.382 & 1.00 & 0.00 & $\mathrm{H}$ \\
\hline TER & & & & & & & & & & \\
\hline ATOM & 122 & $\mathrm{C} 2$ & VIN & 12 & 1.200 & 1.601 & 10.256 & 1.00 & 0.00 & $\mathrm{C}$ \\
\hline ATOM & 123 & H 21 & VIN & 12 & 1.866 & 1.702 & 11.115 & 1.00 & 0.00 & $\mathrm{H}$ \\
\hline ATOM & 124 & $\mathrm{C} 1$ & VIN & 12 & 0.033 & 0.957 & 10.415 & 1.00 & 0.00 & $\mathrm{C}$ \\
\hline ATOM & 125 & $\mathrm{H} 11$ & VIN & 12 & -0.647 & 0.800 & 9.585 & 1.00 & 0.00 & $\mathrm{H}$ \\
\hline ATOM & 126 & $\mathrm{H} 12$ & VIN & 12 & -0.272 & 0.559 & 11.381 & 1.00 & 0.00 & $\mathrm{H}$ \\
\hline TER & & & & & & & & & & \\
\hline ATOM & 127 & $\mathrm{C} 1$ & MET & 13 & -0.484 & 3.284 & 8.052 & 1.00 & 0.00 & \\
\hline ATOM & 128 & H11 & MET & 13 & -0.641 & 4.306 & 7.685 & 1.00 & 0.00 & \\
\hline
\end{tabular}




\begin{tabular}{|c|c|c|c|c|c|c|c|c|c|c|}
\hline ATOM & 129 & $\mathrm{H} 12$ & MET & 13 & -0.917 & 3.219 & 9.057 & 1.00 & 0.00 & $\mathrm{H}$ \\
\hline АTOM & 130 & H13 & MET & 13 & -1.076 & 2.620 & 7.403 & 1.00 & 0.00 & $\mathrm{H}$ \\
\hline \multicolumn{11}{|l|}{ TER } \\
\hline ATOM & 131 & $\mathrm{C} 5$ & $\mathrm{ACP}$ & 14 & 8.604 & 4.366 & 2.161 & 1.00 & 0.00 & $\mathrm{C}$ \\
\hline ATOM & 132 & H51 & $\mathrm{ACP}$ & 14 & 7.865 & 4.377 & 1.349 & 1.00 & 0.00 & $\mathrm{H}$ \\
\hline ATOM & 133 & H52 & $\mathrm{ACP}$ & 14 & 9.445 & 3.758 & 1.798 & 1.00 & 0.00 & $\mathrm{H}$ \\
\hline ATOM & 134 & C4 & $\mathrm{ACP}$ & 14 & 9.082 & 5.803 & 2.434 & 1.00 & 0.00 & C \\
\hline ATOM & 135 & $\mathrm{H} 4 \mathrm{I}$ & $\mathrm{ACP}$ & 14 & 9.640 & 5.865 & 3.382 & 1.00 & 0.00 & $\mathrm{H}$ \\
\hline ATOM & 136 & $\mathrm{H} 42$ & $\mathrm{ACP}$ & 14 & 8.212 & 6.470 & 2.541 & 1.00 & 0.00 & $\mathrm{H}$ \\
\hline ATOM & 137 & $\mathrm{C} 1$ & $\mathrm{ACP}$ & 14 & 9.946 & 6.416 & 1.358 & 1.00 & 0.00 & C \\
\hline ATOM & 138 & $\mathrm{O} 2$ & $\mathrm{ACP}$ & 14 & 10.174 & 5.893 & 0.260 & 1.00 & 0.00 & 0 \\
\hline ATOM & 139 & 03 & $\mathrm{ACP}$ & 14 & 10.462 & 7.595 & 1.722 & 1.00 & 0.00 & 0 \\
\hline ATOM & 140 & H3 & $\mathrm{ACP}$ & 14 & 11.071 & 7.949 & 0.986 & 1.00 & 0.00 & $\mathrm{H}$ \\
\hline \multicolumn{11}{|l|}{ TER } \\
\hline ATOM & 141 & $\mathrm{C} 1$ & MET & 15 & 0.708 & 5.492 & 3.334 & 1.00 & 0.00 & C \\
\hline ATOM & 142 & $\mathrm{H} 11$ & MET & 15 & 0.155 & 4.653 & 2.884 & 1.00 & 0.00 & $\mathrm{H}$ \\
\hline ATOM & 143 & $\mathrm{H} 12$ & $\mathrm{MET}$ & 15 & 0.701 & 6.318 & 2.613 & 1.00 & 0.00 & $\mathrm{H}$ \\
\hline ATOM & 144 & H13 & $\mathrm{MET}$ & 15 & 0.137 & 5.812 & 4.214 & 1.00 & 0.00 & $\mathrm{H}$ \\
\hline \multicolumn{11}{|l|}{ TER } \\
\hline ATOM & 145 & $\mathrm{C} 1$ & MET & 16 & 10.141 & 2.543 & 4.285 & 1.00 & 0.00 & C \\
\hline ATOM & 146 & $\mathrm{H} 1 \mathrm{I}$ & MET & 16 & 10.586 & 2.752 & 3.305 & 1.00 & 0.00 & $\mathrm{H}$ \\
\hline ATOM & 147 & $\mathrm{H} 12$ & MET & 16 & 10.252 & 1.463 & 4.460 & 1.00 & 0.00 & $\mathrm{H}$ \\
\hline ATOM & 148 & $\mathrm{H} 13$ & MET & 16 & 10.742 & 3.069 & 5.042 & 1.00 & 0.00 & $\mathrm{H}$ \\
\hline \multicolumn{11}{|l|}{ TER } \\
\hline ATOM & 149 & $\mathrm{FE}$ & HEM & 17 & 2.346 & 25.104 & 1.491 & 1.00 & 0.00 & $\mathrm{FE}$ \\
\hline ATOM & 150 & $\mathrm{C} 1$ & HEM & 17 & 0.118 & 26.976 & 0.429 & 1.00 & 0.00 & C \\
\hline ATOM & 151 & $\mathrm{C} 2$ & HEM & 17 & -0.240 & 27.767 & -0.742 & 1.00 & 0.00 & C \\
\hline ATOM & 152 & $\mathrm{C} 3$ & HEM & 17 & 0.905 & 27.864 & -1.517 & 1.00 & 0.00 & C \\
\hline ATOM & 153 & C4 & HEM & 17 & 1.935 & 27.146 & -0.809 & 1.00 & 0.00 & C \\
\hline ATOM & 154 & C5 & HEM & 17 & 3.250 & 27.027 & -1.247 & 1.00 & 0.00 & C \\
\hline ATOM & 155 & H5 & HEM & 17 & 3.503 & 27.524 & -2.182 & 1.00 & 0.00 & $\mathrm{H}$ \\
\hline ATOM & 156 & $\mathrm{C} 6$ & HEM & 17 & 4.269 & 26.329 & -0.611 & 1.00 & 0.00 & C \\
\hline ATOM & 157 & C7 & HEM & 17 & 5.638 & 26.256 & -1.081 & 1.00 & 0.00 & C \\
\hline ATOM & 158 & $\mathrm{C} 8$ & HEM & 17 & 6.343 & 25.537 & -0.134 & 1.00 & 0.00 & C \\
\hline ATOM & 159 & C9 & HEM & 17 & 5.392 & 25.172 & 0.891 & 1.00 & 0.00 & C \\
\hline ATOM & 160 & $\mathrm{C} 10$ & HEM & 17 & 5.697 & 24.437 & 2.034 & 1.00 & 0.00 & C \\
\hline ATOM & 161 & $\mathrm{H} 10$ & HEM & 17 & 6.724 & 24.093 & 2.140 & 1.00 & 0.00 & $\mathrm{H}$ \\
\hline ATOM & 162 & $\mathrm{C} 11$ & HEM & 17 & 4.822 & 24.097 & 3.057 & 1.00 & 0.00 & C \\
\hline ATOM & 163 & $\mathrm{C} 12$ & HEM & 17 & 5.203 & 23.371 & 4.247 & 1.00 & 0.00 & C \\
\hline ATOM & 164 & $\mathrm{C} 13$ & HEM & 17 & 4.064 & 23.224 & 5.004 & 1.00 & 0.00 & C \\
\hline ATOM & 165 & $\mathrm{C} 14$ & HEM & 17 & 2.991 & 23.866 & 4.273 & 1.00 & 0.00 & $\mathrm{C}$ \\
\hline ATOM & 166 & C15 & HEM & 17 & 1.663 & 23.920 & 4.694 & 1.00 & 0.00 & C \\
\hline ATOM & 167 & H15 & HEM & 17 & 1.423 & 23.436 & 5.642 & 1.00 & 0.00 & $\mathrm{H}$ \\
\hline ATOM & 168 & C16 & HEM & 17 & 0.633 & 24.602 & 4.051 & 1.00 & 0.00 & C \\
\hline ATOM & 169 & $\mathrm{C} 17$ & HEM & 17 & -0.733 & 24.716 & 4.533 & 1.00 & 0.00 & $\mathrm{C}$ \\
\hline ATOM & 170 & $\mathrm{C} 18$ & HEM & 17 & -1.408 & 25.483 & 3.609 & 1.00 & 0.00 & C \\
\hline ATOM & 171 & C19 & HEM & 17 & -0.459 & 25.835 & 2.573 & 1.00 & 0.00 & C \\
\hline ATOM & 172 & $\mathrm{C} 20$ & HEM & 17 & -0.750 & 26.626 & 1.462 & 1.00 & 0.00 & C \\
\hline ATOM & 173 & $\mathrm{H} 2 \mathrm{O}$ & HEM & 17 & -1.764 & 27.015 & 1.402 & 1.00 & 0.00 & $\mathrm{H}$ \\
\hline ATOM & 174 & N21 & HEM & 17 & 1.440 & 26.613 & 0.367 & 1.00 & 0.00 & $\mathrm{~N}$ \\
\hline ATOM & 175 & N22 & HEM & 17 & 4.145 & 25.666 & 0.590 & 1.00 & 0.00 & $\mathrm{~N}$ \\
\hline ATOM & 176 & N23 & HEM & 17 & 3.474 & 24.403 & 3.090 & 1.00 & 0.00 & $\mathrm{~N}$ \\
\hline ATOM & 177 & N2 4 & HEM & 17 & 0.774 & 25.287 & 2.854 & 1.00 & 0.00 & $\mathrm{~N}$ \\
\hline ATOM & 178 & C99 & HEM & 17 & 1.681 & 22.422 & 0.352 & 1.00 & 0.00 & C \\
\hline ATOM & 179 & 091 & HEM & 17 & 1.841 & 23.736 & 0.365 & 1.00 & 0.00 & 0 \\
\hline ATOM & 180 & 092 & HEM & 17 & 1.526 & 21.805 & -0.703 & 1.00 & 0.00 & O \\
\hline ATOM & 181 & СТ 91 & HEM & 17 & 1.697 & 21.741 & 1.709 & 1.00 & 0.00 & C \\
\hline ATOM & 182 & H911 & HEM & 17 & 2.676 & 21.936 & 2.171 & 1.00 & 0.00 & $\mathrm{H}$ \\
\hline ATOM & 183 & H912 & HEM & 17 & 0.949 & 22.231 & 2.352 & 1.00 & 0.00 & $\mathrm{H}$ \\
\hline ATOM & 184 & СТ92 & HEM & 17 & 1.437 & 20.236 & 1.597 & 1.00 & 0.00 & C \\
\hline ATOM & 185 & H921 & HEM & 17 & 2.234 & 19.776 & 1.000 & 1.00 & 0.00 & $\mathrm{H}$ \\
\hline ATOM & 186 & H922 & HEM & 17 & 0.513 & 20.081 & 1.027 & 1.00 & 0.00 & $\mathrm{H}$ \\
\hline TER & & & & & & & & & & \\
\hline ATOM & 187 & $\mathrm{FE}$ & HEM & 18 & 3.039 & 17.690 & 6.440 & 1.00 & 0.00 & $\mathrm{FE}$ \\
\hline ATOM & 188 & $\mathrm{C} 1$ & HEM & 18 & 5.266 & 15.818 & 7.502 & 1.00 & 0.00 & C \\
\hline ATOM & 189 & $\mathrm{C} 2$ & HEM & 18 & 5.625 & 15.027 & 8.674 & 1.00 & 0.00 & C \\
\hline ATOM & 190 & $\mathrm{C} 3$ & HEM & 18 & 4.480 & 14.930 & 9.448 & 1.00 & 0.00 & C \\
\hline ATOM & 191 & C4 & HEM & 18 & 3.450 & 15.647 & 8.740 & 1.00 & 0.00 & C \\
\hline ATOM & 192 & C5 & HEM & 18 & 2.135 & 15.766 & 9.178 & 1.00 & 0.00 & C \\
\hline ATOM & 193 & H5 & HEM & 18 & 1.882 & 15.270 & 10.113 & 1.00 & 0.00 & $\mathrm{H}$ \\
\hline ATOM & 194 & $\mathrm{C} 6$ & HEM & 18 & 1.116 & 16.465 & 8.542 & 1.00 & 0.00 & C \\
\hline ATOM & 195 & C7 & HEM & 18 & -0.253 & 16.537 & 9.012 & 1.00 & 0.00 & C \\
\hline ATOM & 196 & $\mathrm{C} 8$ & HEM & 18 & -0.958 & 17.257 & 8.065 & 1.00 & 0.00 & C \\
\hline ATOM & 197 & C9 & HEM & 18 & -0.007 & 17.622 & 7.041 & 1.00 & 0.00 & C \\
\hline ATOM & 198 & $\mathrm{C} 10$ & HEM & 18 & -0.312 & 18.357 & 5.897 & 1.00 & 0.00 & C \\
\hline ATOM & 199 & $\mathrm{H} 10$ & HEM & 18 & -1.339 & 18.701 & 5.791 & 1.00 & 0.00 & $\mathrm{H}$ \\
\hline ATOM & 200 & $\mathrm{C} 11$ & HEM & 18 & 0.562 & 18.697 & 4.874 & 1.00 & 0.00 & C \\
\hline ATOM & 201 & $\mathrm{C} 12$ & HEM & 18 & 0.182 & 19.422 & 3.684 & 1.00 & 0.00 & C \\
\hline ATOM & 202 & $\mathrm{C} 13$ & HEM & 18 & 1.321 & 19.569 & 2.927 & 1.00 & 0.00 & 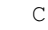 \\
\hline
\end{tabular}




\begin{tabular}{|c|c|c|c|}
\hline ATOM & 203 & C14 & HEM \\
\hline ATOM & 204 & C15 & HEM \\
\hline AтOM & 205 & H15 & HEM \\
\hline ATOM & 206 & C16 & HEM \\
\hline ATOM & 207 & C17 & HEM \\
\hline ATOM & 208 & C18 & HEM \\
\hline ATOM & 209 & C19 & HEM \\
\hline ATOM & 210 & $\mathrm{C} 20$ & HEM \\
\hline ATOM & 211 & H 20 & HEM \\
\hline ATOM & 212 & $\mathrm{~N} 21$ & HEM \\
\hline ATOM & 213 & N22 & HEM \\
\hline ATOM & 214 & N23 & HEM \\
\hline ATOM & 215 & N24 & HEM \\
\hline ATOM & 216 & C99 & HEM \\
\hline ATOM & 217 & 091 & HEM \\
\hline ATOM & 218 & 092 & HEM \\
\hline ATOM & 219 & CT91 & HEM \\
\hline ATOM & 220 & H911 & HEM \\
\hline ATOM & 221 & H912 & HEM \\
\hline ATOM & 222 & СТ92 & HEM \\
\hline ATOM & 223 & H921 & HEM \\
\hline ATOM & 224 & H922 & HEM \\
\hline \multicolumn{4}{|l|}{ TER } \\
\hline ATOM & 225 & $\mathrm{C} 2$ & VIN \\
\hline ATOM & 226 & H 21 & VIN \\
\hline ATOM & 227 & $\mathrm{C} 1$ & VIN \\
\hline ATOM & 228 & H11 & VIN \\
\hline ATOM & 229 & $\mathrm{H} 12$ & VIN \\
\hline \multicolumn{4}{|l|}{ TER } \\
\hline ATOM & 230 & $\mathrm{C} 1$ & MET \\
\hline ATOM & 231 & H11 & MET \\
\hline ATOM & 232 & H12 & MET \\
\hline ATOM & 233 & $\mathrm{H} 13$ & MET \\
\hline \multicolumn{4}{|l|}{ TER } \\
\hline ATOM & 234 & $\mathrm{C} 2$ & VIN \\
\hline ATOM & 235 & H 21 & VIN \\
\hline ATOM & 236 & $\mathrm{C} 1$ & VIN \\
\hline ATOM & 237 & $\mathrm{H} 11$ & VIN \\
\hline ATOM & 238 & $\mathrm{H} 12$ & VIN \\
\hline \multicolumn{4}{|l|}{ TER } \\
\hline ATOM & 239 & $\mathrm{C} 1$ & $\mathrm{MET}$ \\
\hline AтOM & 240 & H11 & MET \\
\hline ATOM & 241 & $\mathrm{H} 12$ & $\mathrm{MET}$ \\
\hline ATOM & 242 & H13 & $\mathrm{MET}$ \\
\hline \multicolumn{4}{|l|}{ TER } \\
\hline ATOM & 243 & $\mathrm{C} 5$ & $\mathrm{ACP}$ \\
\hline ATOM & 244 & H51 & $\mathrm{ACP}$ \\
\hline ATOM & 245 & H52 & $\mathrm{ACP}$ \\
\hline ATOM & 246 & $\mathrm{C} 4$ & $\mathrm{ACP}$ \\
\hline ATOM & 247 & $\mathrm{H} 41$ & $\mathrm{ACP}$ \\
\hline ATOM & 248 & $\mathrm{H} 42$ & $\mathrm{ACP}$ \\
\hline ATOM & 249 & $\mathrm{C} 1$ & $\mathrm{ACP}$ \\
\hline ATOM & 250 & $\mathrm{O} 2$ & $\mathrm{ACP}$ \\
\hline ATOM & 251 & 03 & $\mathrm{ACP}$ \\
\hline ATOM & 252 & H3 & $\mathrm{ACP}$ \\
\hline \multicolumn{4}{|l|}{ TER } \\
\hline ATOM & 253 & $\mathrm{C} 1$ & $\mathrm{MET}$ \\
\hline ATOM & 254 & $\mathrm{H} 11$ & MET \\
\hline ATOM & 255 & $\mathrm{H} 12$ & MET \\
\hline ATOM & 256 & H13 & $\mathrm{MET}$ \\
\hline \multicolumn{4}{|l|}{ TER } \\
\hline ATOM & 257 & $\mathrm{C} 1$ & $\mathrm{MET}$ \\
\hline ATOM & 258 & H11 & $\mathrm{MET}$ \\
\hline ATOM & 259 & H12 & $\mathrm{MET}$ \\
\hline ATOM & 260 & H13 & $\mathrm{MET}$ \\
\hline \multicolumn{4}{|l|}{ TER } \\
\hline ATOM & 261 & $\mathrm{C} 2$ & VIN \\
\hline ATOM & 262 & H21 & VIN \\
\hline ATOM & 263 & $\mathrm{C} 1$ & VIN \\
\hline ATOM & 264 & H11 & VIN \\
\hline ATOM & 265 & H12 & VIN \\
\hline TER & & & \\
\hline ATOM & 266 & $\mathrm{C} 1$ & MET \\
\hline ATOM & 267 & $\mathrm{H} 11$ & $\mathrm{MET}$ \\
\hline ATOM & 268 & H12 & $\mathrm{MET}$ \\
\hline ATOM & 269 & H13 & $\mathrm{MET}$ \\
\hline TER & & & \\
\hline ATOM & 270 & $\mathrm{C} 2$ & VIN \\
\hline ATOM & 271 & H 21 & VIN \\
\hline
\end{tabular}

\begin{tabular}{|c|c|c|c|c|c|}
\hline 2.394 & 18.928 & 3.659 & 1.00 & 0.00 & C \\
\hline 3.722 & 18.873 & 3.237 & 1.00 & 0.00 & C \\
\hline 3.962 & 19.358 & 2.289 & 1.00 & 0.00 & $\mathrm{H}$ \\
\hline 4.752 & 18.192 & 3.880 & 1.00 & 0.00 & c \\
\hline 6.118 & 18.078 & 3.398 & 1.00 & 0.00 & $c$ \\
\hline 6.793 & 17.310 & 4.322 & 1.00 & 0.00 & c \\
\hline 5.844 & 16.959 & 5.358 & 1.00 & 0.00 & c \\
\hline 6.135 & 16.168 & 6.469 & 1.00 & 0.00 & c \\
\hline 7.149 & 15.778 & 6.530 & 1.00 & 0.00 & $\mathrm{H}$ \\
\hline 3.945 & 16.181 & 7.564 & 1.00 & 0.00 & $\mathrm{~N}$ \\
\hline 1.240 & 17.128 & 7.342 & 1.00 & 0.00 & $\mathrm{~N}$ \\
\hline 1.911 & 18.391 & 4.841 & 1.00 & 0.00 & $\mathrm{~N}$ \\
\hline 4.611 & 17.506 & 5.077 & 1.00 & 0.00 & $\mathrm{~N}$ \\
\hline 3.704 & 20.371 & 7.579 & 1.00 & 0.00 & c \\
\hline 3.544 & 19.058 & 7.567 & 1.00 & 0.00 & 0 \\
\hline 3.859 & 20.989 & 8.635 & 1.00 & 0.00 & 0 \\
\hline 3.688 & 21.053 & 6.223 & 1.00 & 0.00 & $c$ \\
\hline 2.709 & 20.858 & 5.760 & 1.00 & 0.00 & $\mathrm{H}$ \\
\hline 4.436 & 20.563 & 5.580 & 1.00 & 0.00 & $\mathrm{H}$ \\
\hline 3.947 & 22.557 & 6.334 & 1.00 & 0.00 & c \\
\hline 4.872 & 22.713 & 6.905 & 1.00 & 0.00 & $\mathrm{H}$ \\
\hline 3.151 & 23.018 & 6.931 & 1.00 & 0.00 & $\mathrm{H}$ \\
\hline-1.572 & 28.269 & -1.002 & 1.00 & 0.00 & c \\
\hline-2.388 & 27.571 & -0.837 & 1.00 & 0.00 & $\mathrm{H}$ \\
\hline-1.905 & 29.494 & -1.403 & 1.00 & 0.00 & c \\
\hline-1.174 & 30.273 & -1.511 & 1.00 & 0.00 & $\mathrm{H}$ \\
\hline-2.947 & 29.763 & -1.570 & 1.00 & 0.00 & $\mathrm{H}$ \\
\hline 1.091 & 28.524 & -2.840 & 1.00 & 0.00 & c \\
\hline 1.326 & 29.595 & -2.720 & 1.00 & 0.00 & $\mathrm{H}$ \\
\hline 1.904 & 28.060 & -3.410 & 1.00 & 0.00 & $\mathrm{H}$ \\
\hline 0.184 & 28.446 & -3.450 & 1.00 & 0.00 & $\mathrm{H}$ \\
\hline 6.102 & 26.846 & -2.325 & 1.00 & 0.00 & c \\
\hline 5.436 & 26.745 & -3.184 & 1.00 & 0.00 & $\mathrm{H}$ \\
\hline 7.268 & 27.490 & -2.484 & 1.00 & 0.00 & c \\
\hline 7.948 & 27.647 & -1.654 & 1.00 & 0.00 & $\mathrm{H}$ \\
\hline 7.573 & 27.888 & -3.450 & 1.00 & 0.00 & $\mathrm{H}$ \\
\hline 7.786 & 25.163 & -0.121 & 1.00 & 0.00 & c \\
\hline 8.377 & 25.827 & 0.528 & 1.00 & 0.00 & $\mathrm{H}$ \\
\hline 7.943 & 24.141 & 0.246 & 1.00 & 0.00 & $\mathrm{H}$ \\
\hline 8.218 & 25.228 & -1.126 & 1.00 & 0.00 & $\mathrm{H}$ \\
\hline-1.302 & 24.081 & 5.770 & 1.00 & 0.00 & c \\
\hline-0.563 & 24.070 & 6.582 & 1.00 & 0.00 & $\mathrm{H}$ \\
\hline-2.143 & 24.689 & 6.134 & 1.00 & 0.00 & $\mathrm{H}$ \\
\hline-1.781 & 22.644 & 5.497 & 1.00 & 0.00 & c \\
\hline-2.338 & 22.583 & 4.549 & 1.00 & 0.00 & $\mathrm{H}$ \\
\hline-0.910 & 21.977 & 5.390 & 1.00 & 0.00 & $\mathrm{H}$ \\
\hline-2.645 & 22.031 & 6.573 & 1.00 & 0.00 & c \\
\hline-2.873 & 22.554 & 7.671 & 1.00 & 0.00 & 0 \\
\hline-3.161 & 20.852 & 6.209 & 1.00 & 0.00 & 0 \\
\hline-3.770 & 20.498 & 6.945 & 1.00 & 0.00 & $\mathrm{H}$ \\
\hline 6.594 & 22.955 & 4.597 & 1.00 & 0.00 & c \\
\hline 7.146 & 23.794 & 5.047 & 1.00 & 0.00 & $\mathrm{H}$ \\
\hline 6.600 & 22.129 & 5.319 & 1.00 & 0.00 & $\mathrm{H}$ \\
\hline 7.165 & 22.635 & 3.717 & 1.00 & 0.00 & $\mathrm{H}$ \\
\hline-2.840 & 25.904 & 3.646 & 1.00 & 0.00 & c \\
\hline-3.285 & 25.695 & 4.626 & 1.00 & 0.00 & $\mathrm{H}$ \\
\hline-2.950 & 26.984 & 3.471 & 1.00 & 0.00 & $\mathrm{H}$ \\
\hline-3.440 & 25.378 & 2.889 & 1.00 & 0.00 & $\mathrm{H}$ \\
\hline 6.957 & 14.525 & 8.933 & 1.00 & 0.00 & c \\
\hline 7.773 & 15.223 & 8.768 & 1.00 & 0.00 & $\mathrm{H}$ \\
\hline 7.290 & 13.300 & 9.335 & 1.00 & 0.00 & c \\
\hline 6.559 & 12.521 & 9.442 & 1.00 & 0.00 & $\mathrm{H}$ \\
\hline 8.332 & 13.030 & 9.501 & 1.00 & 0.00 & $\mathrm{H}$ \\
\hline 4.293 & 14.270 & 10.771 & 1.00 & 0.00 & c \\
\hline 4.059 & 13.199 & 10.652 & 1.00 & 0.00 & $\mathrm{H}$ \\
\hline 3.480 & 14.734 & 11.341 & 1.00 & 0.00 & $\mathrm{H}$ \\
\hline 5.201 & 14.347 & 11.382 & 1.00 & 0.00 & $\mathrm{H}$ \\
\hline-0.717 & 15.948 & 10.256 & 1.00 & 0.00 & c \\
\hline-0.051 & 16.048 & 11.115 & 1.00 & 0.00 & $\mathrm{H}$ \\
\hline
\end{tabular}




\begin{tabular}{|c|c|c|c|c|c|c|c|c|c|c|}
\hline ATOM & 272 & $\mathrm{C} 1$ & VIN & 28 & -1.883 & 15.303 & 10.415 & 1.00 & 0.00 & C \\
\hline ATOM & 273 & $\mathrm{H} 11$ & VIN & 28 & -2.563 & 15.147 & 9.585 & 1.00 & 0.00 & $\mathrm{H}$ \\
\hline ATOM & 274 & $\mathrm{H} 12$ & VIN & 28 & -2.188 & 14.905 & 11.381 & 1.00 & 0.00 & H \\
\hline \multicolumn{10}{|l|}{ TER } & \\
\hline ATOM & 275 & $\mathrm{C} 1$ & $\mathrm{MET}$ & 29 & -2.401 & 17.631 & 8.052 & 1.00 & 0.00 & C \\
\hline ATOM & 276 & $\mathrm{H} 11$ & $\mathrm{MET}$ & 29 & -2.558 & 18.653 & 7.685 & 1.00 & 0.00 & H \\
\hline ATOM & 277 & $\mathrm{H} 12$ & $\mathrm{MET}$ & 29 & -2.834 & 17.565 & 9.057 & 1.00 & 0.00 & $\mathrm{H}$ \\
\hline ATOM & 278 & $\mathrm{H} 13$ & $\mathrm{MET}$ & 29 & -2.993 & 16.967 & 7.403 & 1.00 & 0.00 & $\mathrm{H}$ \\
\hline \multicolumn{10}{|l|}{ TER } & \\
\hline ATOM & 279 & C5 & $\mathrm{ACP}$ & 30 & 6.687 & 18.712 & 2.161 & 1.00 & 0.00 & C \\
\hline ATOM & 280 & H51 & $\mathrm{ACP}$ & 30 & 5.948 & 18.724 & 1.349 & 1.00 & 0.00 & 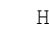 \\
\hline ATOM & 281 & H52 & $\mathrm{ACP}$ & 30 & 7.528 & 18.104 & 1.798 & 1.00 & 0.00 & \\
\hline ATOM & 282 & $\mathrm{C} 4$ & $\mathrm{ACP}$ & 30 & 7.166 & 20.150 & 2.434 & 1.00 & 0.00 & 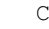 \\
\hline ATOM & 283 & $\mathrm{H} 41$ & $\mathrm{ACP}$ & 30 & 7.723 & 20.211 & 3.382 & 1.00 & 0.00 & 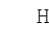 \\
\hline ATOM & 284 & $\mathrm{H} 42$ & $\mathrm{ACP}$ & 30 & 6.295 & 20.817 & 2.541 & 1.00 & 0.00 & \\
\hline ATOM & 285 & $\mathrm{C} 1$ & $\mathrm{ACP}$ & 30 & 8.030 & 20.762 & 1.358 & 1.00 & 0.00 & 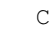 \\
\hline ATOM & 286 & $\mathrm{O} 2$ & $\mathrm{ACP}$ & 30 & 8.258 & 20.239 & 0.260 & 1.00 & 0.00 & 0 \\
\hline ATOM & 287 & 03 & $\mathrm{ACP}$ & 30 & 8.546 & 21.942 & 1.722 & 1.00 & 0.00 & O \\
\hline ATOM & 288 & $\mathrm{H} 3$ & $\mathrm{ACP}$ & 30 & 9.155 & 22.296 & 0.986 & 1.00 & 0.00 & $\mathrm{H}$ \\
\hline \multicolumn{11}{|l|}{ TER } \\
\hline ATOM & 289 & C1 & $\mathrm{MET}$ & 31 & -1.209 & 19.839 & 3.334 & 1.00 & 0.00 & C \\
\hline ATOM & 290 & $\mathrm{H} 11$ & MET & 31 & -1.761 & 18.999 & 2.884 & 1.00 & 0.00 & $\mathrm{H}$ \\
\hline ATOM & 291 & $\mathrm{H} 12$ & MET & 31 & -1.215 & 20.665 & 2.613 & 1.00 & 0.00 & $\mathrm{H}$ \\
\hline ATOM & 292 & $\mathrm{H} 13$ & $\mathrm{MET}$ & 31 & -1.780 & 20.158 & 4.214 & 1.00 & 0.00 & H \\
\hline \multicolumn{11}{|l|}{ TER } \\
\hline ATOM & 293 & $\mathrm{C} 1$ & $\mathrm{MET}$ & 32 & 8.225 & 16.890 & 4.285 & 1.00 & 0.00 & C \\
\hline ATOM & 294 & H11 & $\mathrm{MET}$ & 32 & 8.670 & 17.099 & 3.305 & 1.00 & 0.00 & $\mathrm{H}$ \\
\hline ATOM & 295 & $\mathrm{H} 12$ & $\mathrm{MET}$ & 32 & 8.335 & 15.810 & 4.460 & 1.00 & 0.00 & $\mathrm{H}$ \\
\hline ATOM & 296 & $\mathrm{H} 13$ & $\mathrm{MET}$ & 32 & 8.825 & 17.416 & 5.042 & 1.00 & 0.00 & H \\
\hline TER & & & & & & & & & & \\
\hline ATOM & 297 & $\mathrm{FE}$ & HEM & 33 & 3.316 & 10.511 & 9.423 & 1.00 & 0.00 & $\mathrm{FE}$ \\
\hline ATOM & 298 & $\mathrm{C} 1$ & HEM & 33 & 1.088 & 12.383 & 8.361 & 1.00 & 0.00 & C \\
\hline ATOM & 299 & $\mathrm{C} 2$ & HEM & 33 & 0.729 & 13.174 & 7.189 & 1.00 & 0.00 & C \\
\hline ATOM & 300 & $\mathrm{C} 3$ & HEM & 33 & 1.874 & 13.271 & 6.414 & 1.00 & 0.00 & C \\
\hline ATOM & 301 & $\mathrm{C} 4$ & HEM & 33 & 2.904 & 12.553 & 7.122 & 1.00 & 0.00 & C \\
\hline ATOM & 302 & $\mathrm{C} 5$ & HEM & 33 & 4.219 & 12.435 & 6.684 & 1.00 & 0.00 & C \\
\hline ATOM & 303 & H5 & HEM & 33 & 4.472 & 12.931 & 5.749 & 1.00 & 0.00 & $\mathrm{H}$ \\
\hline ATOM & 304 & $\mathrm{C} 6$ & HEM & 33 & 5.238 & 11.736 & 7.320 & 1.00 & 0.00 & C \\
\hline ATOM & 305 & C7 & HEM & 33 & 6.607 & 11.664 & 6.851 & 1.00 & 0.00 & C \\
\hline ATOM & 306 & C8 & HEM & 33 & 7.313 & 10.944 & 7.798 & 1.00 & 0.00 & C \\
\hline ATOM & 307 & C9 & HEM & 33 & 6.362 & 10.579 & 8.822 & 1.00 & 0.00 & C \\
\hline ATOM & 308 & $\mathrm{C} 10$ & HEM & 33 & 6.666 & 9.844 & 9.965 & 1.00 & 0.00 & C \\
\hline ATOM & 309 & $\mathrm{H} 10$ & HEM & 33 & 7.693 & 9.500 & 10.071 & 1.00 & 0.00 & $\mathrm{H}$ \\
\hline ATOM & 310 & $\mathrm{C} 11$ & HEM & 33 & 5.792 & 9.504 & 10.988 & 1.00 & 0.00 & C \\
\hline ATOM & 311 & $\mathrm{C} 12$ & HEM & 33 & 6.173 & 8.779 & 12.178 & 1.00 & 0.00 & C \\
\hline ATOM & 312 & $\mathrm{C} 13$ & HEM & 33 & 5.033 & 8.631 & 12.935 & 1.00 & 0.00 & C \\
\hline ATOM & 313 & $\mathrm{C} 14$ & HEM & 33 & 3.960 & 9.273 & 12.204 & 1.00 & 0.00 & C \\
\hline ATOM & 314 & C15 & HEM & 33 & 2.632 & 9.328 & 12.625 & 1.00 & 0.00 & C \\
\hline ATOM & 315 & H15 & HEM & 33 & 2.392 & 8.843 & 13.573 & 1.00 & 0.00 & $\mathrm{H}$ \\
\hline ATOM & 316 & $\mathrm{C} 16$ & HEM & 33 & 1.602 & 10.009 & 11.982 & 1.00 & 0.00 & C \\
\hline ATOM & 317 & $\mathrm{C} 17$ & HEM & 33 & 0.236 & 10.123 & 12.464 & 1.00 & 0.00 & C \\
\hline ATOM & 318 & $\mathrm{C} 18$ & HEM & 33 & -0.439 & 10.890 & 11.541 & 1.00 & 0.00 & C \\
\hline ATOM & 319 & C19 & HEM & 33 & 0.510 & 11.242 & 10.504 & 1.00 & 0.00 & C \\
\hline ATOM & 320 & $\mathrm{C} 20$ & HEM & 33 & 0.219 & 12.033 & 9.393 & 1.00 & 0.00 & C \\
\hline ATOM & 321 & H 20 & HEM & 33 & -0.795 & 12.423 & 9.333 & 1.00 & 0.00 & $\mathrm{H}$ \\
\hline ATOM & 322 & $\mathrm{~N} 21$ & HEM & 33 & 2.409 & 12.020 & 8.298 & 1.00 & 0.00 & $\mathrm{~N}$ \\
\hline ATOM & 323 & $\mathrm{~N} 22$ & HEM & 33 & 5.114 & 11.073 & 8.521 & 1.00 & 0.00 & $\mathrm{~N}$ \\
\hline ATOM & 324 & N23 & HEM & 33 & 4.443 & 9.810 & 11.021 & 1.00 & 0.00 & $\mathrm{~N}$ \\
\hline ATOM & 325 & $\mathrm{~N} 24$ & HEM & 33 & 1.743 & 10.694 & 10.786 & 1.00 & 0.00 & $\mathrm{~N}$ \\
\hline ATOM & 326 & C99 & HEM & 33 & 2.650 & 7.829 & 8.283 & 1.00 & 0.00 & C \\
\hline ATOM & 327 & 091 & HEM & 33 & 2.810 & 9.143 & 8.296 & 1.00 & 0.00 & 0 \\
\hline ATOM & 328 & 092 & HEM & 33 & 2.495 & 7.212 & 7.228 & 1.00 & 0.00 & O \\
\hline ATOM & 329 & СТ 91 & HEM & 33 & 2.666 & 7.148 & 9.640 & 1.00 & 0.00 & C \\
\hline ATOM & 330 & H911 & HEM & 33 & 3.645 & 7.343 & 10.103 & 1.00 & 0.00 & $\mathrm{H}$ \\
\hline ATOM & 331 & H912 & HEM & 33 & 1.918 & 7.638 & 10.283 & 1.00 & 0.00 & $\mathrm{H}$ \\
\hline ATOM & 332 & СТ92 & HEM & 33 & 2.407 & 5.644 & 9.528 & 1.00 & 0.00 & C \\
\hline ATOM & 333 & H921 & HEM & 33 & 3.203 & 5.183 & 8.931 & 1.00 & 0.00 & $\mathrm{H}$ \\
\hline ATOM & 334 & H922 & HEM & 33 & 1.482 & 5.488 & 8.958 & 1.00 & 0.00 & $\mathrm{H}$ \\
\hline TER & & & & & & & & & & \\
\hline ATOM & 335 & $\mathrm{FE}$ & HEM & 34 & 4.008 & 3.097 & 14.371 & 1.00 & 0.00 & $\mathrm{FE}$ \\
\hline ATOM & 336 & $\mathrm{C} 1$ & HEM & 34 & 6.236 & 1.225 & 15.433 & 1.00 & 0.00 & \\
\hline AтOM & 337 & $\mathrm{C} 2$ & HEM & 34 & 6.595 & 0.434 & 16.605 & 1.00 & 0.00 & \\
\hline ATOM & 338 & $\mathrm{C} 3$ & HEM & 34 & 5.449 & 0.337 & 17.380 & 1.00 & 0.00 & \\
\hline ATOM & 339 & $\mathrm{C} 4$ & HEM & 34 & 4.419 & 1.055 & 16.672 & 1.00 & 0.00 & \\
\hline ATOM & 340 & $\mathrm{C} 5$ & HEM & 34 & 3.104 & 1.173 & 17.109 & 1.00 & 0.00 & 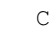 \\
\hline ATOM & 341 & H5 & HEM & 34 & 2.851 & 0.677 & 18.045 & 1.00 & 0.00 & 1 \\
\hline ATOM & 342 & $\mathrm{C} 6$ & HEM & 34 & 2.085 & 1.872 & 16.473 & 1.00 & 0.00 & 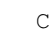 \\
\hline ATOM & 343 & C7 & HEM & 34 & 0.716 & 1.944 & 16.943 & 1.00 & 0.00 & C \\
\hline ATOM & 344 & $\mathrm{C} 8$ & HEM & 34 & 0.011 & 2.664 & 15.996 & 1.00 & 0.00 & \\
\hline
\end{tabular}




\begin{tabular}{|c|c|c|c|}
\hline ATOM & 345 & C9 & HEM \\
\hline ATOM & 346 & C10 & HEM \\
\hline ATOM & 347 & H10 & HEM \\
\hline ATOM & 348 & C11 & HEM \\
\hline ATOM & 349 & C12 & HEM \\
\hline ATOM & 350 & C13 & HEM \\
\hline ATOM & 351 & C14 & HEM \\
\hline ATOM & 352 & C15 & HEM \\
\hline ATOM & 353 & H15 & HEM \\
\hline ATOM & 354 & C16 & HEM \\
\hline ATOM & 355 & $\mathrm{C} 17$ & HEM \\
\hline ATOM & 356 & C18 & HEM \\
\hline ATOM & 357 & C19 & HEM \\
\hline ATOM & 358 & C20 & HEM \\
\hline ATOM & 359 & $\mathrm{H} 20$ & HEM \\
\hline ATOM & 360 & $\mathrm{~N} 21$ & HEM \\
\hline ATOM & 361 & N22 & HEM \\
\hline ATOM & 362 & N23 & HEM \\
\hline ATOM & 363 & N24 & HEM \\
\hline ATOM & 364 & C99 & HEM \\
\hline ATOM & 365 & 091 & HEM \\
\hline ATOM & 366 & 092 & HEM \\
\hline ATOM & 367 & СТ91 & HEM \\
\hline ATOM & 368 & H911 & HEM \\
\hline ATOM & 369 & H912 & HEM \\
\hline ATOM & 370 & СТ 92 & HEM \\
\hline ATOM & 371 & H921 & HEM \\
\hline ATOM & 372 & H922 & HEM \\
\hline \multicolumn{4}{|l|}{ TER } \\
\hline ATOM & 373 & $\mathrm{C} 2$ & VIN \\
\hline ATOM & 374 & $\mathrm{H} 21$ & VIN \\
\hline ATOM & 375 & $\mathrm{C} 1$ & VIN \\
\hline ATOM & 376 & H11 & VIN \\
\hline ATOM & 377 & $\mathrm{H} 12$ & VIN \\
\hline \multicolumn{4}{|l|}{ TER } \\
\hline ATOM & 378 & $\mathrm{C} 1$ & MET \\
\hline ATOM & 379 & $\mathrm{H} 11$ & MET \\
\hline ATOM & 380 & $\mathrm{H} 12$ & MET \\
\hline ATOM & 381 & $\mathrm{H} 13$ & MET \\
\hline \multicolumn{4}{|l|}{ TER } \\
\hline ATOM & 382 & C2 & VIN \\
\hline ATOM & 383 & H 21 & VIN \\
\hline ATOM & 384 & $\mathrm{C} 1$ & VIN \\
\hline ATOM & 385 & H11 & VIN \\
\hline ATOM & 386 & $\mathrm{H} 12$ & VIN \\
\hline \multicolumn{4}{|l|}{ TER } \\
\hline ATOM & 387 & $\mathrm{C} 1$ & MET \\
\hline ATOM & 388 & $\mathrm{H} 11$ & MET \\
\hline ATOM & 389 & H12 & $\mathrm{MET}$ \\
\hline ATOM & 390 & H13 & MET \\
\hline \multicolumn{4}{|l|}{ TER } \\
\hline ATOM & 391 & C5 & $\mathrm{ACP}$ \\
\hline ATOM & 392 & H51 & $\mathrm{ACP}$ \\
\hline ATOM & 393 & H52 & $\mathrm{ACP}$ \\
\hline ATOM & 394 & $\mathrm{C} 4$ & $\mathrm{ACP}$ \\
\hline ATOM & 395 & H 41 & $\mathrm{ACP}$ \\
\hline ATOM & 396 & $\mathrm{H} 42$ & $\mathrm{ACP}$ \\
\hline ATOM & 397 & $\mathrm{C} 1$ & $\mathrm{ACP}$ \\
\hline ATOM & 398 & 02 & $\mathrm{ACP}$ \\
\hline ATOM & 399 & 03 & $\mathrm{ACP}$ \\
\hline ATOM & 400 & $\mathrm{H} 3$ & $\mathrm{ACP}$ \\
\hline \multicolumn{4}{|l|}{ TER } \\
\hline ATOM & 401 & $\mathrm{C} 1$ & $\mathrm{MET}$ \\
\hline ATOM & 402 & $\mathrm{H} 11$ & MET \\
\hline ATOM & 403 & $\mathrm{H} 12$ & $\mathrm{MET}$ \\
\hline ATOM & 404 & $\mathrm{H} 13$ & MET \\
\hline \multicolumn{4}{|l|}{ TER } \\
\hline ATOM & 405 & $\mathrm{C} 1$ & $\mathrm{MET}$ \\
\hline ATOM & 406 & H11 & MET \\
\hline ATOM & 407 & $\mathrm{H} 12$ & MET \\
\hline ATOM & 408 & $\mathrm{H} 13$ & MET \\
\hline \multicolumn{4}{|l|}{ TER } \\
\hline ATOM & 409 & C2 & VIN \\
\hline ATOM & 410 & H 21 & VIN \\
\hline ATOM & 411 & $\mathrm{C} 1$ & VIN \\
\hline ATOM & 412 & H11 & VIN \\
\hline ATOM & 413 & $\mathrm{H} 12$ & VIN \\
\hline TER & & & \\
\hline ATOM & 414 & $\mathrm{C} 1$ & MET \\
\hline
\end{tabular}

\begin{tabular}{|c|c|c|c|c|c|}
\hline 0.962 & 3.029 & 14.972 & 1.00 & 0.00 & C \\
\hline 0.657 & 3.764 & 13.828 & 1.00 & 0.00 & C \\
\hline-0.370 & 4.108 & 13.722 & 1.00 & 0.00 & $\mathrm{H}$ \\
\hline 1.532 & 4.104 & 12.806 & 1.00 & 0.00 & c \\
\hline 1.151 & 4.829 & 11.615 & 1.00 & 0.00 & c \\
\hline 2.290 & 4.977 & 10.858 & 1.00 & 0.00 & 0 \\
\hline 3.363 & 4.335 & 11.590 & 1.00 & 0.00 & 4 \\
\hline 4.691 & 4.280 & 11.168 & 1.00 & 0.00 & \\
\hline 4.931 & 4.765 & 10.221 & 1.00 & 0.00 & $\mathrm{H}$ \\
\hline 5.721 & 3.599 & 11.812 & 1.00 & 0.00 & c \\
\hline 7.087 & 3.485 & 11.329 & 1.00 & 0.00 & c \\
\hline 7.763 & 2.718 & 12.253 & 1.00 & 0.00 & c \\
\hline 6.813 & 2.366 & 13.289 & 1.00 & 0.00 & 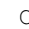 \\
\hline 7.104 & 1.575 & 14.401 & 1.00 & 0.00 & 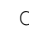 \\
\hline 8.118 & 1.185 & 14.461 & 1.00 & 0.00 & . \\
\hline 4.914 & 1.588 & 15.495 & 1.00 & 0.00 & , \\
\hline 2.209 & 2.535 & 15.273 & 1.00 & 0.00 & $\mathrm{~N}$ \\
\hline 2.880 & 3.798 & 12.772 & 1.00 & 0.00 & $N$ \\
\hline 5.580 & 2.914 & 13.008 & 1.00 & 0.00 & $N$ \\
\hline 4.673 & 5.779 & 15.510 & 1.00 & 0.00 & c \\
\hline 4.514 & 4.465 & 15.498 & 1.00 & 0.00 & 0 \\
\hline 4.828 & 6.396 & 16.566 & 1.00 & 0.00 & o \\
\hline 4.657 & 6.460 & 14.154 & 1.00 & 0.00 & c \\
\hline 3.678 & 6.265 & 13.691 & 1.00 & 0.00 & $\mathrm{H}$ \\
\hline 5.405 & 5.970 & 13.511 & 1.00 & 0.00 & $\mathrm{H}$ \\
\hline 4.917 & 7.964 & 14.265 & 1.00 & 0.00 & c \\
\hline 5.842 & 8.120 & 14.836 & 1.00 & 0.00 & $\mathrm{H}$ \\
\hline 4.120 & 8.425 & 14.862 & 1.00 & 0.00 & $\mathrm{H}$ \\
\hline-0.603 & 13.676 & 6.929 & 1.00 & 0.00 & c \\
\hline-1.419 & 12.978 & 7.094 & 1.00 & 0.00 & $\mathrm{H}$ \\
\hline-0.936 & 14.901 & 6.528 & 1.00 & 0.00 & c \\
\hline-0.205 & 15.680 & 6.421 & 1.00 & 0.00 & $\mathrm{H}$ \\
\hline-1.978 & 15.170 & 6.361 & 1.00 & 0.00 & $\mathrm{H}$ \\
\hline 2.061 & 13.931 & 5.091 & 1.00 & 0.00 & c \\
\hline 2.295 & 15.002 & 5.211 & 1.00 & 0.00 & $\mathrm{H}$ \\
\hline 2.874 & 13.467 & 4.522 & 1.00 & 0.00 & $\mathrm{H}$ \\
\hline 1.153 & 13.854 & 4.481 & 1.00 & 0.00 & $\mathrm{H}$ \\
\hline 7.071 & 12.253 & 5.606 & 1.00 & 0.00 & c \\
\hline 6.405 & 12.152 & 4.747 & 1.00 & 0.00 & $\mathrm{H}$ \\
\hline 8.238 & 12.898 & 5.447 & 1.00 & 0.00 & c \\
\hline 8.917 & 13.054 & 6.277 & 1.00 & 0.00 & $\mathrm{H}$ \\
\hline 8.543 & 13.296 & 4.482 & 1.00 & 0.00 & $\mathrm{H}$ \\
\hline 8.755 & 10.570 & 7.810 & 1.00 & 0.00 & c \\
\hline 9.347 & 11.234 & 8.459 & 1.00 & 0.00 & $\mathrm{H}$ \\
\hline 8.912 & 9.548 & 8.177 & 1.00 & 0.00 & $\mathrm{H}$ \\
\hline 9.188 & 10.636 & 6.805 & 1.00 & 0.00 & $\mathrm{H}$ \\
\hline-0.333 & 9.488 & 13.701 & 1.00 & 0.00 & c \\
\hline 0.406 & 9.477 & 14.514 & 1.00 & 0.00 & $\mathrm{H}$ \\
\hline-1.174 & 10.097 & 14.065 & 1.00 & 0.00 & $\mathrm{H}$ \\
\hline-0.812 & 8.051 & 13.428 & 1.00 & 0.00 & c \\
\hline-1.369 & 7.990 & 12.481 & 1.00 & 0.00 & $\mathrm{H}$ \\
\hline 0.059 & 7.384 & 13.321 & 1.00 & 0.00 & $\mathrm{H}$ \\
\hline-1.676 & 7.439 & 14.504 & 1.00 & 0.00 & c \\
\hline-1.903 & 7.961 & 15.603 & 1.00 & 0.00 & 0 \\
\hline-2.191 & 6.259 & 14.140 & 1.00 & 0.00 & 0 \\
\hline-2.800 & 5.905 & 14.876 & 1.00 & 0.00 & $\mathrm{H}$ \\
\hline 7.563 & 8.362 & 12.528 & 1.00 & 0.00 & c \\
\hline 8.115 & 9.201 & 12.978 & 1.00 & 0.00 & $\mathrm{H}$ \\
\hline 7.570 & 7.536 & 13.250 & 1.00 & 0.00 & $\mathrm{H}$ \\
\hline 8.134 & 8.042 & 11.649 & 1.00 & 0.00 & $\mathrm{H}$ \\
\hline-1.870 & 11.311 & 11.577 & 1.00 & 0.00 & $c$ \\
\hline-2.316 & 11.102 & 12.557 & 1.00 & 0.00 & $\mathrm{H}$ \\
\hline-1.981 & 12.391 & 11.402 & 1.00 & 0.00 & $\mathrm{H}$ \\
\hline-2.471 & 10.785 & 10.821 & 1.00 & 0.00 & $\mathrm{H}$ \\
\hline 7.926 & -0.068 & 16.864 & 1.00 & 0.00 & c \\
\hline 8.742 & 0.630 & 16.699 & 1.00 & 0.00 & $\mathrm{H}$ \\
\hline 8.259 & -1.293 & 17.266 & 1.00 & 0.00 & c \\
\hline 7.528 & -2.072 & 17.373 & 1.00 & 0.00 & $\mathrm{H}$ \\
\hline 9.301 & -1.562 & 17.432 & 1.00 & 0.00 & $\mathrm{H}$ \\
\hline 5.263 & -0.323 & 18.702 & 1.00 & 0.00 & c \\
\hline
\end{tabular}




\begin{tabular}{|c|c|c|c|c|c|c|c|c|c|c|}
\hline ATOM & 415 & $\mathrm{H} 11$ & MET & 43 & 5.028 & -1.394 & 18.583 & 1.00 & 0.00 & $\mathrm{H}$ \\
\hline ATOM & 416 & $\mathrm{H} 12$ & MET & 43 & 4.450 & 0.141 & 19.272 & 1.00 & 0.00 & $\mathrm{H}$ \\
\hline ATOM & 417 & $\mathrm{H} 13$ & MET & 43 & 6.170 & -0.246 & 19.313 & 1.00 & 0.00 & $\mathrm{H}$ \\
\hline \multicolumn{10}{|l|}{ TER } & \\
\hline ATOM & 418 & $\mathrm{C} 2$ & VIN & 44 & 0.252 & 1.355 & 18.188 & 1.00 & 0.00 & $\mathrm{C}$ \\
\hline ATOM & 419 & $\mathrm{H} 21$ & VIN & 44 & 0.918 & 1.456 & 19.046 & 1.00 & 0.00 & $\mathrm{H}$ \\
\hline ATOM & 420 & $\mathrm{C} 1$ & VIN & 44 & -0.914 & 0.710 & 18.346 & 1.00 & 0.00 & C \\
\hline ATOM & 421 & $\mathrm{H} 11$ & VIN & 44 & -1.594 & 0.554 & 17.516 & 1.00 & 0.00 & $\mathrm{H}$ \\
\hline ATOM & 422 & $\mathrm{H} 12$ & VIN & 44 & -1.219 & 0.312 & 19.312 & 1.00 & 0.00 & $\mathrm{H}$ \\
\hline \multicolumn{10}{|l|}{ TER } & \\
\hline ATOM & 423 & $\mathrm{C} 1$ & MET & 45 & -1.432 & 3.038 & 15.984 & 1.00 & 0.00 & C \\
\hline ATOM & 424 & $\mathrm{H} 11$ & MET & 45 & -1.588 & 4.060 & 15.617 & 1.00 & 0.00 & $\mathrm{H}$ \\
\hline ATOM & 425 & $\mathrm{H} 12$ & MET & 45 & -1.864 & 2.972 & 16.989 & 1.00 & 0.00 & $\mathrm{H}$ \\
\hline ATOM & 426 & $\mathrm{H} 13$ & MET & 45 & -2.023 & 2.374 & 15.335 & 1.00 & 0.00 & $\mathrm{H}$ \\
\hline \multicolumn{10}{|l|}{ TER } & \\
\hline ATOM & 427 & C5 & $\mathrm{ACP}$ & 46 & 7.656 & 4.120 & 10.092 & 1.00 & 0.00 & C \\
\hline ATOM & 428 & H51 & $\mathrm{ACP}$ & 46 & 6.917 & 4.131 & 9.280 & 1.00 & 0.00 & $\mathrm{H}$ \\
\hline ATOM & 429 & H52 & $\mathrm{ACP}$ & 46 & 8.497 & 3.511 & 9.729 & 1.00 & 0.00 & $\mathrm{H}$ \\
\hline ATOM & 430 & C4 & $\mathrm{ACP}$ & 46 & 8.135 & 5.557 & 10.365 & 1.00 & 0.00 & C \\
\hline ATOM & 431 & $\mathrm{H} 41$ & $\mathrm{ACP}$ & 46 & 8.693 & 5.618 & 11.313 & 1.00 & 0.00 & $\mathrm{H}$ \\
\hline ATOM & 432 & $\mathrm{H} 42$ & $\mathrm{ACP}$ & 46 & 7.264 & 6.224 & 10.472 & 1.00 & 0.00 & $\mathrm{H}$ \\
\hline ATOM & 433 & $\mathrm{C} 1$ & $\mathrm{ACP}$ & 46 & 8.999 & 6.169 & 9.289 & 1.00 & 0.00 & C \\
\hline ATOM & 434 & $\mathrm{O} 2$ & $\mathrm{ACP}$ & 46 & 9.227 & 5.647 & 8.191 & 1.00 & 0.00 & 0 \\
\hline ATOM & 435 & 03 & $\mathrm{ACP}$ & 46 & 9.515 & 7.349 & 9.653 & 1.00 & 0.00 & O \\
\hline ATOM & 436 & $\mathrm{H} 3$ & $\mathrm{ACP}$ & 46 & 10.124 & 7.703 & 8.917 & 1.00 & 0.00 & $\mathrm{H}$ \\
\hline \multicolumn{10}{|l|}{ TER } & \\
\hline ATOM & 437 & $\mathrm{C} 1$ & MET & 47 & -0.240 & 5.246 & 11.266 & 1.00 & 0.00 & C \\
\hline ATOM & 438 & $\mathrm{H} 11$ & MET & 47 & -0.792 & 4.407 & 10.815 & 1.00 & 0.00 & $\mathrm{H}$ \\
\hline ATOM & 439 & $\mathrm{H} 12$ & MET & 47 & -0.246 & 6.072 & 10.544 & 1.00 & 0.00 & $\mathrm{H}$ \\
\hline ATOM & 440 & $\mathrm{H} 13$ & MET & 47 & -0.811 & 5.565 & 12.145 & 1.00 & 0.00 & $\mathrm{H}$ \\
\hline \multicolumn{10}{|l|}{ TER } & \\
\hline ATOM & 441 & $\mathrm{C} 1$ & MET & 48 & 9.194 & 2.297 & 12.216 & 1.00 & 0.00 & c \\
\hline ATOM & 442 & $\mathrm{H} 11$ & MET & 48 & 9.639 & 2.506 & 11.236 & 1.00 & 0.00 & $\mathrm{H}$ \\
\hline ATOM & 443 & $\mathrm{H} 12$ & MET & 48 & 9.304 & 1.217 & 12.391 & 1.00 & 0.00 & $\mathrm{H}$ \\
\hline ATOM & 444 & $\mathrm{H} 13$ & MET & 48 & 9.794 & 2.823 & 12.973 & 1.00 & 0.00 & $\mathrm{H}$ \\
\hline \multicolumn{10}{|l|}{ TER } & \\
\hline . & & & & & & & & & & \\
\hline
\end{tabular}

\subsection{T3 dimer: A03_T3.pdb file}

$\begin{array}{lrrrl}\text { ATOM } & 1 & \text { FE } & \text { HEM } & 1 \\ \text { ATOM } & 2 & \text { C1 } & \text { HEM } & 1 \\ \text { ATOM } & 3 & \text { C2 } & \text { HEM } & 1 \\ \text { ATOM } & 4 & \text { C3 } & \text { HEM } & 1 \\ \text { ATOM } & 5 & \text { C4 } & \text { HEM } & 1 \\ \text { ATOM } & 6 & \text { C5 } & \text { HEM } & 1 \\ \text { ATOM } & 7 & \text { H5 } & \text { HEM } & 1 \\ \text { ATOM } & 8 & \text { C6 } & \text { HEM } & 1 \\ \text { ATOM } & 9 & \text { C7 } & \text { HEM } & 1 \\ \text { ATOM } & 10 & \text { C8 } & \text { HEM } & 1 \\ \text { ATOM } & 11 & \text { C9 } & \text { HEM } & 1 \\ \text { ATOM } & 12 & \text { C10 } & \text { HEM } & 1 \\ \text { ATOM } & 13 & \text { H10 } & \text { HEM } & 1 \\ \text { ATOM } & 14 & \text { C11 } & \text { HEM } & 1 \\ \text { ATOM } & 15 & \text { C12 } & \text { HEM } & 1 \\ \text { ATOM } & 16 & \text { C13 } & \text { HEM } & 1 \\ \text { ATOM } & 17 & \text { C14 } & \text { HEM } & 1 \\ \text { ATOM } & 18 & \text { C15 } & \text { HEM } & 1 \\ \text { ATOM } & 19 & \text { H15 } & \text { HEM } & 1 \\ \text { ATOM } & 20 & \text { C16 } & \text { HEM } & 1 \\ \text { ATOM } & 21 & \text { C17 } & \text { HEM } & 1 \\ \text { ATOM } & 22 & \text { C18 } & \text { HEM } & 1 \\ \text { ATOM } & 23 & \text { C19 } & \text { HEM } & 1 \\ \text { ATOM } & 24 & \text { C20 } & \text { HEM } & 1 \\ \text { ATOM } & 25 & \text { H20 } & \text { HEM } & 1 \\ \text { ATOM } & 26 & \text { N21 } & \text { HEM } & 1 \\ \text { ATOM } & 27 & \text { N22 } & \text { HEM } & 1 \\ \text { ATOM } & 28 & \text { N23 } & \text { HEM } & 1 \\ \text { ATOM } & 29 & \text { N24 } & \text { HEM } & 1 \\ \text { ATOM } & 30 & \text { C99 } & \text { HEM } & 1 \\ \text { ATOM } & 31 & \text { O91 } & \text { HEM } & 1 \\ \text { ATOM } & 32 & \text { O92 } & \text { HEM } & 1 \\ \text { ATOM } & 33 & \text { CT91 } & \text { HEM } & 1 \\ \text { ATOM } & 34 & \text { H911 } & \text { HEM } & 1 \\ \text { ATOM } & 35 & \text { H912 } & \text { HEM } & 1 \\ \text { ATOM } & 36 & \text { CT92 } & \text { HEM } & 1 \\ \text { ATOM } & 37 & \text { H921 } & \text { HEM } & 1 \\ \text { ATOM } & 38 & \text { H922 } & \text { HEM } & 1 \\ & & & & \end{array}$

$\begin{array}{rrrrr}4.263 & 10.757 & 1.491 & 1.00 & 0.00 \\ 2.035 & 12.629 & 0.429 & 1.00 & 0.00 \\ 1.676 & 13.420 & -0.742 & 1.00 & 0.00 \\ 2.822 & 13.517 & -1.517 & 1.00 & 0.00 \\ 3.851 & 12.800 & -0.809 & 1.00 & 0.00 \\ 5.167 & 12.681 & -1.247 & 1.00 & 0.00 \\ 5.420 & 13.177 & -2.182 & 1.00 & 0.00 \\ 6.186 & 11.982 & -0.611 & 1.00 & 0.00 \\ 7.555 & 11.910 & -1.081 & 1.00 & 0.00 \\ 8.260 & 11.190 & -0.134 & 1.00 & 0.00 \\ 7.309 & 10.825 & 0.891 & 1.00 & 0.00 \\ 7.614 & 10.090 & 2.034 & 1.00 & 0.00 \\ 8.641 & 9.746 & 2.140 & 1.00 & 0.00 \\ 6.739 & 9.750 & 3.057 & 1.00 & 0.00 \\ 7.120 & 9.025 & 4.247 & 1.00 & 0.00 \\ 5.981 & 8.878 & 5.004 & 1.00 & 0.00 \\ 4.908 & 9.519 & 4.273 & 1.00 & 0.00 \\ 3.580 & 9.574 & 4.694 & 1.00 & 0.00 \\ 3.340 & 9.089 & 5.642 & 1.00 & 0.00 \\ 2.550 & 10.255 & 4.051 & 1.00 & 0.00 \\ 1.184 & 10.369 & 4.533 & 1.00 & 0.00 \\ 0.508 & 11.137 & 3.609 & 1.00 & 0.00 \\ 1.458 & 11.489 & 2.573 & 1.00 & 0.00 \\ 1.167 & 12.280 & 1.462 & 1.00 & 0.00 \\ 0.152 & 12.669 & 1.402 & 1.00 & 0.00 \\ 3.357 & 12.266 & 0.367 & 1.00 & 0.00 \\ 6.062 & 11.319 & 0.590 & 1.00 & 0.00 \\ 5.391 & 10.056 & 3.090 & 1.00 & 0.00 \\ 2.691 & 10.941 & 2.854 & 1.00 & 0.00 \\ 3.598 & 8.076 & 0.352 & 1.00 & 0.00 \\ 3.757 & 9.389 & 0.365 & 1.00 & 0.00 \\ 3.443 & 7.458 & -0.703 & 1.00 & 0.00 \\ 3.614 & 7.394 & 1.709 & 1.00 & 0.00 \\ 4.593 & 7.590 & 2.171 & 1.00 & 0.00 \\ 2.866 & 7.884 & 2.352 & 1.00 & 0.00 \\ 3.354 & 5.890 & 1.597 & 1.00 & 0.00 \\ 4.151 & 5.429 & 1.000 & 1.00 & 0.00 \\ 2.429 & 5.734 & 1.027 & 1.00 & 0.00\end{array}$




\begin{tabular}{|c|c|c|c|c|c|c|c|c|c|c|}
\hline \multicolumn{11}{|l|}{ TER } \\
\hline ATOM & 39 & $\mathrm{FE}$ & HEM & 2 & 4.955 & 3.343 & 6.440 & 1.00 & 0.00 & $\mathrm{FE}$ \\
\hline ATOM & 40 & $\mathrm{C} 1$ & HEM & 2 & 7.183 & 1.471 & 7.502 & 1.00 & 0.00 & C \\
\hline ATOM & 41 & $\mathrm{C} 2$ & HEM & 2 & 7.542 & 0.680 & 8.674 & 1.00 & 0.00 & C \\
\hline ATOM & 42 & C3 & HEM & 2 & 6.397 & 0.583 & 9.448 & 1.00 & 0.00 & C \\
\hline ATOM & 43 & $\mathrm{C} 4$ & HEM & 2 & 5.367 & 1.301 & 8.740 & 1.00 & 0.00 & C \\
\hline ATOM & 44 & C5 & HEM & 2 & 4.052 & 1.420 & 9.178 & 1.00 & 0.00 & $\mathrm{C}$ \\
\hline ATOM & 45 & H5 & HEM & 2 & 3.798 & 0.923 & 10.113 & 1.00 & 0.00 & $\mathrm{H}$ \\
\hline ATOM & 46 & $\mathrm{C} 6$ & HEM & 2 & 3.033 & 2.118 & 8.542 & 1.00 & 0.00 & C \\
\hline ATOM & 47 & C7 & HEM & 2 & 1.664 & 2.191 & 9.012 & 1.00 & 0.00 & C \\
\hline ATOM & 48 & $\mathrm{C} 8$ & HEM & 2 & 0.958 & 2.910 & 8.065 & 1.00 & 0.00 & C \\
\hline ATOM & 49 & C9 & HEM & 2 & 1.909 & 3.275 & 7.041 & 1.00 & 0.00 & C \\
\hline ATOM & 50 & $\mathrm{C} 10$ & HEM & 2 & 1.605 & 4.010 & 5.897 & 1.00 & 0.00 & C \\
\hline ATOM & 51 & $\mathrm{H} 10$ & HEM & 2 & 0.578 & 4.354 & 5.791 & 1.00 & 0.00 & $\mathrm{H}$ \\
\hline ATOM & 52 & $\mathrm{C} 11$ & HEM & 2 & 2.479 & 4.350 & 4.874 & 1.00 & 0.00 & C \\
\hline ATOM & 53 & $\mathrm{C} 12$ & HEM & 2 & 2.098 & 5.076 & 3.684 & 1.00 & 0.00 & C \\
\hline ATOM & 54 & $\mathrm{C} 13$ & HEM & 2 & 3.238 & 5.223 & 2.927 & 1.00 & 0.00 & C \\
\hline ATOM & 55 & C14 & HEM & 2 & 4.311 & 4.581 & 3.659 & 1.00 & 0.00 & $\mathrm{C}$ \\
\hline ATOM & 56 & C15 & HEM & 2 & 5.639 & 4.527 & 3.237 & 1.00 & 0.00 & $\mathrm{C}$ \\
\hline ATOM & 57 & H15 & HEM & 2 & 5.879 & 5.011 & 2.289 & 1.00 & 0.00 & $\mathrm{H}$ \\
\hline ATOM & 58 & C16 & HEM & 2 & 6.669 & 3.845 & 3.880 & 1.00 & 0.00 & C \\
\hline ATOM & 59 & $\mathrm{C} 17$ & HEM & 2 & 8.035 & 3.732 & 3.398 & 1.00 & 0.00 & C \\
\hline ATOM & 60 & C18 & HEM & 2 & 8.710 & 2.964 & 4.322 & 1.00 & 0.00 & C \\
\hline ATOM & 61 & C19 & HEM & 2 & 7.761 & 2.612 & 5.358 & 1.00 & 0.00 & C \\
\hline ATOM & 62 & $\mathrm{C} 20$ & HEM & 2 & 8.052 & 1.821 & 6.469 & 1.00 & 0.00 & C \\
\hline ATOM & 63 & $\mathrm{H} 2 \mathrm{O}$ & HEM & 2 & 9.066 & 1.432 & 6.530 & 1.00 & 0.00 & $\mathrm{H}$ \\
\hline ATOM & 64 & $\mathrm{~N} 21$ & HEM & 2 & 5.862 & 1.834 & 7.564 & 1.00 & 0.00 & $\mathrm{~N}$ \\
\hline ATOM & 65 & N22 & HEM & 2 & 3.157 & 2.781 & 7.342 & 1.00 & 0.00 & $\mathrm{~N}$ \\
\hline ATOM & 66 & N23 & HEM & 2 & 3.828 & 4.044 & 4.841 & 1.00 & 0.00 & $\mathrm{~N}$ \\
\hline ATOM & 67 & N2 4 & HEM & 2 & 6.527 & 3.160 & 5.077 & 1.00 & 0.00 & $\mathrm{~N}$ \\
\hline ATOM & 68 & C99 & HEM & 2 & 5.621 & 6.025 & 7.579 & 1.00 & 0.00 & C \\
\hline ATOM & 69 & 091 & HEM & 2 & 5.461 & 4.711 & 7.567 & 1.00 & 0.00 & 0 \\
\hline ATOM & 70 & 092 & HEM & 2 & 5.776 & 6.642 & 8.635 & 1.00 & 0.00 & 0 \\
\hline ATOM & 71 & СТ91 & HEM & 2 & 5.605 & 6.706 & 6.223 & 1.00 & 0.00 & C \\
\hline ATOM & 72 & H911 & HEM & 2 & 4.626 & 6.511 & 5.760 & 1.00 & 0.00 & $\mathrm{H}$ \\
\hline ATOM & 73 & H912 & HEM & 2 & 6.353 & 6.216 & 5.580 & 1.00 & 0.00 & $\mathrm{H}$ \\
\hline ATOM & 74 & СТ92 & HEM & 2 & 5.864 & 8.211 & 6.334 & 1.00 & 0.00 & C \\
\hline ATOM & 75 & H921 & HEM & 2 & 6.789 & 8.367 & 6.905 & 1.00 & 0.00 & $\mathrm{H}$ \\
\hline ATOM & 76 & H922 & HEM & 2 & 5.068 & 8.671 & 6.931 & 1.00 & 0.00 & $\mathrm{H}$ \\
\hline \multicolumn{11}{|l|}{ TER } \\
\hline AтOM & 77 & $\mathrm{C} 2$ & VIN & 3 & 0.345 & 13.923 & -1.002 & 1.00 & 0.00 & C \\
\hline ATOM & 78 & $\mathrm{H} 21$ & VIN & 3 & -0.471 & 13.224 & -0.837 & 1.00 & 0.00 & $\mathrm{H}$ \\
\hline ATOM & 79 & $\mathrm{C} 1$ & VIN & 3 & 0.012 & 15.147 & -1.403 & 1.00 & 0.00 & C \\
\hline ATOM & 80 & $\mathrm{H} 11$ & VIN & 3 & 0.743 & 15.926 & -1.511 & 1.00 & 0.00 & $\mathrm{H}$ \\
\hline ATOM & 81 & $\mathrm{H} 12$ & VIN & 3 & -1.030 & 15.417 & -1.570 & 1.00 & 0.00 & $\mathrm{H}$ \\
\hline \multicolumn{11}{|l|}{ TER } \\
\hline ATOM & 82 & $\mathrm{C} 1$ & $\mathrm{MET}$ & 4 & 3.008 & 14.177 & -2.840 & 1.00 & 0.00 & C \\
\hline ATOM & 83 & $\mathrm{H} 11$ & $\mathrm{MET}$ & 4 & 3.243 & 15.248 & -2.720 & 1.00 & 0.00 & $\mathrm{H}$ \\
\hline ATOM & 84 & $\mathrm{H} 12$ & MET & 4 & 3.821 & 13.713 & -3.410 & 1.00 & 0.00 & $\mathrm{H}$ \\
\hline ATOM & 85 & $\mathrm{H} 13$ & MET & 4 & 2.101 & 14.100 & -3.450 & 1.00 & 0.00 & $\mathrm{H}$ \\
\hline \multicolumn{11}{|l|}{ TER } \\
\hline ATOM & 86 & $\mathrm{C} 2$ & VIN & 5 & 8.018 & 12.499 & -2.325 & 1.00 & 0.00 & C \\
\hline ATOM & 87 & $\mathrm{H} 21$ & VIN & 5 & 7.352 & 12.399 & -3.184 & 1.00 & 0.00 & $\mathrm{H}$ \\
\hline ATOM & 88 & $\mathrm{C} 1$ & VIN & 5 & 9.185 & 13.144 & -2.484 & 1.00 & 0.00 & C \\
\hline ATOM & 89 & $\mathrm{H} 11$ & VIN & 5 & 9.865 & 13.300 & -1.654 & 1.00 & 0.00 & $\mathrm{H}$ \\
\hline ATOM & 90 & $\mathrm{H} 12$ & VIN & 5 & 9.490 & 13.542 & -3.450 & 1.00 & 0.00 & $\mathrm{H}$ \\
\hline TER & & & & & & & & & & \\
\hline AтOM & 91 & $\mathrm{C} 1$ & MET & 6 & 9.703 & 10.816 & -0.121 & 1.00 & 0.00 & C \\
\hline ATOM & 92 & $\mathrm{H} 11$ & $\mathrm{MET}$ & 6 & 10.294 & 11.480 & 0.528 & 1.00 & 0.00 & $\mathrm{H}$ \\
\hline ATOM & 93 & $\mathrm{H} 12$ & $\mathrm{MET}$ & 6 & 9.859 & 9.794 & 0.246 & 1.00 & 0.00 & $\mathrm{H}$ \\
\hline ATOM & 94 & $\mathrm{H} 13$ & $\mathrm{MET}$ & 6 & 10.135 & 10.882 & -1.126 & 1.00 & 0.00 & $\mathrm{H}$ \\
\hline TER & & & & & & & & & & \\
\hline ATOM & 95 & C5 & $\mathrm{ACP}$ & 7 & 0.615 & 9.735 & 5.770 & 1.00 & 0.00 & C \\
\hline ATOM & 96 & H51 & $\mathrm{ACP}$ & 7 & 1.354 & 9.723 & 6.582 & 1.00 & 0.00 & $\mathrm{H}$ \\
\hline ATOM & 97 & $\mathrm{H} 52$ & $\mathrm{ACP}$ & 7 & -0.226 & 10.343 & 6.134 & 1.00 & 0.00 & $\mathrm{H}$ \\
\hline ATOM & 98 & $\mathrm{C} 4$ & $\mathrm{ACP}$ & 7 & 0.136 & 8.297 & 5.497 & 1.00 & 0.00 & C \\
\hline ATOM & 99 & $\mathrm{H} 41$ & $\mathrm{ACP}$ & 7 & -0.422 & 8.236 & 4.549 & 1.00 & 0.00 & $\mathrm{H}$ \\
\hline ATOM & 100 & $\mathrm{H} 42$ & $\mathrm{ACP}$ & 7 & 1.007 & 7.630 & 5.390 & 1.00 & 0.00 & $\mathrm{H}$ \\
\hline ATOM & 101 & $\mathrm{C} 1$ & $\mathrm{ACP}$ & 7 & -0.728 & 7.685 & 6.573 & 1.00 & 0.00 & C \\
\hline ATOM & 102 & $\mathrm{O} 2$ & $\mathrm{ACP}$ & 7 & -0.956 & 8.208 & 7.671 & 1.00 & 0.00 & 0 \\
\hline ATOM & 103 & 03 & $\mathrm{ACP}$ & 7 & -1.244 & 6.505 & 6.209 & 1.00 & 0.00 & 0 \\
\hline ATOM & 104 & H3 & $\mathrm{ACP}$ & 7 & -1.853 & 6.151 & 6.945 & 1.00 & 0.00 & $\mathrm{H}$ \\
\hline TER & & & & & & & & & & \\
\hline ATOM & 105 & $\mathrm{C} 1$ & MET & 8 & 8.511 & 8.608 & 4.597 & 1.00 & 0.00 & C \\
\hline ATOM & 106 & $\mathrm{H} 11$ & MET & 8 & 9.063 & 9.448 & 5.047 & 1.00 & 0.00 & $\mathrm{H}$ \\
\hline ATOM & 107 & $\mathrm{H} 12$ & MET & 8 & 8.517 & 7.782 & 5.319 & 1.00 & 0.00 & $\mathrm{H}$ \\
\hline ATOM & 108 & $\mathrm{H} 13$ & MET & 8 & 9.082 & 8.289 & 3.717 & 1.00 & 0.00 & $\mathrm{H}$ \\
\hline TER & & & & & & & & & & \\
\hline ATOM & 109 & $\mathrm{C} 1$ & $\mathrm{MET}$ & 9 & -0.923 & 11.557 & 3.646 & 1.00 & 0.00 & $\mathrm{C}$ \\
\hline
\end{tabular}




\begin{tabular}{|c|c|c|c|c|c|c|c|c|c|c|}
\hline ATOM & 110 & H11 & MET & 9 & -1.368 & 11.348 & 4.626 & 1.00 & 0.00 & $\mathrm{H}$ \\
\hline ATOM & 111 & H12 & MET & 9 & -1.033 & 12.637 & 3.471 & 1.00 & 0.00 & $\mathrm{H}$ \\
\hline ATOM & 112 & $\mathrm{H} 13$ & MET & 9 & -1.523 & 11.031 & 2.889 & 1.00 & 0.00 & $\mathrm{H}$ \\
\hline \multicolumn{11}{|l|}{ TER } \\
\hline ATOM & 113 & C2 & VIN & 10 & 8.874 & 0.178 & 8.933 & 1.00 & 0.00 & C \\
\hline ATOM & 114 & Н 21 & VIN & 10 & 9.690 & 0.876 & 8.768 & 1.00 & 0.00 & $\mathrm{H}$ \\
\hline ATOM & 115 & $\mathrm{C} 1$ & VIN & 10 & 9.207 & -1.047 & 9.335 & 1.00 & 0.00 & C \\
\hline ATOM & 116 & H11 & VIN & 10 & 8.475 & -1.826 & 9.442 & 1.00 & 0.00 & $\mathrm{H}$ \\
\hline ATOM & 117 & H12 & VIN & 10 & 10.249 & -1.316 & 9.501 & 1.00 & 0.00 & $H$ \\
\hline \multicolumn{11}{|l|}{ TER } \\
\hline ATOM & 118 & $\mathrm{C} 1$ & MET & 11 & 6.210 & -0.077 & 10.771 & 1.00 & 0.00 & 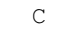 \\
\hline ATOM & 119 & $\mathrm{H} 11$ & MET & 11 & 5.975 & -1.148 & 10.652 & 1.00 & 0.00 & $H$ \\
\hline ATOM & 120 & H12 & MET & 11 & 5.397 & 0.387 & 11.341 & 1.00 & 0.00 & $\mathrm{H}$ \\
\hline ATOM & 121 & H13 & MET & 11 & 7.118 & 0.001 & 11.382 & 1.00 & 0.00 & $\mathrm{H}$ \\
\hline \multicolumn{11}{|l|}{ TER } \\
\hline ATOM & 122 & $\mathrm{C} 2$ & VIN & 12 & 1.200 & 1.601 & 10.256 & 1.00 & 0.00 & C \\
\hline ATOM & 123 & H 21 & VIN & 12 & 1.866 & 1.702 & 11.115 & 1.00 & 0.00 & $\mathrm{H}$ \\
\hline ATOM & 124 & $\mathrm{C} 1$ & VIN & 12 & 0.033 & 0.957 & 10.415 & 1.00 & 0.00 & C \\
\hline ATOM & 125 & H11 & VIN & 12 & -0.647 & 0.800 & 9.585 & 1.00 & 0.00 & $\mathrm{H}$ \\
\hline ATOM & 126 & H12 & VIN & 12 & -0.272 & 0.559 & 11.381 & 1.00 & 0.00 & $\mathrm{H}$ \\
\hline \multicolumn{11}{|l|}{ TER } \\
\hline ATOM & 127 & $\mathrm{C} 1$ & MET & 13 & -0.484 & 3.284 & 8.052 & 1.00 & 0.00 & C \\
\hline ATOM & 128 & H11 & MET & 13 & -0.641 & 4.306 & 7.685 & 1.00 & 0.00 & $\mathrm{H}$ \\
\hline ATOM & 129 & H12 & $\mathrm{MET}$ & 13 & -0.917 & 3.219 & 9.057 & 1.00 & 0.00 & $\mathrm{H}$ \\
\hline ATOM & 130 & H13 & MET & 13 & -1.076 & 2.620 & 7.403 & 1.00 & 0.00 & H \\
\hline TER & & & & & & & & & & \\
\hline ATOM & 131 & C5 & $\mathrm{ACP}$ & 14 & 8.604 & 4.366 & 2.161 & 1.00 & 0.00 & C \\
\hline ATOM & 132 & H51 & $\mathrm{ACP}$ & 14 & 7.865 & 4.377 & 1.349 & 1.00 & 0.00 & H \\
\hline ATOM & 133 & H52 & $\mathrm{ACP}$ & 14 & 9.445 & 3.758 & 1.798 & 1.00 & 0.00 & H \\
\hline ATOM & 134 & C4 & $\mathrm{ACP}$ & 14 & 9.082 & 5.803 & 2.434 & 1.00 & 0.00 & $\mathrm{C}$ \\
\hline ATOM & 135 & H 41 & $\mathrm{ACP}$ & 14 & 9.640 & 5.865 & 3.382 & 1.00 & 0.00 & $\mathrm{H}$ \\
\hline ATOM & 136 & H 42 & $\mathrm{ACP}$ & 14 & 8.212 & 6.470 & 2.541 & 1.00 & 0.00 & $\mathrm{H}$ \\
\hline ATOM & 137 & $\mathrm{C} 1$ & $\mathrm{ACP}$ & 14 & 9.946 & 6.416 & 1.358 & 1.00 & 0.00 & C \\
\hline ATOM & 138 & 02 & $\mathrm{ACP}$ & 14 & 10.174 & 5.893 & 0.260 & 1.00 & 0.00 & 0 \\
\hline ATOM & 139 & 03 & $\mathrm{ACP}$ & 14 & 10.462 & 7.595 & 1.722 & 1.00 & 0.00 & 0 \\
\hline ATOM & 140 & H3 & $\mathrm{ACP}$ & 14 & 11.071 & 7.949 & 0.986 & 1.00 & 0.00 & $\mathrm{H}$ \\
\hline TER & & & & & & & & & & \\
\hline ATOM & 141 & $\mathrm{C} 1$ & $\mathrm{MET}$ & 15 & 0.708 & 5.492 & 3.334 & 1.00 & 0.00 & C \\
\hline ATOM & 142 & H11 & MET & 15 & 0.155 & 4.653 & 2.884 & 1.00 & 0.00 & $\mathrm{H}$ \\
\hline ATOM & 143 & H12 & MET & 15 & 0.701 & 6.318 & 2.613 & 1.00 & 0.00 & $\mathrm{H}$ \\
\hline ATOM & 144 & H13 & MET & 15 & 0.137 & 5.812 & 4.214 & 1.00 & 0.00 & $\mathrm{H}$ \\
\hline TER & & & & & & & & & & \\
\hline ATOM & 145 & $\mathrm{C} 1$ & MET & 16 & 10.141 & 2.543 & 4.285 & 1.00 & 0.00 & C \\
\hline ATOM & 146 & H11 & MET & 16 & 10.586 & 2.752 & 3.305 & 1.00 & 0.00 & $\mathrm{H}$ \\
\hline ATOM & 147 & $\mathrm{H} 12$ & MET & 16 & 10.252 & 1.463 & 4.460 & 1.00 & 0.00 & $\mathrm{H}$ \\
\hline ATOM & 148 & H13 & $\mathrm{MET}$ & 16 & 10.742 & 3.069 & 5.042 & 1.00 & 0.00 & $\mathrm{H}$ \\
\hline TER & & & & & & & & & & \\
\hline ATOM & 149 & $\mathrm{FE}$ & HEM & 17 & 3.316 & 10.511 & 9.423 & 1.00 & 0.00 & $\mathrm{FE}$ \\
\hline ATOM & 150 & $\mathrm{C} 1$ & HEM & 17 & 1.088 & 12.383 & 8.361 & 1.00 & 0.00 & C \\
\hline ATOM & 151 & $\mathrm{C} 2$ & HEM & 17 & 0.729 & 13.174 & 7.189 & 1.00 & 0.00 & C \\
\hline ATOM & 152 & C3 & HEM & 17 & 1.874 & 13.271 & 6.414 & 1.00 & 0.00 & C \\
\hline ATOM & 153 & $\mathrm{C} 4$ & HEM & 17 & 2.904 & 12.553 & 7.122 & 1.00 & 0.00 & C \\
\hline ATOM & 154 & C5 & HEM & 17 & 4.219 & 12.435 & 6.684 & 1.00 & 0.00 & C \\
\hline ATOM & 155 & H5 & HEM & 17 & 4.472 & 12.931 & 5.749 & 1.00 & 0.00 & $\mathrm{H}$ \\
\hline ATOM & 156 & $\mathrm{C} 6$ & HEM & 17 & 5.238 & 11.736 & 7.320 & 1.00 & 0.00 & C \\
\hline ATOM & 157 & $\mathrm{C} 7$ & HEM & 17 & 6.607 & 11.664 & 6.851 & 1.00 & 0.00 & C \\
\hline ATOM & 158 & C8 & HEM & 17 & 7.313 & 10.944 & 7.798 & 1.00 & 0.00 & C \\
\hline ATOM & 159 & C9 & HEM & 17 & 6.362 & 10.579 & 8.822 & 1.00 & 0.00 & C \\
\hline ATOM & 160 & $\mathrm{C} 10$ & HEM & 17 & 6.666 & 9.844 & 9.965 & 1.00 & 0.00 & C \\
\hline ATOM & 161 & $\mathrm{H} 10$ & HEM & 17 & 7.693 & 9.500 & 10.071 & 1.00 & 0.00 & 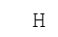 \\
\hline ATOM & 162 & $\mathrm{C} 11$ & HEM & 17 & 5.792 & 9.504 & 10.988 & 1.00 & 0.00 & C \\
\hline ATOM & 163 & $\mathrm{C} 12$ & HEM & 17 & 6.173 & 8.779 & 12.178 & 1.00 & 0.00 & C \\
\hline ATOM & 164 & C13 & HEM & 17 & 5.033 & 8.631 & 12.935 & 1.00 & 0.00 & C \\
\hline ATOM & 165 & C14 & HEM & 17 & 3.960 & 9.273 & 12.204 & 1.00 & 0.00 & C \\
\hline ATOM & 166 & C15 & HEM & 17 & 2.632 & 9.328 & 12.625 & 1.00 & 0.00 & C \\
\hline ATOM & 167 & H15 & HEM & 17 & 2.392 & 8.843 & 13.573 & 1.00 & 0.00 & 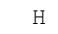 \\
\hline ATOM & 168 & C16 & HEM & 17 & 1.602 & 10.009 & 11.982 & 1.00 & 0.00 & 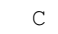 \\
\hline ATOM & 169 & $\mathrm{C} 17$ & HEM & 17 & 0.236 & 10.123 & 12.464 & 1.00 & 0.00 & C \\
\hline ATOM & 170 & C18 & HEM & 17 & -0.439 & 10.890 & 11.541 & 1.00 & 0.00 & \\
\hline ATOM & 171 & C19 & HEM & 17 & 0.510 & 11.242 & 10.504 & 1.00 & 0.00 & \\
\hline ATOM & 172 & $\mathrm{C} 20$ & HEM & 17 & 0.219 & 12.033 & 9.393 & 1.00 & 0.00 & $C$ \\
\hline ATOM & 173 & H 20 & HEM & 17 & -0.795 & 12.423 & 9.333 & 1.00 & 0.00 & H \\
\hline ATOM & 174 & N21 & HEM & 17 & 2.409 & 12.020 & 8.298 & 1.00 & 0.00 & N \\
\hline ATOM & 175 & $\mathrm{~N} 22$ & HEM & 17 & 5.114 & 11.073 & 8.521 & 1.00 & 0.00 & 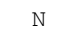 \\
\hline ATOM & 176 & N23 & HEM & 17 & 4.443 & 9.810 & 11.021 & 1.00 & 0.00 & \\
\hline ATOM & 177 & N24 & HEM & 17 & 1.743 & 10.694 & 10.786 & 1.00 & 0.00 & \\
\hline ATOM & 178 & C99 & HEM & 17 & 2.650 & 7.829 & 8.283 & 1.00 & 0.00 & \\
\hline ATOM & 179 & 091 & HEM & 17 & 2.810 & 9.143 & 8.296 & 1.00 & 0.00 & \\
\hline ATOM & 180 & 092 & HEM & 17 & 2.495 & 7.212 & 7.228 & 1.00 & 0.00 & \\
\hline
\end{tabular}




\begin{tabular}{|c|c|c|c|c|c|c|c|c|c|c|}
\hline ATOM & 181 & СТ91 & HEM & 17 & 2.666 & 7.148 & 9.640 & 1.00 & 0.00 & $\mathrm{C}$ \\
\hline ATOM & 182 & H911 & HEM & 17 & 3.645 & 7.343 & 10.103 & 1.00 & 0.00 & $\mathrm{H}$ \\
\hline ATOM & 183 & H912 & HEM & 17 & 1.918 & 7.638 & 10.283 & 1.00 & 0.00 & $\mathrm{H}$ \\
\hline ATOM & 184 & CT92 & HEM & 17 & 2.407 & 5.644 & 9.528 & 1.00 & 0.00 & C \\
\hline ATOM & 185 & H921 & HEM & 17 & 3.203 & 5.183 & 8.931 & 1.00 & 0.00 & $\mathrm{H}$ \\
\hline ATOM & 186 & H922 & HEM & 17 & 1.482 & 5.488 & 8.958 & 1.00 & 0.00 & $\mathrm{H}$ \\
\hline \multicolumn{11}{|l|}{ TER } \\
\hline ATOM & 187 & $\mathrm{FE}$ & HEM & 18 & 4.008 & 3.097 & 14.371 & 1.00 & 0.00 & $\mathrm{FE}$ \\
\hline ATOM & 188 & $\mathrm{C} 1$ & HEM & 18 & 6.236 & 1.225 & 15.433 & 1.00 & 0.00 & r \\
\hline ATOM & 189 & C2 & HEM & 18 & 6.595 & 0.434 & 16.605 & 1.00 & 0.00 & $\mathrm{C}$ \\
\hline ATOM & 190 & C3 & HEM & 18 & 5.449 & 0.337 & 17.380 & 1.00 & 0.00 & 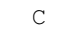 \\
\hline ATOM & 191 & $\mathrm{C} 4$ & HEM & 18 & 4.419 & 1.055 & 16.672 & 1.00 & 0.00 & C \\
\hline ATOM & 192 & C5 & HEM & 18 & 3.104 & 1.173 & 17.109 & 1.00 & 0.00 & C \\
\hline ATOM & 193 & H5 & HEM & 18 & 2.851 & 0.677 & 18.045 & 1.00 & 0.00 & $\mathrm{H}$ \\
\hline ATOM & 194 & $\mathrm{C} 6$ & HEM & 18 & 2.085 & 1.872 & 16.473 & 1.00 & 0.00 & C \\
\hline ATOM & 195 & $\mathrm{C} 7$ & HEM & 18 & 0.716 & 1.944 & 16.943 & 1.00 & 0.00 & C \\
\hline ATOM & 196 & $\mathrm{C} 8$ & HEM & 18 & 0.011 & 2.664 & 15.996 & 1.00 & 0.00 & C \\
\hline ATOM & 197 & C9 & HEM & 18 & 0.962 & 3.029 & 14.972 & 1.00 & 0.00 & C \\
\hline ATOM & 198 & $\mathrm{C} 10$ & HEM & 18 & 0.657 & 3.764 & 13.828 & 1.00 & 0.00 & C \\
\hline ATOM & 199 & $\mathrm{H} 10$ & HEM & 18 & -0.370 & 4.108 & 13.722 & 1.00 & 0.00 & $\mathrm{H}$ \\
\hline ATOM & 200 & $\mathrm{C} 11$ & HEM & 18 & 1.532 & 4.104 & 12.806 & 1.00 & 0.00 & C \\
\hline ATOM & 201 & $\mathrm{C} 12$ & HEM & 18 & 1.151 & 4.829 & 11.615 & 1.00 & 0.00 & C \\
\hline ATOM & 202 & C13 & HEM & 18 & 2.290 & 4.977 & 10.858 & 1.00 & 0.00 & C \\
\hline ATOM & 203 & C14 & HEM & 18 & 3.363 & 4.335 & 11.590 & 1.00 & 0.00 & $\mathrm{C}$ \\
\hline ATOM & 204 & C15 & HEM & 18 & 4.691 & 4.280 & 11.168 & 1.00 & 0.00 & C \\
\hline ATOM & 205 & H15 & HEM & 18 & 4.931 & 4.765 & 10.221 & 1.00 & 0.00 & $\mathrm{H}$ \\
\hline ATOM & 206 & $\mathrm{C} 16$ & HEM & 18 & 5.721 & 3.599 & 11.812 & 1.00 & 0.00 & 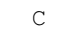 \\
\hline ATOM & 207 & C17 & HEM & 18 & 7.087 & 3.485 & 11.329 & 1.00 & 0.00 & 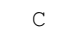 \\
\hline ATOM & 208 & C18 & HEM & 18 & 7.763 & 2.718 & 12.253 & 1.00 & 0.00 & 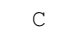 \\
\hline ATOM & 209 & C19 & HEM & 18 & 6.813 & 2.366 & 13.289 & 1.00 & 0.00 & 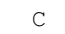 \\
\hline ATOM & 210 & $\mathrm{C} 20$ & HEM & 18 & 7.104 & 1.575 & 14.401 & 1.00 & 0.00 & 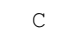 \\
\hline ATOM & 211 & H 20 & HEM & 18 & 8.118 & 1.185 & 14.461 & 1.00 & 0.00 & $\mathrm{H}$ \\
\hline ATOM & 212 & N21 & HEM & 18 & 4.914 & 1.588 & 15.495 & 1.00 & 0.00 & $\mathrm{~N}$ \\
\hline ATOM & 213 & N22 & HEM & 18 & 2.209 & 2.535 & 15.273 & 1.00 & 0.00 & $\mathrm{~N}$ \\
\hline ATOM & 214 & N23 & HEM & 18 & 2.880 & 3.798 & 12.772 & 1.00 & 0.00 & $\mathrm{~N}$ \\
\hline ATOM & 215 & N24 & HEM & 18 & 5.580 & 2.914 & 13.008 & 1.00 & 0.00 & $\mathrm{~N}$ \\
\hline ATOM & 216 & C99 & HEM & 18 & 4.673 & 5.779 & 15.510 & 1.00 & 0.00 & C \\
\hline ATOM & 217 & 091 & HEM & 18 & 4.514 & 4.465 & 15.498 & 1.00 & 0.00 & O \\
\hline ATOM & 218 & 092 & HEM & 18 & 4.828 & 6.396 & 16.566 & 1.00 & 0.00 & 0 \\
\hline ATOM & 219 & СТ91 & HEM & 18 & 4.657 & 6.460 & 14.154 & 1.00 & 0.00 & C \\
\hline ATOM & 220 & H911 & HEM & 18 & 3.678 & 6.265 & 13.691 & 1.00 & 0.00 & $\mathrm{H}$ \\
\hline ATOM & 221 & H912 & HEM & 18 & 5.405 & 5.970 & 13.511 & 1.00 & 0.00 & $\mathrm{H}$ \\
\hline ATOM & 222 & СТ92 & HEM & 18 & 4.917 & 7.964 & 14.265 & 1.00 & 0.00 & $\mathrm{C}$ \\
\hline ATOM & 223 & H921 & HEM & 18 & 5.842 & 8.120 & 14.836 & 1.00 & 0.00 & $\mathrm{H}$ \\
\hline ATOM & 224 & H922 & HEM & 18 & 4.120 & 8.425 & 14.862 & 1.00 & 0.00 & . \\
\hline \multicolumn{11}{|l|}{ TER } \\
\hline ATOM & 225 & $\mathrm{C} 2$ & VIN & 19 & -0.603 & 13.676 & 6.929 & 1.00 & 0.00 & C \\
\hline ATOM & 226 & H 21 & VIN & 19 & -1.419 & 12.978 & 7.094 & 1.00 & 0.00 & $\mathrm{H}$ \\
\hline ATOM & 227 & $\mathrm{C} 1$ & VIN & 19 & -0.936 & 14.901 & 6.528 & 1.00 & 0.00 & C \\
\hline ATOM & 228 & H11 & VIN & 19 & -0.205 & 15.680 & 6.421 & 1.00 & 0.00 & 21 \\
\hline ATOM & 229 & $\mathrm{H} 12$ & VIN & 19 & -1.978 & 15.170 & 6.361 & 1.00 & 0.00 & $\mathrm{H}$ \\
\hline \multicolumn{11}{|l|}{ TER } \\
\hline ATOM & 230 & $\mathrm{C} 1$ & MET & 20 & 2.061 & 13.931 & 5.091 & 1.00 & 0.00 & C \\
\hline ATOM & 231 & H11 & MET & 20 & 2.295 & 15.002 & 5.211 & 1.00 & 0.00 & $\mathrm{H}$ \\
\hline ATOM & 232 & $\mathrm{H} 12$ & MET & 20 & 2.874 & 13.467 & 4.522 & 1.00 & 0.00 & $\mathrm{H}$ \\
\hline ATOM & 233 & H13 & MET & 20 & 1.153 & 13.854 & 4.481 & 1.00 & 0.00 & $\mathrm{H}$ \\
\hline \multicolumn{11}{|l|}{ TER } \\
\hline ATOM & 234 & $\mathrm{C} 2$ & VIN & 21 & 7.071 & 12.253 & 5.606 & 1.00 & 0.00 & C \\
\hline ATOM & 235 & H21 & VIN & 21 & 6.405 & 12.152 & 4.747 & 1.00 & 0.00 & 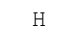 \\
\hline ATOM & 236 & $\mathrm{C} 1$ & VIN & 21 & 8.238 & 12.898 & 5.447 & 1.00 & 0.00 & C \\
\hline ATOM & 237 & H11 & VIN & 21 & 8.917 & 13.054 & 6.277 & 1.00 & 0.00 & $\mathrm{H}$ \\
\hline ATOM & 238 & $\mathrm{H} 12$ & VIN & 21 & 8.543 & 13.296 & 4.482 & 1.00 & 0.00 & $\mathrm{H}$ \\
\hline \multicolumn{11}{|l|}{ TER } \\
\hline ATOM & 239 & $\mathrm{C} 1$ & MET & 22 & 8.755 & 10.570 & 7.810 & 1.00 & 0.00 & 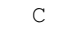 \\
\hline ATOM & 240 & H11 & MET & 22 & 9.347 & 11.234 & 8.459 & 1.00 & 0.00 & $H$ \\
\hline ATOM & 241 & H12 & MET & 22 & 8.912 & 9.548 & 8.177 & 1.00 & 0.00 & 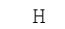 \\
\hline ATOM & 242 & H13 & MET & 22 & 9.188 & 10.636 & 6.805 & 1.00 & 0.00 & $\mathrm{H}$ \\
\hline TER & & & & & & & & & & \\
\hline ATOM & 243 & C5 & $\mathrm{ACP}$ & 23 & -0.333 & 9.488 & 13.701 & 1.00 & 0.00 & \\
\hline ATOM & 244 & H51 & $\mathrm{ACP}$ & 23 & 0.406 & 9.477 & 14.514 & 1.00 & 0.00 & \\
\hline ATOM & 245 & H52 & $\mathrm{ACP}$ & 23 & -1.174 & 10.097 & 14.065 & 1.00 & 0.00 & \\
\hline ATOM & 246 & $\mathrm{C} 4$ & $\mathrm{ACP}$ & 23 & -0.812 & 8.051 & 13.428 & 1.00 & 0.00 & \\
\hline ATOM & 247 & $\mathrm{H} 41$ & $\mathrm{ACP}$ & 23 & -1.369 & 7.990 & 12.481 & 1.00 & 0.00 & \\
\hline ATOM & 248 & $\mathrm{H} 42$ & $\mathrm{ACP}$ & 23 & 0.059 & 7.384 & 13.321 & 1.00 & 0.00 & \\
\hline ATOM & 249 & $\mathrm{C} 1$ & $\mathrm{ACP}$ & 23 & -1.676 & 7.439 & 14.504 & 1.00 & 0.00 & \\
\hline ATOM & 250 & 02 & $\mathrm{ACP}$ & 23 & -1.903 & 7.961 & 15.603 & 1.00 & 0.00 & \\
\hline ATOM & 251 & 03 & $\mathrm{ACP}$ & 23 & -2.191 & 6.259 & 14.140 & 1.00 & 0.00 & \\
\hline ATOM & 252 & H3 & $\mathrm{ACP}$ & 23 & -2.800 & 5.905 & 14.876 & 1.00 & 0.00 & \\
\hline
\end{tabular}




\begin{tabular}{|c|c|c|c|c|c|c|c|c|c|c|}
\hline ATOM & 253 & $\mathrm{C} 1$ & MET & 24 & 7.563 & 8.362 & 12.528 & 1.00 & 0.00 & C \\
\hline ATOM & 254 & H11 & $\mathrm{MET}$ & 24 & 8.115 & 9.201 & 12.978 & 1.00 & 0.00 & $\mathrm{H}$ \\
\hline ATOM & 255 & $\mathrm{H} 12$ & $\mathrm{MET}$ & 24 & 7.570 & 7.536 & 13.250 & 1.00 & 0.00 & $\mathrm{H}$ \\
\hline ATOM & 256 & $\mathrm{H} 13$ & $\mathrm{MET}$ & 24 & 8.134 & 8.042 & 11.649 & 1.00 & 0.00 & $\mathrm{H}$ \\
\hline \multicolumn{11}{|l|}{ TER } \\
\hline ATOM & 257 & $\mathrm{C} 1$ & $\mathrm{MET}$ & 25 & -1.870 & 11.311 & 11.577 & 1.00 & 0.00 & C \\
\hline ATOM & 258 & $\mathrm{H} 11$ & $\mathrm{MET}$ & 25 & -2.316 & 11.102 & 12.557 & 1.00 & 0.00 & H \\
\hline ATOM & 259 & $\mathrm{H} 12$ & $\mathrm{MET}$ & 25 & -1.981 & 12.391 & 11.402 & 1.00 & 0.00 & 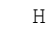 \\
\hline ATOM & 260 & $\mathrm{H} 13$ & $\mathrm{MET}$ & 25 & -2.471 & 10.785 & 10.821 & 1.00 & 0.00 & 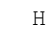 \\
\hline \multicolumn{11}{|l|}{ TER } \\
\hline ATOM & 261 & $\mathrm{C} 2$ & VIN & 26 & 7.926 & -0.068 & 16.864 & 1.00 & 0.00 & C \\
\hline ATOM & 262 & H 21 & VIN & 26 & 8.742 & 0.630 & 16.699 & 1.00 & 0.00 & $\mathrm{H}$ \\
\hline ATOM & 263 & $\mathrm{C} 1$ & VIN & 26 & 8.259 & -1.293 & 17.266 & 1.00 & 0.00 & C \\
\hline ATOM & 264 & $\mathrm{H} 11$ & VIN & 26 & 7.528 & -2.072 & 17.373 & 1.00 & 0.00 & $\mathrm{H}$ \\
\hline ATOM & 265 & $\mathrm{H} 12$ & VIN & 26 & 9.301 & -1.562 & 17.432 & 1.00 & 0.00 & 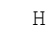 \\
\hline \multicolumn{11}{|l|}{ TER } \\
\hline ATOM & 266 & $\mathrm{C} 1$ & MET & 27 & 5.263 & -0.323 & 18.702 & 1.00 & 0.00 & $C$ \\
\hline ATOM & 267 & H11 & $\mathrm{MET}$ & 27 & 5.028 & -1.394 & 18.583 & 1.00 & 0.00 & 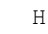 \\
\hline ATOM & 268 & $\mathrm{H} 12$ & $\mathrm{MET}$ & 27 & 4.450 & 0.141 & 19.272 & 1.00 & 0.00 & $\mathrm{H}$ \\
\hline ATOM & 269 & H1 3 & $\mathrm{MET}$ & 27 & 6.170 & -0.246 & 19.313 & 1.00 & 0.00 & $\mathrm{H}$ \\
\hline \multicolumn{11}{|l|}{ TER } \\
\hline ATOM & 270 & $\mathrm{C} 2$ & VIN & 28 & 0.252 & 1.355 & 18.188 & 1.00 & 0.00 & C \\
\hline ATOM & 271 & H 21 & VIN & 28 & 0.918 & 1.456 & 19.046 & 1.00 & 0.00 & $\mathrm{H}$ \\
\hline ATOM & 272 & $\mathrm{C} 1$ & VIN & 28 & -0.914 & 0.710 & 18.346 & 1.00 & 0.00 & C \\
\hline ATOM & 273 & H11 & VIN & 28 & -1.594 & 0.554 & 17.516 & 1.00 & 0.00 & $\mathrm{H}$ \\
\hline ATOM & 274 & $\mathrm{H} 12$ & VIN & 28 & -1.219 & 0.312 & 19.312 & 1.00 & 0.00 & $\mathrm{H}$ \\
\hline TER & & & & & & & & & & \\
\hline ATOM & 275 & $\mathrm{C} 1$ & $\mathrm{MET}$ & 29 & -1.432 & 3.038 & 15.984 & 1.00 & 0.00 & C \\
\hline ATOM & 276 & $\mathrm{H} 11$ & $\mathrm{MET}$ & 29 & -1.588 & 4.060 & 15.617 & 1.00 & 0.00 & $\mathrm{H}$ \\
\hline ATOM & 277 & $\mathrm{H} 12$ & $\mathrm{MET}$ & 29 & -1.864 & 2.972 & 16.989 & 1.00 & 0.00 & $\mathrm{H}$ \\
\hline ATOM & 278 & $\mathrm{H} 13$ & MET & 29 & -2.023 & 2.374 & 15.335 & 1.00 & 0.00 & $\mathrm{H}$ \\
\hline TER & & & & & & & & & & \\
\hline ATOM & 279 & C5 & $\mathrm{ACP}$ & 30 & 7.656 & 4.120 & 10.092 & 1.00 & 0.00 & C \\
\hline ATOM & 280 & H5 1 & $\mathrm{ACP}$ & 30 & 6.917 & 4.131 & 9.280 & 1.00 & 0.00 & $\mathrm{H}$ \\
\hline ATOM & 281 & H52 & $\mathrm{ACP}$ & 30 & 8.497 & 3.511 & 9.729 & 1.00 & 0.00 & $\mathrm{H}$ \\
\hline ATOM & 282 & $\mathrm{C} 4$ & $\mathrm{ACP}$ & 30 & 8.135 & 5.557 & 10.365 & 1.00 & 0.00 & C \\
\hline ATOM & 283 & H 41 & $\mathrm{ACP}$ & 30 & 8.693 & 5.618 & 11.313 & 1.00 & 0.00 & $\mathrm{H}$ \\
\hline ATOM & 284 & $\mathrm{H} 42$ & $\mathrm{ACP}$ & 30 & 7.264 & 6.224 & 10.472 & 1.00 & 0.00 & $\mathrm{H}$ \\
\hline ATOM & 285 & $\mathrm{C} 1$ & $\mathrm{ACP}$ & 30 & 8.999 & 6.169 & 9.289 & 1.00 & 0.00 & C \\
\hline ATOM & 286 & $\mathrm{O} 2$ & $\mathrm{ACP}$ & 30 & 9.227 & 5.647 & 8.191 & 1.00 & 0.00 & O \\
\hline ATOM & 287 & 03 & $\mathrm{ACP}$ & 30 & 9.515 & 7.349 & 9.653 & 1.00 & 0.00 & 0 \\
\hline ATOM & 288 & H3 & $\mathrm{ACP}$ & 30 & 10.124 & 7.703 & 8.917 & 1.00 & 0.00 & $\mathrm{H}$ \\
\hline TER & & & & & & & & & & \\
\hline ATOM & 289 & $\mathrm{C} 1$ & $\mathrm{MET}$ & 31 & -0.240 & 5.246 & 11.266 & 1.00 & 0.00 & $\mathrm{C}$ \\
\hline ATOM & 290 & $\mathrm{H} 11$ & MET & 31 & -0.792 & 4.407 & 10.815 & 1.00 & 0.00 & $\mathrm{H}$ \\
\hline ATOM & 291 & $\mathrm{H} 12$ & $\mathrm{MET}$ & 31 & -0.246 & 6.072 & 10.544 & 1.00 & 0.00 & $\mathrm{H}$ \\
\hline ATOM & 292 & $\mathrm{H} 13$ & $\mathrm{MET}$ & 31 & -0.811 & 5.565 & 12.145 & 1.00 & 0.00 & $\mathrm{H}$ \\
\hline TER & & & & & & & & & & \\
\hline ATOM & 293 & $\mathrm{C} 1$ & $\mathrm{MET}$ & 32 & 9.194 & 2.297 & 12.216 & 1.00 & 0.00 & $\mathrm{C}$ \\
\hline ATOM & 294 & $\mathrm{H} 11$ & MET & 32 & 9.639 & 2.506 & 11.236 & 1.00 & 0.00 & $\mathrm{H}$ \\
\hline ATOM & 295 & $\mathrm{H} 12$ & $\mathrm{MET}$ & 32 & 9.304 & 1.217 & 12.391 & 1.00 & 0.00 & $\mathrm{H}$ \\
\hline ATOM & 296 & $\mathrm{H} 13$ & MET & 32 & 9.794 & 2.823 & 12.973 & 1.00 & 0.00 & $\mathrm{H}$ \\
\hline TER & & & & & & & & & & \\
\hline ATOM & 297 & $\mathrm{FE}$ & HEM & 33 & 3.294 & 25.350 & -6.440 & 1.00 & 0.00 & $\mathrm{FE}$ \\
\hline ATOM & 298 & $\mathrm{C} 1$ & HEM & 33 & 1.066 & 27.222 & -7.502 & 1.00 & 0.00 & C \\
\hline ATOM & 299 & $\mathrm{C} 2$ & HEM & 33 & 0.707 & 28.013 & -8.674 & 1.00 & 0.00 & C \\
\hline ATOM & 300 & $\mathrm{C} 3$ & HEM & 33 & 1.853 & 28.110 & -9.448 & 1.00 & 0.00 & $\mathrm{C}$ \\
\hline ATOM & 301 & $\mathrm{C} 4$ & HEM & 33 & 2.882 & 27.392 & -8.740 & 1.00 & 0.00 & C \\
\hline ATOM & 302 & $\mathrm{C} 5$ & HEM & 33 & 4.197 & 27.274 & -9.178 & 1.00 & 0.00 & $\mathrm{C}$ \\
\hline ATOM & 303 & H5 & HEM & 33 & 4.451 & 27.770 & -10.113 & 1.00 & 0.00 & $\mathrm{H}$ \\
\hline ATOM & 304 & $\mathrm{C} 6$ & HEM & 33 & 5.217 & 26.575 & -8.542 & 1.00 & 0.00 & C \\
\hline ATOM & 305 & C7 & HEM & 33 & 6.586 & 26.503 & -9.012 & 1.00 & 0.00 & C \\
\hline ATOM & 306 & $\mathrm{C} 8$ & HEM & 33 & 7.291 & 25.783 & -8.065 & 1.00 & 0.00 & C \\
\hline ATOM & 307 & $\mathrm{C} 9$ & HEM & 33 & 6.340 & 25.418 & -7.041 & 1.00 & 0.00 & C \\
\hline ATOM & 308 & $\mathrm{C} 10$ & HEM & 33 & 6.645 & 24.683 & -5.897 & 1.00 & 0.00 & C \\
\hline ATOM & 309 & $\mathrm{H} 10$ & HEM & 33 & 7.671 & 24.339 & -5.791 & 1.00 & 0.00 & $\mathrm{H}$ \\
\hline ATOM & 310 & $\mathrm{C} 11$ & HEM & 33 & 5.770 & 24.343 & -4.874 & 1.00 & 0.00 & C \\
\hline ATOM & 311 & $\mathrm{C} 12$ & HEM & 33 & 6.151 & 23.618 & -3.684 & 1.00 & 0.00 & $\mathrm{C}$ \\
\hline ATOM & 312 & $\mathrm{C} 13$ & HEM & 33 & 5.012 & 23.470 & -2.927 & 1.00 & 0.00 & C \\
\hline ATOM & 313 & C14 & HEM & 33 & 3.939 & 24.112 & -3.659 & 1.00 & 0.00 & C \\
\hline ATOM & 314 & C15 & HEM & 33 & 2.611 & 24.167 & -3.237 & 1.00 & 0.00 & C \\
\hline ATOM & 315 & H15 & HEM & 33 & 2.370 & 23.682 & -2.289 & 1.00 & 0.00 & $\mathrm{H}$ \\
\hline ATOM & 316 & $\mathrm{C} 16$ & HEM & 33 & 1.580 & 24.848 & -3.880 & 1.00 & 0.00 & $\mathrm{C}$ \\
\hline ATOM & 317 & $\mathrm{C} 17$ & HEM & 33 & 0.215 & 24.962 & -3.398 & 1.00 & 0.00 & C \\
\hline ATOM & 318 & $\mathrm{C} 18$ & HEM & 33 & -0.461 & 25.729 & -4.322 & 1.00 & 0.00 & C \\
\hline ATOM & 319 & C19 & HEM & 33 & 0.489 & 26.081 & -5.358 & 1.00 & 0.00 & $\mathrm{C}$ \\
\hline ATOM & 320 & $\mathrm{C} 20$ & HEM & 33 & 0.197 & 26.872 & -6.469 & 1.00 & 0.00 & C \\
\hline ATOM & 321 & $\mathrm{H} 20$ & HEM & 33 & -0.817 & 27.262 & -6.530 & 1.00 & 0.00 & $\mathrm{H}$ \\
\hline ATOM & 322 & N21 & HEM & 33 & 2.388 & 26.859 & -7.564 & 1.00 & 0.00 & \\
\hline
\end{tabular}




\begin{tabular}{|c|c|c|c|}
\hline ATOM & 323 & N22 & HEM \\
\hline ATOM & 324 & N23 & HEM \\
\hline AтOM & 325 & N24 & HEM \\
\hline ATOM & 326 & C99 & HEM \\
\hline ATOM & 327 & 091 & HEM \\
\hline ATOM & 328 & 092 & HEM \\
\hline ATOM & 329 & CT91 & HEM \\
\hline ATOM & 330 & H911 & HEM \\
\hline ATOM & 331 & H912 & HEM \\
\hline ATOM & 332 & CT92 & HEM \\
\hline ATOM & 333 & H921 & HEM \\
\hline ATOM & 334 & H922 & HEM \\
\hline \multicolumn{4}{|l|}{ TER } \\
\hline ATOM & 335 & $\mathrm{FE}$ & HEM \\
\hline ATOM & 336 & $\mathrm{C} 1$ & HEM \\
\hline ATOM & 337 & $\mathrm{C} 2$ & HEM \\
\hline ATOM & 338 & $\mathrm{C} 3$ & HEM \\
\hline ATOM & 339 & $\mathrm{C} 4$ & HEM \\
\hline ATOM & 340 & $\mathrm{C} 5$ & HEM \\
\hline ATOM & 341 & H5 & HEM \\
\hline ATOM & 342 & $\mathrm{C} 6$ & HEM \\
\hline ATOM & 343 & C7 & HEM \\
\hline AтOM & 344 & C8 & HEM \\
\hline ATOM & 345 & C9 & HEM \\
\hline ATOM & 346 & C10 & HEM \\
\hline ATOM & 347 & $\mathrm{H} 10$ & HEM \\
\hline ATOM & 348 & $\mathrm{C} 11$ & HEM \\
\hline ATOM & 349 & $\mathrm{C} 12$ & HEM \\
\hline ATOM & 350 & C13 & HEM \\
\hline ATOM & 351 & C14 & HEM \\
\hline ATOM & 352 & C15 & HEM \\
\hline ATOM & 353 & H15 & HEM \\
\hline ATOM & 354 & $\mathrm{C} 16$ & HEM \\
\hline ATOM & 355 & C17 & HEM \\
\hline ATOM & 356 & C18 & HEM \\
\hline ATOM & 357 & C19 & HEM \\
\hline ATOM & 358 & $\mathrm{C} 20$ & HEM \\
\hline ATOM & 359 & H2O & HEM \\
\hline ATOM & 360 & $\mathrm{~N} 21$ & HEM \\
\hline ATOM & 361 & $\mathrm{~N} 22$ & HEM \\
\hline ATOM & 362 & N23 & HEM \\
\hline ATOM & 363 & N24 & HEM \\
\hline ATOM & 364 & C99 & HEM \\
\hline ATOM & 365 & 091 & HEM \\
\hline ATOM & 366 & 092 & HEM \\
\hline ATOM & 367 & CT91 & HEM \\
\hline ATOM & 368 & H911 & HEM \\
\hline ATOM & 369 & H912 & HEM \\
\hline ATOM & 370 & CT92 & HEM \\
\hline ATOM & 371 & H921 & HEM \\
\hline ATOM & 372 & H922 & HEM \\
\hline \multicolumn{4}{|l|}{ TER } \\
\hline ATOM & 373 & $\mathrm{C} 2$ & VIN \\
\hline ATOM & 374 & H 21 & VIN \\
\hline ATOM & 375 & $\mathrm{C} 1$ & VIN \\
\hline ATOM & 376 & $\mathrm{H} 11$ & VIN \\
\hline ATOM & 377 & $\mathrm{H} 12$ & VIN \\
\hline \multicolumn{4}{|l|}{ TER } \\
\hline ATOM & 378 & $\mathrm{C} 1$ & $\mathrm{MET}$ \\
\hline ATOM & 379 & H11 & MET \\
\hline ATOM & 380 & $\mathrm{H} 12$ & MET \\
\hline ATOM & 381 & $\mathrm{H} 13$ & MET \\
\hline \multicolumn{4}{|l|}{ TER } \\
\hline ATOM & 382 & $\mathrm{C} 2$ & VIN \\
\hline ATOM & 383 & H 21 & VIN \\
\hline ATOM & 384 & $\mathrm{C} 1$ & VIN \\
\hline ATOM & 385 & $\mathrm{H} 11$ & VIN \\
\hline ATOM & 386 & $\mathrm{H} 12$ & VIN \\
\hline \multicolumn{4}{|l|}{ TER } \\
\hline ATOM & 387 & $\mathrm{C} 1$ & MET \\
\hline ATOM & 388 & H11 & MET \\
\hline ATOM & 389 & $\mathrm{H} 12$ & MET \\
\hline ATOM & 390 & H13 & MET \\
\hline \multicolumn{4}{|l|}{ TER } \\
\hline ATOM & 391 & C5 & $\mathrm{ACP}$ \\
\hline ATOM & 392 & H51 & $\mathrm{ACP}$ \\
\hline ATOM & 393 & H52 & $\mathrm{ACP}$ \\
\hline ATOM & 394 & $\mathrm{C} 4$ & $\mathrm{ACP}$ \\
\hline ATOM & 395 & H 41 & $\mathrm{ACP}$ \\
\hline
\end{tabular}

\begin{tabular}{|c|c|c|c|c|c|}
\hline 5.092 & 25.912 & -7.342 & 1.00 & 0.00 & $\mathrm{~N}$ \\
\hline 4.422 & 24.649 & -4.841 & 1.00 & 0.00 & $\mathrm{~N}$ \\
\hline 1.722 & 25.533 & -5.077 & 1.00 & 0.00 & $\mathrm{~N}$ \\
\hline 2.629 & 22.668 & -7.579 & 1.00 & 0.00 & C \\
\hline 2.788 & 23.982 & -7.567 & 1.00 & 0.00 & 0 \\
\hline 2.474 & 22.051 & -8.635 & 1.00 & 0.00 & 0 \\
\hline 2.645 & 21.987 & -6.223 & 1.00 & 0.00 & C \\
\hline 3.623 & 22.182 & -5.760 & 1.00 & 0.00 & $\mathrm{H}$ \\
\hline 1.897 & 22.477 & -5.580 & 1.00 & 0.00 & $\mathrm{H}$ \\
\hline 2.385 & 20.483 & -6.334 & 1.00 & 0.00 & C \\
\hline 3.181 & 20.022 & -6.931 & 1.00 & 0.00 & $\mathrm{H}$ \\
\hline 1.460 & 20.327 & -6.905 & 1.00 & 0.00 & $\mathrm{H}$ \\
\hline 3.986 & 17.936 & -1.491 & 1.00 & 0.00 & $\mathrm{FE}$ \\
\hline 6.214 & 16.064 & -0.429 & 1.00 & 0.00 & C \\
\hline 6.573 & 15.273 & 0.742 & 1.00 & 0.00 & C \\
\hline 5.427 & 15.176 & 1.517 & 1.00 & 0.00 & C \\
\hline 4.398 & 15.894 & 0.809 & 1.00 & 0.00 & C \\
\hline 3.083 & 16.012 & 1.247 & 1.00 & 0.00 & C \\
\hline 2.829 & 15.516 & 2.182 & 1.00 & 0.00 & $\mathrm{H}$ \\
\hline 2.063 & 16.711 & 0.611 & 1.00 & 0.00 & C \\
\hline 0.694 & 16.783 & 1.081 & 1.00 & 0.00 & C \\
\hline-0.011 & 17.503 & 0.134 & 1.00 & 0.00 & C \\
\hline 0.940 & 17.868 & -0.891 & 1.00 & 0.00 & C \\
\hline 0.636 & 18.603 & -2.034 & 1.00 & 0.00 & C \\
\hline-0.391 & 18.947 & -2.140 & 1.00 & 0.00 & $\mathrm{H}$ \\
\hline 1.510 & 18.943 & -3.057 & 1.00 & 0.00 & C \\
\hline 1.129 & 19.668 & -4.247 & 1.00 & 0.00 & C \\
\hline 2.269 & 19.816 & -5.004 & 1.00 & 0.00 & C \\
\hline 3.341 & 19.174 & -4.273 & 1.00 & 0.00 & C \\
\hline 4.670 & 19.119 & -4.694 & 1.00 & 0.00 & C \\
\hline 4.910 & 19.604 & -5.642 & 1.00 & 0.00 & $\mathrm{H}$ \\
\hline 5.700 & 18.438 & -4.051 & 1.00 & 0.00 & C \\
\hline 7.066 & 18.324 & -4.533 & 1.00 & 0.00 & C \\
\hline 7.741 & 17.557 & -3.609 & 1.00 & 0.00 & C \\
\hline 6.791 & 17.205 & -2.573 & 1.00 & 0.00 & C \\
\hline 7.083 & 16.414 & -1.462 & 1.00 & 0.00 & C \\
\hline 8.097 & 16.024 & -1.402 & 1.00 & 0.00 & $\mathrm{H}$ \\
\hline 4.892 & 16.427 & -0.367 & 1.00 & 0.00 & $\mathrm{~N}$ \\
\hline 2.188 & 17.374 & -0.590 & 1.00 & 0.00 & $\mathrm{~N}$ \\
\hline 2.859 & 18.637 & -3.090 & 1.00 & 0.00 & $\mathrm{~N}$ \\
\hline 5.558 & 17.753 & -2.854 & 1.00 & 0.00 & $\mathrm{~N}$ \\
\hline 4.652 & 20.618 & -0.352 & 1.00 & 0.00 & C \\
\hline 4.492 & 19.304 & -0.365 & 1.00 & 0.00 & o \\
\hline 4.806 & 21.235 & 0.703 & 1.00 & 0.00 & 0 \\
\hline 4.635 & 21.299 & -1.709 & 1.00 & 0.00 & C \\
\hline 3.657 & 21.104 & -2.171 & 1.00 & 0.00 & $\mathrm{H}$ \\
\hline 5.383 & 20.809 & -2.352 & 1.00 & 0.00 & $\mathrm{H}$ \\
\hline 4.895 & 22.803 & -1.597 & 1.00 & 0.00 & C \\
\hline 5.820 & 22.959 & -1.027 & 1.00 & 0.00 & $\mathrm{H}$ \\
\hline 4.099 & 23.264 & -1.000 & 1.00 & 0.00 & $\mathrm{H}$ \\
\hline-0.625 & 28.515 & -8.933 & 1.00 & 0.00 & C \\
\hline-1.440 & 27.817 & -8.768 & 1.00 & 0.00 & $\mathrm{H}$ \\
\hline-0.958 & 29.740 & -9.335 & 1.00 & 0.00 & C \\
\hline-0.226 & 30.519 & -9.442 & 1.00 & 0.00 & $\mathrm{H}$ \\
\hline-1.999 & 30.010 & -9.501 & 1.00 & 0.00 & $\mathrm{H}$ \\
\hline 2.039 & 28.770 & -10.771 & 1.00 & 0.00 & C \\
\hline 2.274 & 29.841 & -10.652 & 1.00 & 0.00 & $\mathrm{H}$ \\
\hline 2.852 & 28.306 & -11.341 & 1.00 & 0.00 & $\mathrm{H}$ \\
\hline 1.131 & 28.693 & $-11 \cdot 382$ & 1.00 & 0.00 & $\mathrm{H}$ \\
\hline 7.049 & 27.092 & -10.256 & 1.00 & 0.00 & C \\
\hline 6.383 & 26.991 & -11.115 & 1.00 & 0.00 & $\mathrm{H}$ \\
\hline 8.216 & 27.737 & -10.415 & 1.00 & 0.00 & $\mathrm{C}$ \\
\hline 8.896 & 27.893 & -9.585 & 1.00 & 0.00 & $\mathrm{H}$ \\
\hline 8.521 & 28.135 & -11.381 & 1.00 & 0.00 & $\mathrm{H}$ \\
\hline 8.734 & 25.409 & -8.052 & 1.00 & 0.00 & C \\
\hline 9.325 & 26.073 & -7.403 & 1.00 & 0.00 & $\mathrm{H}$ \\
\hline 8.890 & 24.387 & -7.685 & 1.00 & 0.00 & $\mathrm{H}$ \\
\hline 9.166 & 25.475 & -9.057 & 1.00 & 0.00 & $\mathrm{H}$ \\
\hline-0.355 & 24.327 & -2.161 & 1.00 & 0.00 & C \\
\hline 0.384 & 24.316 & -1.349 & 1.00 & 0.00 & $\mathrm{H}$ \\
\hline-1.195 & 24.936 & -1.798 & 1.00 & 0.00 & $\mathrm{H}$ \\
\hline-0.833 & 22.890 & -2.434 & 1.00 & 0.00 & C \\
\hline-1.391 & 22.829 & -3.382 & 1.00 & 0.00 & $\mathrm{H}$ \\
\hline
\end{tabular}




\begin{tabular}{|c|c|c|c|c|c|c|c|c|c|c|}
\hline ATOM & 396 & $\mathrm{H} 42$ & $\mathrm{ACP}$ & 39 & 0.037 & 22.223 & -2.541 & 1.00 & 0.00 & $\mathrm{H}$ \\
\hline ATOM & 397 & $\mathrm{C} 1$ & $\mathrm{ACP}$ & 39 & -1.697 & 22.278 & -1.358 & 1.00 & 0.00 & C \\
\hline ATOM & 398 & $\mathrm{O} 2$ & $\mathrm{ACP}$ & 39 & -1.925 & 22.800 & -0.260 & 1.00 & 0.00 & 0 \\
\hline ATOM & 399 & 03 & $\mathrm{ACP}$ & 39 & -2.213 & 21.098 & -1.722 & 1.00 & 0.00 & o \\
\hline ATOM & 400 & H3 & $\mathrm{ACP}$ & 39 & -2.822 & 20.744 & -0.986 & 1.00 & 0.00 & $\mathrm{H}$ \\
\hline \multicolumn{11}{|l|}{ TER } \\
\hline ATOM & 401 & $\mathrm{C} 1$ & $\mathrm{MET}$ & 40 & 7.541 & 23.201 & -3.334 & 1.00 & 0.00 & $C$ \\
\hline ATOM & 402 & $\mathrm{H} 11$ & $\mathrm{MET}$ & 40 & 8.094 & 24.040 & -2.884 & 1.00 & 0.00 & 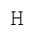 \\
\hline ATOM & 403 & $\mathrm{H} 12$ & MET & 40 & 7.548 & 22.375 & -2.613 & 1.00 & 0.00 & $\mathrm{H}$ \\
\hline ATOM & 404 & $\mathrm{H} 13$ & $\mathrm{MET}$ & 40 & 8.112 & 22.882 & -4.214 & 1.00 & 0.00 & $\mathrm{H}$ \\
\hline \multicolumn{11}{|l|}{ TER } \\
\hline ATOM & 405 & $\mathrm{C} 1$ & $\mathrm{MET}$ & 41 & -1.892 & 26.150 & -4.285 & 1.00 & 0.00 & $C$ \\
\hline ATOM & 406 & $\mathrm{H} 11$ & $\mathrm{MET}$ & 41 & -2.337 & 25.941 & -3.305 & 1.00 & 0.00 & $\mathrm{H}$ \\
\hline ATOM & 407 & $\mathrm{H} 12$ & $\mathrm{MET}$ & 41 & -2.002 & 27.230 & -4.460 & 1.00 & 0.00 & $\mathrm{H}$ \\
\hline ATOM & 408 & $\mathrm{H} 13$ & $\mathrm{MET}$ & 41 & -2.493 & 25.624 & -5.042 & 1.00 & 0.00 & $\mathrm{H}$ \\
\hline \multicolumn{11}{|l|}{ TER } \\
\hline ATOM & 409 & $\mathrm{C} 2$ & VIN & 42 & 7.905 & 14.771 & 1.002 & 1.00 & 0.00 & C \\
\hline ATOM & 410 & H 21 & VIN & 42 & 8.721 & 15.469 & 0.837 & 1.00 & 0.00 & $\mathrm{H}$ \\
\hline ATOM & 411 & $\mathrm{C} 1$ & VIN & 42 & 8.238 & 13.546 & 1.403 & 1.00 & 0.00 & C \\
\hline ATOM & 412 & $\mathrm{H} 11$ & VIN & 42 & 7.506 & 12.767 & 1.511 & 1.00 & 0.00 & $\mathrm{H}$ \\
\hline ATOM & 413 & $\mathrm{H} 12$ & VIN & 42 & 9.279 & 13.277 & 1.570 & 1.00 & 0.00 & $\mathrm{H}$ \\
\hline \multicolumn{11}{|l|}{ TER } \\
\hline ATOM & 414 & $\mathrm{C} 1$ & $\mathrm{MET}$ & 43 & 5.241 & 14.516 & 2.840 & 1.00 & 0.00 & C \\
\hline ATOM & 415 & $\mathrm{H} 11$ & MET & 43 & 5.006 & 13.445 & 2.720 & 1.00 & 0.00 & $\mathrm{H}$ \\
\hline ATOM & 416 & $\mathrm{H} 12$ & $\mathrm{MET}$ & 43 & 4.428 & 14.980 & 3.410 & 1.00 & 0.00 & $\mathrm{H}$ \\
\hline ATOM & 417 & $\mathrm{H} 13$ & $\mathrm{MET}$ & 43 & 6.149 & 14.593 & 3.450 & 1.00 & 0.00 & $\mathrm{H}$ \\
\hline \multicolumn{11}{|l|}{ TER } \\
\hline ATOM & 418 & $\mathrm{C} 2$ & VIN & 44 & 0.231 & 16.194 & 2.325 & 1.00 & 0.00 & C \\
\hline ATOM & 419 & H 21 & VIN & 44 & 0.897 & 16.295 & 3.184 & 1.00 & 0.00 & $\mathrm{H}$ \\
\hline ATOM & 420 & $\mathrm{C} 1$ & VIN & 44 & -0.936 & 15.550 & 2.484 & 1.00 & 0.00 & C \\
\hline ATOM & 421 & $\mathrm{H} 11$ & VIN & 44 & -1.616 & 15.393 & 1.654 & 1.00 & 0.00 & $\mathrm{H}$ \\
\hline ATOM & 422 & $\mathrm{H} 12$ & VIN & 44 & -1.241 & 15.151 & 3.450 & 1.00 & 0.00 & 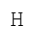 \\
\hline \multicolumn{11}{|l|}{ TER } \\
\hline Атом & 423 & $\mathrm{C} 1$ & MET & 45 & -1.454 & 17.877 & 0.121 & 1.00 & 0.00 & C \\
\hline ATOM & 424 & $\mathrm{H} 11$ & $\mathrm{MET}$ & 45 & -1.610 & 18.899 & -0.246 & 1.00 & 0.00 & $\mathrm{H}$ \\
\hline ATOM & 425 & $\mathrm{H} 12$ & $\mathrm{MET}$ & 45 & -1.886 & 17.811 & 1.126 & 1.00 & 0.00 & $\mathrm{H}$ \\
\hline ATOM & 426 & $\mathrm{H} 13$ & MET & 45 & -2.045 & 17.213 & -0.528 & 1.00 & 0.00 & $\mathrm{H}$ \\
\hline \multicolumn{11}{|l|}{ TER } \\
\hline ATOM & 427 & C5 & $\mathrm{ACP}$ & 46 & 7.635 & 18.959 & -5.770 & 1.00 & 0.00 & C \\
\hline ATOM & 428 & H51 & $\mathrm{ACP}$ & 46 & 6.896 & 18.970 & -6.582 & 1.00 & 0.00 & $\mathrm{H}$ \\
\hline ATOM & 429 & H52 & $\mathrm{ACP}$ & 46 & 8.475 & 18.350 & -6.134 & 1.00 & 0.00 & $\mathrm{H}$ \\
\hline ATOM & 430 & $\mathrm{C} 4$ & $\mathrm{ACP}$ & 46 & 8.113 & 20.396 & -5.497 & 1.00 & 0.00 & C \\
\hline ATOM & 431 & $\mathrm{H} 41$ & $\mathrm{ACP}$ & 46 & 8.671 & 20.457 & -4.549 & 1.00 & 0.00 & $\mathrm{H}$ \\
\hline ATOM & 432 & $\mathrm{H} 42$ & $\mathrm{ACP}$ & 46 & 7.243 & 21.063 & -5.390 & 1.00 & 0.00 & $\mathrm{H}$ \\
\hline ATOM & 433 & $\mathrm{C} 1$ & $\mathrm{ACP}$ & 46 & 8.977 & 21.008 & -6.573 & 1.00 & 0.00 & C \\
\hline ATOM & 434 & $\mathrm{O} 2$ & $\mathrm{ACP}$ & 46 & 9.205 & 20.486 & -7.671 & 1.00 & 0.00 & o \\
\hline ATOM & 435 & $\mathrm{O} 3$ & $\mathrm{ACP}$ & 46 & 9.493 & 22.188 & -6.209 & 1.00 & 0.00 & 0 \\
\hline ATOM & 436 & H3 & $\mathrm{ACP}$ & 46 & 10.102 & 22.542 & -6.945 & 1.00 & 0.00 & $\mathrm{H}$ \\
\hline \multicolumn{11}{|l|}{ TER } \\
\hline ATOM & 437 & $\mathrm{C} 1$ & $\mathrm{MET}$ & 47 & -0.261 & 20.085 & -4.597 & 1.00 & 0.00 & C \\
\hline ATOM & 438 & $\mathrm{H} 11$ & MET & 47 & -0.814 & 19.246 & -5.047 & 1.00 & 0.00 & $\mathrm{H}$ \\
\hline ATOM & 439 & $\mathrm{H} 12$ & MET & 47 & -0.268 & 20.911 & -5.319 & 1.00 & 0.00 & $\mathrm{H}$ \\
\hline ATOM & 440 & $\mathrm{H} 13$ & MET & 47 & -0.832 & 20.405 & -3.717 & 1.00 & 0.00 & $\mathrm{H}$ \\
\hline \multicolumn{11}{|l|}{ TER } \\
\hline ATOM & 441 & $\mathrm{C} 1$ & $\mathrm{MET}$ & 48 & 9.172 & 17.136 & -3.646 & 1.00 & 0.00 & C \\
\hline ATOM & 442 & $\mathrm{H} 11$ & MET & 48 & 9.617 & 17.345 & -4.626 & 1.00 & 0.00 & $\mathrm{H}$ \\
\hline ATOM & 443 & $\mathrm{H} 12$ & MET & 48 & 9.283 & 16.056 & -3.471 & 1.00 & 0.00 & $\mathrm{H}$ \\
\hline ATOM & 444 & $\mathrm{H} 13$ & MET & 48 & 9.773 & 17.662 & -2.889 & 1.00 & 0.00 & 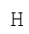 \\
\hline \multicolumn{11}{|l|}{ TER } \\
\hline END & & & & & & & & & & \\
\hline
\end{tabular}

\subsection{T4 dimer: AYR_T4.pdb file}

\begin{tabular}{|c|c|c|c|c|c|c|c|c|c|}
\hline HETATM & 1 & $\mathrm{FE}$ & HEM & 1 & 4.263 & 10.757 & 1.491 & 1.00 & 0.00 \\
\hline HETATM & 2 & $\mathrm{C} 1$ & HEM & 1 & 2.035 & 12.629 & 0.429 & 1.00 & 0.00 \\
\hline HETATM & 3 & C2 & HEM & 1 & 1.676 & 13.420 & -0.742 & 1.00 & 0.00 \\
\hline HETATM & 4 & C3 & HEM & 1 & 2.822 & 13.517 & -1.517 & 1.00 & 0.00 \\
\hline HETATM & 5 & $\mathrm{C} 4$ & HEM & 1 & 3.851 & 12.800 & -0.809 & 1.00 & 0.00 \\
\hline HETATM & 6 & C5 & HEM & 1 & 5.167 & 12.681 & -1.247 & 1.00 & 0.00 \\
\hline HETATM & 7 & H5 & HEM & 1 & 5.420 & 13.177 & -2.182 & 1.00 & 0.00 \\
\hline HETATM & 8 & $\mathrm{C} 6$ & HEM & 1 & 6.186 & 11.982 & -0.611 & 1.00 & 0.00 \\
\hline HETATM & 9 & C7 & HEM & 1 & 7.555 & 11.910 & -1.081 & 1.00 & 0.00 \\
\hline HETATM & 10 & $\mathrm{C} 8$ & HEM & 1 & 8.260 & 11.190 & -0.134 & 1.00 & 0.00 \\
\hline HETATM & 11 & C9 & HEM & 1 & 7.309 & 10.825 & 0.891 & 1.00 & 0.00 \\
\hline HETATM & 12 & C10 & HEM & 1 & 7.614 & 10.090 & 2.034 & 1.00 & .00 \\
\hline HETATM & 13 & H1O & HEM & 1 & 8.641 & 9.746 & 2.140 & 1.00 & .00 \\
\hline HETATM & 14 & $\mathrm{C} 11$ & HEM & 1 & 6.739 & 9.750 & 3.057 & 1.00 & 0.00 \\
\hline HETATM & 15 & $\mathrm{C} 12$ & HEM & 1 & 7.120 & 9.025 & 4.247 & 1.00 & 0.00 \\
\hline
\end{tabular}




\begin{tabular}{|c|c|c|c|}
\hline HETATM & 16 & C13 & HEM \\
\hline HETATM & 17 & C14 & HEM \\
\hline HETATM & 18 & C15 & HEM \\
\hline HETATM & 19 & H15 & HEM \\
\hline HETATM & 20 & C16 & HEM \\
\hline HETATM & 21 & C17 & HEM \\
\hline HETATM & 22 & C18 & HEM \\
\hline HETATM & 23 & C19 & HEM \\
\hline HETATM & 24 & C20 & HEM \\
\hline HETATM & 25 & H2O & HEM \\
\hline HETATM & 26 & N21 & HEM \\
\hline HETATM & 27 & N22 & HEM \\
\hline HETATM & 28 & N23 & HEM \\
\hline HETATM & 29 & N24 & HEM \\
\hline HETATM & 30 & C99 & HEM \\
\hline HETATM & 31 & 091 & HEM \\
\hline HETATM & 32 & 092 & HEM \\
\hline HETATM & 33 & СТ91 & HEM \\
\hline HETATM & 34 & H911 & HEM \\
\hline HETATM & 35 & H912 & HEM \\
\hline HETATM & 36 & СТ92 & HEM \\
\hline HETATM & 37 & H921 & HEM \\
\hline HETATM & 38 & H922 & HEM \\
\hline \multicolumn{4}{|l|}{ TER } \\
\hline HETATM & 39 & $\mathrm{FE}$ & HEM \\
\hline HETATM & 40 & C1 & HEM \\
\hline HETATM & 41 & C2 & HEM \\
\hline HETATM & 42 & C3 & HEM \\
\hline HETATM & 43 & $\mathrm{C} 4$ & HEM \\
\hline HETATM & 44 & $\mathrm{C} 5$ & HEM \\
\hline HETATM & 45 & H5 & HEM \\
\hline HETATM & 46 & C6 & HEM \\
\hline HETATM & 47 & C7 & HEM \\
\hline HETATM & 48 & C8 & HEM \\
\hline HETATM & 49 & C9 & HEM \\
\hline HETATM & 50 & C10 & HEM \\
\hline HETATM & 51 & H10 & HEM \\
\hline HETATM & 52 & C11 & HEM \\
\hline HETATM & 53 & C12 & HEM \\
\hline HETATM & 54 & C13 & HEM \\
\hline HETATM & 55 & C14 & HEM \\
\hline HETATM & 56 & C15 & HEM \\
\hline HETATM & 57 & H15 & HEM \\
\hline HETATM & 58 & C16 & HEM \\
\hline HETATM & 59 & C17 & HEM \\
\hline HETATM & 60 & C18 & HEM \\
\hline HETATM & 61 & C19 & HEM \\
\hline HETATM & 62 & C20 & HEM \\
\hline HETATM & 63 & H2O & HEM \\
\hline HETATM & 64 & N21 & HEM \\
\hline HETATM & 65 & N22 & HEM \\
\hline HETATM & 66 & N23 & HEM \\
\hline HETATM & 67 & N24 & HEM \\
\hline HETATM & 68 & C99 & HEM \\
\hline HETATM & 69 & 091 & HEM \\
\hline HETATM & 70 & 092 & HEM \\
\hline HETATM & 71 & СТ91 & HEM \\
\hline HETATM & 72 & H911 & HEM \\
\hline HETATM & 73 & H912 & HEM \\
\hline HETATM & 74 & СТ92 & HEM \\
\hline HETATM & 75 & H921 & HEM \\
\hline HETATM & 76 & H922 & HEM \\
\hline \multicolumn{4}{|l|}{ TER } \\
\hline HETATM & 77 & $\mathrm{C} 2$ & VIN \\
\hline HETATM & 78 & H 21 & VIN \\
\hline HETATM & 79 & $\mathrm{C} 1$ & VIN \\
\hline HETATM & 80 & H11 & VIN \\
\hline HETATM & 81 & H12 & VIN \\
\hline \multicolumn{4}{|l|}{ TER } \\
\hline HETATM & 82 & $\mathrm{C} 1$ & MET \\
\hline HETATM & 83 & H11 & MET \\
\hline HETATM & 84 & $\mathrm{H} 12$ & $\mathrm{MET}$ \\
\hline HETATM & 85 & H13 & $\mathrm{MET}$ \\
\hline \multicolumn{4}{|l|}{ TER } \\
\hline HETATM & 86 & C2 & VIN \\
\hline HETATM & 87 & H21 & VIN \\
\hline HETATM & 88 & $\mathrm{C} 1$ & VIN \\
\hline HETATM & 89 & H11 & VIN \\
\hline HETATM & 90 & & VIN \\
\hline
\end{tabular}

\begin{tabular}{|c|c|c|c|c|c|}
\hline 5.981 & 8.878 & 5.004 & 1.00 & 0.00 & C \\
\hline 4.908 & 9.519 & 4.273 & 1.00 & 0.00 & C \\
\hline 3.580 & 9.574 & 4.694 & 1.00 & 0.00 & C \\
\hline 3.340 & 9.089 & 5.642 & 1.00 & 0.00 & $\mathrm{H}$ \\
\hline 2.550 & 10.255 & 4.051 & 1.00 & 0.00 & C \\
\hline 1.184 & 10.369 & 4.533 & 1.00 & 0.00 & C \\
\hline 0.508 & 11.137 & 3.609 & 1.00 & 0.00 & $\mathrm{C}$ \\
\hline 1.458 & 11.489 & 2.573 & 1.00 & 0.00 & C \\
\hline 1.167 & 12.280 & 1.462 & 1.00 & 0.00 & C \\
\hline 0.152 & 12.669 & 1.402 & 1.00 & 0.00 & $\mathrm{H}$ \\
\hline 3.357 & 12.266 & 0.367 & 1.00 & 0.00 & $\mathrm{~N}$ \\
\hline 6.062 & 11.319 & 0.590 & 1.00 & 0.00 & $\mathrm{~N}$ \\
\hline 5.391 & 10.056 & 3.090 & 1.00 & 0.00 & $\mathrm{~N}$ \\
\hline 2.691 & 10.941 & 2.854 & 1.00 & 0.00 & $\mathrm{~N}$ \\
\hline 3.598 & 8.076 & 0.352 & 1.00 & 0.00 & $\mathrm{C}$ \\
\hline 3.757 & 9.389 & 0.365 & 1.00 & 0.00 & O \\
\hline 3.443 & 7.458 & -0.703 & 1.00 & 0.00 & O \\
\hline 3.614 & 7.394 & 1.709 & 1.00 & 0.00 & C \\
\hline 4.593 & 7.590 & 2.171 & 1.00 & 0.00 & $\mathrm{H}$ \\
\hline 2.866 & 7.884 & 2.352 & 1.00 & 0.00 & $\mathrm{H}$ \\
\hline 3.354 & 5.890 & 1.597 & 1.00 & 0.00 & $\mathrm{C}$ \\
\hline 4.151 & 5.429 & 1.000 & 1.00 & 0.00 & $\mathrm{H}$ \\
\hline 2.429 & 5.734 & 1.027 & 1.00 & 0.00 & $\mathrm{H}$ \\
\hline 4.955 & 3.343 & 6.440 & 1.00 & 0.00 & $\mathrm{FE}$ \\
\hline 7.183 & 1.471 & 7.502 & 1.00 & 0.00 & C \\
\hline 7.542 & 0.680 & 8.674 & 1.00 & 0.00 & C \\
\hline 6.397 & 0.583 & 9.448 & 1.00 & 0.00 & C \\
\hline 5.367 & 1.301 & 8.740 & 1.00 & 0.00 & C \\
\hline 4.052 & 1.420 & 9.178 & 1.00 & 0.00 & $\mathrm{C}$ \\
\hline 3.798 & 0.923 & 10.113 & 1.00 & 0.00 & $\mathrm{H}$ \\
\hline 3.033 & 2.118 & 8.542 & 1.00 & 0.00 & C \\
\hline 1.664 & 2.191 & 9.012 & 1.00 & 0.00 & $\mathrm{C}$ \\
\hline 0.958 & 2.910 & 8.065 & 1.00 & 0.00 & $\mathrm{C}$ \\
\hline 1.909 & 3.275 & 7.041 & 1.00 & 0.00 & C \\
\hline 1.605 & 4.010 & 5.897 & 1.00 & 0.00 & C \\
\hline 0.578 & 4.354 & 5.791 & 1.00 & 0.00 & $\mathrm{H}$ \\
\hline 2.479 & 4.350 & 4.874 & 1.00 & 0.00 & C \\
\hline 2.098 & 5.076 & 3.684 & 1.00 & 0.00 & C \\
\hline 3.238 & 5.223 & 2.927 & 1.00 & 0.00 & $\mathrm{C}$ \\
\hline 4.311 & 4.581 & 3.659 & 1.00 & 0.00 & C \\
\hline 5.639 & 4.527 & 3.237 & 1.00 & 0.00 & C \\
\hline 5.879 & 5.011 & 2.289 & 1.00 & 0.00 & $\mathrm{H}$ \\
\hline 6.669 & 3.845 & 3.880 & 1.00 & 0.00 & C \\
\hline 8.035 & 3.732 & 3.398 & 1.00 & 0.00 & C \\
\hline 8.710 & 2.964 & 4.322 & 1.00 & 0.00 & C \\
\hline 7.761 & 2.612 & 5.358 & 1.00 & 0.00 & C \\
\hline 8.052 & 1.821 & 6.469 & 1.00 & 0.00 & C \\
\hline 9.066 & 1.432 & 6.530 & 1.00 & 0.00 & $\mathrm{H}$ \\
\hline 5.862 & 1.834 & 7.564 & 1.00 & 0.00 & $\mathrm{~N}$ \\
\hline 3.157 & 2.781 & 7.342 & 1.00 & 0.00 & $\mathrm{~N}$ \\
\hline 3.828 & 4.044 & 4.841 & 1.00 & 0.00 & $\mathrm{~N}$ \\
\hline 6.527 & 3.160 & 5.077 & 1.00 & 0.00 & $\mathrm{~N}$ \\
\hline 5.621 & 6.025 & 7.579 & 1.00 & 0.00 & C \\
\hline 5.461 & 4.711 & 7.567 & 1.00 & 0.00 & 0 \\
\hline 5.776 & 6.642 & 8.635 & 1.00 & 0.00 & O \\
\hline 5.605 & 6.706 & 6.223 & 1.00 & 0.00 & $\mathrm{C}$ \\
\hline 4.626 & 6.511 & 5.760 & 1.00 & 0.00 & $\mathrm{H}$ \\
\hline 6.353 & 6.216 & 5.580 & 1.00 & 0.00 & $\mathrm{H}$ \\
\hline 5.864 & 8.211 & 6.334 & 1.00 & 0.00 & $\mathrm{C}$ \\
\hline 6.789 & 8.367 & 6.905 & 1.00 & 0.00 & $\mathrm{H}$ \\
\hline 5.068 & 8.671 & 6.931 & 1.00 & 0.00 & $\mathrm{H}$ \\
\hline 0.345 & 13.923 & -1.002 & 1.00 & 0.00 & $\mathrm{C}$ \\
\hline-0.471 & 13.224 & -0.837 & 1.00 & 0.00 & $\mathrm{H}$ \\
\hline 0.012 & 15.147 & -1.403 & 1.00 & 0.00 & C \\
\hline 0.743 & 15.926 & -1.511 & 1.00 & 0.00 & $\mathrm{H}$ \\
\hline-1.030 & 15.417 & -1.570 & 1.00 & 0.00 & $\mathrm{H}$ \\
\hline 3.008 & 14.177 & -2.840 & 1.00 & 0.00 & $\mathrm{C}$ \\
\hline 3.243 & 15.248 & -2.720 & 1.00 & 0.00 & $\mathrm{H}$ \\
\hline 3.821 & 13.713 & -3.410 & 1.00 & 0.00 & $\mathrm{H}$ \\
\hline 2.101 & 14.100 & -3.450 & 1.00 & 0.00 & $\mathrm{H}$ \\
\hline 8.018 & 12.499 & -2.325 & 1.00 & 0.00 & C \\
\hline 7.352 & 12.399 & -3.184 & 1.00 & 0.00 & $\mathrm{H}$ \\
\hline 9.185 & 13.144 & -2.484 & 1.00 & 0.00 & C \\
\hline 9.865 & 13.300 & -1.654 & 1.00 & 0.00 & $\mathrm{H}$ \\
\hline 9.490 & 13.542 & -3.450 & 1.00 & 0.00 & $\mathrm{H}$ \\
\hline
\end{tabular}


TER

$\begin{array}{lrrl}\text { HETATM } & 91 & \text { C1 } & \text { MET } \\ \text { HETATM } & 92 & \text { H11 } & \text { MET } \\ \text { HETATM } & 93 & \text { H12 } & \text { MET } \\ \text { HETATM } & 94 & \text { H13 } & \text { MET } \\ \text { TER } & & & \\ \text { HETATM } & 95 & \text { C5 } & \text { ACP } \\ \text { HETATM } & 96 & \text { H51 } & \text { ACP } \\ \text { HETATM } & 97 & \text { H52 } & \text { ACP } \\ \text { HETATM } & 98 & \text { C4 } & \text { ACP } \\ \text { HETATM } & 99 & \text { H41 } & \text { ACP } \\ \text { HETATM } & 100 & \text { H42 } & \text { ACP } \\ \text { HETATM } & 101 & \text { C1 } & \text { ACP } \\ \text { HETATM } & 102 & \text { O2 } & \text { ACP } \\ \text { HETATM } & 103 & \text { O3 } & \text { ACP } \\ \text { HETATM } & 104 & \text { H3 } & \text { ACP } \\ \text { TER } & & & \\ \text { HETATM } & 105 & \text { C1 } & \text { MET } \\ \text { HETATM } & 106 & \text { H11 } & \text { MET } \\ \text { HETATM } & 107 & \text { H12 } & \text { MET } \\ \text { HETATM } & 108 & \text { H13 } & \text { MET } \\ \text { TER } & & & \end{array}$

TER

HETATM 109 C1 MET HETATM 110 H11 MET HETATM 111 H12 MET HETATM 112 H13 MET TER

HETATM 113 C2 VIN HETATM 114 H21 VIN HETATM 115 C1 VIN HETATM 116 H11 VIN HETATM 117 H12 VIN TER

HETATM 118 C1 MET HETATM 119 H11 MET HETATM 120 H12 MET HETATM 121 H13 MET TER

HETATM 122 C2 VIN HETATM 123 H21 VIN HETATM 124 C1 VIN HETATM 125 H11 VIN HETATM 126 H12 VIN TER

HETATM 127 C1 MET HETATM 128 H11 MET HETATM 129 H12 MET HETATM 130 H13 MET TER

HETATM 131 C5 ACP HETATM 132 H51 ACP HETATM 133 H52 ACP HETATM $134 \quad \mathrm{C} 4$ ACP HETATM 135 H41 ACP HETATM 136 H42 ACP HETATM 137 C1 ACP HETATM $138 \quad 02$ ACP HETATM 139 O3 ACP HETATM 140 H3 ACP TER

HETATM 141 C1 MET HETATM 142 H11 MET HETATM 143 H12 MET HETATM 144 H13 MET TER

HETATM 145 C1 MET HETATM 146 H11 MET HETATM 147 H12 MET HETATM 148 H13 MET TER

HETATM 149 FE HEM 17 HETATM $150 \quad$ C1 HEM 17 HETATM 151 C2 HEM 17 HETATM 152 C3 HEM 17 HETATM 153 C4 HEM 17 HETATM 154 C5 HEM 17 HETATM 155 H5 HEM 17 HETATM 156 C6 HEM 17

C7 HEM

\begin{tabular}{|c|c|c|c|c|c|}
\hline 6 & 9.703 & 10.816 & -0.121 & 1.00 & 0.00 \\
\hline 6 & 10.294 & 11.480 & 0.528 & 1.00 & 0.00 \\
\hline 6 & 9.859 & 9.794 & 0.246 & 1.00 & 0.00 \\
\hline 6 & 10.135 & 10.882 & -1.126 & 1.00 & 0.00 \\
\hline 7 & 0.615 & 9.735 & 5.770 & 1.00 & 0.00 \\
\hline 7 & 1.354 & 9.723 & 6.582 & 1.00 & 0.00 \\
\hline 7 & -0.226 & 10.343 & 6.134 & 1.00 & 0.00 \\
\hline 7 & 0.136 & 8.297 & 5.497 & 1.00 & 0.00 \\
\hline 7 & -0.422 & 8.236 & 4.549 & 1.00 & 0.00 \\
\hline 7 & 1.007 & 7.630 & 5.390 & 1.00 & 0.00 \\
\hline 7 & -0.728 & 7.685 & 6.573 & 1.00 & 0.00 \\
\hline 7 & -0.956 & 8.208 & 7.671 & 1.00 & 0.00 \\
\hline 7 & -1.244 & 6.505 & 6.209 & 1.00 & 0.00 \\
\hline 7 & -1.853 & 6.151 & 6.945 & 1.00 & 0.00 \\
\hline 8 & 8.511 & 8.608 & 4.597 & 1.00 & 0.00 \\
\hline 8 & 9.063 & 9.448 & 5.047 & 1.00 & 0.00 \\
\hline 8 & 8.517 & 7.782 & 5.319 & 1.00 & 0.00 \\
\hline 8 & 9.082 & 8.289 & 3.717 & 1.00 & 0.00 \\
\hline 9 & -0.923 & 11.557 & 3.646 & 1.00 & 0.00 \\
\hline 9 & $-1 \cdot 368$ & 11.348 & 4.626 & 1.00 & 0.00 \\
\hline 9 & -1.033 & 12.637 & 3.471 & 1.00 & 0.00 \\
\hline 9 & -1.523 & 11.031 & 2.889 & 1.00 & 0.00 \\
\hline 0 & 8.874 & 0.178 & 8.933 & 1.00 & 0.00 \\
\hline 0 & 9.690 & 0.876 & 8.768 & 1.00 & 0.00 \\
\hline 0 & 9.207 & -1.047 & 9.335 & 1.00 & 0.00 \\
\hline 0 & 8.475 & -1.826 & 9.442 & 1.00 & 0.00 \\
\hline 0 & 10.249 & -1.316 & 9.501 & 1.00 & 0.00 \\
\hline 1 & 6.210 & -0.077 & 10.771 & 1.00 & 0.00 \\
\hline 1 & 5.975 & -1.148 & 10.652 & 1.00 & 0.00 \\
\hline 1 & 5.397 & 0.387 & 11.341 & 1.00 & 0.00 \\
\hline 1 & 7.118 & 0.001 & 11.382 & 1.00 & 0.00 \\
\hline 2 & 1.200 & 1.601 & 10.256 & 1.00 & 0.00 \\
\hline 2 & 1.866 & 1.702 & 11.115 & 1.00 & 0.00 \\
\hline 2 & 0.033 & 0.957 & 10.415 & 1.00 & 0.00 \\
\hline 2 & -0.647 & 0.800 & 9.585 & 1.00 & 0.00 \\
\hline & -0.272 & 0.559 & 11.381 & 1.00 & 0.00 \\
\hline 3 & -0.484 & 3.284 & 8.052 & 1.00 & 0.00 \\
\hline 3 & -0.641 & 4.306 & 7.685 & 1.00 & 0.00 \\
\hline 3 & -0.917 & 3.219 & 9.057 & 1.00 & 0.00 \\
\hline & -1.076 & 2.620 & 7.403 & 1.00 & 0.00 \\
\hline & 8.604 & 4.366 & 2.161 & 1.00 & 0.00 \\
\hline & 7.865 & 4.377 & 1.349 & 1.00 & 0.00 \\
\hline & 9.445 & 3.758 & 1.798 & 1.00 & 0.00 \\
\hline & 9.082 & 5.803 & 2.434 & 1.00 & 0.00 \\
\hline & 9.640 & 5.865 & 3.382 & 1.00 & 0.00 \\
\hline & 8.212 & 6.470 & 2.541 & 1.00 & 0.00 \\
\hline & 9.946 & 6.416 & 1.358 & 1.00 & 0.00 \\
\hline & 10.174 & 5.893 & 0.260 & 1.00 & 0.00 \\
\hline & 10.462 & 7.595 & 1.722 & 1.00 & 0.00 \\
\hline & 11.071 & 7.949 & 0.986 & 1.00 & 0.00 \\
\hline & 0.708 & 5.492 & 3.334 & 1.00 & 0.00 \\
\hline & 0.155 & 4.653 & 2.884 & 1.00 & 0.00 \\
\hline & 0.701 & 6.318 & 2.613 & 1.00 & 0.00 \\
\hline & 0.137 & 5.812 & 4.214 & 1.00 & 0.00 \\
\hline & 10.141 & 2.543 & 4.285 & 1.00 & 0.00 \\
\hline & 10.586 & 2.752 & 3.305 & 1.00 & 0.00 \\
\hline & 10.252 & 1.463 & 4.460 & 1.00 & 0.00 \\
\hline & 10.742 & 3.069 & 5.042 & 1.00 & 0.00 \\
\hline & 5.211 & 11.003 & -6.440 & 1.00 & 0.00 \\
\hline & 2.983 & 12.876 & -7.502 & 1.00 & 0.00 \\
\hline & 2.624 & 13.666 & -8.674 & 1.00 & 0.00 \\
\hline & 3.770 & 13.763 & -9.448 & 1.00 & 0.00 \\
\hline & 4.799 & 13.046 & -8.740 & 1.00 & 0.00 \\
\hline & 6.114 & 12.927 & -9.178 & 1.00 & 0.00 \\
\hline & 6.368 & 13.423 & -10.113 & 1.00 & 0.00 \\
\hline & 7.134 & 12.229 & -8.542 & 1.00 & 0.00 \\
\hline & 8.502 & 12.156 & -9.012 & 1.00 & 0.00 \\
\hline
\end{tabular}




\begin{tabular}{|c|c|c|c|}
\hline HETATM & 158 & C8 & HEM \\
\hline HETATM & 159 & C9 & HEM \\
\hline HETATM & 160 & C10 & HEM \\
\hline HETATM & 161 & H1O & HEM \\
\hline HETATM & 162 & C11 & HEM \\
\hline HETATM & 163 & C12 & HEM \\
\hline HETATM & 164 & C13 & HEM \\
\hline HETATM & 165 & C14 & HEM \\
\hline HETATM & 166 & C15 & HEM \\
\hline HETATM & 167 & H15 & HEM \\
\hline HETATM & 168 & C16 & HEM \\
\hline HETATM & 169 & C17 & HEM \\
\hline HETATM & 170 & C18 & HEM \\
\hline HETATM & 171 & C19 & HEM \\
\hline HETATM & 172 & C20 & HEM \\
\hline HETATM & 173 & $\mathrm{H} 2 \mathrm{O}$ & HEM \\
\hline HETATM & 174 & N21 & HEM \\
\hline HETATM & 175 & N22 & HEM \\
\hline HETATM & 176 & N23 & HEM \\
\hline HETATM & 177 & N24 & HEM \\
\hline HETATM & 178 & C99 & HEM \\
\hline HETATM & 179 & 091 & HEM \\
\hline HETATM & 180 & 092 & HEM \\
\hline HETATM & 181 & СТ91 & HEM \\
\hline HETATM & 182 & H911 & HEM \\
\hline HETATM & 183 & H912 & HEM \\
\hline HETATM & 184 & CT92 & HEM \\
\hline HETATM & 185 & H921 & HEM \\
\hline HETATM & 186 & H922 & HEM \\
\hline \multicolumn{4}{|l|}{ TER } \\
\hline HETATM & 187 & $\mathrm{FE}$ & HEM \\
\hline HETATM & 188 & $\mathrm{C} 1$ & HEM \\
\hline HETATM & 189 & C2 & HEM \\
\hline HETATM & 190 & C3 & HEM \\
\hline HETATM & 191 & C4 & HEM \\
\hline HETATM & 192 & C5 & HEM \\
\hline HETATM & 193 & H5 & HEM \\
\hline HETATM & 194 & $\mathrm{C} 6$ & HEM \\
\hline HETATM & 195 & C7 & HEM \\
\hline HETATM & 196 & C8 & HEM \\
\hline HETATM & 197 & C9 & HEM \\
\hline HETATM & 198 & C10 & HEM \\
\hline HETATM & 199 & $\mathrm{H} 10$ & HEM \\
\hline HETATM & 200 & C11 & HEM \\
\hline HETATM & 201 & C12 & HEM \\
\hline HETATM & 202 & C13 & HEM \\
\hline HETATM & 203 & C14 & HEM \\
\hline HETATM & 204 & C15 & HEM \\
\hline HETATM & 205 & H15 & HEM \\
\hline HETATM & 206 & C16 & HEM \\
\hline HETATM & 207 & C17 & HEM \\
\hline HETATM & 208 & C18 & HEM \\
\hline HETATM & 209 & C19 & HEM \\
\hline HETATM & 210 & $\mathrm{C} 20$ & HEM \\
\hline HETATM & 211 & $\mathrm{H} 2 \mathrm{O}$ & HEM \\
\hline HETATM & 212 & N21 & HEM \\
\hline HETATM & 213 & $\mathrm{~N} 22$ & HEM \\
\hline HETATM & 214 & N23 & HEM \\
\hline HETATM & 215 & $\mathrm{~N} 24$ & HEM \\
\hline HETATM & 216 & C99 & HEM \\
\hline HETATM & 217 & 091 & HEM \\
\hline HETATM & 218 & 092 & HEM \\
\hline HETATM & 219 & CT91 & HEM \\
\hline HETATM & 220 & H911 & HEM \\
\hline HETATM & 221 & H912 & HEM \\
\hline HETATM & 222 & CT 92 & HEM \\
\hline HETATM & 223 & H921 & HEM \\
\hline HETATM & 224 & H922 & HEM \\
\hline \multicolumn{4}{|l|}{ TER } \\
\hline HETATM & 225 & C2 & VIN \\
\hline HETATM & 226 & H 21 & VIN \\
\hline HETATM & 227 & C1 & VIN \\
\hline HETATM & 228 & H11 & VIN \\
\hline HETATM & 229 & H12 & VIN \\
\hline \multicolumn{4}{|l|}{ TER } \\
\hline HETATM & 230 & $\mathrm{C} 1$ & MET \\
\hline HETATM & 231 & H11 & MET \\
\hline HETATM & 232 & H12 & MET \\
\hline $\mathrm{HET}$ & 233 & & MET \\
\hline
\end{tabular}

\begin{tabular}{|c|c|c|c|c|}
\hline 9.208 & 11.436 & -8.065 & 1.00 & 0.00 \\
\hline 8.257 & 11.071 & -7.041 & 1.00 & 0.00 \\
\hline 8.561 & 10.336 & -5.897 & 1.00 & 0.00 \\
\hline 9.588 & 9.993 & -5.791 & 1.00 & 0.00 \\
\hline 7.687 & 9.996 & -4.874 & 1.00 & 0.00 \\
\hline 8.068 & 9.271 & -3.684 & 1.00 & 0.00 \\
\hline 6.928 & 9.124 & -2.927 & 1.00 & 0.00 \\
\hline 5.855 & 9.765 & -3.659 & 1.00 & 0.00 \\
\hline 4.527 & 9.820 & -3.237 & 1.00 & 0.00 \\
\hline 4.287 & 9.335 & -2.289 & 1.00 & 0.00 \\
\hline 3.497 & 10.502 & -3.880 & 1.00 & 0.00 \\
\hline 2.131 & 10.615 & -3.398 & 1.00 & 0.00 \\
\hline 1.456 & 11.383 & -4.322 & 1.00 & 0.00 \\
\hline 2.406 & 11.735 & -5.358 & 1.00 & 0.00 \\
\hline 2.114 & 12.526 & -6.469 & 1.00 & 0.00 \\
\hline 1.100 & 12.915 & -6.530 & 1.00 & 0.00 \\
\hline 4.305 & 12.513 & -7.564 & 1.00 & 0.00 \\
\hline 7.009 & 11.566 & -7.342 & 1.00 & 0.00 \\
\hline 6.338 & 10.303 & -4.841 & 1.00 & 0.00 \\
\hline 3.639 & 11.187 & -5.077 & 1.00 & 0.00 \\
\hline 4.545 & 8.322 & -7.579 & 1.00 & 0.00 \\
\hline 4.705 & 9.635 & -7.567 & 1.00 & 0.00 \\
\hline 4.391 & 7.705 & -8.635 & 1.00 & 0.00 \\
\hline 4.561 & 7.640 & -6.223 & 1.00 & 0.00 \\
\hline 5.540 & 7.836 & -5.760 & 1.00 & 0.00 \\
\hline 3.813 & 8.130 & -5.580 & 1.00 & 0.00 \\
\hline 4.302 & 6.136 & -6.334 & 1.00 & 0.00 \\
\hline 5.098 & 5.676 & -6.931 & 1.00 & 0.00 \\
\hline 3.377 & 5.980 & -6.905 & 1.00 & 0.00 \\
\hline 5.903 & 3.589 & -1.491 & 1.00 & 0.00 \\
\hline 8.131 & 1.717 & -0.429 & 1.00 & 0.00 \\
\hline 8.490 & 0.927 & 0.742 & 1.00 & 0.00 \\
\hline 7.344 & 0.830 & 1.517 & 1.00 & 0.00 \\
\hline 6.315 & 1.547 & 0.809 & 1.00 & 0.00 \\
\hline 5.000 & 1.666 & 1.247 & 1.00 & 0.00 \\
\hline 4.746 & 1.170 & 2.182 & 1.00 & 0.00 \\
\hline 3.980 & 2.364 & 0.611 & 1.00 & 0.00 \\
\hline 2.611 & 2.437 & 1.081 & 1.00 & 0.00 \\
\hline 1.906 & 3.157 & 0.134 & 1.00 & 0.00 \\
\hline 2.857 & 3.522 & -0.891 & 1.00 & 0.00 \\
\hline 2.552 & 4.256 & -2.034 & 1.00 & 0.00 \\
\hline 1.526 & 4.600 & -2.140 & 1.00 & 0.00 \\
\hline 3.427 & 4.596 & -3.057 & 1.00 & 0.00 \\
\hline 3.046 & 5.322 & -4.247 & 1.00 & 0.00 \\
\hline 4.185 & 5.469 & -5.004 & 1.00 & 0.00 \\
\hline 5.258 & 4.828 & -4.273 & 1.00 & 0.00 \\
\hline 6.586 & 4.773 & -4.694 & 1.00 & 0.00 \\
\hline 6.826 & 5.258 & -5.642 & 1.00 & 0.00 \\
\hline 7.617 & 4.091 & -4.051 & 1.00 & 0.00 \\
\hline 8.982 & 3.978 & -4.533 & 1.00 & 0.00 \\
\hline 9.658 & 3.210 & -3.609 & 1.00 & 0.00 \\
\hline 8.708 & 2.858 & -2.573 & 1.00 & 0.00 \\
\hline 8.999 & 2.067 & -1.462 & 1.00 & 0.00 \\
\hline 10.014 & 1.678 & -1.402 & 1.00 & 0.00 \\
\hline 6.809 & 2.080 & -0.367 & 1.00 & 0.00 \\
\hline 4.105 & 3.027 & -0.590 & 1.00 & 0.00 \\
\hline 4.775 & 4.290 & -3.090 & 1.00 & 0.00 \\
\hline 7.475 & 3.406 & -2.854 & 1.00 & 0.00 \\
\hline 6.568 & 6.271 & -0.352 & 1.00 & 0.00 \\
\hline 6.409 & 4.958 & -0.365 & 1.00 & 0.00 \\
\hline 6.723 & 6.888 & 0.703 & 1.00 & 0.00 \\
\hline 6.552 & 6.952 & -1.709 & 1.00 & 0.00 \\
\hline 5.573 & 6.757 & -2.171 & 1.00 & 0.00 \\
\hline 7.300 & 6.463 & -2.352 & 1.00 & 0.00 \\
\hline 6.812 & 8.457 & -1.597 & 1.00 & 0.00 \\
\hline 7.737 & 8.613 & -1.027 & 1.00 & 0.00 \\
\hline 6.015 & 8.917 & -1.000 & 1.00 & 0.00 \\
\hline 1.292 & 14.169 & -8.933 & 1.00 & 0.00 \\
\hline 0.476 & 13.470 & -8.768 & 1.00 & 0.00 \\
\hline 0.959 & 15.394 & -9.335 & 1.00 & 0.00 \\
\hline 1.691 & 16.172 & -9.442 & 1.00 & 0.00 \\
\hline-0.082 & 15.663 & -9.501 & 1.00 & 0.00 \\
\hline 3.956 & 14.424 & -10.771 & 1.00 & 0.00 \\
\hline 4.191 & 15.494 & -10.652 & 1.00 & 0.00 \\
\hline 4.769 & 13.959 & $-11 \cdot 341$ & 1.00 & 0.00 \\
\hline 3.048 & 14.346 & -11.382 & 1.00 & 0.00 \\
\hline
\end{tabular}


TER

HETATM 234 C2 VIN 21 HETATM 235 H21 VIN HETATM 236 C1 VIN HETATM 237 H11 VIN HETATM 238 H12 VIN $T E R$

HETATM 239 C1 MET HETATM 240 H11 MET HETATM 241 H12 MET HETATM 242 H13 MET TER

HETATM 243 C5 ACP HETATM 244 H51 ACP HETATM 245 H52 ACP HETATM $246 \quad$ C4 ACP HETATM 247 H41 ACP HETATM 248 H42 ACP HETATM 249 C1 ACP HETATM 250 O2 ACP HETATM $251 \quad 03$ ACP HETATM 252 H3 ACP TER

HETATM 253 C1 MET HETATM 254 H11 MET HETATM 255 H12 MET HETATM 256 H13 MET TER

HETATM 257 HETATM 258 H11 MET HETATM 259 H12 MET HETATM 260 H13 MET TER

HETATM 261 C2 VIN HETATM 262 H21 VIN HETATM 263 C1 VIN HETATM 264 H11 VIN HETATM 265 H12 VIN TER

HETATM 266 Cl MET HETATM 267 H11 MET HETATM 268 H12 MET HETATM 269 H13 MET TER

HETATM 270 C2 VIN HETATM 271 H21 VIN HETATM 272 C1 VIN HETATM 273 H11 VIN HETATM 274 H12 VIN TER

HETATM 275 HETATM 276 H11 MET HETATM 277 H12 MET HETATM 278 H13 MET

HETATM 279 C5 ACP HETATM 280 H51 ACP HETATM 281 H52 ACP HETATM $282 \quad \mathrm{C} 4 \mathrm{ACP}$ HETATM 283 H41 ACP HETATM 284 H42 ACP HETATM 285 C1 ACP HETATM $286 \quad 02 \mathrm{ACP}$ HETATM $287 \quad 03$ ACP HETATM 288 H3 ACP

HETATM 289 C1 MET HETATM 290 H11 MET HETATM 291 H12 MET HETATM 292 H13 MET TER

HETATM 293 C1 MET HETATM 294 H11 MET HETATM 295 H12 MET HETATM 296 H13 MET

HETATM 297 FE HEM HETATM 298 C1 HEM HETATM 299 C2 HEM

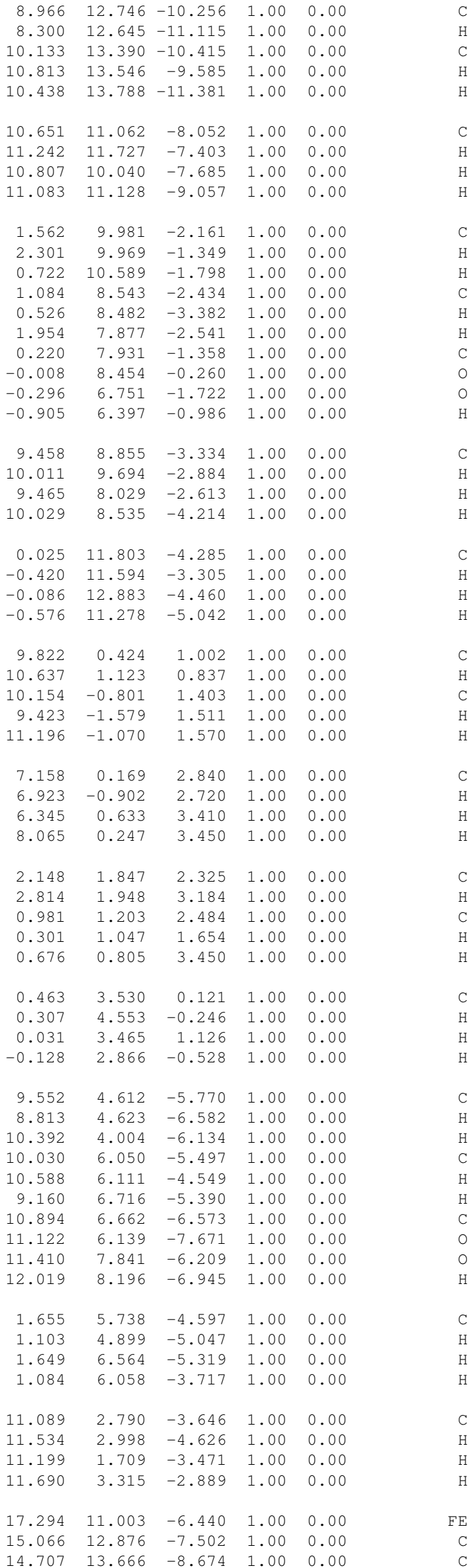




\begin{tabular}{|c|c|c|c|c|c|c|c|c|c|c|}
\hline HETATM & 300 & $\mathrm{C} 3$ & HEM & 33 & 15.852 & 13.763 & -9.448 & 1.00 & 0.00 & C \\
\hline HETATM & 301 & $\mathrm{C} 4$ & HEM & 33 & 16.882 & 13.046 & -8.740 & 1.00 & 0.00 & C \\
\hline HETATM & 302 & C5 & HEM & 33 & 18.197 & 12.927 & -9.178 & 1.00 & 0.00 & C \\
\hline HETATM & 303 & H5 & HEM & 33 & 18.451 & 13.423 & -10.113 & 1.00 & 0.00 & $\mathrm{H}$ \\
\hline HETATM & 304 & $\mathrm{C} 6$ & HEM & 33 & 19.216 & 12.229 & -8.542 & 1.00 & 0.00 & C \\
\hline HETATM & 305 & C7 & HEM & 33 & 20.585 & 12.156 & -9.012 & 1.00 & 0.00 & C \\
\hline HETATM & 306 & $\mathrm{C} 8$ & HEM & 33 & 21.291 & 11.436 & -8.065 & 1.00 & 0.00 & C \\
\hline HETATM & 307 & C9 & HEM & 33 & 20.340 & 11.071 & -7.041 & 1.00 & 0.00 & C \\
\hline HETATM & 308 & $\mathrm{C} 10$ & HEM & 33 & 20.644 & 10.336 & -5.897 & 1.00 & 0.00 & $\mathrm{C}$ \\
\hline HETATM & 309 & $\mathrm{H} 10$ & HEM & 33 & 21.671 & 9.993 & -5.791 & 1.00 & 0.00 & $\mathrm{H}$ \\
\hline HETATM & 310 & C11 & HEM & 33 & 19.770 & 9.996 & -4.874 & 1.00 & 0.00 & C \\
\hline HETATM & 311 & $\mathrm{C} 12$ & HEM & 33 & 20.151 & 9.271 & -3.684 & 1.00 & 0.00 & C \\
\hline HETATM & 312 & $\mathrm{C} 13$ & HEM & 33 & 19.011 & 9.124 & -2.927 & 1.00 & 0.00 & C \\
\hline HETATM & 313 & C14 & HEM & 33 & 17.938 & 9.765 & -3.659 & 1.00 & 0.00 & C \\
\hline HETATM & 314 & C15 & HEM & 33 & 16.610 & 9.820 & -3.237 & 1.00 & 0.00 & C \\
\hline HETATM & 315 & H15 & HEM & 33 & 16.370 & 9.335 & -2.289 & 1.00 & 0.00 & $\mathrm{H}$ \\
\hline HETATM & 316 & $\mathrm{C} 16$ & HEM & 33 & 15.580 & 10.502 & -3.880 & 1.00 & 0.00 & C \\
\hline HETATM & 317 & $\mathrm{C} 17$ & HEM & 33 & 14.214 & 10.615 & -3.398 & 1.00 & 0.00 & C \\
\hline HETATM & 318 & $\mathrm{C} 18$ & HEM & 33 & 13.539 & 11.383 & -4.322 & 1.00 & 0.00 & C \\
\hline HETATM & 319 & C19 & HEM & 33 & 14.488 & 11.735 & -5.358 & 1.00 & 0.00 & C \\
\hline HETATM & 320 & $\mathrm{C} 20$ & HEM & 33 & 14.197 & 12.526 & -6.469 & 1.00 & 0.00 & C \\
\hline HETATM & 321 & $\mathrm{H} 20$ & HEM & 33 & 13.183 & 12.915 & -6.530 & 1.00 & 0.00 & $\mathrm{H}$ \\
\hline HETATM & 322 & $\mathrm{~N} 21$ & HEM & 33 & 16.387 & 12.513 & -7.564 & 1.00 & 0.00 & $\mathrm{~N}$ \\
\hline HETATM & 323 & N22 & HEM & 33 & 19.092 & 11.566 & -7.342 & 1.00 & 0.00 & $\mathrm{~N}$ \\
\hline HETATM & 324 & N23 & HEM & 33 & 18.421 & 10.303 & -4.841 & 1.00 & 0.00 & $\mathrm{~N}$ \\
\hline HETATM & 325 & $\mathrm{~N} 24$ & HEM & 33 & 15.722 & 11.187 & -5.077 & 1.00 & 0.00 & $\mathrm{~N}$ \\
\hline HETATM & 326 & C99 & HEM & 33 & 16.628 & 8.322 & -7.579 & 1.00 & 0.00 & $\mathrm{C}$ \\
\hline HETATM & 327 & 091 & HEM & 33 & 16.788 & 9.635 & -7.567 & 1.00 & 0.00 & 0 \\
\hline HETATM & 328 & 092 & HEM & 33 & 16.473 & 7.705 & -8.635 & 1.00 & 0.00 & 0 \\
\hline HETATM & 329 & СТ91 & HEM & 33 & 16.644 & 7.640 & -6.223 & 1.00 & 0.00 & C \\
\hline HETATM & 330 & H911 & HEM & 33 & 17.623 & 7.836 & -5.760 & 1.00 & 0.00 & $\mathrm{H}$ \\
\hline HETATM & 331 & H912 & HEM & 33 & 15.896 & 8.130 & -5.580 & 1.00 & 0.00 & $\mathrm{H}$ \\
\hline HETATM & 332 & СТ92 & HEM & 33 & 16.385 & 6.136 & -6.334 & 1.00 & 0.00 & C \\
\hline HETATM & 333 & H921 & HEM & 33 & 17.181 & 5.676 & -6.931 & 1.00 & 0.00 & $\mathrm{H}$ \\
\hline HETATM & 334 & H922 & HEM & 33 & 15.460 & 5.980 & -6.905 & 1.00 & 0.00 & $\mathrm{H}$ \\
\hline \multicolumn{11}{|l|}{ TER } \\
\hline HETATM & 335 & $\mathrm{FE}$ & HEM & 34 & 17.986 & 3.589 & -1.491 & 1.00 & 0.00 & $\mathrm{FE}$ \\
\hline HETATM & 336 & $\mathrm{C} 1$ & HEM & 34 & 20.214 & 1.717 & -0.429 & 1.00 & 0.00 & C \\
\hline HETATM & 337 & $\mathrm{C} 2$ & HEM & 34 & 20.573 & 0.927 & 0.742 & 1.00 & 0.00 & C \\
\hline HETATM & 338 & $\mathrm{C} 3$ & HEM & 34 & 19.427 & 0.830 & 1.517 & 1.00 & 0.00 & C \\
\hline HETATM & 339 & C4 & HEM & 34 & 18.398 & 1.547 & 0.809 & 1.00 & 0.00 & C \\
\hline HETATM & 340 & C5 & HEM & 34 & 17.082 & 1.666 & 1.247 & 1.00 & 0.00 & C \\
\hline HETATM & 341 & H5 & HEM & 34 & 16.829 & 1.170 & 2.182 & 1.00 & 0.00 & $\mathrm{H}$ \\
\hline HETATM & 342 & $\mathrm{C} 6$ & HEM & 34 & 16.063 & 2.364 & 0.611 & 1.00 & 0.00 & C \\
\hline HETATM & 343 & C7 & HEM & 34 & 14.694 & 2.437 & 1.081 & 1.00 & 0.00 & $\mathrm{C}$ \\
\hline HETATM & 344 & $\mathrm{C} 8$ & HEM & 34 & 13.989 & 3.157 & 0.134 & 1.00 & 0.00 & C \\
\hline HETATM & 345 & $\mathrm{C} 9$ & HEM & 34 & 14.940 & 3.522 & -0.891 & 1.00 & 0.00 & C \\
\hline HETATM & 346 & $\mathrm{C} 10$ & HEM & 34 & 14.635 & 4.256 & -2.034 & 1.00 & 0.00 & $\mathrm{C}$ \\
\hline HETATM & 347 & $\mathrm{H} 10$ & HEM & 34 & 13.608 & 4.600 & -2.140 & 1.00 & 0.00 & $\mathrm{H}$ \\
\hline HETATM & 348 & $\mathrm{C} 11$ & HEM & 34 & 15.510 & 4.596 & -3.057 & 1.00 & 0.00 & C \\
\hline HETATM & 349 & $\mathrm{C} 12$ & HEM & 34 & 15.129 & 5.322 & -4.247 & 1.00 & 0.00 & C \\
\hline HETATM & 350 & $\mathrm{C} 13$ & HEM & 34 & 16.268 & 5.469 & -5.004 & 1.00 & 0.00 & C \\
\hline HETATM & 351 & C14 & HEM & 34 & 17.341 & 4.828 & -4.273 & 1.00 & 0.00 & C \\
\hline HETATM & 352 & C15 & HEM & 34 & 18.669 & 4.773 & -4.694 & 1.00 & 0.00 & C \\
\hline HETATM & 353 & H15 & HEM & 34 & 18.909 & 5.258 & -5.642 & 1.00 & 0.00 & $\mathrm{H}$ \\
\hline HETATM & 354 & $\mathrm{C} 16$ & HEM & 34 & 19.699 & 4.091 & -4.051 & 1.00 & 0.00 & C \\
\hline HETATM & 355 & $\mathrm{C} 17$ & HEM & 34 & 21.065 & 3.978 & -4.533 & 1.00 & 0.00 & C \\
\hline HETATM & 356 & $\mathrm{C} 18$ & HEM & 34 & 21.741 & 3.210 & -3.609 & 1.00 & 0.00 & C \\
\hline HETATM & 357 & C19 & HEM & 34 & 20.791 & 2.858 & -2.573 & 1.00 & 0.00 & C \\
\hline HETATM & 358 & $\mathrm{C} 20$ & HEM & 34 & 21.082 & 2.067 & -1.462 & 1.00 & 0.00 & C \\
\hline HETATM & 359 & $\mathrm{H} 20$ & HEM & 34 & 22.097 & 1.678 & -1.402 & 1.00 & 0.00 & $\mathrm{H}$ \\
\hline HETATM & 360 & $\mathrm{~N} 21$ & HEM & 34 & 18.892 & 2.080 & -0.367 & 1.00 & 0.00 & $\mathrm{~N}$ \\
\hline HETATM & 361 & $\mathrm{~N} 22$ & HEM & 34 & 16.187 & 3.027 & -0.590 & 1.00 & 0.00 & $\mathrm{~N}$ \\
\hline HETATM & 362 & N23 & HEM & 34 & 16.858 & 4.290 & -3.090 & 1.00 & 0.00 & $\mathrm{~N}$ \\
\hline HETATM & 363 & N24 & HEM & 34 & 19.558 & 3.406 & -2.854 & 1.00 & 0.00 & $\mathrm{~N}$ \\
\hline HETATM & 364 & C99 & HEM & 34 & 18.651 & 6.271 & -0.352 & 1.00 & 0.00 & C \\
\hline HETATM & 365 & 091 & HEM & 34 & 18.492 & 4.958 & -0.365 & 1.00 & 0.00 & o \\
\hline HETATM & 366 & 092 & HEM & 34 & 18.806 & 6.888 & 0.703 & 1.00 & 0.00 & 0 \\
\hline HETATM & 367 & СТ91 & HEM & 34 & 18.635 & 6.952 & -1.709 & 1.00 & 0.00 & C \\
\hline HETATM & 368 & H911 & HEM & 34 & 17.656 & 6.757 & -2.171 & 1.00 & 0.00 & $\mathrm{H}$ \\
\hline HETATM & 369 & H912 & HEM & 34 & 19.383 & 6.463 & -2.352 & 1.00 & 0.00 & $\mathrm{H}$ \\
\hline HETATM & 370 & СТ 92 & HEM & 34 & 18.895 & 8.457 & -1.597 & 1.00 & 0.00 & C \\
\hline HETATM & 371 & H921 & HEM & 34 & 19.820 & 8.613 & -1.027 & 1.00 & 0.00 & $\mathrm{H}$ \\
\hline HETATM & 372 & H922 & HEM & 34 & 18.098 & 8.917 & -1.000 & 1.00 & 0.00 & $\mathrm{H}$ \\
\hline \multicolumn{11}{|l|}{ TER } \\
\hline HETATM & 373 & $\mathrm{C} 2$ & VIN & 35 & 13.375 & 14.169 & -8.933 & 1.00 & 0.00 & C \\
\hline HETATM & 374 & $\mathrm{H} 21$ & VIN & 35 & 12.559 & 13.470 & -8.768 & 1.00 & 0.00 & $\mathrm{H}$ \\
\hline HETATM & 375 & $\mathrm{C} 1$ & VIN & 35 & 13.042 & 15.394 & -9.335 & 1.00 & 0.00 & C \\
\hline HETATM & 376 & H11 & VIN & 35 & 13.774 & 16.172 & -9.442 & 1.00 & 0.00 & $\mathrm{H}$ \\
\hline
\end{tabular}




\begin{tabular}{|c|c|c|c|}
\hline HETATM & 377 & $\mathrm{H} 12$ & VIN \\
\hline \multicolumn{4}{|l|}{ TER } \\
\hline HETATM & 378 & $\mathrm{C} 1$ & MET \\
\hline HETATM & 379 & H11 & MET \\
\hline HETATM & 380 & H12 & MET \\
\hline HETATM & 381 & H13 & MET \\
\hline \multicolumn{4}{|l|}{ TER } \\
\hline HETATM & 382 & $\mathrm{C} 2$ & VIN \\
\hline HETATM & 383 & H 21 & VIN \\
\hline HETATM & 384 & $\mathrm{C} 1$ & VIN \\
\hline HETATM & 385 & H11 & VIN \\
\hline HETATM & 386 & H12 & VIN \\
\hline \multicolumn{4}{|l|}{ TER } \\
\hline HETATM & 387 & $\mathrm{C} 1$ & MET \\
\hline HETATM & 388 & H11 & MET \\
\hline HETATM & 389 & $\mathrm{H} 12$ & $\mathrm{MET}$ \\
\hline HETATM & 390 & H13 & MET \\
\hline \multicolumn{4}{|l|}{ TER } \\
\hline HETATM & 391 & C5 & $\mathrm{ACP}$ \\
\hline HETATM & 392 & H51 & $\mathrm{ACP}$ \\
\hline HETATM & 393 & H52 & $\mathrm{ACP}$ \\
\hline HETATM & 394 & $\mathrm{C} 4$ & $\mathrm{ACP}$ \\
\hline HETATM & 395 & H 41 & $\mathrm{ACP}$ \\
\hline HETATM & 396 & H 42 & $\mathrm{ACP}$ \\
\hline HETATM & 397 & $\mathrm{C} 1$ & $\mathrm{ACP}$ \\
\hline HETATM & 398 & $\mathrm{O} 2$ & $\mathrm{ACP}$ \\
\hline HETATM & 399 & 03 & $\mathrm{ACP}$ \\
\hline HETATM & 400 & H3 & $\mathrm{ACP}$ \\
\hline \multicolumn{4}{|l|}{ TER } \\
\hline HETATM & 401 & $\mathrm{C} 1$ & MET \\
\hline HETATM & 402 & H11 & MET \\
\hline HETATM & 403 & H12 & MET \\
\hline HETATM & 404 & H13 & MET \\
\hline \multicolumn{4}{|l|}{ TER } \\
\hline HETATM & 405 & $\mathrm{C} 1$ & MET \\
\hline HETATM & 406 & $\mathrm{H} 11$ & MET \\
\hline HETATM & 407 & $\mathrm{H} 12$ & MET \\
\hline HETATM & 408 & $\mathrm{H} 13$ & MET \\
\hline \multicolumn{4}{|l|}{ TER } \\
\hline HETATM & 409 & $\mathrm{C} 2$ & VIN \\
\hline HETATM & 410 & H 21 & VIN \\
\hline HETATM & 411 & $\mathrm{C} 1$ & VIN \\
\hline HETATM & 412 & H11 & VIN \\
\hline HETATM & 413 & $\mathrm{H} 12$ & VIN \\
\hline \multicolumn{4}{|l|}{ TER } \\
\hline HETATM & 414 & $\mathrm{C} 1$ & MET \\
\hline HETATM & 415 & H11 & MET \\
\hline HETATM & 416 & H12 & MET \\
\hline HETATM & 417 & H13 & $\mathrm{MET}$ \\
\hline TER & & & \\
\hline HETATM & 418 & $\mathrm{C} 2$ & VIN \\
\hline HETATM & 419 & H 21 & VIN \\
\hline HETATM & 420 & $\mathrm{C} 1$ & VIN \\
\hline HETATM & 421 & H11 & VIN \\
\hline HETATM & 422 & H12 & VIN \\
\hline TER & & & \\
\hline HETATM & 423 & $\mathrm{C} 1$ & MET \\
\hline HETATM & 424 & $\mathrm{H} 11$ & MET \\
\hline HETATM & 425 & H12 & MET \\
\hline HETATM & 426 & H13 & MET \\
\hline TER & & & \\
\hline HETATM & 427 & C5 & $\mathrm{ACP}$ \\
\hline HETATM & 428 & H51 & $\mathrm{ACP}$ \\
\hline HETATM & 429 & H52 & $\mathrm{ACP}$ \\
\hline HETATM & 430 & C4 & $\mathrm{ACP}$ \\
\hline HETATM & 431 & $\mathrm{H} 41$ & $\mathrm{ACP}$ \\
\hline HETATM & 432 & H 42 & $\mathrm{ACP}$ \\
\hline HETATM & 433 & $\mathrm{C} 1$ & $\mathrm{ACP}$ \\
\hline HETATM & 434 & $\mathrm{O} 2$ & $\mathrm{ACP}$ \\
\hline HETATM & 435 & 03 & $\mathrm{ACP}$ \\
\hline HETATM & 436 & H3 & $\mathrm{ACP}$ \\
\hline TER & & & \\
\hline HETATM & 437 & $\mathrm{C} 1$ & MET \\
\hline HETATM & 438 & H11 & MET \\
\hline HETATM & 439 & H12 & MET \\
\hline HETATM & 440 & $\mathrm{H} 13$ & MET \\
\hline TER & & & \\
\hline HETATM & 441 & $\mathrm{C} 1$ & MET \\
\hline HETATM & 442 & H11 & MET \\
\hline
\end{tabular}

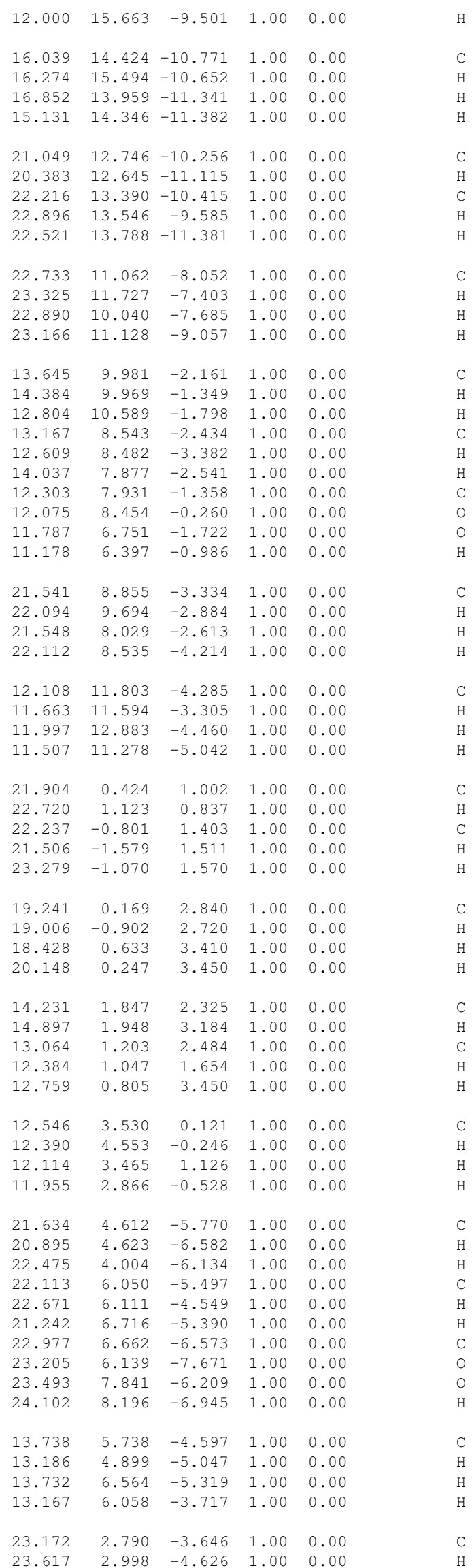


$\begin{array}{lllllllll}\text { HETATM } 444 & \text { H13 MET } & 48 & 23.772 & 3.315 & -2.889 & 1.00 & 0.00\end{array}$

\subsection{T5 dimer: AYM_T5.pdb file}

\begin{tabular}{|c|c|c|c|c|c|c|c|c|c|c|}
\hline HETATM & 1 & $\mathrm{FE}$ & HEM & 1 & 4.263 & 10.757 & 1.491 & 1.00 & 0.00 & $\mathrm{FE}$ \\
\hline HETATM & 2 & $\mathrm{C} 1$ & HEM & 1 & 2.035 & 12.629 & 0.429 & 1.00 & 0.00 & $\mathrm{C}$ \\
\hline HETATM & 3 & $\mathrm{C} 2$ & HEM & 1 & 1.676 & 13.420 & -0.742 & 1.00 & 0.00 & C \\
\hline HETATM & 4 & $\mathrm{C} 3$ & HEM & 1 & 2.822 & 13.517 & -1.517 & 1.00 & 0.00 & C \\
\hline HETATM & 5 & $\mathrm{C} 4$ & HEM & 1 & 3.851 & 12.800 & -0.809 & 1.00 & 0.00 & C \\
\hline HETATM & 6 & C5 & HEM & 1 & 5.167 & 12.681 & -1.247 & 1.00 & 0.00 & C \\
\hline HETATM & 7 & H5 & HEM & 1 & 5.420 & 13.177 & -2.182 & 1.00 & 0.00 & $\mathrm{H}$ \\
\hline HETATM & 8 & $\mathrm{C} 6$ & HEM & 1 & 6.186 & 11.982 & -0.611 & 1.00 & 0.00 & C \\
\hline HETATM & 9 & C7 & HEM & 1 & 7.555 & 11.910 & -1.081 & 1.00 & 0.00 & $\mathrm{C}$ \\
\hline HETATM & 10 & $\mathrm{C} 8$ & HEM & 1 & 8.260 & 11.190 & -0.134 & 1.00 & 0.00 & C \\
\hline HETATM & 11 & $\mathrm{C} 9$ & HEM & 1 & 7.309 & 10.825 & 0.891 & 1.00 & 0.00 & $\mathrm{C}$ \\
\hline HETATM & 12 & $\mathrm{C} 10$ & HEM & 1 & 7.614 & 10.090 & 2.034 & 1.00 & 0.00 & $\mathrm{C}$ \\
\hline HETATM & 13 & $\mathrm{H} 10$ & HEM & 1 & 8.641 & 9.746 & 2.140 & 1.00 & 0.00 & $\mathrm{H}$ \\
\hline HETATM & 14 & $\mathrm{C} 11$ & HEM & 1 & 6.739 & 9.750 & 3.057 & 1.00 & 0.00 & C \\
\hline HETATM & 15 & $\mathrm{C} 12$ & HEM & 1 & 7.120 & 9.025 & 4.247 & 1.00 & 0.00 & C \\
\hline HETATM & 16 & $\mathrm{C} 13$ & HEM & 1 & 5.981 & 8.878 & 5.004 & 1.00 & 0.00 & C \\
\hline HETATM & 17 & $\mathrm{C} 14$ & HEM & 1 & 4.908 & 9.519 & 4.273 & 1.00 & 0.00 & C \\
\hline HETATM & 18 & $\mathrm{C} 15$ & HEM & 1 & 3.580 & 9.574 & 4.694 & 1.00 & 0.00 & C \\
\hline HETATM & 19 & $\mathrm{H} 15$ & HEM & 1 & 3.340 & 9.089 & 5.642 & 1.00 & 0.00 & $\mathrm{H}$ \\
\hline HETATM & 20 & $\mathrm{C} 16$ & HEM & 1 & 2.550 & 10.255 & 4.051 & 1.00 & 0.00 & C \\
\hline HETATM & 21 & $\mathrm{C} 17$ & HEM & 1 & 1.184 & 10.369 & 4.533 & 1.00 & 0.00 & C \\
\hline HETATM & 22 & $\mathrm{C} 18$ & HEM & 1 & 0.508 & 11.137 & 3.609 & 1.00 & 0.00 & C \\
\hline HETATM & 23 & C19 & HEM & 1 & 1.458 & 11.489 & 2.573 & 1.00 & 0.00 & C \\
\hline HETATM & 24 & $\mathrm{C} 20$ & HEM & 1 & 1.167 & 12.280 & 1.462 & 1.00 & 0.00 & C \\
\hline HETATM & 25 & $\mathrm{H} 2 \mathrm{O}$ & HEM & 1 & 0.152 & 12.669 & 1.402 & 1.00 & 0.00 & $\mathrm{H}$ \\
\hline HETATM & 26 & $\mathrm{~N} 21$ & HEM & 1 & 3.357 & 12.266 & 0.367 & 1.00 & 0.00 & N \\
\hline HETATM & 27 & $\mathrm{~N} 22$ & HEM & 1 & 6.062 & 11.319 & 0.590 & 1.00 & 0.00 & $\mathrm{~N}$ \\
\hline HETATM & 28 & $\mathrm{~N} 23$ & HEM & 1 & 5.391 & 10.056 & 3.090 & 1.00 & 0.00 & $\mathrm{~N}$ \\
\hline HETATM & 29 & $\mathrm{~N} 24$ & HEM & 1 & 2.691 & 10.941 & 2.854 & 1.00 & 0.00 & $\mathrm{~N}$ \\
\hline HETATM & 30 & C99 & HEM & 1 & 3.598 & 8.076 & 0.352 & 1.00 & 0.00 & C \\
\hline HETATM & 31 & 091 & HEM & 1 & 3.757 & 9.389 & 0.365 & 1.00 & 0.00 & 0 \\
\hline HETATM & 32 & 092 & HEM & 1 & 3.443 & 7.458 & -0.703 & 1.00 & 0.00 & 0 \\
\hline HETATM & 33 & CT91 & HEM & 1 & 3.614 & 7.394 & 1.709 & 1.00 & 0.00 & $\mathrm{C}$ \\
\hline HETATM & 34 & H911 & HEM & 1 & 4.593 & 7.590 & 2.171 & 1.00 & 0.00 & $\mathrm{H}$ \\
\hline HETATM & 35 & H912 & HEM & 1 & 2.866 & 7.884 & 2.352 & 1.00 & 0.00 & $\mathrm{H}$ \\
\hline HETATM & 36 & CT92 & HEM & 1 & 3.354 & 5.890 & 1.597 & 1.00 & 0.00 & C \\
\hline HETATM & 37 & H921 & HEM & 1 & 4.151 & 5.429 & 1.000 & 1.00 & 0.00 & $\mathrm{H}$ \\
\hline HETATM & 38 & H922 & HEM & 1 & 2.429 & 5.734 & 1.027 & 1.00 & 0.00 & $\mathrm{H}$ \\
\hline TER & & & & & & & & & & \\
\hline HETATM & 39 & $\mathrm{FE}$ & HEM & 2 & 4.955 & 3.343 & 6.440 & 1.00 & 0.00 & $\mathrm{FE}$ \\
\hline HETATM & 40 & $\mathrm{C} 1$ & HEM & 2 & 7.183 & 1.471 & 7.502 & 1.00 & 0.00 & C \\
\hline HETATM & 41 & $\mathrm{C} 2$ & HEM & 2 & 7.542 & 0.680 & 8.674 & 1.00 & 0.00 & C \\
\hline HETATM & 42 & $\mathrm{C} 3$ & HEM & 2 & 6.397 & 0.583 & 9.448 & 1.00 & 0.00 & C \\
\hline HETATM & 43 & $\mathrm{C} 4$ & HEM & 2 & 5.367 & 1.301 & 8.740 & 1.00 & 0.00 & C \\
\hline HETATM & 44 & $\mathrm{C} 5$ & HEM & 2 & 4.052 & 1.420 & 9.178 & 1.00 & 0.00 & C \\
\hline HETATM & 45 & H5 & HEM & 2 & 3.798 & 0.923 & 10.113 & 1.00 & 0.00 & $\mathrm{H}$ \\
\hline HETATM & 46 & $\mathrm{C} 6$ & HEM & 2 & 3.033 & 2.118 & 8.542 & 1.00 & 0.00 & C \\
\hline HETATM & 47 & $\mathrm{C} 7$ & HEM & 2 & 1.664 & 2.191 & 9.012 & 1.00 & 0.00 & C \\
\hline HETATM & 48 & $\mathrm{C} 8$ & HEM & 2 & 0.958 & 2.910 & 8.065 & 1.00 & 0.00 & C \\
\hline HETATM & 49 & $\mathrm{C} 9$ & HEM & 2 & 1.909 & 3.275 & 7.041 & 1.00 & 0.00 & C \\
\hline HETATM & 50 & $\mathrm{C} 10$ & HEM & 2 & 1.605 & 4.010 & 5.897 & 1.00 & 0.00 & C \\
\hline HETATM & 51 & H1O & HEM & 2 & 0.578 & 4.354 & 5.791 & 1.00 & 0.00 & $\mathrm{H}$ \\
\hline HETATM & 52 & $\mathrm{C} 11$ & HEM & 2 & 2.479 & 4.350 & 4.874 & 1.00 & 0.00 & C \\
\hline HETATM & 53 & $\mathrm{C} 12$ & HEM & 2 & 2.098 & 5.076 & 3.684 & 1.00 & 0.00 & C \\
\hline HETATM & 54 & $\mathrm{C} 13$ & HEM & 2 & 3.238 & 5.223 & 2.927 & 1.00 & 0.00 & C \\
\hline HETATM & 55 & $\mathrm{C} 14$ & HEM & 2 & 4.311 & 4.581 & 3.659 & 1.00 & 0.00 & C \\
\hline HETATM & 56 & C15 & HEM & 2 & 5.639 & 4.527 & 3.237 & 1.00 & 0.00 & C \\
\hline HETATM & 57 & H1 5 & HEM & 2 & 5.879 & 5.011 & 2.289 & 1.00 & 0.00 & $\mathrm{H}$ \\
\hline HETATM & 58 & $\mathrm{C} 16$ & HEM & 2 & 6.669 & 3.845 & 3.880 & 1.00 & 0.00 & C \\
\hline HETATM & 59 & C17 & HEM & 2 & 8.035 & 3.732 & 3.398 & 1.00 & 0.00 & C \\
\hline HETATM & 60 & $\mathrm{C} 18$ & HEM & 2 & 8.710 & 2.964 & 4.322 & 1.00 & 0.00 & C \\
\hline HETATM & 61 & C19 & HEM & 2 & 7.761 & 2.612 & 5.358 & 1.00 & 0.00 & C \\
\hline HETATM & 62 & $\mathrm{C} 20$ & HEM & 2 & 8.052 & 1.821 & 6.469 & 1.00 & 0.00 & C \\
\hline HETATM & 63 & $\mathrm{H} 2 \mathrm{O}$ & HEM & 2 & 9.066 & 1.432 & 6.530 & 1.00 & 0.00 & $\mathrm{H}$ \\
\hline HETATM & 64 & N21 & HEM & 2 & 5.862 & 1.834 & 7.564 & 1.00 & 0.00 & $\mathrm{~N}$ \\
\hline HETATM & 65 & N22 & HEM & 2 & 3.157 & 2.781 & 7.342 & 1.00 & 0.00 & $\mathrm{~N}$ \\
\hline HETATM & 66 & N23 & HEM & 2 & 3.828 & 4.044 & 4.841 & 1.00 & 0.00 & $\mathrm{~N}$ \\
\hline HETATM & 67 & $\mathrm{~N} 24$ & HEM & 2 & 6.527 & 3.160 & 5.077 & 1.00 & 0.00 & $\mathrm{~N}$ \\
\hline HETATM & 68 & C99 & HEM & 2 & 5.621 & 6.025 & 7.579 & 1.00 & 0.00 & C \\
\hline HETATM & 69 & 091 & HEM & 2 & 5.461 & 4.711 & 7.567 & 1.00 & 0.00 & 0 \\
\hline HETATM & 70 & 092 & HEM & 2 & 5.776 & 6.642 & 8.635 & 1.00 & 0.00 & 0 \\
\hline
\end{tabular}




\begin{tabular}{|c|c|c|c|}
\hline HETATM & 71 & СТ91 & HEM \\
\hline HETATM & 72 & H911 & HEM \\
\hline HETATM & 73 & H912 & HEM \\
\hline HETATM & 74 & СТ92 & HEM \\
\hline HETATM & 75 & H921 & $\mathrm{HEM}$ \\
\hline HETATM & 76 & H922 & HEM \\
\hline \multicolumn{4}{|l|}{ TER } \\
\hline HETATM & 77 & $\mathrm{C} 2$ & VIN \\
\hline HETATM & 78 & $\mathrm{H} 21$ & VIN \\
\hline HETATM & 79 & $\mathrm{C} 1$ & VIN \\
\hline HETATM & 80 & H11 & VIN \\
\hline HETATM & 81 & $\mathrm{H} 12$ & VIN \\
\hline \multicolumn{4}{|l|}{ TER } \\
\hline HETATM & 82 & $\mathrm{C} 1$ & MET \\
\hline HETATM & 83 & $\mathrm{H} 11$ & MET \\
\hline HETATM & 84 & $\mathrm{H} 12$ & MET \\
\hline HETATM & 85 & H13 & MET \\
\hline \multicolumn{4}{|l|}{ TER } \\
\hline HETATM & 86 & $\mathrm{C} 2$ & VIN \\
\hline HETATM & 87 & $\mathrm{H} 21$ & VIN \\
\hline HETATM & 88 & $\mathrm{C} 1$ & VIN \\
\hline HETATM & 89 & $\mathrm{H} 11$ & VIN \\
\hline HETATM & 90 & $\mathrm{H} 12$ & VIN \\
\hline \multicolumn{4}{|l|}{ TER } \\
\hline HETATM & 91 & $\mathrm{C} 1$ & $\mathrm{MET}$ \\
\hline HETATM & 92 & H11 & MET \\
\hline HETATM & 93 & $\mathrm{H} 12$ & MET \\
\hline HETATM & 94 & $\mathrm{H} 13$ & $\mathrm{MET}$ \\
\hline \multicolumn{4}{|l|}{ TER } \\
\hline HETATM & 95 & C5 & $\mathrm{ACP}$ \\
\hline HETATM & 96 & H51 & $\mathrm{ACP}$ \\
\hline HETATM & 97 & H52 & $\mathrm{ACP}$ \\
\hline HETATM & 98 & C4 & $\mathrm{ACP}$ \\
\hline HETATM & 99 & $\mathrm{H} 41$ & $\mathrm{ACP}$ \\
\hline HETATM & 100 & $\mathrm{H} 42$ & $\mathrm{ACP}$ \\
\hline HETATM & 101 & $\mathrm{C} 1$ & $\mathrm{ACP}$ \\
\hline HETATM & 102 & $\mathrm{O} 2$ & $\mathrm{ACP}$ \\
\hline HETATM & 103 & 03 & $\mathrm{ACP}$ \\
\hline HETATM & 104 & H3 & $\mathrm{ACP}$ \\
\hline \multicolumn{4}{|l|}{ TER } \\
\hline HETATM & 105 & $\mathrm{C} 1$ & MET \\
\hline HETATM & 106 & $\mathrm{H} 11$ & MET \\
\hline HETATM & 107 & $\mathrm{H} 12$ & MET \\
\hline HETATM & 108 & $\mathrm{H} 13$ & MET \\
\hline \multicolumn{4}{|l|}{ TER } \\
\hline HETATM & 109 & $\mathrm{C} 1$ & MET \\
\hline HETATM & 110 & $\mathrm{H} 11$ & $\mathrm{MET}$ \\
\hline HETATM & 111 & $\mathrm{H} 12$ & $\mathrm{MET}$ \\
\hline HETATM & 112 & $\mathrm{H} 13$ & $\mathrm{MET}$ \\
\hline \multicolumn{4}{|l|}{ TER } \\
\hline HETATM & 113 & C2 & VIN \\
\hline HETATM & 114 & H 21 & VIN \\
\hline HETATM & 115 & $\mathrm{C} 1$ & VIN \\
\hline HETATM & 116 & H11 & VIN \\
\hline HETATM & 117 & $\mathrm{H} 12$ & VIN \\
\hline TER & & & \\
\hline HETATM & 118 & $\mathrm{C} 1$ & MET \\
\hline HETATM & 119 & $\mathrm{H} 11$ & $\mathrm{MET}$ \\
\hline HETATM & 120 & $\mathrm{H} 12$ & $\mathrm{MET}$ \\
\hline HETATM & 121 & $\mathrm{H} 13$ & MET \\
\hline TER & & & \\
\hline HETATM & 122 & $\mathrm{C} 2$ & VIN \\
\hline HETATM & 123 & H21 & VIN \\
\hline HETATM & 124 & $\mathrm{C} 1$ & VIN \\
\hline HETATM & 125 & H11 & VIN \\
\hline HETATM & 126 & $\mathrm{H} 12$ & VIN \\
\hline TER & & & \\
\hline HETATM & 127 & $\mathrm{C} 1$ & MET \\
\hline HETATM & 128 & H11 & MET \\
\hline HETATM & 129 & $\mathrm{H} 12$ & MET \\
\hline HETATM & 130 & H13 & MET \\
\hline TER & & & \\
\hline HETATM & 131 & C5 & $\mathrm{ACP}$ \\
\hline HETATM & 132 & H51 & $\mathrm{ACP}$ \\
\hline HETATM & 133 & H52 & $\mathrm{ACP}$ \\
\hline HETATM & 134 & $\mathrm{C} 4$ & $\mathrm{ACP}$ \\
\hline HETATM & 135 & H4 1 & $\mathrm{ACP}$ \\
\hline HETATM & 136 & $\mathrm{H} 42$ & $\mathrm{ACP}$ \\
\hline HETATM & 137 & $\mathrm{C} 1$ & $\mathrm{ACP}$ \\
\hline
\end{tabular}

\begin{tabular}{|c|c|c|c|c|c|}
\hline 5.605 & 6.706 & 6.223 & 1.00 & 0.00 & C \\
\hline 4.626 & 6.511 & 5.760 & 1.00 & 0.00 & $\mathrm{H}$ \\
\hline 6.353 & 6.216 & 5.580 & 1.00 & 0.00 & $\mathrm{H}$ \\
\hline 5.864 & 8.211 & 6.334 & 1.00 & 0.00 & C \\
\hline 6.789 & 8.367 & 6.905 & 1.00 & 0.00 & $\mathrm{H}$ \\
\hline 5.068 & 8.671 & 6.931 & 1.00 & 0.00 & $\mathrm{H}$ \\
\hline 0.345 & 13.923 & -1.002 & 1.00 & 0.00 & C \\
\hline-0.471 & 13.224 & -0.837 & 1.00 & 0.00 & $\mathrm{H}$ \\
\hline 0.012 & 15.147 & -1.403 & 1.00 & 0.00 & C \\
\hline 0.743 & 15.926 & -1.511 & 1.00 & 0.00 & $\mathrm{H}$ \\
\hline-1.030 & 15.417 & -1.570 & 1.00 & 0.00 & $\mathrm{H}$ \\
\hline 3.008 & 14.177 & -2.840 & 1.00 & 0.00 & C \\
\hline 3.243 & 15.248 & -2.720 & 1.00 & 0.00 & $\mathrm{H}$ \\
\hline 3.821 & 13.713 & -3.410 & 1.00 & 0.00 & $\mathrm{H}$ \\
\hline 2.101 & 14.100 & -3.450 & 1.00 & 0.00 & $\mathrm{H}$ \\
\hline 8.018 & 12.499 & -2.325 & 1.00 & 0.00 & C \\
\hline 7.352 & 12.399 & -3.184 & 1.00 & 0.00 & $\mathrm{H}$ \\
\hline 9.185 & 13.144 & -2.484 & 1.00 & 0.00 & C \\
\hline 9.865 & 13.300 & -1.654 & 1.00 & 0.00 & $\mathrm{H}$ \\
\hline 9.490 & 13.542 & -3.450 & 1.00 & 0.00 & $\mathrm{H}$ \\
\hline 9.703 & 10.816 & -0.121 & 1.00 & 0.00 & C \\
\hline 10.294 & 11.480 & 0.528 & 1.00 & 0.00 & $\mathrm{H}$ \\
\hline 9.859 & 9.794 & 0.246 & 1.00 & 0.00 & $\mathrm{H}$ \\
\hline 10.135 & 10.882 & -1.126 & 1.00 & 0.00 & $\mathrm{H}$ \\
\hline 0.615 & 9.735 & 5.770 & 1.00 & 0.00 & C \\
\hline 1.354 & 9.723 & 6.582 & 1.00 & 0.00 & $\mathrm{H}$ \\
\hline-0.226 & 10.343 & 6.134 & 1.00 & 0.00 & $\mathrm{H}$ \\
\hline 0.136 & 8.297 & 5.497 & 1.00 & 0.00 & C \\
\hline-0.422 & 8.236 & 4.549 & 1.00 & 0.00 & $\mathrm{H}$ \\
\hline 1.007 & 7.630 & 5.390 & 1.00 & 0.00 & $\mathrm{H}$ \\
\hline-0.728 & 7.685 & 6.573 & 1.00 & 0.00 & C \\
\hline-0.956 & 8.208 & 7.671 & 1.00 & 0.00 & O \\
\hline-1.244 & 6.505 & 6.209 & 1.00 & 0.00 & O \\
\hline-1.853 & 6.151 & 6.945 & 1.00 & 0.00 & $\mathrm{H}$ \\
\hline 8.511 & 8.608 & 4.597 & 1.00 & 0.00 & C \\
\hline 9.063 & 9.448 & 5.047 & 1.00 & 0.00 & $\mathrm{H}$ \\
\hline 8.517 & 7.782 & 5.319 & 1.00 & 0.00 & $\mathrm{H}$ \\
\hline 9.082 & 8.289 & 3.717 & 1.00 & 0.00 & $\mathrm{H}$ \\
\hline-0.923 & 11.557 & 3.646 & 1.00 & 0.00 & C \\
\hline-1.368 & 11.348 & 4.626 & 1.00 & 0.00 & $\mathrm{H}$ \\
\hline-1.033 & 12.637 & 3.471 & 1.00 & 0.00 & $\mathrm{H}$ \\
\hline-1.523 & 11.031 & 2.889 & 1.00 & 0.00 & $\mathrm{H}$ \\
\hline 8.874 & 0.178 & 8.933 & 1.00 & 0.00 & C \\
\hline 9.690 & 0.876 & 8.768 & 1.00 & 0.00 & $\mathrm{H}$ \\
\hline 9.207 & -1.047 & 9.335 & 1.00 & 0.00 & C \\
\hline 8.475 & -1.826 & 9.442 & 1.00 & 0.00 & $\mathrm{H}$ \\
\hline 10.249 & -1.316 & 9.501 & 1.00 & 0.00 & $\mathrm{H}$ \\
\hline 6.210 & -0.077 & 10.771 & 1.00 & 0.00 & C \\
\hline 5.975 & -1.148 & 10.652 & 1.00 & 0.00 & $\mathrm{H}$ \\
\hline 5.397 & 0.387 & 11.341 & 1.00 & 0.00 & $\mathrm{H}$ \\
\hline 7.118 & 0.001 & 11.382 & 1.00 & 0.00 & $\mathrm{H}$ \\
\hline 1.200 & 1.601 & 10.256 & 1.00 & 0.00 & C \\
\hline 1.866 & 1.702 & 11.115 & 1.00 & 0.00 & $\mathrm{H}$ \\
\hline 0.033 & 0.957 & 10.415 & 1.00 & 0.00 & C \\
\hline-0.647 & 0.800 & 9.585 & 1.00 & 0.00 & $\mathrm{H}$ \\
\hline-0.272 & 0.559 & 11.381 & 1.00 & 0.00 & $\mathrm{H}$ \\
\hline-0.484 & 3.284 & 8.052 & 1.00 & 0.00 & C \\
\hline-0.641 & 4.306 & 7.685 & 1.00 & 0.00 & $\mathrm{H}$ \\
\hline-0.917 & 3.219 & 9.057 & 1.00 & 0.00 & $\mathrm{H}$ \\
\hline-1.076 & 2.620 & 7.403 & 1.00 & 0.00 & $\mathrm{H}$ \\
\hline 8.604 & 4.366 & 2.161 & 1.00 & 0.00 & C \\
\hline 7.865 & 4.377 & 1.349 & 1.00 & 0.00 & $\mathrm{H}$ \\
\hline 9.445 & 3.758 & 1.798 & 1.00 & 0.00 & $\mathrm{H}$ \\
\hline 9.082 & 5.803 & 2.434 & 1.00 & 0.00 & C \\
\hline 9.640 & 5.865 & 3.382 & 1.00 & 0.00 & $\mathrm{H}$ \\
\hline 8.212 & 6.470 & 2.541 & 1.00 & 0.00 & $\mathrm{H}$ \\
\hline 9.946 & 6.416 & 1.358 & 1.00 & 0.00 & C \\
\hline
\end{tabular}




\begin{tabular}{|c|c|c|c|c|c|c|c|c|c|c|}
\hline HETATM & 138 & $\mathrm{O} 2$ & $\mathrm{ACP}$ & 14 & 10.174 & 5.893 & 0.260 & 1.00 & 0.00 & 0 \\
\hline HETATM & 139 & 03 & $\mathrm{ACP}$ & 14 & 10.462 & 7.595 & 1.722 & 1.00 & 0.00 & 0 \\
\hline HETATM & 140 & H3 & $\mathrm{ACP}$ & 14 & 11.071 & 7.949 & 0.986 & 1.00 & 0.00 & $\mathrm{H}$ \\
\hline \multicolumn{11}{|l|}{ TER } \\
\hline HETATM & 141 & $\mathrm{C} 1$ & $\mathrm{MET}$ & 15 & 0.708 & 5.492 & 3.334 & 1.00 & 0.00 & C \\
\hline HETATM & 142 & H11 & MET & 15 & 0.155 & 4.653 & 2.884 & 1.00 & 0.00 & $\mathrm{H}$ \\
\hline HETATM & 143 & $\mathrm{H} 12$ & MET & 15 & 0.701 & 6.318 & 2.613 & 1.00 & 0.00 & $\mathrm{H}$ \\
\hline HETATM & 144 & H13 & MET & 15 & 0.137 & 5.812 & 4.214 & 1.00 & 0.00 & $\mathrm{H}$ \\
\hline \multicolumn{11}{|l|}{ TER } \\
\hline HETATM & 145 & $\mathrm{C} 1$ & MET & 16 & 10.141 & 2.543 & 4.285 & 1.00 & 0.00 & 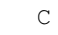 \\
\hline HETATM & 146 & H11 & MET & 16 & 10.586 & 2.752 & 3.305 & 1.00 & 0.00 & 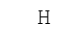 \\
\hline HETATM & 147 & $\mathrm{H} 12$ & MET & 16 & 10.252 & 1.463 & 4.460 & 1.00 & 0.00 & 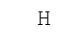 \\
\hline HETATM & 148 & $\mathrm{H} 13$ & $\mathrm{MET}$ & 16 & 10.742 & 3.069 & 5.042 & 1.00 & 0.00 & $\mathrm{H}$ \\
\hline \multicolumn{11}{|l|}{ TER } \\
\hline HETATM & 149 & $\mathrm{FE}$ & HEM & 17 & 17.294 & 11.003 & -6.440 & 1.00 & 0.00 & $\mathrm{FE}$ \\
\hline HETATM & 150 & $\mathrm{C} 1$ & HEM & 17 & 15.066 & 12.876 & -7.502 & 1.00 & 0.00 & C \\
\hline HETATM & 151 & $\mathrm{C} 2$ & HEM & 17 & 14.707 & 13.666 & -8.674 & 1.00 & 0.00 & C \\
\hline HETATM & 152 & C3 & HEM & 17 & 15.852 & 13.763 & -9.448 & 1.00 & 0.00 & C \\
\hline HETATM & 153 & $\mathrm{C} 4$ & HEM & 17 & 16.882 & 13.046 & -8.740 & 1.00 & 0.00 & C \\
\hline HETATM & 154 & C5 & HEM & 17 & 18.197 & 12.927 & -9.178 & 1.00 & 0.00 & C \\
\hline HETATM & 155 & H5 & HEM & 17 & 18.451 & 13.423 & -10.113 & 1.00 & 0.00 & $\mathrm{H}$ \\
\hline HETATM & 156 & $\mathrm{C} 6$ & HEM & 17 & 19.216 & 12.229 & -8.542 & 1.00 & 0.00 & C \\
\hline HETATM & 157 & C7 & HEM & 17 & 20.585 & 12.156 & -9.012 & 1.00 & 0.00 & C \\
\hline HETATM & 158 & $\mathrm{C} 8$ & HEM & 17 & 21.291 & 11.436 & -8.065 & 1.00 & 0.00 & C \\
\hline HETATM & 159 & $\mathrm{C} 9$ & HEM & 17 & 20.340 & 11.071 & -7.041 & 1.00 & 0.00 & C \\
\hline HETATM & 160 & $\mathrm{C} 10$ & HEM & 17 & 20.644 & 10.336 & -5.897 & 1.00 & 0.00 & C \\
\hline HETATM & 161 & $\mathrm{H} 1 \mathrm{O}$ & HEM & 17 & 21.671 & 9.993 & -5.791 & 1.00 & 0.00 & $\mathrm{H}$ \\
\hline HETATM & 162 & C11 & HEM & 17 & 19.770 & 9.996 & -4.874 & 1.00 & 0.00 & $\mathrm{C}$ \\
\hline HETATM & 163 & $\mathrm{C} 12$ & HEM & 17 & 20.151 & 9.271 & -3.684 & 1.00 & 0.00 & C \\
\hline HETATM & 164 & C13 & HEM & 17 & 19.011 & 9.124 & -2.927 & 1.00 & 0.00 & C \\
\hline HETATM & 165 & C14 & HEM & 17 & 17.938 & 9.765 & -3.659 & 1.00 & 0.00 & C \\
\hline HETATM & 166 & C15 & HEM & 17 & 16.610 & 9.820 & -3.237 & 1.00 & 0.00 & C \\
\hline HETATM & 167 & H15 & HEM & 17 & 16.370 & 9.335 & -2.289 & 1.00 & 0.00 & $\mathrm{H}$ \\
\hline HETATM & 168 & C16 & HEM & 17 & 15.580 & 10.502 & -3.880 & 1.00 & 0.00 & C \\
\hline HETATM & 169 & C17 & HEM & 17 & 14.214 & 10.615 & -3.398 & 1.00 & 0.00 & C \\
\hline HETATM & 170 & $\mathrm{C} 18$ & HEM & 17 & 13.539 & 11.383 & -4.322 & 1.00 & 0.00 & C \\
\hline HETATM & 171 & C19 & HEM & 17 & 14.488 & 11.735 & -5.358 & 1.00 & 0.00 & C \\
\hline HETATM & 172 & $\mathrm{C} 20$ & HEM & 17 & 14.197 & 12.526 & -6.469 & 1.00 & 0.00 & C \\
\hline HETATM & 173 & $\mathrm{H} 2 \mathrm{O}$ & HEM & 17 & 13.183 & 12.915 & -6.530 & 1.00 & 0.00 & $\mathrm{H}$ \\
\hline HETATM & 174 & $\mathrm{~N} 21$ & HEM & 17 & 16.387 & 12.513 & -7.564 & 1.00 & 0.00 & $\mathrm{~N}$ \\
\hline HETATM & 175 & N22 & HEM & 17 & 19.092 & 11.566 & -7.342 & 1.00 & 0.00 & $\mathrm{~N}$ \\
\hline HETATM & 176 & N23 & HEM & 17 & 18.421 & 10.303 & -4.841 & 1.00 & 0.00 & $\mathrm{~N}$ \\
\hline HETATM & 177 & $\mathrm{~N} 24$ & HEM & 17 & 15.722 & 11.187 & -5.077 & 1.00 & 0.00 & $\mathrm{~N}$ \\
\hline HETATM & 178 & C99 & HEM & 17 & 16.628 & 8.322 & -7.579 & 1.00 & 0.00 & C \\
\hline HETATM & 179 & 091 & HEM & 17 & 16.788 & 9.635 & -7.567 & 1.00 & 0.00 & 0 \\
\hline HETATM & 180 & 092 & HEM & 17 & 16.473 & 7.705 & -8.635 & 1.00 & 0.00 & 0 \\
\hline HETATM & 181 & СТ 91 & HEM & 17 & 16.644 & 7.640 & -6.223 & 1.00 & 0.00 & C \\
\hline HETATM & 182 & H911 & HEM & 17 & 17.623 & 7.836 & -5.760 & 1.00 & 0.00 & $\mathrm{H}$ \\
\hline HETATM & 183 & H912 & HEM & 17 & 15.896 & 8.130 & -5.580 & 1.00 & 0.00 & $\mathrm{H}$ \\
\hline HETATM & 184 & СТ 92 & HEM & 17 & 16.385 & 6.136 & -6.334 & 1.00 & 0.00 & C \\
\hline HETATM & 185 & H921 & HEM & 17 & 17.181 & 5.676 & -6.931 & 1.00 & 0.00 & $\mathrm{H}$ \\
\hline HETATM & 186 & H922 & HEM & 17 & 15.460 & 5.980 & -6.905 & 1.00 & 0.00 & $\mathrm{H}$ \\
\hline \multicolumn{11}{|l|}{ TER } \\
\hline HETATM & 187 & $\mathrm{FE}$ & HEM & 18 & 17.986 & 3.589 & -1.491 & 1.00 & 0.00 & $\mathrm{FE}$ \\
\hline HETATM & 188 & $\mathrm{C} 1$ & HEM & 18 & 20.214 & 1.717 & -0.429 & 1.00 & 0.00 & C \\
\hline HETATM & 189 & $\mathrm{C} 2$ & HEM & 18 & 20.573 & 0.927 & 0.742 & 1.00 & 0.00 & C \\
\hline HETATM & 190 & $\mathrm{C} 3$ & HEM & 18 & 19.427 & 0.830 & 1.517 & 1.00 & 0.00 & C \\
\hline HETATM & 191 & $\mathrm{C} 4$ & HEM & 18 & 18.398 & 1.547 & 0.809 & 1.00 & 0.00 & C \\
\hline HETATM & 192 & C5 & HEM & 18 & 17.082 & 1.666 & 1.247 & 1.00 & 0.00 & C \\
\hline HETATM & 193 & H5 & HEM & 18 & 16.829 & 1.170 & 2.182 & 1.00 & 0.00 & $\mathrm{H}$ \\
\hline HETATM & 194 & $\mathrm{C} 6$ & HEM & 18 & 16.063 & 2.364 & 0.611 & 1.00 & 0.00 & C \\
\hline HETATM & 195 & C7 & HEM & 18 & 14.694 & 2.437 & 1.081 & 1.00 & 0.00 & C \\
\hline HETATM & 196 & $\mathrm{C} 8$ & HEM & 18 & 13.989 & 3.157 & 0.134 & 1.00 & 0.00 & C \\
\hline HETATM & 197 & $\mathrm{C} 9$ & HEM & 18 & 14.940 & 3.522 & -0.891 & 1.00 & 0.00 & C \\
\hline HETATM & 198 & $\mathrm{C} 10$ & HEM & 18 & 14.635 & 4.256 & -2.034 & 1.00 & 0.00 & C \\
\hline HETATM & 199 & $\mathrm{H} 10$ & HEM & 18 & 13.608 & 4.600 & -2.140 & 1.00 & 0.00 & $\mathrm{H}$ \\
\hline HETATM & 200 & C11 & HEM & 18 & 15.510 & 4.596 & -3.057 & 1.00 & 0.00 & $\mathrm{C}$ \\
\hline HETATM & 201 & $\mathrm{C} 12$ & HEM & 18 & 15.129 & 5.322 & -4.247 & 1.00 & 0.00 & C \\
\hline HETATM & 202 & C13 & HEM & 18 & 16.268 & 5.469 & -5.004 & 1.00 & 0.00 & C \\
\hline HETATM & 203 & C14 & HEM & 18 & 17.341 & 4.828 & -4.273 & 1.00 & 0.00 & C \\
\hline HETATM & 204 & C15 & HEM & 18 & 18.669 & 4.773 & -4.694 & 1.00 & 0.00 & C \\
\hline HETATM & 205 & H1 5 & HEM & 18 & 18.909 & 5.258 & -5.642 & 1.00 & 0.00 & $\mathrm{H}$ \\
\hline HETATM & 206 & C16 & HEM & 18 & 19.699 & 4.091 & -4.051 & 1.00 & 0.00 & C \\
\hline HETATM & 207 & C17 & HEM & 18 & 21.065 & 3.978 & -4.533 & 1.00 & 0.00 & C \\
\hline HETATM & 208 & $\mathrm{C} 18$ & HEM & 18 & 21.741 & 3.210 & -3.609 & 1.00 & 0.00 & C \\
\hline HETATM & 209 & C19 & HEM & 18 & 20.791 & 2.858 & -2.573 & 1.00 & 0.00 & C \\
\hline HETATM & 210 & $\mathrm{C} 20$ & HEM & 18 & 21.082 & 2.067 & -1.462 & 1.00 & 0.00 & C \\
\hline HETATM & 211 & $\mathrm{H} 2 \mathrm{O}$ & HEM & 18 & 22.097 & 1.678 & -1.402 & 1.00 & 0.00 & $\mathrm{H}$ \\
\hline HETATM & 212 & N21 & HEM & 18 & 18.892 & 2.080 & -0.367 & 1.00 & 0.00 & $\mathrm{~N}$ \\
\hline
\end{tabular}




\begin{tabular}{|c|c|c|c|}
\hline HETATM & 213 & N22 & HEM \\
\hline HETATM & 214 & N23 & HEM \\
\hline HETATM & 215 & N24 & HEM \\
\hline HETATM & 216 & C99 & HEM \\
\hline HETATM & 217 & 091 & HEM \\
\hline HETATM & 218 & 092 & HEM \\
\hline HETATM & 219 & CT91 & HEM \\
\hline HETATM & 220 & H911 & HEM \\
\hline HETATM & 221 & H912 & HEM \\
\hline HETATM & 222 & СТ92 & HEM \\
\hline HETATM & 223 & H921 & HEM \\
\hline HETATM & 224 & H922 & HEM \\
\hline \multicolumn{4}{|l|}{ TER } \\
\hline HETATM & 225 & C2 & VIN \\
\hline HETATM & 226 & H 21 & VIN \\
\hline HETATM & 227 & $\mathrm{C} 1$ & VIN \\
\hline HETATM & 228 & H11 & VIN \\
\hline HETATM & 229 & $\mathrm{H} 12$ & VIN \\
\hline \multicolumn{4}{|l|}{ TER } \\
\hline HETATM & 230 & $\mathrm{C} 1$ & MET \\
\hline HETATM & 231 & H11 & $\mathrm{MET}$ \\
\hline HETATM & 232 & $\mathrm{H} 12$ & $\mathrm{MET}$ \\
\hline HETATM & 233 & $\mathrm{H} 13$ & $\mathrm{MET}$ \\
\hline \multicolumn{4}{|l|}{ TER } \\
\hline HETATM & 234 & $\mathrm{C} 2$ & VIN \\
\hline HETATM & 235 & H 21 & VIN \\
\hline HETATM & 236 & $\mathrm{C} 1$ & VIN \\
\hline HETATM & 237 & H11 & VIN \\
\hline HETATM & 238 & $\mathrm{H} 12$ & VIN \\
\hline \multicolumn{4}{|l|}{ TER } \\
\hline HETATM & 239 & $\mathrm{C} 1$ & MET \\
\hline HETATM & 240 & H11 & MET \\
\hline HETATM & 241 & $\mathrm{H} 12$ & MET \\
\hline HETATM & 242 & $\mathrm{H} 13$ & MET \\
\hline \multicolumn{4}{|l|}{ TER } \\
\hline HETATM & 243 & C5 & $\mathrm{ACP}$ \\
\hline HETATM & 244 & H51 & $\mathrm{ACP}$ \\
\hline HETATM & 245 & H52 & $\mathrm{ACP}$ \\
\hline HETATM & 246 & C4 & $\mathrm{ACP}$ \\
\hline HETATM & 247 & $\mathrm{H} 41$ & $\mathrm{ACP}$ \\
\hline HETATM & 248 & $\mathrm{H} 42$ & $\mathrm{ACP}$ \\
\hline HETATM & 249 & $\mathrm{C} 1$ & $\mathrm{ACP}$ \\
\hline HETATM & 250 & $\mathrm{O} 2$ & $\mathrm{ACP}$ \\
\hline HETATM & 251 & 03 & $\mathrm{ACP}$ \\
\hline HETATM & 252 & H3 & $\mathrm{ACP}$ \\
\hline \multicolumn{4}{|l|}{ TER } \\
\hline HETATM & 253 & $\mathrm{C} 1$ & MET \\
\hline HETATM & 254 & H11 & MET \\
\hline HETATM & 255 & $\mathrm{H} 12$ & MET \\
\hline HETATM & 256 & H13 & MET \\
\hline \multicolumn{4}{|l|}{ TER } \\
\hline HETATM & 257 & $\mathrm{C} 1$ & MET \\
\hline HETATM & 258 & $\mathrm{H} 11$ & MET \\
\hline HETATM & 259 & $\mathrm{H} 12$ & $\mathrm{MET}$ \\
\hline HETATM & 260 & $\mathrm{H} 13$ & MET \\
\hline \multicolumn{4}{|l|}{ TER } \\
\hline HETATM & 261 & C2 & VIN \\
\hline HETATM & 262 & H 21 & VIN \\
\hline HETATM & 263 & $\mathrm{C} 1$ & VIN \\
\hline HETATM & 264 & $\mathrm{H} 11$ & VIN \\
\hline HETATM & 265 & $\mathrm{H} 12$ & VIN \\
\hline TER & & & \\
\hline HETATM & 266 & $\mathrm{C} 1$ & MET \\
\hline HETATM & 267 & H11 & MET \\
\hline HETATM & 268 & $\mathrm{H} 12$ & MET \\
\hline HETATM & 269 & H13 & MET \\
\hline TER & & & \\
\hline HETATM & 270 & C2 & VIN \\
\hline HETATM & 271 & H21 & VIN \\
\hline HETATM & 272 & C1 & VIN \\
\hline HETATM & 273 & $\mathrm{H} 11$ & VIN \\
\hline HETATM & 274 & $\mathrm{H} 12$ & VIN \\
\hline TER & & & \\
\hline HETATM & 275 & $\mathrm{C} 1$ & MET \\
\hline HETATM & 276 & $\mathrm{H} 11$ & MET \\
\hline HETATM & 277 & H12 & MET \\
\hline HETATM & 278 & $\mathrm{H} 13$ & MET \\
\hline TER & & & \\
\hline HETATM & 279 & $a_{-}$ & $\mathrm{ACP}$ \\
\hline
\end{tabular}

\begin{tabular}{|c|c|c|c|c|c|}
\hline 16.187 & 3.027 & -0.590 & 1.00 & 0.00 & $\mathrm{~N}$ \\
\hline 16.858 & 4.290 & -3.090 & 1.00 & 0.00 & $\mathrm{~N}$ \\
\hline 19.558 & 3.406 & -2.854 & 1.00 & 0.00 & $\mathrm{~N}$ \\
\hline 18.651 & 6.271 & -0.352 & 1.00 & 0.00 & C \\
\hline 18.492 & 4.958 & -0.365 & 1.00 & 0.00 & 0 \\
\hline 18.806 & 6.888 & 0.703 & 1.00 & 0.00 & 0 \\
\hline 18.635 & 6.952 & -1.709 & 1.00 & 0.00 & C \\
\hline 17.656 & 6.757 & -2.171 & 1.00 & 0.00 & $\mathrm{H}$ \\
\hline 19.383 & 6.463 & -2.352 & 1.00 & 0.00 & $\mathrm{H}$ \\
\hline 18.895 & 8.457 & -1.597 & 1.00 & 0.00 & C \\
\hline 19.820 & 8.613 & -1.027 & 1.00 & 0.00 & $\mathrm{H}$ \\
\hline 18.098 & 8.917 & -1.000 & 1.00 & 0.00 & $\mathrm{H}$ \\
\hline 13.375 & 14.169 & -8.933 & 1.00 & 0.00 & C \\
\hline 12.559 & 13.470 & -8.768 & 1.00 & 0.00 & $\mathrm{H}$ \\
\hline 13.042 & 15.394 & -9.335 & 1.00 & 0.00 & C \\
\hline 13.774 & 16.172 & -9.442 & 1.00 & 0.00 & $\mathrm{H}$ \\
\hline 12.000 & 15.663 & -9.501 & 1.00 & 0.00 & $\mathrm{H}$ \\
\hline 16.039 & 14.424 & -10.771 & 1.00 & 0.00 & C \\
\hline 16.274 & 15.494 & -10.652 & 1.00 & 0.00 & $\mathrm{H}$ \\
\hline 16.852 & 13.959 & -11.341 & 1.00 & 0.00 & $\mathrm{H}$ \\
\hline 15.131 & 14.346 & $-11 \cdot 382$ & 1.00 & 0.00 & $\mathrm{H}$ \\
\hline 21.049 & 12.746 & -10.256 & 1.00 & 0.00 & C \\
\hline 20.383 & 12.645 & -11.115 & 1.00 & 0.00 & $\mathrm{H}$ \\
\hline 22.216 & 13.390 & -10.415 & 1.00 & 0.00 & C \\
\hline 22.896 & 13.546 & -9.585 & 1.00 & 0.00 & $\mathrm{H}$ \\
\hline 22.521 & 13.788 & -11.381 & 1.00 & 0.00 & $\mathrm{H}$ \\
\hline 22.733 & 11.062 & -8.052 & 1.00 & 0.00 & C \\
\hline 23.325 & 11.727 & -7.403 & 1.00 & 0.00 & $\mathrm{H}$ \\
\hline 22.890 & 10.040 & -7.685 & 1.00 & 0.00 & $\mathrm{H}$ \\
\hline 23.166 & 11.128 & -9.057 & 1.00 & 0.00 & $\mathrm{H}$ \\
\hline 13.645 & 9.981 & -2.161 & 1.00 & 0.00 & C \\
\hline 14.384 & 9.969 & -1.349 & 1.00 & 0.00 & $\mathrm{H}$ \\
\hline 12.804 & 10.589 & -1.798 & 1.00 & 0.00 & $\mathrm{H}$ \\
\hline 13.167 & 8.543 & -2.434 & 1.00 & 0.00 & C \\
\hline 12.609 & 8.482 & $-3 \cdot 382$ & 1.00 & 0.00 & $\mathrm{H}$ \\
\hline 14.037 & 7.877 & -2.541 & 1.00 & 0.00 & $\mathrm{H}$ \\
\hline 12.303 & 7.931 & -1.358 & 1.00 & 0.00 & C \\
\hline 12.075 & 8.454 & -0.260 & 1.00 & 0.00 & O \\
\hline 11.787 & 6.751 & -1.722 & 1.00 & 0.00 & o \\
\hline 11.178 & 6.397 & -0.986 & 1.00 & 0.00 & $\mathrm{H}$ \\
\hline 21.541 & 8.855 & -3.334 & 1.00 & 0.00 & C \\
\hline 22.094 & 9.694 & -2.884 & 1.00 & 0.00 & $\mathrm{H}$ \\
\hline 21.548 & 8.029 & -2.613 & 1.00 & 0.00 & $\mathrm{H}$ \\
\hline 22.112 & 8.535 & -4.214 & 1.00 & 0.00 & $\mathrm{H}$ \\
\hline 12.108 & 11.803 & -4.285 & 1.00 & 0.00 & c \\
\hline 11.663 & 11.594 & -3.305 & 1.00 & 0.00 & $\mathrm{H}$ \\
\hline 11.997 & 12.883 & -4.460 & 1.00 & 0.00 & $\mathrm{H}$ \\
\hline 11.507 & 11.278 & -5.042 & 1.00 & 0.00 & $\mathrm{H}$ \\
\hline 21.904 & 0.424 & 1.002 & 1.00 & 0.00 & c \\
\hline 22.720 & 1.123 & 0.837 & 1.00 & 0.00 & $\mathrm{H}$ \\
\hline 22.237 & -0.801 & 1.403 & 1.00 & 0.00 & c \\
\hline 21.506 & -1.579 & 1.511 & 1.00 & 0.00 & $\mathrm{H}$ \\
\hline 23.279 & -1.070 & 1.570 & 1.00 & 0.00 & $\mathrm{H}$ \\
\hline 19.241 & 0.169 & 2.840 & 1.00 & 0.00 & c \\
\hline 19.006 & -0.902 & 2.720 & 1.00 & 0.00 & $\mathrm{H}$ \\
\hline 18.428 & 0.633 & 3.410 & 1.00 & 0.00 & $\mathrm{H}$ \\
\hline 20.148 & 0.247 & 3.450 & 1.00 & 0.00 & $\mathrm{H}$ \\
\hline 14.231 & 1.847 & 2.325 & 1.00 & 0.00 & c \\
\hline 14.897 & 1.948 & 3.184 & 1.00 & 0.00 & $\mathrm{H}$ \\
\hline 13.064 & 1.203 & 2.484 & 1.00 & 0.00 & c \\
\hline 12.384 & 1.047 & 1.654 & 1.00 & 0.00 & $\mathrm{H}$ \\
\hline 12.759 & 0.805 & 3.450 & 1.00 & 0.00 & $\mathrm{H}$ \\
\hline 12.546 & 3.530 & 0.121 & 1.00 & 0.00 & c \\
\hline 12.390 & 4.553 & -0.246 & 1.00 & 0.00 & $\mathrm{H}$ \\
\hline 12.114 & 3.465 & 1.126 & 1.00 & 0.00 & $\mathrm{H}$ \\
\hline 11.955 & 2.866 & -0.528 & 1.00 & 0.00 & $\mathrm{H}$ \\
\hline 21.634 & 4.612 & -5.770 & 1.00 & 0.00 & c \\
\hline
\end{tabular}




\begin{tabular}{|c|c|c|c|c|c|c|c|c|c|c|}
\hline HETATM & 280 & H51 & $\mathrm{ACP}$ & 30 & 20.895 & 4.623 & -6.582 & 1.00 & 0.00 & $\mathrm{H}$ \\
\hline HETATM & 281 & H52 & $\mathrm{ACP}$ & 30 & 22.475 & 4.004 & -6.134 & 1.00 & 0.00 & $\mathrm{H}$ \\
\hline HETATM & 282 & C4 & $\mathrm{ACP}$ & 30 & 22.113 & 6.050 & -5.497 & 1.00 & 0.00 & C \\
\hline HETATM & 283 & H 41 & $\mathrm{ACP}$ & 30 & 22.671 & 6.111 & -4.549 & 1.00 & 0.00 & $\mathrm{H}$ \\
\hline HETATM & 284 & $\mathrm{H} 42$ & $\mathrm{ACP}$ & 30 & 21.242 & 6.716 & -5.390 & 1.00 & 0.00 & $\mathrm{H}$ \\
\hline HETATM & 285 & $\mathrm{C} 1$ & $\mathrm{ACP}$ & 30 & 22.977 & 6.662 & -6.573 & 1.00 & 0.00 & $\mathrm{C}$ \\
\hline HETATM & 286 & $\mathrm{O} 2$ & $\mathrm{ACP}$ & 30 & 23.205 & 6.139 & -7.671 & 1.00 & 0.00 & o \\
\hline HETATM & 287 & 03 & $\mathrm{ACP}$ & 30 & 23.493 & 7.841 & -6.209 & 1.00 & 0.00 & O \\
\hline HETATM & 288 & H3 & $\mathrm{ACP}$ & 30 & 24.102 & 8.196 & -6.945 & 1.00 & 0.00 & $\mathrm{H}$ \\
\hline \multicolumn{11}{|l|}{ TER } \\
\hline HETATM & 289 & $\mathrm{C} 1$ & $\mathrm{MET}$ & 31 & 13.738 & 5.738 & -4.597 & 1.00 & 0.00 & C \\
\hline HETATM & 290 & H11 & $\mathrm{MET}$ & 31 & 13.186 & 4.899 & -5.047 & 1.00 & 0.00 & $\mathrm{H}$ \\
\hline HETATM & 291 & H12 & $\mathrm{MET}$ & 31 & 13.732 & 6.564 & -5.319 & 1.00 & 0.00 & $\mathrm{H}$ \\
\hline HETATM & 292 & $\mathrm{H} 13$ & $\mathrm{MET}$ & 31 & 13.167 & 6.058 & -3.717 & 1.00 & 0.00 & $\mathrm{H}$ \\
\hline \multicolumn{11}{|l|}{ TER } \\
\hline HETATM & 293 & $\mathrm{C} 1$ & MET & 32 & 23.172 & 2.790 & -3.646 & 1.00 & 0.00 & C \\
\hline HETATM & 294 & H11 & MET & 32 & 23.617 & 2.998 & -4.626 & 1.00 & 0.00 & $\mathrm{H}$ \\
\hline HETATM & 295 & H12 & $\mathrm{MET}$ & 32 & 23.282 & 1.709 & -3.471 & 1.00 & 0.00 & $\mathrm{H}$ \\
\hline HETATM & 296 & H13 & $\mathrm{MET}$ & 32 & 23.772 & 3.315 & -2.889 & 1.00 & 0.00 & $\mathrm{H}$ \\
\hline \multicolumn{11}{|l|}{ TER } \\
\hline HETATM & 297 & $\mathrm{FE}$ & HEM & 33 & -8.767 & 10.511 & 9.423 & 1.00 & 0.00 & $\mathrm{FE}$ \\
\hline HETATM & 298 & $\mathrm{C} 1$ & HEM & 33 & -10.995 & 12.383 & 8.361 & 1.00 & 0.00 & C \\
\hline HETATM & 299 & $\mathrm{C} 2$ & HEM & 33 & $-11 \cdot 354$ & 13.174 & 7.189 & 1.00 & 0.00 & C \\
\hline HETATM & 300 & C3 & HEM & 33 & -10.209 & 13.271 & 6.414 & 1.00 & 0.00 & C \\
\hline HETATM & 301 & $\mathrm{C} 4$ & HEM & 33 & -9.179 & 12.553 & 7.122 & 1.00 & 0.00 & C \\
\hline HETATM & 302 & C5 & HEM & 33 & -7.864 & 12.435 & 6.684 & 1.00 & 0.00 & C \\
\hline HETATM & 303 & H5 & HEM & 33 & -7.610 & 12.931 & 5.749 & 1.00 & 0.00 & $\mathrm{H}$ \\
\hline HETATM & 304 & C6 & HEM & 33 & -6.845 & 11.736 & 7.320 & 1.00 & 0.00 & $\mathrm{C}$ \\
\hline HETATM & 305 & C7 & HEM & 33 & -5.476 & 11.664 & 6.851 & 1.00 & 0.00 & C \\
\hline HETATM & 306 & $\mathrm{C} 8$ & HEM & 33 & -4.770 & 10.944 & 7.798 & 1.00 & 0.00 & $\mathrm{C}$ \\
\hline HETATM & 307 & C9 & HEM & 33 & -5.721 & 10.579 & 8.822 & 1.00 & 0.00 & C \\
\hline HETATM & 308 & $\mathrm{C} 10$ & HEM & 33 & -5.417 & 9.844 & 9.965 & 1.00 & 0.00 & C \\
\hline HETATM & 309 & H1O & HEM & 33 & -4.390 & 9.500 & 10.071 & 1.00 & 0.00 & $\mathrm{H}$ \\
\hline HETATM & 310 & $\mathrm{C} 11$ & HEM & 33 & -6.291 & 9.504 & 10.988 & 1.00 & 0.00 & $\mathrm{C}$ \\
\hline HETATM & 311 & $\mathrm{C} 12$ & HEM & 33 & -5.910 & 8.779 & 12.178 & 1.00 & 0.00 & C \\
\hline HETATM & 312 & $\mathrm{C} 13$ & HEM & 33 & -7.050 & 8.631 & 12.935 & 1.00 & 0.00 & C \\
\hline HETATM & 313 & $\mathrm{C} 14$ & HEM & 33 & -8.123 & 9.273 & 12.204 & 1.00 & 0.00 & C \\
\hline HETATM & 314 & C15 & HEM & 33 & -9.451 & 9.328 & 12.625 & 1.00 & 0.00 & C \\
\hline HETATM & 315 & H15 & HEM & 33 & -9.691 & 8.843 & 13.573 & 1.00 & 0.00 & $\mathrm{H}$ \\
\hline HETATM & 316 & $\mathrm{C} 16$ & HEM & 33 & -10.481 & 10.009 & 11.982 & 1.00 & 0.00 & C \\
\hline HETATM & 317 & C17 & HEM & 33 & -11.847 & 10.123 & 12.464 & 1.00 & 0.00 & C \\
\hline HETATM & 318 & C18 & HEM & 33 & -12.522 & 10.890 & 11.541 & 1.00 & 0.00 & C \\
\hline HETATM & 319 & C19 & HEM & 33 & -11.573 & 11.242 & 10.504 & 1.00 & 0.00 & C \\
\hline HETATM & 320 & $\mathrm{C} 20$ & HEM & 33 & -11.864 & 12.033 & 9.393 & 1.00 & 0.00 & $\mathrm{C}$ \\
\hline HETATM & 321 & H 20 & HEM & 33 & -12.878 & 12.423 & 9.333 & 1.00 & 0.00 & $\mathrm{H}$ \\
\hline HETATM & 322 & N21 & HEM & 33 & -9.674 & 12.020 & 8.298 & 1.00 & 0.00 & $\mathrm{~N}$ \\
\hline HETATM & 323 & N22 & HEM & 33 & -6.969 & 11.073 & 8.521 & 1.00 & 0.00 & $\mathrm{~N}$ \\
\hline HETATM & 324 & N23 & HEM & 33 & -7.640 & 9.810 & 11.021 & 1.00 & 0.00 & $\mathrm{~N}$ \\
\hline HETATM & 325 & $\mathrm{~N} 24$ & HEM & 33 & -10.339 & 10.694 & 10.786 & 1.00 & 0.00 & $\mathrm{~N}$ \\
\hline HETATM & 326 & C99 & HEM & 33 & -9.433 & 7.829 & 8.283 & 1.00 & 0.00 & C \\
\hline HETATM & 327 & 091 & HEM & 33 & -9.273 & 9.143 & 8.296 & 1.00 & 0.00 & O \\
\hline HETATM & 328 & 092 & HEM & 33 & -9.588 & 7.212 & 7.228 & 1.00 & 0.00 & O \\
\hline HETATM & 329 & СТ91 & HEM & 33 & -9.417 & 7.148 & 9.640 & 1.00 & 0.00 & $\mathrm{C}$ \\
\hline HETATM & 330 & H911 & HEM & 33 & -8.438 & 7.343 & 10.103 & 1.00 & 0.00 & $\mathrm{H}$ \\
\hline HETATM & 331 & H912 & HEM & 33 & -10.165 & 7.638 & 10.283 & 1.00 & 0.00 & $\mathrm{H}$ \\
\hline HETATM & 332 & CT92 & HEM & 33 & -9.676 & 5.644 & 9.528 & 1.00 & 0.00 & C \\
\hline HETATM & 333 & H921 & HEM & 33 & -8.880 & 5.183 & 8.931 & 1.00 & 0.00 & $\mathrm{H}$ \\
\hline HETATM & 334 & H922 & HEM & 33 & -10.601 & 5.488 & 8.958 & 1.00 & 0.00 & $\mathrm{H}$ \\
\hline \multicolumn{11}{|l|}{ TER } \\
\hline HETATM & 335 & $\mathrm{FE}$ & HEM & 34 & -8.075 & 3.097 & 14.371 & 1.00 & 0.00 & $\mathrm{FE}$ \\
\hline HETATM & 336 & $\mathrm{C} 1$ & HEM & 34 & -5.847 & 1.225 & 15.433 & 1.00 & 0.00 & C \\
\hline HETATM & 337 & $\mathrm{C} 2$ & HEM & 34 & -5.488 & 0.434 & 16.605 & 1.00 & 0.00 & C \\
\hline HETATM & 338 & C3 & HEM & 34 & -6.634 & 0.337 & 17.380 & 1.00 & 0.00 & C \\
\hline HETATM & 339 & $\mathrm{C} 4$ & HEM & 34 & -7.663 & 1.055 & 16.672 & 1.00 & 0.00 & C \\
\hline HETATM & 340 & C5 & HEM & 34 & -8.979 & 1.173 & 17.109 & 1.00 & 0.00 & C \\
\hline HETATM & 341 & H5 & HEM & 34 & -9.232 & 0.677 & 18.045 & 1.00 & 0.00 & $\mathrm{H}$ \\
\hline HETATM & 342 & $\mathrm{C} 6$ & HEM & 34 & -9.998 & 1.872 & 16.473 & 1.00 & 0.00 & C \\
\hline HETATM & 343 & C7 & HEM & 34 & -11.367 & 1.944 & 16.943 & 1.00 & 0.00 & C \\
\hline HETATM & 344 & C8 & HEM & 34 & -12.072 & 2.664 & 15.996 & 1.00 & 0.00 & C \\
\hline HETATM & 345 & C9 & HEM & 34 & $-11 \cdot 121$ & 3.029 & 14.972 & 1.00 & 0.00 & C \\
\hline HETATM & 346 & $\mathrm{C} 10$ & HEM & 34 & -11.426 & 3.764 & 13.828 & 1.00 & 0.00 & C \\
\hline HETATM & 347 & $\mathrm{H} 10$ & HEM & 34 & -12.453 & 4.108 & 13.722 & 1.00 & 0.00 & $\mathrm{H}$ \\
\hline HETATM & 348 & $\mathrm{C} 11$ & HEM & 34 & -10.551 & 4.104 & 12.806 & 1.00 & 0.00 & C \\
\hline HETATM & 349 & $\mathrm{C} 12$ & HEM & 34 & -10.932 & 4.829 & 11.615 & 1.00 & 0.00 & C \\
\hline HETATM & 350 & C13 & HEM & 34 & -9.793 & 4.977 & 10.858 & 1.00 & 0.00 & C \\
\hline HETATM & 351 & C14 & HEM & 34 & -8.720 & 4.335 & 11.590 & 1.00 & 0.00 & C \\
\hline HETATM & 352 & C15 & HEM & 34 & -7.392 & 4.280 & 11.168 & 1.00 & 0.00 & C \\
\hline HETATM & 353 & H15 & HEM & 34 & -7.152 & 4.765 & 10.221 & 1.00 & 0.00 & $\mathrm{H}$ \\
\hline HETATM & 354 & C16 & HEM & 34 & -6.362 & 3.599 & 11.812 & 1.00 & 0.00 & C \\
\hline
\end{tabular}




\begin{tabular}{|c|c|c|c|c|c|c|c|c|c|c|}
\hline HETATM & 355 & C17 & HEM & 34 & -4.996 & 3.485 & 11.329 & 1.00 & 0.00 & C \\
\hline HETATM & 356 & $\mathrm{C} 18$ & HEM & 34 & -4.320 & 2.718 & 12.253 & 1.00 & 0.00 & C \\
\hline HETATM & 357 & C19 & HEM & 34 & -5.270 & 2.366 & 13.289 & 1.00 & 0.00 & C \\
\hline HETATM & 358 & $\mathrm{C} 20$ & HEM & 34 & -4.979 & 1.575 & 14.401 & 1.00 & 0.00 & $\mathrm{C}$ \\
\hline HETATM & 359 & H 20 & HEM & 34 & -3.964 & 1.185 & 14.461 & 1.00 & 0.00 & $\mathrm{H}$ \\
\hline HETATM & 360 & $\mathrm{~N} 21$ & HEM & 34 & -7.169 & 1.588 & 15.495 & 1.00 & 0.00 & $\mathrm{~N}$ \\
\hline HETATM & 361 & N22 & HEM & 34 & -9.874 & 2.535 & 15.273 & 1.00 & 0.00 & $\mathrm{~N}$ \\
\hline HETATM & 362 & N23 & HEM & 34 & -9.203 & 3.798 & 12.772 & 1.00 & 0.00 & $\mathrm{~N}$ \\
\hline HETATM & 363 & $\mathrm{~N} 24$ & HEM & 34 & -6.503 & 2.914 & 13.008 & 1.00 & 0.00 & $\mathrm{~N}$ \\
\hline HETATM & 364 & C99 & HEM & 34 & -7.410 & 5.779 & 15.510 & 1.00 & 0.00 & C \\
\hline HETATM & 365 & 091 & HEM & 34 & -7.569 & 4.465 & 15.498 & 1.00 & 0.00 & 0 \\
\hline HETATM & 366 & 092 & HEM & 34 & -7.255 & 6.396 & 16.566 & 1.00 & 0.00 & o \\
\hline HETATM & 367 & СТ91 & HEM & 34 & -7.426 & 6.460 & 14.154 & 1.00 & 0.00 & C \\
\hline HETATM & 368 & H911 & HEM & 34 & -8.405 & 6.265 & 13.691 & 1.00 & 0.00 & $\mathrm{H}$ \\
\hline HETATM & 369 & H912 & HEM & 34 & -6.678 & 5.970 & 13.511 & 1.00 & 0.00 & $\mathrm{H}$ \\
\hline HETATM & 370 & СТ 92 & HEM & 34 & -7.166 & 7.964 & 14.265 & 1.00 & 0.00 & C \\
\hline HETATM & 371 & H921 & HEM & 34 & -6.241 & 8.120 & 14.836 & 1.00 & 0.00 & $\mathrm{H}$ \\
\hline HETATM & 372 & H922 & HEM & 34 & -7.963 & 8.425 & 14.862 & 1.00 & 0.00 & $\mathrm{H}$ \\
\hline \multicolumn{11}{|l|}{ TER } \\
\hline HETATM & 373 & $\mathrm{C} 2$ & VIN & 35 & -12.686 & 13.676 & 6.929 & 1.00 & 0.00 & $\mathrm{C}$ \\
\hline HETATM & 374 & $\mathrm{H} 21$ & VIN & 35 & -13.502 & 12.978 & 7.094 & 1.00 & 0.00 & $\mathrm{H}$ \\
\hline HETATM & 375 & $\mathrm{C} 1$ & VIN & 35 & -13.019 & 14.901 & 6.528 & 1.00 & 0.00 & C \\
\hline HETATM & 376 & $\mathrm{H} 11$ & VIN & 35 & -12.287 & 15.680 & 6.421 & 1.00 & 0.00 & $\mathrm{H}$ \\
\hline HETATM & 377 & $\mathrm{H} 12$ & VIN & 35 & -14.061 & 15.170 & 6.361 & 1.00 & 0.00 & $\mathrm{H}$ \\
\hline \multicolumn{11}{|l|}{ TER } \\
\hline HETATM & 378 & $\mathrm{C} 1$ & MET & 36 & -10.022 & 13.931 & 5.091 & 1.00 & 0.00 & C \\
\hline HETATM & 379 & H11 & MET & 36 & -9.787 & 15.002 & 5.211 & 1.00 & 0.00 & $\mathrm{H}$ \\
\hline HETATM & 380 & $\mathrm{H} 12$ & MET & 36 & -9.209 & 13.467 & 4.522 & 1.00 & 0.00 & $\mathrm{H}$ \\
\hline HETATM & 381 & $\mathrm{H} 13$ & MET & 36 & -10.930 & 13.854 & 4.481 & 1.00 & 0.00 & $\mathrm{H}$ \\
\hline \multicolumn{11}{|l|}{ TER } \\
\hline HETATM & 382 & $\mathrm{C} 2$ & VIN & 37 & -5.012 & 12.253 & 5.606 & 1.00 & 0.00 & C \\
\hline HETATM & 383 & H 21 & VIN & 37 & -5.678 & 12.152 & 4.747 & 1.00 & 0.00 & $\mathrm{H}$ \\
\hline HETATM & 384 & $\mathrm{C} 1$ & VIN & 37 & -3.845 & 12.898 & 5.447 & 1.00 & 0.00 & C \\
\hline HETATM & 385 & H11 & VIN & 37 & -3.165 & 13.054 & 6.277 & 1.00 & 0.00 & $\mathrm{H}$ \\
\hline HETATM & 386 & $\mathrm{H} 12$ & VIN & 37 & -3.540 & 13.296 & 4.482 & 1.00 & 0.00 & $\mathrm{H}$ \\
\hline \multicolumn{11}{|l|}{ TER } \\
\hline HETATM & 387 & $\mathrm{C} 1$ & MET & 38 & -3.328 & 10.570 & 7.810 & 1.00 & 0.00 & C \\
\hline HETATM & 388 & H11 & MET & 38 & -2.736 & 11.234 & 8.459 & 1.00 & 0.00 & $\mathrm{H}$ \\
\hline HETATM & 389 & $\mathrm{H} 12$ & MET & 38 & -3.171 & 9.548 & 8.177 & 1.00 & 0.00 & $\mathrm{H}$ \\
\hline HETATM & 390 & $\mathrm{H} 13$ & MET & 38 & -2.895 & 10.636 & 6.805 & 1.00 & 0.00 & $\mathrm{H}$ \\
\hline \multicolumn{11}{|l|}{ TER } \\
\hline HETATM & 391 & C5 & $\mathrm{ACP}$ & 39 & -12.416 & 9.488 & 13.701 & 1.00 & 0.00 & C \\
\hline HETATM & 392 & H51 & $\mathrm{ACP}$ & 39 & -11.677 & 9.477 & 14.514 & 1.00 & 0.00 & $\mathrm{H}$ \\
\hline HETATM & 393 & H5 2 & $\mathrm{ACP}$ & 39 & -13.257 & 10.097 & 14.065 & 1.00 & 0.00 & $\mathrm{H}$ \\
\hline HETATM & 394 & $\mathrm{C} 4$ & $\mathrm{ACP}$ & 39 & -12.894 & 8.051 & 13.428 & 1.00 & 0.00 & C \\
\hline HETATM & 395 & $\mathrm{H} 41$ & $\mathrm{ACP}$ & 39 & -13.452 & 7.990 & 12.481 & 1.00 & 0.00 & $\mathrm{H}$ \\
\hline HETATM & 396 & $\mathrm{H} 42$ & $\mathrm{ACP}$ & 39 & -12.024 & 7.384 & 13.321 & 1.00 & 0.00 & $\mathrm{H}$ \\
\hline HETATM & 397 & $\mathrm{C} 1$ & $\mathrm{ACP}$ & 39 & -13.758 & 7.439 & 14.504 & 1.00 & 0.00 & C \\
\hline HETATM & 398 & $\mathrm{O} 2$ & $\mathrm{ACP}$ & 39 & -13.986 & 7.961 & 15.603 & 1.00 & 0.00 & 0 \\
\hline HETATM & 399 & 03 & $\mathrm{ACP}$ & 39 & -14.274 & 6.259 & 14.140 & 1.00 & 0.00 & o \\
\hline HETATM & 400 & H3 & $\mathrm{ACP}$ & 39 & -14.883 & 5.905 & 14.876 & 1.00 & 0.00 & $\mathrm{H}$ \\
\hline TER & & & & & & & & & & \\
\hline HETATM & 401 & $\mathrm{C} 1$ & MET & 40 & -4.520 & 8.362 & 12.528 & 1.00 & 0.00 & C \\
\hline HETATM & 402 & $\mathrm{H} 11$ & MET & 40 & -3.967 & 9.201 & 12.978 & 1.00 & 0.00 & $\mathrm{H}$ \\
\hline HETATM & 403 & $\mathrm{H} 12$ & MET & 40 & -4.513 & 7.536 & 13.250 & 1.00 & 0.00 & $\mathrm{H}$ \\
\hline HETATM & 404 & $\mathrm{H} 13$ & MET & 40 & -3.949 & 8.042 & 11.649 & 1.00 & 0.00 & $\mathrm{H}$ \\
\hline TER & & & & & & & & & & \\
\hline HETATM & 405 & $\mathrm{C} 1$ & MET & 41 & -13.953 & 11.311 & 11.577 & 1.00 & 0.00 & C \\
\hline HETATM & 406 & $\mathrm{H} 11$ & $\mathrm{MET}$ & 41 & -14.398 & 11.102 & 12.557 & 1.00 & 0.00 & $\mathrm{H}$ \\
\hline HETATM & 407 & $\mathrm{H} 12$ & MET & 41 & -14.064 & 12.391 & 11.402 & 1.00 & 0.00 & $\mathrm{H}$ \\
\hline HETATM & 408 & $\mathrm{H} 13$ & MET & 41 & -14.554 & 10.785 & 10.821 & 1.00 & 0.00 & $\mathrm{H}$ \\
\hline TER & & & & & & & & & & \\
\hline HETATM & 409 & $\mathrm{C} 2$ & VIN & 42 & -4.157 & -0.068 & 16.864 & 1.00 & 0.00 & C \\
\hline HETATM & 410 & H 21 & VIN & 42 & -3.341 & 0.630 & 16.699 & 1.00 & 0.00 & $\mathrm{H}$ \\
\hline HETATM & 411 & $\mathrm{C} 1$ & VIN & 42 & -3.824 & -1.293 & 17.266 & 1.00 & 0.00 & C \\
\hline HETATM & 412 & H11 & VIN & 42 & -4.555 & -2.072 & 17.373 & 1.00 & 0.00 & $\mathrm{H}$ \\
\hline HETATM & 413 & $\mathrm{H} 12$ & VIN & 42 & -2.782 & -1.562 & 17.432 & 1.00 & 0.00 & $\mathrm{H}$ \\
\hline TER & & & & & & & & & & \\
\hline HETATM & 414 & $\mathrm{C} 1$ & MET & 43 & -6.820 & -0.323 & 18.702 & 1.00 & 0.00 & C \\
\hline HETATM & 415 & H11 & MET & 43 & -7.055 & -1.394 & 18.583 & 1.00 & 0.00 & $\mathrm{H}$ \\
\hline HETATM & 416 & $\mathrm{H} 12$ & MET & 43 & -7.633 & 0.141 & 19.272 & 1.00 & 0.00 & $\mathrm{H}$ \\
\hline HETATM & 417 & $\mathrm{H} 13$ & MET & 43 & -5.913 & -0.246 & 19.313 & 1.00 & 0.00 & $\mathrm{H}$ \\
\hline TER & & & & & & & & & & \\
\hline HETATM & 418 & $\mathrm{C} 2$ & VIN & 44 & -11.830 & 1.355 & 18.188 & 1.00 & 0.00 & C \\
\hline HETATM & 419 & H2 1 & VIN & 44 & -11.164 & 1.456 & 19.046 & 1.00 & 0.00 & $\mathrm{H}$ \\
\hline HETATM & 420 & $\mathrm{C} 1$ & VIN & 44 & -12.997 & 0.710 & 18.346 & 1.00 & 0.00 & C \\
\hline HETATM & 421 & H11 & VIN & 44 & -13.677 & 0.554 & 17.516 & 1.00 & 0.00 & $\mathrm{H}$ \\
\hline HETATM & 422 & $\mathrm{H} 12$ & VIN & 44 & -13.302 & 0.312 & 19.312 & 1.00 & 0.00 & $\mathrm{H}$ \\
\hline
\end{tabular}




\begin{tabular}{|c|c|c|c|c|c|c|c|c|c|c|}
\hline HETATM & 423 & $\mathrm{C} 1$ & MET & 45 & -13.515 & 3.038 & 15.984 & 1.00 & 0.00 & C \\
\hline HETATM & 424 & H11 & MET & 45 & -13.671 & 4.060 & 15.617 & 1.00 & 0.00 & $\mathrm{H}$ \\
\hline HETATM & 425 & $\mathrm{H} 12$ & MET & 45 & -13.947 & 2.972 & 16.989 & 1.00 & 0.00 & $\mathrm{H}$ \\
\hline HETATM & 426 & $\mathrm{H} 13$ & MET & 45 & -14.106 & 2.374 & 15.335 & 1.00 & 0.00 & $\mathrm{H}$ \\
\hline \multicolumn{11}{|l|}{ TER } \\
\hline HETATM & 427 & C5 & $\mathrm{ACP}$ & 46 & -4.427 & 4.120 & 10.092 & 1.00 & 0.00 & C \\
\hline HETATM & 428 & H51 & $\mathrm{ACP}$ & 46 & -5.166 & 4.131 & 9.280 & 1.00 & 0.00 & $\mathrm{H}$ \\
\hline HETATM & 429 & H52 & $\mathrm{ACP}$ & 46 & -3.586 & 3.511 & 9.729 & 1.00 & 0.00 & $\mathrm{H}$ \\
\hline HETATM & 430 & C4 & $\mathrm{ACP}$ & 46 & -3.948 & 5.557 & 10.365 & 1.00 & 0.00 & C \\
\hline HETATM & 431 & $\mathrm{H} 4 \mathrm{I}$ & $\mathrm{ACP}$ & 46 & -3.390 & 5.618 & 11.313 & 1.00 & 0.00 & $\mathrm{H}$ \\
\hline HETATM & 432 & $\mathrm{H} 42$ & $\mathrm{ACP}$ & 46 & -4.819 & 6.224 & 10.472 & 1.00 & 0.00 & $\mathrm{H}$ \\
\hline HETATM & 433 & $\mathrm{C} 1$ & $\mathrm{ACP}$ & 46 & -3.084 & 6.169 & 9.289 & 1.00 & 0.00 & C \\
\hline HETATM & 434 & $\mathrm{O} 2$ & $\mathrm{ACP}$ & 46 & -2.856 & 5.647 & 8.191 & 1.00 & 0.00 & 0 \\
\hline HETATM & 435 & 03 & $\mathrm{ACP}$ & 46 & -2.568 & 7.349 & 9.653 & 1.00 & 0.00 & o \\
\hline HETATM & 436 & H3 & $\mathrm{ACP}$ & 46 & -1.959 & 7.703 & 8.917 & 1.00 & 0.00 & $\mathrm{H}$ \\
\hline \multicolumn{11}{|l|}{ TER } \\
\hline HETATM & 437 & $\mathrm{C} 1$ & $\mathrm{MET}$ & 47 & $-12 \cdot 323$ & 5.246 & 11.266 & 1.00 & 0.00 & C \\
\hline HETATM & 438 & $\mathrm{H} 11$ & MET & 47 & -12.875 & 4.407 & 10.815 & 1.00 & 0.00 & $\mathrm{H}$ \\
\hline HETATM & 439 & $\mathrm{H} 12$ & MET & 47 & $-12 \cdot 329$ & 6.072 & 10.544 & 1.00 & 0.00 & $\mathrm{H}$ \\
\hline HETATM & 440 & $\mathrm{H} 13$ & MET & 47 & -12.894 & 5.565 & 12.145 & 1.00 & 0.00 & $\mathrm{H}$ \\
\hline \multicolumn{11}{|l|}{ TER } \\
\hline HETATM & 441 & $\mathrm{C} 1$ & MET & 48 & -2.889 & 2.297 & 12.216 & 1.00 & 0.00 & C \\
\hline HETATM & 442 & $\mathrm{H} 11$ & MET & 48 & -2.444 & 2.506 & 11.236 & 1.00 & 0.00 & $\mathrm{H}$ \\
\hline HETATM & 443 & $\mathrm{H} 12$ & MET & 48 & -2.779 & 1.217 & 12.391 & 1.00 & 0.00 & $\mathrm{H}$ \\
\hline HETATM & 444 & H13 & MET & 48 & -2.289 & 2.823 & 12.973 & 1.00 & 0.00 & $\mathrm{H}$ \\
\hline TER & & & & & & & & & & \\
\hline
\end{tabular}

\subsection{Q1 dimer: $A Y W_{-} Q 1 . p d b$ file}

\begin{tabular}{|c|c|c|c|c|c|c|c|c|c|c|}
\hline HETATM & 1 & $\mathrm{FE}$ & HEM & 1 & 4.263 & 10.757 & 1.491 & 1.00 & 0.00 & $\mathrm{FE}$ \\
\hline HETATM & 2 & $\mathrm{C} 1$ & HEM & 1 & 2.035 & 12.629 & 0.429 & 1.00 & 0.00 & C \\
\hline HETATM & 3 & $\mathrm{C} 2$ & HEM & 1 & 1.676 & 13.420 & -0.742 & 1.00 & 0.00 & C \\
\hline HETATM & 4 & $\mathrm{C} 3$ & HEM & 1 & 2.822 & 13.517 & -1.517 & 1.00 & 0.00 & C \\
\hline HETATM & 5 & $\mathrm{C} 4$ & HEM & 1 & 3.851 & 12.800 & -0.809 & 1.00 & 0.00 & C \\
\hline HETATM & 6 & C5 & HEM & 1 & 5.167 & 12.681 & -1.247 & 1.00 & 0.00 & C \\
\hline HETATM & 7 & H5 & HEM & 1 & 5.420 & 13.177 & -2.182 & 1.00 & 0.00 & $\mathrm{H}$ \\
\hline HETATM & 8 & C6 & HEM & 1 & 6.186 & 11.982 & -0.611 & 1.00 & 0.00 & $\mathrm{C}$ \\
\hline HETATM & 9 & C7 & HEM & 1 & 7.555 & 11.910 & -1.081 & 1.00 & 0.00 & $\mathrm{C}$ \\
\hline HETATM & 10 & $\mathrm{C} 8$ & HEM & 1 & 8.260 & 11.190 & -0.134 & 1.00 & 0.00 & C \\
\hline HETATM & 11 & $\mathrm{C} 9$ & HEM & 1 & 7.309 & 10.825 & 0.891 & 1.00 & 0.00 & C \\
\hline HETATM & 12 & $\mathrm{C} 10$ & HEM & 1 & 7.614 & 10.090 & 2.034 & 1.00 & 0.00 & C \\
\hline HETATM & 13 & $\mathrm{H} 10$ & HEM & 1 & 8.641 & 9.746 & 2.140 & 1.00 & 0.00 & $\mathrm{H}$ \\
\hline HETATM & 14 & $\mathrm{C} 11$ & HEM & 1 & 6.739 & 9.750 & 3.057 & 1.00 & 0.00 & C \\
\hline HETATM & 15 & $\mathrm{C} 12$ & HEM & 1 & 7.120 & 9.025 & 4.247 & 1.00 & 0.00 & C \\
\hline HETATM & 16 & $\mathrm{C} 13$ & HEM & 1 & 5.981 & 8.878 & 5.004 & 1.00 & 0.00 & C \\
\hline HETATM & 17 & $\mathrm{C} 14$ & HEM & 1 & 4.908 & 9.519 & 4.273 & 1.00 & 0.00 & C \\
\hline HETATM & 18 & $\mathrm{C} 15$ & HEM & 1 & 3.580 & 9.574 & 4.694 & 1.00 & 0.00 & $\mathrm{C}$ \\
\hline HETATM & 19 & H15 & HEM & 1 & 3.340 & 9.089 & 5.642 & 1.00 & 0.00 & $\mathrm{H}$ \\
\hline HETATM & 20 & $\mathrm{C} 16$ & HEM & 1 & 2.550 & 10.255 & 4.051 & 1.00 & 0.00 & C \\
\hline HETATM & 21 & C17 & HEM & 1 & 1.184 & 10.369 & 4.533 & 1.00 & 0.00 & C \\
\hline HETATM & 22 & $\mathrm{C} 18$ & HEM & 1 & 0.508 & 11.137 & 3.609 & 1.00 & 0.00 & $\mathrm{C}$ \\
\hline HETATM & 23 & C19 & HEM & 1 & 1.458 & 11.489 & 2.573 & 1.00 & 0.00 & C \\
\hline HETATM & 24 & $\mathrm{C} 20$ & HEM & 1 & 1.167 & 12.280 & 1.462 & 1.00 & 0.00 & C \\
\hline HETATM & 25 & $\mathrm{H} 20$ & HEM & 1 & 0.152 & 12.669 & 1.402 & 1.00 & 0.00 & $\mathrm{H}$ \\
\hline HETATM & 26 & $\mathrm{~N} 21$ & HEM & 1 & 3.357 & 12.266 & 0.367 & 1.00 & 0.00 & $\mathrm{~N}$ \\
\hline HETATM & 27 & N22 & HEM & 1 & 6.062 & 11.319 & 0.590 & 1.00 & 0.00 & $\mathrm{~N}$ \\
\hline HETATM & 28 & N23 & HEM & 1 & 5.391 & 10.056 & 3.090 & 1.00 & 0.00 & $\mathrm{~N}$ \\
\hline HETATM & 29 & N24 & HEM & 1 & 2.691 & 10.941 & 2.854 & 1.00 & 0.00 & $\mathrm{~N}$ \\
\hline HETATM & 30 & C99 & HEM & 1 & 3.598 & 8.076 & 0.352 & 1.00 & 0.00 & C \\
\hline HETATM & 31 & 091 & HEM & 1 & 3.757 & 9.389 & 0.365 & 1.00 & 0.00 & 0 \\
\hline HETATM & 32 & 092 & HEM & 1 & 3.443 & 7.458 & -0.703 & 1.00 & 0.00 & O \\
\hline HETATM & 33 & СТ91 & HEM & 1 & 3.614 & 7.394 & 1.709 & 1.00 & 0.00 & $\mathrm{C}$ \\
\hline HETATM & 34 & H911 & HEM & 1 & 4.593 & 7.590 & 2.171 & 1.00 & 0.00 & $\mathrm{H}$ \\
\hline HETATM & 35 & H912 & HEM & 1 & 2.866 & 7.884 & 2.352 & 1.00 & 0.00 & $\mathrm{H}$ \\
\hline HETATM & 36 & СТ92 & HEM & 1 & 3.354 & 5.890 & 1.597 & 1.00 & 0.00 & C \\
\hline HETATM & 37 & H921 & HEM & 1 & 4.151 & 5.429 & 1.000 & 1.00 & 0.00 & $\mathrm{H}$ \\
\hline HETATM & 38 & H922 & HEM & 1 & 2.429 & 5.734 & 1.027 & 1.00 & 0.00 & $\mathrm{H}$ \\
\hline \multicolumn{11}{|l|}{ TER } \\
\hline HETATM & 39 & $\mathrm{FE}$ & HEM & 2 & 4.955 & 3.343 & 6.440 & 1.00 & 0.00 & $\mathrm{FE}$ \\
\hline HETATM & 40 & $\mathrm{C} 1$ & HEM & 2 & 7.183 & 1.471 & 7.502 & 1.00 & 0.00 & C \\
\hline HETATM & 41 & $\mathrm{C} 2$ & HEM & 2 & 7.542 & 0.680 & 8.674 & 1.00 & 0.00 & C \\
\hline HETATM & 42 & C3 & HEM & 2 & 6.397 & 0.583 & 9.448 & 1.00 & 0.00 & C \\
\hline HETATM & 43 & $\mathrm{C} 4$ & HEM & 2 & 5.367 & 1.301 & 8.740 & 1.00 & 0.00 & C \\
\hline HETATM & 44 & C5 & HEM & 2 & 4.052 & 1.420 & 9.178 & 1.00 & 0.00 & C \\
\hline HETATM & 45 & H5 & HEM & 2 & 3.798 & 0.923 & 10.113 & 1.00 & 0.00 & $\mathrm{H}$ \\
\hline HETATM & 46 & $\mathrm{C} 6$ & HEM & 2 & 3.033 & 2.118 & 8.542 & 1.00 & 0.00 & C \\
\hline HETATM & 47 & $\mathrm{C} 7$ & HEM & 2 & 1.664 & 2.191 & 9.012 & 1.00 & 0.00 & C \\
\hline
\end{tabular}




\begin{tabular}{|c|c|c|c|c|c|c|c|c|c|c|}
\hline HETATM & 48 & $\mathrm{C} 8$ & HEM & 2 & 0.958 & 2.910 & 8.065 & 1.00 & 0.00 & C \\
\hline HETATM & 49 & C9 & HEM & 2 & 1.909 & 3.275 & 7.041 & 1.00 & 0.00 & C \\
\hline HETATM & 50 & $\mathrm{C} 10$ & HEM & 2 & 1.605 & 4.010 & 5.897 & 1.00 & 0.00 & C \\
\hline HETATM & 51 & $\mathrm{H} 10$ & HEM & 2 & 0.578 & 4.354 & 5.791 & 1.00 & 0.00 & $\mathrm{H}$ \\
\hline HETATM & 52 & C11 & HEM & 2 & 2.479 & 4.350 & 4.874 & 1.00 & 0.00 & C \\
\hline HETATM & 53 & C12 & HEM & 2 & 2.098 & 5.076 & 3.684 & 1.00 & 0.00 & C \\
\hline HETATM & 54 & $\mathrm{C} 13$ & HEM & 2 & 3.238 & 5.223 & 2.927 & 1.00 & 0.00 & C \\
\hline HETATM & 55 & $\mathrm{C} 14$ & HEM & 2 & 4.311 & 4.581 & 3.659 & 1.00 & 0.00 & C \\
\hline HETATM & 56 & C15 & HEM & 2 & 5.639 & 4.527 & 3.237 & 1.00 & 0.00 & C \\
\hline HETATM & 57 & H15 & HEM & 2 & 5.879 & 5.011 & 2.289 & 1.00 & 0.00 & $\mathrm{H}$ \\
\hline HETATM & 58 & $\mathrm{C} 16$ & HEM & 2 & 6.669 & 3.845 & 3.880 & 1.00 & 0.00 & C \\
\hline HETATM & 59 & C17 & HEM & 2 & 8.035 & 3.732 & 3.398 & 1.00 & 0.00 & C \\
\hline HETATM & 60 & C18 & HEM & 2 & 8.710 & 2.964 & 4.322 & 1.00 & 0.00 & $\mathrm{C}$ \\
\hline HETATM & 61 & C19 & HEM & 2 & 7.761 & 2.612 & 5.358 & 1.00 & 0.00 & $\mathrm{C}$ \\
\hline HETATM & 62 & $\mathrm{C} 20$ & HEM & 2 & 8.052 & 1.821 & 6.469 & 1.00 & 0.00 & $\mathrm{C}$ \\
\hline HETATM & 63 & H2O & HEM & 2 & 9.066 & 1.432 & 6.530 & 1.00 & 0.00 & $\mathrm{H}$ \\
\hline HETATM & 64 & N21 & HEM & 2 & 5.862 & 1.834 & 7.564 & 1.00 & 0.00 & $\mathrm{~N}$ \\
\hline HETATM & 65 & N22 & HEM & 2 & 3.157 & 2.781 & 7.342 & 1.00 & 0.00 & $\mathrm{~N}$ \\
\hline HETATM & 66 & N23 & HEM & 2 & 3.828 & 4.044 & 4.841 & 1.00 & 0.00 & $\mathrm{~N}$ \\
\hline HETATM & 67 & N24 & HEM & 2 & 6.527 & 3.160 & 5.077 & 1.00 & 0.00 & $\mathrm{~N}$ \\
\hline HETATM & 68 & C99 & HEM & 2 & 5.621 & 6.025 & 7.579 & 1.00 & 0.00 & C \\
\hline HETATM & 69 & 091 & HEM & 2 & 5.461 & 4.711 & 7.567 & 1.00 & 0.00 & 0 \\
\hline HETATM & 70 & 092 & HEM & 2 & 5.776 & 6.642 & 8.635 & 1.00 & 0.00 & o \\
\hline HETATM & 71 & CT91 & HEM & 2 & 5.605 & 6.706 & 6.223 & 1.00 & 0.00 & C \\
\hline HETATM & 72 & H911 & HEM & 2 & 4.626 & 6.511 & 5.760 & 1.00 & 0.00 & $\mathrm{H}$ \\
\hline HETATM & 73 & H912 & HEM & 2 & 6.353 & 6.216 & 5.580 & 1.00 & 0.00 & $\mathrm{H}$ \\
\hline HETATM & 74 & CT92 & HEM & 2 & 5.864 & 8.211 & 6.334 & 1.00 & 0.00 & C \\
\hline HETATM & 75 & H921 & HEM & 2 & 6.789 & 8.367 & 6.905 & 1.00 & 0.00 & $\mathrm{H}$ \\
\hline HETATM & 76 & H922 & HEM & 2 & 5.068 & 8.671 & 6.931 & 1.00 & 0.00 & $\mathrm{H}$ \\
\hline \multicolumn{11}{|l|}{ TER } \\
\hline HETATM & 77 & $\mathrm{C} 2$ & VIN & 3 & 0.345 & 13.923 & -1.002 & 1.00 & 0.00 & C \\
\hline HETATM & 78 & $\mathrm{H} 21$ & VIN & 3 & -0.471 & 13.224 & -0.837 & 1.00 & 0.00 & $\mathrm{H}$ \\
\hline HETATM & 79 & $\mathrm{C} 1$ & VIN & 3 & 0.012 & 15.147 & -1.403 & 1.00 & 0.00 & C \\
\hline HETATM & 80 & H11 & VIN & 3 & 0.743 & 15.926 & -1.511 & 1.00 & 0.00 & $\mathrm{H}$ \\
\hline HETATM & 81 & $\mathrm{H} 12$ & VIN & 3 & -1.030 & 15.417 & -1.570 & 1.00 & 0.00 & $\mathrm{H}$ \\
\hline \multicolumn{11}{|l|}{ TER } \\
\hline HETATM & 82 & $\mathrm{C} 1$ & MET & 4 & 3.008 & 14.177 & -2.840 & 1.00 & 0.00 & C \\
\hline HETATM & 83 & H11 & MET & 4 & 3.243 & 15.248 & -2.720 & 1.00 & 0.00 & $\mathrm{H}$ \\
\hline HETATM & 84 & $\mathrm{H} 12$ & MET & 4 & 3.821 & 13.713 & -3.410 & 1.00 & 0.00 & $\mathrm{H}$ \\
\hline HETATM & 85 & $\mathrm{H} 13$ & MET & 4 & 2.101 & 14.100 & -3.450 & 1.00 & 0.00 & $\mathrm{H}$ \\
\hline \multicolumn{11}{|l|}{ TER } \\
\hline HETATM & 86 & $\mathrm{C} 2$ & VIN & 5 & 8.018 & 12.499 & -2.325 & 1.00 & 0.00 & C \\
\hline HETATM & 87 & H 21 & VIN & 5 & 7.352 & 12.399 & -3.184 & 1.00 & 0.00 & $\mathrm{H}$ \\
\hline HETATM & 88 & $\mathrm{C} 1$ & VIN & 5 & 9.185 & 13.144 & -2.484 & 1.00 & 0.00 & C \\
\hline HETATM & 89 & $\mathrm{H} 11$ & VIN & 5 & 9.865 & 13.300 & -1.654 & 1.00 & 0.00 & $\mathrm{H}$ \\
\hline HETATM & 90 & $\mathrm{H} 12$ & VIN & 5 & 9.490 & 13.542 & -3.450 & 1.00 & 0.00 & $\mathrm{H}$ \\
\hline \multicolumn{11}{|l|}{ TER } \\
\hline HETATM & 91 & $\mathrm{C} 1$ & MET & 6 & 9.703 & 10.816 & -0.121 & 1.00 & 0.00 & C \\
\hline HETATM & 92 & H11 & $\mathrm{MET}$ & 6 & 10.294 & 11.480 & 0.528 & 1.00 & 0.00 & $\mathrm{H}$ \\
\hline HETATM & 93 & $\mathrm{H} 12$ & MET & 6 & 9.859 & 9.794 & 0.246 & 1.00 & 0.00 & $\mathrm{H}$ \\
\hline HETATM & 94 & $\mathrm{H} 13$ & MET & 6 & 10.135 & 10.882 & -1.126 & 1.00 & 0.00 & $\mathrm{H}$ \\
\hline \multicolumn{11}{|l|}{ TER } \\
\hline HETATM & 95 & C5 & $\mathrm{ACP}$ & 7 & 0.615 & 9.735 & 5.770 & 1.00 & 0.00 & C \\
\hline HETATM & 96 & H51 & $\mathrm{ACP}$ & 7 & 1.354 & 9.723 & 6.582 & 1.00 & 0.00 & $\mathrm{H}$ \\
\hline HETATM & 97 & H52 & $\mathrm{ACP}$ & 7 & -0.226 & 10.343 & 6.134 & 1.00 & 0.00 & $\mathrm{H}$ \\
\hline HETATM & 98 & $\mathrm{C} 4$ & $\mathrm{ACP}$ & 7 & 0.136 & 8.297 & 5.497 & 1.00 & 0.00 & C \\
\hline HETATM & 99 & $\mathrm{H} 41$ & $\mathrm{ACP}$ & 7 & -0.422 & 8.236 & 4.549 & 1.00 & 0.00 & $\mathrm{H}$ \\
\hline HETATM & 100 & $\mathrm{H} 42$ & $\mathrm{ACP}$ & 7 & 1.007 & 7.630 & 5.390 & 1.00 & 0.00 & $\mathrm{H}$ \\
\hline HETATM & 101 & $\mathrm{C} 1$ & $\mathrm{ACP}$ & 7 & -0.728 & 7.685 & 6.573 & 1.00 & 0.00 & C \\
\hline HETATM & 102 & $\mathrm{O} 2$ & $\mathrm{ACP}$ & 7 & -0.956 & 8.208 & 7.671 & 1.00 & 0.00 & 0 \\
\hline HETATM & 103 & 03 & $\mathrm{ACP}$ & 7 & -1.244 & 6.505 & 6.209 & 1.00 & 0.00 & o \\
\hline HETATM & 104 & $\mathrm{H} 3$ & $\mathrm{ACP}$ & 7 & -1.853 & 6.151 & 6.945 & 1.00 & 0.00 & $\mathrm{H}$ \\
\hline TER & & & & & & & & & & \\
\hline HETATM & 105 & $\mathrm{C} 1$ & MET & 8 & 8.511 & 8.608 & 4.597 & 1.00 & 0.00 & C \\
\hline HETATM & 106 & $\mathrm{H} 11$ & MET & 8 & 9.063 & 9.448 & 5.047 & 1.00 & 0.00 & $\mathrm{H}$ \\
\hline HETATM & 107 & H12 & MET & 8 & 8.517 & 7.782 & 5.319 & 1.00 & 0.00 & $\mathrm{H}$ \\
\hline HETATM & 108 & H13 & MET & 8 & 9.082 & 8.289 & 3.717 & 1.00 & 0.00 & $\mathrm{H}$ \\
\hline TER & & & & & & & & & & \\
\hline HETATM & 109 & $\mathrm{C} 1$ & MET & 9 & -0.923 & 11.557 & 3.646 & 1.00 & 0.00 & C \\
\hline HETATM & 110 & H11 & MET & 9 & -1.368 & 11.348 & 4.626 & 1.00 & 0.00 & $\mathrm{H}$ \\
\hline HETATM & 111 & H12 & MET & 9 & -1.033 & 12.637 & 3.471 & 1.00 & 0.00 & $\mathrm{H}$ \\
\hline HETATM & 112 & H13 & MET & 9 & -1.523 & 11.031 & 2.889 & 1.00 & 0.00 & $\mathrm{H}$ \\
\hline TER & & & & & & & & & & \\
\hline HETATM & 113 & $\mathrm{C} 2$ & VIN & 10 & 8.874 & 0.178 & 8.933 & 1.00 & 0.00 & C \\
\hline HETATM & 114 & H 21 & VIN & 10 & 9.690 & 0.876 & 8.768 & 1.00 & 0.00 & $\mathrm{H}$ \\
\hline HETATM & 115 & $\mathrm{C} 1$ & VIN & 10 & 9.207 & -1.047 & 9.335 & 1.00 & 0.00 & C \\
\hline HETATM & 116 & H11 & VIN & 10 & 8.475 & -1.826 & 9.442 & 1.00 & 0.00 & $\mathrm{H}$ \\
\hline HETATM & 117 & $\mathrm{H} 12$ & VIN & 10 & 10.249 & -1.316 & 9.501 & 1.00 & 0.00 & $\mathrm{H}$ \\
\hline
\end{tabular}




\begin{tabular}{|c|c|c|c|}
\hline HETATM & 118 & $\mathrm{C} 1$ & MET \\
\hline HETATM & 119 & H11 & $\mathrm{MET}$ \\
\hline HETATM & 120 & $\mathrm{H} 12$ & MET \\
\hline HETATM & 121 & $\mathrm{H} 13$ & MET \\
\hline \multicolumn{4}{|l|}{ TER } \\
\hline HETATM & 122 & $\mathrm{C} 2$ & VIN \\
\hline HETATM & 123 & $\mathrm{H} 21$ & VIN \\
\hline HETATM & 124 & $\mathrm{C} 1$ & VIN \\
\hline HETATM & 125 & H11 & VIN \\
\hline HETATM & 126 & $\mathrm{H} 12$ & VIN \\
\hline \multicolumn{4}{|l|}{ TER } \\
\hline HETATM & 127 & $\mathrm{C} 1$ & $\mathrm{MET}$ \\
\hline HETATM & 128 & H11 & $\mathrm{MET}$ \\
\hline HETATM & 129 & $\mathrm{H} 12$ & MET \\
\hline HETATM & 130 & $\mathrm{H} 13$ & MET \\
\hline \multicolumn{4}{|l|}{ TER } \\
\hline HETATM & 131 & C5 & $\mathrm{ACP}$ \\
\hline HETATM & 132 & H51 & $\mathrm{ACP}$ \\
\hline HETATM & 133 & H52 & $\mathrm{ACP}$ \\
\hline HETATM & 134 & $\mathrm{C} 4$ & $\mathrm{ACP}$ \\
\hline HETATM & 135 & $\mathrm{H} 41$ & $\mathrm{ACP}$ \\
\hline HETATM & 136 & $\mathrm{H} 42$ & $\mathrm{ACP}$ \\
\hline HETATM & 137 & C1 & $\mathrm{ACP}$ \\
\hline HETATM & 138 & 02 & $\mathrm{ACP}$ \\
\hline HETATM & 139 & 03 & $\mathrm{ACP}$ \\
\hline HETATM & 140 & H3 & $\mathrm{ACP}$ \\
\hline \multicolumn{4}{|l|}{ TER } \\
\hline HETATM & 141 & $\mathrm{C} 1$ & MET \\
\hline HETATM & 142 & H11 & MET \\
\hline HETATM & 143 & $\mathrm{H} 12$ & MET \\
\hline HETATM & 144 & H13 & MET \\
\hline \multicolumn{4}{|l|}{ TER } \\
\hline HETATM & 145 & $\mathrm{C} 1$ & MET \\
\hline HETATM & 146 & $\mathrm{H} 11$ & MET \\
\hline HETATM & 147 & $\mathrm{H} 12$ & $\mathrm{MET}$ \\
\hline HETATM & 148 & H13 & $\mathrm{MET}$ \\
\hline \multicolumn{4}{|l|}{ TER } \\
\hline HETATM & 149 & $\mathrm{FE}$ & HEM \\
\hline HETATM & 150 & $\mathrm{C} 1$ & HEM \\
\hline HETATM & 151 & $\mathrm{C} 2$ & HEM \\
\hline HETATM & 152 & C3 & HEM \\
\hline HETATM & 153 & C4 & HEM \\
\hline HETATM & 154 & C5 & HEM \\
\hline HETATM & 155 & H5 & HEM \\
\hline HETATM & 156 & C6 & HEM \\
\hline HETATM & 157 & $\mathrm{C} 7$ & HEM \\
\hline HETATM & 158 & $\mathrm{C} 8$ & HEM \\
\hline HETATM & 159 & C9 & HEM \\
\hline HETATM & 160 & C10 & HEM \\
\hline HETATM & 161 & $\mathrm{H} 10$ & HEM \\
\hline HETATM & 162 & C11 & HEM \\
\hline HETATM & 163 & C12 & HEM \\
\hline HETATM & 164 & C13 & HEM \\
\hline HETATM & 165 & C14 & HEM \\
\hline HETATM & 166 & C15 & HEM \\
\hline HETATM & 167 & H15 & HEM \\
\hline HETATM & 168 & C16 & HEM \\
\hline HETATM & 169 & C17 & HEM \\
\hline HETATM & 170 & C18 & HEM \\
\hline HETATM & 171 & C19 & HEM \\
\hline HETATM & 172 & $\mathrm{C} 20$ & HEM \\
\hline HETATM & 173 & $\mathrm{H} 2 \mathrm{O}$ & HEM \\
\hline HETATM & 174 & N21 & HEM \\
\hline HETATM & 175 & N22 & HEM \\
\hline HETATM & 176 & N23 & HEM \\
\hline HETATM & 177 & $\mathrm{~N} 24$ & HEM \\
\hline HETATM & 178 & C99 & HEM \\
\hline HETATM & 179 & 091 & HEM \\
\hline HETATM & 180 & 092 & HEM \\
\hline HETATM & 181 & СТ91 & HEM \\
\hline HETATM & 182 & H911 & HEM \\
\hline HETATM & 183 & H912 & HEM \\
\hline HETATM & 184 & СТ92 & HEM \\
\hline HETATM & 185 & H921 & HEM \\
\hline HETATM & 186 & H922 & HEM \\
\hline \multicolumn{4}{|l|}{ TER } \\
\hline HETATM & 187 & $\mathrm{FE}$ & HEM \\
\hline HETATM & 188 & $\mathrm{C} 1$ & HEM \\
\hline HETATM & 189 & C2 & HEM \\
\hline
\end{tabular}

\begin{tabular}{|c|c|c|c|c|c|}
\hline 6.210 & -0.077 & 10.771 & 1.00 & 0.00 & C \\
\hline 5.975 & -1.148 & 10.652 & 1.00 & 0.00 & $\mathrm{H}$ \\
\hline 5.397 & 0.387 & 11.341 & 1.00 & 0.00 & $\mathrm{H}$ \\
\hline 7.118 & 0.001 & 11.382 & 1.00 & 0.00 & $\mathrm{H}$ \\
\hline 1.200 & 1.601 & 10.256 & 1.00 & 0.00 & C \\
\hline 1.866 & 1.702 & 11.115 & 1.00 & 0.00 & $\mathrm{H}$ \\
\hline 0.033 & 0.957 & 10.415 & 1.00 & 0.00 & C \\
\hline-0.647 & 0.800 & 9.585 & 1.00 & 0.00 & $\mathrm{H}$ \\
\hline-0.272 & 0.559 & 11.381 & 1.00 & 0.00 & $\mathrm{H}$ \\
\hline-0.484 & 3.284 & 8.052 & 1.00 & 0.00 & C \\
\hline-0.641 & 4.306 & 7.685 & 1.00 & 0.00 & $\mathrm{H}$ \\
\hline-0.917 & 3.219 & 9.057 & 1.00 & 0.00 & $\mathrm{H}$ \\
\hline-1.076 & 2.620 & 7.403 & 1.00 & 0.00 & $\mathrm{H}$ \\
\hline 8.604 & 4.366 & 2.161 & 1.00 & 0.00 & C \\
\hline 7.865 & 4.377 & 1.349 & 1.00 & 0.00 & $\mathrm{H}$ \\
\hline 9.445 & 3.758 & 1.798 & 1.00 & 0.00 & $\mathrm{H}$ \\
\hline 9.082 & 5.803 & 2.434 & 1.00 & 0.00 & C \\
\hline 9.640 & 5.865 & 3.382 & 1.00 & 0.00 & $\mathrm{H}$ \\
\hline 8.212 & 6.470 & 2.541 & 1.00 & 0.00 & $\mathrm{H}$ \\
\hline 9.946 & 6.416 & 1.358 & 1.00 & 0.00 & C \\
\hline 10.174 & 5.893 & 0.260 & 1.00 & 0.00 & 0 \\
\hline 10.462 & 7.595 & 1.722 & 1.00 & 0.00 & 0 \\
\hline 11.071 & 7.949 & 0.986 & 1.00 & 0.00 & $\mathrm{H}$ \\
\hline 0.708 & 5.492 & 3.334 & 1.00 & 0.00 & C \\
\hline 0.155 & 4.653 & 2.884 & 1.00 & 0.00 & $\mathrm{H}$ \\
\hline 0.701 & 6.318 & 2.613 & 1.00 & 0.00 & $\mathrm{H}$ \\
\hline 0.137 & 5.812 & 4.214 & 1.00 & 0.00 & $\mathrm{H}$ \\
\hline 10.141 & 2.543 & 4.285 & 1.00 & 0.00 & C \\
\hline 10.586 & 2.752 & 3.305 & 1.00 & 0.00 & $\mathrm{H}$ \\
\hline 10.252 & 1.463 & 4.460 & 1.00 & 0.00 & $\mathrm{H}$ \\
\hline 10.742 & 3.069 & 5.042 & 1.00 & 0.00 & $\mathrm{H}$ \\
\hline 3.316 & 10.511 & 9.423 & 1.00 & 0.00 & $\mathrm{FE}$ \\
\hline 1.088 & 12.383 & 8.361 & 1.00 & 0.00 & C \\
\hline 0.729 & 13.174 & 7.189 & 1.00 & 0.00 & C \\
\hline 1.874 & 13.271 & 6.414 & 1.00 & 0.00 & C \\
\hline 2.904 & 12.553 & 7.122 & 1.00 & 0.00 & C \\
\hline 4.219 & 12.435 & 6.684 & 1.00 & 0.00 & C \\
\hline 4.472 & 12.931 & 5.749 & 1.00 & 0.00 & $\mathrm{H}$ \\
\hline 5.238 & 11.736 & 7.320 & 1.00 & 0.00 & C \\
\hline 6.607 & 11.664 & 6.851 & 1.00 & 0.00 & C \\
\hline 7.313 & 10.944 & 7.798 & 1.00 & 0.00 & C \\
\hline 6.362 & 10.579 & 8.822 & 1.00 & 0.00 & C \\
\hline 6.666 & 9.844 & 9.965 & 1.00 & 0.00 & C \\
\hline 7.693 & 9.500 & 10.071 & 1.00 & 0.00 & $\mathrm{H}$ \\
\hline 5.792 & 9.504 & 10.988 & 1.00 & 0.00 & $\mathrm{C}$ \\
\hline 6.173 & 8.779 & 12.178 & 1.00 & 0.00 & C \\
\hline 5.033 & 8.631 & 12.935 & 1.00 & 0.00 & C \\
\hline 3.960 & 9.273 & 12.204 & 1.00 & 0.00 & C \\
\hline 2.632 & 9.328 & 12.625 & 1.00 & 0.00 & C \\
\hline 2.392 & 8.843 & 13.573 & 1.00 & 0.00 & $\mathrm{H}$ \\
\hline 1.602 & 10.009 & 11.982 & 1.00 & 0.00 & C \\
\hline 0.236 & 10.123 & 12.464 & 1.00 & 0.00 & C \\
\hline-0.439 & 10.890 & 11.541 & 1.00 & 0.00 & C \\
\hline 0.510 & 11.242 & 10.504 & 1.00 & 0.00 & C \\
\hline 0.219 & 12.033 & 9.393 & 1.00 & 0.00 & C \\
\hline-0.795 & 12.423 & 9.333 & 1.00 & 0.00 & $\mathrm{H}$ \\
\hline 2.409 & 12.020 & 8.298 & 1.00 & 0.00 & $\mathrm{~N}$ \\
\hline 5.114 & 11.073 & 8.521 & 1.00 & 0.00 & $\mathrm{~N}$ \\
\hline 4.443 & 9.810 & 11.021 & 1.00 & 0.00 & $\mathrm{~N}$ \\
\hline 1.743 & 10.694 & 10.786 & 1.00 & 0.00 & $\mathrm{~N}$ \\
\hline 2.650 & 7.829 & 8.283 & 1.00 & 0.00 & C \\
\hline 2.810 & 9.143 & 8.296 & 1.00 & 0.00 & O \\
\hline 2.495 & 7.212 & 7.228 & 1.00 & 0.00 & o \\
\hline 2.666 & 7.148 & 9.640 & 1.00 & 0.00 & C \\
\hline 3.645 & 7.343 & 10.103 & 1.00 & 0.00 & $\mathrm{H}$ \\
\hline 1.918 & 7.638 & 10.283 & 1.00 & 0.00 & $\mathrm{H}$ \\
\hline 2.407 & 5.644 & 9.528 & 1.00 & 0.00 & C \\
\hline 3.203 & 5.183 & 8.931 & 1.00 & 0.00 & $\mathrm{H}$ \\
\hline 1.482 & 5.488 & 8.958 & 1.00 & 0.00 & $\mathrm{H}$ \\
\hline 4.008 & 3.097 & 14.371 & 1.00 & 0.00 & $\mathrm{FE}$ \\
\hline 6.236 & 1.225 & 15.433 & 1.00 & 0.00 & C \\
\hline 6.595 & 0.434 & 16.605 & 1.00 & 0.00 & C \\
\hline
\end{tabular}




\begin{tabular}{|c|c|c|c|c|c|c|c|c|c|c|}
\hline HETATM & 190 & C3 & HEM & 18 & 5.449 & 0.337 & 17.380 & 1.00 & 0.00 & C \\
\hline HETATM & 191 & C4 & HEM & 18 & 4.419 & 1.055 & 16.672 & 1.00 & 0.00 & $\mathrm{C}$ \\
\hline HETATM & 192 & C5 & HEM & 18 & 3.104 & 1.173 & 17.109 & 1.00 & 0.00 & C \\
\hline HETATM & 193 & H5 & HEM & 18 & 2.851 & 0.677 & 18.045 & 1.00 & 0.00 & $\mathrm{H}$ \\
\hline HETATM & 194 & $\mathrm{C} 6$ & HEM & 18 & 2.085 & 1.872 & 16.473 & 1.00 & 0.00 & C \\
\hline HETATM & 195 & C7 & HEM & 18 & 0.716 & 1.944 & 16.943 & 1.00 & 0.00 & $\mathrm{C}$ \\
\hline HETATM & 196 & $\mathrm{C} 8$ & HEM & 18 & 0.011 & 2.664 & 15.996 & 1.00 & 0.00 & $\mathrm{C}$ \\
\hline HETATM & 197 & C9 & HEM & 18 & 0.962 & 3.029 & 14.972 & 1.00 & 0.00 & $\mathrm{C}$ \\
\hline HETATM & 198 & $\mathrm{C} 10$ & HEM & 18 & 0.657 & 3.764 & 13.828 & 1.00 & 0.00 & C \\
\hline HETATM & 199 & H1O & HEM & 18 & -0.370 & 4.108 & 13.722 & 1.00 & 0.00 & $\mathrm{H}$ \\
\hline HETATM & 200 & C11 & HEM & 18 & 1.532 & 4.104 & 12.806 & 1.00 & 0.00 & $\mathrm{C}$ \\
\hline HETATM & 201 & $\mathrm{C} 12$ & HEM & 18 & 1.151 & 4.829 & 11.615 & 1.00 & 0.00 & $\mathrm{C}$ \\
\hline HETATM & 202 & $\mathrm{C} 13$ & HEM & 18 & 2.290 & 4.977 & 10.858 & 1.00 & 0.00 & $\mathrm{C}$ \\
\hline HETATM & 203 & C14 & HEM & 18 & 3.363 & 4.335 & 11.590 & 1.00 & 0.00 & $\mathrm{C}$ \\
\hline HETATM & 204 & C15 & HEM & 18 & 4.691 & 4.280 & 11.168 & 1.00 & 0.00 & $\mathrm{C}$ \\
\hline HETATM & 205 & H15 & HEM & 18 & 4.931 & 4.765 & 10.221 & 1.00 & 0.00 & $\mathrm{H}$ \\
\hline HETATM & 206 & $\mathrm{C} 16$ & HEM & 18 & 5.721 & 3.599 & 11.812 & 1.00 & 0.00 & $\mathrm{C}$ \\
\hline HETATM & 207 & $\mathrm{C} 17$ & HEM & 18 & 7.087 & 3.485 & 11.329 & 1.00 & 0.00 & $\mathrm{C}$ \\
\hline HETATM & 208 & $\mathrm{C} 18$ & HEM & 18 & 7.763 & 2.718 & 12.253 & 1.00 & 0.00 & $\mathrm{C}$ \\
\hline HETATM & 209 & C19 & HEM & 18 & 6.813 & 2.366 & 13.289 & 1.00 & 0.00 & C \\
\hline HETATM & 210 & $\mathrm{C} 20$ & HEM & 18 & 7.104 & 1.575 & 14.401 & 1.00 & 0.00 & $\mathrm{C}$ \\
\hline HETATM & 211 & $\mathrm{H} 2 \mathrm{O}$ & HEM & 18 & 8.118 & 1.185 & 14.461 & 1.00 & 0.00 & $\mathrm{H}$ \\
\hline HETATM & 212 & N21 & HEM & 18 & 4.914 & 1.588 & 15.495 & 1.00 & 0.00 & $\mathrm{~N}$ \\
\hline HETATM & 213 & $\mathrm{~N} 22$ & HEM & 18 & 2.209 & 2.535 & 15.273 & 1.00 & 0.00 & $\mathrm{~N}$ \\
\hline HETATM & 214 & N23 & HEM & 18 & 2.880 & 3.798 & 12.772 & 1.00 & 0.00 & $\mathrm{~N}$ \\
\hline HETATM & 215 & $\mathrm{~N} 24$ & HEM & 18 & 5.580 & 2.914 & 13.008 & 1.00 & 0.00 & $\mathrm{~N}$ \\
\hline HETATM & 216 & C99 & HEM & 18 & 4.673 & 5.779 & 15.510 & 1.00 & 0.00 & $\mathrm{C}$ \\
\hline HETATM & 217 & 091 & HEM & 18 & 4.514 & 4.465 & 15.498 & 1.00 & 0.00 & 0 \\
\hline HETATM & 218 & 092 & HEM & 18 & 4.828 & 6.396 & 16.566 & 1.00 & 0.00 & 0 \\
\hline HETATM & 219 & СТ91 & HEM & 18 & 4.657 & 6.460 & 14.154 & 1.00 & 0.00 & $\mathrm{C}$ \\
\hline HETATM & 220 & H911 & HEM & 18 & 3.678 & 6.265 & 13.691 & 1.00 & 0.00 & $\mathrm{H}$ \\
\hline HETATM & 221 & H912 & HEM & 18 & 5.405 & 5.970 & 13.511 & 1.00 & 0.00 & $\mathrm{H}$ \\
\hline HETATM & 222 & СТ 92 & HEM & 18 & 4.917 & 7.964 & 14.265 & 1.00 & 0.00 & $\mathrm{C}$ \\
\hline HETATM & 223 & H921 & HEM & 18 & 5.842 & 8.120 & 14.836 & 1.00 & 0.00 & $\mathrm{H}$ \\
\hline HETATM & 224 & H922 & HEM & 18 & 4.120 & 8.425 & 14.862 & 1.00 & 0.00 & $\mathrm{H}$ \\
\hline \multicolumn{11}{|l|}{ TER } \\
\hline HETATM & 225 & $\mathrm{C} 2$ & VIN & 19 & -0.603 & 13.676 & 6.929 & 1.00 & 0.00 & $\mathrm{C}$ \\
\hline HETATM & 226 & H 21 & VIN & 19 & -1.419 & 12.978 & 7.094 & 1.00 & 0.00 & $\mathrm{H}$ \\
\hline HETATM & 227 & $\mathrm{C} 1$ & VIN & 19 & -0.936 & 14.901 & 6.528 & 1.00 & 0.00 & $\mathrm{C}$ \\
\hline HETATM & 228 & H11 & VIN & 19 & -0.205 & 15.680 & 6.421 & 1.00 & 0.00 & $\mathrm{H}$ \\
\hline HETATM & 229 & $\mathrm{H} 12$ & VIN & 19 & -1.978 & 15.170 & 6.361 & 1.00 & 0.00 & $\mathrm{H}$ \\
\hline \multicolumn{11}{|c|}{ TER } \\
\hline HETATM & 230 & $\mathrm{C} 1$ & $\mathrm{MET}$ & 20 & 2.061 & 13.931 & 5.091 & 1.00 & 0.00 & C \\
\hline HETATM & 231 & H11 & $\mathrm{MET}$ & 20 & 2.295 & 15.002 & 5.211 & 1.00 & 0.00 & $\mathrm{H}$ \\
\hline HETATM & 232 & $\mathrm{H} 12$ & $\mathrm{MET}$ & 20 & 2.874 & 13.467 & 4.522 & 1.00 & 0.00 & $\mathrm{H}$ \\
\hline HETATM & 233 & H13 & $\mathrm{MET}$ & 20 & 1.153 & 13.854 & 4.481 & 1.00 & 0.00 & $\mathrm{H}$ \\
\hline \multicolumn{11}{|l|}{ TER } \\
\hline HETATM & 234 & $\mathrm{C} 2$ & VIN & 21 & 7.071 & 12.253 & 5.606 & 1.00 & 0.00 & C \\
\hline HETATM & 235 & H2 1 & VIN & 21 & 6.405 & 12.152 & 4.747 & 1.00 & 0.00 & $\mathrm{H}$ \\
\hline HETATM & 236 & $\mathrm{C} 1$ & VIN & 21 & 8.238 & 12.898 & 5.447 & 1.00 & 0.00 & C \\
\hline HETATM & 237 & H11 & VIN & 21 & 8.917 & 13.054 & 6.277 & 1.00 & 0.00 & $\mathrm{H}$ \\
\hline HETATM & 238 & $\mathrm{H} 12$ & VIN & 21 & 8.543 & 13.296 & 4.482 & 1.00 & 0.00 & $\mathrm{H}$ \\
\hline \multicolumn{11}{|l|}{ TER } \\
\hline HETATM & 239 & $\mathrm{C} 1$ & $\mathrm{MET}$ & 22 & 8.755 & 10.570 & 7.810 & 1.00 & 0.00 & C \\
\hline HETATM & 240 & H11 & $\mathrm{MET}$ & 22 & 9.347 & 11.234 & 8.459 & 1.00 & 0.00 & $\mathrm{H}$ \\
\hline HETATM & 241 & $\mathrm{H} 12$ & $\mathrm{MET}$ & 22 & 8.912 & 9.548 & 8.177 & 1.00 & 0.00 & $\mathrm{H}$ \\
\hline HETATM & 242 & H13 & $\mathrm{MET}$ & 22 & 9.188 & 10.636 & 6.805 & 1.00 & 0.00 & $\mathrm{H}$ \\
\hline \multicolumn{11}{|l|}{ TER } \\
\hline HETATM & 243 & C5 & $\mathrm{ACP}$ & 23 & -0.333 & 9.488 & 13.701 & 1.00 & 0.00 & C \\
\hline HETATM & 244 & H51 & $\mathrm{ACP}$ & 23 & 0.406 & 9.477 & 14.514 & 1.00 & 0.00 & $\mathrm{H}$ \\
\hline HETATM & 245 & H52 & $\mathrm{ACP}$ & 23 & -1.174 & 10.097 & 14.065 & 1.00 & 0.00 & $\mathrm{H}$ \\
\hline HETATM & 246 & $\mathrm{C} 4$ & $\mathrm{ACP}$ & 23 & -0.812 & 8.051 & 13.428 & 1.00 & 0.00 & C \\
\hline HETATM & 247 & $\mathrm{H} 41$ & $\mathrm{ACP}$ & 23 & -1.369 & 7.990 & 12.481 & 1.00 & 0.00 & $\mathrm{H}$ \\
\hline HETATM & 248 & $\mathrm{H} 42$ & $\mathrm{ACP}$ & 23 & 0.059 & 7.384 & 13.321 & 1.00 & 0.00 & $\mathrm{H}$ \\
\hline HETATM & 249 & $\mathrm{C} 1$ & $\mathrm{ACP}$ & 23 & -1.676 & 7.439 & 14.504 & 1.00 & 0.00 & C \\
\hline HETATM & 250 & $\mathrm{O} 2$ & $\mathrm{ACP}$ & 23 & -1.903 & 7.961 & 15.603 & 1.00 & 0.00 & 0 \\
\hline HETATM & 251 & 03 & $\mathrm{ACP}$ & 23 & -2.191 & 6.259 & 14.140 & 1.00 & 0.00 & 0 \\
\hline HETATM & 252 & H3 & $\mathrm{ACP}$ & 23 & -2.800 & 5.905 & 14.876 & 1.00 & 0.00 & $\mathrm{H}$ \\
\hline TER & & & & & & & & & & \\
\hline HETATM & 253 & $\mathrm{C} 1$ & $\mathrm{MET}$ & 24 & 7.563 & 8.362 & 12.528 & 1.00 & 0.00 & C \\
\hline HETATM & 254 & H11 & MET & 24 & 8.115 & 9.201 & 12.978 & 1.00 & 0.00 & $\mathrm{H}$ \\
\hline HETATM & 255 & $\mathrm{H} 12$ & MET & 24 & 7.570 & 7.536 & 13.250 & 1.00 & 0.00 & $\mathrm{H}$ \\
\hline HETATM & 256 & H13 & $\mathrm{MET}$ & 24 & 8.134 & 8.042 & 11.649 & 1.00 & 0.00 & $\mathrm{H}$ \\
\hline TER & & & & & & & & & & \\
\hline HETATM & 257 & $\mathrm{C} 1$ & $\mathrm{MET}$ & 25 & -1.870 & 11.311 & 11.577 & 1.00 & 0.00 & C \\
\hline HETATM & 258 & H11 & $\mathrm{MET}$ & 25 & -2.316 & 11.102 & 12.557 & 1.00 & 0.00 & $\mathrm{H}$ \\
\hline HETATM & 259 & $\mathrm{H} 12$ & $\mathrm{MET}$ & 25 & -1.981 & 12.391 & 11.402 & 1.00 & 0.00 & $\mathrm{H}$ \\
\hline HETATM & 260 & $\mathrm{H} 13$ & MET & 25 & -2.471 & 10.785 & 10.821 & 1.00 & 0.00 & $\mathrm{H}$ \\
\hline
\end{tabular}




\begin{tabular}{|c|c|c|c|}
\hline HETATM & 261 & $\mathrm{C} 2$ & VIN \\
\hline HETATM & 262 & H21 & VIN \\
\hline HETATM & 263 & $\mathrm{C} 1$ & VIN \\
\hline HETATM & 264 & H11 & VIN \\
\hline HETATM & 265 & H12 & VIN \\
\hline \multicolumn{4}{|l|}{ TER } \\
\hline HETATM & 266 & $\mathrm{C} 1$ & $\mathrm{MET}$ \\
\hline HETATM & 267 & H11 & $\mathrm{MET}$ \\
\hline HETATM & 268 & $\mathrm{H} 12$ & $\mathrm{MET}$ \\
\hline HETATM & 269 & H13 & $\mathrm{MET}$ \\
\hline \multicolumn{4}{|l|}{ TER } \\
\hline HETATM & 270 & $\mathrm{C} 2$ & VIN \\
\hline HETATM & 271 & H 21 & VIN \\
\hline HETATM & 272 & $\mathrm{C} 1$ & VIN \\
\hline HETATM & 273 & $\mathrm{H} 11$ & VIN \\
\hline HETATM & 274 & $\mathrm{H} 12$ & VIN \\
\hline \multicolumn{4}{|l|}{ TER } \\
\hline HETATM & 275 & $\mathrm{C} 1$ & MET \\
\hline HETATM & 276 & H11 & MET \\
\hline HETATM & 277 & $\mathrm{H} 12$ & MET \\
\hline HETATM & 278 & H13 & $\mathrm{MET}$ \\
\hline \multicolumn{4}{|l|}{ TER } \\
\hline HETATM & 279 & C5 & $\mathrm{ACP}$ \\
\hline HETATM & 280 & H51 & $\mathrm{ACP}$ \\
\hline HETATM & 281 & H52 & $\mathrm{ACP}$ \\
\hline HETATM & 282 & $\mathrm{C} 4$ & $\mathrm{ACP}$ \\
\hline HETATM & 283 & $\mathrm{H} 41$ & $\mathrm{ACP}$ \\
\hline HETATM & 284 & $\mathrm{H} 42$ & $\mathrm{ACP}$ \\
\hline HETATM & 285 & $\mathrm{C} 1$ & $\mathrm{ACP}$ \\
\hline HETATM & 286 & $\mathrm{O} 2$ & $\mathrm{ACP}$ \\
\hline HETATM & 287 & 03 & $\mathrm{ACP}$ \\
\hline HETATM & 288 & H3 & $\mathrm{ACP}$ \\
\hline \multicolumn{4}{|l|}{ TER } \\
\hline HETATM & 289 & $\mathrm{C} 1$ & MET \\
\hline HETATM & 290 & H11 & $\mathrm{MET}$ \\
\hline HETATM & 291 & H12 & MET \\
\hline HETATM & 292 & H13 & MET \\
\hline \multicolumn{4}{|l|}{ TER } \\
\hline HETATM & 293 & $\mathrm{C} 1$ & MET \\
\hline HETATM & 294 & H11 & $\mathrm{MET}$ \\
\hline HETATM & 295 & H12 & $\mathrm{MET}$ \\
\hline HETATM & 296 & $\mathrm{H} 13$ & $\mathrm{MET}$ \\
\hline \multicolumn{4}{|l|}{ TER } \\
\hline HETATM & 297 & $\mathrm{FE}$ & HEM \\
\hline HETATM & 298 & $\mathrm{C} 1$ & HEM \\
\hline HETATM & 299 & $\mathrm{C} 2$ & HEM \\
\hline HETATM & 300 & $\mathrm{C} 3$ & HEM \\
\hline HETATM & 301 & $\mathrm{C} 4$ & HEM \\
\hline HETATM & 302 & C5 & HEM \\
\hline HETATM & 303 & H5 & HEM \\
\hline HETATM & 304 & $\mathrm{C} 6$ & HEM \\
\hline HETATM & 305 & C7 & HEM \\
\hline HETATM & 306 & $\mathrm{C} 8$ & HEM \\
\hline HETATM & 307 & $\mathrm{C} 9$ & HEM \\
\hline HETATM & 308 & $\mathrm{C} 10$ & HEM \\
\hline HETATM & 309 & $\mathrm{H} 10$ & HEM \\
\hline HETATM & 310 & $\mathrm{C} 11$ & HEM \\
\hline HETATM & 311 & $\mathrm{C} 12$ & HEM \\
\hline HETATM & 312 & $\mathrm{C} 13$ & HEM \\
\hline HETATM & 313 & $\mathrm{C} 14$ & HEM \\
\hline HETATM & 314 & $\mathrm{C} 15$ & HEM \\
\hline HETATM & 315 & H15 & HEM \\
\hline HETATM & 316 & C16 & HEM \\
\hline HETATM & 317 & $\mathrm{C} 17$ & HEM \\
\hline HETATM & 318 & $\mathrm{C} 18$ & HEM \\
\hline HETATM & 319 & C19 & HEM \\
\hline HETATM & 320 & $\mathrm{C} 20$ & HEM \\
\hline HETATM & 321 & $\mathrm{H} 2 \mathrm{O}$ & HEM \\
\hline HETATM & 322 & $\mathrm{~N} 21$ & HEM \\
\hline HETATM & 323 & N22 & HEM \\
\hline HETATM & 324 & N23 & HEM \\
\hline HETATM & 325 & $\mathrm{~N} 24$ & HEM \\
\hline HETATM & 326 & C99 & HEM \\
\hline HETATM & 327 & 091 & HEM \\
\hline HETATM & 328 & 092 & HEM \\
\hline HETATM & 329 & СТ91 & HEM \\
\hline HETATM & 330 & H911 & HEM \\
\hline HETATM & 331 & H912 & HEM \\
\hline HETATM & 332 & СТ92 & HEM \\
\hline
\end{tabular}

\begin{tabular}{|c|c|c|c|c|c|}
\hline 7.926 & -0.068 & 16.864 & 1.00 & 0.00 & C \\
\hline 8.742 & 0.630 & 16.699 & 1.00 & 0.00 & $\mathrm{H}$ \\
\hline 8.259 & -1.293 & 17.266 & 1.00 & 0.00 & C \\
\hline 7.528 & -2.072 & 17.373 & 1.00 & 0.00 & $\mathrm{H}$ \\
\hline 9.301 & -1.562 & 17.432 & 1.00 & 0.00 & $\mathrm{H}$ \\
\hline 5.263 & -0.323 & 18.702 & 1.00 & 0.00 & C \\
\hline 5.028 & -1.394 & 18.583 & 1.00 & 0.00 & $\mathrm{H}$ \\
\hline 4.450 & 0.141 & 19.272 & 1.00 & 0.00 & $\mathrm{H}$ \\
\hline 6.170 & -0.246 & 19.313 & 1.00 & 0.00 & $\mathrm{H}$ \\
\hline 0.252 & 1.355 & 18.188 & 1.00 & 0.00 & C \\
\hline 0.918 & 1.456 & 19.046 & 1.00 & 0.00 & $\mathrm{H}$ \\
\hline-0.914 & 0.710 & 18.346 & 1.00 & 0.00 & C \\
\hline-1.594 & 0.554 & 17.516 & 1.00 & 0.00 & $\mathrm{H}$ \\
\hline-1.219 & 0.312 & 19.312 & 1.00 & 0.00 & $\mathrm{H}$ \\
\hline-1.432 & 3.038 & 15.984 & 1.00 & 0.00 & C \\
\hline-1.588 & 4.060 & 15.617 & 1.00 & 0.00 & $\mathrm{H}$ \\
\hline-1.864 & 2.972 & 16.989 & 1.00 & 0.00 & $\mathrm{H}$ \\
\hline-2.023 & 2.374 & 15.335 & 1.00 & 0.00 & $\mathrm{H}$ \\
\hline 7.656 & 4.120 & 10.092 & 1.00 & 0.00 & C \\
\hline 6.917 & 4.131 & 9.280 & 1.00 & 0.00 & $\mathrm{H}$ \\
\hline 8.497 & 3.511 & 9.729 & 1.00 & 0.00 & $\mathrm{H}$ \\
\hline 8.135 & 5.557 & 10.365 & 1.00 & 0.00 & C \\
\hline 8.693 & 5.618 & 11.313 & 1.00 & 0.00 & $\mathrm{H}$ \\
\hline 7.264 & 6.224 & 10.472 & 1.00 & 0.00 & $\mathrm{H}$ \\
\hline 8.999 & 6.169 & 9.289 & 1.00 & 0.00 & C \\
\hline 9.227 & 5.647 & 8.191 & 1.00 & 0.00 & O \\
\hline 9.515 & 7.349 & 9.653 & 1.00 & 0.00 & O \\
\hline 10.124 & 7.703 & 8.917 & 1.00 & 0.00 & $\mathrm{H}$ \\
\hline-0.240 & 5.246 & 11.266 & 1.00 & 0.00 & $\mathrm{C}$ \\
\hline-0.792 & 4.407 & 10.815 & 1.00 & 0.00 & $\mathrm{H}$ \\
\hline-0.246 & 6.072 & 10.544 & 1.00 & 0.00 & $\mathrm{H}$ \\
\hline-0.811 & 5.565 & 12.145 & 1.00 & 0.00 & $\mathrm{H}$ \\
\hline 9.194 & 2.297 & 12.216 & 1.00 & 0.00 & $\mathrm{C}$ \\
\hline 9.639 & 2.506 & 11.236 & 1.00 & 0.00 & $\mathrm{H}$ \\
\hline 9.304 & 1.217 & 12.391 & 1.00 & 0.00 & $\mathrm{H}$ \\
\hline 9.794 & 2.823 & 12.973 & 1.00 & 0.00 & $\mathrm{H}$ \\
\hline 5.211 & 11.003 & -6.440 & 1.00 & 0.00 & $\mathrm{FE}$ \\
\hline 2.983 & 12.876 & -7.502 & 1.00 & 0.00 & C \\
\hline 2.624 & 13.666 & -8.674 & 1.00 & 0.00 & C \\
\hline 3.770 & 13.763 & -9.448 & 1.00 & 0.00 & C \\
\hline 4.799 & 13.046 & -8.740 & 1.00 & 0.00 & C \\
\hline 6.114 & 12.927 & -9.178 & 1.00 & 0.00 & C \\
\hline 6.368 & 13.423 & -10.113 & 1.00 & 0.00 & $\mathrm{H}$ \\
\hline 7.134 & 12.229 & -8.542 & 1.00 & 0.00 & C \\
\hline 8.502 & 12.156 & -9.012 & 1.00 & 0.00 & C \\
\hline 9.208 & 11.436 & -8.065 & 1.00 & 0.00 & $\mathrm{C}$ \\
\hline 8.257 & 11.071 & -7.041 & 1.00 & 0.00 & C \\
\hline 8.561 & 10.336 & -5.897 & 1.00 & 0.00 & C \\
\hline 9.588 & 9.993 & -5.791 & 1.00 & 0.00 & $\mathrm{H}$ \\
\hline 7.687 & 9.996 & -4.874 & 1.00 & 0.00 & $\mathrm{C}$ \\
\hline 8.068 & 9.271 & -3.684 & 1.00 & 0.00 & C \\
\hline 6.928 & 9.124 & -2.927 & 1.00 & 0.00 & C \\
\hline 5.855 & 9.765 & -3.659 & 1.00 & 0.00 & C \\
\hline 4.527 & 9.820 & -3.237 & 1.00 & 0.00 & C \\
\hline 4.287 & 9.335 & -2.289 & 1.00 & 0.00 & $\mathrm{H}$ \\
\hline 3.497 & 10.502 & -3.880 & 1.00 & 0.00 & C \\
\hline 2.131 & 10.615 & -3.398 & 1.00 & 0.00 & C \\
\hline 1.456 & 11.383 & -4.322 & 1.00 & 0.00 & C \\
\hline 2.406 & 11.735 & -5.358 & 1.00 & 0.00 & C \\
\hline 2.114 & 12.526 & -6.469 & 1.00 & 0.00 & C \\
\hline 1.100 & 12.915 & -6.530 & 1.00 & 0.00 & $\mathrm{H}$ \\
\hline 4.305 & 12.513 & -7.564 & 1.00 & 0.00 & $\mathrm{~N}$ \\
\hline 7.009 & 11.566 & -7.342 & 1.00 & 0.00 & $\mathrm{~N}$ \\
\hline 6.338 & 10.303 & -4.841 & 1.00 & 0.00 & $\mathrm{~N}$ \\
\hline 3.639 & 11.187 & -5.077 & 1.00 & 0.00 & $\mathrm{~N}$ \\
\hline 4.545 & 8.322 & -7.579 & 1.00 & 0.00 & C \\
\hline 4.705 & 9.635 & -7.567 & 1.00 & 0.00 & O \\
\hline 4.391 & 7.705 & -8.635 & 1.00 & 0.00 & 0 \\
\hline 4.561 & 7.640 & -6.223 & 1.00 & 0.00 & C \\
\hline 5.540 & 7.836 & -5.760 & 1.00 & 0.00 & $\mathrm{H}$ \\
\hline 3.813 & 8.130 & -5.580 & 1.00 & 0.00 & $\mathrm{H}$ \\
\hline 4.302 & 6.136 & -6.334 & 1.00 & 0.00 & C \\
\hline
\end{tabular}




\begin{tabular}{|c|c|c|c|c|c|c|c|c|c|c|}
\hline HETATM & 333 & H921 & HEM & 33 & 5.098 & 5.676 & -6.931 & 1.00 & 0.00 & $\mathrm{H}$ \\
\hline HETATM & 334 & H922 & HEM & 33 & 3.377 & 5.980 & -6.905 & 1.00 & 0.00 & $\mathrm{H}$ \\
\hline \multicolumn{11}{|l|}{ TER } \\
\hline HETATM & 335 & $\mathrm{FE}$ & HEM & 34 & 5.903 & 3.589 & -1.491 & 1.00 & 0.00 & $\mathrm{FE}$ \\
\hline HETATM & 336 & $\mathrm{C} 1$ & HEM & 34 & 8.131 & 1.717 & -0.429 & 1.00 & 0.00 & C \\
\hline HETATM & 337 & $\mathrm{C} 2$ & HEM & 34 & 8.490 & 0.927 & 0.742 & 1.00 & 0.00 & C \\
\hline HETATM & 338 & $\mathrm{C} 3$ & HEM & 34 & 7.344 & 0.830 & 1.517 & 1.00 & 0.00 & C \\
\hline HETATM & 339 & $\mathrm{C} 4$ & HEM & 34 & 6.315 & 1.547 & 0.809 & 1.00 & 0.00 & C \\
\hline HETATM & 340 & C5 & HEM & 34 & 5.000 & 1.666 & 1.247 & 1.00 & 0.00 & $\mathrm{C}$ \\
\hline HETATM & 341 & H5 & HEM & 34 & 4.746 & 1.170 & 2.182 & 1.00 & 0.00 & $\mathrm{H}$ \\
\hline HETATM & 342 & $\mathrm{C} 6$ & HEM & 34 & 3.980 & 2.364 & 0.611 & 1.00 & 0.00 & $\mathrm{C}$ \\
\hline HETATM & 343 & C7 & HEM & 34 & 2.611 & 2.437 & 1.081 & 1.00 & 0.00 & C \\
\hline HETATM & 344 & $\mathrm{C} 8$ & HEM & 34 & 1.906 & 3.157 & 0.134 & 1.00 & 0.00 & C \\
\hline HETATM & 345 & $\mathrm{C} 9$ & HEM & 34 & 2.857 & 3.522 & -0.891 & 1.00 & 0.00 & C \\
\hline HETATM & 346 & $\mathrm{C} 10$ & HEM & 34 & 2.552 & 4.256 & -2.034 & 1.00 & 0.00 & C \\
\hline HETATM & 347 & $\mathrm{H} 10$ & HEM & 34 & 1.526 & 4.600 & -2.140 & 1.00 & 0.00 & $\mathrm{H}$ \\
\hline HETATM & 348 & C11 & HEM & 34 & 3.427 & 4.596 & -3.057 & 1.00 & 0.00 & C \\
\hline HETATM & 349 & $\mathrm{C} 12$ & HEM & 34 & 3.046 & 5.322 & -4.247 & 1.00 & 0.00 & C \\
\hline HETATM & 350 & $\mathrm{C} 13$ & HEM & 34 & 4.185 & 5.469 & -5.004 & 1.00 & 0.00 & C \\
\hline HETATM & 351 & C14 & HEM & 34 & 5.258 & 4.828 & -4.273 & 1.00 & 0.00 & C \\
\hline HETATM & 352 & C15 & HEM & 34 & 6.586 & 4.773 & -4.694 & 1.00 & 0.00 & C \\
\hline HETATM & 353 & H1 5 & HEM & 34 & 6.826 & 5.258 & -5.642 & 1.00 & 0.00 & $\mathrm{H}$ \\
\hline HETATM & 354 & $\mathrm{C} 16$ & HEM & 34 & 7.617 & 4.091 & -4.051 & 1.00 & 0.00 & $\mathrm{C}$ \\
\hline HETATM & 355 & $\mathrm{C} 17$ & HEM & 34 & 8.982 & 3.978 & -4.533 & 1.00 & 0.00 & C \\
\hline HETATM & 356 & $\mathrm{C} 18$ & HEM & 34 & 9.658 & 3.210 & -3.609 & 1.00 & 0.00 & C \\
\hline HETATM & 357 & C19 & HEM & 34 & 8.708 & 2.858 & -2.573 & 1.00 & 0.00 & $\mathrm{C}$ \\
\hline HETATM & 358 & $\mathrm{C} 20$ & HEM & 34 & 8.999 & 2.067 & -1.462 & 1.00 & 0.00 & C \\
\hline HETATM & 359 & $\mathrm{H} 2 \mathrm{O}$ & HEM & 34 & 10.014 & 1.678 & -1.402 & 1.00 & 0.00 & $\mathrm{H}$ \\
\hline HETATM & 360 & N21 & HEM & 34 & 6.809 & 2.080 & -0.367 & 1.00 & 0.00 & $\mathrm{~N}$ \\
\hline HETATM & 361 & N22 & HEM & 34 & 4.105 & 3.027 & -0.590 & 1.00 & 0.00 & $\mathrm{~N}$ \\
\hline HETATM & 362 & N23 & HEM & 34 & 4.775 & 4.290 & -3.090 & 1.00 & 0.00 & $\mathrm{~N}$ \\
\hline HETATM & 363 & $\mathrm{~N} 24$ & HEM & 34 & 7.475 & 3.406 & -2.854 & 1.00 & 0.00 & $\mathrm{~N}$ \\
\hline HETATM & 364 & C99 & HEM & 34 & 6.568 & 6.271 & -0.352 & 1.00 & 0.00 & C \\
\hline HETATM & 365 & 091 & HEM & 34 & 6.409 & 4.958 & -0.365 & 1.00 & 0.00 & 0 \\
\hline HETATM & 366 & 092 & HEM & 34 & 6.723 & 6.888 & 0.703 & 1.00 & 0.00 & 0 \\
\hline HETATM & 367 & СТ91 & HEM & 34 & 6.552 & 6.952 & -1.709 & 1.00 & 0.00 & C \\
\hline HETATM & 368 & H911 & HEM & 34 & 5.573 & 6.757 & -2.171 & 1.00 & 0.00 & $\mathrm{H}$ \\
\hline HETATM & 369 & H912 & HEM & 34 & 7.300 & 6.463 & -2.352 & 1.00 & 0.00 & $\mathrm{H}$ \\
\hline HETATM & 370 & СТ92 & HEM & 34 & 6.812 & 8.457 & -1.597 & 1.00 & 0.00 & C \\
\hline HETATM & 371 & H921 & HEM & 34 & 7.737 & 8.613 & -1.027 & 1.00 & 0.00 & $\mathrm{H}$ \\
\hline HETATM & 372 & H922 & HEM & 34 & 6.015 & 8.917 & -1.000 & 1.00 & 0.00 & $\mathrm{H}$ \\
\hline \multicolumn{11}{|l|}{ TER } \\
\hline HETATM & 373 & $\mathrm{C} 2$ & VIN & 35 & 1.292 & 14.169 & -8.933 & 1.00 & 0.00 & C \\
\hline HETATM & 374 & $\mathrm{H} 21$ & VIN & 35 & 0.476 & 13.470 & -8.768 & 1.00 & 0.00 & $\mathrm{H}$ \\
\hline HETATM & 375 & $\mathrm{C} 1$ & VIN & 35 & 0.959 & 15.394 & -9.335 & 1.00 & 0.00 & $\mathrm{C}$ \\
\hline HETATM & 376 & H11 & VIN & 35 & 1.691 & 16.172 & -9.442 & 1.00 & 0.00 & $\mathrm{H}$ \\
\hline HETATM & 377 & $\mathrm{H} 12$ & VIN & 35 & -0.082 & 15.663 & -9.501 & 1.00 & 0.00 & $\mathrm{H}$ \\
\hline \multicolumn{11}{|l|}{ TER } \\
\hline HETATM & 378 & $\mathrm{C} 1$ & MET & 36 & 3.956 & 14.424 & -10.771 & 1.00 & 0.00 & $\mathrm{C}$ \\
\hline HETATM & 379 & H11 & MET & 36 & 4.191 & 15.494 & -10.652 & 1.00 & 0.00 & $\mathrm{H}$ \\
\hline HETATM & 380 & $\mathrm{H} 12$ & $\mathrm{MET}$ & 36 & 4.769 & 13.959 & -11.341 & 1.00 & 0.00 & $\mathrm{H}$ \\
\hline HETATM & 381 & $\mathrm{H} 13$ & MET & 36 & 3.048 & 14.346 & $-11 \cdot 382$ & 1.00 & 0.00 & $\mathrm{H}$ \\
\hline \multicolumn{11}{|l|}{ TER } \\
\hline HETATM & 382 & $\mathrm{C} 2$ & VIN & 37 & 8.966 & 12.746 & -10.256 & 1.00 & 0.00 & C \\
\hline HETATM & 383 & H 21 & VIN & 37 & 8.300 & 12.645 & -11.115 & 1.00 & 0.00 & $\mathrm{H}$ \\
\hline HETATM & 384 & $\mathrm{C} 1$ & VIN & 37 & 10.133 & 13.390 & -10.415 & 1.00 & 0.00 & C \\
\hline HETATM & 385 & H11 & VIN & 37 & 10.813 & 13.546 & -9.585 & 1.00 & 0.00 & $\mathrm{H}$ \\
\hline HETATM & 386 & $\mathrm{H} 12$ & VIN & 37 & 10.438 & 13.788 & -11.381 & 1.00 & 0.00 & $\mathrm{H}$ \\
\hline TER & & & & & & & & & & \\
\hline HETATM & 387 & $\mathrm{C} 1$ & $\mathrm{MET}$ & 38 & 10.651 & 11.062 & -8.052 & 1.00 & 0.00 & C \\
\hline HETATM & 388 & H11 & $\mathrm{MET}$ & 38 & 11.242 & 11.727 & -7.403 & 1.00 & 0.00 & $\mathrm{H}$ \\
\hline HETATM & 389 & $\mathrm{H} 12$ & MET & 38 & 10.807 & 10.040 & -7.685 & 1.00 & 0.00 & $\mathrm{H}$ \\
\hline HETATM & 390 & $\mathrm{H} 13$ & MET & 38 & 11.083 & 11.128 & -9.057 & 1.00 & 0.00 & $\mathrm{H}$ \\
\hline TER & & & & & & & & & & \\
\hline HETATM & 391 & C5 & $\mathrm{ACP}$ & 39 & 1.562 & 9.981 & -2.161 & 1.00 & 0.00 & C \\
\hline HETATM & 392 & H51 & $\mathrm{ACP}$ & 39 & 2.301 & 9.969 & -1.349 & 1.00 & 0.00 & $\mathrm{H}$ \\
\hline HETATM & 393 & $\mathrm{H} 52$ & $\mathrm{ACP}$ & 39 & 0.722 & 10.589 & -1.798 & 1.00 & 0.00 & $\mathrm{H}$ \\
\hline HETATM & 394 & $\mathrm{C} 4$ & $\mathrm{ACP}$ & 39 & 1.084 & 8.543 & -2.434 & 1.00 & 0.00 & C \\
\hline HETATM & 395 & H 41 & $\mathrm{ACP}$ & 39 & 0.526 & 8.482 & -3.382 & 1.00 & 0.00 & $\mathrm{H}$ \\
\hline HETATM & 396 & H4 2 & $\mathrm{ACP}$ & 39 & 1.954 & 7.877 & -2.541 & 1.00 & 0.00 & $\mathrm{H}$ \\
\hline HETATM & 397 & $\mathrm{C} 1$ & $\mathrm{ACP}$ & 39 & 0.220 & 7.931 & -1.358 & 1.00 & 0.00 & C \\
\hline HETATM & 398 & $\mathrm{O} 2$ & $\mathrm{ACP}$ & 39 & -0.008 & 8.454 & -0.260 & 1.00 & 0.00 & o \\
\hline HETATM & 399 & 03 & $\mathrm{ACP}$ & 39 & -0.296 & 6.751 & -1.722 & 1.00 & 0.00 & 0 \\
\hline HETATM & 400 & $\mathrm{H} 3$ & $\mathrm{ACP}$ & 39 & -0.905 & 6.397 & -0.986 & 1.00 & 0.00 & $\mathrm{H}$ \\
\hline TER & & & & & & & & & & \\
\hline HETATM & 401 & $\mathrm{C} 1$ & $\mathrm{MET}$ & 40 & 9.458 & 8.855 & -3.334 & 1.00 & 0.00 & C \\
\hline HETATM & 402 & H11 & $\mathrm{MET}$ & 40 & 10.011 & 9.694 & -2.884 & 1.00 & 0.00 & $\mathrm{H}$ \\
\hline HETATM & 403 & $\mathrm{H} 12$ & MET & 40 & 9.465 & 8.029 & -2.613 & 1.00 & 0.00 & $\mathrm{H}$ \\
\hline HETATM & 404 & H13 & MET & 40 & 10.029 & 8.535 & -4.214 & 1.00 & 0.00 & 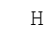 \\
\hline
\end{tabular}


TER

HETATM 405 C1 MET HETATM 406 H11 MET HETATM 407 H12 MET HETATM 408 H13 MET TER

HETATM 409 C2 VI HETATM 410 H21 VIN HETATM 411 C1 VIN HETATM 412 H11 VIN HETATM 413 H12 VIN TER

HETATM 414 C1 MET HETATM 415 H11 MET HETATM 416 H12 MET HETATM 417 H13 MET TER

HETATM 418 C2 VIN HETATM 419 H21 VIN HETATM 420 C1 VIN HETATM 421 H11 VIN HETATM 422 H12 VIN TER

HETATM 423 C1 MET HETATM 424 H11 MET HETATM 425 H12 MET HETATM 426 H13 MET TER

HETATM 427 C5 ACP HETATM 428 H51 ACP HETATM 429 H52 ACP HETATM $430 \quad$ C4 ACP HETATM 431 H41 ACP HETATM 432 H42 ACP HETATM 433 C1 ACP HETATM 434 O2 ACP HETATM 435 O3 ACP HETATM 436 H3 ACP TER

HETATM 437 C1 ME HETATM 438 H11 MET HETATM 439 H12 MET HETATM 440 H13 MET TER

HETATM 441 C1 MET HETATM 442 H11 MET HETATM 443 H12 MET HETATM 444 H13 MET TER

HETATM 445 FE HEM HETATM 446 C1 HEM HETATM 447 C2 HEM HETATM 448 C3 HEM HETATM 449 C4 HEM HETATM 450 C5 HEM HETATM 451 H5 HEM HETATM 452 C6 HEM HETATM $453 \quad$ C7 HEM HETATM 454 C8 HEM HETATM 455 C9 HEM HETATM 456 C10 HEM HETATM 457 H1O HEM HETATM 458 C11 HEM HETATM 459 C12 HEM HETATM 460 C13 HEM HETATM 461 C14 HEM HETATM 462 C15 HEM HETATM 463 H15 HEM HETATM 464 C16 HEM HETATM 465 C17 HEM HETATM 466 C18 HEM HETATM 467 C19 HEM HETATM 468 C20 HEM HETATM 469 H2O HEM HETATM 470 N21 HEM HETATM 471 N22 HEM HETATM 472 N23 HEM HETATM 473 N24 HEM HETATM 474 C99 HEM $\begin{array}{llllll}0.025 & 11.803 & -4.285 & 1.00 & 0.00\end{array}$

$-0.420-11.5$

$\begin{array}{lllll}-0.086 & 12.883 & -4.460 & 1.00 & 0.00\end{array}$

$\begin{array}{lllll}-0.576 & 11.278 & -5.042 & 1.00 & 0.00\end{array}$

$\begin{array}{llllll}9.822 & 0.424 & 1.002 & 1.00 & 0.00\end{array}$

$\begin{array}{lllll}10.637 & 1.123 & 0.837 & 1.00 & 0.00\end{array}$

$\begin{array}{lllll}10.154 & -0.801 & 1.403 & 1.00 & 0.00\end{array}$

$\begin{array}{lllll}9.423 & -1.579 & 1.511 & 1.00 & 0.00\end{array}$

$\begin{array}{lllll}11.196 & -1.070 & 1.570 & 1.00 & 0.00\end{array}$

$\begin{array}{lllll}7.158 & 0.169 & 2.840 & 1.00 & 0.00\end{array}$

$\begin{array}{lllll}6.923 & -0.902 & 2.720 & 1.00 & 0.00\end{array}$

$\begin{array}{lllll}6.345 & 0.633 & 3.410 & 1.00 & 0.00\end{array}$

$\begin{array}{lllll}8.065 & 0.247 & 3.450 & 1.00 & 0.00\end{array}$

$\begin{array}{lllll}2.148 & 1.847 & 2.325 & 1.00 & 0.00\end{array}$

$2.814 \quad 1.948 \quad 3.184 \quad 1.00 \quad 0.00$

$\begin{array}{lllll}0.981 & 1.203 & 2.484 & 1.00 & 0.00\end{array}$

$\begin{array}{lllll}0.301 & 1.047 & 1.654 & 1.00 & 0.00\end{array}$

$\begin{array}{lllll}0.676 & 0.805 & 3.450 & 1.00 & 0.00\end{array}$

$\begin{array}{llllll}0.463 & 3.530 & 0.121 & 1.00 & 0.00\end{array}$

$\begin{array}{llllll}0.307 & 4.553 & -0.246 & 1.00 & 0.00\end{array}$

$\begin{array}{lllll}0.031 & 3.465 & 1.126 & 1.00 & 0.00\end{array}$

$\begin{array}{lllll}-0.128 & 2.866 & -0.528 & 1.00 & 0.00\end{array}$

$\begin{array}{lllll}9.552 & 4.612 & -5.770 & 1.00 & 0.00\end{array}$

$\begin{array}{lllll}8.813 & 4.623 & -6.582 & 1.00 & 0.00\end{array}$

$\begin{array}{lllll}10.392 & 4.004 & -6.134 & 1.00 & 0.00\end{array}$

$\begin{array}{lllll}10.030 & 6.050 & -5.497 & 1.00 & 0.00\end{array}$

$\begin{array}{llllll}10.588 & 6.111 & -4.549 & 1.00 & 0.00\end{array}$

$\begin{array}{lllll}9.160 & 6.716 & -5.390 & 1.00 & 0.00\end{array}$

$\begin{array}{lllll}10.894 & 6.662 & -6.573 & 1.00 & 0.00\end{array}$

$\begin{array}{lllll}11.122 & 6.139 & -7.671 & 1.00 & 0.00\end{array}$

$\begin{array}{lllll}11.410 & 7.841 & -6.209 & 1.00 & 0.00\end{array}$

$\begin{array}{llllll}12.019 & 8.196 & -6.945 & 1.00 & 0.00\end{array}$

$\begin{array}{lllll}1.655 & 5.738 & -4.597 & 1.00 & 0.00\end{array}$

$\begin{array}{lllll}1.103 & 4.899 & -5.047 & 1.00 & 0.00\end{array}$

$\begin{array}{lllll}1.649 & 6.564 & -5.319 & 1.00 & 0.00\end{array}$

$\begin{array}{lllll}1.084 & 6.058 & -3.717 & 1.00 & 0.00\end{array}$

$\begin{array}{llllll}11.089 & 2.790 & -3.646 & 1.00 & 0.00\end{array}$

$\begin{array}{lllll}11.534 & 2.998 & -4.626 & 1.00 & 0.00\end{array}$

$\begin{array}{lllll}11.199 & 1.709 & -3.471 & 1.00 & 0.00\end{array}$

$\begin{array}{lllll}11.690 & 3.315 & -2.889 & 1.00 & 0.00\end{array}$

$\begin{array}{lllll}2.368 & 10.265 & 17.354 & 1.00 & 0.00\end{array}$

$\begin{array}{lllll}0.140 & 12.137 & 16.292 & 1.00 & 0.00\end{array}$

$\begin{array}{lllll}-0.219 & 12.928 & 15.120 & 1.00 & 0.00\end{array}$

$\begin{array}{lllll}0.927 & 13.025 & 14.345 & 1.00 & 0.00\end{array}$

$\begin{array}{lllll}1.956 & 12.307 & 15.053 & 1.00 & 0.00\end{array}$

$\begin{array}{lllll}3.271 & 12.188 & 14.615 & 1.00 & 0.00\end{array}$

$\begin{array}{lllll}3.525 & 12.685 & 13.680 & 1.00 & 0.00\end{array}$

$\begin{array}{lllll}4.291 & 11.490 & 15.251 & 1.00 & 0.00\end{array}$

$\begin{array}{lllll}5.660 & 11.417 & 14.782 & 1.00 & 0.00\end{array}$

$\begin{array}{lllll}6.365 & 10.698 & 15.729 & 1.00 & 0.00\end{array}$

$\begin{array}{lllll}5.414 & 10.333 & 16.753 & 1.00 & 0.00\end{array}$

$\begin{array}{lllll}5.719 & 9.598 & 17.897 & 1.00 & 0.00\end{array}$

$\begin{array}{lllll}6.745 & 9.254 & 18.002 & 1.00 & 0.00\end{array}$

$\begin{array}{lllll}4.844 & 9.258 & 18.919 & 1.00 & 0.00\end{array}$

$\begin{array}{lllll}5.225 & 8.532 & 20.109 & 1.00 & 0.00\end{array}$

$\begin{array}{lllll}4.086 & 8.385 & 20.866 & 1.00 & 0.00\end{array}$

$\begin{array}{lllll}3.013 & 9.027 & 20.135 & 1.00 & 0.00\end{array}$

$\begin{array}{lllll}1.685 & 9.081 & 20.557 & 1.00 & 0.00\end{array}$

$\begin{array}{lllll}1.444 & 8.597 & 21.504 & 1.00 & 0.00\end{array}$

$\begin{array}{llllll}0.654 & 9.763 & 19.913 & 1.00 & 0.00\end{array}$

$\begin{array}{lllll}-0.711 & 9.876 & 20.396 & 1.00 & 0.00\end{array}$

$\begin{array}{lllll}-1.387 & 10.644 & 19.472 & 1.00 & 0.00\end{array}$

$\begin{array}{lllll}-0.437 & 10.996 & 18.435 & 1.00 & 0.00\end{array}$

$\begin{array}{lllll}-0.729 & 11.787 & 17.324 & 1.00 & 0.00\end{array}$

$\begin{array}{lllll}-1.743 & 12.176 & 17.264 & 1.00 & 0.00\end{array}$

$\begin{array}{llllll}1.462 & 11.774 & 16.230 & 1.00 & 0.00\end{array}$

$\begin{array}{lllll}4.166 & 10.827 & 16.452 & 1.00 & 0.00\end{array}$

$\begin{array}{lllll}3.496 & 9.564 & 18.952 & 1.00 & 0.00\end{array}$

$\begin{array}{lllll}0.796 & 10.448 & 18.717 & 1.00 & 0.00\end{array}$

$\begin{array}{lllll}1.703 & 7.583 & 16.214 & 1.00 & 0.00\end{array}$

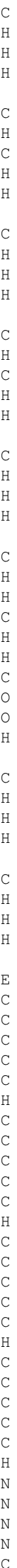




\begin{tabular}{|c|c|c|c|c|c|}
\hline 1.862 & 8.897 & 16.227 & 1.00 & 0.00 & 0 \\
\hline 1.548 & 6.966 & 15.159 & 1.00 & 0.00 & 0 \\
\hline 1.719 & 6.902 & 17.571 & 1.00 & 0.00 & $\mathrm{C}$ \\
\hline 2.697 & 7.097 & 18.034 & 1.00 & 0.00 & $\mathrm{H}$ \\
\hline 0.971 & 7.392 & 18.214 & 1.00 & 0.00 & $\mathrm{H}$ \\
\hline 1.459 & 5.397 & 17.459 & 1.00 & 0.00 & C \\
\hline 2.255 & 4.937 & 16.863 & 1.00 & 0.00 & $\mathrm{H}$ \\
\hline 0.534 & 5.241 & 16.889 & 1.00 & 0.00 & $\mathrm{H}$ \\
\hline 3.060 & 2.851 & 22.302 & 1.00 & 0.00 & $\mathrm{FE}$ \\
\hline 5.288 & 0.979 & 23.364 & 1.00 & 0.00 & C \\
\hline 5.647 & 0.188 & 24.536 & 1.00 & 0.00 & C \\
\hline 4.501 & 0.091 & 25.311 & 1.00 & 0.00 & C \\
\hline 3.472 & 0.808 & 24.603 & 1.00 & 0.00 & C \\
\hline 2.157 & 0.927 & 25.041 & 1.00 & 0.00 & $\mathrm{C}$ \\
\hline 1.903 & 0.431 & 25.976 & 1.00 & 0.00 & $\mathrm{H}$ \\
\hline 1.137 & 1.626 & 24.405 & 1.00 & 0.00 & $\mathrm{C}$ \\
\hline-0.232 & 1.698 & 24.874 & 1.00 & 0.00 & $\mathrm{C}$ \\
\hline-0.937 & 2.418 & 23.927 & 1.00 & 0.00 & C \\
\hline 0.014 & 2.783 & 22.903 & 1.00 & 0.00 & C \\
\hline-0.290 & 3.518 & 21.759 & 1.00 & 0.00 & C \\
\hline-1.317 & 3.862 & 21.654 & 1.00 & 0.00 & $\mathrm{H}$ \\
\hline 0.584 & 3.858 & 20.737 & 1.00 & 0.00 & C \\
\hline 0.203 & 4.583 & 19.547 & 1.00 & 0.00 & C \\
\hline 1.343 & 4.730 & 18.790 & 1.00 & 0.00 & C \\
\hline 2.415 & 4.089 & 19.521 & 1.00 & 0.00 & C \\
\hline 3.744 & 4.034 & 19.099 & 1.00 & 0.00 & C \\
\hline 3.984 & 4.519 & 18.152 & 1.00 & 0.00 & $\mathrm{H}$ \\
\hline 4.774 & 3.353 & 19.743 & 1.00 & 0.00 & C \\
\hline 6.140 & 3.239 & 19.260 & 1.00 & 0.00 & C \\
\hline 6.815 & 2.471 & 20.184 & 1.00 & 0.00 & C \\
\hline 5.865 & 2.119 & 21.221 & 1.00 & 0.00 & C \\
\hline 6.157 & 1.328 & 22.332 & 1.00 & 0.00 & $\mathrm{C}$ \\
\hline 7.171 & 0.939 & 22.392 & 1.00 & 0.00 & $\mathrm{H}$ \\
\hline 3.966 & 1.342 & 23.426 & 1.00 & 0.00 & $\mathrm{~N}$ \\
\hline 1.262 & 2.289 & 23.204 & 1.00 & 0.00 & $\mathrm{~N}$ \\
\hline 1.933 & 3.552 & 20.704 & 1.00 & 0.00 & $\mathrm{~N}$ \\
\hline 4.632 & 2.667 & 20.939 & 1.00 & 0.00 & $\mathrm{~N}$ \\
\hline 3.726 & 5.532 & 23.442 & 1.00 & 0.00 & C \\
\hline 3.566 & 4.219 & 23.429 & 1.00 & 0.00 & o \\
\hline 3.880 & 6.150 & 24.497 & 1.00 & 0.00 & 0 \\
\hline 3.709 & 6.214 & 22.085 & 1.00 & 0.00 & C \\
\hline 2.731 & 6.018 & 21.622 & 1.00 & 0.00 & $\mathrm{H}$ \\
\hline 4.457 & 5.724 & 21.442 & 1.00 & 0.00 & $\mathrm{H}$ \\
\hline 3.969 & 7.718 & 22.197 & 1.00 & 0.00 & C \\
\hline 4.894 & 7.874 & 22.767 & 1.00 & 0.00 & $\mathrm{H}$ \\
\hline 3.173 & 8.179 & 22.793 & 1.00 & 0.00 & $\mathrm{H}$ \\
\hline-1.551 & 13.430 & 14.860 & 1.00 & 0.00 & C \\
\hline-2.366 & 12.732 & 15.025 & 1.00 & 0.00 & $\mathrm{H}$ \\
\hline-1.884 & 14.655 & 14.459 & 1.00 & 0.00 & C \\
\hline-1.152 & 15.434 & 14.352 & 1.00 & 0.00 & $\mathrm{H}$ \\
\hline-2.925 & 14.924 & 14.292 & 1.00 & 0.00 & $\mathrm{H}$ \\
\hline 1.113 & 13.685 & 13.022 & 1.00 & 0.00 & C \\
\hline 1.348 & 14.756 & 13.142 & 1.00 & 0.00 & $\mathrm{H}$ \\
\hline 1.926 & 13.221 & 12.453 & 1.00 & 0.00 & $\mathrm{H}$ \\
\hline 0.205 & 13.607 & 12.412 & 1.00 & 0.00 & $\mathrm{H}$ \\
\hline 6.123 & 12.007 & 13.537 & 1.00 & 0.00 & C \\
\hline 5.457 & 11.906 & 12.679 & 1.00 & 0.00 & $\mathrm{H}$ \\
\hline 7.290 & 12.651 & 13.379 & 1.00 & 0.00 & C \\
\hline 7.970 & 12.808 & 14.209 & 1.00 & 0.00 & $\mathrm{H}$ \\
\hline 7.595 & 13.049 & 12.413 & 1.00 & 0.00 & $\mathrm{H}$ \\
\hline 7.808 & 10.324 & 15.741 & 1.00 & 0.00 & C \\
\hline 8.399 & 10.988 & 16.390 & 1.00 & 0.00 & $\mathrm{H}$ \\
\hline 7.964 & 9.302 & 16.108 & 1.00 & 0.00 & $\mathrm{H}$ \\
\hline 8.240 & 10.389 & 14.736 & 1.00 & 0.00 & $\mathrm{H}$ \\
\hline-1.281 & 9.242 & 21.633 & 1.00 & 0.00 & $\mathrm{C}$ \\
\hline-0.542 & 9.231 & 22.445 & 1.00 & 0.00 & $\mathrm{H}$ \\
\hline-2.121 & 9.850 & 21.996 & 1.00 & 0.00 & $\mathrm{H}$ \\
\hline-1.759 & 7.805 & 21.360 & 1.00 & 0.00 & C \\
\hline-2.317 & 7.743 & 20.412 & 1.00 & 0.00 & $\mathrm{H}$ \\
\hline-0.889 & 7.138 & 21.253 & 1.00 & 0.00 & $\mathrm{H}$ \\
\hline-2.623 & 7.192 & 22.436 & 1.00 & 0.00 & C \\
\hline-2.851 & 7.715 & 23.534 & 1.00 & 0.00 & o \\
\hline-3.139 & 6.013 & 22.071 & 1.00 & 0.00 & 0 \\
\hline
\end{tabular}




\begin{tabular}{|c|c|c|c|}
\hline HETATM & 548 & H3 & $\mathrm{ACP}$ \\
\hline \multicolumn{4}{|l|}{ TER } \\
\hline HETATM & 549 & $\mathrm{C} 1$ & MET \\
\hline HETATM & 550 & $\mathrm{H} 11$ & $\mathrm{MET}$ \\
\hline HETATM & 551 & $\mathrm{H} 12$ & MET \\
\hline HETATM & 552 & $\mathrm{H} 13$ & $\mathrm{MET}$ \\
\hline \multicolumn{4}{|l|}{ TER } \\
\hline HETATM & 553 & $\mathrm{C} 1$ & MET \\
\hline HETATM & 554 & H11 & MET \\
\hline HETATM & 555 & H12 & MET \\
\hline HETATM & 556 & H13 & MET \\
\hline \multicolumn{4}{|l|}{ TER } \\
\hline HETATM & 557 & $\mathrm{C} 2$ & VIN \\
\hline HETATM & 558 & H 21 & VIN \\
\hline HETATM & 559 & $\mathrm{C} 1$ & VIN \\
\hline HETATM & 560 & H11 & VIN \\
\hline HETATM & 561 & $\mathrm{H} 12$ & VIN \\
\hline \multicolumn{4}{|l|}{ TER } \\
\hline HETATM & 562 & $\mathrm{C} 1$ & MET \\
\hline HETATM & 563 & H11 & MET \\
\hline HETATM & 564 & $\mathrm{H} 12$ & MET \\
\hline HETATM & 565 & $\mathrm{H} 13$ & MET \\
\hline \multicolumn{4}{|l|}{ TER } \\
\hline HETATM & 566 & $\mathrm{C} 2$ & VIN \\
\hline HETATM & 567 & H 21 & VIN \\
\hline HETATM & 568 & $\mathrm{C} 1$ & VIN \\
\hline HETATM & 569 & $\mathrm{H} 11$ & VIN \\
\hline HETATM & 570 & $\mathrm{H} 12$ & VIN \\
\hline \multicolumn{4}{|l|}{ TER } \\
\hline HETATM & 571 & $\mathrm{C} 1$ & MET \\
\hline HETATM & 572 & H11 & MET \\
\hline HETATM & 573 & H12 & MET \\
\hline HETATM & 574 & H13 & MET \\
\hline \multicolumn{4}{|l|}{ TER } \\
\hline HETATM & 575 & C5 & $\mathrm{ACP}$ \\
\hline HETATM & 576 & H51 & $\mathrm{ACP}$ \\
\hline HETATM & 577 & H52 & $\mathrm{ACP}$ \\
\hline HETATM & 578 & $\mathrm{C} 4$ & $\mathrm{ACP}$ \\
\hline HETATM & 579 & H 41 & $\mathrm{ACP}$ \\
\hline HETATM & 580 & $\mathrm{H} 42$ & $\mathrm{ACP}$ \\
\hline HETATM & 581 & $\mathrm{C} 1$ & $\mathrm{ACP}$ \\
\hline HETATM & 582 & $\mathrm{O} 2$ & $\mathrm{ACP}$ \\
\hline HETATM & 583 & 03 & $\mathrm{ACP}$ \\
\hline HETATM & 584 & $\mathrm{H} 3$ & $\mathrm{ACP}$ \\
\hline \multicolumn{4}{|l|}{ TER } \\
\hline HETATM & 585 & $\mathrm{C} 1$ & MET \\
\hline HETATM & 586 & H11 & MET \\
\hline HETATM & 587 & $\mathrm{H} 12$ & MET \\
\hline HETATM & 588 & H13 & MET \\
\hline \multicolumn{4}{|l|}{ TER } \\
\hline HETATM & 589 & $\mathrm{C} 1$ & MET \\
\hline HETATM & 590 & H11 & MET \\
\hline HETATM & 591 & $\mathrm{H} 12$ & MET \\
\hline HETATM & 592 & H13 & MET \\
\hline
\end{tabular}

\begin{tabular}{|c|c|c|c|c|c|}
\hline-3.748 & 5.659 & 22.808 & 1.00 & 0.00 & $\mathrm{H}$ \\
\hline 6.615 & 8.116 & 20.459 & 1.00 & 0.00 & C \\
\hline 7.168 & 8.955 & 20.909 & 1.00 & 0.00 & $\mathrm{H}$ \\
\hline 6.622 & 7.290 & 21.181 & 1.00 & 0.00 & $\mathrm{H}$ \\
\hline 7.186 & 7.796 & 19.580 & 1.00 & 0.00 & $\mathrm{H}$ \\
\hline-2.818 & 11.065 & 19.508 & 1.00 & 0.00 & C \\
\hline-3.263 & 10.856 & 20.488 & 1.00 & 0.00 & $\mathrm{H}$ \\
\hline-2.928 & 12.145 & 19.333 & 1.00 & 0.00 & $\mathrm{H}$ \\
\hline-3.419 & 10.539 & 18.752 & 1.00 & 0.00 & $\mathrm{H}$ \\
\hline 6.979 & -0.315 & 24.796 & 1.00 & 0.00 & C \\
\hline 7.795 & 0.384 & 24.630 & 1.00 & 0.00 & $\mathrm{H}$ \\
\hline 7.312 & -1.539 & 25.197 & 1.00 & 0.00 & C \\
\hline 6.580 & -2.318 & 25.304 & 1.00 & 0.00 & $\mathrm{H}$ \\
\hline 8.353 & -1.809 & 25.364 & 1.00 & 0.00 & $\mathrm{H}$ \\
\hline 4.315 & -0.569 & 26.634 & 1.00 & 0.00 & C \\
\hline 4.080 & -1.640 & 26.514 & 1.00 & 0.00 & $\mathrm{H}$ \\
\hline 3.502 & -0.105 & 27.203 & 1.00 & 0.00 & $\mathrm{H}$ \\
\hline 5.223 & -0.492 & 27.244 & 1.00 & 0.00 & $\mathrm{H}$ \\
\hline-0.695 & 1.109 & 26.119 & 1.00 & 0.00 & C \\
\hline-0.029 & 1.209 & 26.977 & 1.00 & 0.00 & $\mathrm{H}$ \\
\hline-1.862 & 0.464 & 26.277 & 1.00 & 0.00 & C \\
\hline-2.542 & 0.308 & 25.447 & 1.00 & 0.00 & $\mathrm{H}$ \\
\hline-2.167 & 0.066 & 27.243 & 1.00 & 0.00 & $\mathrm{H}$ \\
\hline-2.380 & 2.792 & 23.915 & 1.00 & 0.00 & $\mathrm{C}$ \\
\hline-2.536 & 3.814 & 23.548 & 1.00 & 0.00 & $\mathrm{H}$ \\
\hline-2.812 & 2.726 & 24.920 & 1.00 & 0.00 & $\mathrm{H}$ \\
\hline-2.971 & 2.128 & 23.266 & 1.00 & 0.00 & $\mathrm{H}$ \\
\hline 6.709 & 3.873 & 18.023 & 1.00 & 0.00 & C \\
\hline 5.970 & 3.885 & 17.211 & 1.00 & 0.00 & $\mathrm{H}$ \\
\hline 7.549 & 3.265 & 17.660 & 1.00 & 0.00 & $\mathrm{H}$ \\
\hline 7.187 & 5.311 & 18.296 & 1.00 & 0.00 & C \\
\hline 7.745 & 5.372 & 19.244 & 1.00 & 0.00 & $\mathrm{H}$ \\
\hline 6.317 & 5.978 & 18.403 & 1.00 & 0.00 & $\mathrm{H}$ \\
\hline 8.051 & 5.923 & 17.220 & 1.00 & 0.00 & C \\
\hline 8.279 & 5.400 & 16.122 & 1.00 & 0.00 & 0 \\
\hline 8.567 & 7.103 & 17.585 & 1.00 & 0.00 & 0 \\
\hline 9.176 & 7.457 & 16.848 & 1.00 & 0.00 & $\mathrm{H}$ \\
\hline-1.187 & 5.000 & 19.197 & 1.00 & 0.00 & C \\
\hline-1.740 & 4.160 & 18.746 & 1.00 & 0.00 & $\mathrm{H}$ \\
\hline-1.194 & 5.825 & 18.475 & 1.00 & 0.00 & $\mathrm{H}$ \\
\hline-1.758 & 5.319 & 20.076 & 1.00 & 0.00 & $\mathrm{H}$ \\
\hline 8.246 & 2.051 & 20.148 & 1.00 & 0.00 & C \\
\hline 8.691 & 2.260 & 19.168 & 1.00 & 0.00 & $\mathrm{H}$ \\
\hline 8.357 & 0.971 & 20.323 & 1.00 & 0.00 & $\mathrm{H}$ \\
\hline 8.847 & 2.577 & 20.904 & 1.00 & 0.00 & $H$ \\
\hline
\end{tabular}

TER

END

\subsection{Q2 dimer: A04_Q2.pdb file}

$\begin{array}{lrrl}\text { ATOM } & 1 & \text { FE } & \text { HEM } \\ \text { ATOM } & 2 & \text { C1 } & \text { HEM } \\ \text { ATOM } & 3 & \text { C2 } & \text { HEM } \\ \text { ATOM } & 4 & \text { C3 } & \text { HEM } \\ \text { ATOM } & 5 & \text { C4 } & \text { HEM } \\ \text { ATOM } & 6 & \text { C5 } & \text { HEM } \\ \text { ATOM } & 7 & \text { H5 } & \text { HEM } \\ \text { ATOM } & 8 & \text { C6 } & \text { HEM } \\ \text { ATOM } & 9 & \text { C7 } & \text { HEM } \\ \text { ATOM } & 10 & \text { C8 } & \text { HEM } \\ \text { ATOM } & 11 & \text { C9 } & \text { HEM } \\ \text { ATOM } & 12 & \text { C10 } & \text { HEM } \\ \text { ATOM } & 13 & \text { H10 } & \text { HEM } \\ \text { ATOM } & 14 & \text { C11 } & \text { HEM } \\ \text { ATOM } & 15 & \text { C12 } & \text { HEM } \\ \text { ATOM } & 16 & \text { C13 } & \text { HEM } \\ \text { ATOM } & 17 & \text { C14 } & \text { HEM } \\ \text { ATOM } & 18 & \text { C15 } & \text { HEM } \\ \text { ATOM } & 19 & \text { H15 } & \text { HEM }\end{array}$

$\begin{array}{rrrrr}4.263 & 10.757 & 1.491 & 1.00 & 0.00 \\ 2.035 & 12.629 & 0.429 & 1.00 & 0.00 \\ 1.676 & 13.420 & -0.742 & 1.00 & 0.00 \\ 2.822 & 13.517 & -1.517 & 1.00 & 0.00 \\ 3.851 & 12.800 & -0.809 & 1.00 & 0.00 \\ 5.167 & 12.681 & -1.247 & 1.00 & 0.00 \\ 5.420 & 13.177 & -2.182 & 1.00 & 0.00 \\ 6.186 & 11.982 & -0.611 & 1.00 & 0.00 \\ 7.555 & 11.910 & -1.081 & 1.00 & 0.00 \\ 8.260 & 11.190 & -0.134 & 1.00 & 0.00 \\ 7.309 & 10.825 & 0.891 & 1.00 & 0.00 \\ 7.614 & 10.090 & 2.034 & 1.00 & 0.00 \\ 8.641 & 9.746 & 2.140 & 1.00 & 0.00 \\ 6.739 & 9.750 & 3.057 & 1.00 & 0.00 \\ 7.120 & 9.025 & 4.247 & 1.00 & 0.00 \\ 5.981 & 8.878 & 5.004 & 1.00 & 0.00 \\ 4.908 & 9.519 & 4.273 & 1.00 & 0.00 \\ 3.580 & 9.574 & 4.694 & 1.00 & 0.00 \\ 3.340 & 9.089 & 5.642 & 1.00 & 0.00\end{array}$




\begin{tabular}{|c|c|c|c|}
\hline ATOM & 20 & C16 & HEM \\
\hline ATOM & 21 & C17 & HEM \\
\hline ATOM & 22 & $\mathrm{C} 18$ & HEM \\
\hline АTOM & 23 & C19 & HEM \\
\hline ATOM & 24 & C20 & HEM \\
\hline ATOM & 25 & H2O & HEM \\
\hline ATOM & 26 & N21 & HEM \\
\hline ATOM & 27 & N22 & HEM \\
\hline ATOM & 28 & N23 & HEM \\
\hline ATOM & 29 & N24 & HEM \\
\hline ATOM & 30 & C99 & HEM \\
\hline ATOM & 31 & 091 & HEM \\
\hline ATOM & 32 & 092 & HEM \\
\hline ATOM & 33 & CT91 & HEM \\
\hline ATOM & 34 & H911 & HEM \\
\hline ATOM & 35 & H912 & HEM \\
\hline ATOM & 36 & СТ92 & HEM \\
\hline ATOM & 37 & H921 & HEM \\
\hline ATOM & 38 & H922 & HEM \\
\hline \multicolumn{4}{|l|}{ TER } \\
\hline ATOM & 39 & $\mathrm{FE}$ & HEM \\
\hline ATOM & 40 & $\mathrm{C} 1$ & HEM \\
\hline ATOM & 41 & $\mathrm{C} 2$ & HEM \\
\hline АTOM & 42 & C3 & HEM \\
\hline ATOM & 43 & $\mathrm{C} 4$ & HEM \\
\hline ATOM & 44 & C5 & HEM \\
\hline ATOM & 45 & H5 & HEM \\
\hline ATOM & 46 & $\mathrm{C} 6$ & HEM \\
\hline ATOM & 47 & C7 & HEM \\
\hline ATOM & 48 & C8 & HEM \\
\hline ATOM & 49 & $\mathrm{C} 9$ & HEM \\
\hline ATOM & 50 & $\mathrm{C} 10$ & HEM \\
\hline ATOM & 51 & H10 & HEM \\
\hline ATOM & 52 & C11 & HEM \\
\hline ATOM & 53 & C12 & HEM \\
\hline ATOM & 54 & C13 & HEM \\
\hline ATOM & 55 & C14 & HEM \\
\hline ATOM & 56 & C15 & HEM \\
\hline ATOM & 57 & H15 & HEM \\
\hline ATOM & 58 & C16 & HEM \\
\hline ATOM & 59 & $\mathrm{C} 17$ & HEM \\
\hline ATOM & 60 & C18 & HEM \\
\hline ATOM & 61 & C19 & HEM \\
\hline ATOM & 62 & $\mathrm{C} 20$ & HEM \\
\hline ATOM & 63 & H2O & HEM \\
\hline ATOM & 64 & N21 & HEM \\
\hline ATOM & 65 & N22 & HEM \\
\hline ATOM & 66 & N23 & HEM \\
\hline ATOM & 67 & N24 & HEM \\
\hline ATOM & 68 & C99 & HEM \\
\hline ATOM & 69 & 091 & HEM \\
\hline ATOM & 70 & 092 & HEM \\
\hline ATOM & 71 & СТ91 & HEM \\
\hline ATOM & 72 & H911 & HEM \\
\hline ATOM & 73 & H912 & HEM \\
\hline ATOM & 74 & СТ92 & HEM \\
\hline ATOM & 75 & H921 & HEM \\
\hline ATOM & 76 & H922 & HEM \\
\hline \multicolumn{4}{|l|}{ TER } \\
\hline ATOM & 77 & $\mathrm{C} 2$ & VIN \\
\hline ATOM & 78 & $\mathrm{H} 21$ & VIN \\
\hline ATOM & 79 & $\mathrm{C} 1$ & VIN \\
\hline ATOM & 80 & H11 & VIN \\
\hline ATOM & 81 & $\mathrm{H} 12$ & VIN \\
\hline \multicolumn{4}{|l|}{ TER } \\
\hline ATOM & 82 & $\mathrm{C} 1$ & MET \\
\hline ATOM & 83 & $\mathrm{H} 11$ & MET \\
\hline ATOM & 84 & H12 & MET \\
\hline ATOM & 85 & $\mathrm{H} 13$ & MET \\
\hline \multicolumn{4}{|l|}{ TER } \\
\hline ATOM & 86 & $\mathrm{C} 2$ & VIN \\
\hline ATOM & 87 & H 21 & VIN \\
\hline ATOM & 88 & $\mathrm{C} 1$ & VIN \\
\hline ATOM & 89 & H11 & VIN \\
\hline ATOM & 90 & $\mathrm{H} 12$ & VIN \\
\hline \multicolumn{4}{|l|}{ TER } \\
\hline ATOM & 91 & $\mathrm{C} 1$ & MET \\
\hline ATOM & 92 & $\mathrm{H} 11$ & MET \\
\hline АТOM & 93 & H12 & MET \\
\hline
\end{tabular}

\begin{tabular}{|c|c|c|c|c|c|}
\hline 2.550 & 10.255 & 4.051 & 1.00 & 0.00 & C \\
\hline 1.184 & 10.369 & 4.533 & 1.00 & 0.00 & C \\
\hline 0.508 & 11.137 & 3.609 & 1.00 & 0.00 & C \\
\hline 1.458 & 11.489 & 2.573 & 1.00 & 0.00 & C \\
\hline 1.167 & 12.280 & 1.462 & 1.00 & 0.00 & C \\
\hline 0.152 & 12.669 & 1.402 & 1.00 & 0.00 & $\mathrm{H}$ \\
\hline 3.357 & 12.266 & 0.367 & 1.00 & 0.00 & $\mathrm{~N}$ \\
\hline 6.062 & 11.319 & 0.590 & 1.00 & 0.00 & $\mathrm{~N}$ \\
\hline 5.391 & 10.056 & 3.090 & 1.00 & 0.00 & $\mathrm{~N}$ \\
\hline 2.691 & 10.941 & 2.854 & 1.00 & 0.00 & $\mathrm{~N}$ \\
\hline 3.598 & 8.076 & 0.352 & 1.00 & 0.00 & C \\
\hline 3.757 & 9.389 & 0.365 & 1.00 & 0.00 & o \\
\hline 3.443 & 7.458 & -0.703 & 1.00 & 0.00 & O \\
\hline 3.614 & 7.394 & 1.709 & 1.00 & 0.00 & C \\
\hline 4.593 & 7.590 & 2.171 & 1.00 & 0.00 & $\mathrm{H}$ \\
\hline 2.866 & 7.884 & 2.352 & 1.00 & 0.00 & $\mathrm{H}$ \\
\hline 3.354 & 5.890 & 1.597 & 1.00 & 0.00 & C \\
\hline 4.151 & 5.429 & 1.000 & 1.00 & 0.00 & $\mathrm{H}$ \\
\hline 2.429 & 5.734 & 1.027 & 1.00 & 0.00 & $\mathrm{H}$ \\
\hline 4.955 & 3.343 & 6.440 & 1.00 & 0.00 & $\mathrm{FE}$ \\
\hline 7.183 & 1.471 & 7.502 & 1.00 & 0.00 & C \\
\hline 7.542 & 0.680 & 8.674 & 1.00 & 0.00 & C \\
\hline 6.397 & 0.583 & 9.448 & 1.00 & 0.00 & C \\
\hline 5.367 & 1.301 & 8.740 & 1.00 & 0.00 & C \\
\hline 4.052 & 1.420 & 9.178 & 1.00 & 0.00 & C \\
\hline 3.798 & 0.923 & 10.113 & 1.00 & 0.00 & $\mathrm{H}$ \\
\hline 3.033 & 2.118 & 8.542 & 1.00 & 0.00 & C \\
\hline 1.664 & 2.191 & 9.012 & 1.00 & 0.00 & C \\
\hline 0.958 & 2.910 & 8.065 & 1.00 & 0.00 & C \\
\hline 1.909 & 3.275 & 7.041 & 1.00 & 0.00 & C \\
\hline 1.605 & 4.010 & 5.897 & 1.00 & 0.00 & C \\
\hline 0.578 & 4.354 & 5.791 & 1.00 & 0.00 & $\mathrm{H}$ \\
\hline 2.479 & 4.350 & 4.874 & 1.00 & 0.00 & C \\
\hline 2.098 & 5.076 & 3.684 & 1.00 & 0.00 & C \\
\hline 3.238 & 5.223 & 2.927 & 1.00 & 0.00 & C \\
\hline 4.311 & 4.581 & 3.659 & 1.00 & 0.00 & C \\
\hline 5.639 & 4.527 & 3.237 & 1.00 & 0.00 & C \\
\hline 5.879 & 5.011 & 2.289 & 1.00 & 0.00 & $\mathrm{H}$ \\
\hline 6.669 & 3.845 & 3.880 & 1.00 & 0.00 & C \\
\hline 8.035 & 3.732 & 3.398 & 1.00 & 0.00 & C \\
\hline 8.710 & 2.964 & 4.322 & 1.00 & 0.00 & C \\
\hline 7.761 & 2.612 & 5.358 & 1.00 & 0.00 & C \\
\hline 8.052 & 1.821 & 6.469 & 1.00 & 0.00 & C \\
\hline 9.066 & 1.432 & 6.530 & 1.00 & 0.00 & $\mathrm{H}$ \\
\hline 5.862 & 1.834 & 7.564 & 1.00 & 0.00 & $\mathrm{~N}$ \\
\hline 3.157 & 2.781 & 7.342 & 1.00 & 0.00 & $\mathrm{~N}$ \\
\hline 3.828 & 4.044 & 4.841 & 1.00 & 0.00 & $\mathrm{~N}$ \\
\hline 6.527 & 3.160 & 5.077 & 1.00 & 0.00 & $\mathrm{~N}$ \\
\hline 5.621 & 6.025 & 7.579 & 1.00 & 0.00 & C \\
\hline 5.461 & 4.711 & 7.567 & 1.00 & 0.00 & 0 \\
\hline 5.776 & 6.642 & 8.635 & 1.00 & 0.00 & 0 \\
\hline 5.605 & 6.706 & 6.223 & 1.00 & 0.00 & C \\
\hline 4.626 & 6.511 & 5.760 & 1.00 & 0.00 & $\mathrm{H}$ \\
\hline 6.353 & 6.216 & 5.580 & 1.00 & 0.00 & $\mathrm{H}$ \\
\hline 5.864 & 8.211 & 6.334 & 1.00 & 0.00 & C \\
\hline 6.789 & 8.367 & 6.905 & 1.00 & 0.00 & $\mathrm{H}$ \\
\hline 5.068 & 8.671 & 6.931 & 1.00 & 0.00 & $\mathrm{H}$ \\
\hline 0.345 & 13.923 & -1.002 & 1.00 & 0.00 & C \\
\hline-0.471 & 13.224 & -0.837 & 1.00 & 0.00 & $\mathrm{H}$ \\
\hline 0.012 & 15.147 & -1.403 & 1.00 & 0.00 & C \\
\hline 0.743 & 15.926 & -1.511 & 1.00 & 0.00 & $\mathrm{H}$ \\
\hline-1.030 & 15.417 & -1.570 & 1.00 & 0.00 & $\mathrm{H}$ \\
\hline 3.008 & 14.177 & -2.840 & 1.00 & 0.00 & C \\
\hline 3.243 & 15.248 & -2.720 & 1.00 & 0.00 & $\mathrm{H}$ \\
\hline 3.821 & 13.713 & -3.410 & 1.00 & 0.00 & $\mathrm{H}$ \\
\hline 2.101 & 14.100 & -3.450 & 1.00 & 0.00 & $\mathrm{H}$ \\
\hline 8.018 & 12.499 & -2.325 & 1.00 & 0.00 & C \\
\hline 7.352 & 12.399 & -3.184 & 1.00 & 0.00 & $\mathrm{H}$ \\
\hline 9.185 & 13.144 & -2.484 & 1.00 & 0.00 & C \\
\hline 9.865 & 13.300 & -1.654 & 1.00 & 0.00 & $\mathrm{H}$ \\
\hline 9.490 & 13.542 & -3.450 & 1.00 & 0.00 & $\mathrm{H}$ \\
\hline 9.703 & 10.816 & -0.121 & 1.00 & 0.00 & C \\
\hline 10.294 & 11.480 & 0.528 & 1.00 & 0.00 & $\mathrm{H}$ \\
\hline 9.859 & 9.794 & 0.246 & 1.00 & 0.00 & $\mathrm{H}$ \\
\hline
\end{tabular}




\begin{tabular}{|c|c|c|c|c|c|c|c|c|c|c|}
\hline \multicolumn{11}{|c|}{$10.882-1.126$} \\
\hline ATOM & 95 & $\mathrm{C} 5$ & $\mathrm{ACP}$ & 7 & 0.615 & 9.735 & 5.770 & 1.00 & 0.00 & C \\
\hline ATOM & 96 & H51 & $\mathrm{ACP}$ & 7 & 1.354 & 9.723 & 6.582 & 1.00 & 0.00 & $\mathrm{H}$ \\
\hline ATOM & 97 & H5 2 & $\mathrm{ACP}$ & 7 & -0.226 & 10.343 & 6.134 & 1.00 & 0.00 & $\mathrm{H}$ \\
\hline ATOM & 98 & $\mathrm{C} 4$ & $\mathrm{ACP}$ & 7 & 0.136 & 8.297 & 5.497 & 1.00 & 0.00 & C \\
\hline ATOM & 99 & $\mathrm{H} 41$ & $\mathrm{ACP}$ & 7 & -0.422 & 8.236 & 4.549 & 1.00 & 0.00 & $\mathrm{H}$ \\
\hline ATOM & 100 & $\mathrm{H} 42$ & $\mathrm{ACP}$ & 7 & 1.007 & 7.630 & 5.390 & 1.00 & 0.00 & $\mathrm{H}$ \\
\hline ATOM & 101 & $\mathrm{C} 1$ & $\mathrm{ACP}$ & 7 & -0.728 & 7.685 & 6.573 & 1.00 & 0.00 & $\mathrm{C}$ \\
\hline ATOM & 102 & $\mathrm{O} 2$ & $\mathrm{ACP}$ & 7 & -0.956 & 8.208 & 7.671 & 1.00 & 0.00 & 0 \\
\hline ATOM & 103 & 03 & $\mathrm{ACP}$ & 7 & -1.244 & 6.505 & 6.209 & 1.00 & 0.00 & O \\
\hline ATOM & 104 & H3 & $\mathrm{ACP}$ & 7 & -1.853 & 6.151 & 6.945 & 1.00 & 0.00 & $\mathrm{H}$ \\
\hline \multicolumn{11}{|l|}{ TER } \\
\hline ATOM & 105 & $\mathrm{C} 1$ & $\mathrm{MET}$ & 8 & 8.511 & 8.608 & 4.597 & 1.00 & 0.00 & $\mathrm{C}$ \\
\hline ATOM & 106 & $\mathrm{H} 11$ & $\mathrm{MET}$ & 8 & 9.063 & 9.448 & 5.047 & 1.00 & 0.00 & $\mathrm{H}$ \\
\hline ATOM & 107 & $\mathrm{H} 12$ & $\mathrm{MET}$ & 8 & 8.517 & 7.782 & 5.319 & 1.00 & 0.00 & $\mathrm{H}$ \\
\hline ATOM & 108 & $\mathrm{H} 13$ & $\mathrm{MET}$ & 8 & 9.082 & 8.289 & 3.717 & 1.00 & 0.00 & $H$ \\
\hline \multicolumn{11}{|l|}{ TER } \\
\hline ATOM & 109 & $\mathrm{C} 1$ & $\mathrm{MET}$ & 9 & -0.923 & 11.557 & 3.646 & 1.00 & 0.00 & C \\
\hline ATOM & 110 & H11 & $\mathrm{MET}$ & 9 & -1.368 & 11.348 & 4.626 & 1.00 & 0.00 & $\mathrm{H}$ \\
\hline ATOM & 111 & $\mathrm{H} 12$ & $\mathrm{MET}$ & 9 & -1.033 & 12.637 & 3.471 & 1.00 & 0.00 & $\mathrm{H}$ \\
\hline ATOM & 112 & $\mathrm{H} 13$ & $\mathrm{MET}$ & 9 & -1.523 & 11.031 & 2.889 & 1.00 & 0.00 & $\mathrm{H}$ \\
\hline \multicolumn{11}{|l|}{ TER } \\
\hline ATOM & 113 & $\mathrm{C} 2$ & VIN & 10 & 8.874 & 0.178 & 8.933 & 1.00 & 0.00 & C \\
\hline ATOM & 114 & H 21 & VIN & 10 & 9.690 & 0.876 & 8.768 & 1.00 & 0.00 & $\mathrm{H}$ \\
\hline ATOM & 115 & $\mathrm{C} 1$ & VIN & 10 & 9.207 & -1.047 & 9.335 & 1.00 & 0.00 & C \\
\hline ATOM & 116 & H11 & VIN & 10 & 8.475 & -1.826 & 9.442 & 1.00 & 0.00 & $\mathrm{H}$ \\
\hline ATOM & 117 & $\mathrm{H} 12$ & VIN & 10 & 10.249 & -1.316 & 9.501 & 1.00 & 0.00 & $\mathrm{H}$ \\
\hline \multicolumn{11}{|l|}{ TER } \\
\hline ATOM & 118 & $\mathrm{C} 1$ & $\mathrm{MET}$ & 11 & 6.210 & -0.077 & 10.771 & 1.00 & 0.00 & $\mathrm{C}$ \\
\hline ATOM & 119 & $\mathrm{H} 11$ & MET & 11 & 5.975 & -1.148 & 10.652 & 1.00 & 0.00 & $\mathrm{H}$ \\
\hline ATOM & 120 & $\mathrm{H} 12$ & $\mathrm{MET}$ & 11 & 5.397 & 0.387 & 11.341 & 1.00 & 0.00 & $\mathrm{H}$ \\
\hline ATOM & 121 & H13 & $\mathrm{MET}$ & 11 & 7.118 & 0.001 & 11.382 & 1.00 & 0.00 & $\mathrm{H}$ \\
\hline TER & & & & & & & & & & \\
\hline ATOM & 122 & $\mathrm{C} 2$ & VIN & 12 & 1.200 & 1.601 & 10.256 & 1.00 & 0.00 & C \\
\hline ATOM & 123 & H 21 & VIN & 12 & 1.866 & 1.702 & 11.115 & 1.00 & 0.00 & $\mathrm{H}$ \\
\hline ATOM & 124 & $\mathrm{C} 1$ & VIN & 12 & 0.033 & 0.957 & 10.415 & 1.00 & 0.00 & C \\
\hline ATOM & 125 & H11 & VIN & 12 & -0.647 & 0.800 & 9.585 & 1.00 & 0.00 & $\mathrm{H}$ \\
\hline ATOM & 126 & $\mathrm{H} 12$ & VIN & 12 & -0.272 & 0.559 & 11.381 & 1.00 & 0.00 & $\mathrm{H}$ \\
\hline TER & & & & & & & & & & \\
\hline ATOM & 127 & $\mathrm{C} 1$ & $\mathrm{MET}$ & 13 & -0.484 & 3.284 & 8.052 & 1.00 & 0.00 & $\mathrm{C}$ \\
\hline ATOM & 128 & H 11 & $\mathrm{MET}$ & 13 & -0.641 & 4.306 & 7.685 & 1.00 & 0.00 & $\mathrm{H}$ \\
\hline ATOM & 129 & $\mathrm{H} 12$ & $\mathrm{MET}$ & 13 & -0.917 & 3.219 & 9.057 & 1.00 & 0.00 & $\mathrm{H}$ \\
\hline ATOM & 130 & $\mathrm{H} 13$ & $\mathrm{MET}$ & 13 & -1.076 & 2.620 & 7.403 & 1.00 & 0.00 & $\mathrm{H}$ \\
\hline TER & & & & & & & & & & \\
\hline ATOM & 131 & $\mathrm{C} 5$ & $\mathrm{ACP}$ & 14 & 8.604 & 4.366 & 2.161 & 1.00 & 0.00 & C \\
\hline ATOM & 132 & H51 & $\mathrm{ACP}$ & 14 & 7.865 & 4.377 & 1.349 & 1.00 & 0.00 & $\mathrm{H}$ \\
\hline ATOM & 133 & H52 & $\mathrm{ACP}$ & 14 & 9.445 & 3.758 & 1.798 & 1.00 & 0.00 & $\mathrm{H}$ \\
\hline ATOM & 134 & $\mathrm{C} 4$ & $\mathrm{ACP}$ & 14 & 9.082 & 5.803 & 2.434 & 1.00 & 0.00 & $\mathrm{C}$ \\
\hline ATOM & 135 & H 41 & $\mathrm{ACP}$ & 14 & 9.640 & 5.865 & 3.382 & 1.00 & 0.00 & $\mathrm{H}$ \\
\hline ATOM & 136 & $\mathrm{H} 42$ & $\mathrm{ACP}$ & 14 & 8.212 & 6.470 & 2.541 & 1.00 & 0.00 & $\mathrm{H}$ \\
\hline ATOM & 137 & $\mathrm{C} 1$ & $\mathrm{ACP}$ & 14 & 9.946 & 6.416 & 1.358 & 1.00 & 0.00 & C \\
\hline ATOM & 138 & $\mathrm{O} 2$ & $\mathrm{ACP}$ & 14 & 10.174 & 5.893 & 0.260 & 1.00 & 0.00 & O \\
\hline ATOM & 139 & 03 & $\mathrm{ACP}$ & 14 & 10.462 & 7.595 & 1.722 & 1.00 & 0.00 & O \\
\hline ATOM & 140 & H3 & $\mathrm{ACP}$ & 14 & 11.071 & 7.949 & 0.986 & 1.00 & 0.00 & $\mathrm{H}$ \\
\hline TER & & & & & & & & & & \\
\hline ATOM & 141 & $\mathrm{C} 1$ & $\mathrm{MET}$ & 15 & 0.708 & 5.492 & 3.334 & 1.00 & 0.00 & C \\
\hline ATOM & 142 & H11 & $\mathrm{MET}$ & 15 & 0.155 & 4.653 & 2.884 & 1.00 & 0.00 & $\mathrm{H}$ \\
\hline ATOM & 143 & $\mathrm{H} 12$ & $\mathrm{MET}$ & 15 & 0.701 & 6.318 & 2.613 & 1.00 & 0.00 & $\mathrm{H}$ \\
\hline ATOM & 144 & $\mathrm{H} 13$ & $\mathrm{MET}$ & 15 & 0.137 & 5.812 & 4.214 & 1.00 & 0.00 & $\mathrm{H}$ \\
\hline TER & & & & & & & & & & \\
\hline ATOM & 145 & $\mathrm{C} 1$ & $\mathrm{MET}$ & 16 & 10.141 & 2.543 & 4.285 & 1.00 & 0.00 & $\mathrm{C}$ \\
\hline ATOM & 146 & $\mathrm{H} 11$ & $\mathrm{MET}$ & 16 & 10.586 & 2.752 & 3.305 & 1.00 & 0.00 & $\mathrm{H}$ \\
\hline ATOM & 147 & H12 & MET & 16 & 10.252 & 1.463 & 4.460 & 1.00 & 0.00 & $\mathrm{H}$ \\
\hline ATOM & 148 & H13 & MET & 16 & 10.742 & 3.069 & 5.042 & 1.00 & 0.00 & $\mathrm{H}$ \\
\hline TER & & & & & & & & & & \\
\hline ATOM & 149 & $\mathrm{FE}$ & HEM & 17 & 2.346 & 25.104 & 1.491 & 1.00 & 0.00 & $\mathrm{FE}$ \\
\hline ATOM & 150 & $\mathrm{C} 1$ & HEM & 17 & 0.118 & 26.976 & 0.429 & 1.00 & 0.00 & $\mathrm{C}$ \\
\hline ATOM & 151 & $\mathrm{C} 2$ & HEM & 17 & -0.240 & 27.767 & -0.742 & 1.00 & 0.00 & C \\
\hline ATOM & 152 & $\mathrm{C} 3$ & HEM & 17 & 0.905 & 27.864 & -1.517 & 1.00 & 0.00 & C \\
\hline ATOM & 153 & $\mathrm{C} 4$ & HEM & 17 & 1.935 & 27.146 & -0.809 & 1.00 & 0.00 & C \\
\hline ATOM & 154 & $\mathrm{C} 5$ & HEM & 17 & 3.250 & 27.027 & -1.247 & 1.00 & 0.00 & $\mathrm{C}$ \\
\hline ATOM & 155 & H5 & HEM & 17 & 3.503 & 27.524 & -2.182 & 1.00 & 0.00 & $\mathrm{H}$ \\
\hline ATOM & 156 & $\mathrm{C} 6$ & HEM & 17 & 4.269 & 26.329 & -0.611 & 1.00 & 0.00 & C \\
\hline ATOM & 157 & C7 & HEM & 17 & 5.638 & 26.256 & -1.081 & 1.00 & 0.00 & C \\
\hline ATOM & 158 & $\mathrm{C} 8$ & HEM & 17 & 6.343 & 25.537 & -0.134 & 1.00 & 0.00 & 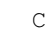 \\
\hline ATOM & 159 & $\mathrm{C} 9$ & HEM & 17 & 5.392 & 25.172 & 0.891 & 1.00 & 0.00 & C \\
\hline ATOM & 160 & $\mathrm{C} 10$ & HEM & 17 & 5.697 & 24.437 & 2.034 & 1.00 & 0.00 & C \\
\hline ATOM & 161 & $\mathrm{H} 10$ & HEM & 17 & 6.724 & 24.093 & 2.140 & 1.00 & 0.00 & \\
\hline
\end{tabular}




\begin{tabular}{|c|c|c|c|c|c|c|c|c|c|c|}
\hline ATOM & 162 & $\mathrm{C} 11$ & HEM & 17 & 4.822 & 24.097 & 3.057 & 1.00 & 0.00 & C \\
\hline ATOM & 163 & $\mathrm{C} 12$ & HEM & 17 & 5.203 & 23.371 & 4.247 & 1.00 & 0.00 & C \\
\hline ATOM & 164 & $\mathrm{C} 13$ & HEM & 17 & 4.064 & 23.224 & 5.004 & 1.00 & 0.00 & C \\
\hline $\mathrm{ATOM}$ & 165 & C14 & HEM & 17 & 2.991 & 23.866 & 4.273 & 1.00 & 0.00 & C \\
\hline ATOM & 166 & C15 & HEM & 17 & 1.663 & 23.920 & 4.694 & 1.00 & 0.00 & C \\
\hline ATOM & 167 & H15 & HEM & 17 & 1.423 & 23.436 & 5.642 & 1.00 & 0.00 & $\mathrm{H}$ \\
\hline ATOM & 168 & $\mathrm{C} 16$ & HEM & 17 & 0.633 & 24.602 & 4.051 & 1.00 & 0.00 & C \\
\hline ATOM & 169 & C17 & HEM & 17 & -0.733 & 24.716 & 4.533 & 1.00 & 0.00 & C \\
\hline ATOM & 170 & C18 & HEM & 17 & -1.408 & 25.483 & 3.609 & 1.00 & 0.00 & C \\
\hline ATOM & 171 & C19 & HEM & 17 & -0.459 & 25.835 & 2.573 & 1.00 & 0.00 & C \\
\hline ATOM & 172 & $\mathrm{C} 20$ & HEM & 17 & -0.750 & 26.626 & 1.462 & 1.00 & 0.00 & C \\
\hline ATOM & 173 & $\mathrm{H} 20$ & HEM & 17 & -1.764 & 27.015 & 1.402 & 1.00 & 0.00 & $\mathrm{H}$ \\
\hline ATOM & 174 & N21 & HEM & 17 & 1.440 & 26.613 & 0.367 & 1.00 & 0.00 & $\mathrm{~N}$ \\
\hline ATOM & 175 & N22 & HEM & 17 & 4.145 & 25.666 & 0.590 & 1.00 & 0.00 & $\mathrm{~N}$ \\
\hline ATOM & 176 & N23 & HEM & 17 & 3.474 & 24.403 & 3.090 & 1.00 & 0.00 & $\mathrm{~N}$ \\
\hline ATOM & 177 & N2 4 & HEM & 17 & 0.774 & 25.287 & 2.854 & 1.00 & 0.00 & $\mathrm{~N}$ \\
\hline ATOM & 178 & C99 & HEM & 17 & 1.681 & 22.422 & 0.352 & 1.00 & 0.00 & C \\
\hline ATOM & 179 & 091 & HEM & 17 & 1.841 & 23.736 & 0.365 & 1.00 & 0.00 & O \\
\hline ATOM & 180 & 092 & HEM & 17 & 1.526 & 21.805 & -0.703 & 1.00 & 0.00 & O \\
\hline ATOM & 181 & CT91 & HEM & 17 & 1.697 & 21.741 & 1.709 & 1.00 & 0.00 & C \\
\hline ATOM & 182 & H911 & HEM & 17 & 2.676 & 21.936 & 2.171 & 1.00 & 0.00 & $\mathrm{H}$ \\
\hline ATOM & 183 & H912 & HEM & 17 & 0.949 & 22.231 & 2.352 & 1.00 & 0.00 & $\mathrm{H}$ \\
\hline ATOM & 184 & CT92 & HEM & 17 & 1.437 & 20.236 & 1.597 & 1.00 & 0.00 & C \\
\hline ATOM & 185 & H921 & HEM & 17 & 2.234 & 19.776 & 1.000 & 1.00 & 0.00 & $\mathrm{H}$ \\
\hline ATOM & 186 & H922 & HEM & 17 & 0.513 & 20.081 & 1.027 & 1.00 & 0.00 & $\mathrm{H}$ \\
\hline \multicolumn{11}{|l|}{ TER } \\
\hline ATOM & 187 & $\mathrm{FE}$ & HEM & 18 & 3.039 & 17.690 & 6.440 & 1.00 & 0.00 & $\mathrm{FE}$ \\
\hline ATOM & 188 & $\mathrm{C} 1$ & HEM & 18 & 5.266 & 15.818 & 7.502 & 1.00 & 0.00 & C \\
\hline ATOM & 189 & $\mathrm{C} 2$ & HEM & 18 & 5.625 & 15.027 & 8.674 & 1.00 & 0.00 & C \\
\hline ATOM & 190 & $\mathrm{C} 3$ & HEM & 18 & 4.480 & 14.930 & 9.448 & 1.00 & 0.00 & C \\
\hline ATOM & 191 & $\mathrm{C} 4$ & HEM & 18 & 3.450 & 15.647 & 8.740 & 1.00 & 0.00 & C \\
\hline ATOM & 192 & C5 & HEM & 18 & 2.135 & 15.766 & 9.178 & 1.00 & 0.00 & C \\
\hline ATOM & 193 & H5 & HEM & 18 & 1.882 & 15.270 & 10.113 & 1.00 & 0.00 & $\mathrm{H}$ \\
\hline ATOM & 194 & $\mathrm{C} 6$ & HEM & 18 & 1.116 & 16.465 & 8.542 & 1.00 & 0.00 & C \\
\hline ATOM & 195 & C7 & HEM & 18 & -0.253 & 16.537 & 9.012 & 1.00 & 0.00 & C \\
\hline ATOM & 196 & $\mathrm{C} 8$ & HEM & 18 & -0.958 & 17.257 & 8.065 & 1.00 & 0.00 & C \\
\hline ATOM & 197 & $\mathrm{C} 9$ & HEM & 18 & -0.007 & 17.622 & 7.041 & 1.00 & 0.00 & C \\
\hline ATOM & 198 & $\mathrm{C} 10$ & HEM & 18 & -0.312 & 18.357 & 5.897 & 1.00 & 0.00 & C \\
\hline ATOM & 199 & $\mathrm{H} 10$ & HEM & 18 & -1.339 & 18.701 & 5.791 & 1.00 & 0.00 & $\mathrm{H}$ \\
\hline ATOM & 200 & $\mathrm{C} 11$ & HEM & 18 & 0.562 & 18.697 & 4.874 & 1.00 & 0.00 & C \\
\hline ATOM & 201 & $\mathrm{C} 12$ & HEM & 18 & 0.182 & 19.422 & 3.684 & 1.00 & 0.00 & C \\
\hline ATOM & 202 & $\mathrm{C} 13$ & HEM & 18 & 1.321 & 19.569 & 2.927 & 1.00 & 0.00 & C \\
\hline ATOM & 203 & C14 & HEM & 18 & 2.394 & 18.928 & 3.659 & 1.00 & 0.00 & C \\
\hline ATOM & 204 & C15 & HEM & 18 & 3.722 & 18.873 & 3.237 & 1.00 & 0.00 & C \\
\hline ATOM & 205 & H15 & HEM & 18 & 3.962 & 19.358 & 2.289 & 1.00 & 0.00 & $\mathrm{H}$ \\
\hline ATOM & 206 & C16 & HEM & 18 & 4.752 & 18.192 & 3.880 & 1.00 & 0.00 & C \\
\hline ATOM & 207 & $\mathrm{C} 17$ & HEM & 18 & 6.118 & 18.078 & 3.398 & 1.00 & 0.00 & C \\
\hline ATOM & 208 & $\mathrm{C} 18$ & HEM & 18 & 6.793 & 17.310 & 4.322 & 1.00 & 0.00 & C \\
\hline ATOM & 209 & C19 & HEM & 18 & 5.844 & 16.959 & 5.358 & 1.00 & 0.00 & C \\
\hline ATOM & 210 & $\mathrm{C} 20$ & HEM & 18 & 6.135 & 16.168 & 6.469 & 1.00 & 0.00 & C \\
\hline ATOM & 211 & $\mathrm{H} 2 \mathrm{O}$ & HEM & 18 & 7.149 & 15.778 & 6.530 & 1.00 & 0.00 & $\mathrm{H}$ \\
\hline ATOM & 212 & $\mathrm{~N} 21$ & HEM & 18 & 3.945 & 16.181 & 7.564 & 1.00 & 0.00 & $\mathrm{~N}$ \\
\hline ATOM & 213 & N22 & HEM & 18 & 1.240 & 17.128 & 7.342 & 1.00 & 0.00 & $\mathrm{~N}$ \\
\hline ATOM & 214 & N23 & HEM & 18 & 1.911 & 18.391 & 4.841 & 1.00 & 0.00 & $\mathrm{~N}$ \\
\hline ATOM & 215 & N24 & HEM & 18 & 4.611 & 17.506 & 5.077 & 1.00 & 0.00 & $\mathrm{~N}$ \\
\hline ATOM & 216 & C99 & HEM & 18 & 3.704 & 20.371 & 7.579 & 1.00 & 0.00 & C \\
\hline ATOM & 217 & 091 & HEM & 18 & 3.544 & 19.058 & 7.567 & 1.00 & 0.00 & O \\
\hline ATOM & 218 & 092 & HEM & 18 & 3.859 & 20.989 & 8.635 & 1.00 & 0.00 & O \\
\hline ATOM & 219 & СТ91 & HEM & 18 & 3.688 & 21.053 & 6.223 & 1.00 & 0.00 & C \\
\hline ATOM & 220 & H911 & HEM & 18 & 2.709 & 20.858 & 5.760 & 1.00 & 0.00 & $\mathrm{H}$ \\
\hline $\mathrm{ATOM}$ & 221 & H912 & HEM & 18 & 4.436 & 20.563 & 5.580 & 1.00 & 0.00 & $\mathrm{H}$ \\
\hline ATOM & 222 & СТ92 & HEM & 18 & 3.947 & 22.557 & 6.334 & 1.00 & 0.00 & C \\
\hline ATOM & 223 & H921 & HEM & 18 & 4.872 & 22.713 & 6.905 & 1.00 & 0.00 & $\mathrm{H}$ \\
\hline ATOM & 224 & H922 & HEM & 18 & 3.151 & 23.018 & 6.931 & 1.00 & 0.00 & $\mathrm{H}$ \\
\hline \multicolumn{11}{|l|}{ TER } \\
\hline ATOM & 225 & $\mathrm{C} 2$ & VIN & 19 & -1.572 & 28.269 & -1.002 & 1.00 & 0.00 & C \\
\hline ATOM & 226 & $\mathrm{H} 21$ & VIN & 19 & -2.388 & 27.571 & -0.837 & 1.00 & 0.00 & $\mathrm{H}$ \\
\hline ATOM & 227 & $\mathrm{C} 1$ & VIN & 19 & -1.905 & 29.494 & -1.403 & 1.00 & 0.00 & C \\
\hline ATOM & 228 & $\mathrm{H} 11$ & VIN & 19 & -1.174 & 30.273 & -1.511 & 1.00 & 0.00 & $\mathrm{H}$ \\
\hline ATOM & 229 & $\mathrm{H} 12$ & VIN & 19 & -2.947 & 29.763 & -1.570 & 1.00 & 0.00 & $\mathrm{H}$ \\
\hline \multicolumn{11}{|l|}{ TER } \\
\hline ATOM & 230 & $\mathrm{C} 1$ & MET & 20 & 1.091 & 28.524 & -2.840 & 1.00 & 0.00 & $\mathrm{C}$ \\
\hline ATOM & 231 & $\mathrm{H} 11$ & $\mathrm{MET}$ & 20 & 1.326 & 29.595 & -2.720 & 1.00 & 0.00 & $\mathrm{H}$ \\
\hline ATOM & 232 & $\mathrm{H} 12$ & $\mathrm{MET}$ & 20 & 1.904 & 28.060 & -3.410 & 1.00 & 0.00 & $\mathrm{H}$ \\
\hline ATOM & 233 & $\mathrm{H} 13$ & MET & 20 & 0.184 & 28.446 & -3.450 & 1.00 & 0.00 & $\mathrm{H}$ \\
\hline \multicolumn{11}{|l|}{ TER } \\
\hline ATOM & 234 & $\mathrm{C} 2$ & VIN & 21 & 6.102 & 26.846 & -2.325 & 1.00 & 0.00 & \\
\hline ATOM & 235 & $\mathrm{H} 21$ & VIN & 21 & 5.436 & 26.745 & -3.184 & 1.00 & 0.00 & \\
\hline ATOM & 236 & $\mathrm{C} 1$ & VIN & 21 & 7.268 & 27.490 & -2.484 & 1.00 & 0.00 & \\
\hline
\end{tabular}




\begin{tabular}{|c|c|c|c|c|c|c|c|c|c|c|}
\hline ATOM & 237 & $\mathrm{H} 11$ & VIN & 21 & 7.948 & 27.647 & -1.654 & 1.00 & 0.00 & $\mathrm{H}$ \\
\hline ATOM & 238 & $\mathrm{H} 12$ & VIN & 21 & 7.573 & 27.888 & -3.450 & 1.00 & 0.00 & $\mathrm{H}$ \\
\hline \multicolumn{10}{|l|}{ TER } & \\
\hline ATOM & 239 & $\mathrm{C} 1$ & MET & 22 & 7.786 & 25.163 & -0.121 & 1.00 & 0.00 & $\mathrm{C}$ \\
\hline ATOM & 240 & H11 & $\mathrm{MET}$ & 22 & 8.377 & 25.827 & 0.528 & 1.00 & 0.00 & $\mathrm{H}$ \\
\hline ATOM & 241 & $\mathrm{H} 12$ & $\mathrm{MET}$ & 22 & 7.943 & 24.141 & 0.246 & 1.00 & 0.00 & $\mathrm{H}$ \\
\hline ATOM & 242 & H13 & MET & 22 & 8.218 & 25.228 & -1.126 & 1.00 & 0.00 & $\mathrm{H}$ \\
\hline \multicolumn{10}{|l|}{ TER } & \\
\hline ATOM & 243 & C5 & $\mathrm{ACP}$ & 23 & -1.302 & 24.081 & 5.770 & 1.00 & 0.00 & C \\
\hline ATOM & 244 & H51 & $\mathrm{ACP}$ & 23 & -0.563 & 24.070 & 6.582 & 1.00 & 0.00 & 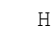 \\
\hline ATOM & 245 & H52 & $\mathrm{ACP}$ & 23 & -2.143 & 24.689 & 6.134 & 1.00 & 0.00 & 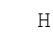 \\
\hline ATOM & 246 & $\mathrm{C} 4$ & $\mathrm{ACP}$ & 23 & -1.781 & 22.644 & 5.497 & 1.00 & 0.00 & 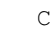 \\
\hline ATOM & 247 & H 41 & $\mathrm{ACP}$ & 23 & -2.338 & 22.583 & 4.549 & 1.00 & 0.00 & $\mathrm{H}$ \\
\hline ATOM & 248 & H 42 & $\mathrm{ACP}$ & 23 & -0.910 & 21.977 & 5.390 & 1.00 & 0.00 & $\mathrm{H}$ \\
\hline ATOM & 249 & $\mathrm{C} 1$ & $\mathrm{ACP}$ & 23 & -2.645 & 22.031 & 6.573 & 1.00 & 0.00 & C \\
\hline ATOM & 250 & $\mathrm{O} 2$ & $\mathrm{ACP}$ & 23 & -2.873 & 22.554 & 7.671 & 1.00 & 0.00 & 0 \\
\hline ATOM & 251 & 03 & $\mathrm{ACP}$ & 23 & -3.161 & 20.852 & 6.209 & 1.00 & 0.00 & o \\
\hline ATOM & 252 & H3 & $\mathrm{ACP}$ & 23 & -3.770 & 20.498 & 6.945 & 1.00 & 0.00 & 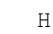 \\
\hline \multicolumn{11}{|l|}{ TER } \\
\hline ATOM & 253 & $\mathrm{C} 1$ & $\mathrm{MET}$ & 24 & 6.594 & 22.955 & 4.597 & 1.00 & 0.00 & $C$ \\
\hline ATOM & 254 & $\mathrm{H} 11$ & MET & 24 & 7.146 & 23.794 & 5.047 & 1.00 & 0.00 & $\mathrm{H}$ \\
\hline ATOM & 255 & $\mathrm{H} 12$ & MET & 24 & 6.600 & 22.129 & 5.319 & 1.00 & 0.00 & $\mathrm{H}$ \\
\hline ATOM & 256 & $\mathrm{H} 13$ & MET & 24 & 7.165 & 22.635 & 3.717 & 1.00 & 0.00 & $\mathrm{H}$ \\
\hline \multicolumn{11}{|l|}{ TER } \\
\hline ATOM & 257 & $\mathrm{C} 1$ & MET & 25 & -2.840 & 25.904 & 3.646 & 1.00 & 0.00 & C \\
\hline ATOM & 258 & $\mathrm{H} 11$ & MET & 25 & -3.285 & 25.695 & 4.626 & 1.00 & 0.00 & $\mathrm{H}$ \\
\hline ATOM & 259 & $\mathrm{H} 12$ & $\mathrm{MET}$ & 25 & -2.950 & 26.984 & 3.471 & 1.00 & 0.00 & $\mathrm{H}$ \\
\hline ATOM & 260 & $\mathrm{H} 13$ & MET & 25 & -3.440 & 25.378 & 2.889 & 1.00 & 0.00 & $\mathrm{H}$ \\
\hline TER & & & & & & & & & & \\
\hline ATOM & 261 & $\mathrm{C} 2$ & VIN & 26 & 6.957 & 14.525 & 8.933 & 1.00 & 0.00 & $\mathrm{C}$ \\
\hline ATOM & 262 & H 21 & VIN & 26 & 7.773 & 15.223 & 8.768 & 1.00 & 0.00 & $\mathrm{H}$ \\
\hline ATOM & 263 & $\mathrm{C} 1$ & VIN & 26 & 7.290 & 13.300 & 9.335 & 1.00 & 0.00 & $\mathrm{C}$ \\
\hline ATOM & 264 & $\mathrm{H} 11$ & VIN & 26 & 6.559 & 12.521 & 9.442 & 1.00 & 0.00 & $\mathrm{H}$ \\
\hline ATOM & 265 & $\mathrm{H} 12$ & VIN & 26 & 8.332 & 13.030 & 9.501 & 1.00 & 0.00 & $H$ \\
\hline TER & & & & & & & & & & \\
\hline ATOM & 266 & $\mathrm{C} 1$ & $\mathrm{MET}$ & 27 & 4.293 & 14.270 & 10.771 & 1.00 & 0.00 & C \\
\hline ATOM & 267 & H11 & $\mathrm{MET}$ & 27 & 4.059 & 13.199 & 10.652 & 1.00 & 0.00 & $\mathrm{H}$ \\
\hline ATOM & 268 & $\mathrm{H} 12$ & MET & 27 & 3.480 & 14.734 & 11.341 & 1.00 & 0.00 & $\mathrm{H}$ \\
\hline ATOM & 269 & $\mathrm{H} 13$ & MET & 27 & 5.201 & 14.347 & 11.382 & 1.00 & 0.00 & $\mathrm{H}$ \\
\hline TER & & & & & & & & & & \\
\hline ATOM & 270 & $\mathrm{C} 2$ & VIN & 28 & -0.717 & 15.948 & 10.256 & 1.00 & 0.00 & C \\
\hline ATOM & 271 & $\mathrm{H} 21$ & VIN & 28 & -0.051 & 16.048 & 11.115 & 1.00 & 0.00 & $\Pi$ \\
\hline ATOM & 272 & $\mathrm{C} 1$ & VIN & 28 & -1.883 & 15.303 & 10.415 & 1.00 & 0.00 & $\mathrm{C}$ \\
\hline ATOM & 273 & H11 & VIN & 28 & -2.563 & 15.147 & 9.585 & 1.00 & 0.00 & $\mathrm{H}$ \\
\hline ATOM & 274 & H12 & VIN & 28 & -2.188 & 14.905 & 11.381 & 1.00 & 0.00 & $\mathrm{H}$ \\
\hline TER & & & & & & & & & & \\
\hline ATOM & 275 & $\mathrm{C} 1$ & MET & 29 & -2.401 & 17.631 & 8.052 & 1.00 & 0.00 & $\mathrm{C}$ \\
\hline ATOM & 276 & $\mathrm{H} 11$ & MET & 29 & -2.558 & 18.653 & 7.685 & 1.00 & 0.00 & $\mathrm{H}$ \\
\hline ATOM & 277 & $\mathrm{H} 12$ & MET & 29 & -2.834 & 17.565 & 9.057 & 1.00 & 0.00 & $\mathrm{H}$ \\
\hline ATOM & 278 & $\mathrm{H} 13$ & MET & 29 & -2.993 & 16.967 & 7.403 & 1.00 & 0.00 & $\mathrm{H}$ \\
\hline TER & & & & & & & & & & \\
\hline ATOM & 279 & $\mathrm{C} 5$ & $\mathrm{ACP}$ & 30 & 6.687 & 18.712 & 2.161 & 1.00 & 0.00 & C \\
\hline ATOM & 280 & H5 1 & $\mathrm{ACP}$ & 30 & 5.948 & 18.724 & 1.349 & 1.00 & 0.00 & $\mathrm{H}$ \\
\hline ATOM & 281 & H52 & $\mathrm{ACP}$ & 30 & 7.528 & 18.104 & 1.798 & 1.00 & 0.00 & $\mathrm{H}$ \\
\hline ATOM & 282 & $\mathrm{C} 4$ & $\mathrm{ACP}$ & 30 & 7.166 & 20.150 & 2.434 & 1.00 & 0.00 & $\mathrm{C}$ \\
\hline ATOM & 283 & H 41 & $\mathrm{ACP}$ & 30 & 7.723 & 20.211 & 3.382 & 1.00 & 0.00 & 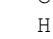 \\
\hline ATOM & 284 & $\mathrm{H} 42$ & $\mathrm{ACP}$ & 30 & 6.295 & 20.817 & 2.541 & 1.00 & 0.00 & $\mathrm{H}$ \\
\hline ATOM & 285 & $\mathrm{C} 1$ & $\mathrm{ACP}$ & 30 & 8.030 & 20.762 & 1.358 & 1.00 & 0.00 & C \\
\hline ATOM & 286 & $\mathrm{O} 2$ & $\mathrm{ACP}$ & 30 & 8.258 & 20.239 & 0.260 & 1.00 & 0.00 & O \\
\hline ATOM & 287 & 03 & $\mathrm{ACP}$ & 30 & 8.546 & 21.942 & 1.722 & 1.00 & 0.00 & 0 \\
\hline ATOM & 288 & $\mathrm{H} 3$ & $\mathrm{ACP}$ & 30 & 9.155 & 22.296 & 0.986 & 1.00 & 0.00 & 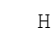 \\
\hline TER & & & & & & & & & & \\
\hline ATOM & 289 & $\mathrm{C} 1$ & MET & 31 & -1.209 & 19.839 & 3.334 & 1.00 & 0.00 & C \\
\hline ATOM & 290 & H11 & MET & 31 & -1.761 & 18.999 & 2.884 & 1.00 & 0.00 & $\mathrm{H}$ \\
\hline ATOM & 291 & $\mathrm{H} 12$ & MET & 31 & -1.215 & 20.665 & 2.613 & 1.00 & 0.00 & $\mathrm{H}$ \\
\hline ATOM & 292 & H13 & $\mathrm{MET}$ & 31 & -1.780 & 20.158 & 4.214 & 1.00 & 0.00 & $\mathrm{H}$ \\
\hline TER & & & & & & & & & & \\
\hline ATOM & 293 & $\mathrm{C} 1$ & $\mathrm{MET}$ & 32 & 8.225 & 16.890 & 4.285 & 1.00 & 0.00 & C \\
\hline ATOM & 294 & $\mathrm{H} 11$ & MET & 32 & 8.670 & 17.099 & 3.305 & 1.00 & 0.00 & $H$ \\
\hline ATOM & 295 & H12 & MET & 32 & 8.335 & 15.810 & 4.460 & 1.00 & 0.00 & $\mathrm{H}$ \\
\hline ATOM & 296 & $\mathrm{H} 13$ & MET & 32 & 8.825 & 17.416 & 5.042 & 1.00 & 0.00 & $\mathrm{H}$ \\
\hline TER & & & & & & & & & & \\
\hline ATOM & 297 & $\mathrm{FE}$ & HEM & 33 & 3.316 & 10.511 & 9.423 & 1.00 & 0.00 & $\mathrm{FE}$ \\
\hline ATOM & 298 & $\mathrm{C} 1$ & HEM & 33 & 1.088 & 12.383 & 8.361 & 1.00 & 0.00 & C \\
\hline ATOM & 299 & $\mathrm{C} 2$ & HEM & 33 & 0.729 & 13.174 & 7.189 & 1.00 & 0.00 & C \\
\hline ATOM & 300 & $\mathrm{C} 3$ & HEM & 33 & 1.874 & 13.271 & 6.414 & 1.00 & 0.00 & $\mathrm{C}$ \\
\hline ATOM & 301 & $\mathrm{C} 4$ & HEM & 33 & 2.904 & 12.553 & 7.122 & 1.00 & 0.00 & 6 \\
\hline ATOM & 302 & $\mathrm{C} 5$ & HEM & 33 & 4.219 & 12.435 & 6.684 & 1.00 & 0.00 & \\
\hline ATOM & 303 & H5 & HEM & 33 & 4.472 & 12.931 & 5.749 & 1.00 & 0.00 & \\
\hline
\end{tabular}




\begin{tabular}{|c|c|c|c|c|c|c|c|c|c|c|}
\hline ATOM & 304 & $\mathrm{C} 6$ & HEM & 33 & 5.238 & 11.736 & 7.320 & 1.00 & 0.00 & C \\
\hline ATOM & 305 & $\mathrm{C} 7$ & HEM & 33 & 6.607 & 11.664 & 6.851 & 1.00 & 0.00 & C \\
\hline ATOM & 306 & $\mathrm{C} 8$ & HEM & 33 & 7.313 & 10.944 & 7.798 & 1.00 & 0.00 & C \\
\hline ATOM & 307 & C9 & HEM & 33 & 6.362 & 10.579 & 8.822 & 1.00 & 0.00 & C \\
\hline ATOM & 308 & $\mathrm{C} 10$ & HEM & 33 & 6.666 & 9.844 & 9.965 & 1.00 & 0.00 & C \\
\hline ATOM & 309 & $\mathrm{H} 10$ & HEM & 33 & 7.693 & 9.500 & 10.071 & 1.00 & 0.00 & $\mathrm{H}$ \\
\hline ATOM & 310 & C11 & HEM & 33 & 5.792 & 9.504 & 10.988 & 1.00 & 0.00 & C \\
\hline ATOM & 311 & $\mathrm{C} 12$ & HEM & 33 & 6.173 & 8.779 & 12.178 & 1.00 & 0.00 & $\mathrm{C}$ \\
\hline ATOM & 312 & C13 & HEM & 33 & 5.033 & 8.631 & 12.935 & 1.00 & 0.00 & C \\
\hline ATOM & 313 & C14 & HEM & 33 & 3.960 & 9.273 & 12.204 & 1.00 & 0.00 & C \\
\hline ATOM & 314 & C15 & HEM & 33 & 2.632 & 9.328 & 12.625 & 1.00 & 0.00 & $\mathrm{C}$ \\
\hline ATOM & 315 & H15 & HEM & 33 & 2.392 & 8.843 & 13.573 & 1.00 & 0.00 & $\mathrm{H}$ \\
\hline ATOM & 316 & C16 & HEM & 33 & 1.602 & 10.009 & 11.982 & 1.00 & 0.00 & C \\
\hline ATOM & 317 & C17 & HEM & 33 & 0.236 & 10.123 & 12.464 & 1.00 & 0.00 & C \\
\hline ATOM & 318 & C18 & HEM & 33 & -0.439 & 10.890 & 11.541 & 1.00 & 0.00 & C \\
\hline ATOM & 319 & C19 & HEM & 33 & 0.510 & 11.242 & 10.504 & 1.00 & 0.00 & C \\
\hline ATOM & 320 & $\mathrm{C} 20$ & HEM & 33 & 0.219 & 12.033 & 9.393 & 1.00 & 0.00 & $\mathrm{C}$ \\
\hline ATOM & 321 & $\mathrm{H} 20$ & HEM & 33 & -0.795 & 12.423 & 9.333 & 1.00 & 0.00 & $\mathrm{H}$ \\
\hline ATOM & 322 & N21 & HEM & 33 & 2.409 & 12.020 & 8.298 & 1.00 & 0.00 & $\mathrm{~N}$ \\
\hline ATOM & 323 & N22 & HEM & 33 & 5.114 & 11.073 & 8.521 & 1.00 & 0.00 & $\mathrm{~N}$ \\
\hline ATOM & 324 & N23 & HEM & 33 & 4.443 & 9.810 & 11.021 & 1.00 & 0.00 & $\mathrm{~N}$ \\
\hline ATOM & 325 & N24 & HEM & 33 & 1.743 & 10.694 & 10.786 & 1.00 & 0.00 & $\mathrm{~N}$ \\
\hline ATOM & 326 & C99 & HEM & 33 & 2.650 & 7.829 & 8.283 & 1.00 & 0.00 & $\mathrm{C}$ \\
\hline ATOM & 327 & 091 & HEM & 33 & 2.810 & 9.143 & 8.296 & 1.00 & 0.00 & O \\
\hline ATOM & 328 & 092 & HEM & 33 & 2.495 & 7.212 & 7.228 & 1.00 & 0.00 & 0 \\
\hline ATOM & 329 & СТ 91 & HEM & 33 & 2.666 & 7.148 & 9.640 & 1.00 & 0.00 & $\mathrm{C}$ \\
\hline ATOM & 330 & H911 & HEM & 33 & 3.645 & 7.343 & 10.103 & 1.00 & 0.00 & $\mathrm{H}$ \\
\hline ATOM & 331 & H912 & HEM & 33 & 1.918 & 7.638 & 10.283 & 1.00 & 0.00 & $\mathrm{H}$ \\
\hline ATOM & 332 & СТ 92 & HEM & 33 & 2.407 & 5.644 & 9.528 & 1.00 & 0.00 & $\mathrm{C}$ \\
\hline ATOM & 333 & H921 & HEM & 33 & 3.203 & 5.183 & 8.931 & 1.00 & 0.00 & $\mathrm{H}$ \\
\hline ATOM & 334 & H922 & HEM & 33 & 1.482 & 5.488 & 8.958 & 1.00 & 0.00 & $\mathrm{H}$ \\
\hline \multicolumn{11}{|l|}{ TER } \\
\hline ATOM & 335 & $\mathrm{FE}$ & HEM & 34 & 4.008 & 3.097 & 14.371 & 1.00 & 0.00 & $\mathrm{FE}$ \\
\hline ATOM & 336 & $\mathrm{C} 1$ & HEM & 34 & 6.236 & 1.225 & 15.433 & 1.00 & 0.00 & C \\
\hline ATOM & 337 & $\mathrm{C} 2$ & HEM & 34 & 6.595 & 0.434 & 16.605 & 1.00 & 0.00 & C \\
\hline ATOM & 338 & $\mathrm{C} 3$ & HEM & 34 & 5.449 & 0.337 & 17.380 & 1.00 & 0.00 & C \\
\hline ATOM & 339 & $\mathrm{C} 4$ & HEM & 34 & 4.419 & 1.055 & 16.672 & 1.00 & 0.00 & C \\
\hline ATOM & 340 & C5 & HEM & 34 & 3.104 & 1.173 & 17.109 & 1.00 & 0.00 & C \\
\hline ATOM & 341 & H5 & HEM & 34 & 2.851 & 0.677 & 18.045 & 1.00 & 0.00 & $\mathrm{H}$ \\
\hline ATOM & 342 & $\mathrm{C} 6$ & HEM & 34 & 2.085 & 1.872 & 16.473 & 1.00 & 0.00 & $\mathrm{C}$ \\
\hline ATOM & 343 & $\mathrm{C} 7$ & HEM & 34 & 0.716 & 1.944 & 16.943 & 1.00 & 0.00 & C \\
\hline ATOM & 344 & $\mathrm{C} 8$ & HEM & 34 & 0.011 & 2.664 & 15.996 & 1.00 & 0.00 & $\mathrm{C}$ \\
\hline ATOM & 345 & $\mathrm{C} 9$ & HEM & 34 & 0.962 & 3.029 & 14.972 & 1.00 & 0.00 & $\mathrm{C}$ \\
\hline ATOM & 346 & $\mathrm{C} 10$ & HEM & 34 & 0.657 & 3.764 & 13.828 & 1.00 & 0.00 & C \\
\hline ATOM & 347 & $\mathrm{H} 10$ & HEM & 34 & -0.370 & 4.108 & 13.722 & 1.00 & 0.00 & $\mathrm{H}$ \\
\hline ATOM & 348 & $\mathrm{C} 11$ & HEM & 34 & 1.532 & 4.104 & 12.806 & 1.00 & 0.00 & C \\
\hline ATOM & 349 & $\mathrm{C} 12$ & HEM & 34 & 1.151 & 4.829 & 11.615 & 1.00 & 0.00 & $\mathrm{C}$ \\
\hline ATOM & 350 & $\mathrm{C} 13$ & HEM & 34 & 2.290 & 4.977 & 10.858 & 1.00 & 0.00 & $\mathrm{C}$ \\
\hline ATOM & 351 & C14 & HEM & 34 & 3.363 & 4.335 & 11.590 & 1.00 & 0.00 & $\mathrm{C}$ \\
\hline ATOM & 352 & C15 & HEM & 34 & 4.691 & 4.280 & 11.168 & 1.00 & 0.00 & C \\
\hline ATOM & 353 & H15 & HEM & 34 & 4.931 & 4.765 & 10.221 & 1.00 & 0.00 & $\mathrm{H}$ \\
\hline ATOM & 354 & $\mathrm{C} 16$ & HEM & 34 & 5.721 & 3.599 & 11.812 & 1.00 & 0.00 & $\mathrm{C}$ \\
\hline ATOM & 355 & $\mathrm{C} 17$ & HEM & 34 & 7.087 & 3.485 & 11.329 & 1.00 & 0.00 & $\mathrm{C}$ \\
\hline ATOM & 356 & C18 & HEM & 34 & 7.763 & 2.718 & 12.253 & 1.00 & 0.00 & C \\
\hline ATOM & 357 & C19 & HEM & 34 & 6.813 & 2.366 & 13.289 & 1.00 & 0.00 & C \\
\hline ATOM & 358 & $\mathrm{C} 20$ & HEM & 34 & 7.104 & 1.575 & 14.401 & 1.00 & 0.00 & C \\
\hline ATOM & 359 & $\mathrm{H} 2 \mathrm{O}$ & HEM & 34 & 8.118 & 1.185 & 14.461 & 1.00 & 0.00 & $\mathrm{H}$ \\
\hline ATOM & 360 & N21 & HEM & 34 & 4.914 & 1.588 & 15.495 & 1.00 & 0.00 & $\mathrm{~N}$ \\
\hline ATOM & 361 & N22 & HEM & 34 & 2.209 & 2.535 & 15.273 & 1.00 & 0.00 & $\mathrm{~N}$ \\
\hline ATOM & 362 & N23 & HEM & 34 & 2.880 & 3.798 & 12.772 & 1.00 & 0.00 & $\mathrm{~N}$ \\
\hline ATOM & 363 & N24 & HEM & 34 & 5.580 & 2.914 & 13.008 & 1.00 & 0.00 & $\mathrm{~N}$ \\
\hline ATOM & 364 & C99 & HEM & 34 & 4.673 & 5.779 & 15.510 & 1.00 & 0.00 & $\mathrm{C}$ \\
\hline ATOM & 365 & 091 & HEM & 34 & 4.514 & 4.465 & 15.498 & 1.00 & 0.00 & 0 \\
\hline ATOM & 366 & 092 & HEM & 34 & 4.828 & 6.396 & 16.566 & 1.00 & 0.00 & 0 \\
\hline ATOM & 367 & СТ91 & HEM & 34 & 4.657 & 6.460 & 14.154 & 1.00 & 0.00 & C \\
\hline ATOM & 368 & H911 & HEM & 34 & 3.678 & 6.265 & 13.691 & 1.00 & 0.00 & $\mathrm{H}$ \\
\hline ATOM & 369 & H912 & HEM & 34 & 5.405 & 5.970 & 13.511 & 1.00 & 0.00 & $\mathrm{H}$ \\
\hline ATOM & 370 & CT 92 & HEM & 34 & 4.917 & 7.964 & 14.265 & 1.00 & 0.00 & $\mathrm{C}$ \\
\hline ATOM & 371 & H921 & HEM & 34 & 5.842 & 8.120 & 14.836 & 1.00 & 0.00 & $\mathrm{H}$ \\
\hline ATOM & 372 & H922 & HEM & 34 & 4.120 & 8.425 & 14.862 & 1.00 & 0.00 & $\mathrm{H}$ \\
\hline \multicolumn{11}{|l|}{ TER } \\
\hline ATOM & 373 & $\mathrm{C} 2$ & VIN & 35 & -0.603 & 13.676 & 6.929 & 1.00 & 0.00 & $\mathrm{C}$ \\
\hline ATOM & 374 & H21 & VIN & 35 & -1.419 & 12.978 & 7.094 & 1.00 & 0.00 & $\mathrm{H}$ \\
\hline ATOM & 375 & $\mathrm{C} 1$ & VIN & 35 & -0.936 & 14.901 & 6.528 & 1.00 & 0.00 & $\mathrm{C}$ \\
\hline ATOM & 376 & $\mathrm{H} 11$ & VIN & 35 & -0.205 & 15.680 & 6.421 & 1.00 & 0.00 & $\mathrm{H}$ \\
\hline ATOM & 377 & $\mathrm{H} 12$ & VIN & 35 & -1.978 & 15.170 & 6.361 & 1.00 & 0.00 & $\mathrm{H}$ \\
\hline \multicolumn{11}{|l|}{ TER } \\
\hline ATOM & 378 & $\mathrm{C} 1$ & MET & 36 & 2.061 & 13.931 & 5.091 & 1.00 & 0.00 & \\
\hline ATOM & 379 & H11 & MET & 36 & 2.295 & 15.002 & 5.211 & 1.00 & 0.00 & \\
\hline
\end{tabular}




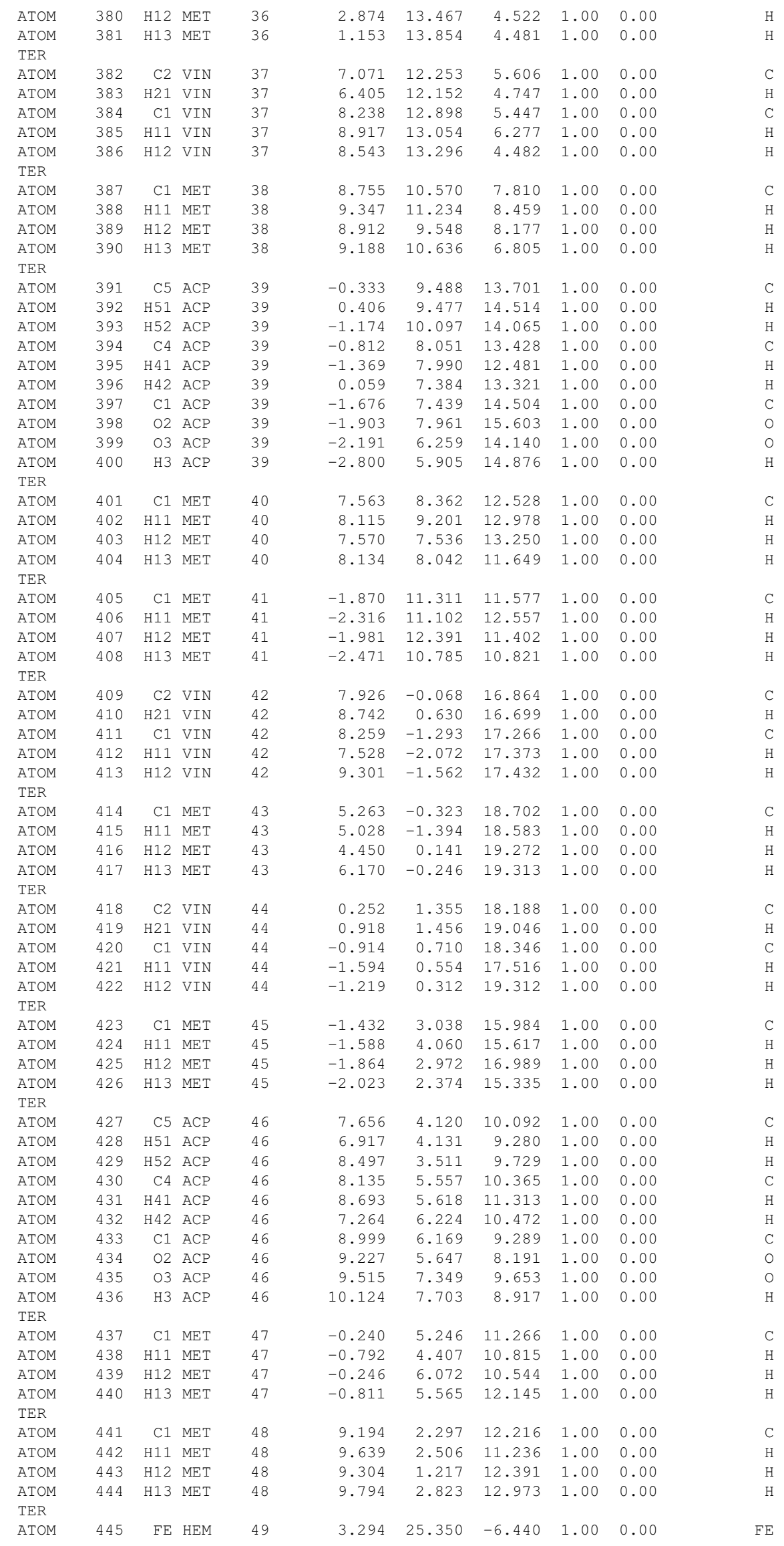




\begin{tabular}{|c|c|c|c|c|c|c|c|c|c|c|}
\hline ATOM & 446 & $\mathrm{C} 1$ & HEM & 49 & 1.066 & 27.222 & -7.502 & 1.00 & 0.00 & C \\
\hline ATOM & 447 & C2 & HEM & 49 & 0.707 & 28.013 & -8.674 & 1.00 & 0.00 & C \\
\hline ATOM & 448 & $\mathrm{C} 3$ & HEM & 49 & 1.853 & 28.110 & -9.448 & 1.00 & 0.00 & C \\
\hline ATOM & 449 & $\mathrm{C} 4$ & HEM & 49 & 2.882 & 27.392 & -8.740 & 1.00 & 0.00 & $\mathrm{C}$ \\
\hline ATOM & 450 & C5 & HEM & 49 & 4.197 & 27.274 & -9.178 & 1.00 & 0.00 & C \\
\hline ATOM & 451 & H5 & HEM & 49 & 4.451 & 27.770 & -10.113 & 1.00 & 0.00 & $\mathrm{H}$ \\
\hline ATOM & 452 & C6 & HEM & 49 & 5.217 & 26.575 & -8.542 & 1.00 & 0.00 & C \\
\hline ATOM & 453 & $\mathrm{C} 7$ & HEM & 49 & 6.586 & 26.503 & -9.012 & 1.00 & 0.00 & $\mathrm{C}$ \\
\hline ATOM & 454 & $\mathrm{C} 8$ & HEM & 49 & 7.291 & 25.783 & -8.065 & 1.00 & 0.00 & C \\
\hline ATOM & 455 & $\mathrm{C} 9$ & HEM & 49 & 6.340 & 25.418 & -7.041 & 1.00 & 0.00 & C \\
\hline ATOM & 456 & $\mathrm{C} 10$ & HEM & 49 & 6.645 & 24.683 & -5.897 & 1.00 & 0.00 & $\mathrm{C}$ \\
\hline ATOM & 457 & $\mathrm{H} 10$ & HEM & 49 & 7.671 & 24.339 & -5.791 & 1.00 & 0.00 & $\mathrm{H}$ \\
\hline ATOM & 458 & C11 & HEM & 49 & 5.770 & 24.343 & -4.874 & 1.00 & 0.00 & C \\
\hline ATOM & 459 & $\mathrm{C} 12$ & HEM & 49 & 6.151 & 23.618 & -3.684 & 1.00 & 0.00 & C \\
\hline ATOM & 460 & $\mathrm{C} 13$ & HEM & 49 & 5.012 & 23.470 & -2.927 & 1.00 & 0.00 & C \\
\hline ATOM & 461 & C14 & HEM & 49 & 3.939 & 24.112 & -3.659 & 1.00 & 0.00 & C \\
\hline ATOM & 462 & C15 & HEM & 49 & 2.611 & 24.167 & -3.237 & 1.00 & 0.00 & C \\
\hline ATOM & 463 & $\mathrm{H} 15$ & HEM & 49 & 2.370 & 23.682 & -2.289 & 1.00 & 0.00 & $\mathrm{H}$ \\
\hline ATOM & 464 & $\mathrm{C} 16$ & HEM & 49 & 1.580 & 24.848 & -3.880 & 1.00 & 0.00 & $\mathrm{C}$ \\
\hline ATOM & 465 & $\mathrm{C} 17$ & HEM & 49 & 0.215 & 24.962 & -3.398 & 1.00 & 0.00 & $\mathrm{C}$ \\
\hline ATOM & 466 & C18 & HEM & 49 & -0.461 & 25.729 & -4.322 & 1.00 & 0.00 & C \\
\hline ATOM & 467 & $\mathrm{C} 19$ & HEM & 49 & 0.489 & 26.081 & -5.358 & 1.00 & 0.00 & C \\
\hline ATOM & 468 & $\mathrm{C} 20$ & HEM & 49 & 0.197 & 26.872 & -6.469 & 1.00 & 0.00 & $\mathrm{C}$ \\
\hline ATOM & 469 & $\mathrm{H} 2 \mathrm{O}$ & HEM & 49 & -0.817 & 27.262 & -6.530 & 1.00 & 0.00 & $\mathrm{H}$ \\
\hline ATOM & 470 & N21 & HEM & 49 & 2.388 & 26.859 & -7.564 & 1.00 & 0.00 & $\mathrm{~N}$ \\
\hline ATOM & 471 & N22 & HEM & 49 & 5.092 & 25.912 & -7.342 & 1.00 & 0.00 & $\mathrm{~N}$ \\
\hline ATOM & 472 & N23 & HEM & 49 & 4.422 & 24.649 & -4.841 & 1.00 & 0.00 & $\mathrm{~N}$ \\
\hline ATOM & 473 & N24 & HEM & 49 & 1.722 & 25.533 & -5.077 & 1.00 & 0.00 & $\mathrm{~N}$ \\
\hline ATOM & 474 & C99 & HEM & 49 & 2.629 & 22.668 & -7.579 & 1.00 & 0.00 & $\mathrm{C}$ \\
\hline ATOM & 475 & 091 & HEM & 49 & 2.788 & 23.982 & -7.567 & 1.00 & 0.00 & 0 \\
\hline ATOM & 476 & 092 & HEM & 49 & 2.474 & 22.051 & -8.635 & 1.00 & 0.00 & 0 \\
\hline ATOM & 477 & СТ91 & HEM & 49 & 2.645 & 21.987 & -6.223 & 1.00 & 0.00 & $\mathrm{C}$ \\
\hline ATOM & 478 & H911 & HEM & 49 & 3.623 & 22.182 & -5.760 & 1.00 & 0.00 & $\mathrm{H}$ \\
\hline ATOM & 479 & H912 & HEM & 49 & 1.897 & 22.477 & -5.580 & 1.00 & 0.00 & $\mathrm{H}$ \\
\hline ATOM & 480 & СТ 92 & HEM & 49 & 2.385 & 20.483 & -6.334 & 1.00 & 0.00 & $\mathrm{C}$ \\
\hline ATOM & 481 & H921 & HEM & 49 & 3.181 & 20.022 & -6.931 & 1.00 & 0.00 & $\mathrm{H}$ \\
\hline ATOM & 482 & H922 & HEM & 49 & 1.460 & 20.327 & -6.905 & 1.00 & 0.00 & $\mathrm{H}$ \\
\hline \multicolumn{11}{|l|}{ TER } \\
\hline ATOM & 483 & $\mathrm{FE}$ & HEM & 50 & 3.986 & 17.936 & -1.491 & 1.00 & 0.00 & $\mathrm{FE}$ \\
\hline ATOM & 484 & $\mathrm{C} 1$ & HEM & 50 & 6.214 & 16.064 & -0.429 & 1.00 & 0.00 & C \\
\hline ATOM & 485 & $\mathrm{C} 2$ & HEM & 50 & 6.573 & 15.273 & 0.742 & 1.00 & 0.00 & C \\
\hline ATOM & 486 & $\mathrm{C} 3$ & HEM & 50 & 5.427 & 15.176 & 1.517 & 1.00 & 0.00 & $\mathrm{C}$ \\
\hline ATOM & 487 & $\mathrm{C} 4$ & HEM & 50 & 4.398 & 15.894 & 0.809 & 1.00 & 0.00 & $\mathrm{C}$ \\
\hline ATOM & 488 & $\mathrm{C} 5$ & HEM & 50 & 3.083 & 16.012 & 1.247 & 1.00 & 0.00 & C \\
\hline ATOM & 489 & H5 & HEM & 50 & 2.829 & 15.516 & 2.182 & 1.00 & 0.00 & $\mathrm{H}$ \\
\hline ATOM & 490 & $\mathrm{C} 6$ & HEM & 50 & 2.063 & 16.711 & 0.611 & 1.00 & 0.00 & $\mathrm{C}$ \\
\hline ATOM & 491 & $\mathrm{C} 7$ & HEM & 50 & 0.694 & 16.783 & 1.081 & 1.00 & 0.00 & $\mathrm{C}$ \\
\hline ATOM & 492 & $\mathrm{C} 8$ & HEM & 50 & -0.011 & 17.503 & 0.134 & 1.00 & 0.00 & $\mathrm{C}$ \\
\hline ATOM & 493 & $\mathrm{C} 9$ & HEM & 50 & 0.940 & 17.868 & -0.891 & 1.00 & 0.00 & $\mathrm{C}$ \\
\hline ATOM & 494 & $\mathrm{C} 10$ & HEM & 50 & 0.636 & 18.603 & -2.034 & 1.00 & 0.00 & C \\
\hline ATOM & 495 & $\mathrm{H} 10$ & HEM & 50 & -0.391 & 18.947 & -2.140 & 1.00 & 0.00 & $\mathrm{H}$ \\
\hline ATOM & 496 & $\mathrm{C} 11$ & HEM & 50 & 1.510 & 18.943 & -3.057 & 1.00 & 0.00 & $\mathrm{C}$ \\
\hline ATOM & 497 & $\mathrm{C} 12$ & HEM & 50 & 1.129 & 19.668 & -4.247 & 1.00 & 0.00 & $\mathrm{C}$ \\
\hline ATOM & 498 & $\mathrm{C} 13$ & HEM & 50 & 2.269 & 19.816 & -5.004 & 1.00 & 0.00 & C \\
\hline ATOM & 499 & C14 & HEM & 50 & 3.341 & 19.174 & -4.273 & 1.00 & 0.00 & C \\
\hline ATOM & 500 & C15 & HEM & 50 & 4.670 & 19.119 & -4.694 & 1.00 & 0.00 & C \\
\hline ATOM & 501 & H15 & HEM & 50 & 4.910 & 19.604 & -5.642 & 1.00 & 0.00 & $\mathrm{H}$ \\
\hline ATOM & 502 & $\mathrm{C} 16$ & HEM & 50 & 5.700 & 18.438 & -4.051 & 1.00 & 0.00 & C \\
\hline ATOM & 503 & $\mathrm{C} 17$ & HEM & 50 & 7.066 & 18.324 & -4.533 & 1.00 & 0.00 & $\mathrm{C}$ \\
\hline ATOM & 504 & $\mathrm{C} 18$ & HEM & 50 & 7.741 & 17.557 & -3.609 & 1.00 & 0.00 & $\mathrm{C}$ \\
\hline ATOM & 505 & C19 & HEM & 50 & 6.791 & 17.205 & -2.573 & 1.00 & 0.00 & $\mathrm{C}$ \\
\hline ATOM & 506 & $\mathrm{C} 20$ & HEM & 50 & 7.083 & 16.414 & -1.462 & 1.00 & 0.00 & $\mathrm{C}$ \\
\hline ATOM & 507 & $\mathrm{H} 2 \mathrm{O}$ & HEM & 50 & 8.097 & 16.024 & -1.402 & 1.00 & 0.00 & $\mathrm{H}$ \\
\hline ATOM & 508 & N21 & HEM & 50 & 4.892 & 16.427 & -0.367 & 1.00 & 0.00 & $\mathrm{~N}$ \\
\hline ATOM & 509 & N22 & HEM & 50 & 2.188 & 17.374 & -0.590 & 1.00 & 0.00 & $\mathrm{~N}$ \\
\hline ATOM & 510 & N23 & HEM & 50 & 2.859 & 18.637 & -3.090 & 1.00 & 0.00 & $\mathrm{~N}$ \\
\hline ATOM & 511 & N24 & HEM & 50 & 5.558 & 17.753 & -2.854 & 1.00 & 0.00 & $\mathrm{~N}$ \\
\hline ATOM & 512 & C99 & HEM & 50 & 4.652 & 20.618 & -0.352 & 1.00 & 0.00 & $\mathrm{C}$ \\
\hline ATOM & 513 & 091 & HEM & 50 & 4.492 & 19.304 & -0.365 & 1.00 & 0.00 & 0 \\
\hline ATOM & 514 & 092 & HEM & 50 & 4.806 & 21.235 & 0.703 & 1.00 & 0.00 & 0 \\
\hline ATOM & 515 & СТ91 & HEM & 50 & 4.635 & 21.299 & -1.709 & 1.00 & 0.00 & C \\
\hline ATOM & 516 & H911 & HEM & 50 & 3.657 & 21.104 & -2.171 & 1.00 & 0.00 & $\mathrm{H}$ \\
\hline ATOM & 517 & H912 & HEM & 50 & 5.383 & 20.809 & -2.352 & 1.00 & 0.00 & $\mathrm{H}$ \\
\hline ATOM & 518 & CT 92 & HEM & 50 & 4.895 & 22.803 & -1.597 & 1.00 & 0.00 & $\mathrm{C}$ \\
\hline ATOM & 519 & H921 & HEM & 50 & 5.820 & 22.959 & -1.027 & 1.00 & 0.00 & $\mathrm{H}$ \\
\hline ATOM & 520 & H922 & HEM & 50 & 4.099 & 23.264 & -1.000 & 1.00 & 0.00 & $\mathrm{H}$ \\
\hline \multicolumn{11}{|c|}{$4.03020 .204 \quad 1.000$} \\
\hline ATOM & 521 & $\mathrm{C} 2$ & VIN & 51 & -0.625 & 28.515 & -8.933 & 1.00 & 0.00 & \\
\hline ATOM & 522 & H21 & VIN & 51 & -1.440 & 27.817 & -8.768 & 1.00 & 0.00 & \\
\hline
\end{tabular}




\begin{tabular}{|c|c|c|c|c|c|c|c|c|c|c|}
\hline AтOM & 523 & $\mathrm{C} 1$ & VIN & 51 & -0.958 & 29.740 & -9.335 & 1.00 & 0.00 & C \\
\hline ATOM & 524 & $\mathrm{H} 11$ & VIN & 51 & -0.226 & 30.519 & -9.442 & 1.00 & 0.00 & $\mathrm{H}$ \\
\hline ATOM & 525 & $\mathrm{H} 12$ & VIN & 51 & -1.999 & 30.010 & -9.501 & 1.00 & 0.00 & 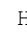 \\
\hline \multicolumn{10}{|l|}{ TER } & \\
\hline ATOM & 526 & $\mathrm{C} 1$ & $\mathrm{MET}$ & 52 & 2.039 & 28.770 & -10.771 & 1.00 & 0.00 & c \\
\hline AтOM & 527 & $\mathrm{H} 11$ & MET & 52 & 2.274 & 29.841 & -10.652 & 1.00 & 0.00 & $\mathrm{H}$ \\
\hline ATOM & 528 & $\mathrm{H} 12$ & MET & 52 & 2.852 & 28.306 & -11.341 & 1.00 & 0.00 & 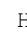 \\
\hline ATOM & 529 & $\mathrm{H} 13$ & MET & 52 & 1.131 & 28.693 & -11.382 & 1.00 & 0.00 & \\
\hline \multicolumn{10}{|l|}{ TER } & \\
\hline ATOM & 530 & $\mathrm{C} 2$ & VIN & 53 & 7.049 & 27.092 & -10.256 & 1.00 & 0.00 & c \\
\hline ATOM & 531 & H 21 & VIN & 53 & 6.383 & 26.991 & -11.115 & 1.00 & 0.00 & 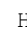 \\
\hline АTOM & 532 & $\mathrm{C} 1$ & VIN & 53 & 8.216 & 27.737 & -10.415 & 1.00 & 0.00 & \\
\hline ATOM & 533 & $\mathrm{H} 11$ & VIN & 53 & 8.896 & 27.893 & -9.585 & 1.00 & 0.00 & \\
\hline ATOM & 534 & $\mathrm{H} 12$ & VIN & 53 & 8.521 & 28.135 & -11.381 & 1.00 & 0.00 & \\
\hline \multicolumn{10}{|l|}{ TER } & \\
\hline ATOM & 535 & $\mathrm{C} 1$ & $\mathrm{MET}$ & 54 & 8.734 & 25.409 & -8.052 & 1.00 & 0.00 & \\
\hline ATOM & 536 & $\mathrm{H} 11$ & $\mathrm{MET}$ & 54 & 9.325 & 26.073 & -7.403 & 1.00 & 0.00 & \\
\hline ATOM & 537 & $\mathrm{H} 12$ & MET & 54 & 8.890 & 24.387 & -7.685 & 1.00 & 0.00 & 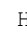 \\
\hline ATOM & 538 & H13 & MET & 54 & 9.166 & 25.475 & -9.057 & 1.00 & 0.00 & \\
\hline \multicolumn{10}{|l|}{ TER } & \\
\hline ATOM & 539 & C5 & $\mathrm{ACP}$ & 55 & -0.355 & 24.327 & -2.161 & 1.00 & 0.00 & c \\
\hline ATOM & 540 & H51 & $\mathrm{ACP}$ & 55 & 0.384 & 24.316 & -1.349 & 1.00 & 0.00 & 8 \\
\hline ATOM & 541 & H5 2 & $\mathrm{ACP}$ & 55 & -1.195 & 24.936 & -1.798 & 1.00 & 0.00 & . \\
\hline ATOM & 542 & $\mathrm{C} 4$ & $\mathrm{ACP}$ & 55 & -0.833 & 22.890 & -2.434 & 1.00 & 0.00 & o \\
\hline AтOM & 543 & $\mathrm{H} 41$ & $\mathrm{ACP}$ & 55 & -1.391 & 22.829 & -3.382 & 1.00 & 0.00 & $\mathrm{H}$ \\
\hline ATOM & 544 & $\mathrm{H} 42$ & $\mathrm{ACP}$ & 55 & 0.037 & 22.223 & -2.541 & 1.00 & 0.00 & $\mathrm{H}$ \\
\hline ATOM & 545 & $\mathrm{C} 1$ & $\mathrm{ACP}$ & 55 & -1.697 & 22.278 & -1.358 & 1.00 & 0.00 & C \\
\hline AтOM & 546 & $\mathrm{O} 2$ & $\mathrm{ACP}$ & 55 & -1.925 & 22.800 & -0.260 & 1.00 & 0.00 & 0 \\
\hline ATOM & 547 & 03 & $\mathrm{ACP}$ & 55 & -2.213 & 21.098 & -1.722 & 1.00 & 0.00 & o \\
\hline ATOM & 548 & $\mathrm{H} 3$ & $\mathrm{ACP}$ & 55 & -2.822 & 20.744 & -0.986 & 1.00 & 0.00 & \\
\hline \multicolumn{10}{|l|}{ TER } & \\
\hline ATOM & 549 & $\mathrm{C} 1$ & MET & 56 & 7.541 & 23.201 & -3.334 & 1.00 & 0.00 & \\
\hline ATOM & 550 & $\mathrm{H} 11$ & MET & 56 & 8.094 & 24.040 & -2.884 & 1.00 & 0.00 & $\mathrm{H}$ \\
\hline AтOM & 551 & $\mathrm{H} 12$ & MET & 56 & 7.548 & 22.375 & -2.613 & 1.00 & 0.00 & $\mathrm{H}$ \\
\hline ATOM & 552 & $\mathrm{H} 13$ & MET & 56 & 8.112 & 22.882 & -4.214 & 1.00 & 0.00 & \\
\hline TER & & & & & & & & & & \\
\hline ATOM & 553 & $\mathrm{C} 1$ & MET & 57 & -1.892 & 26.150 & -4.285 & 1.00 & 0.00 & 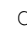 \\
\hline ATOM & 554 & $\mathrm{H} 11$ & MET & 57 & -2.337 & 25.941 & -3.305 & 1.00 & 0.00 & $\mathrm{H}$ \\
\hline ATOM & 555 & $\mathrm{H} 12$ & MET & 57 & -2.002 & 27.230 & -4.460 & 1.00 & 0.00 & $\mathrm{H}$ \\
\hline ATOM & 556 & $\mathrm{H} 13$ & MET & 57 & -2.493 & 25.624 & -5.042 & 1.00 & 0.00 & H \\
\hline TER & & & & & & & & & & \\
\hline ATOM & 557 & $\mathrm{C} 2$ & VIN & 58 & 7.905 & 14.771 & 1.002 & 1.00 & 0.00 & c \\
\hline ATOM & 558 & $\mathrm{H} 21$ & VIN & 58 & 8.721 & 15.469 & 0.837 & 1.00 & 0.00 & $\mathrm{H}$ \\
\hline ATOM & 559 & $\mathrm{C} 1$ & VIN & 58 & 8.238 & 13.546 & 1.403 & 1.00 & 0.00 & c \\
\hline ATOM & 560 & $\mathrm{H} 11$ & VIN & 58 & 7.506 & 12.767 & 1.511 & 1.00 & 0.00 & $\mathrm{H}$ \\
\hline ATOM & 561 & $\mathrm{H} 12$ & VIN & 58 & 9.279 & 13.277 & 1.570 & 1.00 & 0.00 & \\
\hline TER & & & & & & & & & & \\
\hline ATOM & 562 & $\mathrm{C} 1$ & MET & 59 & 5.241 & 14.516 & 2.840 & 1.00 & 0.00 & \\
\hline ATOM & 563 & $\mathrm{H} 11$ & MET & 59 & 5.006 & 13.445 & 2.720 & 1.00 & 0.00 & \\
\hline ATOM & 564 & $\mathrm{H} 12$ & MET & 59 & 4.428 & 14.980 & 3.410 & 1.00 & 0.00 & \\
\hline ATOM & 565 & $\mathrm{H} 13$ & MET & 59 & 6.149 & 14.593 & 3.450 & 1.00 & 0.00 & \\
\hline TER & & & & & & & & & & \\
\hline Атом & 566 & $\mathrm{C} 2$ & VIN & 60 & 0.231 & 16.194 & 2.325 & 1.00 & 0.00 & o \\
\hline ATOM & 567 & $\mathrm{H} 21$ & VIN & 60 & 0.897 & 16.295 & 3.184 & 1.00 & 0.00 & \\
\hline ATOM & 568 & $\mathrm{C} 1$ & VIN & 60 & -0.936 & 15.550 & 2.484 & 1.00 & 0.00 & 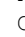 \\
\hline ATOM & 569 & $\mathrm{H} 11$ & VIN & 60 & -1.616 & 15.393 & 1.654 & 1.00 & 0.00 & $\mathrm{H}$ \\
\hline ATOM & 570 & $\mathrm{H} 12$ & VIN & 60 & -1.241 & 15.151 & 3.450 & 1.00 & 0.00 & . \\
\hline TER & & & & & & & & & & \\
\hline ATOM & 571 & $\mathrm{C} 1$ & $\mathrm{MET}$ & 61 & -1.454 & 17.877 & 0.121 & 1.00 & 0.00 & c \\
\hline ATOM & 572 & $\mathrm{H} 11$ & MET & 61 & -1.610 & 18.899 & -0.246 & 1.00 & 0.00 & F \\
\hline AтOM & 573 & $\mathrm{H} 12$ & MET & 61 & -1.886 & 17.811 & 1.126 & 1.00 & 0.00 & F \\
\hline ATOM & 574 & $\mathrm{H} 13$ & MET & 61 & -2.045 & 17.213 & -0.528 & 1.00 & 0.00 & \\
\hline TER & & & & & & & & & & \\
\hline ATOM & 575 & C5 & $\mathrm{ACP}$ & 62 & 7.635 & 18.959 & -5.770 & 1.00 & 0.00 & c \\
\hline ATOM & 576 & H51 & $\mathrm{ACP}$ & 62 & 6.896 & 18.970 & -6.582 & 1.00 & 0.00 & \\
\hline AтOM & 577 & H5 2 & $\mathrm{ACP}$ & 62 & 8.475 & 18.350 & -6.134 & 1.00 & 0.00 & \\
\hline ATOM & 578 & $\mathrm{C} 4$ & $\mathrm{ACP}$ & 62 & 8.113 & 20.396 & -5.497 & 1.00 & 0.00 & \\
\hline ATOM & 579 & $\mathrm{H} 41$ & $\mathrm{ACP}$ & 62 & 8.671 & 20.457 & -4.549 & 1.00 & 0.00 & \\
\hline ATOM & 580 & $\mathrm{H} 42$ & $\mathrm{ACP}$ & 62 & 7.243 & 21.063 & -5.390 & 1.00 & 0.00 & \\
\hline ATOM & 581 & $\mathrm{C} 1$ & $\mathrm{ACP}$ & 62 & 8.977 & 21.008 & -6.573 & 1.00 & 0.00 & \\
\hline ATOM & 582 & $\mathrm{O} 2$ & $\mathrm{ACP}$ & 62 & 9.205 & 20.486 & -7.671 & 1.00 & 0.00 & c \\
\hline Атом & 583 & 03 & $\mathrm{ACP}$ & 62 & 9.493 & 22.188 & -6.209 & 1.00 & 0.00 & c \\
\hline ATOM & 584 & H3 & $\mathrm{ACP}$ & 62 & 10.102 & 22.542 & -6.945 & 1.00 & 0.00 & \\
\hline TER & & & & & & & & & & \\
\hline ATOM & 585 & $\mathrm{C} 1$ & $\mathrm{MET}$ & 63 & -0.261 & 20.085 & -4.597 & 1.00 & 0.00 & \\
\hline ATOM & 586 & $\mathrm{H} 11$ & MET & 63 & -0.814 & 19.246 & -5.047 & 1.00 & 0.00 & \\
\hline AтOM & 587 & $\mathrm{H} 12$ & MET & 63 & -0.268 & 20.911 & -5.319 & 1.00 & 0.00 & \\
\hline ATOM & 588 & H13 & MET & 63 & -0.832 & 20.405 & -3.717 & 1.00 & 0.00 & \\
\hline
\end{tabular}




$\begin{array}{lrrllllllll}\text { ATOM } & 589 & \text { C1 MET } & 64 & 9.172 & 17.136 & -3.646 & 1.00 & 0.00 & \text { C } \\ \text { ATOM } & 590 & \text { H11 } & \text { MET } & 64 & 9.617 & 17.345 & -4.626 & 1.00 & 0.00 & \text { H } \\ \text { ATOM } & 591 & \text { H12 } & \text { MET } & 64 & 9.283 & 16.056 & -3.471 & 1.00 & 0.00 & \text { H } \\ \text { ATOM } & 592 & \text { H13 } & \text { MET } & 64 & 9.773 & 17.662 & -2.889 & 1.00 & 0.00 & \text { H }\end{array}$

TER

END

\subsection{Q3 dimer: AYQ_Q3.pdb file}

\begin{tabular}{|c|c|c|c|c|c|c|c|c|c|c|}
\hline HETATM & 1 & $\mathrm{FE}$ & HEM & 1 & 4.263 & 10.757 & 1.491 & 1.00 & 0.00 & $\mathrm{FE}$ \\
\hline HETATM & 2 & $\mathrm{C} 1$ & HEM & 1 & 2.035 & 12.629 & 0.429 & 1.00 & 0.00 & C \\
\hline HETATM & 3 & $\mathrm{C} 2$ & HEM & 1 & 1.676 & 13.420 & -0.742 & 1.00 & 0.00 & C \\
\hline HETATM & 4 & C3 & HEM & 1 & 2.822 & 13.517 & -1.517 & 1.00 & 0.00 & C \\
\hline HETATM & 5 & C4 & HEM & 1 & 3.851 & 12.800 & -0.809 & 1.00 & 0.00 & C \\
\hline HETATM & 6 & C5 & HEM & 1 & 5.167 & 12.681 & -1.247 & 1.00 & 0.00 & C \\
\hline HETATM & 7 & H5 & HEM & 1 & 5.420 & 13.177 & -2.182 & 1.00 & 0.00 & $\mathrm{H}$ \\
\hline HETATM & 8 & $\mathrm{C} 6$ & HEM & 1 & 6.186 & 11.982 & -0.611 & 1.00 & 0.00 & C \\
\hline HETATM & 9 & C7 & HEM & 1 & 7.555 & 11.910 & -1.081 & 1.00 & 0.00 & C \\
\hline HETATM & 10 & $\mathrm{C} 8$ & HEM & 1 & 8.260 & 11.190 & -0.134 & 1.00 & 0.00 & C \\
\hline HETATM & 11 & $\mathrm{C} 9$ & HEM & 1 & 7.309 & 10.825 & 0.891 & 1.00 & 0.00 & C \\
\hline HETATM & 12 & $\mathrm{C} 10$ & HEM & 1 & 7.614 & 10.090 & 2.034 & 1.00 & 0.00 & C \\
\hline HETATM & 13 & $\mathrm{H} 10$ & HEM & 1 & 8.641 & 9.746 & 2.140 & 1.00 & 0.00 & $\mathrm{H}$ \\
\hline HETATM & 14 & C11 & HEM & 1 & 6.739 & 9.750 & 3.057 & 1.00 & 0.00 & C \\
\hline HETATM & 15 & C12 & HEM & 1 & 7.120 & 9.025 & 4.247 & 1.00 & 0.00 & $\mathrm{C}$ \\
\hline HETATM & 16 & $\mathrm{C} 13$ & HEM & 1 & 5.981 & 8.878 & 5.004 & 1.00 & 0.00 & C \\
\hline HETATM & 17 & C14 & HEM & 1 & 4.908 & 9.519 & 4.273 & 1.00 & 0.00 & C \\
\hline HETATM & 18 & C15 & HEM & 1 & 3.580 & 9.574 & 4.694 & 1.00 & 0.00 & C \\
\hline HETATM & 19 & H15 & HEM & 1 & 3.340 & 9.089 & 5.642 & 1.00 & 0.00 & $\mathrm{H}$ \\
\hline HETATM & 20 & C16 & HEM & 1 & 2.550 & 10.255 & 4.051 & 1.00 & 0.00 & C \\
\hline HETATM & 21 & $\mathrm{C} 17$ & HEM & 1 & 1.184 & 10.369 & 4.533 & 1.00 & 0.00 & C \\
\hline HETATM & 22 & $\mathrm{C} 18$ & HEM & 1 & 0.508 & 11.137 & 3.609 & 1.00 & 0.00 & C \\
\hline HETATM & 23 & C19 & HEM & 1 & 1.458 & 11.489 & 2.573 & 1.00 & 0.00 & C \\
\hline HETATM & 24 & $\mathrm{C} 20$ & HEM & 1 & 1.167 & 12.280 & 1.462 & 1.00 & 0.00 & C \\
\hline HETATM & 25 & $\mathrm{H} 2 \mathrm{O}$ & HEM & 1 & 0.152 & 12.669 & 1.402 & 1.00 & 0.00 & $\mathrm{H}$ \\
\hline HETATM & 26 & N21 & HEM & 1 & 3.357 & 12.266 & 0.367 & 1.00 & 0.00 & $\mathrm{~N}$ \\
\hline HETATM & 27 & N22 & HEM & 1 & 6.062 & 11.319 & 0.590 & 1.00 & 0.00 & $\mathrm{~N}$ \\
\hline HETATM & 28 & N23 & HEM & 1 & 5.391 & 10.056 & 3.090 & 1.00 & 0.00 & $\mathrm{~N}$ \\
\hline HETATM & 29 & N24 & HEM & 1 & 2.691 & 10.941 & 2.854 & 1.00 & 0.00 & $\mathrm{~N}$ \\
\hline HETATM & 30 & C99 & HEM & 1 & 3.598 & 8.076 & 0.352 & 1.00 & 0.00 & C \\
\hline HETATM & 31 & 091 & HEM & 1 & 3.757 & 9.389 & 0.365 & 1.00 & 0.00 & o \\
\hline HETATM & 32 & 092 & HEM & 1 & 3.443 & 7.458 & -0.703 & 1.00 & 0.00 & o \\
\hline HETATM & 33 & CT91 & HEM & 1 & 3.614 & 7.394 & 1.709 & 1.00 & 0.00 & C \\
\hline HETATM & 34 & H911 & HEM & 1 & 4.593 & 7.590 & 2.171 & 1.00 & 0.00 & $\mathrm{H}$ \\
\hline HETATM & 35 & H912 & HEM & 1 & 2.866 & 7.884 & 2.352 & 1.00 & 0.00 & $\mathrm{H}$ \\
\hline HETATM & 36 & CT92 & HEM & 1 & 3.354 & 5.890 & 1.597 & 1.00 & 0.00 & C \\
\hline HETATM & 37 & H921 & HEM & 1 & 4.151 & 5.429 & 1.000 & 1.00 & 0.00 & $\mathrm{H}$ \\
\hline HETATM & 38 & H922 & HEM & 1 & 2.429 & 5.734 & 1.027 & 1.00 & 0.00 & $\mathrm{H}$ \\
\hline \multicolumn{11}{|l|}{ TER } \\
\hline HETATM & 39 & $\mathrm{FE}$ & HEM & 2 & 4.955 & 3.343 & 6.440 & 1.00 & 0.00 & $\mathrm{FE}$ \\
\hline HETATM & 40 & $\mathrm{C} 1$ & HEM & 2 & 7.183 & 1.471 & 7.502 & 1.00 & 0.00 & C \\
\hline HETATM & 41 & $\mathrm{C} 2$ & HEM & 2 & 7.542 & 0.680 & 8.674 & 1.00 & 0.00 & C \\
\hline HETATM & 42 & C3 & HEM & 2 & 6.397 & 0.583 & 9.448 & 1.00 & 0.00 & C \\
\hline HETATM & 43 & C4 & HEM & 2 & 5.367 & 1.301 & 8.740 & 1.00 & 0.00 & C \\
\hline HETATM & 44 & C5 & HEM & 2 & 4.052 & 1.420 & 9.178 & 1.00 & 0.00 & C \\
\hline HETATM & 45 & H5 & HEM & 2 & 3.798 & 0.923 & 10.113 & 1.00 & 0.00 & $\mathrm{H}$ \\
\hline HETATM & 46 & $\mathrm{C} 6$ & HEM & 2 & 3.033 & 2.118 & 8.542 & 1.00 & 0.00 & C \\
\hline HETATM & 47 & C7 & HEM & 2 & 1.664 & 2.191 & 9.012 & 1.00 & 0.00 & C \\
\hline HETATM & 48 & $\mathrm{C} 8$ & HEM & 2 & 0.958 & 2.910 & 8.065 & 1.00 & 0.00 & C \\
\hline HETATM & 49 & C9 & HEM & 2 & 1.909 & 3.275 & 7.041 & 1.00 & 0.00 & C \\
\hline HETATM & 50 & $\mathrm{C} 10$ & HEM & 2 & 1.605 & 4.010 & 5.897 & 1.00 & 0.00 & C \\
\hline HETATM & 51 & $\mathrm{H} 10$ & HEM & 2 & 0.578 & 4.354 & 5.791 & 1.00 & 0.00 & $\mathrm{H}$ \\
\hline HETATM & 52 & $\mathrm{C} 11$ & HEM & 2 & 2.479 & 4.350 & 4.874 & 1.00 & 0.00 & C \\
\hline HETATM & 53 & $\mathrm{C} 12$ & HEM & 2 & 2.098 & 5.076 & 3.684 & 1.00 & 0.00 & C \\
\hline HETATM & 54 & $\mathrm{C} 13$ & HEM & 2 & 3.238 & 5.223 & 2.927 & 1.00 & 0.00 & C \\
\hline HETATM & 55 & $\mathrm{C} 14$ & HEM & 2 & 4.311 & 4.581 & 3.659 & 1.00 & 0.00 & C \\
\hline HETATM & 56 & C15 & HEM & 2 & 5.639 & 4.527 & 3.237 & 1.00 & 0.00 & C \\
\hline HETATM & 57 & H15 & HEM & 2 & 5.879 & 5.011 & 2.289 & 1.00 & 0.00 & $\mathrm{H}$ \\
\hline HETATM & 58 & $\mathrm{C} 16$ & HEM & 2 & 6.669 & 3.845 & 3.880 & 1.00 & 0.00 & C \\
\hline HETATM & 59 & $\mathrm{C} 17$ & HEM & 2 & 8.035 & 3.732 & 3.398 & 1.00 & 0.00 & C \\
\hline HETATM & 60 & $\mathrm{C} 18$ & HEM & 2 & 8.710 & 2.964 & 4.322 & 1.00 & 0.00 & C \\
\hline HETATM & 61 & C19 & HEM & 2 & 7.761 & 2.612 & 5.358 & 1.00 & 0.00 & C \\
\hline HETATM & 62 & $\mathrm{C} 20$ & HEM & 2 & 8.052 & 1.821 & 6.469 & 1.00 & 0.00 & C \\
\hline HETATM & 63 & $\mathrm{H} 2 \mathrm{O}$ & HEM & 2 & 9.066 & 1.432 & 6.530 & 1.00 & 0.00 & $\mathrm{H}$ \\
\hline HETATM & 64 & N21 & HEM & 2 & 5.862 & 1.834 & 7.564 & 1.00 & 0.00 & $\mathrm{~N}$ \\
\hline HETATM & 65 & N22 & HEM & 2 & 3.157 & 2.781 & 7.342 & 1.00 & 0.00 & $\mathrm{~N}$ \\
\hline HETATM & 66 & N23 & HEM & 2 & 3.828 & 4.044 & 4.841 & 1.00 & 0.00 & $\mathrm{~N}$ \\
\hline HETATM & 67 & N24 & HEM & 2 & 6.527 & 3.160 & 5.077 & 1.00 & 0.00 & $\mathrm{~N}$ \\
\hline HETATM & 68 & C99 & HEM & 2 & 5.621 & 6.025 & 7.579 & 1.00 & 0.00 & C \\
\hline
\end{tabular}




\begin{tabular}{|c|c|c|c|}
\hline HETATM & 69 & 091 & HEM \\
\hline HETATM & 70 & 092 & HEM \\
\hline HETATM & 71 & СТ 91 & HEM \\
\hline HETATM & 72 & H911 & HEM \\
\hline HETATM & 73 & H912 & HEM \\
\hline HETATM & 74 & CT92 & HEM \\
\hline HETATM & 75 & H921 & HEM \\
\hline HETATM & 76 & H922 & HEM \\
\hline \multicolumn{4}{|l|}{ TER } \\
\hline HETATM & 77 & C2 & VIN \\
\hline HETATM & 78 & H 21 & VIN \\
\hline HETATM & 79 & $\mathrm{C} 1$ & VIN \\
\hline HETATM & 80 & $\mathrm{H} 11$ & VIN \\
\hline HETATM & 81 & $\mathrm{H} 12$ & VIN \\
\hline \multicolumn{4}{|l|}{ TER } \\
\hline HETATM & 82 & $\mathrm{C} 1$ & MET \\
\hline HETATM & 83 & $\mathrm{H} 11$ & MET \\
\hline HETATM & 84 & $\mathrm{H} 12$ & MET \\
\hline HETATM & 85 & $\mathrm{H} 13$ & MET \\
\hline \multicolumn{4}{|l|}{ TER } \\
\hline HETATM & 86 & $\mathrm{C} 2$ & VIN \\
\hline HETATM & 87 & H 21 & VIN \\
\hline HETATM & 88 & $\mathrm{C} 1$ & VIN \\
\hline HETATM & 89 & $\mathrm{H} 11$ & VIN \\
\hline HETATM & 90 & $\mathrm{H} 12$ & VIN \\
\hline \multicolumn{4}{|l|}{ TER } \\
\hline HETATM & 91 & $\mathrm{C} 1$ & MET \\
\hline HETATM & 92 & $\mathrm{H} 11$ & MET \\
\hline HETATM & 93 & $\mathrm{H} 12$ & MET \\
\hline HETATM & 94 & $\mathrm{H} 13$ & $\mathrm{MET}$ \\
\hline \multicolumn{4}{|l|}{ TER } \\
\hline HETATM & 95 & C5 & $\mathrm{ACP}$ \\
\hline HETATM & 96 & H51 & $\mathrm{ACP}$ \\
\hline HETATM & 97 & H52 & $\mathrm{ACP}$ \\
\hline HETATM & 98 & C4 & $\mathrm{ACP}$ \\
\hline HETATM & 99 & H4 1 & $\mathrm{ACP}$ \\
\hline HETATM & 100 & H42 & $\mathrm{ACP}$ \\
\hline HETATM & 101 & $\mathrm{C} 1$ & $\mathrm{ACP}$ \\
\hline HETATM & 102 & $\mathrm{O} 2$ & $\mathrm{ACP}$ \\
\hline HETATM & 103 & 03 & $\mathrm{ACP}$ \\
\hline HETATM & 104 & H3 & $\mathrm{ACP}$ \\
\hline \multicolumn{4}{|l|}{ TER } \\
\hline HETATM & 105 & $\mathrm{C} 1$ & $\mathrm{MET}$ \\
\hline HETATM & 106 & $\mathrm{H} 11$ & $\mathrm{MET}$ \\
\hline HETATM & 107 & $\mathrm{H} 12$ & MET \\
\hline HETATM & 108 & $\mathrm{H} 13$ & MET \\
\hline \multicolumn{4}{|l|}{ TER } \\
\hline HETATM & 109 & $\mathrm{C} 1$ & MET \\
\hline HETATM & 110 & $\mathrm{H} 11$ & MET \\
\hline HETATM & 111 & $\mathrm{H} 12$ & MET \\
\hline HETATM & 112 & $\mathrm{H} 13$ & MET \\
\hline \multicolumn{4}{|l|}{ TER } \\
\hline HETATM & 113 & $\mathrm{C} 2$ & VIN \\
\hline HETATM & 114 & H21 & VIN \\
\hline HETATM & 115 & $\mathrm{C} 1$ & VIN \\
\hline HETATM & 116 & $\mathrm{H} 11$ & VIN \\
\hline HETATM & 117 & H12 & VIN \\
\hline TER & & & \\
\hline HETATM & 118 & $\mathrm{C} 1$ & $\mathrm{MET}$ \\
\hline HETATM & 119 & $\mathrm{H} 11$ & MET \\
\hline HETATM & 120 & $\mathrm{H} 12$ & MET \\
\hline HETATM & 121 & $\mathrm{H} 13$ & MET \\
\hline TER & & & \\
\hline HETATM & 122 & $\mathrm{C} 2$ & VIN \\
\hline HETATM & 123 & $\mathrm{H} 21$ & VIN \\
\hline HETATM & 124 & $\mathrm{C} 1$ & VIN \\
\hline HETATM & 125 & $\mathrm{H} 11$ & VIN \\
\hline HETATM & 126 & $\mathrm{H} 12$ & VIN \\
\hline TER & & & \\
\hline HETATM & 127 & $\mathrm{C} 1$ & MET \\
\hline HETATM & 128 & $\mathrm{H} 11$ & MET \\
\hline HETATM & 129 & $\mathrm{H} 12$ & MET \\
\hline HETATM & 130 & $\mathrm{H} 13$ & MET \\
\hline TER & & & \\
\hline HETATM & 131 & C5 & $\mathrm{ACP}$ \\
\hline HETATM & 132 & H51 & $\mathrm{ACP}$ \\
\hline HETATM & 133 & H52 & $\mathrm{ACP}$ \\
\hline HETATM & 134 & C4 & $\mathrm{ACP}$ \\
\hline HETATM & 135 & $\mathrm{H} 4 \mathrm{I}$ & $\mathrm{ACP}$ \\
\hline
\end{tabular}

\begin{tabular}{|c|c|c|c|c|c|}
\hline 5.461 & 4.711 & 7.567 & 1.00 & 0.00 & 0 \\
\hline 5.776 & 6.642 & 8.635 & 1.00 & 0.00 & 0 \\
\hline 5.605 & 6.706 & 6.223 & 1.00 & 0.00 & C \\
\hline 4.626 & 6.511 & 5.760 & 1.00 & 0.00 & $\mathrm{H}$ \\
\hline 6.353 & 6.216 & 5.580 & 1.00 & 0.00 & $\mathrm{H}$ \\
\hline 5.864 & 8.211 & 6.334 & 1.00 & 0.00 & C \\
\hline 6.789 & 8.367 & 6.905 & 1.00 & 0.00 & $\mathrm{H}$ \\
\hline 5.068 & 8.671 & 6.931 & 1.00 & 0.00 & $\mathrm{H}$ \\
\hline 0.345 & 13.923 & -1.002 & 1.00 & 0.00 & C \\
\hline-0.471 & 13.224 & -0.837 & 1.00 & 0.00 & $\mathrm{H}$ \\
\hline 0.012 & 15.147 & -1.403 & 1.00 & 0.00 & $\mathrm{C}$ \\
\hline 0.743 & 15.926 & -1.511 & 1.00 & 0.00 & $\mathrm{H}$ \\
\hline-1.030 & 15.417 & -1.570 & 1.00 & 0.00 & $\mathrm{H}$ \\
\hline 3.008 & 14.177 & -2.840 & 1.00 & 0.00 & C \\
\hline 3.243 & 15.248 & -2.720 & 1.00 & 0.00 & $\mathrm{H}$ \\
\hline 3.821 & 13.713 & -3.410 & 1.00 & 0.00 & $\mathrm{H}$ \\
\hline 2.101 & 14.100 & -3.450 & 1.00 & 0.00 & $\mathrm{H}$ \\
\hline 8.018 & 12.499 & -2.325 & 1.00 & 0.00 & C \\
\hline 7.352 & 12.399 & -3.184 & 1.00 & 0.00 & $\mathrm{H}$ \\
\hline 9.185 & 13.144 & -2.484 & 1.00 & 0.00 & C \\
\hline 9.865 & 13.300 & -1.654 & 1.00 & 0.00 & $\mathrm{H}$ \\
\hline 9.490 & 13.542 & -3.450 & 1.00 & 0.00 & $\mathrm{H}$ \\
\hline 9.703 & 10.816 & -0.121 & 1.00 & 0.00 & C \\
\hline 10.294 & 11.480 & 0.528 & 1.00 & 0.00 & $\mathrm{H}$ \\
\hline 9.859 & 9.794 & 0.246 & 1.00 & 0.00 & $\mathrm{H}$ \\
\hline 10.135 & 10.882 & -1.126 & 1.00 & 0.00 & $\mathrm{H}$ \\
\hline 0.615 & 9.735 & 5.770 & 1.00 & 0.00 & C \\
\hline 1.354 & 9.723 & 6.582 & 1.00 & 0.00 & $\mathrm{H}$ \\
\hline-0.226 & 10.343 & 6.134 & 1.00 & 0.00 & $\mathrm{H}$ \\
\hline 0.136 & 8.297 & 5.497 & 1.00 & 0.00 & C \\
\hline-0.422 & 8.236 & 4.549 & 1.00 & 0.00 & $\mathrm{H}$ \\
\hline 1.007 & 7.630 & 5.390 & 1.00 & 0.00 & $\mathrm{H}$ \\
\hline-0.728 & 7.685 & 6.573 & 1.00 & 0.00 & C \\
\hline-0.956 & 8.208 & 7.671 & 1.00 & 0.00 & 0 \\
\hline-1.244 & 6.505 & 6.209 & 1.00 & 0.00 & o \\
\hline-1.853 & 6.151 & 6.945 & 1.00 & 0.00 & $\mathrm{H}$ \\
\hline 8.511 & 8.608 & 4.597 & 1.00 & 0.00 & C \\
\hline 9.063 & 9.448 & 5.047 & 1.00 & 0.00 & $\mathrm{H}$ \\
\hline 8.517 & 7.782 & 5.319 & 1.00 & 0.00 & $\mathrm{H}$ \\
\hline 9.082 & 8.289 & 3.717 & 1.00 & 0.00 & $\mathrm{H}$ \\
\hline-0.923 & 11.557 & 3.646 & 1.00 & 0.00 & C \\
\hline-1.368 & 11.348 & 4.626 & 1.00 & 0.00 & $\mathrm{H}$ \\
\hline-1.033 & 12.637 & 3.471 & 1.00 & 0.00 & $\mathrm{H}$ \\
\hline-1.523 & 11.031 & 2.889 & 1.00 & 0.00 & $\mathrm{H}$ \\
\hline 8.874 & 0.178 & 8.933 & 1.00 & 0.00 & C \\
\hline 9.690 & 0.876 & 8.768 & 1.00 & 0.00 & $\mathrm{H}$ \\
\hline 9.207 & -1.047 & 9.335 & 1.00 & 0.00 & C \\
\hline 8.475 & -1.826 & 9.442 & 1.00 & 0.00 & $\mathrm{H}$ \\
\hline 10.249 & -1.316 & 9.501 & 1.00 & 0.00 & $\mathrm{H}$ \\
\hline 6.210 & -0.077 & 10.771 & 1.00 & 0.00 & C \\
\hline 5.975 & -1.148 & 10.652 & 1.00 & 0.00 & $\mathrm{H}$ \\
\hline 5.397 & 0.387 & 11.341 & 1.00 & 0.00 & $\mathrm{H}$ \\
\hline 7.118 & 0.001 & 11.382 & 1.00 & 0.00 & $\mathrm{H}$ \\
\hline 1.200 & 1.601 & 10.256 & 1.00 & 0.00 & C \\
\hline 1.866 & 1.702 & 11.115 & 1.00 & 0.00 & $\mathrm{H}$ \\
\hline 0.033 & 0.957 & 10.415 & 1.00 & 0.00 & C \\
\hline-0.647 & 0.800 & 9.585 & 1.00 & 0.00 & $\mathrm{H}$ \\
\hline-0.272 & 0.559 & 11.381 & 1.00 & 0.00 & $\mathrm{H}$ \\
\hline-0.484 & 3.284 & 8.052 & 1.00 & 0.00 & C \\
\hline-0.641 & 4.306 & 7.685 & 1.00 & 0.00 & $\mathrm{H}$ \\
\hline-0.917 & 3.219 & 9.057 & 1.00 & 0.00 & $\mathrm{H}$ \\
\hline-1.076 & 2.620 & 7.403 & 1.00 & 0.00 & $\mathrm{H}$ \\
\hline 8.604 & 4.366 & 2.161 & 1.00 & 0.00 & C \\
\hline 7.865 & 4.377 & 1.349 & 1.00 & 0.00 & $\mathrm{H}$ \\
\hline 9.445 & 3.758 & 1.798 & 1.00 & 0.00 & $\mathrm{H}$ \\
\hline 9.082 & 5.803 & 2.434 & 1.00 & 0.00 & C \\
\hline 9.640 & 5.865 & 3.382 & 1.00 & 0.00 & $\mathrm{H}$ \\
\hline
\end{tabular}




\begin{tabular}{|c|c|c|c|c|c|c|c|c|c|c|}
\hline HETATM & 136 & $\mathrm{H} 42$ & $\mathrm{ACP}$ & 14 & 8.212 & 6.470 & 2.541 & 1.00 & 0.00 & $\mathrm{H}$ \\
\hline HETATM & 137 & $\mathrm{C} 1$ & $\mathrm{ACP}$ & 14 & 9.946 & 6.416 & 1.358 & 1.00 & 0.00 & C \\
\hline HETATM & 138 & $\mathrm{O} 2$ & $\mathrm{ACP}$ & 14 & 10.174 & 5.893 & 0.260 & 1.00 & 0.00 & 0 \\
\hline HETATM & 139 & 03 & $\mathrm{ACP}$ & 14 & 10.462 & 7.595 & 1.722 & 1.00 & 0.00 & 0 \\
\hline HETATM & 140 & H3 & $\mathrm{ACP}$ & 14 & 11.071 & 7.949 & 0.986 & 1.00 & 0.00 & $\mathrm{H}$ \\
\hline \multicolumn{11}{|l|}{ TER } \\
\hline HETATM & 141 & $\mathrm{C} 1$ & MET & 15 & 0.708 & 5.492 & 3.334 & 1.00 & 0.00 & C \\
\hline HETATM & 142 & H11 & $\mathrm{MET}$ & 15 & 0.155 & 4.653 & 2.884 & 1.00 & 0.00 & $\mathrm{H}$ \\
\hline HETATM & 143 & H12 & $\mathrm{MET}$ & 15 & 0.701 & 6.318 & 2.613 & 1.00 & 0.00 & $\mathrm{H}$ \\
\hline HETATM & 144 & H13 & $\mathrm{MET}$ & 15 & 0.137 & 5.812 & 4.214 & 1.00 & 0.00 & $\mathrm{H}$ \\
\hline \multicolumn{11}{|l|}{ TER } \\
\hline HETATM & 145 & $\mathrm{C} 1$ & MET & 16 & 10.141 & 2.543 & 4.285 & 1.00 & 0.00 & C \\
\hline HETATM & 146 & H11 & $\mathrm{MET}$ & 16 & 10.586 & 2.752 & 3.305 & 1.00 & 0.00 & $\mathrm{H}$ \\
\hline HETATM & 147 & $\mathrm{H} 12$ & MET & 16 & 10.252 & 1.463 & 4.460 & 1.00 & 0.00 & $\mathrm{H}$ \\
\hline HETATM & 148 & H13 & MET & 16 & 10.742 & 3.069 & 5.042 & 1.00 & 0.00 & $\mathrm{H}$ \\
\hline \multicolumn{11}{|l|}{ TER } \\
\hline HETATM & 149 & $\mathrm{FE}$ & HEM & 17 & 5.211 & 11.003 & -6.440 & 1.00 & 0.00 & $\mathrm{FE}$ \\
\hline HETATM & 150 & $\mathrm{C} 1$ & HEM & 17 & 2.983 & 12.876 & -7.502 & 1.00 & 0.00 & C \\
\hline HETATM & 151 & $\mathrm{C} 2$ & HEM & 17 & 2.624 & 13.666 & -8.674 & 1.00 & 0.00 & C \\
\hline HETATM & 152 & $\mathrm{C} 3$ & HEM & 17 & 3.770 & 13.763 & -9.448 & 1.00 & 0.00 & C \\
\hline HETATM & 153 & $\mathrm{C} 4$ & HEM & 17 & 4.799 & 13.046 & -8.740 & 1.00 & 0.00 & C \\
\hline HETATM & 154 & C5 & HEM & 17 & 6.114 & 12.927 & -9.178 & 1.00 & 0.00 & C \\
\hline HETATM & 155 & H5 & HEM & 17 & 6.368 & 13.423 & -10.113 & 1.00 & 0.00 & $\mathrm{H}$ \\
\hline HETATM & 156 & $\mathrm{C} 6$ & HEM & 17 & 7.134 & 12.229 & -8.542 & 1.00 & 0.00 & C \\
\hline HETATM & 157 & C7 & HEM & 17 & 8.502 & 12.156 & -9.012 & 1.00 & 0.00 & C \\
\hline HETATM & 158 & $\mathrm{C} 8$ & HEM & 17 & 9.208 & 11.436 & -8.065 & 1.00 & 0.00 & C \\
\hline HETATM & 159 & $\mathrm{C} 9$ & HEM & 17 & 8.257 & 11.071 & -7.041 & 1.00 & 0.00 & C \\
\hline HETATM & 160 & C10 & HEM & 17 & 8.561 & 10.336 & -5.897 & 1.00 & 0.00 & C \\
\hline HETATM & 161 & $\mathrm{H} 10$ & HEM & 17 & 9.588 & 9.993 & -5.791 & 1.00 & 0.00 & $\mathrm{H}$ \\
\hline HETATM & 162 & C11 & HEM & 17 & 7.687 & 9.996 & -4.874 & 1.00 & 0.00 & C \\
\hline HETATM & 163 & $\mathrm{C} 12$ & HEM & 17 & 8.068 & 9.271 & -3.684 & 1.00 & 0.00 & C \\
\hline HETATM & 164 & $\mathrm{C} 13$ & HEM & 17 & 6.928 & 9.124 & -2.927 & 1.00 & 0.00 & C \\
\hline HETATM & 165 & C14 & HEM & 17 & 5.855 & 9.765 & -3.659 & 1.00 & 0.00 & C \\
\hline HETATM & 166 & C15 & HEM & 17 & 4.527 & 9.820 & -3.237 & 1.00 & 0.00 & C \\
\hline HETATM & 167 & H15 & HEM & 17 & 4.287 & 9.335 & -2.289 & 1.00 & 0.00 & $\mathrm{H}$ \\
\hline HETATM & 168 & C16 & HEM & 17 & 3.497 & 10.502 & -3.880 & 1.00 & 0.00 & C \\
\hline HETATM & 169 & C17 & HEM & 17 & 2.131 & 10.615 & -3.398 & 1.00 & 0.00 & C \\
\hline HETATM & 170 & C18 & HEM & 17 & 1.456 & 11.383 & -4.322 & 1.00 & 0.00 & C \\
\hline HETATM & 171 & C19 & HEM & 17 & 2.406 & 11.735 & -5.358 & 1.00 & 0.00 & C \\
\hline HETATM & 172 & $\mathrm{C} 20$ & HEM & 17 & 2.114 & 12.526 & -6.469 & 1.00 & 0.00 & C \\
\hline HETATM & 173 & $\mathrm{H} 20$ & HEM & 17 & 1.100 & 12.915 & -6.530 & 1.00 & 0.00 & $\mathrm{H}$ \\
\hline HETATM & 174 & N21 & HEM & 17 & 4.305 & 12.513 & -7.564 & 1.00 & 0.00 & $\mathrm{~N}$ \\
\hline HETATM & 175 & N22 & HEM & 17 & 7.009 & 11.566 & -7.342 & 1.00 & 0.00 & $\mathrm{~N}$ \\
\hline HETATM & 176 & N23 & HEM & 17 & 6.338 & 10.303 & -4.841 & 1.00 & 0.00 & $\mathrm{~N}$ \\
\hline HETATM & 177 & $\mathrm{~N} 24$ & HEM & 17 & 3.639 & 11.187 & -5.077 & 1.00 & 0.00 & $\mathrm{~N}$ \\
\hline HETATM & 178 & C99 & HEM & 17 & 4.545 & 8.322 & -7.579 & 1.00 & 0.00 & C \\
\hline HETATM & 179 & 091 & HEM & 17 & 4.705 & 9.635 & -7.567 & 1.00 & 0.00 & 0 \\
\hline HETATM & 180 & 092 & HEM & 17 & 4.391 & 7.705 & -8.635 & 1.00 & 0.00 & 0 \\
\hline HETATM & 181 & СТ91 & HEM & 17 & 4.561 & 7.640 & -6.223 & 1.00 & 0.00 & C \\
\hline HETATM & 182 & H911 & HEM & 17 & 5.540 & 7.836 & -5.760 & 1.00 & 0.00 & $\mathrm{H}$ \\
\hline HETATM & 183 & H912 & HEM & 17 & 3.813 & 8.130 & -5.580 & 1.00 & 0.00 & $\mathrm{H}$ \\
\hline HETATM & 184 & СТ92 & HEM & 17 & 4.302 & 6.136 & -6.334 & 1.00 & 0.00 & C \\
\hline HETATM & 185 & H921 & HEM & 17 & 5.098 & 5.676 & -6.931 & 1.00 & 0.00 & $\mathrm{H}$ \\
\hline HETATM & 186 & H922 & HEM & 17 & 3.377 & 5.980 & -6.905 & 1.00 & 0.00 & $\mathrm{H}$ \\
\hline \multicolumn{11}{|l|}{ TER } \\
\hline HETATM & 187 & $\mathrm{FE}$ & HEM & 18 & 5.903 & 3.589 & -1.491 & 1.00 & 0.00 & $\mathrm{FE}$ \\
\hline HETATM & 188 & $\mathrm{C} 1$ & HEM & 18 & 8.131 & 1.717 & -0.429 & 1.00 & 0.00 & C \\
\hline HETATM & 189 & $\mathrm{C} 2$ & HEM & 18 & 8.490 & 0.927 & 0.742 & 1.00 & 0.00 & C \\
\hline HETATM & 190 & C3 & HEM & 18 & 7.344 & 0.830 & 1.517 & 1.00 & 0.00 & C \\
\hline HETATM & 191 & $\mathrm{C} 4$ & HEM & 18 & 6.315 & 1.547 & 0.809 & 1.00 & 0.00 & C \\
\hline HETATM & 192 & C5 & HEM & 18 & 5.000 & 1.666 & 1.247 & 1.00 & 0.00 & C \\
\hline HETATM & 193 & H5 & HEM & 18 & 4.746 & 1.170 & 2.182 & 1.00 & 0.00 & $\mathrm{H}$ \\
\hline HETATM & 194 & $\mathrm{C} 6$ & HEM & 18 & 3.980 & 2.364 & 0.611 & 1.00 & 0.00 & C \\
\hline HETATM & 195 & C7 & HEM & 18 & 2.611 & 2.437 & 1.081 & 1.00 & 0.00 & C \\
\hline HETATM & 196 & $\mathrm{C} 8$ & HEM & 18 & 1.906 & 3.157 & 0.134 & 1.00 & 0.00 & C \\
\hline HETATM & 197 & C9 & HEM & 18 & 2.857 & 3.522 & -0.891 & 1.00 & 0.00 & C \\
\hline HETATM & 198 & $\mathrm{C} 10$ & HEM & 18 & 2.552 & 4.256 & -2.034 & 1.00 & 0.00 & C \\
\hline HETATM & 199 & H1O & HEM & 18 & 1.526 & 4.600 & -2.140 & 1.00 & 0.00 & $\mathrm{H}$ \\
\hline HETATM & 200 & $\mathrm{C} 11$ & HEM & 18 & 3.427 & 4.596 & -3.057 & 1.00 & 0.00 & C \\
\hline HETATM & 201 & $\mathrm{C} 12$ & HEM & 18 & 3.046 & 5.322 & -4.247 & 1.00 & 0.00 & C \\
\hline HETATM & 202 & $\mathrm{C} 13$ & HEM & 18 & 4.185 & 5.469 & -5.004 & 1.00 & 0.00 & C \\
\hline HETATM & 203 & C14 & HEM & 18 & 5.258 & 4.828 & -4.273 & 1.00 & 0.00 & C \\
\hline HETATM & 204 & C15 & HEM & 18 & 6.586 & 4.773 & -4.694 & 1.00 & 0.00 & C \\
\hline HETATM & 205 & H15 & HEM & 18 & 6.826 & 5.258 & -5.642 & 1.00 & 0.00 & $\mathrm{H}$ \\
\hline HETATM & 206 & $\mathrm{C} 16$ & HEM & 18 & 7.617 & 4.091 & -4.051 & 1.00 & 0.00 & C \\
\hline HETATM & 207 & C17 & HEM & 18 & 8.982 & 3.978 & -4.533 & 1.00 & 0.00 & C \\
\hline HETATM & 208 & $\mathrm{C} 18$ & HEM & 18 & 9.658 & 3.210 & -3.609 & 1.00 & 0.00 & C \\
\hline HETATM & 209 & C19 & HEM & 18 & 8.708 & 2.858 & -2.573 & 1.00 & 0.00 & C \\
\hline HETATM & 210 & $\mathrm{C} 20$ & HEM & 18 & 8.999 & 2.067 & -1.462 & 1.00 & 0.00 & $\mathrm{C}$ \\
\hline
\end{tabular}




\begin{tabular}{|c|c|c|c|c|c|}
\hline 10.014 & 1.678 & -1.402 & 1.00 & 0.00 & $\mathrm{H}$ \\
\hline 6.809 & 2.080 & -0.367 & 1.00 & 0.00 & $\mathrm{~N}$ \\
\hline 4.105 & 3.027 & -0.590 & 1.00 & 0.00 & $\mathrm{~N}$ \\
\hline 4.775 & 4.290 & -3.090 & 1.00 & 0.00 & $\mathrm{~N}$ \\
\hline 7.475 & 3.406 & -2.854 & 1.00 & 0.00 & $\mathrm{~N}$ \\
\hline 6.568 & 6.271 & -0.352 & 1.00 & 0.00 & C \\
\hline 6.409 & 4.958 & -0.365 & 1.00 & 0.00 & o \\
\hline 6.723 & 6.888 & 0.703 & 1.00 & 0.00 & O \\
\hline 6.552 & 6.952 & -1.709 & 1.00 & 0.00 & C \\
\hline 5.573 & 6.757 & -2.171 & 1.00 & 0.00 & $\mathrm{H}$ \\
\hline 7.300 & 6.463 & -2.352 & 1.00 & 0.00 & $\mathrm{H}$ \\
\hline 6.812 & 8.457 & -1.597 & 1.00 & 0.00 & C \\
\hline 7.737 & 8.613 & -1.027 & 1.00 & 0.00 & $\mathrm{H}$ \\
\hline 6.015 & 8.917 & -1.000 & 1.00 & 0.00 & $\mathrm{H}$ \\
\hline 1.292 & 14.169 & -8.933 & 1.00 & 0.00 & $\mathrm{C}$ \\
\hline 0.476 & 13.470 & -8.768 & 1.00 & 0.00 & $\mathrm{H}$ \\
\hline 0.959 & 15.394 & -9.335 & 1.00 & 0.00 & $\mathrm{C}$ \\
\hline 1.691 & 16.172 & -9.442 & 1.00 & 0.00 & $\mathrm{H}$ \\
\hline-0.082 & 15.663 & -9.501 & 1.00 & 0.00 & $\mathrm{H}$ \\
\hline 3.956 & 14.424 & -10.771 & 1.00 & 0.00 & C \\
\hline 4.191 & 15.494 & -10.652 & 1.00 & 0.00 & $\mathrm{H}$ \\
\hline 4.769 & 13.959 & $-11 \cdot 341$ & 1.00 & 0.00 & $\mathrm{H}$ \\
\hline 3.048 & 14.346 & $-11 \cdot 382$ & 1.00 & 0.00 & $\mathrm{H}$ \\
\hline 8.966 & 12.746 & -10.256 & 1.00 & 0.00 & C \\
\hline 8.300 & 12.645 & -11.115 & 1.00 & 0.00 & $\mathrm{H}$ \\
\hline 10.133 & 13.390 & -10.415 & 1.00 & 0.00 & C \\
\hline 10.813 & 13.546 & -9.585 & 1.00 & 0.00 & $\mathrm{H}$ \\
\hline 10.438 & 13.788 & -11.381 & 1.00 & 0.00 & $\mathrm{H}$ \\
\hline 10.651 & 11.062 & -8.052 & 1.00 & 0.00 & C \\
\hline 11.242 & 11.727 & -7.403 & 1.00 & 0.00 & $\mathrm{H}$ \\
\hline 10.807 & 10.040 & -7.685 & 1.00 & 0.00 & $\mathrm{H}$ \\
\hline 11.083 & 11.128 & -9.057 & 1.00 & 0.00 & $\mathrm{H}$ \\
\hline 1.562 & 9.981 & -2.161 & 1.00 & 0.00 & $\mathrm{C}$ \\
\hline 2.301 & 9.969 & -1.349 & 1.00 & 0.00 & $\mathrm{H}$ \\
\hline 0.722 & 10.589 & -1.798 & 1.00 & 0.00 & $\mathrm{H}$ \\
\hline 1.084 & 8.543 & -2.434 & 1.00 & 0.00 & C \\
\hline 0.526 & 8.482 & -3.382 & 1.00 & 0.00 & $\mathrm{H}$ \\
\hline 1.954 & 7.877 & -2.541 & 1.00 & 0.00 & $\mathrm{H}$ \\
\hline 0.220 & 7.931 & -1.358 & 1.00 & 0.00 & C \\
\hline-0.008 & 8.454 & -0.260 & 1.00 & 0.00 & o \\
\hline-0.296 & 6.751 & -1.722 & 1.00 & 0.00 & o \\
\hline-0.905 & 6.397 & -0.986 & 1.00 & 0.00 & $\mathrm{H}$ \\
\hline 9.458 & 8.855 & -3.334 & 1.00 & 0.00 & $\mathrm{C}$ \\
\hline 10.011 & 9.694 & -2.884 & 1.00 & 0.00 & $\mathrm{H}$ \\
\hline 9.465 & 8.029 & -2.613 & 1.00 & 0.00 & $\mathrm{H}$ \\
\hline 10.029 & 8.535 & -4.214 & 1.00 & 0.00 & $\mathrm{H}$ \\
\hline 0.025 & 11.803 & -4.285 & 1.00 & 0.00 & C \\
\hline-0.420 & 11.594 & -3.305 & 1.00 & 0.00 & $\mathrm{H}$ \\
\hline-0.086 & 12.883 & -4.460 & 1.00 & 0.00 & $\mathrm{H}$ \\
\hline-0.576 & 11.278 & -5.042 & 1.00 & 0.00 & $\mathrm{H}$ \\
\hline 9.822 & 0.424 & 1.002 & 1.00 & 0.00 & C \\
\hline 10.637 & 1.123 & 0.837 & 1.00 & 0.00 & $\mathrm{H}$ \\
\hline 10.154 & -0.801 & 1.403 & 1.00 & 0.00 & C \\
\hline 9.423 & -1.579 & 1.511 & 1.00 & 0.00 & $\mathrm{H}$ \\
\hline 11.196 & -1.070 & 1.570 & 1.00 & 0.00 & $\mathrm{H}$ \\
\hline 7.158 & 0.169 & 2.840 & 1.00 & 0.00 & C \\
\hline 6.923 & -0.902 & 2.720 & 1.00 & 0.00 & $\mathrm{H}$ \\
\hline 6.345 & 0.633 & 3.410 & 1.00 & 0.00 & $\mathrm{H}$ \\
\hline 8.065 & 0.247 & 3.450 & 1.00 & 0.00 & $\mathrm{H}$ \\
\hline 2.148 & 1.847 & 2.325 & 1.00 & 0.00 & C \\
\hline 2.814 & 1.948 & 3.184 & 1.00 & 0.00 & $\mathrm{H}$ \\
\hline 0.981 & 1.203 & 2.484 & 1.00 & 0.00 & C \\
\hline 0.301 & 1.047 & 1.654 & 1.00 & 0.00 & $\mathrm{H}$ \\
\hline 0.676 & 0.805 & 3.450 & 1.00 & 0.00 & $\mathrm{H}$ \\
\hline 0.463 & 3.530 & 0.121 & 1.00 & 0.00 & C \\
\hline 0.307 & 4.553 & -0.246 & 1.00 & 0.00 & $\mathrm{H}$ \\
\hline 0.031 & 3.465 & 1.126 & 1.00 & 0.00 & $\mathrm{H}$ \\
\hline-0.128 & 2.866 & -0.528 & 1.00 & 0.00 & $\mathrm{H}$ \\
\hline
\end{tabular}


TER

\begin{tabular}{|c|c|c|c|c|c|c|c|c|c|c|}
\hline HETATM & 279 & C5 & $\mathrm{ACP}$ & 30 & 9.552 & 4.612 & -5.770 & 1.00 & 0.00 & C \\
\hline HETATM & 280 & H51 & $\mathrm{ACP}$ & 30 & 8.813 & 4.623 & -6.582 & 1.00 & 0.00 & $\mathrm{H}$ \\
\hline HETATM & 281 & H52 & $\mathrm{ACP}$ & 30 & 10.392 & 4.004 & -6.134 & 1.00 & 0.00 & $\mathrm{H}$ \\
\hline HETATM & 282 & $\mathrm{C} 4$ & $\mathrm{ACP}$ & 30 & 10.030 & 6.050 & -5.497 & 1.00 & 0.00 & C \\
\hline HETATM & 283 & H4 1 & $\mathrm{ACP}$ & 30 & 10.588 & 6.111 & -4.549 & 1.00 & 0.00 & $\mathrm{H}$ \\
\hline HETATM & 284 & $\mathrm{H} 42$ & $\mathrm{ACP}$ & 30 & 9.160 & 6.716 & -5.390 & 1.00 & 0.00 & $\mathrm{H}$ \\
\hline HETATM & 285 & $\mathrm{C} 1$ & $\mathrm{ACP}$ & 30 & 10.894 & 6.662 & -6.573 & 1.00 & 0.00 & C \\
\hline HETATM & 286 & $\mathrm{O} 2$ & $\mathrm{ACP}$ & 30 & 11.122 & 6.139 & -7.671 & 1.00 & 0.00 & 0 \\
\hline HETATM & 287 & 03 & $\mathrm{ACP}$ & 30 & 11.410 & 7.841 & -6.209 & 1.00 & 0.00 & 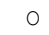 \\
\hline HETATM & 288 & H3 & $\mathrm{ACP}$ & 30 & 12.019 & 8.196 & -6.945 & 1.00 & 0.00 & $\mathrm{H}$ \\
\hline \multicolumn{11}{|l|}{ TER } \\
\hline HETATM & 289 & $\mathrm{C} 1$ & $\mathrm{MET}$ & 31 & 1.655 & 5.738 & -4.597 & 1.00 & 0.00 & C \\
\hline HETATM & 290 & H11 & MET & 31 & 1.103 & 4.899 & -5.047 & 1.00 & 0.00 & $\mathrm{H}$ \\
\hline HETATM & 291 & H12 & $\mathrm{MET}$ & 31 & 1.649 & 6.564 & -5.319 & 1.00 & 0.00 & $\mathrm{H}$ \\
\hline HETATM & 292 & H13 & $\mathrm{MET}$ & 31 & 1.084 & 6.058 & -3.717 & 1.00 & 0.00 & $\mathrm{H}$ \\
\hline \multicolumn{11}{|l|}{ TER } \\
\hline HETATM & 293 & $\mathrm{C} 1$ & MET & 32 & 11.089 & 2.790 & -3.646 & 1.00 & 0.00 & C \\
\hline HETATM & 294 & H11 & $\mathrm{MET}$ & 32 & 11.534 & 2.998 & -4.626 & 1.00 & 0.00 & $\mathrm{H}$ \\
\hline HETATM & 295 & $\mathrm{H} 12$ & $\mathrm{MET}$ & 32 & 11.199 & 1.709 & -3.471 & 1.00 & 0.00 & $\mathrm{H}$ \\
\hline HETATM & 296 & H13 & $\mathrm{MET}$ & 32 & 11.690 & 3.315 & -2.889 & 1.00 & 0.00 & $\mathrm{H}$ \\
\hline \multicolumn{11}{|c|}{ TER } \\
\hline HETATM & 297 & $\mathrm{FE}$ & HEM & 33 & 16.346 & 10.757 & 1.491 & 1.00 & 0.00 & $\mathrm{FE}$ \\
\hline HETATM & 298 & $\mathrm{C} 1$ & HEM & 33 & 14.118 & 12.629 & 0.429 & 1.00 & 0.00 & C \\
\hline HETATM & 299 & $\mathrm{C} 2$ & HEM & 33 & 13.759 & 13.420 & -0.742 & 1.00 & 0.00 & C \\
\hline HETATM & 300 & C3 & HEM & 33 & 14.905 & 13.517 & -1.517 & 1.00 & 0.00 & C \\
\hline HETATM & 301 & $\mathrm{C} 4$ & HEM & 33 & 15.934 & 12.800 & -0.809 & 1.00 & 0.00 & C \\
\hline HETATM & 302 & C5 & HEM & 33 & 17.249 & 12.681 & -1.247 & 1.00 & 0.00 & C \\
\hline HETATM & 303 & H5 & HEM & 33 & 17.503 & 13.177 & -2.182 & 1.00 & 0.00 & $\mathrm{H}$ \\
\hline HETATM & 304 & $\mathrm{C} 6$ & HEM & 33 & 18.269 & 11.982 & -0.611 & 1.00 & 0.00 & C \\
\hline HETATM & 305 & C7 & HEM & 33 & 19.638 & 11.910 & -1.081 & 1.00 & 0.00 & C \\
\hline HETATM & 306 & $\mathrm{C} 8$ & HEM & 33 & 20.343 & 11.190 & -0.134 & 1.00 & 0.00 & C \\
\hline HETATM & 307 & C9 & HEM & 33 & 19.392 & 10.825 & 0.891 & 1.00 & 0.00 & C \\
\hline HETATM & 308 & $\mathrm{C} 10$ & HEM & 33 & 19.697 & 10.090 & 2.034 & 1.00 & 0.00 & C \\
\hline HETATM & 309 & H1O & HEM & 33 & 20.723 & 9.746 & 2.140 & 1.00 & 0.00 & $\mathrm{H}$ \\
\hline HETATM & 310 & C11 & HEM & 33 & 18.822 & 9.750 & 3.057 & 1.00 & 0.00 & C \\
\hline HETATM & 311 & $\mathrm{C} 12$ & HEM & 33 & 19.203 & 9.025 & 4.247 & 1.00 & 0.00 & C \\
\hline HETATM & 312 & C13 & HEM & 33 & 18.064 & 8.878 & 5.004 & 1.00 & 0.00 & C \\
\hline HETATM & 313 & C14 & HEM & 33 & 16.991 & 9.519 & 4.273 & 1.00 & 0.00 & C \\
\hline HETATM & 314 & C15 & HEM & 33 & 15.663 & 9.574 & 4.694 & 1.00 & 0.00 & C \\
\hline HETATM & 315 & H15 & HEM & 33 & 15.423 & 9.089 & 5.642 & 1.00 & 0.00 & $\mathrm{H}$ \\
\hline HETATM & 316 & C16 & HEM & 33 & 14.632 & 10.255 & 4.051 & 1.00 & 0.00 & C \\
\hline HETATM & 317 & $\mathrm{C} 17$ & HEM & 33 & 13.267 & 10.369 & 4.533 & 1.00 & 0.00 & C \\
\hline HETATM & 318 & $\mathrm{C} 18$ & HEM & 33 & 12.591 & 11.137 & 3.609 & 1.00 & 0.00 & C \\
\hline HETATM & 319 & C19 & HEM & 33 & 13.541 & 11.489 & 2.573 & 1.00 & 0.00 & C \\
\hline HETATM & 320 & $\mathrm{C} 20$ & HEM & 33 & 13.250 & 12.280 & 1.462 & 1.00 & 0.00 & C \\
\hline HETATM & 321 & $\mathrm{H} 20$ & HEM & 33 & 12.235 & 12.669 & 1.402 & 1.00 & 0.00 & $\mathrm{H}$ \\
\hline HETATM & 322 & N21 & HEM & 33 & 15.440 & 12.266 & 0.367 & 1.00 & 0.00 & $\mathrm{~N}$ \\
\hline HETATM & 323 & N22 & HEM & 33 & 18.144 & 11.319 & 0.590 & 1.00 & 0.00 & $\mathrm{~N}$ \\
\hline HETATM & 324 & N23 & HEM & 33 & 17.474 & 10.056 & 3.090 & 1.00 & 0.00 & $\mathrm{~N}$ \\
\hline HETATM & 325 & N24 & HEM & 33 & 14.774 & 10.941 & 2.854 & 1.00 & 0.00 & $\mathrm{~N}$ \\
\hline HETATM & 326 & C99 & HEM & 33 & 15.681 & 8.076 & 0.352 & 1.00 & 0.00 & C \\
\hline HETATM & 327 & 091 & HEM & 33 & 15.840 & 9.389 & 0.365 & 1.00 & 0.00 & 0 \\
\hline HETATM & 328 & 092 & HEM & 33 & 15.526 & 7.458 & -0.703 & 1.00 & 0.00 & 0 \\
\hline HETATM & 329 & CT91 & HEM & 33 & 15.697 & 7.394 & 1.709 & 1.00 & 0.00 & C \\
\hline HETATM & 330 & H911 & HEM & 33 & 16.676 & 7.590 & 2.171 & 1.00 & 0.00 & $\mathrm{H}$ \\
\hline HETATM & 331 & H912 & HEM & 33 & 14.949 & 7.884 & 2.352 & 1.00 & 0.00 & $\mathrm{H}$ \\
\hline HETATM & 332 & CT92 & HEM & 33 & 15.437 & 5.890 & 1.597 & 1.00 & 0.00 & C \\
\hline HETATM & 333 & H921 & HEM & 33 & 16.234 & 5.429 & 1.000 & 1.00 & 0.00 & $\mathrm{H}$ \\
\hline HETATM & 334 & H922 & HEM & 33 & 14.512 & 5.734 & 1.027 & 1.00 & 0.00 & $\mathrm{H}$ \\
\hline \multicolumn{11}{|c|}{ TER } \\
\hline HETATM & 335 & $\mathrm{FE}$ & HEM & 34 & 17.038 & 3.343 & 6.440 & 1.00 & 0.00 & $\mathrm{FE}$ \\
\hline HETATM & 336 & $\mathrm{C} 1$ & HEM & 34 & 19.266 & 1.471 & 7.502 & 1.00 & 0.00 & $\mathrm{C}$ \\
\hline HETATM & 337 & $\mathrm{C} 2$ & HEM & 34 & 19.625 & 0.680 & 8.674 & 1.00 & 0.00 & $\mathrm{C}$ \\
\hline HETATM & 338 & C3 & HEM & 34 & 18.479 & 0.583 & 9.448 & 1.00 & 0.00 & C \\
\hline HETATM & 339 & $\mathrm{C} 4$ & HEM & 34 & 17.450 & 1.301 & 8.740 & 1.00 & 0.00 & C \\
\hline HETATM & 340 & C5 & HEM & 34 & 16.135 & 1.420 & 9.178 & 1.00 & 0.00 & C \\
\hline HETATM & 341 & H5 & HEM & 34 & 15.881 & 0.923 & 10.113 & 1.00 & 0.00 & $\mathrm{H}$ \\
\hline HETATM & 342 & $\mathrm{C} 6$ & HEM & 34 & 15.115 & 2.118 & 8.542 & 1.00 & 0.00 & C \\
\hline HETATM & 343 & C7 & HEM & 34 & 13.747 & 2.191 & 9.012 & 1.00 & 0.00 & C \\
\hline HETATM & 344 & $\mathrm{C} 8$ & HEM & 34 & 13.041 & 2.910 & 8.065 & 1.00 & 0.00 & C \\
\hline HETATM & 345 & C9 & HEM & 34 & 13.992 & 3.275 & 7.041 & 1.00 & 0.00 & C \\
\hline HETATM & 346 & $\mathrm{C} 10$ & HEM & 34 & 13.688 & 4.010 & 5.897 & 1.00 & 0.00 & C \\
\hline HETATM & 347 & $\mathrm{H} 10$ & HEM & 34 & 12.661 & 4.354 & 5.791 & 1.00 & 0.00 & $\mathrm{H}$ \\
\hline HETATM & 348 & $\mathrm{C} 11$ & HEM & 34 & 14.562 & 4.350 & 4.874 & 1.00 & 0.00 & C \\
\hline HETATM & 349 & $\mathrm{C} 12$ & HEM & 34 & 14.181 & 5.076 & 3.684 & 1.00 & 0.00 & C \\
\hline HETATM & 350 & C13 & HEM & 34 & 15.321 & 5.223 & 2.927 & 1.00 & 0.00 & C \\
\hline HETATM & 351 & C14 & HEM & 34 & 16.394 & 4.581 & 3.659 & 1.00 & 0.00 & C \\
\hline HETATM & 352 & C15 & HEM & 34 & 17.722 & 4.527 & 3.237 & 1.00 & 0.00 & C \\
\hline
\end{tabular}




\begin{tabular}{|c|c|c|c|c|c|c|c|c|c|c|}
\hline HETATM & 353 & H15 & HEM & 34 & 17.962 & 5.011 & 2.289 & 1.00 & 0.00 & $\mathrm{H}$ \\
\hline HETATM & 354 & $\mathrm{C} 16$ & HEM & 34 & 18.752 & 3.845 & 3.880 & 1.00 & 0.00 & C \\
\hline HETATM & 355 & C17 & HEM & 34 & 20.118 & 3.732 & 3.398 & 1.00 & 0.00 & $\mathrm{C}$ \\
\hline HETATM & 356 & $\mathrm{C} 18$ & HEM & 34 & 20.793 & 2.964 & 4.322 & 1.00 & 0.00 & C \\
\hline HETATM & 357 & C19 & HEM & 34 & 19.843 & 2.612 & 5.358 & 1.00 & 0.00 & C \\
\hline HETATM & 358 & $\mathrm{C} 20$ & HEM & 34 & 20.135 & 1.821 & 6.469 & 1.00 & 0.00 & C \\
\hline HETATM & 359 & $\mathrm{H} 20$ & HEM & 34 & 21.149 & 1.432 & 6.530 & 1.00 & 0.00 & $\mathrm{H}$ \\
\hline HETATM & 360 & N21 & HEM & 34 & 17.944 & 1.834 & 7.564 & 1.00 & 0.00 & $\mathrm{~N}$ \\
\hline HETATM & 361 & N22 & HEM & 34 & 15.240 & 2.781 & 7.342 & 1.00 & 0.00 & $\mathrm{~N}$ \\
\hline HETATM & 362 & N23 & HEM & 34 & 15.911 & 4.044 & 4.841 & 1.00 & 0.00 & $\mathrm{~N}$ \\
\hline HETATM & 363 & $\mathrm{~N} 24$ & HEM & 34 & 18.610 & 3.160 & 5.077 & 1.00 & 0.00 & $\mathrm{~N}$ \\
\hline HETATM & 364 & C99 & HEM & 34 & 17.704 & 6.025 & 7.579 & 1.00 & 0.00 & C \\
\hline HETATM & 365 & 091 & HEM & 34 & 17.544 & 4.711 & 7.567 & 1.00 & 0.00 & 0 \\
\hline HETATM & 366 & 092 & HEM & 34 & 17.858 & 6.642 & 8.635 & 1.00 & 0.00 & 0 \\
\hline HETATM & 367 & СТ 91 & HEM & 34 & 17.688 & 6.706 & 6.223 & 1.00 & 0.00 & C \\
\hline HETATM & 368 & H911 & HEM & 34 & 16.709 & 6.511 & 5.760 & 1.00 & 0.00 & $\mathrm{H}$ \\
\hline HETATM & 369 & H912 & HEM & 34 & 18.436 & 6.216 & 5.580 & 1.00 & 0.00 & $\mathrm{H}$ \\
\hline HETATM & 370 & СТ 92 & HEM & 34 & 17.947 & 8.211 & 6.334 & 1.00 & 0.00 & C \\
\hline HETATM & 371 & H921 & HEM & 34 & 18.872 & 8.367 & 6.905 & 1.00 & 0.00 & $\mathrm{H}$ \\
\hline HETATM & 372 & H922 & HEM & 34 & 17.151 & 8.671 & 6.931 & 1.00 & 0.00 & $\mathrm{H}$ \\
\hline \multicolumn{11}{|l|}{ TER } \\
\hline HETATM & 373 & $\mathrm{C} 2$ & VIN & 35 & 12.427 & 13.923 & -1.002 & 1.00 & 0.00 & C \\
\hline HETATM & 374 & H2 1 & VIN & 35 & 11.612 & 13.224 & -0.837 & 1.00 & 0.00 & $\mathrm{H}$ \\
\hline HETATM & 375 & $\mathrm{C} 1$ & VIN & 35 & 12.095 & 15.147 & -1.403 & 1.00 & 0.00 & C \\
\hline HETATM & 376 & H11 & VIN & 35 & 12.826 & 15.926 & -1.511 & 1.00 & 0.00 & $\mathrm{H}$ \\
\hline HETATM & 377 & $\mathrm{H} 12$ & VIN & 35 & 11.053 & 15.417 & -1.570 & 1.00 & 0.00 & $\mathrm{H}$ \\
\hline \multicolumn{11}{|l|}{ TER } \\
\hline HETATM & 378 & $\mathrm{C} 1$ & MET & 36 & 15.091 & 14.177 & -2.840 & 1.00 & 0.00 & $\mathrm{C}$ \\
\hline HETATM & 379 & H11 & MET & 36 & 15.326 & 15.248 & -2.720 & 1.00 & 0.00 & $\mathrm{H}$ \\
\hline HETATM & 380 & $\mathrm{H} 12$ & MET & 36 & 15.904 & 13.713 & -3.410 & 1.00 & 0.00 & $\mathrm{H}$ \\
\hline HETATM & 381 & $\mathrm{H} 13$ & MET & 36 & 14.184 & 14.100 & -3.450 & 1.00 & 0.00 & $\mathrm{H}$ \\
\hline \multicolumn{11}{|l|}{ TER } \\
\hline HETATM & 382 & $\mathrm{C} 2$ & VIN & 37 & 20.101 & 12.499 & -2.325 & 1.00 & 0.00 & C \\
\hline HETATM & 383 & H 21 & VIN & 37 & 19.435 & 12.399 & -3.184 & 1.00 & 0.00 & $\mathrm{H}$ \\
\hline HETATM & 384 & $\mathrm{C} 1$ & VIN & 37 & 21.268 & 13.144 & -2.484 & 1.00 & 0.00 & C \\
\hline HETATM & 385 & H11 & VIN & 37 & 21.948 & 13.300 & -1.654 & 1.00 & 0.00 & $\mathrm{H}$ \\
\hline HETATM & 386 & $\mathrm{H} 12$ & VIN & 37 & 21.573 & 13.542 & -3.450 & 1.00 & 0.00 & $\mathrm{H}$ \\
\hline \multicolumn{11}{|l|}{ TER } \\
\hline HETATM & 387 & $\mathrm{C} 1$ & MET & 38 & 21.786 & 10.816 & -0.121 & 1.00 & 0.00 & C \\
\hline HETATM & 388 & H11 & MET & 38 & 22.377 & 11.480 & 0.528 & 1.00 & 0.00 & $\mathrm{H}$ \\
\hline HETATM & 389 & $\mathrm{H} 12$ & MET & 38 & 21.942 & 9.794 & 0.246 & 1.00 & 0.00 & $\mathrm{H}$ \\
\hline HETATM & 390 & H13 & MET & 38 & 22.218 & 10.882 & -1.126 & 1.00 & 0.00 & $\mathrm{H}$ \\
\hline TER & & & & & & & & & & \\
\hline HETATM & 391 & C5 & $\mathrm{ACP}$ & 39 & 12.697 & 9.735 & 5.770 & 1.00 & 0.00 & C \\
\hline HETATM & 392 & H51 & $\mathrm{ACP}$ & 39 & 13.436 & 9.723 & 6.582 & 1.00 & 0.00 & $\mathrm{H}$ \\
\hline HETATM & 393 & H52 & $\mathrm{ACP}$ & 39 & 11.857 & 10.343 & 6.134 & 1.00 & 0.00 & $\mathrm{H}$ \\
\hline HETATM & 394 & $\mathrm{C} 4$ & $\mathrm{ACP}$ & 39 & 12.219 & 8.297 & 5.497 & 1.00 & 0.00 & C \\
\hline HETATM & 395 & $\mathrm{H} 41$ & $\mathrm{ACP}$ & 39 & 11.661 & 8.236 & 4.549 & 1.00 & 0.00 & $\mathrm{H}$ \\
\hline HETATM & 396 & $\mathrm{H} 42$ & $\mathrm{ACP}$ & 39 & 13.089 & 7.630 & 5.390 & 1.00 & 0.00 & $\mathrm{H}$ \\
\hline HETATM & 397 & $\mathrm{C} 1$ & $\mathrm{ACP}$ & 39 & 11.355 & 7.685 & 6.573 & 1.00 & 0.00 & C \\
\hline HETATM & 398 & $\mathrm{O} 2$ & $\mathrm{ACP}$ & 39 & 11.127 & 8.208 & 7.671 & 1.00 & 0.00 & 0 \\
\hline HETATM & 399 & 03 & $\mathrm{ACP}$ & 39 & 10.839 & 6.505 & 6.209 & 1.00 & 0.00 & 0 \\
\hline HETATM & 400 & H3 & $\mathrm{ACP}$ & 39 & 10.230 & 6.151 & 6.945 & 1.00 & 0.00 & $\mathrm{H}$ \\
\hline TER & & & & & & & & & & \\
\hline HETATM & 401 & $\mathrm{C} 1$ & MET & 40 & 20.594 & 8.608 & 4.597 & 1.00 & 0.00 & C \\
\hline HETATM & 402 & $\mathrm{H} 11$ & MET & 40 & 21.146 & 9.448 & 5.047 & 1.00 & 0.00 & $\mathrm{H}$ \\
\hline HETATM & 403 & $\mathrm{H} 12$ & MET & 40 & 20.600 & 7.782 & 5.319 & 1.00 & 0.00 & $\mathrm{H}$ \\
\hline HETATM & 404 & $\mathrm{H} 13$ & MET & 40 & 21.165 & 8.289 & 3.717 & 1.00 & 0.00 & $\mathrm{H}$ \\
\hline TER & & & & & & & & & & \\
\hline HETATM & 405 & $\mathrm{C} 1$ & MET & 41 & 11.160 & 11.557 & 3.646 & 1.00 & 0.00 & C \\
\hline HETATM & 406 & H11 & MET & 41 & 10.715 & 11.348 & 4.626 & 1.00 & 0.00 & $\mathrm{H}$ \\
\hline HETATM & 407 & $\mathrm{H} 12$ & MET & 41 & 11.050 & 12.637 & 3.471 & 1.00 & 0.00 & $\mathrm{H}$ \\
\hline HETATM & 408 & $\mathrm{H} 13$ & MET & 41 & 10.559 & 11.031 & 2.889 & 1.00 & 0.00 & $\mathrm{H}$ \\
\hline TER & & & & & & & & & & \\
\hline HETATM & 409 & $\mathrm{C} 2$ & VIN & 42 & 20.957 & 0.178 & 8.933 & 1.00 & 0.00 & C \\
\hline HETATM & 410 & H2 1 & VIN & 42 & 21.773 & 0.876 & 8.768 & 1.00 & 0.00 & $\mathrm{H}$ \\
\hline HETATM & 411 & $\mathrm{C} 1$ & VIN & 42 & 21.290 & -1.047 & 9.335 & 1.00 & 0.00 & C \\
\hline HETATM & 412 & $\mathrm{H} 11$ & VIN & 42 & 20.558 & -1.826 & 9.442 & 1.00 & 0.00 & $\mathrm{H}$ \\
\hline HETATM & 413 & $\mathrm{H} 12$ & VIN & 42 & 22.331 & -1.316 & 9.501 & 1.00 & 0.00 & $\mathrm{H}$ \\
\hline TER & & & & & & & & & & \\
\hline HETATM & 414 & $\mathrm{C} 1$ & MET & 43 & 18.293 & -0.077 & 10.771 & 1.00 & 0.00 & C \\
\hline HETATM & 415 & $\mathrm{H} 11$ & MET & 43 & 18.058 & -1.148 & 10.652 & 1.00 & 0.00 & $\mathrm{H}$ \\
\hline HETATM & 416 & $\mathrm{H} 12$ & MET & 43 & 17.480 & 0.387 & 11.341 & 1.00 & 0.00 & $\mathrm{H}$ \\
\hline HETATM & 417 & H13 & MET & 43 & 19.201 & 0.001 & 11.382 & 1.00 & 0.00 & $\mathrm{H}$ \\
\hline TER & & & & & & & & & & \\
\hline HETATM & 418 & $\mathrm{C} 2$ & VIN & 44 & 13.283 & 1.601 & 10.256 & 1.00 & 0.00 & C \\
\hline HETATM & 419 & $\mathrm{H} 21$ & VIN & 44 & 13.949 & 1.702 & 11.115 & 1.00 & 0.00 & $\mathrm{H}$ \\
\hline HETATM & 420 & $\mathrm{C} 1$ & VIN & 44 & 12.116 & 0.957 & 10.415 & 1.00 & 0.00 & C \\
\hline HETATM & 421 & H11 & VIN & 44 & 11.436 & 0.800 & 9.585 & 1.00 & 0.00 & 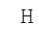 \\
\hline
\end{tabular}




\begin{tabular}{|c|c|c|c|c|c|c|c|c|c|c|}
\hline $\begin{array}{l}\text { HETATM } \\
\text { TER }\end{array}$ & 422 & $\mathrm{H} 12$ & VIN & 44 & 11.811 & 0.559 & 11.381 & 1.00 & 0.00 & $\mathrm{H}$ \\
\hline HETATM & 423 & $\mathrm{C} 1$ & MET & 45 & 11.598 & 3.284 & 8.052 & 1.00 & 0.00 & $\mathrm{C}$ \\
\hline HETATM & 424 & H11 & MET & 45 & 11.442 & 4.306 & 7.685 & 1.00 & 0.00 & $\mathrm{H}$ \\
\hline HETATM & 425 & $\mathrm{H} 12$ & $\mathrm{MET}$ & 45 & 11.166 & 3.219 & 9.057 & 1.00 & 0.00 & $\mathrm{H}$ \\
\hline HETATM & 426 & H13 & MET & 45 & 11.007 & 2.620 & 7.403 & 1.00 & 0.00 & 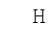 \\
\hline \multicolumn{11}{|l|}{ TER } \\
\hline HETATM & 427 & C5 & $\mathrm{ACP}$ & 46 & 20.687 & 4.366 & 2.161 & 1.00 & 0.00 & C \\
\hline HETATM & 428 & H51 & $\mathrm{ACP}$ & 46 & 19.948 & 4.377 & 1.349 & 1.00 & 0.00 & $\mathrm{H}$ \\
\hline HETATM & 429 & H52 & $\mathrm{ACP}$ & 46 & 21.527 & 3.758 & 1.798 & 1.00 & 0.00 & $\mathrm{H}$ \\
\hline HETATM & 430 & $\mathrm{C} 4$ & $\mathrm{ACP}$ & 46 & 21.165 & 5.803 & 2.434 & 1.00 & 0.00 & C \\
\hline HETATM & 431 & $\mathrm{H} 41$ & $\mathrm{ACP}$ & 46 & 21.723 & 5.865 & 3.382 & 1.00 & 0.00 & $\mathrm{H}$ \\
\hline HETATM & 432 & $\mathrm{H} 42$ & $\mathrm{ACP}$ & 46 & 20.295 & 6.470 & 2.541 & 1.00 & 0.00 & $\mathrm{H}$ \\
\hline HETATM & 433 & $\mathrm{C} 1$ & $\mathrm{ACP}$ & 46 & 22.029 & 6.416 & 1.358 & 1.00 & 0.00 & C \\
\hline HETATM & 434 & $\mathrm{O} 2$ & $\mathrm{ACP}$ & 46 & 22.257 & 5.893 & 0.260 & 1.00 & 0.00 & o \\
\hline HETATM & 435 & 03 & $\mathrm{ACP}$ & 46 & 22.545 & 7.595 & 1.722 & 1.00 & 0.00 & O \\
\hline HETATM & 436 & H3 & $\mathrm{ACP}$ & 46 & 23.154 & 7.949 & 0.986 & 1.00 & 0.00 & $\mathrm{H}$ \\
\hline \multicolumn{11}{|l|}{ TER } \\
\hline HETATM & 437 & $\mathrm{C} 1$ & MET & 47 & 12.791 & 5.492 & 3.334 & 1.00 & 0.00 & C \\
\hline HETATM & 438 & H11 & MET & 47 & 12.238 & 4.653 & 2.884 & 1.00 & 0.00 & $\mathrm{H}$ \\
\hline HETATM & 439 & $\mathrm{H} 12$ & MET & 47 & 12.784 & 6.318 & 2.613 & 1.00 & 0.00 & $\mathrm{H}$ \\
\hline HETATM & 440 & $\mathrm{H} 13$ & MET & 47 & 12.220 & 5.812 & 4.214 & 1.00 & 0.00 & $\mathrm{H}$ \\
\hline \multicolumn{11}{|c|}{ TER } \\
\hline HETATM & 441 & $\mathrm{C} 1$ & MET & 48 & 22.224 & 2.543 & 4.285 & 1.00 & 0.00 & C \\
\hline HETATM & 442 & H11 & MET & 48 & 22.669 & 2.752 & 3.305 & 1.00 & 0.00 & $\mathrm{H}$ \\
\hline HETATM & 443 & $\mathrm{H} 12$ & MET & 48 & 22.335 & 1.463 & 4.460 & 1.00 & 0.00 & $\mathrm{H}$ \\
\hline HETATM & 444 & $\mathrm{H} 13$ & MET & 48 & 22.825 & 3.069 & 5.042 & 1.00 & 0.00 & $\mathrm{H}$ \\
\hline \multicolumn{11}{|c|}{ TER } \\
\hline HETATM & 445 & $\mathrm{FE}$ & HEM & 49 & 17.294 & 11.003 & -6.440 & 1.00 & 0.00 & $\mathrm{FE}$ \\
\hline HETATM & 446 & $\mathrm{C} 1$ & HEM & 49 & 15.066 & 12.876 & -7.502 & 1.00 & 0.00 & C \\
\hline HETATM & 447 & $\mathrm{C} 2$ & HEM & 49 & 14.707 & 13.666 & -8.674 & 1.00 & 0.00 & C \\
\hline HETATM & 448 & $\mathrm{C} 3$ & HEM & 49 & 15.852 & 13.763 & -9.448 & 1.00 & 0.00 & $\mathrm{C}$ \\
\hline HETATM & 449 & $\mathrm{C} 4$ & HEM & 49 & 16.882 & 13.046 & -8.740 & 1.00 & 0.00 & $\mathrm{C}$ \\
\hline HETATM & 450 & C5 & HEM & 49 & 18.197 & 12.927 & -9.178 & 1.00 & 0.00 & C \\
\hline HETATM & 451 & H5 & HEM & 49 & 18.451 & 13.423 & -10.113 & 1.00 & 0.00 & $\mathrm{H}$ \\
\hline HETATM & 452 & $\mathrm{C} 6$ & HEM & 49 & 19.216 & 12.229 & -8.542 & 1.00 & 0.00 & C \\
\hline HETATM & 453 & $\mathrm{C} 7$ & HEM & 49 & 20.585 & 12.156 & -9.012 & 1.00 & 0.00 & C \\
\hline HETATM & 454 & $\mathrm{C} 8$ & HEM & 49 & 21.291 & 11.436 & -8.065 & 1.00 & 0.00 & C \\
\hline HETATM & 455 & $\mathrm{C} 9$ & HEM & 49 & 20.340 & 11.071 & -7.041 & 1.00 & 0.00 & C \\
\hline HETATM & 456 & $\mathrm{C} 10$ & HEM & 49 & 20.644 & 10.336 & -5.897 & 1.00 & 0.00 & C \\
\hline HETATM & 457 & $\mathrm{H} 10$ & HEM & 49 & 21.671 & 9.993 & -5.791 & 1.00 & 0.00 & $\mathrm{H}$ \\
\hline HETATM & 458 & $\mathrm{C} 11$ & HEM & 49 & 19.770 & 9.996 & -4.874 & 1.00 & 0.00 & C \\
\hline HETATM & 459 & $\mathrm{C} 12$ & HEM & 49 & 20.151 & 9.271 & -3.684 & 1.00 & 0.00 & C \\
\hline HETATM & 460 & $\mathrm{C} 13$ & HEM & 49 & 19.011 & 9.124 & -2.927 & 1.00 & 0.00 & C \\
\hline HETATM & 461 & C14 & HEM & 49 & 17.938 & 9.765 & -3.659 & 1.00 & 0.00 & C \\
\hline HETATM & 462 & $\mathrm{C} 15$ & HEM & 49 & 16.610 & 9.820 & -3.237 & 1.00 & 0.00 & C \\
\hline HETATM & 463 & H15 & HEM & 49 & 16.370 & 9.335 & -2.289 & 1.00 & 0.00 & $\mathrm{H}$ \\
\hline HETATM & 464 & $\mathrm{C} 16$ & HEM & 49 & 15.580 & 10.502 & -3.880 & 1.00 & 0.00 & C \\
\hline HETATM & 465 & $\mathrm{C} 17$ & HEM & 49 & 14.214 & 10.615 & -3.398 & 1.00 & 0.00 & $\mathrm{C}$ \\
\hline HETATM & 466 & $\mathrm{C} 18$ & HEM & 49 & 13.539 & 11.383 & -4.322 & 1.00 & 0.00 & C \\
\hline HETATM & 467 & C19 & HEM & 49 & 14.488 & 11.735 & -5.358 & 1.00 & 0.00 & C \\
\hline HETATM & 468 & $\mathrm{C} 20$ & HEM & 49 & 14.197 & 12.526 & -6.469 & 1.00 & 0.00 & C \\
\hline HETATM & 469 & $\mathrm{H} 20$ & HEM & 49 & 13.183 & 12.915 & -6.530 & 1.00 & 0.00 & $\mathrm{H}$ \\
\hline HETATM & 470 & $\mathrm{~N} 21$ & HEM & 49 & 16.387 & 12.513 & -7.564 & 1.00 & 0.00 & $\mathrm{~N}$ \\
\hline HETATM & 471 & $\mathrm{~N} 22$ & HEM & 49 & 19.092 & 11.566 & -7.342 & 1.00 & 0.00 & $\mathrm{~N}$ \\
\hline HETATM & 472 & $\mathrm{~N} 23$ & HEM & 49 & 18.421 & 10.303 & -4.841 & 1.00 & 0.00 & $\mathrm{~N}$ \\
\hline HETATM & 473 & $\mathrm{~N} 24$ & HEM & 49 & 15.722 & 11.187 & -5.077 & 1.00 & 0.00 & $\mathrm{~N}$ \\
\hline HETATM & 474 & C99 & HEM & 49 & 16.628 & 8.322 & -7.579 & 1.00 & 0.00 & C \\
\hline HETATM & 475 & 091 & HEM & 49 & 16.788 & 9.635 & -7.567 & 1.00 & 0.00 & 0 \\
\hline HETATM & 476 & 092 & HEM & 49 & 16.473 & 7.705 & -8.635 & 1.00 & 0.00 & O \\
\hline HETATM & 477 & СТ91 & HEM & 49 & 16.644 & 7.640 & -6.223 & 1.00 & 0.00 & C \\
\hline HETATM & 478 & H911 & HEM & 49 & 17.623 & 7.836 & -5.760 & 1.00 & 0.00 & $\mathrm{H}$ \\
\hline HETATM & 479 & H912 & HEM & 49 & 15.896 & 8.130 & -5.580 & 1.00 & 0.00 & $\mathrm{H}$ \\
\hline HETATM & 480 & СТ 92 & HEM & 49 & 16.385 & 6.136 & -6.334 & 1.00 & 0.00 & C \\
\hline HETATM & 481 & H921 & HEM & 49 & 17.181 & 5.676 & -6.931 & 1.00 & 0.00 & $\mathrm{H}$ \\
\hline HETATM & 482 & H922 & HEM & 49 & 15.460 & 5.980 & -6.905 & 1.00 & 0.00 & $\mathrm{H}$ \\
\hline \multicolumn{11}{|c|}{ TER } \\
\hline HETATM & 483 & $\mathrm{FE}$ & HEM & 50 & 17.986 & 3.589 & -1.491 & 1.00 & 0.00 & $\mathrm{FE}$ \\
\hline HETATM & 484 & $\mathrm{C} 1$ & HEM & 50 & 20.214 & 1.717 & -0.429 & 1.00 & 0.00 & $\mathrm{C}$ \\
\hline HETATM & 485 & $\mathrm{C} 2$ & HEM & 50 & 20.573 & 0.927 & 0.742 & 1.00 & 0.00 & $\mathrm{C}$ \\
\hline HETATM & 486 & $\mathrm{C} 3$ & HEM & 50 & 19.427 & 0.830 & 1.517 & 1.00 & 0.00 & $\mathrm{C}$ \\
\hline HETATM & 487 & $\mathrm{C} 4$ & HEM & 50 & 18.398 & 1.547 & 0.809 & 1.00 & 0.00 & $\mathrm{C}$ \\
\hline HETATM & 488 & C5 & HEM & 50 & 17.082 & 1.666 & 1.247 & 1.00 & 0.00 & $\mathrm{C}$ \\
\hline HETATM & 489 & H5 & HEM & 50 & 16.829 & 1.170 & 2.182 & 1.00 & 0.00 & $\mathrm{H}$ \\
\hline HETATM & 490 & $\mathrm{C} 6$ & HEM & 50 & 16.063 & 2.364 & 0.611 & 1.00 & 0.00 & C \\
\hline HETATM & 491 & $\mathrm{C} 7$ & HEM & 50 & 14.694 & 2.437 & 1.081 & 1.00 & 0.00 & C \\
\hline HETATM & 492 & $\mathrm{C} 8$ & HEM & 50 & 13.989 & 3.157 & 0.134 & 1.00 & 0.00 & C \\
\hline HETATM & 493 & $\mathrm{C} 9$ & HEM & 50 & 14.940 & 3.522 & -0.891 & 1.00 & 0.00 & $\mathrm{C}$ \\
\hline HETATM & 494 & $\mathrm{C} 10$ & HEM & 50 & 14.635 & 4.256 & -2.034 & 1.00 & 0.00 & C \\
\hline
\end{tabular}




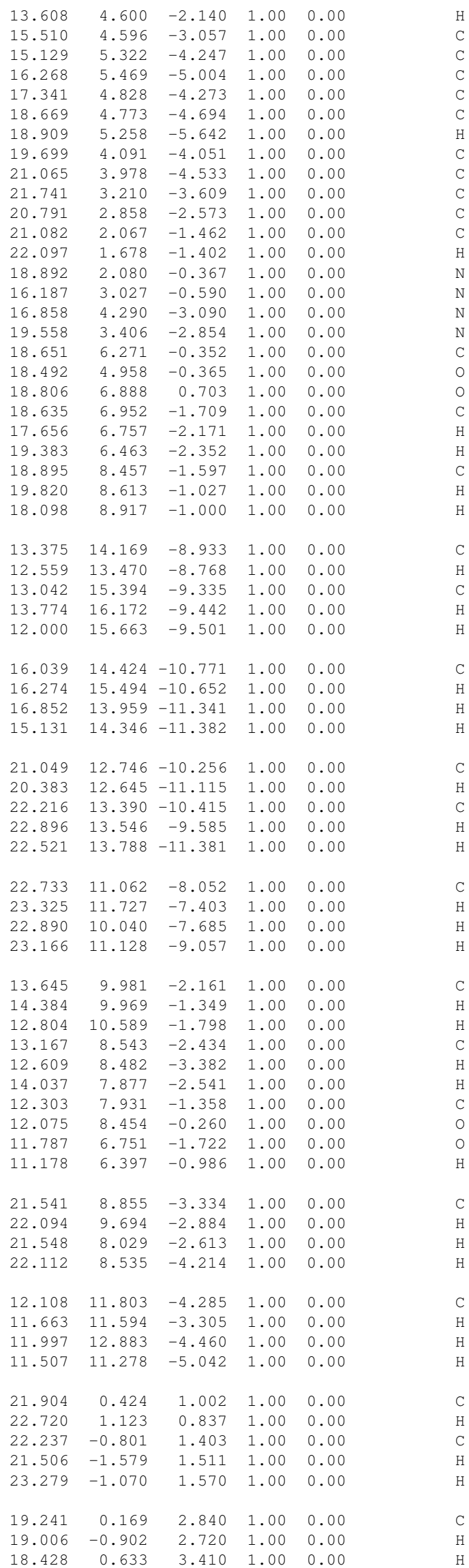




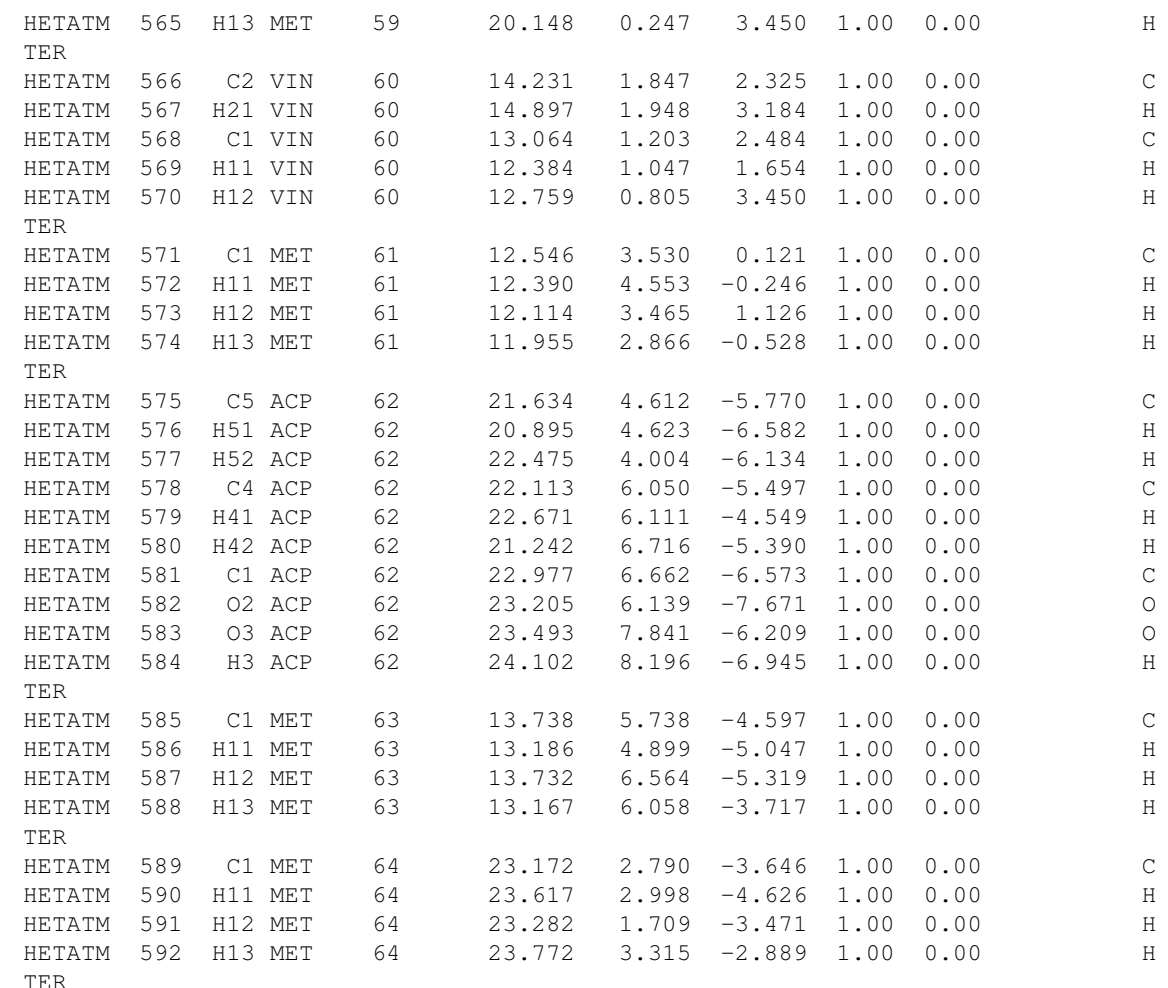

\subsection{Q4 dimer: $A Y V \_Q 4 . p d b$ file}

\begin{tabular}{|c|c|c|c|c|c|c|c|c|c|c|}
\hline HETATM & 1 & $\mathrm{FE}$ & HEM & 1 & 4.263 & 10.757 & 1.491 & 1.00 & 0.00 & $\mathrm{FE}$ \\
\hline HETATM & 2 & $\mathrm{C} 1$ & HEM & 1 & 2.035 & 12.629 & 0.429 & 1.00 & 0.00 & C \\
\hline HETATM & 3 & $\mathrm{C} 2$ & HEM & 1 & 1.676 & 13.420 & -0.742 & 1.00 & 0.00 & C \\
\hline HETATM & 4 & $\mathrm{C} 3$ & HEM & 1 & 2.822 & 13.517 & -1.517 & 1.00 & 0.00 & C \\
\hline HETATM & 5 & $\mathrm{C} 4$ & HEM & 1 & 3.851 & 12.800 & -0.809 & 1.00 & 0.00 & C \\
\hline HETATM & 6 & C5 & HEM & 1 & 5.167 & 12.681 & -1.247 & 1.00 & 0.00 & C \\
\hline HETATM & 7 & H5 & HEM & 1 & 5.420 & 13.177 & -2.182 & 1.00 & 0.00 & $\mathrm{H}$ \\
\hline HETATM & 8 & $\mathrm{C} 6$ & HEM & 1 & 6.186 & 11.982 & -0.611 & 1.00 & 0.00 & C \\
\hline HETATM & 9 & C7 & HEM & 1 & 7.555 & 11.910 & -1.081 & 1.00 & 0.00 & C \\
\hline HETATM & 10 & $\mathrm{C} 8$ & HEM & 1 & 8.260 & 11.190 & -0.134 & 1.00 & 0.00 & C \\
\hline HETATM & 11 & $\mathrm{C} 9$ & HEM & 1 & 7.309 & 10.825 & 0.891 & 1.00 & 0.00 & C \\
\hline HETATM & 12 & $\mathrm{C} 10$ & HEM & 1 & 7.614 & 10.090 & 2.034 & 1.00 & 0.00 & C \\
\hline HETATM & 13 & $\mathrm{H} 10$ & HEM & 1 & 8.641 & 9.746 & 2.140 & 1.00 & 0.00 & $\mathrm{H}$ \\
\hline HETATM & 14 & $\mathrm{C} 11$ & HEM & 1 & 6.739 & 9.750 & 3.057 & 1.00 & 0.00 & $\mathrm{C}$ \\
\hline HETATM & 15 & $\mathrm{C} 12$ & HEM & 1 & 7.120 & 9.025 & 4.247 & 1.00 & 0.00 & C \\
\hline HETATM & 16 & $\mathrm{C} 13$ & HEM & 1 & 5.981 & 8.878 & 5.004 & 1.00 & 0.00 & C \\
\hline HETATM & 17 & $\mathrm{C} 14$ & HEM & 1 & 4.908 & 9.519 & 4.273 & 1.00 & 0.00 & C \\
\hline HETATM & 18 & $\mathrm{C} 15$ & HEM & 1 & 3.580 & 9.574 & 4.694 & 1.00 & 0.00 & $\mathrm{C}$ \\
\hline HETATM & 19 & H15 & HEM & 1 & 3.340 & 9.089 & 5.642 & 1.00 & 0.00 & $\mathrm{H}$ \\
\hline HETATM & 20 & $\mathrm{C} 16$ & HEM & 1 & 2.550 & 10.255 & 4.051 & 1.00 & 0.00 & $\mathrm{C}$ \\
\hline HETATM & 21 & $\mathrm{C} 17$ & HEM & 1 & 1.184 & 10.369 & 4.533 & 1.00 & 0.00 & C \\
\hline HETATM & 22 & $\mathrm{C} 18$ & HEM & 1 & 0.508 & 11.137 & 3.609 & 1.00 & 0.00 & $\mathrm{C}$ \\
\hline HETATM & 23 & C19 & HEM & 1 & 1.458 & 11.489 & 2.573 & 1.00 & 0.00 & C \\
\hline HETATM & 24 & $\mathrm{C} 20$ & HEM & 1 & 1.167 & 12.280 & 1.462 & 1.00 & 0.00 & $\mathrm{C}$ \\
\hline HETATM & 25 & $\mathrm{H} 2 \mathrm{O}$ & HEM & 1 & 0.152 & 12.669 & 1.402 & 1.00 & 0.00 & $\mathrm{H}$ \\
\hline HETATM & 26 & $\mathrm{~N} 21$ & HEM & 1 & 3.357 & 12.266 & 0.367 & 1.00 & 0.00 & $\mathrm{~N}$ \\
\hline HETATM & 27 & $\mathrm{~N} 22$ & HEM & 1 & 6.062 & 11.319 & 0.590 & 1.00 & 0.00 & $\mathrm{~N}$ \\
\hline HETATM & 28 & N23 & HEM & 1 & 5.391 & 10.056 & 3.090 & 1.00 & 0.00 & $\mathrm{~N}$ \\
\hline HETATM & 29 & N2 4 & HEM & 1 & 2.691 & 10.941 & 2.854 & 1.00 & 0.00 & $\mathrm{~N}$ \\
\hline HETATM & 30 & C99 & HEM & 1 & 3.598 & 8.076 & 0.352 & 1.00 & 0.00 & C \\
\hline HETATM & 31 & 091 & HEM & 1 & 3.757 & 9.389 & 0.365 & 1.00 & 0.00 & 0 \\
\hline HETATM & 32 & 092 & HEM & 1 & 3.443 & 7.458 & -0.703 & 1.00 & 0.00 & o \\
\hline HETATM & 33 & CT91 & HEM & 1 & 3.614 & 7.394 & 1.709 & 1.00 & 0.00 & $\mathrm{C}$ \\
\hline HETATM & 34 & H911 & HEM & 1 & 4.593 & 7.590 & 2.171 & 1.00 & 0.00 & $\mathrm{H}$ \\
\hline HETATM & 35 & H912 & HEM & 1 & 2.866 & 7.884 & 2.352 & 1.00 & 0.00 & $\mathrm{H}$ \\
\hline HETATM & 36 & СТ92 & HEM & 1 & 3.354 & 5.890 & 1.597 & 1.00 & 0.00 & C \\
\hline HETATM & 37 & H921 & HEM & 1 & 4.151 & 5.429 & 1.000 & 1.00 & 0.00 & $\mathrm{H}$ \\
\hline HETATM & 38 & H922 & HEM & 1 & 2.429 & 5.734 & 1.027 & 1.00 & 0.00 & $\mathrm{H}$ \\
\hline \multicolumn{11}{|l|}{ TER } \\
\hline HETATM & 39 & $\mathrm{FE}$ & HEM & 2 & 4.955 & 3.343 & 6.440 & 1.00 & 0.00 & $\mathrm{FE}$ \\
\hline
\end{tabular}




\begin{tabular}{|c|c|c|c|c|c|c|c|c|c|c|}
\hline HETATM & 40 & $\mathrm{C} 1$ & HEM & 2 & 7.183 & 1.471 & 7.502 & 1.00 & 0.00 & C \\
\hline HETATM & 41 & $\mathrm{C} 2$ & HEM & 2 & 7.542 & 0.680 & 8.674 & 1.00 & 0.00 & C \\
\hline HETATM & 42 & $\mathrm{C} 3$ & HEM & 2 & 6.397 & 0.583 & 9.448 & 1.00 & 0.00 & C \\
\hline HETATM & 43 & $\mathrm{C} 4$ & HEM & 2 & 5.367 & 1.301 & 8.740 & 1.00 & 0.00 & C \\
\hline HETATM & 44 & C5 & HEM & 2 & 4.052 & 1.420 & 9.178 & 1.00 & 0.00 & C \\
\hline HETATM & 45 & H5 & HEM & 2 & 3.798 & 0.923 & 10.113 & 1.00 & 0.00 & $\mathrm{H}$ \\
\hline HETATM & 46 & $\mathrm{C} 6$ & HEM & 2 & 3.033 & 2.118 & 8.542 & 1.00 & 0.00 & C \\
\hline HETATM & 47 & C7 & HEM & 2 & 1.664 & 2.191 & 9.012 & 1.00 & 0.00 & C \\
\hline HETATM & 48 & $\mathrm{C} 8$ & HEM & 2 & 0.958 & 2.910 & 8.065 & 1.00 & 0.00 & $\mathrm{C}$ \\
\hline HETATM & 49 & C9 & HEM & 2 & 1.909 & 3.275 & 7.041 & 1.00 & 0.00 & $\mathrm{C}$ \\
\hline HETATM & 50 & $\mathrm{C} 10$ & HEM & 2 & 1.605 & 4.010 & 5.897 & 1.00 & 0.00 & C \\
\hline HETATM & 51 & $\mathrm{H} 10$ & HEM & 2 & 0.578 & 4.354 & 5.791 & 1.00 & 0.00 & $\mathrm{H}$ \\
\hline HETATM & 52 & $\mathrm{C} 11$ & HEM & 2 & 2.479 & 4.350 & 4.874 & 1.00 & 0.00 & C \\
\hline HETATM & 53 & $\mathrm{C} 12$ & HEM & 2 & 2.098 & 5.076 & 3.684 & 1.00 & 0.00 & C \\
\hline HETATM & 54 & $\mathrm{C} 13$ & HEM & 2 & 3.238 & 5.223 & 2.927 & 1.00 & 0.00 & C \\
\hline HETATM & 55 & C14 & HEM & 2 & 4.311 & 4.581 & 3.659 & 1.00 & 0.00 & C \\
\hline HETATM & 56 & C15 & HEM & 2 & 5.639 & 4.527 & 3.237 & 1.00 & 0.00 & C \\
\hline HETATM & 57 & H1 5 & HEM & 2 & 5.879 & 5.011 & 2.289 & 1.00 & 0.00 & $\mathrm{H}$ \\
\hline HETATM & 58 & C16 & HEM & 2 & 6.669 & 3.845 & 3.880 & 1.00 & 0.00 & C \\
\hline HETATM & 59 & C17 & HEM & 2 & 8.035 & 3.732 & 3.398 & 1.00 & 0.00 & C \\
\hline HETATM & 60 & C18 & HEM & 2 & 8.710 & 2.964 & 4.322 & 1.00 & 0.00 & C \\
\hline HETATM & 61 & C19 & HEM & 2 & 7.761 & 2.612 & 5.358 & 1.00 & 0.00 & C \\
\hline HETATM & 62 & $\mathrm{C} 20$ & HEM & 2 & 8.052 & 1.821 & 6.469 & 1.00 & 0.00 & C \\
\hline HETATM & 63 & $\mathrm{H} 20$ & HEM & 2 & 9.066 & 1.432 & 6.530 & 1.00 & 0.00 & $\mathrm{H}$ \\
\hline HETATM & 64 & N21 & HEM & 2 & 5.862 & 1.834 & 7.564 & 1.00 & 0.00 & $\mathrm{~N}$ \\
\hline HETATM & 65 & $\mathrm{~N} 22$ & HEM & 2 & 3.157 & 2.781 & 7.342 & 1.00 & 0.00 & $\mathrm{~N}$ \\
\hline HETATM & 66 & N23 & HEM & 2 & 3.828 & 4.044 & 4.841 & 1.00 & 0.00 & $\mathrm{~N}$ \\
\hline HETATM & 67 & N2 4 & HEM & 2 & 6.527 & 3.160 & 5.077 & 1.00 & 0.00 & $\mathrm{~N}$ \\
\hline HETATM & 68 & C99 & HEM & 2 & 5.621 & 6.025 & 7.579 & 1.00 & 0.00 & $\mathrm{C}$ \\
\hline HETATM & 69 & 091 & HEM & 2 & 5.461 & 4.711 & 7.567 & 1.00 & 0.00 & 0 \\
\hline HETATM & 70 & 092 & HEM & 2 & 5.776 & 6.642 & 8.635 & 1.00 & 0.00 & 0 \\
\hline HETATM & 71 & СТ91 & HEM & 2 & 5.605 & 6.706 & 6.223 & 1.00 & 0.00 & C \\
\hline HETATM & 72 & H911 & HEM & 2 & 4.626 & 6.511 & 5.760 & 1.00 & 0.00 & $\mathrm{H}$ \\
\hline HETATM & 73 & H912 & HEM & 2 & 6.353 & 6.216 & 5.580 & 1.00 & 0.00 & $\mathrm{H}$ \\
\hline HETATM & 74 & СТ 92 & HEM & 2 & 5.864 & 8.211 & 6.334 & 1.00 & 0.00 & C \\
\hline HETATM & 75 & H921 & HEM & 2 & 6.789 & 8.367 & 6.905 & 1.00 & 0.00 & $\mathrm{H}$ \\
\hline HETATM & 76 & H922 & HEM & 2 & 5.068 & 8.671 & 6.931 & 1.00 & 0.00 & $\mathrm{H}$ \\
\hline \multicolumn{11}{|l|}{ TER } \\
\hline HETATM & 77 & $\mathrm{C} 2$ & VIN & 3 & 0.345 & 13.923 & -1.002 & 1.00 & 0.00 & C \\
\hline HETATM & 78 & $\mathrm{H} 21$ & VIN & 3 & -0.471 & 13.224 & -0.837 & 1.00 & 0.00 & $\mathrm{H}$ \\
\hline HETATM & 79 & $\mathrm{C} 1$ & VIN & 3 & 0.012 & 15.147 & -1.403 & 1.00 & 0.00 & C \\
\hline HETATM & 80 & $\mathrm{H} 11$ & VIN & 3 & 0.743 & 15.926 & -1.511 & 1.00 & 0.00 & $\mathrm{H}$ \\
\hline HETATM & 81 & $\mathrm{H} 12$ & VIN & 3 & -1.030 & 15.417 & -1.570 & 1.00 & 0.00 & $\mathrm{H}$ \\
\hline \multicolumn{11}{|l|}{ TER } \\
\hline HETATM & 82 & $\mathrm{C} 1$ & MET & 4 & 3.008 & 14.177 & -2.840 & 1.00 & 0.00 & C \\
\hline HETATM & 83 & H11 & $\mathrm{MET}$ & 4 & 3.243 & 15.248 & -2.720 & 1.00 & 0.00 & $\mathrm{H}$ \\
\hline HETATM & 84 & $\mathrm{H} 12$ & $\mathrm{MET}$ & 4 & 3.821 & 13.713 & -3.410 & 1.00 & 0.00 & $\mathrm{H}$ \\
\hline HETATM & 85 & $\mathrm{H} 13$ & $\mathrm{MET}$ & 4 & 2.101 & 14.100 & -3.450 & 1.00 & 0.00 & $\mathrm{H}$ \\
\hline \multicolumn{11}{|l|}{ TER } \\
\hline HETATM & 86 & $\mathrm{C} 2$ & VIN & 5 & 8.018 & 12.499 & -2.325 & 1.00 & 0.00 & C \\
\hline HETATM & 87 & H 21 & VIN & 5 & 7.352 & 12.399 & -3.184 & 1.00 & 0.00 & $\mathrm{H}$ \\
\hline HETATM & 88 & $\mathrm{C} 1$ & VIN & 5 & 9.185 & 13.144 & -2.484 & 1.00 & 0.00 & C \\
\hline HETATM & 89 & H11 & VIN & 5 & 9.865 & 13.300 & -1.654 & 1.00 & 0.00 & $\mathrm{H}$ \\
\hline HETATM & 90 & $\mathrm{H} 12$ & VIN & 5 & 9.490 & 13.542 & -3.450 & 1.00 & 0.00 & $\mathrm{H}$ \\
\hline \multicolumn{11}{|l|}{ TER } \\
\hline HETATM & 91 & $\mathrm{C} 1$ & MET & 6 & 9.703 & 10.816 & -0.121 & 1.00 & 0.00 & C \\
\hline HETATM & 92 & H11 & MET & 6 & 10.294 & 11.480 & 0.528 & 1.00 & 0.00 & $\mathrm{H}$ \\
\hline HETATM & 93 & $\mathrm{H} 12$ & $\mathrm{MET}$ & 6 & 9.859 & 9.794 & 0.246 & 1.00 & 0.00 & $\mathrm{H}$ \\
\hline HETATM & 94 & $\mathrm{H} 13$ & $\mathrm{MET}$ & 6 & 10.135 & 10.882 & -1.126 & 1.00 & 0.00 & $\mathrm{H}$ \\
\hline TER & & & & & & & & & & \\
\hline HETATM & 95 & $\mathrm{C} 5$ & $\mathrm{ACP}$ & 7 & 0.615 & 9.735 & 5.770 & 1.00 & 0.00 & C \\
\hline HETATM & 96 & H51 & $\mathrm{ACP}$ & 7 & 1.354 & 9.723 & 6.582 & 1.00 & 0.00 & $\mathrm{H}$ \\
\hline HETATM & 97 & H5 2 & $\mathrm{ACP}$ & 7 & -0.226 & 10.343 & 6.134 & 1.00 & 0.00 & $\mathrm{H}$ \\
\hline HETATM & 98 & $\mathrm{C} 4$ & $\mathrm{ACP}$ & 7 & 0.136 & 8.297 & 5.497 & 1.00 & 0.00 & C \\
\hline HETATM & 99 & $\mathrm{H} 41$ & $\mathrm{ACP}$ & 7 & -0.422 & 8.236 & 4.549 & 1.00 & 0.00 & $\mathrm{H}$ \\
\hline HETATM & 100 & $\mathrm{H} 42$ & $\mathrm{ACP}$ & 7 & 1.007 & 7.630 & 5.390 & 1.00 & 0.00 & $\mathrm{H}$ \\
\hline HETATM & 101 & $\mathrm{C} 1$ & $\mathrm{ACP}$ & 7 & -0.728 & 7.685 & 6.573 & 1.00 & 0.00 & C \\
\hline HETATM & 102 & $\mathrm{O} 2$ & $\mathrm{ACP}$ & 7 & -0.956 & 8.208 & 7.671 & 1.00 & 0.00 & 0 \\
\hline HETATM & 103 & 03 & $\mathrm{ACP}$ & 7 & -1.244 & 6.505 & 6.209 & 1.00 & 0.00 & 0 \\
\hline HETATM & 104 & H3 & $\mathrm{ACP}$ & 7 & -1.853 & 6.151 & 6.945 & 1.00 & 0.00 & $\mathrm{H}$ \\
\hline TER & & & & & & & & & & \\
\hline HETATM & 105 & $\mathrm{C} 1$ & MET & 8 & 8.511 & 8.608 & 4.597 & 1.00 & 0.00 & C \\
\hline HETATM & 106 & H11 & $\mathrm{MET}$ & 8 & 9.063 & 9.448 & 5.047 & 1.00 & 0.00 & $\mathrm{H}$ \\
\hline HETATM & 107 & $\mathrm{H} 12$ & MET & 8 & 8.517 & 7.782 & 5.319 & 1.00 & 0.00 & $\mathrm{H}$ \\
\hline HETATM & 108 & $\mathrm{H} 13$ & MET & 8 & 9.082 & 8.289 & 3.717 & 1.00 & 0.00 & $\mathrm{H}$ \\
\hline TER & & & & & & & & & & \\
\hline HETATM & 109 & $\mathrm{C} 1$ & $\mathrm{MET}$ & 9 & -0.923 & 11.557 & 3.646 & 1.00 & 0.00 & C \\
\hline HETATM & 110 & $\mathrm{H} 11$ & MET & 9 & -1.368 & 11.348 & 4.626 & 1.00 & 0.00 & $\mathrm{H}$ \\
\hline HETATM & 111 & $\mathrm{H} 12$ & $\mathrm{MET}$ & 9 & -1.033 & 12.637 & 3.471 & 1.00 & 0.00 & $\mathrm{H}$ \\
\hline
\end{tabular}




\begin{tabular}{|c|c|c|c|c|c|}
\hline-1.523 & 11.031 & 2.889 & 1.00 & 0.00 & $\mathrm{H}$ \\
\hline 8.874 & 0.178 & 8.933 & 1.00 & 0.00 & C \\
\hline 9.690 & 0.876 & 8.768 & 1.00 & 0.00 & $\mathrm{H}$ \\
\hline 9.207 & -1.047 & 9.335 & 1.00 & 0.00 & C \\
\hline 8.475 & -1.826 & 9.442 & 1.00 & 0.00 & $\mathrm{H}$ \\
\hline 10.249 & -1.316 & 9.501 & 1.00 & 0.00 & $\mathrm{H}$ \\
\hline 6.210 & -0.077 & 10.771 & 1.00 & 0.00 & C \\
\hline 5.975 & -1.148 & 10.652 & 1.00 & 0.00 & $\mathrm{H}$ \\
\hline 5.397 & 0.387 & 11.341 & 1.00 & 0.00 & $\mathrm{H}$ \\
\hline 7.118 & 0.001 & 11.382 & 1.00 & 0.00 & $\mathrm{H}$ \\
\hline 1.200 & 1.601 & 10.256 & 1.00 & 0.00 & C \\
\hline 1.866 & 1.702 & 11.115 & 1.00 & 0.00 & $\mathrm{H}$ \\
\hline 0.033 & 0.957 & 10.415 & 1.00 & 0.00 & $\mathrm{C}$ \\
\hline-0.647 & 0.800 & 9.585 & 1.00 & 0.00 & $\mathrm{H}$ \\
\hline-0.272 & 0.559 & 11.381 & 1.00 & 0.00 & $\mathrm{H}$ \\
\hline-0.484 & 3.284 & 8.052 & 1.00 & 0.00 & C \\
\hline-0.641 & 4.306 & 7.685 & 1.00 & 0.00 & $\mathrm{H}$ \\
\hline-0.917 & 3.219 & 9.057 & 1.00 & 0.00 & $\mathrm{H}$ \\
\hline-1.076 & 2.620 & 7.403 & 1.00 & 0.00 & $\mathrm{H}$ \\
\hline 8.604 & 4.366 & 2.161 & 1.00 & 0.00 & C \\
\hline 7.865 & 4.377 & 1.349 & 1.00 & 0.00 & $\mathrm{H}$ \\
\hline 9.445 & 3.758 & 1.798 & 1.00 & 0.00 & $\mathrm{H}$ \\
\hline 9.082 & 5.803 & 2.434 & 1.00 & 0.00 & $\mathrm{C}$ \\
\hline 9.640 & 5.865 & 3.382 & 1.00 & 0.00 & $\mathrm{H}$ \\
\hline 8.212 & 6.470 & 2.541 & 1.00 & 0.00 & $\mathrm{H}$ \\
\hline 9.946 & 6.416 & 1.358 & 1.00 & 0.00 & C \\
\hline 10.174 & 5.893 & 0.260 & 1.00 & 0.00 & o \\
\hline 10.462 & 7.595 & 1.722 & 1.00 & 0.00 & 0 \\
\hline 11.071 & 7.949 & 0.986 & 1.00 & 0.00 & $\mathrm{H}$ \\
\hline 0.708 & 5.492 & 3.334 & 1.00 & 0.00 & $\mathrm{C}$ \\
\hline 0.155 & 4.653 & 2.884 & 1.00 & 0.00 & $\mathrm{H}$ \\
\hline 0.701 & 6.318 & 2.613 & 1.00 & 0.00 & $\mathrm{H}$ \\
\hline 0.137 & 5.812 & 4.214 & 1.00 & 0.00 & $\mathrm{H}$ \\
\hline 10.141 & 2.543 & 4.285 & 1.00 & 0.00 & C \\
\hline 10.586 & 2.752 & 3.305 & 1.00 & 0.00 & $\mathrm{H}$ \\
\hline 10.252 & 1.463 & 4.460 & 1.00 & 0.00 & $\mathrm{H}$ \\
\hline 10.742 & 3.069 & 5.042 & 1.00 & 0.00 & $\mathrm{H}$ \\
\hline 3.316 & 10.511 & 9.423 & 1.00 & 0.00 & $\mathrm{FE}$ \\
\hline 1.088 & 12.383 & 8.361 & 1.00 & 0.00 & C \\
\hline 0.729 & 13.174 & 7.189 & 1.00 & 0.00 & C \\
\hline 1.874 & 13.271 & 6.414 & 1.00 & 0.00 & C \\
\hline 2.904 & 12.553 & 7.122 & 1.00 & 0.00 & C \\
\hline 4.219 & 12.435 & 6.684 & 1.00 & 0.00 & C \\
\hline 4.472 & 12.931 & 5.749 & 1.00 & 0.00 & $\mathrm{H}$ \\
\hline 5.238 & 11.736 & 7.320 & 1.00 & 0.00 & C \\
\hline 6.607 & 11.664 & 6.851 & 1.00 & 0.00 & C \\
\hline 7.313 & 10.944 & 7.798 & 1.00 & 0.00 & C \\
\hline 6.362 & 10.579 & 8.822 & 1.00 & 0.00 & C \\
\hline 6.666 & 9.844 & 9.965 & 1.00 & 0.00 & $\mathrm{C}$ \\
\hline 7.693 & 9.500 & 10.071 & 1.00 & 0.00 & $\mathrm{H}$ \\
\hline 5.792 & 9.504 & 10.988 & 1.00 & 0.00 & C \\
\hline 6.173 & 8.779 & 12.178 & 1.00 & 0.00 & C \\
\hline 5.033 & 8.631 & 12.935 & 1.00 & 0.00 & C \\
\hline 3.960 & 9.273 & 12.204 & 1.00 & 0.00 & C \\
\hline 2.632 & 9.328 & 12.625 & 1.00 & 0.00 & C \\
\hline 2.392 & 8.843 & 13.573 & 1.00 & 0.00 & $\mathrm{H}$ \\
\hline 1.602 & 10.009 & 11.982 & 1.00 & 0.00 & C \\
\hline 0.236 & 10.123 & 12.464 & 1.00 & 0.00 & C \\
\hline-0.439 & 10.890 & 11.541 & 1.00 & 0.00 & C \\
\hline 0.510 & 11.242 & 10.504 & 1.00 & 0.00 & C \\
\hline 0.219 & 12.033 & 9.393 & 1.00 & 0.00 & C \\
\hline-0.795 & 12.423 & 9.333 & 1.00 & 0.00 & $\mathrm{H}$ \\
\hline 2.409 & 12.020 & 8.298 & 1.00 & 0.00 & $\mathrm{~N}$ \\
\hline 5.114 & 11.073 & 8.521 & 1.00 & 0.00 & $\mathrm{~N}$ \\
\hline 4.443 & 9.810 & 11.021 & 1.00 & 0.00 & $\mathrm{~N}$ \\
\hline 1.743 & 10.694 & 10.786 & 1.00 & 0.00 & $\mathrm{~N}$ \\
\hline 2.650 & 7.829 & 8.283 & 1.00 & 0.00 & C \\
\hline 2.810 & 9.143 & 8.296 & 1.00 & 0.00 & 0 \\
\hline 2.495 & 7.212 & 7.228 & 1.00 & 0.00 & o \\
\hline 2.666 & 7.148 & 9.640 & 1.00 & 0.00 & C \\
\hline 3.645 & 7.343 & 10.103 & 1.00 & 0.00 & $\mathrm{H}$ \\
\hline
\end{tabular}




\begin{tabular}{|c|c|c|c|c|c|c|c|c|c|c|}
\hline HETATM & 183 & H912 & HEM & 17 & 1.918 & 7.638 & 10.283 & 1.00 & 0.00 & $\mathrm{H}$ \\
\hline HETATM & 184 & СТ92 & HEM & 17 & 2.407 & 5.644 & 9.528 & 1.00 & 0.00 & C \\
\hline HETATM & 185 & H921 & HEM & 17 & 3.203 & 5.183 & 8.931 & 1.00 & 0.00 & $\mathrm{H}$ \\
\hline HETATM & 186 & H922 & HEM & 17 & 1.482 & 5.488 & 8.958 & 1.00 & 0.00 & $\mathrm{H}$ \\
\hline \multicolumn{11}{|l|}{ TER } \\
\hline HETATM & 187 & $\mathrm{FE}$ & HEM & 18 & 4.008 & 3.097 & 14.371 & 1.00 & 0.00 & $\mathrm{FE}$ \\
\hline HETATM & 188 & $\mathrm{C} 1$ & HEM & 18 & 6.236 & 1.225 & 15.433 & 1.00 & 0.00 & C \\
\hline HETATM & 189 & $\mathrm{C} 2$ & HEM & 18 & 6.595 & 0.434 & 16.605 & 1.00 & 0.00 & C \\
\hline HETATM & 190 & C3 & HEM & 18 & 5.449 & 0.337 & 17.380 & 1.00 & 0.00 & C \\
\hline HETATM & 191 & C4 & HEM & 18 & 4.419 & 1.055 & 16.672 & 1.00 & 0.00 & C \\
\hline HETATM & 192 & C5 & HEM & 18 & 3.104 & 1.173 & 17.109 & 1.00 & 0.00 & C \\
\hline HETATM & 193 & H5 & HEM & 18 & 2.851 & 0.677 & 18.045 & 1.00 & 0.00 & $\mathrm{H}$ \\
\hline HETATM & 194 & $\mathrm{C} 6$ & HEM & 18 & 2.085 & 1.872 & 16.473 & 1.00 & 0.00 & C \\
\hline HETATM & 195 & C7 & HEM & 18 & 0.716 & 1.944 & 16.943 & 1.00 & 0.00 & C \\
\hline HETATM & 196 & $\mathrm{C} 8$ & HEM & 18 & 0.011 & 2.664 & 15.996 & 1.00 & 0.00 & C \\
\hline HETATM & 197 & C9 & HEM & 18 & 0.962 & 3.029 & 14.972 & 1.00 & 0.00 & C \\
\hline HETATM & 198 & $\mathrm{C} 10$ & HEM & 18 & 0.657 & 3.764 & 13.828 & 1.00 & 0.00 & C \\
\hline HETATM & 199 & $\mathrm{H} 10$ & HEM & 18 & -0.370 & 4.108 & 13.722 & 1.00 & 0.00 & $\mathrm{H}$ \\
\hline HETATM & 200 & $\mathrm{C} 11$ & HEM & 18 & 1.532 & 4.104 & 12.806 & 1.00 & 0.00 & C \\
\hline HETATM & 201 & $\mathrm{C} 12$ & HEM & 18 & 1.151 & 4.829 & 11.615 & 1.00 & 0.00 & C \\
\hline HETATM & 202 & C13 & HEM & 18 & 2.290 & 4.977 & 10.858 & 1.00 & 0.00 & C \\
\hline HETATM & 203 & C14 & HEM & 18 & 3.363 & 4.335 & 11.590 & 1.00 & 0.00 & C \\
\hline HETATM & 204 & C15 & HEM & 18 & 4.691 & 4.280 & 11.168 & 1.00 & 0.00 & C \\
\hline HETATM & 205 & H15 & HEM & 18 & 4.931 & 4.765 & 10.221 & 1.00 & 0.00 & $\mathrm{H}$ \\
\hline HETATM & 206 & $\mathrm{C} 16$ & HEM & 18 & 5.721 & 3.599 & 11.812 & 1.00 & 0.00 & $\mathrm{C}$ \\
\hline HETATM & 207 & $\mathrm{C} 17$ & HEM & 18 & 7.087 & 3.485 & 11.329 & 1.00 & 0.00 & C \\
\hline HETATM & 208 & C18 & HEM & 18 & 7.763 & 2.718 & 12.253 & 1.00 & 0.00 & C \\
\hline HETATM & 209 & C19 & HEM & 18 & 6.813 & 2.366 & 13.289 & 1.00 & 0.00 & C \\
\hline HETATM & 210 & $\mathrm{C} 20$ & HEM & 18 & 7.104 & 1.575 & 14.401 & 1.00 & 0.00 & C \\
\hline HETATM & 211 & $\mathrm{H} 2 \mathrm{O}$ & HEM & 18 & 8.118 & 1.185 & 14.461 & 1.00 & 0.00 & $\mathrm{H}$ \\
\hline HETATM & 212 & N21 & HEM & 18 & 4.914 & 1.588 & 15.495 & 1.00 & 0.00 & $\mathrm{~N}$ \\
\hline HETATM & 213 & N22 & HEM & 18 & 2.209 & 2.535 & 15.273 & 1.00 & 0.00 & $\mathrm{~N}$ \\
\hline HETATM & 214 & N23 & HEM & 18 & 2.880 & 3.798 & 12.772 & 1.00 & 0.00 & $\mathrm{~N}$ \\
\hline HETATM & 215 & N24 & HEM & 18 & 5.580 & 2.914 & 13.008 & 1.00 & 0.00 & $\mathrm{~N}$ \\
\hline HETATM & 216 & C99 & HEM & 18 & 4.673 & 5.779 & 15.510 & 1.00 & 0.00 & C \\
\hline HETATM & 217 & 091 & HEM & 18 & 4.514 & 4.465 & 15.498 & 1.00 & 0.00 & o \\
\hline HETATM & 218 & 092 & HEM & 18 & 4.828 & 6.396 & 16.566 & 1.00 & 0.00 & O \\
\hline HETATM & 219 & СТ91 & HEM & 18 & 4.657 & 6.460 & 14.154 & 1.00 & 0.00 & C \\
\hline HETATM & 220 & H911 & HEM & 18 & 3.678 & 6.265 & 13.691 & 1.00 & 0.00 & $\mathrm{H}$ \\
\hline HETATM & 221 & H912 & HEM & 18 & 5.405 & 5.970 & 13.511 & 1.00 & 0.00 & $\mathrm{H}$ \\
\hline HETATM & 222 & CT92 & HEM & 18 & 4.917 & 7.964 & 14.265 & 1.00 & 0.00 & C \\
\hline HETATM & 223 & H921 & HEM & 18 & 5.842 & 8.120 & 14.836 & 1.00 & 0.00 & $\mathrm{H}$ \\
\hline HETATM & 224 & H922 & HEM & 18 & 4.120 & 8.425 & 14.862 & 1.00 & 0.00 & $\mathrm{H}$ \\
\hline \multicolumn{11}{|l|}{ TER } \\
\hline HETATM & 225 & $\mathrm{C} 2$ & VIN & 19 & -0.603 & 13.676 & 6.929 & 1.00 & 0.00 & C \\
\hline HETATM & 226 & H 21 & VIN & 19 & -1.419 & 12.978 & 7.094 & 1.00 & 0.00 & $\mathrm{H}$ \\
\hline HETATM & 227 & $\mathrm{C} 1$ & VIN & 19 & -0.936 & 14.901 & 6.528 & 1.00 & 0.00 & $\mathrm{C}$ \\
\hline HETATM & 228 & H11 & VIN & 19 & -0.205 & 15.680 & 6.421 & 1.00 & 0.00 & $\mathrm{H}$ \\
\hline HETATM & 229 & $\mathrm{H} 12$ & VIN & 19 & -1.978 & 15.170 & 6.361 & 1.00 & 0.00 & $\mathrm{H}$ \\
\hline \multicolumn{11}{|l|}{ TER } \\
\hline HETATM & 230 & $\mathrm{C} 1$ & $\mathrm{MET}$ & 20 & 2.061 & 13.931 & 5.091 & 1.00 & 0.00 & C \\
\hline HETATM & 231 & H11 & $\mathrm{MET}$ & 20 & 2.295 & 15.002 & 5.211 & 1.00 & 0.00 & $\mathrm{H}$ \\
\hline HETATM & 232 & $\mathrm{H} 12$ & $\mathrm{MET}$ & 20 & 2.874 & 13.467 & 4.522 & 1.00 & 0.00 & $\mathrm{H}$ \\
\hline HETATM & 233 & $\mathrm{H} 13$ & $\mathrm{MET}$ & 20 & 1.153 & 13.854 & 4.481 & 1.00 & 0.00 & $\mathrm{H}$ \\
\hline \multicolumn{11}{|l|}{ TER } \\
\hline HETATM & 234 & C2 & VIN & 21 & 7.071 & 12.253 & 5.606 & 1.00 & 0.00 & C \\
\hline HETATM & 235 & H 21 & VIN & 21 & 6.405 & 12.152 & 4.747 & 1.00 & 0.00 & $\mathrm{H}$ \\
\hline HETATM & 236 & $\mathrm{C} 1$ & VIN & 21 & 8.238 & 12.898 & 5.447 & 1.00 & 0.00 & C \\
\hline HETATM & 237 & $\mathrm{H} 11$ & VIN & 21 & 8.917 & 13.054 & 6.277 & 1.00 & 0.00 & $\mathrm{H}$ \\
\hline HETATM & 238 & H12 & VIN & 21 & 8.543 & 13.296 & 4.482 & 1.00 & 0.00 & $\mathrm{H}$ \\
\hline TER & & & & & & & & & & \\
\hline HETATM & 239 & $\mathrm{C} 1$ & $\mathrm{MET}$ & 22 & 8.755 & 10.570 & 7.810 & 1.00 & 0.00 & C \\
\hline HETATM & 240 & $\mathrm{H} 11$ & MET & 22 & 9.347 & 11.234 & 8.459 & 1.00 & 0.00 & $\mathrm{H}$ \\
\hline HETATM & 241 & $\mathrm{H} 12$ & MET & 22 & 8.912 & 9.548 & 8.177 & 1.00 & 0.00 & $\mathrm{H}$ \\
\hline HETATM & 242 & H13 & $\mathrm{MET}$ & 22 & 9.188 & 10.636 & 6.805 & 1.00 & 0.00 & $\mathrm{H}$ \\
\hline TER & & & & & & & & & & \\
\hline HETATM & 243 & C5 & $\mathrm{ACP}$ & 23 & -0.333 & 9.488 & 13.701 & 1.00 & 0.00 & C \\
\hline HETATM & 244 & H51 & $\mathrm{ACP}$ & 23 & 0.406 & 9.477 & 14.514 & 1.00 & 0.00 & $\mathrm{H}$ \\
\hline HETATM & 245 & H52 & $\mathrm{ACP}$ & 23 & -1.174 & 10.097 & 14.065 & 1.00 & 0.00 & $\mathrm{H}$ \\
\hline HETATM & 246 & C4 & $\mathrm{ACP}$ & 23 & -0.812 & 8.051 & 13.428 & 1.00 & 0.00 & C \\
\hline HETATM & 247 & H4 1 & $\mathrm{ACP}$ & 23 & -1.369 & 7.990 & 12.481 & 1.00 & 0.00 & $\mathrm{H}$ \\
\hline HETATM & 248 & $\mathrm{H} 42$ & $\mathrm{ACP}$ & 23 & 0.059 & 7.384 & 13.321 & 1.00 & 0.00 & $\mathrm{H}$ \\
\hline HETATM & 249 & $\mathrm{C} 1$ & $\mathrm{ACP}$ & 23 & -1.676 & 7.439 & 14.504 & 1.00 & 0.00 & C \\
\hline HETATM & 250 & $\mathrm{O} 2$ & $\mathrm{ACP}$ & 23 & -1.903 & 7.961 & 15.603 & 1.00 & 0.00 & 0 \\
\hline HETATM & 251 & 03 & $\mathrm{ACP}$ & 23 & -2.191 & 6.259 & 14.140 & 1.00 & 0.00 & O \\
\hline HETATM & 252 & $\mathrm{H} 3$ & $\mathrm{ACP}$ & 23 & -2.800 & 5.905 & 14.876 & 1.00 & 0.00 & $\mathrm{H}$ \\
\hline TER & & & & & & & & & & \\
\hline HETATM & 253 & $\mathrm{C} 1$ & MET & 24 & 7.563 & 8.362 & 12.528 & 1.00 & 0.00 & C \\
\hline HETATM & 254 & $\mathrm{H} 11$ & $\mathrm{MET}$ & 24 & 8.115 & 9.201 & 12.978 & 1.00 & 0.00 & H \\
\hline
\end{tabular}




\begin{tabular}{|c|c|c|c|c|c|c|c|c|c|c|}
\hline HETATM & 255 & $\mathrm{H} 12$ & MET & 24 & 7.570 & 7.536 & 13.250 & 1.00 & 0.00 & $\mathrm{H}$ \\
\hline HETATM & 256 & $\mathrm{H} 13$ & MET & 24 & 8.134 & 8.042 & 11.649 & 1.00 & 0.00 & $\mathrm{H}$ \\
\hline \multicolumn{11}{|l|}{ TER } \\
\hline HETATM & 257 & $\mathrm{C} 1$ & MET & 25 & -1.870 & 11.311 & 11.577 & 1.00 & 0.00 & $\mathrm{C}$ \\
\hline HETATM & 258 & $\mathrm{H} 11$ & $\mathrm{MET}$ & 25 & -2.316 & 11.102 & 12.557 & 1.00 & 0.00 & $\mathrm{H}$ \\
\hline HETATM & 259 & $\mathrm{H} 12$ & MET & 25 & -1.981 & 12.391 & 11.402 & 1.00 & 0.00 & $\mathrm{H}$ \\
\hline HETATM & 260 & H13 & MET & 25 & -2.471 & 10.785 & 10.821 & 1.00 & 0.00 & $\mathrm{H}$ \\
\hline \multicolumn{11}{|c|}{ TER } \\
\hline HETATM & 261 & $\mathrm{C} 2$ & VIN & 26 & 7.926 & -0.068 & 16.864 & 1.00 & 0.00 & $\mathrm{C}$ \\
\hline HETATM & 262 & H21 & VIN & 26 & 8.742 & 0.630 & 16.699 & 1.00 & 0.00 & $\mathrm{H}$ \\
\hline HETATM & 263 & $\mathrm{C} 1$ & VIN & 26 & 8.259 & -1.293 & 17.266 & 1.00 & 0.00 & $\mathrm{C}$ \\
\hline HETATM & 264 & H11 & VIN & 26 & 7.528 & -2.072 & 17.373 & 1.00 & 0.00 & $\mathrm{H}$ \\
\hline HETATM & 265 & $\mathrm{H} 12$ & VIN & 26 & 9.301 & -1.562 & 17.432 & 1.00 & 0.00 & $\mathrm{H}$ \\
\hline \multicolumn{11}{|l|}{ TER } \\
\hline HETATM & 266 & $\mathrm{C} 1$ & MET & 27 & 5.263 & -0.323 & 18.702 & 1.00 & 0.00 & C \\
\hline HETATM & 267 & $\mathrm{H} 11$ & MET & 27 & 5.028 & -1.394 & 18.583 & 1.00 & 0.00 & $\mathrm{H}$ \\
\hline HETATM & 268 & $\mathrm{H} 12$ & MET & 27 & 4.450 & 0.141 & 19.272 & 1.00 & 0.00 & $\mathrm{H}$ \\
\hline HETATM & 269 & $\mathrm{H} 13$ & $\mathrm{MET}$ & 27 & 6.170 & -0.246 & 19.313 & 1.00 & 0.00 & $\mathrm{H}$ \\
\hline \multicolumn{11}{|l|}{ TER } \\
\hline HETATM & 270 & $\mathrm{C} 2$ & VIN & 28 & 0.252 & 1.355 & 18.188 & 1.00 & 0.00 & C \\
\hline HETATM & 271 & H 21 & VIN & 28 & 0.918 & 1.456 & 19.046 & 1.00 & 0.00 & $\mathrm{H}$ \\
\hline HETATM & 272 & $\mathrm{C} 1$ & VIN & 28 & -0.914 & 0.710 & 18.346 & 1.00 & 0.00 & C \\
\hline HETATM & 273 & H11 & VIN & 28 & -1.594 & 0.554 & 17.516 & 1.00 & 0.00 & $\mathrm{H}$ \\
\hline HETATM & 274 & $\mathrm{H} 12$ & VIN & 28 & -1.219 & 0.312 & 19.312 & 1.00 & 0.00 & $\mathrm{H}$ \\
\hline TER & & & & & & & & & & \\
\hline HETATM & 275 & $\mathrm{C} 1$ & MET & 29 & -1.432 & 3.038 & 15.984 & 1.00 & 0.00 & C \\
\hline HETATM & 276 & $\mathrm{H} 11$ & MET & 29 & -1.588 & 4.060 & 15.617 & 1.00 & 0.00 & $\mathrm{H}$ \\
\hline HETATM & 277 & $\mathrm{H} 12$ & MET & 29 & -1.864 & 2.972 & 16.989 & 1.00 & 0.00 & $\mathrm{H}$ \\
\hline HETATM & 278 & H13 & MET & 29 & -2.023 & 2.374 & 15.335 & 1.00 & 0.00 & $\mathrm{H}$ \\
\hline TER & & & & & & & & & & \\
\hline HETATM & 279 & $\mathrm{C} 5$ & $\mathrm{ACP}$ & 30 & 7.656 & 4.120 & 10.092 & 1.00 & 0.00 & C \\
\hline HETATM & 280 & H51 & $\mathrm{ACP}$ & 30 & 6.917 & 4.131 & 9.280 & 1.00 & 0.00 & $\mathrm{H}$ \\
\hline HETATM & 281 & H52 & $\mathrm{ACP}$ & 30 & 8.497 & 3.511 & 9.729 & 1.00 & 0.00 & $\mathrm{H}$ \\
\hline HETATM & 282 & C4 & $\mathrm{ACP}$ & 30 & 8.135 & 5.557 & 10.365 & 1.00 & 0.00 & C \\
\hline HETATM & 283 & H 41 & $\mathrm{ACP}$ & 30 & 8.693 & 5.618 & 11.313 & 1.00 & 0.00 & $\mathrm{H}$ \\
\hline HETATM & 284 & $\mathrm{H} 42$ & $\mathrm{ACP}$ & 30 & 7.264 & 6.224 & 10.472 & 1.00 & 0.00 & $\mathrm{H}$ \\
\hline HETATM & 285 & $\mathrm{C} 1$ & $\mathrm{ACP}$ & 30 & 8.999 & 6.169 & 9.289 & 1.00 & 0.00 & C \\
\hline HETATM & 286 & $\mathrm{O} 2$ & $\mathrm{ACP}$ & 30 & 9.227 & 5.647 & 8.191 & 1.00 & 0.00 & o \\
\hline HETATM & 287 & 03 & $\mathrm{ACP}$ & 30 & 9.515 & 7.349 & 9.653 & 1.00 & 0.00 & 0 \\
\hline HETATM & 288 & H3 & $\mathrm{ACP}$ & 30 & 10.124 & 7.703 & 8.917 & 1.00 & 0.00 & $\mathrm{H}$ \\
\hline TER & & & & & & & & & & \\
\hline HETATM & 289 & $\mathrm{C} 1$ & MET & 31 & -0.240 & 5.246 & 11.266 & 1.00 & 0.00 & C \\
\hline HETATM & 290 & H11 & MET & 31 & -0.792 & 4.407 & 10.815 & 1.00 & 0.00 & $\mathrm{H}$ \\
\hline HETATM & 291 & $\mathrm{H} 12$ & MET & 31 & -0.246 & 6.072 & 10.544 & 1.00 & 0.00 & $\mathrm{H}$ \\
\hline HETATM & 292 & $\mathrm{H} 13$ & MET & 31 & -0.811 & 5.565 & 12.145 & 1.00 & 0.00 & $\mathrm{H}$ \\
\hline TER & & & & & & & & & & \\
\hline HETATM & 293 & $\mathrm{C} 1$ & MET & 32 & 9.194 & 2.297 & 12.216 & 1.00 & 0.00 & $\mathrm{C}$ \\
\hline HETATM & 294 & $\mathrm{H} 11$ & MET & 32 & 9.639 & 2.506 & 11.236 & 1.00 & 0.00 & $\mathrm{H}$ \\
\hline HETATM & 295 & $\mathrm{H} 12$ & MET & 32 & 9.304 & 1.217 & 12.391 & 1.00 & 0.00 & $\mathrm{H}$ \\
\hline HETATM & 296 & H1 3 & MET & 32 & 9.794 & 2.823 & 12.973 & 1.00 & 0.00 & $\mathrm{H}$ \\
\hline TER & & & & & & & & & & \\
\hline HETATM & 297 & $\mathrm{FE}$ & HEM & 33 & 16.346 & 10.757 & 1.491 & 1.00 & 0.00 & $\mathrm{FE}$ \\
\hline HETATM & 298 & $\mathrm{C} 1$ & HEM & 33 & 14.118 & 12.629 & 0.429 & 1.00 & 0.00 & C \\
\hline HETATM & 299 & $\mathrm{C} 2$ & HEM & 33 & 13.759 & 13.420 & -0.742 & 1.00 & 0.00 & C \\
\hline HETATM & 300 & $\mathrm{C} 3$ & HEM & 33 & 14.905 & 13.517 & -1.517 & 1.00 & 0.00 & C \\
\hline HETATM & 301 & $\mathrm{C} 4$ & HEM & 33 & 15.934 & 12.800 & -0.809 & 1.00 & 0.00 & C \\
\hline HETATM & 302 & $\mathrm{C} 5$ & HEM & 33 & 17.249 & 12.681 & -1.247 & 1.00 & 0.00 & C \\
\hline HETATM & 303 & H5 & HEM & 33 & 17.503 & 13.177 & -2.182 & 1.00 & 0.00 & $\mathrm{H}$ \\
\hline HETATM & 304 & $\mathrm{C} 6$ & HEM & 33 & 18.269 & 11.982 & -0.611 & 1.00 & 0.00 & C \\
\hline HETATM & 305 & C7 & HEM & 33 & 19.638 & 11.910 & -1.081 & 1.00 & 0.00 & C \\
\hline HETATM & 306 & $\mathrm{C} 8$ & HEM & 33 & 20.343 & 11.190 & -0.134 & 1.00 & 0.00 & C \\
\hline HETATM & 307 & $\mathrm{C} 9$ & HEM & 33 & 19.392 & 10.825 & 0.891 & 1.00 & 0.00 & C \\
\hline HETATM & 308 & $\mathrm{C} 10$ & HEM & 33 & 19.697 & 10.090 & 2.034 & 1.00 & 0.00 & C \\
\hline HETATM & 309 & $\mathrm{H} 10$ & HEM & 33 & 20.723 & 9.746 & 2.140 & 1.00 & 0.00 & $\mathrm{H}$ \\
\hline HETATM & 310 & $\mathrm{C} 11$ & HEM & 33 & 18.822 & 9.750 & 3.057 & 1.00 & 0.00 & C \\
\hline HETATM & 311 & $\mathrm{C} 12$ & HEM & 33 & 19.203 & 9.025 & 4.247 & 1.00 & 0.00 & C \\
\hline HETATM & 312 & $\mathrm{C} 13$ & HEM & 33 & 18.064 & 8.878 & 5.004 & 1.00 & 0.00 & C \\
\hline HETATM & 313 & C14 & HEM & 33 & 16.991 & 9.519 & 4.273 & 1.00 & 0.00 & C \\
\hline HETATM & 314 & C15 & HEM & 33 & 15.663 & 9.574 & 4.694 & 1.00 & 0.00 & C \\
\hline HETATM & 315 & H15 & HEM & 33 & 15.423 & 9.089 & 5.642 & 1.00 & 0.00 & $\mathrm{H}$ \\
\hline HETATM & 316 & $\mathrm{C} 16$ & HEM & 33 & 14.632 & 10.255 & 4.051 & 1.00 & 0.00 & C \\
\hline HETATM & 317 & $\mathrm{C} 17$ & HEM & 33 & 13.267 & 10.369 & 4.533 & 1.00 & 0.00 & C \\
\hline HETATM & 318 & $\mathrm{C} 18$ & HEM & 33 & 12.591 & 11.137 & 3.609 & 1.00 & 0.00 & C \\
\hline HETATM & 319 & C19 & HEM & 33 & 13.541 & 11.489 & 2.573 & 1.00 & 0.00 & C \\
\hline HETATM & 320 & $\mathrm{C} 20$ & HEM & 33 & 13.250 & 12.280 & 1.462 & 1.00 & 0.00 & C \\
\hline HETATM & 321 & $\mathrm{H} 20$ & HEM & 33 & 12.235 & 12.669 & 1.402 & 1.00 & 0.00 & $\mathrm{H}$ \\
\hline HETATM & 322 & N21 & HEM & 33 & 15.440 & 12.266 & 0.367 & 1.00 & 0.00 & $\mathrm{~N}$ \\
\hline HETATM & 323 & N22 & HEM & 33 & 18.144 & 11.319 & 0.590 & 1.00 & 0.00 & $\mathrm{~N}$ \\
\hline HETATM & 324 & N23 & HEM & 33 & 17.474 & 10.056 & 3.090 & 1.00 & 0.00 & $\mathrm{~N}$ \\
\hline
\end{tabular}




\begin{tabular}{|c|c|c|c|c|c|c|c|c|c|c|}
\hline HETATM & 325 & N24 & HEM & 33 & 14.774 & 10.941 & 2.854 & 1.00 & 0.00 & $\mathrm{~N}$ \\
\hline HETATM & 326 & C99 & HEM & 33 & 15.681 & 8.076 & 0.352 & 1.00 & 0.00 & C \\
\hline HETATM & 327 & 091 & HEM & 33 & 15.840 & 9.389 & 0.365 & 1.00 & 0.00 & 0 \\
\hline HETATM & 328 & 092 & HEM & 33 & 15.526 & 7.458 & -0.703 & 1.00 & 0.00 & 0 \\
\hline HETATM & 329 & СТ91 & HEM & 33 & 15.697 & 7.394 & 1.709 & 1.00 & 0.00 & $\mathrm{C}$ \\
\hline HETATM & 330 & H911 & HEM & 33 & 16.676 & 7.590 & 2.171 & 1.00 & 0.00 & $\mathrm{H}$ \\
\hline HETATM & 331 & H912 & HEM & 33 & 14.949 & 7.884 & 2.352 & 1.00 & 0.00 & $\mathrm{H}$ \\
\hline HETATM & 332 & CT 92 & HEM & 33 & 15.437 & 5.890 & 1.597 & 1.00 & 0.00 & C \\
\hline HETATM & 333 & H921 & HEM & 33 & 16.234 & 5.429 & 1.000 & 1.00 & 0.00 & $\mathrm{H}$ \\
\hline HETATM & 334 & H922 & HEM & 33 & 14.512 & 5.734 & 1.027 & 1.00 & 0.00 & $\mathrm{H}$ \\
\hline \multicolumn{11}{|l|}{ TER } \\
\hline HETATM & 335 & $\mathrm{FE}$ & HEM & 34 & 17.038 & 3.343 & 6.440 & 1.00 & 0.00 & $\mathrm{FE}$ \\
\hline HETATM & 336 & $\mathrm{C} 1$ & HEM & 34 & 19.266 & 1.471 & 7.502 & 1.00 & 0.00 & $\mathrm{C}$ \\
\hline HETATM & 337 & C2 & HEM & 34 & 19.625 & 0.680 & 8.674 & 1.00 & 0.00 & $\mathrm{C}$ \\
\hline HETATM & 338 & C3 & HEM & 34 & 18.479 & 0.583 & 9.448 & 1.00 & 0.00 & $\mathrm{C}$ \\
\hline HETATM & 339 & C4 & HEM & 34 & 17.450 & 1.301 & 8.740 & 1.00 & 0.00 & C \\
\hline HETATM & 340 & C5 & HEM & 34 & 16.135 & 1.420 & 9.178 & 1.00 & 0.00 & C \\
\hline HETATM & 341 & H5 & HEM & 34 & 15.881 & 0.923 & 10.113 & 1.00 & 0.00 & $\mathrm{H}$ \\
\hline HETATM & 342 & $\mathrm{C} 6$ & HEM & 34 & 15.115 & 2.118 & 8.542 & 1.00 & 0.00 & C \\
\hline HETATM & 343 & C7 & HEM & 34 & 13.747 & 2.191 & 9.012 & 1.00 & 0.00 & C \\
\hline HETATM & 344 & $\mathrm{C} 8$ & HEM & 34 & 13.041 & 2.910 & 8.065 & 1.00 & 0.00 & C \\
\hline HETATM & 345 & C9 & HEM & 34 & 13.992 & 3.275 & 7.041 & 1.00 & 0.00 & C \\
\hline HETATM & 346 & C10 & HEM & 34 & 13.688 & 4.010 & 5.897 & 1.00 & 0.00 & C \\
\hline HETATM & 347 & $\mathrm{H} 10$ & HEM & 34 & 12.661 & 4.354 & 5.791 & 1.00 & 0.00 & $\mathrm{H}$ \\
\hline HETATM & 348 & $\mathrm{C} 11$ & HEM & 34 & 14.562 & 4.350 & 4.874 & 1.00 & 0.00 & C \\
\hline HETATM & 349 & C12 & HEM & 34 & 14.181 & 5.076 & 3.684 & 1.00 & 0.00 & $\mathrm{C}$ \\
\hline HETATM & 350 & C13 & HEM & 34 & 15.321 & 5.223 & 2.927 & 1.00 & 0.00 & C \\
\hline HETATM & 351 & C14 & HEM & 34 & 16.394 & 4.581 & 3.659 & 1.00 & 0.00 & C \\
\hline HETATM & 352 & C15 & HEM & 34 & 17.722 & 4.527 & 3.237 & 1.00 & 0.00 & C \\
\hline HETATM & 353 & H1 5 & HEM & 34 & 17.962 & 5.011 & 2.289 & 1.00 & 0.00 & $\mathrm{H}$ \\
\hline HETATM & 354 & C16 & HEM & 34 & 18.752 & 3.845 & 3.880 & 1.00 & 0.00 & C \\
\hline HETATM & 355 & $\mathrm{C} 17$ & HEM & 34 & 20.118 & 3.732 & 3.398 & 1.00 & 0.00 & C \\
\hline HETATM & 356 & $\mathrm{C} 18$ & HEM & 34 & 20.793 & 2.964 & 4.322 & 1.00 & 0.00 & C \\
\hline HETATM & 357 & C19 & HEM & 34 & 19.843 & 2.612 & 5.358 & 1.00 & 0.00 & C \\
\hline HETATM & 358 & $\mathrm{C} 20$ & HEM & 34 & 20.135 & 1.821 & 6.469 & 1.00 & 0.00 & C \\
\hline HETATM & 359 & $\mathrm{H} 2 \mathrm{O}$ & HEM & 34 & 21.149 & 1.432 & 6.530 & 1.00 & 0.00 & $\mathrm{H}$ \\
\hline HETATM & 360 & N21 & HEM & 34 & 17.944 & 1.834 & 7.564 & 1.00 & 0.00 & $\mathrm{~N}$ \\
\hline HETATM & 361 & N22 & HEM & 34 & 15.240 & 2.781 & 7.342 & 1.00 & 0.00 & $\mathrm{~N}$ \\
\hline HETATM & 362 & N23 & HEM & 34 & 15.911 & 4.044 & 4.841 & 1.00 & 0.00 & $\mathrm{~N}$ \\
\hline HETATM & 363 & N24 & HEM & 34 & 18.610 & 3.160 & 5.077 & 1.00 & 0.00 & $\mathrm{~N}$ \\
\hline HETATM & 364 & C99 & HEM & 34 & 17.704 & 6.025 & 7.579 & 1.00 & 0.00 & C \\
\hline HETATM & 365 & 091 & HEM & 34 & 17.544 & 4.711 & 7.567 & 1.00 & 0.00 & O \\
\hline HETATM & 366 & 092 & HEM & 34 & 17.858 & 6.642 & 8.635 & 1.00 & 0.00 & 0 \\
\hline HETATM & 367 & CT91 & HEM & 34 & 17.688 & 6.706 & 6.223 & 1.00 & 0.00 & C \\
\hline HETATM & 368 & H911 & HEM & 34 & 16.709 & 6.511 & 5.760 & 1.00 & 0.00 & $\mathrm{H}$ \\
\hline HETATM & 369 & H912 & HEM & 34 & 18.436 & 6.216 & 5.580 & 1.00 & 0.00 & $\mathrm{H}$ \\
\hline HETATM & 370 & СТ92 & HEM & 34 & 17.947 & 8.211 & 6.334 & 1.00 & 0.00 & C \\
\hline HETATM & 371 & H921 & HEM & 34 & 18.872 & 8.367 & 6.905 & 1.00 & 0.00 & $\mathrm{H}$ \\
\hline HETATM & 372 & H922 & HEM & 34 & 17.151 & 8.671 & 6.931 & 1.00 & 0.00 & $\mathrm{H}$ \\
\hline \multicolumn{11}{|l|}{ TER } \\
\hline HETATM & 373 & C2 & VIN & 35 & 12.427 & 13.923 & -1.002 & 1.00 & 0.00 & C \\
\hline HETATM & 374 & H 21 & VIN & 35 & 11.612 & 13.224 & -0.837 & 1.00 & 0.00 & $\mathrm{H}$ \\
\hline HETATM & 375 & $\mathrm{C} 1$ & VIN & 35 & 12.095 & 15.147 & -1.403 & 1.00 & 0.00 & C \\
\hline HETATM & 376 & H11 & VIN & 35 & 12.826 & 15.926 & -1.511 & 1.00 & 0.00 & $\mathrm{H}$ \\
\hline HETATM & 377 & $\mathrm{H} 12$ & VIN & 35 & 11.053 & 15.417 & -1.570 & 1.00 & 0.00 & $\mathrm{H}$ \\
\hline \multicolumn{11}{|l|}{ TER } \\
\hline HETATM & 378 & $\mathrm{C} 1$ & $\mathrm{MET}$ & 36 & 15.091 & 14.177 & -2.840 & 1.00 & 0.00 & C \\
\hline HETATM & 379 & H11 & $\mathrm{MET}$ & 36 & 15.326 & 15.248 & -2.720 & 1.00 & 0.00 & $\mathrm{H}$ \\
\hline HETATM & 380 & $\mathrm{H} 12$ & $\mathrm{MET}$ & 36 & 15.904 & 13.713 & -3.410 & 1.00 & 0.00 & $\mathrm{H}$ \\
\hline HETATM & 381 & H13 & $\mathrm{MET}$ & 36 & 14.184 & 14.100 & -3.450 & 1.00 & 0.00 & $\mathrm{H}$ \\
\hline \multicolumn{11}{|l|}{ TER } \\
\hline HETATM & 382 & $\mathrm{C} 2$ & VIN & 37 & 20.101 & 12.499 & -2.325 & 1.00 & 0.00 & C \\
\hline HETATM & 383 & H 21 & VIN & 37 & 19.435 & 12.399 & -3.184 & 1.00 & 0.00 & $\mathrm{H}$ \\
\hline HETATM & 384 & $\mathrm{C} 1$ & VIN & 37 & 21.268 & 13.144 & -2.484 & 1.00 & 0.00 & C \\
\hline HETATM & 385 & H11 & VIN & 37 & 21.948 & 13.300 & -1.654 & 1.00 & 0.00 & $\mathrm{H}$ \\
\hline HETATM & 386 & $\mathrm{H} 12$ & VIN & 37 & 21.573 & 13.542 & -3.450 & 1.00 & 0.00 & $\mathrm{H}$ \\
\hline TER & & & & & & & & & & \\
\hline HETATM & 387 & $\mathrm{C} 1$ & $\mathrm{MET}$ & 38 & 21.786 & 10.816 & -0.121 & 1.00 & 0.00 & C \\
\hline HETATM & 388 & H11 & $\mathrm{MET}$ & 38 & 22.377 & 11.480 & 0.528 & 1.00 & 0.00 & $\mathrm{H}$ \\
\hline HETATM & 389 & $\mathrm{H} 12$ & $\mathrm{MET}$ & 38 & 21.942 & 9.794 & 0.246 & 1.00 & 0.00 & $\mathrm{H}$ \\
\hline HETATM & 390 & $\mathrm{H} 13$ & $\mathrm{MET}$ & 38 & 22.218 & 10.882 & -1.126 & 1.00 & 0.00 & $\mathrm{H}$ \\
\hline TER & & & & & & & & & & \\
\hline HETATM & 391 & C5 & $\mathrm{ACP}$ & 39 & 12.697 & 9.735 & 5.770 & 1.00 & 0.00 & C \\
\hline HETATM & 392 & H51 & $\mathrm{ACP}$ & 39 & 13.436 & 9.723 & 6.582 & 1.00 & 0.00 & $\mathrm{H}$ \\
\hline HETATM & 393 & H52 & $\mathrm{ACP}$ & 39 & 11.857 & 10.343 & 6.134 & 1.00 & 0.00 & $\mathrm{H}$ \\
\hline HETATM & 394 & C4 & $\mathrm{ACP}$ & 39 & 12.219 & 8.297 & 5.497 & 1.00 & 0.00 & C \\
\hline HETATM & 395 & $\mathrm{H} 41$ & $\mathrm{ACP}$ & 39 & 11.661 & 8.236 & 4.549 & 1.00 & 0.00 & $\mathrm{H}$ \\
\hline HETATM & 396 & $\mathrm{H} 42$ & $\mathrm{ACP}$ & 39 & 13.089 & 7.630 & 5.390 & 1.00 & 0.00 & $\mathrm{H}$ \\
\hline HETATM & 397 & $\mathrm{C} 1$ & $\mathrm{ACP}$ & 39 & 11.355 & 7.685 & 6.573 & 1.00 & 0.00 & C \\
\hline
\end{tabular}




\begin{tabular}{|c|c|c|c|c|c|c|c|c|c|c|}
\hline HETATM & 398 & $\mathrm{O} 2$ & $\mathrm{ACP}$ & 39 & 11.127 & 8.208 & 7.671 & 1.00 & 0.00 & 0 \\
\hline HETATM & 399 & 03 & $\mathrm{ACP}$ & 39 & 10.839 & 6.505 & 6.209 & 1.00 & 0.00 & 0 \\
\hline HETATM & 400 & H3 & $\mathrm{ACP}$ & 39 & 10.230 & 6.151 & 6.945 & 1.00 & 0.00 & $\mathrm{H}$ \\
\hline \multicolumn{11}{|l|}{ TER } \\
\hline HETATM & 401 & $\mathrm{C} 1$ & $\mathrm{MET}$ & 40 & 20.594 & 8.608 & 4.597 & 1.00 & 0.00 & C \\
\hline HETATM & 402 & $\mathrm{H} 11$ & MET & 40 & 21.146 & 9.448 & 5.047 & 1.00 & 0.00 & $\mathrm{H}$ \\
\hline HETATM & 403 & $\mathrm{H} 12$ & MET & 40 & 20.600 & 7.782 & 5.319 & 1.00 & 0.00 & $\mathrm{H}$ \\
\hline HETATM & 404 & $\mathrm{H} 13$ & MET & 40 & 21.165 & 8.289 & 3.717 & 1.00 & 0.00 & $\mathrm{H}$ \\
\hline \multicolumn{11}{|l|}{ TER } \\
\hline HETATM & 405 & $\mathrm{C} 1$ & MET & 41 & 11.160 & 11.557 & 3.646 & 1.00 & 0.00 & $\mathrm{C}$ \\
\hline HETATM & 406 & $\mathrm{H} 11$ & MET & 41 & 10.715 & 11.348 & 4.626 & 1.00 & 0.00 & $\mathrm{H}$ \\
\hline HETATM & 407 & $\mathrm{H} 12$ & MET & 41 & 11.050 & 12.637 & 3.471 & 1.00 & 0.00 & $\mathrm{H}$ \\
\hline HETATM & 408 & $\mathrm{H} 13$ & $\mathrm{MET}$ & 41 & 10.559 & 11.031 & 2.889 & 1.00 & 0.00 & $\mathrm{H}$ \\
\hline \multicolumn{11}{|l|}{ TER } \\
\hline HETATM & 409 & $\mathrm{C} 2$ & VIN & 42 & 20.957 & 0.178 & 8.933 & 1.00 & 0.00 & C \\
\hline HETATM & 410 & H 21 & VIN & 42 & 21.773 & 0.876 & 8.768 & 1.00 & 0.00 & $\mathrm{H}$ \\
\hline HETATM & 411 & C1 & VIN & 42 & 21.290 & -1.047 & 9.335 & 1.00 & 0.00 & C \\
\hline HETATM & 412 & $\mathrm{H} 11$ & VIN & 42 & 20.558 & -1.826 & 9.442 & 1.00 & 0.00 & $\mathrm{H}$ \\
\hline HETATM & 413 & $\mathrm{H} 12$ & VIN & 42 & 22.331 & -1.316 & 9.501 & 1.00 & 0.00 & $\mathrm{H}$ \\
\hline \multicolumn{11}{|l|}{ TER } \\
\hline HETATM & 414 & $\mathrm{C} 1$ & MET & 43 & 18.293 & -0.077 & 10.771 & 1.00 & 0.00 & C \\
\hline HETATM & 415 & $\mathrm{H} 11$ & MET & 43 & 18.058 & -1.148 & 10.652 & 1.00 & 0.00 & $\mathrm{H}$ \\
\hline HETATM & 416 & $\mathrm{H} 12$ & MET & 43 & 17.480 & 0.387 & 11.341 & 1.00 & 0.00 & $\mathrm{H}$ \\
\hline HETATM & 417 & $\mathrm{H} 13$ & $\mathrm{MET}$ & 43 & 19.201 & 0.001 & 11.382 & 1.00 & 0.00 & $\mathrm{H}$ \\
\hline TER & & & & & & & & & & \\
\hline HETATM & 418 & $\mathrm{C} 2$ & VIN & 44 & 13.283 & 1.601 & 10.256 & 1.00 & 0.00 & C \\
\hline HETATM & 419 & H21 & VIN & 44 & 13.949 & 1.702 & 11.115 & 1.00 & 0.00 & $\mathrm{H}$ \\
\hline HETATM & 420 & $\mathrm{C} 1$ & VIN & 44 & 12.116 & 0.957 & 10.415 & 1.00 & 0.00 & $\mathrm{C}$ \\
\hline HETATM & 421 & $\mathrm{H} 11$ & VIN & 44 & 11.436 & 0.800 & 9.585 & 1.00 & 0.00 & $\mathrm{H}$ \\
\hline HETATM & 422 & $\mathrm{H} 12$ & VIN & 44 & 11.811 & 0.559 & 11.381 & 1.00 & 0.00 & $\mathrm{H}$ \\
\hline TER & & & & & & & & & & \\
\hline HETATM & 423 & $\mathrm{C} 1$ & MET & 45 & 11.598 & 3.284 & 8.052 & 1.00 & 0.00 & C \\
\hline HETATM & 424 & $\mathrm{H} 11$ & MET & 45 & 11.442 & 4.306 & 7.685 & 1.00 & 0.00 & $\mathrm{H}$ \\
\hline HETATM & 425 & $\mathrm{H} 12$ & MET & 45 & 11.166 & 3.219 & 9.057 & 1.00 & 0.00 & $\mathrm{H}$ \\
\hline HETATM & 426 & $\mathrm{H} 13$ & MET & 45 & 11.007 & 2.620 & 7.403 & 1.00 & 0.00 & $\mathrm{H}$ \\
\hline TER & & & & & & & & & & \\
\hline HETATM & 427 & C5 & $\mathrm{ACP}$ & 46 & 20.687 & 4.366 & 2.161 & 1.00 & 0.00 & C \\
\hline HETATM & 428 & H51 & $\mathrm{ACP}$ & 46 & 19.948 & 4.377 & 1.349 & 1.00 & 0.00 & $\mathrm{H}$ \\
\hline HETATM & 429 & H52 & $\mathrm{ACP}$ & 46 & 21.527 & 3.758 & 1.798 & 1.00 & 0.00 & $\mathrm{H}$ \\
\hline HETATM & 430 & $\mathrm{C} 4$ & $\mathrm{ACP}$ & 46 & 21.165 & 5.803 & 2.434 & 1.00 & 0.00 & C \\
\hline HETATM & 431 & H4 1 & $\mathrm{ACP}$ & 46 & 21.723 & 5.865 & 3.382 & 1.00 & 0.00 & $\mathrm{H}$ \\
\hline HETATM & 432 & $\mathrm{H} 42$ & $\mathrm{ACP}$ & 46 & 20.295 & 6.470 & 2.541 & 1.00 & 0.00 & $\mathrm{H}$ \\
\hline HETATM & 433 & $\mathrm{C} 1$ & $\mathrm{ACP}$ & 46 & 22.029 & 6.416 & 1.358 & 1.00 & 0.00 & C \\
\hline HETATM & 434 & $\mathrm{O} 2$ & $\mathrm{ACP}$ & 46 & 22.257 & 5.893 & 0.260 & 1.00 & 0.00 & 0 \\
\hline HETATM & 435 & 03 & $\mathrm{ACP}$ & 46 & 22.545 & 7.595 & 1.722 & 1.00 & 0.00 & 0 \\
\hline HETATM & 436 & H3 & $\mathrm{ACP}$ & 46 & 23.154 & 7.949 & 0.986 & 1.00 & 0.00 & $\mathrm{H}$ \\
\hline TER & & & & & & & & & & \\
\hline HETATM & 437 & $\mathrm{C} 1$ & MET & 47 & 12.791 & 5.492 & 3.334 & 1.00 & 0.00 & C \\
\hline HETATM & 438 & $\mathrm{H} 11$ & MET & 47 & 12.238 & 4.653 & 2.884 & 1.00 & 0.00 & $\mathrm{H}$ \\
\hline HETATM & 439 & $\mathrm{H} 12$ & MET & 47 & 12.784 & 6.318 & 2.613 & 1.00 & 0.00 & $\mathrm{H}$ \\
\hline HETATM & 440 & $\mathrm{H} 13$ & MET & 47 & 12.220 & 5.812 & 4.214 & 1.00 & 0.00 & $\mathrm{H}$ \\
\hline TER & & & & & & & & & & \\
\hline HETATM & 441 & $\mathrm{C} 1$ & $\mathrm{MET}$ & 48 & 22.224 & 2.543 & 4.285 & 1.00 & 0.00 & C \\
\hline HETATM & 442 & $\mathrm{H} 11$ & MET & 48 & 22.669 & 2.752 & 3.305 & 1.00 & 0.00 & $\mathrm{H}$ \\
\hline HETATM & 443 & $\mathrm{H} 12$ & MET & 48 & 22.335 & 1.463 & 4.460 & 1.00 & 0.00 & $\mathrm{H}$ \\
\hline HETATM & 444 & $\mathrm{H} 13$ & MET & 48 & 22.825 & 3.069 & 5.042 & 1.00 & 0.00 & $\mathrm{H}$ \\
\hline TER & & & & & & & & & & \\
\hline HETATM & 445 & $\mathrm{FE}$ & HEM & 49 & 17.294 & 11.003 & -6.440 & 1.00 & 0.00 & $\mathrm{FE}$ \\
\hline HETATM & 446 & $\mathrm{C} 1$ & HEM & 49 & 15.066 & 12.876 & -7.502 & 1.00 & 0.00 & C \\
\hline HETATM & 447 & $\mathrm{C} 2$ & HEM & 49 & 14.707 & 13.666 & -8.674 & 1.00 & 0.00 & C \\
\hline HETATM & 448 & $\mathrm{C} 3$ & HEM & 49 & 15.852 & 13.763 & -9.448 & 1.00 & 0.00 & C \\
\hline HETATM & 449 & $\mathrm{C} 4$ & HEM & 49 & 16.882 & 13.046 & -8.740 & 1.00 & 0.00 & C \\
\hline HETATM & 450 & C5 & HEM & 49 & 18.197 & 12.927 & -9.178 & 1.00 & 0.00 & C \\
\hline HETATM & 451 & H5 & HEM & 49 & 18.451 & 13.423 & -10.113 & 1.00 & 0.00 & $\mathrm{H}$ \\
\hline HETATM & 452 & $\mathrm{C} 6$ & HEM & 49 & 19.216 & 12.229 & -8.542 & 1.00 & 0.00 & C \\
\hline HETATM & 453 & $\mathrm{C} 7$ & HEM & 49 & 20.585 & 12.156 & -9.012 & 1.00 & 0.00 & C \\
\hline HETATM & 454 & C8 & HEM & 49 & 21.291 & 11.436 & -8.065 & 1.00 & 0.00 & $\mathrm{C}$ \\
\hline HETATM & 455 & $\mathrm{C} 9$ & HEM & 49 & 20.340 & 11.071 & -7.041 & 1.00 & 0.00 & C \\
\hline HETATM & 456 & $\mathrm{C} 10$ & HEM & 49 & 20.644 & 10.336 & -5.897 & 1.00 & 0.00 & C \\
\hline HETATM & 457 & $\mathrm{H} 10$ & HEM & 49 & 21.671 & 9.993 & -5.791 & 1.00 & 0.00 & $\mathrm{H}$ \\
\hline HETATM & 458 & $\mathrm{C} 11$ & HEM & 49 & 19.770 & 9.996 & -4.874 & 1.00 & 0.00 & C \\
\hline HETATM & 459 & C12 & HEM & 49 & 20.151 & 9.271 & -3.684 & 1.00 & 0.00 & C \\
\hline HETATM & 460 & C13 & HEM & 49 & 19.011 & 9.124 & -2.927 & 1.00 & 0.00 & C \\
\hline HETATM & 461 & C14 & HEM & 49 & 17.938 & 9.765 & -3.659 & 1.00 & 0.00 & C \\
\hline HETATM & 462 & C15 & HEM & 49 & 16.610 & 9.820 & -3.237 & 1.00 & 0.00 & C \\
\hline HETATM & 463 & H15 & HEM & 49 & 16.370 & 9.335 & -2.289 & 1.00 & 0.00 & $\mathrm{H}$ \\
\hline HETATM & 464 & C16 & HEM & 49 & 15.580 & 10.502 & -3.880 & 1.00 & 0.00 & C \\
\hline HETATM & 465 & $\mathrm{C} 17$ & HEM & 49 & 14.214 & 10.615 & -3.398 & 1.00 & 0.00 & C \\
\hline HETATM & 466 & $\mathrm{C} 18$ & HEM & 49 & 13.539 & 11.383 & -4.322 & 1.00 & 0.00 & C \\
\hline
\end{tabular}


$\begin{array}{lllll}17.986 & 3.589 & -1.491 & 1.00 & 0.00\end{array}$

$\begin{array}{lllll}20.214 & 1.717 & -0.429 & 1.00 & 0.00\end{array}$

$\begin{array}{lllll}20.573 & 0.927 & 0.742 & 1.00 & 0.00\end{array}$

$\begin{array}{lllll}19.427 & 0.830 & 1.517 & 1.00 & 0.00\end{array}$

$\begin{array}{lllll}18.398 & 1.547 & 0.809 & 1.00 & 0.00\end{array}$

$\begin{array}{lllll}17.082 & 1.666 & 1.247 & 1.00 & 0.00\end{array}$

$\begin{array}{lllll}16.829 & 1.170 & 2.182 & 1.00 & 0.00\end{array}$

$\begin{array}{lllll}16.063 & 2.364 & 0.611 & 1.00 & 0.00\end{array}$

$\begin{array}{lllll}14.694 & 2.437 & 1.081 & 1.00 & 0.00\end{array}$

$\begin{array}{lllll}13.989 & 3.157 & 0.134 & 1.00 & 0.00\end{array}$

$\begin{array}{lllll}14.940 & 3.522 & -0.891 & 1.00 & 0.00\end{array}$

$\begin{array}{lllll}14.635 & 4.256 & -2.034 & 1.00 & 0.00\end{array}$

$\begin{array}{lllll}13.608 & 4.600 & -2.140 & 1.00 & 0.00\end{array}$

$\begin{array}{llllll}15.510 & 4.596 & -3.057 & 1.00 & 0.00\end{array}$

$\begin{array}{lllll}15.129 & 5.322 & -4.247 & 1.00 & 0.00\end{array}$

$\begin{array}{lllll}16.268 & 5.469 & -5.004 & 1.00 & 0.00\end{array}$

$\begin{array}{llllll}17.341 & 4.828 & -4.273 & 1.00 & 0.00\end{array}$

$\begin{array}{lllll}18.669 & 4.773 & -4.694 & 1.00 & 0.00\end{array}$

$\begin{array}{lllll}18.909 & 5.258 & -5.642 & 1.00 & 0.00\end{array}$

$\begin{array}{lllll}19.699 & 4.091 & -4.051 & 1.00 & 0.00\end{array}$

$\begin{array}{lllll}21.065 & 3.978 & -4.533 & 1.00 & 0.00\end{array}$

$\begin{array}{lllll}21.741 & 3.210 & -3.609 & 1.00 & 0.00\end{array}$

$\begin{array}{lllll}20.791 & 2.858 & -2.573 & 1.00 & 0.00\end{array}$

$\begin{array}{lllll}21.082 & 2.067 & -1.462 & 1.00 & 0.00\end{array}$

$\begin{array}{lllll}22.097 & 1.678 & -1.402 & 1.00 & 0.00\end{array}$

$\begin{array}{lllll}18.892 & 2.080 & -0.367 & 1.00 & 0.00\end{array}$

$\begin{array}{lllll}16.187 & 3.027 & -0.590 & 1.00 & 0.00\end{array}$

$\begin{array}{llllll}16.858 & 4.290 & -3.090 & 1.00 & 0.00\end{array}$

$\begin{array}{lllll}19.558 & 3.406 & -2.854 & 1.00 & 0.00\end{array}$

$\begin{array}{lllll}18.651 & 6.271 & -0.352 & 1.00 & 0.00\end{array}$

$\begin{array}{lllll}18.492 & 4.958 & -0.365 & 1.00 & 0.00\end{array}$

$\begin{array}{lllll}18.806 & 6.888 & 0.703 & 1.00 & 0.00\end{array}$

$\begin{array}{lllll}18.635 & 6.952 & -1.709 & 1.00 & 0.00\end{array}$

$\begin{array}{lllll}17.656 & 6.757 & -2.171 & 1.00 & 0.00\end{array}$

$\begin{array}{lllll}19.383 & 6.463 & -2.352 & 1.00 & 0.00\end{array}$

$\begin{array}{llllll}18.895 & 8.457 & -1.597 & 1.00 & 0.00\end{array}$

$\begin{array}{lllll}19.820 & 8.613 & -1.027 & 1.00 & 0.00\end{array}$

$\begin{array}{lllll}18.098 & 8.917 & -1.000 & 1.00 & 0.00\end{array}$

$\begin{array}{lllll}13.375 & 14.169 & -8.933 & 1.00 & 0.00\end{array}$

$\begin{array}{lllll}12.559 & 13.470 & -8.768 & 1.00 & 0.00\end{array}$

$\begin{array}{lllll}13.042 & 15.394 & -9.335 & 1.00 & 0.00\end{array}$

$\begin{array}{lllll}13.774 & 16.172 & -9.442 & 1.00 & 0.00\end{array}$

$\begin{array}{lllll}12.000 & 15.663 & -9.501 & 1.00 & 0.00\end{array}$

$\begin{array}{llllll}16.039 & 14.424 & -10.771 & 1.00 & 0.00\end{array}$

$\begin{array}{lllll}16.274 & 15.494 & -10.652 & 1.00 & 0.00\end{array}$

$\begin{array}{lllll}16.852 & 13.959 & -11.341 & 1.00 & 0.00\end{array}$

$\begin{array}{lllll}15.131 & 14.346 & -11.382 & 1.00 & 0.00\end{array}$

$\begin{array}{llllll}21.049 & 12.746 & -10.256 & 1.00 & 0.00\end{array}$

$20.383 \quad 12.645-11.115 \quad 1.00 \quad 0.00$

$22.216 \quad 13.390-10.415 \quad 1.00 \quad 0.00$

$\begin{array}{lllll}22.896 & 13.546 & -9.585 & 1.00 & 0.00\end{array}$

$\begin{array}{lllll}22.521 & 13.788 & -11.381 & 1.00 & 0.00\end{array}$

$\begin{array}{lllll}22.733 & 11.062 & -8.052 & 1.00 & 0.00\end{array}$

$\begin{array}{llllll}23.325 & 11.727 & -7.403 & 1.00 & 0.00\end{array}$

$\begin{array}{lllll}22.890 & 10.040 & -7.685 & 1.00 & 0.00\end{array}$

$\begin{array}{lllll}23.166 & 11.128 & -9.057 & 1.00 & 0.00\end{array}$ 


\begin{tabular}{|c|c|c|c|}
\hline HETATM & 540 & H51 & $\mathrm{ACP}$ \\
\hline HETATM & 541 & H52 & $\mathrm{ACP}$ \\
\hline HETATM & 542 & C4 & $\mathrm{ACP}$ \\
\hline HETATM & 543 & H 41 & $\mathrm{ACP}$ \\
\hline HETATM & 544 & H 42 & $\mathrm{ACP}$ \\
\hline HETATM & 545 & $\mathrm{C} 1$ & $\mathrm{ACP}$ \\
\hline HETATM & 546 & 02 & $\mathrm{ACP}$ \\
\hline HETATM & 547 & 03 & $\mathrm{ACP}$ \\
\hline HETATM & 548 & H3 & $\mathrm{ACP}$ \\
\hline \multicolumn{4}{|l|}{ TER } \\
\hline HETATM & 549 & $\mathrm{C} 1$ & MET \\
\hline HETATM & 550 & H11 & MET \\
\hline HETATM & 551 & H12 & MET \\
\hline HETATM & 552 & H13 & MET \\
\hline \multicolumn{4}{|l|}{ TER } \\
\hline HETATM & 553 & $\mathrm{C} 1$ & MET \\
\hline HETATM & 554 & $\mathrm{H} 11$ & MET \\
\hline HETATM & 555 & $\mathrm{H} 12$ & MET \\
\hline HETATM & 556 & $\mathrm{H} 13$ & MET \\
\hline \multicolumn{4}{|l|}{ TER } \\
\hline HETATM & 557 & $\mathrm{C} 2$ & VIN \\
\hline HETATM & 558 & H 21 & VIN \\
\hline HETATM & 559 & $\mathrm{C} 1$ & VIN \\
\hline HETATM & 560 & $\mathrm{H} 11$ & VIN \\
\hline HETATM & 561 & $\mathrm{H} 12$ & VIN \\
\hline \multicolumn{4}{|l|}{ TER } \\
\hline HETATM & 562 & $\mathrm{C} 1$ & MET \\
\hline HETATM & 563 & $\mathrm{H} 11$ & MET \\
\hline HETATM & 564 & $\mathrm{H} 12$ & MET \\
\hline HETATM & 565 & $\mathrm{H} 13$ & MET \\
\hline \multicolumn{4}{|l|}{ TER } \\
\hline HETATM & 566 & C2 & VIN \\
\hline HETATM & 567 & H 21 & VIN \\
\hline HETATM & 568 & $\mathrm{C} 1$ & VIN \\
\hline HETATM & 569 & H11 & VIN \\
\hline HETATM & 570 & $\mathrm{H} 12$ & VIN \\
\hline \multicolumn{4}{|l|}{ TER } \\
\hline HETATM & 571 & $\mathrm{C} 1$ & MET \\
\hline HETATM & 572 & $\mathrm{H} 11$ & MET \\
\hline HETATM & 573 & $\mathrm{H} 12$ & MET \\
\hline HETATM & 574 & $\mathrm{H} 13$ & MET \\
\hline \multicolumn{4}{|l|}{ TER } \\
\hline HETATM & 575 & C5 & $\mathrm{ACP}$ \\
\hline HETATM & 576 & H51 & $\mathrm{ACP}$ \\
\hline HETATM & 577 & H52 & $\mathrm{ACP}$ \\
\hline HETATM & 578 & C4 & $\mathrm{ACP}$ \\
\hline HETATM & 579 & $\mathrm{H} 4 \mathrm{I}$ & $\mathrm{ACP}$ \\
\hline HETATM & 580 & $\mathrm{H} 42$ & $\mathrm{ACP}$ \\
\hline HETATM & 581 & $\mathrm{C} 1$ & $\mathrm{ACP}$ \\
\hline HETATM & 582 & $\mathrm{O} 2$ & $\mathrm{ACP}$ \\
\hline HETATM & 583 & 03 & $\mathrm{ACP}$ \\
\hline HETATM & 584 & H3 & $\mathrm{ACP}$ \\
\hline \multicolumn{4}{|l|}{ TER } \\
\hline HETATM & 585 & $\mathrm{C} 1$ & MET \\
\hline HETATM & 586 & H11 & MET \\
\hline HETATM & 587 & H12 & MET \\
\hline HETATM & 588 & H13 & MET \\
\hline \multicolumn{4}{|l|}{ TER } \\
\hline HETATM & 589 & $\mathrm{C} 1$ & MET \\
\hline HETATM & 590 & H11 & MET \\
\hline HETATM & 591 & H12 & MET \\
\hline HETATM & 592 & H13 & MET \\
\hline TER & & & \\
\hline
\end{tabular}

\begin{tabular}{|c|c|c|c|c|c|}
\hline 14.384 & 9.969 & -1.349 & 1.00 & 0.00 & $\mathrm{H}$ \\
\hline 12.804 & 10.589 & -1.798 & 1.00 & 0.00 & $\mathrm{H}$ \\
\hline 13.167 & 8.543 & -2.434 & 1.00 & 0.00 & C \\
\hline 12.609 & 8.482 & -3.382 & 1.00 & 0.00 & $\mathrm{H}$ \\
\hline 14.037 & 7.877 & -2.541 & 1.00 & 0.00 & $\mathrm{H}$ \\
\hline 12.303 & 7.931 & -1.358 & 1.00 & 0.00 & C \\
\hline 12.075 & 8.454 & -0.260 & 1.00 & 0.00 & 0 \\
\hline 11.787 & 6.751 & -1.722 & 1.00 & 0.00 & 0 \\
\hline 11.178 & 6.397 & -0.986 & 1.00 & 0.00 & $\mathrm{H}$ \\
\hline 21.541 & 8.855 & -3.334 & 1.00 & 0.00 & C \\
\hline 22.094 & 9.694 & -2.884 & 1.00 & 0.00 & $\mathrm{H}$ \\
\hline 21.548 & 8.029 & -2.613 & 1.00 & 0.00 & $\mathrm{H}$ \\
\hline 22.112 & 8.535 & -4.214 & 1.00 & 0.00 & $\mathrm{H}$ \\
\hline 12.108 & 11.803 & -4.285 & 1.00 & 0.00 & C \\
\hline 11.663 & 11.594 & -3.305 & 1.00 & 0.00 & $\mathrm{H}$ \\
\hline 11.997 & 12.883 & -4.460 & 1.00 & 0.00 & $\mathrm{H}$ \\
\hline 11.507 & 11.278 & -5.042 & 1.00 & 0.00 & $\mathrm{H}$ \\
\hline 21.904 & 0.424 & 1.002 & 1.00 & 0.00 & $\mathrm{C}$ \\
\hline 22.720 & 1.123 & 0.837 & 1.00 & 0.00 & $\mathrm{H}$ \\
\hline 22.237 & -0.801 & 1.403 & 1.00 & 0.00 & C \\
\hline 21.506 & -1.579 & 1.511 & 1.00 & 0.00 & $\mathrm{H}$ \\
\hline 23.279 & -1.070 & 1.570 & 1.00 & 0.00 & $\mathrm{H}$ \\
\hline 19.241 & 0.169 & 2.840 & 1.00 & 0.00 & C \\
\hline 19.006 & -0.902 & 2.720 & 1.00 & 0.00 & $\mathrm{H}$ \\
\hline 18.428 & 0.633 & 3.410 & 1.00 & 0.00 & $\mathrm{H}$ \\
\hline 20.148 & 0.247 & 3.450 & 1.00 & 0.00 & $\mathrm{H}$ \\
\hline 14.231 & 1.847 & 2.325 & 1.00 & 0.00 & C \\
\hline 14.897 & 1.948 & 3.184 & 1.00 & 0.00 & $\mathrm{H}$ \\
\hline 13.064 & 1.203 & 2.484 & 1.00 & 0.00 & C \\
\hline 12.384 & 1.047 & 1.654 & 1.00 & 0.00 & $\mathrm{H}$ \\
\hline 12.759 & 0.805 & 3.450 & 1.00 & 0.00 & $\mathrm{H}$ \\
\hline 12.546 & 3.530 & 0.121 & 1.00 & 0.00 & C \\
\hline 12.390 & 4.553 & -0.246 & 1.00 & 0.00 & $\mathrm{H}$ \\
\hline 12.114 & 3.465 & 1.126 & 1.00 & 0.00 & $\mathrm{H}$ \\
\hline 11.955 & 2.866 & -0.528 & 1.00 & 0.00 & $\mathrm{H}$ \\
\hline 21.634 & 4.612 & -5.770 & 1.00 & 0.00 & $\mathrm{C}$ \\
\hline 20.895 & 4.623 & -6.582 & 1.00 & 0.00 & $\mathrm{H}$ \\
\hline 22.475 & 4.004 & -6.134 & 1.00 & 0.00 & $\mathrm{H}$ \\
\hline 22.113 & 6.050 & -5.497 & 1.00 & 0.00 & C \\
\hline 22.671 & 6.111 & -4.549 & 1.00 & 0.00 & $\mathrm{H}$ \\
\hline 21.242 & 6.716 & -5.390 & 1.00 & 0.00 & $\mathrm{H}$ \\
\hline 22.977 & 6.662 & -6.573 & 1.00 & 0.00 & C \\
\hline 23.205 & 6.139 & -7.671 & 1.00 & 0.00 & 0 \\
\hline 23.493 & 7.841 & -6.209 & 1.00 & 0.00 & O \\
\hline 24.102 & 8.196 & -6.945 & 1.00 & 0.00 & $\mathrm{H}$ \\
\hline 13.738 & 5.738 & -4.597 & 1.00 & 0.00 & C \\
\hline 13.186 & 4.899 & -5.047 & 1.00 & 0.00 & $\mathrm{H}$ \\
\hline 13.732 & 6.564 & -5.319 & 1.00 & 0.00 & $\mathrm{H}$ \\
\hline 13.167 & 6.058 & -3.717 & 1.00 & 0.00 & $\mathrm{H}$ \\
\hline 23.172 & 2.790 & -3.646 & 1.00 & 0.00 & C \\
\hline 23.617 & 2.998 & -4.626 & 1.00 & 0.00 & $\mathrm{H}$ \\
\hline 23.282 & 1.709 & -3.471 & 1.00 & 0.00 & $\mathrm{H}$ \\
\hline 23.772 & 3.315 & -2.889 & 1.00 & 0.00 & $\mathrm{H}$ \\
\hline
\end{tabular}

\subsection{Q5 dimer: Q05_Q5.pdb file}

\begin{tabular}{|c|c|c|c|c|c|c|c|c|c|}
\hline HETATM & 1 & $\mathrm{FE}$ & HEM & 1 & 4.263 & 10.757 & 1.491 & 1.00 & 0.00 \\
\hline HETATM & 2 & $\mathrm{C} 1$ & HEM & 1 & 2.035 & 12.629 & 0.429 & 1.00 & 0.00 \\
\hline HETATM & 3 & $\mathrm{C} 2$ & HEM & 1 & 1.676 & 13.420 & -0.742 & 1.00 & 0.00 \\
\hline HETATM & 4 & C3 & HEM & 1 & 2.822 & 13.517 & -1.517 & 1.00 & 0.00 \\
\hline HETATM & 5 & $\mathrm{C} 4$ & HEM & 1 & 3.851 & 12.800 & -0.809 & 1.00 & 0.00 \\
\hline HETATM & 6 & C5 & HEM & 1 & 5.167 & 12.681 & -1.247 & 1.00 & 0.00 \\
\hline HETATM & 7 & H5 & HEM & 1 & 5.420 & 13.177 & -2.182 & 1.00 & 0.00 \\
\hline HETATM & 8 & C6 & HEM & 1 & 6.186 & 11.982 & -0.611 & 1.00 & .00 \\
\hline HETATM & 9 & C7 & HEM & 1 & 7.555 & 11.910 & -1.081 & 1.00 & .00 \\
\hline HETATM & 10 & $\mathrm{C} 8$ & HEM & 1 & 8.260 & 11.190 & -0.134 & 1.00 & .00 \\
\hline HETATM & 11 & C9 & HEM & 1 & 7.309 & 10.825 & 0.891 & 1.00 & 0.00 \\
\hline
\end{tabular}




\begin{tabular}{|c|c|c|c|c|c|c|c|c|c|c|}
\hline HETATM & 12 & $\mathrm{C} 10$ & HEM & 1 & 7.614 & 10.090 & 2.034 & 1.00 & 0.00 & C \\
\hline HETATM & 13 & $\mathrm{H} 10$ & HEM & 1 & 8.641 & 9.746 & 2.140 & 1.00 & 0.00 & $\mathrm{H}$ \\
\hline HETATM & 14 & $\mathrm{C} 11$ & HEM & 1 & 6.739 & 9.750 & 3.057 & 1.00 & 0.00 & C \\
\hline HETATM & 15 & $\mathrm{C} 12$ & HEM & 1 & 7.120 & 9.025 & 4.247 & 1.00 & 0.00 & C \\
\hline HETATM & 16 & C13 & HEM & 1 & 5.981 & 8.878 & 5.004 & 1.00 & 0.00 & C \\
\hline HETATM & 17 & $\mathrm{C} 14$ & HEM & 1 & 4.908 & 9.519 & 4.273 & 1.00 & 0.00 & C \\
\hline HETATM & 18 & C15 & HEM & 1 & 3.580 & 9.574 & 4.694 & 1.00 & 0.00 & C \\
\hline HETATM & 19 & H15 & HEM & 1 & 3.340 & 9.089 & 5.642 & 1.00 & 0.00 & $\mathrm{H}$ \\
\hline HETATM & 20 & $\mathrm{C} 16$ & HEM & 1 & 2.550 & 10.255 & 4.051 & 1.00 & 0.00 & C \\
\hline HETATM & 21 & $\mathrm{C} 17$ & HEM & 1 & 1.184 & 10.369 & 4.533 & 1.00 & 0.00 & C \\
\hline HETATM & 22 & $\mathrm{C} 18$ & HEM & 1 & 0.508 & 11.137 & 3.609 & 1.00 & 0.00 & C \\
\hline HETATM & 23 & C19 & HEM & 1 & 1.458 & 11.489 & 2.573 & 1.00 & 0.00 & C \\
\hline HETATM & 24 & $\mathrm{C} 20$ & HEM & 1 & 1.167 & 12.280 & 1.462 & 1.00 & 0.00 & C \\
\hline HETATM & 25 & $\mathrm{H} 2 \mathrm{O}$ & HEM & 1 & 0.152 & 12.669 & 1.402 & 1.00 & 0.00 & $\mathrm{H}$ \\
\hline HETATM & 26 & N21 & HEM & 1 & 3.357 & 12.266 & 0.367 & 1.00 & 0.00 & $\mathrm{~N}$ \\
\hline HETATM & 27 & N22 & HEM & 1 & 6.062 & 11.319 & 0.590 & 1.00 & 0.00 & $\mathrm{~N}$ \\
\hline HETATM & 28 & N23 & HEM & 1 & 5.391 & 10.056 & 3.090 & 1.00 & 0.00 & $\mathrm{~N}$ \\
\hline HETATM & 29 & N24 & HEM & 1 & 2.691 & 10.941 & 2.854 & 1.00 & 0.00 & $\mathrm{~N}$ \\
\hline HETATM & 30 & C99 & HEM & 1 & 3.598 & 8.076 & 0.352 & 1.00 & 0.00 & C \\
\hline HETATM & 31 & 091 & HEM & 1 & 3.757 & 9.389 & 0.365 & 1.00 & 0.00 & o \\
\hline HETATM & 32 & 092 & HEM & 1 & 3.443 & 7.458 & -0.703 & 1.00 & 0.00 & O \\
\hline HETATM & 33 & СТ91 & HEM & 1 & 3.614 & 7.394 & 1.709 & 1.00 & 0.00 & C \\
\hline HETATM & 34 & H911 & HEM & 1 & 4.593 & 7.590 & 2.171 & 1.00 & 0.00 & $\mathrm{H}$ \\
\hline HETATM & 35 & H912 & HEM & 1 & 2.866 & 7.884 & 2.352 & 1.00 & 0.00 & $\mathrm{H}$ \\
\hline HETATM & 36 & СТ92 & HEM & 1 & 3.354 & 5.890 & 1.597 & 1.00 & 0.00 & C \\
\hline HETATM & 37 & H921 & HEM & 1 & 4.151 & 5.429 & 1.000 & 1.00 & 0.00 & $\mathrm{H}$ \\
\hline HETATM & 38 & H922 & HEM & 1 & 2.429 & 5.734 & 1.027 & 1.00 & 0.00 & $\mathrm{H}$ \\
\hline \multicolumn{11}{|l|}{ TER } \\
\hline HETATM & 39 & $\mathrm{FE}$ & HEM & 2 & 4.955 & 3.343 & 6.440 & 1.00 & 0.00 & $\mathrm{Fe}$ \\
\hline HETATM & 40 & $\mathrm{C} 1$ & HEM & 2 & 7.183 & 1.471 & 7.502 & 1.00 & 0.00 & C \\
\hline HETATM & 41 & $\mathrm{C} 2$ & HEM & 2 & 7.542 & 0.680 & 8.674 & 1.00 & 0.00 & C \\
\hline HETATM & 42 & C3 & HEM & 2 & 6.397 & 0.583 & 9.448 & 1.00 & 0.00 & C \\
\hline HETATM & 43 & $\mathrm{C} 4$ & HEM & 2 & 5.367 & 1.301 & 8.740 & 1.00 & 0.00 & C \\
\hline HETATM & 44 & C5 & HEM & 2 & 4.052 & 1.420 & 9.178 & 1.00 & 0.00 & C \\
\hline HETATM & 45 & H5 & HEM & 2 & 3.798 & 0.923 & 10.113 & 1.00 & 0.00 & $\mathrm{H}$ \\
\hline HETATM & 46 & C6 & HEM & 2 & 3.033 & 2.118 & 8.542 & 1.00 & 0.00 & C \\
\hline HETATM & 47 & C7 & HEM & 2 & 1.664 & 2.191 & 9.012 & 1.00 & 0.00 & C \\
\hline HETATM & 48 & $\mathrm{C} 8$ & HEM & 2 & 0.958 & 2.910 & 8.065 & 1.00 & 0.00 & C \\
\hline HETATM & 49 & $\mathrm{C} 9$ & HEM & 2 & 1.909 & 3.275 & 7.041 & 1.00 & 0.00 & C \\
\hline HETATM & 50 & $\mathrm{C} 10$ & HEM & 2 & 1.605 & 4.010 & 5.897 & 1.00 & 0.00 & C \\
\hline HETATM & 51 & $\mathrm{H} 10$ & HEM & 2 & 0.578 & 4.354 & 5.791 & 1.00 & 0.00 & $\mathrm{H}$ \\
\hline HETATM & 52 & $\mathrm{C} 11$ & HEM & 2 & 2.479 & 4.350 & 4.874 & 1.00 & 0.00 & C \\
\hline HETATM & 53 & $\mathrm{C} 12$ & HEM & 2 & 2.098 & 5.076 & 3.684 & 1.00 & 0.00 & C \\
\hline HETATM & 54 & $\mathrm{C} 13$ & HEM & 2 & 3.238 & 5.223 & 2.927 & 1.00 & 0.00 & C \\
\hline HETATM & 55 & $\mathrm{C} 14$ & HEM & 2 & 4.311 & 4.581 & 3.659 & 1.00 & 0.00 & $\mathrm{C}$ \\
\hline HETATM & 56 & C15 & HEM & 2 & 5.639 & 4.527 & 3.237 & 1.00 & 0.00 & C \\
\hline HETATM & 57 & H15 & HEM & 2 & 5.879 & 5.011 & 2.289 & 1.00 & 0.00 & $\mathrm{H}$ \\
\hline HETATM & 58 & $\mathrm{C} 16$ & HEM & 2 & 6.669 & 3.845 & 3.880 & 1.00 & 0.00 & C \\
\hline HETATM & 59 & $\mathrm{C} 17$ & HEM & 2 & 8.035 & 3.732 & 3.398 & 1.00 & 0.00 & $\mathrm{C}$ \\
\hline HETATM & 60 & $\mathrm{C} 18$ & HEM & 2 & 8.710 & 2.964 & 4.322 & 1.00 & 0.00 & C \\
\hline HETATM & 61 & C19 & HEM & 2 & 7.761 & 2.612 & 5.358 & 1.00 & 0.00 & $\mathrm{C}$ \\
\hline HETATM & 62 & $\mathrm{C} 20$ & HEM & 2 & 8.052 & 1.821 & 6.469 & 1.00 & 0.00 & C \\
\hline HETATM & 63 & $\mathrm{H} 2 \mathrm{O}$ & HEM & 2 & 9.066 & 1.432 & 6.530 & 1.00 & 0.00 & $\mathrm{H}$ \\
\hline HETATM & 64 & N21 & HEM & 2 & 5.862 & 1.834 & 7.564 & 1.00 & 0.00 & $\mathrm{~N}$ \\
\hline HETATM & 65 & N22 & HEM & 2 & 3.157 & 2.781 & 7.342 & 1.00 & 0.00 & $\mathrm{~N}$ \\
\hline HETATM & 66 & N23 & HEM & 2 & 3.828 & 4.044 & 4.841 & 1.00 & 0.00 & $\mathrm{~N}$ \\
\hline HETATM & 67 & N24 & HEM & 2 & 6.527 & 3.160 & 5.077 & 1.00 & 0.00 & $\mathrm{~N}$ \\
\hline HETATM & 68 & C99 & HEM & 2 & 5.621 & 6.025 & 7.579 & 1.00 & 0.00 & C \\
\hline HETATM & 69 & 091 & HEM & 2 & 5.461 & 4.711 & 7.567 & 1.00 & 0.00 & O \\
\hline HETATM & 70 & 092 & HEM & 2 & 5.776 & 6.642 & 8.635 & 1.00 & 0.00 & 0 \\
\hline HETATM & 71 & СТ91 & HEM & 2 & 5.605 & 6.706 & 6.223 & 1.00 & 0.00 & C \\
\hline HETATM & 72 & H911 & HEM & 2 & 4.626 & 6.511 & 5.760 & 1.00 & 0.00 & $\mathrm{H}$ \\
\hline HETATM & 73 & H912 & HEM & 2 & 6.353 & 6.216 & 5.580 & 1.00 & 0.00 & $\mathrm{H}$ \\
\hline HETATM & 74 & СТ92 & HEM & 2 & 5.864 & 8.211 & 6.334 & 1.00 & 0.00 & C \\
\hline HETATM & 75 & H921 & HEM & 2 & 6.789 & 8.367 & 6.905 & 1.00 & 0.00 & $\mathrm{H}$ \\
\hline HETATM & 76 & H922 & HEM & 2 & 5.068 & 8.671 & 6.931 & 1.00 & 0.00 & $\mathrm{H}$ \\
\hline \multicolumn{11}{|l|}{ TER } \\
\hline HETATM & 77 & $\mathrm{C} 2$ & VIN & 3 & 0.345 & 13.923 & -1.002 & 1.00 & 0.00 & C \\
\hline HETATM & 78 & H 21 & VIN & 3 & -0.471 & 13.224 & -0.837 & 1.00 & 0.00 & $\mathrm{H}$ \\
\hline HETATM & 79 & $\mathrm{C} 1$ & VIN & 3 & 0.012 & 15.147 & -1.403 & 1.00 & 0.00 & $\mathrm{C}$ \\
\hline HETATM & 80 & $\mathrm{H} 11$ & VIN & 3 & 0.743 & 15.926 & -1.511 & 1.00 & 0.00 & $\mathrm{H}$ \\
\hline HETATM & 81 & $\mathrm{H} 12$ & VIN & 3 & -1.030 & 15.417 & -1.570 & 1.00 & 0.00 & $\mathrm{H}$ \\
\hline \multicolumn{11}{|l|}{ TER } \\
\hline HETATM & 82 & $\mathrm{C} 1$ & MET & 4 & 3.008 & 14.177 & -2.840 & 1.00 & 0.00 & C \\
\hline HETATM & 83 & $\mathrm{H} 11$ & MET & 4 & 3.243 & 15.248 & -2.720 & 1.00 & 0.00 & $\mathrm{H}$ \\
\hline HETATM & 84 & $\mathrm{H} 12$ & MET & 4 & 3.821 & 13.713 & -3.410 & 1.00 & 0.00 & $\mathrm{H}$ \\
\hline HETATM & 85 & H13 & $\mathrm{MET}$ & 4 & 2.101 & 14.100 & -3.450 & 1.00 & 0.00 & $\mathrm{H}$ \\
\hline \multicolumn{11}{|l|}{ TER } \\
\hline HETATM & 86 & $\mathrm{C} 2$ & VIN & 5 & 8.018 & 12.499 & -2.325 & 1.00 & 0.00 & C \\
\hline
\end{tabular}




\begin{tabular}{|c|c|c|c|}
\hline HETATM & 87 & H 21 & VIN \\
\hline HETATM & 88 & $\mathrm{C} 1$ & VIN \\
\hline HETATM & 89 & $\mathrm{H} 11$ & VIN \\
\hline HETATM & 90 & $\mathrm{H} 12$ & VIN \\
\hline \multicolumn{4}{|l|}{ TER } \\
\hline HETATM & 91 & $\mathrm{C} 1$ & MET \\
\hline HETATM & 92 & H11 & MET \\
\hline HETATM & 93 & $\mathrm{H} 12$ & MET \\
\hline HETATM & 94 & H13 & MET \\
\hline \multicolumn{4}{|l|}{ TER } \\
\hline HETATM & 95 & C5 & $\mathrm{ACP}$ \\
\hline HETATM & 96 & H51 & $\mathrm{ACP}$ \\
\hline HETATM & 97 & H52 & $\mathrm{ACP}$ \\
\hline HETATM & 98 & C4 & $\mathrm{ACP}$ \\
\hline HETATM & 99 & $\mathrm{H} 41$ & $\mathrm{ACP}$ \\
\hline HETATM & 100 & $\mathrm{H} 42$ & $\mathrm{ACP}$ \\
\hline HETATM & 101 & $\mathrm{C} 1$ & $\mathrm{ACP}$ \\
\hline HETATM & 102 & $\mathrm{O} 2$ & $\mathrm{ACP}$ \\
\hline HETATM & 103 & 03 & $\mathrm{ACP}$ \\
\hline HETATM & 104 & H3 & $\mathrm{ACP}$ \\
\hline \multicolumn{4}{|l|}{ TER } \\
\hline HETATM & 105 & $\mathrm{C} 1$ & $\mathrm{MET}$ \\
\hline HETATM & 106 & $\mathrm{H} 11$ & $\mathrm{MET}$ \\
\hline HETATM & 107 & $\mathrm{H} 12$ & $\mathrm{MET}$ \\
\hline HETATM & 108 & $\mathrm{H} 13$ & MET \\
\hline \multicolumn{4}{|l|}{ TER } \\
\hline HETATM & 109 & $\mathrm{C} 1$ & $\mathrm{MET}$ \\
\hline HETATM & 110 & $\mathrm{H} 11$ & MET \\
\hline HETATM & 111 & $\mathrm{H} 12$ & MET \\
\hline HETATM & 112 & H13 & MET \\
\hline \multicolumn{4}{|l|}{ TER } \\
\hline HETATM & 113 & $\mathrm{C} 2$ & VIN \\
\hline HETATM & 114 & H 21 & VIN \\
\hline HETATM & 115 & $\mathrm{C} 1$ & VIN \\
\hline HETATM & 116 & $\mathrm{H} 11$ & VIN \\
\hline HETATM & 117 & $\mathrm{H} 12$ & VIN \\
\hline \multicolumn{4}{|l|}{ TER } \\
\hline HETATM & 118 & $\mathrm{C} 1$ & MET \\
\hline HETATM & 119 & $\mathrm{H} 11$ & MET \\
\hline HETATM & 120 & $\mathrm{H} 12$ & $\mathrm{MET}$ \\
\hline HETATM & 121 & $\mathrm{H} 13$ & $\mathrm{MET}$ \\
\hline \multicolumn{4}{|l|}{ TER } \\
\hline HETATM & 122 & $\mathrm{C} 2$ & VIN \\
\hline HETATM & 123 & $\mathrm{H} 21$ & VIN \\
\hline HETATM & 124 & $\mathrm{C} 1$ & VIN \\
\hline HETATM & 125 & $\mathrm{H} 11$ & VIN \\
\hline HETATM & 126 & $\mathrm{H} 12$ & VIN \\
\hline \multicolumn{4}{|l|}{ TER } \\
\hline HETATM & 127 & $\mathrm{C} 1$ & $\mathrm{MET}$ \\
\hline HETATM & 128 & H11 & MET \\
\hline HETATM & 129 & $\mathrm{H} 12$ & $\mathrm{MET}$ \\
\hline HETATM & 130 & $\mathrm{H} 13$ & MET \\
\hline TER & & & \\
\hline HETATM & 131 & C5 & $\mathrm{ACP}$ \\
\hline HETATM & 132 & H51 & $\mathrm{ACP}$ \\
\hline HETATM & 133 & H52 & $\mathrm{ACP}$ \\
\hline HETATM & 134 & $\mathrm{C} 4$ & $\mathrm{ACP}$ \\
\hline HETATM & 135 & $\mathrm{H} 41$ & $\mathrm{ACP}$ \\
\hline HETATM & 136 & $\mathrm{H} 42$ & $\mathrm{ACP}$ \\
\hline HETATM & 137 & $\mathrm{C} 1$ & $\mathrm{ACP}$ \\
\hline HETATM & 138 & 02 & $\mathrm{ACP}$ \\
\hline HETATM & 139 & 03 & $\mathrm{ACP}$ \\
\hline HETATM & 140 & H3 & $\mathrm{ACP}$ \\
\hline TER & & & \\
\hline HETATM & 141 & $\mathrm{C} 1$ & $\mathrm{MET}$ \\
\hline HETATM & 142 & $\mathrm{H} 11$ & MET \\
\hline HETATM & 143 & $\mathrm{H} 12$ & MET \\
\hline HETATM & 144 & H13 & MET \\
\hline TER & & & \\
\hline HETATM & 145 & C1 & $\mathrm{MET}$ \\
\hline HETATM & 146 & H11 & MET \\
\hline HETATM & 147 & $\mathrm{H} 12$ & MET \\
\hline HETATM & 148 & $\mathrm{H} 13$ & $\mathrm{MET}$ \\
\hline TER & & & \\
\hline HETATM & 149 & $\mathrm{FE}$ & HEM \\
\hline HETATM & 150 & $\mathrm{C} 1$ & HEM \\
\hline HETATM & 151 & C2 & HEM \\
\hline HETATM & 152 & C3 & HEM \\
\hline HETATM & 153 & C4 & HEM \\
\hline
\end{tabular}

\begin{tabular}{|c|c|c|c|c|c|}
\hline 7.352 & 12.399 & -3.184 & 1.00 & 0.00 & $\mathrm{H}$ \\
\hline 9.185 & 13.144 & -2.484 & 1.00 & 0.00 & C \\
\hline 9.865 & 13.300 & -1.654 & 1.00 & 0.00 & $\mathrm{H}$ \\
\hline 9.490 & 13.542 & -3.450 & 1.00 & 0.00 & $\mathrm{H}$ \\
\hline 9.703 & 10.816 & -0.121 & 1.00 & 0.00 & C \\
\hline 10.294 & 11.480 & 0.528 & 1.00 & 0.00 & $\mathrm{H}$ \\
\hline 9.859 & 9.794 & 0.246 & 1.00 & 0.00 & $\mathrm{H}$ \\
\hline 10.135 & 10.882 & -1.126 & 1.00 & 0.00 & $\mathrm{H}$ \\
\hline 0.615 & 9.735 & 5.770 & 1.00 & 0.00 & C \\
\hline 1.354 & 9.723 & 6.582 & 1.00 & 0.00 & $\mathrm{H}$ \\
\hline-0.226 & 10.343 & 6.134 & 1.00 & 0.00 & $\mathrm{H}$ \\
\hline 0.136 & 8.297 & 5.497 & 1.00 & 0.00 & $\mathrm{C}$ \\
\hline-0.422 & 8.236 & 4.549 & 1.00 & 0.00 & $\mathrm{H}$ \\
\hline 1.007 & 7.630 & 5.390 & 1.00 & 0.00 & $\mathrm{H}$ \\
\hline-0.728 & 7.685 & 6.573 & 1.00 & 0.00 & $\mathrm{C}$ \\
\hline-0.956 & 8.208 & 7.671 & 1.00 & 0.00 & O \\
\hline-1.244 & 6.505 & 6.209 & 1.00 & 0.00 & $\mathrm{O}$ \\
\hline-1.853 & 6.151 & 6.945 & 1.00 & 0.00 & $\mathrm{H}$ \\
\hline 8.511 & 8.608 & 4.597 & 1.00 & 0.00 & C \\
\hline 9.063 & 9.448 & 5.047 & 1.00 & 0.00 & $\mathrm{H}$ \\
\hline 8.517 & 7.782 & 5.319 & 1.00 & 0.00 & $\mathrm{H}$ \\
\hline 9.082 & 8.289 & 3.717 & 1.00 & 0.00 & $\mathrm{H}$ \\
\hline-0.923 & 11.557 & 3.646 & 1.00 & 0.00 & C \\
\hline-1.368 & 11.348 & 4.626 & 1.00 & 0.00 & $\mathrm{H}$ \\
\hline-1.033 & 12.637 & 3.471 & 1.00 & 0.00 & $\mathrm{H}$ \\
\hline-1.523 & 11.031 & 2.889 & 1.00 & 0.00 & $\mathrm{H}$ \\
\hline 8.874 & 0.178 & 8.933 & 1.00 & 0.00 & C \\
\hline 9.690 & 0.876 & 8.768 & 1.00 & 0.00 & $\mathrm{H}$ \\
\hline 9.207 & -1.047 & 9.335 & 1.00 & 0.00 & $\mathrm{C}$ \\
\hline 8.475 & -1.826 & 9.442 & 1.00 & 0.00 & $\mathrm{H}$ \\
\hline 10.249 & -1.316 & 9.501 & 1.00 & 0.00 & $\mathrm{H}$ \\
\hline 6.210 & -0.077 & 10.771 & 1.00 & 0.00 & C \\
\hline 5.975 & -1.148 & 10.652 & 1.00 & 0.00 & $\mathrm{H}$ \\
\hline 5.397 & 0.387 & 11.341 & 1.00 & 0.00 & $\mathrm{H}$ \\
\hline 7.118 & 0.001 & 11.382 & 1.00 & 0.00 & $\mathrm{H}$ \\
\hline 1.200 & 1.601 & 10.256 & 1.00 & 0.00 & $\mathrm{C}$ \\
\hline 1.866 & 1.702 & 11.115 & 1.00 & 0.00 & $\mathrm{H}$ \\
\hline 0.033 & 0.957 & 10.415 & 1.00 & 0.00 & C \\
\hline-0.647 & 0.800 & 9.585 & 1.00 & 0.00 & $\mathrm{H}$ \\
\hline-0.272 & 0.559 & 11.381 & 1.00 & 0.00 & $\mathrm{H}$ \\
\hline-0.484 & 3.284 & 8.052 & 1.00 & 0.00 & $\mathrm{C}$ \\
\hline-0.641 & 4.306 & 7.685 & 1.00 & 0.00 & $\mathrm{H}$ \\
\hline-0.917 & 3.219 & 9.057 & 1.00 & 0.00 & $\mathrm{H}$ \\
\hline-1.076 & 2.620 & 7.403 & 1.00 & 0.00 & $\mathrm{H}$ \\
\hline 8.604 & 4.366 & 2.161 & 1.00 & 0.00 & C \\
\hline 7.865 & 4.377 & 1.349 & 1.00 & 0.00 & $\mathrm{H}$ \\
\hline 9.445 & 3.758 & 1.798 & 1.00 & 0.00 & $\mathrm{H}$ \\
\hline 9.082 & 5.803 & 2.434 & 1.00 & 0.00 & C \\
\hline 9.640 & 5.865 & 3.382 & 1.00 & 0.00 & $\mathrm{H}$ \\
\hline 8.212 & 6.470 & 2.541 & 1.00 & 0.00 & $\mathrm{H}$ \\
\hline 9.946 & 6.416 & 1.358 & 1.00 & 0.00 & C \\
\hline 10.174 & 5.893 & 0.260 & 1.00 & 0.00 & 0 \\
\hline 10.462 & 7.595 & 1.722 & 1.00 & 0.00 & 0 \\
\hline 11.071 & 7.949 & 0.986 & 1.00 & 0.00 & $\mathrm{H}$ \\
\hline 0.708 & 5.492 & 3.334 & 1.00 & 0.00 & C \\
\hline 0.155 & 4.653 & 2.884 & 1.00 & 0.00 & $\mathrm{H}$ \\
\hline 0.701 & 6.318 & 2.613 & 1.00 & 0.00 & $\mathrm{H}$ \\
\hline 0.137 & 5.812 & 4.214 & 1.00 & 0.00 & $\mathrm{H}$ \\
\hline 10.141 & 2.543 & 4.285 & 1.00 & 0.00 & C \\
\hline 10.586 & 2.752 & 3.305 & 1.00 & 0.00 & $\mathrm{H}$ \\
\hline 10.252 & 1.463 & 4.460 & 1.00 & 0.00 & $\mathrm{H}$ \\
\hline 10.742 & 3.069 & 5.042 & 1.00 & 0.00 & $\mathrm{H}$ \\
\hline 17.294 & 11.003 & -6.440 & 1.00 & 0.00 & $\mathrm{Fe}$ \\
\hline 15.066 & 12.876 & -7.502 & 1.00 & 0.00 & C \\
\hline 14.707 & 13.666 & -8.674 & 1.00 & 0.00 & C \\
\hline 15.852 & 13.763 & -9.448 & 1.00 & 0.00 & C \\
\hline 16.882 & 13.046 & -8.740 & 1.00 & 0.00 & C \\
\hline
\end{tabular}




\begin{tabular}{|c|c|c|c|c|c|c|c|c|c|c|}
\hline HETATM & 154 & C5 & HEM & 17 & 18.197 & 12.927 & -9.178 & 1.00 & 0.00 & $\mathrm{C}$ \\
\hline HETATM & 155 & H5 & HEM & 17 & 18.451 & 13.423 & -10.113 & 1.00 & 0.00 & $\mathrm{H}$ \\
\hline HETATM & 156 & $\mathrm{C} 6$ & HEM & 17 & 19.216 & 12.229 & -8.542 & 1.00 & 0.00 & C \\
\hline HETATM & 157 & C7 & HEM & 17 & 20.585 & 12.156 & -9.012 & 1.00 & 0.00 & C \\
\hline HETATM & 158 & $\mathrm{C} 8$ & HEM & 17 & 21.291 & 11.436 & -8.065 & 1.00 & 0.00 & C \\
\hline HETATM & 159 & $\mathrm{C} 9$ & HEM & 17 & 20.340 & 11.071 & -7.041 & 1.00 & 0.00 & C \\
\hline HETATM & 160 & $\mathrm{C} 10$ & HEM & 17 & 20.644 & 10.336 & -5.897 & 1.00 & 0.00 & C \\
\hline HETATM & 161 & $\mathrm{H} 10$ & HEM & 17 & 21.671 & 9.993 & -5.791 & 1.00 & 0.00 & $\mathrm{H}$ \\
\hline HETATM & 162 & C11 & HEM & 17 & 19.770 & 9.996 & -4.874 & 1.00 & 0.00 & $\mathrm{C}$ \\
\hline HETATM & 163 & $\mathrm{C} 12$ & HEM & 17 & 20.151 & 9.271 & -3.684 & 1.00 & 0.00 & $\mathrm{C}$ \\
\hline HETATM & 164 & $\mathrm{C} 13$ & HEM & 17 & 19.011 & 9.124 & -2.927 & 1.00 & 0.00 & $\mathrm{C}$ \\
\hline HETATM & 165 & C14 & HEM & 17 & 17.938 & 9.765 & -3.659 & 1.00 & 0.00 & $\mathrm{C}$ \\
\hline HETATM & 166 & $\mathrm{C} 15$ & HEM & 17 & 16.610 & 9.820 & -3.237 & 1.00 & 0.00 & C \\
\hline HETATM & 167 & H15 & HEM & 17 & 16.370 & 9.335 & -2.289 & 1.00 & 0.00 & $\mathrm{H}$ \\
\hline HETATM & 168 & $\mathrm{C} 16$ & HEM & 17 & 15.580 & 10.502 & -3.880 & 1.00 & 0.00 & $\mathrm{C}$ \\
\hline HETATM & 169 & C17 & HEM & 17 & 14.214 & 10.615 & -3.398 & 1.00 & 0.00 & C \\
\hline HETATM & 170 & $\mathrm{C} 18$ & HEM & 17 & 13.539 & 11.383 & -4.322 & 1.00 & 0.00 & C \\
\hline HETATM & 171 & C19 & HEM & 17 & 14.488 & 11.735 & -5.358 & 1.00 & 0.00 & C \\
\hline HETATM & 172 & $\mathrm{C} 20$ & HEM & 17 & 14.197 & 12.526 & -6.469 & 1.00 & 0.00 & C \\
\hline HETATM & 173 & $\mathrm{H} 20$ & HEM & 17 & 13.183 & 12.915 & -6.530 & 1.00 & 0.00 & $\mathrm{H}$ \\
\hline HETATM & 174 & $\mathrm{~N} 21$ & HEM & 17 & 16.387 & 12.513 & -7.564 & 1.00 & 0.00 & $\mathrm{~N}$ \\
\hline HETATM & 175 & N22 & HEM & 17 & 19.092 & 11.566 & -7.342 & 1.00 & 0.00 & $\mathrm{~N}$ \\
\hline HETATM & 176 & $\mathrm{~N} 23$ & HEM & 17 & 18.421 & 10.303 & -4.841 & 1.00 & 0.00 & $\mathrm{~N}$ \\
\hline HETATM & 177 & $\mathrm{~N} 24$ & HEM & 17 & 15.722 & 11.187 & -5.077 & 1.00 & 0.00 & $\mathrm{~N}$ \\
\hline HETATM & 178 & C99 & HEM & 17 & 16.628 & 8.322 & -7.579 & 1.00 & 0.00 & C \\
\hline HETATM & 179 & 091 & HEM & 17 & 16.788 & 9.635 & -7.567 & 1.00 & 0.00 & 0 \\
\hline HETATM & 180 & 092 & HEM & 17 & 16.473 & 7.705 & -8.635 & 1.00 & 0.00 & 0 \\
\hline HETATM & 181 & СТ 91 & HEM & 17 & 16.644 & 7.640 & -6.223 & 1.00 & 0.00 & $\mathrm{C}$ \\
\hline HETATM & 182 & H911 & HEM & 17 & 17.623 & 7.836 & -5.760 & 1.00 & 0.00 & $\mathrm{H}$ \\
\hline HETATM & 183 & H912 & HEM & 17 & 15.896 & 8.130 & -5.580 & 1.00 & 0.00 & $\mathrm{H}$ \\
\hline HETATM & 184 & СТ92 & HEM & 17 & 16.385 & 6.136 & -6.334 & 1.00 & 0.00 & C \\
\hline HETATM & 185 & H921 & HEM & 17 & 17.181 & 5.676 & -6.931 & 1.00 & 0.00 & $\mathrm{H}$ \\
\hline HETATM & 186 & H922 & HEM & 17 & 15.460 & 5.980 & -6.905 & 1.00 & 0.00 & $\mathrm{H}$ \\
\hline \multicolumn{11}{|l|}{ TER } \\
\hline HETATM & 187 & $\mathrm{FE}$ & HEM & 18 & 17.986 & 3.589 & -1.491 & 1.00 & 0.00 & $\mathrm{Fe}$ \\
\hline HETATM & 188 & $\mathrm{C} 1$ & HEM & 18 & 20.214 & 1.717 & -0.429 & 1.00 & 0.00 & C \\
\hline HETATM & 189 & $\mathrm{C} 2$ & HEM & 18 & 20.573 & 0.927 & 0.742 & 1.00 & 0.00 & C \\
\hline HETATM & 190 & $\mathrm{C} 3$ & HEM & 18 & 19.427 & 0.830 & 1.517 & 1.00 & 0.00 & C \\
\hline HETATM & 191 & $\mathrm{C} 4$ & HEM & 18 & 18.398 & 1.547 & 0.809 & 1.00 & 0.00 & C \\
\hline HETATM & 192 & C5 & HEM & 18 & 17.082 & 1.666 & 1.247 & 1.00 & 0.00 & C \\
\hline HETATM & 193 & H5 & HEM & 18 & 16.829 & 1.170 & 2.182 & 1.00 & 0.00 & $\mathrm{H}$ \\
\hline HETATM & 194 & $\mathrm{C} 6$ & HEM & 18 & 16.063 & 2.364 & 0.611 & 1.00 & 0.00 & C \\
\hline HETATM & 195 & $\mathrm{C} 7$ & HEM & 18 & 14.694 & 2.437 & 1.081 & 1.00 & 0.00 & C \\
\hline HETATM & 196 & $\mathrm{C} 8$ & HEM & 18 & 13.989 & 3.157 & 0.134 & 1.00 & 0.00 & C \\
\hline HETATM & 197 & $\mathrm{C} 9$ & HEM & 18 & 14.940 & 3.522 & -0.891 & 1.00 & 0.00 & C \\
\hline HETATM & 198 & $\mathrm{C} 10$ & HEM & 18 & 14.635 & 4.256 & -2.034 & 1.00 & 0.00 & C \\
\hline HETATM & 199 & $\mathrm{H} 10$ & HEM & 18 & 13.608 & 4.600 & -2.140 & 1.00 & 0.00 & $\mathrm{H}$ \\
\hline HETATM & 200 & $\mathrm{C} 11$ & HEM & 18 & 15.510 & 4.596 & -3.057 & 1.00 & 0.00 & C \\
\hline HETATM & 201 & $\mathrm{C} 12$ & HEM & 18 & 15.129 & 5.322 & -4.247 & 1.00 & 0.00 & $\mathrm{C}$ \\
\hline HETATM & 202 & $\mathrm{C} 13$ & HEM & 18 & 16.268 & 5.469 & -5.004 & 1.00 & 0.00 & C \\
\hline HETATM & 203 & $\mathrm{C} 14$ & HEM & 18 & 17.341 & 4.828 & -4.273 & 1.00 & 0.00 & C \\
\hline HETATM & 204 & $\mathrm{C} 15$ & HEM & 18 & 18.669 & 4.773 & -4.694 & 1.00 & 0.00 & C \\
\hline HETATM & 205 & $\mathrm{H} 15$ & HEM & 18 & 18.909 & 5.258 & -5.642 & 1.00 & 0.00 & $\mathrm{H}$ \\
\hline HETATM & 206 & $\mathrm{C} 16$ & HEM & 18 & 19.699 & 4.091 & -4.051 & 1.00 & 0.00 & $\mathrm{C}$ \\
\hline HETATM & 207 & C17 & HEM & 18 & 21.065 & 3.978 & -4.533 & 1.00 & 0.00 & C \\
\hline HETATM & 208 & $\mathrm{C} 18$ & HEM & 18 & 21.741 & 3.210 & -3.609 & 1.00 & 0.00 & C \\
\hline HETATM & 209 & C19 & HEM & 18 & 20.791 & 2.858 & -2.573 & 1.00 & 0.00 & C \\
\hline HETATM & 210 & $\mathrm{C} 20$ & HEM & 18 & 21.082 & 2.067 & -1.462 & 1.00 & 0.00 & C \\
\hline HETATM & 211 & $\mathrm{H} 20$ & HEM & 18 & 22.097 & 1.678 & -1.402 & 1.00 & 0.00 & $\mathrm{H}$ \\
\hline HETATM & 212 & $\mathrm{~N} 21$ & HEM & 18 & 18.892 & 2.080 & -0.367 & 1.00 & 0.00 & $\mathrm{~N}$ \\
\hline HETATM & 213 & $\mathrm{~N} 22$ & HEM & 18 & 16.187 & 3.027 & -0.590 & 1.00 & 0.00 & $\mathrm{~N}$ \\
\hline HETATM & 214 & $\mathrm{~N} 23$ & HEM & 18 & 16.858 & 4.290 & -3.090 & 1.00 & 0.00 & $\mathrm{~N}$ \\
\hline HETATM & 215 & $\mathrm{~N} 24$ & HEM & 18 & 19.558 & 3.406 & -2.854 & 1.00 & 0.00 & $\mathrm{~N}$ \\
\hline HETATM & 216 & C99 & HEM & 18 & 18.651 & 6.271 & -0.352 & 1.00 & 0.00 & C \\
\hline HETATM & 217 & 091 & HEM & 18 & 18.492 & 4.958 & -0.365 & 1.00 & 0.00 & 0 \\
\hline HETATM & 218 & 092 & HEM & 18 & 18.806 & 6.888 & 0.703 & 1.00 & 0.00 & 0 \\
\hline HETATM & 219 & СТ 91 & HEM & 18 & 18.635 & 6.952 & -1.709 & 1.00 & 0.00 & C \\
\hline HETATM & 220 & H911 & HEM & 18 & 17.656 & 6.757 & -2.171 & 1.00 & 0.00 & $\mathrm{H}$ \\
\hline HETATM & 221 & H912 & HEM & 18 & 19.383 & 6.463 & -2.352 & 1.00 & 0.00 & $\mathrm{H}$ \\
\hline HETATM & 222 & СТ92 & HEM & 18 & 18.895 & 8.457 & -1.597 & 1.00 & 0.00 & C \\
\hline HETATM & 223 & H921 & HEM & 18 & 19.820 & 8.613 & -1.027 & 1.00 & 0.00 & $\mathrm{H}$ \\
\hline HETATM & 224 & H922 & HEM & 18 & 18.098 & 8.917 & -1.000 & 1.00 & 0.00 & $\mathrm{H}$ \\
\hline \multicolumn{11}{|l|}{ TER } \\
\hline HETATM & 225 & $\mathrm{C} 2$ & VIN & 19 & 13.375 & 14.169 & -8.933 & 1.00 & 0.00 & C \\
\hline HETATM & 226 & H2 1 & VIN & 19 & 12.559 & 13.470 & -8.768 & 1.00 & 0.00 & $\mathrm{H}$ \\
\hline HETATM & 227 & $\mathrm{C} 1$ & VIN & 19 & 13.042 & 15.394 & -9.335 & 1.00 & 0.00 & C \\
\hline HETATM & 228 & H11 & VIN & 19 & 13.774 & 16.172 & -9.442 & 1.00 & 0.00 & $\mathrm{H}$ \\
\hline HETATM & 229 & $\mathrm{H} 12$ & VIN & 19 & 12.000 & 15.663 & -9.501 & 1.00 & 0.00 & $\mathrm{H}$ \\
\hline
\end{tabular}




\begin{tabular}{|c|c|c|c|c|c|c|c|c|c|c|}
\hline HETATM & 230 & $\mathrm{C} 1$ & MET & 20 & 16.039 & 14.424 & -10.771 & 1.00 & 0.00 & C \\
\hline HETATM & 231 & H11 & MET & 20 & 16.274 & 15.494 & -10.652 & 1.00 & 0.00 & $\mathrm{H}$ \\
\hline HETATM & 232 & $\mathrm{H} 12$ & MET & 20 & 16.852 & 13.959 & -11.341 & 1.00 & 0.00 & $\mathrm{H}$ \\
\hline HETATM & 233 & $\mathrm{H} 13$ & MET & 20 & 15.131 & 14.346 & $-11 \cdot 382$ & 1.00 & 0.00 & $\mathrm{H}$ \\
\hline \multicolumn{11}{|l|}{ TER } \\
\hline HETATM & 234 & $\mathrm{C} 2$ & VIN & 21 & 21.049 & 12.746 & -10.256 & 1.00 & 0.00 & C \\
\hline HETATM & 235 & H 21 & VIN & 21 & 20.383 & 12.645 & -11.115 & 1.00 & 0.00 & $\mathrm{H}$ \\
\hline HETATM & 236 & $\mathrm{C} 1$ & VIN & 21 & 22.216 & 13.390 & -10.415 & 1.00 & 0.00 & C \\
\hline HETATM & 237 & H11 & VIN & 21 & 22.896 & 13.546 & -9.585 & 1.00 & 0.00 & $\mathrm{H}$ \\
\hline HETATM & 238 & $\mathrm{H} 12$ & VIN & 21 & 22.521 & 13.788 & -11.381 & 1.00 & 0.00 & $\mathrm{H}$ \\
\hline \multicolumn{11}{|l|}{ TER } \\
\hline HETATM & 239 & $\mathrm{C} 1$ & MET & 22 & 22.733 & 11.062 & -8.052 & 1.00 & 0.00 & C \\
\hline HETATM & 240 & H11 & MET & 22 & 23.325 & 11.727 & -7.403 & 1.00 & 0.00 & $\mathrm{H}$ \\
\hline HETATM & 241 & $\mathrm{H} 12$ & MET & 22 & 22.890 & 10.040 & -7.685 & 1.00 & 0.00 & $\mathrm{H}$ \\
\hline HETATM & 242 & H13 & MET & 22 & 23.166 & 11.128 & -9.057 & 1.00 & 0.00 & $\mathrm{H}$ \\
\hline \multicolumn{11}{|l|}{ TER } \\
\hline HETATM & 243 & C5 & $\mathrm{ACP}$ & 23 & 13.645 & 9.981 & -2.161 & 1.00 & 0.00 & C \\
\hline HETATM & 244 & H5 1 & $\mathrm{ACP}$ & 23 & 14.384 & 9.969 & -1.349 & 1.00 & 0.00 & $\mathrm{H}$ \\
\hline HETATM & 245 & H52 & $\mathrm{ACP}$ & 23 & 12.804 & 10.589 & -1.798 & 1.00 & 0.00 & $\mathrm{H}$ \\
\hline HETATM & 246 & $\mathrm{C} 4$ & $\mathrm{ACP}$ & 23 & 13.167 & 8.543 & -2.434 & 1.00 & 0.00 & C \\
\hline HETATM & 247 & $\mathrm{H} 41$ & $\mathrm{ACP}$ & 23 & 12.609 & 8.482 & -3.382 & 1.00 & 0.00 & $\mathrm{H}$ \\
\hline HETATM & 248 & $\mathrm{H} 42$ & $\mathrm{ACP}$ & 23 & 14.037 & 7.877 & -2.541 & 1.00 & 0.00 & $\mathrm{H}$ \\
\hline HETATM & 249 & C1 & $\mathrm{ACP}$ & 23 & 12.303 & 7.931 & -1.358 & 1.00 & 0.00 & $\mathrm{C}$ \\
\hline HETATM & 250 & $\mathrm{O} 2$ & $\mathrm{ACP}$ & 23 & 12.075 & 8.454 & -0.260 & 1.00 & 0.00 & 0 \\
\hline HETATM & 251 & 03 & $\mathrm{ACP}$ & 23 & 11.787 & 6.751 & -1.722 & 1.00 & 0.00 & 0 \\
\hline HETATM & 252 & H3 & $\mathrm{ACP}$ & 23 & 11.178 & 6.397 & -0.986 & 1.00 & 0.00 & $\mathrm{H}$ \\
\hline \multicolumn{11}{|l|}{ TER } \\
\hline HETATM & 253 & $\mathrm{C} 1$ & MET & 24 & 21.541 & 8.855 & -3.334 & 1.00 & 0.00 & $\mathrm{C}$ \\
\hline HETATM & 254 & H11 & MET & 24 & 22.094 & 9.694 & -2.884 & 1.00 & 0.00 & $\mathrm{H}$ \\
\hline HETATM & 255 & $\mathrm{H} 12$ & MET & 24 & 21.548 & 8.029 & -2.613 & 1.00 & 0.00 & $\mathrm{H}$ \\
\hline HETATM & 256 & $\mathrm{H} 13$ & MET & 24 & 22.112 & 8.535 & -4.214 & 1.00 & 0.00 & $\mathrm{H}$ \\
\hline TER & & & & & & & & & & \\
\hline HETATM & 257 & $\mathrm{C} 1$ & MET & 25 & 12.108 & 11.803 & -4.285 & 1.00 & 0.00 & C \\
\hline HETATM & 258 & H 11 & MET & 25 & 11.663 & 11.594 & -3.305 & 1.00 & 0.00 & $\mathrm{H}$ \\
\hline HETATM & 259 & $\mathrm{H} 12$ & MET & 25 & 11.997 & 12.883 & -4.460 & 1.00 & 0.00 & $\mathrm{H}$ \\
\hline HETATM & 260 & H13 & MET & 25 & 11.507 & 11.278 & -5.042 & 1.00 & 0.00 & $\mathrm{H}$ \\
\hline TER & & & & & & & & & & \\
\hline HETATM & 261 & $\mathrm{C} 2$ & VIN & 26 & 21.904 & 0.424 & 1.002 & 1.00 & 0.00 & $\mathrm{C}$ \\
\hline HETATM & 262 & H 21 & VIN & 26 & 22.720 & 1.123 & 0.837 & 1.00 & 0.00 & $\mathrm{H}$ \\
\hline HETATM & 263 & $\mathrm{C} 1$ & VIN & 26 & 22.237 & -0.801 & 1.403 & 1.00 & 0.00 & C \\
\hline HETATM & 264 & H11 & VIN & 26 & 21.506 & -1.579 & 1.511 & 1.00 & 0.00 & $\mathrm{H}$ \\
\hline HETATM & 265 & $\mathrm{H} 12$ & VIN & 26 & 23.279 & -1.070 & 1.570 & 1.00 & 0.00 & $\mathrm{H}$ \\
\hline TER & & & & & & & & & & \\
\hline HETATM & 266 & $\mathrm{C} 1$ & MET & 27 & 19.241 & 0.169 & 2.840 & 1.00 & 0.00 & C \\
\hline HETATM & 267 & H11 & MET & 27 & 19.006 & -0.902 & 2.720 & 1.00 & 0.00 & $\mathrm{H}$ \\
\hline HETATM & 268 & $\mathrm{H} 12$ & MET & 27 & 18.428 & 0.633 & 3.410 & 1.00 & 0.00 & $\mathrm{H}$ \\
\hline HETATM & 269 & $\mathrm{H} 13$ & MET & 27 & 20.148 & 0.247 & 3.450 & 1.00 & 0.00 & $\mathrm{H}$ \\
\hline TER & & & & & & & & & & \\
\hline HETATM & 270 & $\mathrm{C} 2$ & VIN & 28 & 14.231 & 1.847 & 2.325 & 1.00 & 0.00 & $\mathrm{C}$ \\
\hline HETATM & 271 & H 21 & VIN & 28 & 14.897 & 1.948 & 3.184 & 1.00 & 0.00 & $\mathrm{H}$ \\
\hline HETATM & 272 & $\mathrm{C} 1$ & VIN & 28 & 13.064 & 1.203 & 2.484 & 1.00 & 0.00 & C \\
\hline HETATM & 273 & $\mathrm{H} 11$ & VIN & 28 & 12.384 & 1.047 & 1.654 & 1.00 & 0.00 & $\mathrm{H}$ \\
\hline HETATM & 274 & $\mathrm{H} 12$ & VIN & 28 & 12.759 & 0.805 & 3.450 & 1.00 & 0.00 & $\mathrm{H}$ \\
\hline TER & & & & & & & & & & \\
\hline HETATM & 275 & $\mathrm{C} 1$ & MET & 29 & 12.546 & 3.530 & 0.121 & 1.00 & 0.00 & C \\
\hline HETATM & 276 & H11 & MET & 29 & 12.390 & 4.553 & -0.246 & 1.00 & 0.00 & $\mathrm{H}$ \\
\hline HETATM & 277 & $\mathrm{H} 12$ & MET & 29 & 12.114 & 3.465 & 1.126 & 1.00 & 0.00 & $\mathrm{H}$ \\
\hline HETATM & 278 & $\mathrm{H} 13$ & MET & 29 & 11.955 & 2.866 & -0.528 & 1.00 & 0.00 & $\mathrm{H}$ \\
\hline TER & & & & & & & & & & \\
\hline HETATM & 279 & $\mathrm{C} 5$ & $\mathrm{ACP}$ & 30 & 21.634 & 4.612 & -5.770 & 1.00 & 0.00 & C \\
\hline HETATM & 280 & H51 & $\mathrm{ACP}$ & 30 & 20.895 & 4.623 & -6.582 & 1.00 & 0.00 & $\mathrm{H}$ \\
\hline HETATM & 281 & H5 2 & $\mathrm{ACP}$ & 30 & 22.475 & 4.004 & -6.134 & 1.00 & 0.00 & $\mathrm{H}$ \\
\hline HETATM & 282 & $\mathrm{C} 4$ & $\mathrm{ACP}$ & 30 & 22.113 & 6.050 & -5.497 & 1.00 & 0.00 & C \\
\hline HETATM & 283 & H 41 & $\mathrm{ACP}$ & 30 & 22.671 & 6.111 & -4.549 & 1.00 & 0.00 & $\mathrm{H}$ \\
\hline HETATM & 284 & $\mathrm{H} 42$ & $\mathrm{ACP}$ & 30 & 21.242 & 6.716 & -5.390 & 1.00 & 0.00 & $\mathrm{H}$ \\
\hline HETATM & 285 & $\mathrm{C} 1$ & $\mathrm{ACP}$ & 30 & 22.977 & 6.662 & -6.573 & 1.00 & 0.00 & C \\
\hline HETATM & 286 & $\mathrm{O} 2$ & $\mathrm{ACP}$ & 30 & 23.205 & 6.139 & -7.671 & 1.00 & 0.00 & 0 \\
\hline HETATM & 287 & 03 & $\mathrm{ACP}$ & 30 & 23.493 & 7.841 & -6.209 & 1.00 & 0.00 & O \\
\hline HETATM & 288 & H3 & $\mathrm{ACP}$ & 30 & 24.102 & 8.196 & -6.945 & 1.00 & 0.00 & $\mathrm{H}$ \\
\hline TER & & & & & & & & & & \\
\hline HETATM & 289 & $\mathrm{C} 1$ & MET & 31 & 13.738 & 5.738 & -4.597 & 1.00 & 0.00 & C \\
\hline HETATM & 290 & $\mathrm{H} 11$ & MET & 31 & 13.186 & 4.899 & -5.047 & 1.00 & 0.00 & $\mathrm{H}$ \\
\hline HETATM & 291 & $\mathrm{H} 12$ & MET & 31 & 13.732 & 6.564 & -5.319 & 1.00 & 0.00 & $\mathrm{H}$ \\
\hline HETATM & 292 & H13 & MET & 31 & 13.167 & 6.058 & -3.717 & 1.00 & 0.00 & $\mathrm{H}$ \\
\hline TER & & & & & & & & & & \\
\hline HETATM & 293 & $\mathrm{C} 1$ & MET & 32 & 23.172 & 2.790 & -3.646 & 1.00 & 0.00 & C \\
\hline HETATM & 294 & $\mathrm{H} 11$ & MET & 32 & 23.617 & 2.998 & -4.626 & 1.00 & 0.00 & $\mathrm{H}$ \\
\hline HETATM & 295 & $\mathrm{H} 12$ & MET & 32 & 23.282 & 1.709 & -3.471 & 1.00 & 0.00 & $\mathrm{H}$ \\
\hline HETATM & 296 & H13 & MET & 32 & 23.772 & 3.315 & -2.889 & 1.00 & 0.00 & $\mathrm{H}$ \\
\hline
\end{tabular}


TER

\begin{tabular}{|c|c|c|c|c|c|c|c|c|c|c|}
\hline HETATM & 297 & $\mathrm{FE}$ & HEM & 33 & 30.324 & 11.250 & -14.371 & 1.00 & 0.00 & $\mathrm{Fe}$ \\
\hline HETATM & 298 & $\mathrm{C} 1$ & HEM & 33 & 28.096 & 13.122 & -15.433 & 1.00 & 0.00 & C \\
\hline HETATM & 299 & $\mathrm{C} 2$ & HEM & 33 & 27.737 & 13.912 & -16.605 & 1.00 & 0.00 & C \\
\hline HETATM & 300 & C3 & HEM & 33 & 28.883 & 14.009 & -17.380 & 1.00 & 0.00 & C \\
\hline HETATM & 301 & C4 & HEM & 33 & 29.912 & 13.292 & -16.672 & 1.00 & 0.00 & C \\
\hline HETATM & 302 & C5 & HEM & 33 & 31.228 & 13.173 & -17.109 & 1.00 & 0.00 & C \\
\hline HETATM & 303 & H5 & HEM & 33 & 31.481 & 13.670 & -18.045 & 1.00 & 0.00 & $\mathrm{H}$ \\
\hline HETATM & 304 & C6 & HEM & 33 & 32.247 & 12.475 & -16.473 & 1.00 & 0.00 & C \\
\hline HETATM & 305 & C7 & HEM & 33 & 33.616 & 12.402 & -16.943 & 1.00 & 0.00 & C \\
\hline HETATM & 306 & $\mathrm{C} 8$ & HEM & 33 & 34.321 & 11.682 & -15.996 & 1.00 & 0.00 & C \\
\hline HETATM & 307 & $\mathrm{C} 9$ & HEM & 33 & 33.370 & 11.317 & -14.972 & 1.00 & 0.00 & C \\
\hline HETATM & 308 & $\mathrm{C} 10$ & HEM & 33 & 33.675 & 10.583 & -13.828 & 1.00 & 0.00 & C \\
\hline HETATM & 309 & H1O & HEM & 33 & 34.702 & 10.239 & $-13 \cdot 722$ & 1.00 & 0.00 & $\mathrm{H}$ \\
\hline HETATM & 310 & $\mathrm{C} 11$ & HEM & 33 & 32.800 & 10.243 & -12.806 & 1.00 & 0.00 & C \\
\hline HETATM & 311 & $\mathrm{C} 12$ & HEM & 33 & 33.181 & 9.517 & -11.615 & 1.00 & 0.00 & C \\
\hline HETATM & 312 & $\mathrm{C} 13$ & HEM & 33 & 32.042 & 9.370 & -10.858 & 1.00 & 0.00 & C \\
\hline HETATM & 313 & C14 & HEM & 33 & 30.969 & 10.011 & -11.590 & 1.00 & 0.00 & C \\
\hline HETATM & 314 & C15 & HEM & 33 & 29.641 & 10.066 & -11.168 & 1.00 & 0.00 & C \\
\hline HETATM & 315 & H15 & HEM & 33 & 29.401 & 9.581 & -10.221 & 1.00 & 0.00 & $\mathrm{H}$ \\
\hline HETATM & 316 & C16 & HEM & 33 & 28.611 & 10.748 & -11.812 & 1.00 & 0.00 & C \\
\hline HETATM & 317 & C17 & HEM & 33 & 27.245 & 10.861 & -11.329 & 1.00 & 0.00 & C \\
\hline HETATM & 318 & $\mathrm{C} 18$ & HEM & 33 & 26.569 & 11.629 & -12.253 & 1.00 & 0.00 & C \\
\hline HETATM & 319 & C19 & HEM & 33 & 27.519 & 11.981 & -13.289 & 1.00 & 0.00 & C \\
\hline HETATM & 320 & $\mathrm{C} 20$ & HEM & 33 & 27.228 & 12.772 & -14.401 & 1.00 & 0.00 & C \\
\hline HETATM & 321 & $\mathrm{H} 20$ & HEM & 33 & 26.213 & 13.161 & -14.461 & 1.00 & 0.00 & $\mathrm{H}$ \\
\hline HETATM & 322 & N21 & HEM & 33 & 29.418 & 12.759 & -15.495 & 1.00 & 0.00 & $\mathrm{~N}$ \\
\hline HETATM & 323 & N22 & HEM & 33 & 32.123 & 11.812 & -15.273 & 1.00 & 0.00 & $\mathrm{~N}$ \\
\hline HETATM & 324 & $\mathrm{~N} 23$ & HEM & 33 & 31.452 & 10.549 & -12.772 & 1.00 & 0.00 & $\mathrm{~N}$ \\
\hline HETATM & 325 & N24 & HEM & 33 & 28.752 & 11.433 & -13.008 & 1.00 & 0.00 & $\mathrm{~N}$ \\
\hline HETATM & 326 & C99 & HEM & 33 & 29.659 & 8.568 & -15.510 & 1.00 & 0.00 & C \\
\hline HETATM & 327 & 091 & HEM & 33 & 29.818 & 9.881 & -15.498 & 1.00 & 0.00 & o \\
\hline HETATM & 328 & 092 & HEM & 33 & 29.504 & 7.951 & -16.566 & 1.00 & 0.00 & o \\
\hline HETATM & 329 & СТ91 & HEM & 33 & 29.675 & 7.887 & -14.154 & 1.00 & 0.00 & C \\
\hline HETATM & 330 & H911 & HEM & 33 & 30.654 & 8.082 & -13.691 & 1.00 & 0.00 & $\mathrm{H}$ \\
\hline HETATM & 331 & H912 & HEM & 33 & 28.927 & 8.376 & -13.511 & 1.00 & 0.00 & $\mathrm{H}$ \\
\hline HETATM & 332 & CT 92 & HEM & 33 & 29.415 & 6.382 & -14.265 & 1.00 & 0.00 & C \\
\hline HETATM & 333 & H921 & HEM & 33 & 30.212 & 5.922 & -14.862 & 1.00 & 0.00 & $\mathrm{H}$ \\
\hline HETATM & 334 & H922 & HEM & 33 & 28.490 & 6.226 & -14.836 & 1.00 & 0.00 & $\mathrm{H}$ \\
\hline \multicolumn{11}{|c|}{ TER } \\
\hline HETATM & 335 & $\mathrm{FE}$ & HEM & 34 & 31.016 & 3.836 & -9.423 & 1.00 & 0.00 & $\mathrm{Fe}$ \\
\hline HETATM & 336 & $\mathrm{C} 1$ & HEM & 34 & 33.244 & 1.963 & -8.361 & 1.00 & 0.00 & C \\
\hline HETATM & 337 & $\mathrm{C} 2$ & HEM & 34 & 33.603 & 1.173 & -7.189 & 1.00 & 0.00 & C \\
\hline HETATM & 338 & C3 & HEM & 34 & 32.458 & 1.076 & -6.414 & 1.00 & 0.00 & C \\
\hline HETATM & 339 & $\mathrm{C} 4$ & HEM & 34 & 31.428 & 1.793 & -7.122 & 1.00 & 0.00 & C \\
\hline HETATM & 340 & C5 & HEM & 34 & 30.113 & 1.912 & -6.684 & 1.00 & 0.00 & C \\
\hline HETATM & 341 & H5 & HEM & 34 & 29.859 & 1.416 & -5.749 & 1.00 & 0.00 & $\mathrm{H}$ \\
\hline HETATM & 342 & C6 & HEM & 34 & 29.094 & 2.610 & -7.320 & 1.00 & 0.00 & C \\
\hline HETATM & 343 & $\mathrm{C} 7$ & HEM & 34 & 27.725 & 2.683 & -6.851 & 1.00 & 0.00 & C \\
\hline HETATM & 344 & $\mathrm{C} 8$ & HEM & 34 & 27.019 & 3.403 & -7.798 & 1.00 & 0.00 & C \\
\hline HETATM & 345 & $\mathrm{C} 9$ & HEM & 34 & 27.970 & 3.768 & -8.822 & 1.00 & 0.00 & C \\
\hline HETATM & 346 & $\mathrm{C} 10$ & HEM & 34 & 27.666 & 4.503 & -9.965 & 1.00 & 0.00 & C \\
\hline HETATM & 347 & $\mathrm{H} 10$ & HEM & 34 & 26.639 & 4.846 & -10.071 & 1.00 & 0.00 & $\mathrm{H}$ \\
\hline HETATM & 348 & $\mathrm{C} 11$ & HEM & 34 & 28.540 & 4.843 & -10.988 & 1.00 & 0.00 & C \\
\hline HETATM & 349 & $\mathrm{C} 12$ & HEM & 34 & 28.159 & 5.568 & -12.178 & 1.00 & 0.00 & C \\
\hline HETATM & 350 & C13 & HEM & 34 & 29.299 & 5.715 & -12.935 & 1.00 & 0.00 & C \\
\hline HETATM & 351 & $\mathrm{C} 14$ & HEM & 34 & 30.372 & 5.074 & -12.204 & 1.00 & 0.00 & C \\
\hline HETATM & 352 & C15 & HEM & 34 & 31.700 & 5.019 & -12.625 & 1.00 & 0.00 & C \\
\hline HETATM & 353 & H15 & HEM & 34 & 31.940 & 5.504 & -13.573 & 1.00 & 0.00 & $\mathrm{H}$ \\
\hline HETATM & 354 & $\mathrm{C} 16$ & HEM & 34 & 32.730 & 4.337 & -11.982 & 1.00 & 0.00 & C \\
\hline HETATM & 355 & C17 & HEM & 34 & 34.096 & 4.224 & -12.464 & 1.00 & 0.00 & C \\
\hline HETATM & 356 & $\mathrm{C} 18$ & HEM & 34 & 34.771 & 3.456 & -11.541 & 1.00 & 0.00 & C \\
\hline HETATM & 357 & C19 & HEM & 34 & 33.822 & 3.104 & -10.504 & 1.00 & 0.00 & $\mathrm{C}$ \\
\hline HETATM & 358 & $\mathrm{C} 20$ & HEM & 34 & 34.113 & 2.313 & -9.393 & 1.00 & 0.00 & $\mathrm{C}$ \\
\hline HETATM & 359 & $\mathrm{H} 20$ & HEM & 34 & 35.127 & 1.924 & -9.333 & 1.00 & 0.00 & $\mathrm{H}$ \\
\hline HETATM & 360 & N21 & HEM & 34 & 31.923 & 2.326 & -8.298 & 1.00 & 0.00 & $\mathrm{~N}$ \\
\hline HETATM & 361 & N22 & HEM & 34 & 29.218 & 3.273 & -8.521 & 1.00 & 0.00 & $\mathrm{~N}$ \\
\hline HETATM & 362 & N23 & HEM & 34 & 29.889 & 4.536 & -11.021 & 1.00 & 0.00 & $\mathrm{~N}$ \\
\hline HETATM & 363 & N2 4 & HEM & 34 & 32.588 & 3.652 & -10.786 & 1.00 & 0.00 & $\mathrm{~N}$ \\
\hline HETATM & 364 & C99 & HEM & 34 & 31.682 & 6.517 & -8.283 & 1.00 & 0.00 & C \\
\hline HETATM & 365 & 091 & HEM & 34 & 31.522 & 5.204 & -8.296 & 1.00 & 0.00 & 0 \\
\hline HETATM & 366 & 092 & HEM & 34 & 31.837 & 7.134 & -7.228 & 1.00 & 0.00 & O \\
\hline HETATM & 367 & СТ 91 & HEM & 34 & 31.666 & 7.199 & -9.640 & 1.00 & 0.00 & C \\
\hline HETATM & 368 & H911 & HEM & 34 & 30.687 & 7.003 & -10.103 & 1.00 & 0.00 & $\mathrm{H}$ \\
\hline HETATM & 369 & H912 & HEM & 34 & 32.414 & 6.709 & -10.283 & 1.00 & 0.00 & $\mathrm{H}$ \\
\hline HETATM & 370 & СТ92 & HEM & 34 & 31.925 & 8.703 & -9.528 & 1.00 & 0.00 & C \\
\hline HETATM & 371 & H921 & HEM & 34 & 32.850 & 8.859 & -8.958 & 1.00 & 0.00 & $\mathrm{H}$ \\
\hline HETATM & 372 & H922 & HEM & 34 & 31.129 & 9.163 & -8.931 & 1.00 & 0.00 & $\mathrm{H}$ \\
\hline
\end{tabular}

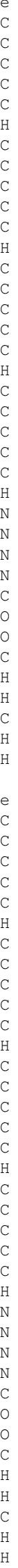




\begin{tabular}{|c|c|c|c|}
\hline HETATM & 373 & $\mathrm{C} 2$ & VIN \\
\hline HETATM & 374 & H 21 & VIN \\
\hline HETATM & 375 & $\mathrm{C} 1$ & VIN \\
\hline HETATM & 376 & H11 & VIN \\
\hline HETATM & 377 & H12 & VIN \\
\hline \multicolumn{4}{|l|}{ TER } \\
\hline HETATM & 378 & $\mathrm{C} 1$ & MET \\
\hline HETATM & 379 & H11 & MET \\
\hline HETATM & 380 & H12 & MET \\
\hline HETATM & 381 & H13 & MET \\
\hline \multicolumn{4}{|l|}{ TER } \\
\hline HETATM & 382 & C2 & VIN \\
\hline HETATM & 383 & $\mathrm{H} 21$ & VIN \\
\hline HETATM & 384 & $\mathrm{C} 1$ & VIN \\
\hline HETATM & 385 & H11 & VIN \\
\hline HETATM & 386 & H12 & VIN \\
\hline \multicolumn{4}{|l|}{ TER } \\
\hline HETATM & 387 & $\mathrm{C} 1$ & MET \\
\hline HETATM & 388 & H11 & MET \\
\hline HETATM & 389 & H12 & MET \\
\hline HETATM & 390 & H13 & MET \\
\hline \multicolumn{4}{|l|}{ TER } \\
\hline HETATM & 391 & C5 & $\mathrm{ACP}$ \\
\hline HETATM & 392 & H51 & $\mathrm{ACP}$ \\
\hline HETATM & 393 & H52 & $\mathrm{ACP}$ \\
\hline HETATM & 394 & $\mathrm{C} 4$ & $\mathrm{ACP}$ \\
\hline HETATM & 395 & $\mathrm{H} 4 \mathrm{I}$ & $\mathrm{ACP}$ \\
\hline HETATM & 396 & $\mathrm{H} 42$ & $\mathrm{ACP}$ \\
\hline HETATM & 397 & $\mathrm{C} 1$ & $\mathrm{ACP}$ \\
\hline HETATM & 398 & 02 & $\mathrm{ACP}$ \\
\hline HETATM & 399 & 03 & $\mathrm{ACP}$ \\
\hline HETATM & 400 & H3 & $\mathrm{ACP}$ \\
\hline \multicolumn{4}{|l|}{$\mathrm{TER}$} \\
\hline HETATM & 401 & $\mathrm{C} 1$ & MET \\
\hline HETATM & 402 & H11 & MET \\
\hline HETATM & 403 & H12 & $\mathrm{MET}$ \\
\hline HETATM & 404 & H13 & MET \\
\hline \multicolumn{4}{|l|}{ TER } \\
\hline HETATM & 405 & $\mathrm{C} 1$ & MET \\
\hline HETATM & 406 & H11 & MET \\
\hline HETATM & 407 & H12 & $\mathrm{MET}$ \\
\hline HETATM & 408 & H13 & MET \\
\hline \multicolumn{4}{|l|}{ TER } \\
\hline HETATM & 409 & $\mathrm{C} 2$ & VIN \\
\hline HETATM & 410 & Н 21 & VIN \\
\hline HETATM & 411 & $\mathrm{C} 1$ & VIN \\
\hline HETATM & 412 & H11 & VIN \\
\hline HETATM & 413 & H12 & VIN \\
\hline \multicolumn{4}{|l|}{ TER } \\
\hline HETATM & 414 & $\mathrm{C} 1$ & MET \\
\hline HETATM & 415 & H11 & MET \\
\hline HETATM & 416 & H12 & MET \\
\hline HETATM & 417 & H13 & MET \\
\hline TER & & & \\
\hline HETATM & 418 & C2 & VIN \\
\hline HETATM & 419 & H 21 & VIN \\
\hline HETATM & 420 & $\mathrm{C} 1$ & VIN \\
\hline HETATM & 421 & H11 & VIN \\
\hline HETATM & 422 & H12 & VIN \\
\hline TER & & & \\
\hline HETATM & 423 & $\mathrm{C} 1$ & MET \\
\hline HETATM & 424 & H11 & MET \\
\hline HETATM & 425 & H12 & MET \\
\hline HETATM & 426 & H13 & MET \\
\hline TER & & & \\
\hline HETATM & 427 & C5 & $\mathrm{ACP}$ \\
\hline HETATM & 428 & H51 & $\mathrm{ACP}$ \\
\hline HETATM & 429 & H52 & $\mathrm{ACP}$ \\
\hline HETATM & 430 & $\mathrm{C} 4$ & $\mathrm{ACP}$ \\
\hline HETATM & 431 & $\mathrm{H} 4 \mathrm{I}$ & $\mathrm{ACP}$ \\
\hline HETATM & 432 & H4 2 & $\mathrm{ACP}$ \\
\hline HETATM & 433 & $\mathrm{C} 1$ & $\mathrm{ACP}$ \\
\hline HETATM & 434 & $\mathrm{O} 2$ & $\mathrm{ACP}$ \\
\hline HETATM & 435 & 03 & $\mathrm{ACP}$ \\
\hline HETATM & 436 & H3 & $\mathrm{ACP}$ \\
\hline TER & & & \\
\hline HETATM & 437 & $\mathrm{C} 1$ & MET \\
\hline HETATM & 438 & H11 & MET \\
\hline HETATM & 439 & H12 & MET \\
\hline
\end{tabular}

\begin{tabular}{|c|c|c|c|c|c|}
\hline 26.406 & 14.415 & -16.864 & 1.00 & 0.00 & C \\
\hline 25.590 & 13.716 & -16.699 & 1.00 & 0.00 & $\mathrm{H}$ \\
\hline 26.073 & 15.640 & -17.266 & 1.00 & 0.00 & C \\
\hline 26.804 & 16.418 & -17.373 & 1.00 & 0.00 & $\mathrm{H}$ \\
\hline 25.031 & 15.909 & -17.432 & 1.00 & 0.00 & $\mathrm{H}$ \\
\hline 29.069 & 14.670 & -18.702 & 1.00 & 0.00 & C \\
\hline 29.304 & 15.741 & -18.583 & 1.00 & 0.00 & $\mathrm{H}$ \\
\hline 29.882 & 14.206 & -19.272 & 1.00 & 0.00 & $\mathrm{H}$ \\
\hline 28.162 & 14.592 & -19.313 & 1.00 & 0.00 & $\mathrm{H}$ \\
\hline 34.079 & 12.992 & -18.188 & 1.00 & 0.00 & C \\
\hline 33.413 & 12.891 & -19.046 & 1.00 & 0.00 & $\mathrm{H}$ \\
\hline 35.246 & 13.636 & $-18 \cdot 346$ & 1.00 & 0.00 & C \\
\hline 35.926 & 13.792 & -17.516 & 1.00 & 0.00 & $\mathrm{H}$ \\
\hline 35.551 & 14.034 & $-19 \cdot 312$ & 1.00 & 0.00 & $\mathrm{H}$ \\
\hline 35.764 & 11.309 & -15.984 & 1.00 & 0.00 & C \\
\hline 36.355 & 11.973 & -15.335 & 1.00 & 0.00 & $\mathrm{H}$ \\
\hline 35.920 & 10.286 & -15.617 & 1.00 & 0.00 & $\mathrm{H}$ \\
\hline 36.196 & 11.374 & -16.989 & 1.00 & 0.00 & $\mathrm{H}$ \\
\hline 26.676 & 10.227 & -10.092 & 1.00 & 0.00 & C \\
\hline 27.415 & 10.216 & -9.280 & 1.00 & 0.00 & $\mathrm{H}$ \\
\hline 25.835 & 10.835 & -9.729 & 1.00 & 0.00 & $\mathrm{H}$ \\
\hline 26.197 & 8.789 & -10.365 & 1.00 & 0.00 & C \\
\hline 25.639 & 8.728 & -11.313 & 1.00 & 0.00 & $\mathrm{H}$ \\
\hline 27.068 & 8.123 & -10.472 & 1.00 & 0.00 & H \\
\hline 25.333 & 8.177 & -9.289 & 1.00 & 0.00 & C \\
\hline 25.105 & 8.700 & -8.191 & 1.00 & 0.00 & 0 \\
\hline 24.817 & 6.998 & -9.653 & 1.00 & 0.00 & O \\
\hline 24.208 & 6.643 & -8.917 & 1.00 & 0.00 & $\mathrm{H}$ \\
\hline 34.572 & 9.101 & -11.266 & 1.00 & 0.00 & C \\
\hline 35.124 & 9.940 & -10.815 & 1.00 & 0.00 & $\mathrm{H}$ \\
\hline 34.578 & 8.275 & -10.544 & 1.00 & 0.00 & $\mathrm{H}$ \\
\hline 35.143 & 8.781 & -12.145 & 1.00 & 0.00 & $\mathrm{H}$ \\
\hline 25.138 & 12.049 & -12.216 & 1.00 & 0.00 & C \\
\hline 24.693 & 11.841 & -11.236 & 1.00 & 0.00 & $\mathrm{H}$ \\
\hline 25.028 & 13.130 & -12.391 & 1.00 & 0.00 & $\mathrm{H}$ \\
\hline 24.538 & 11.524 & -12.973 & 1.00 & 0.00 & $\mathrm{H}$ \\
\hline 34.935 & 0.670 & -6.929 & 1.00 & 0.00 & c \\
\hline 35.751 & 1.369 & -7.094 & 1.00 & 0.00 & $\mathrm{H}$ \\
\hline 35.268 & -0.555 & -6.528 & 1.00 & 0.00 & c \\
\hline 34.536 & -1.333 & -6.421 & 1.00 & 0.00 & $\mathrm{H}$ \\
\hline 36.310 & -0.824 & -6.361 & 1.00 & 0.00 & $\mathrm{H}$ \\
\hline 32.271 & 0.415 & -5.091 & 1.00 & 0.00 & c \\
\hline 32.036 & -0.655 & -5.211 & 1.00 & 0.00 & $\mathrm{H}$ \\
\hline 31.458 & 0.880 & -4.522 & 1.00 & 0.00 & $\mathrm{H}$ \\
\hline 33.179 & 0.493 & -4.481 & 1.00 & 0.00 & $\mathrm{H}$ \\
\hline 27.261 & 2.093 & -5.606 & 1.00 & 0.00 & c \\
\hline 27.927 & 2.194 & -4.747 & 1.00 & 0.00 & $\mathrm{H}$ \\
\hline 26.094 & 1.449 & -5.447 & 1.00 & 0.00 & c \\
\hline 25.414 & 1.293 & -6.277 & 1.00 & 0.00 & $\mathrm{H}$ \\
\hline 25.789 & 1.051 & -4.482 & 1.00 & 0.00 & $\mathrm{H}$ \\
\hline 25.577 & 3.777 & -7.810 & 1.00 & 0.00 & c \\
\hline 25.420 & 4.799 & -8.177 & 1.00 & 0.00 & $\mathrm{H}$ \\
\hline 25.144 & 3.711 & -6.805 & 1.00 & 0.00 & $\mathrm{H}$ \\
\hline 24.985 & 3.112 & -8.459 & 1.00 & 0.00 & $\mathrm{H}$ \\
\hline 34.665 & 4.858 & -13.701 & 1.00 & 0.00 & c \\
\hline 33.926 & 4.870 & -14.514 & 1.00 & 0.00 & $\mathrm{H}$ \\
\hline 35.506 & 4.250 & -14.065 & 1.00 & 0.00 & $\mathrm{H}$ \\
\hline 35.143 & 6.296 & -13.428 & 1.00 & 0.00 & c \\
\hline 35.701 & 6.357 & -12.481 & 1.00 & 0.00 & $\mathrm{H}$ \\
\hline 34.273 & 6.962 & -13.321 & 1.00 & 0.00 & $\mathrm{H}$ \\
\hline 36.007 & 6.908 & -14.504 & 1.00 & 0.00 & c \\
\hline 36.235 & 6.385 & -15.603 & 1.00 & 0.00 & 0 \\
\hline 36.523 & 8.088 & -14.140 & 1.00 & 0.00 & 0 \\
\hline 37.132 & 8.442 & -14.876 & 1.00 & 0.00 & $\mathrm{H}$ \\
\hline 26.769 & 5.985 & -12.528 & 1.00 & 0.00 & c \\
\hline 26.216 & 5.145 & -12.978 & 1.00 & 0.00 & $\mathrm{H}$ \\
\hline 26.762 & 6.810 & -13.250 & 1.00 & 0.00 & $\mathrm{H}$ \\
\hline
\end{tabular}




\begin{tabular}{|c|c|c|c|c|c|c|c|c|c|c|}
\hline $\begin{array}{l}\text { HETATM } \\
\text { TER }\end{array}$ & 440 & H13 & MET & 47 & 26.198 & 6.304 & -11.649 & 1.00 & 0.00 & $\mathrm{H}$ \\
\hline HETATM & 441 & $\mathrm{C} 1$ & $\mathrm{MET}$ & 48 & 36.202 & 3.036 & -11.577 & 1.00 & 0.00 & C \\
\hline HETATM & 442 & H11 & $\mathrm{MET}$ & 48 & 36.647 & 3.245 & -12.557 & 1.00 & 0.00 & $\mathrm{H}$ \\
\hline HETATM & 443 & $\mathrm{H} 12$ & $\mathrm{MET}$ & 48 & 36.313 & 1.956 & -11.402 & 1.00 & 0.00 & $\mathrm{H}$ \\
\hline HETATM & 444 & H13 & $\mathrm{MET}$ & 48 & 36.803 & 3.561 & -10.821 & 1.00 & 0.00 & 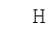 \\
\hline \multicolumn{11}{|l|}{ TER } \\
\hline HETATM & 445 & $\mathrm{FE}$ & HEM & 49 & -8.767 & 10.511 & 9.423 & 1.00 & 0.00 & $\mathrm{Fe}$ \\
\hline HETATM & 446 & $\mathrm{C} 1$ & HEM & 49 & -10.995 & 12.383 & 8.361 & 1.00 & 0.00 & C \\
\hline HETATM & 447 & $\mathrm{C} 2$ & HEM & 49 & $-11 \cdot 354$ & 13.174 & 7.189 & 1.00 & 0.00 & C \\
\hline HETATM & 448 & $\mathrm{C} 3$ & HEM & 49 & -10.209 & 13.271 & 6.414 & 1.00 & 0.00 & C \\
\hline HETATM & 449 & C4 & HEM & 49 & -9.179 & 12.553 & 7.122 & 1.00 & 0.00 & C \\
\hline HETATM & 450 & C5 & HEM & 49 & -7.864 & 12.435 & 6.684 & 1.00 & 0.00 & $\mathrm{C}$ \\
\hline HETATM & 451 & H5 & HEM & 49 & -7.610 & 12.931 & 5.749 & 1.00 & 0.00 & $\mathrm{H}$ \\
\hline HETATM & 452 & C6 & HEM & 49 & -6.845 & 11.736 & 7.320 & 1.00 & 0.00 & C \\
\hline HETATM & 453 & C7 & HEM & 49 & -5.476 & 11.664 & 6.851 & 1.00 & 0.00 & $\mathrm{C}$ \\
\hline HETATM & 454 & $\mathrm{C} 8$ & HEM & 49 & -4.770 & 10.944 & 7.798 & 1.00 & 0.00 & C \\
\hline HETATM & 455 & $\mathrm{C} 9$ & HEM & 49 & -5.721 & 10.579 & 8.822 & 1.00 & 0.00 & C \\
\hline HETATM & 456 & $\mathrm{C} 10$ & HEM & 49 & -5.417 & 9.844 & 9.965 & 1.00 & 0.00 & C \\
\hline HETATM & 457 & $\mathrm{H} 10$ & HEM & 49 & -4.390 & 9.500 & 10.071 & 1.00 & 0.00 & $\mathrm{H}$ \\
\hline HETATM & 458 & C11 & HEM & 49 & -6.291 & 9.504 & 10.988 & 1.00 & 0.00 & C \\
\hline HETATM & 459 & $\mathrm{C} 12$ & HEM & 49 & -5.910 & 8.779 & 12.178 & 1.00 & 0.00 & C \\
\hline HETATM & 460 & $\mathrm{C} 13$ & HEM & 49 & -7.050 & 8.631 & 12.935 & 1.00 & 0.00 & C \\
\hline HETATM & 461 & C14 & HEM & 49 & -8.123 & 9.273 & 12.204 & 1.00 & 0.00 & C \\
\hline HETATM & 462 & C15 & HEM & 49 & -9.451 & 9.328 & 12.625 & 1.00 & 0.00 & C \\
\hline HETATM & 463 & H15 & HEM & 49 & -9.691 & 8.843 & 13.573 & 1.00 & 0.00 & $\mathrm{H}$ \\
\hline HETATM & 464 & $\mathrm{C} 16$ & HEM & 49 & -10.481 & 10.009 & 11.982 & 1.00 & 0.00 & C \\
\hline HETATM & 465 & $\mathrm{C} 17$ & HEM & 49 & -11.847 & 10.123 & 12.464 & 1.00 & 0.00 & C \\
\hline HETATM & 466 & $\mathrm{C} 18$ & HEM & 49 & -12.522 & 10.890 & 11.541 & 1.00 & 0.00 & $\mathrm{C}$ \\
\hline HETATM & 467 & C19 & HEM & 49 & -11.573 & 11.242 & 10.504 & 1.00 & 0.00 & $\mathrm{C}$ \\
\hline HETATM & 468 & $\mathrm{C} 20$ & HEM & 49 & -11.864 & 12.033 & 9.393 & 1.00 & 0.00 & C \\
\hline HETATM & 469 & $\mathrm{H} 20$ & HEM & 49 & -12.878 & 12.423 & 9.333 & 1.00 & 0.00 & $\mathrm{H}$ \\
\hline HETATM & 470 & $\mathrm{~N} 21$ & HEM & 49 & -9.674 & 12.020 & 8.298 & 1.00 & 0.00 & $\mathrm{~N}$ \\
\hline HETATM & 471 & $\mathrm{~N} 22$ & HEM & 49 & -6.969 & 11.073 & 8.521 & 1.00 & 0.00 & $\mathrm{~N}$ \\
\hline HETATM & 472 & N23 & HEM & 49 & -7.640 & 9.810 & 11.021 & 1.00 & 0.00 & $\mathrm{~N}$ \\
\hline HETATM & 473 & N2 4 & HEM & 49 & -10.339 & 10.694 & 10.786 & 1.00 & 0.00 & $\mathrm{~N}$ \\
\hline HETATM & 474 & C99 & HEM & 49 & -9.433 & 7.829 & 8.283 & 1.00 & 0.00 & C \\
\hline HETATM & 475 & 091 & HEM & 49 & -9.273 & 9.143 & 8.296 & 1.00 & 0.00 & O \\
\hline HETATM & 476 & 092 & HEM & 49 & -9.588 & 7.212 & 7.228 & 1.00 & 0.00 & 0 \\
\hline HETATM & 477 & СТ91 & HEM & 49 & -9.417 & 7.148 & 9.640 & 1.00 & 0.00 & $\mathrm{C}$ \\
\hline HETATM & 478 & H911 & HEM & 49 & -8.438 & 7.343 & 10.103 & 1.00 & 0.00 & $\mathrm{H}$ \\
\hline HETATM & 479 & H912 & HEM & 49 & -10.165 & 7.638 & 10.283 & 1.00 & 0.00 & $\mathrm{H}$ \\
\hline HETATM & 480 & СТ 92 & HEM & 49 & -9.676 & 5.644 & 9.528 & 1.00 & 0.00 & C \\
\hline HETATM & 481 & H921 & HEM & 49 & -8.880 & 5.183 & 8.931 & 1.00 & 0.00 & $\mathrm{H}$ \\
\hline HETATM & 482 & H922 & HEM & 49 & -10.601 & 5.488 & 8.958 & 1.00 & 0.00 & $\mathrm{H}$ \\
\hline \multicolumn{11}{|c|}{ TER } \\
\hline HETATM & 483 & $\mathrm{FE}$ & HEM & 50 & -8.075 & 3.097 & 14.371 & 1.00 & 0.00 & $\mathrm{Fe}$ \\
\hline HETATM & 484 & $\mathrm{C} 1$ & HEM & 50 & -5.847 & 1.225 & 15.433 & 1.00 & 0.00 & $\mathrm{C}$ \\
\hline HETATM & 485 & $\mathrm{C} 2$ & HEM & 50 & -5.488 & 0.434 & 16.605 & 1.00 & 0.00 & $\mathrm{C}$ \\
\hline HETATM & 486 & $\mathrm{C} 3$ & HEM & 50 & -6.634 & 0.337 & 17.380 & 1.00 & 0.00 & $\mathrm{C}$ \\
\hline HETATM & 487 & $\mathrm{C} 4$ & HEM & 50 & -7.663 & 1.055 & 16.672 & 1.00 & 0.00 & $\mathrm{C}$ \\
\hline HETATM & 488 & C5 & HEM & 50 & -8.979 & 1.173 & 17.109 & 1.00 & 0.00 & $\mathrm{C}$ \\
\hline HETATM & 489 & H5 & HEM & 50 & -9.232 & 0.677 & 18.045 & 1.00 & 0.00 & $\mathrm{H}$ \\
\hline HETATM & 490 & $\mathrm{C} 6$ & HEM & 50 & -9.998 & 1.872 & 16.473 & 1.00 & 0.00 & C \\
\hline HETATM & 491 & C7 & HEM & 50 & -11.367 & 1.944 & 16.943 & 1.00 & 0.00 & C \\
\hline HETATM & 492 & $\mathrm{C} 8$ & HEM & 50 & -12.072 & 2.664 & 15.996 & 1.00 & 0.00 & C \\
\hline HETATM & 493 & $\mathrm{C} 9$ & HEM & 50 & -11.121 & 3.029 & 14.972 & 1.00 & 0.00 & C \\
\hline HETATM & 494 & $\mathrm{C} 10$ & HEM & 50 & -11.426 & 3.764 & 13.828 & 1.00 & 0.00 & C \\
\hline HETATM & 495 & $\mathrm{H} 10$ & HEM & 50 & -12.453 & 4.108 & 13.722 & 1.00 & 0.00 & $\mathrm{H}$ \\
\hline HETATM & 496 & $\mathrm{C} 11$ & HEM & 50 & -10.551 & 4.104 & 12.806 & 1.00 & 0.00 & C \\
\hline HETATM & 497 & $\mathrm{C} 12$ & HEM & 50 & -10.932 & 4.829 & 11.615 & 1.00 & 0.00 & C \\
\hline HETATM & 498 & $\mathrm{C} 13$ & HEM & 50 & -9.793 & 4.977 & 10.858 & 1.00 & 0.00 & C \\
\hline HETATM & 499 & C14 & HEM & 50 & -8.720 & 4.335 & 11.590 & 1.00 & 0.00 & C \\
\hline HETATM & 500 & C15 & HEM & 50 & -7.392 & 4.280 & 11.168 & 1.00 & 0.00 & C \\
\hline HETATM & 501 & H15 & HEM & 50 & -7.152 & 4.765 & 10.221 & 1.00 & 0.00 & $\mathrm{H}$ \\
\hline HETATM & 502 & C16 & HEM & 50 & -6.362 & 3.599 & 11.812 & 1.00 & 0.00 & C \\
\hline HETATM & 503 & C17 & HEM & 50 & -4.996 & 3.485 & 11.329 & 1.00 & 0.00 & C \\
\hline HETATM & 504 & $\mathrm{C} 18$ & HEM & 50 & -4.320 & 2.718 & 12.253 & 1.00 & 0.00 & C \\
\hline HETATM & 505 & C19 & HEM & 50 & -5.270 & 2.366 & 13.289 & 1.00 & 0.00 & C \\
\hline HETATM & 506 & $\mathrm{C} 20$ & HEM & 50 & -4.979 & 1.575 & 14.401 & 1.00 & 0.00 & C \\
\hline HETATM & 507 & $\mathrm{H} 20$ & HEM & 50 & -3.964 & 1.185 & 14.461 & 1.00 & 0.00 & $\mathrm{H}$ \\
\hline HETATM & 508 & $\mathrm{~N} 21$ & HEM & 50 & -7.169 & 1.588 & 15.495 & 1.00 & 0.00 & $\mathrm{~N}$ \\
\hline HETATM & 509 & $\mathrm{~N} 22$ & HEM & 50 & -9.874 & 2.535 & 15.273 & 1.00 & 0.00 & $\mathrm{~N}$ \\
\hline HETATM & 510 & N23 & HEM & 50 & -9.203 & 3.798 & 12.772 & 1.00 & 0.00 & $\mathrm{~N}$ \\
\hline HETATM & 511 & $\mathrm{~N} 24$ & HEM & 50 & -6.503 & 2.914 & 13.008 & 1.00 & 0.00 & $\mathrm{~N}$ \\
\hline HETATM & 512 & C99 & HEM & 50 & -7.410 & 5.779 & 15.510 & 1.00 & 0.00 & C \\
\hline HETATM & 513 & 091 & HEM & 50 & -7.569 & 4.465 & 15.498 & 1.00 & 0.00 & 0 \\
\hline HETATM & 514 & 092 & HEM & 50 & -7.255 & 6.396 & 16.566 & 1.00 & 0.00 & O \\
\hline HETATM & 515 & СТ91 & HEM & 50 & -7.426 & 6.460 & 14.154 & 1.00 & 0.00 & C \\
\hline
\end{tabular}




\begin{tabular}{|c|c|c|c|c|}
\hline-8.405 & 6.265 & 13.691 & 1.00 & 0.00 \\
\hline-6.678 & 5.970 & 13.511 & 1.00 & 0.00 \\
\hline-7.166 & 7.964 & 14.265 & 1.00 & 0.00 \\
\hline-6.241 & 8.120 & 14.836 & 1.00 & 0.00 \\
\hline-7.963 & 8.425 & 14.862 & 1.00 & 0.00 \\
\hline-12.686 & 13.676 & 6.929 & 1.00 & 0.00 \\
\hline-13.502 & 12.978 & 7.094 & 1.00 & 0.00 \\
\hline-13.019 & 14.901 & 6.528 & 1.00 & 0.00 \\
\hline-12.287 & 15.680 & 6.421 & 1.00 & 0.00 \\
\hline-14.061 & 15.170 & 6.361 & 1.00 & 0.00 \\
\hline-10.022 & 13.931 & 5.091 & 1.00 & 0.00 \\
\hline-9.787 & 15.002 & 5.211 & 1.00 & 0.00 \\
\hline-9.209 & 13.467 & 4.522 & 1.00 & 0.00 \\
\hline-10.930 & 13.854 & 4.481 & 1.00 & 0.00 \\
\hline-5.012 & 12.253 & 5.606 & 1.00 & 0.00 \\
\hline-5.678 & 12.152 & 4.747 & 1.00 & 0.00 \\
\hline-3.845 & 12.898 & 5.447 & 1.00 & 0.00 \\
\hline-3.165 & 13.054 & 6.277 & 1.00 & 0.00 \\
\hline-3.540 & 13.296 & 4.482 & 1.00 & 0.00 \\
\hline-3.328 & 10.570 & 7.810 & 1.00 & 0.00 \\
\hline-2.736 & 11.234 & 8.459 & 1.00 & 0.00 \\
\hline-3.171 & 9.548 & 8.177 & 1.00 & 0.00 \\
\hline-2.895 & 10.636 & 6.805 & 1.00 & 0.00 \\
\hline-12.416 & 9.488 & 13.701 & 1.00 & 0.00 \\
\hline-11.677 & 9.477 & 14.514 & 1.00 & 0.00 \\
\hline-13.257 & 10.097 & 14.065 & 1.00 & 0.00 \\
\hline-12.894 & 8.051 & 13.428 & 1.00 & 0.00 \\
\hline-13.452 & 7.990 & 12.481 & 1.00 & 0.00 \\
\hline-12.024 & 7.384 & 13.321 & 1.00 & 0.00 \\
\hline-13.758 & 7.439 & 14.504 & 1.00 & 0.00 \\
\hline-13.986 & 7.961 & 15.603 & 1.00 & 0.00 \\
\hline-14.274 & 6.259 & 14.140 & 1.00 & 0.00 \\
\hline-14.883 & 5.905 & 14.876 & 1.00 & 0.00 \\
\hline-4.520 & 8.362 & 12.528 & 1.00 & 0.00 \\
\hline-3.967 & 9.201 & 12.978 & 1.00 & 0.00 \\
\hline-4.513 & 7.536 & 13.250 & 1.00 & 0.00 \\
\hline-3.949 & 8.042 & 11.649 & 1.00 & 0.00 \\
\hline-13.953 & 11.311 & 11.577 & 1.00 & 0.00 \\
\hline-14.398 & 11.102 & 12.557 & 1.00 & 0.00 \\
\hline-14.064 & 12.391 & 11.402 & 1.00 & 0.00 \\
\hline-14.554 & 10.785 & 10.821 & 1.00 & 0.00 \\
\hline-4.157 & -0.068 & 16.864 & 1.00 & 0.00 \\
\hline-3.341 & 0.630 & 16.699 & 1.00 & 0.00 \\
\hline-3.824 & -1.293 & 17.266 & 1.00 & 0.00 \\
\hline-4.555 & -2.072 & 17.373 & 1.00 & 0.00 \\
\hline-2.782 & -1.562 & 17.432 & 1.00 & 0.00 \\
\hline-6.820 & -0.323 & 18.702 & 1.00 & 0.00 \\
\hline-7.055 & -1.394 & 18.583 & 1.00 & 0.00 \\
\hline-7.633 & 0.141 & 19.272 & 1.00 & 0.00 \\
\hline-5.913 & -0.246 & 19.313 & 1.00 & 0.00 \\
\hline-11.830 & 1.355 & 18.188 & 1.00 & 0.00 \\
\hline-11.164 & 1.456 & 19.046 & 1.00 & 0.00 \\
\hline-12.997 & 0.710 & 18.346 & 1.00 & 0.00 \\
\hline-13.677 & 0.554 & 17.516 & 1.00 & 0.00 \\
\hline-13.302 & 0.312 & 19.312 & 1.00 & 0.00 \\
\hline-13.515 & 3.038 & 15.984 & 1.00 & 0.00 \\
\hline-13.671 & 4.060 & 15.617 & 1.00 & 0.00 \\
\hline-13.947 & 2.972 & 16.989 & 1.00 & 0.00 \\
\hline-14.106 & 2.374 & 15.335 & 1.00 & 0.00 \\
\hline-4.427 & 4.120 & 10.092 & 1.00 & 0.00 \\
\hline-5.166 & 4.131 & 9.280 & 1.00 & 0.00 \\
\hline-3.586 & 3.511 & 9.729 & 1.00 & 0.00 \\
\hline-3.948 & 5.557 & 10.365 & 1.00 & 0.00 \\
\hline-3.390 & 5.618 & 11.313 & 1.00 & 0.00 \\
\hline-4.819 & 6.224 & 10.472 & 1.00 & 0.00 \\
\hline-3.084 & 6.169 & 9.289 & 1.00 & 0.00 \\
\hline-2.856 & 5.647 & 8.191 & 1.00 & 0.00 \\
\hline
\end{tabular}




\begin{tabular}{|c|c|c|c|c|c|c|c|c|c|c|}
\hline HETATM & 583 & 03 & $\mathrm{ACP}$ & 62 & -2.568 & 7.349 & 9.653 & 1.00 & 0.00 & o \\
\hline HETATM & 584 & H3 & $\mathrm{ACP}$ & 62 & -1.959 & 7.703 & 8.917 & 1.00 & 0.00 & $\mathrm{H}$ \\
\hline \multicolumn{11}{|l|}{ TER } \\
\hline HETATM & 585 & $\mathrm{C} 1$ & MET & 63 & $-12 \cdot 323$ & 5.246 & 11.266 & 1.00 & 0.00 & C \\
\hline HETATM & 586 & $\mathrm{H} 11$ & MET & 63 & -12.875 & 4.407 & 10.815 & 1.00 & 0.00 & n \\
\hline HETATM & 587 & $\mathrm{H} 12$ & MET & 63 & -12.329 & 6.072 & 10.544 & 1.00 & 0.00 & $\mathrm{H}$ \\
\hline HETATM & 588 & $\mathrm{H} 13$ & MET & 63 & -12.894 & 5.565 & 12.145 & 1.00 & 0.00 & $\mathrm{H}$ \\
\hline \multicolumn{11}{|l|}{ TER } \\
\hline HETATM & 589 & $\mathrm{C} 1$ & MET & 64 & -2.889 & 2.297 & 12.216 & 1.00 & 0.00 & c \\
\hline HETATM & 590 & $\mathrm{H} 11$ & MET & 64 & -2.444 & 2.506 & 11.236 & 1.00 & 0.00 & \\
\hline HETATM & 591 & $\mathrm{H} 12$ & $\mathrm{MET}$ & 64 & -2.779 & 1.217 & 12.391 & 1.00 & 0.00 & \\
\hline HETATM & 592 & $\mathrm{H} 13$ & $\mathrm{MET}$ & 64 & -2.289 & 2.823 & 12.973 & 1.00 & 0.00 & \\
\hline
\end{tabular}

TER

END

\subsection{Crystal model: Q05_Q5.pdb file}

\begin{tabular}{|c|c|c|c|c|c|c|c|c|c|c|}
\hline HETATM & 1 & $\mathrm{FE}$ & HEM & 1 & 4.263 & 10.757 & 1.491 & 1.00 & 0.00 & $\mathrm{Fe}$ \\
\hline HETATM & 2 & $\mathrm{C} 1$ & HEM & 1 & 2.035 & 12.629 & 0.429 & 1.00 & 0.00 & C \\
\hline HETATM & 3 & $\mathrm{C} 2$ & HEM & 1 & 1.676 & 13.420 & -0.742 & 1.00 & 0.00 & C \\
\hline HETATM & 4 & $\mathrm{C} 3$ & HEM & 1 & 2.822 & 13.517 & -1.517 & 1.00 & 0.00 & C \\
\hline HETATM & 5 & $\mathrm{C} 4$ & HEM & 1 & 3.851 & 12.800 & -0.809 & 1.00 & 0.00 & C \\
\hline HETATM & 6 & C5 & HEM & 1 & 5.167 & 12.681 & -1.247 & 1.00 & 0.00 & C \\
\hline HETATM & 7 & H5 & HEM & 1 & 5.420 & 13.177 & -2.182 & 1.00 & 0.00 & $\mathrm{H}$ \\
\hline HETATM & 8 & C6 & HEM & 1 & 6.186 & 11.982 & -0.611 & 1.00 & 0.00 & C \\
\hline HETATM & 9 & $\mathrm{C} 7$ & HEM & 1 & 7.555 & 11.910 & -1.081 & 1.00 & 0.00 & C \\
\hline HETATM & 10 & C8 & HEM & 1 & 8.260 & 11.190 & -0.134 & 1.00 & 0.00 & C \\
\hline HETATM & 11 & C9 & HEM & 1 & 7.309 & 10.825 & 0.891 & 1.00 & 0.00 & C \\
\hline HETATM & 12 & $\mathrm{C} 10$ & HEM & 1 & 7.614 & 10.090 & 2.034 & 1.00 & 0.00 & C \\
\hline HETATM & 13 & $\mathrm{H} 1 \mathrm{O}$ & HEM & 1 & 8.641 & 9.746 & 2.140 & 1.00 & 0.00 & $\mathrm{H}$ \\
\hline HETATM & 14 & $\mathrm{C} 11$ & HEM & 1 & 6.739 & 9.750 & 3.057 & 1.00 & 0.00 & C \\
\hline HETATM & 15 & $\mathrm{C} 12$ & HEM & 1 & 7.120 & 9.025 & 4.247 & 1.00 & 0.00 & C \\
\hline HETATM & 16 & C13 & HEM & 1 & 5.981 & 8.878 & 5.004 & 1.00 & 0.00 & C \\
\hline HETATM & 17 & $\mathrm{C} 14$ & HEM & 1 & 4.908 & 9.519 & 4.273 & 1.00 & 0.00 & C \\
\hline HETATM & 18 & C15 & HEM & 1 & 3.580 & 9.574 & 4.694 & 1.00 & 0.00 & C \\
\hline HETATM & 19 & H15 & HEM & 1 & 3.340 & 9.089 & 5.642 & 1.00 & 0.00 & $\mathrm{H}$ \\
\hline HETATM & 20 & $\mathrm{C} 16$ & HEM & 1 & 2.550 & 10.255 & 4.051 & 1.00 & 0.00 & C \\
\hline HETATM & 21 & $\mathrm{C} 17$ & HEM & 1 & 1.184 & 10.369 & 4.533 & 1.00 & 0.00 & C \\
\hline HETATM & 22 & $\mathrm{C} 18$ & HEM & 1 & 0.508 & 11.137 & 3.609 & 1.00 & 0.00 & C \\
\hline HETATM & 23 & C19 & HEM & 1 & 1.458 & 11.489 & 2.573 & 1.00 & 0.00 & C \\
\hline HETATM & 24 & $\mathrm{C} 20$ & HEM & 1 & 1.167 & 12.280 & 1.462 & 1.00 & 0.00 & C \\
\hline HETATM & 25 & $\mathrm{H} 2 \mathrm{O}$ & HEM & 1 & 0.152 & 12.669 & 1.402 & 1.00 & 0.00 & $\mathrm{H}$ \\
\hline HETATM & 26 & N21 & HEM & 1 & 3.357 & 12.266 & 0.367 & 1.00 & 0.00 & $\mathrm{~N}$ \\
\hline HETATM & 27 & $\mathrm{~N} 22$ & HEM & 1 & 6.062 & 11.319 & 0.590 & 1.00 & 0.00 & $\mathrm{~N}$ \\
\hline HETATM & 28 & N23 & HEM & 1 & 5.391 & 10.056 & 3.090 & 1.00 & 0.00 & $\mathrm{~N}$ \\
\hline HETATM & 29 & N24 & HEM & 1 & 2.691 & 10.941 & 2.854 & 1.00 & 0.00 & $\mathrm{~N}$ \\
\hline HETATM & 30 & C99 & HEM & 1 & 3.598 & 8.076 & 0.352 & 1.00 & 0.00 & C \\
\hline HETATM & 31 & 091 & HEM & 1 & 3.757 & 9.389 & 0.365 & 1.00 & 0.00 & O \\
\hline HETATM & 32 & 092 & HEM & 1 & 3.443 & 7.458 & -0.703 & 1.00 & 0.00 & O \\
\hline HETATM & 33 & CT91 & HEM & 1 & 3.614 & 7.394 & 1.709 & 1.00 & 0.00 & C \\
\hline HETATM & 34 & H911 & HEM & 1 & 4.593 & 7.590 & 2.171 & 1.00 & 0.00 & $\mathrm{H}$ \\
\hline HETATM & 35 & H912 & HEM & 1 & 2.866 & 7.884 & 2.352 & 1.00 & 0.00 & $\mathrm{H}$ \\
\hline HETATM & 36 & CT92 & HEM & 1 & 3.354 & 5.890 & 1.597 & 1.00 & 0.00 & C \\
\hline HETATM & 37 & H921 & HEM & 1 & 4.151 & 5.429 & 1.000 & 1.00 & 0.00 & $\mathrm{H}$ \\
\hline HETATM & 38 & H922 & HEM & 1 & 2.429 & 5.734 & 1.027 & 1.00 & 0.00 & $\mathrm{H}$ \\
\hline TER & & & & & & & & & & \\
\hline HETATM & 39 & $\mathrm{FE}$ & HEM & 2 & 4.955 & 3.343 & 6.440 & 1.00 & 0.00 & $\mathrm{Fe}$ \\
\hline HETATM & 40 & $\mathrm{C} 1$ & HEM & 2 & 7.183 & 1.471 & 7.502 & 1.00 & 0.00 & $\mathrm{C}$ \\
\hline HETATM & 41 & $\mathrm{C} 2$ & HEM & 2 & 7.542 & 0.680 & 8.674 & 1.00 & 0.00 & C \\
\hline HETATM & 42 & $\mathrm{C} 3$ & HEM & 2 & 6.397 & 0.583 & 9.448 & 1.00 & 0.00 & C \\
\hline HETATM & 43 & $\mathrm{C} 4$ & HEM & 2 & 5.367 & 1.301 & 8.740 & 1.00 & 0.00 & C \\
\hline HETATM & 44 & C5 & HEM & 2 & 4.052 & 1.420 & 9.178 & 1.00 & 0.00 & C \\
\hline HETATM & 45 & H5 & HEM & 2 & 3.798 & 0.923 & 10.113 & 1.00 & 0.00 & $\mathrm{H}$ \\
\hline HETATM & 46 & $\mathrm{C} 6$ & HEM & 2 & 3.033 & 2.118 & 8.542 & 1.00 & 0.00 & C \\
\hline HETATM & 47 & $\mathrm{C} 7$ & HEM & 2 & 1.664 & 2.191 & 9.012 & 1.00 & 0.00 & C \\
\hline HETATM & 48 & $\mathrm{C} 8$ & HEM & 2 & 0.958 & 2.910 & 8.065 & 1.00 & 0.00 & $\mathrm{C}$ \\
\hline HETATM & 49 & $\mathrm{Cg}$ & HEM & 2 & 1.909 & 3.275 & 7.041 & 1.00 & 0.00 & C \\
\hline HETATM & 50 & $\mathrm{C} 10$ & HEM & 2 & 1.605 & 4.010 & 5.897 & 1.00 & 0.00 & C \\
\hline HETATM & 51 & $\mathrm{H} 10$ & HEM & 2 & 0.578 & 4.354 & 5.791 & 1.00 & 0.00 & $\mathrm{H}$ \\
\hline HETATM & 52 & $\mathrm{C} 11$ & HEM & 2 & 2.479 & 4.350 & 4.874 & 1.00 & 0.00 & C \\
\hline HETATM & 53 & $\mathrm{C} 12$ & HEM & 2 & 2.098 & 5.076 & 3.684 & 1.00 & 0.00 & C \\
\hline HETATM & 54 & $\mathrm{C} 13$ & HEM & 2 & 3.238 & 5.223 & 2.927 & 1.00 & 0.00 & C \\
\hline HETATM & 55 & C14 & HEM & 2 & 4.311 & 4.581 & 3.659 & 1.00 & 0.00 & C \\
\hline HETATM & 56 & C15 & HEM & 2 & 5.639 & 4.527 & 3.237 & 1.00 & 0.00 & $\mathrm{C}$ \\
\hline HETATM & 57 & $\mathrm{H} 15$ & HEM & 2 & 5.879 & 5.011 & 2.289 & 1.00 & 0.00 & $\mathrm{H}$ \\
\hline HETATM & 58 & $\mathrm{C} 16$ & HEM & 2 & 6.669 & 3.845 & 3.880 & 1.00 & 0.00 & C \\
\hline HETATM & 59 & $\mathrm{C} 17$ & HEM & 2 & 8.035 & 3.732 & 3.398 & 1.00 & 0.00 & $\mathrm{C}$ \\
\hline HETATM & 60 & $\mathrm{C} 18$ & HEM & 2 & 8.710 & 2.964 & 4.322 & 1.00 & 0.00 & C \\
\hline
\end{tabular}




\begin{tabular}{|c|c|c|c|}
\hline HETATM & 61 & C19 & HEM \\
\hline HETATM & 62 & C20 & HEM \\
\hline HETATM & 63 & H 20 & HEM \\
\hline HETATM & 64 & N21 & HEM \\
\hline HETATM & 65 & N22 & HEM \\
\hline HETATM & 66 & N23 & HEM \\
\hline HETATM & 67 & N24 & HEM \\
\hline HETATM & 68 & C99 & HEM \\
\hline HETATM & 69 & 091 & HEM \\
\hline HETATM & 70 & 092 & HEM \\
\hline HETATM & 71 & CT91 & HEM \\
\hline HETATM & 72 & H911 & HEM \\
\hline HETATM & 73 & H912 & HEM \\
\hline HETATM & 74 & СТ92 & HEM \\
\hline HETATM & 75 & H921 & HEM \\
\hline HETATM & 76 & H922 & HEM \\
\hline \multicolumn{4}{|l|}{ TER } \\
\hline HETATM & 77 & C2 & VIN \\
\hline HETATM & 78 & H 21 & VIN \\
\hline HETATM & 79 & $\mathrm{C} 1$ & VIN \\
\hline HETATM & 80 & H11 & VIN \\
\hline HETATM & 81 & $\mathrm{H} 12$ & VIN \\
\hline \multicolumn{4}{|l|}{ TER } \\
\hline HETATM & 82 & $\mathrm{C} 1$ & MET \\
\hline HETATM & 83 & H11 & MET \\
\hline HETATM & 84 & $\mathrm{H} 12$ & MET \\
\hline HETATM & 85 & $\mathrm{H} 13$ & MET \\
\hline \multicolumn{4}{|l|}{ TER } \\
\hline HETATM & 86 & C2 & VIN \\
\hline HETATM & 87 & H 21 & VIN \\
\hline HETATM & 88 & $\mathrm{C} 1$ & VIN \\
\hline HETATM & 89 & H11 & VIN \\
\hline HETATM & 90 & $\mathrm{H} 12$ & VIN \\
\hline \multicolumn{4}{|l|}{ TER } \\
\hline HETATM & 91 & $\mathrm{C} 1$ & $\mathrm{MET}$ \\
\hline HETATM & 92 & $\mathrm{H} 11$ & MET \\
\hline HETATM & 93 & $\mathrm{H} 12$ & MET \\
\hline HETATM & 94 & H13 & MET \\
\hline \multicolumn{4}{|l|}{ TER } \\
\hline HETATM & 95 & C5 & $\mathrm{ACP}$ \\
\hline HETATM & 96 & H51 & $\mathrm{ACP}$ \\
\hline HETATM & 97 & H52 & $\mathrm{ACP}$ \\
\hline HETATM & 98 & $\mathrm{C} 4$ & $\mathrm{ACP}$ \\
\hline HETATM & 99 & $\mathrm{H} 4 \mathrm{I}$ & $\mathrm{ACP}$ \\
\hline HETATM & 100 & $\mathrm{H} 42$ & $\mathrm{ACP}$ \\
\hline HETATM & 101 & $\mathrm{C} 1$ & $\mathrm{ACP}$ \\
\hline HETATM & 102 & $\mathrm{O} 2$ & $\mathrm{ACP}$ \\
\hline HETATM & 103 & 03 & $\mathrm{ACP}$ \\
\hline HETATM & 104 & H3 & $\mathrm{ACP}$ \\
\hline \multicolumn{4}{|l|}{ TER } \\
\hline HETATM & 105 & $\mathrm{C} 1$ & MET \\
\hline HETATM & 106 & $\mathrm{H} 11$ & MET \\
\hline HETATM & 107 & $\mathrm{H} 12$ & MET \\
\hline HETATM & 108 & $\mathrm{H} 13$ & $\mathrm{MET}$ \\
\hline \multicolumn{4}{|l|}{ TER } \\
\hline HETATM & 109 & $\mathrm{C} 1$ & MET \\
\hline HETATM & 110 & $\mathrm{H} 11$ & MET \\
\hline HETATM & 111 & $\mathrm{H} 12$ & MET \\
\hline HETATM & 112 & H13 & MET \\
\hline \multicolumn{4}{|l|}{ TER } \\
\hline HETATM & 113 & $\mathrm{C} 2$ & VIN \\
\hline HETATM & 114 & H 21 & VIN \\
\hline HETATM & 115 & $\mathrm{C} 1$ & VIN \\
\hline HETATM & 116 & H11 & VIN \\
\hline HETATM & 117 & $\mathrm{H} 12$ & VIN \\
\hline TER & & & \\
\hline HETATM & 118 & $\mathrm{C} 1$ & MET \\
\hline HETATM & 119 & H11 & MET \\
\hline HETATM & 120 & $\mathrm{H} 12$ & MET \\
\hline HETATM & 121 & H13 & MET \\
\hline TER & & & \\
\hline HETATM & 122 & $\mathrm{C} 2$ & VIN \\
\hline HETATM & 123 & H 21 & VIN \\
\hline HETATM & 124 & $\mathrm{C} 1$ & VIN \\
\hline HETATM & 125 & H11 & VIN \\
\hline HETATM & 126 & $\mathrm{H} 12$ & VIN \\
\hline TER & & & \\
\hline HETATM & 127 & $\mathrm{C} 1$ & MET \\
\hline HETATM & 128 & H11 & MET \\
\hline
\end{tabular}

\begin{tabular}{|c|c|c|c|c|c|}
\hline 7.761 & 2.612 & 5.358 & 1.00 & 0.00 & C \\
\hline 8.052 & 1.821 & 6.469 & 1.00 & 0.00 & C \\
\hline 9.066 & 1.432 & 6.530 & 1.00 & 0.00 & $\mathrm{H}$ \\
\hline 5.862 & 1.834 & 7.564 & 1.00 & 0.00 & $\mathrm{~N}$ \\
\hline 3.157 & 2.781 & 7.342 & 1.00 & 0.00 & $\mathrm{~N}$ \\
\hline 3.828 & 4.044 & 4.841 & 1.00 & 0.00 & $\mathrm{~N}$ \\
\hline 6.527 & 3.160 & 5.077 & 1.00 & 0.00 & $\mathrm{~N}$ \\
\hline 5.621 & 6.025 & 7.579 & 1.00 & 0.00 & C \\
\hline 5.461 & 4.711 & 7.567 & 1.00 & 0.00 & o \\
\hline 5.776 & 6.642 & 8.635 & 1.00 & 0.00 & o \\
\hline 5.605 & 6.706 & 6.223 & 1.00 & 0.00 & C \\
\hline 4.626 & 6.511 & 5.760 & 1.00 & 0.00 & $\mathrm{H}$ \\
\hline 6.353 & 6.216 & 5.580 & 1.00 & 0.00 & $\mathrm{H}$ \\
\hline 5.864 & 8.211 & 6.334 & 1.00 & 0.00 & C \\
\hline 6.789 & 8.367 & 6.905 & 1.00 & 0.00 & $\mathrm{H}$ \\
\hline 5.068 & 8.671 & 6.931 & 1.00 & 0.00 & $\mathrm{H}$ \\
\hline 0.345 & 13.923 & -1.002 & 1.00 & 0.00 & C \\
\hline-0.471 & 13.224 & -0.837 & 1.00 & 0.00 & $\mathrm{H}$ \\
\hline 0.012 & 15.147 & -1.403 & 1.00 & 0.00 & C \\
\hline 0.743 & 15.926 & -1.511 & 1.00 & 0.00 & $\mathrm{H}$ \\
\hline-1.030 & 15.417 & -1.570 & 1.00 & 0.00 & $\mathrm{H}$ \\
\hline 3.008 & 14.177 & -2.840 & 1.00 & 0.00 & C \\
\hline 3.243 & 15.248 & -2.720 & 1.00 & 0.00 & $\mathrm{H}$ \\
\hline 3.821 & 13.713 & -3.410 & 1.00 & 0.00 & $\mathrm{H}$ \\
\hline 2.101 & 14.100 & -3.450 & 1.00 & 0.00 & $\mathrm{H}$ \\
\hline 8.018 & 12.499 & -2.325 & 1.00 & 0.00 & C \\
\hline 7.352 & 12.399 & -3.184 & 1.00 & 0.00 & $\mathrm{H}$ \\
\hline 9.185 & 13.144 & -2.484 & 1.00 & 0.00 & C \\
\hline 9.865 & 13.300 & -1.654 & 1.00 & 0.00 & $\mathrm{H}$ \\
\hline 9.490 & 13.542 & -3.450 & 1.00 & 0.00 & $\mathrm{H}$ \\
\hline 9.703 & 10.816 & -0.121 & 1.00 & 0.00 & C \\
\hline 10.294 & 11.480 & 0.528 & 1.00 & 0.00 & $\mathrm{H}$ \\
\hline 9.859 & 9.794 & 0.246 & 1.00 & 0.00 & $\mathrm{H}$ \\
\hline 10.135 & 10.882 & -1.126 & 1.00 & 0.00 & $\mathrm{H}$ \\
\hline 0.615 & 9.735 & 5.770 & 1.00 & 0.00 & C \\
\hline 1.354 & 9.723 & 6.582 & 1.00 & 0.00 & $\mathrm{H}$ \\
\hline-0.226 & 10.343 & 6.134 & 1.00 & 0.00 & $\mathrm{H}$ \\
\hline 0.136 & 8.297 & 5.497 & 1.00 & 0.00 & C \\
\hline-0.422 & 8.236 & 4.549 & 1.00 & 0.00 & $\mathrm{H}$ \\
\hline 1.007 & 7.630 & 5.390 & 1.00 & 0.00 & $\mathrm{H}$ \\
\hline-0.728 & 7.685 & 6.573 & 1.00 & 0.00 & C \\
\hline-0.956 & 8.208 & 7.671 & 1.00 & 0.00 & 0 \\
\hline-1.244 & 6.505 & 6.209 & 1.00 & 0.00 & 0 \\
\hline-1.853 & 6.151 & 6.945 & 1.00 & 0.00 & $\mathrm{H}$ \\
\hline 8.511 & 8.608 & 4.597 & 1.00 & 0.00 & C \\
\hline 9.063 & 9.448 & 5.047 & 1.00 & 0.00 & $\mathrm{H}$ \\
\hline 8.517 & 7.782 & 5.319 & 1.00 & 0.00 & $\mathrm{H}$ \\
\hline 9.082 & 8.289 & 3.717 & 1.00 & 0.00 & $\mathrm{H}$ \\
\hline-0.923 & 11.557 & 3.646 & 1.00 & 0.00 & C \\
\hline-1.368 & 11.348 & 4.626 & 1.00 & 0.00 & $\mathrm{H}$ \\
\hline-1.033 & 12.637 & 3.471 & 1.00 & 0.00 & $\mathrm{H}$ \\
\hline-1.523 & 11.031 & 2.889 & 1.00 & 0.00 & $\mathrm{H}$ \\
\hline 8.874 & 0.178 & 8.933 & 1.00 & 0.00 & C \\
\hline 9.690 & 0.876 & 8.768 & 1.00 & 0.00 & $\mathrm{H}$ \\
\hline 9.207 & -1.047 & 9.335 & 1.00 & 0.00 & C \\
\hline 8.475 & -1.826 & 9.442 & 1.00 & 0.00 & $\mathrm{H}$ \\
\hline 10.249 & -1.316 & 9.501 & 1.00 & 0.00 & $\mathrm{H}$ \\
\hline 6.210 & -0.077 & 10.771 & 1.00 & 0.00 & C \\
\hline 5.975 & -1.148 & 10.652 & 1.00 & 0.00 & $\mathrm{H}$ \\
\hline 5.397 & 0.387 & 11.341 & 1.00 & 0.00 & $\mathrm{H}$ \\
\hline 7.118 & 0.001 & 11.382 & 1.00 & 0.00 & $\mathrm{H}$ \\
\hline 1.200 & 1.601 & 10.256 & 1.00 & 0.00 & C \\
\hline 1.866 & 1.702 & 11.115 & 1.00 & 0.00 & $\mathrm{H}$ \\
\hline 0.033 & 0.957 & 10.415 & 1.00 & 0.00 & C \\
\hline-0.647 & 0.800 & 9.585 & 1.00 & 0.00 & $\mathrm{H}$ \\
\hline-0.272 & 0.559 & 11.381 & 1.00 & 0.00 & $\mathrm{H}$ \\
\hline-0.484 & 3.284 & 8.052 & 1.00 & 0.00 & C \\
\hline-0.641 & 4.306 & 7.685 & 1.00 & 0.00 & . \\
\hline
\end{tabular}




\begin{tabular}{|c|c|c|c|c|c|c|c|c|c|c|}
\hline HETATM & 129 & $\mathrm{H} 12$ & $\mathrm{MET}$ & 13 & -0.917 & 3.219 & 9.057 & 1.00 & 0.00 & $\mathrm{H}$ \\
\hline HETATM & 130 & H13 & $\mathrm{MET}$ & 13 & -1.076 & 2.620 & 7.403 & 1.00 & 0.00 & $\mathrm{H}$ \\
\hline \multicolumn{11}{|l|}{ TER } \\
\hline HETATM & 131 & C5 & $\mathrm{ACP}$ & 14 & 8.604 & 4.366 & 2.161 & 1.00 & 0.00 & C \\
\hline HETATM & 132 & H51 & $\mathrm{ACP}$ & 14 & 7.865 & 4.377 & 1.349 & 1.00 & 0.00 & $\mathrm{H}$ \\
\hline HETATM & 133 & H52 & $\mathrm{ACP}$ & 14 & 9.445 & 3.758 & 1.798 & 1.00 & 0.00 & $\mathrm{H}$ \\
\hline HETATM & 134 & $\mathrm{C} 4$ & $\mathrm{ACP}$ & 14 & 9.082 & 5.803 & 2.434 & 1.00 & 0.00 & C \\
\hline HETATM & 135 & $\mathrm{H} 41$ & $\mathrm{ACP}$ & 14 & 9.640 & 5.865 & 3.382 & 1.00 & 0.00 & $\mathrm{H}$ \\
\hline HETATM & 136 & H 42 & $\mathrm{ACP}$ & 14 & 8.212 & 6.470 & 2.541 & 1.00 & 0.00 & $\mathrm{H}$ \\
\hline HETATM & 137 & $\mathrm{C} 1$ & $\mathrm{ACP}$ & 14 & 9.946 & 6.416 & 1.358 & 1.00 & 0.00 & C \\
\hline HETATM & 138 & $\mathrm{O} 2$ & $\mathrm{ACP}$ & 14 & 10.174 & 5.893 & 0.260 & 1.00 & 0.00 & 0 \\
\hline HETATM & 139 & 03 & $\mathrm{ACP}$ & 14 & 10.462 & 7.595 & 1.722 & 1.00 & 0.00 & 0 \\
\hline HETATM & 140 & H3 & $\mathrm{ACP}$ & 14 & 11.071 & 7.949 & 0.986 & 1.00 & 0.00 & $\mathrm{H}$ \\
\hline \multicolumn{11}{|l|}{ TER } \\
\hline HETATM & 141 & $\mathrm{C} 1$ & $\mathrm{MET}$ & 15 & 0.708 & 5.492 & 3.334 & 1.00 & 0.00 & C \\
\hline HETATM & 142 & H11 & $\mathrm{MET}$ & 15 & 0.155 & 4.653 & 2.884 & 1.00 & 0.00 & $\mathrm{H}$ \\
\hline HETATM & 143 & $\mathrm{H} 12$ & $\mathrm{MET}$ & 15 & 0.701 & 6.318 & 2.613 & 1.00 & 0.00 & $\mathrm{H}$ \\
\hline HETATM & 144 & H13 & $\mathrm{MET}$ & 15 & 0.137 & 5.812 & 4.214 & 1.00 & 0.00 & $\mathrm{H}$ \\
\hline \multicolumn{11}{|l|}{ TER } \\
\hline HETATM & 145 & $\mathrm{C} 1$ & $\mathrm{MET}$ & 16 & 10.141 & 2.543 & 4.285 & 1.00 & 0.00 & C \\
\hline HETATM & 146 & H11 & $\mathrm{MET}$ & 16 & 10.586 & 2.752 & 3.305 & 1.00 & 0.00 & $\mathrm{H}$ \\
\hline HETATM & 147 & $\mathrm{H} 12$ & $\mathrm{MET}$ & 16 & 10.252 & 1.463 & 4.460 & 1.00 & 0.00 & $\mathrm{H}$ \\
\hline HETATM & 148 & H13 & $\mathrm{MET}$ & 16 & 10.742 & 3.069 & 5.042 & 1.00 & 0.00 & $\mathrm{H}$ \\
\hline \multicolumn{11}{|c|}{ TER } \\
\hline HETATM & 149 & $\mathrm{FE}$ & HEM & 17 & 2.346 & 25.104 & 1.491 & 1.00 & 0.00 & $\mathrm{Fe}$ \\
\hline HETATM & 150 & $\mathrm{C} 1$ & HEM & 17 & 0.118 & 26.976 & 0.429 & 1.00 & 0.00 & C \\
\hline HETATM & 151 & $\mathrm{C} 2$ & HEM & 17 & -0.240 & 27.767 & -0.742 & 1.00 & 0.00 & C \\
\hline HETATM & 152 & $\mathrm{C} 3$ & HEM & 17 & 0.905 & 27.864 & -1.517 & 1.00 & 0.00 & C \\
\hline HETATM & 153 & $\mathrm{C} 4$ & HEM & 17 & 1.935 & 27.146 & -0.809 & 1.00 & 0.00 & C \\
\hline HETATM & 154 & C5 & HEM & 17 & 3.250 & 27.027 & -1.247 & 1.00 & 0.00 & C \\
\hline HETATM & 155 & H5 & HEM & 17 & 3.503 & 27.524 & -2.182 & 1.00 & 0.00 & $\mathrm{H}$ \\
\hline HETATM & 156 & $\mathrm{C} 6$ & HEM & 17 & 4.269 & 26.329 & -0.611 & 1.00 & 0.00 & $\mathrm{C}$ \\
\hline HETATM & 157 & C7 & HEM & 17 & 5.638 & 26.256 & -1.081 & 1.00 & 0.00 & $\mathrm{C}$ \\
\hline HETATM & 158 & $\mathrm{C} 8$ & HEM & 17 & 6.343 & 25.537 & -0.134 & 1.00 & 0.00 & C \\
\hline HETATM & 159 & $\mathrm{C} 9$ & HEM & 17 & 5.392 & 25.172 & 0.891 & 1.00 & 0.00 & C \\
\hline HETATM & 160 & $\mathrm{C} 10$ & HEM & 17 & 5.697 & 24.437 & 2.034 & 1.00 & 0.00 & C \\
\hline HETATM & 161 & $\mathrm{H} 10$ & HEM & 17 & 6.724 & 24.093 & 2.140 & 1.00 & 0.00 & $\mathrm{H}$ \\
\hline HETATM & 162 & $\mathrm{C} 11$ & HEM & 17 & 4.822 & 24.097 & 3.057 & 1.00 & 0.00 & C \\
\hline HETATM & 163 & $\mathrm{C} 12$ & HEM & 17 & 5.203 & 23.371 & 4.247 & 1.00 & 0.00 & C \\
\hline HETATM & 164 & $\mathrm{C} 13$ & HEM & 17 & 4.064 & 23.224 & 5.004 & 1.00 & 0.00 & C \\
\hline HETATM & 165 & C14 & HEM & 17 & 2.991 & 23.866 & 4.273 & 1.00 & 0.00 & C \\
\hline HETATM & 166 & C15 & HEM & 17 & 1.663 & 23.920 & 4.694 & 1.00 & 0.00 & C \\
\hline HETATM & 167 & H15 & HEM & 17 & 1.423 & 23.436 & 5.642 & 1.00 & 0.00 & $\mathrm{H}$ \\
\hline HETATM & 168 & $\mathrm{C} 16$ & HEM & 17 & 0.633 & 24.602 & 4.051 & 1.00 & 0.00 & C \\
\hline HETATM & 169 & $\mathrm{C} 17$ & HEM & 17 & -0.733 & 24.716 & 4.533 & 1.00 & 0.00 & C \\
\hline HETATM & 170 & $\mathrm{C} 18$ & HEM & 17 & -1.408 & 25.483 & 3.609 & 1.00 & 0.00 & C \\
\hline HETATM & 171 & C19 & HEM & 17 & -0.459 & 25.835 & 2.573 & 1.00 & 0.00 & C \\
\hline HETATM & 172 & $\mathrm{C} 20$ & HEM & 17 & -0.750 & 26.626 & 1.462 & 1.00 & 0.00 & C \\
\hline HETATM & 173 & $\mathrm{H} 2 \mathrm{O}$ & HEM & 17 & -1.764 & 27.015 & 1.402 & 1.00 & 0.00 & $\mathrm{H}$ \\
\hline HETATM & 174 & $\mathrm{~N} 21$ & HEM & 17 & 1.440 & 26.613 & 0.367 & 1.00 & 0.00 & $\mathrm{~N}$ \\
\hline HETATM & 175 & $\mathrm{~N} 22$ & HEM & 17 & 4.145 & 25.666 & 0.590 & 1.00 & 0.00 & $\mathrm{~N}$ \\
\hline HETATM & 176 & $\mathrm{~N} 23$ & HEM & 17 & 3.474 & 24.403 & 3.090 & 1.00 & 0.00 & $\mathrm{~N}$ \\
\hline HETATM & 177 & $\mathrm{~N} 24$ & HEM & 17 & 0.774 & 25.287 & 2.854 & 1.00 & 0.00 & $\mathrm{~N}$ \\
\hline HETATM & 178 & C99 & HEM & 17 & 1.681 & 22.422 & 0.352 & 1.00 & 0.00 & C \\
\hline HETATM & 179 & 091 & HEM & 17 & 1.841 & 23.736 & 0.365 & 1.00 & 0.00 & 0 \\
\hline HETATM & 180 & 092 & HEM & 17 & 1.526 & 21.805 & -0.703 & 1.00 & 0.00 & o \\
\hline HETATM & 181 & СТ91 & HEM & 17 & 1.697 & 21.741 & 1.709 & 1.00 & 0.00 & C \\
\hline HETATM & 182 & H911 & HEM & 17 & 2.676 & 21.936 & 2.171 & 1.00 & 0.00 & $\mathrm{H}$ \\
\hline HETATM & 183 & $\mathrm{H} 912$ & HEM & 17 & 0.949 & 22.231 & 2.352 & 1.00 & 0.00 & $\mathrm{H}$ \\
\hline HETATM & 184 & СТ 92 & HEM & 17 & 1.437 & 20.236 & 1.597 & 1.00 & 0.00 & C \\
\hline HETATM & 185 & H921 & HEM & 17 & 2.234 & 19.776 & 1.000 & 1.00 & 0.00 & $\mathrm{H}$ \\
\hline HETATM & 186 & H922 & HEM & 17 & 0.513 & 20.081 & 1.027 & 1.00 & 0.00 & $\mathrm{H}$ \\
\hline TER & & & & & & & & & & \\
\hline HETATM & 187 & $\mathrm{FE}$ & HEM & 18 & 3.039 & 17.690 & 6.440 & 1.00 & 0.00 & $\mathrm{Fe}$ \\
\hline HETATM & 188 & $\mathrm{C} 1$ & HEM & 18 & 5.266 & 15.818 & 7.502 & 1.00 & 0.00 & C \\
\hline HETATM & 189 & $\mathrm{C} 2$ & HEM & 18 & 5.625 & 15.027 & 8.674 & 1.00 & 0.00 & C \\
\hline HETATM & 190 & $\mathrm{C} 3$ & HEM & 18 & 4.480 & 14.930 & 9.448 & 1.00 & 0.00 & C \\
\hline HETATM & 191 & $\mathrm{C} 4$ & HEM & 18 & 3.450 & 15.647 & 8.740 & 1.00 & 0.00 & $\mathrm{C}$ \\
\hline HETATM & 192 & $\mathrm{C} 5$ & HEM & 18 & 2.135 & 15.766 & 9.178 & 1.00 & 0.00 & C \\
\hline HETATM & 193 & H5 & HEM & 18 & 1.882 & 15.270 & 10.113 & 1.00 & 0.00 & $\mathrm{H}$ \\
\hline HETATM & 194 & $\mathrm{C} 6$ & HEM & 18 & 1.116 & 16.465 & 8.542 & 1.00 & 0.00 & C \\
\hline HETATM & 195 & $\mathrm{C} 7$ & HEM & 18 & -0.253 & 16.537 & 9.012 & 1.00 & 0.00 & C \\
\hline HETATM & 196 & $\mathrm{C} 8$ & HEM & 18 & -0.958 & 17.257 & 8.065 & 1.00 & 0.00 & C \\
\hline HETATM & 197 & $\mathrm{C} 9$ & HEM & 18 & -0.007 & 17.622 & 7.041 & 1.00 & 0.00 & C \\
\hline HETATM & 198 & $\mathrm{C} 10$ & HEM & 18 & -0.312 & 18.357 & 5.897 & 1.00 & 0.00 & C \\
\hline HETATM & 199 & $\mathrm{H} 10$ & HEM & 18 & -1.339 & 18.701 & 5.791 & 1.00 & 0.00 & $\mathrm{H}$ \\
\hline HETATM & 200 & $\mathrm{C} 11$ & HEM & 18 & 0.562 & 18.697 & 4.874 & 1.00 & 0.00 & C \\
\hline HETATM & 201 & $\mathrm{C} 12$ & HEM & 18 & 0.182 & 19.422 & 3.684 & 1.00 & 0.00 & C \\
\hline HETATM & 202 & C13 & HEM & 18 & 1.321 & 19.569 & 2.927 & 1.00 & 0.00 & C \\
\hline
\end{tabular}




\begin{tabular}{|c|c|c|c|}
\hline HETATM & 203 & C14 & HEM \\
\hline HETATM & 204 & C15 & HEM \\
\hline HETATM & 205 & H15 & HEM \\
\hline HETATM & 206 & C16 & HEM \\
\hline HETATM & 207 & C17 & HEM \\
\hline HETATM & 208 & C18 & HEM \\
\hline HETATM & 209 & C19 & HEM \\
\hline HETATM & 210 & C20 & HEM \\
\hline HETATM & 211 & H2O & HEM \\
\hline HETATM & 212 & N21 & HEM \\
\hline HETATM & 213 & N22 & HEM \\
\hline HETATM & 214 & N23 & HEM \\
\hline HETATM & 215 & N2 4 & HEM \\
\hline HETATM & 216 & C99 & HEM \\
\hline HETATM & 217 & 091 & HEM \\
\hline HETATM & 218 & 092 & HEM \\
\hline HETATM & 219 & CT91 & HEM \\
\hline HETATM & 220 & H911 & HEM \\
\hline HETATM & 221 & H912 & HEM \\
\hline HETATM & 222 & СT92 & HEM \\
\hline HETATM & 223 & H921 & HEM \\
\hline HETATM & 224 & H922 & HEM \\
\hline \multicolumn{4}{|l|}{ TER } \\
\hline HETATM & 225 & $\mathrm{C} 2$ & VIN \\
\hline HETATM & 226 & H21 & VIN \\
\hline HETATM & 227 & C1 & VIN \\
\hline HETATM & 228 & H11 & VIN \\
\hline HETATM & 229 & H12 & VIN \\
\hline \multicolumn{4}{|l|}{ TER } \\
\hline HETATM & 230 & C1 & MET \\
\hline HETATM & 231 & H11 & MET \\
\hline HETATM & 232 & H12 & MET \\
\hline HETATM & 233 & H13 & $\mathrm{MET}$ \\
\hline \multicolumn{4}{|l|}{ TER } \\
\hline HETATM & 234 & $\mathrm{C} 2$ & VIN \\
\hline HETATM & 235 & H 21 & VIN \\
\hline HETATM & 236 & $\mathrm{C} 1$ & VIN \\
\hline HETATM & 237 & H11 & VIN \\
\hline HETATM & 238 & H12 & VIN \\
\hline \multicolumn{4}{|l|}{ TER } \\
\hline HETATM & 239 & $\mathrm{C} 1$ & MET \\
\hline HETATM & 240 & H11 & MET \\
\hline HETATM & 241 & H12 & MET \\
\hline HETATM & 242 & H13 & $\mathrm{MET}$ \\
\hline \multicolumn{4}{|l|}{ TER } \\
\hline HETATM & 243 & C5 & $\mathrm{ACP}$ \\
\hline HETATM & 244 & H51 & $\mathrm{ACP}$ \\
\hline HETATM & 245 & H52 & $\mathrm{ACP}$ \\
\hline HETATM & 246 & C4 & $\mathrm{ACP}$ \\
\hline HETATM & 247 & H4 1 & $\mathrm{ACP}$ \\
\hline HETATM & 248 & $\mathrm{H} 42$ & $\mathrm{ACP}$ \\
\hline HETATM & 249 & $\mathrm{C} 1$ & $\mathrm{ACP}$ \\
\hline HETATM & 250 & $\mathrm{O} 2$ & $\mathrm{ACP}$ \\
\hline HETATM & 251 & 03 & $\mathrm{ACP}$ \\
\hline HETATM & 252 & H3 & $\mathrm{ACP}$ \\
\hline \multicolumn{4}{|l|}{ TER } \\
\hline HETATM & 253 & C1 & $\mathrm{MET}$ \\
\hline HETATM & 254 & H11 & MET \\
\hline HETATM & 255 & H12 & $\mathrm{MET}$ \\
\hline HETATM & 256 & $\mathrm{H} 13$ & MET \\
\hline \multicolumn{4}{|l|}{ TER } \\
\hline HETATM & 257 & C1 & MET \\
\hline HETATM & 258 & H11 & MET \\
\hline HETATM & 259 & $\mathrm{H} 12$ & MET \\
\hline HETATM & 260 & H13 & MET \\
\hline \multicolumn{4}{|l|}{ TER } \\
\hline HETATM & 261 & $\mathrm{C} 2$ & VIN \\
\hline HETATM & 262 & H21 & VIN \\
\hline HETATM & 263 & $\mathrm{C} 1$ & VIN \\
\hline HETATM & 264 & H11 & VIN \\
\hline HETATM & 265 & $\mathrm{H} 12$ & VIN \\
\hline TER & & & \\
\hline HETATM & 266 & $\mathrm{C} 1$ & MET \\
\hline HETATM & 267 & H11 & MET \\
\hline HETATM & 268 & $\mathrm{H} 12$ & $\mathrm{MET}$ \\
\hline HETATM & 269 & H13 & MET \\
\hline TER & & & \\
\hline HETATM & 270 & $\mathrm{C} 2$ & VIN \\
\hline HETATM & 271 & H21 & VIN \\
\hline
\end{tabular}

\begin{tabular}{|c|c|c|c|c|c|}
\hline 2.394 & 18.928 & 3.659 & 1.00 & 0.00 & C \\
\hline 3.722 & 18.873 & 3.237 & 1.00 & 0.00 & C \\
\hline 3.962 & 19.358 & 2.289 & 1.00 & 0.00 & $\mathrm{H}$ \\
\hline 4.752 & 18.192 & 3.880 & 1.00 & 0.00 & C \\
\hline 6.118 & 18.078 & 3.398 & 1.00 & 0.00 & C \\
\hline 6.793 & 17.310 & 4.322 & 1.00 & 0.00 & C \\
\hline 5.844 & 16.959 & 5.358 & 1.00 & 0.00 & C \\
\hline 6.135 & 16.168 & 6.469 & 1.00 & 0.00 & C \\
\hline 7.149 & 15.778 & 6.530 & 1.00 & 0.00 & $\mathrm{H}$ \\
\hline 3.945 & 16.181 & 7.564 & 1.00 & 0.00 & $\mathrm{~N}$ \\
\hline 1.240 & 17.128 & 7.342 & 1.00 & 0.00 & $\mathrm{~N}$ \\
\hline 1.911 & 18.391 & 4.841 & 1.00 & 0.00 & $\mathrm{~N}$ \\
\hline 4.611 & 17.506 & 5.077 & 1.00 & 0.00 & $\mathrm{~N}$ \\
\hline 3.704 & 20.371 & 7.579 & 1.00 & 0.00 & $\mathrm{C}$ \\
\hline 3.544 & 19.058 & 7.567 & 1.00 & 0.00 & 0 \\
\hline 3.859 & 20.989 & 8.635 & 1.00 & 0.00 & 0 \\
\hline 3.688 & 21.053 & 6.223 & 1.00 & 0.00 & C \\
\hline 2.709 & 20.858 & 5.760 & 1.00 & 0.00 & $\mathrm{H}$ \\
\hline 4.436 & 20.563 & 5.580 & 1.00 & 0.00 & $\mathrm{H}$ \\
\hline 3.947 & 22.557 & 6.334 & 1.00 & 0.00 & C \\
\hline 4.872 & 22.713 & 6.905 & 1.00 & 0.00 & $\mathrm{H}$ \\
\hline 3.151 & 23.018 & 6.931 & 1.00 & 0.00 & $\mathrm{H}$ \\
\hline-1.572 & 28.269 & -1.002 & 1.00 & 0.00 & C \\
\hline-2.388 & 27.571 & -0.837 & 1.00 & 0.00 & $\mathrm{H}$ \\
\hline-1.905 & 29.494 & -1.403 & 1.00 & 0.00 & C \\
\hline-1.174 & 30.273 & -1.511 & 1.00 & 0.00 & $\mathrm{H}$ \\
\hline-2.947 & 29.763 & -1.570 & 1.00 & 0.00 & $\mathrm{H}$ \\
\hline 1.091 & 28.524 & -2.840 & 1.00 & 0.00 & C \\
\hline 1.326 & 29.595 & -2.720 & 1.00 & 0.00 & $\mathrm{H}$ \\
\hline 1.904 & 28.060 & -3.410 & 1.00 & 0.00 & $\mathrm{H}$ \\
\hline 0.184 & 28.446 & -3.450 & 1.00 & 0.00 & $\mathrm{H}$ \\
\hline 6.102 & 26.846 & -2.325 & 1.00 & 0.00 & C \\
\hline 5.436 & 26.745 & -3.184 & 1.00 & 0.00 & $\mathrm{H}$ \\
\hline 7.268 & 27.490 & -2.484 & 1.00 & 0.00 & C \\
\hline 7.948 & 27.647 & -1.654 & 1.00 & 0.00 & $\mathrm{H}$ \\
\hline 7.573 & 27.888 & -3.450 & 1.00 & 0.00 & $\mathrm{H}$ \\
\hline 7.786 & 25.163 & -0.121 & 1.00 & 0.00 & C \\
\hline 8.377 & 25.827 & 0.528 & 1.00 & 0.00 & $\mathrm{H}$ \\
\hline 7.943 & 24.141 & 0.246 & 1.00 & 0.00 & $\mathrm{H}$ \\
\hline 8.218 & 25.228 & -1.126 & 1.00 & 0.00 & $\mathrm{H}$ \\
\hline-1.302 & 24.081 & 5.770 & 1.00 & 0.00 & C \\
\hline-0.563 & 24.070 & 6.582 & 1.00 & 0.00 & $\mathrm{H}$ \\
\hline-2.143 & 24.689 & 6.134 & 1.00 & 0.00 & $\mathrm{H}$ \\
\hline-1.781 & 22.644 & 5.497 & 1.00 & 0.00 & C \\
\hline-2.338 & 22.583 & 4.549 & 1.00 & 0.00 & $\mathrm{H}$ \\
\hline-0.910 & 21.977 & 5.390 & 1.00 & 0.00 & $\mathrm{H}$ \\
\hline-2.645 & 22.031 & 6.573 & 1.00 & 0.00 & $\mathrm{C}$ \\
\hline-2.873 & 22.554 & 7.671 & 1.00 & 0.00 & 0 \\
\hline-3.161 & 20.852 & 6.209 & 1.00 & 0.00 & o \\
\hline-3.770 & 20.498 & 6.945 & 1.00 & 0.00 & $\mathrm{H}$ \\
\hline 6.594 & 22.955 & 4.597 & 1.00 & 0.00 & C \\
\hline 7.146 & 23.794 & 5.047 & 1.00 & 0.00 & $\mathrm{H}$ \\
\hline 6.600 & 22.129 & 5.319 & 1.00 & 0.00 & $\mathrm{H}$ \\
\hline 7.165 & 22.635 & 3.717 & 1.00 & 0.00 & $\mathrm{H}$ \\
\hline-2.840 & 25.904 & 3.646 & 1.00 & 0.00 & C \\
\hline-3.285 & 25.695 & 4.626 & 1.00 & 0.00 & $\mathrm{H}$ \\
\hline-2.950 & 26.984 & 3.471 & 1.00 & 0.00 & $\mathrm{H}$ \\
\hline-3.440 & 25.378 & 2.889 & 1.00 & 0.00 & $\mathrm{H}$ \\
\hline 6.957 & 14.525 & 8.933 & 1.00 & 0.00 & C \\
\hline 7.773 & 15.223 & 8.768 & 1.00 & 0.00 & $\mathrm{H}$ \\
\hline 7.290 & 13.300 & 9.335 & 1.00 & 0.00 & C \\
\hline 6.559 & 12.521 & 9.442 & 1.00 & 0.00 & $\mathrm{H}$ \\
\hline 8.332 & 13.030 & 9.501 & 1.00 & 0.00 & $\mathrm{H}$ \\
\hline 4.293 & 14.270 & 10.771 & 1.00 & 0.00 & C \\
\hline 4.059 & 13.199 & 10.652 & 1.00 & 0.00 & $\mathrm{H}$ \\
\hline 3.480 & 14.734 & 11.341 & 1.00 & 0.00 & $\mathrm{H}$ \\
\hline 5.201 & 14.347 & 11.382 & 1.00 & 0.00 & $\mathrm{H}$ \\
\hline-0.717 & 15.948 & 10.256 & 1.00 & 0.00 & C \\
\hline-0.051 & 16.048 & 11.115 & 1.00 & 0.00 & $\mathrm{H}$ \\
\hline
\end{tabular}




\begin{tabular}{|c|c|c|c|}
\hline HETATM & 272 & $\mathrm{C} 1$ & VIN \\
\hline HETATM & 273 & H11 & VIN \\
\hline HETATM & 274 & $\mathrm{H} 12$ & VIN \\
\hline \multicolumn{4}{|l|}{ TER } \\
\hline HETATM & 275 & C1 & MET \\
\hline HETATM & 276 & H11 & MET \\
\hline HETATM & 277 & $\mathrm{H} 12$ & MET \\
\hline HETATM & 278 & $\mathrm{H} 13$ & MET \\
\hline \multicolumn{4}{|l|}{ TER } \\
\hline HETATM & 279 & C5 & $\mathrm{ACP}$ \\
\hline HETATM & 280 & H51 & $\mathrm{ACP}$ \\
\hline HETATM & 281 & H52 & $\mathrm{ACP}$ \\
\hline HETATM & 282 & C4 & $\mathrm{ACP}$ \\
\hline HETATM & 283 & H4 1 & $\mathrm{ACP}$ \\
\hline HETATM & 284 & $\mathrm{H} 42$ & $\mathrm{ACP}$ \\
\hline HETATM & 285 & $\mathrm{C} 1$ & $\mathrm{ACP}$ \\
\hline HETATM & 286 & $\mathrm{O} 2$ & $\mathrm{ACP}$ \\
\hline HETATM & 287 & 03 & $\mathrm{ACP}$ \\
\hline HETATM & 288 & H3 & $\mathrm{ACP}$ \\
\hline \multicolumn{4}{|l|}{ TER } \\
\hline HETATM & 289 & $\mathrm{C} 1$ & MET \\
\hline HETATM & 290 & H11 & MET \\
\hline HETATM & 291 & $\mathrm{H} 12$ & MET \\
\hline HETATM & 292 & $\mathrm{H} 13$ & MET \\
\hline \multicolumn{4}{|l|}{ TER } \\
\hline HETATM & 293 & $\mathrm{C} 1$ & MET \\
\hline HETATM & 294 & $\mathrm{H} 11$ & MET \\
\hline HETATM & 295 & $\mathrm{H} 12$ & MET \\
\hline HETATM & 296 & $\mathrm{H} 13$ & MET \\
\hline \multicolumn{4}{|l|}{ TER } \\
\hline HETATM & 297 & $\mathrm{FE}$ & HEM \\
\hline HETATM & 298 & $\mathrm{C} 1$ & HEM \\
\hline HETATM & 299 & C2 & HEM \\
\hline HETATM & 300 & C3 & HEM \\
\hline HETATM & 301 & $\mathrm{C} 4$ & HEM \\
\hline HETATM & 302 & C5 & HEM \\
\hline HETATM & 303 & H5 & HEM \\
\hline HETATM & 304 & $\mathrm{C} 6$ & HEM \\
\hline HETATM & 305 & $\mathrm{C} 7$ & HEM \\
\hline HETATM & 306 & $\mathrm{C} 8$ & HEM \\
\hline HETATM & 307 & C9 & HEM \\
\hline HETATM & 308 & C10 & HEM \\
\hline HETATM & 309 & $\mathrm{H} 10$ & HEM \\
\hline HETATM & 310 & C11 & HEM \\
\hline HETATM & 311 & $\mathrm{C} 12$ & HEM \\
\hline HETATM & 312 & C13 & HEM \\
\hline HETATM & 313 & $\mathrm{C} 14$ & HEM \\
\hline HETATM & 314 & C15 & HEM \\
\hline HETATM & 315 & H15 & HEM \\
\hline HETATM & 316 & C16 & HEM \\
\hline HETATM & 317 & $\mathrm{C} 17$ & HEM \\
\hline HETATM & 318 & C18 & HEM \\
\hline HETATM & 319 & C19 & HEM \\
\hline HETATM & 320 & $\mathrm{C} 20$ & HEM \\
\hline HETATM & 321 & $\mathrm{H} 2 \mathrm{O}$ & HEM \\
\hline HETATM & 322 & $\mathrm{~N} 21$ & HEM \\
\hline HETATM & 323 & N22 & HEM \\
\hline HETATM & 324 & N23 & HEM \\
\hline HETATM & 325 & $\mathrm{~N} 24$ & HEM \\
\hline HETATM & 326 & C99 & HEM \\
\hline HETATM & 327 & 091 & HEM \\
\hline HETATM & 328 & 092 & HEM \\
\hline HETATM & 329 & СТ91 & HEM \\
\hline HETATM & 330 & H911 & HEM \\
\hline HETATM & 331 & H912 & HEM \\
\hline HETATM & 332 & CT92 & HEM \\
\hline HETATM & 333 & H921 & HEM \\
\hline HETATM & 334 & H922 & HEM \\
\hline \multicolumn{4}{|l|}{ TER } \\
\hline HETATM & 335 & $\mathrm{FE}$ & HEM \\
\hline HETATM & 336 & $\mathrm{C} 1$ & HEM \\
\hline HETATM & 337 & $\mathrm{C} 2$ & HEM \\
\hline HETATM & 338 & C3 & HEM \\
\hline HETATM & 339 & C4 & HEM \\
\hline HETATM & 340 & C5 & HEM \\
\hline HETATM & 341 & H5 & HEM \\
\hline HETATM & 342 & $\mathrm{C} 6$ & HEM \\
\hline HETATM & 343 & C7 & HEM \\
\hline HETATM & 344 & $\mathrm{C} 8$ & HEM \\
\hline
\end{tabular}

\begin{tabular}{|c|c|c|c|c|c|}
\hline-1.883 & 15.303 & 10.415 & 1.00 & 0.00 & C \\
\hline-2.563 & 15.147 & 9.585 & 1.00 & 0.00 & $\mathrm{H}$ \\
\hline-2.188 & 14.905 & 11.381 & 1.00 & 0.00 & $\mathrm{H}$ \\
\hline-2.401 & 17.631 & 8.052 & 1.00 & 0.00 & C \\
\hline-2.558 & 18.653 & 7.685 & 1.00 & 0.00 & $\mathrm{H}$ \\
\hline-2.834 & 17.565 & 9.057 & 1.00 & 0.00 & $\mathrm{H}$ \\
\hline-2.993 & 16.967 & 7.403 & 1.00 & 0.00 & $\mathrm{H}$ \\
\hline 6.687 & 18.712 & 2.161 & 1.00 & 0.00 & C \\
\hline 5.948 & 18.724 & 1.349 & 1.00 & 0.00 & $\mathrm{H}$ \\
\hline 7.528 & 18.104 & 1.798 & 1.00 & 0.00 & $\mathrm{H}$ \\
\hline 7.166 & 20.150 & 2.434 & 1.00 & 0.00 & C \\
\hline 7.723 & 20.211 & 3.382 & 1.00 & 0.00 & $\mathrm{H}$ \\
\hline 6.295 & 20.817 & 2.541 & 1.00 & 0.00 & $\mathrm{H}$ \\
\hline 8.030 & 20.762 & 1.358 & 1.00 & 0.00 & C \\
\hline 8.258 & 20.239 & 0.260 & 1.00 & 0.00 & O \\
\hline 8.546 & 21.942 & 1.722 & 1.00 & 0.00 & O \\
\hline 9.155 & 22.296 & 0.986 & 1.00 & 0.00 & $\mathrm{H}$ \\
\hline-1.209 & 19.839 & 3.334 & 1.00 & 0.00 & C \\
\hline-1.761 & 18.999 & 2.884 & 1.00 & 0.00 & $\mathrm{H}$ \\
\hline-1.215 & 20.665 & 2.613 & 1.00 & 0.00 & $\mathrm{H}$ \\
\hline-1.780 & 20.158 & 4.214 & 1.00 & 0.00 & $\mathrm{H}$ \\
\hline 8.225 & 16.890 & 4.285 & 1.00 & 0.00 & C \\
\hline 8.670 & 17.099 & 3.305 & 1.00 & 0.00 & $\mathrm{H}$ \\
\hline 8.335 & 15.810 & 4.460 & 1.00 & 0.00 & $\mathrm{H}$ \\
\hline 8.825 & 17.416 & 5.042 & 1.00 & 0.00 & $\mathrm{H}$ \\
\hline 6.180 & -3.589 & 1.491 & 1.00 & 0.00 & $\mathrm{Fe}$ \\
\hline 3.952 & -1.717 & 0.429 & 1.00 & 0.00 & C \\
\hline 3.593 & -0.927 & -0.742 & 1.00 & 0.00 & C \\
\hline 4.739 & -0.830 & -1.517 & 1.00 & 0.00 & C \\
\hline 5.768 & -1.547 & -0.809 & 1.00 & 0.00 & C \\
\hline 7.083 & -1.666 & -1.247 & 1.00 & 0.00 & C \\
\hline 7.337 & -1.170 & -2.182 & 1.00 & 0.00 & $\mathrm{H}$ \\
\hline 8.103 & -2.364 & -0.611 & 1.00 & 0.00 & C \\
\hline 9.472 & -2.437 & -1.081 & 1.00 & 0.00 & C \\
\hline 10.177 & -3.157 & -0.134 & 1.00 & 0.00 & C \\
\hline 9.226 & -3.522 & 0.891 & 1.00 & 0.00 & C \\
\hline 9.531 & -4.256 & 2.034 & 1.00 & 0.00 & C \\
\hline 10.557 & -4.600 & 2.140 & 1.00 & 0.00 & $\mathrm{H}$ \\
\hline 8.656 & -4.596 & 3.057 & 1.00 & 0.00 & C \\
\hline 9.037 & -5.322 & 4.247 & 1.00 & 0.00 & C \\
\hline 7.898 & -5.469 & 5.004 & 1.00 & 0.00 & C \\
\hline 6.825 & -4.828 & 4.273 & 1.00 & 0.00 & C \\
\hline 5.497 & -4.773 & 4.694 & 1.00 & 0.00 & C \\
\hline 5.256 & -5.258 & 5.642 & 1.00 & 0.00 & $\mathrm{H}$ \\
\hline 4.466 & -4.091 & 4.051 & 1.00 & 0.00 & C \\
\hline 3.100 & -3.978 & 4.533 & 1.00 & 0.00 & C \\
\hline 2.425 & -3.210 & 3.609 & 1.00 & 0.00 & C \\
\hline 3.375 & -2.858 & 2.573 & 1.00 & 0.00 & C \\
\hline 3.083 & -2.067 & 1.462 & 1.00 & 0.00 & C \\
\hline 2.069 & -1.678 & 1.402 & 1.00 & 0.00 & $\mathrm{H}$ \\
\hline 5.274 & -2.080 & 0.367 & 1.00 & 0.00 & $\mathrm{~N}$ \\
\hline 7.978 & -3.027 & 0.590 & 1.00 & 0.00 & $\mathrm{~N}$ \\
\hline 7.308 & -4.290 & 3.090 & 1.00 & 0.00 & $\mathrm{~N}$ \\
\hline 4.608 & -3.406 & 2.854 & 1.00 & 0.00 & $\mathrm{~N}$ \\
\hline 5.515 & -6.271 & 0.352 & 1.00 & 0.00 & C \\
\hline 5.674 & -4.958 & 0.365 & 1.00 & 0.00 & o \\
\hline 5.360 & -6.888 & -0.703 & 1.00 & 0.00 & o \\
\hline 5.531 & -6.952 & 1.709 & 1.00 & 0.00 & C \\
\hline 6.509 & -6.757 & 2.171 & 1.00 & 0.00 & $\mathrm{H}$ \\
\hline 4.783 & -6.463 & 2.352 & 1.00 & 0.00 & $\mathrm{H}$ \\
\hline 5.271 & -8.457 & 1.597 & 1.00 & 0.00 & C \\
\hline 6.067 & -8.917 & 1.000 & 1.00 & 0.00 & $\mathrm{H}$ \\
\hline 4.346 & -8.613 & 1.027 & 1.00 & 0.00 & $\mathrm{H}$ \\
\hline 6.872 & -11.003 & 6.440 & 1.00 & 0.00 & $\mathrm{Fe}$ \\
\hline 9.100 & -12.876 & 7.502 & 1.00 & 0.00 & C \\
\hline 9.459 & -13.666 & 8.674 & 1.00 & 0.00 & C \\
\hline 8.313 & -13.763 & 9.448 & 1.00 & 0.00 & C \\
\hline 7.284 & -13.046 & 8.740 & 1.00 & 0.00 & C \\
\hline 5.969 & -12.927 & 9.178 & 1.00 & 0.00 & C \\
\hline 5.715 & -13.423 & 10.113 & 1.00 & 0.00 & $\mathrm{H}$ \\
\hline 4.949 & -12.229 & 8.542 & 1.00 & 0.00 & C \\
\hline 3.580 & -12.156 & 9.012 & 1.00 & 0.00 & C \\
\hline 2.875 & -11.436 & 8.065 & 1.00 & 0.00 & C \\
\hline
\end{tabular}




\begin{tabular}{|c|c|c|c|}
\hline HETATM & 345 & C9 & HEM \\
\hline HETATM & 346 & C10 & HEM \\
\hline HETATM & 347 & H10 & HEM \\
\hline HETATM & 348 & C11 & HEM \\
\hline HETATM & 349 & C12 & HEM \\
\hline HETATM & 350 & C13 & HEM \\
\hline HETATM & 351 & C14 & HEM \\
\hline HETATM & 352 & C15 & HEM \\
\hline HETATM & 353 & H15 & HEM \\
\hline HETATM & 354 & C16 & HEM \\
\hline HETATM & 355 & C17 & HEM \\
\hline HETATM & 356 & C18 & HEM \\
\hline HETATM & 357 & C19 & HEM \\
\hline HETATM & 358 & C20 & HEM \\
\hline HETATM & 359 & H2O & HEM \\
\hline HETATM & 360 & N21 & HEM \\
\hline HETATM & 361 & N22 & HEM \\
\hline HETATM & 362 & N23 & HEM \\
\hline HETATM & 363 & N24 & HEM \\
\hline HETATM & 364 & C99 & HEM \\
\hline HETATM & 365 & 091 & HEM \\
\hline HETATM & 366 & 092 & HEM \\
\hline HETATM & 367 & СТ91 & HEM \\
\hline HETATM & 368 & H911 & HEM \\
\hline HETATM & 369 & H912 & HEM \\
\hline HETATM & 370 & СТ92 & HEM \\
\hline HETATM & 371 & H921 & HEM \\
\hline HETATM & 372 & H922 & HEM \\
\hline \multicolumn{4}{|l|}{ TER } \\
\hline HETATM & 373 & C2 & VIN \\
\hline HETATM & 374 & H 21 & VIN \\
\hline HETATM & 375 & C1 & VIN \\
\hline HETATM & 376 & H11 & VIN \\
\hline HETATM & 377 & $\mathrm{H} 12$ & VIN \\
\hline \multicolumn{4}{|l|}{ TER } \\
\hline HETATM & 378 & $\mathrm{C} 1$ & MET \\
\hline HETATM & 379 & H11 & MET \\
\hline HETATM & 380 & $\mathrm{H} 12$ & MET \\
\hline HETATM & 381 & $\mathrm{H} 13$ & MET \\
\hline \multicolumn{4}{|l|}{ TER } \\
\hline HETATM & 382 & C2 & VIN \\
\hline HETATM & 383 & H 21 & VIN \\
\hline HETATM & 384 & C1 & VIN \\
\hline HETATM & 385 & H11 & VIN \\
\hline HETATM & 386 & $\mathrm{H} 12$ & VIN \\
\hline \multicolumn{4}{|l|}{ TER } \\
\hline HETATM & 387 & $\mathrm{C} 1$ & MET \\
\hline HETATM & 388 & H11 & MET \\
\hline HETATM & 389 & $\mathrm{H} 12$ & MET \\
\hline HETATM & 390 & H13 & MET \\
\hline \multicolumn{4}{|l|}{ TER } \\
\hline HETATM & 391 & C5 & $\mathrm{ACP}$ \\
\hline HETATM & 392 & H51 & $\mathrm{ACP}$ \\
\hline HETATM & 393 & H52 & $\mathrm{ACP}$ \\
\hline HETATM & 394 & C4 & $\mathrm{ACP}$ \\
\hline HETATM & 395 & H4 1 & $\mathrm{ACP}$ \\
\hline HETATM & 396 & H4 2 & $\mathrm{ACP}$ \\
\hline HETATM & 397 & $\mathrm{C} 1$ & $\mathrm{ACP}$ \\
\hline HETATM & 398 & 02 & $\mathrm{ACP}$ \\
\hline HETATM & 399 & 03 & $\mathrm{ACP}$ \\
\hline HETATM & 400 & H3 & $\mathrm{ACP}$ \\
\hline \multicolumn{4}{|l|}{ TER } \\
\hline HETATM & 401 & $\mathrm{C} 1$ & MET \\
\hline HETATM & 402 & H11 & MET \\
\hline HETATM & 403 & H12 & MET \\
\hline HETATM & 404 & H13 & MET \\
\hline \multicolumn{4}{|l|}{ TER } \\
\hline HETATM & 405 & $\mathrm{C} 1$ & MET \\
\hline HETATM & 406 & H11 & MET \\
\hline HETATM & 407 & H12 & MET \\
\hline HETATM & 408 & H13 & MET \\
\hline \multicolumn{4}{|l|}{ TER } \\
\hline HETATM & 409 & C2 & VIN \\
\hline HETATM & 410 & H 21 & VIN \\
\hline HETATM & 411 & $\mathrm{C} 1$ & VIN \\
\hline HETATM & 412 & H11 & VIN \\
\hline HETATM & 413 & H12 & VIN \\
\hline TER & & & \\
\hline HETATM & 414 & $C 1$ & ME \\
\hline
\end{tabular}

\begin{tabular}{|c|c|c|c|c|c|}
\hline 3.826 & -11.071 & 7.041 & 1.00 & 0.00 & C \\
\hline 3.522 & -10.336 & 5.897 & 1.00 & 0.00 & C \\
\hline 2.495 & -9.993 & 5.791 & 1.00 & 0.00 & $\mathrm{H}$ \\
\hline 4.396 & -9.996 & 4.874 & 1.00 & 0.00 & C \\
\hline 4.015 & -9.271 & 3.684 & 1.00 & 0.00 & C \\
\hline 5.155 & -9.124 & 2.927 & 1.00 & 0.00 & C \\
\hline 6.227 & -9.765 & 3.659 & 1.00 & 0.00 & C \\
\hline 7.556 & -9.820 & 3.237 & 1.00 & 0.00 & C \\
\hline 7.796 & -9.335 & 2.289 & 1.00 & 0.00 & $\mathrm{H}$ \\
\hline 8.586 & -10.502 & 3.880 & 1.00 & 0.00 & C \\
\hline 9.952 & -10.615 & 3.398 & 1.00 & 0.00 & C \\
\hline 10.627 & -11.383 & 4.322 & 1.00 & 0.00 & C \\
\hline 9.677 & -11.735 & 5.358 & 1.00 & 0.00 & C \\
\hline 9.969 & -12.526 & 6.469 & 1.00 & 0.00 & C \\
\hline 10.983 & -12.915 & 6.530 & 1.00 & 0.00 & $\mathrm{H}$ \\
\hline 7.778 & -12.513 & 7.564 & 1.00 & 0.00 & $\mathrm{~N}$ \\
\hline 5.074 & -11.566 & 7.342 & 1.00 & 0.00 & $\mathrm{~N}$ \\
\hline 5.745 & -10.303 & 4.841 & 1.00 & 0.00 & $\mathrm{~N}$ \\
\hline 8.444 & -11.187 & 5.077 & 1.00 & 0.00 & $\mathrm{~N}$ \\
\hline 7.538 & -8.322 & 7.579 & 1.00 & 0.00 & C \\
\hline 7.378 & -9.635 & 7.567 & 1.00 & 0.00 & 0 \\
\hline 7.692 & -7.705 & 8.635 & 1.00 & 0.00 & o \\
\hline 7.521 & -7.640 & 6.223 & 1.00 & 0.00 & C \\
\hline 6.543 & -7.836 & 5.760 & 1.00 & 0.00 & $\mathrm{H}$ \\
\hline 8.269 & -8.130 & 5.580 & 1.00 & 0.00 & $\mathrm{H}$ \\
\hline 7.781 & -6.136 & 6.334 & 1.00 & 0.00 & C \\
\hline 8.706 & -5.980 & 6.905 & 1.00 & 0.00 & $\mathrm{H}$ \\
\hline 6.985 & -5.676 & 6.931 & 1.00 & 0.00 & $\mathrm{H}$ \\
\hline 2.261 & -0.424 & -1.002 & 1.00 & 0.00 & C \\
\hline 1.446 & -1.123 & -0.837 & 1.00 & 0.00 & $\mathrm{H}$ \\
\hline 1.928 & 0.801 & -1.403 & 1.00 & 0.00 & C \\
\hline 2.660 & 1.579 & -1.511 & 1.00 & 0.00 & $\mathrm{H}$ \\
\hline 0.887 & 1.070 & -1.570 & 1.00 & 0.00 & $\mathrm{H}$ \\
\hline 4.925 & -0.169 & -2.840 & 1.00 & 0.00 & C \\
\hline 5.160 & 0.902 & -2.720 & 1.00 & 0.00 & $\mathrm{H}$ \\
\hline 5.738 & -0.633 & -3.410 & 1.00 & 0.00 & $\mathrm{H}$ \\
\hline 4.017 & -0.247 & -3.450 & 1.00 & 0.00 & $\mathrm{H}$ \\
\hline 9.935 & -1.847 & -2.325 & 1.00 & 0.00 & C \\
\hline 9.269 & -1.948 & -3.184 & 1.00 & 0.00 & $\mathrm{H}$ \\
\hline 11.102 & -1.203 & -2.484 & 1.00 & 0.00 & C \\
\hline 11.782 & -1.047 & -1.654 & 1.00 & 0.00 & $\mathrm{H}$ \\
\hline 11.407 & -0.805 & -3.450 & 1.00 & 0.00 & $\mathrm{H}$ \\
\hline 11.620 & -3.530 & -0.121 & 1.00 & 0.00 & C \\
\hline 12.211 & -2.866 & 0.528 & 1.00 & 0.00 & $\mathrm{H}$ \\
\hline 11.776 & -4.553 & 0.246 & 1.00 & 0.00 & $\mathrm{H}$ \\
\hline 12.052 & -3.465 & -1.126 & 1.00 & 0.00 & $\mathrm{H}$ \\
\hline 2.531 & -4.612 & 5.770 & 1.00 & 0.00 & C \\
\hline 3.270 & -4.623 & 6.582 & 1.00 & 0.00 & $\mathrm{H}$ \\
\hline 1.691 & -4.004 & 6.134 & 1.00 & 0.00 & $\mathrm{H}$ \\
\hline 2.053 & -6.050 & 5.497 & 1.00 & 0.00 & C \\
\hline 1.495 & -6.111 & 4.549 & 1.00 & 0.00 & $\mathrm{H}$ \\
\hline 2.923 & -6.716 & 5.390 & 1.00 & 0.00 & $\mathrm{H}$ \\
\hline 1.189 & -6.662 & 6.573 & 1.00 & 0.00 & C \\
\hline 0.961 & -6.139 & 7.671 & 1.00 & 0.00 & o \\
\hline 0.673 & -7.841 & 6.209 & 1.00 & 0.00 & o \\
\hline 0.064 & -8.196 & 6.945 & 1.00 & 0.00 & $\mathrm{H}$ \\
\hline 10.427 & -5.738 & 4.597 & 1.00 & 0.00 & C \\
\hline 10.980 & -4.899 & 5.047 & 1.00 & 0.00 & $\mathrm{H}$ \\
\hline 10.434 & -6.564 & 5.319 & 1.00 & 0.00 & $\mathrm{H}$ \\
\hline 10.998 & -6.058 & 3.717 & 1.00 & 0.00 & $\mathrm{H}$ \\
\hline 0.994 & -2.790 & 3.646 & 1.00 & 0.00 & C \\
\hline 0.549 & -2.998 & 4.626 & 1.00 & 0.00 & $\mathrm{H}$ \\
\hline 0.884 & -1.709 & 3.471 & 1.00 & 0.00 & $\mathrm{H}$ \\
\hline 0.393 & -3.315 & 2.889 & 1.00 & 0.00 & $\mathrm{H}$ \\
\hline 10.791 & -14.169 & 8.933 & 1.00 & 0.00 & c \\
\hline 11.607 & -13.470 & 8.768 & 1.00 & 0.00 & $\mathrm{H}$ \\
\hline 11.124 & -15.394 & 9.335 & 1.00 & 0.00 & c \\
\hline 10.392 & -16.172 & 9.442 & 1.00 & 0.00 & $\mathrm{H}$ \\
\hline 12.165 & -15.663 & 9.501 & 1.00 & 0.00 & $\mathrm{H}$ \\
\hline 8. & -14.424 & 10.771 & 1.00 & 0.00 & , \\
\hline
\end{tabular}




\begin{tabular}{|c|c|c|c|}
\hline HETATM & 415 & H11 & MET \\
\hline HETATM & 416 & $\mathrm{H} 12$ & MET \\
\hline HETATM & 417 & $\mathrm{H} 13$ & MET \\
\hline \multicolumn{4}{|l|}{ TER } \\
\hline HETATM & 418 & $\mathrm{C} 2$ & VIN \\
\hline HETATM & 419 & $\mathrm{H} 21$ & VIN \\
\hline HETATM & 420 & $\mathrm{C} 1$ & VIN \\
\hline HETATM & 421 & $\mathrm{H} 11$ & VIN \\
\hline HETATM & 422 & $\mathrm{H} 12$ & VIN \\
\hline \multicolumn{4}{|l|}{ TER } \\
\hline HETATM & 423 & $\mathrm{C} 1$ & MET \\
\hline HETATM & 424 & $\mathrm{H} 11$ & MET \\
\hline HETATM & 425 & $\mathrm{H} 12$ & MET \\
\hline HETATM & 426 & $\mathrm{H} 13$ & $\mathrm{MET}$ \\
\hline \multicolumn{4}{|l|}{ TER } \\
\hline HETATM & 427 & C5 & $\mathrm{ACP}$ \\
\hline HETATM & 428 & H51 & $\mathrm{ACP}$ \\
\hline HETATM & 429 & H52 & $\mathrm{ACP}$ \\
\hline HETATM & 430 & $\mathrm{C} 4$ & $\mathrm{ACP}$ \\
\hline HETATM & 431 & $\mathrm{H} 41$ & $\mathrm{ACP}$ \\
\hline HETATM & 432 & $\mathrm{H} 42$ & $\mathrm{ACP}$ \\
\hline HETATM & 433 & $\mathrm{C} 1$ & $\mathrm{ACP}$ \\
\hline HETATM & 434 & 02 & $\mathrm{ACP}$ \\
\hline HETATM & 435 & 03 & $\mathrm{ACP}$ \\
\hline HETATM & 436 & H3 & $\mathrm{ACP}$ \\
\hline \multicolumn{4}{|l|}{ TER } \\
\hline HETATM & 437 & $\mathrm{C} 1$ & $\mathrm{MET}$ \\
\hline HETATM & 438 & H11 & $\mathrm{MET}$ \\
\hline HETATM & 439 & $\mathrm{H} 12$ & MET \\
\hline HETATM & 440 & H13 & MET \\
\hline \multicolumn{4}{|l|}{ TER } \\
\hline HETATM & 441 & $\mathrm{C} 1$ & $\mathrm{MET}$ \\
\hline HETATM & 442 & $\mathrm{H} 11$ & MET \\
\hline HETATM & 443 & H12 & MET \\
\hline HETATM & 444 & H13 & MET \\
\hline \multicolumn{4}{|l|}{ TER } \\
\hline HETATM & 445 & $\mathrm{FE}$ & HEM \\
\hline HETATM & 446 & $\mathrm{C} 1$ & HEM \\
\hline HETATM & 447 & $\mathrm{C} 2$ & HEM \\
\hline HETATM & 448 & C3 & HEM \\
\hline HETATM & 449 & $\mathrm{C} 4$ & HEM \\
\hline HETATM & 450 & $\mathrm{C} 5$ & HEM \\
\hline HETATM & 451 & H5 & HEM \\
\hline HETATM & 452 & $\mathrm{C} 6$ & HEM \\
\hline HETATM & 453 & C7 & HEM \\
\hline HETATM & 454 & $\mathrm{C} 8$ & HEM \\
\hline HETATM & 455 & $\mathrm{C} 9$ & HEM \\
\hline HETATM & 456 & $\mathrm{C} 10$ & HEM \\
\hline HETATM & 457 & $\mathrm{H} 10$ & HEM \\
\hline HETATM & 458 & $\mathrm{C} 11$ & HEM \\
\hline HETATM & 459 & $\mathrm{C} 12$ & HEM \\
\hline HETATM & 460 & C13 & HEM \\
\hline HETATM & 461 & C14 & HEM \\
\hline HETATM & 462 & C15 & HEM \\
\hline HETATM & 463 & H15 & HEM \\
\hline HETATM & 464 & $\mathrm{C} 16$ & HEM \\
\hline HETATM & 465 & $\mathrm{C} 17$ & HEM \\
\hline HETATM & 466 & $\mathrm{C} 18$ & HEM \\
\hline HETATM & 467 & C19 & HEM \\
\hline HETATM & 468 & $\mathrm{C} 20$ & HEM \\
\hline HETATM & 469 & $\mathrm{H} 2 \mathrm{O}$ & HEM \\
\hline HETATM & 470 & N21 & HEM \\
\hline HETATM & 471 & N22 & HEM \\
\hline HETATM & 472 & N23 & HEM \\
\hline HETATM & 473 & N24 & HEM \\
\hline HETATM & 474 & C99 & HEM \\
\hline HETATM & 475 & 091 & HEM \\
\hline HETATM & 476 & 092 & HEM \\
\hline HETATM & 477 & СТ91 & HEM \\
\hline HETATM & 478 & H911 & HEM \\
\hline HETATM & 479 & H912 & HEM \\
\hline HETATM & 480 & СТ92 & HEM \\
\hline HETATM & 481 & H921 & HEM \\
\hline HETATM & 482 & H922 & HEM \\
\hline \multicolumn{4}{|l|}{ TER } \\
\hline HETATM & 483 & $\mathrm{FE}$ & HEM \\
\hline HETATM & 484 & $\mathrm{C} 1$ & HEM \\
\hline HETATM & 485 & $\mathrm{C} 2$ & HEM \\
\hline HETATM & 40 & & מקחע \\
\hline
\end{tabular}

$\begin{array}{llllll}7.892 & -15.494 & 10.652 & 1.00 & 0.00\end{array}$

$\begin{array}{lll}11.382 & 1.00 & 0.00\end{array}$

$\begin{array}{lllll}3.117 & -12.746 & 10.256 & 1.00 & 0.00\end{array}$

$\begin{array}{lllll}3.783 & -12.645 & 11.115 & 1.00 & 0.00\end{array}$

$\begin{array}{lllll}1.950 & -13.390 & 10.415 & 1.00 & 0.00\end{array}$

$\begin{array}{rrrrr}1.270 & -13.546 & 9.585 & 1.00 & 0.00\end{array}$

$\begin{array}{lllll}1.645 & -13.788 & 11.381 & 1.00 & 0.00\end{array}$

$1.432-11.062$

$\begin{array}{lllll}1.276 & -10.040 & 7.685 & 1.00 & 0.00\end{array}$

$\begin{array}{lllll}1.000 & -11.128 & 9.057 & 1.00 & 0.00\end{array}$

$\begin{array}{lllll}0.841 & -11.727 & 7.403 & 1.00 & 0.00\end{array}$

$\begin{array}{lllll}10.521 & -9.981 & 2.161 & 1.00 & 0.00\end{array}$

$\begin{array}{lllll}9.782 & -9.969 & 1.349 & 1.00 & 0.00\end{array}$

$\begin{array}{lllll}11.361 & -10.589 & 1.798 & 1.00 & 0.00\end{array}$

$\begin{array}{lllll}10.999 & -8.543 & 2.434 & 1.00 & 0.00\end{array}$

$\begin{array}{lllll}11.557 & -8.482 & 3.382 & 1.00 & 0.00\end{array}$

$\begin{array}{lllll}10.129 & -7.877 & 2.541 & 1.00 & 0.00\end{array}$

$\begin{array}{lllll}11.863 & -7.931 & 1.358 & 1.00 & 0.00\end{array}$

$\begin{array}{lllll}12.091 & -8.454 & 0.260 & 1.00 & 0.00\end{array}$

$\begin{array}{lllll}12.379 & -6.751 & 1.722 & 1.00 & 0.00\end{array}$

$\begin{array}{lllll}12.988 & -6.397 & 0.986 & 1.00 & 0.00\end{array}$

$\begin{array}{llllll}2.625 & -8.855 & 3.334 & 1.00 & 0.00\end{array}$

$\begin{array}{lllll}2.072 & -9.694 & 2.884 & 1.00 & 0.00\end{array}$

$\begin{array}{lllll}2.618 & -8.029 & 2.613 & 1.00 & 0.00\end{array}$

$\begin{array}{lllll}2.054 & -8.535 & 4.214 & 1.00 & 0.00\end{array}$

$\begin{array}{lllll}12.058 & -11.803 & 4.285 & 1.00 & 0.00\end{array}$

$\begin{array}{lllll}12.503 & -11.594 & 3.305 & 1.00 & 0.00\end{array}$

$\begin{array}{lllll}12.168 & -12.883 & 4.460 & 1.00 & 0.00\end{array}$

$\begin{array}{lllll}12.659 & -11.278 & 5.042 & 1.00 & 0.00\end{array}$

$3.316 \quad 10.511$

$1.088 \quad 12.383$

$9.423 \quad 1.00 \quad 0.00$

$0.729 \quad 13.174$

$1.874 \quad 13.271$

$2.904 \quad 12.553$

$4.219 \quad 12.435$

$8.361 \quad 1.00 \quad 0.00$

$\begin{array}{lll}7.189 & 1.00 & 0.00\end{array}$

$\begin{array}{lll}6.414 & 1.00 & 0.00\end{array}$

$\begin{array}{lll}7.122 & 1.00 \quad 0.00\end{array}$

$\begin{array}{lll}6.684 & 1.00 & 0.00\end{array}$

$\begin{array}{lllll}4.472 & 12.931 & 5.749 & 1.00 & 0.00\end{array}$

$\begin{array}{lllll}5.238 & 11.736 & 7.320 & 1.00 & 0.00\end{array}$

$\begin{array}{lllll}6.607 & 11.664 & 6.851 & 1.00 & 0.00\end{array}$

$\begin{array}{lllll}7.313 & 10.944 & 7.798 & 1.00 & 0.00\end{array}$

$\begin{array}{lllll}6.362 & 10.579 & 8.822 & 1.00 & 0.00\end{array}$

$\begin{array}{lllll}6.666 & 9.844 & 9.965 & 1.00 & 0.00\end{array}$

$\begin{array}{lllll}7.693 & 9.500 & 10.071 & 1.00 & 0.00\end{array}$

$\begin{array}{lllll}5.792 & 9.504 & 10.988 & 1.00 & 0.00\end{array}$

$\begin{array}{lllll}6.173 & 8.779 & 12.178 & 1.00 & 0.00\end{array}$

$\begin{array}{lllll}5.033 & 8.631 & 12.935 & 1.00 & 0.00\end{array}$

$\begin{array}{lllll}3.960 & 9.273 & 12.204 & 1.00 & 0.00\end{array}$

$\begin{array}{lllll}2.632 & 9.328 & 12.625 & 1.00 & 0.00\end{array}$

$\begin{array}{lllll}2.392 & 8.843 & 13.573 & 1.00 & 0.00\end{array}$

$\begin{array}{lllll}1.602 & 10.009 & 11.982 & 1.00 & 0.00\end{array}$

$\begin{array}{lllll}0.236 & 10.123 & 12.464 & 1.00 & 0.00\end{array}$

$\begin{array}{lllll}-0.439 & 10.890 & 11.541 & 1.00 & 0.00\end{array}$

$\begin{array}{lllll}0.510 & 11.242 & 10.504 & 1.00 & 0.00\end{array}$

$\begin{array}{lllll}0.219 & 12.033 & 9.393 & 1.00 & 0.00\end{array}$

$\begin{array}{lllll}-0.795 & 12.423 & 9.333 & 1.00 & 0.00\end{array}$

$\begin{array}{lllll}2.409 & 12.020 & 8.298 & 1.00 & 0.00\end{array}$

$\begin{array}{lllll}5.114 & 11.073 & 8.521 & 1.00 & 0.00\end{array}$

$\begin{array}{lllll}4.443 & 9.810 & 11.021 & 1.00 & 0.00\end{array}$

$\begin{array}{lllll}1.743 & 10.694 & 10.786 & 1.00 & 0.00\end{array}$

$\begin{array}{lllll}2.650 & 7.829 & 8.283 & 1.00 & 0.00\end{array}$

$\begin{array}{lllll}2.810 & 9.143 & 8.296 & 1.00 & 0.00\end{array}$

$\begin{array}{lllll}2.495 & 7.212 & 7.228 & 1.00 & 0.00\end{array}$

$\begin{array}{lllll}2.666 & 7.148 & 9.640 & 1.00 & 0.00\end{array}$

$\begin{array}{lllll}3.645 & 7.343 & 10.103 & 1.00 & 0.00\end{array}$

$\begin{array}{lllll}1.918 & 7.638 & 10.283 & 1.00 & 0.00\end{array}$

$\begin{array}{lllll}2.407 & 5.644 & 9.528 & 1.00 & 0.00\end{array}$

$\begin{array}{lllll}3.203 & 5.183 & 8.931 & 1.00 & 0.00\end{array}$

$\begin{array}{lllll}1.482 & 5.488 & 8.958 & 1.00 & 0.00\end{array}$

$\begin{array}{lllll}4.008 & 3.097 & 14.371 & 1.00 & 0.00\end{array}$

$\begin{array}{lllll}6.236 & 1.225 & 15.433 & 1.00 & 0.00\end{array}$

$\begin{array}{lllll}6.595 & 0.434 & 16.605 & 1.00 & 0.00\end{array}$

$\begin{array}{lllll}5.449 & 0.337 & 17.380 & 1.00 & 0.00\end{array}$ 


\begin{tabular}{|c|c|c|c|}
\hline HETATM & 487 & C4 & HEM \\
\hline HETATM & 488 & C5 & HEM \\
\hline HETATM & 489 & H5 & HEM \\
\hline HETATM & 490 & $\mathrm{C} 6$ & HEM \\
\hline HETATM & 491 & C7 & HEM \\
\hline HETATM & 492 & C8 & HEM \\
\hline HETATM & 493 & C9 & HEM \\
\hline HETATM & 494 & C10 & HEM \\
\hline HETATM & 495 & H10 & HEM \\
\hline HETATM & 496 & C11 & HEM \\
\hline HETATM & 497 & C12 & HEM \\
\hline HETATM & 498 & C13 & HEM \\
\hline HETATM & 499 & C14 & HEM \\
\hline HETATM & 500 & C15 & HEM \\
\hline HETATM & 501 & H15 & HEM \\
\hline HETATM & 502 & C16 & HEM \\
\hline HETATM & 503 & C17 & HEM \\
\hline HETATM & 504 & C18 & HEM \\
\hline HETATM & 505 & C19 & HEM \\
\hline HETATM & 506 & $\mathrm{C} 20$ & HEM \\
\hline HETATM & 507 & $\mathrm{H} 20$ & HEM \\
\hline HETATM & 508 & $\mathrm{~N} 21$ & HEM \\
\hline HETATM & 509 & $\mathrm{~N} 22$ & HEM \\
\hline HETATM & 510 & N23 & HEM \\
\hline HETATM & 511 & N2 4 & HEM \\
\hline HETATM & 512 & C99 & HEM \\
\hline HETATM & 513 & 091 & HEM \\
\hline HETATM & 514 & 092 & HEM \\
\hline HETATM & 515 & СТ91 & HEM \\
\hline HETATM & 516 & H911 & HEM \\
\hline HETATM & 517 & H912 & HEM \\
\hline HETATM & 518 & CT92 & HEM \\
\hline HETATM & 519 & H921 & HEM \\
\hline HETATM & 520 & H922 & HEM \\
\hline \multicolumn{4}{|l|}{ TER } \\
\hline HETATM & 521 & $\mathrm{C} 2$ & VIN \\
\hline HETATM & 522 & H 21 & VIN \\
\hline HETATM & 523 & $\mathrm{C} 1$ & VIN \\
\hline HETATM & 524 & $\mathrm{H} 11$ & VIN \\
\hline HETATM & 525 & $\mathrm{H} 12$ & VIN \\
\hline \multicolumn{4}{|l|}{ TER } \\
\hline HETATM & 526 & $\mathrm{C} 1$ & MET \\
\hline HETATM & 527 & H11 & MET \\
\hline HETATM & 528 & $\mathrm{H} 12$ & MET \\
\hline HETATM & 529 & $\mathrm{H} 13$ & MET \\
\hline \multicolumn{4}{|l|}{ TER } \\
\hline HETATM & 530 & $\mathrm{C} 2$ & VIN \\
\hline HETATM & 531 & H 21 & VIN \\
\hline HETATM & 532 & $\mathrm{C} 1$ & VIN \\
\hline HETATM & 533 & H11 & VIN \\
\hline HETATM & 534 & $\mathrm{H} 12$ & VIN \\
\hline \multicolumn{4}{|l|}{ TER } \\
\hline HETATM & 535 & $\mathrm{C} 1$ & MET \\
\hline HETATM & 536 & H11 & MET \\
\hline HETATM & 537 & H12 & MET \\
\hline HETATM & 538 & H13 & $\mathrm{MET}$ \\
\hline \multicolumn{4}{|l|}{ TER } \\
\hline HETATM & 539 & $\mathrm{C} 5$ & $\mathrm{ACP}$ \\
\hline HETATM & 540 & H51 & $\mathrm{ACP}$ \\
\hline HETATM & 541 & H52 & $\mathrm{ACP}$ \\
\hline HETATM & 542 & $\mathrm{C} 4$ & $\mathrm{ACP}$ \\
\hline HETATM & 543 & $\mathrm{H} 41$ & $\mathrm{ACP}$ \\
\hline HETATM & 544 & $\mathrm{H} 42$ & $\mathrm{ACP}$ \\
\hline HETATM & 545 & C1 & $\mathrm{ACP}$ \\
\hline HETATM & 546 & $\mathrm{O} 2$ & $\mathrm{ACP}$ \\
\hline HETATM & 547 & 03 & $\mathrm{ACP}$ \\
\hline HETATM & 548 & H3 & $\mathrm{ACP}$ \\
\hline \multicolumn{4}{|c|}{ TER } \\
\hline HETATM & 549 & $\mathrm{C} 1$ & $\mathrm{MET}$ \\
\hline HETATM & 550 & H11 & MET \\
\hline HETATM & 551 & $\mathrm{H} 12$ & MET \\
\hline HETATM & 552 & H13 & MET \\
\hline \multicolumn{4}{|l|}{ TER } \\
\hline HETATM & 553 & $\mathrm{C} 1$ & $\mathrm{MET}$ \\
\hline HETATM & 554 & H11 & MET \\
\hline HETATM & 555 & $\mathrm{H} 12$ & MET \\
\hline HETATM & 556 & H13 & MET \\
\hline TER & & & \\
\hline HET & J & & VIN \\
\hline
\end{tabular}

\begin{tabular}{|c|c|c|c|c|c|}
\hline 4.419 & 1.055 & 16.672 & 1.00 & 0.00 & C \\
\hline 3.104 & 1.173 & 17.109 & 1.00 & 0.00 & C \\
\hline 2.851 & 0.677 & 18.045 & 1.00 & 0.00 & $\mathrm{H}$ \\
\hline 2.085 & 1.872 & 16.473 & 1.00 & 0.00 & c \\
\hline 0.716 & 1.944 & 16.943 & 1.00 & 0.00 & c \\
\hline 0.011 & 2.664 & 15.996 & 1.00 & 0.00 & c \\
\hline 0.962 & 3.029 & 14.972 & 1.00 & 0.00 & c \\
\hline 0.657 & 3.764 & 13.828 & 1.00 & 0.00 & c \\
\hline-0.370 & 4.108 & 13.722 & 1.00 & 0.00 & $\mathrm{~F}$ \\
\hline 1.532 & 4.104 & 12.806 & 1.00 & 0.00 & 0 \\
\hline 1.151 & 4.829 & 11.615 & 1.00 & 0.00 & e \\
\hline 2.290 & 4.977 & 10.858 & 1.00 & 0.00 & c \\
\hline 3.363 & 4.335 & 11.590 & 1.00 & 0.00 & c \\
\hline 4.691 & 4.280 & 11.168 & 1.00 & 0.00 & c \\
\hline 4.931 & 4.765 & 10.221 & 1.00 & 0.00 & $\mathrm{H}$ \\
\hline 5.721 & 3.599 & 11.812 & 1.00 & 0.00 & c \\
\hline 7.087 & 3.485 & 11.329 & 1.00 & 0.00 & c \\
\hline 7.763 & 2.718 & 12.253 & 1.00 & 0.00 & c \\
\hline 6.813 & 2.366 & 13.289 & 1.00 & 0.00 & 0 \\
\hline 7.104 & 1.575 & 14.401 & 1.00 & 0.00 & c \\
\hline 8.118 & 1.185 & 14.461 & 1.00 & 0.00 & $\mathrm{H}$ \\
\hline 4.914 & 1.588 & 15.495 & 1.00 & 0.00 & $\mathrm{~N}$ \\
\hline 2.209 & 2.535 & 15.273 & 1.00 & 0.00 & $N$ \\
\hline 2.880 & 3.798 & 12.772 & 1.00 & 0.00 & $\mathrm{~N}$ \\
\hline 5.580 & 2.914 & 13.008 & 1.00 & 0.00 & $N$ \\
\hline 4.673 & 5.779 & 15.510 & 1.00 & 0.00 & c \\
\hline 4.514 & 4.465 & 15.498 & 1.00 & 0.00 & 0 \\
\hline 4.828 & 6.396 & 16.566 & 1.00 & 0.00 & o \\
\hline 4.657 & 6.460 & 14.154 & 1.00 & 0.00 & c \\
\hline 3.678 & 6.265 & 13.691 & 1.00 & 0.00 & $\mathrm{H}$ \\
\hline 5.405 & 5.970 & 13.511 & 1.00 & 0.00 & H \\
\hline 4.917 & 7.964 & 14.265 & 1.00 & 0.00 & c \\
\hline 5.842 & 8.120 & 14.836 & 1.00 & 0.00 & $\mathrm{H}$ \\
\hline 4.120 & 8.425 & 14.862 & 1.00 & 0.00 & $\mathrm{H}$ \\
\hline-0.603 & 13.676 & 6.929 & 1.00 & 0.00 & c \\
\hline-1.419 & 12.978 & 7.094 & 1.00 & 0.00 & $\mathrm{H}$ \\
\hline-0.936 & 14.901 & 6.528 & 1.00 & 0.00 & c \\
\hline-0.205 & 15.680 & 6.421 & 1.00 & 0.00 & $\mathrm{H}$ \\
\hline-1.978 & 15.170 & 6.361 & 1.00 & 0.00 & $\mathrm{H}$ \\
\hline 2.061 & 13.931 & 5.091 & 1.00 & 0.00 & c \\
\hline 2.295 & 15.002 & 5.211 & 1.00 & 0.00 & $\mathrm{H}$ \\
\hline 2.874 & 13.467 & 4.522 & 1.00 & 0.00 & $\mathrm{H}$ \\
\hline 1.153 & 13.854 & 4.481 & 1.00 & 0.00 & $\mathrm{H}$ \\
\hline 7.071 & 12.253 & 5.606 & 1.00 & 0.00 & c \\
\hline 6.405 & 12.152 & 4.747 & 1.00 & 0.00 & H \\
\hline 8.238 & 12.898 & 5.447 & 1.00 & 0.00 & c \\
\hline 8.917 & 13.054 & 6.277 & 1.00 & 0.00 & H \\
\hline 8.543 & 13.296 & 4.482 & 1.00 & 0.00 & $\mathrm{H}$ \\
\hline 8.755 & 10.570 & 7.810 & 1.00 & 0.00 & c \\
\hline 9.347 & 11.234 & 8.459 & 1.00 & 0.00 & $\mathrm{H}$ \\
\hline 8.912 & 9.548 & 8.177 & 1.00 & 0.00 & H \\
\hline 9.188 & 10.636 & 6.805 & 1.00 & 0.00 & $\mathrm{H}$ \\
\hline-0.333 & 9.488 & 13.701 & 1.00 & 0.00 & c \\
\hline 0.406 & 9.477 & 14.514 & 1.00 & 0.00 & $\mathrm{H}$ \\
\hline-1.174 & 10.097 & 14.065 & 1.00 & 0.00 & H \\
\hline-0.812 & 8.051 & 13.428 & 1.00 & 0.00 & $c$ \\
\hline-1.369 & 7.990 & 12.481 & 1.00 & 0.00 & $\mathrm{H}$ \\
\hline 0.059 & 7.384 & 13.321 & 1.00 & 0.00 & $\mathrm{t}$ \\
\hline-1.676 & 7.439 & 14.504 & 1.00 & 0.00 & c \\
\hline-1.903 & 7.961 & 15.603 & 1.00 & 0.00 & o \\
\hline-2.191 & 6.259 & 14.140 & 1.00 & 0.00 & o \\
\hline-2.800 & 5.905 & 14.876 & 1.00 & 0.00 & $\mathrm{H}$ \\
\hline 7.563 & 8.362 & 12.528 & 1.00 & 0.00 & 4 \\
\hline 8.115 & 9.201 & 12.978 & 1.00 & 0.00 & $\mathrm{H}$ \\
\hline 7.570 & 7.536 & 13.250 & 1.00 & 0.00 & $\mathrm{H}$ \\
\hline 8.134 & 8.042 & 11.649 & 1.00 & 0.00 & $\mathrm{H}$ \\
\hline-1.870 & 11.311 & 11.577 & 1.00 & 0.00 & 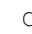 \\
\hline-2.316 & 11.102 & 12.557 & 1.00 & 0.00 & H \\
\hline-1.981 & 12.391 & 11.402 & 1.00 & 0.00 & 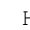 \\
\hline-2.471 & 10.785 & 10.821 & 1.00 & 0.00 & $\mathrm{H}$ \\
\hline 7. & -0.068 & 16.864 & 1.00 & 0.00 & \\
\hline
\end{tabular}




\begin{tabular}{|c|c|c|c|}
\hline HETATM & 558 & H21 & VIN \\
\hline HETATM & 559 & $\mathrm{C} 1$ & VIN \\
\hline HETATM & 560 & H11 & VIN \\
\hline HETATM & 561 & $\mathrm{H} 12$ & VIN \\
\hline \multicolumn{4}{|l|}{ TER } \\
\hline HETATM & 562 & $\mathrm{C} 1$ & MET \\
\hline HETATM & 563 & H11 & MET \\
\hline HETATM & 564 & $\mathrm{H} 12$ & MET \\
\hline HETATM & 565 & $\mathrm{H} 13$ & MET \\
\hline \multicolumn{4}{|l|}{ TER } \\
\hline HETATM & 566 & $\mathrm{C} 2$ & VIN \\
\hline HETATM & 567 & H 21 & VIN \\
\hline HETATM & 568 & $\mathrm{C} 1$ & VIN \\
\hline HETATM & 569 & H11 & VIN \\
\hline HETATM & 570 & $\mathrm{H} 12$ & VIN \\
\hline \multicolumn{4}{|l|}{ TER } \\
\hline HETATM & 571 & $\mathrm{C} 1$ & MET \\
\hline HETATM & 572 & H11 & MET \\
\hline HETATM & 573 & $\mathrm{H} 12$ & MET \\
\hline HETATM & 574 & $\mathrm{H} 13$ & MET \\
\hline \multicolumn{4}{|l|}{ TER } \\
\hline HETATM & 575 & C5 & $\mathrm{ACP}$ \\
\hline HETATM & 576 & H51 & $\mathrm{ACP}$ \\
\hline HETATM & 577 & H52 & $\mathrm{ACP}$ \\
\hline HETATM & 578 & C4 & $\mathrm{ACP}$ \\
\hline HETATM & 579 & H 41 & $\mathrm{ACP}$ \\
\hline HETATM & 580 & $\mathrm{H} 42$ & $\mathrm{ACP}$ \\
\hline HETATM & 581 & $\mathrm{C} 1$ & $\mathrm{ACP}$ \\
\hline HETATM & 582 & $\mathrm{O} 2$ & $\mathrm{ACP}$ \\
\hline HETATM & 583 & 03 & $\mathrm{ACP}$ \\
\hline HETATM & 584 & H3 & $\mathrm{ACP}$ \\
\hline \multicolumn{4}{|l|}{ TER } \\
\hline HETATM & 585 & $\mathrm{C} 1$ & MET \\
\hline HETATM & 586 & H11 & MET \\
\hline HETATM & 587 & $\mathrm{H} 12$ & MET \\
\hline HETATM & 588 & H13 & MET \\
\hline \multicolumn{4}{|l|}{ TER } \\
\hline HETATM & 589 & $\mathrm{C} 1$ & MET \\
\hline HETATM & 590 & H11 & MET \\
\hline HETATM & 591 & $\mathrm{H} 12$ & MET \\
\hline HETATM & 592 & H13 & MET \\
\hline \multicolumn{4}{|l|}{ TER } \\
\hline HETATM & 593 & $\mathrm{FE}$ & HEM \\
\hline HETATM & 594 & $\mathrm{C} 1$ & HEM \\
\hline HETATM & 595 & $\mathrm{C} 2$ & HEM \\
\hline HETATM & 596 & $\mathrm{C} 3$ & HEM \\
\hline HETATM & 597 & $\mathrm{C} 4$ & HEM \\
\hline HETATM & 598 & $\mathrm{C} 5$ & HEM \\
\hline HETATM & 599 & H5 & HEM \\
\hline HETATM & 600 & $\mathrm{C} 6$ & HEM \\
\hline HETATM & 601 & C7 & HEM \\
\hline HETATM & 602 & $\mathrm{C} 8$ & HEM \\
\hline HETATM & 603 & C9 & HEM \\
\hline HETATM & 604 & $\mathrm{C} 10$ & HEM \\
\hline HETATM & 605 & H1O & HEM \\
\hline HETATM & 606 & C11 & HEM \\
\hline HETATM & 607 & $\mathrm{C} 12$ & HEM \\
\hline HETATM & 608 & C13 & HEM \\
\hline HETATM & 609 & C14 & HEM \\
\hline HETATM & 610 & C15 & HEM \\
\hline HETATM & 611 & H15 & HEM \\
\hline HETATM & 612 & C16 & HEM \\
\hline HETATM & 613 & C17 & HEM \\
\hline HETATM & 614 & C18 & HEM \\
\hline HETATM & 615 & C19 & HEM \\
\hline HETATM & 616 & $\mathrm{C} 20$ & HEM \\
\hline HETATM & 617 & $\mathrm{H} 2 \mathrm{O}$ & HEM \\
\hline HETATM & 618 & N21 & HEM \\
\hline HETATM & 619 & N22 & HEM \\
\hline HETATM & 620 & N23 & HEM \\
\hline HETATM & 621 & $\mathrm{~N} 24$ & HEM \\
\hline HETATM & 622 & C99 & HEM \\
\hline HETATM & 623 & 091 & HEM \\
\hline HETATM & 624 & 092 & HEM \\
\hline HETATM & 625 & СТ91 & HEM \\
\hline HETATM & 626 & H911 & HEM \\
\hline HETATM & 627 & H912 & HEM \\
\hline HETATM & 628 & СТ92 & HEM \\
\hline HETATM & 629 & H921 & HEM \\
\hline
\end{tabular}

\begin{tabular}{|c|c|c|c|c|c|}
\hline 8.742 & 0.630 & 16.699 & 1.00 & 0.00 & $\mathrm{H}$ \\
\hline 8.259 & -1.293 & 17.266 & 1.00 & 0.00 & C \\
\hline 7.528 & -2.072 & 17.373 & 1.00 & 0.00 & $\mathrm{H}$ \\
\hline 9.301 & -1.562 & 17.432 & 1.00 & 0.00 & $\mathrm{H}$ \\
\hline 5.263 & -0.323 & 18.702 & 1.00 & 0.00 & C \\
\hline 5.028 & -1.394 & 18.583 & 1.00 & 0.00 & $\mathrm{H}$ \\
\hline 4.450 & 0.141 & 19.272 & 1.00 & 0.00 & $\mathrm{H}$ \\
\hline 6.170 & -0.246 & 19.313 & 1.00 & 0.00 & H \\
\hline 0.252 & 1.355 & 18.188 & 1.00 & 0.00 & C \\
\hline 0.918 & 1.456 & 19.046 & 1.00 & 0.00 & $\mathrm{H}$ \\
\hline-0.914 & 0.710 & 18.346 & 1.00 & 0.00 & C \\
\hline-1.594 & 0.554 & 17.516 & 1.00 & 0.00 & $\mathrm{H}$ \\
\hline-1.219 & 0.312 & 19.312 & 1.00 & 0.00 & $\mathrm{H}$ \\
\hline-1.432 & 3.038 & 15.984 & 1.00 & 0.00 & C \\
\hline-1.588 & 4.060 & 15.617 & 1.00 & 0.00 & $\mathrm{H}$ \\
\hline-1.864 & 2.972 & 16.989 & 1.00 & 0.00 & $\mathrm{H}$ \\
\hline-2.023 & 2.374 & 15.335 & 1.00 & 0.00 & $\mathrm{H}$ \\
\hline 7.656 & 4.120 & 10.092 & 1.00 & 0.00 & C \\
\hline 6.917 & 4.131 & 9.280 & 1.00 & 0.00 & $\mathrm{H}$ \\
\hline 8.497 & 3.511 & 9.729 & 1.00 & 0.00 & $\mathrm{H}$ \\
\hline 8.135 & 5.557 & 10.365 & 1.00 & 0.00 & C \\
\hline 8.693 & 5.618 & 11.313 & 1.00 & 0.00 & $\mathrm{H}$ \\
\hline 7.264 & 6.224 & 10.472 & 1.00 & 0.00 & $\mathrm{H}$ \\
\hline 8.999 & 6.169 & 9.289 & 1.00 & 0.00 & C \\
\hline 9.227 & 5.647 & 8.191 & 1.00 & 0.00 & O \\
\hline 9.515 & 7.349 & 9.653 & 1.00 & 0.00 & 0 \\
\hline 10.124 & 7.703 & 8.917 & 1.00 & 0.00 & $\mathrm{H}$ \\
\hline-0.240 & 5.246 & 11.266 & 1.00 & 0.00 & C \\
\hline-0.792 & 4.407 & 10.815 & 1.00 & 0.00 & $\mathrm{H}$ \\
\hline-0.246 & 6.072 & 10.544 & 1.00 & 0.00 & $\mathrm{H}$ \\
\hline-0.811 & 5.565 & 12.145 & 1.00 & 0.00 & $\mathrm{H}$ \\
\hline 9.194 & 2.297 & 12.216 & 1.00 & 0.00 & C \\
\hline 9.639 & 2.506 & 11.236 & 1.00 & 0.00 & $\mathrm{H}$ \\
\hline 9.304 & 1.217 & 12.391 & 1.00 & 0.00 & $\mathrm{H}$ \\
\hline 9.794 & 2.823 & 12.973 & 1.00 & 0.00 & $\mathrm{H}$ \\
\hline 5.232 & -3.836 & 9.423 & 1.00 & 0.00 & $\mathrm{Fe}$ \\
\hline 3.004 & -1.963 & 8.361 & 1.00 & 0.00 & C \\
\hline 2.646 & -1.173 & 7.189 & 1.00 & 0.00 & C \\
\hline 3.791 & -1.076 & 6.414 & 1.00 & 0.00 & C \\
\hline 4.821 & -1.793 & 7.122 & 1.00 & 0.00 & C \\
\hline 6.136 & -1.912 & 6.684 & 1.00 & 0.00 & C \\
\hline 6.389 & -1.416 & 5.749 & 1.00 & 0.00 & $\mathrm{H}$ \\
\hline 7.155 & -2.610 & 7.320 & 1.00 & 0.00 & C \\
\hline 8.524 & -2.683 & 6.851 & 1.00 & 0.00 & C \\
\hline 9.229 & -3.403 & 7.798 & 1.00 & 0.00 & C \\
\hline 8.278 & -3.768 & 8.822 & 1.00 & 0.00 & C \\
\hline 8.583 & -4.503 & 9.965 & 1.00 & 0.00 & C \\
\hline 9.610 & -4.846 & 10.071 & 1.00 & 0.00 & $\mathrm{H}$ \\
\hline 7.708 & -4.843 & 10.988 & 1.00 & 0.00 & C \\
\hline 8.089 & -5.568 & 12.178 & 1.00 & 0.00 & C \\
\hline 6.950 & -5.715 & 12.935 & 1.00 & 0.00 & C \\
\hline 5.877 & -5.074 & 12.204 & 1.00 & 0.00 & C \\
\hline 4.549 & -5.019 & 12.625 & 1.00 & 0.00 & C \\
\hline 4.309 & -5.504 & 13.573 & 1.00 & 0.00 & $\mathrm{H}$ \\
\hline 3.519 & -4.337 & 11.982 & 1.00 & 0.00 & c \\
\hline 2.153 & -4.224 & 12.464 & 1.00 & 0.00 & c \\
\hline 1.478 & -3.456 & 11.541 & 1.00 & 0.00 & c \\
\hline 2.427 & -3.104 & 10.504 & 1.00 & 0.00 & c \\
\hline 2.136 & -2.313 & 9.393 & 1.00 & 0.00 & c \\
\hline 1.122 & -1.924 & 9.333 & 1.00 & 0.00 & $\mathrm{H}$ \\
\hline 4.326 & -2.326 & 8.298 & 1.00 & 0.00 & $\mathrm{~N}$ \\
\hline 7.031 & -3.273 & 8.521 & 1.00 & 0.00 & $\mathrm{~N}$ \\
\hline 6.360 & -4.536 & 11.021 & 1.00 & 0.00 & $\mathrm{~N}$ \\
\hline 3.660 & -3.652 & 10.786 & 1.00 & 0.00 & $\mathrm{~N}$ \\
\hline 4.567 & -6.517 & 8.283 & 1.00 & 0.00 & c \\
\hline 4.727 & -5.204 & 8.296 & 1.00 & 0.00 & 0 \\
\hline 4.412 & -7.134 & 7.228 & 1.00 & 0.00 & 0 \\
\hline 4.583 & -7.199 & 9.640 & 1.00 & 0.00 & c \\
\hline 5.562 & -7.003 & 10.103 & 1.00 & 0.00 & $\mathrm{H}$ \\
\hline 3.835 & -6.709 & 10.283 & 1.00 & 0.00 & $\mathrm{H}$ \\
\hline 4.323 & -8.703 & 9.528 & 1.00 & 0.00 & c \\
\hline 5.120 & -9.163 & 8.931 & 1.00 & 0.00 & $\mathrm{H}$ \\
\hline
\end{tabular}




\begin{tabular}{|c|c|c|c|c|c|c|c|c|c|c|}
\hline $\begin{array}{l}\text { HETATM } \\
\text { TER }\end{array}$ & 630 & H922 & HEM & 65 & 3.399 & -8.859 & 8.958 & 1.00 & 0.00 & $\mathrm{H}$ \\
\hline HETATM & 631 & $\mathrm{FE}$ & HEM & 66 & 5.925 & -11.250 & 14.371 & 1.00 & 0.00 & $\mathrm{Fe}$ \\
\hline HETATM & 632 & $\mathrm{C} 1$ & HEM & 66 & 8.152 & $-13 \cdot 122$ & 15.433 & 1.00 & 0.00 & C \\
\hline HETATM & 633 & $\mathrm{C} 2$ & HEM & 66 & 8.511 & -13.912 & 16.605 & 1.00 & 0.00 & $\mathrm{C}$ \\
\hline HETATM & 634 & C3 & HEM & 66 & 7.366 & -14.009 & 17.380 & 1.00 & 0.00 & C \\
\hline HETATM & 635 & $\mathrm{C} 4$ & HEM & 66 & 6.336 & -13.292 & 16.672 & 1.00 & 0.00 & C \\
\hline HETATM & 636 & C5 & HEM & 66 & 5.021 & -13.173 & 17.109 & 1.00 & 0.00 & C \\
\hline HETATM & 637 & H5 & HEM & 66 & 4.768 & -13.670 & 18.045 & 1.00 & 0.00 & $\mathrm{H}$ \\
\hline HETATM & 638 & $\mathrm{C} 6$ & HEM & 66 & 4.002 & -12.475 & 16.473 & 1.00 & 0.00 & C \\
\hline HETATM & 639 & C7 & HEM & 66 & 2.633 & -12.402 & 16.943 & 1.00 & 0.00 & C \\
\hline HETATM & 640 & C8 & HEM & 66 & 1.928 & -11.682 & 15.996 & 1.00 & 0.00 & C \\
\hline HETATM & 641 & C9 & HEM & 66 & 2.879 & -11.317 & 14.972 & 1.00 & 0.00 & $\mathrm{C}$ \\
\hline HETATM & 642 & $\mathrm{C} 10$ & HEM & 66 & 2.574 & -10.583 & 13.828 & 1.00 & 0.00 & $\mathrm{C}$ \\
\hline HETATM & 643 & $\mathrm{H} 10$ & HEM & 66 & 1.547 & -10.239 & 13.722 & 1.00 & 0.00 & $\mathrm{H}$ \\
\hline HETATM & 644 & $\mathrm{C} 11$ & HEM & 66 & 3.448 & -10.243 & 12.806 & 1.00 & 0.00 & $\mathrm{C}$ \\
\hline HETATM & 645 & $\mathrm{C} 12$ & HEM & 66 & 3.068 & -9.517 & 11.615 & 1.00 & 0.00 & C \\
\hline HETATM & 646 & $\mathrm{C} 13$ & HEM & 66 & 4.207 & -9.370 & 10.858 & 1.00 & 0.00 & C \\
\hline HETATM & 647 & $\mathrm{C} 14$ & HEM & 66 & 5.280 & -10.011 & 11.590 & 1.00 & 0.00 & C \\
\hline HETATM & 648 & C15 & HEM & 66 & 6.608 & -10.066 & 11.168 & 1.00 & 0.00 & C \\
\hline HETATM & 649 & H15 & HEM & 66 & 6.848 & -9.581 & 10.221 & 1.00 & 0.00 & $\mathrm{H}$ \\
\hline HETATM & 650 & $\mathrm{C} 16$ & HEM & 66 & 7.638 & $-10 \cdot 748$ & 11.812 & 1.00 & 0.00 & C \\
\hline HETATM & 651 & C17 & HEM & 66 & 9.004 & -10.861 & 11.329 & 1.00 & 0.00 & C \\
\hline HETATM & 652 & $\mathrm{C} 18$ & HEM & 66 & 9.679 & -11.629 & 12.253 & 1.00 & 0.00 & C \\
\hline HETATM & 653 & C19 & HEM & 66 & 8.730 & -11.981 & 13.289 & 1.00 & 0.00 & C \\
\hline HETATM & 654 & $\mathrm{C} 20$ & HEM & 66 & 9.021 & -12.772 & 14.401 & 1.00 & 0.00 & C \\
\hline HETATM & 655 & $\mathrm{H} 20$ & HEM & 66 & 10.035 & -13.161 & 14.461 & 1.00 & 0.00 & $\mathrm{H}$ \\
\hline HETATM & 656 & $\mathrm{~N} 21$ & HEM & 66 & 6.831 & -12.759 & 15.495 & 1.00 & 0.00 & $\mathrm{~N}$ \\
\hline HETATM & 657 & N22 & HEM & 66 & 4.126 & -11.812 & 15.273 & 1.00 & 0.00 & $\mathrm{~N}$ \\
\hline HETATM & 658 & N23 & HEM & 66 & 4.797 & -10.549 & 12.772 & 1.00 & 0.00 & $\mathrm{~N}$ \\
\hline HETATM & 659 & N2 4 & HEM & 66 & 7.497 & -11.433 & 13.008 & 1.00 & 0.00 & $\mathrm{~N}$ \\
\hline HETATM & 660 & C99 & HEM & 66 & 6.590 & -8.568 & 15.510 & 1.00 & 0.00 & C \\
\hline HETATM & 661 & 091 & HEM & 66 & 6.430 & -9.881 & 15.498 & 1.00 & 0.00 & 0 \\
\hline HETATM & 662 & 092 & HEM & 66 & 6.745 & -7.951 & 16.566 & 1.00 & 0.00 & 0 \\
\hline HETATM & 663 & СТ 91 & HEM & 66 & 6.574 & -7.887 & 14.154 & 1.00 & 0.00 & $\mathrm{C}$ \\
\hline HETATM & 664 & H911 & HEM & 66 & 5.595 & -8.082 & 13.691 & 1.00 & 0.00 & $\mathrm{H}$ \\
\hline HETATM & 665 & H912 & HEM & 66 & 7.322 & -8.376 & 13.511 & 1.00 & 0.00 & $\mathrm{H}$ \\
\hline HETATM & 666 & СТ92 & HEM & 66 & 6.833 & -6.382 & 14.265 & 1.00 & 0.00 & C \\
\hline HETATM & 667 & H921 & HEM & 66 & 7.758 & -6.226 & 14.836 & 1.00 & 0.00 & $\mathrm{H}$ \\
\hline HETATM & 668 & H922 & HEM & 66 & 6.037 & -5.922 & 14.862 & 1.00 & 0.00 & $\mathrm{H}$ \\
\hline \multicolumn{11}{|l|}{ TER } \\
\hline HETATM & 669 & $\mathrm{C} 2$ & VIN & 67 & 1.314 & -0.670 & 6.929 & 1.00 & 0.00 & C \\
\hline HETATM & 670 & H 21 & VIN & 67 & 0.498 & -1.369 & 7.094 & 1.00 & 0.00 & $\mathrm{H}$ \\
\hline HETATM & 671 & $\mathrm{C} 1$ & VIN & 67 & 0.981 & 0.555 & 6.528 & 1.00 & 0.00 & C \\
\hline HETATM & 672 & H11 & VIN & 67 & 1.712 & 1.333 & 6.421 & 1.00 & 0.00 & $\mathrm{H}$ \\
\hline HETATM & 673 & $\mathrm{H} 12$ & VIN & 67 & -0.061 & 0.824 & 6.361 & 1.00 & 0.00 & $\mathrm{H}$ \\
\hline \multicolumn{11}{|l|}{ TER } \\
\hline HETATM & 674 & $\mathrm{C} 1$ & $\mathrm{MET}$ & 68 & 3.977 & -0.415 & 5.091 & 1.00 & 0.00 & C \\
\hline HETATM & 675 & $\mathrm{H} 11$ & MET & 68 & 4.212 & 0.655 & 5.211 & 1.00 & 0.00 & $\mathrm{H}$ \\
\hline HETATM & 676 & $\mathrm{H} 12$ & $\mathrm{MET}$ & 68 & 4.790 & -0.880 & 4.522 & 1.00 & 0.00 & $\mathrm{H}$ \\
\hline HETATM & 677 & $\mathrm{H} 13$ & $\mathrm{MET}$ & 68 & 3.070 & -0.493 & 4.481 & 1.00 & 0.00 & $\mathrm{H}$ \\
\hline \multicolumn{11}{|l|}{ TER } \\
\hline HETATM & 678 & $\mathrm{C} 2$ & VIN & 69 & 8.988 & -2.093 & 5.606 & 1.00 & 0.00 & C \\
\hline HETATM & 679 & $\mathrm{H} 21$ & VIN & 69 & 8.322 & -2.194 & 4.747 & 1.00 & 0.00 & $\mathrm{H}$ \\
\hline HETATM & 680 & $\mathrm{C} 1$ & VIN & 69 & 10.154 & -1.449 & 5.447 & 1.00 & 0.00 & C \\
\hline HETATM & 681 & H11 & VIN & 69 & 10.834 & -1.293 & 6.277 & 1.00 & 0.00 & $\mathrm{H}$ \\
\hline HETATM & 682 & H12 & VIN & 69 & 10.459 & -1.051 & 4.482 & 1.00 & 0.00 & $\mathrm{H}$ \\
\hline \multicolumn{11}{|l|}{ TER } \\
\hline HETATM & 683 & $\mathrm{C} 1$ & $\mathrm{MET}$ & 70 & 10.672 & -3.777 & 7.810 & 1.00 & 0.00 & C \\
\hline HETATM & 684 & H11 & MET & 70 & 11.263 & -3.112 & 8.459 & 1.00 & 0.00 & $\mathrm{H}$ \\
\hline HETATM & 685 & $\mathrm{H} 12$ & $\mathrm{MET}$ & 70 & 10.829 & -4.799 & 8.177 & 1.00 & 0.00 & $\mathrm{H}$ \\
\hline HETATM & 686 & H13 & $\mathrm{MET}$ & 70 & 11.104 & -3.711 & 6.805 & 1.00 & 0.00 & $\mathrm{H}$ \\
\hline \multicolumn{11}{|l|}{ TER } \\
\hline HETATM & 687 & C5 & $\mathrm{ACP}$ & 71 & 1.584 & -4.858 & 13.701 & 1.00 & 0.00 & C \\
\hline HETATM & 688 & H51 & $\mathrm{ACP}$ & 71 & 2.323 & -4.870 & 14.514 & 1.00 & 0.00 & $\mathrm{H}$ \\
\hline HETATM & 689 & H52 & $\mathrm{ACP}$ & 71 & 0.743 & -4.250 & 14.065 & 1.00 & 0.00 & $\mathrm{H}$ \\
\hline HETATM & 690 & C4 & $\mathrm{ACP}$ & 71 & 1.105 & -6.296 & 13.428 & 1.00 & 0.00 & C \\
\hline HETATM & 691 & $\mathrm{H} 41$ & $\mathrm{ACP}$ & 71 & 0.548 & -6.357 & 12.481 & 1.00 & 0.00 & $\mathrm{H}$ \\
\hline HETATM & 692 & $\mathrm{H} 42$ & $\mathrm{ACP}$ & 71 & 1.976 & -6.962 & 13.321 & 1.00 & 0.00 & $\mathrm{H}$ \\
\hline HETATM & 693 & $\mathrm{C} 1$ & $\mathrm{ACP}$ & 71 & 0.241 & -6.908 & 14.504 & 1.00 & 0.00 & C \\
\hline HETATM & 694 & $\mathrm{O} 2$ & $\mathrm{ACP}$ & 71 & 0.013 & -6.385 & 15.603 & 1.00 & 0.00 & 0 \\
\hline HETATM & 695 & 03 & $\mathrm{ACP}$ & 71 & -0.275 & -8.088 & 14.140 & 1.00 & 0.00 & 0 \\
\hline HETATM & 696 & H3 & $\mathrm{ACP}$ & 71 & -0.884 & -8.442 & 14.876 & 1.00 & 0.00 & $\mathrm{H}$ \\
\hline TER & & & & & & & & & & \\
\hline HETATM & 697 & $\mathrm{C} 1$ & $\mathrm{MET}$ & 72 & 9.480 & -5.985 & 12.528 & 1.00 & 0.00 & C \\
\hline HETATM & 698 & $\mathrm{H} 11$ & MET & 72 & 10.032 & -5.145 & 12.978 & 1.00 & 0.00 & $\mathrm{H}$ \\
\hline HETATM & 699 & $\mathrm{H} 12$ & $\mathrm{MET}$ & 72 & 9.486 & -6.810 & 13.250 & 1.00 & 0.00 & $\mathrm{H}$ \\
\hline HETATM & 700 & H13 & MET & 72 & 10.051 & -6.304 & 11.649 & 1.00 & 0.00 & $\mathrm{H}$ \\
\hline
\end{tabular}




\begin{tabular}{|c|c|c|c|}
\hline HETATM & 701 & $\mathrm{C} 1$ & MET \\
\hline HETATM & 702 & H11 & MET \\
\hline HETATM & 703 & $\mathrm{H} 12$ & MET \\
\hline HETATM & 704 & $\mathrm{H} 13$ & $\mathrm{MET}$ \\
\hline \multicolumn{4}{|l|}{ TER } \\
\hline HETATM & 705 & $\mathrm{C} 2$ & VIN \\
\hline HETATM & 706 & $\mathrm{H} 21$ & VIN \\
\hline HETATM & 707 & $\mathrm{C} 1$ & VIN \\
\hline HETATM & 708 & H11 & VIN \\
\hline HETATM & 709 & $\mathrm{H} 12$ & VIN \\
\hline \multicolumn{4}{|l|}{ TER } \\
\hline HETATM & 710 & $\mathrm{C} 1$ & MET \\
\hline HETATM & 711 & H11 & $\mathrm{MET}$ \\
\hline HETATM & 712 & $\mathrm{H} 12$ & MET \\
\hline HETATM & 713 & $\mathrm{H} 13$ & MET \\
\hline \multicolumn{4}{|l|}{ TER } \\
\hline HETATM & 714 & C2 & VIN \\
\hline HETATM & 715 & H 21 & VIN \\
\hline HETATM & 716 & $\mathrm{C} 1$ & VIN \\
\hline HETATM & 717 & $\mathrm{H} 11$ & VIN \\
\hline HETATM & 718 & $\mathrm{H} 12$ & VIN \\
\hline \multicolumn{4}{|l|}{ TER } \\
\hline HETATM & 719 & $\mathrm{C} 1$ & $\mathrm{MET}$ \\
\hline HETATM & 720 & H11 & $\mathrm{MET}$ \\
\hline HETATM & 721 & $\mathrm{H} 12$ & MET \\
\hline HETATM & 722 & $\mathrm{H} 13$ & $\mathrm{MET}$ \\
\hline \multicolumn{4}{|l|}{ TER } \\
\hline HETATM & 723 & C5 & $\mathrm{ACP}$ \\
\hline HETATM & 724 & H51 & $\mathrm{ACP}$ \\
\hline HETATM & 725 & H52 & $\mathrm{ACP}$ \\
\hline HETATM & 726 & C4 & $\mathrm{ACP}$ \\
\hline HETATM & 727 & H4 1 & $\mathrm{ACP}$ \\
\hline HETATM & 728 & $\mathrm{H} 42$ & $\mathrm{ACP}$ \\
\hline HETATM & 729 & $\mathrm{C} 1$ & $\mathrm{ACP}$ \\
\hline HETATM & 730 & 02 & $\mathrm{ACP}$ \\
\hline HETATM & 731 & 03 & $\mathrm{ACP}$ \\
\hline HETATM & 732 & H3 & $\mathrm{ACP}$ \\
\hline \multicolumn{4}{|l|}{ TER } \\
\hline HETATM & 733 & $\mathrm{C} 1$ & MET \\
\hline HETATM & 734 & $\mathrm{H} 11$ & MET \\
\hline HETATM & 735 & $\mathrm{H} 12$ & MET \\
\hline HETATM & 736 & $\mathrm{H} 13$ & $\mathrm{MET}$ \\
\hline \multicolumn{4}{|l|}{ TER } \\
\hline HETATM & 737 & $\mathrm{C} 1$ & MET \\
\hline HETATM & 738 & $\mathrm{H} 11$ & MET \\
\hline HETATM & 739 & $\mathrm{H} 12$ & $\mathrm{MET}$ \\
\hline HETATM & 740 & $\mathrm{H} 13$ & $\mathrm{MET}$ \\
\hline \multicolumn{4}{|l|}{ TER } \\
\hline HETATM & 741 & $\mathrm{FE}$ & HEM \\
\hline HETATM & 742 & $\mathrm{C} 1$ & HEM \\
\hline HETATM & 743 & $\mathrm{C} 2$ & HEM \\
\hline HETATM & 744 & $\mathrm{C} 3$ & HEM \\
\hline HETATM & 745 & C4 & HEM \\
\hline HETATM & 746 & C5 & HEM \\
\hline HETATM & 747 & H5 & HEM \\
\hline HETATM & 748 & C6 & HEM \\
\hline HETATM & 749 & C7 & HEM \\
\hline HETATM & 750 & C8 & HEM \\
\hline HETATM & 751 & C9 & HEM \\
\hline HETATM & 752 & $\mathrm{C} 10$ & HEM \\
\hline HETATM & 753 & $\mathrm{H} 10$ & HEM \\
\hline HETATM & 754 & C11 & HEM \\
\hline HETATM & 755 & $\mathrm{C} 12$ & HEM \\
\hline HETATM & 756 & $\mathrm{C} 13$ & HEM \\
\hline HETATM & 757 & C14 & HEM \\
\hline HETATM & 758 & C15 & HEM \\
\hline HETATM & 759 & H15 & HEM \\
\hline HETATM & 760 & $\mathrm{C} 16$ & HEM \\
\hline HETATM & 761 & $\mathrm{C} 17$ & HEM \\
\hline HETATM & 762 & C18 & HEM \\
\hline HETATM & 763 & C19 & HEM \\
\hline HETATM & 764 & $\mathrm{C} 20$ & HEM \\
\hline HETATM & 765 & $\mathrm{H} 2 \mathrm{O}$ & HEM \\
\hline HETATM & 766 & N21 & HEM \\
\hline HETATM & 767 & N22 & HEM \\
\hline HETATM & 768 & N23 & HEM \\
\hline HETATM & 769 & N24 & HEM \\
\hline HETATM & 770 & C99 & HEM \\
\hline HETATM & 771 & 091 & HEM \\
\hline
\end{tabular}

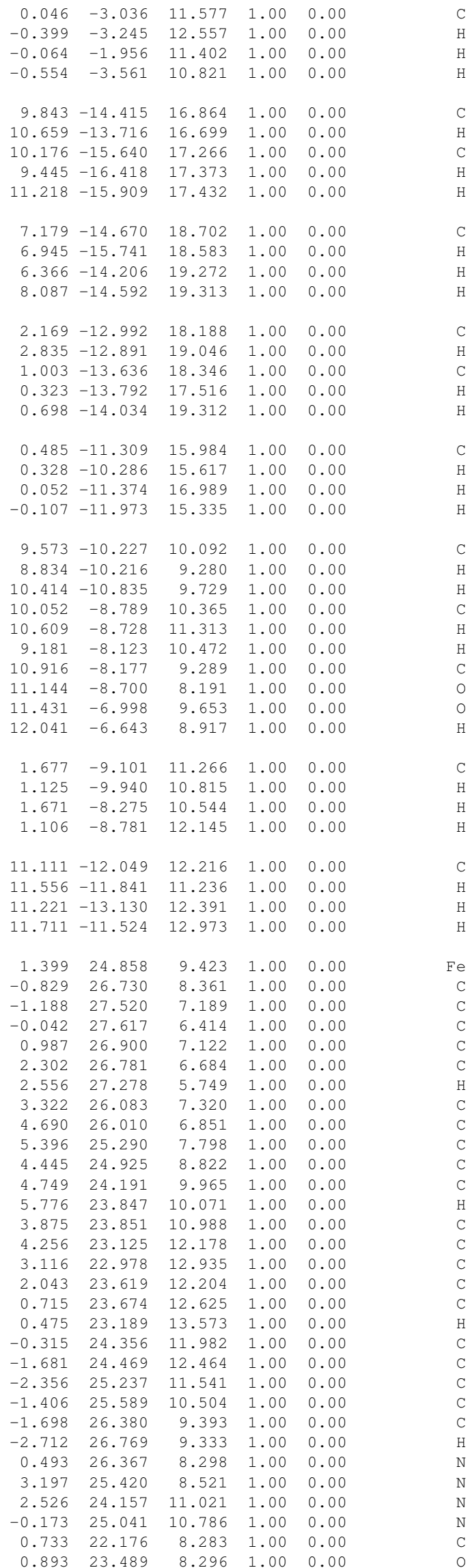




\begin{tabular}{|c|c|c|c|}
\hline HETATM & 772 & 092 & HEM \\
\hline HETATM & 773 & СТ91 & HEM \\
\hline HETATM & 774 & H911 & HEM \\
\hline HETATM & 775 & H912 & HEM \\
\hline HETATM & 776 & CT92 & HEM \\
\hline HETATM & 777 & H921 & HEM \\
\hline HETATM & 778 & H922 & HEM \\
\hline \multicolumn{4}{|l|}{ TER } \\
\hline HETATM & 779 & $\mathrm{FE}$ & HEM \\
\hline HETATM & 780 & $\mathrm{C} 1$ & HEM \\
\hline HETATM & 781 & C2 & HEM \\
\hline HETATM & 782 & C3 & HEM \\
\hline HETATM & 783 & C4 & HEM \\
\hline HETATM & 784 & C5 & HEM \\
\hline HETATM & 785 & H5 & HEM \\
\hline HETATM & 786 & $\mathrm{C} 6$ & HEM \\
\hline HETATM & 787 & C7 & HEM \\
\hline HETATM & 788 & $\mathrm{C} 8$ & HEM \\
\hline HETATM & 789 & C9 & HEM \\
\hline HETATM & 790 & C10 & HEM \\
\hline HETATM & 791 & $\mathrm{H} 10$ & HEM \\
\hline HETATM & 792 & C11 & HEM \\
\hline HETATM & 793 & C12 & HEM \\
\hline HETATM & 794 & C13 & HEM \\
\hline HETATM & 795 & C14 & HEM \\
\hline HETATM & 796 & C15 & HEM \\
\hline HETATM & 797 & H15 & HEM \\
\hline HETATM & 798 & C16 & HEM \\
\hline HETATM & 799 & C17 & HEM \\
\hline HETATM & 800 & C18 & HEM \\
\hline HETATM & 801 & C19 & HEM \\
\hline HETATM & 802 & C20 & HEM \\
\hline HETATM & 803 & $\mathrm{H} 2 \mathrm{O}$ & HEM \\
\hline HETATM & 804 & N21 & HEM \\
\hline HETATM & 805 & N22 & HEM \\
\hline HETATM & 806 & N23 & HEM \\
\hline HETATM & 807 & N24 & HEM \\
\hline HETATM & 808 & C99 & HEM \\
\hline HETATM & 809 & 091 & HEM \\
\hline HETATM & 810 & 092 & HEM \\
\hline HETATM & 811 & CT91 & HEM \\
\hline HETATM & 812 & H911 & HEM \\
\hline HETATM & 813 & H912 & HEM \\
\hline HETATM & 814 & CT92 & HEM \\
\hline HETATM & 815 & H921 & HEM \\
\hline HETATM & 816 & H922 & HEM \\
\hline \multicolumn{4}{|l|}{ TER } \\
\hline HETATM & 817 & C2 & VIN \\
\hline HETATM & 818 & H 21 & VIN \\
\hline HETATM & 819 & $\mathrm{C} 1$ & VIN \\
\hline HETATM & 820 & H11 & VIN \\
\hline HETATM & 821 & $\mathrm{H} 12$ & VIN \\
\hline \multicolumn{4}{|l|}{ TER } \\
\hline HETATM & 822 & $\mathrm{C} 1$ & MET \\
\hline HETATM & 823 & $\mathrm{H} 11$ & MET \\
\hline HETATM & 824 & $\mathrm{H} 12$ & MET \\
\hline HETATM & 825 & H13 & MET \\
\hline \multicolumn{4}{|l|}{ TER } \\
\hline HETATM & 826 & $\mathrm{C} 2$ & VIN \\
\hline HETATM & 827 & H 21 & VIN \\
\hline HETATM & 828 & $\mathrm{C} 1$ & VIN \\
\hline HETATM & 829 & $\mathrm{H} 11$ & VIN \\
\hline HETATM & 830 & $\mathrm{H} 12$ & VIN \\
\hline \multicolumn{4}{|l|}{ TER } \\
\hline HETATM & 831 & $\mathrm{C} 1$ & MET \\
\hline HETATM & 832 & H11 & MET \\
\hline HETATM & 833 & H12 & MET \\
\hline HETATM & 834 & H13 & MET \\
\hline \multicolumn{4}{|l|}{ TER } \\
\hline HETATM & 835 & C5 & $\mathrm{ACP}$ \\
\hline HETATM & 836 & H51 & $\mathrm{ACP}$ \\
\hline HETATM & 837 & H52 & $\mathrm{ACP}$ \\
\hline HETATM & 838 & C4 & $\mathrm{ACP}$ \\
\hline HETATM & 839 & $\mathrm{H} 4 \mathrm{I}$ & $\mathrm{ACP}$ \\
\hline HETATM & 840 & $\mathrm{H} 42$ & $\mathrm{ACP}$ \\
\hline HETATM & 841 & $\mathrm{C} 1$ & $\mathrm{ACP}$ \\
\hline HETATM & 842 & 02 & $\mathrm{ACP}$ \\
\hline HETATM & 843 & 03 & $\mathrm{ACP}$ \\
\hline HETATM & 844 & 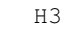 & $\mathrm{ACP}$ \\
\hline
\end{tabular}

\begin{tabular}{|c|c|c|c|c|c|}
\hline 0.579 & 21.559 & 7.228 & 1.00 & 0.00 & o \\
\hline 0.749 & 21.495 & 9.640 & 1.00 & 0.00 & C \\
\hline 1.728 & 21.690 & 10.103 & 1.00 & 0.00 & $\mathrm{H}$ \\
\hline 0.001 & 21.984 & 10.283 & 1.00 & 0.00 & $\mathrm{H}$ \\
\hline 0.490 & 19.990 & 9.528 & 1.00 & 0.00 & C \\
\hline 1.286 & 19.530 & 8.931 & 1.00 & 0.00 & $\mathrm{H}$ \\
\hline-0.435 & 19.834 & 8.958 & 1.00 & 0.00 & $\mathrm{H}$ \\
\hline 2.091 & 17.444 & 14.371 & 1.00 & 0.00 & $\mathrm{Fe}$ \\
\hline 4.319 & 15.571 & 15.433 & 1.00 & 0.00 & C \\
\hline 4.678 & 14.781 & 16.605 & 1.00 & 0.00 & C \\
\hline 3.532 & 14.684 & 17.380 & 1.00 & 0.00 & C \\
\hline 2.503 & 15.401 & 16.672 & 1.00 & 0.00 & C \\
\hline 1.188 & 15.520 & 17.109 & 1.00 & 0.00 & C \\
\hline 0.934 & 15.024 & 18.045 & 1.00 & 0.00 & $\mathrm{H}$ \\
\hline 0.168 & 16.218 & 16.473 & 1.00 & 0.00 & C \\
\hline-1.201 & 16.291 & 16.943 & 1.00 & 0.00 & C \\
\hline-1.906 & 17.011 & 15.996 & 1.00 & 0.00 & C \\
\hline-0.955 & 17.376 & 14.972 & 1.00 & 0.00 & C \\
\hline-1.260 & 18.111 & 13.828 & 1.00 & 0.00 & C \\
\hline-2.286 & 18.454 & 13.722 & 1.00 & 0.00 & $\mathrm{H}$ \\
\hline-0.385 & 18.451 & 12.806 & 1.00 & 0.00 & C \\
\hline-0.766 & 19.176 & 11.615 & 1.00 & 0.00 & C \\
\hline 0.373 & 19.323 & 10.858 & 1.00 & 0.00 & C \\
\hline 1.446 & 18.682 & 11.590 & 1.00 & 0.00 & C \\
\hline 2.774 & 18.627 & 11.168 & 1.00 & 0.00 & C \\
\hline 3.014 & 19.112 & 10.221 & 1.00 & 0.00 & $\mathrm{H}$ \\
\hline 3.805 & 17.945 & 11.812 & 1.00 & 0.00 & C \\
\hline 5.170 & 17.832 & 11.329 & 1.00 & 0.00 & C \\
\hline 5.846 & 17.064 & 12.253 & 1.00 & 0.00 & C \\
\hline 4.896 & 16.712 & 13.289 & 1.00 & 0.00 & C \\
\hline 5.187 & 15.921 & 14.401 & 1.00 & 0.00 & C \\
\hline 6.202 & 15.532 & 14.461 & 1.00 & 0.00 & $\mathrm{H}$ \\
\hline 2.997 & 15.934 & 15.495 & 1.00 & 0.00 & $\mathrm{~N}$ \\
\hline 0.293 & 16.881 & 15.273 & 1.00 & 0.00 & $\mathrm{~N}$ \\
\hline 0.963 & 18.144 & 12.772 & 1.00 & 0.00 & $\mathrm{~N}$ \\
\hline 3.663 & 17.260 & 13.008 & 1.00 & 0.00 & $\mathrm{~N}$ \\
\hline 2.756 & 20.125 & 15.510 & 1.00 & 0.00 & C \\
\hline 2.597 & 18.812 & 15.498 & 1.00 & 0.00 & 0 \\
\hline 2.911 & 20.742 & 16.566 & 1.00 & 0.00 & 0 \\
\hline 2.740 & 20.807 & 14.154 & 1.00 & 0.00 & c \\
\hline 1.761 & 20.611 & 13.691 & 1.00 & 0.00 & $\mathrm{H}$ \\
\hline 3.488 & 20.317 & 13.511 & 1.00 & 0.00 & $\mathrm{H}$ \\
\hline 3.000 & 22.311 & 14.265 & 1.00 & 0.00 & c \\
\hline 3.925 & 22.467 & 14.836 & 1.00 & 0.00 & $\mathrm{H}$ \\
\hline 2.204 & 22.771 & 14.862 & 1.00 & 0.00 & $\mathrm{H}$ \\
\hline-2.520 & 28.023 & 6.929 & 1.00 & 0.00 & c \\
\hline-3.336 & 27.324 & 7.094 & 1.00 & 0.00 & $\mathrm{H}$ \\
\hline-2.853 & 29.248 & 6.528 & 1.00 & 0.00 & c \\
\hline-2.121 & 30.026 & 6.421 & 1.00 & 0.00 & $\mathrm{H}$ \\
\hline-3.894 & 29.517 & 6.361 & 1.00 & 0.00 & $\mathrm{H}$ \\
\hline 0.144 & 28.278 & 5.091 & 1.00 & 0.00 & c \\
\hline 0.379 & 29.349 & 5.211 & 1.00 & 0.00 & $\mathrm{H}$ \\
\hline 0.957 & 27.814 & 4.522 & 1.00 & 0.00 & $\mathrm{H}$ \\
\hline-0.764 & 28.200 & 4.481 & 1.00 & 0.00 & $\mathrm{H}$ \\
\hline 5.154 & 26.600 & 5.606 & 1.00 & 0.00 & c \\
\hline 4.488 & 26.499 & 4.747 & 1.00 & 0.00 & $\mathrm{H}$ \\
\hline 6.321 & 27.244 & 5.447 & 1.00 & 0.00 & c \\
\hline 7.001 & 27.400 & 6.277 & 1.00 & 0.00 & $\mathrm{H}$ \\
\hline 6.626 & 27.642 & 4.482 & 1.00 & 0.00 & $\mathrm{H}$ \\
\hline 6.839 & 24.917 & 7.810 & 1.00 & 0.00 & c \\
\hline 7.430 & 25.581 & 8.459 & 1.00 & 0.00 & $\mathrm{H}$ \\
\hline 6.995 & 23.894 & 8.177 & 1.00 & 0.00 & $\mathrm{H}$ \\
\hline 7.271 & 24.982 & 6.805 & 1.00 & 0.00 & $\mathrm{H}$ \\
\hline-2.250 & 23.835 & 13.701 & 1.00 & 0.00 & c \\
\hline-1.511 & 23.824 & 14.514 & 1.00 & 0.00 & $\mathrm{H}$ \\
\hline-3.090 & 24.443 & 14.065 & 1.00 & 0.00 & $\mathrm{H}$ \\
\hline-2.728 & 22.397 & 13.428 & 1.00 & 0.00 & c \\
\hline-3.286 & 22.336 & 12.481 & 1.00 & 0.00 & $\mathrm{H}$ \\
\hline-1.858 & 21.731 & 13.321 & 1.00 & 0.00 & $\mathrm{H}$ \\
\hline-3.592 & 21.785 & 14.504 & 1.00 & 0.00 & c \\
\hline-3.820 & 22.308 & 15.603 & 1.00 & 0.00 & o \\
\hline-4.108 & 20.606 & 14.140 & 1.00 & 0.00 & 0 \\
\hline-4.717 & 20.251 & 14.876 & 1.00 & 0.00 & $\mathrm{H}$ \\
\hline
\end{tabular}


TER

HETATM $845 \quad$ C1 MET 88

HETATM 846

HETATM 847 H12 MET

HETATM 848 H13 MET

TER

HETATM 849 C1 MET

HETATM 850 H11 MET

HETATM 851 H12 MET

HETATM 852 H13 MET

TER

HETATM 853 C2 VIN

HETATM 854 H21 VIN

HETATM 855 C1 VIN

HETATM 856 H11 VIN

HETATM 857 H12 VIN

HETATM 858

HETATM 859 H11 MET

HETATM 860 H12 MET

HETATM 861 H13 MET TER

HETATM 862 C2 VIN

HETATM 863 H21 VIN

HETATM 864 C1 VIN

HETATM 865 H11 VIN

HETATM 866 H12 VIN

HETATM $867 \quad$ C1 MET

HETATM 868 H11 MET

HETATM 869 H12 MET

HETATM 870 H13 MET TER

HETATM $871 \quad$ C5 ACP

HETATM 872 H51 ACP

HETATM 873 H52 ACP

HETATM $874 \quad$ C4 ACP

HETATM 875 H41 ACP

HETATM 876 H42 ACP

HETATM $877 \quad$ C1 ACP

HETATM $878 \quad 02 \mathrm{ACP}$

HETATM 879 O3 ACP

HETATM 880 H3 ACP

TER

HETATM 881 C1 MET

HETATM 882 H11 MET

HETATM 883 H12 MET

HETATM 884 H13 MET

TER

HETATM 885 C1 MET

HETATM 886 H11 MET

HETATM 887 H12 MET

HETATM 888 H13 MET

TER

HETATM 889 FE HEM

HETATM $890 \quad$ C1 HEM

HETATM 891 C2 HEM

HETATM 892 C3 HEM

HETATM 893 C4 HEM

HETATM 894 C5 HEM

HETATM 895 H5 HEM

HETATM 896 C6 HEM

HETATM $897 \quad$ C7 HEM

HETATM 898 C8 HEM

HETATM 899 C9 HEM

HETATM 900 C10 HEM

HETATM 901 H1O HEM

HETATM 902 C11 HEM

HETATM 903 C12 HEM

HETATM 904 C13 HEM

HETATM 905 C14 HEM

HETATM 906 C15 HEM

HETATM 907 H15 HEM

HETATM 908 C16 HEM

HETATM 909 C17 HEM

HETATM 910 C18 HEM

HETATM 911 C19 HEM

HETATM 912 C20 HEM

HETATM 913 H20 HEM $\begin{array}{lllll}5.646 & 22.709 & 12.528 & 1.00 & 0.00\end{array}$

$\begin{array}{llllll}6.199 & 23.548 & 12.978 & 1.00 & 0.00\end{array}$

$\begin{array}{lllll}5.653 & 21.883 & 13.250 & 1.00 & 0.00\end{array}$

$\begin{array}{lllll}6.217 & 22.389 & 11.649 & 1.00 & 0.00\end{array}$

$\begin{array}{llllll}-3.787 & 25.657 & 11.577 & 1.00 & 0.00\end{array}$

$\begin{array}{lllll}-4.232 & 25.449 & 12.557 & 1.00 & 0.00\end{array}$

$\begin{array}{lllll}-3.898 & 26.738 & 11.402 & 1.00 & 0.00\end{array}$

$\begin{array}{llllll}-4.388 & 25.132 & 10.821 & 1.00 & 0.00\end{array}$

$\begin{array}{lllll}6.010 & 14.278 & 16.864 & 1.00 & 0.00\end{array}$

$\begin{array}{lllll}6.825 & 14.977 & 16.699 & 1.00 & 0.00\end{array}$

$\begin{array}{lllll}6.342 & 13.053 & 17.266 & 1.00 & 0.00\end{array}$

$\begin{array}{llllll}5.611 & 12.275 & 17.373 & 1.00 & 0.00\end{array}$

$\begin{array}{lllll}7.384 & 12.784 & 17.432 & 1.00 & 0.00\end{array}$

$\begin{array}{lllll}3.346 & 14.023 & 18.702 & 1.00 & 0.00\end{array}$

$\begin{array}{lllll}3.111 & 12.953 & 18.583 & 1.00 & 0.00\end{array}$

$\begin{array}{lllll}2.533 & 14.488 & 19.272 & 1.00 & 0.00\end{array}$

$\begin{array}{lllll}4.253 & 14.101 & 19.313 & 1.00 & 0.00\end{array}$

$\begin{array}{lllll}-1.664 & 15.701 & 18.188 & 1.00 & 0.00\end{array}$

$\begin{array}{llllll}-0.998 & 15.802 & 19.046 & 1.00 & 0.00\end{array}$

$\begin{array}{lllll}-2.831 & 15.057 & 18.346 & 1.00 & 0.00\end{array}$

$\begin{array}{lllll}-3.511 & 14.901 & 17.516 & 1.00 & 0.00\end{array}$

$\begin{array}{lllll}-3.136 & 14.659 & 19.312 & 1.00 & 0.00\end{array}$

$\begin{array}{lllll}-3.349 & 17.385 & 15.984 & 1.00 & 0.00\end{array}$

$\begin{array}{lllll}-3.505 & 18.407 & 15.617 & 1.00 & 0.00\end{array}$

$\begin{array}{lllll}-3.781 & 17.319 & 16.989 & 1.00 & 0.00\end{array}$

$\begin{array}{lllll}-3.940 & 16.720 & 15.335 & 1.00 & 0.00\end{array}$

$\begin{array}{lllll}5.740 & 18.466 & 10.092 & 1.00 & 0.00\end{array}$

$\begin{array}{lllll}5.001 & 18.478 & 9.280 & 1.00 & 0.00\end{array}$

$\begin{array}{llllll}6.580 & 17.858 & 9.729 & 1.00 & 0.00\end{array}$

$\begin{array}{lllll}6.218 & 19.904 & 10.365 & 1.00 & 0.00\end{array}$

$\begin{array}{lllll}6.776 & 19.965 & 11.313 & 1.00 & 0.00\end{array}$

$\begin{array}{lllll}5.348 & 20.570 & 10.472 & 1.00 & 0.00\end{array}$

$\begin{array}{lllll}7.082 & 20.516 & 9.289 & 1.00 & 0.00\end{array}$

$\begin{array}{lllll}7.310 & 19.993 & 8.191 & 1.00 & 0.00\end{array}$

$\begin{array}{lllll}7.598 & 21.696 & 9.653 & 1.00 & 0.00\end{array}$

$\begin{array}{lllll}8.207 & 22.050 & 8.917 & 1.00 & 0.00\end{array}$

$\begin{array}{lllll}-2.157 & 19.593 & 11.266 & 1.00 & 0.00\end{array}$

$\begin{array}{lllll}-2.709 & 18.753 & 10.815 & 1.00 & 0.00\end{array}$

$\begin{array}{lllll}-2.163 & 20.418 & 10.544 & 1.00 & 0.00\end{array}$

$\begin{array}{lllll}-2.728 & 19.912 & 12.145 & 1.00 & 0.00\end{array}$

$\begin{array}{lllll}7.277 & 16.644 & 12.216 & 1.00 & 0.00\end{array}$

$\begin{array}{lllll}7.722 & 16.853 & 11.236 & 1.00 & 0.00\end{array}$

$\begin{array}{llllll}7.387 & 15.564 & 12.391 & 1.00 & 0.00\end{array}$

$\begin{array}{lllll}7.878 & 17.169 & 12.973 & 1.00 & 0.00\end{array}$

$\begin{array}{llllll}5.211 & 11.003 & -6.440 & 1.00 & 0.00\end{array}$

$\begin{array}{lllll}2.983 & 12.876 & -7.502 & 1.00 & 0.00\end{array}$

$\begin{array}{lllll}2.624 & 13.666 & -8.674 & 1.00 & 0.00\end{array}$

$\begin{array}{llllll}3.770 & 13.763 & -9.448 & 1.00 & 0.00\end{array}$

$\begin{array}{llllll}4.799 & 13.046 & -8.740 & 1.00 & 0.00\end{array}$

$\begin{array}{llllll}6.114 & 12.927 & -9.178 & 1.00 & 0.00\end{array}$

$\begin{array}{lllll}6.368 & 13.423 & -10.113 & 1.00 & 0.00\end{array}$

$\begin{array}{lllll}7.134 & 12.229 & -8.542 & 1.00 & 0.00\end{array}$

$\begin{array}{lllll}8.502 & 12.156 & -9.012 & 1.00 & 0.00\end{array}$

$\begin{array}{llllll}9.208 & 11.436 & -8.065 & 1.00 & 0.00\end{array}$

$\begin{array}{lllll}8.257 & 11.071 & -7.041 & 1.00 & 0.00\end{array}$

$\begin{array}{llllll}8.561 & 10.336 & -5.897 & 1.00 & 0.00\end{array}$

$\begin{array}{rrrrr}9.588 & 9.993 & -5.791 & 1.00 & 0.00\end{array}$

$\begin{array}{lllll}7.687 & 9.996 & -4.874 & 1.00 & 0.00\end{array}$

$\begin{array}{llllll}8.068 & 9.271 & -3.684 & 1.00 & 0.00\end{array}$

$\begin{array}{lllll}6.928 & 9.124 & -2.927 & 1.00 & 0.00\end{array}$

$\begin{array}{lllll}5.855 & 9.765 & -3.659 & 1.00 & 0.00\end{array}$

$\begin{array}{lllll}4.527 & 9.820 & -3.237 & 1.00 & 0.00\end{array}$

$\begin{array}{lllll}4.287 & 9.335 & -2.289 & 1.00 & 0.00\end{array}$

$\begin{array}{lllll}3.497 & 10.502 & -3.880 & 1.00 & 0.00\end{array}$

$\begin{array}{lllll}2.131 & 10.615 & -3.398 & 1.00 & 0.00\end{array}$

$\begin{array}{lllll}1.456 & 11.383 & -4.322 & 1.00 & 0.00\end{array}$

$\begin{array}{lllll}2.406 & 11.735 & -5.358 & 1.00 & 0.00\end{array}$

$\begin{array}{lllll}2.114 & 12.526 & -6.469 & 1.00 & 0.00\end{array}$

$\begin{array}{lllll}1.100 & 12.915 & -6.530 & 1.00 & 0.00\end{array}$
C

$\mathrm{H}$

$\mathrm{H}$

C

O

$\mathrm{H}$

$\mathrm{Fe}$

C

C

C

$\mathrm{H}$
$\mathrm{C}$

C

C

$\mathrm{H}$
$\mathrm{C}$

C

C

H

C

C
C

$\mathrm{H}$ 


\begin{tabular}{|c|c|c|c|c|c|c|c|c|c|c|}
\hline HETATM & 914 & N21 & HEM & 97 & 4.305 & 12.513 & -7.564 & 1.00 & 0.00 & $\mathrm{~N}$ \\
\hline HETATM & 915 & N22 & HEM & 97 & 7.009 & 11.566 & $-7 \cdot 342$ & 1.00 & 0.00 & $\mathrm{~N}$ \\
\hline HETATM & 916 & N23 & HEM & 97 & 6.338 & 10.303 & -4.841 & 1.00 & 0.00 & $\mathrm{~N}$ \\
\hline HETATM & 917 & N24 & HEM & 97 & 3.639 & 11.187 & -5.077 & 1.00 & 0.00 & $\mathrm{~N}$ \\
\hline HETATM & 918 & C99 & HEM & 97 & 4.545 & 8.322 & -7.579 & 1.00 & 0.00 & C \\
\hline HETATM & 919 & 091 & HEM & 97 & 4.705 & 9.635 & -7.567 & 1.00 & 0.00 & 0 \\
\hline HETATM & 920 & 092 & HEM & 97 & 4.391 & 7.705 & -8.635 & 1.00 & 0.00 & O \\
\hline HETATM & 921 & СТ91 & HEM & 97 & 4.561 & 7.640 & -6.223 & 1.00 & 0.00 & C \\
\hline HETATM & 922 & H911 & HEM & 97 & 5.540 & 7.836 & -5.760 & 1.00 & 0.00 & $\mathrm{H}$ \\
\hline HETATM & 923 & H912 & HEM & 97 & 3.813 & 8.130 & -5.580 & 1.00 & 0.00 & $\mathrm{H}$ \\
\hline HETATM & 924 & CT92 & HEM & 97 & 4.302 & 6.136 & -6.334 & 1.00 & 0.00 & C \\
\hline HETATM & 925 & H921 & HEM & 97 & 5.098 & 5.676 & -6.931 & 1.00 & 0.00 & $\mathrm{H}$ \\
\hline HETATM & 926 & H922 & HEM & 97 & 3.377 & 5.980 & -6.905 & 1.00 & 0.00 & $\mathrm{H}$ \\
\hline \multicolumn{11}{|l|}{ TER } \\
\hline HETATM & 927 & $\mathrm{FE}$ & HEM & 98 & 5.903 & 3.589 & -1.491 & 1.00 & 0.00 & $\mathrm{Fe}$ \\
\hline HETATM & 928 & $\mathrm{C} 1$ & HEM & 98 & 8.131 & 1.717 & -0.429 & 1.00 & 0.00 & C \\
\hline HETATM & 929 & C2 & HEM & 98 & 8.490 & 0.927 & 0.742 & 1.00 & 0.00 & C \\
\hline HETATM & 930 & C3 & HEM & 98 & 7.344 & 0.830 & 1.517 & 1.00 & 0.00 & C \\
\hline HETATM & 931 & C4 & HEM & 98 & 6.315 & 1.547 & 0.809 & 1.00 & 0.00 & C \\
\hline HETATM & 932 & C5 & HEM & 98 & 5.000 & 1.666 & 1.247 & 1.00 & 0.00 & C \\
\hline HETATM & 933 & H5 & HEM & 98 & 4.746 & 1.170 & 2.182 & 1.00 & 0.00 & $\mathrm{H}$ \\
\hline HETATM & 934 & $\mathrm{C} 6$ & HEM & 98 & 3.980 & 2.364 & 0.611 & 1.00 & 0.00 & C \\
\hline HETATM & 935 & C7 & HEM & 98 & 2.611 & 2.437 & 1.081 & 1.00 & 0.00 & C \\
\hline HETATM & 936 & $\mathrm{C} 8$ & HEM & 98 & 1.906 & 3.157 & 0.134 & 1.00 & 0.00 & C \\
\hline HETATM & 937 & C9 & HEM & 98 & 2.857 & 3.522 & -0.891 & 1.00 & 0.00 & C \\
\hline HETATM & 938 & $\mathrm{C} 10$ & HEM & 98 & 2.552 & 4.256 & -2.034 & 1.00 & 0.00 & C \\
\hline HETATM & 939 & $\mathrm{H} 10$ & HEM & 98 & 1.526 & 4.600 & -2.140 & 1.00 & 0.00 & $\mathrm{H}$ \\
\hline HETATM & 940 & $\mathrm{C} 11$ & HEM & 98 & 3.427 & 4.596 & -3.057 & 1.00 & 0.00 & C \\
\hline HETATM & 941 & $\mathrm{C} 12$ & HEM & 98 & 3.046 & 5.322 & -4.247 & 1.00 & 0.00 & C \\
\hline HETATM & 942 & $\mathrm{C} 13$ & HEM & 98 & 4.185 & 5.469 & -5.004 & 1.00 & 0.00 & C \\
\hline HETATM & 943 & C14 & HEM & 98 & 5.258 & 4.828 & -4.273 & 1.00 & 0.00 & C \\
\hline HETATM & 944 & C15 & HEM & 98 & 6.586 & 4.773 & -4.694 & 1.00 & 0.00 & C \\
\hline HETATM & 945 & H15 & HEM & 98 & 6.826 & 5.258 & -5.642 & 1.00 & 0.00 & $\mathrm{H}$ \\
\hline HETATM & 946 & $\mathrm{C} 16$ & HEM & 98 & 7.617 & 4.091 & -4.051 & 1.00 & 0.00 & $\mathrm{C}$ \\
\hline HETATM & 947 & $\mathrm{C} 17$ & HEM & 98 & 8.982 & 3.978 & -4.533 & 1.00 & 0.00 & C \\
\hline HETATM & 948 & $\mathrm{C} 18$ & HEM & 98 & 9.658 & 3.210 & -3.609 & 1.00 & 0.00 & C \\
\hline HETATM & 949 & C19 & HEM & 98 & 8.708 & 2.858 & -2.573 & 1.00 & 0.00 & C \\
\hline HETATM & 950 & $\mathrm{C} 20$ & HEM & 98 & 8.999 & 2.067 & -1.462 & 1.00 & 0.00 & C \\
\hline HETATM & 951 & $\mathrm{H} 2 \mathrm{O}$ & HEM & 98 & 10.014 & 1.678 & -1.402 & 1.00 & 0.00 & $\mathrm{H}$ \\
\hline HETATM & 952 & N21 & HEM & 98 & 6.809 & 2.080 & -0.367 & 1.00 & 0.00 & $\mathrm{~N}$ \\
\hline HETATM & 953 & N22 & HEM & 98 & 4.105 & 3.027 & -0.590 & 1.00 & 0.00 & $\mathrm{~N}$ \\
\hline HETATM & 954 & N23 & HEM & 98 & 4.775 & 4.290 & -3.090 & 1.00 & 0.00 & $\mathrm{~N}$ \\
\hline HETATM & 955 & N2 4 & HEM & 98 & 7.475 & 3.406 & -2.854 & 1.00 & 0.00 & $\mathrm{~N}$ \\
\hline HETATM & 956 & C99 & HEM & 98 & 6.568 & 6.271 & -0.352 & 1.00 & 0.00 & C \\
\hline HETATM & 957 & 091 & HEM & 98 & 6.409 & 4.958 & -0.365 & 1.00 & 0.00 & O \\
\hline HETATM & 958 & 092 & HEM & 98 & 6.723 & 6.888 & 0.703 & 1.00 & 0.00 & O \\
\hline HETATM & 959 & CT 91 & HEM & 98 & 6.552 & 6.952 & -1.709 & 1.00 & 0.00 & C \\
\hline HETATM & 960 & H911 & HEM & 98 & 5.573 & 6.757 & -2.171 & 1.00 & 0.00 & $\mathrm{H}$ \\
\hline HETATM & 961 & H912 & HEM & 98 & 7.300 & 6.463 & -2.352 & 1.00 & 0.00 & $\mathrm{H}$ \\
\hline HETATM & 962 & CT92 & HEM & 98 & 6.812 & 8.457 & -1.597 & 1.00 & 0.00 & C \\
\hline HETATM & 963 & H921 & HEM & 98 & 7.737 & 8.613 & -1.027 & 1.00 & 0.00 & $\mathrm{H}$ \\
\hline HETATM & 964 & H922 & HEM & 98 & 6.015 & 8.917 & -1.000 & 1.00 & 0.00 & $\mathrm{H}$ \\
\hline \multicolumn{11}{|l|}{ TER } \\
\hline HETATM & 965 & $\mathrm{C} 2$ & VIN & 99 & 1.292 & 14.169 & -8.933 & 1.00 & 0.00 & C \\
\hline HETATM & 966 & H 21 & VIN & 99 & 0.476 & 13.470 & -8.768 & 1.00 & 0.00 & $\mathrm{H}$ \\
\hline HETATM & 967 & $\mathrm{C} 1$ & VIN & 99 & 0.959 & 15.394 & -9.335 & 1.00 & 0.00 & C \\
\hline HETATM & 968 & H11 & VIN & 99 & 1.691 & 16.172 & -9.442 & 1.00 & 0.00 & $\mathrm{H}$ \\
\hline HETATM & 969 & $\mathrm{H} 12$ & VIN & 99 & -0.082 & 15.663 & -9.501 & 1.00 & 0.00 & $\mathrm{H}$ \\
\hline \multicolumn{11}{|l|}{ TER } \\
\hline HETATM & 970 & $\mathrm{C} 1$ & $\mathrm{MET}$ & 100 & 3.956 & 14.424 & -10.771 & 1.00 & 0.00 & C \\
\hline HETATM & 971 & H11 & $\mathrm{MET}$ & 100 & 4.191 & 15.494 & -10.652 & 1.00 & 0.00 & $\mathrm{H}$ \\
\hline HETATM & 972 & $\mathrm{H} 12$ & $\mathrm{MET}$ & 100 & 4.769 & 13.959 & $-11 \cdot 341$ & 1.00 & 0.00 & $\mathrm{H}$ \\
\hline HETATM & 973 & H13 & $\mathrm{MET}$ & 100 & 3.048 & 14.346 & $-11 \cdot 382$ & 1.00 & 0.00 & $\mathrm{H}$ \\
\hline \multicolumn{11}{|l|}{ TER } \\
\hline HETATM & 974 & C2 & VIN & 101 & 8.966 & 12.746 & -10.256 & 1.00 & 0.00 & C \\
\hline HETATM & 975 & H2 1 & VIN & 101 & 8.300 & 12.645 & -11.115 & 1.00 & 0.00 & $\mathrm{H}$ \\
\hline HETATM & 976 & $\mathrm{C} 1$ & VIN & 101 & 10.133 & 13.390 & -10.415 & 1.00 & 0.00 & C \\
\hline HETATM & 977 & H11 & VIN & 101 & 10.813 & 13.546 & -9.585 & 1.00 & 0.00 & $\mathrm{H}$ \\
\hline HETATM & 978 & H12 & VIN & 101 & 10.438 & 13.788 & -11.381 & 1.00 & 0.00 & $\mathrm{H}$ \\
\hline TER & & & & & & & & & & \\
\hline HETATM & 979 & $\mathrm{C} 1$ & MET & 102 & 10.651 & 11.062 & -8.052 & 1.00 & 0.00 & C \\
\hline HETATM & 980 & $\mathrm{H} 11$ & $\mathrm{MET}$ & 102 & 11.242 & 11.727 & -7.403 & 1.00 & 0.00 & $\mathrm{H}$ \\
\hline HETATM & 981 & $\mathrm{H} 12$ & $\mathrm{MET}$ & 102 & 10.807 & 10.040 & -7.685 & 1.00 & 0.00 & $\mathrm{H}$ \\
\hline HETATM & 982 & H13 & $\mathrm{MET}$ & 102 & 11.083 & 11.128 & -9.057 & 1.00 & 0.00 & $\mathrm{H}$ \\
\hline TER & & & & & & & & & & \\
\hline HETATM & 983 & C5 & $\mathrm{ACP}$ & 103 & 1.562 & 9.981 & -2.161 & 1.00 & 0.00 & C \\
\hline HETATM & 984 & H51 & $\mathrm{ACP}$ & 103 & 2.301 & 9.969 & -1.349 & 1.00 & 0.00 & $\mathrm{H}$ \\
\hline HETATM & 985 & H52 & $\mathrm{ACP}$ & 103 & 0.722 & 10.589 & -1.798 & 1.00 & 0.00 & $\mathrm{H}$ \\
\hline HETATM & 986 & C4 & $\mathrm{ACP}$ & 103 & 1.084 & 8.543 & -2.434 & 1.00 & 0.00 & $C$ \\
\hline
\end{tabular}




\begin{tabular}{|c|c|c|c|c|c|c|c|c|c|c|}
\hline HETATM & 987 & $\mathrm{H} 41$ & $\mathrm{ACP}$ & 103 & 0.526 & 8.482 & -3.382 & 1.00 & 0.00 & $\mathrm{H}$ \\
\hline HETATM & 988 & $\mathrm{H} 42$ & $\mathrm{ACP}$ & 103 & 1.954 & 7.877 & -2.541 & 1.00 & 0.00 & $\mathrm{H}$ \\
\hline HETATM & 989 & $\mathrm{C} 1$ & $\mathrm{ACP}$ & 103 & 0.220 & 7.931 & -1.358 & 1.00 & 0.00 & C \\
\hline HETATM & 990 & $\mathrm{O} 2$ & $\mathrm{ACP}$ & 103 & -0.008 & 8.454 & -0.260 & 1.00 & 0.00 & 0 \\
\hline HETATM & 991 & 03 & $\mathrm{ACP}$ & 103 & -0.296 & 6.751 & -1.722 & 1.00 & 0.00 & 0 \\
\hline HETATM & 992 & H3 & $\mathrm{ACP}$ & 103 & -0.905 & 6.397 & -0.986 & 1.00 & 0.00 & $\mathrm{H}$ \\
\hline \multicolumn{11}{|l|}{ TER } \\
\hline HETATM & 993 & $\mathrm{C} 1$ & MET & 104 & 9.458 & 8.855 & -3.334 & 1.00 & 0.00 & C \\
\hline HETATM & 994 & $\mathrm{H} 11$ & $\mathrm{MET}$ & 104 & 10.011 & 9.694 & -2.884 & 1.00 & 0.00 & $\mathrm{H}$ \\
\hline HETATM & 995 & $\mathrm{H} 12$ & $\mathrm{MET}$ & 104 & 9.465 & 8.029 & -2.613 & 1.00 & 0.00 & $\mathrm{H}$ \\
\hline HETATM & 996 & $\mathrm{H} 13$ & $\mathrm{MET}$ & 104 & 10.029 & 8.535 & -4.214 & 1.00 & 0.00 & $\mathrm{H}$ \\
\hline \multicolumn{11}{|l|}{ TER } \\
\hline HETATM & 997 & $\mathrm{C} 1$ & MET & 105 & 0.025 & 11.803 & -4.285 & 1.00 & 0.00 & C \\
\hline HETATM & 998 & H11 & MET & 105 & -0.420 & 11.594 & -3.305 & 1.00 & 0.00 & $\mathrm{H}$ \\
\hline HETATM & 999 & $\mathrm{H} 12$ & $\mathrm{MET}$ & 105 & -0.086 & 12.883 & -4.460 & 1.00 & 0.00 & $\mathrm{H}$ \\
\hline HETATM & 1000 & $\mathrm{H} 13$ & $\mathrm{MET}$ & 105 & -0.576 & 11.278 & -5.042 & 1.00 & 0.00 & $\mathrm{H}$ \\
\hline \multicolumn{11}{|l|}{ TER } \\
\hline HETATM & 1001 & $\mathrm{C} 2$ & VIN & 106 & 9.822 & 0.424 & 1.002 & 1.00 & 0.00 & C \\
\hline HETATM & 1002 & H21 & VIN & 106 & 10.637 & 1.123 & 0.837 & 1.00 & 0.00 & $\mathrm{H}$ \\
\hline HETATM & 1003 & $\mathrm{C} 1$ & VIN & 106 & 10.154 & -0.801 & 1.403 & 1.00 & 0.00 & C \\
\hline HETATM & 1004 & $\mathrm{H} 11$ & VIN & 106 & 9.423 & -1.579 & 1.511 & 1.00 & 0.00 & $\mathrm{H}$ \\
\hline HETATM & 1005 & $\mathrm{H} 12$ & VIN & 106 & 11.196 & -1.070 & 1.570 & 1.00 & 0.00 & $\mathrm{H}$ \\
\hline \multicolumn{11}{|l|}{ TER } \\
\hline HETATM & 1006 & $\mathrm{C} 1$ & $\mathrm{MET}$ & 107 & 7.158 & 0.169 & 2.840 & 1.00 & 0.00 & C \\
\hline HETATM & 1007 & $\mathrm{H} 11$ & $\mathrm{MET}$ & 107 & 6.923 & -0.902 & 2.720 & 1.00 & 0.00 & $\mathrm{H}$ \\
\hline HETATM & 1008 & $\mathrm{H} 12$ & $\mathrm{MET}$ & 107 & 6.345 & 0.633 & 3.410 & 1.00 & 0.00 & $\mathrm{H}$ \\
\hline HETATM & 1009 & $\mathrm{H} 13$ & $\mathrm{MET}$ & 107 & 8.065 & 0.247 & 3.450 & 1.00 & 0.00 & $\mathrm{H}$ \\
\hline TER & & & & & & & & & & \\
\hline HETATM & 1010 & $\mathrm{C} 2$ & VIN & 108 & 2.148 & 1.847 & 2.325 & 1.00 & 0.00 & C \\
\hline HETATM & 1011 & H21 & VIN & 108 & 2.814 & 1.948 & 3.184 & 1.00 & 0.00 & $\mathrm{H}$ \\
\hline HETATM & 1012 & $\mathrm{C} 1$ & VIN & 108 & 0.981 & 1.203 & 2.484 & 1.00 & 0.00 & C \\
\hline HETATM & 1013 & $\mathrm{H} 11$ & VIN & 108 & 0.301 & 1.047 & 1.654 & 1.00 & 0.00 & $\mathrm{H}$ \\
\hline HETATM & 1014 & $\mathrm{H} 12$ & VIN & 108 & 0.676 & 0.805 & 3.450 & 1.00 & 0.00 & $\mathrm{H}$ \\
\hline TER & & & & & & & & & & \\
\hline HETATM & 1015 & $\mathrm{C} 1$ & $\mathrm{MET}$ & 109 & 0.463 & 3.530 & 0.121 & 1.00 & 0.00 & C \\
\hline HETATM & 1016 & $\mathrm{H} 11$ & $\mathrm{MET}$ & 109 & 0.307 & 4.553 & -0.246 & 1.00 & 0.00 & $\mathrm{H}$ \\
\hline HETATM & 1017 & $\mathrm{H} 12$ & MET & 109 & 0.031 & 3.465 & 1.126 & 1.00 & 0.00 & $\mathrm{H}$ \\
\hline HETATM & 1018 & $\mathrm{H} 13$ & $\mathrm{MET}$ & 109 & -0.128 & 2.866 & -0.528 & 1.00 & 0.00 & $\mathrm{H}$ \\
\hline TER & & & & & & & & & & \\
\hline HETATM & 1019 & C5 & $\mathrm{ACP}$ & 110 & 9.552 & 4.612 & -5.770 & 1.00 & 0.00 & C \\
\hline HETATM & 1020 & H51 & $\mathrm{ACP}$ & 110 & 8.813 & 4.623 & -6.582 & 1.00 & 0.00 & $\mathrm{H}$ \\
\hline HETATM & 1021 & H52 & $\mathrm{ACP}$ & 110 & 10.392 & 4.004 & -6.134 & 1.00 & 0.00 & $\mathrm{H}$ \\
\hline HETATM & 1022 & $\mathrm{C} 4$ & $\mathrm{ACP}$ & 110 & 10.030 & 6.050 & -5.497 & 1.00 & 0.00 & C \\
\hline HETATM & 1023 & H4 1 & $\mathrm{ACP}$ & 110 & 10.588 & 6.111 & -4.549 & 1.00 & 0.00 & $\mathrm{H}$ \\
\hline HETATM & 1024 & $\mathrm{H} 42$ & $\mathrm{ACP}$ & 110 & 9.160 & 6.716 & -5.390 & 1.00 & 0.00 & $\mathrm{H}$ \\
\hline HETATM & 1025 & $\mathrm{C} 1$ & $\mathrm{ACP}$ & 110 & 10.894 & 6.662 & -6.573 & 1.00 & 0.00 & C \\
\hline HETATM & 1026 & $\mathrm{O} 2$ & $\mathrm{ACP}$ & 110 & 11.122 & 6.139 & -7.671 & 1.00 & 0.00 & o \\
\hline HETATM & 1027 & 03 & $\mathrm{ACP}$ & 110 & 11.410 & 7.841 & -6.209 & 1.00 & 0.00 & O \\
\hline HETATM & 1028 & H3 & $\mathrm{ACP}$ & 110 & 12.019 & 8.196 & -6.945 & 1.00 & 0.00 & $\mathrm{H}$ \\
\hline TER & & & & & & & & & & \\
\hline HETATM & 1029 & $\mathrm{C} 1$ & $\mathrm{MET}$ & 111 & 1.655 & 5.738 & -4.597 & 1.00 & 0.00 & C \\
\hline HETATM & 1030 & H11 & $\mathrm{MET}$ & 111 & 1.103 & 4.899 & -5.047 & 1.00 & 0.00 & $\mathrm{H}$ \\
\hline HETATM & 1031 & $\mathrm{H} 12$ & $\mathrm{MET}$ & 111 & 1.649 & 6.564 & -5.319 & 1.00 & 0.00 & $\mathrm{H}$ \\
\hline HETATM & 1032 & $\mathrm{H} 13$ & $\mathrm{MET}$ & 111 & 1.084 & 6.058 & -3.717 & 1.00 & 0.00 & $\mathrm{H}$ \\
\hline TER & & & & & & & & & & \\
\hline HETATM & 1033 & $\mathrm{C} 1$ & MET & 112 & 11.089 & 2.790 & -3.646 & 1.00 & 0.00 & C \\
\hline HETATM & 1034 & H11 & MET & 112 & 11.534 & 2.998 & -4.626 & 1.00 & 0.00 & $\mathrm{H}$ \\
\hline HETATM & 1035 & $\mathrm{H} 12$ & $\mathrm{MET}$ & 112 & 11.199 & 1.709 & -3.471 & 1.00 & 0.00 & $\mathrm{H}$ \\
\hline HETATM & 1036 & $\mathrm{H} 13$ & MET & 112 & 11.690 & 3.315 & -2.889 & 1.00 & 0.00 & $\mathrm{H}$ \\
\hline TER & & & & & & & & & & \\
\hline HETATM & 1037 & $\mathrm{FE}$ & HEM & 113 & 7.128 & -3.343 & -6.440 & 1.00 & 0.00 & $\mathrm{Fe}$ \\
\hline HETATM & 1038 & $\mathrm{C} 1$ & HEM & 113 & 4.900 & -1.471 & -7.502 & 1.00 & 0.00 & C \\
\hline HETATM & 1039 & $\mathrm{C} 2$ & HEM & 113 & 4.541 & -0.680 & -8.674 & 1.00 & 0.00 & C \\
\hline HETATM & 1040 & $\mathrm{C} 3$ & HEM & 113 & 5.686 & -0.583 & -9.448 & 1.00 & 0.00 & C \\
\hline HETATM & 1041 & C4 & HEM & 113 & 6.716 & -1.301 & -8.740 & 1.00 & 0.00 & C \\
\hline HETATM & 1042 & C5 & HEM & 113 & 8.031 & -1.420 & -9.178 & 1.00 & 0.00 & C \\
\hline HETATM & 1043 & H5 & HEM & 113 & 8.284 & -0.923 & -10.113 & 1.00 & 0.00 & $\mathrm{H}$ \\
\hline HETATM & 1044 & $\mathrm{C} 6$ & HEM & 113 & 9.050 & -2.118 & -8.542 & 1.00 & 0.00 & C \\
\hline HETATM & 1045 & C7 & HEM & 113 & 10.419 & -2.191 & -9.012 & 1.00 & 0.00 & C \\
\hline HETATM & 1046 & $\mathrm{C} 8$ & HEM & 113 & 11.125 & -2.910 & -8.065 & 1.00 & 0.00 & C \\
\hline HETATM & 1047 & $\mathrm{C} 9$ & HEM & 113 & 10.174 & -3.275 & -7.041 & 1.00 & 0.00 & C \\
\hline HETATM & 1048 & $\mathrm{C} 10$ & HEM & 113 & 10.478 & -4.010 & -5.897 & 1.00 & 0.00 & $\mathrm{C}$ \\
\hline HETATM & 1049 & $\mathrm{H} 10$ & HEM & 113 & 11.505 & -4.354 & -5.791 & 1.00 & 0.00 & $\mathrm{H}$ \\
\hline HETATM & 1050 & C11 & HEM & 113 & 9.604 & -4.350 & -4.874 & 1.00 & 0.00 & C \\
\hline HETATM & 1051 & $\mathrm{C} 12$ & HEM & 113 & 9.985 & -5.076 & -3.684 & 1.00 & 0.00 & C \\
\hline HETATM & 1052 & $\mathrm{C} 13$ & HEM & 113 & 8.845 & -5.223 & -2.927 & 1.00 & 0.00 & C \\
\hline HETATM & 1053 & C14 & HEM & 113 & 7.772 & -4.581 & -3.659 & 1.00 & 0.00 & C \\
\hline HETATM & 1054 & C15 & HEM & 113 & 6.444 & -4.527 & -3.237 & 1.00 & 0.00 & C \\
\hline HETATM & 1055 & H15 & HEM & 113 & 6.204 & -5.011 & -2.289 & 1.00 & 0.00 & 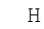 \\
\hline
\end{tabular}


$\begin{array}{lrrll}\text { HETATM } & 1056 & \text { C16 } & \text { HEM } & 113 \\ \text { HETATM } & 1057 & \text { C17 } & \text { HEM } & 113 \\ \text { HETATM } & 1058 & \text { C18 } & \text { HEM } & 113 \\ \text { HETATM } & 1059 & \text { C19 } & \text { HEM } & 113 \\ \text { HETATM } & 1060 & \text { C20 } & \text { HEM } & 113 \\ \text { HETATM } & 1061 & \text { H20 } & \text { HEM } & 113 \\ \text { HETATM } & 1062 & \text { N21 } & \text { HEM } & 113 \\ \text { HETATM } & 1063 & \text { N22 } & \text { HEM } & 113 \\ \text { HETATM } & 1064 & \text { N23 } & \text { HEM } & 113 \\ \text { HETATM } & 1065 & \text { N24 } & \text { HEM } & 113 \\ \text { HETATM } & 1066 & \text { C99 } & \text { HEM } & 113 \\ \text { HETATM } & 1067 & \text { O91 } & \text { HEM } & 113 \\ \text { HETATM } & 1068 & \text { O92 } & \text { HEM } & 113 \\ \text { HETATM } & 1069 & \text { CT91 } & \text { HEM } & 113 \\ \text { HETATM } & 1070 & \text { H911 } & \text { HEM } & 113 \\ \text { HETATM } & 1071 & \text { H912 } & \text { HEM } & 113 \\ \text { HETATM } & 1072 & \text { CT92 } & \text { HEM } & 113 \\ \text { HETATM } & 1073 & \text { H921 } & \text { HEM } & 113 \\ \text { HETATM } & 1074 & \text { H922 } & \text { HEM } & 113 \\ \text { TER } & & & & \end{array}$

HETATM 1075 FE HEM 114

HETATM 1076 C1 HEM 114

$\begin{array}{lll}\text { HETATM } 1077 \text { C2 HEM } & 114\end{array}$

HETATM 1078 C3 HEM 114

HETATM 1079 C4 HEM 114

HETATM 1080 C5 HEM 114

HETATM 1081 H5 HEM 114

HETATM 1082 C6 HEM 114

HETATM $1083 \quad$ C7 HEM 114

HETATM 1084 C8 HEM 114

HETATM 1085 C9 HEM 114

HETATM $1086 \quad$ C10 HEM 114

HETATM 1087 H10 HEM 114

HETATM 1088 C11 HEM 114

HETATM 1089 C12 HEM 114

HETATM $1090 \quad$ C13 HEM 114

HETATM 1091 C14 HEM 114

HETATM 1092 C15 HEM 114

HETATM 1093 H15 HEM 114

HETATM 1094 C16 HEM 114

HETATM 1095 C17 HEM 114

HETATM 1096 C18 HEM 114

HETATM 1097 C19 HEM 114

HETATM 1098 C20 HEM 114

HETATM 1099 H2O HEM 114

HETATM 1100 N21 HEM 114

HETATM 1101 N22 HEM 114

HETATM 1102 N23 HEM 114

HETATM 1103 N24 HEM 114

HETATM 1104 C99 HEM 114

HETATM 1105091 HEM 114

HETATM 1106 O92 HEM 114

HETATM 1107 CT91 HEM 114

HETATM 1108 H911 HEM 114

HETATM 1109 H912 HEM 114

HETATM 1110 CT92 HEM 114

HETATM 1111 H921 HEM 114

HETATM 1112 H922 HEM 114

TER

HETATM 1113 C2 VIN 115

HETATM 1114 H21 VIN 115

HETATM 1115 C1 VIN 115

HETATM 1116 H11 VIN 115

HETATM 1117 H12 VIN 115

TER

HETATM 1118 C1 MET 116

HETATM 1119 H11 MET 116

HETATM 1120 H12 MET 116

HETATM 1121 H13 MET 116

TER

HETATM 1122 C2 VIN 117

HETATM 1123 H21 VIN 117

HETATM 1124 C1 VIN 117

HETATM 1125 H11 VIN 117

HETATM 1126 H12 VIN 117

TER

HETATM 1127 C1 MET 118

HETATM 1128 H11 MET 118

HETATM 1129 H12 MET 118

\begin{tabular}{|c|c|c|c|c|c|}
\hline 5.414 & -3.845 & -3.880 & 1.00 & 0.00 & C \\
\hline 4.048 & -3.732 & -3.398 & 1.00 & 0.00 & C \\
\hline 3.373 & -2.964 & -4.322 & 1.00 & 0.00 & C \\
\hline 4.322 & -2.612 & -5.358 & 1.00 & 0.00 & C \\
\hline 4.031 & -1.821 & -6.469 & 1.00 & 0.00 & C \\
\hline 3.017 & -1.432 & -6.530 & 1.00 & 0.00 & $\mathrm{H}$ \\
\hline 6.221 & -1.834 & -7.564 & 1.00 & 0.00 & $\mathrm{~N}$ \\
\hline 8.926 & -2.781 & -7.342 & 1.00 & 0.00 & $\mathrm{~N}$ \\
\hline 8.255 & -4.044 & -4.841 & 1.00 & 0.00 & $\mathrm{~N}$ \\
\hline 5.555 & -3.160 & -5.077 & 1.00 & 0.00 & $\mathrm{~N}$ \\
\hline 6.462 & -6.025 & -7.579 & 1.00 & 0.00 & C \\
\hline 6.622 & -4.711 & -7.567 & 1.00 & 0.00 & 0 \\
\hline 6.307 & -6.642 & -8.635 & 1.00 & 0.00 & 0 \\
\hline 6.478 & -6.706 & -6.223 & 1.00 & 0.00 & C \\
\hline 7.457 & -6.511 & -5.760 & 1.00 & 0.00 & $\mathrm{H}$ \\
\hline 5.730 & -6.216 & -5.580 & 1.00 & 0.00 & $\mathrm{H}$ \\
\hline 6.219 & -8.211 & -6.334 & 1.00 & 0.00 & C \\
\hline 7.015 & -8.671 & -6.931 & 1.00 & 0.00 & $\mathrm{H}$ \\
\hline 5.294 & -8.367 & -6.905 & 1.00 & 0.00 & $\mathrm{H}$ \\
\hline 7.820 & -10.757 & -1.491 & 1.00 & 0.00 & $\mathrm{Fe}$ \\
\hline 10.048 & -12.629 & -0.429 & 1.00 & 0.00 & C \\
\hline 10.407 & -13.420 & 0.742 & 1.00 & 0.00 & $\mathrm{C}$ \\
\hline 9.261 & -13.517 & 1.517 & 1.00 & 0.00 & C \\
\hline 8.231 & -12.800 & 0.809 & 1.00 & 0.00 & $\mathrm{C}$ \\
\hline 6.916 & -12.681 & 1.247 & 1.00 & 0.00 & C \\
\hline 6.663 & -13.177 & 2.182 & 1.00 & 0.00 & $\mathrm{H}$ \\
\hline 5.897 & -11.982 & 0.611 & 1.00 & 0.00 & C \\
\hline 4.528 & -11.910 & 1.081 & 1.00 & 0.00 & C \\
\hline 3.823 & -11.190 & 0.134 & 1.00 & 0.00 & C \\
\hline 4.774 & -10.825 & -0.891 & 1.00 & 0.00 & C \\
\hline 4.469 & -10.090 & -2.034 & 1.00 & 0.00 & C \\
\hline 3.442 & -9.746 & -2.140 & 1.00 & 0.00 & $\mathrm{H}$ \\
\hline 5.344 & -9.750 & -3.057 & 1.00 & 0.00 & $\mathrm{C}$ \\
\hline 4.963 & -9.025 & -4.247 & 1.00 & 0.00 & C \\
\hline 6.102 & -8.878 & -5.004 & 1.00 & 0.00 & $\mathrm{C}$ \\
\hline 7.175 & -9.519 & -4.273 & 1.00 & 0.00 & $\mathrm{C}$ \\
\hline 8.503 & -9.574 & -4.694 & 1.00 & 0.00 & $\mathrm{C}$ \\
\hline 8.743 & -9.089 & -5.642 & 1.00 & 0.00 & $\mathrm{H}$ \\
\hline 9.533 & -10.255 & -4.051 & 1.00 & 0.00 & C \\
\hline 10.899 & -10.369 & -4.533 & 1.00 & 0.00 & C \\
\hline 11.575 & -11.137 & -3.609 & 1.00 & 0.00 & $\mathrm{C}$ \\
\hline 10.625 & -11.489 & -2.573 & 1.00 & 0.00 & C \\
\hline 10.916 & -12.280 & -1.462 & 1.00 & 0.00 & C \\
\hline 11.930 & -12.669 & -1.402 & 1.00 & 0.00 & $\mathrm{H}$ \\
\hline 8.726 & -12.266 & -0.367 & 1.00 & 0.00 & $\mathrm{~N}$ \\
\hline 6.021 & -11.319 & -0.590 & 1.00 & 0.00 & $\mathrm{~N}$ \\
\hline 6.692 & -10.056 & -3.090 & 1.00 & 0.00 & $\mathrm{~N}$ \\
\hline 9.392 & -10.941 & -2.854 & 1.00 & 0.00 & $\mathrm{~N}$ \\
\hline 8.485 & -8.076 & -0.352 & 1.00 & 0.00 & C \\
\hline 8.326 & -9.389 & -0.365 & 1.00 & 0.00 & O \\
\hline 8.640 & -7.458 & 0.703 & 1.00 & 0.00 & 0 \\
\hline 8.469 & -7.394 & -1.709 & 1.00 & 0.00 & $\mathrm{C}$ \\
\hline 7.490 & -7.590 & -2.171 & 1.00 & 0.00 & $\mathrm{H}$ \\
\hline 9.217 & -7.884 & -2.352 & 1.00 & 0.00 & $\mathrm{H}$ \\
\hline 8.729 & -5.890 & -1.597 & 1.00 & 0.00 & $\mathrm{C}$ \\
\hline 9.654 & -5.734 & -1.027 & 1.00 & 0.00 & $\mathrm{H}$ \\
\hline 7.932 & -5.429 & -1.000 & 1.00 & 0.00 & $\mathrm{H}$ \\
\hline 3.209 & -0.178 & -8.933 & 1.00 & 0.00 & C \\
\hline 2.393 & -0.876 & -8.768 & 1.00 & 0.00 & $\mathrm{H}$ \\
\hline 2.876 & 1.047 & -9.335 & 1.00 & 0.00 & C \\
\hline 3.607 & 1.826 & -9.442 & 1.00 & 0.00 & $\mathrm{H}$ \\
\hline 1.834 & 1.316 & -9.501 & 1.00 & 0.00 & $\mathrm{H}$ \\
\hline 5.873 & 0.077 & -10.771 & 1.00 & 0.00 & C \\
\hline 6.107 & 1.148 & -10.652 & 1.00 & 0.00 & $\mathrm{H}$ \\
\hline 6.686 & -0.387 & -11.341 & 1.00 & 0.00 & $\mathrm{H}$ \\
\hline 4.965 & -0.001 & $-11 \cdot 382$ & 1.00 & 0.00 & $\mathrm{H}$ \\
\hline 10.883 & -1.601 & -10.256 & 1.00 & 0.00 & C \\
\hline 10.217 & -1.702 & -11.115 & 1.00 & 0.00 & $\mathrm{H}$ \\
\hline 12.050 & -0.957 & -10.415 & 1.00 & 0.00 & C \\
\hline 12.729 & -0.800 & -9.585 & 1.00 & 0.00 & $\mathrm{H}$ \\
\hline 12.354 & -0.559 & -11.381 & 1.00 & 0.00 & $\mathrm{H}$ \\
\hline 12.567 & -3.284 & -8.052 & 1.00 & 0.00 & C \\
\hline 13.159 & -2.620 & -7.403 & 1.00 & 0.00 & $\mathrm{H}$ \\
\hline 12.724 & -4.306 & -7.685 & 1.00 & 0.00 & $\mathrm{H}$ \\
\hline
\end{tabular}




\begin{tabular}{|c|c|c|c|c|c|c|c|c|c|c|}
\hline $\begin{array}{l}\text { HETATM } \\
\text { TER }\end{array}$ & 1130 & $\mathrm{H} 13$ & MET & 118 & 13.000 & -3.219 & -9.057 & 1.00 & 0.00 & $\mathrm{H}$ \\
\hline HETATM & 1131 & $\mathrm{C} 5$ & $\mathrm{ACP}$ & 119 & 3.479 & -4.366 & -2.161 & 1.00 & 0.00 & C \\
\hline HETATM & 1132 & H51 & $\mathrm{ACP}$ & 119 & 4.218 & -4.377 & -1.349 & 1.00 & 0.00 & $\mathrm{H}$ \\
\hline HETATM & 1133 & H52 & $\mathrm{ACP}$ & 119 & 2.638 & -3.758 & -1.798 & 1.00 & 0.00 & $\mathrm{H}$ \\
\hline HETATM & 1134 & $\mathrm{C} 4$ & $\mathrm{ACP}$ & 119 & 3.000 & -5.803 & -2.434 & 1.00 & 0.00 & C \\
\hline HETATM & 1135 & H 41 & $\mathrm{ACP}$ & 119 & 2.443 & -5.865 & -3.382 & 1.00 & 0.00 & $\mathrm{H}$ \\
\hline HETATM & 1136 & $\mathrm{H} 42$ & $\mathrm{ACP}$ & 119 & 3.871 & -6.470 & -2.541 & 1.00 & 0.00 & $\mathrm{H}$ \\
\hline HETATM & 1137 & $\mathrm{C} 1$ & $\mathrm{ACP}$ & 119 & 2.136 & -6.416 & -1.358 & 1.00 & 0.00 & C \\
\hline HETATM & 1138 & $\mathrm{O} 2$ & $\mathrm{ACP}$ & 119 & 1.909 & -5.893 & -0.260 & 1.00 & 0.00 & 0 \\
\hline HETATM & 1139 & 03 & $\mathrm{ACP}$ & 119 & 1.621 & -7.595 & -1.722 & 1.00 & 0.00 & 0 \\
\hline HETATM & 1140 & H3 & $\mathrm{ACP}$ & 119 & 1.012 & -7.949 & -0.986 & 1.00 & 0.00 & $\mathrm{H}$ \\
\hline \multicolumn{11}{|c|}{ TER } \\
\hline HETATM & 1141 & $\mathrm{C} 1$ & MET & 120 & 11.375 & -5.492 & -3.334 & 1.00 & 0.00 & C \\
\hline HETATM & 1142 & $\mathrm{H} 11$ & MET & 120 & 11.927 & -4.653 & -2.884 & 1.00 & 0.00 & $\mathrm{H}$ \\
\hline HETATM & 1143 & $\mathrm{H} 12$ & $\mathrm{MET}$ & 120 & 11.382 & -6.318 & -2.613 & 1.00 & 0.00 & $\mathrm{H}$ \\
\hline HETATM & 1144 & $\mathrm{H} 13$ & $\mathrm{MET}$ & 120 & 11.946 & -5.812 & -4.214 & 1.00 & 0.00 & $\mathrm{H}$ \\
\hline \multicolumn{11}{|l|}{ TER } \\
\hline HETATM & 1145 & $\mathrm{C} 1$ & $\mathrm{MET}$ & 121 & 1.942 & -2.543 & -4.285 & 1.00 & 0.00 & C \\
\hline HETATM & 1146 & $\mathrm{H} 11$ & $\mathrm{MET}$ & 121 & 1.496 & -2.752 & -3.305 & 1.00 & 0.00 & $\mathrm{H}$ \\
\hline HETATM & 1147 & $\mathrm{H} 12$ & $\mathrm{MET}$ & 121 & 1.831 & -1.463 & -4.460 & 1.00 & 0.00 & $\mathrm{H}$ \\
\hline HETATM & 1148 & $\mathrm{H} 13$ & $\mathrm{MET}$ & 121 & 1.341 & -3.069 & -5.042 & 1.00 & 0.00 & $\mathrm{H}$ \\
\hline \multicolumn{11}{|l|}{ TER } \\
\hline HETATM & 1149 & $\mathrm{C} 2$ & VIN & 122 & 11.738 & -13.923 & 1.002 & 1.00 & 0.00 & C \\
\hline HETATM & 1150 & H 21 & VIN & 122 & 12.554 & -13.224 & 0.837 & 1.00 & 0.00 & $\mathrm{H}$ \\
\hline HETATM & 1151 & $\mathrm{C} 1$ & VIN & 122 & 12.071 & -15.147 & 1.403 & 1.00 & 0.00 & $\mathrm{C}$ \\
\hline HETATM & 1152 & $\mathrm{H} 11$ & VIN & 122 & 11.340 & -15.926 & 1.511 & 1.00 & 0.00 & $\mathrm{H}$ \\
\hline HETATM & 1153 & $\mathrm{H} 12$ & VIN & 122 & 13.113 & -15.417 & 1.570 & 1.00 & 0.00 & $\mathrm{H}$ \\
\hline \multicolumn{11}{|l|}{ TER } \\
\hline HETATM & 1154 & $\mathrm{C} 1$ & $\mathrm{MET}$ & 123 & 9.075 & -14.177 & 2.840 & 1.00 & 0.00 & $\mathrm{C}$ \\
\hline HETATM & 1155 & $\mathrm{H} 11$ & MET & 123 & 8.840 & -15.248 & 2.720 & 1.00 & 0.00 & $\mathrm{H}$ \\
\hline HETATM & 1156 & $\mathrm{H} 12$ & $\mathrm{MET}$ & 123 & 8.262 & -13.713 & 3.410 & 1.00 & 0.00 & $\mathrm{H}$ \\
\hline HETATM & 1157 & $\mathrm{H} 13$ & MET & 123 & 9.982 & -14.100 & 3.450 & 1.00 & 0.00 & $\mathrm{H}$ \\
\hline \multicolumn{11}{|c|}{ TER } \\
\hline HETATM & 1158 & $\mathrm{C} 2$ & VIN & 124 & 4.064 & -12.499 & 2.325 & 1.00 & 0.00 & C \\
\hline HETATM & 1159 & $\mathrm{H} 21$ & VIN & 124 & 4.730 & -12.399 & 3.184 & 1.00 & 0.00 & $\mathrm{H}$ \\
\hline HETATM & 1160 & $\mathrm{C} 1$ & VIN & 124 & 2.898 & -13.144 & 2.484 & 1.00 & 0.00 & C \\
\hline HETATM & 1161 & $\mathrm{H} 11$ & VIN & 124 & 2.218 & -13.300 & 1.654 & 1.00 & 0.00 & $\mathrm{H}$ \\
\hline HETATM & 1162 & $\mathrm{H} 12$ & VIN & 124 & 2.593 & -13.542 & 3.450 & 1.00 & 0.00 & $\mathrm{H}$ \\
\hline TER & & & & & & & & & & \\
\hline HETATM & 1163 & $\mathrm{C} 1$ & MET & 125 & 2.380 & -10.816 & 0.121 & 1.00 & 0.00 & C \\
\hline HETATM & 1164 & $\mathrm{H} 11$ & $\mathrm{MET}$ & 125 & 2.224 & -9.794 & -0.246 & 1.00 & 0.00 & $\mathrm{H}$ \\
\hline HETATM & 1165 & $\mathrm{H} 12$ & $\mathrm{MET}$ & 125 & 1.948 & -10.882 & 1.126 & 1.00 & 0.00 & $\mathrm{H}$ \\
\hline HETATM & 1166 & $\mathrm{H} 13$ & MET & 125 & 1.789 & -11.480 & -0.528 & 1.00 & 0.00 & $\mathrm{H}$ \\
\hline TER & & & & & & & & & & \\
\hline HETATM & 1167 & $\mathrm{C} 5$ & $\mathrm{ACP}$ & 126 & 11.468 & -9.735 & -5.770 & 1.00 & 0.00 & C \\
\hline HETATM & 1168 & H51 & $\mathrm{ACP}$ & 126 & 10.729 & -9.723 & -6.582 & 1.00 & 0.00 & $\mathrm{H}$ \\
\hline HETATM & 1169 & H52 & $\mathrm{ACP}$ & 126 & 12.309 & -10.343 & -6.134 & 1.00 & 0.00 & $\mathrm{H}$ \\
\hline HETATM & 1170 & $\mathrm{C} 4$ & $\mathrm{ACP}$ & 126 & 11.947 & -8.297 & -5.497 & 1.00 & 0.00 & $\mathrm{C}$ \\
\hline HETATM & 1171 & H 41 & $\mathrm{ACP}$ & 126 & 12.505 & -8.236 & -4.549 & 1.00 & 0.00 & $\mathrm{H}$ \\
\hline HETATM & 1172 & $\mathrm{H} 42$ & $\mathrm{ACP}$ & 126 & 11.076 & -7.630 & -5.390 & 1.00 & 0.00 & $\mathrm{H}$ \\
\hline HETATM & 1173 & $\mathrm{C} 1$ & $\mathrm{ACP}$ & 126 & 12.811 & -7.685 & -6.573 & 1.00 & 0.00 & C \\
\hline HETATM & 1174 & $\mathrm{O} 2$ & $\mathrm{ACP}$ & 126 & 13.039 & -8.208 & -7.671 & 1.00 & 0.00 & o \\
\hline HETATM & 1175 & 03 & $\mathrm{ACP}$ & 126 & 13.327 & -6.505 & -6.209 & 1.00 & 0.00 & o \\
\hline HETATM & 1176 & H3 & $\mathrm{ACP}$ & 126 & 13.936 & -6.151 & -6.945 & 1.00 & 0.00 & $\mathrm{H}$ \\
\hline TER & & & & & & & & & & \\
\hline HETATM & 1177 & $\mathrm{C} 1$ & $\mathrm{MET}$ & 127 & 3.572 & -8.608 & -4.597 & 1.00 & 0.00 & C \\
\hline HETATM & 1178 & $\mathrm{H} 11$ & $\mathrm{MET}$ & 127 & 3.020 & -9.448 & -5.047 & 1.00 & 0.00 & $\mathrm{H}$ \\
\hline HETATM & 1179 & $\mathrm{H} 12$ & $\mathrm{MET}$ & 127 & 3.566 & -7.782 & -5.319 & 1.00 & 0.00 & $\mathrm{H}$ \\
\hline HETATM & 1180 & H13 & $\mathrm{MET}$ & 127 & 3.001 & -8.289 & -3.717 & 1.00 & 0.00 & $\mathrm{H}$ \\
\hline TER & & & & & & & & & & \\
\hline HETATM & 1181 & $\mathrm{C} 1$ & $\mathrm{MET}$ & 128 & 13.006 & -11.557 & -3.646 & 1.00 & 0.00 & C \\
\hline HETATM & 1182 & $\mathrm{H} 11$ & MET & 128 & 13.451 & -11.348 & -4.626 & 1.00 & 0.00 & $\mathrm{H}$ \\
\hline HETATM & 1183 & $\mathrm{H} 12$ & MET & 128 & 13.116 & -12.637 & -3.471 & 1.00 & 0.00 & $\mathrm{H}$ \\
\hline HETATM & 1184 & $\mathrm{H} 13$ & $\mathrm{MET}$ & 128 & 13.606 & -11.031 & -2.889 & 1.00 & 0.00 & $\mathrm{H}$ \\
\hline TER & & & & & & & & & & \\
\hline HETATM & 1185 & $\mathrm{FE}$ & HEM & 129 & 3.294 & 25.350 & -6.440 & 1.00 & 0.00 & $\mathrm{Fe}$ \\
\hline HETATM & 1186 & $\mathrm{C} 1$ & HEM & 129 & 1.066 & 27.222 & -7.502 & 1.00 & 0.00 & C \\
\hline HETATM & 1187 & $\mathrm{C} 2$ & HEM & 129 & 0.707 & 28.013 & -8.674 & 1.00 & 0.00 & C \\
\hline HETATM & 1188 & C3 & HEM & 129 & 1.853 & 28.110 & -9.448 & 1.00 & 0.00 & C \\
\hline HETATM & 1189 & $\mathrm{C} 4$ & HEM & 129 & 2.882 & 27.392 & -8.740 & 1.00 & 0.00 & C \\
\hline HETATM & 1190 & C5 & HEM & 129 & 4.197 & 27.274 & -9.178 & 1.00 & 0.00 & C \\
\hline HETATM & 1191 & H5 & HEM & 129 & 4.451 & 27.770 & -10.113 & 1.00 & 0.00 & $\mathrm{H}$ \\
\hline HETATM & 1192 & $\mathrm{C} 6$ & HEM & 129 & 5.217 & 26.575 & -8.542 & 1.00 & 0.00 & C \\
\hline HETATM & 1193 & C7 & HEM & 129 & 6.586 & 26.503 & -9.012 & 1.00 & 0.00 & C \\
\hline HETATM & 1194 & $\mathrm{C} 8$ & HEM & 129 & 7.291 & 25.783 & -8.065 & 1.00 & 0.00 & C \\
\hline HETATM & 1195 & $\mathrm{C} 9$ & HEM & 129 & 6.340 & 25.418 & -7.041 & 1.00 & 0.00 & C \\
\hline HETATM & 1196 & $\mathrm{C} 10$ & HEM & 129 & 6.645 & 24.683 & -5.897 & 1.00 & 0.00 & C \\
\hline HETATM & 1197 & H1O & HEM & 129 & 7.671 & 24.339 & -5.791 & 1.00 & 0.00 & $\mathrm{H}$ \\
\hline
\end{tabular}




$\begin{array}{lrrll}\text { HETATM } & 1198 & \text { C11 } & \text { HEM } & 129 \\ \text { HETATM } & 1199 & \text { C12 } & \text { HEM } & 129 \\ \text { HETATM } & 1200 & \text { C13 } & \text { HEM } & 129 \\ \text { HETATM } & 1201 & \text { C14 } & \text { HEM } & 129 \\ \text { HETATM } & 1202 & \text { C15 } & \text { HEM } & 129 \\ \text { HETATM } & 1203 & \text { H15 } & \text { HEM } & 129 \\ \text { HETATM } & 1204 & \text { C16 } & \text { HEM } & 129 \\ \text { HETATM } & 1205 & \text { C17 } & \text { HEM } & 129 \\ \text { HETATM } & 1206 & \text { C18 } & \text { HEM } & 129 \\ \text { HETATM } & 1207 & \text { C19 } & \text { HEM } & 129 \\ \text { HETATM } & 1208 & \text { C20 } & \text { HEM } & 129 \\ \text { HETATM } & 1209 & \text { H20 } & \text { HEM } & 129 \\ \text { HETATM } & 1210 & \text { N21 } & \text { HEM } & 129 \\ \text { HETATM } & 1211 & \text { N22 } & \text { HEM } & 129 \\ \text { HETATM } & 1212 & \text { N23 } & \text { HEM } & 129 \\ \text { HETATM } & 1213 & \text { N24 } & \text { HEM } & 129 \\ \text { HETATM } & 1214 & \text { C99 } & \text { HEM } & 129 \\ \text { HETATM } & 1215 & 091 & \text { HEM } & 129 \\ \text { HETATM } & 1216 & 092 & \text { HEM } & 129 \\ \text { HETATM } & 1217 & \text { CT91 } & \text { HEM } & 129 \\ \text { HETATM } & 1218 & \text { H911 } & \text { HEM } & 129 \\ \text { HETATM } & 1219 & \text { H912 } & \text { HEM } & 129 \\ \text { HETATM } & 1220 & \text { CT92 } & \text { HEM } & 129 \\ \text { HETATM } & 1221 & \text { H921 } & \text { HEM } & 129 \\ \text { HETATM } & 1222 & \text { H922 } & \text { HEM } & 129 \\ \text { TER } & & & & \\ \text { HETATM } & 1223 & & \text { HEM } & 130\end{array}$
TER

HETATM 1223 FE HEM 130 HETATM 1224 C1 HEM 130 HETATM 1225 C2 HEM 130 HETATM 1226 C3 HEM 130 HETATM 1227 C4 HEM 130 HETATM 1228 C5 HEM 130 HETATM 1229 H5 HEM 130 HETATM 1230 C6 HEM 130 HETATM 1231 C7 HEM 130 HETATM 1232 C8 HEM 130 HETATM 1233 C9 HEM 130 HETATM 1234 C10 HEM 130 HETATM 1235 H10 HEM 130 HETATM 1236 C11 HEM 130 HETATM 1237 C12 HEM 130 HETATM 1238 C13 HEM 130 HETATM 1239 C14 HEM 130 HETATM 1240 C15 HEM 130 HETATM 1241 H15 HEM 130 HETATM 1242 C16 HEM 130 HETATM 1243 C17 HEM 130 HETATM 1244 C18 HEM 130 HETATM 1245 C19 HEM 130 HETATM 1246 C20 HEM 130 HETATM 1247 H2O HEM 130 HETATM 1248 N21 HEM 130 HETATM 1249 N22 HEM 130 HETATM 1250 N23 HEM 130 HETATM 1251 N24 HEM 130 HETATM 1252 C99 HEM 130 HETATM 1253091 HEM 130 HETATM $1254 \quad 092$ HEM 130 HETATM 1255 CT91 HEM 130 HETATM 1256 H911 HEM 130 HETATM 1257 H912 HEM 130 HETATM 1258 CT92 HEM 130 HETATM 1259 H921 HEM 130 HETATM 1260 H922 HEM 130 TER

HETATM 1261 C2 VIN 131 HETATM 1262 H21 VIN 131 HETATM 1263 C1 VIN 131 HETATM 1264 H11 VIN 131 HETATM 1265 H12 VIN 131 TER

HETATM 1266 C1 MET 132 HETATM 1267 H11 MET 132 HETATM 1268 H12 MET 132 HETATM 1269 H13 MET 132 TER

HETATM 1270 C2 VIN 133 HETATM 1271 H21 VIN 133 HETATM 1272 C1 VIN 133

\begin{tabular}{|c|c|c|c|c|c|}
\hline 5.770 & 24.343 & -4.874 & 1.00 & 0.00 & C \\
\hline 6.151 & 23.618 & -3.684 & 1.00 & 0.00 & C \\
\hline 5.012 & 23.470 & -2.927 & 1.00 & 0.00 & C \\
\hline 3.939 & 24.112 & -3.659 & 1.00 & 0.00 & $\mathrm{C}$ \\
\hline 2.611 & 24.167 & -3.237 & 1.00 & 0.00 & C \\
\hline 2.370 & 23.682 & -2.289 & 1.00 & 0.00 & $\mathrm{H}$ \\
\hline 1.580 & 24.848 & -3.880 & 1.00 & 0.00 & C \\
\hline 0.215 & 24.962 & -3.398 & 1.00 & 0.00 & C \\
\hline-0.461 & 25.729 & -4.322 & 1.00 & 0.00 & C \\
\hline 0.489 & 26.081 & -5.358 & 1.00 & 0.00 & C \\
\hline 0.197 & 26.872 & -6.469 & 1.00 & 0.00 & C \\
\hline-0.817 & 27.262 & -6.530 & 1.00 & 0.00 & $\mathrm{H}$ \\
\hline 2.388 & 26.859 & -7.564 & 1.00 & 0.00 & $\mathrm{~N}$ \\
\hline 5.092 & 25.912 & -7.342 & 1.00 & 0.00 & $\mathrm{~N}$ \\
\hline 4.422 & 24.649 & -4.841 & 1.00 & 0.00 & $\mathrm{~N}$ \\
\hline 1.722 & 25.533 & -5.077 & 1.00 & 0.00 & $\mathrm{~N}$ \\
\hline 2.629 & 22.668 & -7.579 & 1.00 & 0.00 & C \\
\hline 2.788 & 23.982 & -7.567 & 1.00 & 0.00 & 0 \\
\hline 2.474 & 22.051 & -8.635 & 1.00 & 0.00 & 0 \\
\hline 2.645 & 21.987 & -6.223 & 1.00 & 0.00 & $\mathrm{C}$ \\
\hline 3.623 & 22.182 & -5.760 & 1.00 & 0.00 & $\mathrm{H}$ \\
\hline 1.897 & 22.477 & -5.580 & 1.00 & 0.00 & $\mathrm{H}$ \\
\hline 2.385 & 20.483 & -6.334 & 1.00 & 0.00 & $\mathrm{C}$ \\
\hline 3.181 & 20.022 & -6.931 & 1.00 & 0.00 & $\mathrm{H}$ \\
\hline 1.460 & 20.327 & -6.905 & 1.00 & 0.00 & $\mathrm{H}$ \\
\hline 3.986 & 17.936 & -1.491 & 1.00 & 0.00 & $\mathrm{Fe}$ \\
\hline 6.214 & 16.064 & -0.429 & 1.00 & 0.00 & C \\
\hline 6.573 & 15.273 & 0.742 & 1.00 & 0.00 & C \\
\hline 5.427 & 15.176 & 1.517 & 1.00 & 0.00 & C \\
\hline 4.398 & 15.894 & 0.809 & 1.00 & 0.00 & C \\
\hline 3.083 & 16.012 & 1.247 & 1.00 & 0.00 & C \\
\hline 2.829 & 15.516 & 2.182 & 1.00 & 0.00 & $\mathrm{H}$ \\
\hline 2.063 & 16.711 & 0.611 & 1.00 & 0.00 & $\mathrm{C}$ \\
\hline 0.694 & 16.783 & 1.081 & 1.00 & 0.00 & C \\
\hline-0.011 & 17.503 & 0.134 & 1.00 & 0.00 & $\mathrm{C}$ \\
\hline 0.940 & 17.868 & -0.891 & 1.00 & 0.00 & $\mathrm{C}$ \\
\hline 0.636 & 18.603 & -2.034 & 1.00 & 0.00 & $\mathrm{C}$ \\
\hline-0.391 & 18.947 & -2.140 & 1.00 & 0.00 & $\mathrm{H}$ \\
\hline 1.510 & 18.943 & -3.057 & 1.00 & 0.00 & C \\
\hline 1.129 & 19.668 & -4.247 & 1.00 & 0.00 & $\mathrm{C}$ \\
\hline 2.269 & 19.816 & -5.004 & 1.00 & 0.00 & $\mathrm{C}$ \\
\hline 3.341 & 19.174 & -4.273 & 1.00 & 0.00 & C \\
\hline 4.670 & 19.119 & -4.694 & 1.00 & 0.00 & $\mathrm{C}$ \\
\hline 4.910 & 19.604 & -5.642 & 1.00 & 0.00 & $\mathrm{H}$ \\
\hline 5.700 & 18.438 & -4.051 & 1.00 & 0.00 & C \\
\hline 7.066 & 18.324 & -4.533 & 1.00 & 0.00 & $\mathrm{C}$ \\
\hline 7.741 & 17.557 & -3.609 & 1.00 & 0.00 & C \\
\hline 6.791 & 17.205 & -2.573 & 1.00 & 0.00 & C \\
\hline 7.083 & 16.414 & -1.462 & 1.00 & 0.00 & C \\
\hline 8.097 & 16.024 & -1.402 & 1.00 & 0.00 & $\mathrm{H}$ \\
\hline 4.892 & 16.427 & -0.367 & 1.00 & 0.00 & $\mathrm{~N}$ \\
\hline 2.188 & 17.374 & -0.590 & 1.00 & 0.00 & $\mathrm{~N}$ \\
\hline 2.859 & 18.637 & -3.090 & 1.00 & 0.00 & $\mathrm{~N}$ \\
\hline 5.558 & 17.753 & -2.854 & 1.00 & 0.00 & $\mathrm{~N}$ \\
\hline 4.652 & 20.618 & -0.352 & 1.00 & 0.00 & C \\
\hline 4.492 & 19.304 & -0.365 & 1.00 & 0.00 & 0 \\
\hline 4.806 & 21.235 & 0.703 & 1.00 & 0.00 & 0 \\
\hline 4.635 & 21.299 & -1.709 & 1.00 & 0.00 & C \\
\hline 3.657 & 21.104 & -2.171 & 1.00 & 0.00 & $\mathrm{H}$ \\
\hline 5.383 & 20.809 & -2.352 & 1.00 & 0.00 & $\mathrm{H}$ \\
\hline 4.895 & 22.803 & -1.597 & 1.00 & 0.00 & $\mathrm{C}$ \\
\hline 5.820 & 22.959 & -1.027 & 1.00 & 0.00 & $\mathrm{H}$ \\
\hline 4.099 & 23.264 & -1.000 & 1.00 & 0.00 & $\mathrm{H}$ \\
\hline-0.625 & 28.515 & -8.933 & 1.00 & 0.00 & $\mathrm{C}$ \\
\hline-1.440 & 27.817 & -8.768 & 1.00 & 0.00 & $\mathrm{H}$ \\
\hline-0.958 & 29.740 & -9.335 & 1.00 & 0.00 & C \\
\hline-0.226 & 30.519 & -9.442 & 1.00 & 0.00 & $\mathrm{H}$ \\
\hline-1.999 & 30.010 & -9.501 & 1.00 & 0.00 & $\mathrm{H}$ \\
\hline 2.039 & 28.770 & -10.771 & 1.00 & 0.00 & $\mathrm{C}$ \\
\hline 2.274 & 29.841 & -10.652 & 1.00 & 0.00 & $\mathrm{H}$ \\
\hline 2.852 & 28.306 & -11.341 & 1.00 & 0.00 & $\mathrm{H}$ \\
\hline 1.131 & 28.693 & $-11 \cdot 382$ & 1.00 & 0.00 & $\mathrm{H}$ \\
\hline 7.049 & 27.092 & -10.256 & 1.00 & 0.00 & C \\
\hline 6.383 & 26.991 & -11.115 & 1.00 & 0.00 & $\mathrm{H}$ \\
\hline 8.216 & 27.737 & -10.415 & 1.00 & 0.00 & C \\
\hline
\end{tabular}


HETATM 1273 H11 VIN 133 HETATM 1274 H12 VIN 133 TER

HETATM 1275 C1 MET 134 HETATM 1276 H11 MET 134 HETATM 1277 H12 MET 134 HETATM 1278 H13 MET 134 TER

HETATM 1279 C5 ACP 135 HETATM 1280 H51 ACP 135 HETATM 1281 H52 ACP 135 HETATM 1282 C4 ACP 135 HETATM 1283 H41 ACP 135 HETATM 1284 H42 ACP 135 HETATM 1285 C1 ACP 135 HETATM $1286 \quad 02$ ACP 135 HETATM $1287 \quad 03$ ACP 135 HETATM 1288 H3 ACP 135 TER

HETATM 1289 C1 MET 136 HETATM 1290 H11 MET 136 HETATM 1291 H12 MET 136 HETATM 1292 H13 MET 136 TER

HETATM 1293 C1 MET 137 HETATM 1294 H11 MET 137 HETATM 1295 H12 MET 137 HETATM 1296 H13 MET 137 TER

HETATM 1297 C2 VIN 138 HETATM 1298 H21 VIN 138 HETATM $1299 \quad$ C1 VIN 138 HETATM 1300 H11 VIN 138 HETATM 1301 H12 VIN 138 TER

HETATM 1302 C1 MET 139 HETATM 1303 H11 MET 139 HETATM 1304 H12 MET 139 HETATM 1305 H13 MET 139 TER

HETATM 1306 C2 VIN 140 HETATM 1307 H21 VIN 140 HETATM 1308 C1 VIN 140 HETATM 1309 H11 VIN 140 HETATM 1310 H12 VIN 140 TER

HETATM 1311 C1 MET 141 HETATM 1312 H11 MET 14 HETATM 1313 H12 MET 141 HETATM 1314 H13 MET 141 TER

HETATM 1315 C5 ACP 142 HETATM 1316 H51 ACP 142 HETATM 1317 H52 ACP 142 HETATM $1318 \quad \mathrm{C} 4 \mathrm{ACP} \quad 142$ HETATM 1319 H41 ACP 142 HETATM 1320 H42 ACP 142 HETATM $1321 \quad$ C1 ACP 142 HETATM 1322 O2 ACP 142 HETATM 1323 O3 ACP 142 HETATM 1324 H3 ACP 142 $T E R$

HETATM 1325 C1 MET 143 HETATM 1326 H11 MET 143 HETATM 1327 H12 MET 143 HETATM 1328 H13 MET 143 TER

HETATM 1329 C1 MET 144 HETATM 1330 H11 MET 144 HETATM 1331 H12 MET 144 HETATM 1332 H13 MET 144 TER

HETATM 1333 EE HEM 145 HETATM 1334 C1 HEM 145 HETATM 1335 C2 HEM 145 HETATM 1336 C3 HEM 145 HETATM 1337 C4 HEM 145 HETATM 1338 C5 HEM 145 HETATM 1339 H5 HEM 145

\begin{tabular}{|c|c|c|c|c|c|}
\hline 8.896 & 27.893 & -9.585 & 1.00 & 0.00 & $\mathrm{H}$ \\
\hline 8.521 & 28.135 & -11.381 & 1.00 & 0.00 & $\mathrm{H}$ \\
\hline 8.734 & 25.409 & -8.052 & 1.00 & 0.00 & $\mathrm{C}$ \\
\hline 9.325 & 26.073 & -7.403 & 1.00 & 0.00 & $\mathrm{H}$ \\
\hline 8.890 & 24.387 & -7.685 & 1.00 & 0.00 & $\mathrm{H}$ \\
\hline 9.166 & 25.475 & -9.057 & 1.00 & 0.00 & $\mathrm{H}$ \\
\hline-0.355 & 24.327 & -2.161 & 1.00 & 0.00 & $\mathrm{C}$ \\
\hline 0.384 & 24.316 & -1.349 & 1.00 & 0.00 & $\mathrm{H}$ \\
\hline-1.195 & 24.936 & -1.798 & 1.00 & 0.00 & $\mathrm{H}$ \\
\hline-0.833 & 22.890 & -2.434 & 1.00 & 0.00 & C \\
\hline-1.391 & 22.829 & -3.382 & 1.00 & 0.00 & $\mathrm{H}$ \\
\hline 0.037 & 22.223 & -2.541 & 1.00 & 0.00 & $\mathrm{H}$ \\
\hline-1.697 & 22.278 & -1.358 & 1.00 & 0.00 & C \\
\hline-1.925 & 22.800 & -0.260 & 1.00 & 0.00 & O \\
\hline-2.213 & 21.098 & -1.722 & 1.00 & 0.00 & 0 \\
\hline-2.822 & 20.744 & -0.986 & 1.00 & 0.00 & $\mathrm{H}$ \\
\hline 7.541 & 23.201 & -3.334 & 1.00 & 0.00 & $\mathrm{C}$ \\
\hline 8.094 & 24.040 & -2.884 & 1.00 & 0.00 & $\mathrm{H}$ \\
\hline 7.548 & 22.375 & -2.613 & 1.00 & 0.00 & $\mathrm{H}$ \\
\hline 8.112 & 22.882 & -4.214 & 1.00 & 0.00 & $\mathrm{H}$ \\
\hline-1.892 & 26.150 & -4.285 & 1.00 & 0.00 & $\mathrm{C}$ \\
\hline-2.337 & 25.941 & -3.305 & 1.00 & 0.00 & $\mathrm{H}$ \\
\hline-2.002 & 27.230 & -4.460 & 1.00 & 0.00 & $\mathrm{H}$ \\
\hline-2.493 & 25.624 & -5.042 & 1.00 & 0.00 & $\mathrm{H}$ \\
\hline 7.905 & 14.771 & 1.002 & 1.00 & 0.00 & C \\
\hline 8.721 & 15.469 & 0.837 & 1.00 & 0.00 & $\mathrm{H}$ \\
\hline 8.238 & 13.546 & 1.403 & 1.00 & 0.00 & C \\
\hline 7.506 & 12.767 & 1.511 & 1.00 & 0.00 & $\mathrm{H}$ \\
\hline 9.279 & 13.277 & 1.570 & 1.00 & 0.00 & $\mathrm{H}$ \\
\hline 5.241 & 14.516 & 2.840 & 1.00 & 0.00 & C \\
\hline 5.006 & 13.445 & 2.720 & 1.00 & 0.00 & $\mathrm{H}$ \\
\hline 4.428 & 14.980 & 3.410 & 1.00 & 0.00 & $\mathrm{H}$ \\
\hline 6.149 & 14.593 & 3.450 & 1.00 & 0.00 & $\mathrm{H}$ \\
\hline 0.231 & 16.194 & 2.325 & 1.00 & 0.00 & C \\
\hline 0.897 & 16.295 & 3.184 & 1.00 & 0.00 & $\mathrm{H}$ \\
\hline-0.936 & 15.550 & 2.484 & 1.00 & 0.00 & C \\
\hline-1.616 & 15.393 & 1.654 & 1.00 & 0.00 & $\mathrm{H}$ \\
\hline-1.241 & 15.151 & 3.450 & 1.00 & 0.00 & $\mathrm{H}$ \\
\hline-1.454 & 17.877 & 0.121 & 1.00 & 0.00 & C \\
\hline-1.610 & 18.899 & -0.246 & 1.00 & 0.00 & $\mathrm{H}$ \\
\hline-1.886 & 17.811 & 1.126 & 1.00 & 0.00 & $\mathrm{H}$ \\
\hline-2.045 & 17.213 & -0.528 & 1.00 & 0.00 & $\mathrm{H}$ \\
\hline 7.635 & 18.959 & -5.770 & 1.00 & 0.00 & c \\
\hline 6.896 & 18.970 & -6.582 & 1.00 & 0.00 & $\mathrm{H}$ \\
\hline 8.475 & 18.350 & -6.134 & 1.00 & 0.00 & $\mathrm{H}$ \\
\hline 8.113 & 20.396 & -5.497 & 1.00 & 0.00 & C \\
\hline 8.671 & 20.457 & -4.549 & 1.00 & 0.00 & $\mathrm{H}$ \\
\hline 7.243 & 21.063 & -5.390 & 1.00 & 0.00 & $\mathrm{H}$ \\
\hline 8.977 & 21.008 & -6.573 & 1.00 & 0.00 & C \\
\hline 9.205 & 20.486 & -7.671 & 1.00 & 0.00 & O \\
\hline 9.493 & 22.188 & -6.209 & 1.00 & 0.00 & 0 \\
\hline 10.102 & 22.542 & -6.945 & 1.00 & 0.00 & $\mathrm{H}$ \\
\hline-0.261 & 20.085 & -4.597 & 1.00 & 0.00 & C \\
\hline-0.814 & 19.246 & -5.047 & 1.00 & 0.00 & $\mathrm{H}$ \\
\hline-0.268 & 20.911 & -5.319 & 1.00 & 0.00 & $\mathrm{H}$ \\
\hline-0.832 & 20.405 & -3.717 & 1.00 & 0.00 & $\mathrm{H}$ \\
\hline 9.172 & 17.136 & -3.646 & 1.00 & 0.00 & C \\
\hline 9.617 & 17.345 & -4.626 & 1.00 & 0.00 & $\mathrm{H}$ \\
\hline 9.283 & 16.056 & -3.471 & 1.00 & 0.00 & $\mathrm{H}$ \\
\hline 9.773 & 17.662 & -2.889 & 1.00 & 0.00 & $\mathrm{H}$ \\
\hline 15.377 & 25.350 & -6.440 & 1.00 & 0.00 & $\mathrm{Fe}$ \\
\hline 13.149 & 27.222 & -7.502 & 1.00 & 0.00 & C \\
\hline 12.790 & 28.013 & -8.674 & 1.00 & 0.00 & C \\
\hline 13.936 & 28.110 & -9.448 & 1.00 & 0.00 & C \\
\hline 14.965 & 27.392 & -8.740 & 1.00 & 0.00 & C \\
\hline 16.280 & 27.274 & -9.178 & 1.00 & 0.00 & C \\
\hline 16.534 & 27.770 & -10.113 & 1.00 & 0.00 & $\mathrm{H}$ \\
\hline
\end{tabular}




\begin{tabular}{|c|c|c|c|c|c|c|c|c|c|c|}
\hline HETATM & 1340 & $\mathrm{C} 6$ & HEM & 145 & 17.300 & 26.575 & -8.542 & 1.00 & 0.00 & C \\
\hline HETATM & 1341 & C7 & HEM & 145 & 18.669 & 26.503 & -9.012 & 1.00 & 0.00 & C \\
\hline HETATM & 1342 & C8 & HEM & 145 & 19.374 & 25.783 & -8.065 & 1.00 & 0.00 & C \\
\hline HETATM & 1343 & $\mathrm{C} 9$ & HEM & 145 & 18.423 & 25.418 & -7.041 & 1.00 & 0.00 & $\mathrm{C}$ \\
\hline HETATM & 1344 & $\mathrm{C} 10$ & HEM & 145 & 18.727 & 24.683 & -5.897 & 1.00 & 0.00 & C \\
\hline HETATM & 1345 & $\mathrm{H} 10$ & HEM & 145 & 19.754 & 24.339 & -5.791 & 1.00 & 0.00 & $\mathrm{H}$ \\
\hline HETATM & 1346 & $\mathrm{C} 11$ & HEM & 145 & 17.853 & 24.343 & -4.874 & 1.00 & 0.00 & C \\
\hline HETATM & 1347 & $\mathrm{C} 12$ & HEM & 145 & 18.234 & 23.618 & -3.684 & 1.00 & 0.00 & C \\
\hline HETATM & 1348 & C13 & HEM & 145 & 17.094 & 23.470 & -2.927 & 1.00 & 0.00 & C \\
\hline HETATM & 1349 & C14 & HEM & 145 & 16.022 & 24.112 & -3.659 & 1.00 & 0.00 & C \\
\hline HETATM & 1350 & C15 & HEM & 145 & 14.694 & 24.167 & -3.237 & 1.00 & 0.00 & C \\
\hline HETATM & 1351 & H15 & HEM & 145 & 14.453 & 23.682 & -2.289 & 1.00 & 0.00 & $\mathrm{H}$ \\
\hline HETATM & 1352 & C16 & HEM & 145 & 13.663 & 24.848 & -3.880 & 1.00 & 0.00 & C \\
\hline HETATM & 1353 & $\mathrm{C} 17$ & HEM & 145 & 12.297 & 24.962 & -3.398 & 1.00 & 0.00 & C \\
\hline HETATM & 1354 & C18 & HEM & 145 & 11.622 & 25.729 & -4.322 & 1.00 & 0.00 & C \\
\hline HETATM & 1355 & C19 & HEM & 145 & 12.572 & 26.081 & -5.358 & 1.00 & 0.00 & C \\
\hline HETATM & 1356 & $\mathrm{C} 20$ & HEM & 145 & 12.280 & 26.872 & -6.469 & 1.00 & 0.00 & C \\
\hline HETATM & 1357 & $\mathrm{H} 2 \mathrm{O}$ & HEM & 145 & 11.266 & 27.262 & -6.530 & 1.00 & 0.00 & $\mathrm{H}$ \\
\hline HETATM & 1358 & $\mathrm{~N} 21$ & HEM & 145 & 14.471 & 26.859 & -7.564 & 1.00 & 0.00 & $\mathrm{~N}$ \\
\hline HETATM & 1359 & N22 & HEM & 145 & 17.175 & 25.912 & -7.342 & 1.00 & 0.00 & $\mathrm{~N}$ \\
\hline HETATM & 1360 & N23 & HEM & 145 & 16.504 & 24.649 & -4.841 & 1.00 & 0.00 & $\mathrm{~N}$ \\
\hline HETATM & 1361 & N24 & HEM & 145 & 13.805 & 25.533 & -5.077 & 1.00 & 0.00 & $\mathrm{~N}$ \\
\hline HETATM & 1362 & C99 & HEM & 145 & 14.711 & 22.668 & -7.579 & 1.00 & 0.00 & C \\
\hline HETATM & 1363 & 091 & HEM & 145 & 14.871 & 23.982 & -7.567 & 1.00 & 0.00 & 0 \\
\hline HETATM & 1364 & 092 & HEM & 145 & 14.557 & 22.051 & -8.635 & 1.00 & 0.00 & 0 \\
\hline HETATM & 1365 & CT91 & HEM & 145 & 14.728 & 21.987 & -6.223 & 1.00 & 0.00 & $\mathrm{C}$ \\
\hline HETATM & 1366 & H911 & HEM & 145 & 15.706 & 22.182 & -5.760 & 1.00 & 0.00 & $\mathrm{H}$ \\
\hline HETATM & 1367 & H912 & HEM & 145 & 13.980 & 22.477 & -5.580 & 1.00 & 0.00 & $\mathrm{H}$ \\
\hline HETATM & 1368 & СТ92 & HEM & 145 & 14.468 & 20.483 & -6.334 & 1.00 & 0.00 & C \\
\hline HETATM & 1369 & H921 & HEM & 145 & 15.264 & 20.022 & -6.931 & 1.00 & 0.00 & $\mathrm{H}$ \\
\hline HETATM & 1370 & H922 & HEM & 145 & 13.543 & 20.327 & -6.905 & 1.00 & 0.00 & $\mathrm{H}$ \\
\hline \multicolumn{11}{|l|}{ TER } \\
\hline HETATM & 1371 & $\mathrm{FE}$ & HEM & 146 & 16.069 & 17.936 & -1.491 & 1.00 & 0.00 & $\mathrm{Fe}$ \\
\hline HETATM & 1372 & $\mathrm{C} 1$ & HEM & 146 & 18.297 & 16.064 & -0.429 & 1.00 & 0.00 & C \\
\hline HETATM & 1373 & $\mathrm{C} 2$ & HEM & 146 & 18.656 & 15.273 & 0.742 & 1.00 & 0.00 & C \\
\hline HETATM & 1374 & $\mathrm{C} 3$ & HEM & 146 & 17.510 & 15.176 & 1.517 & 1.00 & 0.00 & C \\
\hline HETATM & 1375 & $\mathrm{C} 4$ & HEM & 146 & 16.481 & 15.894 & 0.809 & 1.00 & 0.00 & C \\
\hline HETATM & 1376 & $\mathrm{C} 5$ & HEM & 146 & 15.166 & 16.012 & 1.247 & 1.00 & 0.00 & C \\
\hline HETATM & 1377 & H5 & HEM & 146 & 14.912 & 15.516 & 2.182 & 1.00 & 0.00 & $\mathrm{H}$ \\
\hline HETATM & 1378 & $\mathrm{C} 6$ & HEM & 146 & 14.146 & 16.711 & 0.611 & 1.00 & 0.00 & C \\
\hline HETATM & 1379 & C7 & HEM & 146 & 12.777 & 16.783 & 1.081 & 1.00 & 0.00 & C \\
\hline HETATM & 1380 & $\mathrm{C} 8$ & HEM & 146 & 12.072 & 17.503 & 0.134 & 1.00 & 0.00 & C \\
\hline HETATM & 1381 & $\mathrm{C} 9$ & HEM & 146 & 13.023 & 17.868 & -0.891 & 1.00 & 0.00 & C \\
\hline HETATM & 1382 & $\mathrm{C} 10$ & HEM & 146 & 12.718 & 18.603 & -2.034 & 1.00 & 0.00 & C \\
\hline HETATM & 1383 & $\mathrm{H} 10$ & HEM & 146 & 11.692 & 18.947 & -2.140 & 1.00 & 0.00 & $\mathrm{H}$ \\
\hline HETATM & 1384 & $\mathrm{C} 11$ & HEM & 146 & 13.593 & 18.943 & -3.057 & 1.00 & 0.00 & C \\
\hline HETATM & 1385 & $\mathrm{C} 12$ & HEM & 146 & 13.212 & 19.668 & -4.247 & 1.00 & 0.00 & C \\
\hline HETATM & 1386 & C13 & HEM & 146 & 14.351 & 19.816 & -5.004 & 1.00 & 0.00 & C \\
\hline HETATM & 1387 & C14 & HEM & 146 & 15.424 & 19.174 & -4.273 & 1.00 & 0.00 & C \\
\hline HETATM & 1388 & $\mathrm{C} 15$ & HEM & 146 & 16.752 & 19.119 & -4.694 & 1.00 & 0.00 & C \\
\hline HETATM & 1389 & H1 5 & HEM & 146 & 16.993 & 19.604 & -5.642 & 1.00 & 0.00 & $\mathrm{H}$ \\
\hline HETATM & 1390 & C16 & HEM & 146 & 17.783 & 18.438 & -4.051 & 1.00 & 0.00 & C \\
\hline HETATM & 1391 & $\mathrm{C} 17$ & HEM & 146 & 19.149 & 18.324 & -4.533 & 1.00 & 0.00 & C \\
\hline HETATM & 1392 & C18 & HEM & 146 & 19.824 & 17.557 & -3.609 & 1.00 & 0.00 & C \\
\hline HETATM & 1393 & C19 & HEM & 146 & 18.874 & 17.205 & -2.573 & 1.00 & 0.00 & C \\
\hline HETATM & 1394 & $\mathrm{C} 20$ & HEM & 146 & 19.166 & 16.414 & -1.462 & 1.00 & 0.00 & C \\
\hline HETATM & 1395 & $\mathrm{H} 2 \mathrm{O}$ & HEM & 146 & 20.180 & 16.024 & -1.402 & 1.00 & 0.00 & $\mathrm{H}$ \\
\hline HETATM & 1396 & $\mathrm{~N} 21$ & HEM & 146 & 16.975 & 16.427 & -0.367 & 1.00 & 0.00 & $\mathrm{~N}$ \\
\hline HETATM & 1397 & N22 & HEM & 146 & 14.271 & 17.374 & -0.590 & 1.00 & 0.00 & $\mathrm{~N}$ \\
\hline HETATM & 1398 & N23 & HEM & 146 & 14.941 & 18.637 & -3.090 & 1.00 & 0.00 & $\mathrm{~N}$ \\
\hline HETATM & 1399 & N24 & HEM & 146 & 17.641 & 17.753 & -2.854 & 1.00 & 0.00 & $\mathrm{~N}$ \\
\hline HETATM & 1400 & C99 & HEM & 146 & 16.734 & 20.618 & -0.352 & 1.00 & 0.00 & C \\
\hline HETATM & 1401 & 091 & HEM & 146 & 16.575 & 19.304 & -0.365 & 1.00 & 0.00 & 0 \\
\hline HETATM & 1402 & 092 & HEM & 146 & 16.889 & 21.235 & 0.703 & 1.00 & 0.00 & 0 \\
\hline HETATM & 1403 & СТ91 & HEM & 146 & 16.718 & 21.299 & -1.709 & 1.00 & 0.00 & C \\
\hline HETATM & 1404 & H911 & HEM & 146 & 15.740 & 21.104 & -2.171 & 1.00 & 0.00 & $\mathrm{H}$ \\
\hline HETATM & 1405 & H912 & HEM & 146 & 17.466 & 20.809 & -2.352 & 1.00 & 0.00 & $\mathrm{H}$ \\
\hline HETATM & 1406 & СТ92 & HEM & 146 & 16.978 & 22.803 & -1.597 & 1.00 & 0.00 & $\mathrm{C}$ \\
\hline HETATM & 1407 & H921 & HEM & 146 & 17.903 & 22.959 & -1.027 & 1.00 & 0.00 & $\mathrm{H}$ \\
\hline HETATM & 1408 & H922 & HEM & 146 & 16.182 & 23.264 & -1.000 & 1.00 & 0.00 & $\mathrm{H}$ \\
\hline \multicolumn{11}{|l|}{ TER } \\
\hline HETATM & 1409 & $\mathrm{C} 2$ & VIN & 147 & 11.458 & 28.515 & -8.933 & 1.00 & 0.00 & C \\
\hline HETATM & 1410 & H 21 & VIN & 147 & 10.642 & 27.817 & -8.768 & 1.00 & 0.00 & $\mathrm{H}$ \\
\hline HETATM & 1411 & $\mathrm{C} 1$ & VIN & 147 & 11.125 & 29.740 & -9.335 & 1.00 & 0.00 & C \\
\hline HETATM & 1412 & $\mathrm{H} 11$ & VIN & 147 & 11.857 & 30.519 & -9.442 & 1.00 & 0.00 & $\mathrm{H}$ \\
\hline HETATM & 1413 & $\mathrm{H} 12$ & VIN & 147 & 10.084 & 30.010 & -9.501 & 1.00 & 0.00 & $\mathrm{H}$ \\
\hline \multicolumn{11}{|l|}{ TER } \\
\hline HETATM & 1414 & $\mathrm{C} 1$ & MET & 148 & 14.122 & 28.770 & -10.771 & 1.00 & 0.00 & $C$ \\
\hline HETATM & 1415 & $\mathrm{H} 11$ & MET & 148 & 14.357 & 29.841 & -10.652 & 1.00 & 0.00 & \\
\hline
\end{tabular}




\begin{tabular}{|c|c|c|c|c|c|c|c|c|c|}
\hline HETATM & 1416 & $\mathrm{H} 12$ & MET & 148 & 14.935 & 28.306 & -11.341 & 1.00 & 0.00 \\
\hline HETATM & 1417 & $\mathrm{H} 13$ & MET & 148 & 13.214 & 28.693 & -11.382 & 1.00 & 0.00 \\
\hline \multicolumn{10}{|l|}{ TER } \\
\hline HETATM & 1418 & $\mathrm{C} 2$ & VIN & 149 & 19.132 & 27.092 & -10.256 & 1.00 & 0.00 \\
\hline HETATM & 1419 & H 21 & VIN & 149 & 18.466 & 26.991 & -11.115 & 1.00 & 0.00 \\
\hline HETATM & 1420 & $\mathrm{C} 1$ & VIN & 149 & 20.299 & 27.737 & -10.415 & 1.00 & 0.00 \\
\hline HETATM & 1421 & H11 & VIN & 149 & 20.979 & 27.893 & -9.585 & 1.00 & 0.00 \\
\hline HETATM & 1422 & $\mathrm{H} 12$ & VIN & 149 & 20.604 & 28.135 & -11.381 & 1.00 & 0.00 \\
\hline \multicolumn{10}{|l|}{ TER } \\
\hline HETATM & 1423 & $\mathrm{C} 1$ & MET & 150 & 20.817 & 25.409 & -8.052 & 1.00 & 0.00 \\
\hline HETATM & 1424 & $\mathrm{H} 11$ & MET & 150 & 21.408 & 26.073 & -7.403 & 1.00 & 0.00 \\
\hline HETATM & 1425 & $\mathrm{H} 12$ & MET & 150 & 20.973 & 24.387 & -7.685 & 1.00 & 0.00 \\
\hline HETATM & 1426 & $\mathrm{H} 13$ & MET & 150 & 21.249 & 25.475 & -9.057 & 1.00 & 0.00 \\
\hline \multicolumn{10}{|l|}{ TER } \\
\hline HETATM & 1427 & C5 & $\mathrm{ACP}$ & 151 & 11.728 & 24.327 & -2.161 & 1.00 & 0.00 \\
\hline HETATM & 1428 & H51 & $\mathrm{ACP}$ & 151 & 12.467 & 24.316 & -1.349 & 1.00 & 0.00 \\
\hline HETATM & 1429 & H52 & $\mathrm{ACP}$ & 151 & 10.888 & 24.936 & -1.798 & 1.00 & 0.00 \\
\hline HETATM & 1430 & $\mathrm{C} 4$ & $\mathrm{ACP}$ & 151 & 11.250 & 22.890 & -2.434 & 1.00 & 0.00 \\
\hline HETATM & 1431 & $\mathrm{H} 41$ & $\mathrm{ACP}$ & 151 & 10.692 & 22.829 & -3.382 & 1.00 & 0.00 \\
\hline HETATM & 1432 & $\mathrm{H} 42$ & $\mathrm{ACP}$ & 151 & 12.120 & 22.223 & -2.541 & 1.00 & 0.00 \\
\hline HETATM & 1433 & $\mathrm{C} 1$ & $\mathrm{ACP}$ & 151 & 10.386 & 22.278 & -1.358 & 1.00 & 0.00 \\
\hline HETATM & 1434 & $\mathrm{O} 2$ & $\mathrm{ACP}$ & 151 & 10.158 & 22.800 & -0.260 & 1.00 & 0.00 \\
\hline HETATM & 1435 & 03 & $\mathrm{ACP}$ & 151 & 9.870 & 21.098 & -1.722 & 1.00 & 0.00 \\
\hline HETATM & 1436 & H3 & $\mathrm{ACP}$ & 151 & 9.261 & 20.744 & -0.986 & 1.00 & 0.00 \\
\hline \multicolumn{10}{|l|}{ TER } \\
\hline HETATM & 1437 & $\mathrm{C} 1$ & MET & 152 & 19.624 & 23.201 & -3.334 & 1.00 & 0.00 \\
\hline HETATM & 1438 & $\mathrm{H} 11$ & $\mathrm{MET}$ & 152 & 20.177 & 24.040 & -2.884 & 1.00 & 0.00 \\
\hline HETATM & 1439 & $\mathrm{H} 12$ & $\mathrm{MET}$ & 152 & 19.631 & 22.375 & -2.613 & 1.00 & 0.00 \\
\hline HETATM & 1440 & $\mathrm{H} 13$ & MET & 152 & 20.195 & 22.882 & -4.214 & 1.00 & 0.00 \\
\hline \multicolumn{10}{|l|}{ TER } \\
\hline HETATM & 1441 & $\mathrm{C} 1$ & MET & 153 & 10.191 & 26.150 & -4.285 & 1.00 & 0.00 \\
\hline HETATM & 1442 & $\mathrm{H} 11$ & MET & 153 & 9.746 & 25.941 & -3.305 & 1.00 & 0.00 \\
\hline HETATM & 1443 & H12 & MET & 153 & 10.081 & 27.230 & -4.460 & 1.00 & 0.00 \\
\hline HETATM & 1444 & H13 & MET & 153 & 9.590 & 25.624 & -5.042 & 1.00 & 0.00 \\
\hline TER & & & & & & & & & \\
\hline HETATM & 1445 & $\mathrm{C} 2$ & VIN & 154 & 19.988 & 14.771 & 1.002 & 1.00 & 0.00 \\
\hline HETATM & 1446 & $\mathrm{H} 21$ & VIN & 154 & 20.803 & 15.469 & 0.837 & 1.00 & 0.00 \\
\hline HETATM & 1447 & $\mathrm{C} 1$ & VIN & 154 & 20.321 & 13.546 & 1.403 & 1.00 & 0.00 \\
\hline HETATM & 1448 & $\mathrm{H} 11$ & VIN & 154 & 19.589 & 12.767 & 1.511 & 1.00 & 0.00 \\
\hline HETATM & 1449 & $\mathrm{H} 12$ & VIN & 154 & 21.362 & 13.277 & 1.570 & 1.00 & 0.00 \\
\hline TER & & & & & & & & & \\
\hline HETATM & 1450 & $\mathrm{C} 1$ & $\mathrm{MET}$ & 155 & 17.324 & 14.516 & 2.840 & 1.00 & 0.00 \\
\hline HETATM & 1451 & $\mathrm{H} 11$ & MET & 155 & 17.089 & 13.445 & 2.720 & 1.00 & 0.00 \\
\hline HETATM & 1452 & $\mathrm{H} 12$ & MET & 155 & 16.511 & 14.980 & 3.410 & 1.00 & 0.00 \\
\hline HETATM & 1453 & $\mathrm{H} 13$ & MET & 155 & 18.232 & 14.593 & 3.450 & 1.00 & 0.00 \\
\hline TER & & & & & & & & & \\
\hline HETATM & 1454 & $\mathrm{C} 2$ & VIN & 156 & 12.314 & 16.194 & 2.325 & 1.00 & 0.00 \\
\hline HETATM & 1455 & $\mathrm{H} 21$ & VIN & 156 & 12.980 & 16.295 & 3.184 & 1.00 & 0.00 \\
\hline HETATM & 1456 & $\mathrm{C} 1$ & VIN & 156 & 11.147 & 15.550 & 2.484 & 1.00 & 0.00 \\
\hline HETATM & 1457 & H11 & VIN & 156 & 10.467 & 15.393 & 1.654 & 1.00 & 0.00 \\
\hline HETATM & 1458 & $\mathrm{H} 12$ & VIN & 156 & 10.842 & 15.151 & 3.450 & 1.00 & 0.00 \\
\hline TER & & & & & & & & & \\
\hline HETATM & 1459 & $\mathrm{C} 1$ & $\mathrm{MET}$ & 157 & 10.629 & 17.877 & 0.121 & 1.00 & 0.00 \\
\hline HETATM & 1460 & H11 & $\mathrm{MET}$ & 157 & 10.473 & 18.899 & -0.246 & 1.00 & 0.00 \\
\hline HETATM & 1461 & $\mathrm{H} 12$ & $\mathrm{MET}$ & 157 & 10.197 & 17.811 & 1.126 & 1.00 & 0.00 \\
\hline HETATM & 1462 & $\mathrm{H} 13$ & MET & 157 & 10.038 & 17.213 & -0.528 & 1.00 & 0.00 \\
\hline TER & & & & & & & & & \\
\hline HETATM & 1463 & $\mathrm{C} 5$ & $\mathrm{ACP}$ & 158 & 19.718 & 18.959 & -5.770 & 1.00 & 0.00 \\
\hline HETATM & 1464 & H5 1 & $\mathrm{ACP}$ & 158 & 18.979 & 18.970 & -6.582 & 1.00 & 0.00 \\
\hline HETATM & 1465 & H52 & $\mathrm{ACP}$ & 158 & 20.558 & 18.350 & -6.134 & 1.00 & 0.00 \\
\hline HETATM & 1466 & C4 & $\mathrm{ACP}$ & 158 & 20.196 & 20.396 & -5.497 & 1.00 & 0.00 \\
\hline HETATM & 1467 & H 41 & $\mathrm{ACP}$ & 158 & 20.754 & 20.457 & -4.549 & 1.00 & 0.00 \\
\hline HETATM & 1468 & $\mathrm{H} 42$ & $\mathrm{ACP}$ & 158 & 19.326 & 21.063 & -5.390 & 1.00 & 0.00 \\
\hline HETATM & 1469 & $\mathrm{C} 1$ & $\mathrm{ACP}$ & 158 & 21.060 & 21.008 & -6.573 & 1.00 & 0.00 \\
\hline HETATM & 1470 & $\mathrm{O} 2$ & $\mathrm{ACP}$ & 158 & 21.288 & 20.486 & -7.671 & 1.00 & 0.00 \\
\hline HETATM & 1471 & 03 & $\mathrm{ACP}$ & 158 & 21.576 & 22.188 & -6.209 & 1.00 & 0.00 \\
\hline HETATM & 1472 & $\mathrm{H} 3$ & $\mathrm{ACP}$ & 158 & 22.185 & 22.542 & -6.945 & 1.00 & 0.00 \\
\hline TER & & & & & & & & & \\
\hline HETATM & 1473 & $\mathrm{C} 1$ & MET & 159 & 11.822 & 20.085 & -4.597 & 1.00 & 0.00 \\
\hline HETATM & 1474 & H11 & MET & 159 & 11.269 & 19.246 & -5.047 & 1.00 & 0.00 \\
\hline HETATM & 1475 & $\mathrm{H} 12$ & $\mathrm{MET}$ & 159 & 11.815 & 20.911 & -5.319 & 1.00 & 0.00 \\
\hline HETATM & 1476 & $\mathrm{H} 13$ & MET & 159 & 11.251 & 20.405 & -3.717 & 1.00 & 0.00 \\
\hline TER & & & & & & & & & \\
\hline HETATM & 1477 & $\mathrm{C} 1$ & $\mathrm{MET}$ & 160 & 21.255 & 17.136 & -3.646 & 1.00 & 0.00 \\
\hline HETATM & 1478 & $\mathrm{H} 11$ & MET & 160 & 21.700 & 17.345 & -4.626 & 1.00 & 0.00 \\
\hline HETATM & 1479 & H12 & MET & 160 & 21.365 & 16.056 & -3.471 & 1.00 & 0.00 \\
\hline HETATM & 1480 & $\mathrm{H} 13$ & MET & 160 & 21.856 & 17.662 & -2.889 & 1.00 & 0.00 \\
\hline TER & & & & & & & & & \\
\hline HETATM & 1481 & $\mathrm{FE}$ & HEM & 161 & 16.346 & 10.757 & 1.491 & 1.00 & 0.00 \\
\hline
\end{tabular}




\begin{tabular}{|c|c|c|c|c|c|c|c|c|c|c|}
\hline HETATM & 1482 & $\mathrm{C} 1$ & HEM & 161 & 14.118 & 12.629 & 0.429 & 1.00 & 0.00 & C \\
\hline HETATM & 1483 & $\mathrm{C} 2$ & HEM & 161 & 13.759 & 13.420 & -0.742 & 1.00 & 0.00 & C \\
\hline HETATM & 1484 & C3 & HEM & 161 & 14.905 & 13.517 & -1.517 & 1.00 & 0.00 & C \\
\hline HETATM & 1485 & $\mathrm{C} 4$ & HEM & 161 & 15.934 & 12.800 & -0.809 & 1.00 & 0.00 & $\mathrm{C}$ \\
\hline HETATM & 1486 & C5 & HEM & 161 & 17.249 & 12.681 & -1.247 & 1.00 & 0.00 & C \\
\hline HETATM & 1487 & H5 & HEM & 161 & 17.503 & 13.177 & -2.182 & 1.00 & 0.00 & $\mathrm{H}$ \\
\hline HETATM & 1488 & $\mathrm{C} 6$ & HEM & 161 & 18.269 & 11.982 & -0.611 & 1.00 & 0.00 & C \\
\hline HETATM & 1489 & C7 & HEM & 161 & 19.638 & 11.910 & -1.081 & 1.00 & 0.00 & C \\
\hline HETATM & 1490 & $\mathrm{C} 8$ & HEM & 161 & 20.343 & 11.190 & -0.134 & 1.00 & 0.00 & C \\
\hline HETATM & 1491 & C9 & HEM & 161 & 19.392 & 10.825 & 0.891 & 1.00 & 0.00 & C \\
\hline HETATM & 1492 & $\mathrm{C} 10$ & HEM & 161 & 19.697 & 10.090 & 2.034 & 1.00 & 0.00 & C \\
\hline HETATM & 1493 & $\mathrm{H} 1 \mathrm{O}$ & HEM & 161 & 20.723 & 9.746 & 2.140 & 1.00 & 0.00 & $\mathrm{H}$ \\
\hline HETATM & 1494 & $\mathrm{C} 11$ & HEM & 161 & 18.822 & 9.750 & 3.057 & 1.00 & 0.00 & C \\
\hline HETATM & 1495 & $\mathrm{C} 12$ & HEM & 161 & 19.203 & 9.025 & 4.247 & 1.00 & 0.00 & C \\
\hline HETATM & 1496 & $\mathrm{C} 13$ & HEM & 161 & 18.064 & 8.878 & 5.004 & 1.00 & 0.00 & C \\
\hline HETATM & 1497 & $\mathrm{C} 14$ & HEM & 161 & 16.991 & 9.519 & 4.273 & 1.00 & 0.00 & C \\
\hline HETATM & 1498 & C15 & HEM & 161 & 15.663 & 9.574 & 4.694 & 1.00 & 0.00 & C \\
\hline HETATM & 1499 & H15 & HEM & 161 & 15.423 & 9.089 & 5.642 & 1.00 & 0.00 & $\mathrm{H}$ \\
\hline HETATM & 1500 & $\mathrm{C} 16$ & HEM & 161 & 14.632 & 10.255 & 4.051 & 1.00 & 0.00 & C \\
\hline HETATM & 1501 & $\mathrm{C} 17$ & HEM & 161 & 13.267 & 10.369 & 4.533 & 1.00 & 0.00 & C \\
\hline HETATM & 1502 & $\mathrm{C} 18$ & HEM & 161 & 12.591 & 11.137 & 3.609 & 1.00 & 0.00 & C \\
\hline HETATM & 1503 & C19 & HEM & 161 & 13.541 & 11.489 & 2.573 & 1.00 & 0.00 & C \\
\hline HETATM & 1504 & $\mathrm{C} 20$ & HEM & 161 & 13.250 & 12.280 & 1.462 & 1.00 & 0.00 & C \\
\hline HETATM & 1505 & $\mathrm{H} 2 \mathrm{O}$ & HEM & 161 & 12.235 & 12.669 & 1.402 & 1.00 & 0.00 & $\mathrm{H}$ \\
\hline HETATM & 1506 & N21 & HEM & 161 & 15.440 & 12.266 & 0.367 & 1.00 & 0.00 & $\mathrm{~N}$ \\
\hline HETATM & 1507 & $\mathrm{~N} 22$ & HEM & 161 & 18.144 & 11.319 & 0.590 & 1.00 & 0.00 & $\mathrm{~N}$ \\
\hline HETATM & 1508 & N23 & HEM & 161 & 17.474 & 10.056 & 3.090 & 1.00 & 0.00 & $\mathrm{~N}$ \\
\hline HETATM & 1509 & N2 4 & HEM & 161 & 14.774 & 10.941 & 2.854 & 1.00 & 0.00 & $\mathrm{~N}$ \\
\hline HETATM & 1510 & C99 & HEM & 161 & 15.681 & 8.076 & 0.352 & 1.00 & 0.00 & C \\
\hline HETATM & 1511 & 091 & HEM & 161 & 15.840 & 9.389 & 0.365 & 1.00 & 0.00 & 0 \\
\hline HETATM & 1512 & 092 & HEM & 161 & 15.526 & 7.458 & -0.703 & 1.00 & 0.00 & O \\
\hline HETATM & 1513 & СТ91 & HEM & 161 & 15.697 & 7.394 & 1.709 & 1.00 & 0.00 & C \\
\hline HETATM & 1514 & H911 & HEM & 161 & 16.676 & 7.590 & 2.171 & 1.00 & 0.00 & $\mathrm{H}$ \\
\hline HETATM & 1515 & H912 & HEM & 161 & 14.949 & 7.884 & 2.352 & 1.00 & 0.00 & $\mathrm{H}$ \\
\hline HETATM & 1516 & СТ92 & HEM & 161 & 15.437 & 5.890 & 1.597 & 1.00 & 0.00 & C \\
\hline HETATM & 1517 & H921 & HEM & 161 & 16.234 & 5.429 & 1.000 & 1.00 & 0.00 & $\mathrm{H}$ \\
\hline HETATM & 1518 & H922 & HEM & 161 & 14.512 & 5.734 & 1.027 & 1.00 & 0.00 & $\mathrm{H}$ \\
\hline \multicolumn{11}{|l|}{ TER } \\
\hline HETATM & 1519 & $\mathrm{FE}$ & HEM & 162 & 17.038 & 3.343 & 6.440 & 1.00 & 0.00 & $\mathrm{Fe}$ \\
\hline HETATM & 1520 & $\mathrm{C} 1$ & HEM & 162 & 19.266 & 1.471 & 7.502 & 1.00 & 0.00 & C \\
\hline HETATM & 1521 & $\mathrm{C} 2$ & HEM & 162 & 19.625 & 0.680 & 8.674 & 1.00 & 0.00 & C \\
\hline HETATM & 1522 & C3 & HEM & 162 & 18.479 & 0.583 & 9.448 & 1.00 & 0.00 & C \\
\hline HETATM & 1523 & $\mathrm{C} 4$ & HEM & 162 & 17.450 & 1.301 & 8.740 & 1.00 & 0.00 & C \\
\hline HETATM & 1524 & $\mathrm{C} 5$ & HEM & 162 & 16.135 & 1.420 & 9.178 & 1.00 & 0.00 & C \\
\hline HETATM & 1525 & H5 & HEM & 162 & 15.881 & 0.923 & 10.113 & 1.00 & 0.00 & $\mathrm{H}$ \\
\hline HETATM & 1526 & $\mathrm{C} 6$ & HEM & 162 & 15.115 & 2.118 & 8.542 & 1.00 & 0.00 & C \\
\hline HETATM & 1527 & C7 & HEM & 162 & 13.747 & 2.191 & 9.012 & 1.00 & 0.00 & C \\
\hline HETATM & 1528 & $\mathrm{C} 8$ & HEM & 162 & 13.041 & 2.910 & 8.065 & 1.00 & 0.00 & C \\
\hline HETATM & 1529 & C9 & HEM & 162 & 13.992 & 3.275 & 7.041 & 1.00 & 0.00 & $\mathrm{C}$ \\
\hline HETATM & 1530 & $\mathrm{C} 10$ & HEM & 162 & 13.688 & 4.010 & 5.897 & 1.00 & 0.00 & C \\
\hline HETATM & 1531 & $\mathrm{H} 10$ & HEM & 162 & 12.661 & 4.354 & 5.791 & 1.00 & 0.00 & $\mathrm{H}$ \\
\hline HETATM & 1532 & $\mathrm{C} 11$ & HEM & 162 & 14.562 & 4.350 & 4.874 & 1.00 & 0.00 & C \\
\hline HETATM & 1533 & $\mathrm{C} 12$ & HEM & 162 & 14.181 & 5.076 & 3.684 & 1.00 & 0.00 & C \\
\hline HETATM & 1534 & $\mathrm{C} 13$ & HEM & 162 & 15.321 & 5.223 & 2.927 & 1.00 & 0.00 & C \\
\hline HETATM & 1535 & $\mathrm{C} 14$ & HEM & 162 & 16.394 & 4.581 & 3.659 & 1.00 & 0.00 & C \\
\hline HETATM & 1536 & $\mathrm{C} 15$ & HEM & 162 & 17.722 & 4.527 & 3.237 & 1.00 & 0.00 & C \\
\hline HETATM & 1537 & H1 5 & HEM & 162 & 17.962 & 5.011 & 2.289 & 1.00 & 0.00 & $\mathrm{H}$ \\
\hline HETATM & 1538 & $\mathrm{C} 16$ & HEM & 162 & 18.752 & 3.845 & 3.880 & 1.00 & 0.00 & C \\
\hline HETATM & 1539 & $\mathrm{C} 17$ & HEM & 162 & 20.118 & 3.732 & 3.398 & 1.00 & 0.00 & C \\
\hline HETATM & 1540 & $\mathrm{C} 18$ & HEM & 162 & 20.793 & 2.964 & 4.322 & 1.00 & 0.00 & C \\
\hline HETATM & 1541 & C19 & HEM & 162 & 19.843 & 2.612 & 5.358 & 1.00 & 0.00 & C \\
\hline HETATM & 1542 & $\mathrm{C} 20$ & HEM & 162 & 20.135 & 1.821 & 6.469 & 1.00 & 0.00 & C \\
\hline HETATM & 1543 & $\mathrm{H} 2 \mathrm{O}$ & HEM & 162 & 21.149 & 1.432 & 6.530 & 1.00 & 0.00 & $\mathrm{H}$ \\
\hline HETATM & 1544 & $\mathrm{~N} 21$ & HEM & 162 & 17.944 & 1.834 & 7.564 & 1.00 & 0.00 & $\mathrm{~N}$ \\
\hline HETATM & 1545 & N22 & HEM & 162 & 15.240 & 2.781 & 7.342 & 1.00 & 0.00 & $\mathrm{~N}$ \\
\hline HETATM & 1546 & N23 & HEM & 162 & 15.911 & 4.044 & 4.841 & 1.00 & 0.00 & $\mathrm{~N}$ \\
\hline HETATM & 1547 & $\mathrm{~N} 24$ & HEM & 162 & 18.610 & 3.160 & 5.077 & 1.00 & 0.00 & $\mathrm{~N}$ \\
\hline HETATM & 1548 & C99 & HEM & 162 & 17.704 & 6.025 & 7.579 & 1.00 & 0.00 & C \\
\hline HETATM & 1549 & 091 & HEM & 162 & 17.544 & 4.711 & 7.567 & 1.00 & 0.00 & o \\
\hline HETATM & 1550 & 092 & HEM & 162 & 17.858 & 6.642 & 8.635 & 1.00 & 0.00 & 0 \\
\hline HETATM & 1551 & СТ91 & HEM & 162 & 17.688 & 6.706 & 6.223 & 1.00 & 0.00 & C \\
\hline HETATM & 1552 & H911 & HEM & 162 & 16.709 & 6.511 & 5.760 & 1.00 & 0.00 & $\mathrm{H}$ \\
\hline HETATM & 1553 & H912 & HEM & 162 & 18.436 & 6.216 & 5.580 & 1.00 & 0.00 & $\mathrm{H}$ \\
\hline HETATM & 1554 & СТ92 & HEM & 162 & 17.947 & 8.211 & 6.334 & 1.00 & 0.00 & C \\
\hline HETATM & 1555 & H921 & HEM & 162 & 18.872 & 8.367 & 6.905 & 1.00 & 0.00 & $\mathrm{H}$ \\
\hline HETATM & 1556 & H922 & HEM & 162 & 17.151 & 8.671 & 6.931 & 1.00 & 0.00 & $\mathrm{H}$ \\
\hline \multicolumn{11}{|l|}{ TER } \\
\hline HETATM & 1557 & $\mathrm{C} 2$ & VIN & 163 & 12.427 & 13.923 & -1.002 & 1.00 & 0.00 & $c$ \\
\hline HETATM & 1558 & H 21 & VIN & 163 & 11.612 & 13.224 & -0.837 & 1.00 & 0.00 & $\mathrm{H}$ \\
\hline
\end{tabular}




\begin{tabular}{|c|c|c|c|c|c|c|c|c|c|c|}
\hline HETATM & 1559 & $\mathrm{C} 1$ & VIN & 163 & 12.095 & 15.147 & -1.403 & 1.00 & 0.00 & C \\
\hline HETATM & 1560 & H11 & VIN & 163 & 12.826 & 15.926 & -1.511 & 1.00 & 0.00 & $\mathrm{H}$ \\
\hline HETATM & 1561 & H12 & VIN & 163 & 11.053 & 15.417 & -1.570 & 1.00 & 0.00 & $\mathrm{H}$ \\
\hline \multicolumn{11}{|l|}{ TER } \\
\hline HETATM & 1562 & $\mathrm{C} 1$ & MET & 164 & 15.091 & 14.177 & -2.840 & 1.00 & 0.00 & C \\
\hline HETATM & 1563 & H11 & MET & 164 & 15.326 & 15.248 & -2.720 & 1.00 & 0.00 & 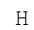 \\
\hline HETATM & 1564 & $\mathrm{H} 12$ & MET & 164 & 15.904 & 13.713 & -3.410 & 1.00 & 0.00 & $\mathrm{H}$ \\
\hline HETATM & 1565 & $\mathrm{H} 13$ & MET & 164 & 14.184 & 14.100 & -3.450 & 1.00 & 0.00 & $\mathrm{H}$ \\
\hline \multicolumn{11}{|l|}{ TER } \\
\hline HETATM & 1566 & $\mathrm{C} 2$ & VIN & 165 & 20.101 & 12.499 & -2.325 & 1.00 & 0.00 & $\mathrm{C}$ \\
\hline HETATM & 1567 & H21 & VIN & 165 & 19.435 & 12.399 & -3.184 & 1.00 & 0.00 & $\mathrm{H}$ \\
\hline HETATM & 1568 & $\mathrm{C} 1$ & VIN & 165 & 21.268 & 13.144 & -2.484 & 1.00 & 0.00 & C \\
\hline HETATM & 1569 & $\mathrm{H} 11$ & VIN & 165 & 21.948 & 13.300 & -1.654 & 1.00 & 0.00 & $\mathrm{H}$ \\
\hline HETATM & 1570 & H12 & VIN & 165 & 21.573 & 13.542 & -3.450 & 1.00 & 0.00 & $\mathrm{H}$ \\
\hline \multicolumn{11}{|c|}{ TER } \\
\hline HETATM & 1571 & $\mathrm{C} 1$ & MET & 166 & 21.786 & 10.816 & -0.121 & 1.00 & 0.00 & C \\
\hline HETATM & 1572 & H11 & MET & 166 & 22.377 & 11.480 & 0.528 & 1.00 & 0.00 & $\mathrm{H}$ \\
\hline HETATM & 1573 & H12 & MET & 166 & 21.942 & 9.794 & 0.246 & 1.00 & 0.00 & $\mathrm{H}$ \\
\hline HETATM & 1574 & H13 & MET & 166 & 22.218 & 10.882 & -1.126 & 1.00 & 0.00 & $\mathrm{H}$ \\
\hline \multicolumn{11}{|l|}{ TER } \\
\hline HETATM & 1575 & C5 & $\mathrm{ACP}$ & 167 & 12.697 & 9.735 & 5.770 & 1.00 & 0.00 & C \\
\hline HETATM & 1576 & H51 & $\mathrm{ACP}$ & 167 & 13.436 & 9.723 & 6.582 & 1.00 & 0.00 & $\mathrm{H}$ \\
\hline HETATM & 1577 & H52 & $\mathrm{ACP}$ & 167 & 11.857 & 10.343 & 6.134 & 1.00 & 0.00 & $\mathrm{H}$ \\
\hline HETATM & 1578 & $\mathrm{C} 4$ & $\mathrm{ACP}$ & 167 & 12.219 & 8.297 & 5.497 & 1.00 & 0.00 & C \\
\hline HETATM & 1579 & $\mathrm{H} 41$ & $\mathrm{ACP}$ & 167 & 11.661 & 8.236 & 4.549 & 1.00 & 0.00 & $\mathrm{H}$ \\
\hline HETATM & 1580 & H 42 & $\mathrm{ACP}$ & 167 & 13.089 & 7.630 & 5.390 & 1.00 & 0.00 & $\mathrm{H}$ \\
\hline HETATM & 1581 & $\mathrm{C} 1$ & $\mathrm{ACP}$ & 167 & 11.355 & 7.685 & 6.573 & 1.00 & 0.00 & C \\
\hline HETATM & 1582 & $\mathrm{O} 2$ & $\mathrm{ACP}$ & 167 & 11.127 & 8.208 & 7.671 & 1.00 & 0.00 & 0 \\
\hline HETATM & 1583 & 03 & $\mathrm{ACP}$ & 167 & 10.839 & 6.505 & 6.209 & 1.00 & 0.00 & 0 \\
\hline HETATM & 1584 & H3 & $\mathrm{ACP}$ & 167 & 10.230 & 6.151 & 6.945 & 1.00 & 0.00 & $\mathrm{H}$ \\
\hline \multicolumn{11}{|c|}{ TER } \\
\hline HETATM & 1585 & $\mathrm{C} 1$ & MET & 168 & 20.594 & 8.608 & 4.597 & 1.00 & 0.00 & C \\
\hline HETATM & 1586 & $\mathrm{H} 11$ & MET & 168 & 21.146 & 9.448 & 5.047 & 1.00 & 0.00 & $\mathrm{H}$ \\
\hline HETATM & 1587 & $\mathrm{H} 12$ & MET & 168 & 20.600 & 7.782 & 5.319 & 1.00 & 0.00 & $\mathrm{H}$ \\
\hline HETATM & 1588 & $\mathrm{H} 13$ & MET & 168 & 21.165 & 8.289 & 3.717 & 1.00 & 0.00 & $\mathrm{H}$ \\
\hline TER & & & & & & & & & & \\
\hline HETATM & 1589 & $\mathrm{C} 1$ & MET & 169 & 11.160 & 11.557 & 3.646 & 1.00 & 0.00 & $\mathrm{C}$ \\
\hline HETATM & 1590 & H11 & MET & 169 & 10.715 & 11.348 & 4.626 & 1.00 & 0.00 & $\mathrm{H}$ \\
\hline HETATM & 1591 & $\mathrm{H} 12$ & MET & 169 & 11.050 & 12.637 & 3.471 & 1.00 & 0.00 & $\mathrm{H}$ \\
\hline HETATM & 1592 & $\mathrm{H} 13$ & MET & 169 & 10.559 & 11.031 & 2.889 & 1.00 & 0.00 & $\mathrm{H}$ \\
\hline TER & & & & & & & & & & \\
\hline HETATM & 1593 & $\mathrm{C} 2$ & VIN & 170 & 20.957 & 0.178 & 8.933 & 1.00 & 0.00 & $\mathrm{C}$ \\
\hline HETATM & 1594 & $\mathrm{H} 21$ & VIN & 170 & 21.773 & 0.876 & 8.768 & 1.00 & 0.00 & $\mathrm{H}$ \\
\hline HETATM & 1595 & $\mathrm{C} 1$ & VIN & 170 & 21.290 & -1.047 & 9.335 & 1.00 & 0.00 & C \\
\hline HETATM & 1596 & H11 & VIN & 170 & 20.558 & -1.826 & 9.442 & 1.00 & 0.00 & $\mathrm{H}$ \\
\hline HETATM & 1597 & H12 & VIN & 170 & 22.331 & -1.316 & 9.501 & 1.00 & 0.00 & $\mathrm{H}$ \\
\hline TER & & & & & & & & & & \\
\hline HETATM & 1598 & $\mathrm{C} 1$ & MET & 171 & 18.293 & -0.077 & 10.771 & 1.00 & 0.00 & $\mathrm{C}$ \\
\hline HETATM & 1599 & $\mathrm{H} 11$ & MET & 171 & 18.058 & -1.148 & 10.652 & 1.00 & 0.00 & $\mathrm{H}$ \\
\hline HETATM & 1600 & $\mathrm{H} 12$ & MET & 171 & 17.480 & 0.387 & 11.341 & 1.00 & 0.00 & $\mathrm{H}$ \\
\hline HETATM & 1601 & $\mathrm{H} 13$ & MET & 171 & 19.201 & 0.001 & 11.382 & 1.00 & 0.00 & $\mathrm{H}$ \\
\hline TER & & & & & & & & & & \\
\hline HETATM & 1602 & $\mathrm{C} 2$ & VIN & 172 & 13.283 & 1.601 & 10.256 & 1.00 & 0.00 & $\mathrm{C}$ \\
\hline HETATM & 1603 & $\mathrm{H} 21$ & VIN & 172 & 13.949 & 1.702 & 11.115 & 1.00 & 0.00 & $\mathrm{H}$ \\
\hline HETATM & 1604 & $\mathrm{C} 1$ & VIN & 172 & 12.116 & 0.957 & 10.415 & 1.00 & 0.00 & C \\
\hline HETATM & 1605 & $\mathrm{H} 11$ & VIN & 172 & 11.436 & 0.800 & 9.585 & 1.00 & 0.00 & $\mathrm{H}$ \\
\hline HETATM & 1606 & $\mathrm{H} 12$ & VIN & 172 & 11.811 & 0.559 & 11.381 & 1.00 & 0.00 & $\mathrm{H}$ \\
\hline TER & & & & & & & & & & \\
\hline HETATM & 1607 & $\mathrm{C} 1$ & MET & 173 & 11.598 & 3.284 & 8.052 & 1.00 & 0.00 & C \\
\hline HETATM & 1608 & $\mathrm{H} 11$ & MET & 173 & 11.442 & 4.306 & 7.685 & 1.00 & 0.00 & $\mathrm{H}$ \\
\hline HETATM & 1609 & $\mathrm{H} 12$ & MET & 173 & 11.166 & 3.219 & 9.057 & 1.00 & 0.00 & $\mathrm{H}$ \\
\hline HETATM & 1610 & $\mathrm{H} 13$ & MET & 173 & 11.007 & 2.620 & 7.403 & 1.00 & 0.00 & $\mathrm{H}$ \\
\hline TER & & & & & & & & & & \\
\hline HETATM & 1611 & C5 & $\mathrm{ACP}$ & 174 & 20.687 & 4.366 & 2.161 & 1.00 & 0.00 & C \\
\hline HETATM & 1612 & H51 & $\mathrm{ACP}$ & 174 & 19.948 & 4.377 & 1.349 & 1.00 & 0.00 & $\mathrm{H}$ \\
\hline HETATM & 1613 & H52 & $\mathrm{ACP}$ & 174 & 21.527 & 3.758 & 1.798 & 1.00 & 0.00 & $\mathrm{H}$ \\
\hline HETATM & 1614 & C4 & $\mathrm{ACP}$ & 174 & 21.165 & 5.803 & 2.434 & 1.00 & 0.00 & C \\
\hline HETATM & 1615 & H 41 & $\mathrm{ACP}$ & 174 & 21.723 & 5.865 & 3.382 & 1.00 & 0.00 & $\mathrm{H}$ \\
\hline HETATM & 1616 & H 42 & $\mathrm{ACP}$ & 174 & 20.295 & 6.470 & 2.541 & 1.00 & 0.00 & $\mathrm{H}$ \\
\hline HETATM & 1617 & $\mathrm{C} 1$ & $\mathrm{ACP}$ & 174 & 22.029 & 6.416 & 1.358 & 1.00 & 0.00 & $\mathrm{C}$ \\
\hline HETATM & 1618 & $\mathrm{O} 2$ & $\mathrm{ACP}$ & 174 & 22.257 & 5.893 & 0.260 & 1.00 & 0.00 & 0 \\
\hline HETATM & 1619 & 03 & $\mathrm{ACP}$ & 174 & 22.545 & 7.595 & 1.722 & 1.00 & 0.00 & 0 \\
\hline HETATM & 1620 & $\mathrm{H} 3$ & $\mathrm{ACP}$ & 174 & 23.154 & 7.949 & 0.986 & 1.00 & 0.00 & $\mathrm{H}$ \\
\hline TER & & & & & & & & & & \\
\hline HETATM & 1621 & $\mathrm{C} 1$ & MET & 175 & 12.791 & 5.492 & 3.334 & 1.00 & 0.00 & $\mathrm{C}$ \\
\hline HETATM & 1622 & $\mathrm{H} 11$ & MET & 175 & 12.238 & 4.653 & 2.884 & 1.00 & 0.00 & $\mathrm{H}$ \\
\hline HETATM & 1623 & H12 & MET & 175 & 12.784 & 6.318 & 2.613 & 1.00 & 0.00 & $\mathrm{H}$ \\
\hline HETATM & 1624 & H13 & MET & 175 & 12.220 & 5.812 & 4.214 & 1.00 & 0.00 & $\mathrm{H}$ \\
\hline
\end{tabular}




\begin{tabular}{|c|c|c|c|c|c|c|c|c|c|c|}
\hline HETATM & 1625 & $\mathrm{C} 1$ & MET & 176 & 22.224 & 2.543 & 4.285 & 1.00 & 0.00 & C \\
\hline HETATM & 1626 & H11 & MET & 176 & 22.669 & 2.752 & 3.305 & 1.00 & 0.00 & $\mathrm{H}$ \\
\hline HETATM & 1627 & H12 & MET & 176 & 22.335 & 1.463 & 4.460 & 1.00 & 0.00 & $\mathrm{H}$ \\
\hline HETATM & 1628 & $\mathrm{H} 13$ & $\mathrm{MET}$ & 176 & 22.825 & 3.069 & 5.042 & 1.00 & 0.00 & $\mathrm{H}$ \\
\hline \multicolumn{11}{|l|}{ TER } \\
\hline HETATM & 1629 & $\mathrm{FE}$ & HEM & 177 & 15.398 & 10.511 & 9.423 & 1.00 & 0.00 & $\mathrm{Fe}$ \\
\hline HETATM & 1630 & $\mathrm{C} 1$ & HEM & 177 & 13.171 & 12.383 & 8.361 & 1.00 & 0.00 & C \\
\hline HETATM & 1631 & $\mathrm{C} 2$ & HEM & 177 & 12.812 & 13.174 & 7.189 & 1.00 & 0.00 & C \\
\hline HETATM & 1632 & C3 & HEM & 177 & 13.957 & 13.271 & 6.414 & 1.00 & 0.00 & $\mathrm{C}$ \\
\hline HETATM & 1633 & C4 & HEM & 177 & 14.987 & 12.553 & 7.122 & 1.00 & 0.00 & $\mathrm{C}$ \\
\hline HETATM & 1634 & C5 & HEM & 177 & 16.302 & 12.435 & 6.684 & 1.00 & 0.00 & $\mathrm{C}$ \\
\hline HETATM & 1635 & H5 & HEM & 177 & 16.555 & 12.931 & 5.749 & 1.00 & 0.00 & $\mathrm{H}$ \\
\hline HETATM & 1636 & C6 & HEM & 177 & 17.321 & 11.736 & 7.320 & 1.00 & 0.00 & C \\
\hline HETATM & 1637 & C7 & HEM & 177 & 18.690 & 11.664 & 6.851 & 1.00 & 0.00 & C \\
\hline HETATM & 1638 & $\mathrm{C} 8$ & HEM & 177 & 19.395 & 10.944 & 7.798 & 1.00 & 0.00 & C \\
\hline HETATM & 1639 & C9 & HEM & 177 & 18.445 & 10.579 & 8.822 & 1.00 & 0.00 & C \\
\hline HETATM & 1640 & C10 & HEM & 177 & 18.749 & 9.844 & 9.965 & 1.00 & 0.00 & C \\
\hline HETATM & 1641 & H1O & HEM & 177 & 19.776 & 9.500 & 10.071 & 1.00 & 0.00 & $\mathrm{H}$ \\
\hline HETATM & 1642 & C11 & HEM & 177 & 17.875 & 9.504 & 10.988 & 1.00 & 0.00 & C \\
\hline HETATM & 1643 & $\mathrm{C} 12$ & HEM & 177 & 18.255 & 8.779 & 12.178 & 1.00 & 0.00 & C \\
\hline HETATM & 1644 & C13 & HEM & 177 & 17.116 & 8.631 & 12.935 & 1.00 & 0.00 & C \\
\hline HETATM & 1645 & C14 & HEM & 177 & 16.043 & 9.273 & 12.204 & 1.00 & 0.00 & C \\
\hline HETATM & 1646 & C15 & HEM & 177 & 14.715 & 9.328 & 12.625 & 1.00 & 0.00 & C \\
\hline HETATM & 1647 & H15 & HEM & 177 & 14.475 & 8.843 & 13.573 & 1.00 & 0.00 & $\mathrm{H}$ \\
\hline HETATM & 1648 & C16 & HEM & 177 & 13.685 & 10.009 & 11.982 & 1.00 & 0.00 & C \\
\hline HETATM & 1649 & $\mathrm{C} 17$ & HEM & 177 & 12.319 & 10.123 & 12.464 & 1.00 & 0.00 & C \\
\hline HETATM & 1650 & C18 & HEM & 177 & 11.644 & 10.890 & 11.541 & 1.00 & 0.00 & C \\
\hline HETATM & 1651 & C19 & HEM & 177 & 12.593 & 11.242 & 10.504 & 1.00 & 0.00 & $\mathrm{C}$ \\
\hline HETATM & 1652 & $\mathrm{C} 20$ & HEM & 177 & 12.302 & 12.033 & 9.393 & 1.00 & 0.00 & $\mathrm{C}$ \\
\hline HETATM & 1653 & $\mathrm{H} 20$ & HEM & 177 & 11.288 & 12.423 & 9.333 & 1.00 & 0.00 & $\mathrm{H}$ \\
\hline HETATM & 1654 & $\mathrm{~N} 21$ & HEM & 177 & 14.492 & 12.020 & 8.298 & 1.00 & 0.00 & $\mathrm{~N}$ \\
\hline HETATM & 1655 & N22 & HEM & 177 & 17.197 & 11.073 & 8.521 & 1.00 & 0.00 & $\mathrm{~N}$ \\
\hline HETATM & 1656 & N23 & HEM & 177 & 16.526 & 9.810 & 11.021 & 1.00 & 0.00 & $\mathrm{~N}$ \\
\hline HETATM & 1657 & N24 & HEM & 177 & 13.826 & 10.694 & 10.786 & 1.00 & 0.00 & $\mathrm{~N}$ \\
\hline HETATM & 1658 & C99 & HEM & 177 & 14.733 & 7.829 & 8.283 & 1.00 & 0.00 & C \\
\hline HETATM & 1659 & 091 & HEM & 177 & 14.893 & 9.143 & 8.296 & 1.00 & 0.00 & 0 \\
\hline HETATM & 1660 & 092 & HEM & 177 & 14.578 & 7.212 & 7.228 & 1.00 & 0.00 & 0 \\
\hline HETATM & 1661 & СТ91 & HEM & 177 & 14.749 & 7.148 & 9.640 & 1.00 & 0.00 & C \\
\hline HETATM & 1662 & H911 & HEM & 177 & 15.728 & 7.343 & 10.103 & 1.00 & 0.00 & $\mathrm{H}$ \\
\hline HETATM & 1663 & H912 & HEM & 177 & 14.001 & 7.638 & 10.283 & 1.00 & 0.00 & $\mathrm{H}$ \\
\hline HETATM & 1664 & CT 92 & HEM & 177 & 14.490 & 5.644 & 9.528 & 1.00 & 0.00 & C \\
\hline HETATM & 1665 & H921 & HEM & 177 & 15.286 & 5.183 & 8.931 & 1.00 & 0.00 & $\mathrm{H}$ \\
\hline HETATM & 1666 & H922 & HEM & 177 & 13.565 & 5.488 & 8.958 & 1.00 & 0.00 & $\mathrm{H}$ \\
\hline \multicolumn{11}{|c|}{ TER } \\
\hline HETATM & 1667 & $\mathrm{FE}$ & HEM & 178 & 16.091 & 3.097 & 14.371 & 1.00 & 0.00 & $\mathrm{Fe}$ \\
\hline HETATM & 1668 & $\mathrm{C} 1$ & HEM & 178 & 18.319 & 1.225 & 15.433 & 1.00 & 0.00 & C \\
\hline HETATM & 1669 & $\mathrm{C} 2$ & HEM & 178 & 18.678 & 0.434 & 16.605 & 1.00 & 0.00 & C \\
\hline HETATM & 1670 & C3 & HEM & 178 & 17.532 & 0.337 & 17.380 & 1.00 & 0.00 & C \\
\hline HETATM & 1671 & C4 & HEM & 178 & 16.502 & 1.055 & 16.672 & 1.00 & 0.00 & $\mathrm{C}$ \\
\hline HETATM & 1672 & C5 & HEM & 178 & 15.187 & 1.173 & 17.109 & 1.00 & 0.00 & C \\
\hline HETATM & 1673 & H5 & HEM & 178 & 14.934 & 0.677 & 18.045 & 1.00 & 0.00 & $\mathrm{H}$ \\
\hline HETATM & 1674 & C6 & HEM & 178 & 14.168 & 1.872 & 16.473 & 1.00 & 0.00 & C \\
\hline HETATM & 1675 & C7 & HEM & 178 & 12.799 & 1.944 & 16.943 & 1.00 & 0.00 & C \\
\hline HETATM & 1676 & $\mathrm{C} 8$ & HEM & 178 & 12.094 & 2.664 & 15.996 & 1.00 & 0.00 & C \\
\hline HETATM & 1677 & C9 & HEM & 178 & 13.045 & 3.029 & 14.972 & 1.00 & 0.00 & C \\
\hline HETATM & 1678 & C10 & HEM & 178 & 12.740 & 3.764 & 13.828 & 1.00 & 0.00 & C \\
\hline HETATM & 1679 & H1O & HEM & 178 & 11.713 & 4.108 & 13.722 & 1.00 & 0.00 & $\mathrm{H}$ \\
\hline HETATM & 1680 & $\mathrm{C} 11$ & HEM & 178 & 13.615 & 4.104 & 12.806 & 1.00 & 0.00 & C \\
\hline HETATM & 1681 & $\mathrm{C} 12$ & HEM & 178 & 13.234 & 4.829 & 11.615 & 1.00 & 0.00 & C \\
\hline HETATM & 1682 & C13 & HEM & 178 & 14.373 & 4.977 & 10.858 & 1.00 & 0.00 & C \\
\hline HETATM & 1683 & C14 & HEM & 178 & 15.446 & 4.335 & 11.590 & 1.00 & 0.00 & C \\
\hline HETATM & 1684 & C15 & HEM & 178 & 16.774 & 4.280 & 11.168 & 1.00 & 0.00 & C \\
\hline HETATM & 1685 & H1 5 & HEM & 178 & 17.014 & 4.765 & 10.221 & 1.00 & 0.00 & $\mathrm{H}$ \\
\hline HETATM & 1686 & C16 & HEM & 178 & 17.804 & 3.599 & 11.812 & 1.00 & 0.00 & C \\
\hline HETATM & 1687 & C17 & HEM & 178 & 19.170 & 3.485 & 11.329 & 1.00 & 0.00 & C \\
\hline HETATM & 1688 & C18 & HEM & 178 & 19.845 & 2.718 & 12.253 & 1.00 & 0.00 & C \\
\hline HETATM & 1689 & C19 & HEM & 178 & 18.896 & 2.366 & 13.289 & 1.00 & 0.00 & C \\
\hline HETATM & 1690 & $\mathrm{C} 20$ & HEM & 178 & 19.187 & 1.575 & 14.401 & 1.00 & 0.00 & $\mathrm{C}$ \\
\hline HETATM & 1691 & $\mathrm{H} 2 \mathrm{O}$ & HEM & 178 & 20.201 & 1.185 & 14.461 & 1.00 & 0.00 & $\mathrm{H}$ \\
\hline HETATM & 1692 & N21 & HEM & 178 & 16.997 & 1.588 & 15.495 & 1.00 & 0.00 & $\mathrm{~N}$ \\
\hline HETATM & 1693 & N22 & HEM & 178 & 14.292 & 2.535 & 15.273 & 1.00 & 0.00 & $\mathrm{~N}$ \\
\hline HETATM & 1694 & N23 & HEM & 178 & 14.963 & 3.798 & 12.772 & 1.00 & 0.00 & $\mathrm{~N}$ \\
\hline HETATM & 1695 & N24 & HEM & 178 & 17.663 & 2.914 & 13.008 & 1.00 & 0.00 & $\mathrm{~N}$ \\
\hline HETATM & 1696 & C99 & HEM & 178 & 16.756 & 5.779 & 15.510 & 1.00 & 0.00 & C \\
\hline HETATM & 1697 & 091 & HEM & 178 & 16.596 & 4.465 & 15.498 & 1.00 & 0.00 & 0 \\
\hline HETATM & 1698 & 092 & HEM & 178 & 16.911 & 6.396 & 16.566 & 1.00 & 0.00 & 0 \\
\hline HETATM & 1699 & СТ91 & HEM & 178 & 16.740 & 6.460 & 14.154 & 1.00 & 0.00 & C \\
\hline HETATM & 1700 & H911 & HEM & 178 & 15.761 & 6.265 & 13.691 & 1.00 & 0.00 & $\mathrm{H}$ \\
\hline HETATM & 1701 & H912 & HEM & 178 & 17.488 & 5.970 & 13.511 & 1.00 & 0.00 & $\mathrm{H}$ \\
\hline
\end{tabular}


HETATM 1702 CT92 HEM 178

HETATM 1703 H921 HEM 178

HETATM 1704 H922 HEM 178

HETATM 1705 C2 VIN 179

HETATM 1706 H21 VIN 179

HETATM 1707 C1 VIN 179

HETATM 1708 H11 VIN 179

HETATM 1709 H12 VIN 179

TER

HETATM 1710 C1 MET 180

HETATM 1711 H11 MET 180

HETATM 1712 H12 MET 180

HETATM 1713 H13 MET 180

HETATM 1714 C2 VIN 181

HETATM 1715 H21 VIN 181

HETATM 1716 C1 VIN 181

HETATM 1717 H11 VIN 18

HETATM 1718 H12 VIN 18

TER

HETATM 1719 C1 MET 182

HETATM 1720 H11 MET 182

$\begin{array}{llll}\text { HETATM } 1721 & \text { H12 } & \text { MET } & 182\end{array}$

HETATM 1722 H13 MET 182

TER

HETATM 1723 C5 ACP 183

HETATM 1724 H51 ACP 183

HETATM 1725 H52 ACP 183

HETATM 1726 C4 ACP 183

HETATM 1727 H41 ACP 183

HETATM 1728 H42 ACP 183

HETATM 1729 C1 ACP 183

HETATM $1730 \quad 02$ ACP 183

HETATM $1731 \quad 03$ ACP 183

HETATM 1732 H3 ACP 183

TER

HETATM 1733 C1 MET 184

HETATM 1734 H11 MET 184

HETATM 1735 H12 MET 184

HETATM 1736 H13 MET 184

TER

HETATM 1737 C1 MET 185

HETATM 1738 H11 MET 185

HETATM 1739 H12 MET 185

HETATM 1740 H13 MET 185

TER

HETATM 1741 C2 VIN 186

HETATM 1742 H21 VIN 186

HETATM 1743 C1 VIN 186

HETATM 1744 H11 VIN 186

HETATM 1745 H12 VIN 186

TER

HETATM 1746 C1 MET 187

HETATM 1747 H11 MET 187

HETATM 1748 H12 MET 187

HETATM 1749 H13 MET 187

TER

HETATM 1750 C2 VIN 188

HETATM 1751 H21 VIN 188

HETATM 1752 C1 VIN 188

HETATM 1753 H11 VIN 188

HETATM 1754 H12 VIN 188

TER

HETATM 1755 C1 MET 189

HETATM 1756 H11 MET 189

HETATM 1757 H12 MET 189

HETATM 1758 H13 MET 189

TER

HETATM 1759 C5 ACP 190

HETATM 1760 H51 ACP 190

$\begin{array}{llll}\text { HETATM } 1761 & \text { H52 ACP } & 190\end{array}$

HETATM 1762 C4 ACP 190

HETATM 1763 H41 ACP 190

HETATM 1764 H42 ACP 190

HETATM $1765 \quad C 1$ ACP 190

HETATM $1766 \quad 02 \mathrm{ACP} 190$

HETATM 1767 O3 ACP 190

HETATM 1768 H3 ACP 190

\begin{tabular}{|c|c|c|c|c|c|}
\hline 17.000 & 7.964 & 14.265 & 1.00 & 0.00 & C \\
\hline 17.925 & 8.120 & 14.836 & 1.00 & 0.00 & $\mathrm{H}$ \\
\hline 16.203 & 8.425 & 14.862 & 1.00 & 0.00 & $\mathrm{H}$ \\
\hline 11.480 & 13.676 & 6.929 & 1.00 & 0.00 & C \\
\hline 10.664 & 12.978 & 7.094 & 1.00 & 0.00 & $\mathrm{H}$ \\
\hline 11.147 & 14.901 & 6.528 & 1.00 & 0.00 & C \\
\hline 11.878 & 15.680 & 6.421 & 1.00 & 0.00 & $\mathrm{H}$ \\
\hline 10.105 & 15.170 & 6.361 & 1.00 & 0.00 & $\mathrm{H}$ \\
\hline 14.144 & 13.931 & 5.091 & 1.00 & 0.00 & C \\
\hline 14.378 & 15.002 & 5.211 & 1.00 & 0.00 & $\mathrm{H}$ \\
\hline 14.957 & 13.467 & 4.522 & 1.00 & 0.00 & $\mathrm{H}$ \\
\hline 13.236 & 13.854 & 4.481 & 1.00 & 0.00 & $\mathrm{H}$ \\
\hline 19.154 & 12.253 & 5.606 & 1.00 & 0.00 & C \\
\hline 18.488 & 12.152 & 4.747 & 1.00 & 0.00 & $\mathrm{H}$ \\
\hline 20.320 & 12.898 & 5.447 & 1.00 & 0.00 & C \\
\hline 21.000 & 13.054 & 6.277 & 1.00 & 0.00 & $\mathrm{H}$ \\
\hline 20.625 & 13.296 & 4.482 & 1.00 & 0.00 & $\mathrm{H}$ \\
\hline 20.838 & 10.570 & 7.810 & 1.00 & 0.00 & C \\
\hline 21.430 & 11.234 & 8.459 & 1.00 & 0.00 & $\mathrm{H}$ \\
\hline 20.995 & 9.548 & 8.177 & 1.00 & 0.00 & $\mathrm{H}$ \\
\hline 21.271 & 10.636 & 6.805 & 1.00 & 0.00 & $\mathrm{H}$ \\
\hline 11.750 & 9.488 & 13.701 & 1.00 & 0.00 & C \\
\hline 12.489 & 9.477 & 14.514 & 1.00 & 0.00 & $\mathrm{H}$ \\
\hline 10.909 & 10.097 & 14.065 & 1.00 & 0.00 & $\mathrm{H}$ \\
\hline 11.271 & 8.051 & 13.428 & 1.00 & 0.00 & C \\
\hline 10.714 & 7.990 & 12.481 & 1.00 & 0.00 & $\mathrm{H}$ \\
\hline 12.142 & 7.384 & 13.321 & 1.00 & 0.00 & $\mathrm{H}$ \\
\hline 10.407 & 7.439 & 14.504 & 1.00 & 0.00 & C \\
\hline 10.179 & 7.961 & 15.603 & 1.00 & 0.00 & 0 \\
\hline 9.892 & 6.259 & 14.140 & 1.00 & 0.00 & 0 \\
\hline 9.282 & 5.905 & 14.876 & 1.00 & 0.00 & $\mathrm{H}$ \\
\hline 19.646 & 8.362 & 12.528 & 1.00 & 0.00 & C \\
\hline 20.198 & 9.201 & 12.978 & 1.00 & 0.00 & $\mathrm{H}$ \\
\hline 19.652 & 7.536 & 13.250 & 1.00 & 0.00 & $\mathrm{H}$ \\
\hline 20.217 & 8.042 & 11.649 & 1.00 & 0.00 & $\mathrm{H}$ \\
\hline 10.212 & 11.311 & 11.577 & 1.00 & 0.00 & c \\
\hline 9.767 & 11.102 & 12.557 & 1.00 & 0.00 & $\mathrm{H}$ \\
\hline 10.102 & 12.391 & 11.402 & 1.00 & 0.00 & $\mathrm{H}$ \\
\hline 9.612 & 10.785 & 10.821 & 1.00 & 0.00 & $\mathrm{H}$ \\
\hline 20.009 & -0.068 & 16.864 & 1.00 & 0.00 & c \\
\hline 20.825 & 0.630 & 16.699 & 1.00 & 0.00 & $\mathrm{H}$ \\
\hline 20.342 & -1.293 & 17.266 & 1.00 & 0.00 & $c$ \\
\hline 19.611 & -2.072 & 17.373 & 1.00 & 0.00 & $\mathrm{H}$ \\
\hline 21.384 & -1.562 & 17.432 & 1.00 & 0.00 & $\mathrm{H}$ \\
\hline 17.346 & -0.323 & 18.702 & 1.00 & 0.00 & c \\
\hline 17.111 & -1.394 & 18.583 & 1.00 & 0.00 & $\mathrm{H}$ \\
\hline 16.533 & 0.141 & 19.272 & 1.00 & 0.00 & $\mathrm{H}$ \\
\hline 18.253 & -0.246 & 19.313 & 1.00 & 0.00 & $\mathrm{H}$ \\
\hline 12.335 & 1.355 & 18.188 & 1.00 & 0.00 & c \\
\hline 13.001 & 1.456 & 19.046 & 1.00 & 0.00 & $\mathrm{H}$ \\
\hline 11.169 & 0.710 & 18.346 & 1.00 & 0.00 & c \\
\hline 10.489 & 0.554 & 17.516 & 1.00 & 0.00 & $\mathrm{H}$ \\
\hline 10.864 & 0.312 & 19.312 & 1.00 & 0.00 & $\mathrm{H}$ \\
\hline 10.651 & 3.038 & 15.984 & 1.00 & 0.00 & c \\
\hline 10.494 & 4.060 & 15.617 & 1.00 & 0.00 & $\mathrm{H}$ \\
\hline 10.219 & 2.972 & 16.989 & 1.00 & 0.00 & $\mathrm{H}$ \\
\hline 10.060 & 2.374 & 15.335 & 1.00 & 0.00 & $\mathrm{H}$ \\
\hline 19.739 & 4.120 & 10.092 & 1.00 & 0.00 & c \\
\hline 19.000 & 4.131 & 9.280 & 1.00 & 0.00 & $\mathrm{H}$ \\
\hline 20.580 & 3.511 & 9.729 & 1.00 & 0.00 & $\mathrm{H}$ \\
\hline 20.218 & 5.557 & 10.365 & 1.00 & 0.00 & c \\
\hline 20.775 & 5.618 & 11.313 & 1.00 & 0.00 & $\mathrm{H}$ \\
\hline 19.347 & 6.224 & 10.472 & 1.00 & 0.00 & $\mathrm{H}$ \\
\hline 21.082 & 6.169 & 9.289 & 1.00 & 0.00 & c \\
\hline 21.310 & 5.647 & 8.191 & 1.00 & 0.00 & 0 \\
\hline 21.598 & 7.349 & 9.653 & 1.00 & 0.00 & 0 \\
\hline 22.207 & 7.703 & 8.917 & 1.00 & 0.00 & $\mathrm{H}$ \\
\hline
\end{tabular}

S152 
TER

$\begin{array}{llrll}\text { HETATM } & 1769 & \text { C1 } & \text { MET } & 191 \\ \text { HETATM } & 1770 & \text { H11 } & \text { MET } & 191\end{array}$

HETATM 1771 H12 MET 191

HETATM 1772 H13 MET 191

TER

HETATM 1773 C1 MET 192

HETATM 1774 H11 MET 192

HETATM 1775 H12 MET 192

HETATM 1776 H13 MET 192

TER

HETATM 1777 FE HEM 193

HETATM 1778 C1 HEM 193

HETATM 1779 C2 HEM 193

HETATM 1780 C3 HEM 193

HETATM 1781 C4 HEM 193

HETATM 1782 C5 HEM 193

HETATM 1783 H5 HEM 193

HETATM 1784 C6 HEM 193

HETATM $1785 \quad$ C7 HEM 193

HETATM 1786 C8 HEM 193

HETATM 1787 C9 HEM 193

HETATM 1788 C10 HEM 193

HETATM 1789 H1O HEM 193

HETATM 1790 C11 HEM 193

HETATM 1791 C12 HEM 193

HETATM 1792 C13 HEM 193

HETATM 1793 C14 HEM 193

HETATM 1794 C15 HEM 193

HETATM 1795 H15 HEM 193

HETATM 1796 C16 HEM 193

HETATM 1797 C17 HEM 193

HETATM 1798 C18 HEM 193

HETATM 1799 C19 HEM 193

HETATM $1800 \quad$ C20 HEM 193

HETATM 1801 H2O HEM 193

HETATM 1802 N21 HEM 193

HETATM 1803 N22 HEM 193

HETATM $1804 \quad$ N23 HEM 193

HETATM 1805 N24 HEM 193

HETATM 1806 C99 HEM 193

HETATM 1807 O91 HEM 193

HETATM 1808092 HEM 193

HETATM 1809 CT91 HEM 193

HETATM 1810 H911 HEM 193

HETATM 1811 H912 HEM 193

HETATM 1812 CT92 HEM 193

HETATM 1813 H921 HEM 193

HETATM 1814 H922 HEM 193

TER

HETATM 1815 FE HEM 194

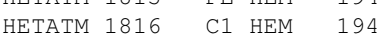

HETATM $1817 \quad$ C2 HEM 194

HETATM 1818 C3 HEM 194

HETATM 1819 C4 HEM 194

HETATM 1820 C5 HEM 194

HETATM 1821 H5 HEM 194

HETATM 1822 C6 HEM 194

HETATM $1823 \quad$ C7 HEM 194

HETATM $1824 \quad$ C8 HEM 194

HETATM $1825 \quad$ C9 HEM 194

HETATM 1826 C10 HEM 194

HETATM 1827 H1O HEM 194

HETATM 1828 C11 HEM 194

HETATM 1829 C12 HEM 194

HETATM 1830 C13 HEM 194

HETATM 1831 C14 HEM 194

HETATM 1832 C15 HEM 194

HETATM 1833 H15 HEM 194

HETATM 1834 C16 HEM 194

HETATM 1835 C17 HEM 194

HETATM 1836 C18 HEM 194

HETATM 1837 C19 HEM 194

HETATM 1838 C20 HEM 194

HETATM 1839 H2O HEM 194

HETATM 1840 N21 HEM 194

HETATM 1841 N22 HEM 194

HETATM 1842 N23 HEM 194

HETATM 1843 N24 HEM 194

\begin{tabular}{|c|c|c|c|c|c|}
\hline 11.843 & 5.246 & 11.266 & 1.00 & 0.00 & C \\
\hline 11.291 & 4.407 & 10.815 & 1.00 & 0.00 & $\mathrm{H}$ \\
\hline 11.837 & 6.072 & 10.544 & 1.00 & 0.00 & $\mathrm{H}$ \\
\hline 11.272 & 5.565 & 12.145 & 1.00 & 0.00 & $\mathrm{H}$ \\
\hline 21.277 & 2.297 & 12.216 & 1.00 & 0.00 & C \\
\hline 21.722 & 2.506 & 11.236 & 1.00 & 0.00 & $\mathrm{H}$ \\
\hline 21.387 & 1.217 & 12.391 & 1.00 & 0.00 & $\mathrm{H}$ \\
\hline 21.877 & 2.823 & 12.973 & 1.00 & 0.00 & $\mathrm{H}$ \\
\hline 17.315 & -3.836 & 9.423 & 1.00 & 0.00 & $\mathrm{Fe}$ \\
\hline 15.087 & -1.963 & 8.361 & 1.00 & 0.00 & c \\
\hline 14.728 & -1.173 & 7.189 & 1.00 & 0.00 & $\mathrm{C}$ \\
\hline 15.874 & -1.076 & 6.414 & 1.00 & 0.00 & $\mathrm{C}$ \\
\hline 16.904 & -1.793 & 7.122 & 1.00 & 0.00 & C \\
\hline 18.219 & -1.912 & 6.684 & 1.00 & 0.00 & C \\
\hline 18.472 & -1.416 & 5.749 & 1.00 & 0.00 & $\mathrm{H}$ \\
\hline 19.238 & -2.610 & 7.320 & 1.00 & 0.00 & C \\
\hline 20.607 & -2.683 & 6.851 & 1.00 & 0.00 & C \\
\hline 21.312 & -3.403 & 7.798 & 1.00 & 0.00 & C \\
\hline 20.361 & -3.768 & 8.822 & 1.00 & 0.00 & C \\
\hline 20.666 & -4.503 & 9.965 & 1.00 & 0.00 & C \\
\hline 21.693 & -4.846 & 10.071 & 1.00 & 0.00 & $\mathrm{H}$ \\
\hline 19.791 & -4.843 & 10.988 & 1.00 & 0.00 & C \\
\hline 20.172 & -5.568 & 12.178 & 1.00 & 0.00 & C \\
\hline 19.033 & -5.715 & 12.935 & 1.00 & 0.00 & C \\
\hline 17.960 & -5.074 & 12.204 & 1.00 & 0.00 & C \\
\hline 16.632 & -5.019 & 12.625 & 1.00 & 0.00 & C \\
\hline 16.392 & -5.504 & 13.573 & 1.00 & 0.00 & $\mathrm{H}$ \\
\hline 15.602 & -4.337 & 11.982 & 1.00 & 0.00 & C \\
\hline 14.236 & -4.224 & 12.464 & 1.00 & 0.00 & C \\
\hline 13.560 & -3.456 & 11.541 & 1.00 & 0.00 & $\mathrm{C}$ \\
\hline 14.510 & -3.104 & 10.504 & 1.00 & 0.00 & $\mathrm{C}$ \\
\hline 14.219 & $-2 \cdot 313$ & 9.393 & 1.00 & 0.00 & C \\
\hline 13.205 & -1.924 & 9.333 & 1.00 & 0.00 & $\mathrm{H}$ \\
\hline 16.409 & -2.326 & 8.298 & 1.00 & 0.00 & $\mathrm{~N}$ \\
\hline 19.114 & -3.273 & 8.521 & 1.00 & 0.00 & $\mathrm{~N}$ \\
\hline 18.443 & -4.536 & 11.021 & 1.00 & 0.00 & $\mathrm{~N}$ \\
\hline 15.743 & -3.652 & 10.786 & 1.00 & 0.00 & $\mathrm{~N}$ \\
\hline 16.650 & -6.517 & 8.283 & 1.00 & 0.00 & C \\
\hline 16.809 & -5.204 & 8.296 & 1.00 & 0.00 & O \\
\hline 16.495 & -7.134 & 7.228 & 1.00 & 0.00 & o \\
\hline 16.666 & -7.199 & 9.640 & 1.00 & 0.00 & C \\
\hline 17.645 & -7.003 & 10.103 & 1.00 & 0.00 & $\mathrm{H}$ \\
\hline 15.918 & -6.709 & 10.283 & 1.00 & 0.00 & $\mathrm{H}$ \\
\hline 16.406 & -8.703 & 9.528 & 1.00 & 0.00 & C \\
\hline 17.203 & -9.163 & 8.931 & 1.00 & 0.00 & $\mathrm{H}$ \\
\hline 15.481 & -8.859 & 8.958 & 1.00 & 0.00 & $\mathrm{H}$ \\
\hline 18.007 & -11.250 & 14.371 & 1.00 & 0.00 & $\mathrm{Fe}$ \\
\hline 20.235 & $-13 \cdot 122$ & 15.433 & 1.00 & 0.00 & $\mathrm{C}$ \\
\hline 20.594 & $-13 \cdot 912$ & 16.605 & 1.00 & 0.00 & $\mathrm{C}$ \\
\hline 19.449 & -14.009 & 17.380 & 1.00 & 0.00 & C \\
\hline 18.419 & -13.292 & 16.672 & 1.00 & 0.00 & C \\
\hline 17.104 & -13.173 & 17.109 & 1.00 & 0.00 & C \\
\hline 16.851 & -13.670 & 18.045 & 1.00 & 0.00 & $\mathrm{H}$ \\
\hline 16.085 & -12.475 & 16.473 & 1.00 & 0.00 & C \\
\hline 14.716 & -12.402 & 16.943 & 1.00 & 0.00 & C \\
\hline 14.010 & -11.682 & 15.996 & 1.00 & 0.00 & C \\
\hline 14.961 & $-11 \cdot 317$ & 14.972 & 1.00 & 0.00 & C \\
\hline 14.657 & -10.583 & 13.828 & 1.00 & 0.00 & C \\
\hline 13.630 & -10.239 & 13.722 & 1.00 & 0.00 & $\mathrm{H}$ \\
\hline 15.531 & -10.243 & 12.806 & 1.00 & 0.00 & C \\
\hline 15.150 & -9.517 & 11.615 & 1.00 & 0.00 & C \\
\hline 16.290 & -9.370 & 10.858 & 1.00 & 0.00 & C \\
\hline 17.363 & -10.011 & 11.590 & 1.00 & 0.00 & C \\
\hline 18.691 & -10.066 & 11.168 & 1.00 & 0.00 & C \\
\hline 18.931 & -9.581 & 10.221 & 1.00 & 0.00 & $\mathrm{H}$ \\
\hline 19.721 & -10.748 & 11.812 & 1.00 & 0.00 & C \\
\hline 21.087 & -10.861 & 11.329 & 1.00 & 0.00 & C \\
\hline 21.762 & -11.629 & 12.253 & 1.00 & 0.00 & $\mathrm{C}$ \\
\hline 20.813 & -11.981 & 13.289 & 1.00 & 0.00 & C \\
\hline 21.104 & -12.772 & 14.401 & 1.00 & 0.00 & C \\
\hline 22.118 & -13.161 & 14.461 & 1.00 & 0.00 & $\mathrm{H}$ \\
\hline 18.914 & -12.759 & 15.495 & 1.00 & 0.00 & $\mathrm{~N}$ \\
\hline 16.209 & -11.812 & 15.273 & 1.00 & 0.00 & $\mathrm{~N}$ \\
\hline 16.880 & -10.549 & 12.772 & 1.00 & 0.00 & $\mathrm{~N}$ \\
\hline 19.580 & -11.433 & 13.008 & 1.00 & 0.00 & $\mathrm{~N}$ \\
\hline
\end{tabular}


HETATM 1844 C99 HEM 194 HETATM 1845091 HEM 194 HETATM 1846092 HEM HETATM 1847 CT91 HEM 194 HETATM 1848 H911 HEM 194 HETATM 1849 H912 HEM 194 HETATM 1850 CT92 HEM 194 HETATM 1851 H921 HEM 194 HETATM 1852 H922 HEM 194 TER

HETATM 1853 C2 VIN 195 HETATM 1854 H21 VIN 195 HETATM 1855 C1 VIN 195 HETATM 1856 H11 VIN 195 HETATM 1857 H12 VIN 19 TER

HETATM 1858 C1 MET 196 HETATM 1859 H11 MET 196 HETATM 1860 H12 MET 196 HETATM 1861 H13 MET 19 TER

HETATM $1862 \quad \mathrm{C2}$ VTN 197 HETATM 1863 H21 VIN 197 HETATM 1864 C1 VIN 197 HETATM 1865 H11 VIN 197 HETATM 1866 H12 VIN 197 TER

HETATM $1867 \quad$ C1 MET 198 HETATM 1868 H11 MET 198 HETATM 1869 H12 MET 19 HETATM 1870 H13 MET 198 TER

HETATM 1871 C5 ACP 199 HETATM 1872 H51 ACP 199 HETATM 1873 H52 ACP 199 HETATM 1874 C4 ACP 199 HETATM 1875 H41 ACP 199 HETATM 1876 H42 ACP 199 HETATM $1877 \quad$ C1 ACP 199 HETATM $1878 \quad 02$ ACP 199 HETATM $1879 \quad 03 \mathrm{ACP} 199$ HETATM 1880 H3 ACP 199 TER

HETATM 1881 C1 MET 200 HETATM 1882 H11 MET 200 HETATM $1883 \quad$ H12 MET 200 HETATM 1884 H13 MET 200 TER

HETATM 1885 C1 MET 201 HETATM 1886 H11 MET 201 HETATM 1887 H12 MET 201 HETATM 1888 H13 MET 20 TER

HETATM 1889 C2 VIN 202 HETATM 1890 H21 VIN 202 HETATM 1891 C1 VIN 202 HETATM 1892 H11 VIN 202 HETATM 1893 H12 VIN 202

HETATM $1894 \quad$ C1 MET 203 HETATM 1895 H11 MET 203 HETATM 1896 H12 MET 203 HETATM 1897 H13 MET 203 TER

HETATM 1898 C2 VIN 204 HETATM 1899 H21 VIN 204 HETATM 1900 C1 VIN 204 HETATM 1901 H11 VIN 204 HETATM 1902 H12 VIN 204

HETATM 1903 C1 MET 205 HETATM 1904 H11 MET 205 HETATM 1905 H12 MET 205 HETATM 1906 H13 MET 205 TER

HETATM 1907 C5 ACP 206 HETATM 1908 H51 ACP 206 HETATM 1909 H52 ACP 206 HETATM $1910 \quad$ C4 ACP 206

\begin{tabular}{|c|c|c|c|c|c|}
\hline 18.673 & -8.568 & 15.510 & 1.00 & 0.00 & C \\
\hline 18.513 & -9.881 & 15.498 & 1.00 & 0.00 & o \\
\hline 18.828 & -7.951 & 16.566 & 1.00 & 0.00 & 0 \\
\hline 18.657 & -7.887 & 14.154 & 1.00 & 0.00 & $\mathrm{C}$ \\
\hline 17.678 & -8.082 & 13.691 & 1.00 & 0.00 & $\mathrm{H}$ \\
\hline 19.405 & -8.376 & 13.511 & 1.00 & 0.00 & $\mathrm{H}$ \\
\hline 18.916 & -6.382 & 14.265 & 1.00 & 0.00 & C \\
\hline 19.841 & -6.226 & 14.836 & 1.00 & 0.00 & $\mathrm{H}$ \\
\hline 18.120 & -5.922 & 14.862 & 1.00 & 0.00 & $\mathrm{H}$ \\
\hline 13.397 & -0.670 & 6.929 & 1.00 & 0.00 & $\mathrm{C}$ \\
\hline 12.581 & -1.369 & 7.094 & 1.00 & 0.00 & $\mathrm{H}$ \\
\hline 13.064 & 0.555 & 6.528 & 1.00 & 0.00 & $\mathrm{C}$ \\
\hline 13.795 & 1.333 & 6.421 & 1.00 & 0.00 & $\mathrm{H}$ \\
\hline 12.022 & 0.824 & 6.361 & 1.00 & 0.00 & $\mathrm{H}$ \\
\hline 16.060 & -0.415 & 5.091 & 1.00 & 0.00 & $\mathrm{C}$ \\
\hline 16.295 & 0.655 & 5.211 & 1.00 & 0.00 & $\mathrm{H}$ \\
\hline 16.873 & -0.880 & 4.522 & 1.00 & 0.00 & $\mathrm{H}$ \\
\hline 15.153 & -0.493 & 4.481 & 1.00 & 0.00 & $\mathrm{H}$ \\
\hline 21.071 & -2.093 & 5.606 & 1.00 & 0.00 & $\mathrm{C}$ \\
\hline 20.405 & -2.194 & 4.747 & 1.00 & 0.00 & $\mathrm{H}$ \\
\hline 22.237 & -1.449 & 5.447 & 1.00 & 0.00 & C \\
\hline 22.917 & -1.293 & 6.277 & 1.00 & 0.00 & $\mathrm{H}$ \\
\hline 22.542 & -1.051 & 4.482 & 1.00 & 0.00 & $\mathrm{H}$ \\
\hline 22.755 & -3.777 & 7.810 & 1.00 & 0.00 & C \\
\hline 23.346 & -3.112 & 8.459 & 1.00 & 0.00 & $\mathrm{H}$ \\
\hline 22.911 & -4.799 & 8.177 & 1.00 & 0.00 & $\mathrm{H}$ \\
\hline 23.187 & -3.711 & 6.805 & 1.00 & 0.00 & $\mathrm{H}$ \\
\hline 13.667 & -4.858 & 13.701 & 1.00 & 0.00 & C \\
\hline 14.406 & -4.870 & 14.514 & 1.00 & 0.00 & $\mathrm{H}$ \\
\hline 12.826 & -4.250 & 14.065 & 1.00 & 0.00 & $\mathrm{H}$ \\
\hline 13.188 & -6.296 & 13.428 & 1.00 & 0.00 & $\mathrm{C}$ \\
\hline 12.630 & -6.357 & 12.481 & 1.00 & 0.00 & $\mathrm{H}$ \\
\hline 14.059 & -6.962 & 13.321 & 1.00 & 0.00 & $\mathrm{H}$ \\
\hline 12.324 & -6.908 & 14.504 & 1.00 & 0.00 & C \\
\hline 12.096 & -6.385 & 15.603 & 1.00 & 0.00 & 0 \\
\hline 11.808 & -8.088 & 14.140 & 1.00 & 0.00 & 0 \\
\hline 11.199 & -8.442 & 14.876 & 1.00 & 0.00 & $\mathrm{H}$ \\
\hline 21.563 & -5.985 & 12.528 & 1.00 & 0.00 & C \\
\hline 22.115 & -5.145 & 12.978 & 1.00 & 0.00 & $\mathrm{H}$ \\
\hline 21.569 & -6.810 & 13.250 & 1.00 & 0.00 & $\mathrm{H}$ \\
\hline 22.134 & -6.304 & 11.649 & 1.00 & 0.00 & $\mathrm{H}$ \\
\hline 12.129 & -3.036 & 11.577 & 1.00 & 0.00 & C \\
\hline 11.684 & -3.245 & 12.557 & 1.00 & 0.00 & $\mathrm{H}$ \\
\hline 12.019 & -1.956 & 11.402 & 1.00 & 0.00 & $\mathrm{H}$ \\
\hline 11.529 & -3.561 & 10.821 & 1.00 & 0.00 & $\mathrm{H}$ \\
\hline 21.926 & -14.415 & 16.864 & 1.00 & 0.00 & C \\
\hline 22.742 & -13.716 & 16.699 & 1.00 & 0.00 & $\mathrm{H}$ \\
\hline 22.259 & -15.640 & 17.266 & 1.00 & 0.00 & C \\
\hline 21.528 & -16.418 & 17.373 & 1.00 & 0.00 & $\mathrm{H}$ \\
\hline 23.301 & -15.909 & 17.432 & 1.00 & 0.00 & $\mathrm{H}$ \\
\hline 19.262 & -14.670 & 18.702 & 1.00 & 0.00 & C \\
\hline 19.028 & -15.741 & 18.583 & 1.00 & 0.00 & $\mathrm{H}$ \\
\hline 18.449 & -14.206 & 19.272 & 1.00 & 0.00 & $\mathrm{H}$ \\
\hline 20.170 & -14.592 & 19.313 & 1.00 & 0.00 & $\mathrm{H}$ \\
\hline 14.252 & -12.992 & 18.188 & 1.00 & 0.00 & C \\
\hline 14.918 & -12.891 & 19.046 & 1.00 & 0.00 & $\mathrm{H}$ \\
\hline 13.085 & -13.636 & 18.346 & 1.00 & 0.00 & C \\
\hline 12.406 & -13.792 & 17.516 & 1.00 & 0.00 & $\mathrm{H}$ \\
\hline 12.781 & -14.034 & 19.312 & 1.00 & 0.00 & $\mathrm{H}$ \\
\hline 12.568 & -11.309 & 15.984 & 1.00 & 0.00 & C \\
\hline 12.411 & -10.286 & 15.617 & 1.00 & 0.00 & $\mathrm{H}$ \\
\hline 12.135 & -11.374 & 16.989 & 1.00 & 0.00 & $\mathrm{H}$ \\
\hline 11.976 & -11.973 & 15.335 & 1.00 & 0.00 & $\mathrm{H}$ \\
\hline 21.656 & -10.227 & 10.092 & 1.00 & 0.00 & C \\
\hline 20.917 & -10.216 & 9.280 & 1.00 & 0.00 & $\mathrm{H}$ \\
\hline 22.497 & -10.835 & 9.729 & 1.00 & 0.00 & $\mathrm{H}$ \\
\hline 22.135 & -8.789 & 10.365 & 1.00 & 0.00 & 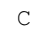 \\
\hline
\end{tabular}


HETATM 1911 H41 ACP HETATM 1912 H42 ACP HETATM 1 HETATM $1914 \quad 02$ ACP HETATM 1915 O3 ACP HETATM 1916 H3 ACP $T E R$

HETATM 1917 C1 MET HETATM 1918 H11 MET HETATM 1919 H12 MET HETATM 1920 H13 MET TER

HETATM 1921 C1 MET HETATM 1922 H11 MET HETATM 1923 H12 MET HETATM 1924 H13 MET TER

HETATM 1925 EE HEM HETATM 1926 C1 HEM 209 HETATM 1927 C2 HEM 209 HETATM 1928 C3 HEM 209 HETATM 1929 C4 HEM 209 HETATM 1930 C5 HEM 20 HETATM 1931 H5 HEM 209 HETATM 1932 C6 HEM 209 HETATM 1933 C7 HEM 209 HETATM 1934 C8 HEM 209 HETATM 1935 C9 HEM 209 HETATM 1936 C10 HEM 209 HETATM 1937 H1O HEM 20 HETATM 1938 C11 HEM 209 HETATM 1939 C12 HEM 209 HETATM 1940 C13 HEM 209 HETATM 1941 C14 HEM 20 HETATM 1942 C15 HEM 209 HETATM 1943 H15 HEM 209 HETATM 1944 C16 HEM 209 HETATM 1945 C17 HEM 209 HETATM 1946 C18 HEM 209 HETATM 1947 C19 HEM 209 HETATM 1948 C20 HEM 209 HETATM 1949 H2O HEM 209 HETATM 1950 N21 HEM 209 HETATM 1951 N22 HEM 209 HETATM 1952 N23 HEM 209 HETATM 1953 N24 HEM 209 HETATM 1954 C99 HEM 209 HETATM 1955091 HEM 209 HETATM 1956092 HEM 209 HETATM 1957 CT91 HEM 209 HETATM 1958 H911 HEM 209 HETATM 1959 H912 HEM 20 HETATM 1960 CT92 HEM 209 HETATM 1961 H921 HEM 209 HETATM 1962 H922 HEM 209 TER

HETATM 1963 FE HEM 210

HETATM 1964 HETATM 1965 C2 HEM 210 HETATM 1966 C3 HEM 210 HETATM 1967 C4 HEM 210 HETATM 1968 C5 HEM 210 HETATM 1969 H5 HEM 21 HETATM 1970 C6 HEM 210 HETATM 1971 C7 HEM 210 HETATM 1972 C8 HEM 210 HETATM 1973 C9 HEM 21 HETATM 1974 C10 HEM 210 HETATM 1975 H10 HEM 210 HETATM 1976 C11 HEM 210 HETATM 1977 C12 HEM 21 HETATM 1978 C13 HEM 210 HETATM 1979 C14 HEM 210 HETATM 1980 C15 HEM 21 HETATM 1981 H15 HEM 210 HETATM 1982 C16 HEM 210 HETATM 1983 C17 HEM 210 HETATM 1984 C18 HEM 210 HETATM 1985 C19 HEM

\begin{tabular}{|c|c|c|c|c|c|}
\hline 22.692 & -8.728 & 11.313 & 1.00 & 0.00 & $\mathrm{H}$ \\
\hline 21.264 & -8.123 & 10.472 & 1.00 & 0.00 & $\mathrm{H}$ \\
\hline 22.999 & -8.177 & 9.289 & 1.00 & 0.00 & C \\
\hline 23.226 & -8.700 & 8.191 & 1.00 & 0.00 & O \\
\hline 23.514 & -6.998 & 9.653 & 1.00 & 0.00 & o \\
\hline 24.123 & -6.643 & 8.917 & 1.00 & 0.00 & $\mathrm{H}$ \\
\hline 13.760 & -9.101 & 11.266 & 1.00 & 0.00 & C \\
\hline 13.208 & -9.940 & 10.815 & 1.00 & 0.00 & $\mathrm{H}$ \\
\hline 13.753 & -8.275 & 10.544 & 1.00 & 0.00 & $\mathrm{H}$ \\
\hline 13.189 & -8.781 & 12.145 & 1.00 & 0.00 & $\mathrm{H}$ \\
\hline 23.193 & -12.049 & 12.216 & 1.00 & 0.00 & C \\
\hline 23.639 & -11.841 & 11.236 & 1.00 & 0.00 & $\mathrm{H}$ \\
\hline 23.304 & -13.130 & 12.391 & 1.00 & 0.00 & $\mathrm{H}$ \\
\hline 23.794 & -11.524 & 12.973 & 1.00 & 0.00 & H \\
\hline 17.294 & 11.003 & -6.440 & 1.00 & 0.00 & $\mathrm{Fe}$ \\
\hline 15.066 & 12.876 & -7.502 & 1.00 & 0.00 & C \\
\hline 14.707 & 13.666 & -8.674 & 1.00 & 0.00 & C \\
\hline 15.852 & 13.763 & -9.448 & 1.00 & 0.00 & C \\
\hline 16.882 & 13.046 & -8.740 & 1.00 & 0.00 & C \\
\hline 18.197 & 12.927 & -9.178 & 1.00 & 0.00 & C \\
\hline 18.451 & 13.423 & -10.113 & 1.00 & 0.00 & $\mathrm{H}$ \\
\hline 19.216 & 12.229 & -8.542 & 1.00 & 0.00 & C \\
\hline 20.585 & 12.156 & -9.012 & 1.00 & 0.00 & C \\
\hline 21.291 & 11.436 & -8.065 & 1.00 & 0.00 & C \\
\hline 20.340 & 11.071 & -7.041 & 1.00 & 0.00 & C \\
\hline 20.644 & 10.336 & -5.897 & 1.00 & 0.00 & C \\
\hline 21.671 & 9.993 & -5.791 & 1.00 & 0.00 & $\mathrm{H}$ \\
\hline 19.770 & 9.996 & -4.874 & 1.00 & 0.00 & C \\
\hline 20.151 & 9.271 & -3.684 & 1.00 & 0.00 & C \\
\hline 19.011 & 9.124 & -2.927 & 1.00 & 0.00 & C \\
\hline 17.938 & 9.765 & -3.659 & 1.00 & 0.00 & C \\
\hline 16.610 & 9.820 & -3.237 & 1.00 & 0.00 & C \\
\hline 16.370 & 9.335 & -2.289 & 1.00 & 0.00 & $\mathrm{H}$ \\
\hline 15.580 & 10.502 & -3.880 & 1.00 & 0.00 & C \\
\hline 14.214 & 10.615 & -3.398 & 1.00 & 0.00 & C \\
\hline 13.539 & 11.383 & -4.322 & 1.00 & 0.00 & C \\
\hline 14.488 & 11.735 & -5.358 & 1.00 & 0.00 & C \\
\hline 14.197 & 12.526 & -6.469 & 1.00 & 0.00 & C \\
\hline 13.183 & 12.915 & -6.530 & 1.00 & 0.00 & $\mathrm{H}$ \\
\hline 16.387 & 12.513 & -7.564 & 1.00 & 0.00 & $\mathrm{~N}$ \\
\hline 19.092 & 11.566 & -7.342 & 1.00 & 0.00 & $\mathrm{~N}$ \\
\hline 18.421 & 10.303 & -4.841 & 1.00 & 0.00 & $\mathrm{~N}$ \\
\hline 15.722 & 11.187 & -5.077 & 1.00 & 0.00 & $\mathrm{~N}$ \\
\hline 16.628 & 8.322 & -7.579 & 1.00 & 0.00 & C \\
\hline 16.788 & 9.635 & -7.567 & 1.00 & 0.00 & 0 \\
\hline 16.473 & 7.705 & -8.635 & 1.00 & 0.00 & 0 \\
\hline 16.644 & 7.640 & -6.223 & 1.00 & 0.00 & C \\
\hline 17.623 & 7.836 & -5.760 & 1.00 & 0.00 & $\mathrm{H}$ \\
\hline 15.896 & 8.130 & -5.580 & 1.00 & 0.00 & $\mathrm{H}$ \\
\hline 16.385 & 6.136 & -6.334 & 1.00 & 0.00 & C \\
\hline 17.181 & 5.676 & -6.931 & 1.00 & 0.00 & $\mathrm{H}$ \\
\hline 15.460 & 5.980 & -6.905 & 1.00 & 0.00 & $\mathrm{H}$ \\
\hline 17.986 & 3.589 & -1.491 & 1.00 & 0.00 & $\mathrm{Fe}$ \\
\hline 20.214 & 1.717 & -0.429 & 1.00 & 0.00 & C \\
\hline 20.573 & 0.927 & 0.742 & 1.00 & 0.00 & C \\
\hline 19.427 & 0.830 & 1.517 & 1.00 & 0.00 & C \\
\hline 18.398 & 1.547 & 0.809 & 1.00 & 0.00 & c \\
\hline 17.082 & 1.666 & 1.247 & 1.00 & 0.00 & c \\
\hline 16.829 & 1.170 & 2.182 & 1.00 & 0.00 & $\mathrm{H}$ \\
\hline 16.063 & 2.364 & 0.611 & 1.00 & 0.00 & c \\
\hline 14.694 & 2.437 & 1.081 & 1.00 & 0.00 & c \\
\hline 13.989 & 3.157 & 0.134 & 1.00 & 0.00 & c \\
\hline 14.940 & 3.522 & -0.891 & 1.00 & 0.00 & c \\
\hline 14.635 & 4.256 & -2.034 & 1.00 & 0.00 & c \\
\hline 13.608 & 4.600 & -2.140 & 1.00 & 0.00 & $\mathrm{H}$ \\
\hline 15.510 & 4.596 & -3.057 & 1.00 & 0.00 & c \\
\hline 15.129 & 5.322 & -4.247 & 1.00 & 0.00 & c \\
\hline 16.268 & 5.469 & -5.004 & 1.00 & 0.00 & c \\
\hline 17.341 & 4.828 & -4.273 & 1.00 & 0.00 & c \\
\hline 18.669 & 4.773 & -4.694 & 1.00 & 0.00 & c \\
\hline 18.909 & 5.258 & -5.642 & 1.00 & 0.00 & $\mathrm{H}$ \\
\hline 19.699 & 4.091 & -4.051 & 1.00 & 0.00 & c \\
\hline 21.065 & 3.978 & -4.533 & 1.00 & 0.00 & c \\
\hline 21.741 & 3.210 & -3.609 & 1.00 & 0.00 & c \\
\hline 20.791 & 2.858 & -2.573 & 1.00 & 0.00 & c \\
\hline
\end{tabular}




\begin{tabular}{|c|c|c|c|c|c|c|c|c|c|c|}
\hline HETATM & 1986 & $\mathrm{C} 20$ & HEM & 210 & 21.082 & 2.067 & -1.462 & 1.00 & 0.00 & C \\
\hline HETATM & 1987 & $\mathrm{H} 2 \mathrm{O}$ & HEM & 210 & 22.097 & 1.678 & -1.402 & 1.00 & 0.00 & $\mathrm{H}$ \\
\hline HETATM & 1988 & N21 & HEM & 210 & 18.892 & 2.080 & -0.367 & 1.00 & 0.00 & $\mathrm{~N}$ \\
\hline HETATM & 1989 & N22 & HEM & 210 & 16.187 & 3.027 & -0.590 & 1.00 & 0.00 & $\mathrm{~N}$ \\
\hline HETATM & 1990 & N23 & HEM & 210 & 16.858 & 4.290 & -3.090 & 1.00 & 0.00 & $\mathrm{~N}$ \\
\hline HETATM & 1991 & N2 4 & HEM & 210 & 19.558 & 3.406 & -2.854 & 1.00 & 0.00 & $\mathrm{~N}$ \\
\hline HETATM & 1992 & C99 & HEM & 210 & 18.651 & 6.271 & -0.352 & 1.00 & 0.00 & C \\
\hline HETATM & 1993 & 091 & HEM & 210 & 18.492 & 4.958 & -0.365 & 1.00 & 0.00 & 0 \\
\hline HETATM & 1994 & 092 & HEM & 210 & 18.806 & 6.888 & 0.703 & 1.00 & 0.00 & 0 \\
\hline HETATM & 1995 & СТ91 & HEM & 210 & 18.635 & 6.952 & -1.709 & 1.00 & 0.00 & C \\
\hline HETATM & 1996 & H911 & HEM & 210 & 17.656 & 6.757 & -2.171 & 1.00 & 0.00 & $\mathrm{H}$ \\
\hline HETATM & 1997 & H912 & HEM & 210 & 19.383 & 6.463 & -2.352 & 1.00 & 0.00 & $\mathrm{H}$ \\
\hline HETATM & 1998 & СТ92 & HEM & 210 & 18.895 & 8.457 & -1.597 & 1.00 & 0.00 & C \\
\hline HETATM & 1999 & H921 & HEM & 210 & 19.820 & 8.613 & -1.027 & 1.00 & 0.00 & $\mathrm{H}$ \\
\hline $\begin{array}{l}\text { HETATM } \\
\text { TER }\end{array}$ & 2000 & H922 & HEM & \multicolumn{7}{|c|}{ TER } \\
\hline HETATM & 2001 & $\mathrm{C} 2$ & VIN & 211 & 13.375 & 14.169 & -8.933 & 1.00 & 0.00 & C \\
\hline HETATM & 2002 & H 21 & VIN & 211 & 12.559 & 13.470 & -8.768 & 1.00 & 0.00 & $\mathrm{H}$ \\
\hline HETATM & 2003 & $\mathrm{C} 1$ & VIN & 211 & 13.042 & 15.394 & -9.335 & 1.00 & 0.00 & C \\
\hline HETATM & 2004 & H11 & VIN & 211 & 13.774 & 16.172 & -9.442 & 1.00 & 0.00 & $\mathrm{H}$ \\
\hline HETATM & 2005 & $\mathrm{H} 12$ & VIN & 211 & 12.000 & 15.663 & -9.501 & 1.00 & 0.00 & $\mathrm{H}$ \\
\hline \multicolumn{11}{|c|}{ TER } \\
\hline HETATM & 2006 & $\mathrm{C} 1$ & MET & 212 & 16.039 & 14.424 & -10.771 & 1.00 & 0.00 & C \\
\hline HETATM & 2007 & $\mathrm{H} 11$ & $\mathrm{MET}$ & 212 & 16.274 & 15.494 & -10.652 & 1.00 & 0.00 & $\mathrm{H}$ \\
\hline HETATM & 2008 & $\mathrm{H} 12$ & $\mathrm{MET}$ & 212 & 16.852 & 13.959 & -11.341 & 1.00 & 0.00 & $\mathrm{H}$ \\
\hline HETATM & 2009 & $\mathrm{H} 13$ & $\mathrm{MET}$ & 212 & 15.131 & 14.346 & $-11 \cdot 382$ & 1.00 & 0.00 & $\mathrm{H}$ \\
\hline \multicolumn{11}{|c|}{ TER } \\
\hline HETATM & 2010 & $\mathrm{C} 2$ & VIN & 213 & 21.049 & 12.746 & -10.256 & 1.00 & 0.00 & C \\
\hline HETATM & 2011 & $\mathrm{H} 21$ & VIN & 213 & 20.383 & 12.645 & -11.115 & 1.00 & 0.00 & $\mathrm{H}$ \\
\hline HETATM & 2012 & $\mathrm{C} 1$ & VIN & 213 & 22.216 & 13.390 & -10.415 & 1.00 & 0.00 & C \\
\hline HETATM & 2013 & H11 & VIN & 213 & 22.896 & 13.546 & -9.585 & 1.00 & 0.00 & $\mathrm{H}$ \\
\hline HETATM & 2014 & $\mathrm{H} 12$ & VIN & 213 & 22.521 & 13.788 & -11.381 & 1.00 & 0.00 & $\mathrm{H}$ \\
\hline \multicolumn{11}{|l|}{ TER } \\
\hline HETATM & 2015 & $\mathrm{C} 1$ & $\mathrm{MET}$ & 214 & 22.733 & 11.062 & -8.052 & 1.00 & 0.00 & C \\
\hline HETATM & 2016 & $\mathrm{H} 11$ & $\mathrm{MET}$ & 214 & 23.325 & 11.727 & -7.403 & 1.00 & 0.00 & $\mathrm{H}$ \\
\hline HETATM & 2017 & $\mathrm{H} 12$ & $\mathrm{MET}$ & 214 & 22.890 & 10.040 & -7.685 & 1.00 & 0.00 & $\mathrm{H}$ \\
\hline HETATM & 2018 & $\mathrm{H} 13$ & $\mathrm{MET}$ & 214 & 23.166 & 11.128 & -9.057 & 1.00 & 0.00 & $\mathrm{H}$ \\
\hline \multicolumn{11}{|l|}{ TER } \\
\hline HETATM & 2019 & $\mathrm{C} 5$ & $\mathrm{ACP}$ & 215 & 13.645 & 9.981 & -2.161 & 1.00 & 0.00 & C \\
\hline HETATM & 2020 & H51 & $\mathrm{ACP}$ & 215 & 14.384 & 9.969 & -1.349 & 1.00 & 0.00 & $\mathrm{H}$ \\
\hline HETATM & 2021 & H52 & $\mathrm{ACP}$ & 215 & 12.804 & 10.589 & -1.798 & 1.00 & 0.00 & $\mathrm{H}$ \\
\hline HETATM & 2022 & $\mathrm{C} 4$ & $\mathrm{ACP}$ & 215 & 13.167 & 8.543 & -2.434 & 1.00 & 0.00 & C \\
\hline HETATM & 2023 & $\mathrm{H} 41$ & $\mathrm{ACP}$ & 215 & 12.609 & 8.482 & -3.382 & 1.00 & 0.00 & $\mathrm{H}$ \\
\hline HETATM & 2024 & $\mathrm{H} 42$ & $\mathrm{ACP}$ & 215 & 14.037 & 7.877 & -2.541 & 1.00 & 0.00 & $\mathrm{H}$ \\
\hline HETATM & 2025 & $\mathrm{C} 1$ & $\mathrm{ACP}$ & 215 & 12.303 & 7.931 & -1.358 & 1.00 & 0.00 & C \\
\hline HETATM & 2026 & $\mathrm{O} 2$ & $\mathrm{ACP}$ & 215 & 12.075 & 8.454 & -0.260 & 1.00 & 0.00 & 0 \\
\hline HETATM & 2027 & 03 & $\mathrm{ACP}$ & 215 & 11.787 & 6.751 & -1.722 & 1.00 & 0.00 & 0 \\
\hline HETATM & 2028 & H3 & $\mathrm{ACP}$ & 215 & 11.178 & 6.397 & -0.986 & 1.00 & 0.00 & $\mathrm{H}$ \\
\hline TER & & & & & & & & & & \\
\hline HETATM & 2029 & $\mathrm{C} 1$ & $\mathrm{MET}$ & 216 & 21.541 & 8.855 & -3.334 & 1.00 & 0.00 & C \\
\hline HETATM & 2030 & $\mathrm{H} 11$ & $\mathrm{MET}$ & 216 & 22.094 & 9.694 & -2.884 & 1.00 & 0.00 & $\mathrm{H}$ \\
\hline HETATM & 2031 & $\mathrm{H} 12$ & $\mathrm{MET}$ & 216 & 21.548 & 8.029 & -2.613 & 1.00 & 0.00 & $\mathrm{H}$ \\
\hline HETATM & 2032 & $\mathrm{H} 13$ & $\mathrm{MET}$ & 216 & 22.112 & 8.535 & -4.214 & 1.00 & 0.00 & $\mathrm{H}$ \\
\hline TER & & & & & & & & & & \\
\hline HETATM & 2033 & $\mathrm{C} 1$ & $\mathrm{MET}$ & 217 & 12.108 & 11.803 & -4.285 & 1.00 & 0.00 & C \\
\hline HETATM & 2034 & H11 & $\mathrm{MET}$ & 217 & 11.663 & 11.594 & -3.305 & 1.00 & 0.00 & $\mathrm{H}$ \\
\hline HETATM & 2035 & $\mathrm{H} 12$ & $\mathrm{MET}$ & 217 & 11.997 & 12.883 & -4.460 & 1.00 & 0.00 & $\mathrm{H}$ \\
\hline HETATM & 2036 & $\mathrm{H} 13$ & $\mathrm{MET}$ & 217 & 11.507 & 11.278 & -5.042 & 1.00 & 0.00 & $\mathrm{H}$ \\
\hline TER & & & & & & & & & & \\
\hline HETATM & 2037 & $\mathrm{C} 2$ & VIN & 218 & 21.904 & 0.424 & 1.002 & 1.00 & 0.00 & C \\
\hline HETATM & 2038 & $\mathrm{H} 21$ & VIN & 218 & 22.720 & 1.123 & 0.837 & 1.00 & 0.00 & $\mathrm{H}$ \\
\hline HETATM & 2039 & $\mathrm{C} 1$ & VIN & 218 & 22.237 & -0.801 & 1.403 & 1.00 & 0.00 & C \\
\hline HETATM & 2040 & $\mathrm{H} 11$ & VIN & 218 & 21.506 & -1.579 & 1.511 & 1.00 & 0.00 & $\mathrm{H}$ \\
\hline HETATM & 2041 & $\mathrm{H} 12$ & VIN & 218 & 23.279 & -1.070 & 1.570 & 1.00 & 0.00 & $\mathrm{H}$ \\
\hline TER & & & & & & & & & & \\
\hline HETATM & 2042 & $\mathrm{C} 1$ & $\mathrm{MET}$ & 219 & 19.241 & 0.169 & 2.840 & 1.00 & 0.00 & C \\
\hline HETATM & 2043 & $\mathrm{H} 11$ & $\mathrm{MET}$ & 219 & 19.006 & -0.902 & 2.720 & 1.00 & 0.00 & $\mathrm{H}$ \\
\hline HETATM & 2044 & $\mathrm{H} 12$ & $\mathrm{MET}$ & 219 & 18.428 & 0.633 & 3.410 & 1.00 & 0.00 & $\mathrm{H}$ \\
\hline HETATM & 2045 & $\mathrm{H} 13$ & $\mathrm{MET}$ & 219 & 20.148 & 0.247 & 3.450 & 1.00 & 0.00 & $\mathrm{H}$ \\
\hline TER & & & & & & & & & & \\
\hline HETATM & 2046 & $\mathrm{C} 2$ & VIN & 220 & 14.231 & 1.847 & 2.325 & 1.00 & 0.00 & C \\
\hline HETATM & 2047 & H 21 & VIN & 220 & 14.897 & 1.948 & 3.184 & 1.00 & 0.00 & $\mathrm{H}$ \\
\hline HETATM & 2048 & $\mathrm{C} 1$ & VIN & 220 & 13.064 & 1.203 & 2.484 & 1.00 & 0.00 & C \\
\hline HETATM & 2049 & $\mathrm{H} 11$ & VIN & 220 & 12.384 & 1.047 & 1.654 & 1.00 & 0.00 & $\mathrm{H}$ \\
\hline HETATM & 2050 & $\mathrm{H} 12$ & VIN & 220 & 12.759 & 0.805 & 3.450 & 1.00 & 0.00 & $\mathrm{H}$ \\
\hline TER & & & & & & & & & & \\
\hline HETATM & 2051 & $\mathrm{C} 1$ & $\mathrm{MET}$ & 221 & 12.546 & 3.530 & 0.121 & 1.00 & 0.00 & C \\
\hline HETATM & 2052 & H11 & $\mathrm{MET}$ & 221 & 12.390 & 4.553 & -0.246 & 1.00 & 0.00 & $\mathrm{H}$ \\
\hline HETATM & 2053 & $\mathrm{H} 12$ & $\mathrm{MET}$ & 221 & 12.114 & 3.465 & 1.126 & 1.00 & 0.00 & $\mathrm{H}$ \\
\hline
\end{tabular}




\begin{tabular}{|c|c|c|c|c|c|c|c|c|c|c|}
\hline $\begin{array}{l}\text { HETATM } \\
\text { TER }\end{array}$ & 2054 & $\mathrm{H} 13$ & $\mathrm{MET}$ & 221 & 11.955 & 2.866 & -0.528 & 1.00 & 0.00 & $\mathrm{H}$ \\
\hline HETATM & 2055 & $\mathrm{C} 5$ & $\mathrm{ACP}$ & 222 & 21.634 & 4.612 & -5.770 & 1.00 & 0.00 & C \\
\hline HETATM & 2056 & H51 & $\mathrm{ACP}$ & 222 & 20.895 & 4.623 & -6.582 & 1.00 & 0.00 & $\mathrm{H}$ \\
\hline HETATM & 2057 & H52 & $\mathrm{ACP}$ & 222 & 22.475 & 4.004 & -6.134 & 1.00 & 0.00 & $\mathrm{H}$ \\
\hline HETATM & 2058 & $\mathrm{C} 4$ & $\mathrm{ACP}$ & 222 & 22.113 & 6.050 & -5.497 & 1.00 & 0.00 & C \\
\hline HETATM & 2059 & $\mathrm{H} 41$ & $\mathrm{ACP}$ & 222 & 22.671 & 6.111 & -4.549 & 1.00 & 0.00 & $\mathrm{H}$ \\
\hline HETATM & 2060 & $\mathrm{H} 42$ & $\mathrm{ACP}$ & 222 & 21.242 & 6.716 & -5.390 & 1.00 & 0.00 & $\mathrm{H}$ \\
\hline HETATM & 2061 & $\mathrm{C} 1$ & $\mathrm{ACP}$ & 222 & 22.977 & 6.662 & -6.573 & 1.00 & 0.00 & C \\
\hline HETATM & 2062 & $\mathrm{O} 2$ & $\mathrm{ACP}$ & 222 & 23.205 & 6.139 & -7.671 & 1.00 & 0.00 & 0 \\
\hline HETATM & 2063 & 03 & $\mathrm{ACP}$ & 222 & 23.493 & 7.841 & -6.209 & 1.00 & 0.00 & 0 \\
\hline HETATM & 2064 & H3 & $\mathrm{ACP}$ & 222 & 24.102 & 8.196 & -6.945 & 1.00 & 0.00 & $\mathrm{H}$ \\
\hline \multicolumn{11}{|l|}{ TER } \\
\hline HETATM & 2065 & $\mathrm{C} 1$ & MET & 223 & 13.738 & 5.738 & -4.597 & 1.00 & 0.00 & C \\
\hline HETATM & 2066 & H11 & $\mathrm{MET}$ & 223 & 13.186 & 4.899 & -5.047 & 1.00 & 0.00 & $\mathrm{H}$ \\
\hline HETATM & 2067 & $\mathrm{H} 12$ & $\mathrm{MET}$ & 223 & 13.732 & 6.564 & -5.319 & 1.00 & 0.00 & $\mathrm{H}$ \\
\hline HETATM & 2068 & $\mathrm{H} 13$ & $\mathrm{MET}$ & 223 & 13.167 & 6.058 & -3.717 & 1.00 & 0.00 & $\mathrm{H}$ \\
\hline \multicolumn{11}{|l|}{ TER } \\
\hline HETATM & 2069 & $\mathrm{C} 1$ & MET & 224 & 23.172 & 2.790 & -3.646 & 1.00 & 0.00 & C \\
\hline HETATM & 2070 & $\mathrm{H} 11$ & $\mathrm{MET}$ & 224 & 23.617 & 2.998 & -4.626 & 1.00 & 0.00 & $\mathrm{H}$ \\
\hline HETATM & 2071 & $\mathrm{H} 12$ & MET & 224 & 23.282 & 1.709 & -3.471 & 1.00 & 0.00 & $\mathrm{H}$ \\
\hline HETATM & 2072 & H13 & $\mathrm{MET}$ & 224 & 23.772 & 3.315 & -2.889 & 1.00 & 0.00 & $\mathrm{H}$ \\
\hline \multicolumn{11}{|l|}{ TER } \\
\hline HETATM & 2073 & $\mathrm{FE}$ & HEM & 225 & 19.210 & -3.343 & -6.440 & 1.00 & 0.00 & $\mathrm{Fe}$ \\
\hline HETATM & 2074 & $\mathrm{C} 1$ & HEM & 225 & 16.983 & -1.471 & -7.502 & 1.00 & 0.00 & C \\
\hline HETATM & 2075 & $\mathrm{C} 2$ & HEM & 225 & 16.624 & -0.680 & -8.674 & 1.00 & 0.00 & C \\
\hline HETATM & 2076 & C3 & HEM & 225 & 17.769 & -0.583 & -9.448 & 1.00 & 0.00 & C \\
\hline HETATM & 2077 & $\mathrm{C} 4$ & HEM & 225 & 18.799 & -1.301 & -8.740 & 1.00 & 0.00 & C \\
\hline HETATM & 2078 & $\mathrm{C} 5$ & HEM & 225 & 20.114 & -1.420 & -9.178 & 1.00 & 0.00 & C \\
\hline HETATM & 2079 & H5 & HEM & 225 & 20.367 & -0.923 & -10.113 & 1.00 & 0.00 & $\mathrm{H}$ \\
\hline HETATM & 2080 & $\mathrm{C} 6$ & HEM & 225 & 21.133 & -2.118 & -8.542 & 1.00 & 0.00 & C \\
\hline HETATM & 2081 & C7 & HEM & 225 & 22.502 & -2.191 & -9.012 & 1.00 & 0.00 & C \\
\hline HETATM & 2082 & $\mathrm{C} 8$ & HEM & 225 & 23.207 & -2.910 & -8.065 & 1.00 & 0.00 & C \\
\hline HETATM & 2083 & $\mathrm{C} 9$ & HEM & 225 & 22.256 & -3.275 & -7.041 & 1.00 & 0.00 & C \\
\hline HETATM & 2084 & $\mathrm{C} 10$ & HEM & 225 & 22.561 & -4.010 & -5.897 & 1.00 & 0.00 & C \\
\hline HETATM & 2085 & $\mathrm{H} 10$ & HEM & 225 & 23.588 & -4.354 & -5.791 & 1.00 & 0.00 & $\mathrm{H}$ \\
\hline HETATM & 2086 & $\mathrm{C} 11$ & HEM & 225 & 21.687 & -4.350 & -4.874 & 1.00 & 0.00 & C \\
\hline HETATM & 2087 & $\mathrm{C} 12$ & HEM & 225 & 22.067 & -5.076 & -3.684 & 1.00 & 0.00 & C \\
\hline HETATM & 2088 & C13 & HEM & 225 & 20.928 & -5.223 & -2.927 & 1.00 & 0.00 & C \\
\hline HETATM & 2089 & C14 & HEM & 225 & 19.855 & -4.581 & -3.659 & 1.00 & 0.00 & C \\
\hline HETATM & 2090 & C15 & HEM & 225 & 18.527 & -4.527 & -3.237 & 1.00 & 0.00 & C \\
\hline HETATM & 2091 & H15 & HEM & 225 & 18.287 & -5.011 & -2.289 & 1.00 & 0.00 & $\mathrm{H}$ \\
\hline HETATM & 2092 & C16 & HEM & 225 & 17.497 & -3.845 & -3.880 & 1.00 & 0.00 & C \\
\hline HETATM & 2093 & $\mathrm{C} 17$ & HEM & 225 & 16.131 & -3.732 & -3.398 & 1.00 & 0.00 & C \\
\hline HETATM & 2094 & C18 & HEM & 225 & 15.456 & -2.964 & -4.322 & 1.00 & 0.00 & C \\
\hline HETATM & 2095 & C19 & HEM & 225 & 16.405 & -2.612 & -5.358 & 1.00 & 0.00 & C \\
\hline HETATM & 2096 & $\mathrm{C} 20$ & HEM & 225 & 16.114 & -1.821 & -6.469 & 1.00 & 0.00 & C \\
\hline HETATM & 2097 & $\mathrm{H} 2 \mathrm{O}$ & HEM & 225 & 15.100 & -1.432 & -6.530 & 1.00 & 0.00 & $\mathrm{H}$ \\
\hline HETATM & 2098 & $\mathrm{~N} 21$ & HEM & 225 & 18.304 & -1.834 & -7.564 & 1.00 & 0.00 & $\mathrm{~N}$ \\
\hline HETATM & 2099 & $\mathrm{~N} 22$ & HEM & 225 & 21.009 & -2.781 & -7.342 & 1.00 & 0.00 & $\mathrm{~N}$ \\
\hline HETATM & 2100 & N23 & HEM & 225 & 20.338 & -4.044 & -4.841 & 1.00 & 0.00 & $\mathrm{~N}$ \\
\hline HETATM & 2101 & $\mathrm{~N} 24$ & HEM & 225 & 17.638 & -3.160 & -5.077 & 1.00 & 0.00 & $\mathrm{~N}$ \\
\hline HETATM & 2102 & C99 & HEM & 225 & 18.545 & -6.025 & -7.579 & 1.00 & 0.00 & C \\
\hline HETATM & 2103 & 091 & HEM & 225 & 18.705 & -4.711 & -7.567 & 1.00 & 0.00 & O \\
\hline HETATM & 2104 & 092 & HEM & 225 & 18.390 & -6.642 & -8.635 & 1.00 & 0.00 & O \\
\hline HETATM & 2105 & СТ91 & HEM & 225 & 18.561 & -6.706 & -6.223 & 1.00 & 0.00 & C \\
\hline HETATM & 2106 & H911 & HEM & 225 & 19.540 & -6.511 & -5.760 & 1.00 & 0.00 & $\mathrm{H}$ \\
\hline HETATM & 2107 & H912 & HEM & 225 & 17.813 & -6.216 & -5.580 & 1.00 & 0.00 & $\mathrm{H}$ \\
\hline HETATM & 2108 & СТ92 & HEM & 225 & 18.302 & -8.211 & -6.334 & 1.00 & 0.00 & C \\
\hline HETATM & 2109 & H921 & HEM & 225 & 19.098 & -8.671 & -6.931 & 1.00 & 0.00 & $\mathrm{H}$ \\
\hline HETATM & 2110 & H922 & HEM & 225 & 17.377 & -8.367 & -6.905 & 1.00 & 0.00 & $\mathrm{H}$ \\
\hline \multicolumn{11}{|l|}{ TER } \\
\hline HETATM & 2111 & $\mathrm{FE}$ & HEM & 226 & 19.903 & -10.757 & -1.491 & 1.00 & 0.00 & $\mathrm{Fe}$ \\
\hline HETATM & 2112 & $\mathrm{C} 1$ & HEM & 226 & 22.131 & -12.629 & -0.429 & 1.00 & 0.00 & C \\
\hline HETATM & 2113 & $\mathrm{C} 2$ & HEM & 226 & 22.489 & -13.420 & 0.742 & 1.00 & 0.00 & C \\
\hline HETATM & 2114 & C3 & HEM & 226 & 21.344 & -13.517 & 1.517 & 1.00 & 0.00 & C \\
\hline HETATM & 2115 & C4 & HEM & 226 & 20.314 & -12.800 & 0.809 & 1.00 & 0.00 & C \\
\hline HETATM & 2116 & C5 & HEM & 226 & 18.999 & -12.681 & 1.247 & 1.00 & 0.00 & C \\
\hline HETATM & 2117 & H5 & HEM & 226 & 18.746 & -13.177 & 2.182 & 1.00 & 0.00 & $\mathrm{H}$ \\
\hline HETATM & 2118 & $\mathrm{C} 6$ & HEM & 226 & 17.980 & -11.982 & 0.611 & 1.00 & 0.00 & C \\
\hline HETATM & 2119 & $\mathrm{C} 7$ & HEM & 226 & 16.611 & -11.910 & 1.081 & 1.00 & 0.00 & C \\
\hline HETATM & 2120 & $\mathrm{C} 8$ & HEM & 226 & 15.906 & -11.190 & 0.134 & 1.00 & 0.00 & C \\
\hline HETATM & 2121 & $\mathrm{C} 9$ & HEM & 226 & 16.857 & -10.825 & -0.891 & 1.00 & 0.00 & C \\
\hline HETATM & 2122 & C10 & HEM & 226 & 16.552 & -10.090 & -2.034 & 1.00 & 0.00 & C \\
\hline HETATM & 2123 & $\mathrm{H} 10$ & HEM & 226 & 15.525 & -9.746 & -2.140 & 1.00 & 0.00 & $\mathrm{H}$ \\
\hline HETATM & 2124 & $\mathrm{C} 11$ & HEM & 226 & 17.427 & -9.750 & -3.057 & 1.00 & 0.00 & C \\
\hline HETATM & 2125 & $\mathrm{C} 12$ & HEM & 226 & 17.046 & -9.025 & -4.247 & 1.00 & 0.00 & C \\
\hline HETATM & 2126 & C13 & HEM & 226 & 18.185 & -8.878 & -5.004 & 1.00 & 0.00 & C \\
\hline HETATM & 2127 & C14 & HEM & 226 & 19.258 & -9.519 & -4.273 & 1.00 & 0.00 & C \\
\hline
\end{tabular}


HETATM 2128 C15 HEM 226 HETATM 2129 H15 HEM 226

HETATM 2130 C16 HEM

HETATM 2131 C17 HEM 226

HETATM 2132 C18 HEM 226

HETATM 2133 C19 HEM 226

HETATM $2134 \quad$ C20 HEM 226

HETATM 2135 H20 HEM 226

HETATM 2136 N21 HEM 226

HETATM 2137 N22 HEM 226

HETATM 2138 N23 HEM 226

HETATM 2139 N24 HEM 226

HETATM 2140 C99 HEM 226

HETATM 2141091 HEM 226

HETATM 2142092 HEM 22

HETATM 2143 CT91 HEM 226

HETATM 2144 H911 HEM 226

HETATM 2145 H912 HEM 226

HETATM 2146 CT92 HEM 226

HETATM 2147 H921 HEM 226

HETATM 2148 H922 HEM 226

TER

HETATM 2149 C2 VIN 227

HETATM 2150 H21 VIN 227

HETATM 2151 C1 VIN 227

HETATM 2152 H11 VIN 22

HETATM 2153 H12 VIN 22

HETATM 2154 C1 MET 228

HETATM 2155 H11 MET 228

HETATM 2156 H12 MET 228

HETATM 2157 H13 MET 228

TER

HETATM 2158 C2 VIN 229

HETATM 2159 H21 VIN 229

HETATM 2160 C1 VIN 229

HETATM 2161 H11 VIN 22

HETATM 2162 H12 VIN 22

TER

HETATM 2163 C1 MET 230

HETATM 2164 H11 MET 230

HETATM 2165 H12 MET 230

HETATM 2166 H13 MET 230

TER

HETATM $2167 \quad$ C5 ACP 231

HETATM 2168 H51 ACP 231

HETATM 2169 H52 ACP 231

HETATM $2170 \quad$ C4 ACP 231

HETATM 2171 H41 ACP 231

HETATM 2172 H42 ACP 231

HETATM 2173 C1 ACP 231

HETATM $2174 \quad 02$ ACP 231

HETATM $2175 \quad 03$ ACP 231

HETATM 2176 H3 ACP 231

TER

HETATM 2177 C1 MET 232

HETATM 2178 H11 MET 232

HETATM 2179 H12 MET 232

HETATM 2180 H13 MET 232

TER

HETATM 2181 C1 MET 233

HETATM 2182 H11 MET 233

HETATM 2183 H12 MET 23

HETATM 2184 H13 MET 233

TER

HETATM 2185 C2 VIN 234

HETATM 2186 H21 VIN 234

HETATM $2187 \quad$ C1 VIN 234

HETATM 2188 H11 VIN 234

HETATM 2189 H12 VIN 23

TER

HETATM 2190 C1 MET 235

HETATM 2191 H11 MET 235

HETATM 2192 H12 MET 235

HETATM 2193 H13 MET 235

HETATM 2194 C2 VIN 236

HETATM 2195 H21 VIN 236

HETATM 2196 C1 VIN 236 $\begin{array}{lllll}20.586 & -9.574 & -4.694 & 1.00 & 0.00\end{array}$

$\begin{array}{lllll}20.826 & -9.089 & -5.642 & 1.00 & 0.00\end{array}$

$\begin{array}{lllll}21.616 & -10.255 & -4.051 & 1.00 & 0.00\end{array}$

$\begin{array}{lllll}22.982 & -10.369 & -4.533 & 1.00 & 0.00\end{array}$

$\begin{array}{lllll}23.657 & -11.137 & -3.609 & 1.00 & 0.00\end{array}$

$22.708-11.489-2.573 \quad 1.00 \quad 0.00$

$\begin{array}{lllll}22.999 & -12.280 & -1.462 & 1.00 & 0.00\end{array}$

$\begin{array}{lllll}24.013 & -12.669 & -1.402 & 1.00 & 0.00\end{array}$

$\begin{array}{lllll}20.809 & -12.266 & -0.367 & 1.00 & 0.00\end{array}$

$\begin{array}{lllll}18.104 & -11.319 & -0.590 & 1.00 & 0.00\end{array}$

$\begin{array}{lllll}18.775 & -10.056 & -3.090 & 1.00 & 0.00\end{array}$

$21.475-10.941 \quad-2.854 \quad 1.00 \quad 0.00$

$\begin{array}{llllll}20.568 & -8.076 & -0.352 & 1.00 & 0.00\end{array}$

$\begin{array}{lllll}20.408 & -9.389 & -0.365 & 1.00 & 0.00\end{array}$

$\begin{array}{lllll}20.723 & -7.458 & 0.703 & 1.00 & 0.00\end{array}$

$\begin{array}{lllll}20.552 & -7.394 & -1.709 & 1.00 & 0.00\end{array}$

$\begin{array}{lllll}19.573 & -7.590 & -2.171 & 1.00 & 0.00\end{array}$

$\begin{array}{lllll}21.300 & -7.884 & -2.352 & 1.00 & 0.00\end{array}$

$\begin{array}{lllll}20.812 & -5.890 & -1.597 & 1.00 & 0.00\end{array}$

$\begin{array}{lllll}21.736 & -5.734 & -1.027 & 1.00 & 0.00\end{array}$

$20.015 \quad-5.429 \quad-1.000 \quad 1.00 \quad 0.00$

$\begin{array}{llllll}15.292 & -0.178 & -8.933 & 1.00 & 0.00\end{array}$

$\begin{array}{lllll}14.476 & -0.876 & -8.768 & 1.00 & 0.00\end{array}$

$\begin{array}{llllll}14.959 & 1.047 & -9.335 & 1.00 & 0.00\end{array}$

$\begin{array}{lllll}15.690 & 1.826 & -9.442 & 1.00 & 0.00\end{array}$

$\begin{array}{lllll}13.917 & 1.316 & -9.501 & 1.00 & 0.00\end{array}$

$\begin{array}{lllll}17.956 & 0.077 & -10.771 & 1.00 & 0.00\end{array}$

$\begin{array}{lllll}18.190 & 1.148 & -10.652 & 1.00 & 0.00\end{array}$

$\begin{array}{lllll}18.769 & -0.387 & -11.341 & 1.00 & 0.00\end{array}$

$\begin{array}{lllll}17.048 & -0.001 & -11.382 & 1.00 & 0.00\end{array}$

$22.966-1.601-10.256 \quad 1.00 \quad 0.00$

$\begin{array}{lllll}22.300 & -1.702 & -11.115 & 1.00 & 0.00\end{array}$

$\begin{array}{llllll}24.132 & -0.957 & -10.415 & 1.00 & 0.00\end{array}$

$\begin{array}{lllll}24.812 & -0.800 & -9.585 & 1.00 & 0.00\end{array}$

$\begin{array}{llllll}24.437 & -0.559 & -11.381 & 1.00 & 0.00\end{array}$

$\begin{array}{lllll}24.650 & -3.284 & -8.052 & 1.00 & 0.00\end{array}$

$\begin{array}{lllll}25.242 & -2.620 & -7.403 & 1.00 & 0.00\end{array}$

$\begin{array}{lllll}24.807 & -4.306 & -7.685 & 1.00 & 0.00\end{array}$

$\begin{array}{llllll}25.083 & -3.219 & -9.057 & 1.00 & 0.00\end{array}$

$\begin{array}{lllll}15.562 & -4.366 & -2.161 & 1.00 & 0.00\end{array}$

$\begin{array}{lllll}16.301 & -4.377 & -1.349 & 1.00 & 0.00\end{array}$

$\begin{array}{lllll}14.721 & -3.758 & -1.798 & 1.00 & 0.00\end{array}$

$\begin{array}{lllll}15.083 & -5.803 & -2.434 & 1.00 & 0.00\end{array}$

$\begin{array}{lllll}14.526 & -5.865 & -3.382 & 1.00 & 0.00\end{array}$

$\begin{array}{lllll}15.954 & -6.470 & -2.541 & 1.00 & 0.00\end{array}$

$\begin{array}{lllll}14.219 & -6.416 & -1.358 & 1.00 & 0.00\end{array}$

$\begin{array}{lllll}13.991 & -5.893 & -0.260 & 1.00 & 0.00\end{array}$

$\begin{array}{lllll}13.703 & -7.595 & -1.722 & 1.00 & 0.00\end{array}$

$\begin{array}{lllll}13.094 & -7.949 & -0.986 & 1.00 & 0.00\end{array}$

$\begin{array}{llllll}23.458 & -5.492 & -3.334 & 1.00 & 0.00\end{array}$

$\begin{array}{lllll}24.010 & -4.653 & -2.884 & 1.00 & 0.00\end{array}$

$\begin{array}{lllll}23.464 & -6.318 & -2.613 & 1.00 & 0.00\end{array}$

$\begin{array}{lllll}24.029 & -5.812 & -4.214 & 1.00 & 0.00\end{array}$

$\begin{array}{lllll}14.024 & -2.543 & -4.285 & 1.00 & 0.00\end{array}$

$\begin{array}{lllll}13.579 & -2.752 & -3.305 & 1.00 & 0.00\end{array}$

$\begin{array}{lllll}13.914 & -1.463 & -4.460 & 1.00 & 0.00\end{array}$

$\begin{array}{lllll}13.424 & -3.069 & -5.042 & 1.00 & 0.00\end{array}$

$23.821-13.923$

$24.637-13.224$

$24.154-15.147$

$23.423-15.926$

$25.196-15.417$

$\begin{array}{lll}1.002 & 1.00 & 0.00\end{array}$

$0.837 \quad 1.00 \quad 0.00$

$\begin{array}{lll}1.403 & 1.00 & 0.00\end{array}$

$\begin{array}{lll}1.511 & 1.00 & 0.00\end{array}$

$1.570 \quad 1.00 \quad 0.00$

$21.158-14.177$

$20.923-15.248$

$20.345-13.713$

$2.840 \quad 1.00 \quad 0.00$

$2.720 \quad 1.00 \quad 0.00$

$\begin{array}{lll}3.410 & 1.00 \quad 0.00\end{array}$

$3.450 \quad 1.00 \quad 0.00$

$16.147-12.499$

$16.813-12.399$

$\begin{array}{llll}2.325 & 1.00 & 0.00\end{array}$

$\begin{array}{lll}3.184 & 1.00 & 0.00\end{array}$

$\begin{array}{llllll}14.981 & -13.144 & 2.484 & 1.00 & 0.00 & \text { C }\end{array}$

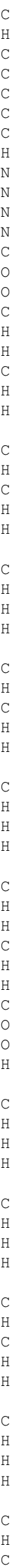

S158 


\begin{tabular}{|c|c|c|c|c|c|c|c|c|c|c|}
\hline HETATM & 2197 & H11 & VIN & 236 & 14.301 & $-13 \cdot 300$ & 1.654 & 1.00 & 0.00 & $\mathrm{H}$ \\
\hline HETATM & 2198 & $\mathrm{H} 12$ & VIN & 236 & 14.676 & $-13 \cdot 542$ & 3.450 & 1.00 & 0.00 & $\mathrm{H}$ \\
\hline \multicolumn{11}{|l|}{ TER } \\
\hline HETATM & 2199 & $\mathrm{C} 1$ & $\mathrm{MET}$ & 237 & 14.463 & -10.816 & 0.121 & 1.00 & 0.00 & C \\
\hline HETATM & 2200 & H11 & $\mathrm{MET}$ & 237 & 14.306 & -9.794 & -0.246 & 1.00 & 0.00 & 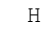 \\
\hline HETATM & 2201 & $\mathrm{H} 12$ & $\mathrm{MET}$ & 237 & 14.031 & -10.882 & 1.126 & 1.00 & 0.00 & $\mathrm{H}$ \\
\hline HETATM & 2202 & H13 & MET & 237 & 13.872 & -11.480 & -0.528 & 1.00 & 0.00 & 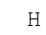 \\
\hline \multicolumn{11}{|l|}{ TER } \\
\hline HETATM & 2203 & C5 & $\mathrm{ACP}$ & 238 & 23.551 & -9.735 & -5.770 & 1.00 & 0.00 & C \\
\hline HETATM & 2204 & H51 & $\mathrm{ACP}$ & 238 & 22.812 & -9.723 & -6.582 & 1.00 & 0.00 & $\mathrm{H}$ \\
\hline HETATM & 2205 & H52 & $\mathrm{ACP}$ & 238 & 24.392 & $-10 \cdot 343$ & -6.134 & 1.00 & 0.00 & $\mathrm{H}$ \\
\hline HETATM & 2206 & C4 & $\mathrm{ACP}$ & 238 & 24.030 & -8.297 & -5.497 & 1.00 & 0.00 & C \\
\hline HETATM & 2207 & H4 1 & $\mathrm{ACP}$ & 238 & 24.587 & -8.236 & -4.549 & 1.00 & 0.00 & $\mathrm{H}$ \\
\hline HETATM & 2208 & $\mathrm{H} 42$ & $\mathrm{ACP}$ & 238 & 23.159 & -7.630 & -5.390 & 1.00 & 0.00 & $\mathrm{H}$ \\
\hline HETATM & 2209 & $\mathrm{C} 1$ & $\mathrm{ACP}$ & 238 & 24.894 & -7.685 & -6.573 & 1.00 & 0.00 & C \\
\hline HETATM & 2210 & $\mathrm{O} 2$ & $\mathrm{ACP}$ & 238 & 25.122 & -8.208 & -7.671 & 1.00 & 0.00 & 0 \\
\hline HETATM & 2211 & 03 & $\mathrm{ACP}$ & 238 & 25.410 & -6.505 & -6.209 & 1.00 & 0.00 & 0 \\
\hline HETATM & 2212 & $\mathrm{H} 3$ & $\mathrm{ACP}$ & 238 & 26.019 & -6.151 & -6.945 & 1.00 & 0.00 & $\mathrm{H}$ \\
\hline \multicolumn{11}{|l|}{ TER } \\
\hline HETATM & 2213 & $\mathrm{C} 1$ & $\mathrm{MET}$ & 239 & 15.655 & -8.608 & -4.597 & 1.00 & 0.00 & C \\
\hline HETATM & 2214 & H11 & MET & 239 & 15.103 & -9.448 & -5.047 & 1.00 & 0.00 & $\mathrm{H}$ \\
\hline HETATM & 2215 & $\mathrm{H} 12$ & $\mathrm{MET}$ & 239 & 15.649 & -7.782 & -5.319 & 1.00 & 0.00 & $\mathrm{H}$ \\
\hline HETATM & 2216 & $\mathrm{H} 13$ & $\mathrm{MET}$ & 239 & 15.084 & -8.289 & -3.717 & 1.00 & 0.00 & $\mathrm{H}$ \\
\hline \multicolumn{11}{|l|}{ TER } \\
\hline HETATM & 2217 & $\mathrm{C} 1$ & $\mathrm{MET}$ & 240 & 25.089 & -11.557 & -3.646 & 1.00 & 0.00 & C \\
\hline HETATM & 2218 & H11 & $\mathrm{MET}$ & 240 & 25.534 & $-11 \cdot 348$ & -4.626 & 1.00 & 0.00 & $\mathrm{H}$ \\
\hline HETATM & 2219 & $\mathrm{H} 12$ & $\mathrm{MET}$ & 240 & 25.199 & -12.637 & -3.471 & 1.00 & 0.00 & $\mathrm{H}$ \\
\hline HETATM & 2220 & $\mathrm{H} 13$ & $\mathrm{MET}$ & 240 & 25.689 & -11.031 & -2.889 & 1.00 & 0.00 & $\mathrm{H}$ \\
\hline TER & & & & & & & & & & \\
\hline HETATM & 2221 & $\mathrm{FE}$ & HEM & 241 & 13.482 & 24.858 & 9.423 & 1.00 & 0.00 & $\mathrm{Fe}$ \\
\hline HETATM & 2222 & $\mathrm{C} 1$ & HEM & 241 & 11.254 & 26.730 & 8.361 & 1.00 & 0.00 & C \\
\hline HETATM & 2223 & $\mathrm{C} 2$ & HEM & 241 & 10.895 & 27.520 & 7.189 & 1.00 & 0.00 & C \\
\hline HETATM & 2224 & C3 & HEM & 241 & 12.040 & 27.617 & 6.414 & 1.00 & 0.00 & C \\
\hline HETATM & 2225 & C4 & HEM & 241 & 13.070 & 26.900 & 7.122 & 1.00 & 0.00 & C \\
\hline HETATM & 2226 & C5 & HEM & 241 & 14.385 & 26.781 & 6.684 & 1.00 & 0.00 & C \\
\hline HETATM & 2227 & H5 & HEM & 241 & 14.639 & 27.278 & 5.749 & 1.00 & 0.00 & $\mathrm{H}$ \\
\hline HETATM & 2228 & $\mathrm{C} 6$ & HEM & 241 & 15.404 & 26.083 & 7.320 & 1.00 & 0.00 & C \\
\hline HETATM & 2229 & $\mathrm{C} 7$ & HEM & 241 & 16.773 & 26.010 & 6.851 & 1.00 & 0.00 & C \\
\hline HETATM & 2230 & C8 & HEM & 241 & 17.479 & 25.290 & 7.798 & 1.00 & 0.00 & C \\
\hline HETATM & 2231 & $\mathrm{C} 9$ & HEM & 241 & 16.528 & 24.925 & 8.822 & 1.00 & 0.00 & C \\
\hline HETATM & 2232 & $\mathrm{C} 10$ & HEM & 241 & 16.832 & 24.191 & 9.965 & 1.00 & 0.00 & C \\
\hline HETATM & 2233 & $\mathrm{H} 10$ & HEM & 241 & 17.859 & 23.847 & 10.071 & 1.00 & 0.00 & $\mathrm{H}$ \\
\hline HETATM & 2234 & C11 & HEM & 241 & 15.958 & 23.851 & 10.988 & 1.00 & 0.00 & C \\
\hline HETATM & 2235 & $\mathrm{C} 12$ & HEM & 241 & 16.339 & 23.125 & 12.178 & 1.00 & 0.00 & C \\
\hline HETATM & 2236 & $\mathrm{C} 13$ & HEM & 241 & 15.199 & 22.978 & 12.935 & 1.00 & 0.00 & C \\
\hline HETATM & 2237 & C14 & HEM & 241 & 14.126 & 23.619 & 12.204 & 1.00 & 0.00 & C \\
\hline HETATM & 2238 & C15 & HEM & 241 & 12.798 & 23.674 & 12.625 & 1.00 & 0.00 & C \\
\hline HETATM & 2239 & H1 5 & HEM & 241 & 12.558 & 23.189 & 13.573 & 1.00 & 0.00 & $\mathrm{H}$ \\
\hline HETATM & 2240 & C16 & HEM & 241 & 11.768 & 24.356 & 11.982 & 1.00 & 0.00 & C \\
\hline HETATM & 2241 & C17 & HEM & 241 & 10.402 & 24.469 & 12.464 & 1.00 & 0.00 & C \\
\hline HETATM & 2242 & $\mathrm{C} 18$ & HEM & 241 & 9.727 & 25.237 & 11.541 & 1.00 & 0.00 & C \\
\hline HETATM & 2243 & C19 & HEM & 241 & 10.676 & 25.589 & 10.504 & 1.00 & 0.00 & C \\
\hline HETATM & 2244 & $\mathrm{C} 20$ & HEM & 241 & 10.385 & 26.380 & 9.393 & 1.00 & 0.00 & C \\
\hline HETATM & 2245 & $\mathrm{H} 20$ & HEM & 241 & 9.371 & 26.769 & 9.333 & 1.00 & 0.00 & $\mathrm{H}$ \\
\hline HETATM & 2246 & N21 & HEM & 241 & 12.575 & 26.367 & 8.298 & 1.00 & 0.00 & $\mathrm{~N}$ \\
\hline HETATM & 2247 & N22 & HEM & 241 & 15.280 & 25.420 & 8.521 & 1.00 & 0.00 & $\mathrm{~N}$ \\
\hline HETATM & 2248 & N23 & HEM & 241 & 14.609 & 24.157 & 11.021 & 1.00 & 0.00 & $\mathrm{~N}$ \\
\hline HETATM & 2249 & N2 4 & HEM & 241 & 11.910 & 25.041 & 10.786 & 1.00 & 0.00 & $\mathrm{~N}$ \\
\hline HETATM & 2250 & C99 & HEM & 241 & 12.816 & 22.176 & 8.283 & 1.00 & 0.00 & C \\
\hline HETATM & 2251 & 091 & HEM & 241 & 12.976 & 23.489 & 8.296 & 1.00 & 0.00 & O \\
\hline HETATM & 2252 & 092 & HEM & 241 & 12.661 & 21.559 & 7.228 & 1.00 & 0.00 & 0 \\
\hline HETATM & 2253 & СТ91 & HEM & 241 & 12.832 & 21.495 & 9.640 & 1.00 & 0.00 & C \\
\hline HETATM & 2254 & H911 & HEM & 241 & 13.811 & 21.690 & 10.103 & 1.00 & 0.00 & $\mathrm{H}$ \\
\hline HETATM & 2255 & H912 & HEM & 241 & 12.084 & 21.984 & 10.283 & 1.00 & 0.00 & $\mathrm{H}$ \\
\hline HETATM & 2256 & СТ 92 & HEM & 241 & 12.573 & 19.990 & 9.528 & 1.00 & 0.00 & C \\
\hline HETATM & 2257 & H921 & HEM & 241 & 13.369 & 19.530 & 8.931 & 1.00 & 0.00 & $\mathrm{H}$ \\
\hline HETATM & 2258 & H922 & HEM & 241 & 11.648 & 19.834 & 8.958 & 1.00 & 0.00 & $\mathrm{H}$ \\
\hline TER & & & & & & & & & & \\
\hline HETATM & 2259 & $\mathrm{FE}$ & HEM & 242 & 14.174 & 17.444 & 14.371 & 1.00 & 0.00 & $\mathrm{Fe}$ \\
\hline HETATM & 2260 & $\mathrm{C} 1$ & HEM & 242 & 16.402 & 15.571 & 15.433 & 1.00 & 0.00 & C \\
\hline HETATM & 2261 & $\mathrm{C} 2$ & HEM & 242 & 16.761 & 14.781 & 16.605 & 1.00 & 0.00 & C \\
\hline HETATM & 2262 & C3 & HEM & 242 & 15.615 & 14.684 & 17.380 & 1.00 & 0.00 & C \\
\hline HETATM & 2263 & $\mathrm{C} 4$ & HEM & 242 & 14.586 & 15.401 & 16.672 & 1.00 & 0.00 & C \\
\hline HETATM & 2264 & C5 & HEM & 242 & 13.270 & 15.520 & 17.109 & 1.00 & 0.00 & C \\
\hline HETATM & 2265 & H5 & HEM & 242 & 13.017 & 15.024 & 18.045 & 1.00 & 0.00 & $\mathrm{H}$ \\
\hline HETATM & 2266 & $\mathrm{C} 6$ & HEM & 242 & 12.251 & 16.218 & 16.473 & 1.00 & 0.00 & C \\
\hline HETATM & 2267 & C7 & HEM & 242 & 10.882 & 16.291 & 16.943 & 1.00 & 0.00 & C \\
\hline HETATM & 2268 & $\mathrm{C} 8$ & HEM & 242 & 10.177 & 17.011 & 15.996 & 1.00 & 0.00 & C \\
\hline HETATM & 2269 & C9 & HEM & 242 & 11.128 & 17.376 & 14.972 & 1.00 & 0.00 & C \\
\hline
\end{tabular}


HETATM 2270 C10 HEM 242 HETATM 2271 H1O HEM 242 HETATM 2272 C11 HEM HETATM 2273 C12 HEM 242 HETATM 2274 C13 HEM 242 HETATM 2275 C14 HEM 242 HETATM 2276 C15 HEM 242 HETATM 2277 H15 HEM 242 HETATM 2278 C16 HEM 242 HETATM 2279 C17 HEM 242 HETATM 2280 C18 HEM 242 HETATM 2281 C19 HEM 242 HETATM 2282 C20 HEM 242 HETATM 2283 H2O HEM 242 HETATM 2284 N21 HEM 242 HETATM 2285 N22 HEM 242 HETATM 2286 N23 HEM 242 HETATM 2287 N24 HEM 242 HETATM 2288 C99 HEM 242 HETATM 2289091 HEM 242 HETATM $2290 \quad 092$ HEM 242 HETATM 2291 CT91 HEM 242 HETATM 2292 H911 HEM 242 HETATM 2293 H912 HEM 242 HETATM 2294 CT92 HEM 242 HETATM 2295 H921 HEM 24 HETATM 2296 H922 HEM 242 HETATM 2298 H21 VIN HETATM 2299 C1 VIN HETATM 2300 H11 VIN HETATM 2301 H12 VIN TER

HETATM 2302 C1 MET 244 HETATM 23 HETATM 2304 H12 MET HETATM 2305 H13 MET TER

HETATM 2306 C2 VIN HETATM 2307 H21 VIN HETATM 2308 C1 VIN HETATM 2309 H11 VIN HETATM 2310 H12 VIN TER

HETATM 2311 C1 MET HETATM 2312 H11 MET HETATM 2313 H12 MET HETATM 2314 H13 MET TER

HETATM 2315 HETATM 2316 H51 ACP HETATM 2317 H52 ACP HETATM 2318 C4 ACP

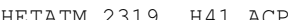
HETATM 2320 H42 ACP HETATM $2321 \quad \mathrm{C} 1 \mathrm{ACP}$ HETATM $2322 \quad 02$ ACP HETATM 2323 O3 ACP HETATM 2324 H3 ACP TER

HETATM 2325 HETATM 2326 H11 MET HETATM 2327 H12 MET HETATM 2328 H13 MET TER

HETATM 2329 C1 MET 249 HETATM 2330 H11 MET 249 HETATM 2331 H12 MET 249 HETATM 2332 H13 MET 249 TER

HETATM 2333 C2 VIN 250 HETATM 2334 H21 VIN 250 HETATM 2335 C1 VIN 250 HETATM 2336 H11 VIN 250 HETATM 2337 H12 VIN 250

HETATM 2338 C1 MET 251 HETATM 2339 H11 MET 251

\begin{tabular}{|c|c|c|c|c|c|}
\hline 10.823 & 18.111 & 13.828 & 1.00 & 0.00 & C \\
\hline 9.796 & 18.454 & 13.722 & 1.00 & 0.00 & $\mathrm{H}$ \\
\hline 11.698 & 18.451 & 12.806 & 1.00 & 0.00 & C \\
\hline 11.317 & 19.176 & 11.615 & 1.00 & 0.00 & C \\
\hline 12.456 & 19.323 & 10.858 & 1.00 & 0.00 & C \\
\hline 13.529 & 18.682 & 11.590 & 1.00 & 0.00 & C \\
\hline 14.857 & 18.627 & 11.168 & 1.00 & 0.00 & C \\
\hline 15.097 & 19.112 & 10.221 & 1.00 & 0.00 & $\mathrm{H}$ \\
\hline 15.887 & 17.945 & 11.812 & 1.00 & 0.00 & C \\
\hline 17.253 & 17.832 & 11.329 & 1.00 & 0.00 & C \\
\hline 17.929 & 17.064 & 12.253 & 1.00 & 0.00 & C \\
\hline 16.979 & 16.712 & 13.289 & 1.00 & 0.00 & C \\
\hline 17.270 & 15.921 & 14.401 & 1.00 & 0.00 & C \\
\hline 18.285 & 15.532 & 14.461 & 1.00 & 0.00 & $\mathrm{H}$ \\
\hline 15.080 & 15.934 & 15.495 & 1.00 & 0.00 & $\mathrm{~N}$ \\
\hline 12.375 & 16.881 & 15.273 & 1.00 & 0.00 & $\mathrm{~N}$ \\
\hline 13.046 & 18.144 & 12.772 & 1.00 & 0.00 & $\mathrm{~N}$ \\
\hline 15.746 & 17.260 & 13.008 & 1.00 & 0.00 & $\mathrm{~N}$ \\
\hline 14.839 & 20.125 & 15.510 & 1.00 & 0.00 & C \\
\hline 14.680 & 18.812 & 15.498 & 1.00 & 0.00 & o \\
\hline 14.994 & 20.742 & 16.566 & 1.00 & 0.00 & o \\
\hline 14.823 & 20.807 & 14.154 & 1.00 & 0.00 & C \\
\hline 13.844 & 20.611 & 13.691 & 1.00 & 0.00 & $\mathrm{H}$ \\
\hline 15.571 & 20.317 & 13.511 & 1.00 & 0.00 & $\mathrm{H}$ \\
\hline 15.083 & 22.311 & 14.265 & 1.00 & 0.00 & C \\
\hline 16.008 & 22.467 & 14.836 & 1.00 & 0.00 & H \\
\hline 14.286 & 22.771 & 14.862 & 1.00 & 0.00 & $\mathrm{H}$ \\
\hline 9.563 & 28.023 & 6.929 & 1.00 & 0.00 & C \\
\hline 8.747 & 27.324 & 7.094 & 1.00 & 0.00 & $\mathrm{H}$ \\
\hline 9.230 & 29.248 & 6.528 & 1.00 & 0.00 & C \\
\hline 9.962 & 30.026 & 6.421 & 1.00 & 0.00 & H \\
\hline 8.188 & 29.517 & 6.361 & 1.00 & 0.00 & $\mathrm{H}$ \\
\hline 12.227 & 28.278 & 5.091 & 1.00 & 0.00 & C \\
\hline 12.462 & 29.349 & 5.211 & 1.00 & 0.00 & $\mathrm{H}$ \\
\hline 13.040 & 27.814 & 4.522 & 1.00 & 0.00 & $\mathrm{H}$ \\
\hline 11.319 & 28.200 & 4.481 & 1.00 & 0.00 & $\mathrm{H}$ \\
\hline 17.237 & 26.600 & 5.606 & 1.00 & 0.00 & C \\
\hline 16.571 & 26.499 & 4.747 & 1.00 & 0.00 & $\mathrm{H}$ \\
\hline 18.404 & 27.244 & 5.447 & 1.00 & 0.00 & c \\
\hline 19.084 & 27.400 & 6.277 & 1.00 & 0.00 & $\mathrm{H}$ \\
\hline 18.709 & 27.642 & 4.482 & 1.00 & 0.00 & $\mathrm{H}$ \\
\hline 18.921 & 24.917 & 7.810 & 1.00 & 0.00 & c \\
\hline 19.513 & 25.581 & 8.459 & 1.00 & 0.00 & $\mathrm{H}$ \\
\hline 19.078 & 23.894 & 8.177 & 1.00 & 0.00 & $\mathrm{H}$ \\
\hline 19.354 & 24.982 & 6.805 & 1.00 & 0.00 & $\mathrm{H}$ \\
\hline 9.833 & 23.835 & 13.701 & 1.00 & 0.00 & c \\
\hline 10.572 & 23.824 & 14.514 & 1.00 & 0.00 & $\mathrm{H}$ \\
\hline 8.992 & 24.443 & 14.065 & 1.00 & 0.00 & $\mathrm{H}$ \\
\hline 9.355 & 22.397 & 13.428 & 1.00 & 0.00 & c \\
\hline 8.797 & 22.336 & 12.481 & 1.00 & 0.00 & $\mathrm{H}$ \\
\hline 10.225 & 21.731 & 13.321 & 1.00 & 0.00 & $\mathrm{H}$ \\
\hline 8.491 & 21.785 & 14.504 & 1.00 & 0.00 & C \\
\hline 8.263 & 22.308 & 15.603 & 1.00 & 0.00 & 0 \\
\hline 7.975 & 20.606 & 14.140 & 1.00 & 0.00 & o \\
\hline 7.366 & 20.251 & 14.876 & 1.00 & 0.00 & $\mathrm{H}$ \\
\hline 17.729 & 22.709 & 12.528 & 1.00 & 0.00 & c \\
\hline 18.282 & 23.548 & 12.978 & 1.00 & 0.00 & $\mathrm{H}$ \\
\hline 17.736 & 21.883 & 13.250 & 1.00 & 0.00 & $\mathrm{H}$ \\
\hline 18.300 & 22.389 & 11.649 & 1.00 & 0.00 & $\mathrm{H}$ \\
\hline 8.296 & 25.657 & 11.577 & 1.00 & 0.00 & c \\
\hline 7.851 & 25.449 & 12.557 & 1.00 & 0.00 & $\mathrm{H}$ \\
\hline 8.185 & 26.738 & 11.402 & 1.00 & 0.00 & $\mathrm{H}$ \\
\hline 7.695 & 25.132 & 10.821 & 1.00 & 0.00 & $\mathrm{H}$ \\
\hline 18.092 & 14.278 & 16.864 & 1.00 & 0.00 & c \\
\hline 18.908 & 14.977 & 16.699 & 1.00 & 0.00 & $\mathrm{H}$ \\
\hline 18.425 & 13.053 & 17.266 & 1.00 & 0.00 & c \\
\hline 17.694 & 12.275 & 17.373 & 1.00 & 0.00 & $\mathrm{H}$ \\
\hline 19.467 & 12.784 & 17.432 & 1.00 & 0.00 & $\mathrm{H}$ \\
\hline 15.429 & 14.023 & 18.702 & 1.00 & 0.00 & c \\
\hline 15.194 & 12.953 & 18.583 & 1.00 & 0.00 & $\mathrm{H}$ \\
\hline
\end{tabular}




\begin{tabular}{|c|c|c|c|c|c|c|c|c|c|c|}
\hline HETATM & 2340 & $\mathrm{H} 12$ & $\mathrm{MET}$ & 251 & 14.616 & 14.488 & 19.272 & 1.00 & 0.00 & $\mathrm{H}$ \\
\hline HETATM & 2341 & H13 & MET & 251 & 16.336 & 14.101 & 19.313 & 1.00 & 0.00 & $\mathrm{H}$ \\
\hline \multicolumn{11}{|l|}{ TER } \\
\hline HETATM & 2342 & C2 & VIN & 252 & 10.419 & 15.701 & 18.188 & 1.00 & 0.00 & C \\
\hline HETATM & 2343 & H 21 & VIN & 252 & 11.085 & 15.802 & 19.046 & 1.00 & 0.00 & $\mathrm{H}$ \\
\hline HETATM & 2344 & $\mathrm{C} 1$ & VIN & 252 & 9.252 & 15.057 & 18.346 & 1.00 & 0.00 & C \\
\hline HETATM & 2345 & H11 & VIN & 252 & 8.572 & 14.901 & 17.516 & 1.00 & 0.00 & $\mathrm{H}$ \\
\hline HETATM & 2346 & H12 & VIN & 252 & 8.947 & 14.659 & 19.312 & 1.00 & 0.00 & $H$ \\
\hline \multicolumn{11}{|l|}{ TER } \\
\hline HETATM & 2347 & $\mathrm{C} 1$ & MET & 253 & 8.734 & 17.385 & 15.984 & 1.00 & 0.00 & C \\
\hline HETATM & 2348 & H11 & MET & 253 & 8.578 & 18.407 & 15.617 & 1.00 & 0.00 & H \\
\hline HETATM & 2349 & H12 & MET & 253 & 8.302 & 17.319 & 16.989 & 1.00 & 0.00 & $\mathrm{H}$ \\
\hline HETATM & 2350 & H13 & MET & 253 & 8.143 & 16.720 & 15.335 & 1.00 & 0.00 & $\mathrm{H}$ \\
\hline \multicolumn{11}{|l|}{ TER } \\
\hline HETATM & 2351 & C5 & $\mathrm{ACP}$ & 254 & 17.822 & 18.466 & 10.092 & 1.00 & 0.00 & 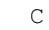 \\
\hline HETATM & 2352 & H51 & $\mathrm{ACP}$ & 254 & 17.083 & 18.478 & 9.280 & 1.00 & 0.00 & $\mathrm{H}$ \\
\hline HETATM & 2353 & H52 & $\mathrm{ACP}$ & 254 & 18.663 & 17.858 & 9.729 & 1.00 & 0.00 & $\mathrm{H}$ \\
\hline HETATM & 2354 & C4 & $\mathrm{ACP}$ & 254 & 18.301 & 19.904 & 10.365 & 1.00 & 0.00 & C \\
\hline HETATM & 2355 & $\mathrm{H} 4 \mathrm{I}$ & $\mathrm{ACP}$ & 254 & 18.859 & 19.965 & 11.313 & 1.00 & 0.00 & $\mathrm{H}$ \\
\hline HETATM & 2356 & $\mathrm{H} 42$ & $\mathrm{ACP}$ & 254 & 17.430 & 20.570 & 10.472 & 1.00 & 0.00 & $\mathrm{H}$ \\
\hline HETATM & 2357 & $\mathrm{C} 1$ & $\mathrm{ACP}$ & 254 & 19.165 & 20.516 & 9.289 & 1.00 & 0.00 & C \\
\hline HETATM & 2358 & 02 & $\mathrm{ACP}$ & 254 & 19.393 & 19.993 & 8.191 & 1.00 & 0.00 & 0 \\
\hline HETATM & 2359 & 03 & $\mathrm{ACP}$ & 254 & 19.681 & 21.696 & 9.653 & 1.00 & 0.00 & 0 \\
\hline HETATM & 2360 & H3 & $\mathrm{ACP}$ & 254 & 20.290 & 22.050 & 8.917 & 1.00 & 0.00 & $\mathrm{H}$ \\
\hline \multicolumn{11}{|l|}{ TER } \\
\hline HETATM & 2361 & $\mathrm{C} 1$ & MET & 255 & 9.926 & 19.593 & 11.266 & 1.00 & 0.00 & C \\
\hline HETATM & 2362 & H11 & MET & 255 & 9.374 & 18.753 & 10.815 & 1.00 & 0.00 & $\mathrm{H}$ \\
\hline HETATM & 2363 & $\mathrm{H} 12$ & $\mathrm{MET}$ & 255 & 9.920 & 20.418 & 10.544 & 1.00 & 0.00 & $\mathrm{H}$ \\
\hline HETATM & 2364 & H13 & $\mathrm{MET}$ & 255 & 9.355 & 19.912 & 12.145 & 1.00 & 0.00 & $\mathrm{H}$ \\
\hline TER & & & & & & & & & & \\
\hline HETATM & 2365 & $\mathrm{C} 1$ & MET & 256 & 19.360 & 16.644 & 12.216 & 1.00 & 0.00 & C \\
\hline HETATM & 2366 & H11 & $\mathrm{MET}$ & 256 & 19.805 & 16.853 & 11.236 & 1.00 & 0.00 & $\mathrm{H}$ \\
\hline HETATM & 2367 & H12 & MET & 256 & 19.470 & 15.564 & 12.391 & 1.00 & 0.00 & $\mathrm{H}$ \\
\hline HETATM & 2368 & H13 & $\mathrm{MET}$ & 256 & 19.960 & 17.169 & 12.973 & 1.00 & 0.00 & $\mathrm{H}$ \\
\hline TER & & & & & & & & & & \\
\hline HETATM & 2369 & $\mathrm{FE}$ & HEM & 257 & 18.263 & -3.589 & 1.491 & 1.00 & 0.00 & $\mathrm{Fe}$ \\
\hline HETATM & 2370 & $\mathrm{C} 1$ & HEM & 257 & 16.035 & -1.717 & 0.429 & 1.00 & 0.00 & C \\
\hline HETATM & 2371 & $\mathrm{C} 2$ & HEM & 257 & 15.676 & -0.927 & -0.742 & 1.00 & 0.00 & C \\
\hline HETATM & 2372 & C3 & HEM & 257 & 16.822 & -0.830 & -1.517 & 1.00 & 0.00 & C \\
\hline HETATM & 2373 & $\mathrm{C} 4$ & HEM & 257 & 17.851 & -1.547 & -0.809 & 1.00 & 0.00 & C \\
\hline HETATM & 2374 & C5 & HEM & 257 & 19.166 & -1.666 & -1.247 & 1.00 & 0.00 & C \\
\hline HETATM & 2375 & H5 & HEM & 257 & 19.420 & -1.170 & -2.182 & 1.00 & 0.00 & $\mathrm{H}$ \\
\hline HETATM & 2376 & C6 & HEM & 257 & 20.186 & $-2 \cdot 364$ & -0.611 & 1.00 & 0.00 & C \\
\hline HETATM & 2377 & C7 & HEM & 257 & 21.555 & -2.437 & -1.081 & 1.00 & 0.00 & C \\
\hline HETATM & 2378 & $\mathrm{C} 8$ & HEM & 257 & 22.260 & -3.157 & -0.134 & 1.00 & 0.00 & C \\
\hline HETATM & 2379 & C9 & HEM & 257 & 21.309 & -3.522 & 0.891 & 1.00 & 0.00 & C \\
\hline HETATM & 2380 & $\mathrm{C} 10$ & HEM & 257 & 21.613 & -4.256 & 2.034 & 1.00 & 0.00 & C \\
\hline HETATM & 2381 & H1O & HEM & 257 & 22.640 & -4.600 & 2.140 & 1.00 & 0.00 & $\mathrm{H}$ \\
\hline HETATM & 2382 & C11 & HEM & 257 & 20.739 & -4.596 & 3.057 & 1.00 & 0.00 & C \\
\hline HETATM & 2383 & $\mathrm{C} 12$ & HEM & 257 & 21.120 & -5.322 & 4.247 & 1.00 & 0.00 & C \\
\hline HETATM & 2384 & C13 & HEM & 257 & 19.980 & -5.469 & 5.004 & 1.00 & 0.00 & C \\
\hline HETATM & 2385 & C14 & HEM & 257 & 18.908 & -4.828 & 4.273 & 1.00 & 0.00 & $\mathrm{C}$ \\
\hline HETATM & 2386 & C15 & HEM & 257 & 17.579 & -4.773 & 4.694 & 1.00 & 0.00 & C \\
\hline HETATM & 2387 & H15 & HEM & 257 & 17.339 & -5.258 & 5.642 & 1.00 & 0.00 & $\mathrm{H}$ \\
\hline HETATM & 2388 & C16 & HEM & 257 & 16.549 & -4.091 & 4.051 & 1.00 & 0.00 & C \\
\hline HETATM & 2389 & C17 & HEM & 257 & 15.183 & -3.978 & 4.533 & 1.00 & 0.00 & C \\
\hline HETATM & 2390 & C18 & HEM & 257 & 14.508 & -3.210 & 3.609 & 1.00 & 0.00 & C \\
\hline HETATM & 2391 & C19 & HEM & 257 & 15.458 & -2.858 & 2.573 & 1.00 & 0.00 & C \\
\hline HETATM & 2392 & $\mathrm{C} 20$ & HEM & 257 & 15.166 & -2.067 & 1.462 & 1.00 & 0.00 & C \\
\hline HETATM & 2393 & H 20 & HEM & 257 & 14.152 & -1.678 & 1.402 & 1.00 & 0.00 & $\mathrm{H}$ \\
\hline HETATM & 2394 & $\mathrm{~N} 21$ & HEM & 257 & 17.357 & -2.080 & 0.367 & 1.00 & 0.00 & $\mathrm{~N}$ \\
\hline HETATM & 2395 & N22 & HEM & 257 & 20.061 & -3.027 & 0.590 & 1.00 & 0.00 & $\mathrm{~N}$ \\
\hline HETATM & 2396 & N23 & HEM & 257 & 19.390 & -4.290 & 3.090 & 1.00 & 0.00 & $\mathrm{~N}$ \\
\hline HETATM & 2397 & N24 & HEM & 257 & 16.691 & -3.406 & 2.854 & 1.00 & 0.00 & $\mathrm{~N}$ \\
\hline HETATM & 2398 & C99 & HEM & 257 & 17.597 & -6.271 & 0.352 & 1.00 & 0.00 & C \\
\hline HETATM & 2399 & 091 & HEM & 257 & 17.757 & -4.958 & 0.365 & 1.00 & 0.00 & 0 \\
\hline HETATM & 2400 & 092 & HEM & 257 & 17.443 & -6.888 & -0.703 & 1.00 & 0.00 & 0 \\
\hline HETATM & 2401 & СТ91 & HEM & 257 & 17.614 & -6.952 & 1.709 & 1.00 & 0.00 & C \\
\hline HETATM & 2402 & H911 & HEM & 257 & 18.592 & -6.757 & 2.171 & 1.00 & 0.00 & $\mathrm{H}$ \\
\hline HETATM & 2403 & H912 & HEM & 257 & 16.866 & -6.463 & 2.352 & 1.00 & 0.00 & $\mathrm{H}$ \\
\hline HETATM & 2404 & CT92 & HEM & 257 & 17.354 & -8.457 & 1.597 & 1.00 & 0.00 & C \\
\hline HETATM & 2405 & H921 & HEM & 257 & 18.150 & -8.917 & 1.000 & 1.00 & 0.00 & $\mathrm{H}$ \\
\hline HETATM & 2406 & H922 & HEM & 257 & 16.429 & -8.613 & 1.027 & 1.00 & 0.00 & $\mathrm{H}$ \\
\hline TER & & & & & & & & & & \\
\hline HETATM & 2407 & $\mathrm{FE}$ & HEM & 258 & 18.955 & -11.003 & 6.440 & 1.00 & 0.00 & $\mathrm{Fe}$ \\
\hline HETATM & 2408 & $\mathrm{C} 1$ & HEM & 258 & 21.183 & -12.876 & 7.502 & 1.00 & 0.00 & C \\
\hline HETATM & 2409 & $\mathrm{C} 2$ & HEM & 258 & 21.542 & -13.666 & 8.674 & 1.00 & 0.00 & C \\
\hline HETATM & 2410 & C3 & HEM & 258 & 20.396 & -13.763 & 9.448 & 1.00 & 0.00 & C \\
\hline HETATM & 2411 & C4 & HEM & 258 & 19.367 & -13.046 & 8.740 & 1.00 & 0.00 & 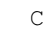 \\
\hline
\end{tabular}




\begin{tabular}{|c|c|c|c|c|c|c|c|c|c|c|}
\hline HETATM & 2412 & $\mathrm{C} 5$ & HEM & 258 & 18.052 & -12.927 & 9.178 & 1.00 & 0.00 & C \\
\hline HETATM & 2413 & H5 & HEM & 258 & 17.798 & -13.423 & 10.113 & 1.00 & 0.00 & $\mathrm{H}$ \\
\hline HETATM & 2414 & $\mathrm{C} 6$ & HEM & 258 & 17.032 & -12.229 & 8.542 & 1.00 & 0.00 & C \\
\hline HETATM & 2415 & $\mathrm{C} 7$ & HEM & 258 & 15.663 & -12.156 & 9.012 & 1.00 & 0.00 & C \\
\hline HETATM & 2416 & $\mathrm{C} 8$ & HEM & 258 & 14.958 & -11.436 & 8.065 & 1.00 & 0.00 & C \\
\hline HETATM & 2417 & $\mathrm{C} 9$ & HEM & 258 & 15.909 & -11.071 & 7.041 & 1.00 & 0.00 & C \\
\hline HETATM & 2418 & $\mathrm{C} 10$ & HEM & 258 & 15.604 & -10.336 & 5.897 & 1.00 & 0.00 & C \\
\hline HETATM & 2419 & $\mathrm{H} 10$ & HEM & 258 & 14.578 & -9.993 & 5.791 & 1.00 & 0.00 & $\mathrm{H}$ \\
\hline HETATM & 2420 & C11 & HEM & 258 & 16.479 & -9.996 & 4.874 & 1.00 & 0.00 & $\mathrm{C}$ \\
\hline HETATM & 2421 & $\mathrm{C} 12$ & HEM & 258 & 16.098 & -9.271 & 3.684 & 1.00 & 0.00 & C \\
\hline HETATM & 2422 & C13 & HEM & 258 & 17.237 & -9.124 & 2.927 & 1.00 & 0.00 & C \\
\hline HETATM & 2423 & C14 & HEM & 258 & 18.310 & -9.765 & 3.659 & 1.00 & 0.00 & C \\
\hline HETATM & 2424 & C15 & HEM & 258 & 19.638 & -9.820 & 3.237 & 1.00 & 0.00 & C \\
\hline HETATM & 2425 & H15 & HEM & 258 & 19.879 & -9.335 & 2.289 & 1.00 & 0.00 & $\mathrm{H}$ \\
\hline HETATM & 2426 & $\mathrm{C} 16$ & HEM & 258 & 20.669 & -10.502 & 3.880 & 1.00 & 0.00 & $\mathrm{C}$ \\
\hline HETATM & 2427 & C17 & HEM & 258 & 22.034 & -10.615 & 3.398 & 1.00 & 0.00 & C \\
\hline HETATM & 2428 & C18 & HEM & 258 & 22.710 & -11.383 & 4.322 & 1.00 & 0.00 & C \\
\hline HETATM & 2429 & C19 & HEM & 258 & 21.760 & -11.735 & 5.358 & 1.00 & 0.00 & C \\
\hline HETATM & 2430 & $\mathrm{C} 20$ & HEM & 258 & 22.052 & -12.526 & 6.469 & 1.00 & 0.00 & C \\
\hline HETATM & 2431 & $\mathrm{H} 2 \mathrm{O}$ & HEM & 258 & 23.066 & -12.915 & 6.530 & 1.00 & 0.00 & $\mathrm{H}$ \\
\hline HETATM & 2432 & N21 & HEM & 258 & 19.861 & -12.513 & 7.564 & 1.00 & 0.00 & $\mathrm{~N}$ \\
\hline HETATM & 2433 & N22 & HEM & 258 & 17.157 & -11.566 & 7.342 & 1.00 & 0.00 & $\mathrm{~N}$ \\
\hline HETATM & 2434 & $\mathrm{~N} 23$ & HEM & 258 & 17.827 & -10.303 & 4.841 & 1.00 & 0.00 & $\mathrm{~N}$ \\
\hline HETATM & 2435 & N2 4 & HEM & 258 & 20.527 & -11.187 & 5.077 & 1.00 & 0.00 & $\mathrm{~N}$ \\
\hline HETATM & 2436 & C99 & HEM & 258 & 19.620 & -8.322 & 7.579 & 1.00 & 0.00 & C \\
\hline HETATM & 2437 & 091 & HEM & 258 & 19.461 & -9.635 & 7.567 & 1.00 & 0.00 & 0 \\
\hline HETATM & 2438 & 092 & HEM & 258 & 19.775 & -7.705 & 8.635 & 1.00 & 0.00 & 0 \\
\hline HETATM & 2439 & CT91 & HEM & 258 & 19.604 & -7.640 & 6.223 & 1.00 & 0.00 & $\mathrm{C}$ \\
\hline HETATM & 2440 & H911 & HEM & 258 & 18.626 & -7.836 & 5.760 & 1.00 & 0.00 & $\mathrm{H}$ \\
\hline HETATM & 2441 & H912 & HEM & 258 & 20.352 & -8.130 & 5.580 & 1.00 & 0.00 & $\mathrm{H}$ \\
\hline HETATM & 2442 & CT92 & HEM & 258 & 19.864 & -6.136 & 6.334 & 1.00 & 0.00 & C \\
\hline HETATM & 2443 & H921 & HEM & 258 & 20.789 & -5.980 & 6.905 & 1.00 & 0.00 & $\mathrm{H}$ \\
\hline HETATM & 2444 & H922 & HEM & 258 & 19.068 & -5.676 & 6.931 & 1.00 & 0.00 & $\mathrm{H}$ \\
\hline \multicolumn{11}{|l|}{ TER } \\
\hline HETATM & 2445 & $\mathrm{C} 2$ & VIN & 259 & 14.344 & -0.424 & -1.002 & 1.00 & 0.00 & C \\
\hline HETATM & 2446 & H 21 & VIN & 259 & 13.528 & -1.123 & -0.837 & 1.00 & 0.00 & $\mathrm{H}$ \\
\hline HETATM & 2447 & $\mathrm{C} 1$ & VIN & 259 & 14.011 & 0.801 & -1.403 & 1.00 & 0.00 & C \\
\hline HETATM & 2448 & $\mathrm{H} 11$ & VIN & 259 & 14.743 & 1.579 & -1.511 & 1.00 & 0.00 & $\mathrm{H}$ \\
\hline HETATM & 2449 & $\mathrm{H} 12$ & VIN & 259 & 12.970 & 1.070 & -1.570 & 1.00 & 0.00 & $\mathrm{H}$ \\
\hline \multicolumn{11}{|l|}{ TER } \\
\hline HETATM & 2450 & $\mathrm{C} 1$ & MET & 260 & 17.008 & -0.169 & -2.840 & 1.00 & 0.00 & C \\
\hline HETATM & 2451 & $\mathrm{H} 11$ & MET & 260 & 17.243 & 0.902 & -2.720 & 1.00 & 0.00 & $\mathrm{H}$ \\
\hline HETATM & 2452 & $\mathrm{H} 12$ & $\mathrm{MET}$ & 260 & 17.821 & -0.633 & -3.410 & 1.00 & 0.00 & $\mathrm{H}$ \\
\hline HETATM & 2453 & H13 & $\mathrm{MET}$ & 260 & 16.100 & -0.247 & -3.450 & 1.00 & 0.00 & $\mathrm{H}$ \\
\hline \multicolumn{11}{|c|}{ TER } \\
\hline HETATM & 2454 & $\mathrm{C} 2$ & VIN & 261 & 22.018 & -1.847 & -2.325 & 1.00 & 0.00 & C \\
\hline HETATM & 2455 & $\mathrm{H} 21$ & VIN & 261 & 21.352 & -1.948 & -3.184 & 1.00 & 0.00 & $\mathrm{H}$ \\
\hline HETATM & 2456 & $\mathrm{C} 1$ & VIN & 261 & 23.185 & -1.203 & -2.484 & 1.00 & 0.00 & $\mathrm{C}$ \\
\hline HETATM & 2457 & $\mathrm{H} 11$ & VIN & 261 & 23.865 & -1.047 & -1.654 & 1.00 & 0.00 & $\mathrm{H}$ \\
\hline HETATM & 2458 & $\mathrm{H} 12$ & VIN & 261 & 23.490 & -0.805 & -3.450 & 1.00 & 0.00 & $\mathrm{H}$ \\
\hline \multicolumn{11}{|l|}{ TER } \\
\hline HETATM & 2459 & $\mathrm{C} 1$ & MET & 262 & 23.703 & -3.530 & -0.121 & 1.00 & 0.00 & C \\
\hline HETATM & 2460 & $\mathrm{H} 11$ & $\mathrm{MET}$ & 262 & 24.294 & -2.866 & 0.528 & 1.00 & 0.00 & $\mathrm{H}$ \\
\hline HETATM & 2461 & $\mathrm{H} 12$ & $\mathrm{MET}$ & 262 & 23.859 & -4.553 & 0.246 & 1.00 & 0.00 & $\mathrm{H}$ \\
\hline HETATM & 2462 & H13 & $\mathrm{MET}$ & 262 & 24.135 & -3.465 & -1.126 & 1.00 & 0.00 & $\mathrm{H}$ \\
\hline TER & & & & & & & & & & \\
\hline HETATM & 2463 & C5 & $\mathrm{ACP}$ & 263 & 14.614 & -4.612 & 5.770 & 1.00 & 0.00 & C \\
\hline HETATM & 2464 & H51 & $\mathrm{ACP}$ & 263 & 15.353 & -4.623 & 6.582 & 1.00 & 0.00 & $\mathrm{H}$ \\
\hline HETATM & 2465 & H52 & $\mathrm{ACP}$ & 263 & 13.774 & -4.004 & 6.134 & 1.00 & 0.00 & $\mathrm{H}$ \\
\hline HETATM & 2466 & $\mathrm{C} 4$ & $\mathrm{ACP}$ & 263 & 14.136 & -6.050 & 5.497 & 1.00 & 0.00 & C \\
\hline HETATM & 2467 & $\mathrm{H} 41$ & $\mathrm{ACP}$ & 263 & 13.578 & -6.111 & 4.549 & 1.00 & 0.00 & $\mathrm{H}$ \\
\hline HETATM & 2468 & $\mathrm{H} 42$ & $\mathrm{ACP}$ & 263 & 15.006 & -6.716 & 5.390 & 1.00 & 0.00 & $\mathrm{H}$ \\
\hline HETATM & 2469 & $\mathrm{C} 1$ & $\mathrm{ACP}$ & 263 & 13.272 & -6.662 & 6.573 & 1.00 & 0.00 & C \\
\hline HETATM & 2470 & $\mathrm{O} 2$ & $\mathrm{ACP}$ & 263 & 13.044 & -6.139 & 7.671 & 1.00 & 0.00 & 0 \\
\hline HETATM & 2471 & 03 & $\mathrm{ACP}$ & 263 & 12.756 & -7.841 & 6.209 & 1.00 & 0.00 & 0 \\
\hline HETATM & 2472 & H3 & $\mathrm{ACP}$ & 263 & 12.147 & -8.196 & 6.945 & 1.00 & 0.00 & $\mathrm{H}$ \\
\hline TER & & & & & & & & & & \\
\hline HETATM & 2473 & $\mathrm{C} 1$ & $\mathrm{MET}$ & 264 & 22.510 & -5.738 & 4.597 & 1.00 & 0.00 & C \\
\hline HETATM & 2474 & $\mathrm{H} 11$ & $\mathrm{MET}$ & 264 & 23.063 & -4.899 & 5.047 & 1.00 & 0.00 & $\mathrm{H}$ \\
\hline HETATM & 2475 & $\mathrm{H} 12$ & $\mathrm{MET}$ & 264 & 22.517 & -6.564 & 5.319 & 1.00 & 0.00 & $\mathrm{H}$ \\
\hline HETATM & 2476 & H13 & MET & 264 & 23.081 & -6.058 & 3.717 & 1.00 & 0.00 & $\mathrm{H}$ \\
\hline TER & & & & & & & & & & \\
\hline HETATM & 2477 & $\mathrm{C} 1$ & $\mathrm{MET}$ & 265 & 13.077 & -2.790 & 3.646 & 1.00 & 0.00 & C \\
\hline HETATM & 2478 & H11 & $\mathrm{MET}$ & 265 & 12.632 & -2.998 & 4.626 & 1.00 & 0.00 & $\mathrm{H}$ \\
\hline HETATM & 2479 & $\mathrm{H} 12$ & $\mathrm{MET}$ & 265 & 12.967 & -1.709 & 3.471 & 1.00 & 0.00 & $\mathrm{H}$ \\
\hline HETATM & 2480 & $\mathrm{H} 13$ & $\mathrm{MET}$ & 265 & 12.476 & -3.315 & 2.889 & 1.00 & 0.00 & $\mathrm{H}$ \\
\hline TER & & & & & & & & & & \\
\hline HETATM & 2481 & $\mathrm{C} 2$ & VIN & 266 & 22.874 & -14.169 & 8.933 & 1.00 & 0.00 & C \\
\hline HETATM & 2482 & $\mathrm{H} 21$ & VIN & 266 & 23.689 & -13.470 & 8.768 & 1.00 & 0.00 & $H$ \\
\hline
\end{tabular}




\begin{tabular}{|c|c|c|c|c|c|c|c|c|c|c|}
\hline HETATM & 2483 & $\mathrm{C} 1$ & VIN & 266 & 23.207 & $-15 \cdot 394$ & 9.335 & 1.00 & 0.00 & C \\
\hline HETATM & 2484 & H11 & VIN & 266 & 22.475 & -16.172 & 9.442 & 1.00 & 0.00 & $\mathrm{H}$ \\
\hline HETATM & 2485 & H12 & VIN & 266 & 24.248 & -15.663 & 9.501 & 1.00 & 0.00 & $\mathrm{H}$ \\
\hline \multicolumn{11}{|l|}{ TER } \\
\hline HETATM & 2486 & $\mathrm{C} 1$ & MET & 267 & 20.210 & -14.424 & 10.771 & 1.00 & 0.00 & C \\
\hline HETATM & 2487 & H11 & MET & 267 & 19.975 & -15.494 & 10.652 & 1.00 & 0.00 & $\mathrm{H}$ \\
\hline HETATM & 2488 & $\mathrm{H} 12$ & MET & 267 & 19.397 & -13.959 & 11.341 & 1.00 & 0.00 & $\mathrm{H}$ \\
\hline HETATM & 2489 & H13 & MET & 267 & 21.118 & -14.346 & 11.382 & 1.00 & 0.00 & $\mathrm{H}$ \\
\hline \multicolumn{11}{|l|}{ TER } \\
\hline HETATM & 2490 & C2 & VIN & 268 & 15.200 & -12.746 & 10.256 & 1.00 & 0.00 & C \\
\hline HETATM & 2491 & H 21 & VIN & 268 & 15.866 & -12.645 & 11.115 & 1.00 & 0.00 & $\mathrm{H}$ \\
\hline HETATM & 2492 & $\mathrm{C} 1$ & VIN & 268 & 14.033 & -13.390 & 10.415 & 1.00 & 0.00 & C \\
\hline HETATM & 2493 & H11 & VIN & 268 & 13.353 & -13.546 & 9.585 & 1.00 & 0.00 & $\mathrm{H}$ \\
\hline HETATM & 2494 & $\mathrm{H} 12$ & VIN & 268 & 13.728 & $-13 \cdot 788$ & 11.381 & 1.00 & 0.00 & $\mathrm{H}$ \\
\hline \multicolumn{11}{|l|}{ TER } \\
\hline HETATM & 2495 & $\mathrm{C} 1$ & MET & 269 & 13.515 & -11.062 & 8.052 & 1.00 & 0.00 & C \\
\hline HETATM & 2496 & H11 & MET & 269 & 13.359 & -10.040 & 7.685 & 1.00 & 0.00 & $\mathrm{H}$ \\
\hline HETATM & 2497 & $\mathrm{H} 12$ & MET & 269 & 13.083 & $-11 \cdot 128$ & 9.057 & 1.00 & 0.00 & $\mathrm{H}$ \\
\hline HETATM & 2498 & H13 & MET & 269 & 12.924 & -11.727 & 7.403 & 1.00 & 0.00 & $\mathrm{H}$ \\
\hline \multicolumn{11}{|c|}{ TER } \\
\hline HETATM & 2499 & C5 & $\mathrm{ACP}$ & 270 & 22.604 & -9.981 & 2.161 & 1.00 & 0.00 & C \\
\hline HETATM & 2500 & H51 & $\mathrm{ACP}$ & 270 & 21.865 & -9.969 & 1.349 & 1.00 & 0.00 & $\mathrm{H}$ \\
\hline HETATM & 2501 & H52 & $\mathrm{ACP}$ & 270 & 23.444 & -10.589 & 1.798 & 1.00 & 0.00 & $\mathrm{H}$ \\
\hline HETATM & 2502 & $\mathrm{C} 4$ & $\mathrm{ACP}$ & 270 & 23.082 & -8.543 & 2.434 & 1.00 & 0.00 & C \\
\hline HETATM & 2503 & $\mathrm{H} 41$ & $\mathrm{ACP}$ & 270 & 23.640 & -8.482 & 3.382 & 1.00 & 0.00 & $\mathrm{H}$ \\
\hline HETATM & 2504 & $\mathrm{H} 42$ & $\mathrm{ACP}$ & 270 & 22.212 & -7.877 & 2.541 & 1.00 & 0.00 & $\mathrm{H}$ \\
\hline HETATM & 2505 & $\mathrm{C} 1$ & $\mathrm{ACP}$ & 270 & 23.946 & -7.931 & 1.358 & 1.00 & 0.00 & $\mathrm{C}$ \\
\hline HETATM & 2506 & $\mathrm{O} 2$ & $\mathrm{ACP}$ & 270 & 24.174 & -8.454 & 0.260 & 1.00 & 0.00 & 0 \\
\hline HETATM & 2507 & 03 & $\mathrm{ACP}$ & 270 & 24.462 & -6.751 & 1.722 & 1.00 & 0.00 & 0 \\
\hline HETATM & 2508 & H3 & $\mathrm{ACP}$ & 270 & 25.071 & -6.397 & 0.986 & 1.00 & 0.00 & $\mathrm{H}$ \\
\hline TER & & & & & & & & & & \\
\hline HETATM & 2509 & $\mathrm{C} 1$ & MET & 271 & 14.708 & -8.855 & 3.334 & 1.00 & 0.00 & C \\
\hline HETATM & 2510 & H11 & MET & 271 & 14.155 & -9.694 & 2.884 & 1.00 & 0.00 & $\mathrm{H}$ \\
\hline HETATM & 2511 & $\mathrm{H} 12$ & MET & 271 & 14.701 & -8.029 & 2.613 & 1.00 & 0.00 & $\mathrm{H}$ \\
\hline HETATM & 2512 & $\mathrm{H} 13$ & MET & 271 & 14.137 & -8.535 & 4.214 & 1.00 & 0.00 & $\mathrm{H}$ \\
\hline TER & & & & & & & & & & \\
\hline HETATM & 2513 & $\mathrm{C} 1$ & $\mathrm{MET}$ & 272 & 24.141 & -11.803 & 4.285 & 1.00 & 0.00 & C \\
\hline HETATM & 2514 & H11 & MET & 272 & 24.586 & -11.594 & 3.305 & 1.00 & 0.00 & $\mathrm{H}$ \\
\hline HETATM & 2515 & $\mathrm{H} 12$ & MET & 272 & 24.251 & -12.883 & 4.460 & 1.00 & 0.00 & $\mathrm{H}$ \\
\hline HETATM & 2516 & H13 & MET & 272 & 24.742 & -11.278 & 5.042 & 1.00 & 0.00 & $\mathrm{H}$ \\
\hline TER & & & & & & & & & & \\
\hline HETATM & 2517 & $\mathrm{FE}$ & HEM & 273 & 14.429 & 25.104 & 1.491 & 1.00 & 0.00 & $\mathrm{Fe}$ \\
\hline HETATM & 2518 & $\mathrm{C} 1$ & HEM & 273 & 12.201 & 26.976 & 0.429 & 1.00 & 0.00 & C \\
\hline HETATM & 2519 & $\mathrm{C} 2$ & HEM & 273 & 11.842 & 27.767 & -0.742 & 1.00 & 0.00 & C \\
\hline HETATM & 2520 & $\mathrm{C} 3$ & HEM & 273 & 12.988 & 27.864 & -1.517 & 1.00 & 0.00 & $\mathrm{C}$ \\
\hline HETATM & 2521 & C4 & HEM & 273 & 14.018 & 27.146 & -0.809 & 1.00 & 0.00 & $\mathrm{C}$ \\
\hline HETATM & 2522 & C5 & HEM & 273 & 15.333 & 27.027 & -1.247 & 1.00 & 0.00 & $\mathrm{C}$ \\
\hline HETATM & 2523 & H5 & HEM & 273 & 15.586 & 27.524 & -2.182 & 1.00 & 0.00 & $\mathrm{H}$ \\
\hline HETATM & 2524 & $\mathrm{C} 6$ & HEM & 273 & 16.352 & 26.329 & -0.611 & 1.00 & 0.00 & C \\
\hline HETATM & 2525 & C7 & HEM & 273 & 17.721 & 26.256 & -1.081 & 1.00 & 0.00 & C \\
\hline HETATM & 2526 & $\mathrm{C} 8$ & HEM & 273 & 18.426 & 25.537 & -0.134 & 1.00 & 0.00 & C \\
\hline HETATM & 2527 & $\mathrm{C} 9$ & HEM & 273 & 17.475 & 25.172 & 0.891 & 1.00 & 0.00 & C \\
\hline HETATM & 2528 & $\mathrm{C} 10$ & HEM & 273 & 17.780 & 24.437 & 2.034 & 1.00 & 0.00 & C \\
\hline HETATM & 2529 & $\mathrm{H} 10$ & HEM & 273 & 18.807 & 24.093 & 2.140 & 1.00 & 0.00 & $\mathrm{H}$ \\
\hline HETATM & 2530 & C11 & HEM & 273 & 16.905 & 24.097 & 3.057 & 1.00 & 0.00 & C \\
\hline HETATM & 2531 & $\mathrm{C} 12$ & HEM & 273 & 17.286 & 23.371 & 4.247 & 1.00 & 0.00 & C \\
\hline HETATM & 2532 & $\mathrm{C} 13$ & HEM & 273 & 16.147 & 23.224 & 5.004 & 1.00 & 0.00 & C \\
\hline HETATM & 2533 & C14 & HEM & 273 & 15.074 & 23.866 & 4.273 & 1.00 & 0.00 & C \\
\hline HETATM & 2534 & C15 & HEM & 273 & 13.746 & 23.920 & 4.694 & 1.00 & 0.00 & C \\
\hline HETATM & 2535 & H15 & HEM & 273 & 13.506 & 23.436 & 5.642 & 1.00 & 0.00 & $\mathrm{H}$ \\
\hline HETATM & 2536 & C16 & HEM & 273 & 12.716 & 24.602 & 4.051 & 1.00 & 0.00 & C \\
\hline HETATM & 2537 & C17 & HEM & 273 & 11.350 & 24.716 & 4.533 & 1.00 & 0.00 & C \\
\hline HETATM & 2538 & $\mathrm{C} 18$ & HEM & 273 & 10.674 & 25.483 & 3.609 & 1.00 & 0.00 & C \\
\hline HETATM & 2539 & C19 & HEM & 273 & 11.624 & 25.835 & 2.573 & 1.00 & 0.00 & $\mathrm{C}$ \\
\hline HETATM & 2540 & $\mathrm{C} 20$ & HEM & 273 & 11.333 & 26.626 & 1.462 & 1.00 & 0.00 & C \\
\hline HETATM & 2541 & $\mathrm{H} 20$ & HEM & 273 & 10.319 & 27.015 & 1.402 & 1.00 & 0.00 & $\mathrm{H}$ \\
\hline HETATM & 2542 & N21 & HEM & 273 & 13.523 & 26.613 & 0.367 & 1.00 & 0.00 & $\mathrm{~N}$ \\
\hline HETATM & 2543 & $\mathrm{~N} 22$ & HEM & 273 & 16.228 & 25.666 & 0.590 & 1.00 & 0.00 & $\mathrm{~N}$ \\
\hline HETATM & 2544 & N23 & HEM & 273 & 15.557 & 24.403 & 3.090 & 1.00 & 0.00 & $\mathrm{~N}$ \\
\hline HETATM & 2545 & $\mathrm{~N} 24$ & HEM & 273 & 12.857 & 25.287 & 2.854 & 1.00 & 0.00 & $\mathrm{~N}$ \\
\hline HETATM & 2546 & C99 & HEM & 273 & 13.764 & 22.422 & 0.352 & 1.00 & 0.00 & C \\
\hline HETATM & 2547 & 091 & HEM & 273 & 13.923 & 23.736 & 0.365 & 1.00 & 0.00 & o \\
\hline HETATM & 2548 & 092 & HEM & 273 & 13.609 & 21.805 & -0.703 & 1.00 & 0.00 & 0 \\
\hline HETATM & 2549 & СТ91 & HEM & 273 & 13.780 & 21.741 & 1.709 & 1.00 & 0.00 & C \\
\hline HETATM & 2550 & H911 & HEM & 273 & 14.759 & 21.936 & 2.171 & 1.00 & 0.00 & $\mathrm{H}$ \\
\hline HETATM & 2551 & H912 & HEM & 273 & 13.032 & 22.231 & 2.352 & 1.00 & 0.00 & $\mathrm{H}$ \\
\hline HETATM & 2552 & СТ92 & HEM & 273 & 13.520 & 20.236 & 1.597 & 1.00 & 0.00 & C \\
\hline HETATM & 2553 & H921 & HEM & 273 & 14.317 & 19.776 & 1.000 & 1.00 & 0.00 & $\mathrm{H}$ \\
\hline HETATM & 2554 & H922 & HEM & 273 & 12.595 & 20.081 & 1.027 & 1.00 & 0.00 & $\mathrm{H}$ \\
\hline
\end{tabular}


TER

HETATM 2555

HETATM 2556

HETATM 2557

HETATM 2558

HETATM 2559

HETATM 2560

HETATM 2561

HETATM 2562

HETATM 2563

HETATM 2564

HETATM 2565 C9 HEM

HETATM 2566 C10 HEM

HETATM 2567 H1O HEM

HETATM 2568 C11 HEM

HETATM 2569 C12 HEM

HETATM 2570 C13 HEM

HETATM 2571 C14 HEM

HETATM 2572 C15 HEM

HETATM 2573 H15 HEM

HETATM 2574 C16 HEM

HETATM 2575 C17 HEM

HETATM 2576 C18 HEM

HETATM 2577 C19 HEM

HETATM 2578 C20 HEM

HETATM 2579 H2O HEM

HETATM 2580 N21 HEM

HETATM 2581 N22 HEM

HETATM 2582 N23 HEM

HETATM 2583 N24 HEM

HETATM 2584 C99 HEM

HETATM 2585091 HEM

HETATM $2586 \quad 092$ HEM

HETATM 2587 CT91 HEM

HETATM 2588 H911 HEM

HETATM 2589 H912 HEM

HETATM 2590 CT92 HEM

HETATM 2591 H921 HEM

HETATM 2592 H922 HEM

TER

HETATM 2593 C2 VIN 275

HETATM 2594 H21 VIN 275

HETATM 2595 C1 VIN 275

HETATM 2596 H11 VIN 275

HETATM 2597 H12 VIN 275

TER

HETATM $2598 \quad$ C1 MET 276

HETATM 2599 H11 MET 276

HETATM 2600 H12 MET 276

HETATM 2601 H13 MET 276

TER

HETATM 2602 C2 VIN 277

HETATM 2603 H21 VIN 277

HETATM 2604 C1 VIN 277

HETATM 2605 H11 VIN 277

HETATM 2606 H12 VIN 277

TER

HETATM 2607 C1 MET 278

HETATM 2608 H11 MET 278

HETATM 2609 H12 MET 278

HETATM 2610 H13 MET 278

$T E R$

HETATM 2611 C5 ACP 279

HETATM 2612 H51 ACP 279

HETATM 2613 H52 ACP 279

HETATM $2614 \quad$ C4 ACP 279

HETATM 2615 H41 ACP 279

HETATM 2616 H42 ACP 279

HETATM 2617 C1 ACP 279

HETATM 2618 O2 ACP 279

HETATM 2619 O3 ACP 279

HETATM 2620 H3 ACP 279

TER

HETATM 2621 C1 MET 280

HETATM 2622 H11 MET 280

HETATM 2623 H12 MET 280

HETATM 2624 H13 MET 280

TER

HETATM 2625 C1 MET 281

$\begin{array}{lllll}15.121 & 17.690 & 6.440 & 1.00 & 0.00\end{array}$

$\begin{array}{lllll}17.349 & 15.818 & 7.502 & 1.00 & 0.00\end{array}$

$\begin{array}{lllll}17.708 & 15.027 & 8.674 & 1.00 & 0.00\end{array}$

$\begin{array}{lllll}16.563 & 14.930 & 9.448 & 1.00 & 0.00\end{array}$

$\begin{array}{lllll}15.533 & 15.647 & 8.740 & 1.00 & 0.00\end{array}$

$\begin{array}{lllll}14.218 & 15.766 & 9.178 & 1.00 & 0.00\end{array}$

$\begin{array}{lllll}13.965 & 15.270 & 10.113 & 1.00 & 0.00\end{array}$

$\begin{array}{lllll}13.199 & 16.465 & 8.542 & 1.00 & 0.00\end{array}$

$\begin{array}{lllll}11.830 & 16.537 & 9.012 & 1.00 & 0.00\end{array}$

$\begin{array}{lllll}11.124 & 17.257 & 8.065 & 1.00 & 0.00\end{array}$

$\begin{array}{lllll}12.075 & 17.622 & 7.041 & 1.00 & 0.00\end{array}$

$\begin{array}{lllll}11.771 & 18.357 & 5.897 & 1.00 & 0.00\end{array}$

$\begin{array}{lllll}10.744 & 18.701 & 5.791 & 1.00 & 0.00\end{array}$

$\begin{array}{lllll}12.645 & 18.697 & 4.874 & 1.00 & 0.00\end{array}$

$\begin{array}{lllll}12.264 & 19.422 & 3.684 & 1.00 & 0.00\end{array}$

$\begin{array}{lllll}13.404 & 19.569 & 2.927 & 1.00 & 0.00\end{array}$

$\begin{array}{lllll}14.477 & 18.928 & 3.659 & 1.00 & 0.00\end{array}$

$\begin{array}{lllll}15.805 & 18.873 & 3.237 & 1.00 & 0.00\end{array}$

$\begin{array}{lllll}16.045 & 19.358 & 2.289 & 1.00 & 0.00\end{array}$

$\begin{array}{lllll}16.835 & 18.192 & 3.880 & 1.00 & 0.00\end{array}$

$\begin{array}{lllll}18.201 & 18.078 & 3.398 & 1.00 & 0.00\end{array}$

$\begin{array}{lllll}18.876 & 17.310 & 4.322 & 1.00 & 0.00\end{array}$

$\begin{array}{lllll}17.927 & 16.959 & 5.358 & 1.00 & 0.00\end{array}$

$\begin{array}{lllll}18.218 & 16.168 & 6.469 & 1.00 & 0.00\end{array}$

$\begin{array}{lllll}19.232 & 15.778 & 6.530 & 1.00 & 0.00\end{array}$

$\begin{array}{lllll}16.028 & 16.181 & 7.564 & 1.00 & 0.00\end{array}$

$\begin{array}{lllll}13.323 & 17.128 & 7.342 & 1.00 & 0.00\end{array}$

$\begin{array}{lllll}13.994 & 18.391 & 4.841 & 1.00 & 0.00\end{array}$

$\begin{array}{lllll}16.694 & 17.506 & 5.077 & 1.00 & 0.00\end{array}$

$\begin{array}{lllll}15.787 & 20.371 & 7.579 & 1.00 & 0.00\end{array}$

$\begin{array}{lllll}15.627 & 19.058 & 7.567 & 1.00 & 0.00\end{array}$

$\begin{array}{lllll}15.942 & 20.989 & 8.635 & 1.00 & 0.00\end{array}$

$\begin{array}{lllll}15.771 & 21.053 & 6.223 & 1.00 & 0.00\end{array}$

$\begin{array}{lllll}14.792 & 20.858 & 5.760 & 1.00 & 0.00\end{array}$

$\begin{array}{lllll}16.519 & 20.563 & 5.580 & 1.00 & 0.00\end{array}$

$\begin{array}{lllll}16.030 & 22.557 & 6.334 & 1.00 & 0.00\end{array}$

$\begin{array}{llllll}16.955 & 22.713 & 6.905 & 1.00 & 0.00\end{array}$

$\begin{array}{lllll}15.234 & 23.018 & 6.931 & 1.00 & 0.00\end{array}$

$\begin{array}{lllll}10.511 & 28.269 & -1.002 & 1.00 & 0.00\end{array}$

$\begin{array}{lllll}9.695 & 27.571 & -0.837 & 1.00 & 0.00\end{array}$

$\begin{array}{lllll}10.178 & 29.494 & -1.403 & 1.00 & 0.00\end{array}$

$\begin{array}{lllll}10.909 & 30.273 & -1.511 & 1.00 & 0.00\end{array}$

$\begin{array}{lllll}9.136 & 29.763 & -1.570 & 1.00 & 0.00\end{array}$

$\begin{array}{lllll}13.174 & 28.524 & -2.840 & 1.00 & 0.00\end{array}$

$\begin{array}{lllll}13.409 & 29.595 & -2.720 & 1.00 & 0.00\end{array}$

$\begin{array}{lllll}13.987 & 28.060 & -3.410 & 1.00 & 0.00\end{array}$

$\begin{array}{lllll}12.267 & 28.446 & -3.450 & 1.00 & 0.00\end{array}$

$\begin{array}{lllll}18.185 & 26.846 & -2.325 & 1.00 & 0.00\end{array}$

$\begin{array}{lllll}17.519 & 26.745 & -3.184 & 1.00 & 0.00\end{array}$

$\begin{array}{lllll}19.351 & 27.490 & -2.484 & 1.00 & 0.00\end{array}$

$\begin{array}{lllll}20.031 & 27.647 & -1.654 & 1.00 & 0.00\end{array}$

$\begin{array}{lllll}19.656 & 27.888 & -3.450 & 1.00 & 0.00\end{array}$

$\begin{array}{llllll}19.869 & 25.163 & -0.121 & 1.00 & 0.00\end{array}$

$\begin{array}{lllll}20.460 & 25.827 & 0.528 & 1.00 & 0.00\end{array}$

$\begin{array}{llllll}20.026 & 24.141 & 0.246 & 1.00 & 0.00\end{array}$

$20.301 \quad 25.228 \quad-1.126 \quad 1.00 \quad 0.00$

$10.781 \quad 24.081$

$11.520 \quad 24.070$

$\begin{array}{lll}5.770 & 1.00 \quad 0.00\end{array}$

$\begin{array}{llll}24.689 & 6.134 & 1.00 & 0.00\end{array}$

$\begin{array}{lllll}10.302 & 22.644 & 5.497 & 1.00 & 0.00\end{array}$

$\begin{array}{lllll}9.744 & 22.583 & 4.549 & 1.00 & 0.00\end{array}$

$\begin{array}{lllll}11.173 & 21.977 & 5.390 & 1.00 & 0.00\end{array}$

$\begin{array}{lllll}9.438 & 22.031 & 6.573 & 1.00 & 0.00\end{array}$

$\begin{array}{lllll}9.210 & 22.554 & 7.671 & 1.00 & 0.00\end{array}$

$\begin{array}{lllll}8.922 & 20.852 & 6.209 & 1.00 & 0.00\end{array}$

$\begin{array}{lllll}8.313 & 20.498 & 6.945 & 1.00 & 0.00\end{array}$

$18.677 \quad 22.955$

$19.229 \quad 23.794$

$\begin{array}{lll}4.597 & 1.00 \quad 0.00\end{array}$

$\begin{array}{lll}5.047 & 1.00 & 0.00\end{array}$

$\begin{array}{lll}5.319 & 1.00 & 0.00\end{array}$

$\begin{array}{lll}3.717 & 1.00 & 0.00\end{array}$

$\begin{array}{lllll}9.243 & 25.904 & 3.646 & 1.00 & 0.00\end{array}$

$\mathrm{Fe}$

C

C

$\mathrm{N}$

$\mathrm{N}$

$\mathrm{H}$

C

$\mathrm{H}$

H

$\mathrm{H}$

H

$\mathrm{H}$

H

H

C

$\mathrm{H}$
$\mathrm{H}$

C

o

H 


\begin{tabular}{|c|c|c|c|c|c|c|c|c|c|c|}
\hline HETATM & 2626 & $\mathrm{H} 11$ & MET & 281 & 8.798 & 25.695 & 4.626 & 1.00 & 0.00 & $\mathrm{H}$ \\
\hline HETATM & 2627 & $\mathrm{H} 12$ & MET & 281 & 9.133 & 26.984 & 3.471 & 1.00 & 0.00 & $\mathrm{H}$ \\
\hline HETATM & 2628 & $\mathrm{H} 13$ & $\mathrm{MET}$ & 281 & 8.643 & 25.378 & 2.889 & 1.00 & 0.00 & $\mathrm{H}$ \\
\hline \multicolumn{11}{|l|}{ TER } \\
\hline HETATM & 2629 & $\mathrm{C} 2$ & VIN & 282 & 19.040 & 14.525 & 8.933 & 1.00 & 0.00 & r \\
\hline HETATM & 2630 & H21 & VIN & 282 & 19.856 & 15.223 & 8.768 & 1.00 & 0.00 & 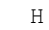 \\
\hline HETATM & 2631 & $\mathrm{C} 1$ & VIN & 282 & 19.373 & 13.300 & 9.335 & 1.00 & 0.00 & 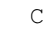 \\
\hline HETATM & 2632 & H11 & VIN & 282 & 18.642 & 12.521 & 9.442 & 1.00 & 0.00 & $\mathrm{H}$ \\
\hline HETATM & 2633 & $\mathrm{H} 12$ & VIN & 282 & 20.415 & 13.030 & 9.501 & 1.00 & 0.00 & $\mathrm{H}$ \\
\hline \multicolumn{11}{|l|}{ TER } \\
\hline HETATM & 2634 & $\mathrm{C} 1$ & MET & 283 & 16.376 & 14.270 & 10.771 & 1.00 & 0.00 & C \\
\hline HETATM & 2635 & $\mathrm{H} 11$ & MET & 283 & 16.142 & 13.199 & 10.652 & 1.00 & 0.00 & 11 \\
\hline HETATM & 2636 & $\mathrm{H} 12$ & MET & 283 & 15.563 & 14.734 & 11.341 & 1.00 & 0.00 & 11 \\
\hline HETATM & 2637 & $\mathrm{H} 13$ & MET & 283 & 17.284 & 14.347 & 11.382 & 1.00 & 0.00 & 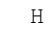 \\
\hline \multicolumn{11}{|l|}{ TER } \\
\hline HETATM & 2638 & $\mathrm{C} 2$ & VIN & 284 & 11.366 & 15.948 & 10.256 & 1.00 & 0.00 & $\mathrm{C}$ \\
\hline HETATM & 2639 & H21 & VIN & 284 & 12.032 & 16.048 & 11.115 & 1.00 & 0.00 & $\mathrm{H}$ \\
\hline HETATM & 2640 & $\mathrm{C} 1$ & VIN & 284 & 10.199 & 15.303 & 10.415 & 1.00 & 0.00 & C \\
\hline HETATM & 2641 & H11 & VIN & 284 & 9.520 & 15.147 & 9.585 & 1.00 & 0.00 & $\mathrm{H}$ \\
\hline HETATM & 2642 & $\mathrm{H} 12$ & VIN & 284 & 9.895 & 14.905 & 11.381 & 1.00 & 0.00 & $\mathrm{H}$ \\
\hline \multicolumn{11}{|l|}{ TER } \\
\hline HETATM & 2643 & $\mathrm{C} 1$ & MET & 285 & 9.682 & 17.631 & 8.052 & 1.00 & 0.00 & C \\
\hline HETATM & 2644 & $\mathrm{H} 11$ & MET & 285 & 9.525 & 18.653 & 7.685 & 1.00 & 0.00 & $\mathrm{H}$ \\
\hline HETATM & 2645 & $\mathrm{H} 12$ & $\mathrm{MET}$ & 285 & 9.249 & 17.565 & 9.057 & 1.00 & 0.00 & $\mathrm{H}$ \\
\hline HETATM & 2646 & $\mathrm{H} 13$ & MET & 285 & 9.090 & 16.967 & 7.403 & 1.00 & 0.00 & $\mathrm{H}$ \\
\hline TER & & & & & & & & & & \\
\hline HETATM & 2647 & C5 & $\mathrm{ACP}$ & 286 & 18.770 & 18.712 & 2.161 & 1.00 & 0.00 & C \\
\hline HETATM & 2648 & H51 & $\mathrm{ACP}$ & 286 & 18.031 & 18.724 & 1.349 & 1.00 & 0.00 & $\mathrm{H}$ \\
\hline HETATM & 2649 & H5 2 & $\mathrm{ACP}$ & 286 & 19.611 & 18.104 & 1.798 & 1.00 & 0.00 & $\mathrm{H}$ \\
\hline HETATM & 2650 & C4 & $\mathrm{ACP}$ & 286 & 19.249 & 20.150 & 2.434 & 1.00 & 0.00 & C \\
\hline HETATM & 2651 & H4 1 & $\mathrm{ACP}$ & 286 & 19.806 & 20.211 & 3.382 & 1.00 & 0.00 & $\mathrm{H}$ \\
\hline HETATM & 2652 & $\mathrm{H} 42$ & $\mathrm{ACP}$ & 286 & 18.378 & 20.817 & 2.541 & 1.00 & 0.00 & $\mathrm{H}$ \\
\hline HETATM & 2653 & $\mathrm{C} 1$ & $\mathrm{ACP}$ & 286 & 20.113 & 20.762 & 1.358 & 1.00 & 0.00 & C \\
\hline HETATM & 2654 & $\mathrm{O} 2$ & $\mathrm{ACP}$ & 286 & 20.340 & 20.239 & 0.260 & 1.00 & 0.00 & 0 \\
\hline HETATM & 2655 & 03 & $\mathrm{ACP}$ & 286 & 20.628 & 21.942 & 1.722 & 1.00 & 0.00 & 0 \\
\hline HETATM & 2656 & H3 & $\mathrm{ACP}$ & 286 & 21.237 & 22.296 & 0.986 & 1.00 & 0.00 & $\mathrm{H}$ \\
\hline TER & & & & & & & & & & \\
\hline HETATM & 2657 & $\mathrm{C} 1$ & MET & 287 & 10.874 & 19.839 & 3.334 & 1.00 & 0.00 & C \\
\hline HETATM & 2658 & $\mathrm{H} 11$ & MET & 287 & 10.322 & 18.999 & 2.884 & 1.00 & 0.00 & $\mathrm{H}$ \\
\hline HETATM & 2659 & $\mathrm{H} 12$ & MET & 287 & 10.867 & 20.665 & 2.613 & 1.00 & 0.00 & $\mathrm{H}$ \\
\hline HETATM & 2660 & $\mathrm{H} 13$ & MET & 287 & 10.303 & 20.158 & 4.214 & 1.00 & 0.00 & $\mathrm{H}$ \\
\hline TER & & & & & & & & & & \\
\hline HETATM & 2661 & $\mathrm{C} 1$ & MET & 288 & 20.307 & 16.890 & 4.285 & 1.00 & 0.00 & C \\
\hline HETATM & 2662 & H11 & MET & 288 & 20.753 & 17.099 & 3.305 & 1.00 & 0.00 & $\mathrm{H}$ \\
\hline HETATM & 2663 & $\mathrm{H} 12$ & MET & 288 & 20.418 & 15.810 & 4.460 & 1.00 & 0.00 & $\mathrm{H}$ \\
\hline HETATM & 2664 & $\mathrm{H} 13$ & $\mathrm{MET}$ & 288 & 20.908 & 17.416 & 5.042 & 1.00 & 0.00 & $\mathrm{H}$ \\
\hline TER & & & & & & & & & & \\
\hline HETATM & 2665 & $\mathrm{FE}$ & HEM & 289 & -8.789 & 25.350 & -6.440 & 1.00 & 0.00 & $\mathrm{Fe}$ \\
\hline HETATM & 2666 & $\mathrm{C} 1$ & HEM & 289 & -11.017 & 27.222 & -7.502 & 1.00 & 0.00 & C \\
\hline HETATM & 2667 & $\mathrm{C} 2$ & HEM & 289 & -11.376 & 28.013 & -8.674 & 1.00 & 0.00 & C \\
\hline HETATM & 2668 & C3 & HEM & 289 & -10.230 & 28.110 & -9.448 & 1.00 & 0.00 & C \\
\hline HETATM & 2669 & C4 & HEM & 289 & -9.201 & 27.392 & -8.740 & 1.00 & 0.00 & C \\
\hline HETATM & 2670 & C5 & HEM & 289 & -7.886 & 27.274 & -9.178 & 1.00 & 0.00 & C \\
\hline HETATM & 2671 & H5 & HEM & 289 & -7.632 & 27.770 & -10.113 & 1.00 & 0.00 & $\mathrm{H}$ \\
\hline HETATM & 2672 & C6 & HEM & 289 & -6.866 & 26.575 & -8.542 & 1.00 & 0.00 & C \\
\hline HETATM & 2673 & C7 & HEM & 289 & -5.497 & 26.503 & -9.012 & 1.00 & 0.00 & C \\
\hline HETATM & 2674 & $\mathrm{C} 8$ & HEM & 289 & -4.792 & 25.783 & -8.065 & 1.00 & 0.00 & C \\
\hline HETATM & 2675 & $\mathrm{C} 9$ & HEM & 289 & -5.743 & 25.418 & -7.041 & 1.00 & 0.00 & C \\
\hline HETATM & 2676 & $\mathrm{C} 10$ & HEM & 289 & -5.438 & 24.683 & -5.897 & 1.00 & 0.00 & C \\
\hline HETATM & 2677 & $\mathrm{H} 10$ & HEM & 289 & -4.412 & 24.339 & -5.791 & 1.00 & 0.00 & $\mathrm{H}$ \\
\hline HETATM & 2678 & C11 & HEM & 289 & -6.313 & 24.343 & -4.874 & 1.00 & 0.00 & C \\
\hline HETATM & 2679 & C12 & HEM & 289 & -5.932 & 23.618 & -3.684 & 1.00 & 0.00 & C \\
\hline HETATM & 2680 & $\mathrm{C} 13$ & HEM & 289 & -7.071 & 23.470 & -2.927 & 1.00 & 0.00 & C \\
\hline HETATM & 2681 & C14 & HEM & 289 & -8.144 & 24.112 & -3.659 & 1.00 & 0.00 & C \\
\hline HETATM & 2682 & C15 & HEM & 289 & -9.472 & 24.167 & -3.237 & 1.00 & 0.00 & C \\
\hline HETATM & 2683 & H15 & HEM & 289 & -9.712 & 23.682 & -2.289 & 1.00 & 0.00 & $\mathrm{H}$ \\
\hline HETATM & 2684 & $\mathrm{C} 16$ & HEM & 289 & -10.503 & 24.848 & -3.880 & 1.00 & 0.00 & C \\
\hline HETATM & 2685 & $\mathrm{C} 17$ & HEM & 289 & -11.868 & 24.962 & -3.398 & 1.00 & 0.00 & C \\
\hline HETATM & 2686 & $\mathrm{C} 18$ & HEM & 289 & -12.544 & 25.729 & -4.322 & 1.00 & 0.00 & C \\
\hline HETATM & 2687 & C19 & HEM & 289 & -11.594 & 26.081 & -5.358 & 1.00 & 0.00 & C \\
\hline HETATM & 2688 & $\mathrm{C} 20$ & HEM & 289 & -11.885 & 26.872 & -6.469 & 1.00 & 0.00 & C \\
\hline HETATM & 2689 & $\mathrm{H} 2 \mathrm{O}$ & HEM & 289 & -12.900 & 27.262 & -6.530 & 1.00 & 0.00 & $\mathrm{H}$ \\
\hline HETATM & 2690 & N21 & HEM & 289 & -9.695 & 26.859 & -7.564 & 1.00 & 0.00 & $\mathrm{~N}$ \\
\hline HETATM & 2691 & $\mathrm{~N} 22$ & HEM & 289 & -6.991 & 25.912 & -7.342 & 1.00 & 0.00 & $\mathrm{~N}$ \\
\hline HETATM & 2692 & N23 & HEM & 289 & -7.661 & 24.649 & -4.841 & 1.00 & 0.00 & $\mathrm{~N}$ \\
\hline HETATM & 2693 & N24 & HEM & 289 & -10.361 & 25.533 & -5.077 & 1.00 & 0.00 & $\mathrm{~N}$ \\
\hline HETATM & 2694 & C99 & HEM & 289 & -9.454 & 22.668 & -7.579 & 1.00 & 0.00 & C \\
\hline HETATM & 2695 & 091 & HEM & 289 & -9.295 & 23.982 & -7.567 & 1.00 & 0.00 & 0 \\
\hline HETATM & 2696 & 092 & HEM & 289 & -9.609 & 22.051 & -8.635 & 1.00 & 0.00 & 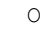 \\
\hline
\end{tabular}


HETATM 2697 CT91 HEM 289 HETATM 2698 H911 HEM HETATM 2699 H912 HEM HETATM 2700 CT92 HEM HETATM 2701 H921 HEM HETATM 2702 H922 HEM

HETATM 2703 UE HETATM 2704 C1 HEM 290 HETATM 2705 C2 HEM 290 HETATM 2706 C3 HEM 290 HETATM 2707 C4 HEM 290 HETATM 2708 C5 HEM 290 HETATM 2709 H5 HEM 290 HETATM 2710 C6 HEM 290 HETATM 2711 C7 HEM 290 HETATM 2712 C8 HEM 290 HETATM 2713 C9 HEM 290 HETATM 2714 C10 HEM 290 HETATM 2715 H1O HEM 290 HETATM 2716 C11 HEM 290 HETATM 2717 C12 HEM 290 HETATM 2718 C13 HEM 290 HETATM 2719 C14 HEM 290 HETATM 2720 C15 HEM 290 HETATM 2721 H15 HEM 290 HETATM 2722 C16 HEM 290 HETATM 2723 C17 HEM 290 HETATM 2724 C18 HEM 290 HETATM 2725 C19 HEM 290 HETATM 2726 C20 HEM 290 HETATM 2727 H20 HEM 290 HETATM 2728 N21 HEM 290 HETATM 2729 N22 HEM 290 HETATM 2730 N23 HEM 290 HETATM 2731 N24 HEM 290 HETATM 2732 C99 HEM 290 HETATM 2733091 HEM 290 HETATM 2734092 HEM 290 HETATM 2735 CT91 HEM 290 HETATM 2736 H911 HEM 290 HETATM 2737 H912 HEM 290 HETATM 2738 CT92 HEM 290 HETATM 2739 H921 HEM 290 HETATM 2740 H922 HEM 29 TER

HETATM 2741 C2 VIN 291 HETATM 2742 H21 VIN 29 HETATM 2743 C1 VIN 29 HETATM 2744 H11 VIN 291 HETATM 2745 H12 VIN 291 (T)

HETATM 2746 C1 MET 292 HETATM 2747 H11 MET 292 HETATM 2748 H12 MET 292 HETATM 2749 H13 MET 292 TER

HETATM 2750 C2 VIN 293 HETATM 2751 H21 VIN 293 HETATM 2752 C1 VIN 293 HETATM 2753 H11 VIN 293 HETATM 2754 H12 VIN 293 TER

HETATM $2755 \quad$ C1 MET 294 HETATM 2756 H11 MET 294 HETATM 2757 H12 MET 294 HETATM 2758 H13 MET 294 TER

HETATM $2759-C 5$ ACP HETATM $2760 \quad$ H51 ACP 295 HETATM 2761 H52 ACP 295 HETATM $2762 \quad$ C4 ACP 295 HETATM 2763 H41 ACP 295 HETATM 2764 H42 ACP 295 HETATM 2765 C1 ACP 295 HETATM 2766 O2 ACP 295 HETATM $2767 \quad 03$ ACP 295 HETATM 2768 H3 ACP 295

\begin{tabular}{|c|c|c|c|c|c|}
\hline-9.438 & 21.987 & -6.223 & 1.00 & 0.00 & C \\
\hline-8.459 & 22.182 & -5.760 & 1.00 & 0.00 & $\mathrm{H}$ \\
\hline-10.186 & 22.477 & -5.580 & 1.00 & 0.00 & $\mathrm{H}$ \\
\hline-9.698 & 20.483 & -6.334 & 1.00 & 0.00 & $\mathrm{C}$ \\
\hline-8.901 & 20.022 & -6.931 & 1.00 & 0.00 & $\mathrm{H}$ \\
\hline-10.623 & 20.327 & -6.905 & 1.00 & 0.00 & $\mathrm{H}$ \\
\hline-8.097 & 17.936 & -1.491 & 1.00 & 0.00 & $\mathrm{Fe}$ \\
\hline-5.869 & 16.064 & -0.429 & 1.00 & 0.00 & C \\
\hline-5.510 & 15.273 & 0.742 & 1.00 & 0.00 & C \\
\hline-6.656 & 15.176 & 1.517 & 1.00 & 0.00 & C \\
\hline-7.685 & 15.894 & 0.809 & 1.00 & 0.00 & C \\
\hline-9.000 & 16.012 & 1.247 & 1.00 & 0.00 & C \\
\hline-9.254 & 15.516 & 2.182 & 1.00 & 0.00 & $\mathrm{H}$ \\
\hline-10.020 & 16.711 & 0.611 & 1.00 & 0.00 & C \\
\hline-11.388 & 16.783 & 1.081 & 1.00 & 0.00 & C \\
\hline-12.094 & 17.503 & 0.134 & 1.00 & 0.00 & C \\
\hline-11.143 & 17.868 & -0.891 & 1.00 & 0.00 & C \\
\hline-11.447 & 18.603 & -2.034 & 1.00 & 0.00 & C \\
\hline-12.474 & 18.947 & -2.140 & 1.00 & 0.00 & $\mathrm{H}$ \\
\hline-10.573 & 18.943 & -3.057 & 1.00 & 0.00 & $\mathrm{C}$ \\
\hline-10.954 & 19.668 & -4.247 & 1.00 & 0.00 & $\mathrm{C}$ \\
\hline-9.814 & 19.816 & -5.004 & 1.00 & 0.00 & C \\
\hline-8.741 & 19.174 & -4.273 & 1.00 & 0.00 & C \\
\hline-7.413 & 19.119 & -4.694 & 1.00 & 0.00 & C \\
\hline-7.173 & 19.604 & -5.642 & 1.00 & 0.00 & $\mathrm{H}$ \\
\hline-6.383 & 18.438 & -4.051 & 1.00 & 0.00 & C \\
\hline-5.017 & 18.324 & -4.533 & 1.00 & 0.00 & C \\
\hline-4.342 & 17.557 & -3.609 & 1.00 & 0.00 & C \\
\hline-5.292 & 17.205 & -2.573 & 1.00 & 0.00 & $\mathrm{C}$ \\
\hline-5.000 & 16.414 & -1.462 & 1.00 & 0.00 & C \\
\hline-3.986 & 16.024 & -1.402 & 1.00 & 0.00 & $\mathrm{H}$ \\
\hline-7.191 & 16.427 & -0.367 & 1.00 & 0.00 & $\mathrm{~N}$ \\
\hline-9.895 & 17.374 & -0.590 & 1.00 & 0.00 & $\mathrm{~N}$ \\
\hline-9.224 & 18.637 & -3.090 & 1.00 & 0.00 & $\mathrm{~N}$ \\
\hline-6.525 & 17.753 & -2.854 & 1.00 & 0.00 & $\mathrm{~N}$ \\
\hline-7.431 & 20.618 & -0.352 & 1.00 & 0.00 & C \\
\hline-7.591 & 19.304 & -0.365 & 1.00 & 0.00 & 0 \\
\hline-7.277 & 21.235 & 0.703 & 1.00 & 0.00 & 0 \\
\hline-7.447 & 21.299 & -1.709 & 1.00 & 0.00 & C \\
\hline-8.426 & 21.104 & -2.171 & 1.00 & 0.00 & $\mathrm{H}$ \\
\hline-6.699 & 20.809 & -2.352 & 1.00 & 0.00 & $\mathrm{H}$ \\
\hline-7.188 & 22.803 & -1.597 & 1.00 & 0.00 & $\mathrm{C}$ \\
\hline-6.263 & 22.959 & -1.027 & 1.00 & 0.00 & $\mathrm{H}$ \\
\hline-7.984 & 23.264 & -1.000 & 1.00 & 0.00 & $\mathrm{H}$ \\
\hline-12.708 & 28.515 & -8.933 & 1.00 & 0.00 & C \\
\hline-13.523 & 27.817 & -8.768 & 1.00 & 0.00 & $\mathrm{H}$ \\
\hline-13.040 & 29.740 & -9.335 & 1.00 & 0.00 & $\mathrm{C}$ \\
\hline-12.309 & 30.519 & -9.442 & 1.00 & 0.00 & $\mathrm{H}$ \\
\hline-14.082 & 30.010 & -9.501 & 1.00 & 0.00 & $\mathrm{H}$ \\
\hline-10.044 & 28.770 & -10.771 & 1.00 & 0.00 & C \\
\hline-9.809 & 29.841 & -10.652 & 1.00 & 0.00 & $\mathrm{H}$ \\
\hline-9.231 & 28.306 & -11.341 & 1.00 & 0.00 & $\mathrm{H}$ \\
\hline-10.951 & 28.693 & -11.382 & 1.00 & 0.00 & $\mathrm{H}$ \\
\hline-5.034 & 27.092 & -10.256 & 1.00 & 0.00 & C \\
\hline-5.700 & 26.991 & -11.115 & 1.00 & 0.00 & $\mathrm{H}$ \\
\hline-3.867 & 27.737 & -10.415 & 1.00 & 0.00 & C \\
\hline-3.187 & 27.893 & -9.585 & 1.00 & 0.00 & $\mathrm{H}$ \\
\hline-3.562 & 28.135 & -11.381 & 1.00 & 0.00 & $\mathrm{H}$ \\
\hline-3.349 & 25.409 & -8.052 & 1.00 & 0.00 & C \\
\hline-2.758 & 26.073 & -7.403 & 1.00 & 0.00 & $\mathrm{H}$ \\
\hline-3.193 & 24.387 & -7.685 & 1.00 & 0.00 & $\mathrm{H}$ \\
\hline-2.917 & 25.475 & -9.057 & 1.00 & 0.00 & $\mathrm{H}$ \\
\hline-12.438 & 24.327 & -2.161 & 1.00 & 0.00 & C \\
\hline-11.699 & 24.316 & -1.349 & 1.00 & 0.00 & $\mathrm{H}$ \\
\hline-13.278 & 24.936 & -1.798 & 1.00 & 0.00 & $\mathrm{H}$ \\
\hline-12.916 & 22.890 & -2.434 & 1.00 & 0.00 & $\mathrm{C}$ \\
\hline-13.474 & 22.829 & -3.382 & 1.00 & 0.00 & $\mathrm{H}$ \\
\hline-12.046 & 22.223 & -2.541 & 1.00 & 0.00 & $\mathrm{H}$ \\
\hline-13.780 & 22.278 & -1.358 & 1.00 & 0.00 & C \\
\hline-14.008 & 22.800 & -0.260 & 1.00 & 0.00 & 0 \\
\hline-14.296 & 21.098 & -1.722 & 1.00 & 0.00 & 0 \\
\hline-14.905 & 20.744 & -0.986 & 1.00 & 0.00 & $\mathrm{H}$ \\
\hline
\end{tabular}




\begin{tabular}{|c|c|c|c|c|c|c|c|c|c|c|}
\hline HETATM & 2769 & $\mathrm{C} 1$ & MET & 296 & -4.541 & 23.201 & -3.334 & 1.00 & 0.00 & C \\
\hline HETATM & 2770 & H11 & MET & 296 & -3.989 & 24.040 & -2.884 & 1.00 & 0.00 & $\mathrm{H}$ \\
\hline HETATM & 2771 & $\mathrm{H} 12$ & MET & 296 & -4.535 & 22.375 & -2.613 & 1.00 & 0.00 & $\mathrm{H}$ \\
\hline HETATM & 2772 & $\mathrm{H} 13$ & $\mathrm{MET}$ & 296 & -3.970 & 22.882 & -4.214 & 1.00 & 0.00 & $\mathrm{H}$ \\
\hline \multicolumn{11}{|l|}{ TER } \\
\hline HETATM & 2773 & $\mathrm{C} 1$ & MET & 297 & -13.975 & 26.150 & -4.285 & 1.00 & 0.00 & C \\
\hline HETATM & 2774 & $\mathrm{H} 11$ & MET & 297 & -14.420 & 25.941 & -3.305 & 1.00 & 0.00 & $\mathrm{H}$ \\
\hline HETATM & 2775 & $\mathrm{H} 12$ & MET & 297 & -14.085 & 27.230 & -4.460 & 1.00 & 0.00 & $\mathrm{H}$ \\
\hline HETATM & 2776 & H13 & MET & 297 & -14.576 & 25.624 & -5.042 & 1.00 & 0.00 & $\mathrm{H}$ \\
\hline \multicolumn{11}{|l|}{ TER } \\
\hline HETATM & 2777 & $\mathrm{C} 2$ & VIN & 298 & -4.178 & 14.771 & 1.002 & 1.00 & 0.00 & C \\
\hline HETATM & 2778 & H 21 & VIN & 298 & -3.362 & 15.469 & 0.837 & 1.00 & 0.00 & $\mathrm{H}$ \\
\hline HETATM & 2779 & $\mathrm{C} 1$ & VIN & 298 & -3.845 & 13.546 & 1.403 & 1.00 & 0.00 & C \\
\hline HETATM & 2780 & H11 & VIN & 298 & -4.577 & 12.767 & 1.511 & 1.00 & 0.00 & $\mathrm{H}$ \\
\hline HETATM & 2781 & $\mathrm{H} 12$ & VIN & 298 & -2.804 & 13.277 & 1.570 & 1.00 & 0.00 & $\mathrm{H}$ \\
\hline \multicolumn{11}{|l|}{ TER } \\
\hline HETATM & 2782 & $\mathrm{C} 1$ & MET & 299 & -6.842 & 14.516 & 2.840 & 1.00 & 0.00 & C \\
\hline HETATM & 2783 & $\mathrm{H} 11$ & MET & 299 & -7.077 & 13.445 & 2.720 & 1.00 & 0.00 & $\mathrm{H}$ \\
\hline HETATM & 2784 & $\mathrm{H} 12$ & $\mathrm{MET}$ & 299 & -7.655 & 14.980 & 3.410 & 1.00 & 0.00 & $\mathrm{H}$ \\
\hline HETATM & 2785 & $\mathrm{H} 13$ & MET & 299 & -5.934 & 14.593 & 3.450 & 1.00 & 0.00 & $\mathrm{H}$ \\
\hline \multicolumn{11}{|l|}{ TER } \\
\hline HETATM & 2786 & $\mathrm{C} 2$ & VIN & 300 & -11.852 & 16.194 & 2.325 & 1.00 & 0.00 & C \\
\hline HETATM & 2787 & H21 & VIN & 300 & -11.186 & 16.295 & 3.184 & 1.00 & 0.00 & $\mathrm{H}$ \\
\hline HETATM & 2788 & $\mathrm{C} 1$ & VIN & 300 & -13.019 & 15.550 & 2.484 & 1.00 & 0.00 & C \\
\hline HETATM & 2789 & $\mathrm{H} 11$ & VIN & 300 & -13.699 & 15.393 & 1.654 & 1.00 & 0.00 & $\mathrm{H}$ \\
\hline HETATM & 2790 & $\mathrm{H} 12$ & VIN & 300 & $-13 \cdot 324$ & 15.151 & 3.450 & 1.00 & 0.00 & $\mathrm{H}$ \\
\hline TER & & & & & & & & & & \\
\hline HETATM & 2791 & $\mathrm{C} 1$ & MET & 301 & -13.537 & 17.877 & 0.121 & 1.00 & 0.00 & C \\
\hline HETATM & 2792 & H11 & MET & 301 & -13.693 & 18.899 & -0.246 & 1.00 & 0.00 & $\mathrm{H}$ \\
\hline HETATM & 2793 & $\mathrm{H} 12$ & MET & 301 & -13.969 & 17.811 & 1.126 & 1.00 & 0.00 & $\mathrm{H}$ \\
\hline HETATM & 2794 & $\mathrm{H} 13$ & $\mathrm{MET}$ & 301 & -14.128 & 17.213 & -0.528 & 1.00 & 0.00 & $\mathrm{H}$ \\
\hline TER & & & & & & & & & & \\
\hline HETATM & 2795 & C5 & $\mathrm{ACP}$ & 302 & -4.448 & 18.959 & -5.770 & 1.00 & 0.00 & C \\
\hline HETATM & 2796 & H51 & $\mathrm{ACP}$ & 302 & -5.187 & 18.970 & -6.582 & 1.00 & 0.00 & $\mathrm{H}$ \\
\hline HETATM & 2797 & H52 & $\mathrm{ACP}$ & 302 & -3.608 & 18.350 & -6.134 & 1.00 & 0.00 & $\mathrm{H}$ \\
\hline HETATM & 2798 & $\mathrm{C} 4$ & $\mathrm{ACP}$ & 302 & -3.970 & 20.396 & -5.497 & 1.00 & 0.00 & C \\
\hline HETATM & 2799 & $\mathrm{H} 41$ & $\mathrm{ACP}$ & 302 & -3.412 & 20.457 & -4.549 & 1.00 & 0.00 & $\mathrm{H}$ \\
\hline HETATM & 2800 & $\mathrm{H} 42$ & $\mathrm{ACP}$ & 302 & -4.840 & 21.063 & -5.390 & 1.00 & 0.00 & $\mathrm{H}$ \\
\hline HETATM & 2801 & $\mathrm{C} 1$ & $\mathrm{ACP}$ & 302 & -3.106 & 21.008 & -6.573 & 1.00 & 0.00 & C \\
\hline HETATM & 2802 & $\mathrm{O} 2$ & $\mathrm{ACP}$ & 302 & -2.878 & 20.486 & -7.671 & 1.00 & 0.00 & O \\
\hline HETATM & 2803 & 03 & $\mathrm{ACP}$ & 302 & -2.590 & 22.188 & -6.209 & 1.00 & 0.00 & 0 \\
\hline HETATM & 2804 & H3 & $\mathrm{ACP}$ & 302 & -1.981 & 22.542 & -6.945 & 1.00 & 0.00 & $\mathrm{H}$ \\
\hline TER & & & & & & & & & & \\
\hline HETATM & 2805 & $\mathrm{C} 1$ & MET & 303 & $-12 \cdot 344$ & 20.085 & -4.597 & 1.00 & 0.00 & C \\
\hline HETATM & 2806 & $\mathrm{H} 11$ & MET & 303 & -12.897 & 19.246 & -5.047 & 1.00 & 0.00 & $\mathrm{H}$ \\
\hline HETATM & 2807 & $\mathrm{H} 12$ & $\mathrm{MET}$ & 303 & $-12 \cdot 351$ & 20.911 & -5.319 & 1.00 & 0.00 & $\mathrm{H}$ \\
\hline HETATM & 2808 & $\mathrm{H} 13$ & MET & 303 & -12.915 & 20.405 & -3.717 & 1.00 & 0.00 & $\mathrm{H}$ \\
\hline TER & & & & & & & & & & \\
\hline HETATM & 2809 & $\mathrm{C} 1$ & MET & 304 & -2.911 & 17.136 & -3.646 & 1.00 & 0.00 & C \\
\hline HETATM & 2810 & H11 & MET & 304 & -2.466 & 17.345 & -4.626 & 1.00 & 0.00 & $\mathrm{H}$ \\
\hline HETATM & 2811 & $\mathrm{H} 12$ & MET & 304 & -2.800 & 16.056 & -3.471 & 1.00 & 0.00 & $\mathrm{H}$ \\
\hline HETATM & 2812 & $\mathrm{H} 13$ & MET & 304 & -2.310 & 17.662 & -2.889 & 1.00 & 0.00 & $\mathrm{H}$ \\
\hline TER & & & & & & & & & & \\
\hline HETATM & 2813 & $\mathrm{FE}$ & HEM & 305 & -7.820 & 10.757 & 1.491 & 1.00 & 0.00 & $\mathrm{Fe}$ \\
\hline HETATM & 2814 & $\mathrm{C} 1$ & HEM & 305 & -10.048 & 12.629 & 0.429 & 1.00 & 0.00 & C \\
\hline HETATM & 2815 & C2 & HEM & 305 & -10.407 & 13.420 & -0.742 & 1.00 & 0.00 & C \\
\hline HETATM & 2816 & C3 & HEM & 305 & -9.261 & 13.517 & -1.517 & 1.00 & 0.00 & C \\
\hline HETATM & 2817 & C4 & HEM & 305 & -8.231 & 12.800 & -0.809 & 1.00 & 0.00 & C \\
\hline HETATM & 2818 & C5 & HEM & 305 & -6.916 & 12.681 & -1.247 & 1.00 & 0.00 & C \\
\hline HETATM & 2819 & H5 & HEM & 305 & -6.663 & 13.177 & -2.182 & 1.00 & 0.00 & $\mathrm{H}$ \\
\hline HETATM & 2820 & $\mathrm{C} 6$ & HEM & 305 & -5.897 & 11.982 & -0.611 & 1.00 & 0.00 & C \\
\hline HETATM & 2821 & C7 & HEM & 305 & -4.528 & 11.910 & -1.081 & 1.00 & 0.00 & C \\
\hline HETATM & 2822 & $\mathrm{C} 8$ & HEM & 305 & -3.823 & 11.190 & -0.134 & 1.00 & 0.00 & C \\
\hline HETATM & 2823 & C9 & HEM & 305 & -4.774 & 10.825 & 0.891 & 1.00 & 0.00 & C \\
\hline HETATM & 2824 & $\mathrm{C} 10$ & HEM & 305 & -4.469 & 10.090 & 2.034 & 1.00 & 0.00 & C \\
\hline HETATM & 2825 & $\mathrm{H} 10$ & HEM & 305 & -3.442 & 9.746 & 2.140 & 1.00 & 0.00 & $\mathrm{H}$ \\
\hline HETATM & 2826 & C11 & HEM & 305 & -5.344 & 9.750 & 3.057 & 1.00 & 0.00 & C \\
\hline HETATM & 2827 & $\mathrm{C} 12$ & HEM & 305 & -4.963 & 9.025 & 4.247 & 1.00 & 0.00 & C \\
\hline HETATM & 2828 & C13 & HEM & 305 & -6.102 & 8.878 & 5.004 & 1.00 & 0.00 & C \\
\hline HETATM & 2829 & C14 & HEM & 305 & -7.175 & 9.519 & 4.273 & 1.00 & 0.00 & C \\
\hline HETATM & 2830 & C15 & HEM & 305 & -8.503 & 9.574 & 4.694 & 1.00 & 0.00 & C \\
\hline HETATM & 2831 & H15 & HEM & 305 & -8.743 & 9.089 & 5.642 & 1.00 & 0.00 & $\mathrm{H}$ \\
\hline HETATM & 2832 & C16 & HEM & 305 & -9.533 & 10.255 & 4.051 & 1.00 & 0.00 & C \\
\hline HETATM & 2833 & C17 & HEM & 305 & -10.899 & 10.369 & 4.533 & 1.00 & 0.00 & C \\
\hline HETATM & 2834 & $\mathrm{C} 18$ & HEM & 305 & -11.575 & 11.137 & 3.609 & 1.00 & 0.00 & C \\
\hline HETATM & 2835 & C19 & HEM & 305 & -10.625 & 11.489 & 2.573 & 1.00 & 0.00 & C \\
\hline HETATM & 2836 & $\mathrm{C} 20$ & HEM & 305 & -10.916 & 12.280 & 1.462 & 1.00 & 0.00 & $\mathrm{C}$ \\
\hline HETATM & 2837 & $\mathrm{H} 2 \mathrm{O}$ & HEM & 305 & -11.930 & 12.669 & 1.402 & 1.00 & 0.00 & $\mathrm{H}$ \\
\hline HETATM & 2838 & N21 & HEM & 305 & -8.726 & 12.266 & 0.367 & 1.00 & 0.00 & $\mathrm{~N}$ \\
\hline
\end{tabular}



HETATM 2840 N23 HEM HETATM 2841 N24 HEM HETATM 2842 C99 HEM 305 HETATM $2843 \quad 091$ HEM 305 HETATM 2844092 HEM 305 HETATM HETATM 2846 H911 HEM HETATM 2847 H912 HEM HETATM 2848 CT92 HEM HETATM 2849 H921 HEM HETATM 2850 H922 HEM TER

HETATM 2851 EE HEM HETATM 2852 C1 HEM 306 HETATM 2853 C2 HEM 306 HETATM 2854 C3 HEM 306 HETATM 2855 C4 HEM 306 HETATM 2856 C5 HEM 306 HETATM 2857 H5 HEM 306 HETATM 2858 C6 HEM 306 HETATM $2859 \quad$ C7 HEM 306 HETATM 2860 C 8 HEM 306 HETATM $2861 \quad$ C9 HEM 306 HETATM 2862 C10 HEM 306 HETATM 2863 H1O HEM 306 HETATM 2864 C11 HEM 306 HETATM 2865 C12 HEM 306 HETATM 2866 C13 HEM 306 HETATM 2867 C14 HEM 30 HETATM 2868 C15 HEM 306 HETATM 2869 H15 HEM 306 HETATM 2870 C16 HEM 306 HETATM 2871 C17 HEM 306 HETATM 2872 C18 HEM 306 HETATM 2873 C19 HEM 306 HETATM $2874 \quad$ C20 HEM 306 HETATM 2875 H20 HEM 30 HETATM 2876 N21 HEM 306 HETATM 2877 N22 HEM 306 HETATM 2878 N23 HEM 30 HETATM 2879 N24 HEM 306 HETATM 2880 C99 HEM 306 HETATM $2881 \quad 091$ HEM 306 HETATM 2882 O92 HEM 306 HETATM 2883 CT91 HEM 306 HETATM 2884 H911 HEM 306 HETATM 2885 H912 HEM 30 HETATM 2886 CT92 HEM 30 HETATM 2887 H921 HEM 306 HETATM 2888 H922 HEM 306 (T)

HETATM 2889 C2 VIN 307 HETATM 2890 H21 VIN 307 HETATM $2891 \quad$ C1 VIN 307 HETATM 2892 H11 VIN 307 HETATM 2893 H12 VIN 307 TER

HETATM $2894 \quad$ C1 MET 308 HETATM 2895 H11 MET 308 HETATM 2896 H12 MET 308 HETATM 2897 H13 MET 308 (T)

HETATM 2898 C2 VIN 309 HETATM 2899 H21 VIN 309 HETATM 2900 C1 VIN 309 HETATM 2901 H11 VIN 309 HETATM 2902 H12 VIN 309 TER

HETATM 2903 C1 MET 310 HETATM 2904 H11 MET 310 HETATM 2905 H12 MET 310 HETATM 2906 H13 MET 310 TER

HETATM 2907 C5 ACP 31 HETATM 2908 H51 ACP 311 HETATM 2909 H52 ACP 311 HETATM $2910 \quad$ C4 ACP 311 HETATM 2911 H41 ACP 31

\begin{tabular}{|c|c|c|c|c|c|}
\hline-6.021 & 11.319 & 0.590 & 1.00 & 0.00 & $\mathrm{~N}$ \\
\hline-6.692 & 10.056 & 3.090 & 1.00 & 0.00 & $\mathrm{~N}$ \\
\hline-9.392 & 10.941 & 2.854 & 1.00 & 0.00 & $\mathrm{~N}$ \\
\hline-8.485 & 8.076 & 0.352 & 1.00 & 0.00 & C \\
\hline-8.326 & 9.389 & 0.365 & 1.00 & 0.00 & 0 \\
\hline-8.640 & 7.458 & -0.703 & 1.00 & 0.00 & 0 \\
\hline-8.469 & 7.394 & 1.709 & 1.00 & 0.00 & C \\
\hline-7.490 & 7.590 & 2.171 & 1.00 & 0.00 & $\mathrm{H}$ \\
\hline-9.217 & 7.884 & 2.352 & 1.00 & 0.00 & $\mathrm{H}$ \\
\hline-8.729 & 5.890 & 1.597 & 1.00 & 0.00 & C \\
\hline-7.932 & 5.429 & 1.000 & 1.00 & 0.00 & $\mathrm{H}$ \\
\hline-9.654 & 5.734 & 1.027 & 1.00 & 0.00 & $\mathrm{H}$ \\
\hline-7.128 & 3.343 & 6.440 & 1.00 & 0.00 & $\mathrm{Fe}$ \\
\hline-4.900 & 1.471 & 7.502 & 1.00 & 0.00 & C \\
\hline-4.541 & 0.680 & 8.674 & 1.00 & 0.00 & C \\
\hline-5.686 & 0.583 & 9.448 & 1.00 & 0.00 & C \\
\hline-6.716 & 1.301 & 8.740 & 1.00 & 0.00 & C \\
\hline-8.031 & 1.420 & 9.178 & 1.00 & 0.00 & C \\
\hline-8.284 & 0.923 & 10.113 & 1.00 & 0.00 & $\mathrm{H}$ \\
\hline-9.050 & 2.118 & 8.542 & 1.00 & 0.00 & C \\
\hline-10.419 & 2.191 & 9.012 & 1.00 & 0.00 & C \\
\hline-11.125 & 2.910 & 8.065 & 1.00 & 0.00 & C \\
\hline-10.174 & 3.275 & 7.041 & 1.00 & 0.00 & C \\
\hline-10.478 & 4.010 & 5.897 & 1.00 & 0.00 & C \\
\hline-11.505 & 4.354 & 5.791 & 1.00 & 0.00 & $\mathrm{H}$ \\
\hline-9.604 & 4.350 & 4.874 & 1.00 & 0.00 & C \\
\hline-9.985 & 5.076 & 3.684 & 1.00 & 0.00 & C \\
\hline-8.845 & 5.223 & 2.927 & 1.00 & 0.00 & C \\
\hline-7.772 & 4.581 & 3.659 & 1.00 & 0.00 & C \\
\hline-6.444 & 4.527 & 3.237 & 1.00 & 0.00 & C \\
\hline-6.204 & 5.011 & 2.289 & 1.00 & 0.00 & $\mathrm{H}$ \\
\hline-5.414 & 3.845 & 3.880 & 1.00 & 0.00 & $\mathrm{C}$ \\
\hline-4.048 & 3.732 & 3.398 & 1.00 & 0.00 & C \\
\hline-3.373 & 2.964 & 4.322 & 1.00 & 0.00 & C \\
\hline-4.322 & 2.612 & 5.358 & 1.00 & 0.00 & C \\
\hline-4.031 & 1.821 & 6.469 & 1.00 & 0.00 & C \\
\hline-3.017 & 1.432 & 6.530 & 1.00 & 0.00 & $\mathrm{H}$ \\
\hline-6.221 & 1.834 & 7.564 & 1.00 & 0.00 & $\mathrm{~N}$ \\
\hline-8.926 & 2.781 & 7.342 & 1.00 & 0.00 & $\mathrm{~N}$ \\
\hline-8.255 & 4.044 & 4.841 & 1.00 & 0.00 & $\mathrm{~N}$ \\
\hline-5.555 & 3.160 & 5.077 & 1.00 & 0.00 & $\mathrm{~N}$ \\
\hline-6.462 & 6.025 & 7.579 & 1.00 & 0.00 & C \\
\hline-6.622 & 4.711 & 7.567 & 1.00 & 0.00 & 0 \\
\hline-6.307 & 6.642 & 8.635 & 1.00 & 0.00 & 0 \\
\hline-6.478 & 6.706 & 6.223 & 1.00 & 0.00 & C \\
\hline-7.457 & 6.511 & 5.760 & 1.00 & 0.00 & $\mathrm{H}$ \\
\hline-5.730 & 6.216 & 5.580 & 1.00 & 0.00 & $\mathrm{H}$ \\
\hline-6.219 & 8.211 & 6.334 & 1.00 & 0.00 & C \\
\hline-5.294 & 8.367 & 6.905 & 1.00 & 0.00 & $\mathrm{H}$ \\
\hline-7.015 & 8.671 & 6.931 & 1.00 & 0.00 & $\mathrm{H}$ \\
\hline-11.738 & 13.923 & -1.002 & 1.00 & 0.00 & $\mathrm{C}$ \\
\hline-12.554 & 13.224 & -0.837 & 1.00 & 0.00 & $\mathrm{H}$ \\
\hline-12.071 & 15.147 & -1.403 & 1.00 & 0.00 & C \\
\hline-11.340 & 15.926 & -1.511 & 1.00 & 0.00 & $\mathrm{H}$ \\
\hline-13.113 & 15.417 & -1.570 & 1.00 & 0.00 & $\mathrm{H}$ \\
\hline-9.075 & 14.177 & -2.840 & 1.00 & 0.00 & C \\
\hline-8.840 & 15.248 & -2.720 & 1.00 & 0.00 & $\mathrm{H}$ \\
\hline-8.262 & 13.713 & -3.410 & 1.00 & 0.00 & $\mathrm{H}$ \\
\hline-9.982 & 14.100 & -3.450 & 1.00 & 0.00 & $\mathrm{H}$ \\
\hline-4.064 & 12.499 & -2.325 & 1.00 & 0.00 & C \\
\hline-4.730 & 12.399 & -3.184 & 1.00 & 0.00 & $\mathrm{H}$ \\
\hline-2.898 & 13.144 & -2.484 & 1.00 & 0.00 & C \\
\hline-2.218 & 13.300 & -1.654 & 1.00 & 0.00 & $\mathrm{H}$ \\
\hline-2.593 & 13.542 & -3.450 & 1.00 & 0.00 & $\mathrm{H}$ \\
\hline$-2 \cdot 380$ & 10.816 & -0.121 & 1.00 & 0.00 & C \\
\hline-1.789 & 11.480 & 0.528 & 1.00 & 0.00 & $\mathrm{H}$ \\
\hline-2.224 & 9.794 & 0.246 & 1.00 & 0.00 & $\mathrm{H}$ \\
\hline-1.948 & 10.882 & -1.126 & 1.00 & 0.00 & $\mathrm{H}$ \\
\hline-11.468 & 9.735 & 5.770 & 1.00 & 0.00 & C \\
\hline-10.729 & 9.723 & 6.582 & 1.00 & 0.00 & $\mathrm{H}$ \\
\hline-12.309 & 10.343 & 6.134 & 1.00 & 0.00 & $\mathrm{H}$ \\
\hline-11.947 & 8.297 & 5.497 & 1.00 & 0.00 & C \\
\hline-12.505 & 8.236 & 4.549 & 1.00 & 0.00 & $\mathrm{H}$ \\
\hline
\end{tabular}

S168 


\begin{tabular}{|c|c|c|c|c|c|c|c|c|c|c|}
\hline HETATM & 2912 & $\mathrm{H} 42$ & $\mathrm{ACP}$ & 311 & -11.076 & 7.630 & 5.390 & 1.00 & 0.00 & $\mathrm{H}$ \\
\hline HETATM & 2913 & $\mathrm{C} 1$ & $\mathrm{ACP}$ & 311 & -12.811 & 7.685 & 6.573 & 1.00 & 0.00 & C \\
\hline HETATM & 2914 & 02 & $\mathrm{ACP}$ & 311 & -13.039 & 8.208 & 7.671 & 1.00 & 0.00 & 0 \\
\hline HETATM & 2915 & 03 & $\mathrm{ACP}$ & 311 & $-13 \cdot 327$ & 6.505 & 6.209 & 1.00 & 0.00 & O \\
\hline HETATM & 2916 & H3 & $\mathrm{ACP}$ & 311 & -13.936 & 6.151 & 6.945 & 1.00 & 0.00 & $\mathrm{H}$ \\
\hline \multicolumn{11}{|l|}{ TER } \\
\hline HETATM & 2917 & $\mathrm{C} 1$ & $\mathrm{MET}$ & 312 & -3.572 & 8.608 & 4.597 & 1.00 & 0.00 & C \\
\hline HETATM & 2918 & H11 & $\mathrm{MET}$ & 312 & -3.020 & 9.448 & 5.047 & 1.00 & 0.00 & $\mathrm{H}$ \\
\hline HETATM & 2919 & H12 & $\mathrm{MET}$ & 312 & -3.566 & 7.782 & 5.319 & 1.00 & 0.00 & $\mathrm{H}$ \\
\hline HETATM & 2920 & $\mathrm{H} 13$ & $\mathrm{MET}$ & 312 & -3.001 & 8.289 & 3.717 & 1.00 & 0.00 & $\mathrm{H}$ \\
\hline \multicolumn{11}{|l|}{ TER } \\
\hline HETATM & 2921 & $\mathrm{C} 1$ & $\mathrm{MET}$ & 313 & -13.006 & 11.557 & 3.646 & 1.00 & 0.00 & C \\
\hline HETATM & 2922 & H11 & $\mathrm{MET}$ & 313 & -13.451 & 11.348 & 4.626 & 1.00 & 0.00 & $\mathrm{H}$ \\
\hline HETATM & 2923 & $\mathrm{H} 12$ & MET & 313 & -13.116 & 12.637 & 3.471 & 1.00 & 0.00 & $\mathrm{H}$ \\
\hline HETATM & 2924 & $\mathrm{H} 13$ & $\mathrm{MET}$ & 313 & -13.606 & 11.031 & 2.889 & 1.00 & 0.00 & $\mathrm{H}$ \\
\hline \multicolumn{11}{|l|}{ TER } \\
\hline HETATM & 2925 & C2 & VIN & 314 & -3.209 & 0.178 & 8.933 & 1.00 & 0.00 & C \\
\hline HETATM & 2926 & H 21 & VIN & 314 & -2.393 & 0.876 & 8.768 & 1.00 & 0.00 & $\mathrm{H}$ \\
\hline HETATM & 2927 & $\mathrm{C} 1$ & VIN & 314 & -2.876 & -1.047 & 9.335 & 1.00 & 0.00 & C \\
\hline HETATM & 2928 & H11 & VIN & 314 & -3.607 & -1.826 & 9.442 & 1.00 & 0.00 & $\mathrm{H}$ \\
\hline HETATM & 2929 & $\mathrm{H} 12$ & VIN & 314 & -1.834 & -1.316 & 9.501 & 1.00 & 0.00 & $\mathrm{H}$ \\
\hline \multicolumn{11}{|l|}{ TER } \\
\hline HETATM & 2930 & $\mathrm{C} 1$ & $\mathrm{MET}$ & 315 & -5.873 & -0.077 & 10.771 & 1.00 & 0.00 & C \\
\hline HETATM & 2931 & H11 & $\mathrm{MET}$ & 315 & -6.107 & -1.148 & 10.652 & 1.00 & 0.00 & $\mathrm{H}$ \\
\hline HETATM & 2932 & H12 & $\mathrm{MET}$ & 315 & -6.686 & 0.387 & 11.341 & 1.00 & 0.00 & $\mathrm{H}$ \\
\hline HETATM & 2933 & H13 & $\mathrm{MET}$ & 315 & -4.965 & 0.001 & 11.382 & 1.00 & 0.00 & $\mathrm{H}$ \\
\hline TER & & & & & & & & & & \\
\hline HETATM & 2934 & $\mathrm{C} 2$ & VIN & 316 & -10.883 & 1.601 & 10.256 & 1.00 & 0.00 & C \\
\hline HETATM & 2935 & H 21 & VIN & 316 & -10.217 & 1.702 & 11.115 & 1.00 & 0.00 & $\mathrm{H}$ \\
\hline HETATM & 2936 & $\mathrm{C} 1$ & VIN & 316 & -12.050 & 0.957 & 10.415 & 1.00 & 0.00 & C \\
\hline HETATM & 2937 & H11 & VIN & 316 & $-12 \cdot 729$ & 0.800 & 9.585 & 1.00 & 0.00 & $\mathrm{H}$ \\
\hline HETATM & 2938 & $\mathrm{H} 12$ & VIN & 316 & -12.354 & 0.559 & 11.381 & 1.00 & 0.00 & $\mathrm{H}$ \\
\hline TER & & & & & & & & & & \\
\hline HETATM & 2939 & $\mathrm{C} 1$ & $\mathrm{MET}$ & 317 & -12.567 & 3.284 & 8.052 & 1.00 & 0.00 & $\mathrm{C}$ \\
\hline HETATM & 2940 & H11 & $\mathrm{MET}$ & 317 & $-12 \cdot 724$ & 4.306 & 7.685 & 1.00 & 0.00 & $\mathrm{H}$ \\
\hline HETATM & 2941 & $\mathrm{H} 12$ & $\mathrm{MET}$ & 317 & -13.000 & 3.219 & 9.057 & 1.00 & 0.00 & $\mathrm{H}$ \\
\hline HETATM & 2942 & H13 & $\mathrm{MET}$ & 317 & -13.159 & 2.620 & 7.403 & 1.00 & 0.00 & $\mathrm{H}$ \\
\hline TER & & & & & & & & & & \\
\hline HETATM & 2943 & C5 & $\mathrm{ACP}$ & 318 & -3.479 & 4.366 & 2.161 & 1.00 & 0.00 & C \\
\hline HETATM & 2944 & H51 & $\mathrm{ACP}$ & 318 & -4.218 & 4.377 & 1.349 & 1.00 & 0.00 & $\mathrm{H}$ \\
\hline HETATM & 2945 & H52 & $\mathrm{ACP}$ & 318 & -2.638 & 3.758 & 1.798 & 1.00 & 0.00 & $\mathrm{H}$ \\
\hline HETATM & 2946 & C4 & $\mathrm{ACP}$ & 318 & -3.000 & 5.803 & 2.434 & 1.00 & 0.00 & C \\
\hline HETATM & 2947 & $\mathrm{H} 4 \mathrm{I}$ & $\mathrm{ACP}$ & 318 & -2.443 & 5.865 & 3.382 & 1.00 & 0.00 & $\mathrm{H}$ \\
\hline HETATM & 2948 & $\mathrm{H} 42$ & $\mathrm{ACP}$ & 318 & -3.871 & 6.470 & 2.541 & 1.00 & 0.00 & $\mathrm{H}$ \\
\hline HETATM & 2949 & $\mathrm{C} 1$ & $\mathrm{ACP}$ & 318 & -2.136 & 6.416 & 1.358 & 1.00 & 0.00 & C \\
\hline HETATM & 2950 & 02 & $\mathrm{ACP}$ & 318 & -1.909 & 5.893 & 0.260 & 1.00 & 0.00 & O \\
\hline HETATM & 2951 & 03 & $\mathrm{ACP}$ & 318 & -1.621 & 7.595 & 1.722 & 1.00 & 0.00 & 0 \\
\hline HETATM & 2952 & H3 & $\mathrm{ACP}$ & 318 & -1.012 & 7.949 & 0.986 & 1.00 & 0.00 & $\mathrm{H}$ \\
\hline TER & & & & & & & & & & \\
\hline HETATM & 2953 & $\mathrm{C} 1$ & $\mathrm{MET}$ & 319 & -11.375 & 5.492 & 3.334 & 1.00 & 0.00 & C \\
\hline HETATM & 2954 & H11 & $\mathrm{MET}$ & 319 & -11.927 & 4.653 & 2.884 & 1.00 & 0.00 & $\mathrm{H}$ \\
\hline HETATM & 2955 & $\mathrm{H} 12$ & MET & 319 & $-11 \cdot 382$ & 6.318 & 2.613 & 1.00 & 0.00 & $\mathrm{H}$ \\
\hline HETATM & 2956 & H13 & $\mathrm{MET}$ & 319 & -11.946 & 5.812 & 4.214 & 1.00 & 0.00 & $\mathrm{H}$ \\
\hline TER & & & & & & & & & & \\
\hline HETATM & 2957 & $\mathrm{C} 1$ & $\mathrm{MET}$ & 320 & -1.942 & 2.543 & 4.285 & 1.00 & 0.00 & C \\
\hline HETATM & 2958 & H11 & $\mathrm{MET}$ & 320 & -1.496 & 2.752 & 3.305 & 1.00 & 0.00 & $\mathrm{H}$ \\
\hline HETATM & 2959 & H12 & $\mathrm{MET}$ & 320 & -1.831 & 1.463 & 4.460 & 1.00 & 0.00 & $\mathrm{H}$ \\
\hline HETATM & 2960 & H13 & $\mathrm{MET}$ & 320 & -1.341 & 3.069 & 5.042 & 1.00 & 0.00 & $\mathrm{H}$ \\
\hline TER & & & & & & & & & & \\
\hline HETATM & 2961 & $\mathrm{FE}$ & HEM & 321 & -8.767 & 10.511 & 9.423 & 1.00 & 0.00 & $\mathrm{Fe}$ \\
\hline HETATM & 2962 & $\mathrm{C} 1$ & HEM & 321 & -10.995 & 12.383 & 8.361 & 1.00 & 0.00 & C \\
\hline HETATM & 2963 & $\mathrm{C} 2$ & HEM & 321 & -11.354 & 13.174 & 7.189 & 1.00 & 0.00 & $\mathrm{C}$ \\
\hline HETATM & 2964 & C3 & HEM & 321 & -10.209 & 13.271 & 6.414 & 1.00 & 0.00 & C \\
\hline HETATM & 2965 & $\mathrm{C} 4$ & HEM & 321 & -9.179 & 12.553 & 7.122 & 1.00 & 0.00 & C \\
\hline HETATM & 2966 & C5 & HEM & 321 & -7.864 & 12.435 & 6.684 & 1.00 & 0.00 & C \\
\hline HETATM & 2967 & H5 & HEM & 321 & -7.610 & 12.931 & 5.749 & 1.00 & 0.00 & $\mathrm{H}$ \\
\hline HETATM & 2968 & C6 & HEM & 321 & -6.845 & 11.736 & 7.320 & 1.00 & 0.00 & C \\
\hline HETATM & 2969 & $\mathrm{C} 7$ & HEM & 321 & -5.476 & 11.664 & 6.851 & 1.00 & 0.00 & C \\
\hline HETATM & 2970 & $\mathrm{C} 8$ & HEM & 321 & -4.770 & 10.944 & 7.798 & 1.00 & 0.00 & C \\
\hline HETATM & 2971 & C9 & HEM & 321 & -5.721 & 10.579 & 8.822 & 1.00 & 0.00 & C \\
\hline HETATM & 2972 & C10 & HEM & 321 & -5.417 & 9.844 & 9.965 & 1.00 & 0.00 & C \\
\hline HETATM & 2973 & $\mathrm{H} 1 \mathrm{O}$ & HEM & 321 & -4.390 & 9.500 & 10.071 & 1.00 & 0.00 & $\mathrm{H}$ \\
\hline HETATM & 2974 & $\mathrm{C} 11$ & HEM & 321 & -6.291 & 9.504 & 10.988 & 1.00 & 0.00 & C \\
\hline HETATM & 2975 & $\mathrm{C} 12$ & HEM & 321 & -5.910 & 8.779 & 12.178 & 1.00 & 0.00 & C \\
\hline HETATM & 2976 & $\mathrm{C} 13$ & HEM & 321 & -7.050 & 8.631 & 12.935 & 1.00 & 0.00 & C \\
\hline HETATM & 2977 & C14 & HEM & 321 & -8.123 & 9.273 & 12.204 & 1.00 & 0.00 & C \\
\hline HETATM & 2978 & C15 & HEM & 321 & -9.451 & 9.328 & 12.625 & 1.00 & 0.00 & C \\
\hline HETATM & 2979 & H15 & HEM & 321 & -9.691 & 8.843 & 13.573 & 1.00 & 0.00 & $\mathrm{H}$ \\
\hline HETATM & 2980 & C16 & HEM & 321 & -10.481 & 10.009 & 11.982 & 1.00 & 0.00 & C \\
\hline
\end{tabular}


HETATM 2981 C17 HEM 321 HETATM 2982 C18 HEM 321 HETATM 2983 C19 HEM HETATM 2984 C20 HEM 321 HETATM 2985 H20 HEM 321 HETATM 2986 N21 HEM 321 HETATM 2987 N22 HEM 321 HETATM 2988 N23 HEM 32 HETATM 2989 N24 HEM 321 HETATM 2990 C99 HEM 321 HETATM 2991091 HEM 321 HETATM 2992092 HEM 32 HETATM 2993 CT91 HEM 32 HETATM 2994 H911 HEM 321 HETATM 2995 H912 HEM 32 HETATM 2996 CT92 HEM 321 HETATM 2997 H921 HEM 321 HETATM 2998 H922 HEM 321 TER

HETATM 2999 FE HEM 322 HETATM 3000 C1 HEM 322 HETATM 3001 C2 HEM 322 HETATM 3002 C3 HEM 322 HETATM $3003 \quad$ C4 HEM 322 HETATM 3004 C5 HEM 322 HETATM 3005 H5 HEM 322 HETATM 3006 C6 HEM 322 HETATM $3007 \quad$ C7 HEM 322 HETATM 3008 C8 HEM 322 HETATM 3009 C9 HEM 32 HETATM 3010 C10 HEM 322 HETATM 3011 H1O HEM 322 HETATM 3012 C11 HEM 32 HETATM 3013 C12 HEM 322 HETATM 3014 C13 HEM 322 HETATM 3015 C14 HEM 322 HETATM 3016 C15 HEM 32 HETATM 3017 H15 HEM 322 HETATM 3018 C16 HEM 322 HETATM 3019 C17 HEM 32 HETATM 3020 C18 HEM 322 HETATM 3021 C19 HEM 322 HETATM 3022 C20 HEM 322 HETATM 3023 H2O HEM 32 HETATM 3024 N21 HEM 32 HETATM 3025 N22 HEM 322 HETATM 3026 N23 HEM 322 HETATM 3027 N24 HEM 32 HETATM 3028 C99 HEM 32 HETATM 3029091 HEM 322 HETATM $3030 \quad 092$ HEM 322 HETATM 3031 CT91 HEM 322 HETATM 3032 H911 HEM 322 HETATM 3033 H912 HEM 322 HETATM 3034 CT92 HEM 322 HETATM 3035 H921 HEM 322 HETATM 3036 H922 HEM 322 TER

HETATM 3037 C2 VIN 323 HETATM 3038 H21 VIN 32 HETATM 3039 C1 VIN 323 HETATM 3040 H11 VIN 323 HETATM 3041 H12 VIN 32 TER

HETATM $3042 \quad$ C1 MET 324 HETATM 3043 H11 MET 324 HETATM 3044 H12 MET 32 HETATM 3045 H13 MET 32 TER

HETATM 3046 C2 VIN 325 HETATM 3047 H21 VIN 325 HETATM 3048 C1 VIN 325 HETATM 3049 H11 VIN 325 HETATM 3050 H12 VIN 32 TER

HETATM $3051 \quad$ C1 MET 326 HETATM 3052 H11 MET 326 HETATM 3053 H12 MET 326 HETATM 3054 H13 MET 326

\begin{tabular}{|c|c|c|c|c|c|}
\hline-11.847 & 10.123 & 12.464 & 1.00 & 0.00 & C \\
\hline-12.522 & 10.890 & 11.541 & 1.00 & 0.00 & C \\
\hline-11.573 & 11.242 & 10.504 & 1.00 & 0.00 & C \\
\hline-11.864 & 12.033 & 9.393 & 1.00 & 0.00 & C \\
\hline-12.878 & 12.423 & 9.333 & 1.00 & 0.00 & $\mathrm{H}$ \\
\hline-9.674 & 12.020 & 8.298 & 1.00 & 0.00 & $\mathrm{~N}$ \\
\hline-6.969 & 11.073 & 8.521 & 1.00 & 0.00 & $\mathrm{~N}$ \\
\hline-7.640 & 9.810 & 11.021 & 1.00 & 0.00 & $\mathrm{~N}$ \\
\hline-10.339 & 10.694 & 10.786 & 1.00 & 0.00 & $\mathrm{~N}$ \\
\hline-9.433 & 7.829 & 8.283 & 1.00 & 0.00 & C \\
\hline-9.273 & 9.143 & 8.296 & 1.00 & 0.00 & o \\
\hline-9.588 & 7.212 & 7.228 & 1.00 & 0.00 & o \\
\hline-9.417 & 7.148 & 9.640 & 1.00 & 0.00 & $\mathrm{C}$ \\
\hline-8.438 & 7.343 & 10.103 & 1.00 & 0.00 & $\mathrm{H}$ \\
\hline-10.165 & 7.638 & 10.283 & 1.00 & 0.00 & $\mathrm{H}$ \\
\hline-9.676 & 5.644 & 9.528 & 1.00 & 0.00 & C \\
\hline-8.880 & 5.183 & 8.931 & 1.00 & 0.00 & $\mathrm{H}$ \\
\hline-10.601 & 5.488 & 8.958 & 1.00 & 0.00 & $\mathrm{H}$ \\
\hline-8.075 & 3.097 & 14.371 & 1.00 & 0.00 & $\mathrm{Fe}$ \\
\hline-5.847 & 1.225 & 15.433 & 1.00 & 0.00 & C \\
\hline-5.488 & 0.434 & 16.605 & 1.00 & 0.00 & C \\
\hline-6.634 & 0.337 & 17.380 & 1.00 & 0.00 & C \\
\hline-7.663 & 1.055 & 16.672 & 1.00 & 0.00 & C \\
\hline-8.979 & 1.173 & 17.109 & 1.00 & 0.00 & C \\
\hline-9.232 & 0.677 & 18.045 & 1.00 & 0.00 & $\mathrm{H}$ \\
\hline-9.998 & 1.872 & 16.473 & 1.00 & 0.00 & C \\
\hline-11.367 & 1.944 & 16.943 & 1.00 & 0.00 & C \\
\hline-12.072 & 2.664 & 15.996 & 1.00 & 0.00 & C \\
\hline-11.121 & 3.029 & 14.972 & 1.00 & 0.00 & C \\
\hline-11.426 & 3.764 & 13.828 & 1.00 & 0.00 & C \\
\hline-12.453 & 4.108 & 13.722 & 1.00 & 0.00 & $\mathrm{H}$ \\
\hline-10.551 & 4.104 & 12.806 & 1.00 & 0.00 & $\mathrm{C}$ \\
\hline-10.932 & 4.829 & 11.615 & 1.00 & 0.00 & C \\
\hline-9.793 & 4.977 & 10.858 & 1.00 & 0.00 & C \\
\hline-8.720 & 4.335 & 11.590 & 1.00 & 0.00 & C \\
\hline-7.392 & 4.280 & 11.168 & 1.00 & 0.00 & C \\
\hline-7.152 & 4.765 & 10.221 & 1.00 & 0.00 & $\mathrm{H}$ \\
\hline-6.362 & 3.599 & 11.812 & 1.00 & 0.00 & C \\
\hline-4.996 & 3.485 & 11.329 & 1.00 & 0.00 & C \\
\hline-4.320 & 2.718 & 12.253 & 1.00 & 0.00 & C \\
\hline-5.270 & 2.366 & 13.289 & 1.00 & 0.00 & C \\
\hline-4.979 & 1.575 & 14.401 & 1.00 & 0.00 & C \\
\hline-3.964 & 1.185 & 14.461 & 1.00 & 0.00 & $\mathrm{H}$ \\
\hline-7.169 & 1.588 & 15.495 & 1.00 & 0.00 & $\mathrm{~N}$ \\
\hline-9.874 & 2.535 & 15.273 & 1.00 & 0.00 & $\mathrm{~N}$ \\
\hline-9.203 & 3.798 & 12.772 & 1.00 & 0.00 & $\mathrm{~N}$ \\
\hline-6.503 & 2.914 & 13.008 & 1.00 & 0.00 & $\mathrm{~N}$ \\
\hline-7.410 & 5.779 & 15.510 & 1.00 & 0.00 & C \\
\hline-7.569 & 4.465 & 15.498 & 1.00 & 0.00 & o \\
\hline-7.255 & 6.396 & 16.566 & 1.00 & 0.00 & o \\
\hline-7.426 & 6.460 & 14.154 & 1.00 & 0.00 & C \\
\hline-8.405 & 6.265 & 13.691 & 1.00 & 0.00 & $\mathrm{H}$ \\
\hline-6.678 & 5.970 & 13.511 & 1.00 & 0.00 & $\mathrm{H}$ \\
\hline-7.166 & 7.964 & 14.265 & 1.00 & 0.00 & C \\
\hline-6.241 & 8.120 & 14.836 & 1.00 & 0.00 & $\mathrm{H}$ \\
\hline-7.963 & 8.425 & 14.862 & 1.00 & 0.00 & $\mathrm{H}$ \\
\hline-12.686 & 13.676 & 6.929 & 1.00 & 0.00 & C \\
\hline-13.502 & 12.978 & 7.094 & 1.00 & 0.00 & $\mathrm{H}$ \\
\hline-13.019 & 14.901 & 6.528 & 1.00 & 0.00 & C \\
\hline-12.287 & 15.680 & 6.421 & 1.00 & 0.00 & $\mathrm{H}$ \\
\hline-14.061 & 15.170 & 6.361 & 1.00 & 0.00 & $\mathrm{H}$ \\
\hline-10.022 & 13.931 & 5.091 & 1.00 & 0.00 & C \\
\hline-9.787 & 15.002 & 5.211 & 1.00 & 0.00 & $\mathrm{H}$ \\
\hline-9.209 & 13.467 & 4.522 & 1.00 & 0.00 & $\mathrm{H}$ \\
\hline-10.930 & 13.854 & 4.481 & 1.00 & 0.00 & $\mathrm{H}$ \\
\hline-5.012 & 12.253 & 5.606 & 1.00 & 0.00 & C \\
\hline-5.678 & 12.152 & 4.747 & 1.00 & 0.00 & $\mathrm{H}$ \\
\hline-3.845 & 12.898 & 5.447 & 1.00 & 0.00 & $\mathrm{C}$ \\
\hline-3.165 & 13.054 & 6.277 & 1.00 & 0.00 & $\mathrm{H}$ \\
\hline-3.540 & 13.296 & 4.482 & 1.00 & 0.00 & $\mathrm{H}$ \\
\hline-3.328 & 10.570 & 7.810 & 1.00 & 0.00 & C \\
\hline-2.736 & 11.234 & 8.459 & 1.00 & 0.00 & $\mathrm{H}$ \\
\hline-3.171 & 9.548 & 8.177 & 1.00 & 0.00 & $\mathrm{H}$ \\
\hline-2.895 & 10.636 & 6.805 & 1.00 & 0.00 & $\mathrm{H}$ \\
\hline
\end{tabular}


TER

HETATM 3055 C5 ACP 327 HETATM 30 HETATM 3057 H52 ACP 327 HETATM 3058 C4 ACP 327 HETATM 3059 H41 ACP 327 HETATM 3060 H42 ACP 327 HETATM 3061 C1 ACP 327 HETATM $3062 \quad 02$ ACP 327 HETATM $3063 \quad 03$ ACP 327 HETATM 3064 H3 ACP 327

TER

HETATM 3065 C1 MET 328

$\begin{array}{llll} & \end{array}$

HETATM 3067 H12 MET 328

HETATM 3068 H13 MET 328

HETATM 3069 C1 MET 329

HETATM 3070 H11 MET 329

HETATM 3071 H12 MET 329

HETATM 3072 H13 MET 329

TER

HETATM 3073 C2 VIN 330

HETATM 3074 H21 VIN 330

HETATM 3075 C1 VIN 330

HETATM 3076 H11 VIN 330

HETATM 3077 H12 VIN 330

HETATM 3078 C1 MET 331

HETATM 3079 H11 MET 33

HETATM 3080 H12 MET 331

HETATM 3081 H13 MET 331

TER

HETATM $3082 \quad$ C2 VIN 332

HETATM 3083 H21 VIN 332

HETATM $3084 \quad$ C1 VIN 332

HETATM 3085 H11 VIN 332

HETATM 3086 H12 VIN 332

TER

HETATM 3087 C1 MET 333

HETATM 3088 H11 MET 333

HETATM 3089 H12 MET 333

HETATM 3090 H13 MET 333

TER

HETATM $3091 \quad$ C5 ACP 334

HETATM 3092 H51 ACP 334

HETATM 3093 H52 ACP 334

HETATM 3094 C4 ACP 334

HETATM 3095 H41 ACP 334

$\begin{array}{llll} & & & \end{array}$

HETATM 3097 C1 ACP 334

HETATM $3098 \quad 02$ ACP 33

HETATM $3099 \quad 03$ ACP 334

HETATM 3100 H3 ACP 334

TER

HETATM 3101 C1 MET 335

HETATM 3102 H11 MET 335

HETATM 3103 H12 MET 335

HETATM 3104 H13 MET 335

TER

HETATM 3105 C1 MET 336

HETATM 3106 H11 MET 336

HETATM 3107 H12 MET 336

HETATM 3108 H13 MET 336

HETATM 3109 FE HEM 337

HETATM 3110 C1 HEM 337

HETATM 3111 C2 HEM 337

HETATM 3112 C3 HEM 337

HETATM 3113 C4 HEM 337

HETATM 3114 C5 HEM 337

HETATM 3115 H5 HEM 337

HETATM 3116 C6 HEM 337

HETATM 3117 C7 HEM 337

HETATM 3118 C8 HEM 337

HETATM 3119 C9 HEM 337

HETATM $3120 \quad$ C10 HEM 337

HETATM 3121 H1O HEM 337

HETATM 3122 C11 HEM 337

\begin{tabular}{|c|c|c|c|c|c|}
\hline-12.416 & 9.488 & 13.701 & 1.00 & 0.00 & C \\
\hline-11.677 & 9.477 & 14.514 & 1.00 & 0.00 & $\mathrm{H}$ \\
\hline-13.257 & 10.097 & 14.065 & 1.00 & 0.00 & $\mathrm{H}$ \\
\hline-12.894 & 8.051 & 13.428 & 1.00 & 0.00 & C \\
\hline-13.452 & 7.990 & 12.481 & 1.00 & 0.00 & $\mathrm{H}$ \\
\hline-12.024 & 7.384 & 13.321 & 1.00 & 0.00 & $\mathrm{H}$ \\
\hline-13.758 & 7.439 & 14.504 & 1.00 & 0.00 & C \\
\hline-13.986 & 7.961 & 15.603 & 1.00 & 0.00 & o \\
\hline-14.274 & 6.259 & 14.140 & 1.00 & 0.00 & o \\
\hline-14.883 & 5.905 & 14.876 & 1.00 & 0.00 & 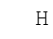 \\
\hline-4.520 & 8.362 & 12.528 & 1.00 & 0.00 & C \\
\hline-3.967 & 9.201 & 12.978 & 1.00 & 0.00 & 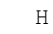 \\
\hline-4.513 & 7.536 & 13.250 & 1.00 & 0.00 & 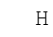 \\
\hline-3.949 & 8.042 & 11.649 & 1.00 & 0.00 & $H$ \\
\hline-13.953 & 11.311 & 11.577 & 1.00 & 0.00 & C \\
\hline-14.398 & 11.102 & 12.557 & 1.00 & 0.00 & $\mathrm{H}$ \\
\hline-14.064 & 12.391 & 11.402 & 1.00 & 0.00 & $\mathrm{H}$ \\
\hline-14.554 & 10.785 & 10.821 & 1.00 & 0.00 & $\mathrm{H}$ \\
\hline-4.157 & -0.068 & 16.864 & 1.00 & 0.00 & 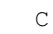 \\
\hline-3.341 & 0.630 & 16.699 & 1.00 & 0.00 & $\mathrm{H}$ \\
\hline-3.824 & -1.293 & 17.266 & 1.00 & 0.00 & C \\
\hline-4.555 & -2.072 & 17.373 & 1.00 & 0.00 & $\mathrm{H}$ \\
\hline-2.782 & -1.562 & 17.432 & 1.00 & 0.00 & $\mathrm{H}$ \\
\hline-6.820 & -0.323 & 18.702 & 1.00 & 0.00 & C \\
\hline-7.055 & -1.394 & 18.583 & 1.00 & 0.00 & $\mathrm{H}$ \\
\hline-7.633 & 0.141 & 19.272 & 1.00 & 0.00 & $\mathrm{H}$ \\
\hline-5.913 & -0.246 & 19.313 & 1.00 & 0.00 & $\mathrm{H}$ \\
\hline-11.830 & 1.355 & 18.188 & 1.00 & 0.00 & C \\
\hline-11.164 & 1.456 & 19.046 & 1.00 & 0.00 & $\mathrm{H}$ \\
\hline-12.997 & 0.710 & 18.346 & 1.00 & 0.00 & C \\
\hline-13.677 & 0.554 & 17.516 & 1.00 & 0.00 & $\mathrm{H}$ \\
\hline$-13 \cdot 302$ & 0.312 & 19.312 & 1.00 & 0.00 & $\mathrm{H}$ \\
\hline-13.515 & 3.038 & 15.984 & 1.00 & 0.00 & C \\
\hline-13.671 & 4.060 & 15.617 & 1.00 & 0.00 & $\mathrm{H}$ \\
\hline-13.947 & 2.972 & 16.989 & 1.00 & 0.00 & $\mathrm{H}$ \\
\hline-14.106 & 2.374 & 15.335 & 1.00 & 0.00 & $\mathrm{H}$ \\
\hline-4.427 & 4.120 & 10.092 & 1.00 & 0.00 & C \\
\hline-5.166 & 4.131 & 9.280 & 1.00 & 0.00 & $\mathrm{H}$ \\
\hline-3.586 & 3.511 & 9.729 & 1.00 & 0.00 & $\mathrm{H}$ \\
\hline-3.948 & 5.557 & 10.365 & 1.00 & 0.00 & C \\
\hline-3.390 & 5.618 & 11.313 & 1.00 & 0.00 & $\mathrm{H}$ \\
\hline-4.819 & 6.224 & 10.472 & 1.00 & 0.00 & $\mathrm{H}$ \\
\hline-3.084 & 6.169 & 9.289 & 1.00 & 0.00 & C \\
\hline-2.856 & 5.647 & 8.191 & 1.00 & 0.00 & 0 \\
\hline-2.568 & 7.349 & 9.653 & 1.00 & 0.00 & 0 \\
\hline-1.959 & 7.703 & 8.917 & 1.00 & 0.00 & $\mathrm{H}$ \\
\hline$-12 \cdot 323$ & 5.246 & 11.266 & 1.00 & 0.00 & C \\
\hline-12.875 & 4.407 & 10.815 & 1.00 & 0.00 & $\mathrm{H}$ \\
\hline-12.329 & 6.072 & 10.544 & 1.00 & 0.00 & $\mathrm{H}$ \\
\hline-12.894 & 5.565 & 12.145 & 1.00 & 0.00 & $\mathrm{H}$ \\
\hline-2.889 & 2.297 & 12.216 & 1.00 & 0.00 & C \\
\hline-2.444 & 2.506 & 11.236 & 1.00 & 0.00 & $\mathrm{H}$ \\
\hline-2.779 & 1.217 & 12.391 & 1.00 & 0.00 & $\mathrm{H}$ \\
\hline-2.289 & 2.823 & 12.973 & 1.00 & 0.00 & $\mathrm{H}$ \\
\hline-6.851 & -3.836 & 9.423 & 1.00 & 0.00 & $\mathrm{Fe}$ \\
\hline-9.078 & -1.963 & 8.361 & 1.00 & 0.00 & $\mathrm{C}$ \\
\hline-9.437 & -1.173 & 7.189 & 1.00 & 0.00 & C \\
\hline-8.292 & -1.076 & 6.414 & 1.00 & 0.00 & C \\
\hline-7.262 & -1.793 & 7.122 & 1.00 & 0.00 & C \\
\hline-5.947 & -1.912 & 6.684 & 1.00 & 0.00 & C \\
\hline-5.694 & -1.416 & 5.749 & 1.00 & 0.00 & $\mathrm{H}$ \\
\hline-4.928 & -2.610 & 7.320 & 1.00 & 0.00 & C \\
\hline-3.559 & -2.683 & 6.851 & 1.00 & 0.00 & C \\
\hline-2.854 & -3.403 & 7.798 & 1.00 & 0.00 & C \\
\hline-3.805 & -3.768 & 8.822 & 1.00 & 0.00 & C \\
\hline-3.500 & -4.503 & 9.965 & 1.00 & 0.00 & C \\
\hline-2.473 & -4.846 & 10.071 & 1.00 & 0.00 & $\mathrm{H}$ \\
\hline-4.374 & -4.843 & 10.988 & 1.00 & 0.00 & 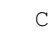 \\
\hline
\end{tabular}


HETATM $3123 \quad$ C12 HEM 337 HETATM 3124 C13 HEM 337 HETATM 3125 C14 HEM HETATM 3126 C15 HEM 33 HETATM 3127 H15 HEM 337 HETATM 3128 C16 HEM 337 HETATM 3129 C17 HEM 337 HETATM $3130 \quad$ C18 HEM 337 HETATM 3131 C19 HEM 337 HETATM 3132 C20 HEM 337 HETATM 3133 H20 HEM 337 HETATM 3134 N21 HEM 337 HETATM 3135 N22 HEM 337 HETATM 3136 N23 HEM 337 HETATM $3137 \quad$ N24 HEM 337 HETATM 3138 C99 HEM 337 HETATM 3139091 HEM 337 HETATM $3140 \quad 092$ HEM 337 HETATM 3141 CT91 HEM 337 HETATM 3142 H911 HEM 337 HETATM 3143 H912 HEM 337 HETATM 3144 CT92 HEM 337 HETATM 3145 H921 HEM 337 HETATM 3146 H922 HEM 337 TER

HETATM 3147 FE HEM 338 HETATM 3148 C1 HEM 338 HETATM 3149 C2 HEM 338 HETATM 3150 C3 HEM 338 HETATM 3151 C4 HEM 338 HETATM $3152 \quad$ C5 HEM 338 HETATM 3153 H5 HEM 338 HETATM 3154 C6 HEM 338 HETATM 3155 C7 HEM 338 HETATM 3156 C8 HEM 338 HETATM 3157 C9 HEM 338 HETATM 3158 C10 HEM 338 HETATM 3159 H1O HEM 338 HETATM 3160 C11 HEM 338 HETATM 3161 C12 HEM 338 HETATM 3162 C13 HEM 33 HETATM 3163 C14 HEM 338 HETATM 3164 C15 HEM 338 HETATM 3165 H15 HEM 338 HETATM 3166 C16 HEM 338 HETATM $3167 \quad$ C17 HEM 338 HETATM 3168 C18 HEM 338 HETATM 3169 C19 HEM 338 HETATM 3170 C20 HEM 338 HETATM 3171 H2O HEM 338 HETATM 3172 N21 HEM 338 HETATM 3173 N22 HEM 33 HETATM 3174 N23 HEM 338 HETATM 3175 N24 HEM 338 HETATM 3176 C99 HEM 338 HETATM 3177091 HEM 338 HETATM 3178092 HEM 338 HETATM 3179 CT91 HEM 338 HETATM 3180 H911 HEM 338 HETATM 3181 H912 HEM 33 HETATM 3182 CT92 HEM 338 HETATM 3183 H921 HEM 338 HETATM 3184 H922 HEM 338 TER

HETATM 3185 C2 VIN 339 HETATM 3186 H21 VIN 339 HETATM 3187 C1 VIN 339 HETATM 3188 H11 VIN 339 HETATM 3189 H12 VIN 339

HETATM $3190 \quad$ C1 MET 340 HETATM 3191 H11 MET 340 HETATM 3192 H12 MET 340 HETATM 3193 H13 MET 340 TER

HETATM 3194 C2 VIN 341 HETATM 3195 H21 VIN 341 HETATM 3196 C1 VIN 341 HETATM 3197 H11 VIN 341

\begin{tabular}{|c|c|c|c|c|c|}
\hline-3.994 & -5.568 & 12.178 & 1.00 & 0.00 & C \\
\hline-5.133 & -5.715 & 12.935 & 1.00 & 0.00 & C \\
\hline-6.206 & -5.074 & 12.204 & 1.00 & 0.00 & C \\
\hline-7.534 & -5.019 & 12.625 & 1.00 & 0.00 & C \\
\hline-7.774 & -5.504 & 13.573 & 1.00 & 0.00 & $\mathrm{H}$ \\
\hline-8.564 & -4.337 & 11.982 & 1.00 & 0.00 & C \\
\hline-9.930 & -4.224 & 12.464 & 1.00 & 0.00 & C \\
\hline-10.605 & -3.456 & 11.541 & 1.00 & 0.00 & C \\
\hline-9.656 & -3.104 & 10.504 & 1.00 & 0.00 & C \\
\hline-9.947 & -2.313 & 9.393 & 1.00 & 0.00 & C \\
\hline-10.961 & -1.924 & 9.333 & 1.00 & 0.00 & $\mathrm{H}$ \\
\hline-7.757 & -2.326 & 8.298 & 1.00 & 0.00 & $\mathrm{~N}$ \\
\hline-5.052 & -3.273 & 8.521 & 1.00 & 0.00 & $\mathrm{~N}$ \\
\hline-5.723 & -4.536 & 11.021 & 1.00 & 0.00 & $\mathrm{~N}$ \\
\hline-8.423 & -3.652 & 10.786 & 1.00 & 0.00 & $\mathrm{~N}$ \\
\hline-7.516 & -6.517 & 8.283 & 1.00 & 0.00 & C \\
\hline-7.356 & -5.204 & 8.296 & 1.00 & 0.00 & o \\
\hline-7.671 & -7.134 & 7.228 & 1.00 & 0.00 & o \\
\hline-7.500 & -7.199 & 9.640 & 1.00 & 0.00 & C \\
\hline-6.521 & -7.003 & 10.103 & 1.00 & 0.00 & $\mathrm{H}$ \\
\hline-8.248 & -6.709 & 10.283 & 1.00 & 0.00 & $\mathrm{H}$ \\
\hline-7.759 & -8.703 & 9.528 & 1.00 & 0.00 & C \\
\hline-6.963 & -9.163 & 8.931 & 1.00 & 0.00 & $\mathrm{H}$ \\
\hline-8.684 & -8.859 & 8.958 & 1.00 & 0.00 & $\mathrm{H}$ \\
\hline-6.158 & -11.250 & 14.371 & 1.00 & 0.00 & $\mathrm{Fe}$ \\
\hline-3.930 & $-13 \cdot 122$ & 15.433 & 1.00 & 0.00 & C \\
\hline-3.572 & -13.912 & 16.605 & 1.00 & 0.00 & C \\
\hline-4.717 & -14.009 & 17.380 & 1.00 & 0.00 & C \\
\hline-5.747 & -13.292 & 16.672 & 1.00 & 0.00 & C \\
\hline-7.062 & -13.173 & 17.109 & 1.00 & 0.00 & C \\
\hline-7.315 & -13.670 & 18.045 & 1.00 & 0.00 & $\mathrm{H}$ \\
\hline-8.081 & -12.475 & 16.473 & 1.00 & 0.00 & C \\
\hline-9.450 & -12.402 & 16.943 & 1.00 & 0.00 & C \\
\hline-10.155 & -11.682 & 15.996 & 1.00 & 0.00 & C \\
\hline-9.204 & -11.317 & 14.972 & 1.00 & 0.00 & C \\
\hline-9.509 & -10.583 & 13.828 & 1.00 & 0.00 & C \\
\hline-10.536 & -10.239 & 13.722 & 1.00 & 0.00 & $\mathrm{H}$ \\
\hline-8.634 & -10.243 & 12.806 & 1.00 & 0.00 & C \\
\hline-9.015 & -9.517 & 11.615 & 1.00 & 0.00 & C \\
\hline-7.876 & -9.370 & 10.858 & 1.00 & 0.00 & C \\
\hline-6.803 & -10.011 & 11.590 & 1.00 & 0.00 & C \\
\hline-5.475 & -10.066 & 11.168 & 1.00 & 0.00 & C \\
\hline-5.235 & -9.581 & 10.221 & 1.00 & 0.00 & $\mathrm{H}$ \\
\hline-4.445 & -10.748 & 11.812 & 1.00 & 0.00 & C \\
\hline-3.079 & -10.861 & 11.329 & 1.00 & 0.00 & C \\
\hline-2.404 & -11.629 & 12.253 & 1.00 & 0.00 & C \\
\hline-3.353 & -11.981 & 13.289 & 1.00 & 0.00 & C \\
\hline-3.062 & -12.772 & 14.401 & 1.00 & 0.00 & C \\
\hline-2.048 & -13.161 & 14.461 & 1.00 & 0.00 & $\mathrm{H}$ \\
\hline-5.252 & -12.759 & 15.495 & 1.00 & 0.00 & $\mathrm{~N}$ \\
\hline-7.957 & -11.812 & 15.273 & 1.00 & 0.00 & $\mathrm{~N}$ \\
\hline-7.286 & -10.549 & 12.772 & 1.00 & 0.00 & $\mathrm{~N}$ \\
\hline-4.586 & -11.433 & 13.008 & 1.00 & 0.00 & $\mathrm{~N}$ \\
\hline-5.493 & -8.568 & 15.510 & 1.00 & 0.00 & C \\
\hline-5.653 & -9.881 & 15.498 & 1.00 & 0.00 & 0 \\
\hline-5.338 & -7.951 & 16.566 & 1.00 & 0.00 & 0 \\
\hline-5.509 & -7.887 & 14.154 & 1.00 & 0.00 & C \\
\hline-6.488 & -8.082 & 13.691 & 1.00 & 0.00 & $\mathrm{H}$ \\
\hline-4.761 & -8.376 & 13.511 & 1.00 & 0.00 & $\mathrm{H}$ \\
\hline-5.249 & -6.382 & 14.265 & 1.00 & 0.00 & C \\
\hline-4.325 & -6.226 & 14.836 & 1.00 & 0.00 & $\mathrm{H}$ \\
\hline-6.046 & -5.922 & 14.862 & 1.00 & 0.00 & $\mathrm{H}$ \\
\hline-10.769 & -0.670 & 6.929 & 1.00 & 0.00 & C \\
\hline-11.585 & -1.369 & 7.094 & 1.00 & 0.00 & $\mathrm{H}$ \\
\hline-11.102 & 0.555 & 6.528 & 1.00 & 0.00 & C \\
\hline-10.371 & 1.333 & 6.421 & 1.00 & 0.00 & $\mathrm{H}$ \\
\hline-12.144 & 0.824 & 6.361 & 1.00 & 0.00 & $\mathrm{H}$ \\
\hline-8.105 & -0.415 & 5.091 & 1.00 & 0.00 & C \\
\hline-7.871 & 0.655 & 5.211 & 1.00 & 0.00 & $\mathrm{H}$ \\
\hline-7.292 & -0.880 & 4.522 & 1.00 & 0.00 & $\mathrm{H}$ \\
\hline-9.013 & -0.493 & 4.481 & 1.00 & 0.00 & $\mathrm{H}$ \\
\hline-3.095 & -2.093 & 5.606 & 1.00 & 0.00 & C \\
\hline-3.761 & -2.194 & 4.747 & 1.00 & 0.00 & $\mathrm{H}$ \\
\hline-1.929 & -1.449 & 5.447 & 1.00 & 0.00 & C \\
\hline-1.249 & -1.293 & 6.277 & 1.00 & 0.00 & $\mathrm{H}$ \\
\hline
\end{tabular}




\begin{tabular}{|c|c|c|c|c|c|c|c|c|c|c|}
\hline $\begin{array}{l}\text { HETATM } \\
\text { TER }\end{array}$ & 3198 & $\mathrm{H} 12$ & VIN & 341 & -1.624 & -1.051 & 4.482 & 1.00 & 0.00 & $\mathrm{H}$ \\
\hline HETATM & 3199 & $\mathrm{C} 1$ & $\mathrm{MET}$ & 342 & -1.411 & -3.777 & 7.810 & 1.00 & 0.00 & C \\
\hline HETATM & 3200 & $\mathrm{H} 11$ & $\mathrm{MET}$ & 342 & -0.819 & -3.112 & 8.459 & 1.00 & 0.00 & $\mathrm{H}$ \\
\hline HETATM & 3201 & $\mathrm{H} 12$ & $\mathrm{MET}$ & 342 & -1.254 & -4.799 & 8.177 & 1.00 & 0.00 & $\mathrm{H}$ \\
\hline HETATM & 3202 & $\mathrm{H} 13$ & $\mathrm{MET}$ & 342 & -0.978 & -3.711 & 6.805 & 1.00 & 0.00 & $\mathrm{H}$ \\
\hline \multicolumn{11}{|l|}{ TER } \\
\hline HETATM & 3203 & C5 & $\mathrm{ACP}$ & 343 & -10.499 & -4.858 & 13.701 & 1.00 & 0.00 & C \\
\hline HETATM & 3204 & H5 1 & $\mathrm{ACP}$ & 343 & -9.760 & -4.870 & 14.514 & 1.00 & 0.00 & $\mathrm{H}$ \\
\hline HETATM & 3205 & H5 2 & $\mathrm{ACP}$ & 343 & $-11 \cdot 340$ & -4.250 & 14.065 & 1.00 & 0.00 & $\mathrm{H}$ \\
\hline HETATM & 3206 & $\mathrm{C} 4$ & $\mathrm{ACP}$ & 343 & -10.978 & -6.296 & 13.428 & 1.00 & 0.00 & C \\
\hline HETATM & 3207 & H 41 & $\mathrm{ACP}$ & 343 & -11.535 & -6.357 & 12.481 & 1.00 & 0.00 & $\mathrm{H}$ \\
\hline HETATM & 3208 & $\mathrm{H} 42$ & $\mathrm{ACP}$ & 343 & -10.107 & -6.962 & 13.321 & 1.00 & 0.00 & $\mathrm{H}$ \\
\hline HETATM & 3209 & $\mathrm{C} 1$ & $\mathrm{ACP}$ & 343 & -11.842 & -6.908 & 14.504 & 1.00 & 0.00 & $\mathrm{C}$ \\
\hline HETATM & 3210 & $\mathrm{O} 2$ & $\mathrm{ACP}$ & 343 & -12.070 & -6.385 & 15.603 & 1.00 & 0.00 & 0 \\
\hline HETATM & 3211 & 03 & $\mathrm{ACP}$ & 343 & -12.357 & -8.088 & 14.140 & 1.00 & 0.00 & o \\
\hline HETATM & 3212 & H3 & $\mathrm{ACP}$ & 343 & -12.967 & -8.442 & 14.876 & 1.00 & 0.00 & $\mathrm{H}$ \\
\hline \multicolumn{11}{|l|}{ TER } \\
\hline HETATM & 3213 & $\mathrm{C} 1$ & MET & 344 & -2.603 & -5.985 & 12.528 & 1.00 & 0.00 & C \\
\hline HETATM & 3214 & H11 & MET & 344 & -2.051 & -5.145 & 12.978 & 1.00 & 0.00 & $\mathrm{H}$ \\
\hline HETATM & 3215 & $\mathrm{H} 12$ & $\mathrm{MET}$ & 344 & -2.597 & -6.810 & 13.250 & 1.00 & 0.00 & $\mathrm{H}$ \\
\hline HETATM & 3216 & $\mathrm{H} 13$ & $\mathrm{MET}$ & 344 & -2.032 & -6.304 & 11.649 & 1.00 & 0.00 & $\mathrm{H}$ \\
\hline \multicolumn{11}{|l|}{ TER } \\
\hline HETATM & 3217 & $\mathrm{C} 1$ & MET & 345 & -12.037 & -3.036 & 11.577 & 1.00 & 0.00 & C \\
\hline HETATM & 3218 & $\mathrm{H} 11$ & $\mathrm{MET}$ & 345 & -12.482 & -3.245 & 12.557 & 1.00 & 0.00 & $\mathrm{H}$ \\
\hline HETATM & 3219 & $\mathrm{H} 12$ & $\mathrm{MET}$ & 345 & -12.147 & -1.956 & 11.402 & 1.00 & 0.00 & $\mathrm{H}$ \\
\hline HETATM & 3220 & $\mathrm{H} 13$ & $\mathrm{MET}$ & 345 & -12.637 & -3.561 & 10.821 & 1.00 & 0.00 & $\mathrm{H}$ \\
\hline \multicolumn{11}{|l|}{ TER } \\
\hline HETATM & 3221 & $\mathrm{C} 2$ & VIN & 346 & -2.240 & -14.415 & 16.864 & 1.00 & 0.00 & C \\
\hline HETATM & 3222 & $\mathrm{H} 21$ & VIN & 346 & -1.424 & -13.716 & 16.699 & 1.00 & 0.00 & $\mathrm{H}$ \\
\hline HETATM & 3223 & $\mathrm{C} 1$ & VIN & 346 & -1.907 & -15.640 & 17.266 & 1.00 & 0.00 & C \\
\hline HETATM & 3224 & $\mathrm{H} 11$ & VIN & 346 & -2.638 & -16.418 & 17.373 & 1.00 & 0.00 & $\mathrm{H}$ \\
\hline HETATM & 3225 & $\mathrm{H} 12$ & VIN & 346 & -0.865 & -15.909 & 17.432 & 1.00 & 0.00 & $\mathrm{H}$ \\
\hline \multicolumn{11}{|l|}{ TER } \\
\hline HETATM & 3226 & $\mathrm{C} 1$ & $\mathrm{MET}$ & 347 & -4.903 & -14.670 & 18.702 & 1.00 & 0.00 & C \\
\hline HETATM & 3227 & H11 & $\mathrm{MET}$ & 347 & -5.138 & -15.741 & 18.583 & 1.00 & 0.00 & $\mathrm{H}$ \\
\hline HETATM & 3228 & $\mathrm{H} 12$ & MET & 347 & -5.716 & -14.206 & 19.272 & 1.00 & 0.00 & $\mathrm{H}$ \\
\hline HETATM & 3229 & $\mathrm{H} 13$ & MET & 347 & -3.996 & -14.592 & 19.313 & 1.00 & 0.00 & $\mathrm{H}$ \\
\hline TER & & & & & & & & & & \\
\hline HETATM & 3230 & $\mathrm{C} 2$ & VIN & 348 & -9.914 & -12.992 & 18.188 & 1.00 & 0.00 & C \\
\hline HETATM & 3231 & H 21 & VIN & 348 & -9.248 & -12.891 & 19.046 & 1.00 & 0.00 & $\mathrm{H}$ \\
\hline HETATM & 3232 & $\mathrm{C} 1$ & VIN & 348 & -11.080 & -13.636 & 18.346 & 1.00 & 0.00 & C \\
\hline HETATM & 3233 & $\mathrm{H} 11$ & VIN & 348 & -11.760 & -13.792 & 17.516 & 1.00 & 0.00 & $\mathrm{H}$ \\
\hline HETATM & 3234 & $\mathrm{H} 12$ & VIN & 348 & -11.385 & -14.034 & 19.312 & 1.00 & 0.00 & $\mathrm{H}$ \\
\hline TER & & & & & & & & & & \\
\hline HETATM & 3235 & $\mathrm{C} 1$ & $\mathrm{MET}$ & 349 & -11.598 & -11.309 & 15.984 & 1.00 & 0.00 & C \\
\hline HETATM & 3236 & $\mathrm{H} 11$ & $\mathrm{MET}$ & 349 & -11.755 & -10.286 & 15.617 & 1.00 & 0.00 & $\mathrm{H}$ \\
\hline HETATM & 3237 & $\mathrm{H} 12$ & MET & 349 & -12.030 & -11.374 & 16.989 & 1.00 & 0.00 & $\mathrm{H}$ \\
\hline HETATM & 3238 & H13 & $\mathrm{MET}$ & 349 & -12.189 & -11.973 & 15.335 & 1.00 & 0.00 & $\mathrm{H}$ \\
\hline TER & & & & & & & & & & \\
\hline HETATM & 3239 & $\mathrm{C} 5$ & $\mathrm{ACP}$ & 350 & -2.510 & -10.227 & 10.092 & 1.00 & 0.00 & C \\
\hline HETATM & 3240 & H51 & $\mathrm{ACP}$ & 350 & -3.249 & -10.216 & 9.280 & 1.00 & 0.00 & $\mathrm{H}$ \\
\hline HETATM & 3241 & H52 & $\mathrm{ACP}$ & 350 & -1.669 & -10.835 & 9.729 & 1.00 & 0.00 & $\mathrm{H}$ \\
\hline HETATM & 3242 & $\mathrm{C} 4$ & $\mathrm{ACP}$ & 350 & -2.031 & -8.789 & 10.365 & 1.00 & 0.00 & C \\
\hline HETATM & 3243 & H 41 & $\mathrm{ACP}$ & 350 & -1.474 & -8.728 & 11.313 & 1.00 & 0.00 & $\mathrm{H}$ \\
\hline HETATM & 3244 & $\mathrm{H} 42$ & $\mathrm{ACP}$ & 350 & -2.902 & -8.123 & 10.472 & 1.00 & 0.00 & $\mathrm{H}$ \\
\hline HETATM & 3245 & $\mathrm{C} 1$ & $\mathrm{ACP}$ & 350 & -1.167 & -8.177 & 9.289 & 1.00 & 0.00 & C \\
\hline HETATM & 3246 & $\mathrm{O} 2$ & $\mathrm{ACP}$ & 350 & -0.939 & -8.700 & 8.191 & 1.00 & 0.00 & o \\
\hline HETATM & 3247 & 03 & $\mathrm{ACP}$ & 350 & -0.651 & -6.998 & 9.653 & 1.00 & 0.00 & O \\
\hline HETATM & 3248 & H3 & $\mathrm{ACP}$ & 350 & -0.042 & -6.643 & 8.917 & 1.00 & 0.00 & $\mathrm{H}$ \\
\hline TER & & & & & & & & & & \\
\hline HETATM & 3249 & $\mathrm{C} 1$ & MET & 351 & -10.406 & -9.101 & 11.266 & 1.00 & 0.00 & C \\
\hline HETATM & 3250 & $\mathrm{H} 11$ & $\mathrm{MET}$ & 351 & -10.958 & -9.940 & 10.815 & 1.00 & 0.00 & $\mathrm{H}$ \\
\hline HETATM & 3251 & $\mathrm{H} 12$ & MET & 351 & -10.412 & -8.275 & 10.544 & 1.00 & 0.00 & $\mathrm{H}$ \\
\hline HETATM & 3252 & $\mathrm{H} 13$ & MET & 351 & -10.977 & -8.781 & 12.145 & 1.00 & 0.00 & $\mathrm{H}$ \\
\hline TER & & & & & & & & & & \\
\hline HETATM & 3253 & $\mathrm{C} 1$ & MET & 352 & -0.972 & -12.049 & 12.216 & 1.00 & 0.00 & C \\
\hline HETATM & 3254 & H11 & MET & 352 & -0.527 & -11.841 & 11.236 & 1.00 & 0.00 & $\mathrm{H}$ \\
\hline HETATM & 3255 & H12 & MET & 352 & -0.862 & -13.130 & 12.391 & 1.00 & 0.00 & $\mathrm{H}$ \\
\hline HETATM & 3256 & H13 & $\mathrm{MET}$ & 352 & -0.372 & -11.524 & 12.973 & 1.00 & 0.00 & $\mathrm{H}$ \\
\hline TER & & & & & & & & & & \\
\hline HETATM & 3257 & $\mathrm{FE}$ & HEM & 353 & -6.872 & 11.003 & -6.440 & 1.00 & 0.00 & $\mathrm{Fe}$ \\
\hline HETATM & 3258 & $\mathrm{C} 1$ & HEM & 353 & -9.100 & 12.876 & -7.502 & 1.00 & 0.00 & C \\
\hline HETATM & 3259 & $\mathrm{C} 2$ & HEM & 353 & -9.459 & 13.666 & -8.674 & 1.00 & 0.00 & C \\
\hline HETATM & 3260 & $\mathrm{C} 3$ & HEM & 353 & -8.313 & 13.763 & -9.448 & 1.00 & 0.00 & C \\
\hline HETATM & 3261 & $\mathrm{C} 4$ & HEM & 353 & -7.284 & 13.046 & -8.740 & 1.00 & 0.00 & C \\
\hline HETATM & 3262 & $\mathrm{C} 5$ & HEM & 353 & -5.969 & 12.927 & -9.178 & 1.00 & 0.00 & C \\
\hline HETATM & 3263 & H5 & HEM & 353 & -5.715 & 13.423 & -10.113 & 1.00 & 0.00 & H \\
\hline HETATM & 3264 & $\mathrm{C} 6$ & HEM & 353 & -4.949 & 12.229 & -8.542 & 1.00 & 0.00 & C \\
\hline
\end{tabular}




\begin{tabular}{|c|c|c|c|c|c|c|c|c|c|c|}
\hline HETATM & 3265 & C7 & HEM & 353 & -3.580 & 12.156 & -9.012 & 1.00 & 0.00 & C \\
\hline HETATM & 3266 & C8 & HEM & 353 & -2.875 & 11.436 & -8.065 & 1.00 & 0.00 & C \\
\hline HETATM & 3267 & C9 & HEM & 353 & -3.826 & 11.071 & -7.041 & 1.00 & 0.00 & C \\
\hline HETATM & 3268 & $\mathrm{C} 10$ & HEM & 353 & -3.522 & 10.336 & -5.897 & 1.00 & 0.00 & C \\
\hline HETATM & 3269 & $\mathrm{H} 10$ & HEM & 353 & -2.495 & 9.993 & -5.791 & 1.00 & 0.00 & $\mathrm{H}$ \\
\hline HETATM & 3270 & $\mathrm{C} 11$ & HEM & 353 & -4.396 & 9.996 & -4.874 & 1.00 & 0.00 & C \\
\hline HETATM & 3271 & $\mathrm{C} 12$ & HEM & 353 & -4.015 & 9.271 & -3.684 & 1.00 & 0.00 & C \\
\hline HETATM & 3272 & $\mathrm{C} 13$ & HEM & 353 & -5.155 & 9.124 & -2.927 & 1.00 & 0.00 & C \\
\hline HETATM & 3273 & $\mathrm{C} 14$ & HEM & 353 & -6.227 & 9.765 & -3.659 & 1.00 & 0.00 & C \\
\hline HETATM & 3274 & C15 & HEM & 353 & -7.556 & 9.820 & -3.237 & 1.00 & 0.00 & C \\
\hline HETATM & 3275 & H15 & HEM & 353 & -7.796 & 9.335 & -2.289 & 1.00 & 0.00 & $H$ \\
\hline HETATM & 3276 & C16 & HEM & 353 & -8.586 & 10.502 & -3.880 & 1.00 & 0.00 & C \\
\hline HETATM & 3277 & $\mathrm{C} 17$ & HEM & 353 & -9.952 & 10.615 & -3.398 & 1.00 & 0.00 & C \\
\hline HETATM & 3278 & $\mathrm{C} 18$ & HEM & 353 & -10.627 & 11.383 & -4.322 & 1.00 & 0.00 & C \\
\hline HETATM & 3279 & C19 & HEM & 353 & -9.677 & 11.735 & -5.358 & 1.00 & 0.00 & C \\
\hline HETATM & 3280 & $\mathrm{C} 20$ & HEM & 353 & -9.969 & 12.526 & -6.469 & 1.00 & 0.00 & C \\
\hline HETATM & 3281 & $\mathrm{H} 20$ & HEM & 353 & -10.983 & 12.915 & -6.530 & 1.00 & 0.00 & $\mathrm{H}$ \\
\hline HETATM & 3282 & $\mathrm{~N} 21$ & HEM & 353 & -7.778 & 12.513 & -7.564 & 1.00 & 0.00 & $\mathrm{~N}$ \\
\hline HETATM & 3283 & N22 & HEM & 353 & -5.074 & 11.566 & -7.342 & 1.00 & 0.00 & $\mathrm{~N}$ \\
\hline HETATM & 3284 & N23 & HEM & 353 & -5.745 & 10.303 & -4.841 & 1.00 & 0.00 & $\mathrm{~N}$ \\
\hline HETATM & 3285 & $\mathrm{~N} 24$ & HEM & 353 & -8.444 & 11.187 & -5.077 & 1.00 & 0.00 & $\mathrm{~N}$ \\
\hline HETATM & 3286 & C99 & HEM & 353 & -7.538 & 8.322 & -7.579 & 1.00 & 0.00 & C \\
\hline HETATM & 3287 & 091 & HEM & 353 & -7.378 & 9.635 & -7.567 & 1.00 & 0.00 & 0 \\
\hline HETATM & 3288 & 092 & HEM & 353 & -7.692 & 7.705 & -8.635 & 1.00 & 0.00 & 0 \\
\hline HETATM & 3289 & СТ91 & HEM & 353 & -7.521 & 7.640 & -6.223 & 1.00 & 0.00 & C \\
\hline HETATM & 3290 & H911 & HEM & 353 & -6.543 & 7.836 & -5.760 & 1.00 & 0.00 & $\mathrm{H}$ \\
\hline HETATM & 3291 & H912 & HEM & 353 & -8.269 & 8.130 & -5.580 & 1.00 & 0.00 & $\mathrm{H}$ \\
\hline HETATM & 3292 & СТ92 & HEM & 353 & -7.781 & 6.136 & -6.334 & 1.00 & 0.00 & C \\
\hline HETATM & 3293 & H921 & HEM & 353 & -6.985 & 5.676 & -6.931 & 1.00 & 0.00 & $\mathrm{H}$ \\
\hline HETATM & 3294 & H922 & HEM & 353 & -8.706 & 5.980 & -6.905 & 1.00 & 0.00 & $\mathrm{H}$ \\
\hline \multicolumn{11}{|l|}{ TER } \\
\hline HETATM & 3295 & $\mathrm{FE}$ & HEM & 354 & -6.180 & 3.589 & -1.491 & 1.00 & 0.00 & $\mathrm{Fe}$ \\
\hline HETATM & 3296 & $\mathrm{C} 1$ & HEM & 354 & -3.952 & 1.717 & -0.429 & 1.00 & 0.00 & C \\
\hline HETATM & 3297 & $\mathrm{C} 2$ & HEM & 354 & -3.593 & 0.927 & 0.742 & 1.00 & 0.00 & C \\
\hline HETATM & 3298 & C3 & HEM & 354 & -4.739 & 0.830 & 1.517 & 1.00 & 0.00 & C \\
\hline HETATM & 3299 & $\mathrm{C} 4$ & HEM & 354 & -5.768 & 1.547 & 0.809 & 1.00 & 0.00 & C \\
\hline HETATM & 3300 & C5 & HEM & 354 & -7.083 & 1.666 & 1.247 & 1.00 & 0.00 & C \\
\hline HETATM & 3301 & H5 & HEM & 354 & -7.337 & 1.170 & 2.182 & 1.00 & 0.00 & $\mathrm{H}$ \\
\hline HETATM & 3302 & $\mathrm{C} 6$ & HEM & 354 & -8.103 & 2.364 & 0.611 & 1.00 & 0.00 & C \\
\hline HETATM & 3303 & C7 & HEM & 354 & -9.472 & 2.437 & 1.081 & 1.00 & 0.00 & C \\
\hline HETATM & 3304 & $\mathrm{C} 8$ & HEM & 354 & -10.177 & 3.157 & 0.134 & 1.00 & 0.00 & C \\
\hline HETATM & 3305 & C9 & HEM & 354 & -9.226 & 3.522 & -0.891 & 1.00 & 0.00 & C \\
\hline HETATM & 3306 & $\mathrm{C} 10$ & HEM & 354 & -9.531 & 4.256 & -2.034 & 1.00 & 0.00 & C \\
\hline HETATM & 3307 & $\mathrm{H} 10$ & HEM & 354 & -10.557 & 4.600 & -2.140 & 1.00 & 0.00 & $\mathrm{H}$ \\
\hline HETATM & 3308 & C11 & HEM & 354 & -8.656 & 4.596 & -3.057 & 1.00 & 0.00 & C \\
\hline HETATM & 3309 & $\mathrm{C} 12$ & HEM & 354 & -9.037 & 5.322 & -4.247 & 1.00 & 0.00 & C \\
\hline HETATM & 3310 & C13 & HEM & 354 & -7.898 & 5.469 & -5.004 & 1.00 & 0.00 & C \\
\hline HETATM & 3311 & C14 & HEM & 354 & -6.825 & 4.828 & -4.273 & 1.00 & 0.00 & C \\
\hline HETATM & 3312 & C15 & HEM & 354 & -5.497 & 4.773 & -4.694 & 1.00 & 0.00 & C \\
\hline HETATM & 3313 & H1 5 & HEM & 354 & -5.256 & 5.258 & -5.642 & 1.00 & 0.00 & $\mathrm{H}$ \\
\hline HETATM & 3314 & C16 & HEM & 354 & -4.466 & 4.091 & -4.051 & 1.00 & 0.00 & C \\
\hline HETATM & 3315 & C17 & HEM & 354 & -3.100 & 3.978 & -4.533 & 1.00 & 0.00 & C \\
\hline HETATM & 3316 & $\mathrm{C} 18$ & HEM & 354 & -2.425 & 3.210 & -3.609 & 1.00 & 0.00 & C \\
\hline HETATM & 3317 & C19 & HEM & 354 & -3.375 & 2.858 & -2.573 & 1.00 & 0.00 & C \\
\hline HETATM & 3318 & $\mathrm{C} 20$ & HEM & 354 & -3.083 & 2.067 & -1.462 & 1.00 & 0.00 & C \\
\hline HETATM & 3319 & H 20 & HEM & 354 & -2.069 & 1.678 & -1.402 & 1.00 & 0.00 & $\mathrm{H}$ \\
\hline HETATM & 3320 & $\mathrm{~N} 21$ & HEM & 354 & -5.274 & 2.080 & -0.367 & 1.00 & 0.00 & $\mathrm{~N}$ \\
\hline HETATM & 3321 & $\mathrm{~N} 22$ & HEM & 354 & -7.978 & 3.027 & -0.590 & 1.00 & 0.00 & $\mathrm{~N}$ \\
\hline HETATM & 3322 & $\mathrm{~N} 23$ & HEM & 354 & -7.308 & 4.290 & -3.090 & 1.00 & 0.00 & $\mathrm{~N}$ \\
\hline HETATM & 3323 & N24 & HEM & 354 & -4.608 & 3.406 & -2.854 & 1.00 & 0.00 & $\mathrm{~N}$ \\
\hline HETATM & 3324 & C99 & HEM & 354 & -5.515 & 6.271 & -0.352 & 1.00 & 0.00 & C \\
\hline HETATM & 3325 & 091 & HEM & 354 & -5.674 & 4.958 & -0.365 & 1.00 & 0.00 & o \\
\hline HETATM & 3326 & 092 & HEM & 354 & -5.360 & 6.888 & 0.703 & 1.00 & 0.00 & o \\
\hline HETATM & 3327 & CT 91 & HEM & 354 & -5.531 & 6.952 & -1.709 & 1.00 & 0.00 & C \\
\hline HETATM & 3328 & H911 & HEM & 354 & -6.509 & 6.757 & -2.171 & 1.00 & 0.00 & $\mathrm{H}$ \\
\hline HETATM & 3329 & H912 & HEM & 354 & -4.783 & 6.463 & -2.352 & 1.00 & 0.00 & $\mathrm{H}$ \\
\hline HETATM & 3330 & СТ 92 & HEM & 354 & -5.271 & 8.457 & -1.597 & 1.00 & 0.00 & C \\
\hline HETATM & 3331 & H921 & HEM & 354 & -4.346 & 8.613 & -1.027 & 1.00 & 0.00 & $\mathrm{H}$ \\
\hline HETATM & 3332 & H922 & HEM & 354 & -6.067 & 8.917 & -1.000 & 1.00 & 0.00 & $\mathrm{H}$ \\
\hline \multicolumn{11}{|l|}{ TER } \\
\hline HETATM & 3333 & $\mathrm{C} 2$ & VIN & 355 & -10.791 & 14.169 & -8.933 & 1.00 & 0.00 & C \\
\hline HETATM & 3334 & Н 21 & VIN & 355 & -11.607 & 13.470 & -8.768 & 1.00 & 0.00 & $\mathrm{H}$ \\
\hline HETATM & 3335 & $\mathrm{C} 1$ & VIN & 355 & -11.124 & 15.394 & -9.335 & 1.00 & 0.00 & C \\
\hline HETATM & 3336 & H11 & VIN & 355 & $-10 \cdot 392$ & 16.172 & -9.442 & 1.00 & 0.00 & $\mathrm{H}$ \\
\hline HETATM & 3337 & H12 & VIN & 355 & -12.165 & 15.663 & -9.501 & 1.00 & 0.00 & $\mathrm{H}$ \\
\hline \multicolumn{11}{|l|}{ TER } \\
\hline HETATM & 3338 & $\mathrm{C} 1$ & MET & 356 & -8.127 & 14.424 & -10.771 & 1.00 & 0.00 & 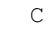 \\
\hline HETATM & 3339 & H11 & MET & 356 & -7.892 & 15.494 & -10.652 & 1.00 & 0.00 & 11 \\
\hline HETATM & 3340 & $\mathrm{H} 12$ & MET & 356 & -7.314 & 13.959 & -11.341 & 1.00 & 0.00 & H \\
\hline
\end{tabular}


HETATM 3341 H13 MET 35

TER

HETATM 3342 C2 VIN 357 HETATM 3343 H21 VIN 357 HETATM 3344 C1 VIN 357 HETATM 3345 H11 VIN 357 HETATM 3346 H12 VIN 357 TER

HETATM 3347 C1 MET 358 HETATM 3348 H11 MET 358 HETATM 3349 H12 MET 358 HETATM 3350 H13 MET 358 TER

HETATM $3351 \quad$ C5 ACP 359 HETATM 3352 H51 ACP 359 HETATM 3353 H52 ACP 359 HETATM $3354 \quad$ C4 ACP 359 HETATM 3355 H41 ACP 359 HETATM 3356 H42 ACP 359 HETATM 3357 C1 ACP 359 HETATM $3358 \quad$ O2 ACP 359 HETATM $3359 \quad 03$ ACP 359 HETATM 3360 H3 ACP 359 TER

HETATM 3361 C1 MET 360 HETATM 3362 H11 MET 360 HETATM 3363 H12 MET 360 HETATM 3364 H13 MET 360 TER

HETATM 3365 C1 MET 361 HETATM 3366 H11 MET 361 HETATM 3367 H12 MET 361 HETATM 3368 H13 MET 361 TER

HETATM 3369 C2 VIN 362 HETATM HETATM 3371 C1 VIN 362 HETATM 3372 H11 VIN 362 HETATM 3373 H12 VIN 362 TER

HETATM 3374 C1 MET 363 HETATM 3375 H11 MET 363 HETATM 3376 H12 MET 363 HETATM 3377 H13 MET 363 TER

HETATM 3378 C2 VIN 364 HETATM 3379 H21 VIN 364 HETATM 3380 C1 VIN 364 HETATM 3381 H11 VIN 364 HETATM 3382 H12 VIN 364 TER

HETATM 3383 C1 MET 365 HETATM 3384 H11 MET 365 HETATM 3385 H12 MET 365 HETATM 3386 H13 MET 365 TER

HETATM 3387 C5 ACP 366 HETATM 3388 H51 ACP HETATM 3389 H52 ACP 366 HETATM $3390 \quad$ C4 ACP 366

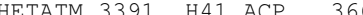
$\begin{array}{llll}H E T A T M & 3392 & \text { H4 } & \text { ACP } 366\end{array}$ HETATM 3393 C1 ACP 36 HETATM $3394 \quad$ O2 ACP 366 $\begin{array}{lll} & \end{array}$ HETATM 3396 H3 ACP 366 TER

HETATM 3397 C1 MET 367 HETATM 3398 H11 MET 367 HETATM 3399 H12 MET 367 HETATM 3400 H13 MET 36 TER

HETATM $3401 \quad C 1$ MET 368 HETATM 3402 H11 MET 368 HETATM 3403 H12 MET 368 HETATM 3404 H13 MET 368 TER

HETATM 3405 FE HEM 369 HETATM 3406 C1 HEM 369

\begin{tabular}{|c|c|c|c|c|c|}
\hline-9.035 & 14.346 & -11.382 & 1.00 & 0.00 & $\mathrm{H}$ \\
\hline-3.117 & 12.746 & -10.256 & 1.00 & 0.00 & C \\
\hline-3.783 & 12.645 & -11.115 & 1.00 & 0.00 & $\mathrm{H}$ \\
\hline-1.950 & 13.390 & -10.415 & 1.00 & 0.00 & C \\
\hline-1.270 & 13.546 & -9.585 & 1.00 & 0.00 & $\mathrm{H}$ \\
\hline-1.645 & 13.788 & -11.381 & 1.00 & 0.00 & $\mathrm{H}$ \\
\hline-1.432 & 11.062 & -8.052 & 1.00 & 0.00 & C \\
\hline-0.841 & 11.727 & -7.403 & 1.00 & 0.00 & $\mathrm{H}$ \\
\hline-1.276 & 10.040 & -7.685 & 1.00 & 0.00 & $\mathrm{H}$ \\
\hline-1.000 & 11.128 & -9.057 & 1.00 & 0.00 & $\mathrm{H}$ \\
\hline-10.521 & 9.981 & -2.161 & 1.00 & 0.00 & $\mathrm{C}$ \\
\hline-9.782 & 9.969 & -1.349 & 1.00 & 0.00 & $\mathrm{H}$ \\
\hline$-11 \cdot 361$ & 10.589 & -1.798 & 1.00 & 0.00 & $\mathrm{H}$ \\
\hline-10.999 & 8.543 & -2.434 & 1.00 & 0.00 & C \\
\hline-11.557 & 8.482 & -3.382 & 1.00 & 0.00 & $\mathrm{H}$ \\
\hline-10.129 & 7.877 & -2.541 & 1.00 & 0.00 & $\mathrm{H}$ \\
\hline-11.863 & 7.931 & -1.358 & 1.00 & 0.00 & C \\
\hline-12.091 & 8.454 & -0.260 & 1.00 & 0.00 & O \\
\hline-12.379 & 6.751 & -1.722 & 1.00 & 0.00 & 0 \\
\hline-12.988 & 6.397 & -0.986 & 1.00 & 0.00 & $\mathrm{H}$ \\
\hline-2.625 & 8.855 & -3.334 & 1.00 & 0.00 & C \\
\hline-2.072 & 9.694 & -2.884 & 1.00 & 0.00 & $\mathrm{H}$ \\
\hline-2.618 & 8.029 & -2.613 & 1.00 & 0.00 & $\mathrm{H}$ \\
\hline-2.054 & 8.535 & -4.214 & 1.00 & 0.00 & $\mathrm{H}$ \\
\hline-12.058 & 11.803 & -4.285 & 1.00 & 0.00 & $\mathrm{C}$ \\
\hline-12.503 & 11.594 & -3.305 & 1.00 & 0.00 & $\mathrm{H}$ \\
\hline-12.168 & 12.883 & -4.460 & 1.00 & 0.00 & $\mathrm{H}$ \\
\hline-12.659 & 11.278 & -5.042 & 1.00 & 0.00 & $\mathrm{H}$ \\
\hline-2.261 & 0.424 & 1.002 & 1.00 & 0.00 & C \\
\hline-1.446 & 1.123 & 0.837 & 1.00 & 0.00 & $\mathrm{H}$ \\
\hline-1.928 & -0.801 & 1.403 & 1.00 & 0.00 & C \\
\hline-2.660 & -1.579 & 1.511 & 1.00 & 0.00 & $\mathrm{H}$ \\
\hline-0.887 & -1.070 & 1.570 & 1.00 & 0.00 & $\mathrm{H}$ \\
\hline-4.925 & 0.169 & 2.840 & 1.00 & 0.00 & C \\
\hline-5.160 & -0.902 & 2.720 & 1.00 & 0.00 & $\mathrm{H}$ \\
\hline-5.738 & 0.633 & 3.410 & 1.00 & 0.00 & $\mathrm{H}$ \\
\hline-4.017 & 0.247 & 3.450 & 1.00 & 0.00 & $\mathrm{H}$ \\
\hline-9.935 & 1.847 & 2.325 & 1.00 & 0.00 & C \\
\hline-9.269 & 1.948 & 3.184 & 1.00 & 0.00 & $\mathrm{H}$ \\
\hline-11.102 & 1.203 & 2.484 & 1.00 & 0.00 & C \\
\hline-11.782 & 1.047 & 1.654 & 1.00 & 0.00 & $\mathrm{H}$ \\
\hline-11.407 & 0.805 & 3.450 & 1.00 & 0.00 & $\mathrm{H}$ \\
\hline-11.620 & 3.530 & 0.121 & 1.00 & 0.00 & C \\
\hline-11.776 & 4.553 & -0.246 & 1.00 & 0.00 & $\mathrm{H}$ \\
\hline-12.052 & 3.465 & 1.126 & 1.00 & 0.00 & $\mathrm{H}$ \\
\hline-12.211 & 2.866 & -0.528 & 1.00 & 0.00 & $\mathrm{H}$ \\
\hline-2.531 & 4.612 & -5.770 & 1.00 & 0.00 & C \\
\hline-3.270 & 4.623 & -6.582 & 1.00 & 0.00 & $\mathrm{H}$ \\
\hline-1.691 & 4.004 & -6.134 & 1.00 & 0.00 & $\mathrm{H}$ \\
\hline-2.053 & 6.050 & -5.497 & 1.00 & 0.00 & C \\
\hline-1.495 & 6.111 & -4.549 & 1.00 & 0.00 & $\mathrm{H}$ \\
\hline-2.923 & 6.716 & -5.390 & 1.00 & 0.00 & $\mathrm{H}$ \\
\hline-1.189 & 6.662 & -6.573 & 1.00 & 0.00 & C \\
\hline-0.961 & 6.139 & -7.671 & 1.00 & 0.00 & 0 \\
\hline-0.673 & 7.841 & -6.209 & 1.00 & 0.00 & O \\
\hline-0.064 & 8.196 & -6.945 & 1.00 & 0.00 & $\mathrm{H}$ \\
\hline-10.427 & 5.738 & -4.597 & 1.00 & 0.00 & $\mathrm{C}$ \\
\hline-10.980 & 4.899 & -5.047 & 1.00 & 0.00 & $\mathrm{H}$ \\
\hline-10.434 & 6.564 & -5.319 & 1.00 & 0.00 & $\mathrm{H}$ \\
\hline-10.998 & 6.058 & -3.717 & 1.00 & 0.00 & $\mathrm{H}$ \\
\hline-0.994 & 2.790 & -3.646 & 1.00 & 0.00 & C \\
\hline-0.549 & 2.998 & -4.626 & 1.00 & 0.00 & $\mathrm{H}$ \\
\hline-0.884 & 1.709 & -3.471 & 1.00 & 0.00 & $\mathrm{H}$ \\
\hline-0.393 & 3.315 & -2.889 & 1.00 & 0.00 & $\mathrm{H}$ \\
\hline-4.955 & -3.343 & -6.440 & 1.00 & 0.00 & $\mathrm{Fe}$ \\
\hline-7.183 & -1.471 & -7.502 & 1.00 & 0.00 & C \\
\hline
\end{tabular}




\begin{tabular}{|c|c|c|c|c|c|c|c|c|c|c|}
\hline HETATM & 3407 & $\mathrm{C} 2$ & HEM & 369 & -7.542 & -0.680 & -8.674 & 1.00 & 0.00 & C \\
\hline HETATM & 3408 & $\mathrm{C} 3$ & HEM & 369 & -6.397 & -0.583 & -9.448 & 1.00 & 0.00 & C \\
\hline HETATM & 3409 & $\mathrm{C} 4$ & HEM & 369 & -5.367 & -1.301 & -8.740 & 1.00 & 0.00 & C \\
\hline HETATM & 3410 & C5 & HEM & 369 & -4.052 & -1.420 & -9.178 & 1.00 & 0.00 & C \\
\hline HETATM & 3411 & H5 & HEM & 369 & -3.798 & -0.923 & -10.113 & 1.00 & 0.00 & $\mathrm{H}$ \\
\hline HETATM & 3412 & $\mathrm{C} 6$ & HEM & 369 & -3.033 & -2.118 & -8.542 & 1.00 & 0.00 & C \\
\hline HETATM & 3413 & C7 & HEM & 369 & -1.664 & -2.191 & -9.012 & 1.00 & 0.00 & C \\
\hline HETATM & 3414 & $\mathrm{C} 8$ & HEM & 369 & -0.958 & -2.910 & -8.065 & 1.00 & 0.00 & C \\
\hline HETATM & 3415 & $\mathrm{C} 9$ & HEM & 369 & -1.909 & -3.275 & -7.041 & 1.00 & 0.00 & C \\
\hline HETATM & 3416 & $\mathrm{C} 10$ & HEM & 369 & -1.605 & -4.010 & -5.897 & 1.00 & 0.00 & C \\
\hline HETATM & 3417 & $\mathrm{H} 1 \mathrm{O}$ & HEM & 369 & -0.578 & -4.354 & -5.791 & 1.00 & 0.00 & $\mathrm{H}$ \\
\hline HETATM & 3418 & C11 & HEM & 369 & -2.479 & -4.350 & -4.874 & 1.00 & 0.00 & C \\
\hline HETATM & 3419 & $\mathrm{C} 12$ & HEM & 369 & -2.098 & -5.076 & -3.684 & 1.00 & 0.00 & C \\
\hline HETATM & 3420 & $\mathrm{C} 13$ & HEM & 369 & -3.238 & -5.223 & -2.927 & 1.00 & 0.00 & C \\
\hline HETATM & 3421 & C14 & HEM & 369 & -4.311 & -4.581 & -3.659 & 1.00 & 0.00 & C \\
\hline HETATM & 3422 & $\mathrm{C} 15$ & HEM & 369 & -5.639 & -4.527 & -3.237 & 1.00 & 0.00 & C \\
\hline HETATM & 3423 & H15 & HEM & 369 & -5.879 & -5.011 & -2.289 & 1.00 & 0.00 & $\mathrm{H}$ \\
\hline HETATM & 3424 & $\mathrm{C} 16$ & HEM & 369 & -6.669 & -3.845 & -3.880 & 1.00 & 0.00 & C \\
\hline HETATM & 3425 & C17 & HEM & 369 & -8.035 & -3.732 & -3.398 & 1.00 & 0.00 & C \\
\hline HETATM & 3426 & $\mathrm{C} 18$ & HEM & 369 & -8.710 & -2.964 & -4.322 & 1.00 & 0.00 & C \\
\hline HETATM & 3427 & C19 & HEM & 369 & -7.761 & -2.612 & -5.358 & 1.00 & 0.00 & C \\
\hline HETATM & 3428 & $\mathrm{C} 20$ & HEM & 369 & -8.052 & -1.821 & -6.469 & 1.00 & 0.00 & C \\
\hline HETATM & 3429 & $\mathrm{H} 20$ & HEM & 369 & -9.066 & -1.432 & -6.530 & 1.00 & 0.00 & $\mathrm{H}$ \\
\hline HETATM & 3430 & N21 & HEM & 369 & -5.862 & -1.834 & -7.564 & 1.00 & 0.00 & $\mathrm{~N}$ \\
\hline HETATM & 3431 & N22 & HEM & 369 & -3.157 & -2.781 & -7.342 & 1.00 & 0.00 & $\mathrm{~N}$ \\
\hline HETATM & 3432 & N23 & HEM & 369 & -3.828 & -4.044 & -4.841 & 1.00 & 0.00 & $\mathrm{~N}$ \\
\hline HETATM & 3433 & N2 4 & HEM & 369 & -6.527 & -3.160 & -5.077 & 1.00 & 0.00 & $\mathrm{~N}$ \\
\hline HETATM & 3434 & C99 & HEM & 369 & -5.621 & -6.025 & -7.579 & 1.00 & 0.00 & C \\
\hline HETATM & 3435 & 091 & HEM & 369 & -5.461 & -4.711 & -7.567 & 1.00 & 0.00 & 0 \\
\hline HETATM & 3436 & 092 & HEM & 369 & -5.776 & -6.642 & -8.635 & 1.00 & 0.00 & o \\
\hline HETATM & 3437 & СТ91 & HEM & 369 & -5.605 & -6.706 & -6.223 & 1.00 & 0.00 & C \\
\hline HETATM & 3438 & H911 & HEM & 369 & -4.626 & -6.511 & -5.760 & 1.00 & 0.00 & $\mathrm{H}$ \\
\hline HETATM & 3439 & H912 & HEM & 369 & -6.353 & -6.216 & -5.580 & 1.00 & 0.00 & $\mathrm{H}$ \\
\hline HETATM & 3440 & СТ92 & HEM & 369 & -5.864 & -8.211 & -6.334 & 1.00 & 0.00 & C \\
\hline HETATM & 3441 & H921 & HEM & 369 & -5.068 & -8.671 & -6.931 & 1.00 & 0.00 & $\mathrm{H}$ \\
\hline HETATM & 3442 & H922 & HEM & 369 & -6.789 & -8.367 & -6.905 & 1.00 & 0.00 & $\mathrm{H}$ \\
\hline \multicolumn{11}{|l|}{ TER } \\
\hline HETATM & 3443 & $\mathrm{FE}$ & HEM & 370 & -4.263 & -10.757 & -1.491 & 1.00 & 0.00 & $\mathrm{Fe}$ \\
\hline HETATM & 3444 & $\mathrm{C} 1$ & HEM & 370 & -2.035 & -12.629 & -0.429 & 1.00 & 0.00 & C \\
\hline HETATM & 3445 & $\mathrm{C} 2$ & HEM & 370 & -1.676 & -13.420 & 0.742 & 1.00 & 0.00 & C \\
\hline HETATM & 3446 & C3 & HEM & 370 & -2.822 & -13.517 & 1.517 & 1.00 & 0.00 & C \\
\hline HETATM & 3447 & $\mathrm{C} 4$ & HEM & 370 & -3.851 & -12.800 & 0.809 & 1.00 & 0.00 & C \\
\hline HETATM & 3448 & C5 & HEM & 370 & -5.167 & -12.681 & 1.247 & 1.00 & 0.00 & C \\
\hline HETATM & 3449 & H5 & HEM & 370 & -5.420 & -13.177 & 2.182 & 1.00 & 0.00 & $\mathrm{H}$ \\
\hline HETATM & 3450 & $\mathrm{C} 6$ & HEM & 370 & -6.186 & $-11 \cdot 982$ & 0.611 & 1.00 & 0.00 & C \\
\hline HETATM & 3451 & C7 & HEM & 370 & -7.555 & -11.910 & 1.081 & 1.00 & 0.00 & C \\
\hline HETATM & 3452 & $\mathrm{C} 8$ & HEM & 370 & -8.260 & -11.190 & 0.134 & 1.00 & 0.00 & C \\
\hline HETATM & 3453 & $\mathrm{C} 9$ & HEM & 370 & -7.309 & -10.825 & -0.891 & 1.00 & 0.00 & C \\
\hline HETATM & 3454 & $\mathrm{C} 10$ & HEM & 370 & -7.614 & -10.090 & -2.034 & 1.00 & 0.00 & C \\
\hline HETATM & 3455 & $\mathrm{H} 10$ & HEM & 370 & -8.641 & -9.746 & -2.140 & 1.00 & 0.00 & $\mathrm{H}$ \\
\hline HETATM & 3456 & $\mathrm{C} 11$ & HEM & 370 & -6.739 & -9.750 & -3.057 & 1.00 & 0.00 & C \\
\hline HETATM & 3457 & $\mathrm{C} 12$ & HEM & 370 & -7.120 & -9.025 & -4.247 & 1.00 & 0.00 & C \\
\hline HETATM & 3458 & $\mathrm{C} 13$ & HEM & 370 & -5.981 & -8.878 & -5.004 & 1.00 & 0.00 & $\mathrm{C}$ \\
\hline HETATM & 3459 & C14 & HEM & 370 & -4.908 & -9.519 & -4.273 & 1.00 & 0.00 & C \\
\hline HETATM & 3460 & C15 & HEM & 370 & -3.580 & -9.574 & -4.694 & 1.00 & 0.00 & C \\
\hline HETATM & 3461 & H1 5 & HEM & 370 & -3.340 & -9.089 & -5.642 & 1.00 & 0.00 & $\mathrm{H}$ \\
\hline HETATM & 3462 & C16 & HEM & 370 & -2.550 & -10.255 & -4.051 & 1.00 & 0.00 & C \\
\hline HETATM & 3463 & $\mathrm{C} 17$ & HEM & 370 & -1.184 & -10.369 & -4.533 & 1.00 & 0.00 & C \\
\hline HETATM & 3464 & $\mathrm{C} 18$ & HEM & 370 & -0.508 & -11.137 & -3.609 & 1.00 & 0.00 & C \\
\hline HETATM & 3465 & C19 & HEM & 370 & -1.458 & -11.489 & -2.573 & 1.00 & 0.00 & C \\
\hline HETATM & 3466 & $\mathrm{C} 20$ & HEM & 370 & -1.167 & $-12 \cdot 280$ & -1.462 & 1.00 & 0.00 & C \\
\hline HETATM & 3467 & $\mathrm{H} 2 \mathrm{O}$ & HEM & 370 & -0.152 & -12.669 & -1.402 & 1.00 & 0.00 & $\mathrm{H}$ \\
\hline HETATM & 3468 & N21 & HEM & 370 & -3.357 & -12.266 & -0.367 & 1.00 & 0.00 & $\mathrm{~N}$ \\
\hline HETATM & 3469 & N22 & HEM & 370 & -6.062 & -11.319 & -0.590 & 1.00 & 0.00 & $\mathrm{~N}$ \\
\hline HETATM & 3470 & N23 & HEM & 370 & -5.391 & -10.056 & -3.090 & 1.00 & 0.00 & $\mathrm{~N}$ \\
\hline HETATM & 3471 & N24 & HEM & 370 & -2.691 & -10.941 & -2.854 & 1.00 & 0.00 & $\mathrm{~N}$ \\
\hline HETATM & 3472 & C99 & HEM & 370 & -3.598 & -8.076 & -0.352 & 1.00 & 0.00 & C \\
\hline HETATM & 3473 & 091 & HEM & 370 & -3.757 & -9.389 & -0.365 & 1.00 & 0.00 & $\mathrm{O}$ \\
\hline HETATM & 3474 & 092 & HEM & 370 & -3.443 & -7.458 & 0.703 & 1.00 & 0.00 & o \\
\hline HETATM & 3475 & СТ91 & HEM & 370 & -3.614 & -7.394 & -1.709 & 1.00 & 0.00 & C \\
\hline HETATM & 3476 & H911 & HEM & 370 & -4.593 & -7.590 & -2.171 & 1.00 & 0.00 & $\mathrm{H}$ \\
\hline HETATM & 3477 & H 912 & HEM & 370 & -2.866 & -7.884 & -2.352 & 1.00 & 0.00 & $\mathrm{H}$ \\
\hline HETATM & 3478 & CT92 & HEM & 370 & -3.354 & -5.890 & -1.597 & 1.00 & 0.00 & C \\
\hline HETATM & 3479 & H921 & HEM & 370 & -2.429 & -5.734 & -1.027 & 1.00 & 0.00 & $\mathrm{H}$ \\
\hline HETATM & 3480 & H922 & HEM & 370 & -4.151 & -5.429 & -1.000 & 1.00 & 0.00 & $\mathrm{H}$ \\
\hline \multicolumn{11}{|l|}{ TER } \\
\hline HETATM & 3481 & $\mathrm{C} 2$ & VIN & 371 & -8.874 & -0.178 & -8.933 & 1.00 & 0.00 & $c$ \\
\hline HETATM & 3482 & $\mathrm{H} 21$ & VIN & 371 & -9.690 & -0.876 & -8.768 & 1.00 & 0.00 & H \\
\hline HETATM & 3483 & $\mathrm{C} 1$ & VIN & 371 & -9.207 & 1.047 & -9.335 & 1.00 & 0.00 & 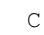 \\
\hline
\end{tabular}




\begin{tabular}{|c|c|c|c|c|c|c|c|c|c|c|}
\hline HETATM & 3484 & $\mathrm{H} 11$ & VIN & 371 & -8.475 & 1.826 & -9.442 & 1.00 & 0.00 & $\mathrm{H}$ \\
\hline HETATM & 3485 & $\mathrm{H} 12$ & VIN & 371 & -10.249 & 1.316 & -9.501 & 1.00 & 0.00 & $\mathrm{H}$ \\
\hline \multicolumn{11}{|l|}{ TER } \\
\hline HETATM & 3486 & $\mathrm{C} 1$ & MET & 372 & -6.210 & 0.077 & -10.771 & 1.00 & 0.00 & C \\
\hline HETATM & 3487 & H11 & $\mathrm{MET}$ & 372 & -5.975 & 1.148 & -10.652 & 1.00 & 0.00 & $\mathrm{H}$ \\
\hline HETATM & 3488 & $\mathrm{H} 12$ & MET & 372 & -5.397 & -0.387 & -11.341 & 1.00 & 0.00 & $\mathrm{H}$ \\
\hline HETATM & 3489 & H13 & MET & 372 & -7.118 & -0.001 & -11.382 & 1.00 & 0.00 & $\mathrm{H}$ \\
\hline \multicolumn{11}{|l|}{ TER } \\
\hline HETATM & 3490 & $\mathrm{C} 2$ & VIN & 373 & -1.200 & -1.601 & -10.256 & 1.00 & 0.00 & $\mathrm{C}$ \\
\hline HETATM & 3491 & H21 & VIN & 373 & -1.866 & -1.702 & -11.115 & 1.00 & 0.00 & $\mathrm{H}$ \\
\hline HETATM & 3492 & $\mathrm{C} 1$ & VIN & 373 & -0.033 & -0.957 & -10.415 & 1.00 & 0.00 & $\mathrm{C}$ \\
\hline HETATM & 3493 & H11 & VIN & 373 & 0.647 & -0.800 & -9.585 & 1.00 & 0.00 & $\mathrm{H}$ \\
\hline HETATM & 3494 & $\mathrm{H} 12$ & VIN & 373 & 0.272 & -0.559 & -11.381 & 1.00 & 0.00 & $\mathrm{H}$ \\
\hline \multicolumn{11}{|l|}{ TER } \\
\hline HETATM & 3495 & $\mathrm{C} 1$ & MET & 374 & 0.484 & -3.284 & -8.052 & 1.00 & 0.00 & C \\
\hline HETATM & 3496 & H11 & MET & 374 & 1.076 & -2.620 & -7.403 & 1.00 & 0.00 & $\mathrm{H}$ \\
\hline HETATM & 3497 & $\mathrm{H} 12$ & MET & 374 & 0.641 & -4.306 & -7.685 & 1.00 & 0.00 & $\mathrm{H}$ \\
\hline HETATM & 3498 & $\mathrm{H} 13$ & MET & 374 & 0.917 & -3.219 & -9.057 & 1.00 & 0.00 & $\mathrm{H}$ \\
\hline \multicolumn{11}{|l|}{ TER } \\
\hline HETATM & 3499 & C5 & $\mathrm{ACP}$ & 375 & -8.604 & -4.366 & -2.161 & 1.00 & 0.00 & C \\
\hline HETATM & 3500 & H51 & $\mathrm{ACP}$ & 375 & -7.865 & -4.377 & -1.349 & 1.00 & 0.00 & $\mathrm{H}$ \\
\hline HETATM & 3501 & H52 & $\mathrm{ACP}$ & 375 & -9.445 & -3.758 & -1.798 & 1.00 & 0.00 & $\mathrm{H}$ \\
\hline HETATM & 3502 & $\mathrm{C} 4$ & $\mathrm{ACP}$ & 375 & -9.082 & -5.803 & -2.434 & 1.00 & 0.00 & C \\
\hline HETATM & 3503 & $\mathrm{H} 41$ & $\mathrm{ACP}$ & 375 & -9.640 & -5.865 & -3.382 & 1.00 & 0.00 & $\mathrm{H}$ \\
\hline HETATM & 3504 & $\mathrm{H} 42$ & $\mathrm{ACP}$ & 375 & -8.212 & -6.470 & -2.541 & 1.00 & 0.00 & $\mathrm{H}$ \\
\hline HETATM & 3505 & $\mathrm{C} 1$ & $\mathrm{ACP}$ & 375 & -9.946 & -6.416 & -1.358 & 1.00 & 0.00 & C \\
\hline HETATM & 3506 & $\mathrm{O} 2$ & $\mathrm{ACP}$ & 375 & -10.174 & -5.893 & -0.260 & 1.00 & 0.00 & 0 \\
\hline HETATM & 3507 & 03 & $\mathrm{ACP}$ & 375 & -10.462 & -7.595 & -1.722 & 1.00 & 0.00 & 0 \\
\hline HETATM & 3508 & H3 & $\mathrm{ACP}$ & 375 & -11.071 & -7.949 & -0.986 & 1.00 & 0.00 & $\mathrm{H}$ \\
\hline TER & & & & & & & & & & \\
\hline HETATM & 3509 & $\mathrm{C} 1$ & MET & 376 & -0.708 & -5.492 & -3.334 & 1.00 & 0.00 & C \\
\hline HETATM & 3510 & $\mathrm{H} 11$ & MET & 376 & -0.155 & -4.653 & -2.884 & 1.00 & 0.00 & $\mathrm{H}$ \\
\hline HETATM & 3511 & $\mathrm{H} 12$ & MET & 376 & -0.701 & -6.318 & -2.613 & 1.00 & 0.00 & $\mathrm{H}$ \\
\hline HETATM & 3512 & H13 & MET & 376 & -0.137 & -5.812 & -4.214 & 1.00 & 0.00 & $\mathrm{H}$ \\
\hline TER & & & & & & & & & & \\
\hline HETATM & 3513 & $\mathrm{C} 1$ & MET & 377 & -10.141 & -2.543 & -4.285 & 1.00 & 0.00 & C \\
\hline HETATM & 3514 & $\mathrm{H} 11$ & MET & 377 & -10.586 & -2.752 & -3.305 & 1.00 & 0.00 & $\mathrm{H}$ \\
\hline HETATM & 3515 & $\mathrm{H} 12$ & MET & 377 & -10.252 & -1.463 & -4.460 & 1.00 & 0.00 & $\mathrm{H}$ \\
\hline HETATM & 3516 & $\mathrm{H} 13$ & MET & 377 & -10.742 & -3.069 & -5.042 & 1.00 & 0.00 & $\mathrm{H}$ \\
\hline TER & & & & & & & & & & \\
\hline HETATM & 3517 & $\mathrm{C} 2$ & VIN & 378 & -0.345 & -13.923 & 1.002 & 1.00 & 0.00 & C \\
\hline HETATM & 3518 & H2 1 & VIN & 378 & 0.471 & -13.224 & 0.837 & 1.00 & 0.00 & $\mathrm{H}$ \\
\hline HETATM & 3519 & $\mathrm{C} 1$ & VIN & 378 & -0.012 & -15.147 & 1.403 & 1.00 & 0.00 & C \\
\hline HETATM & 3520 & $\mathrm{H} 11$ & VIN & 378 & -0.743 & -15.926 & 1.511 & 1.00 & 0.00 & $\mathrm{H}$ \\
\hline HETATM & 3521 & $\mathrm{H} 12$ & VIN & 378 & 1.030 & -15.417 & 1.570 & 1.00 & 0.00 & $\mathrm{H}$ \\
\hline TER & & & & & & & & & & \\
\hline HETATM & 3522 & $\mathrm{C} 1$ & MET & 379 & -3.008 & -14.177 & 2.840 & 1.00 & 0.00 & $\mathrm{C}$ \\
\hline HETATM & 3523 & H11 & MET & 379 & -3.243 & $-15 \cdot 248$ & 2.720 & 1.00 & 0.00 & $\mathrm{H}$ \\
\hline HETATM & 3524 & $\mathrm{H} 12$ & MET & 379 & -3.821 & -13.713 & 3.410 & 1.00 & 0.00 & $\mathrm{H}$ \\
\hline HETATM & 3525 & $\mathrm{H} 13$ & MET & 379 & -2.101 & -14.100 & 3.450 & 1.00 & 0.00 & $\mathrm{H}$ \\
\hline TER & & & & & & & & & & \\
\hline HETATM & 3526 & $\mathrm{C} 2$ & VIN & 380 & -8.018 & -12.499 & 2.325 & 1.00 & 0.00 & C \\
\hline HETATM & 3527 & H 21 & VIN & 380 & -7.352 & -12.399 & 3.184 & 1.00 & 0.00 & $\mathrm{H}$ \\
\hline HETATM & 3528 & $\mathrm{C} 1$ & VIN & 380 & -9.185 & $-13 \cdot 144$ & 2.484 & 1.00 & 0.00 & C \\
\hline HETATM & 3529 & H11 & VIN & 380 & -9.865 & $-13 \cdot 300$ & 1.654 & 1.00 & 0.00 & $\mathrm{H}$ \\
\hline HETATM & 3530 & $\mathrm{H} 12$ & VIN & 380 & -9.490 & -13.542 & 3.450 & 1.00 & 0.00 & $\mathrm{H}$ \\
\hline TER & & & & & & & & & & \\
\hline HETATM & 3531 & $\mathrm{C} 1$ & MET & 381 & -9.703 & -10.816 & 0.121 & 1.00 & 0.00 & C \\
\hline HETATM & 3532 & H11 & MET & 381 & -9.859 & -9.794 & -0.246 & 1.00 & 0.00 & $\mathrm{H}$ \\
\hline HETATM & 3533 & $\mathrm{H} 12$ & MET & 381 & -10.135 & -10.882 & 1.126 & 1.00 & 0.00 & $\mathrm{H}$ \\
\hline HETATM & 3534 & $\mathrm{H} 13$ & MET & 381 & -10.294 & -11.480 & -0.528 & 1.00 & 0.00 & $\mathrm{H}$ \\
\hline TER & & & & & & & & & & \\
\hline HETATM & 3535 & $\mathrm{C} 5$ & $\mathrm{ACP}$ & 382 & -0.615 & -9.735 & -5.770 & 1.00 & 0.00 & C \\
\hline HETATM & 3536 & H51 & $\mathrm{ACP}$ & 382 & -1.354 & -9.723 & -6.582 & 1.00 & 0.00 & $\mathrm{H}$ \\
\hline HETATM & 3537 & H52 & $\mathrm{ACP}$ & 382 & 0.226 & $-10 \cdot 343$ & -6.134 & 1.00 & 0.00 & $\mathrm{H}$ \\
\hline HETATM & 3538 & $\mathrm{C} 4$ & $\mathrm{ACP}$ & 382 & -0.136 & -8.297 & -5.497 & 1.00 & 0.00 & C \\
\hline HETATM & 3539 & H 41 & $\mathrm{ACP}$ & 382 & 0.422 & -8.236 & -4.549 & 1.00 & 0.00 & $\mathrm{H}$ \\
\hline HETATM & 3540 & $\mathrm{H} 42$ & $\mathrm{ACP}$ & 382 & -1.007 & -7.630 & -5.390 & 1.00 & 0.00 & $\mathrm{H}$ \\
\hline HETATM & 3541 & $\mathrm{C} 1$ & $\mathrm{ACP}$ & 382 & 0.728 & -7.685 & -6.573 & 1.00 & 0.00 & C \\
\hline HETATM & 3542 & $\mathrm{O} 2$ & $\mathrm{ACP}$ & 382 & 0.956 & -8.208 & -7.671 & 1.00 & 0.00 & 0 \\
\hline HETATM & 3543 & 03 & $\mathrm{ACP}$ & 382 & 1.244 & -6.505 & -6.209 & 1.00 & 0.00 & 0 \\
\hline HETATM & 3544 & H3 & $\mathrm{ACP}$ & 382 & 1.853 & -6.151 & -6.945 & 1.00 & 0.00 & $\mathrm{H}$ \\
\hline TER & & & & & & & & & & \\
\hline HETATM & 3545 & $\mathrm{C} 1$ & MET & 383 & -8.511 & -8.608 & -4.597 & 1.00 & 0.00 & C \\
\hline HETATM & 3546 & H11 & MET & 383 & -9.063 & -9.448 & -5.047 & 1.00 & 0.00 & $\mathrm{H}$ \\
\hline HETATM & 3547 & $\mathrm{H} 12$ & MET & 383 & -8.517 & -7.782 & -5.319 & 1.00 & 0.00 & $\mathrm{H}$ \\
\hline HETATM & 3548 & $\mathrm{H} 13$ & MET & 383 & -9.082 & -8.289 & -3.717 & 1.00 & 0.00 & $\mathrm{H}$ \\
\hline TER & & & & & & & & & & \\
\hline HETATM & 3549 & $\mathrm{C} 1$ & MET & 384 & 0.923 & -11.557 & -3.646 & 1.00 & 0.00 & C \\
\hline
\end{tabular}


HETATM 3550 H11 MET 384 HETATM 3551 H12 MET 384 HETATM 3552 H13 MET 384 TER HETATM 3554 C1 HEM 385 HETATM 3555 C2 HEM 385 HETATM 3556 C3 HEM 385 HETATM 3557 C4 HEM 385 HETATM 3558 C5 HEM 385 HETATM 3559 H5 HEM 385 HETATM 3560 C6 HEM 385 HETATM $3561 \quad$ C7 HEM 385 HETATM 3562 C8 HEM 385 HETATM 3563 C9 HEM 385 HETATM 3564 C10 HEM 385 HETATM 3565 H1O HEM 385 HETATM 3566 C11 HEM 385 HETATM 3567 C12 HEM 38 HETATM 3568 C13 HEM 385 HETATM 3569 C14 HEM 385 HETATM 3570 C15 HEM 385 HETATM 3571 H15 HEM 38 HETATM 3572 C16 HEM 385 HETATM 3573 C17 HEM 385 HETATM $3574 \quad$ C18 HEM 385 HETATM 3575 C19 HEM 385 HETATM 3576 C20 HEM 385 HETATM 3577 H2O HEM 385 HETATM 3578 N21 HEM 38 HETATM 3579 N22 HEM 385 HETATM 3580 N23 HEM 385 HETATM 3581 N24 HEM 385 HETATM 3582 C99 HEM 38 HETATM $3583 \quad 091$ HEM 385 HETATM $3584 \quad 092$ HEM 385 HETATM 3585 CT91 HEM 385 HETATM 3586 H911 HEM 38 HETATM 3587 H912 HEM 385 HETATM 3588 CT92 HEM 385 HETATM 3589 H921 HEM 38 HETATM 3590 H922 HEM 385

HETATM $3591 \quad$ FE HEM 386 HETATM 3592 C1 HEM 38 HETATM $3593 \quad$ C2 HEM 386 HETATM 3594 C3 HEM 386 HETATM 3595 C4 HEM 386 HETATM 3596 C5 HEM 386 HETATM 3597 H5 HEM 386 HETATM 3598 C6 HEM 386 HETATM 3599 C7 HEM 38 HETATM $3600 \quad$ C 8 HEM 386 HETATM $3601 \quad$ C9 HEM 386 HETATM 3602 C10 HEM 386 HETATM 3603 H10 HEM 386 HETATM 3604 C11 HEM 386 HETATM 3605 C12 HEM 386 HETATM 3606 C13 HEM 38 HETATM 3607 C14 HEM 386 HETATM 3608 C15 HEM 386 HETATM 3609 H15 HEM 386 HETATM 3610 C16 HEM 38 HETATM 3611 C17 HEM 386 HETATM 3612 C18 HEM 386 HETATM 3613 C19 HEM 386 HETATM 3614 C20 HEM 38 HETATM 3615 H20 HEM 386 HETATM 3616 N21 HEM 386 HETATM 3617 N22 HEM 386 HETATM 3618 N23 HEM 38 HETATM 3619 N24 HEM 386 HETATM 3620 C99 HEM 386 HETATM $3621 \quad 091$ HEM 386 HETATM 3622092 HEM 386 HETATM 3623 CT91 HEM 386 HETATM 3624 H911 HEM 386 HETATM 3625 H912 HEM 386 HETATM 3626 CT92 HEM 38 $\begin{array}{llllll}1.368 & -11.348 & -4.626 & 1.00 & 0.00 \quad \mathrm{H}\end{array}$

$\begin{array}{lllll}1.033 & -12.637 & -3.471 & 1.00 & 0.00\end{array}$

$\begin{array}{lllll}1.523 & -11.031 & -2.889 & 1.00 & 0.00\end{array}$

$9.423 \quad 1.00 \quad 0.00$

$8.361 \quad 1.00 \quad 0.00$

$\begin{array}{lll}7.189 & 1.00 & 0.00\end{array}$

$6.414 \quad 1.00 \quad 0.00$

$\begin{array}{lll}7.122 & 1.00 & 0.00\end{array}$

$6.684 \quad 1.00 \quad 0.00$

$5.749 \quad 1.00 \quad 0.00$

$\begin{array}{lll}7.320 & 1.00 & 0.00\end{array}$

$\begin{array}{lll}6.851 & 1.00 & 0.00\end{array}$

$\begin{array}{lll}7.798 & 1.00 & 0.00\end{array}$

$8.822 \quad 1.00 \quad 0.00$

$9.965 \quad 1.00 \quad 0.00$

$\begin{array}{lll}10.071 & 1.00 & 0.00\end{array}$

$10.988 \quad 1.00 \quad 0.00$

$\begin{array}{lll}12.178 & 1.00 & 0.00\end{array}$

$12.9351 .00 \quad 0.00$

$\begin{array}{lll}12.204 & 1.00 & 0.00\end{array}$

$12.625 \quad 1.00 \quad 0.00$

$\begin{array}{lllll}-11.367 & 23.674 & 12.625 & 1.00 & 0.00 \\ -11.608 & 23.189 & 13.573 & 1.00 & 0.00\end{array}$

$\begin{array}{lllll}-12.398 & 24.356 & 11.982 & 1.00 & 0.00\end{array}$

$\begin{array}{lllll}-14.439 & 25.237 & 11.541 & 1.00 & 0.00\end{array}$

$\begin{array}{lllll}-13.489 & 25.589 & 10.504 & 1.00 & 0.00\end{array}$

$\begin{array}{lllll}-13.781 & 26.380 & 9.393 & 1.00 & 0.00\end{array}$

$\begin{array}{lllll}-14.795 & 26.769 & 9.333 & 1.00 & 0.00\end{array}$

$\begin{array}{lllll}-11.590 & 26.367 & 8.298 & 1.00 & 0.00\end{array}$

$\begin{array}{lllll}-8.886 & 25.420 & 8.521 & 1.00 & 0.00\end{array}$

$\begin{array}{lllll}-9.557 & 24.157 & 11.021 & 1.00 & 0.00\end{array}$

$\begin{array}{llllll}-12.256 & 25.041 & 10.786 & 1.00 & 0.00\end{array}$

$\begin{array}{lllll}-11.350 & 22.176 & 8.283 & 1.00 & 0.00\end{array}$

$\begin{array}{lllll}-11.190 & 23.489 & 8.296 & 1.00 & 0.00\end{array}$

$\begin{array}{lllll}-11.504 & 21.559 & 7.228 & 1.00 & 0.00\end{array}$

$\begin{array}{lllll}-11.333 & 21.495 & 9.640 & 1.00 & 0.00\end{array}$

$\begin{array}{lllll}-10.355 & 21.690 & 10.103 & 1.00 & 0.00\end{array}$

$\begin{array}{lllll}-12.081 & 21.984 & 10.283 & 1.00 & 0.00\end{array}$

$\begin{array}{lllll}-11.593 & 19.990 & 9.528 & 1.00 & 0.00\end{array}$

$\begin{array}{lllll}-10.797 & 19.530 & 8.931 & 1.00 & 0.00\end{array}$

$\begin{array}{lllll}-12.518 & 19.834 & 8.958 & 1.00 & 0.00\end{array}$

$\begin{array}{lllll}-9.992 & 17.444 & 14.371 & 1.00 & 0.00\end{array}$

$\begin{array}{lllll}-7.764 & 15.571 & 15.433 & 1.00 & 0.00\end{array}$

$\begin{array}{lllll}-7.405 & 14.781 & 16.605 & 1.00 & 0.00\end{array}$

$\begin{array}{lllll}-8.551 & 14.684 & 17.380 & 1.00 & 0.00\end{array}$

$\begin{array}{lllll}-9.580 & 15.401 & 16.672 & 1.00 & 0.00\end{array}$

$\begin{array}{lllll}-10.895 & 15.520 & 17.109 & 1.00 & 0.00\end{array}$

$\begin{array}{lllll}-11.149 & 15.024 & 18.045 & 1.00 & 0.00\end{array}$

$\begin{array}{lllll}-11.915 & 16.218 & 16.473 & 1.00 & 0.00\end{array}$

$\begin{array}{llllll}-13.284 & 16.291 & 16.943 & 1.00 & 0.00\end{array}$

$\begin{array}{lllll}-13.989 & 17.011 & 15.996 & 1.00 & 0.00\end{array}$

$\begin{array}{lllll}-13.038 & 17.376 & 14.972 & 1.00 & 0.00\end{array}$

$\begin{array}{lllll}-13.343 & 18.111 & 13.828 & 1.00 & 0.00\end{array}$

$\begin{array}{lllll}-14.369 & 18.454 & 13.722 & 1.00 & 0.00\end{array}$

$\begin{array}{lllll}-12.468 & 18.451 & 12.806 & 1.00 & 0.00\end{array}$

$\begin{array}{lllll}-12.849 & 19.176 & 11.615 & 1.00 & 0.00\end{array}$

$\begin{array}{lllll}-11.710 & 19.323 & 10.858 & 1.00 & 0.00\end{array}$

$\begin{array}{llllll}-10.637 & 18.682 & 11.590 & 1.00 & 0.00\end{array}$

$\begin{array}{lllll}-9.309 & 18.627 & 11.168 & 1.00 & 0.00\end{array}$

$\begin{array}{lllll}-9.068 & 19.112 & 10.221 & 1.00 & 0.00\end{array}$

$\begin{array}{lllll}-8.278 & 17.945 & 11.812 & 1.00 & 0.00\end{array}$

$\begin{array}{lllll}-6.912 & 17.832 & 11.329 & 1.00 & 0.00\end{array}$

$\begin{array}{lllll}-6.237 & 17.064 & 12.253 & 1.00 & 0.00\end{array}$

$\begin{array}{lllll}-7.187 & 16.712 & 13.289 & 1.00 & 0.00\end{array}$

$\begin{array}{lllll}-6.895 & 15.921 & 14.401 & 1.00 & 0.00\end{array}$

$\begin{array}{lllll}-5.881 & 15.532 & 14.461 & 1.00 & 0.00\end{array}$

$\begin{array}{lllll}-9.086 & 15.934 & 15.495 & 1.00 & 0.00\end{array}$

$\begin{array}{lllll}-11.790 & 16.881 & 15.273 & 1.00 & 0.00\end{array}$

$\begin{array}{lllll}-11.120 & 18.144 & 12.772 & 1.00 & 0.00\end{array}$

$\begin{array}{lllll}-8.420 & 17.260 & 13.008 & 1.00 & 0.00\end{array}$

$\begin{array}{lllll}-9.327 & 20.125 & 15.510 & 1.00 & 0.00\end{array}$

$\begin{array}{lllll}-9.486 & 18.812 & 15.498 & 1.00 & 0.00\end{array}$

$\begin{array}{lllll}-9.172 & 20.742 & 16.566 & 1.00 & 0.00\end{array}$

$\begin{array}{lllll}-9.343 & 20.807 & 14.154 & 1.00 & 0.00\end{array}$

$\begin{array}{lllll}-10.321 & 20.611 & 13.691 & 1.00 & 0.00\end{array}$

$\begin{array}{llllll}-8.595 & 20.317 & 13.511 & 1.00 & 0.00\end{array}$

$\begin{array}{lllll}-9.083 & 22.311 & 14.265 & 1.00 & 0.00\end{array}$

C 
$\begin{array}{lllll}\text { HETATM } & 3627 & \text { H921 } & \text { HEM } & 386 \\ \text { HETATM } & 3628 & \text { H922 } & \text { HEM } & 386\end{array}$ TER

HERTM 3629 C2 VIN 387

HETATM 3630 H21 VIN 38

HETATM 3631 C1 VIN 387

HETATM 3632 H11 VIN 387

HETATM 3633 H12 VIN 387

TER

HETATM 3634 C1 MET 388

HETATM 3635 H11 MET 388

HETATM 3636 H12 MET 38

HETATM 3637 H13 MET 388

$T E R$

HETATM 3638 C2 VIN 389

HETATM 3639 H21 VIN 389

HETATM $3640 \quad$ C1 VIN 389

HETATM 3641 H11 VIN 389

HETATM 3642 H12 VIN 389

TER

HETATM 3643 C1 MET

HETATM 3644 H11 MET

HETATM 3645 H12 MET 390

HETATM 3646 H13 MET 390

TER

HETATM $3647 \quad$ C5 ACP 391

HETATM 3648 H51 ACP 391

HETATM 3649 H52 ACP 391

HETATM 3650 C4 ACP 391

HETATM 3651 H41 ACP 39

HETATM 3652 H42 ACP 391

HETATM 3653 C1 ACP 391

HETATM 3654 O2 ACP 391

HETATM 3655 O3 ACP 391

HETATM 3656 H3 ACP 391

TER

HETATM 3657 C1 MET 392

HETATM 3658 H11 MET 392

HETATM 3659 H12 MET 392

HETATM 3660 H13 MET 392

HETATM 3661 C1 MET 393

HETATM 3662 H11 MET 393

HETATM 3663 H12 MET 393

HETATM 3664 H13 MET 393

TER

HETATM 3665 C2 VIN 394

HETATM 3666 H21 VIN 394

HETATM 3667 C1 VIN 394

HETATM 3668 H11 VIN 394

HETATM 3669 H12 VIN 394

(T)

HETATM $3670 \quad$ C1 MET 395

HETATM 3671 H11 MET 395

HETATM 3672 H12 MET 395

HETATM 3673 H13 MET 395

TER

HETATM $3674 \quad$ C2 VIN 396

HETATM 3675 H21 VIN 396

HETATM $3676 \quad$ C1 VIN 396

HETATM 3677 H11 VIN 396

HETATM 3678 H12 VIN 396

TER

HETATM 3679 C1 MET 397

HETATM 3680 H11 MET 397

HETATM 3681 H12 MET 397

HETATM 3682 H13 MET 397

TER

HETATM $3683 \quad C 5 \mathrm{ACP} \quad 398$

HETATM 3684 H51 ACP 398

HETATM 3685 H52 ACP 398

HETATM $3686 \quad$ C4 ACP 398

HETATM 3687 H41 ACP 398

HETATM 3688 H42 ACP 398

HETATM 3689 C1 ACP 398

HETATM $3690 \quad 02 \mathrm{ACP} \quad 398$

HETATM $3691 \quad 03$ ACP 398

HETATM 3692 H3 ACP 398

\begin{tabular}{|c|c|c|c|c|c|}
\hline-8.158 & 22.467 & 14.836 & 1.00 & 0.00 & $\mathrm{H}$ \\
\hline-9.879 & 22.771 & 14.862 & 1.00 & 0.00 & $\mathrm{H}$ \\
\hline-14.603 & 28.023 & 6.929 & 1.00 & 0.00 & C \\
\hline-15.419 & 27.324 & 7.094 & 1.00 & 0.00 & $\mathrm{H}$ \\
\hline-14.936 & 29.248 & 6.528 & 1.00 & 0.00 & C \\
\hline-14.204 & 30.026 & 6.421 & 1.00 & 0.00 & $\mathrm{H}$ \\
\hline-15.977 & 29.517 & 6.361 & 1.00 & 0.00 & $\mathrm{H}$ \\
\hline-11.939 & 28.278 & 5.091 & 1.00 & 0.00 & C \\
\hline-11.704 & 29.349 & 5.211 & 1.00 & 0.00 & $\mathrm{H}$ \\
\hline-11.126 & 27.814 & 4.522 & 1.00 & 0.00 & $\mathrm{H}$ \\
\hline-12.847 & 28.200 & 4.481 & 1.00 & 0.00 & $\mathrm{H}$ \\
\hline-6.929 & 26.600 & 5.606 & 1.00 & 0.00 & C \\
\hline-7.595 & 26.499 & 4.747 & 1.00 & 0.00 & $\mathrm{H}$ \\
\hline-5.762 & 27.244 & 5.447 & 1.00 & 0.00 & C \\
\hline-5.082 & 27.400 & 6.277 & 1.00 & 0.00 & $\mathrm{H}$ \\
\hline-5.457 & 27.642 & 4.482 & 1.00 & 0.00 & $\mathrm{H}$ \\
\hline-5.244 & 24.917 & 7.810 & 1.00 & 0.00 & C \\
\hline-4.653 & 25.581 & 8.459 & 1.00 & 0.00 & $\mathrm{H}$ \\
\hline-5.088 & 23.894 & 8.177 & 1.00 & 0.00 & $\mathrm{H}$ \\
\hline-4.812 & 24.982 & 6.805 & 1.00 & 0.00 & $\mathrm{H}$ \\
\hline-14.333 & 23.835 & 13.701 & 1.00 & 0.00 & C \\
\hline-13.594 & 23.824 & 14.514 & 1.00 & 0.00 & $\mathrm{H}$ \\
\hline-15.173 & 24.443 & 14.065 & 1.00 & 0.00 & $\mathrm{H}$ \\
\hline-14.811 & 22.397 & 13.428 & 1.00 & 0.00 & C \\
\hline-15.369 & 22.336 & 12.481 & 1.00 & 0.00 & $\mathrm{H}$ \\
\hline-13.941 & 21.731 & 13.321 & 1.00 & 0.00 & $\mathrm{H}$ \\
\hline-15.675 & 21.785 & 14.504 & 1.00 & 0.00 & C \\
\hline-15.903 & 22.308 & 15.603 & 1.00 & 0.00 & 0 \\
\hline-16.191 & 20.606 & 14.140 & 1.00 & 0.00 & o \\
\hline-16.800 & 20.251 & 14.876 & 1.00 & 0.00 & $\mathrm{H}$ \\
\hline-6.437 & 22.709 & 12.528 & 1.00 & 0.00 & C \\
\hline-5.884 & 23.548 & 12.978 & 1.00 & 0.00 & $\mathrm{H}$ \\
\hline-6.430 & 21.883 & 13.250 & 1.00 & 0.00 & $\mathrm{H}$ \\
\hline-5.866 & 22.389 & 11.649 & 1.00 & 0.00 & $\mathrm{H}$ \\
\hline-15.870 & 25.657 & 11.577 & 1.00 & 0.00 & C \\
\hline-16.315 & 25.449 & 12.557 & 1.00 & 0.00 & $\mathrm{H}$ \\
\hline-15.980 & 26.738 & 11.402 & 1.00 & 0.00 & $\mathrm{H}$ \\
\hline-16.471 & 25.132 & 10.821 & 1.00 & 0.00 & $\mathrm{H}$ \\
\hline-6.073 & 14.278 & 16.864 & 1.00 & 0.00 & C \\
\hline-5.258 & 14.977 & 16.699 & 1.00 & 0.00 & $\mathrm{H}$ \\
\hline-5.740 & 13.053 & 17.266 & 1.00 & 0.00 & C \\
\hline-6.472 & 12.275 & 17.373 & 1.00 & 0.00 & $\mathrm{H}$ \\
\hline-4.699 & 12.784 & 17.432 & 1.00 & 0.00 & $\mathrm{H}$ \\
\hline-8.737 & 14.023 & 18.702 & 1.00 & 0.00 & C \\
\hline-8.972 & 12.953 & 18.583 & 1.00 & 0.00 & $\mathrm{H}$ \\
\hline-9.550 & 14.488 & 19.272 & 1.00 & 0.00 & $\mathrm{H}$ \\
\hline-7.829 & 14.101 & 19.313 & 1.00 & 0.00 & $\mathrm{H}$ \\
\hline-13.747 & 15.701 & 18.188 & 1.00 & 0.00 & C \\
\hline-13.081 & 15.802 & 19.046 & 1.00 & 0.00 & $\mathrm{H}$ \\
\hline-14.914 & 15.057 & 18.346 & 1.00 & 0.00 & C \\
\hline-15.594 & 14.901 & 17.516 & 1.00 & 0.00 & $\mathrm{H}$ \\
\hline-15.219 & 14.659 & 19.312 & 1.00 & 0.00 & $\mathrm{H}$ \\
\hline-15.432 & 17.385 & 15.984 & 1.00 & 0.00 & C \\
\hline-15.588 & 18.407 & 15.617 & 1.00 & 0.00 & $\mathrm{H}$ \\
\hline-15.864 & 17.319 & 16.989 & 1.00 & 0.00 & $\mathrm{H}$ \\
\hline-16.023 & 16.720 & 15.335 & 1.00 & 0.00 & $\mathrm{H}$ \\
\hline-6.343 & 18.466 & 10.092 & 1.00 & 0.00 & C \\
\hline-7.082 & 18.478 & 9.280 & 1.00 & 0.00 & $\mathrm{H}$ \\
\hline-5.503 & 17.858 & 9.729 & 1.00 & 0.00 & $\mathrm{H}$ \\
\hline-5.865 & 19.904 & 10.365 & 1.00 & 0.00 & C \\
\hline-5.307 & 19.965 & 11.313 & 1.00 & 0.00 & $\mathrm{H}$ \\
\hline-6.735 & 20.570 & 10.472 & 1.00 & 0.00 & $\mathrm{H}$ \\
\hline-5.001 & 20.516 & 9.289 & 1.00 & 0.00 & C \\
\hline-4.773 & 19.993 & 8.191 & 1.00 & 0.00 & 0 \\
\hline-4.485 & 21.696 & 9.653 & 1.00 & 0.00 & 0 \\
\hline-3.876 & 22.050 & 8.917 & 1.00 & 0.00 & $\mathrm{H}$ \\
\hline
\end{tabular}




\begin{tabular}{|c|c|c|c|c|c|c|c|c|c|c|}
\hline HETATM & 3693 & $\mathrm{C} 1$ & $\mathrm{MET}$ & 399 & -14.239 & 19.593 & 11.266 & 1.00 & 0.00 & C \\
\hline HETATM & 3694 & H11 & $\mathrm{MET}$ & 399 & -14.792 & 18.753 & 10.815 & 1.00 & 0.00 & $\mathrm{H}$ \\
\hline HETATM & 3695 & H12 & $\mathrm{MET}$ & 399 & -14.246 & 20.418 & 10.544 & 1.00 & 0.00 & $\mathrm{H}$ \\
\hline HETATM & 3696 & H13 & MET & 399 & -14.810 & 19.912 & 12.145 & 1.00 & 0.00 & $\mathrm{H}$ \\
\hline \multicolumn{11}{|l|}{ TER } \\
\hline HETATM & 3697 & $\mathrm{C} 1$ & $\mathrm{MET}$ & 400 & -4.806 & 16.644 & 12.216 & 1.00 & 0.00 & C \\
\hline HETATM & 3698 & H11 & $\mathrm{MET}$ & 400 & -4.361 & 16.853 & 11.236 & 1.00 & 0.00 & $\mathrm{H}$ \\
\hline HETATM & 3699 & H12 & $\mathrm{MET}$ & 400 & -4.696 & 15.564 & 12.391 & 1.00 & 0.00 & $\mathrm{H}$ \\
\hline HETATM & 3700 & H13 & $\mathrm{MET}$ & 400 & -4.205 & 17.169 & 12.973 & 1.00 & 0.00 & $\mathrm{H}$ \\
\hline \multicolumn{11}{|l|}{ TER } \\
\hline HETATM & 3701 & $\mathrm{FE}$ & HEM & 401 & -5.903 & -3.589 & 1.491 & 1.00 & 0.00 & $\mathrm{Fe}$ \\
\hline HETATM & 3702 & $\mathrm{C} 1$ & HEM & 401 & -8.131 & -1.717 & 0.429 & 1.00 & 0.00 & C \\
\hline HETATM & 3703 & $\mathrm{C} 2$ & HEM & 401 & -8.490 & -0.927 & -0.742 & 1.00 & 0.00 & C \\
\hline HETATM & 3704 & C3 & HEM & 401 & -7.344 & -0.830 & -1.517 & 1.00 & 0.00 & C \\
\hline HETATM & 3705 & C4 & HEM & 401 & -6.315 & -1.547 & -0.809 & 1.00 & 0.00 & $\mathrm{C}$ \\
\hline HETATM & 3706 & C5 & HEM & 401 & -5.000 & -1.666 & -1.247 & 1.00 & 0.00 & C \\
\hline HETATM & 3707 & H5 & HEM & 401 & -4.746 & -1.170 & -2.182 & 1.00 & 0.00 & $\mathrm{H}$ \\
\hline HETATM & 3708 & $\mathrm{C} 6$ & HEM & 401 & -3.980 & -2.364 & -0.611 & 1.00 & 0.00 & C \\
\hline HETATM & 3709 & C7 & HEM & 401 & -2.611 & -2.437 & -1.081 & 1.00 & 0.00 & C \\
\hline HETATM & 3710 & $\mathrm{C} 8$ & HEM & 401 & -1.906 & -3.157 & -0.134 & 1.00 & 0.00 & C \\
\hline HETATM & 3711 & C9 & HEM & 401 & -2.857 & -3.522 & 0.891 & 1.00 & 0.00 & C \\
\hline HETATM & 3712 & $\mathrm{C} 10$ & HEM & 401 & -2.552 & -4.256 & 2.034 & 1.00 & 0.00 & C \\
\hline HETATM & 3713 & H1O & HEM & 401 & -1.526 & -4.600 & 2.140 & 1.00 & 0.00 & $\mathrm{H}$ \\
\hline HETATM & 3714 & $\mathrm{C} 11$ & HEM & 401 & -3.427 & -4.596 & 3.057 & 1.00 & 0.00 & C \\
\hline HETATM & 3715 & $\mathrm{C} 12$ & HEM & 401 & -3.046 & -5.322 & 4.247 & 1.00 & 0.00 & C \\
\hline HETATM & 3716 & $\mathrm{C} 13$ & HEM & 401 & -4.185 & -5.469 & 5.004 & 1.00 & 0.00 & C \\
\hline HETATM & 3717 & $\mathrm{C} 14$ & HEM & 401 & -5.258 & -4.828 & 4.273 & 1.00 & 0.00 & C \\
\hline HETATM & 3718 & C15 & HEM & 401 & -6.586 & -4.773 & 4.694 & 1.00 & 0.00 & C \\
\hline HETATM & 3719 & H15 & HEM & 401 & -6.826 & -5.258 & 5.642 & 1.00 & 0.00 & $\mathrm{H}$ \\
\hline HETATM & 3720 & C16 & HEM & 401 & -7.617 & -4.091 & 4.051 & 1.00 & 0.00 & C \\
\hline HETATM & 3721 & $\mathrm{C} 17$ & HEM & 401 & -8.982 & -3.978 & 4.533 & 1.00 & 0.00 & C \\
\hline HETATM & 3722 & $\mathrm{C} 18$ & HEM & 401 & -9.658 & -3.210 & 3.609 & 1.00 & 0.00 & C \\
\hline HETATM & 3723 & C19 & HEM & 401 & -8.708 & -2.858 & 2.573 & 1.00 & 0.00 & $\mathrm{C}$ \\
\hline HETATM & 3724 & $\mathrm{C} 20$ & HEM & 401 & -8.999 & -2.067 & 1.462 & 1.00 & 0.00 & $\mathrm{C}$ \\
\hline HETATM & 3725 & $\mathrm{H} 2 \mathrm{O}$ & HEM & 401 & -10.014 & -1.678 & 1.402 & 1.00 & 0.00 & $\mathrm{H}$ \\
\hline HETATM & 3726 & N21 & HEM & 401 & -6.809 & -2.080 & 0.367 & 1.00 & 0.00 & $\mathrm{~N}$ \\
\hline HETATM & 3727 & N22 & HEM & 401 & -4.105 & -3.027 & 0.590 & 1.00 & 0.00 & $\mathrm{~N}$ \\
\hline HETATM & 3728 & N23 & HEM & 401 & -4.775 & -4.290 & 3.090 & 1.00 & 0.00 & $\mathrm{~N}$ \\
\hline HETATM & 3729 & N24 & HEM & 401 & -7.475 & -3.406 & 2.854 & 1.00 & 0.00 & $\mathrm{~N}$ \\
\hline HETATM & 3730 & C99 & HEM & 401 & -6.568 & -6.271 & 0.352 & 1.00 & 0.00 & C \\
\hline HETATM & 3731 & 091 & HEM & 401 & -6.409 & -4.958 & 0.365 & 1.00 & 0.00 & 0 \\
\hline HETATM & 3732 & 092 & HEM & 401 & -6.723 & -6.888 & -0.703 & 1.00 & 0.00 & O \\
\hline HETATM & 3733 & СТ91 & HEM & 401 & -6.552 & -6.952 & 1.709 & 1.00 & 0.00 & C \\
\hline HETATM & 3734 & H911 & HEM & 401 & -5.573 & -6.757 & 2.171 & 1.00 & 0.00 & $\mathrm{H}$ \\
\hline HETATM & 3735 & H912 & HEM & 401 & -7.300 & -6.463 & 2.352 & 1.00 & 0.00 & $\mathrm{H}$ \\
\hline HETATM & 3736 & СТ92 & HEM & 401 & -6.812 & -8.457 & 1.597 & 1.00 & 0.00 & C \\
\hline HETATM & 3737 & H921 & HEM & 401 & -6.015 & -8.917 & 1.000 & 1.00 & 0.00 & $\mathrm{H}$ \\
\hline HETATM & 3738 & H922 & HEM & 401 & -7.737 & -8.613 & 1.027 & 1.00 & 0.00 & $\mathrm{H}$ \\
\hline \multicolumn{11}{|l|}{ TER } \\
\hline HETATM & 3739 & $\mathrm{FE}$ & HEM & 402 & -5.211 & -11.003 & 6.440 & 1.00 & 0.00 & $\mathrm{Fe}$ \\
\hline HETATM & 3740 & $\mathrm{C} 1$ & HEM & 402 & -2.983 & -12.876 & 7.502 & 1.00 & 0.00 & C \\
\hline HETATM & 3741 & $\mathrm{C} 2$ & HEM & 402 & -2.624 & -13.666 & 8.674 & 1.00 & 0.00 & C \\
\hline HETATM & 3742 & C3 & HEM & 402 & -3.770 & -13.763 & 9.448 & 1.00 & 0.00 & C \\
\hline HETATM & 3743 & $\mathrm{C} 4$ & HEM & 402 & -4.799 & -13.046 & 8.740 & 1.00 & 0.00 & C \\
\hline HETATM & 3744 & C5 & HEM & 402 & -6.114 & -12.927 & 9.178 & 1.00 & 0.00 & C \\
\hline HETATM & 3745 & H5 & HEM & 402 & -6.368 & -13.423 & 10.113 & 1.00 & 0.00 & $\mathrm{H}$ \\
\hline HETATM & 3746 & $\mathrm{C} 6$ & HEM & 402 & -7.134 & -12.229 & 8.542 & 1.00 & 0.00 & C \\
\hline HETATM & 3747 & C7 & HEM & 402 & -8.502 & -12.156 & 9.012 & 1.00 & 0.00 & C \\
\hline HETATM & 3748 & $\mathrm{C} 8$ & HEM & 402 & -9.208 & -11.436 & 8.065 & 1.00 & 0.00 & C \\
\hline HETATM & 3749 & C9 & HEM & 402 & -8.257 & -11.071 & 7.041 & 1.00 & 0.00 & C \\
\hline HETATM & 3750 & $\mathrm{C} 10$ & HEM & 402 & -8.561 & -10.336 & 5.897 & 1.00 & 0.00 & C \\
\hline HETATM & 3751 & $\mathrm{H} 10$ & HEM & 402 & -9.588 & -9.993 & 5.791 & 1.00 & 0.00 & $\mathrm{H}$ \\
\hline HETATM & 3752 & $\mathrm{C} 11$ & HEM & 402 & -7.687 & -9.996 & 4.874 & 1.00 & 0.00 & C \\
\hline HETATM & 3753 & $\mathrm{C} 12$ & HEM & 402 & -8.068 & -9.271 & 3.684 & 1.00 & 0.00 & C \\
\hline HETATM & 3754 & $\mathrm{C} 13$ & HEM & 402 & -6.928 & -9.124 & 2.927 & 1.00 & 0.00 & C \\
\hline HETATM & 3755 & C14 & HEM & 402 & -5.855 & -9.765 & 3.659 & 1.00 & 0.00 & C \\
\hline HETATM & 3756 & C15 & HEM & 402 & -4.527 & -9.820 & 3.237 & 1.00 & 0.00 & C \\
\hline HETATM & 3757 & H15 & HEM & 402 & -4.287 & -9.335 & 2.289 & 1.00 & 0.00 & $\mathrm{H}$ \\
\hline HETATM & 3758 & C16 & HEM & 402 & -3.497 & -10.502 & 3.880 & 1.00 & 0.00 & C \\
\hline HETATM & 3759 & $\mathrm{C} 17$ & HEM & 402 & -2.131 & -10.615 & 3.398 & 1.00 & 0.00 & C \\
\hline HETATM & 3760 & $\mathrm{C} 18$ & HEM & 402 & -1.456 & -11.383 & 4.322 & 1.00 & 0.00 & C \\
\hline HETATM & 3761 & C19 & HEM & 402 & -2.406 & -11.735 & 5.358 & 1.00 & 0.00 & C \\
\hline HETATM & 3762 & $\mathrm{C} 20$ & HEM & 402 & -2.114 & -12.526 & 6.469 & 1.00 & 0.00 & C \\
\hline HETATM & 3763 & $\mathrm{H} 2 \mathrm{O}$ & HEM & 402 & -1.100 & -12.915 & 6.530 & 1.00 & 0.00 & $\mathrm{H}$ \\
\hline HETATM & 3764 & N21 & HEM & 402 & -4.305 & -12.513 & 7.564 & 1.00 & 0.00 & $\mathrm{~N}$ \\
\hline HETATM & 3765 & N22 & HEM & 402 & -7.009 & -11.566 & 7.342 & 1.00 & 0.00 & $\mathrm{~N}$ \\
\hline HETATM & 3766 & N23 & HEM & 402 & -6.338 & -10.303 & 4.841 & 1.00 & 0.00 & $\mathrm{~N}$ \\
\hline HETATM & 3767 & N24 & HEM & 402 & -3.639 & -11.187 & 5.077 & 1.00 & 0.00 & $\mathrm{~N}$ \\
\hline HETATM & 3768 & C99 & HEM & 402 & -4.545 & -8.322 & 7.579 & 1.00 & 0.00 & C \\
\hline
\end{tabular}


HETATM 3769091 HEM 402 HETATM $3770 \quad 092$ HEM HETATM 3771 CT91 HEM HETATM 3772 H911 HEM HETATM 3773 H912 HEM HETATM 3774 CT92 HEM HETATM 3775 H921 HEM HETATM 3776 H922 HEM TER

HETATM 3777 C2 VIN HETATM 3778 H21 VIN HETATM 3779 C1 VIN HETATM 3780 H11 VIN HETATM 3781 H12 VIN

HETATM 3782 C1 MET HETATM 3783 H11 MET HETATM 3784 H12 MET HETATM 3785 H13 MET TER

HETATM 3786 C2 VIN HETATM 3787 H21 VIN HETATM 3788 C1 VIN HETATM 3789 H11 VIN HETATM 3790 H12 VIN TER

HETATM $3791 \quad$ C1 MET HETATM 3792 H11 MET HETATM 3793 H12 MET HETATM 3794 H13 MET TER

HETATM 3795 C5 ACP HETATM 3796 H51 ACP HETATM 3797 H52 ACP HETATM 3798 C4 ACP HETATM 3799 H41 ACP HETATM $3800 \quad$ H42 ACP HETATM $3801 \quad$ C1 ACP HETATM $3802 \quad 02$ ACP HETATM $3803 \quad 03$ ACP HETATM 3804 H3 ACP TER

HETATM 3805 C1 MET HETATM 3806 H11 MET HETATM 3807 H12 MET HETATM 3808 H13 MET TER

HETATM 3809 C1 MET HETATM 3810 H11 MET HETATM 3811 H12 MET HETATM 3812 H13 MET TER

HETATM 3813 C2 VIN HETATM 3814 H21 VIN HETATM 3815 C1 VIN HETATM 3816 H11 VIN HETATM 3817 H12 VIN TER

HETATM 3818 C1 MET HETATM 3819 H11 MET HETATM 3820 H12 MET HETATM 3821 H13 MET TER

HETATM 3822 C2 VIN HETATM 3823 H21 VIN HETATM 3824 C1 VIN HETATM 3825 H11 VIN HETATM 3826 H12 VIN TER

HETATM 3827 C1 MET HETATM 3828 H11 MET HETATM 3829 H12 MET HETATM 3830 H13 MET TER

HETATM 3831 C5 ACP HETATM 3832 H51 ACP HETATM 3833 H52 ACP HETATM 3834 C4 ACP HETATM 3835 H41 ACP

\begin{tabular}{|c|c|c|c|c|c|}
\hline-4.705 & -9.635 & 7.567 & 1.00 & 0.00 & o \\
\hline-4.391 & -7.705 & 8.635 & 1.00 & 0.00 & 0 \\
\hline-4.561 & -7.640 & 6.223 & 1.00 & 0.00 & C \\
\hline-5.540 & -7.836 & 5.760 & 1.00 & 0.00 & $\mathrm{H}$ \\
\hline-3.813 & -8.130 & 5.580 & 1.00 & 0.00 & $\mathrm{H}$ \\
\hline-4.302 & -6.136 & 6.334 & 1.00 & 0.00 & C \\
\hline-3.377 & -5.980 & 6.905 & 1.00 & 0.00 & $\mathrm{H}$ \\
\hline-5.098 & -5.676 & 6.931 & 1.00 & 0.00 & $\mathrm{H}$ \\
\hline-9.822 & -0.424 & -1.002 & 1.00 & 0.00 & C \\
\hline-10.637 & -1.123 & -0.837 & 1.00 & 0.00 & $\mathrm{H}$ \\
\hline-10.154 & 0.801 & -1.403 & 1.00 & 0.00 & C \\
\hline-9.423 & 1.579 & -1.511 & 1.00 & 0.00 & $\mathrm{H}$ \\
\hline-11.196 & 1.070 & -1.570 & 1.00 & 0.00 & $\mathrm{H}$ \\
\hline-7.158 & -0.169 & -2.840 & 1.00 & 0.00 & C \\
\hline-6.923 & 0.902 & -2.720 & 1.00 & 0.00 & $\mathrm{H}$ \\
\hline-6.345 & -0.633 & -3.410 & 1.00 & 0.00 & $\mathrm{H}$ \\
\hline-8.065 & -0.247 & -3.450 & 1.00 & 0.00 & $\mathrm{H}$ \\
\hline-2.148 & -1.847 & -2.325 & 1.00 & 0.00 & C \\
\hline-2.814 & -1.948 & -3.184 & 1.00 & 0.00 & $\mathrm{H}$ \\
\hline-0.981 & -1.203 & -2.484 & 1.00 & 0.00 & C \\
\hline-0.301 & -1.047 & -1.654 & 1.00 & 0.00 & $\mathrm{H}$ \\
\hline-0.676 & -0.805 & -3.450 & 1.00 & 0.00 & $\mathrm{H}$ \\
\hline-0.463 & -3.530 & -0.121 & 1.00 & 0.00 & C \\
\hline 0.128 & -2.866 & 0.528 & 1.00 & 0.00 & $\mathrm{H}$ \\
\hline-0.307 & -4.553 & 0.246 & 1.00 & 0.00 & $\mathrm{H}$ \\
\hline-0.031 & -3.465 & -1.126 & 1.00 & 0.00 & $\mathrm{H}$ \\
\hline-9.552 & -4.612 & 5.770 & 1.00 & 0.00 & C \\
\hline-8.813 & -4.623 & 6.582 & 1.00 & 0.00 & $\mathrm{H}$ \\
\hline-10.392 & -4.004 & 6.134 & 1.00 & 0.00 & $\mathrm{H}$ \\
\hline-10.030 & -6.050 & 5.497 & 1.00 & 0.00 & C \\
\hline-10.588 & -6.111 & 4.549 & 1.00 & 0.00 & $\mathrm{H}$ \\
\hline-9.160 & -6.716 & 5.390 & 1.00 & 0.00 & $\mathrm{H}$ \\
\hline-10.894 & -6.662 & 6.573 & 1.00 & 0.00 & C \\
\hline$-11 \cdot 122$ & -6.139 & 7.671 & 1.00 & 0.00 & o \\
\hline-11.410 & -7.841 & 6.209 & 1.00 & 0.00 & O \\
\hline-12.019 & -8.196 & 6.945 & 1.00 & 0.00 & $\mathrm{H}$ \\
\hline-1.655 & -5.738 & 4.597 & 1.00 & 0.00 & C \\
\hline-1.103 & -4.899 & 5.047 & 1.00 & 0.00 & $\mathrm{H}$ \\
\hline-1.649 & -6.564 & 5.319 & 1.00 & 0.00 & $\mathrm{H}$ \\
\hline-1.084 & -6.058 & 3.717 & 1.00 & 0.00 & $\mathrm{H}$ \\
\hline-11.089 & -2.790 & 3.646 & 1.00 & 0.00 & C \\
\hline-11.534 & -2.998 & 4.626 & 1.00 & 0.00 & $\mathrm{H}$ \\
\hline-11.199 & -1.709 & 3.471 & 1.00 & 0.00 & $\mathrm{H}$ \\
\hline-11.690 & -3.315 & 2.889 & 1.00 & 0.00 & $\mathrm{H}$ \\
\hline-1.292 & -14.169 & 8.933 & 1.00 & 0.00 & C \\
\hline-0.476 & -13.470 & 8.768 & 1.00 & 0.00 & $\mathrm{H}$ \\
\hline-0.959 & -15.394 & 9.335 & 1.00 & 0.00 & C \\
\hline-1.691 & -16.172 & 9.442 & 1.00 & 0.00 & $\mathrm{H}$ \\
\hline 0.082 & -15.663 & 9.501 & 1.00 & 0.00 & $\mathrm{H}$ \\
\hline-3.956 & -14.424 & 10.771 & 1.00 & 0.00 & C \\
\hline-4.191 & -15.494 & 10.652 & 1.00 & 0.00 & $\mathrm{H}$ \\
\hline-4.769 & -13.959 & 11.341 & 1.00 & 0.00 & $\mathrm{H}$ \\
\hline-3.048 & -14.346 & 11.382 & 1.00 & 0.00 & $\mathrm{H}$ \\
\hline-8.966 & -12.746 & 10.256 & 1.00 & 0.00 & C \\
\hline-8.300 & -12.645 & 11.115 & 1.00 & 0.00 & $\mathrm{H}$ \\
\hline-10.133 & -13.390 & 10.415 & 1.00 & 0.00 & C \\
\hline-10.813 & -13.546 & 9.585 & 1.00 & 0.00 & $\mathrm{H}$ \\
\hline-10.438 & -13.788 & 11.381 & 1.00 & 0.00 & $\mathrm{H}$ \\
\hline-10.651 & -11.062 & 8.052 & 1.00 & 0.00 & C \\
\hline-10.807 & -10.040 & 7.685 & 1.00 & 0.00 & $\mathrm{H}$ \\
\hline-11.083 & -11.128 & 9.057 & 1.00 & 0.00 & $\mathrm{H}$ \\
\hline-11.242 & -11.727 & 7.403 & 1.00 & 0.00 & $\mathrm{H}$ \\
\hline-1.562 & -9.981 & 2.161 & 1.00 & 0.00 & C \\
\hline-2.301 & -9.969 & 1.349 & 1.00 & 0.00 & $\mathrm{H}$ \\
\hline-0.722 & -10.589 & 1.798 & 1.00 & 0.00 & $\mathrm{H}$ \\
\hline-1.084 & -8.543 & 2.434 & 1.00 & 0.00 & C \\
\hline-0.526 & -8.482 & 3.382 & 1.00 & 0.00 & $\mathrm{H}$ \\
\hline
\end{tabular}


HETATM 3836 H42 ACP HETATM $3837-\quad \mathrm{C} 1 \mathrm{ACP}$ HETATM HETATM 3839 O3 ACP HETATM 3840 H3 ACP TER

HETATM 3841 C1 MET HETATM 3842 H11 MET HETATM 3843 H12 MET HETATM 3844 H13 MET

HETATM 3845 C1 MET HETATM 3846 H11 MET HETATM 38 HETATM 3848 H13 MET TER

HETATM 3849 FE HEM 417 HETATM 3850 C1 HEM 417 HETATM 3851 C2 HEM 417 HETATM 3852 C3 HEM 417 HETATM $3853 \quad C 4$ HEM 417 HETATM 3854 C5 HEM 417 HETATM 3855 H5 HEM 41 HETATM 3856 C6 HEM 417 HETATM 3857 C7 HEM 417 HETATM 3858 C8 HEM 417 HETATM 3859 C9 HEM 417 HETATM 3860 C10 HEM 417 HETATM 3861 H1O HEM 417 HETATM 3862 C11 HEM 41 HETATM 3863 C12 HEM 417 HETATM 3864 C13 HEM 417 HETATM 3865 C14 HEM 417 HETATM 3866 C15 HEM 417 HETATM 3867 H15 HEM 417 HETATM 3868 C16 HEM 417 HETATM 3869 C17 HEM 417 HETATM 3870 C18 HEM 41 HETATM 3871 C19 HEM 417 HETATM 3872 C20 HEM 417 HETATM 3873 H2O HEM 417 HETATM 3874 N21 HEM 417 HETATM 3875 N22 HEM 417 HETATM 3876 N23 HEM 417 HETATM 3877 N24 HEM 417 HETATM 3878 C99 HEM 417 HETATM 3879091 HEM 417 HETATM $3880 \quad 092$ HEM 417 HETATM 3881 CT91 HEM 417 HETATM 3882 H911 HEM 417 HETATM 3883 H912 HEM 417 HETATM 3884 CT92 HEM 41 HETATM 3885 H921 HEM HETATM 3886 H922 HEM HETATM 3888 C1 HEM 418 HETATM 3889 HETATM 3890 C3 HEM 418 HETATM 3891 C4 HEM 418 HETATM $3892 \quad$ C5 HEM 418 HETATM 3893 H5 HEM 418 HETATM 3894 C6 HEM 41 HETATM 3895 C7 HEM 418 HETATM 3896 C8 HEM 418 HETATM 3897 C9 HEM 418 HETATM 3898 C10 HEM 418 HETATM 3899 H1O HEM 418 HETATM 3900 C11 HEM 418 HETATM 3901 C12 HEM 418 HETATM 3902 C13 HEM 41 HETATM 3903 C14 HEM 418 HETATM 3904 C15 HEM 418 HETATM 3905 H15 HEM 41 HETATM 3906 C16 HEM 418 HETATM 3907 C17 HEM 418 HETATM 3908 C18 HEM 418 HETATM 3909 C19 HEM 418 HETATM 3910 C20 HEM

\begin{tabular}{|c|c|c|c|c|c|}
\hline-1.954 & -7.877 & 2.541 & 1.00 & 0.00 & $\mathrm{H}$ \\
\hline-0.220 & -7.931 & 1.358 & 1.00 & 0.00 & C \\
\hline 0.008 & -8.454 & 0.260 & 1.00 & 0.00 & 0 \\
\hline 0.296 & -6.751 & 1.722 & 1.00 & 0.00 & 0 \\
\hline 0.905 & -6.397 & 0.986 & 1.00 & 0.00 & $\mathrm{H}$ \\
\hline-9.458 & -8.855 & 3.334 & 1.00 & 0.00 & 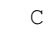 \\
\hline-10.011 & -9.694 & 2.884 & 1.00 & 0.00 & $\mathrm{H}$ \\
\hline-9.465 & -8.029 & 2.613 & 1.00 & 0.00 & $\mathrm{H}$ \\
\hline-10.029 & -8.535 & 4.214 & 1.00 & 0.00 & $\mathrm{H}$ \\
\hline-0.025 & -11.803 & 4.285 & 1.00 & 0.00 & C \\
\hline 0.420 & -11.594 & 3.305 & 1.00 & 0.00 & $\mathrm{H}$ \\
\hline 0.086 & -12.883 & 4.460 & 1.00 & 0.00 & $\mathrm{H}$ \\
\hline 0.576 & -11.278 & 5.042 & 1.00 & 0.00 & $\mathrm{H}$ \\
\hline-9.737 & 25.104 & 1.491 & 1.00 & 0.00 & $\mathrm{Fe}$ \\
\hline-11.964 & 26.976 & 0.429 & 1.00 & 0.00 & C \\
\hline$-12 \cdot 323$ & 27.767 & -0.742 & 1.00 & 0.00 & C \\
\hline-11.178 & 27.864 & -1.517 & 1.00 & 0.00 & C \\
\hline-10.148 & 27.146 & -0.809 & 1.00 & 0.00 & C \\
\hline-8.833 & 27.027 & -1.247 & 1.00 & 0.00 & C \\
\hline-8.580 & 27.524 & -2.182 & 1.00 & 0.00 & $\mathrm{H}$ \\
\hline-7.814 & 26.329 & -0.611 & 1.00 & 0.00 & C \\
\hline-6.445 & 26.256 & -1.081 & 1.00 & 0.00 & C \\
\hline-5.740 & 25.537 & -0.134 & 1.00 & 0.00 & C \\
\hline-6.690 & 25.172 & 0.891 & 1.00 & 0.00 & C \\
\hline-6.386 & 24.437 & 2.034 & 1.00 & 0.00 & C \\
\hline-5.359 & 24.093 & 2.140 & 1.00 & 0.00 & $\mathrm{H}$ \\
\hline-7.260 & 24.097 & 3.057 & 1.00 & 0.00 & $\mathrm{C}$ \\
\hline-6.880 & 23.371 & 4.247 & 1.00 & 0.00 & C \\
\hline-8.019 & 23.224 & 5.004 & 1.00 & 0.00 & C \\
\hline-9.092 & 23.866 & 4.273 & 1.00 & 0.00 & C \\
\hline-10.420 & 23.920 & 4.694 & 1.00 & 0.00 & C \\
\hline-10.660 & 23.436 & 5.642 & 1.00 & 0.00 & $\mathrm{H}$ \\
\hline-11.450 & 24.602 & 4.051 & 1.00 & 0.00 & C \\
\hline-12.816 & 24.716 & 4.533 & 1.00 & 0.00 & C \\
\hline-13.491 & 25.483 & 3.609 & 1.00 & 0.00 & C \\
\hline-12.542 & 25.835 & 2.573 & 1.00 & 0.00 & C \\
\hline-12.833 & 26.626 & 1.462 & 1.00 & 0.00 & C \\
\hline-13.847 & 27.015 & 1.402 & 1.00 & 0.00 & $\mathrm{H}$ \\
\hline-10.643 & 26.613 & 0.367 & 1.00 & 0.00 & $\mathrm{~N}$ \\
\hline-7.938 & 25.666 & 0.590 & 1.00 & 0.00 & $\mathrm{~N}$ \\
\hline-8.609 & 24.403 & 3.090 & 1.00 & 0.00 & $\mathrm{~N}$ \\
\hline-11.309 & 25.287 & 2.854 & 1.00 & 0.00 & $\mathrm{~N}$ \\
\hline-10.402 & 22.422 & 0.352 & 1.00 & 0.00 & C \\
\hline-10.242 & 23.736 & 0.365 & 1.00 & 0.00 & 0 \\
\hline-10.557 & 21.805 & -0.703 & 1.00 & 0.00 & o \\
\hline-10.386 & 21.741 & 1.709 & 1.00 & 0.00 & C \\
\hline-9.407 & 21.936 & 2.171 & 1.00 & 0.00 & $\mathrm{H}$ \\
\hline-11.134 & 22.231 & 2.352 & 1.00 & 0.00 & $\mathrm{H}$ \\
\hline-10.645 & 20.236 & 1.597 & 1.00 & 0.00 & C \\
\hline-9.849 & 19.776 & 1.000 & 1.00 & 0.00 & $\mathrm{H}$ \\
\hline-11.570 & 20.081 & 1.027 & 1.00 & 0.00 & $\mathrm{H}$ \\
\hline-9.044 & 17.690 & 6.440 & 1.00 & 0.00 & $\mathrm{Fe}$ \\
\hline-6.816 & 15.818 & 7.502 & 1.00 & 0.00 & C \\
\hline-6.457 & 15.027 & 8.674 & 1.00 & 0.00 & C \\
\hline-7.603 & 14.930 & 9.448 & 1.00 & 0.00 & C \\
\hline-8.633 & 15.647 & 8.740 & 1.00 & 0.00 & $\mathrm{C}$ \\
\hline-9.948 & 15.766 & 9.178 & 1.00 & 0.00 & C \\
\hline-10.201 & 15.270 & 10.113 & 1.00 & 0.00 & $\mathrm{H}$ \\
\hline-10.967 & 16.465 & 8.542 & 1.00 & 0.00 & C \\
\hline-12.336 & 16.537 & 9.012 & 1.00 & 0.00 & C \\
\hline-13.041 & 17.257 & 8.065 & 1.00 & 0.00 & C \\
\hline-12.090 & 17.622 & 7.041 & 1.00 & 0.00 & C \\
\hline-12.395 & 18.357 & 5.897 & 1.00 & 0.00 & C \\
\hline-13.422 & 18.701 & 5.791 & 1.00 & 0.00 & $\mathrm{H}$ \\
\hline-11.520 & 18.697 & 4.874 & 1.00 & 0.00 & $\mathrm{C}$ \\
\hline-11.901 & 19.422 & 3.684 & 1.00 & 0.00 & C \\
\hline-10.762 & 19.569 & 2.927 & 1.00 & 0.00 & C \\
\hline-9.689 & 18.928 & 3.659 & 1.00 & 0.00 & $\mathrm{C}$ \\
\hline-8.361 & 18.873 & 3.237 & 1.00 & 0.00 & C \\
\hline-8.121 & 19.358 & 2.289 & 1.00 & 0.00 & $\mathrm{H}$ \\
\hline-7.331 & 18.192 & 3.880 & 1.00 & 0.00 & C \\
\hline-5.965 & 18.078 & 3.398 & 1.00 & 0.00 & C \\
\hline-5.290 & 17.310 & 4.322 & 1.00 & 0.00 & C \\
\hline-6.239 & 16.959 & 5.358 & 1.00 & 0.00 & C \\
\hline-5.948 & 16.168 & 6.469 & 1.00 & 0.00 & C \\
\hline
\end{tabular}


HETATM 3911 H2O HEM 418 HETATM 3912 N21 HEM HETATM 3913 N22 HEM HETATM 3914 N23 HEM HETATM 3915 N24 HEM HETATM 3916 C99 HEM HETATM $3917 \quad 091$ HEM HETATM 3918092 HEM HETATM 3919 CT91 HEM HETATM 3920 H911 HEM HETATM 3921 H912 HEM HETATM 3922 CT92 HEM HETATM 3923 H921 HEM HETATM 3924 H922 HEM

HETATM 3925 C2 VIN HETATM 3926 H21 VIN HETATM 3927 C1 VIN HETATM 3928 H11 VIN HETATM 3929 H12 VIN TER

HERATM 3930 C1 MET HETATM 3931 H11 MET HETATM 3932 H12 MET HETATM 3933 H13 MET TER

HETATM 3934 C2 VIN HETATM 3935 H21 VIN HETATM 3936 C1 VIN HETATM 3937 H11 VIN HETATM 3938 H12 VIN TER

IER 3939 C1 MET HETATM 3940 H11 MET HETATM 3941 H12 MET HETATM 3942 H13 MET TER

HETATM 3943 C5 ACP HETATM 3944 H51 ACP HETATM 3945 H52 ACP HETATM 3946 C4 ACP HETATM 3947 H41 ACP HETATM 3948 H42 ACP HETATM 3949 C1 ACP HETATM $3950 \quad 02$ ACP HETATM 3951 O3 ACP HETATM 3952 H3 ACP TER

HETATM 3953 C1 MET HETATM 3954 H11 MET HETATM 3955 H12 MET HETATM 3956 H13 MET TER

HETATM 3957 C1 MET HETATM 3958 H11 MET HETATM 3959 H12 MET HETATM 3960 H13 MET TER

HETATM 3961 C2 VIN HETATM 3962 H21 VIN HETATM $3963 \quad$ C1 VIN HETATM 3964 H11 VIN HETATM 3965 H12 VIN TER

HETATM 3966 C1 MET HETATM 3967 H11 MET HETATM 3968 H12 MET HETATM 3969 H13 MET TER

HETATM 3970 C2 VIN HETATM 3971 H21 VIN HETATM 3972 C1 VIN HETATM 3973 H11 VIN HETATM 3974 H12 VIN TER

HETATM 3975 C1 MET HETATM 3976 H11 MET HETATM 3977 H12 MET HETATM 3978 H13 MET

\begin{tabular}{|c|c|c|c|c|c|}
\hline-4.934 & 15.778 & 6.530 & 1.00 & 0.00 & $\mathrm{H}$ \\
\hline-8.138 & 16.181 & 7.564 & 1.00 & 0.00 & $\mathrm{~N}$ \\
\hline-10.843 & 17.128 & 7.342 & 1.00 & 0.00 & $\mathrm{~N}$ \\
\hline-10.172 & 18.391 & 4.841 & 1.00 & 0.00 & $\mathrm{~N}$ \\
\hline-7.472 & 17.506 & 5.077 & 1.00 & 0.00 & $\mathrm{~N}$ \\
\hline-8.379 & 20.371 & 7.579 & 1.00 & 0.00 & C \\
\hline-8.539 & 19.058 & 7.567 & 1.00 & 0.00 & 0 \\
\hline-8.224 & 20.989 & 8.635 & 1.00 & 0.00 & 0 \\
\hline-8.395 & 21.053 & 6.223 & 1.00 & 0.00 & $\mathrm{C}$ \\
\hline-9.374 & 20.858 & 5.760 & 1.00 & 0.00 & $\mathrm{H}$ \\
\hline-7.647 & 20.563 & 5.580 & 1.00 & 0.00 & $\mathrm{H}$ \\
\hline-8.135 & 22.557 & 6.334 & 1.00 & 0.00 & C \\
\hline-7.210 & 22.713 & 6.905 & 1.00 & 0.00 & $\mathrm{H}$ \\
\hline-8.932 & 23.018 & 6.931 & 1.00 & 0.00 & $\mathrm{H}$ \\
\hline-13.655 & 28.269 & -1.002 & 1.00 & 0.00 & C \\
\hline-14.471 & 27.571 & -0.837 & 1.00 & 0.00 & $\mathrm{H}$ \\
\hline-13.988 & 29.494 & -1.403 & 1.00 & 0.00 & $\mathrm{C}$ \\
\hline-13.257 & 30.273 & -1.511 & 1.00 & 0.00 & $\mathrm{H}$ \\
\hline-15.030 & 29.763 & -1.570 & 1.00 & 0.00 & $\mathrm{H}$ \\
\hline-10.991 & 28.524 & -2.840 & 1.00 & 0.00 & C \\
\hline-10.757 & 29.595 & -2.720 & 1.00 & 0.00 & $\mathrm{H}$ \\
\hline-10.178 & 28.060 & -3.410 & 1.00 & 0.00 & $\mathrm{H}$ \\
\hline-11.899 & 28.446 & -3.450 & 1.00 & 0.00 & $\mathrm{H}$ \\
\hline-5.981 & 26.846 & -2.325 & 1.00 & 0.00 & C \\
\hline-6.647 & 26.745 & -3.184 & 1.00 & 0.00 & $\mathrm{H}$ \\
\hline-4.815 & 27.490 & -2.484 & 1.00 & 0.00 & C \\
\hline-4.135 & 27.647 & -1.654 & 1.00 & 0.00 & $\mathrm{H}$ \\
\hline-4.510 & 27.888 & -3.450 & 1.00 & 0.00 & $\mathrm{H}$ \\
\hline-4.297 & 25.163 & -0.121 & 1.00 & 0.00 & $\mathrm{C}$ \\
\hline-3.705 & 25.827 & 0.528 & 1.00 & 0.00 & $\mathrm{H}$ \\
\hline-4.140 & 24.141 & 0.246 & 1.00 & 0.00 & $\mathrm{H}$ \\
\hline-3.864 & 25.228 & -1.126 & 1.00 & 0.00 & $\mathrm{H}$ \\
\hline-13.385 & 24.081 & 5.770 & 1.00 & 0.00 & C \\
\hline-12.646 & 24.070 & 6.582 & 1.00 & 0.00 & $\mathrm{H}$ \\
\hline-14.226 & 24.689 & 6.134 & 1.00 & 0.00 & $\mathrm{H}$ \\
\hline-13.864 & 22.644 & 5.497 & 1.00 & 0.00 & C \\
\hline-14.421 & 22.583 & 4.549 & 1.00 & 0.00 & $\mathrm{H}$ \\
\hline-12.993 & 21.977 & 5.390 & 1.00 & 0.00 & $\mathrm{H}$ \\
\hline-14.728 & 22.031 & 6.573 & 1.00 & 0.00 & C \\
\hline-14.956 & 22.554 & 7.671 & 1.00 & 0.00 & 0 \\
\hline-15.243 & 20.852 & 6.209 & 1.00 & 0.00 & 0 \\
\hline-15.853 & 20.498 & 6.945 & 1.00 & 0.00 & $\mathrm{H}$ \\
\hline-5.489 & 22.955 & 4.597 & 1.00 & 0.00 & C \\
\hline-4.937 & 23.794 & 5.047 & 1.00 & 0.00 & $\mathrm{H}$ \\
\hline-5.483 & 22.129 & 5.319 & 1.00 & 0.00 & $\mathrm{H}$ \\
\hline-4.918 & 22.635 & 3.717 & 1.00 & 0.00 & $\mathrm{H}$ \\
\hline-14.923 & 25.904 & 3.646 & 1.00 & 0.00 & $\mathrm{C}$ \\
\hline-15.368 & 25.695 & 4.626 & 1.00 & 0.00 & $\mathrm{H}$ \\
\hline-15.033 & 26.984 & 3.471 & 1.00 & 0.00 & $\mathrm{H}$ \\
\hline-15.523 & 25.378 & 2.889 & 1.00 & 0.00 & $\mathrm{H}$ \\
\hline-5.126 & 14.525 & 8.933 & 1.00 & 0.00 & C \\
\hline-4.310 & 15.223 & 8.768 & 1.00 & 0.00 & $\mathrm{H}$ \\
\hline-4.793 & 13.300 & 9.335 & 1.00 & 0.00 & C \\
\hline-5.524 & 12.521 & 9.442 & 1.00 & 0.00 & $\mathrm{H}$ \\
\hline-3.751 & 13.030 & 9.501 & 1.00 & 0.00 & $\mathrm{H}$ \\
\hline-7.789 & 14.270 & 10.771 & 1.00 & 0.00 & C \\
\hline-8.024 & 13.199 & 10.652 & 1.00 & 0.00 & $\mathrm{H}$ \\
\hline-8.602 & 14.734 & 11.341 & 1.00 & 0.00 & $\mathrm{H}$ \\
\hline-6.882 & 14.347 & 11.382 & 1.00 & 0.00 & $\mathrm{H}$ \\
\hline-12.800 & 15.948 & 10.256 & 1.00 & 0.00 & C \\
\hline-12.134 & 16.048 & 11.115 & 1.00 & 0.00 & $\mathrm{H}$ \\
\hline-13.966 & 15.303 & 10.415 & 1.00 & 0.00 & C \\
\hline-14.646 & 15.147 & 9.585 & 1.00 & 0.00 & $\mathrm{H}$ \\
\hline-14.271 & 14.905 & 11.381 & 1.00 & 0.00 & $\mathrm{H}$ \\
\hline-14.484 & 17.631 & 8.052 & 1.00 & 0.00 & C \\
\hline-14.641 & 18.653 & 7.685 & 1.00 & 0.00 & $\mathrm{H}$ \\
\hline-14.916 & 17.565 & 9.057 & 1.00 & 0.00 & $\mathrm{H}$ \\
\hline-15.075 & 16.967 & 7.403 & 1.00 & 0.00 & $\mathrm{H}$ \\
\hline
\end{tabular}


TER

\begin{tabular}{|c|c|c|c|}
\hline HETATM & 3979 & $\mathrm{C} 5$ & $\mathrm{ACP}$ \\
\hline HETATM & 3980 & H51 & $\mathrm{ACP}$ \\
\hline HETATM & 3981 & H52 & $\mathrm{ACP}$ \\
\hline HETATM & 3982 & $\mathrm{C} 4$ & $\mathrm{ACP}$ \\
\hline HETATM & 3983 & $\mathrm{H} 4 \mathrm{I}$ & $\mathrm{ACP}$ \\
\hline HETATM & 3984 & H 42 & $\mathrm{ACP}$ \\
\hline HETATM & 3985 & $\mathrm{C} 1$ & $\mathrm{ACP}$ \\
\hline HETATM & 3986 & $\mathrm{O} 2$ & $\mathrm{ACP}$ \\
\hline HETATM & 3987 & 03 & $\mathrm{ACP}$ \\
\hline HETATM & 3988 & H3 & $\mathrm{ACP}$ \\
\hline TER & & & \\
\hline TATM & 3989 & $\mathrm{C} 1$ & MET \\
\hline ETATM & 3990 & $\mathrm{H} 11$ & MET \\
\hline HETATM & 3991 & $\mathrm{H} 12$ & MET \\
\hline HETATM & 3992 & H13 & MET \\
\hline IER & & & \\
\hline ETATM & 39 & C1 & MET \\
\hline TATM & 399 & H11 & MET \\
\hline ГATM & 39 & $\mathrm{H} 12$ & MET \\
\hline TATM & P0 & H13 & MET \\
\hline
\end{tabular}

HETATM 3996 H13 MET TER

END

$\begin{array}{ll}-5.396 & 18.712 \\ -6.135 & 18.724 \\ -4.555 & 18.104 \\ -4.917 & 20.150 \\ -4.360 & 20.211 \\ -5.788 & 20.817 \\ -4.053 & 20.762 \\ -3.825 & 20.239 \\ -3.537 & 21.942 \\ -2.928 & 22.296 \\ -13.292 & 19.839 \\ -13.844 & 18.999 \\ -13.298 & 20.665 \\ -13.863 & 20.158 \\ -3.858 & 16.890 \\ -3.413 & 17.099 \\ -3.748 & 15.810 \\ -3.258 & 17.416\end{array}$

$\begin{array}{lll}2.161 & 1.00 & 0.00\end{array}$

$\begin{array}{lll}1.349 & 1.00 & 0.00\end{array}$

$1.798 \quad 1.00 \quad 0.00$

$2.434 \quad 1.00 \quad 0.00$

$3.382 \quad 1.00=0.00$

$\begin{array}{lll}2.541 & 1.00 & 0.00\end{array}$

$\begin{array}{lll}1.358 & 1.00 \quad 0.00\end{array}$

$0.260 \quad 1.00 \quad 0.00$

$\begin{array}{lll}1.722 & 1.00 & 0.00\end{array}$

$0.986 \quad 1.00 \quad 0.00$

$3.334 \quad 1.00 \quad 0.00$

$2.884 \quad 1.00 \quad 0.00$

$2.613 \quad 1.00 \quad 0.00$

$4.214 \quad 1.00 \quad 0.00$

$\begin{array}{lll}4.285 & 1.00 & 0.00\end{array}$

$3.305 \quad 1.00 \quad 0.00$

$\begin{array}{lll}4.460 & 1.00 & 0.00\end{array}$

$5.042 \quad 1.00 \quad 0.00$

\section{Introduction to supporting figures}

Apart from the computation of the occupancy rate of hydrogen bonds, Postprocessing of trajectories and analyses were conducted with the CPPTRAJ analysis tool which is distributed as part of AmberTools13. [1, 2] Data were plotted with Grace (http://plasma-gate.weizmann.ac.il/Grace/). The occupancy rates of the hydrogen bonds were computed with the PTRAJ analysis tool distributed as part of AmberTools13. [1, 2] The data related to hydrogen bonds presented in this document are the output files generated by PTRAJ, hence it is important to note that the nomenclature related to hydrogen bond donors and acceptors used in the charts is opposite to the one commonly used and recommended in the IUPAC definition of hydrogen bonds. A typical hydrogen bond may be depicted as $\mathrm{X}-\mathrm{H} \cdots \mathrm{Y}-\mathrm{Z}$, where the three dots denote the bond. Here, $\mathrm{X}-\mathrm{H}$ represents the hydrogen bond acceptor and the donor may be an atom or an anion $Y$, or a fragment or a molecule $Y-Z$, where $Y$ is bonded to $Z$. [3] The term residue stands for a group of atoms which corresponds to a molecular fragment i.e. to a force field library. Each residue is described in the molecular dynamics input structures provided to the PDB file format in the Supporting Information (Residues are separated by the TER keyword in this file format). It is noteworthy to make clear that in the Becker et al. force field a porphyrin ring and a cyclic dimer of ferriprotoporphyrin IX are composed of more than one residue. Indeed, in the PyRED building block approach a cyclic dimer of ferriprotoporphyrin IX is decomposed into 16 molecular fragments or residues. The solvent accessible surface area is computed with the molsurf routine implemented in CPPTRAJ and corresponds to the Connoly surface area determined with a $1.4 \AA$ probe radius. [4] The distances and dihedral angles presented in this document are labeled with the atom names defined in the force field libraries and are also accessible through the input structures provided to the PDB file format in the Supporting Information.

\section{References}

[1] D. R. Roe and T. E. Cheatham. PTRAJ and CPPTRAJ: software for processing and analysis of molecular dynamics trajectory data. J. Chem. Theory Comput., 14(9):3084-3095, 2013.

[2] D. A. Case, T. A. Dardena, T. E. Cheatham III, C. L. Simmerling, J. Wang, R. E. Duke, R. Luo, R. C. Walker, W. Zhang, K. M. Merz, B. Roberts, S. Hayik, A. Roitberg, G. Seabra, J. Swails, A. W. Götz, I. Kolossváry, K. F. Wong, F. Paesani, J. Vanicek, R. M. Wolf, J. Liu, X. Wu, S. R. Brozell, T. Steinbrecher, H. Gohlke, Q. Cai, X. Ye, J. Wang, M.-J. Hsieh, G. Cui, D. R. Roe, D.H. Mathews, M. G. Seetin, R. Salomon-Ferrer, C. Sagui, V. Babin, T. Luchko, S. Gusarov, A. Kovalenko, and P. A. Kollman. AMBER 12, University of California, San Francisco, 2012. 
[3] E. Arunan, G. R. Desiraju, R. A. Klein, J. Sadlej, S. Scheiner, I. Alkorta, D. C. Clary, R. H. Crabtree, J. J. Dannenberg, P. Hobza, H. G. Kjaergaard, A. C. Legon, B. Mennucci, and D. J. Nesbitt. Definition of the hydrogen bond (IUPAC Recommendations 2011). Pure Appl. Chem., 83(8):1637-1641, 2011.

[4] M. L. Connolly. Analytical molecular surface calculation. J. Appl. Cryst., 16:548-558, 1983.

\section{List of abbreviations used in the Supporting Information}

$\begin{array}{ll}\text { FF } & \text { force field } \\ \text { FPD } & \text { cyclic dimer of ferriprotoporphyrin IX } \\ \text { HB } & \text { hydrogen bond } \\ \text { MD } & \text { molecular dynamics } \\ \text { RG } & \text { radius of gyration } \\ \text { RMSD } & \text { root mean square deviation } \\ \text { SASA } & \text { solvent accessible surface area }\end{array}$




\section{List of Figures}

S1 - RMSD of the single FPD entity and of the central FPD entity within the crystal model . . 188

S2 - C-C-C-C dihedral angles of the single FPD entity . . . . . . . . . . . . . . . . . . 189

S3 - Distance between the iron atoms of the single FPD entity . . . . . . . . . . . . . . 190

S4 - Distances between oxygen atoms O3 and O92 of the single FPD entity . . . . . . . . . . 191

S5 - C-C-C-O dihedral angles of the single FPD entity . . . . . . . . . . . . . . 192

S6 - N21-N22-FE-N23 improper dihedral angles of the single FPD entity $\ldots \ldots \ldots$. . . . . . 193

S7 - RMSD of the crystal model composed of 27 FPD entities . . . . . . . . . . . . . . . . . 194

S8 - C-C-C-C dihedral angles of the central FPD entity in the crystal model . . . . . . . . . 195

S9 - Distances between the iron atoms of the central FPD entity in the crystal model . . . . . 196

S10a - N21-N22-FE-N23 dihedral angles of the central FPD entity in the crystal model . . . 197

$\mathrm{S} 10 \mathrm{~b}$ - Distances between oxygen atoms O3 and O92 of the central FPD entity in the crystal

model . . . . . . . . . . . . . . . . . . . . . . . . . . . . 198

S10c - Distances between nitrogen atom N21 and iron atom FE of the central FPD entity in the

crystal model . . . . . . . . . . . . . . . . . . . . . . . . . 199

S11 - Occupancy rate of the HB of the crystal model . . . . . . . . . . . . . . . 200

S12 - Distance between the oxygen atoms O3 and O92 in the case of an inter-FPD HB . . . . . 201

S13-C-C-C-O dihedral angle in the case of an inter-FPD HB . . . . . . . . . . . 202

S14-RMSD of the D1 dimer . . . . . . . . . . . . . . . . . . . . . . . . . . . 203

S15 - RG of the D1 dimer . . . . . . . . . . . . . . . . . . . . . . . . . . . 204

S16 - Distances between the two iron atoms of each FPD entity in the D1 dimer . . . . . . . 205

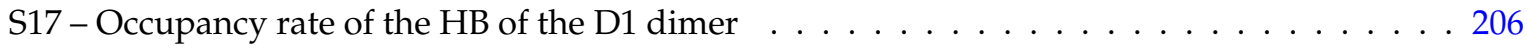

S18 - RMSD of the D2 dimer . . . . . . . . . . . . . . . . . . . . . . . . . . 207

$\mathrm{S} 19-\mathrm{RG}$ of the $\mathrm{D} 2$ dimer . . . . . . . . . . . . . . . . . . . . . . . . . 208

S20 - Distance between the iron atoms of residue 1 and residue 18 in the D2 dimer . . . . . . 209

S21 - SASA of the D2 dimer . . . . . . . . . . . . . . . . . . . . . . 210

S22 - FE-FE-FE-FE dihedral angle of the D2 dimer . . . . . . . . . . . . . . . . . 211

S23 - RMSD of the D3 dimer . . . . . . . . . . . . . . . . . . . . . . . . . . . . . 212

S24-RMSD of the D4 dimer . . . . . . . . . . . . . . . . . . . . . . . . . 213

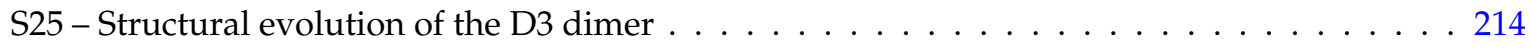

S26 - Structural evolution of the D4 dimer . . . . . . . . . . . . . . . . . . . . 215

S27 - RMSD of the D5 dimer . . . . . . . . . . . . . . . . . . . . . . 216

$\mathrm{S} 28$ - RG of the $\mathrm{D} 5$ dimer . . . . . . . . . . . . . . . . . . . . . . . . . . . . 217

S29 - Structural evolution of the $\mathrm{D} 5$ dimer . . . . . . . . . . . . . . . . . . . . 218

$\mathrm{S} 30$ - RMSD of the $\mathrm{T} 1$ trimer . . . . . . . . . . . . . . . . . . . . . . . . . . 219

S31 - Distances between the two iron atoms of each FPD entity in the T1 trimer . . . . . . . 220

$\mathrm{S} 32-\mathrm{C}-\mathrm{C}-\mathrm{C}-\mathrm{C}$ dihedral angles of each FPD entity in the $\mathrm{T} 1$ trimer $\ldots \ldots \ldots . \ldots 221$

$\mathrm{S} 33$ - Occupancy rate of the $\mathrm{HB}$ in the $\mathrm{T} 1$ dimer . . . . . . . . . . . . . . . . 222

S34-RMSD of the T2 trimer . . . . . . . . . . . . . . . . . . . . . . . . 223

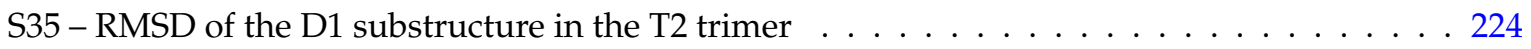

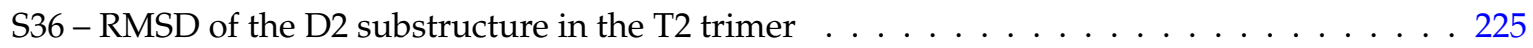

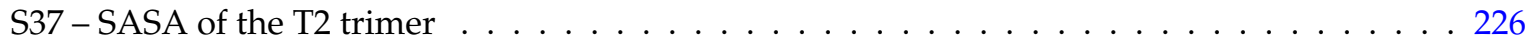

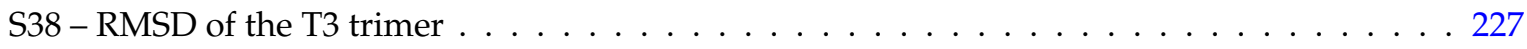

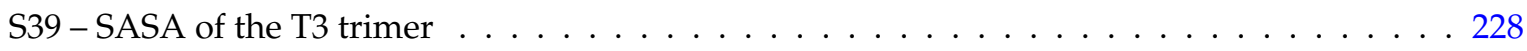

S40 - RMSD of the $\mathrm{T} 4$ trimer . . . . . . . . . . . . . . . . . . . . . . . . . . . . 229

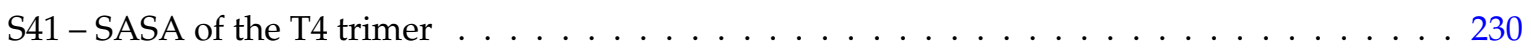

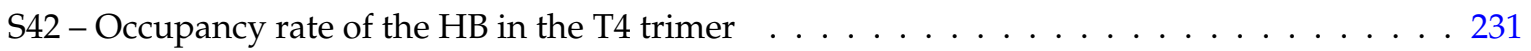

S43-RMSD of the $\mathrm{T} 5$ trimer . . . . . . . . . . . . . . . . . . . . . . 232

S44-Structural evolution of the $\mathrm{T} 5$ trimer . . . . . . . . . . . . . . . . . 233

S45 - RMSD of the Q1 tetramer . . . . . . . . . . . . . . . . . . . . . 234

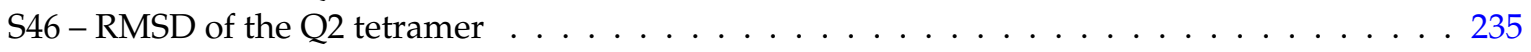

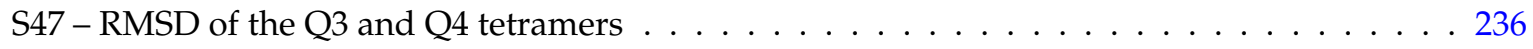




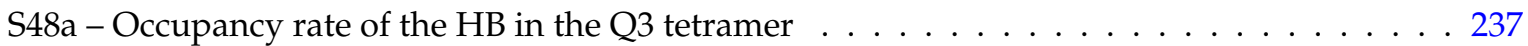

$\mathrm{S} 48 \mathrm{~b}$ - Occupancy rate of the HB between the Q3 tetramer and solvent molecules . . . . . . . 238

S48c - Occupancy rate of the HB in the Q4 tetramer . . . . . . . . . . . . . . . . . . . 239

S48d - Occupancy rate of the HB between the Q4 tetramer and solvent molecules . . . . . . . 240

S49 - Structural evolution of the Q5 tetramer . . . . . . . . . . . . . . . . . . . . 241 


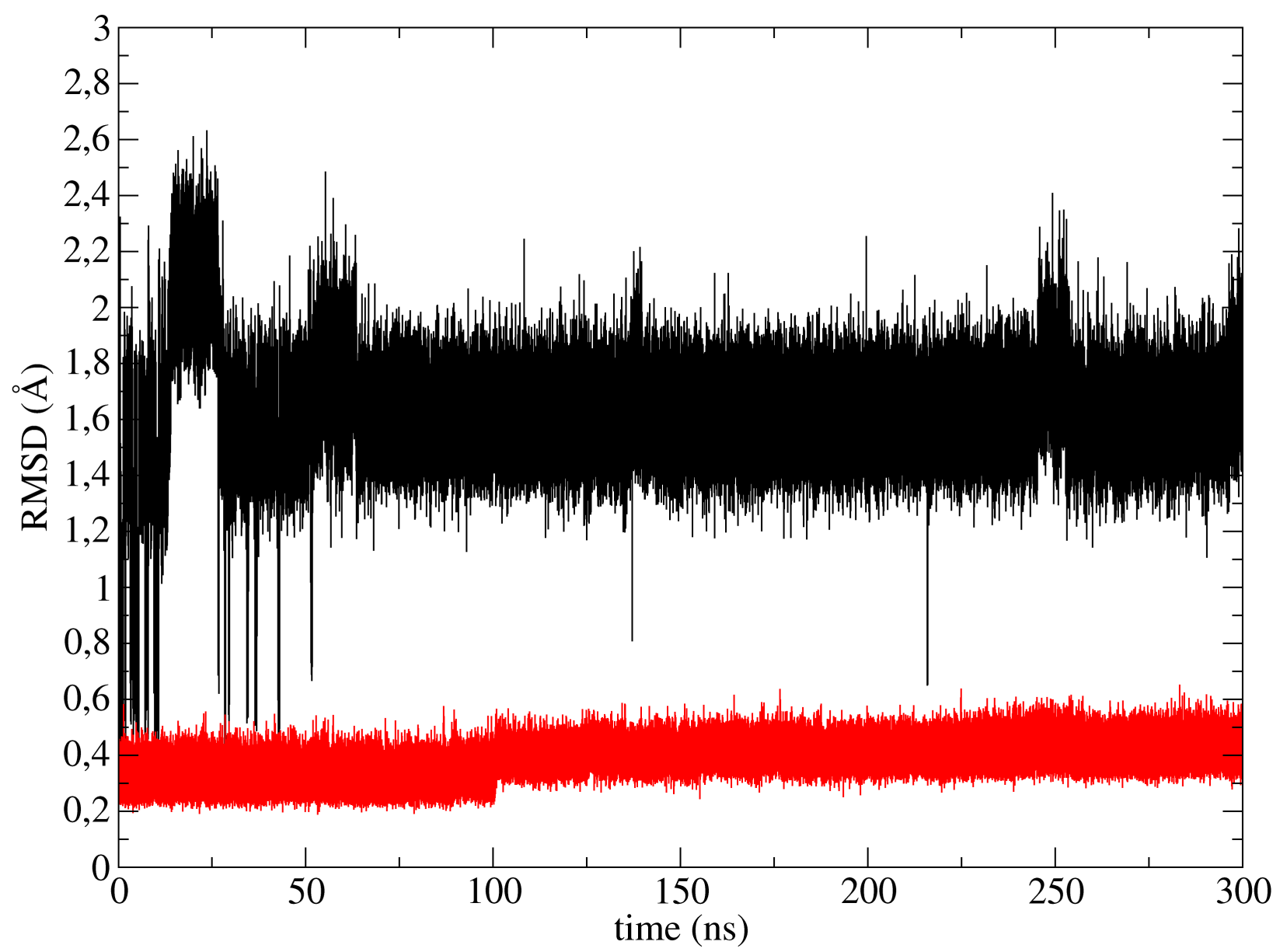

Figure S1 - Values of the RMSD in Ångström $(\AA)$ as a function of time in ns computed on the heavy atoms of the single FPD entity (in black) and of the central FPD entity within the crystal model (in red). The RMSD values are reported for the first $300 \mathrm{~ns}$ of the MD trajectories after equilibration. The slight increase of the RMSD values of the central FPD entity within the crystal model occurring after $100 \mathrm{~ns}$ results from the formation of an inter-FPD HB. The reference structure used to compute the RMSD is the initial configuration of the MD simulation (i.e. the Cartesian coordinates taken from the crystallographic structure). The values represented correspond to MD trajectories representative of the trends shown in the MD trajectories performed in this work. 


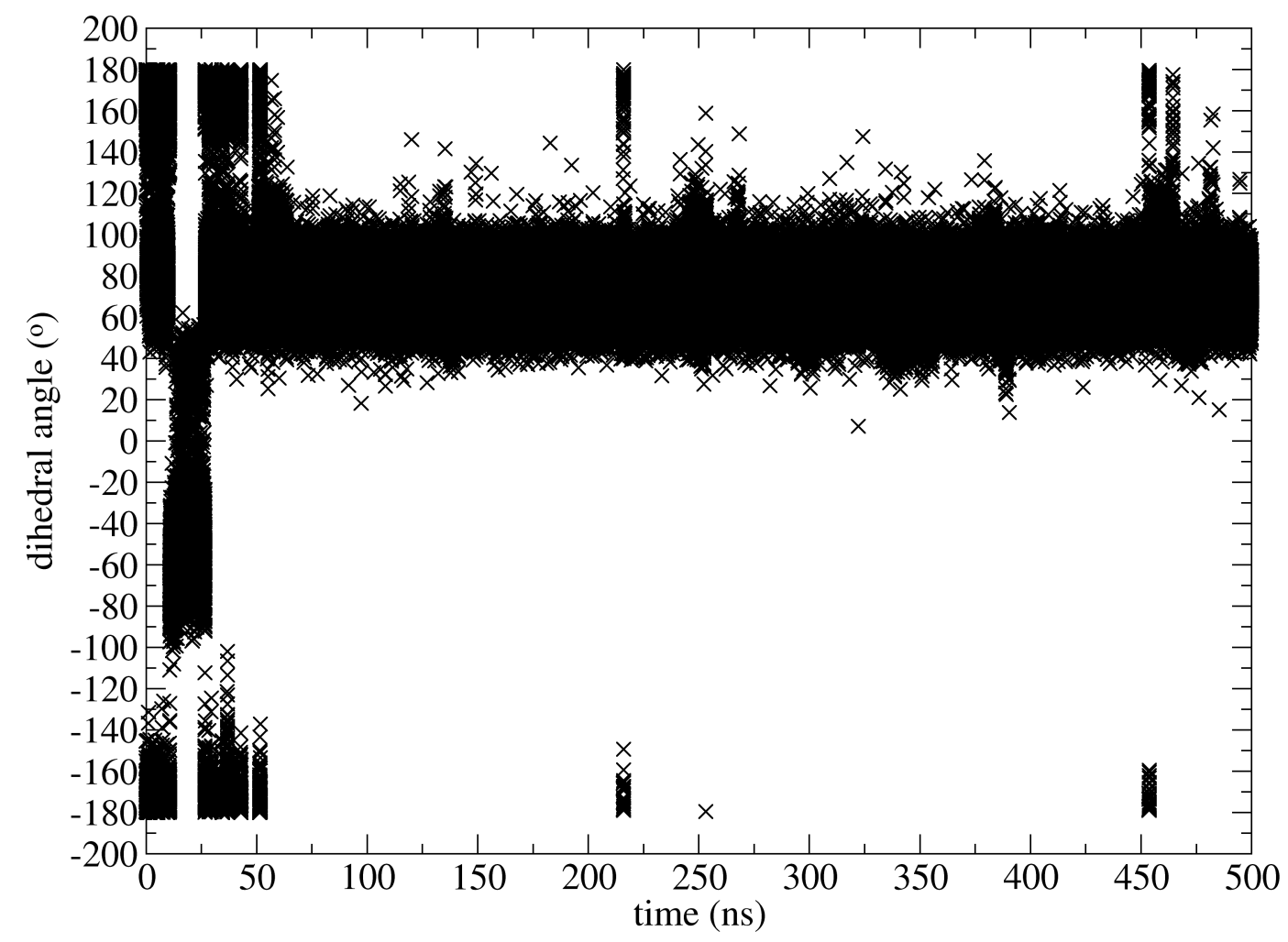

Dihedral angle defined by the sequence of atoms C99, CT91, CT92 in residue 1, and C13 in residue 2

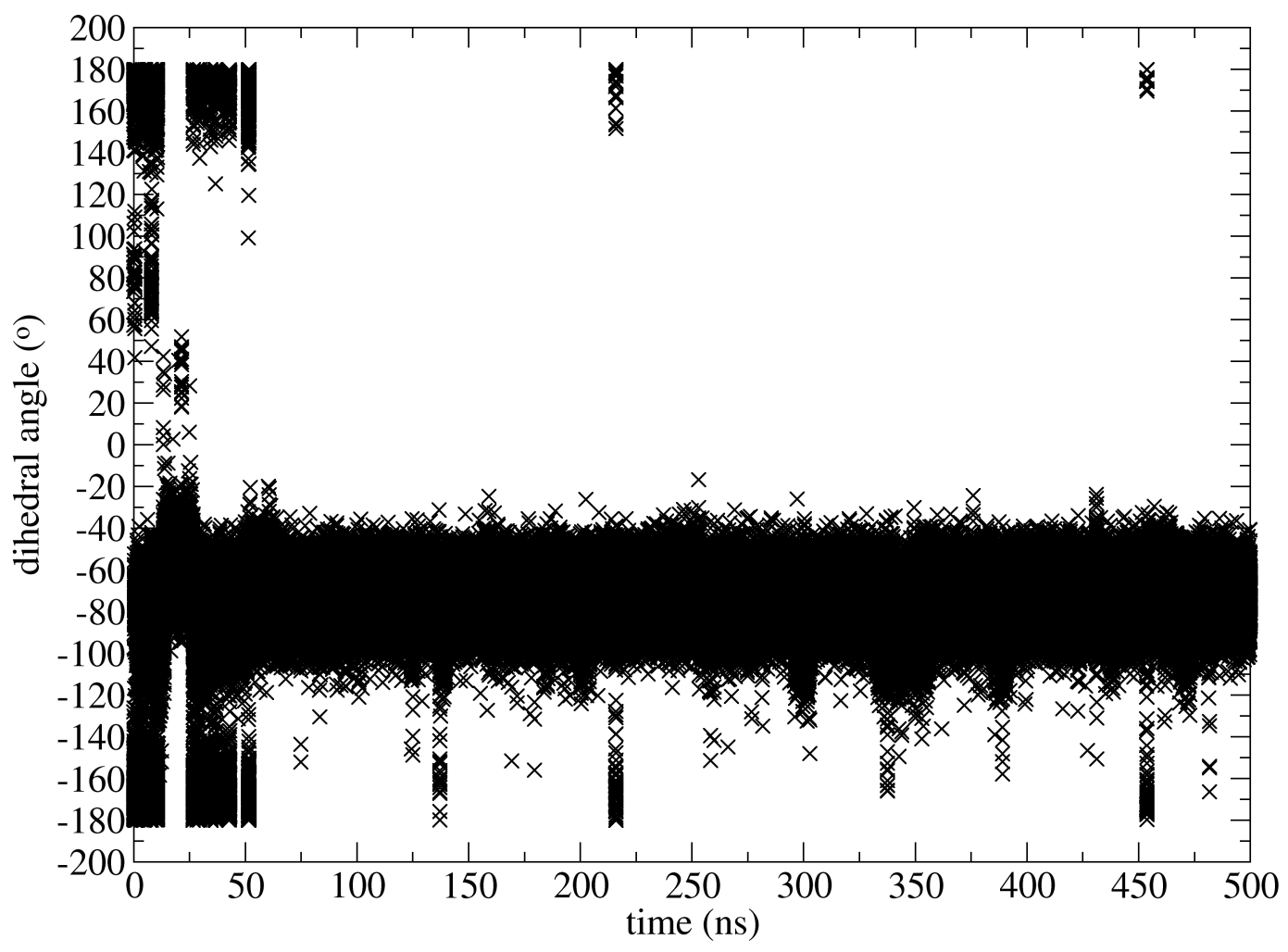

Dihedral angle defined by the sequence of atoms C99, CT91, CT92 in residue 2, and C13 in residue 1

Figure S2 - Values of the two C-C-C-C dihedral angles of the carboxylate-iron bonds connecting the two heminic rings of the single FPD entity (in degrees) as a function of time (in ns). The values represented correspond to a MD trajectory representative of the trends shown in the MD trajectories performed in this work. 


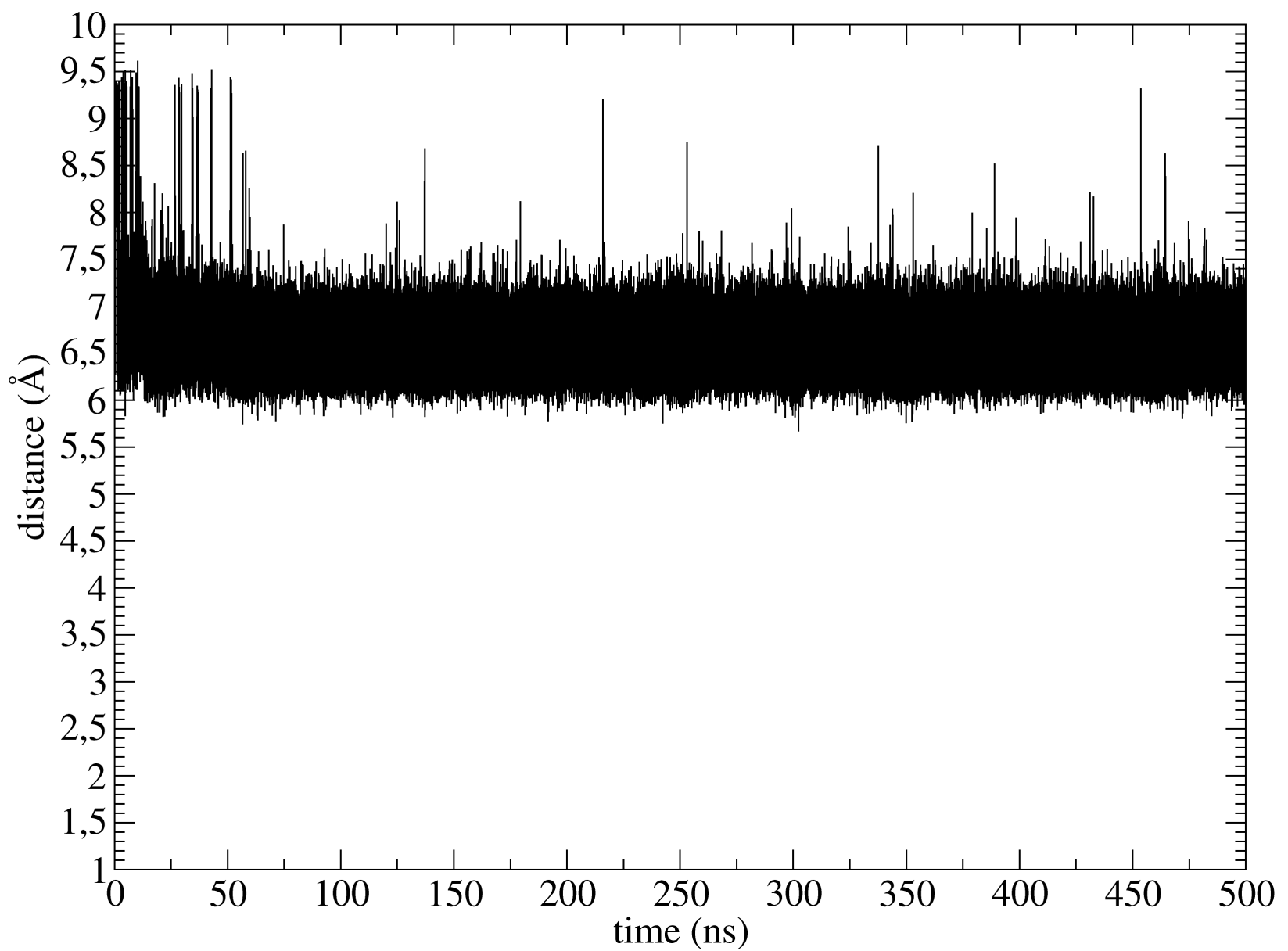

Figure S3 - Values of the distance between the two iron atoms of the single FPD entity (in $\AA$ ) as a function of time (in ns). The values represented correspond to a MD trajectory representative of the trends shown in the MD trajectories performed in this work. 


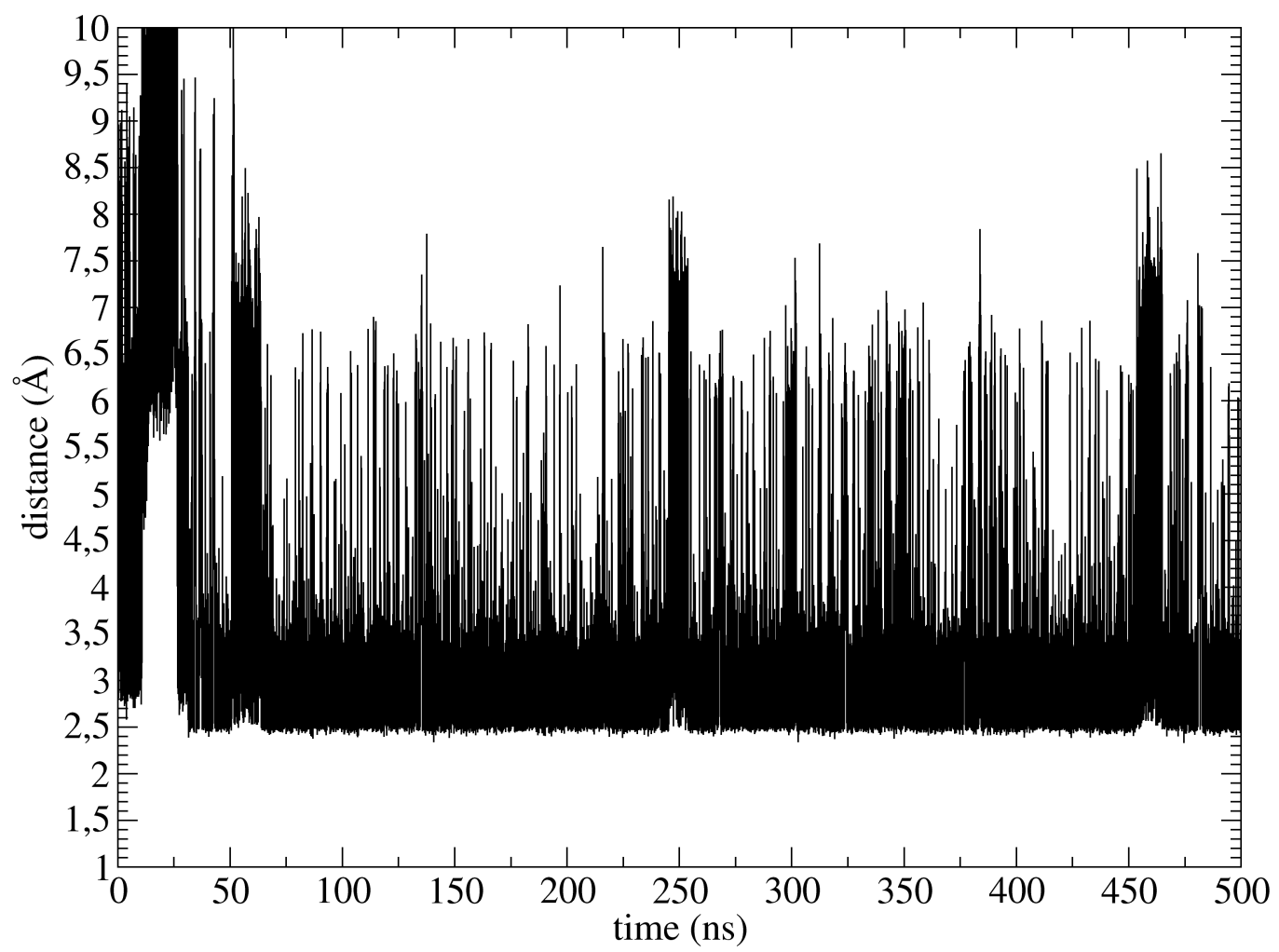

Distance between atom $\mathrm{O} 92$ of residue 1 and atom $\mathrm{O} 3$ of residue 14

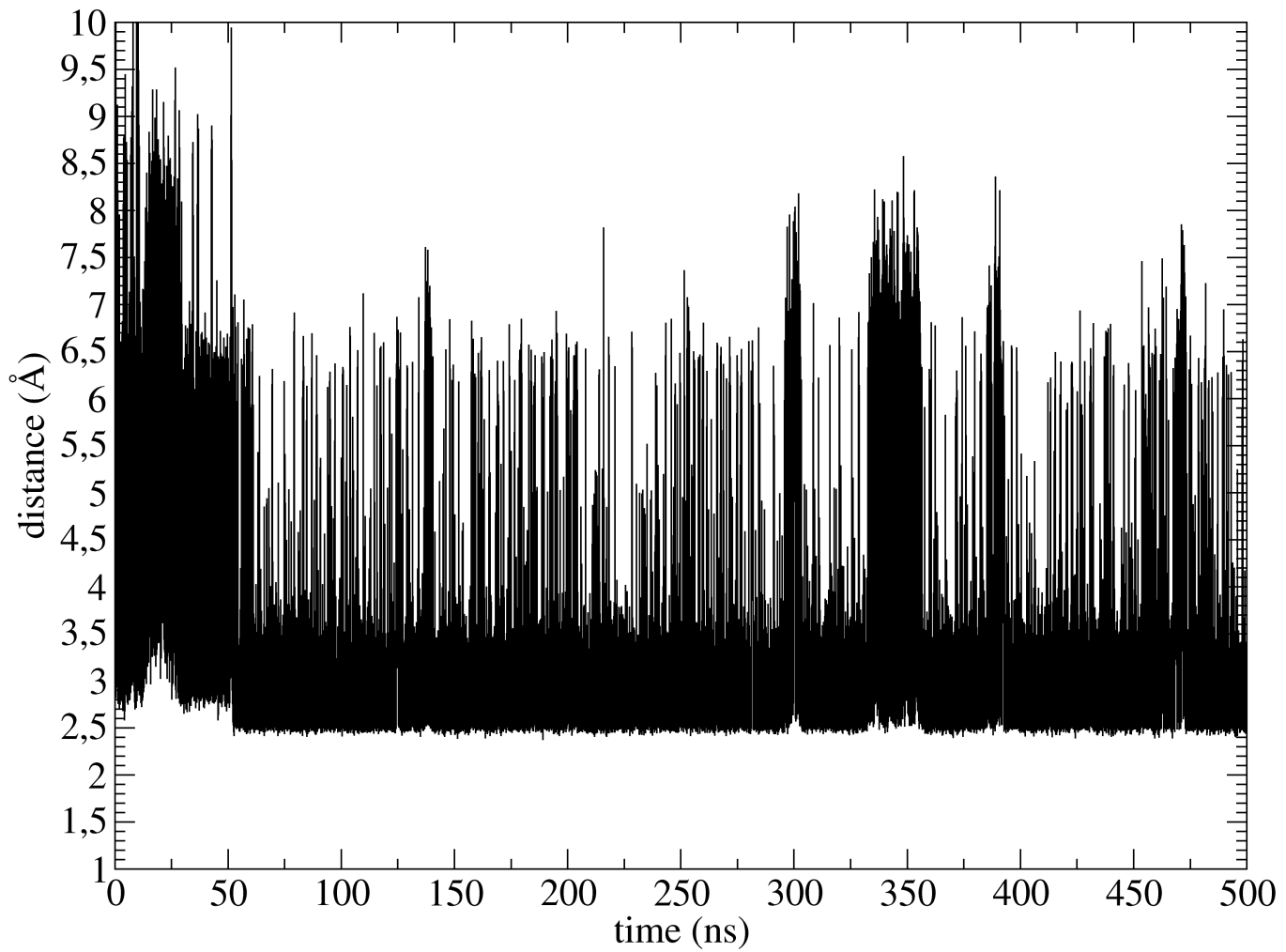

Distance between atom $\mathrm{O} 92$ of residue 2 and atom $\mathrm{O} 3$ of residue 7

Figure S4 - Values of the distance between each pair of oxygen atoms of the single FPD entity involved in intra-FPD HB (in $\AA$ ) as a function of time (in ns). The values represented correspond to a MD trajectory representative of the trends shown in the MD trajectories performed in this work. 


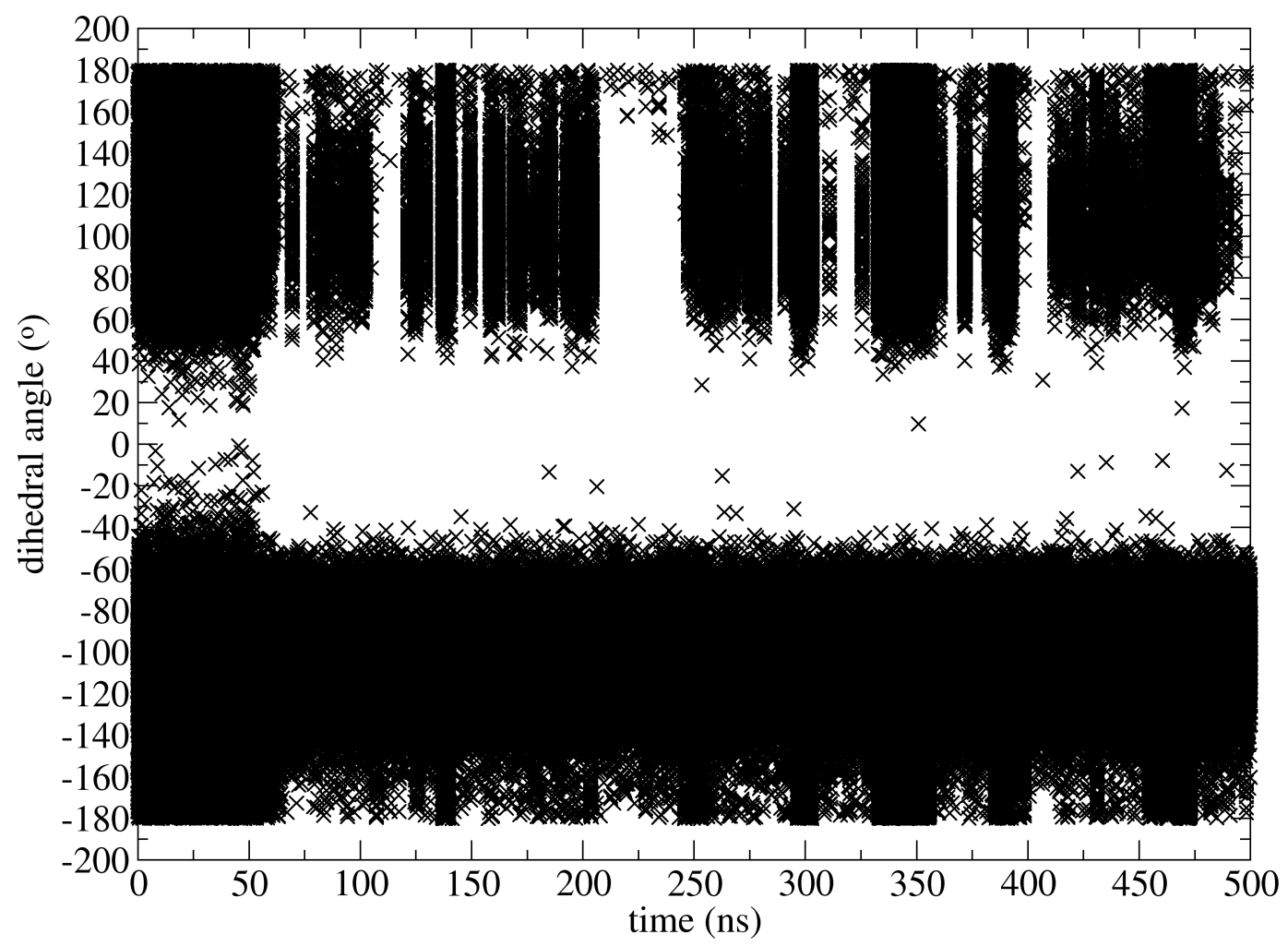

Dihedral angle defined by the sequence of atoms $\mathrm{C} 5, \mathrm{C} 4, \mathrm{C} 1$, and $\mathrm{O} 3$ in residue 7

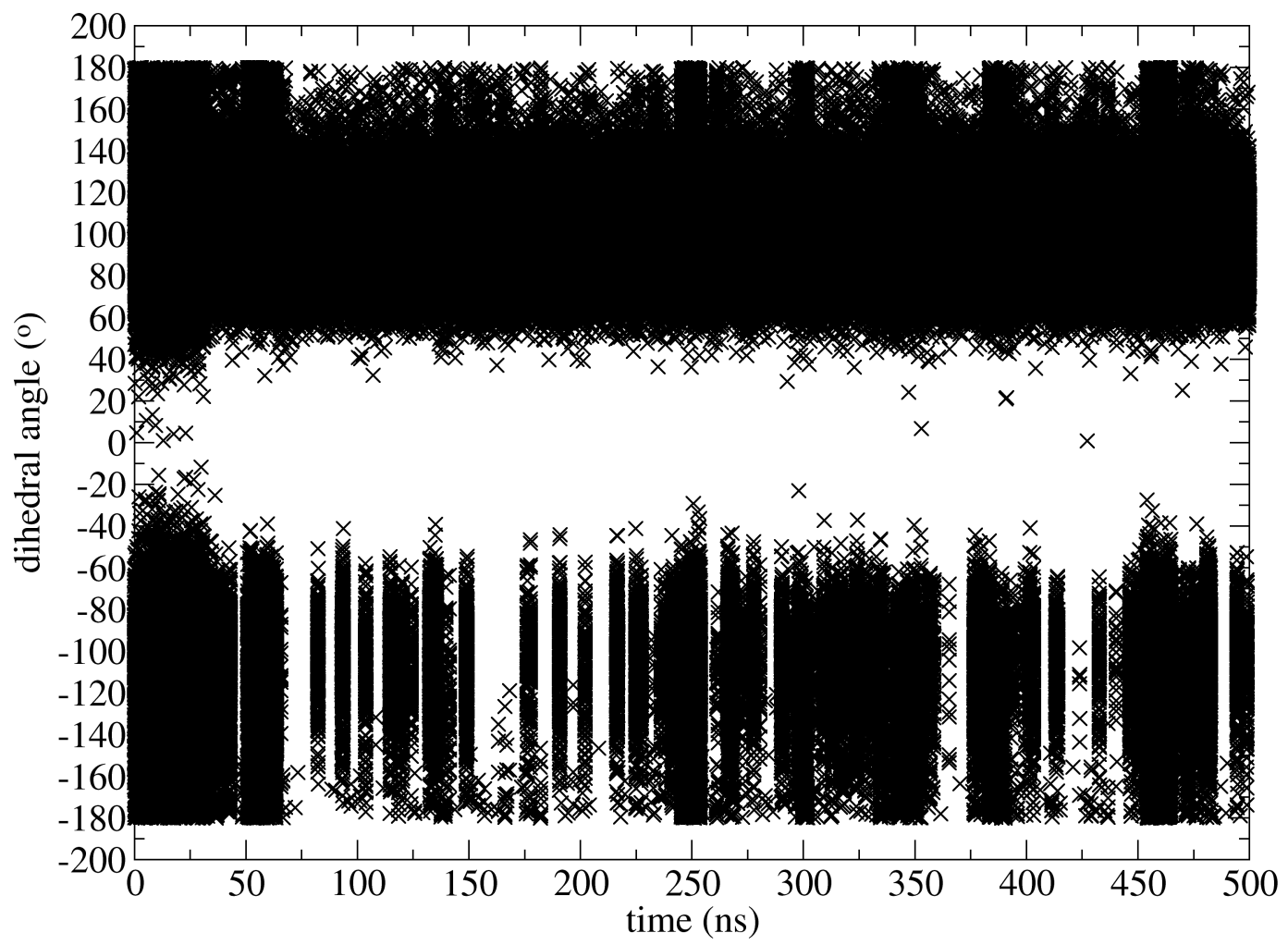

Dihedral angle defined by the sequence of atoms C5, C4, C1, and O3 in residue 14

Figure S5 - Values of the two C-C-C-O dihedral angles of the carboxylic acid groups of the single FPD entity (in degrees) as a function of time (in ns). The values represented correspond to a MD trajectory representative of the trends shown in the MD trajectories performed in this work. 


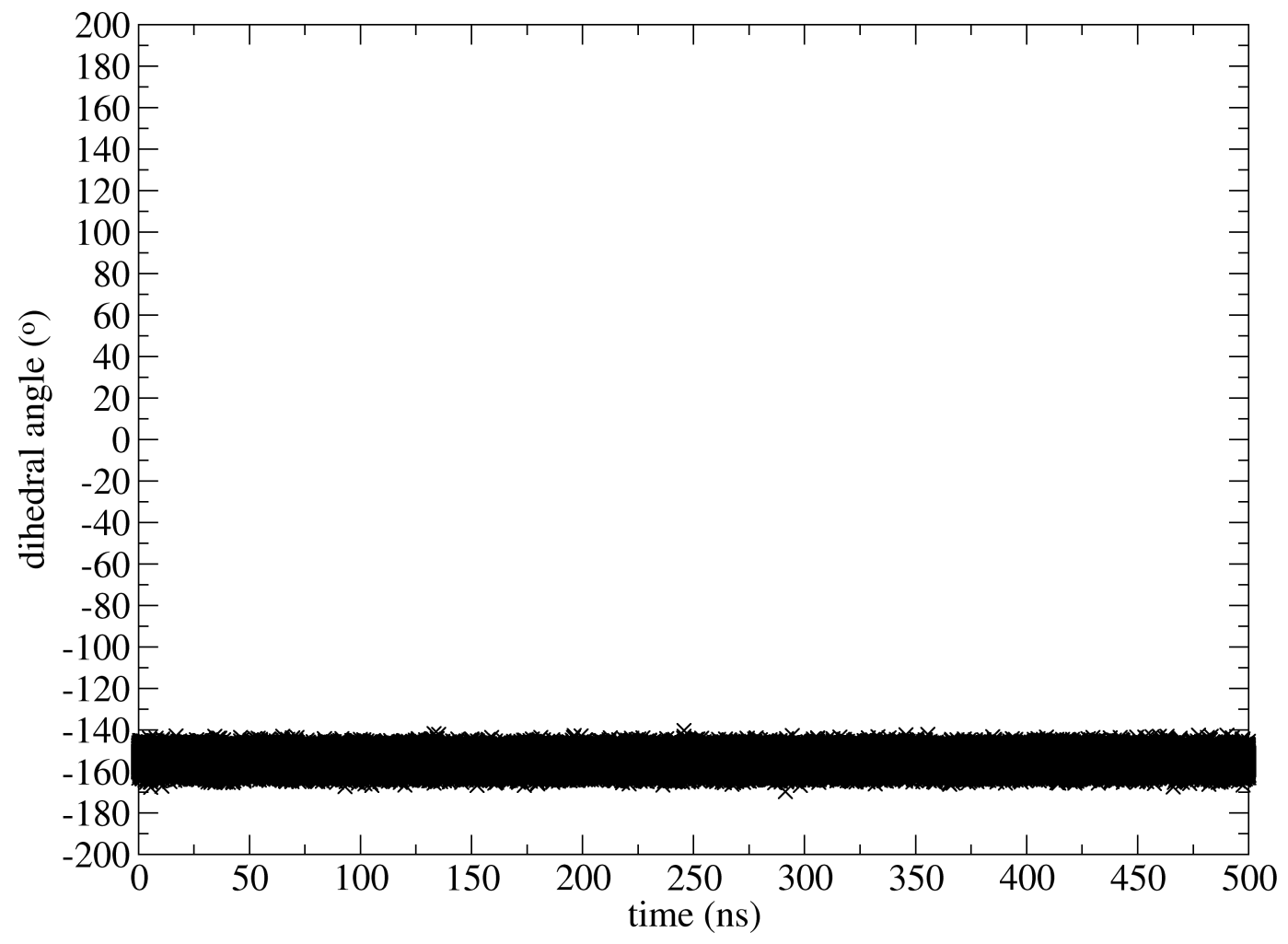

Improper dihedral angle defined by the sequence of atoms N21, N22, FE, and N23 in residue 1

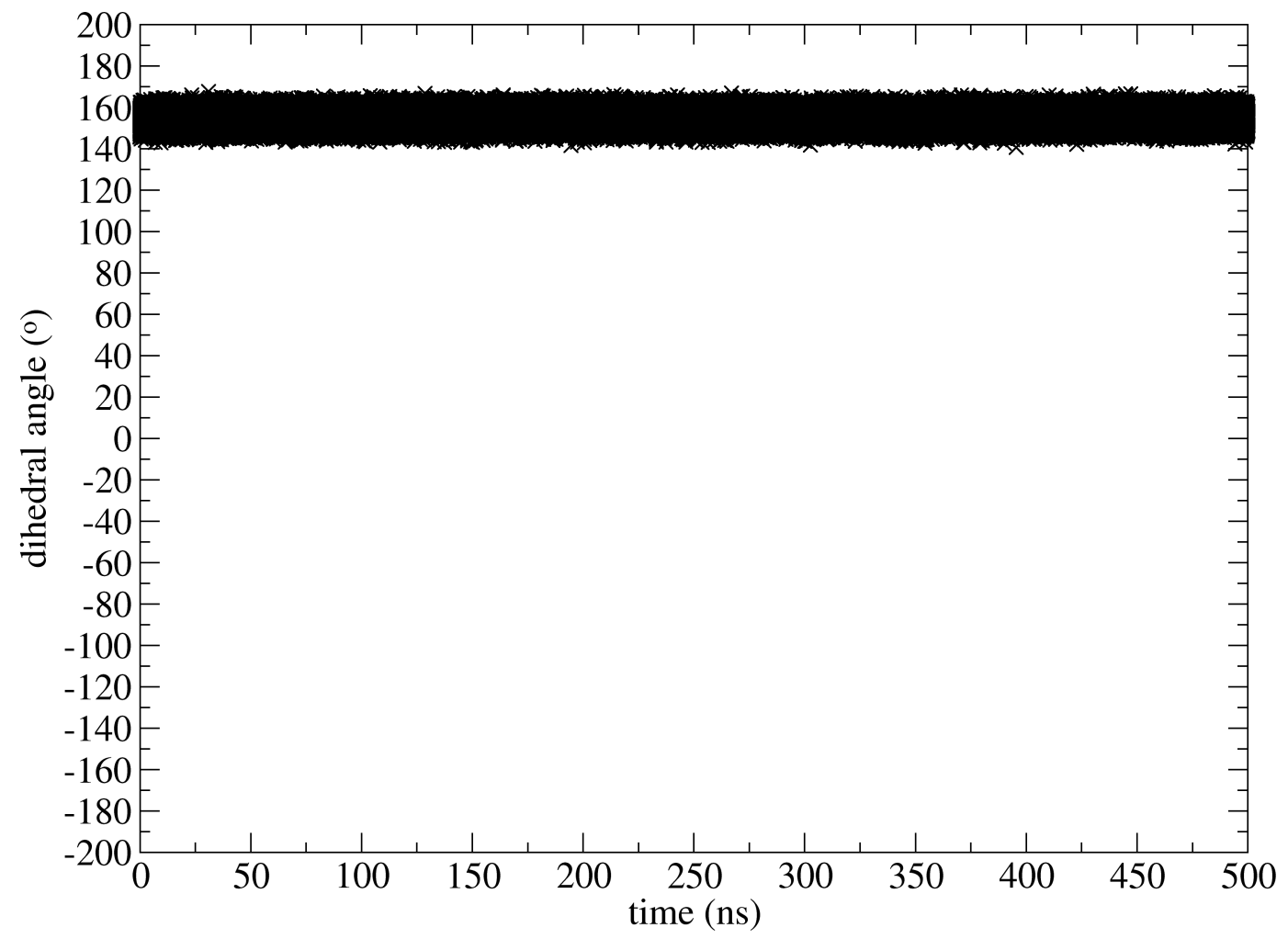

Improper dihedral angle defined by the sequence of atoms N21, N22, FE, and N23 in residue 2

Figure S6 - Values of the two N21-N22-FE-N23 improper dihedral angles of the single FPD entity (in degrees) as a function of time (in ns). Improper dihedral angles are defined based on the alphabetical order of the names of the involved atoms. In the crystallographic reference, the value of this angle is 154 degrees. The values represented correspond to a MD trajectory representative of the trends shown in the MD trajectories performed in this work. 


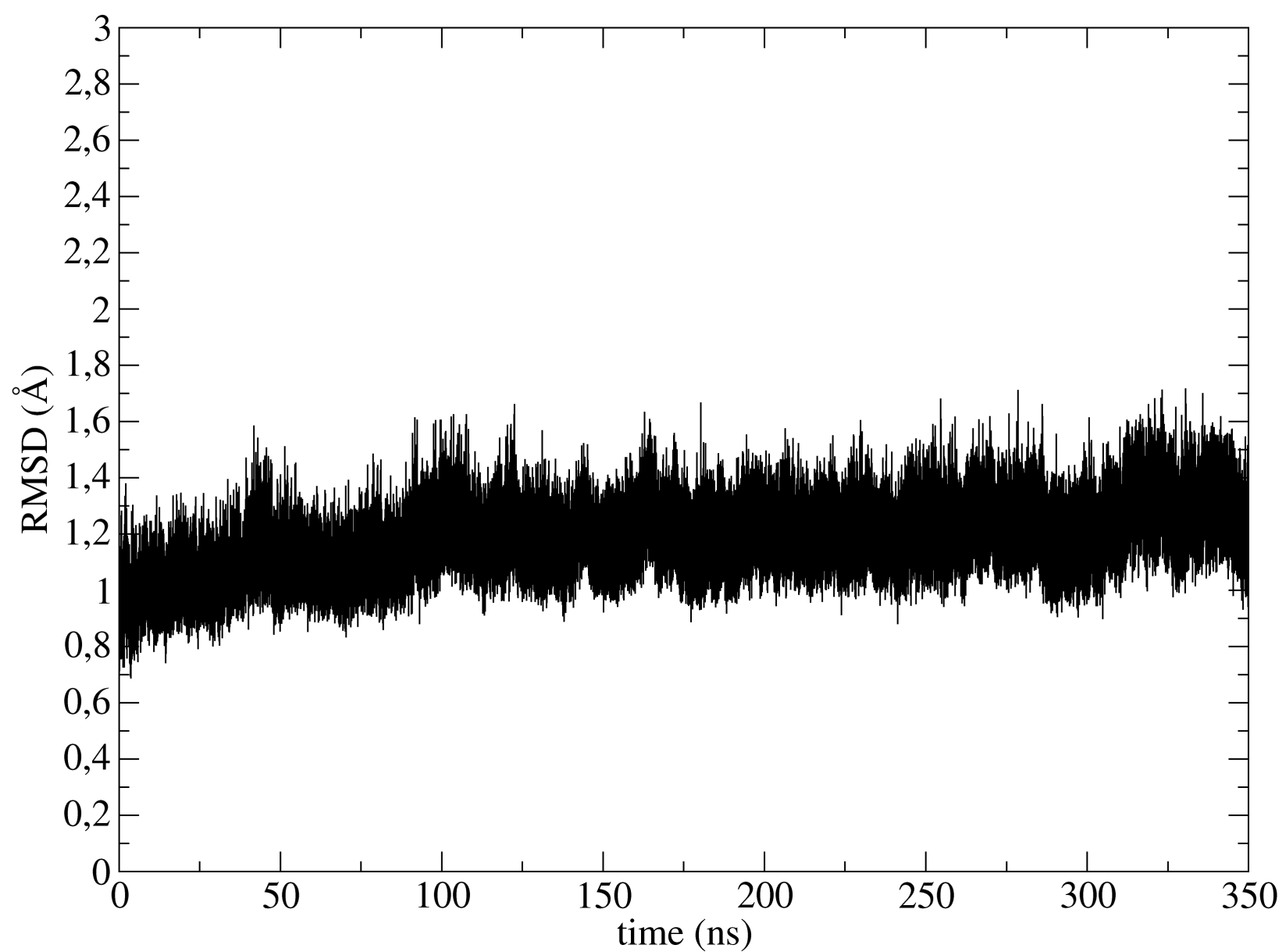

Figure S7 - Values of the RMSD (in $\AA$ ) as a function of time (in ns) computed on the heavy atoms of the crystal model composed of 27 FPD entities. The reference structure used to compute the RMSD is the initial configuration of the MD simulation (i.e. the Cartesian coordinates taken from the crystallographic structure). The values represented correspond to a MD trajectory representative of the trends shown in the MD trajectories performed in this work. 


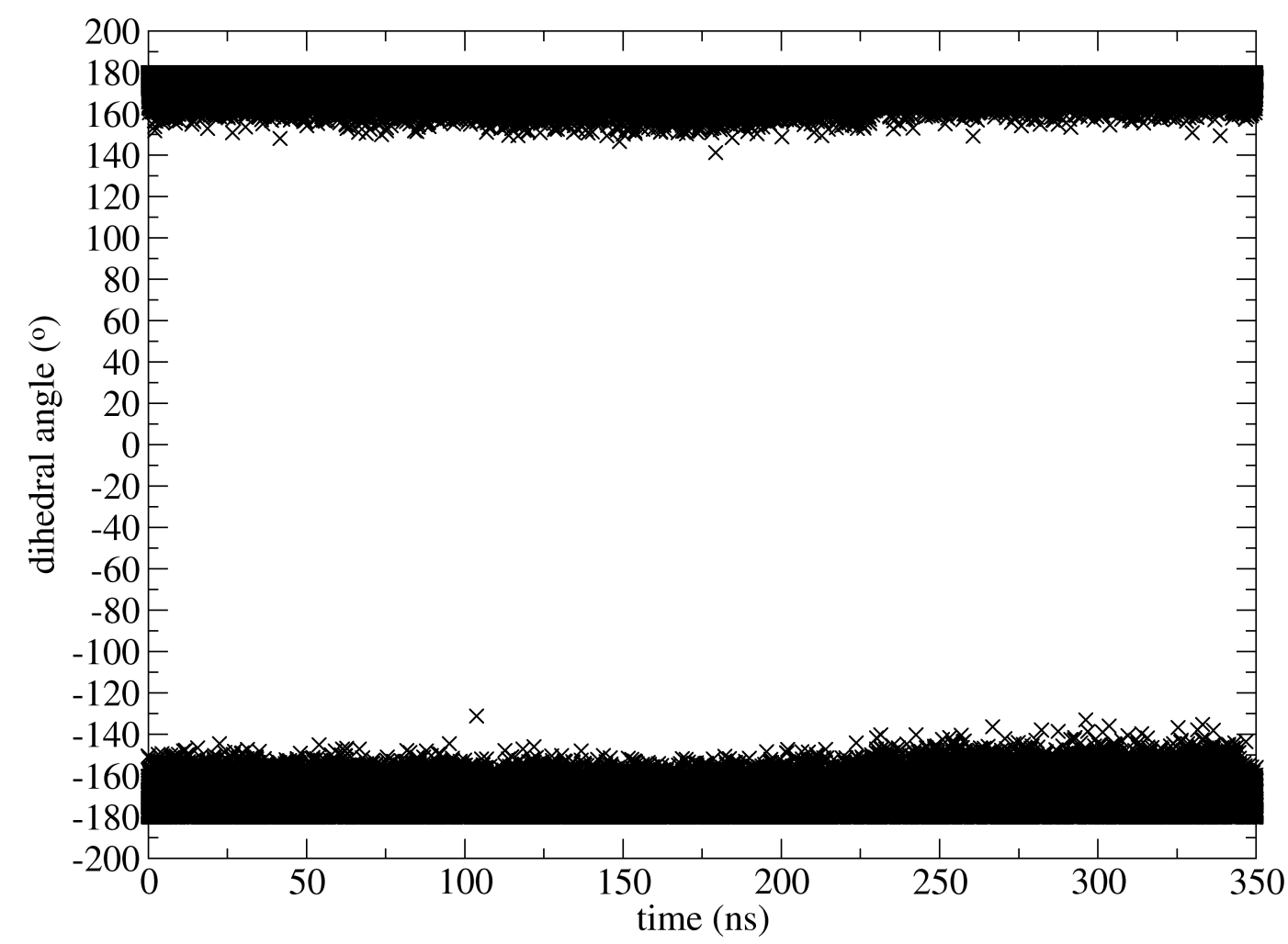

Dihedral angle defined by the sequence of atoms C99, CT91, CT92 in residue 1, and C13 in residue 2

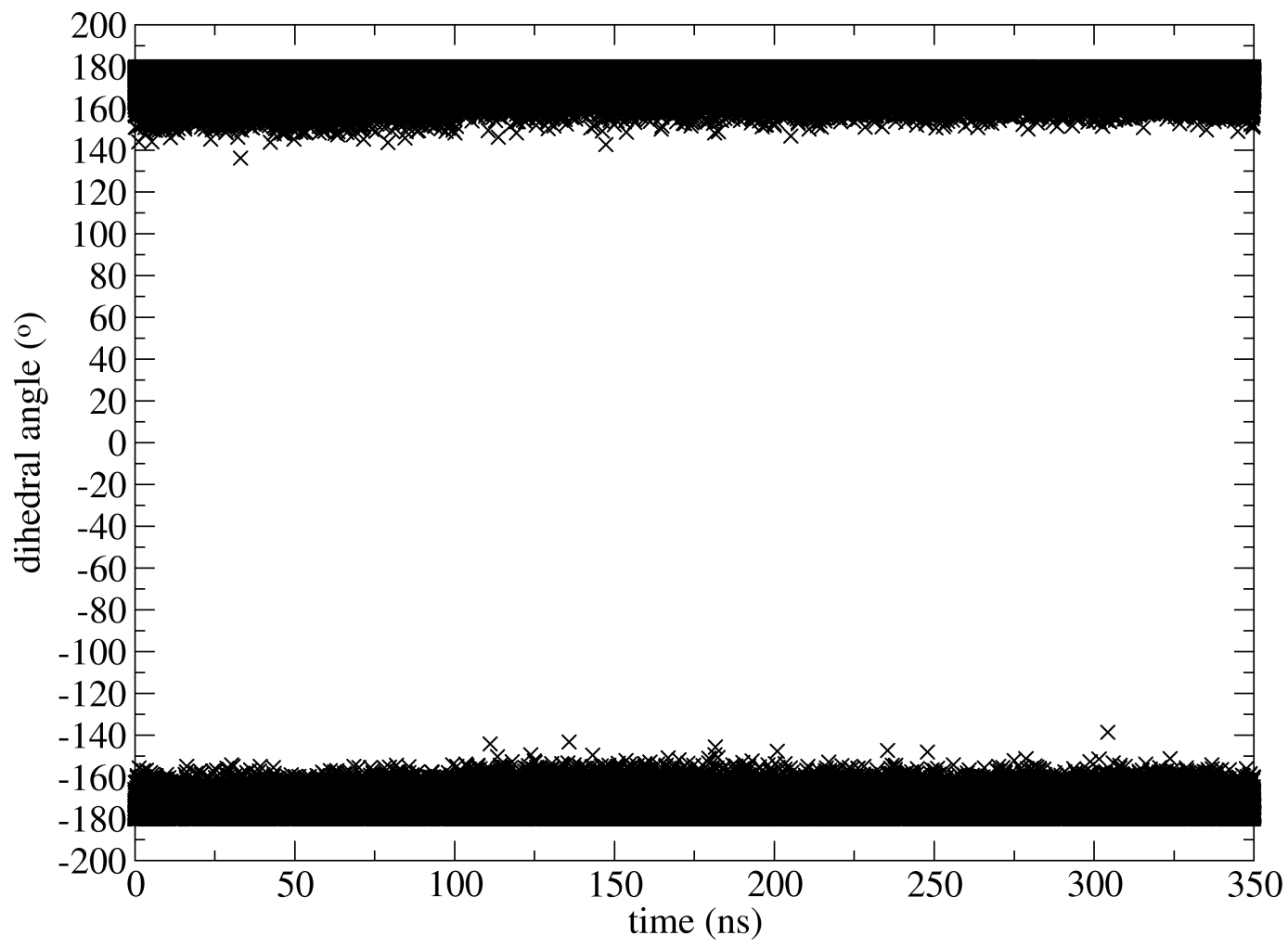

Dihedral angle defined by the sequence of atoms C99, CT91, CT92 in residue 2, and C13 in residue 1

Figure S8 - Values of the two $\mathrm{C}-\mathrm{C}-\mathrm{C}-\mathrm{C}$ dihedral angles of the carboxylate-iron bonds connecting the two heminic rings of the central FPD entity in the crystal model composed of 27 FPD entities (in degrees) as a function of time (in ns). The values represented correspond to a MD trajectory representative of the trends shown in the MD trajectories performed in this work. 


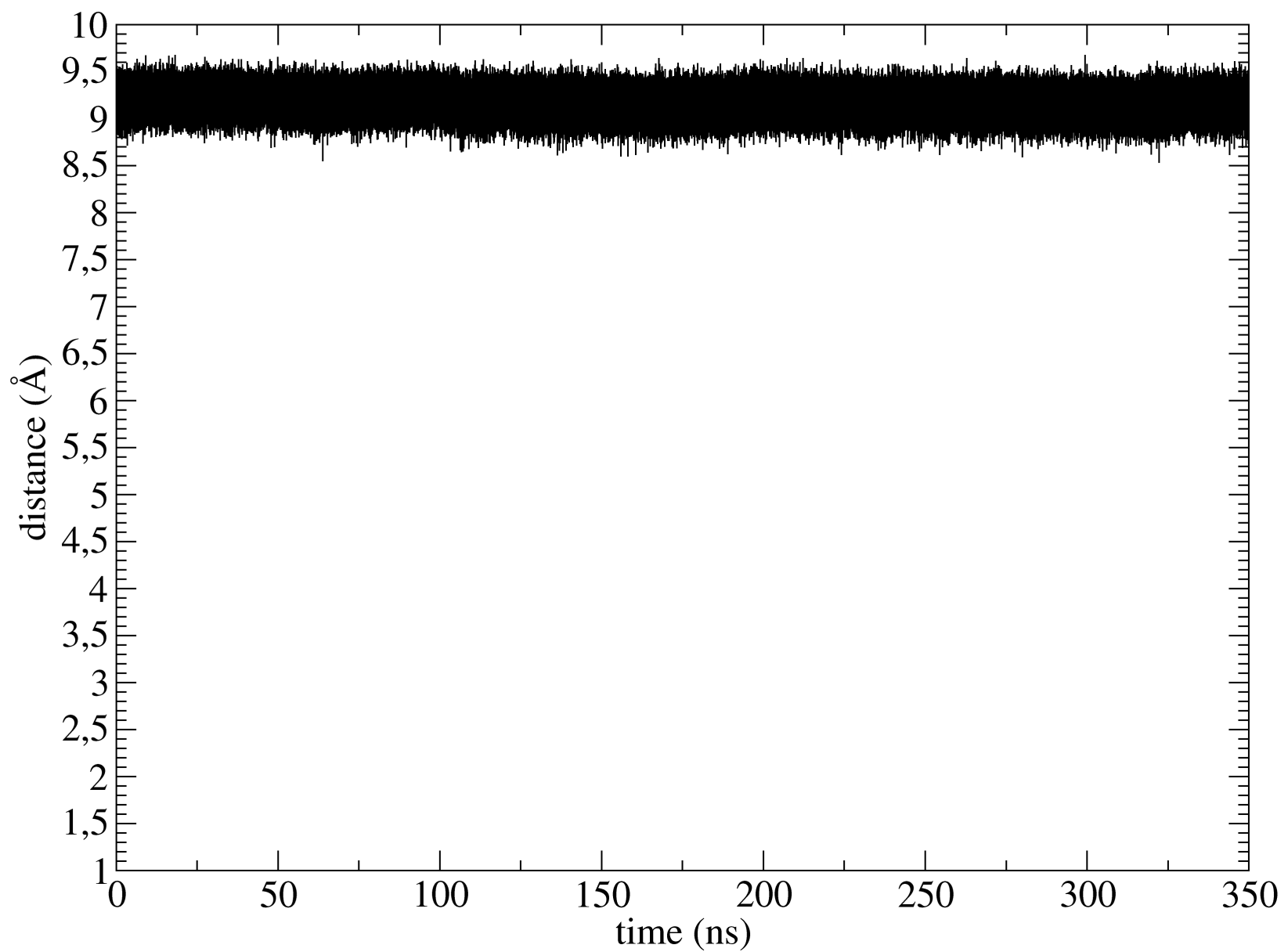

Figure S9 - Values of the distance between the two iron atoms of the central FPD entity in the crystal model made of 27 FPD entities (in $\AA$ ) as a function of time (in ns). The values represented correspond to a MD trajectory representative of the trends shown in the MD trajectories performed in this work. 


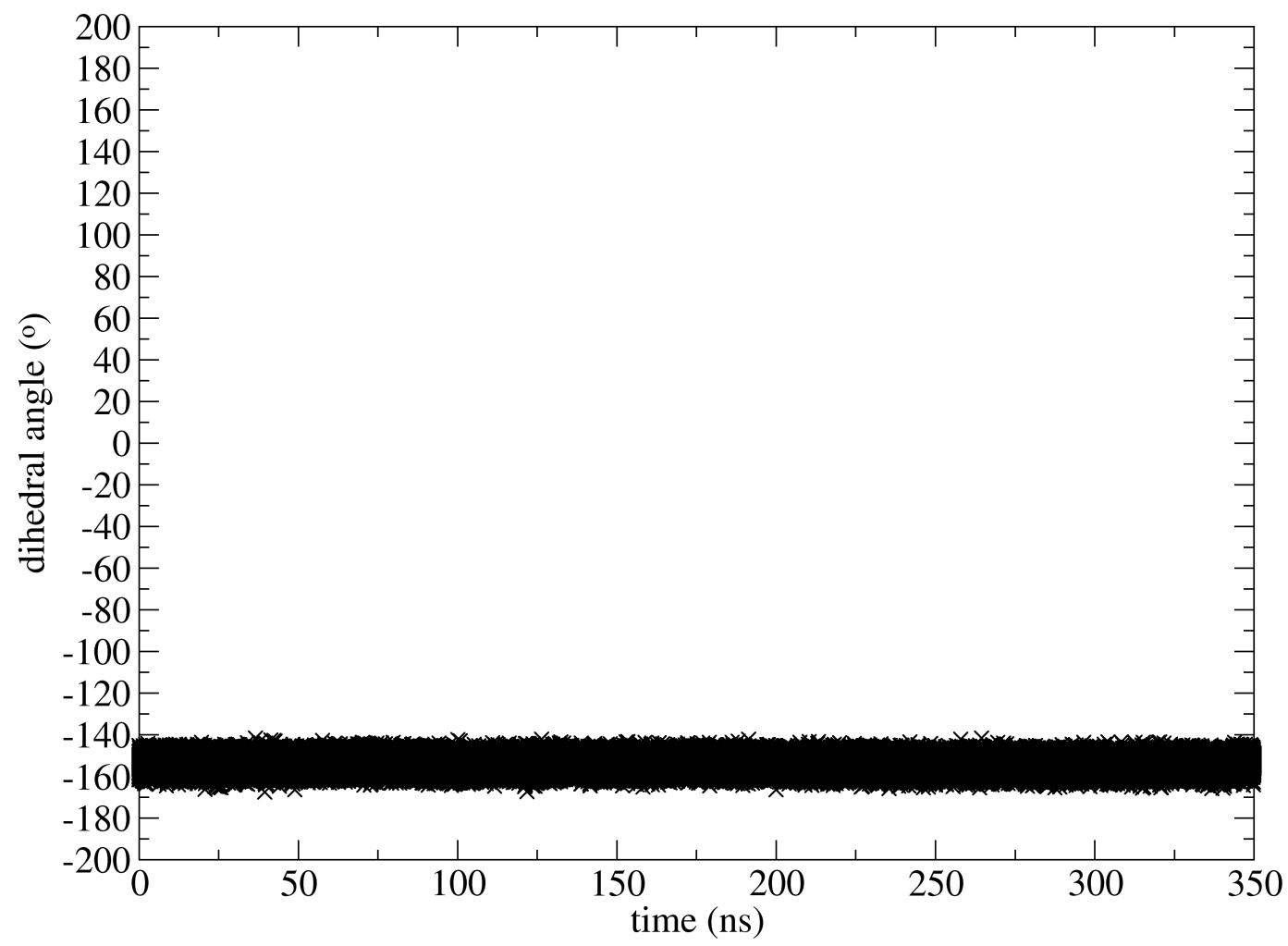

Improper dihedral angle defined by the sequence of atoms N21, N22, FE, N23 in residue 1

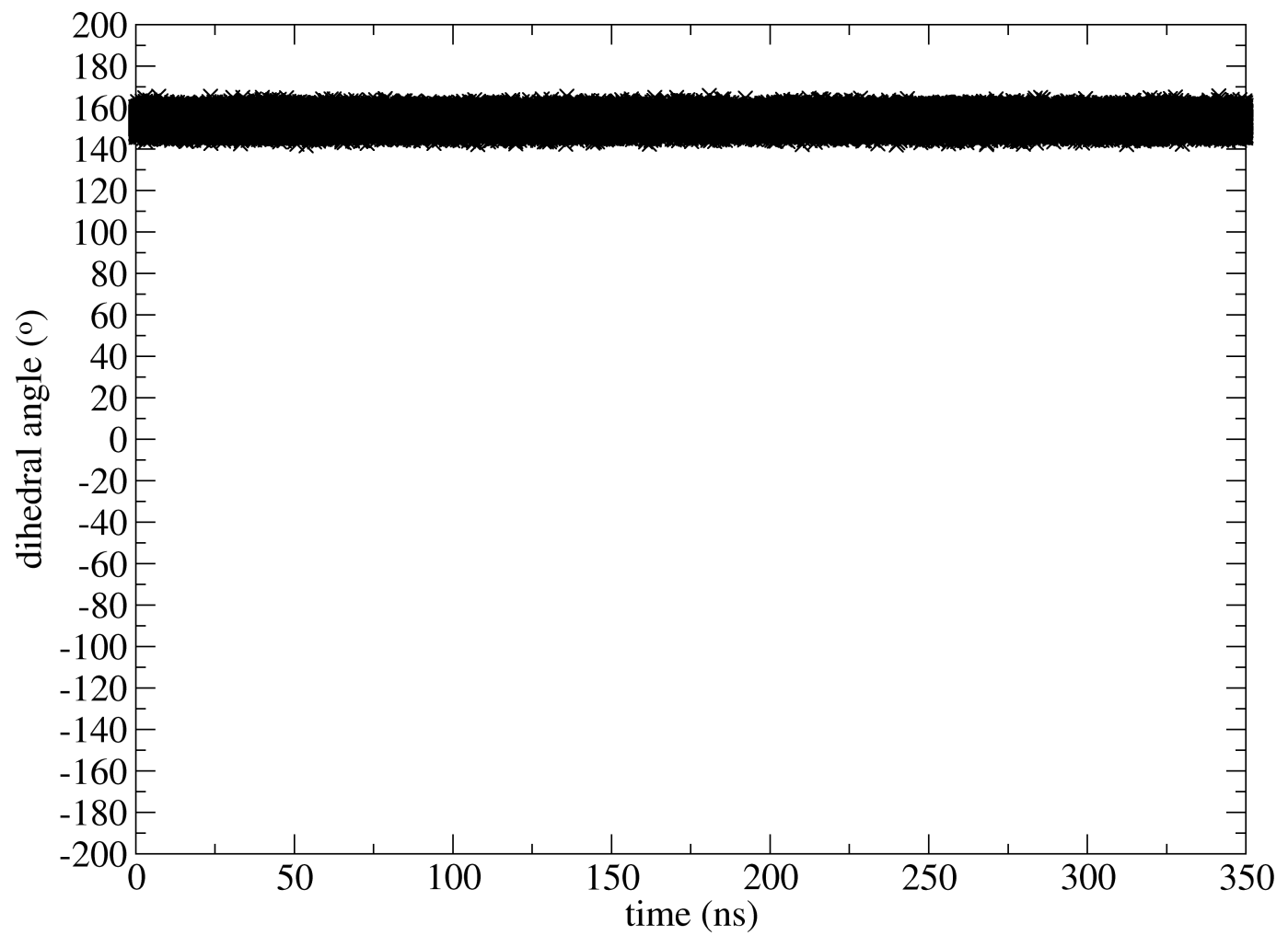

Improper dihedral angle defined by the sequence of atoms N21, N22, FE, N23 in residue 2

Figure S10a - Values of the two N21-N22-FE-N23 improper dihedral angles of the central FPD entity in the crystal model made of 27 FPD entities (in degrees) as a function of time (in ns). Improper dihedral angles are defined based on the alphabetical order of the names of the involved atoms. In the crystallographic reference, the value of this angle is 154 degrees. The values represented correspond to a MD trajectory representative of the trends shown in the MD trajectories performed in this work. 


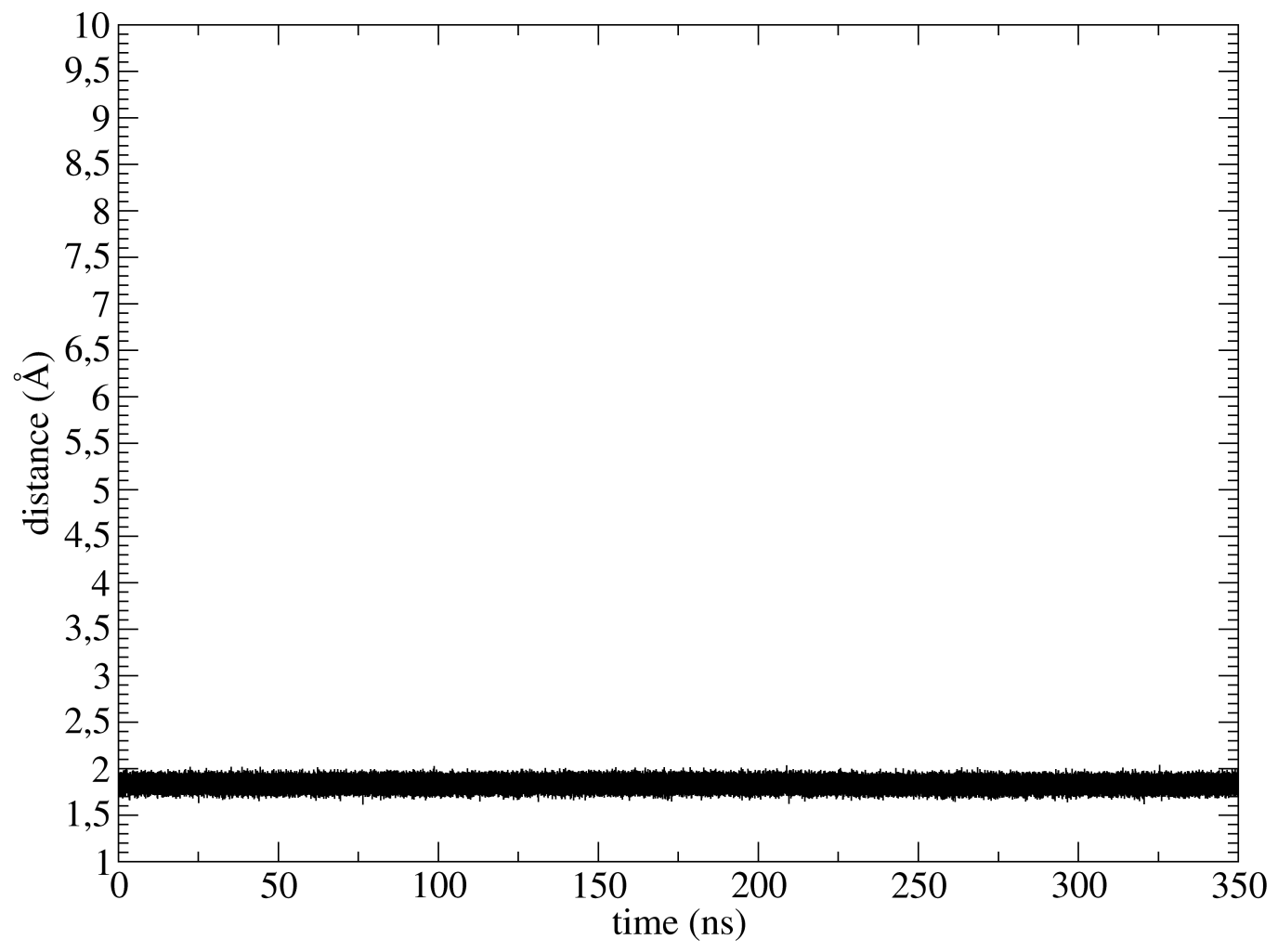

Distance between atom FE and atom $\mathrm{O} 91$ of residue 1

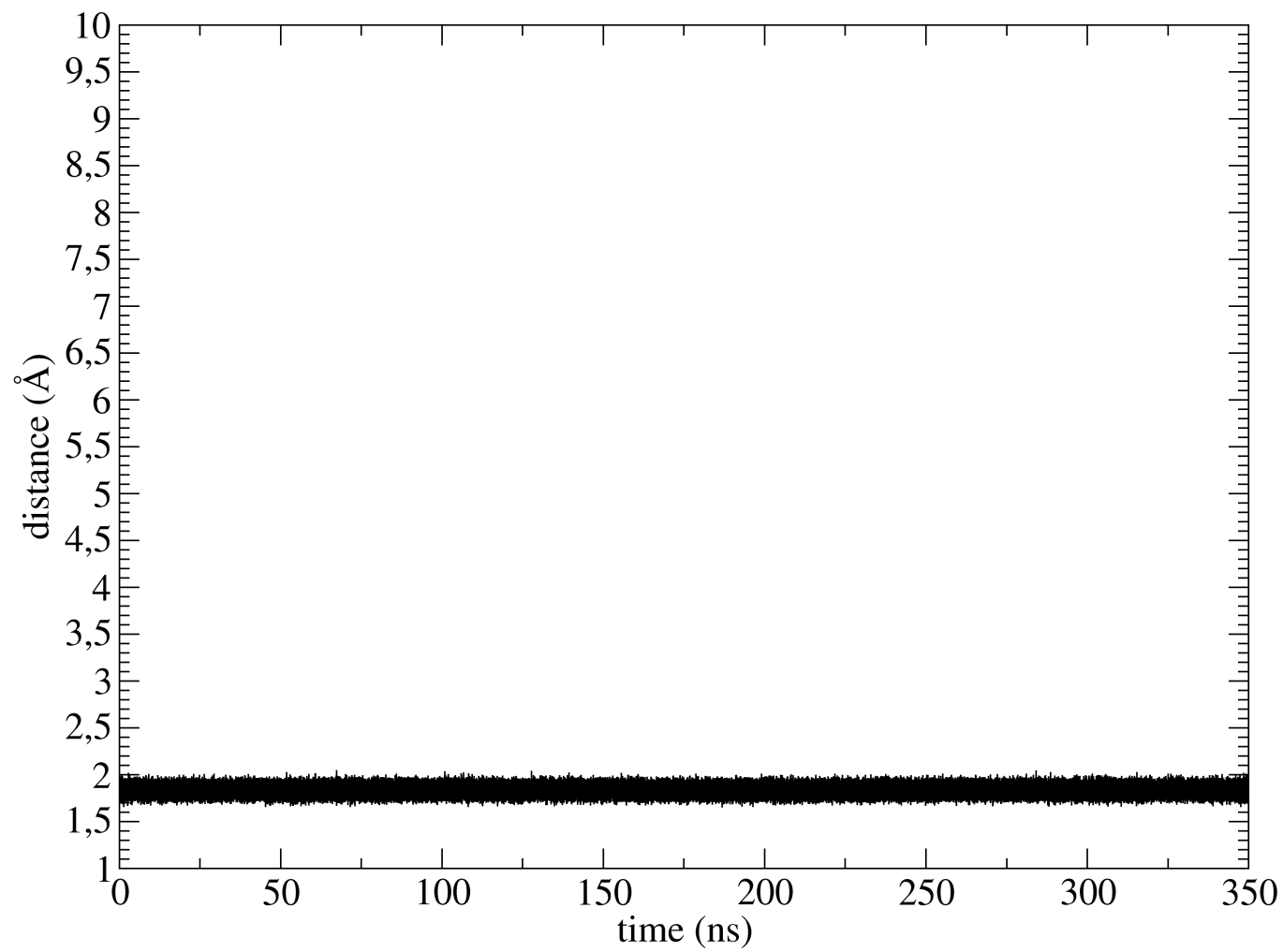

Distance between atom FE and atom O91 of residue 2

Figure S10b - Values of the distance between the iron atom FE and the oxygen atom O91 of residue 1 (top) and of residue 2 (bottom) of the central FPD entity (in $\AA$ ) as a function of time (in ns). In the crystallographic reference, the value of this distance is $1.8 \AA$. The values represented correspond to a MD trajectory representative of the trends shown in the MD trajectories performed in this work. 


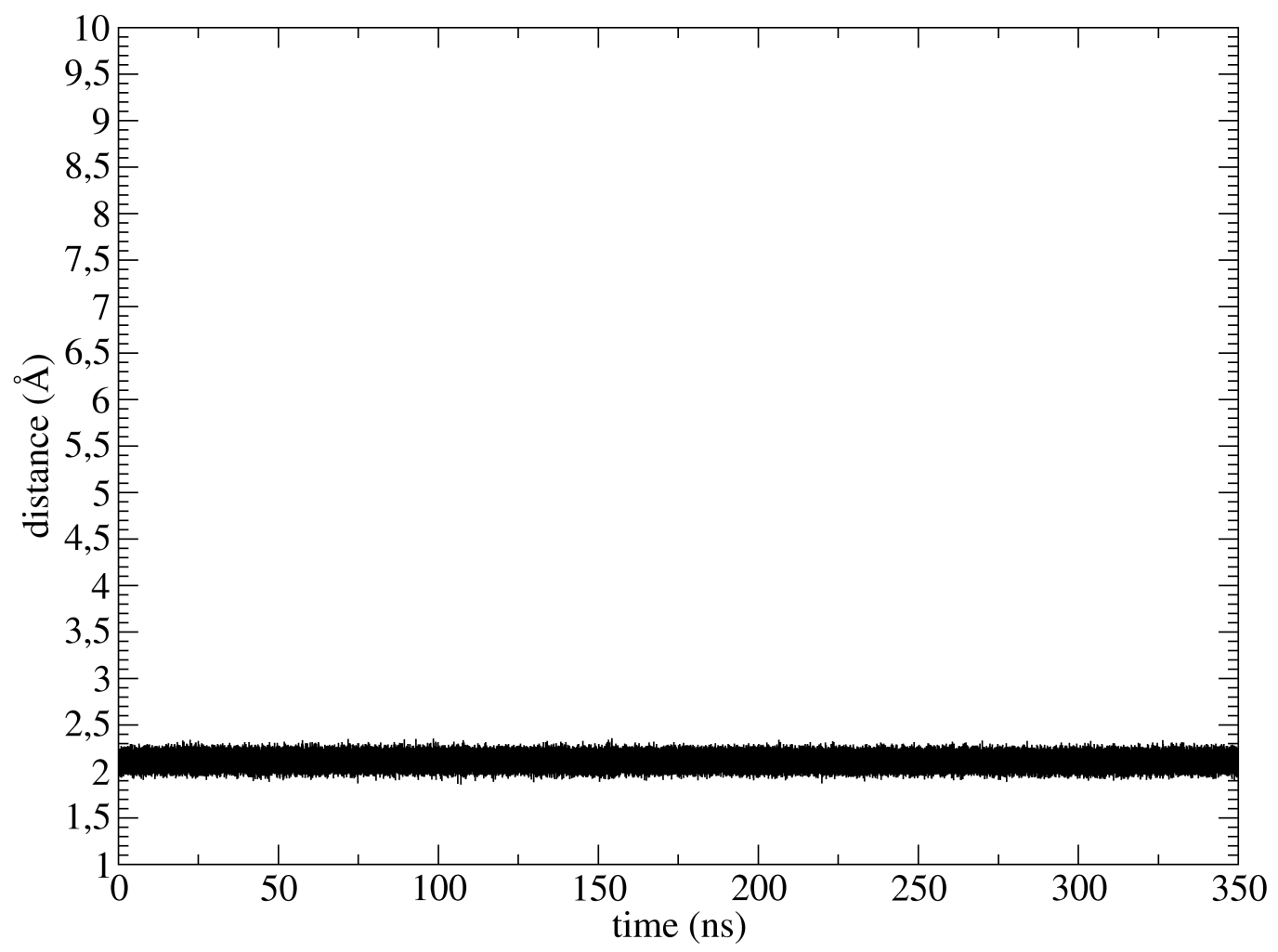

Distance between atom FE and atom N21 of residue 1

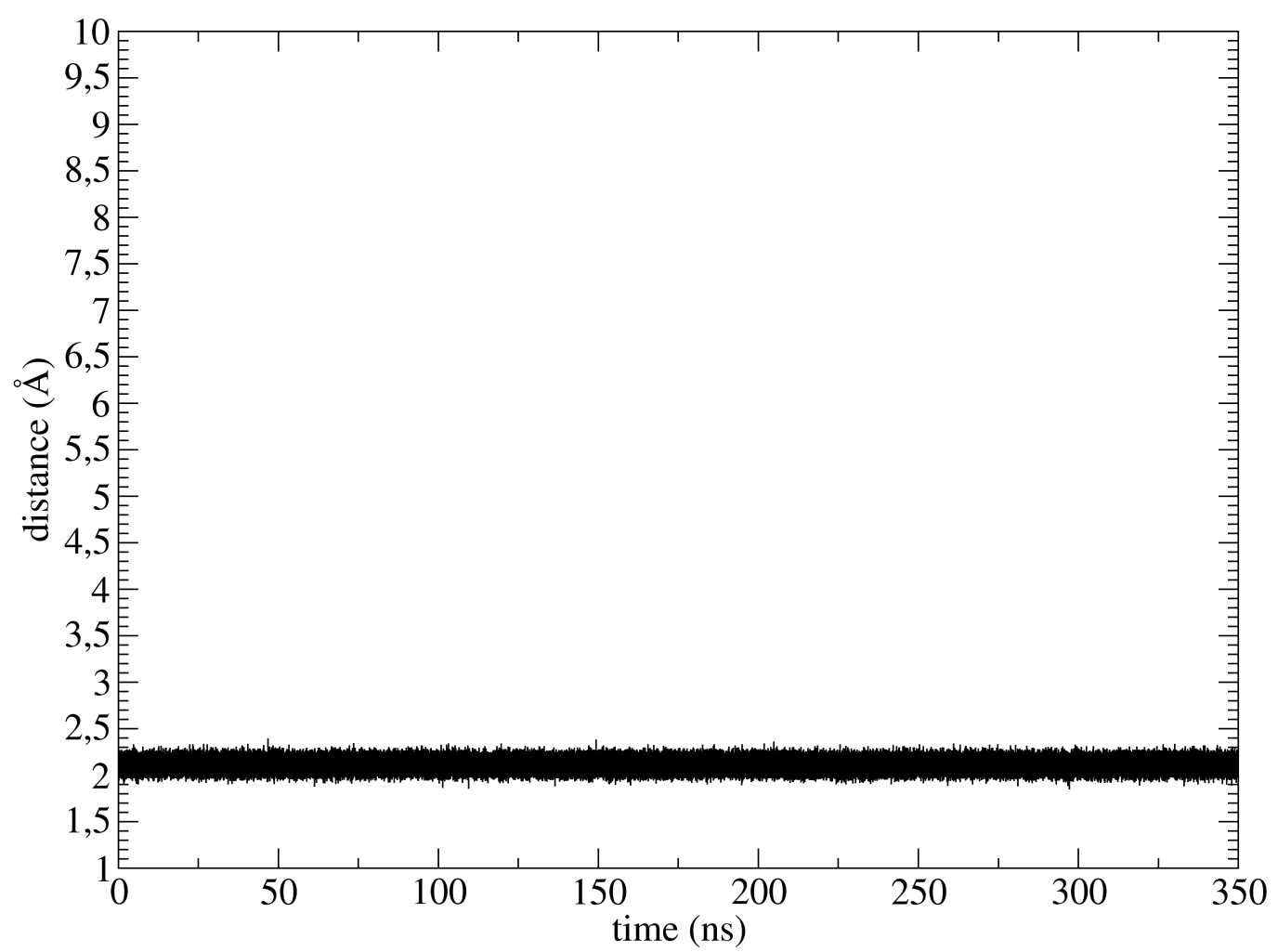

Distance between atom FE and atom N21 of residue 2

Figure S10c - Values of the distance between the iron atom FE and the nitrogen atom N21 of residue 1 (top) and of residue 2 (bottom) of the central FPD entity (in $\AA$ ) as a function of time (in ns). In the crystallographic reference, the value of this distance is $2.1 \AA$. The distances between the iron atoms FE and nitrogen atoms N22, N23, or N24 are similar. The values represented correspond to a MD trajectory representative of the trends shown in the MD trajectories performed in this work. 
data was sorted, intra-residue interactions are NOT included,

Distance cutoff is 3.25 angstroms, angle cutoff is 120.00 degrees

Hydrogen bond information dumped for occupancies $>0.00$

Dumping schematic of time series after each h-bond, key follows:

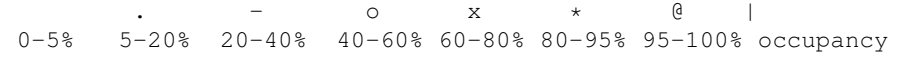

DONOR

atom\# :rescato

$3686: 398 @ 02$

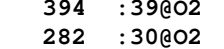

$986: 103002$

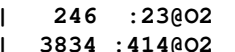

$726 \quad: 78 @ 02$
2022

$2022: 215 @ 02$

\begin{tabular}{rc|rc}
32 & $: 1 @ 092$ & 984 & $: 103 @ \mathrm{H} 3$ \\
134 & $: 14 @ 02$ & 2020 & $: 215 @ \mathrm{H} 3$ \\
\hline 1282 & $: 135002$ & 3980 & $: 430 \mathrm{H}$
\end{tabular}

: $578: 13502$ / $3980 \quad: 430 @ \mathrm{H} 3$

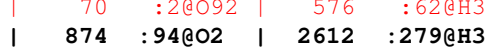

\begin{tabular}{ll|rl}
2844 & $: 305 @ 092$ & 3352 & $: 359 @ H 3$ \\
1134 & $: 119 @ 02$ & 3832 & $: 414 @ H 3$
\end{tabular}

\begin{tabular}{|l|ll|l}
1134 & $: 119 @ 02$ & 3832 & $: 414 @ \mathrm{H} 3$ \\
2438 & $: 258 @ 092$ & 1908 & $: 206 @ \mathrm{H}$
\end{tabular}

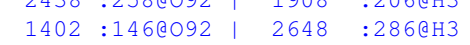

$3880: 4170092$

$2992: 3210092$

$2142: 2260092$

3584
$476: 3850092$
$: 490092$

$1994: 2100092$

$1254: 1300092$

$3732: 4010092$

$3140: 337 @ 092$

\begin{tabular}{rc|r}
2586 & $: 2740092$ & 2352 \\
218 & $: 180092$ & 872 \\
$\mathbf{9 8}$ & $: \mathbf{7 0 0 2}$ & $\mathbf{3 0 9 2}$
\end{tabular}

$2466: 2630202 \quad 7092: 334 \mathrm{QH} 3$

$3982: 430 @ 02 \quad$ | $1280: 135 \Subset \mathrm{H} 3$

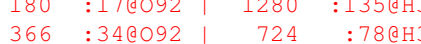

\begin{tabular}{r|r|r|} 
& $1430: 151 @ 02$ & $280 \quad: 30 @ \mathrm{H} 3$
\end{tabular}

$2614: 279 @ 02 \quad$ । $872: 94 @ \mathrm{H}$

$: 94 \mathrm{eH} 3$ 


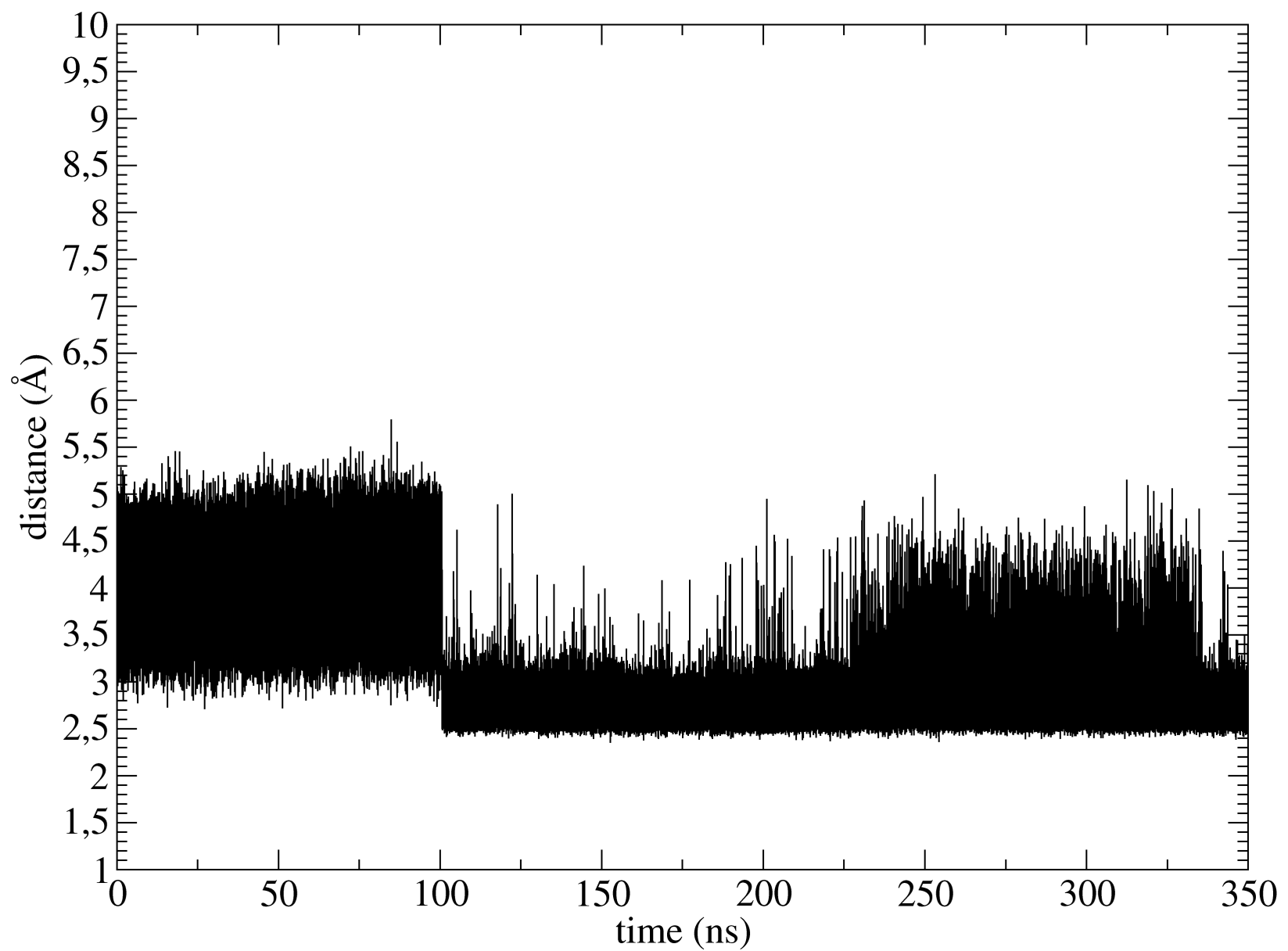

Figure S12 - Example of values of the distance between the oxygen atoms involved in an inter-FPD HB (in $\AA$ ) as a function of time (in ns). The values reported here correspond to the distance between the oxygen atom $\mathrm{O} 3$ of residue 7 and the oxygen atom $\mathrm{O} 92$ of residue 49. The values represented correspond to a MD trajectory representative of the trends shown in the MD trajectories performed in this work. 


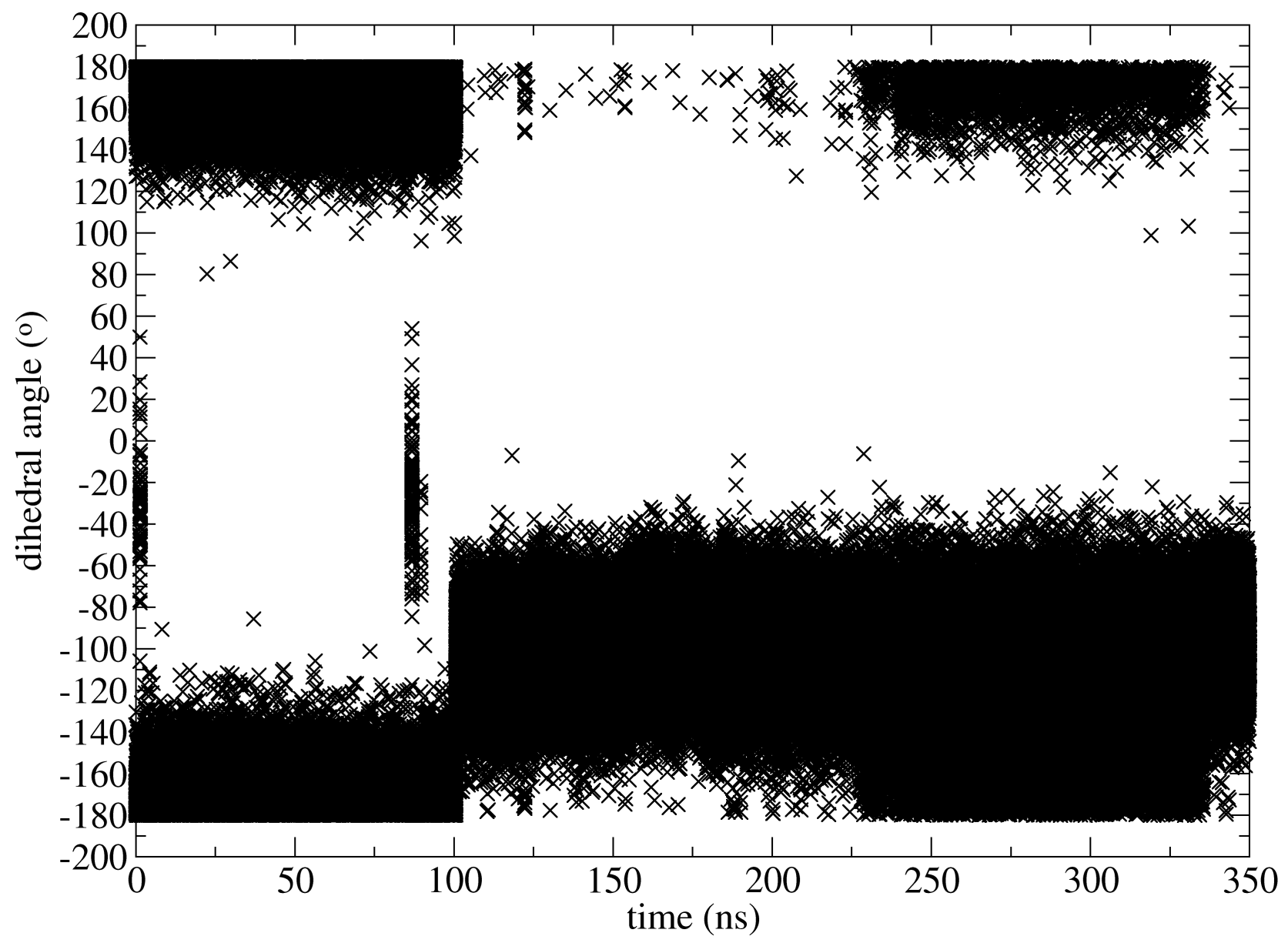

Figure S13 - Example of Values of the $\mathrm{C}-\mathrm{C}-\mathrm{C}-\mathrm{O}$ dihedral angle of the carboxylic acid groups of a FPD entity involved in an inter-FPD HB (in degrees) as a function of time (in ns). The values reported here correspond to the dihedral angle defined by the sequence of atoms C5, C4, C1, and O3 in residue 7. The values represented correspond to a MD trajectory representative of the trends shown in the MD trajectories performed in this work. 


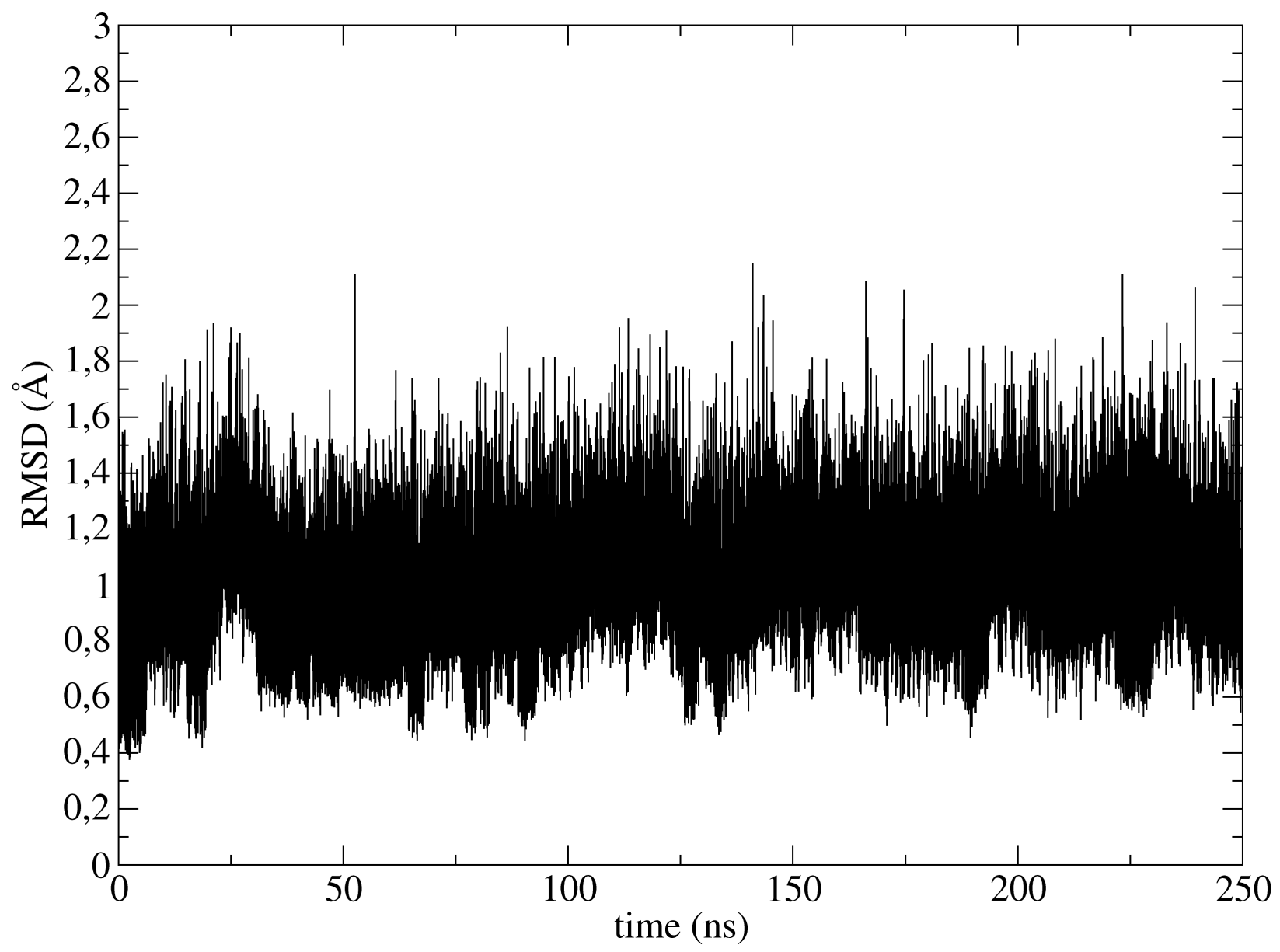

Figure S14 - Values of the RMSD (in $\AA$ ) as a function of time (in ns) computed on the heavy atoms of the D1 dimer. The reference structure used to compute the RMSD is the initial configuration of the MD simulation (i.e. the Cartesian coordinates taken from the crystallographic structure). The values represented correspond to a MD trajectory representative of the trends shown in the MD trajectories performed in this work. 


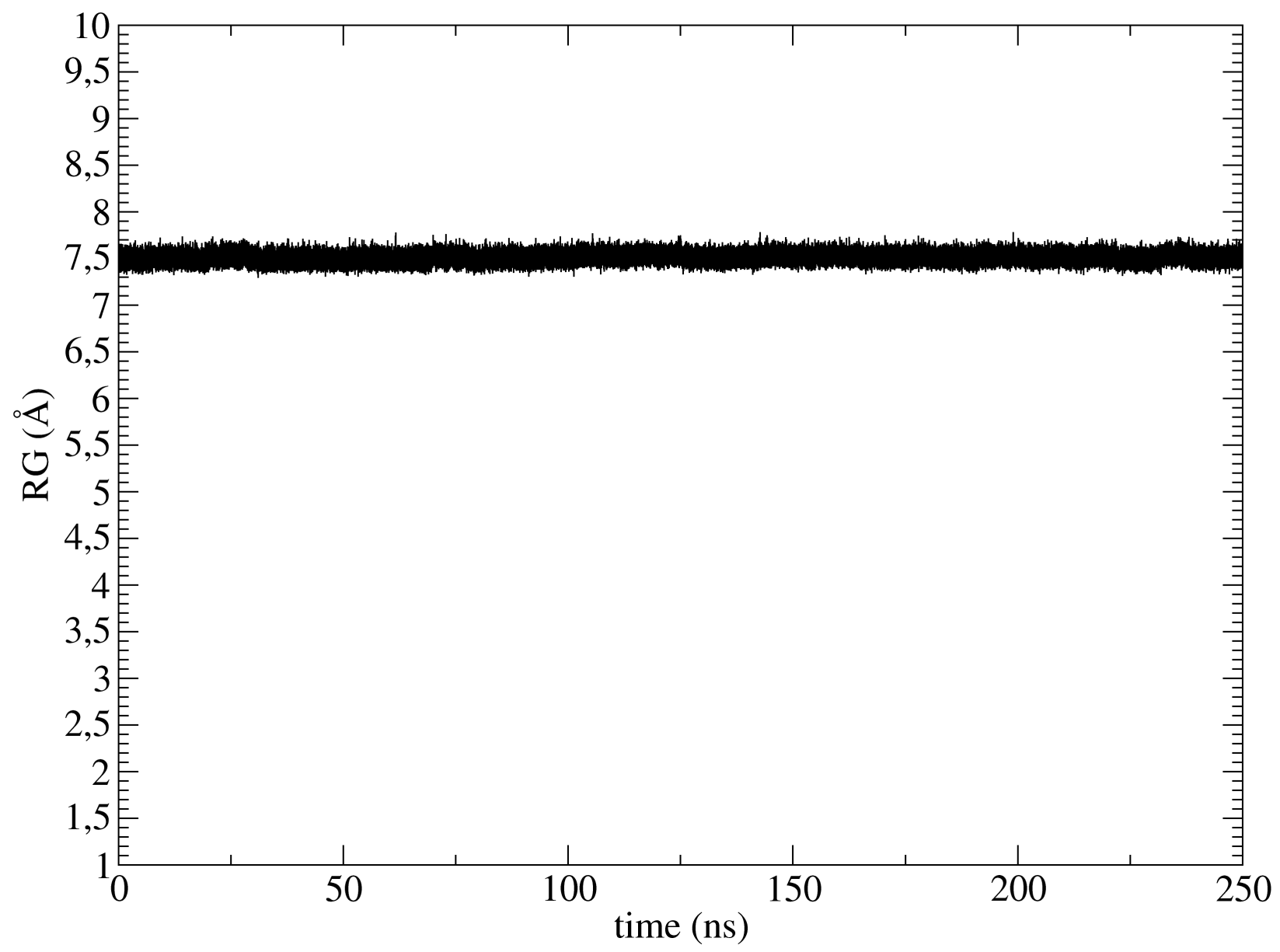

Figure S15 - Values of the RG (in $\AA$ ) as a function of time (in ns) of the D1 dimer. The values represented correspond to a MD trajectory representative of the trends shown in the MD trajectories performed in this work. 


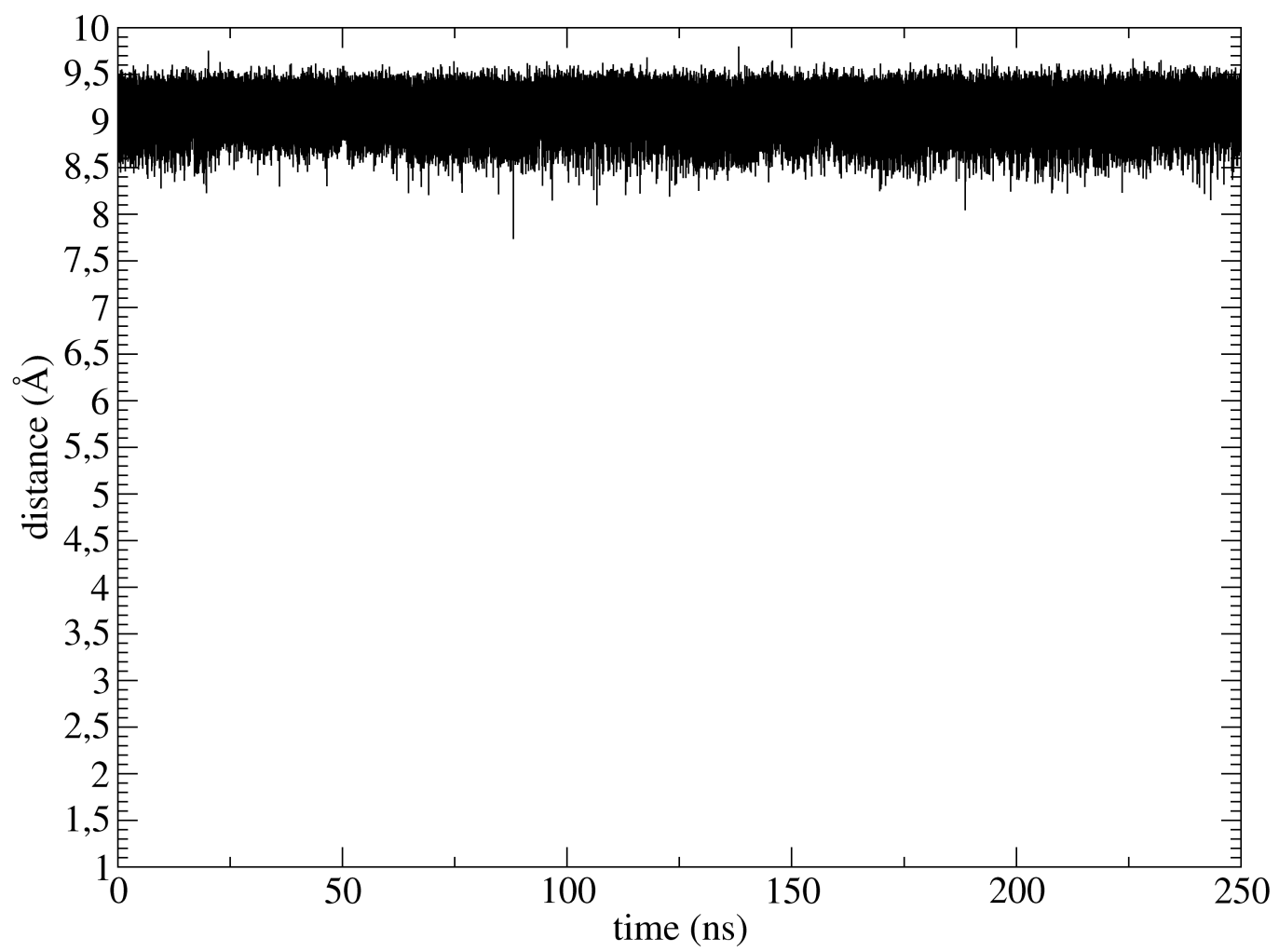

Distance between atom FE of residue 1 and atom FE of residue 2

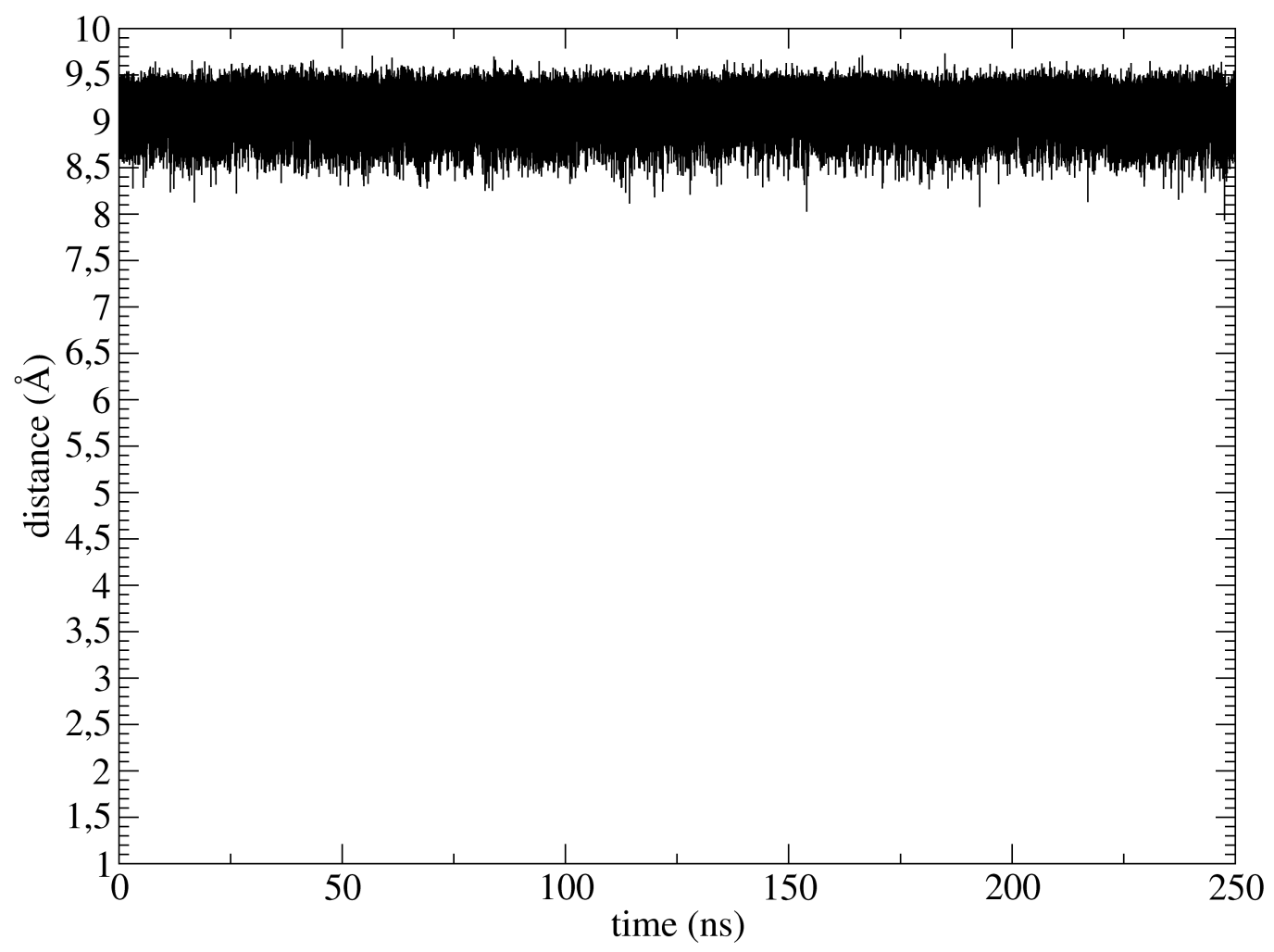

Distance between atom FE of residue 17 and atom FE of residue 18

Figure S16 - Values of the distances between the two iron atoms in each FPD entity of the D1 dimer (in $\AA$ ) as a function of time (in ns). The values represented correspond to a MD trajectory representative of the trends shown in the MD trajectories performed in this work. 
HBOND SUMMARY:

Data was saved to series hbond, output to file HBond_info_intraF_325.txt,

data was sorted, intra-residue interactions are NOT included,

Distance cutoff is 3.25 angstroms, angle cutoff is 120.00 degrees

Hydrogen bond information dumped for occupancies $>0.00$

Dumping schematic of time series after each h-bond, key follows:

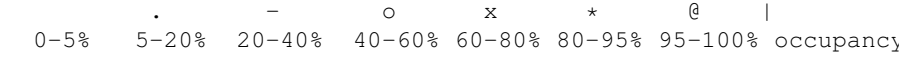

DONOR

atom\# :res@atom atom\# :res@atom atom\# :res@atom

70 :

\begin{tabular}{rr|rrrrrrrrrr}
180 & $: 20092$ & 280 & $: 30 @ \mathrm{H} 3$ & 279 & $: 30 @ 03$ & 81.36 & 2.748 & $(0.13)$ & 24.24 & $(12.99)$ \\
180 & $: 17 @ 092$ & 96 & $: 7 @ \mathrm{H} 3$ & 95 & $: 7 @ 03$ & 52.69 & 2.750 & $(0.13)$ & 24.65 & $(13.10$
\end{tabular}

11fetime maxocc

$19.6(-22.5) \quad 466$

58 ।

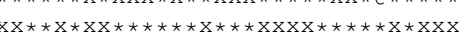

Figure S17 - Occupancy rate of the HB in the D1 dimer. The data reveal the formation of one HB between atom O92 of residue 2 and O3 of residue 30 and one $\mathrm{HB}$ between atom $\mathrm{O} 92$ of residue 17 and $\mathrm{O} 3$ of residue 7. Both of these HB bonds are inter-FPD HB as described in the manuscript. The formation of $\mathrm{HB}$ was followed along the $250 \mathrm{~ns}$ ) of the whole trajectory. The data reported come from one MD trajectory representative of all the MD trajectories performed in this work. 


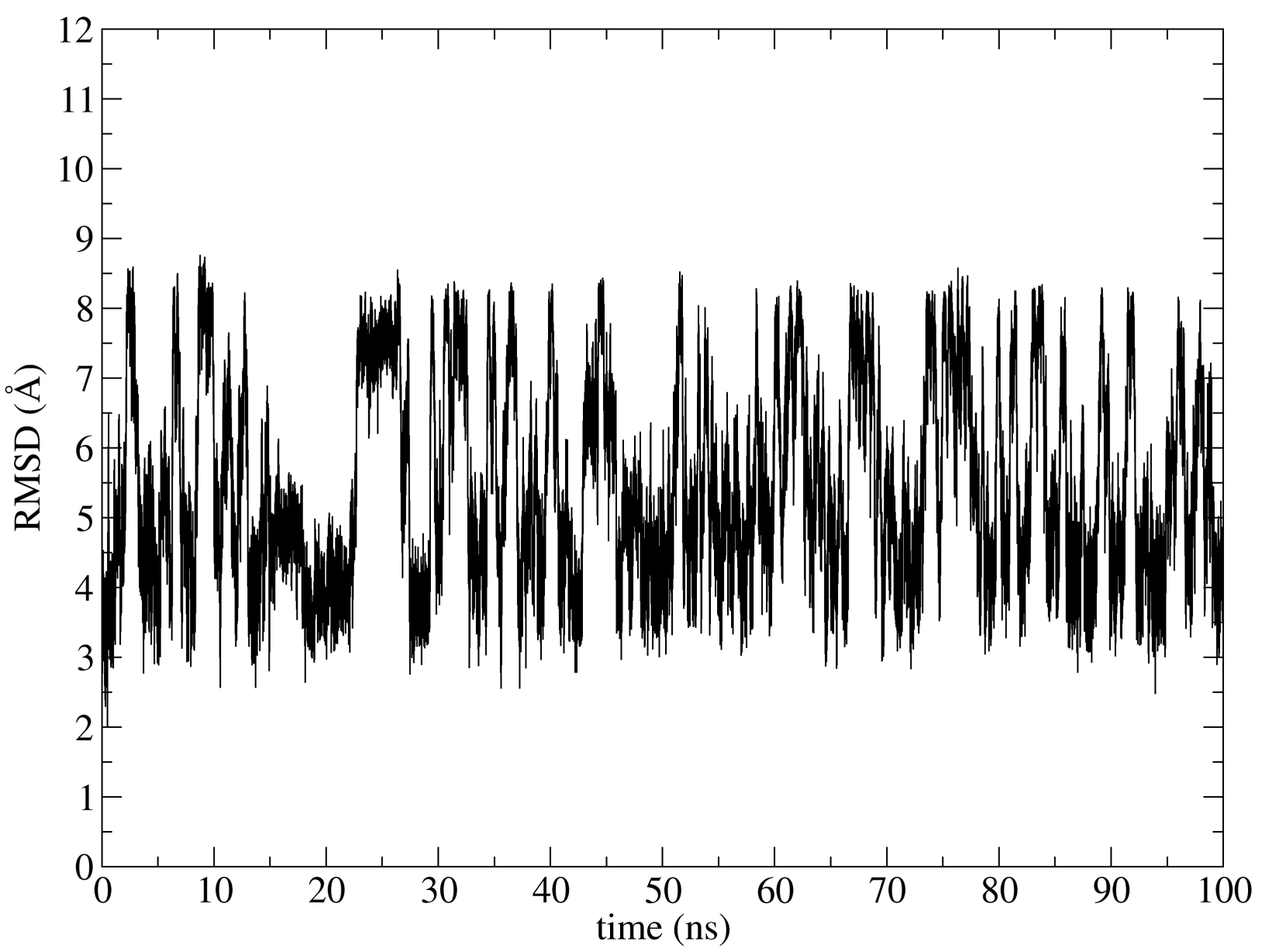

Figure S18 - Values of the RMSD (in $\AA$ ) as a function of time (in ns) computed on the heavy atoms of the D2 dimer. The reference structure used to compute the RMSD is the initial configuration of the MD simulation (i.e. the Cartesian coordinates taken from the crystallographic structure). The values represented correspond to a MD trajectory representative of the trends shown in the MD trajectories performed in this work. 


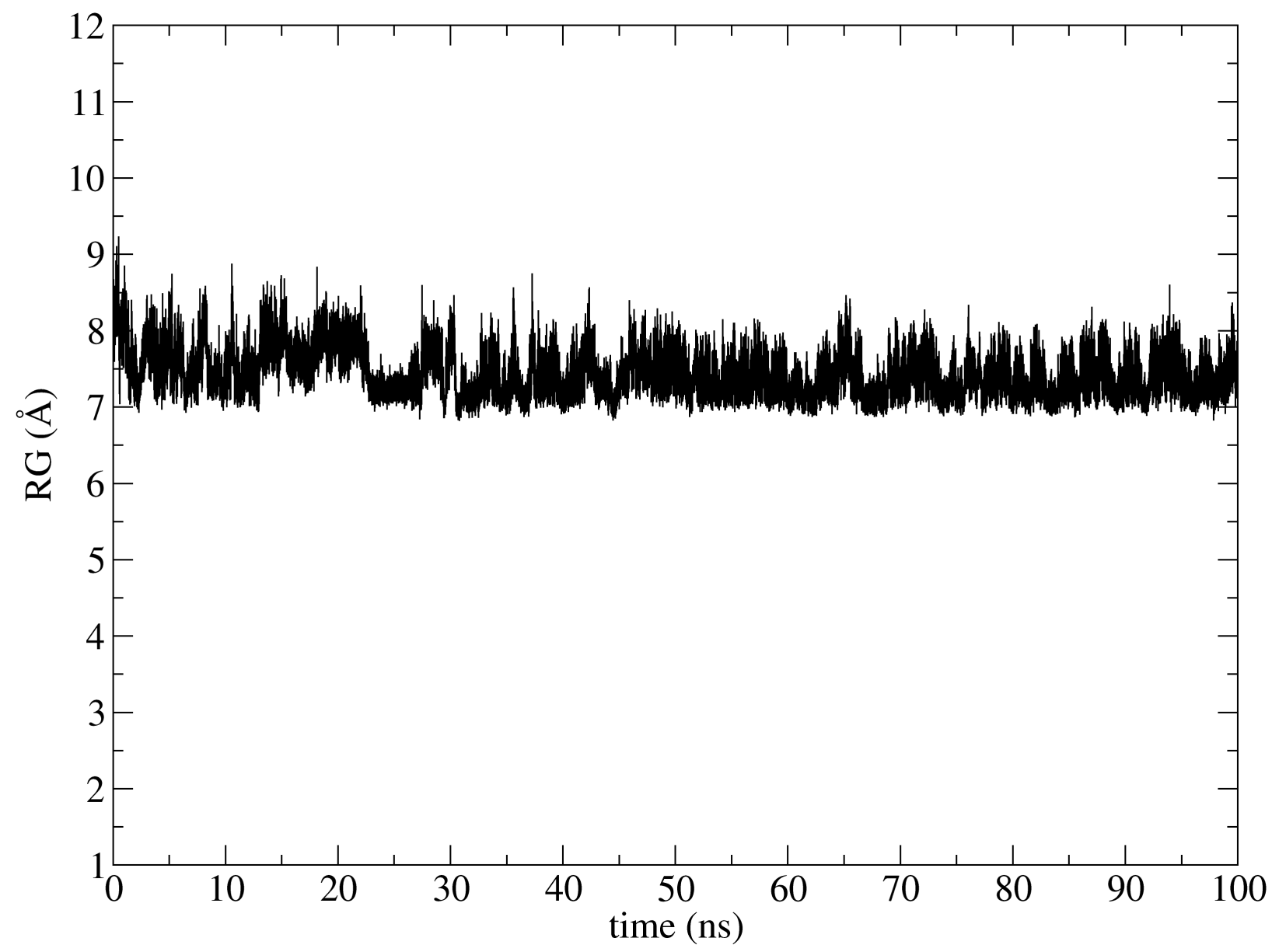

Figure S19 - Values of the RG (in $\AA$ ) as a function of time (in ns) of the D2 dimer. The values represented correspond to a MD trajectory representative of the trends shown in the MD trajectories performed in this work. 


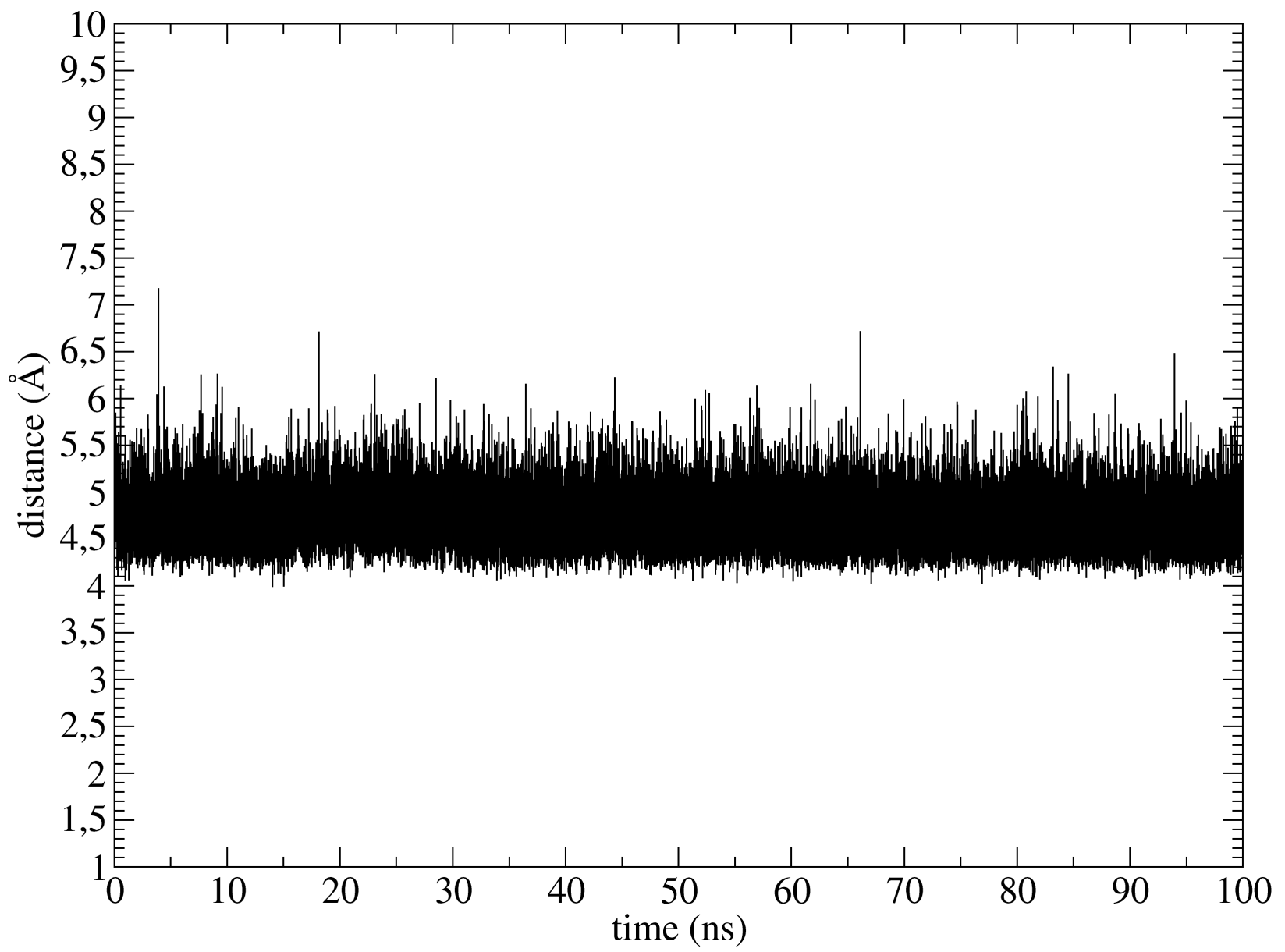

Figure S20 - Values of the distance between the two closest FE atoms (atom FE in residue 1 and atom FE in residue 18) of the two FPD entities of D2 dimer (in $\AA$ ) as function of time (in ns). The values represented correspond to a MD trajectory representative of the trends shown in the MD trajectories performed in this work. 


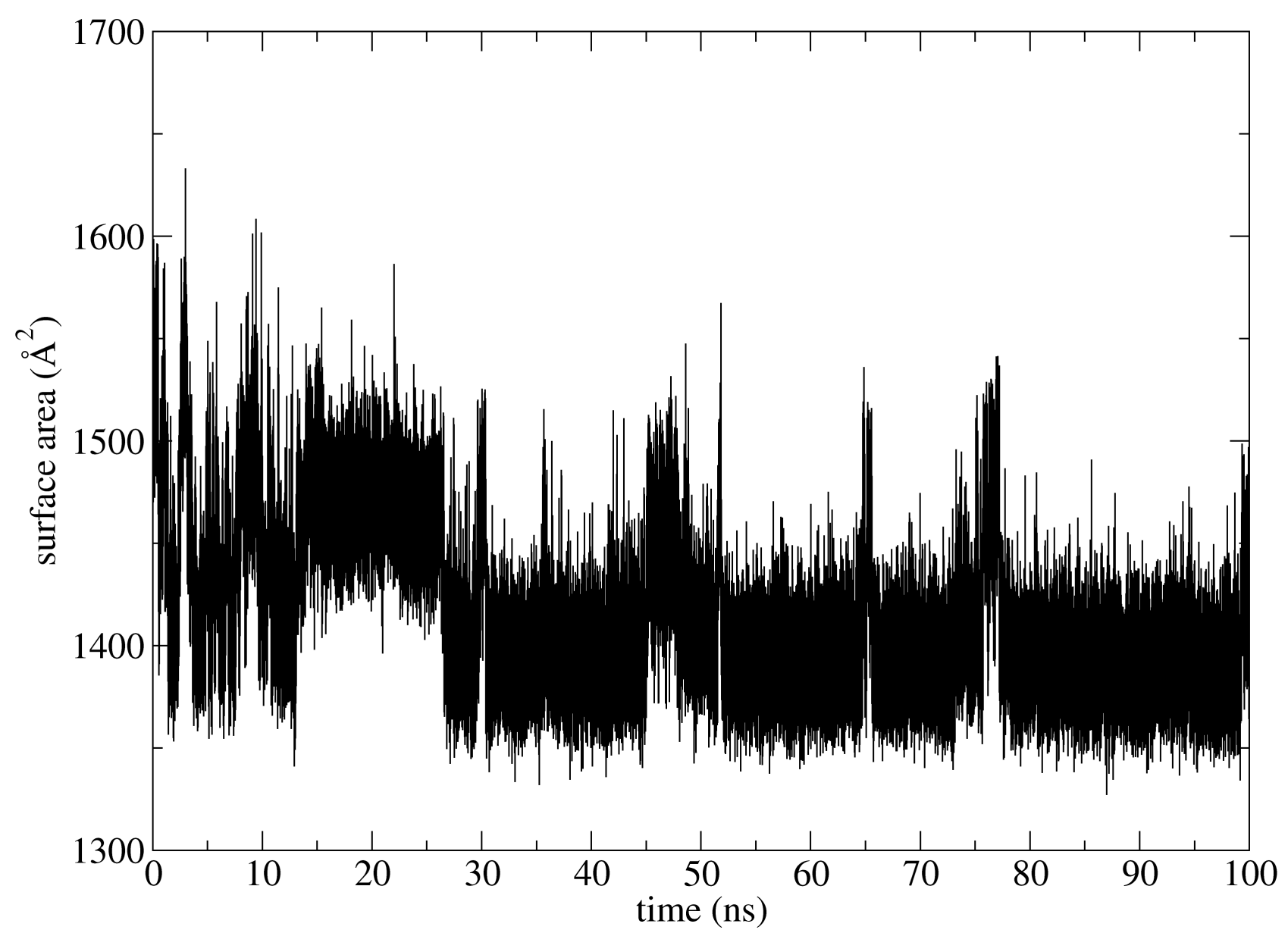

Figure S21 - SASA of the D2 dimer (in $\AA^{2}$ ) as a function of time (in ns). The SASA of the D2 dimer in the initial configuration of the MD is $1689 \AA^{2}$. The values represented correspond to a MD trajectory representative of the trends shown in the MD trajectories performed in this work. 


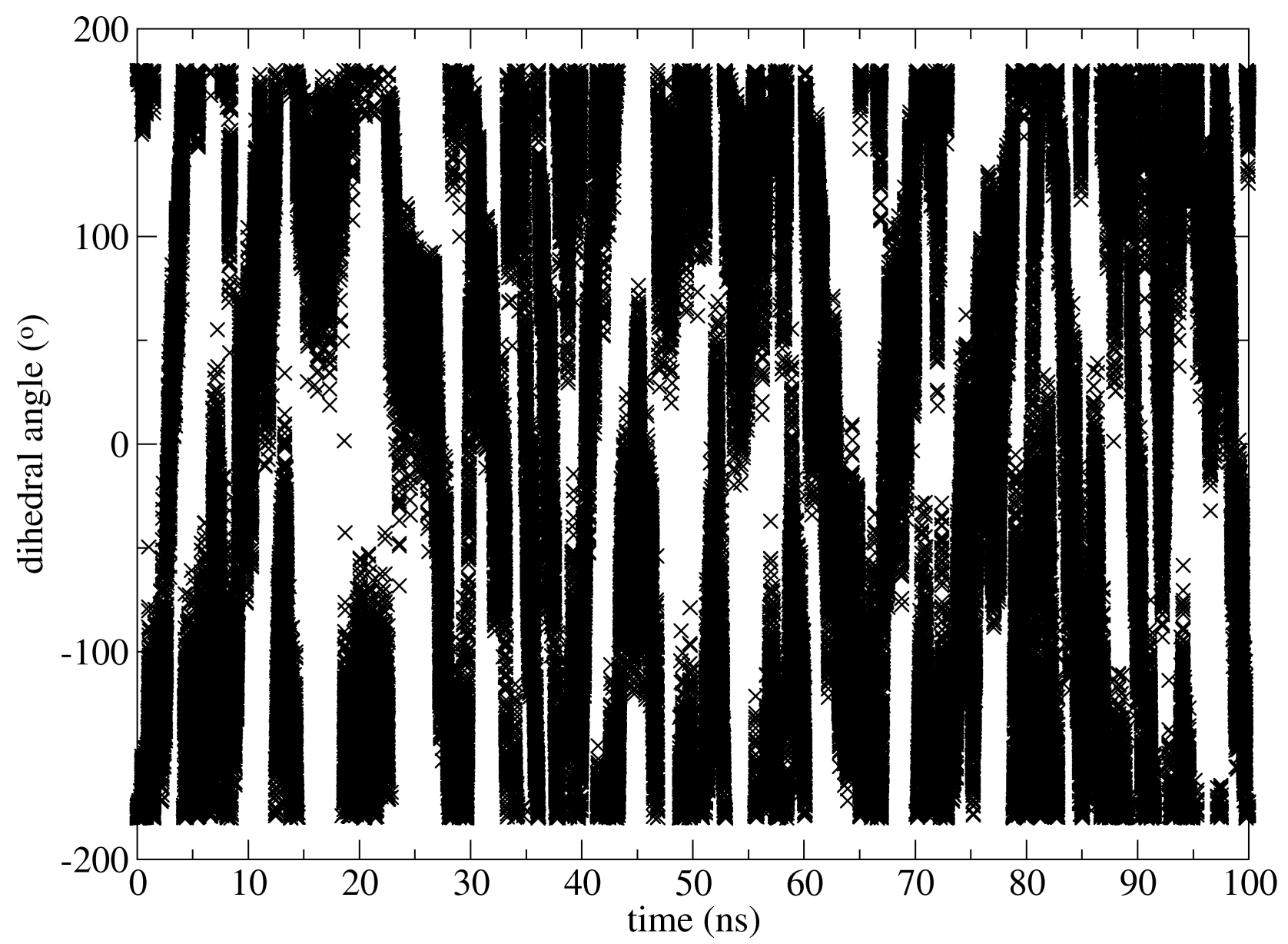

Figure S22 - Values of the FE-FE-FE-FE dihedral angle (i.e. the dihedral angle defined by the sequence of atoms FE in residue 2, FE in residue 1, FE in residue 18, and FE in residue 17) of the dimer D2 (in degrees) as a function of time (in ns). The values represented correspond to a MD trajectory representative of the trends shown in the MD trajectories performed in this work. 


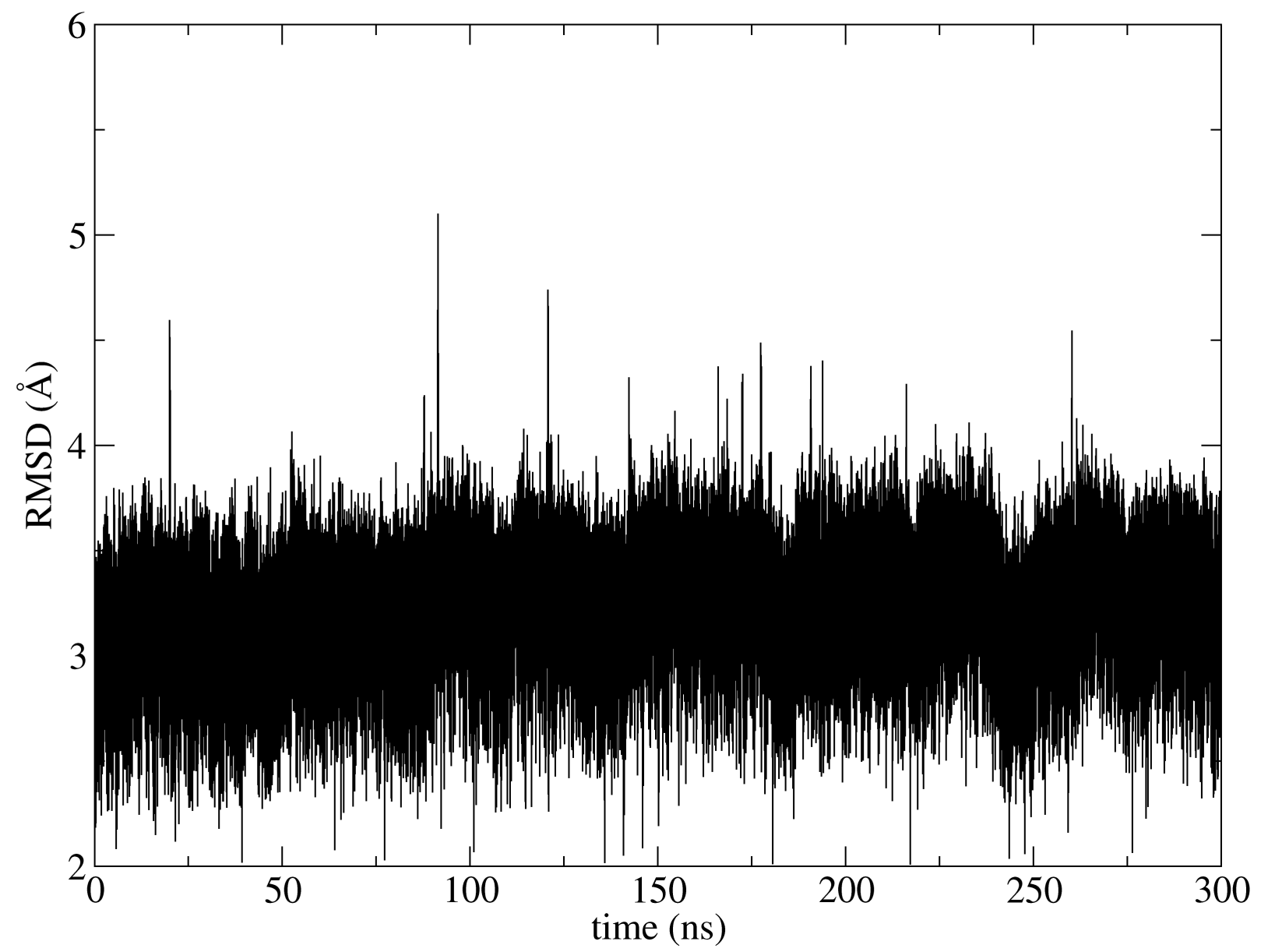

Figure S23 - Values of the RMSD (in $\AA$ ) as a function of time (in ns) computed on the heavy atoms of the D3 dimer. The reference structure used to compute the RMSD is the initial configuration of the MD simulation (i.e. the Cartesian coordinates taken from the crystallographic structure). The values represented correspond to a MD trajectory representative of the trends shown in the MD trajectories performed in this work. 


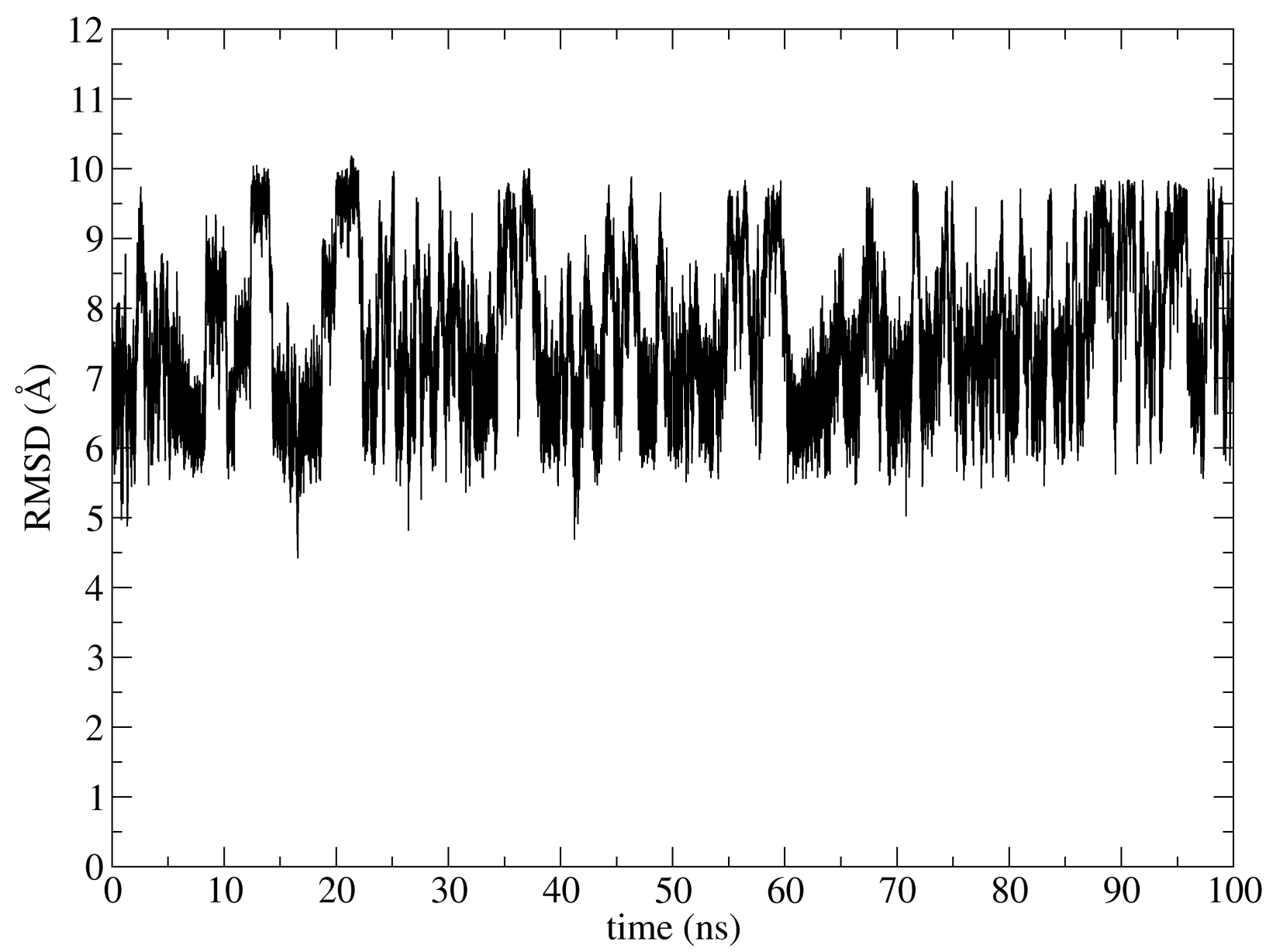

Figure S24 - Values of the RMSD (in $\AA$ ) as a function of time (in ns) computed on the heavy atoms of the D4 dimer. The reference structure used to compute the RMSD is the initial configuration of the MD simulation (i.e. the Cartesian coordinates taken from the crystallographic structure). The values represented correspond to a MD trajectory representative of the trends shown in the MD trajectories performed in this work. 

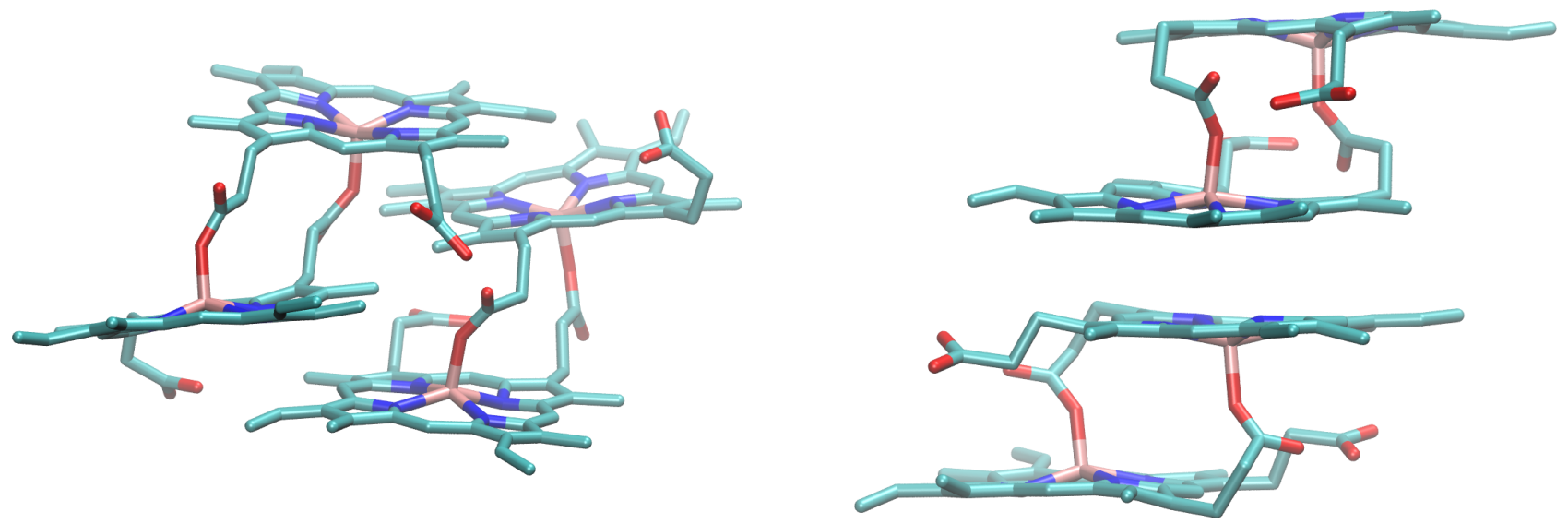

Figure S25 - Illustration of the evolution of the D3 dimer toward molecular structures with reduced SASA (left) and akin to D2 dimer (right). FPD entities are represented by sticks, carbon, nitrogen, oxygen, and iron atoms are colored in green, blue, red, and pink respectively. 

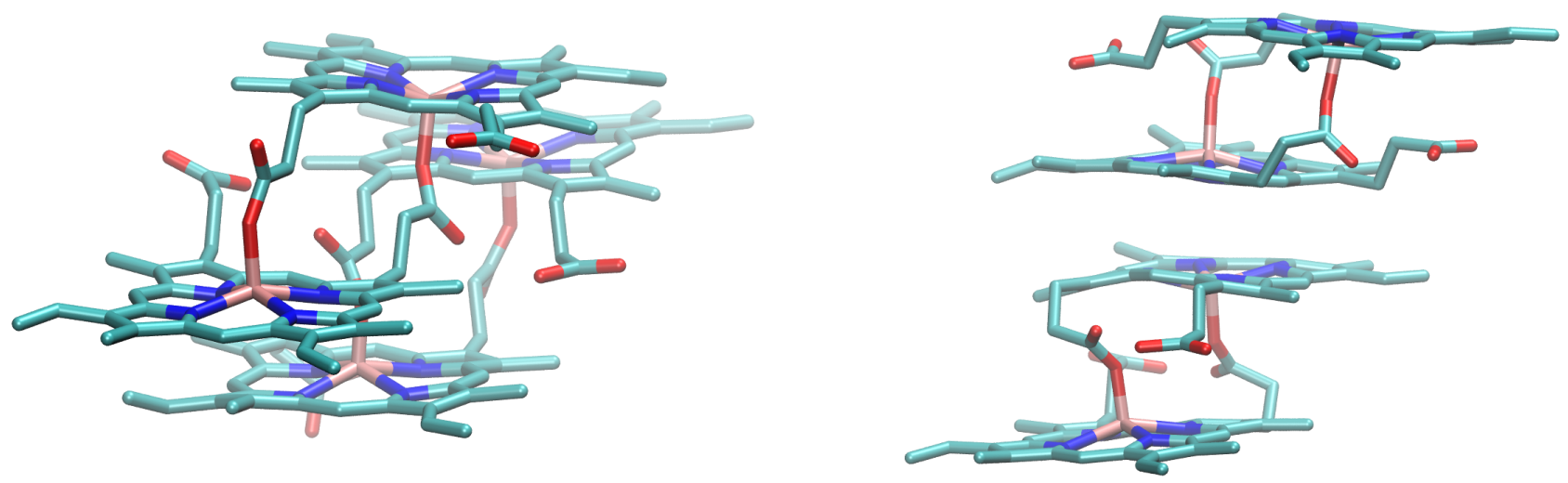

Figure S26 - Illustration of the evolution of the D4 dimer, occurring within 5 to 50 ns of MD simulations, toward molecular structures akin to D1 dimer (left) or D2 dimer (right). FPD entities are represented by sticks, carbon, nitrogen, oxygen, and iron atoms are colored in green, blue, red, and pink respectively. 


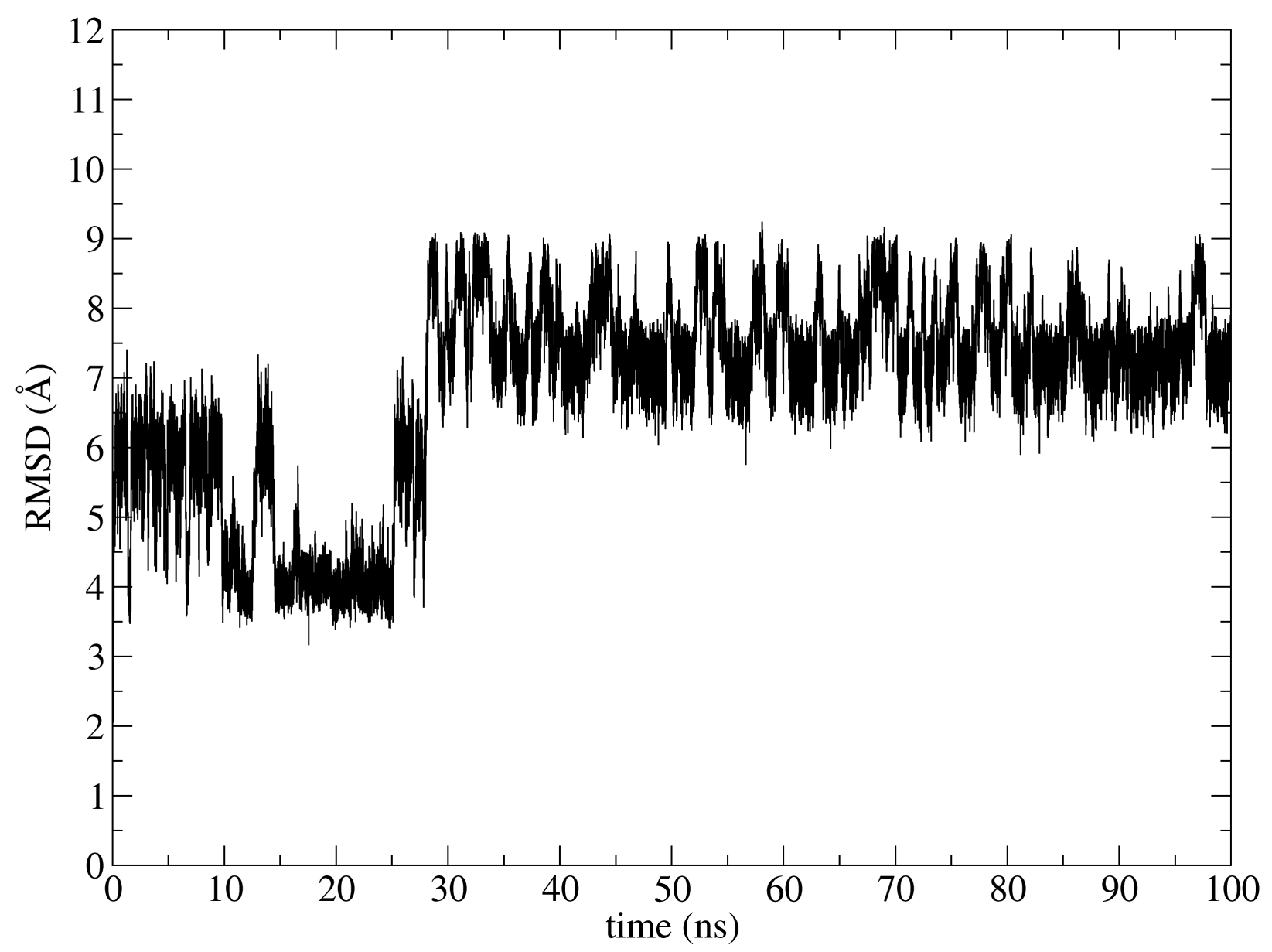

Figure S27 - Values of the RMSD (in $\AA$ ) as a function of time (in ns) computed on the heavy atoms of the D5 dimer. The reference structure used to compute the RMSD is the initial configuration of the MD simulation (i.e. the Cartesian coordinates taken from the crystallographic structure). The values represented correspond to a MD trajectory representative of the trends shown in the MD trajectories performed in this work. 


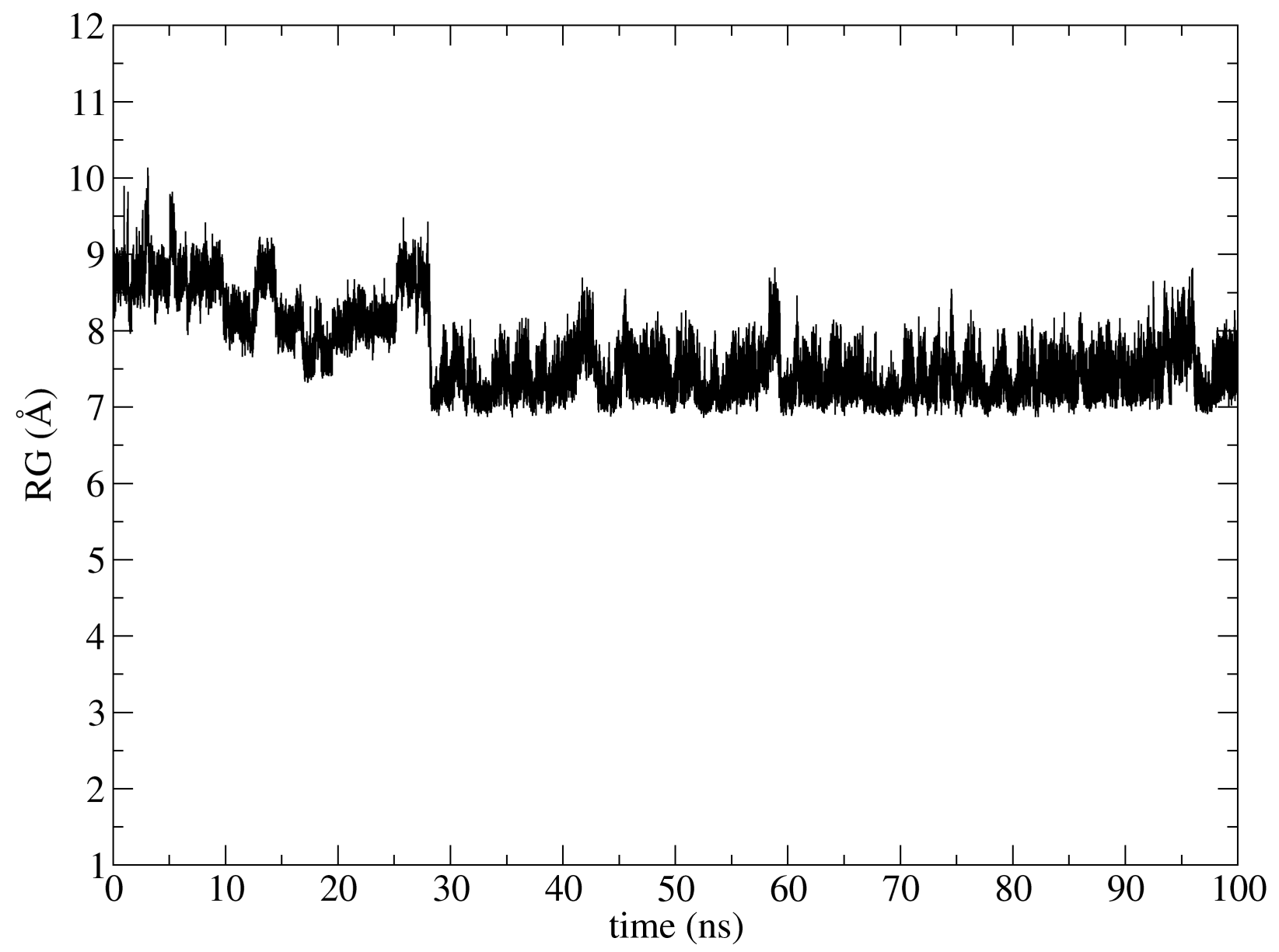

Figure S28 - Values of the RG (in $\AA$ ) as a function of time (in ns) of the D5 dimer. The values represented correspond to a MD trajectory representative of the trends shown in the MD trajectories performed in this work. 

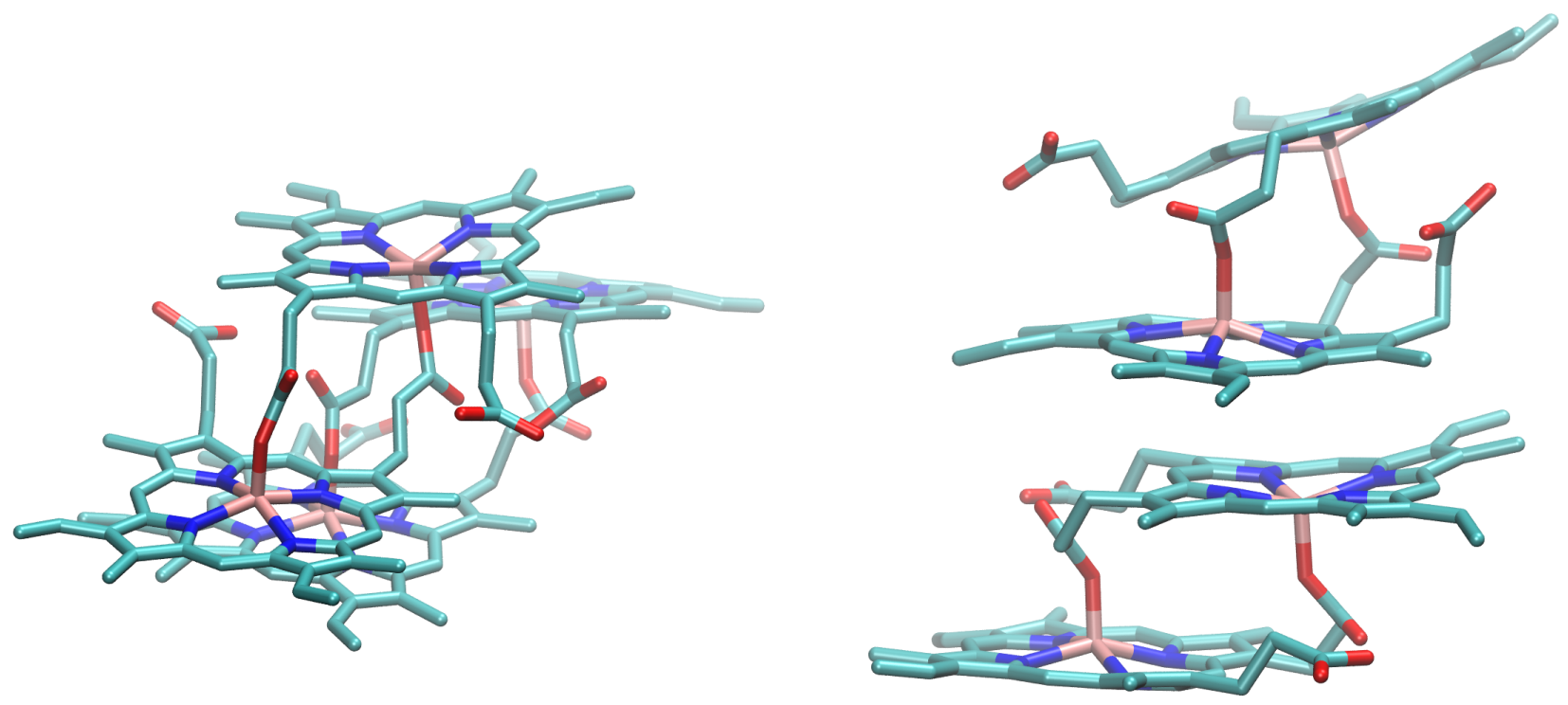

Figure S29 - Illustration of the evolution of the D5 dimer, occurring within 5 to $40 \mathrm{~ns}$ of MD simulations, toward molecular structures akin to D1 dimer (left) or D2 dimer (right). FPD entities are represented by sticks, carbon, nitrogen, oxygen, and iron atoms are colored in green, blue, red, and pink respectively. 


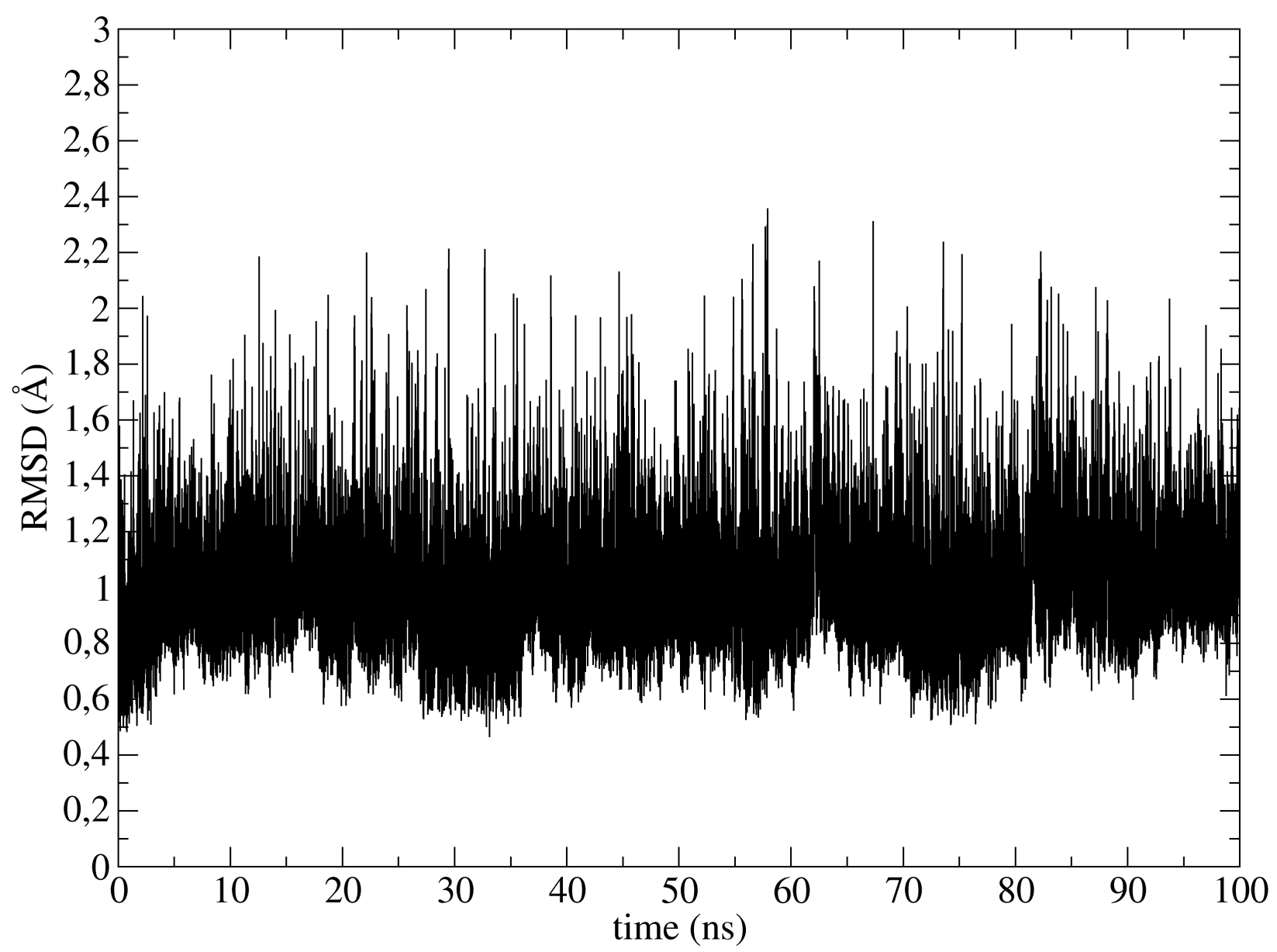

Figure S30 - Values of the RMSD (in $\AA$ ) as a function of time (in ns) computed on the heavy atoms of the T1 trimer. The reference structure used to compute the RMSD is the initial configuration of the MD simulation (i.e. the Cartesian coordinates taken from the crystallographic structure). The values represented correspond to a MD trajectory representative of the trends shown in the MD trajectories performed in this work. 


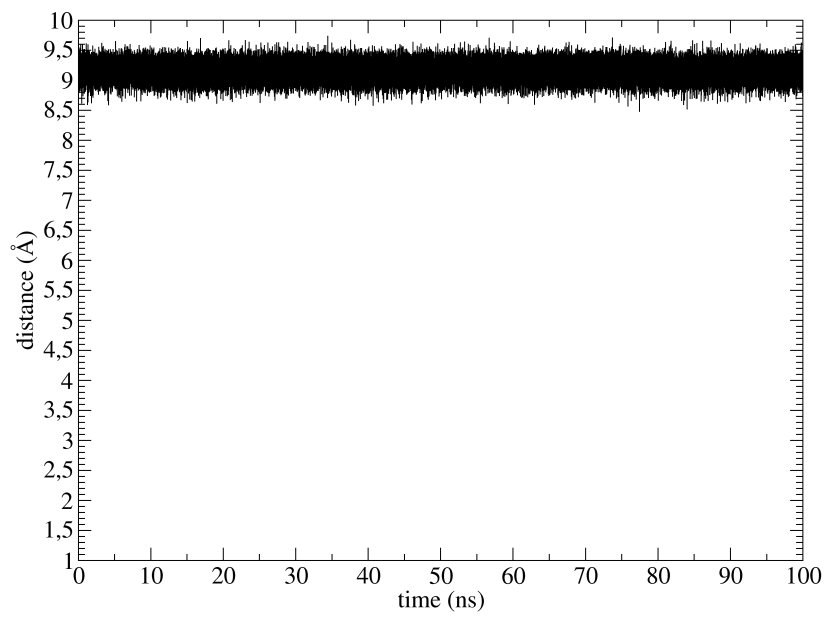

Distance between atom FE of residue 1 and atom FE of residue 2

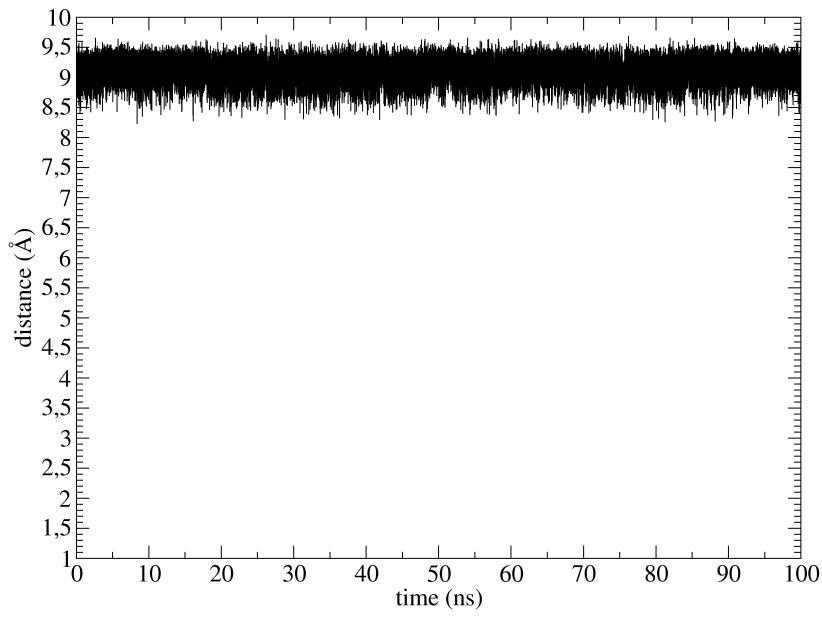

Distance between atom FE of residue 17 and atom FE of residue 18

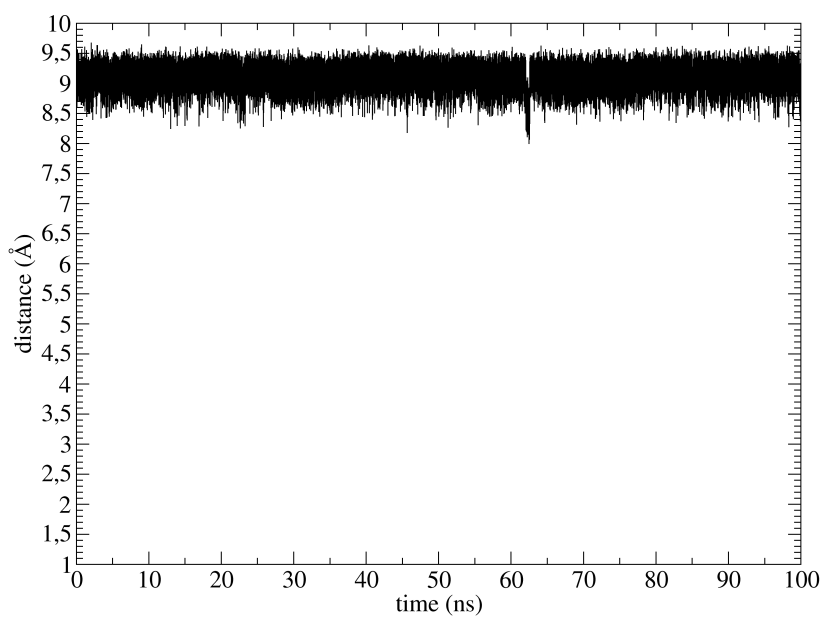

Distance between atom FE of residue 33 and atom FE of residue 34

Figure S31 - Values of the distances between the two iron atoms in each FPD entity of the T1 trimer (in $\AA$ ) as a function of time (in ns). The values represented correspond to a MD trajectory representative of the trends shown in the MD trajectories performed in this work. 


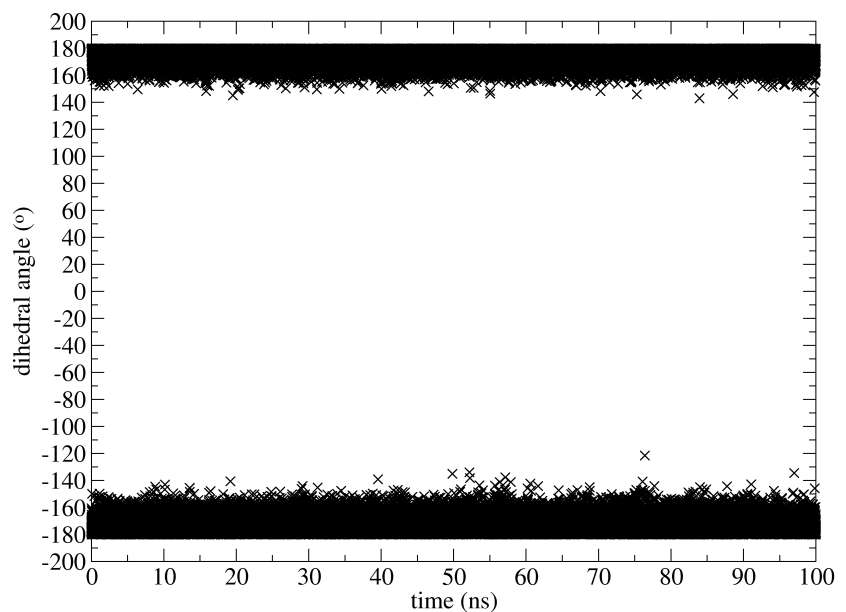

Dihedral angle defined by the sequence of atoms C99, CT91, CT92 in residue 1 , and C13 in residue 2

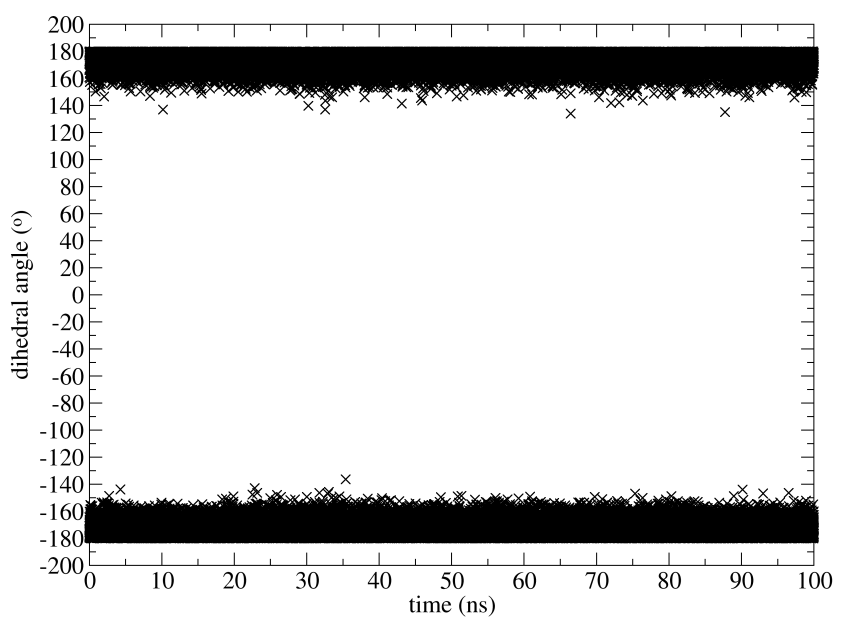

Dihedral angle defined by the sequence of atoms C99, CT91, CT92 in residue 18, and C13 in residue 17

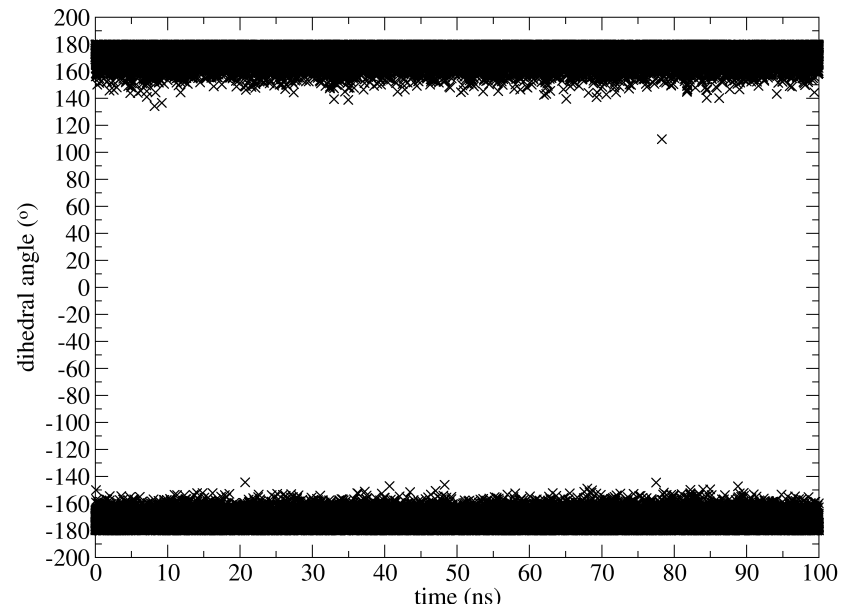

Dihedral angle defined by the sequence of atoms C99, CT91, СT92 in residue 2, and C13 in residue 1

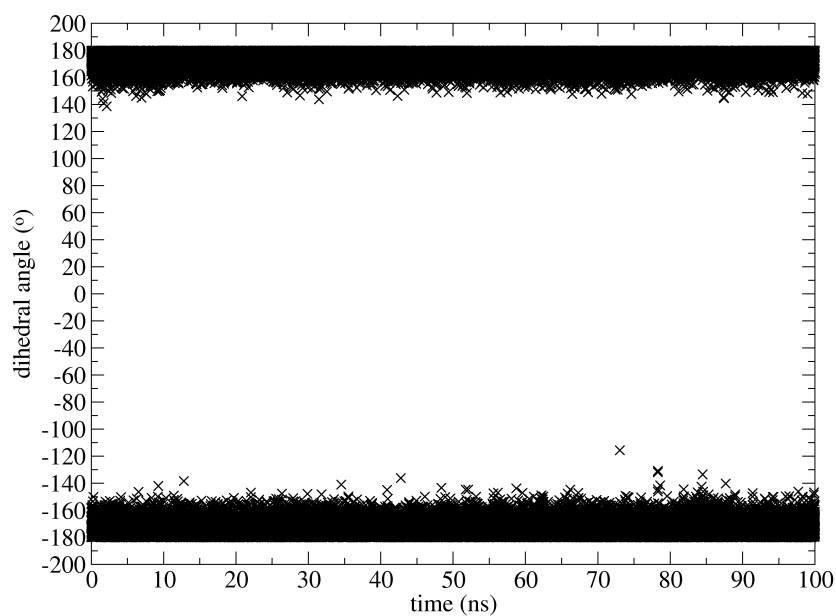

Dihedral angle defined by the sequence of atoms C99, CT91, CT92 in residue 33 , and C13 in residue 34

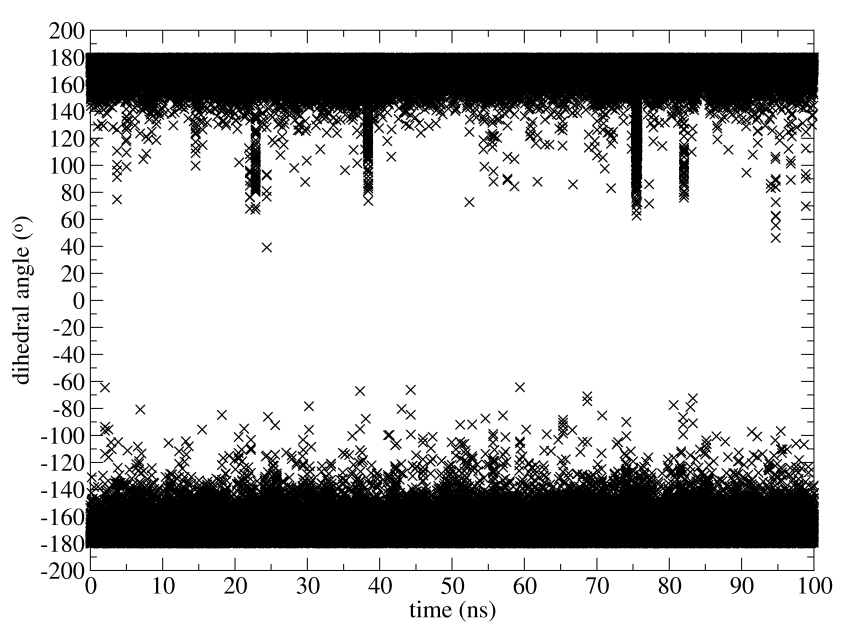

Dihedral angle defined by the sequence of atoms C99, CT91, CT92 in residue 17, and C13 in residue 18

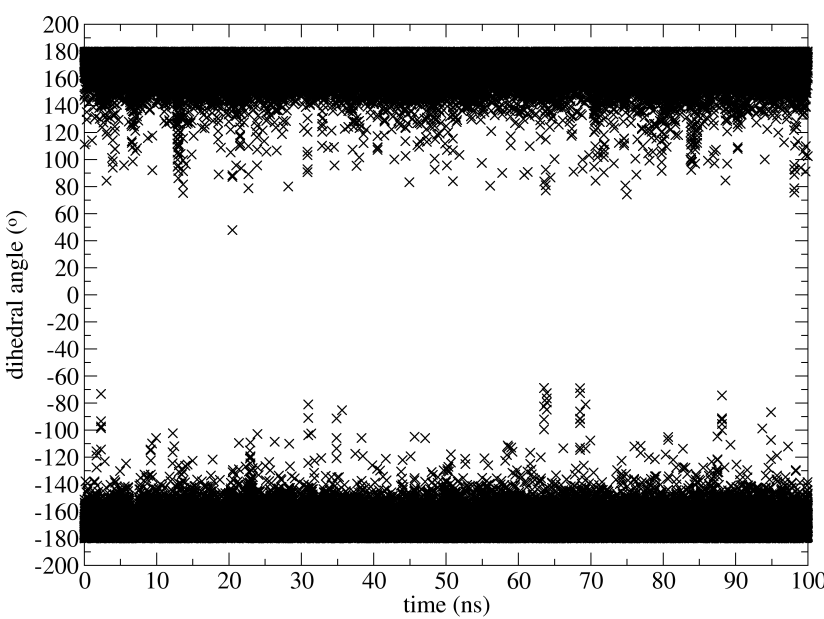

Dihedral angle defined by the sequence of atoms C99, CT91, CT92 in residue 34 , and C13 in residue 33

Figure S32 - Values of the two C-C-C-C dihedral angles of the carboxylate-iron bonds connecting the two heminic rings of each of the FPD entities forming T1 trimer (in degrees) as a function of time (in ns). The values represented correspond to a MD trajectory representative of the trends shown in the MD trajectories performed in this work. 
HBOND SUMMARY:

Data was saved to series hbond, output to file HBond_info_intraF_325.txt,

data was sorted, intra-residue interactions are NOT included,

Distance cutoff is 3.25 angstroms, angle cutoff is 120.00 degrees

Hydrogen bond information dumped for occupancies $>0.00$

Dumping schematic of time series after each h-bond, key follows:

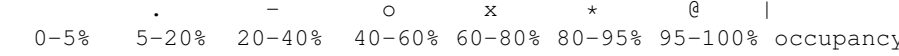

Donos

$\begin{array}{ccc}\text { DONOR } & \text { ACCEPTORH ACCEPTOR } \\ \text { atom\# } & \text { :res@atom atom\# }: \text { res@atom atom\# :res@atom \%occupied distance }\end{array}$

$218: 18092+132 \quad: 14 \mathrm{eH} 3 \quad 131: 14003 \quad 79.2902 .758(0.14)$ angle

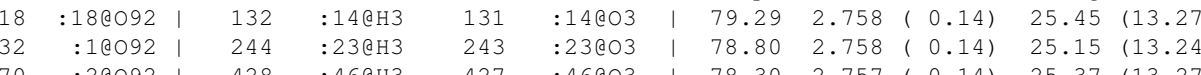

$\begin{array}{llllllllllll}328 & : 33 @ 092 & 96 & : 7 @ \mathrm{H} 3 & 95 & : 7 @ 03 & 73.67 & 2.758 & (0.14) & 25.16 & (13.16)\end{array}$

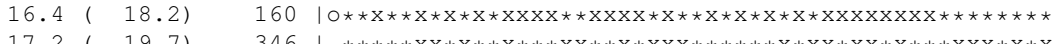

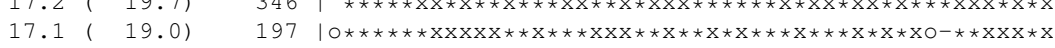

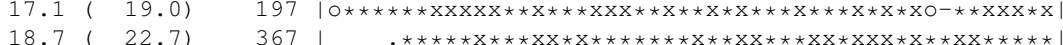

Figure S33 - Occupancy rate of the HB in the T1 trimer. The data reveal the formation of four inter-FPD HB as described in the manuscript. The formation of $\mathrm{HB}$ was followed along the $100 \mathrm{~ns}$ of the whole trajectory. The data reported come from one MD trajectory representative of all the MD trajectories performed in this work. 


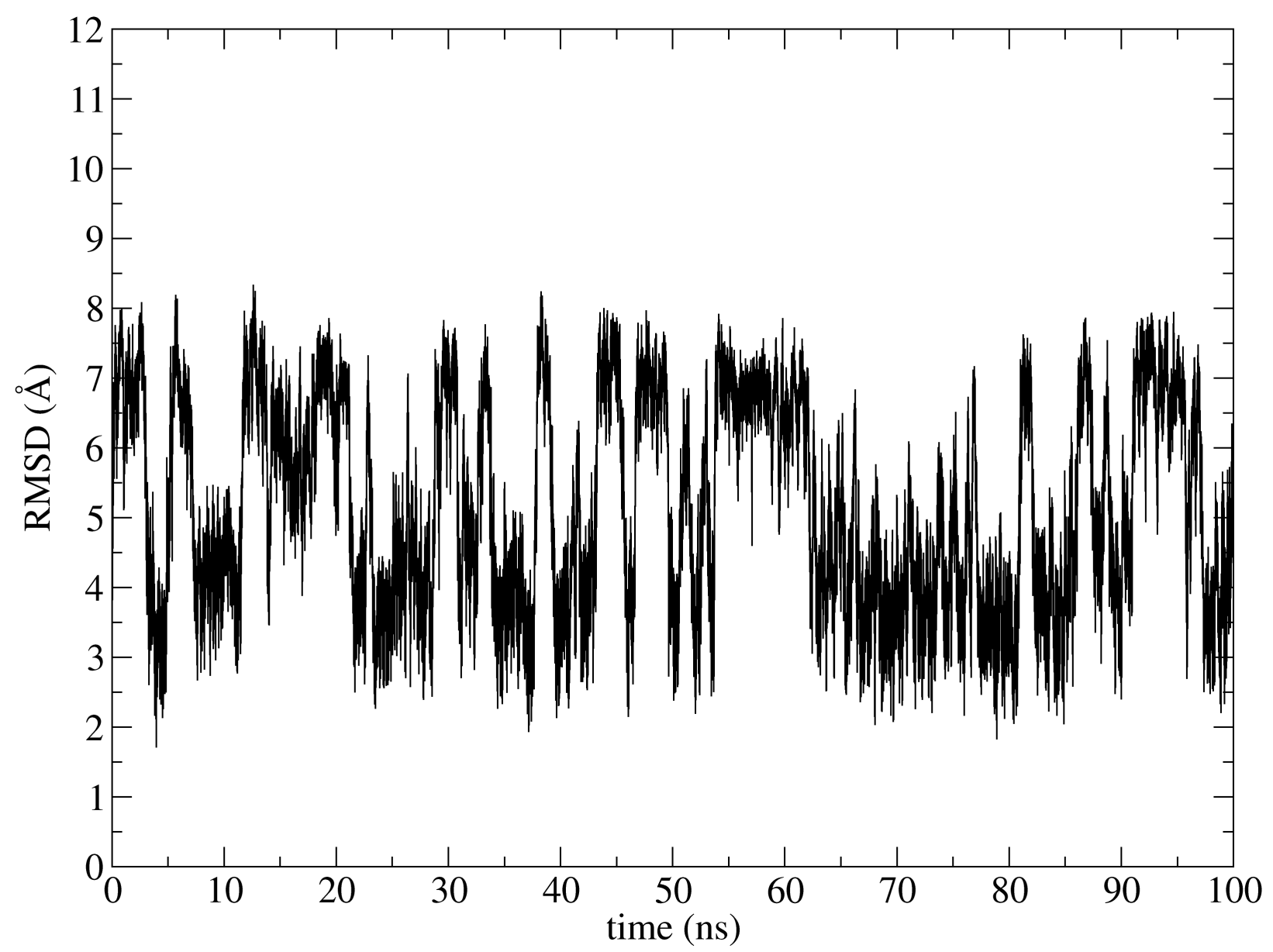

Figure S34 - Values of the RMSD (in $\AA$ ) as a function of time (in ns) computed on the heavy atoms of the T2 trimer. The reference structure used to compute the RMSD is the initial configuration of the MD simulation (i.e. the Cartesian coordinates taken from the crystallographic structure). The values represented correspond to a MD trajectory representative of the trends shown in the MD trajectories performed in this work. 


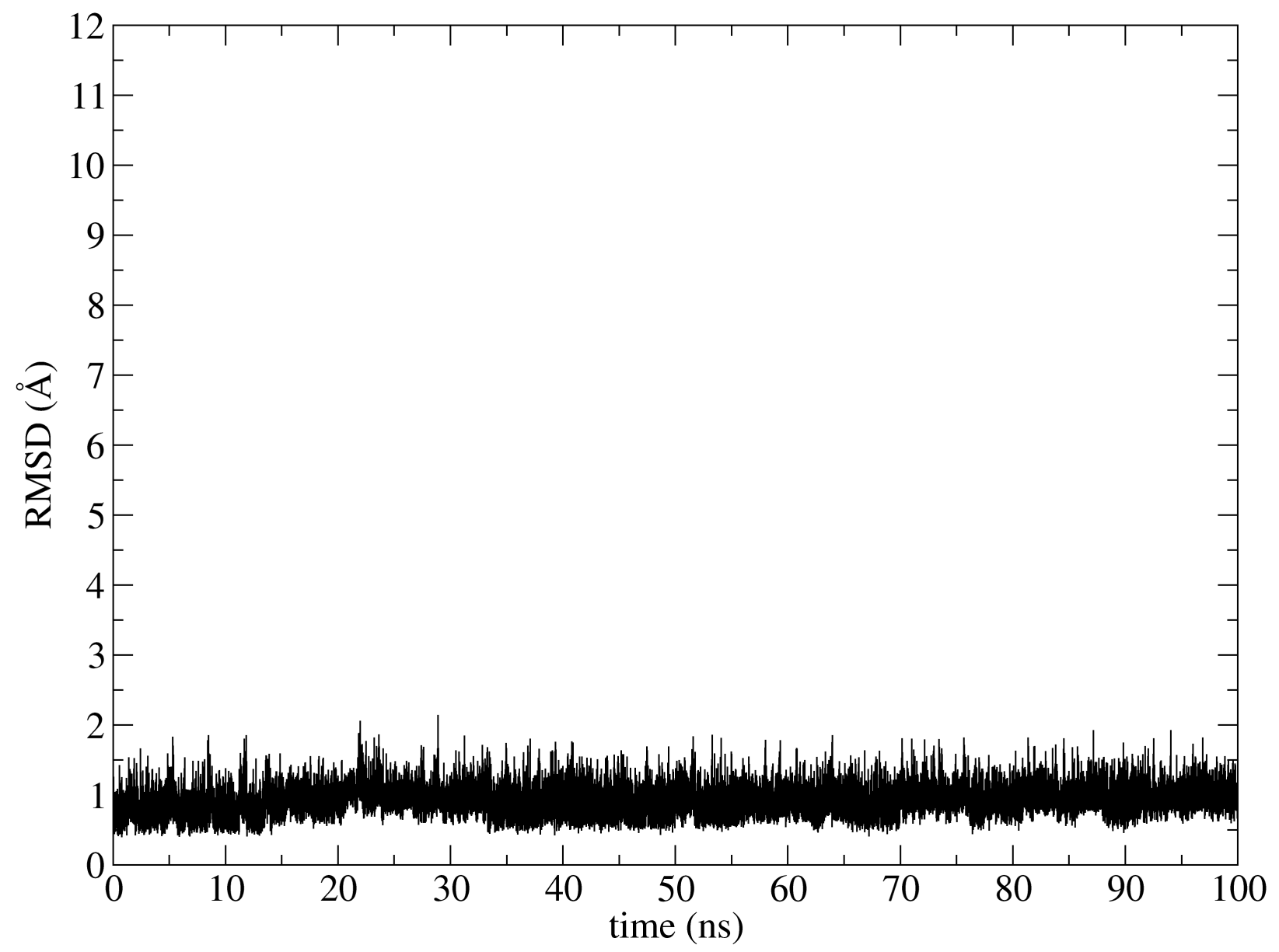

Figure S35 - Values of the RMSD (in $\AA$ ) as a function of time (in ns) computed on the heavy atoms of the D1 substructure in the T2 trimer. The reference structure used to compute the RMSD is the initial configuration of the MD simulation (i.e. the Cartesian coordinates taken from the crystallographic structure). The values represented correspond to a MD trajectory representative of the trends shown in the MD trajectories performed in this work. 


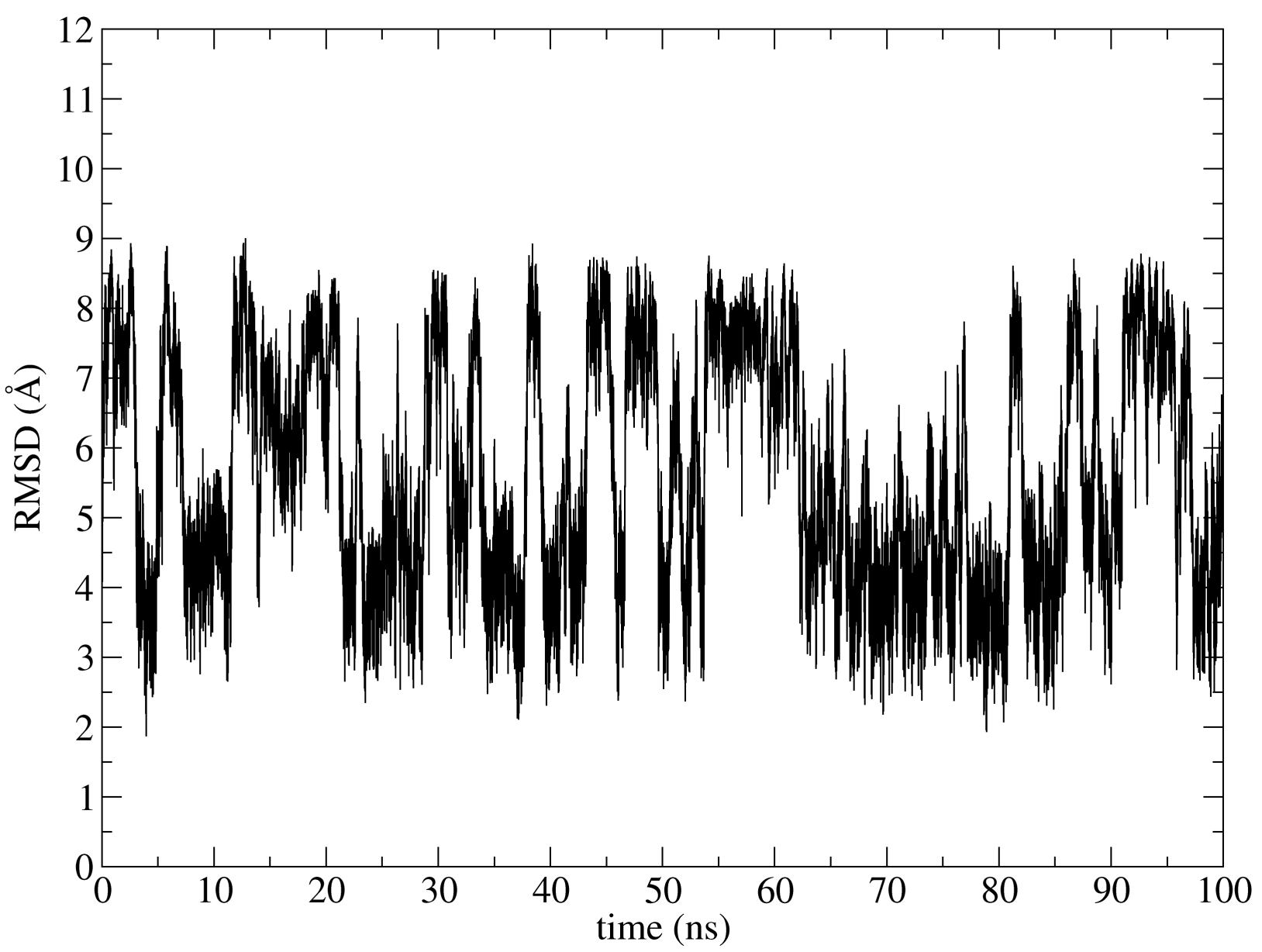

Figure S36 - Values of the RMSD (in $\AA$ ) as a function of time (in ns) computed on the heavy atoms of the D2 substructure in the T2 trimer. The reference structure used to compute the RMSD is the initial configuration of the MD simulation (i.e. the Cartesian coordinates taken from the crystallographic structure). The values represented correspond to a MD trajectory representative of the trends shown in the MD trajectories performed in this work. 


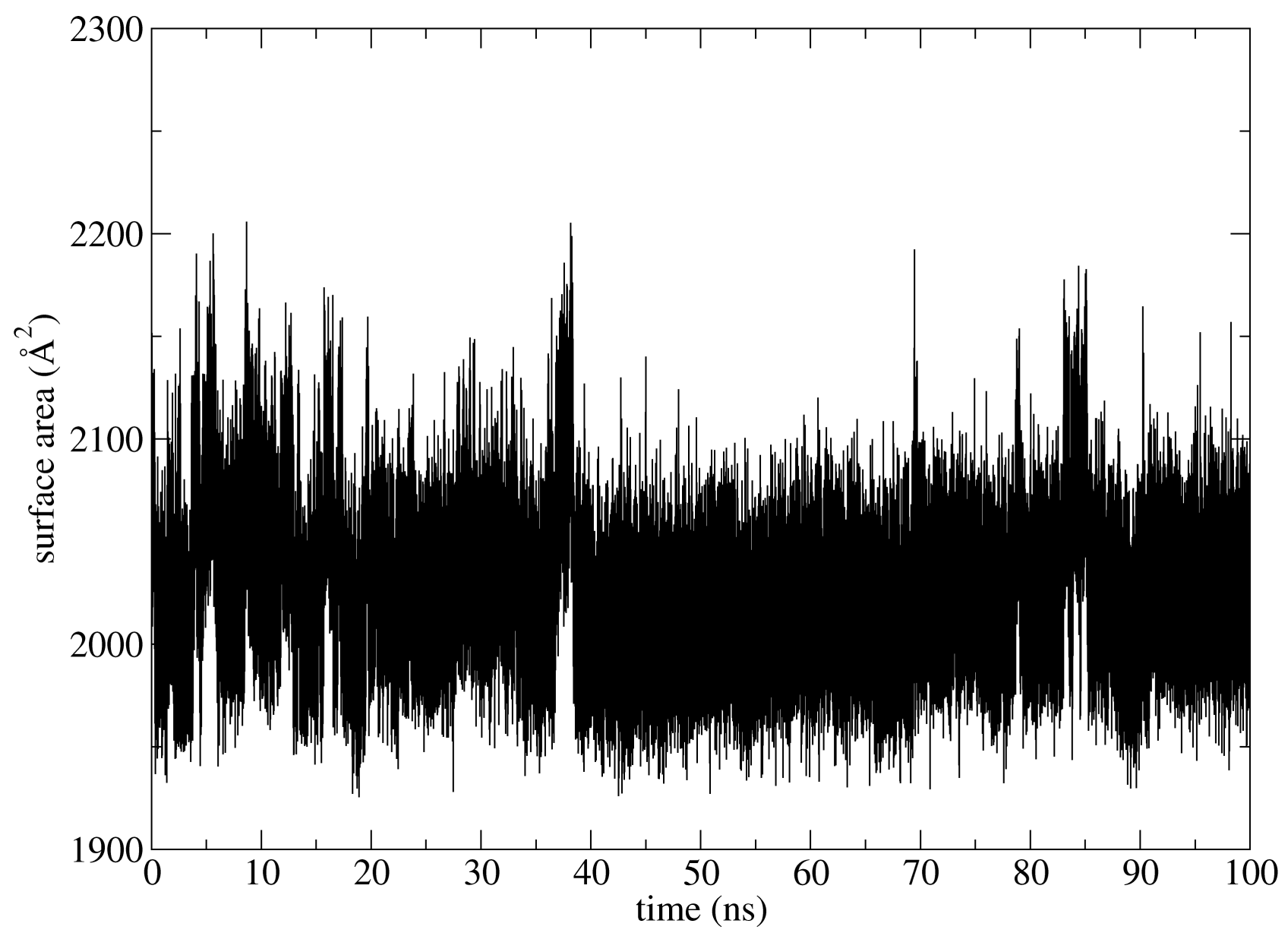

Figure S37 - SASA of the T2 trimer (in $\AA^{2}$ ) as a function of time (in ns). The SASA of the T2 trimer in the initial configuration of the MD is $2217 \AA^{2}$. The values represented correspond to a MD trajectory representative of the trends shown in the MD trajectories performed in this work. 


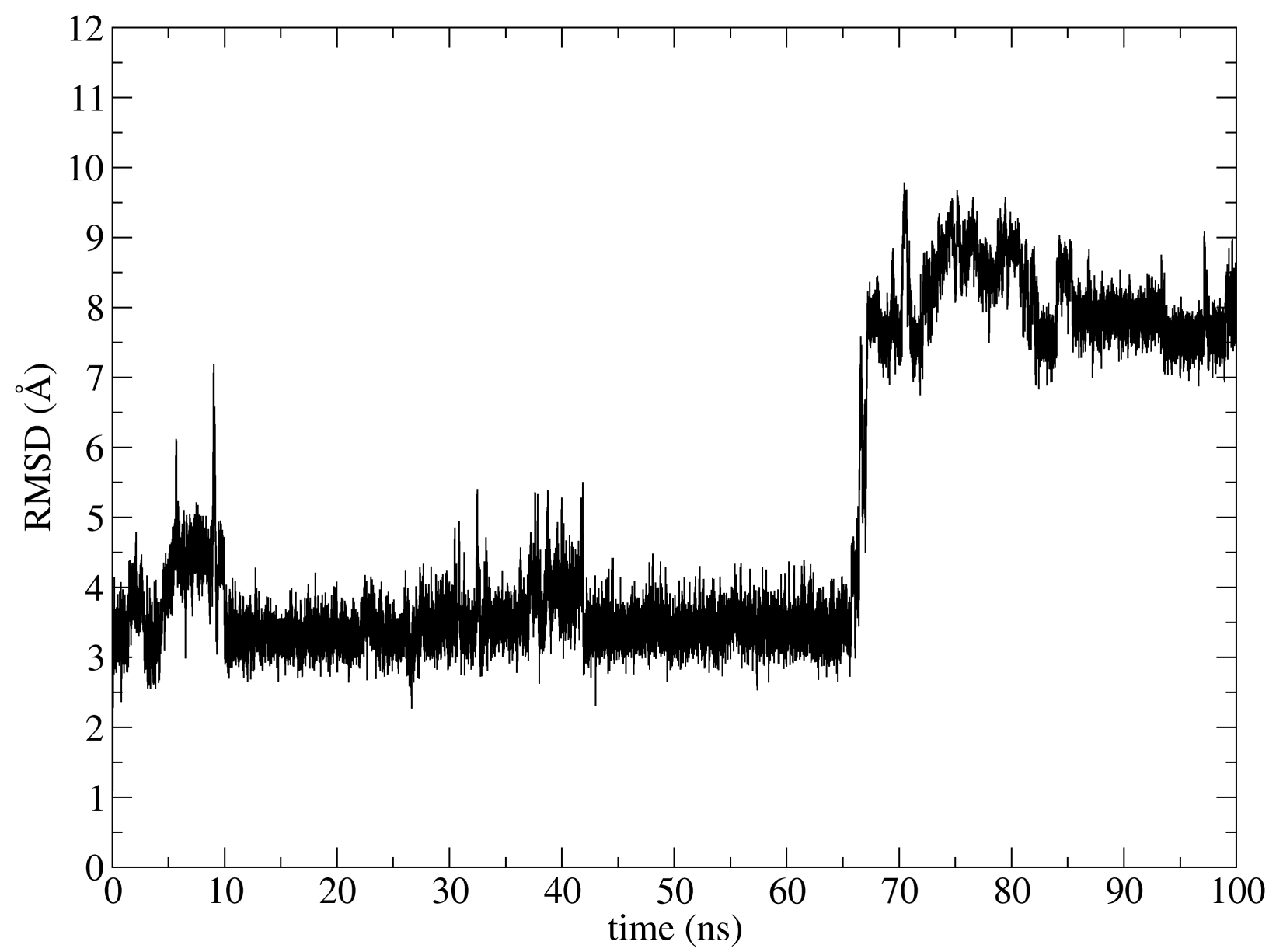

Figure S38 - Values of the RMSD (in $\AA$ ) as a function of time (in ns) computed on the heavy atoms of the T3 trimer. The reference structure used to compute the RMSD is the initial configuration of the MD simulation (i.e. the Cartesian coordinates taken from the crystallographic structure). The values represented correspond to a MD trajectory representative of the trends shown in the MD trajectories performed in this work. 


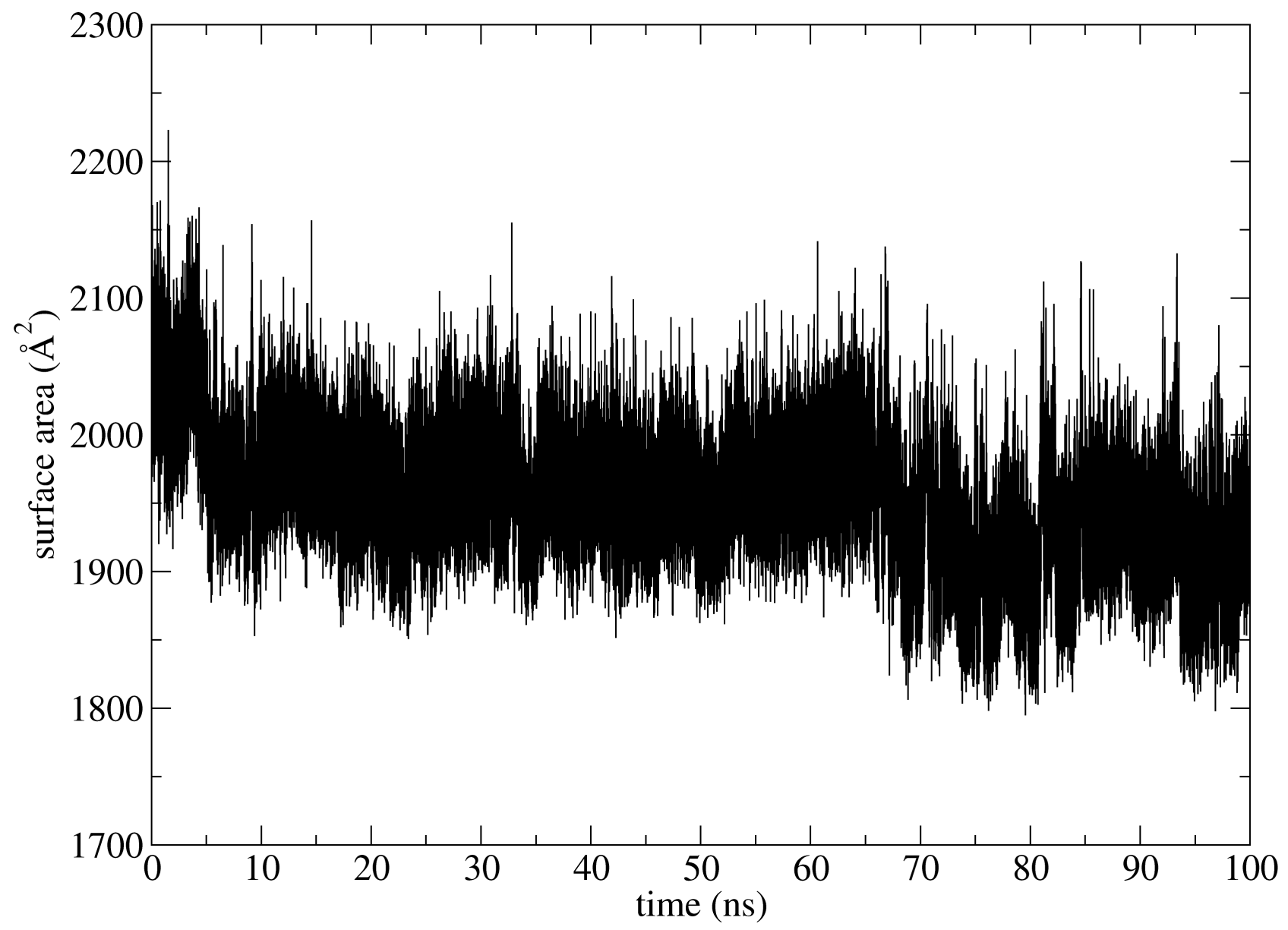

Figure S39 - SASA of the T3 trimer (in $\AA^{2}$ ) as a function of time (in ns). The SASA of the T3 trimer in the initial configuration of the MD is $2158 \AA^{2}$. The values represented correspond to a MD trajectory representative of the trends shown in the MD trajectories performed in this work. 


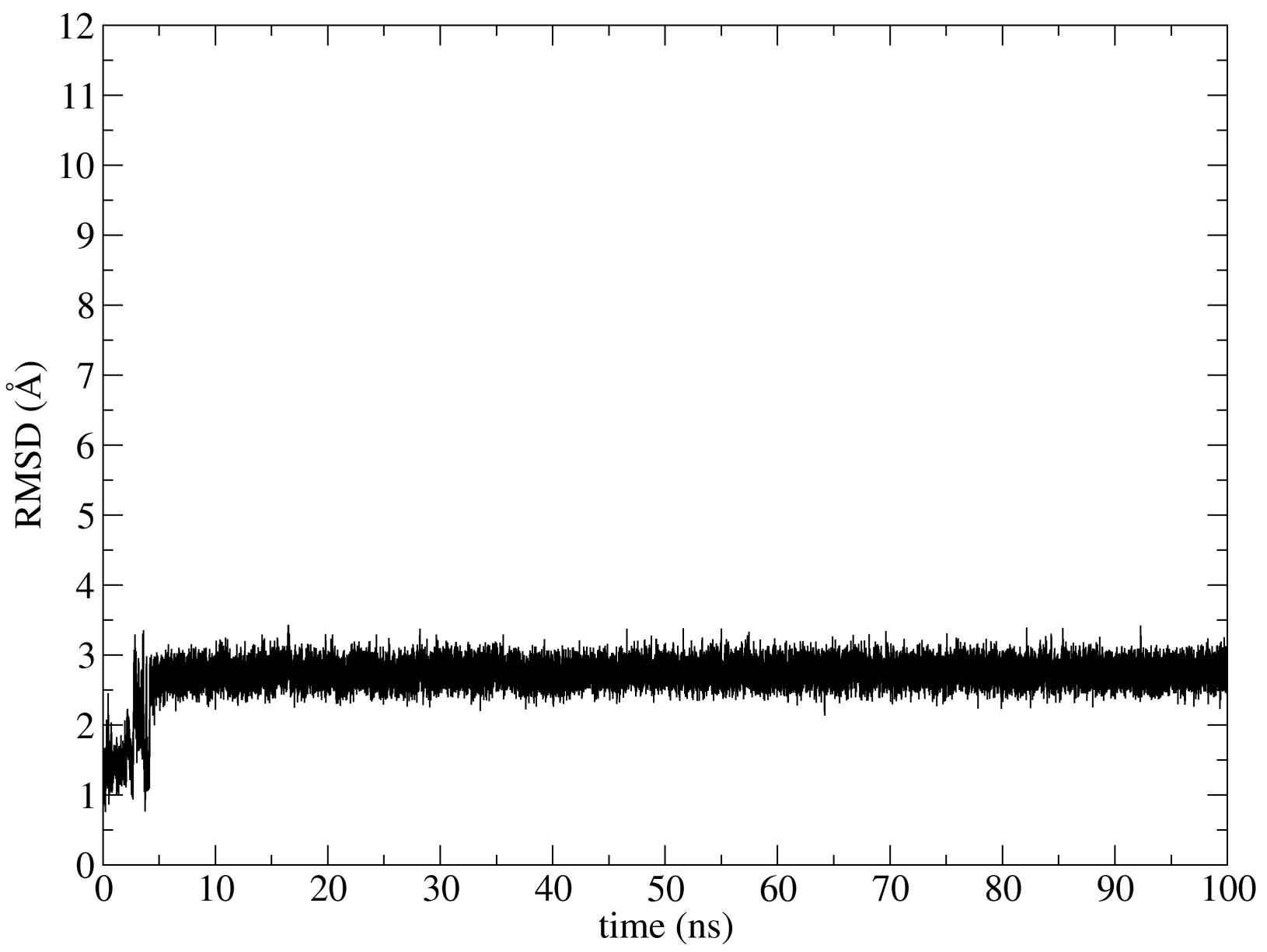

Figure S40 - Values of the RMSD (in $\AA$ ) as a function of time (in ns) computed on the heavy atoms of the T4 trimer. The reference structure used to compute the RMSD is the initial configuration of the MD simulation (i.e. the Cartesian coordinates taken from the crystallographic structure). The values represented correspond to a MD trajectory representative of the trends shown in the MD trajectories performed in this work. 


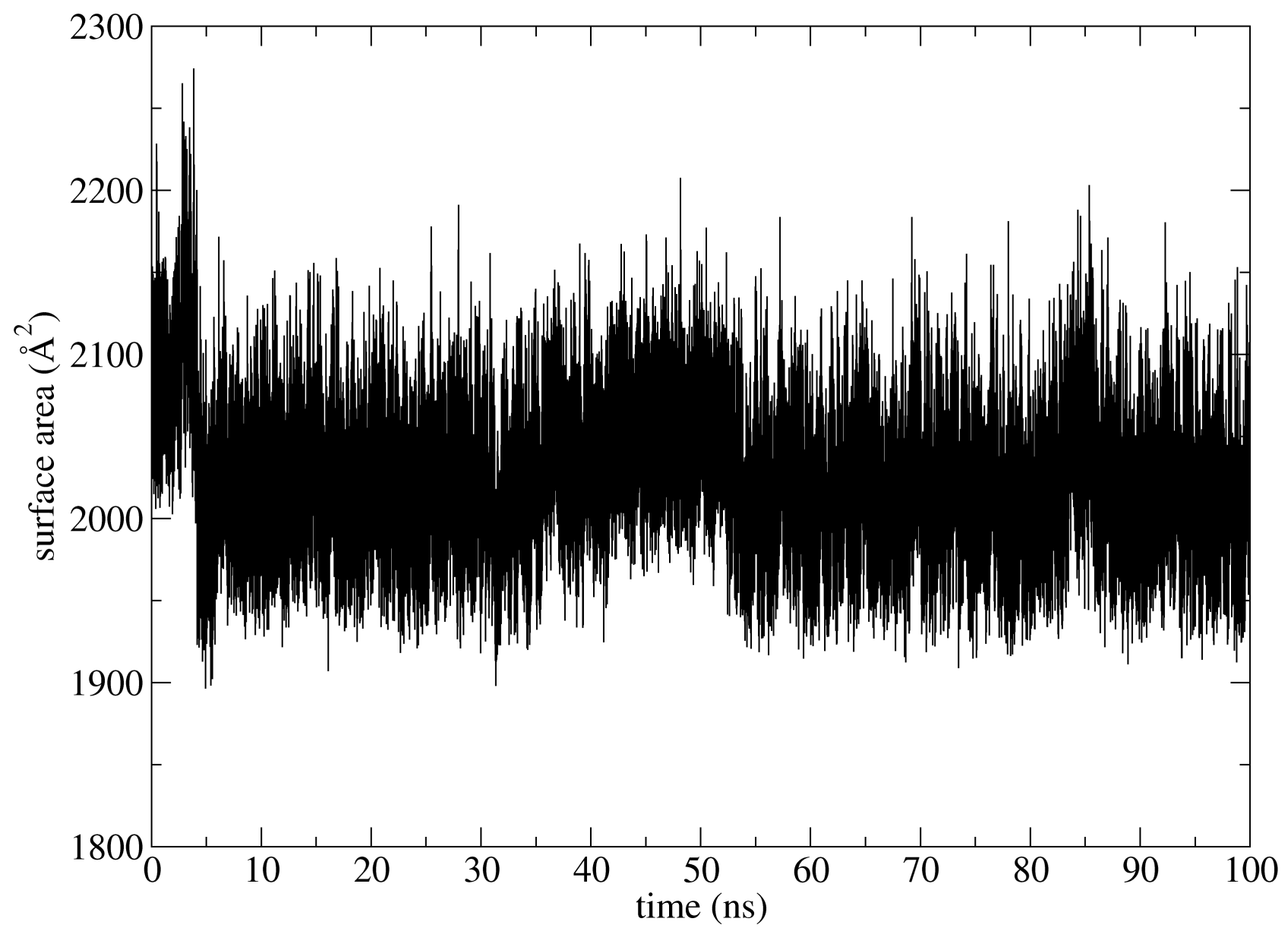

Figure S41 - SASA of the T4 trimer (in $\AA^{2}$ ) as a function of time (in ns). The SASA of the T3 trimer in the initial configuration of the MD is $2155 \AA^{2}$. The values represented correspond to a MD trajectory representative of the trends shown in the MD trajectories performed in this work. 
HBOND SUMMARY:

Data was saved to series hbond, output to file HBond_info_intraF_325.txt,

data was sorted, intra-residue interactions are NOT included,

Distance cutoff is 3.25 angstroms, angle cutoff is 120.00 degrees

Hydrogen bond information dumped for occupancies $>0.00$

Dumping schematic of time series after each h-bond, key follows:

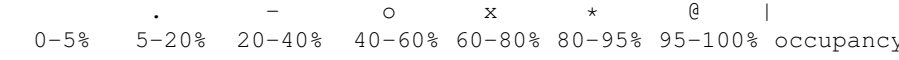

Donos

atom\# ACCEPTORH ACCEPTOR

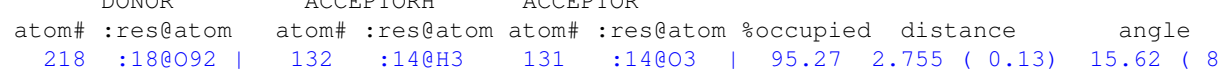

$\begin{array}{llllllllllll}32 & : 10092 & 244 & : 23 \mathrm{AH} 3 & 131 & : 14003 & 95.27 & 2.755 & (0.13) & 15.62 & (8.47)\end{array}$

$\begin{array}{lllllllllll}394 & : 39002 & 132 & : 14 \mathrm{gH} & 131 & : 23003 & 64.30 & 2.790 & (0.14) & 23.06 & (12.25)\end{array}$

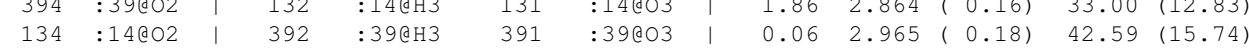

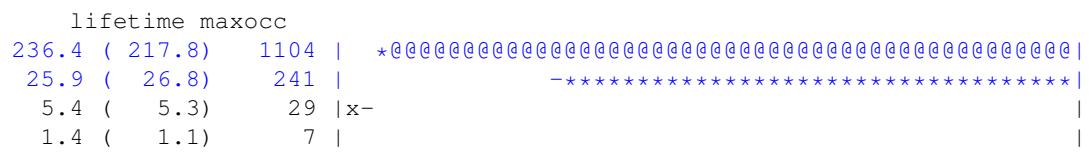

Figure S42 - Occupancy rate of the HB in the T4 trimer. The data reveal the formation of two HB inter-FPD HB (colored in blue) as described in the manuscript between atom $\mathrm{O} 92$ of residue 18 and atom O3 of residue 14 and atom $\mathrm{O} 92$ of residue 1 and atom O3 of residue 23. The pair of HB found between sheets (colored in black) involving atom O2 of residue 39 and atom O3 of residue 14 and atom O2 of residue 14 and atom O3 of residue 39 is rapidly broken. The formation of $\mathrm{HB}$ was followed along the $100 \mathrm{~ns}$ of the whole trajectory. The data reported come from one MD trajectory representative of all the MD trajectories performed in this work. 


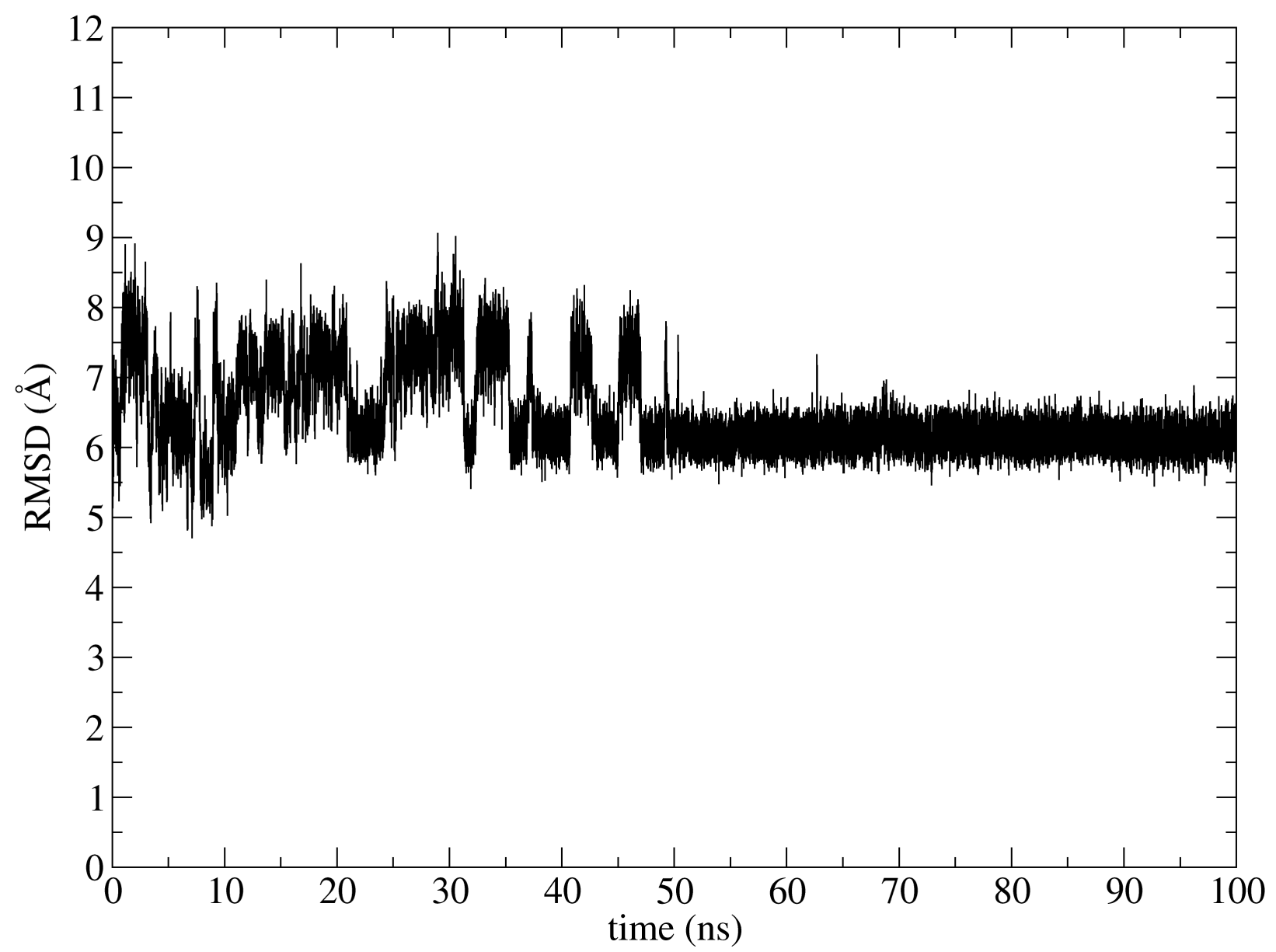

Figure S43 - Values of the RMSD (in $\AA$ ) as a function of time (in ns) computed on the heavy atoms of the T5 trimer. The reference structure used to compute the RMSD is the initial configuration of the MD simulation (i.e. the Cartesian coordinates taken from the crystallographic structure). The values represented correspond to a MD trajectory representative of the trends shown in the MD trajectories performed in this work. 


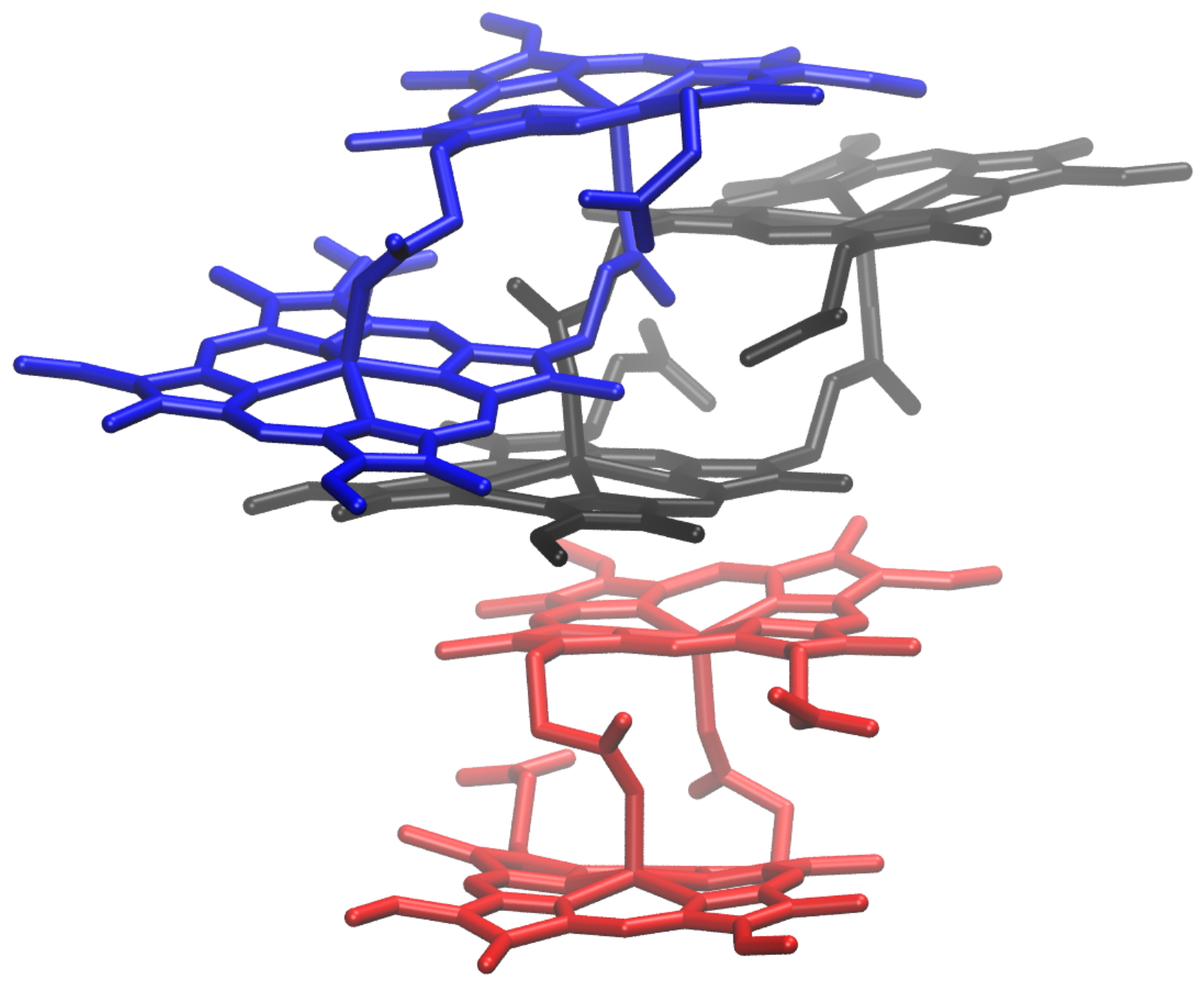

Figure S44 - Illustration of the evolution of the T5 trimer, occurring after $50 \mathrm{ns,} \mathrm{toward} \mathrm{molecular} \mathrm{sub-}$ structures akin to D1 dimer and D2 dimer. FPD entities are represented by sticks. The black FPD entity participates in a substructure of type D1 with the blue FPD entity as well as in a substructure of type D2 with the red FPD entity. 


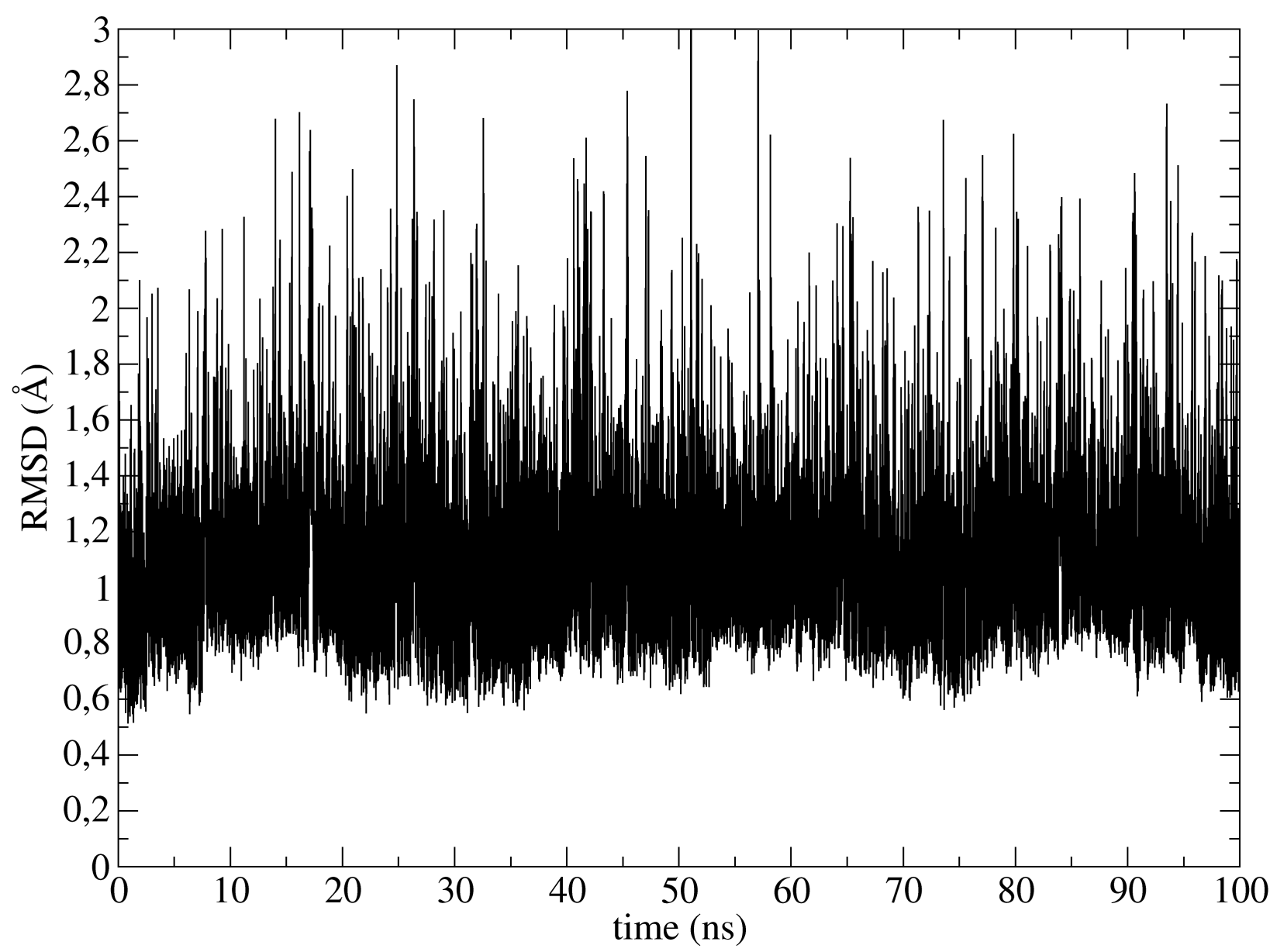

Figure S45 - Values of the RMSD (in $\AA$ ) as a function of time (in ns) computed on the heavy atoms of the Q1 tetramer. The reference structure used to compute the RMSD is the initial configuration of the MD simulation (i.e. the Cartesian coordinates taken from the crystallographic structure). The values represented correspond to a MD trajectory representative of the trends shown in the MD trajectories performed in this work. 


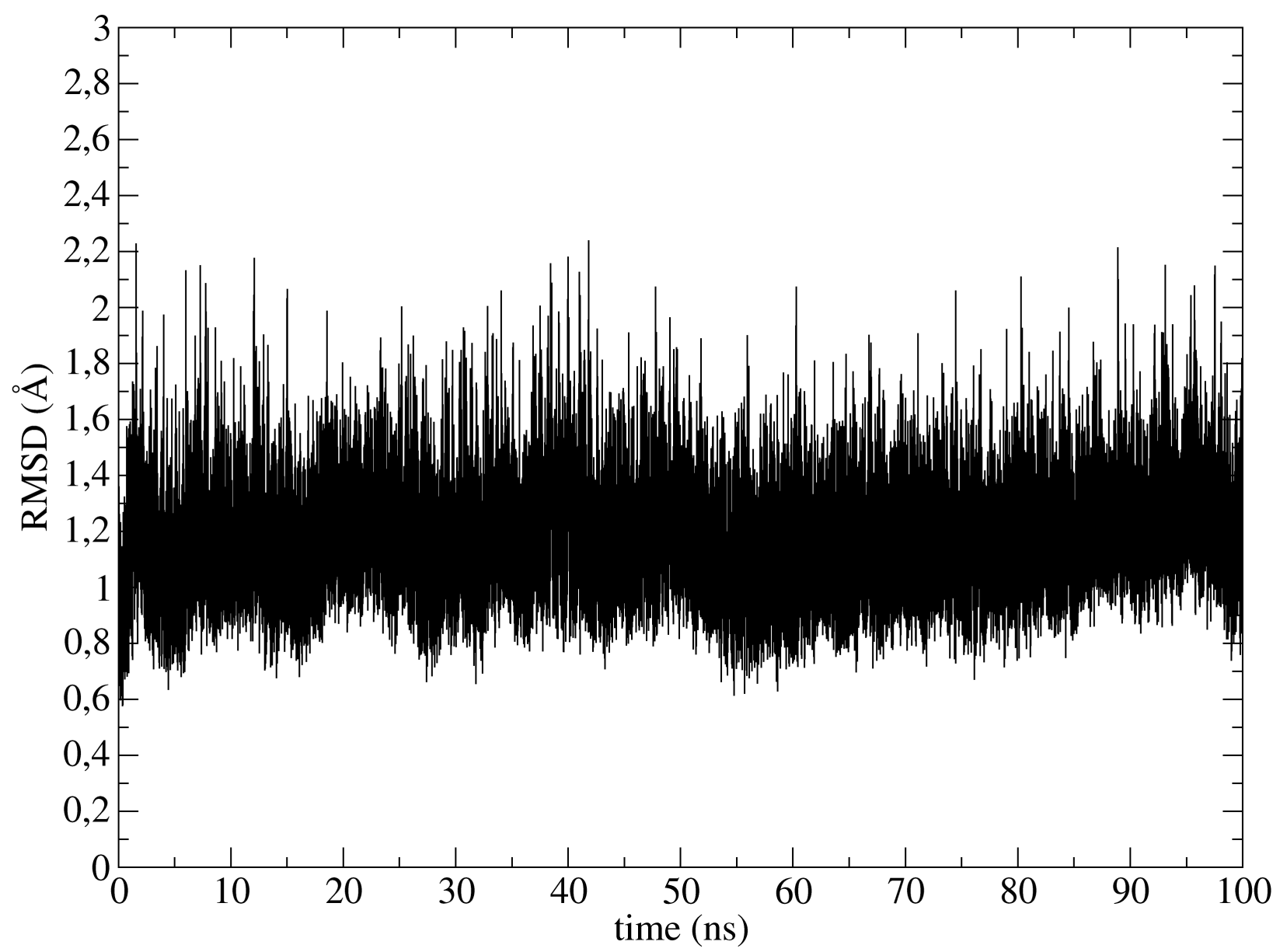

Figure S46 - Values of the RMSD (in $\AA$ ) as a function of time (in ns) computed on the heavy atoms of the Q2 tetramer. The reference structure used to compute the RMSD is the initial configuration of the MD simulation (i.e. the Cartesian coordinates taken from the crystallographic structure). The values represented correspond to a MD trajectory representative of the trends shown in the MD trajectories performed in this work. 

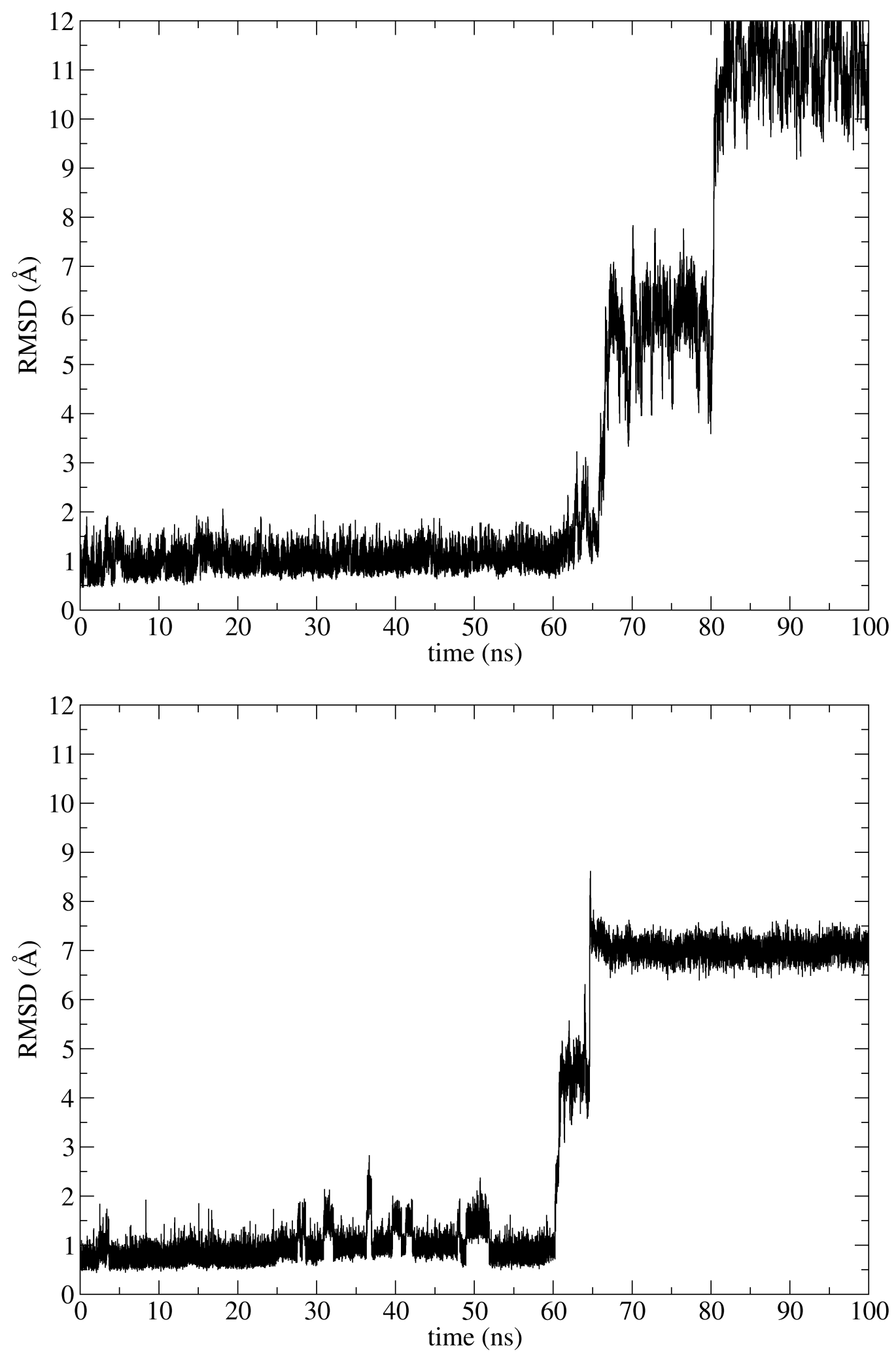

Figure S47 - Values of the RMSD (in $\AA$ ) as a function of time (in ns) computed on the heavy atoms of the Q3 tetramer (top) and of the Q4 tetramer (bottom). The reference structures used to compute the RMSD are the initial configurations of the MD simulations (i.e. the Cartesian coordinates taken from the crystallographic structure). The values represented correspond to a MD trajectory representative of the trends shown in the MD trajectories performed in this work. 
HBOND SUMMARY:

Data was saved to series hbond, output to file HBond_info_intraf_325.txt,

data was sorted, intra-residue interactions are NOT included,

Distance cutoff is 3.25 angstroms, angle cutoff is 120.00 degrees

Hydrogen bond information dumped for occupancies $>0.00$

Dumping schematic of time series after each h-bond, key follows:

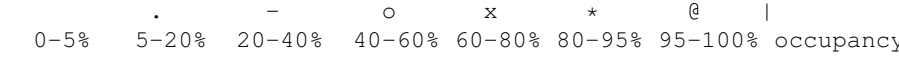

Donos

ACCEPTORH ACCEPTOR

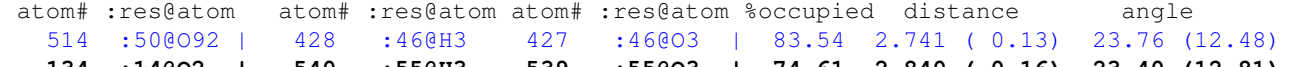

$\begin{array}{lllllllllllll}134 & : 14 @ 02 & \mid & 540 & : 55 @ \mathrm{H} 3 & 539 & : 55 @ 03 & \mathbf{7 4} .61 & \mathbf{2 . 8 4 0} & (0.16) & 23.40 & (12.81)\end{array}$

\begin{tabular}{ll|llllllllll}
32 & $: 10092$ & 244 & $: 230 \mathrm{H} 3$ & 243 & $: 23003$ & 73.47 & 2.749 & $(0.13)$ & 24.46 & $(12.70)$
\end{tabular}

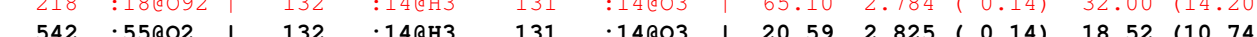

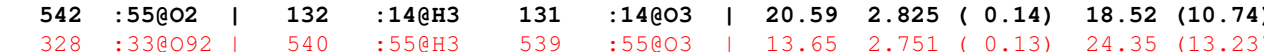

\begin{tabular}{ll|llllllllll}
328 & $: 33 @ 092$ & 540 & $: 55 \Subset \mathrm{H} 3$ & 539 & $: 55 \Subset 03$ & 13.65 & 2.751 & $(0.13)$ & 24.35 & $(13.23)$ \\
131 & $: 14 @ 03$ & 540 & $: 55 \Subset \mathrm{H} 3$ & 539 & $: 55 @ 03$ & 12.64 & 3.051 & $(0.14)$ & 37.55 & $(13.62)$
\end{tabular} $31.3(435.8)$

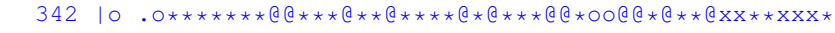
$26.2(31.0)$ $34.5(43.9)$

Figure S48a - Occupancy rate of the HB in the Q3 tetramer. First, the intersheet HB (in bold) between atom O2 of residue 55 and atom O3 of residue 14 breaks and an inter-FPD HB (colored in red) forms between atom O92 of residue 18 and atom O3 of residue 14 then the intersheet HB between atom $\mathrm{O} 2$ of residue 14 and atom $\mathrm{O} 3$ of residue 55 breaks and an inter-FPD HB forms between atom O92 of residue 33 and atom O3 of residue 55 . Atom O92 of residue 1 and atom $\mathrm{O} 3$ of residue 23 on the one side and atom O92 of residue 50 and atom O3 of residue 46 on the other side of Q3 structure form inter-FPD HB (colored in blue) as well. The formation of $\mathrm{HB}$ was followed along the $100 \mathrm{~ns}$ of the whole trajectory. The data reported come from one MD trajectory representative of all the MD trajectories performed in this work. 
HBOND SUMMARY:

Data was saved to series hbond, output to file HBond_info_325_wat.txt,

data was sorted, intra-residue interactions are NOT included,

Distance cutoff is 3.25 angstroms, angle cutoff is 120.00 degrees

Hydrogen bond information dumped for occupancies $>0.00$

Dumping schematic of time series after each h-bond, key follows:

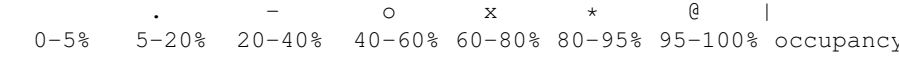

DONOR ACCEPTORH ACCEPTOR

atom\# :res@atom atom\# :res@atom atom\# :res@atom ooccupied distance

\begin{tabular}{ll|l|lllll}
$542: 55 @ 02$ & solvent acceptor & 18.89 & 2.898 & $(0.17)$ & 28.21 & $(14.54)$
\end{tabular}

\begin{tabular}{ll|l|lllll}
134 & $: 14 @ 02$ & solvent acceptor & 16.14 & 2.875 & $(0.17)$ & 24.99 & $(13.43)$
\end{tabular}

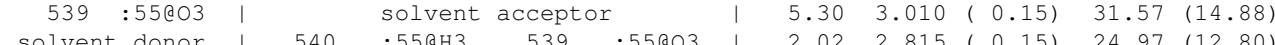

solvent donor | 540 :55लH3 $539: 55003 \quad 2.02 \quad 2.815(0.15) 24.97(12.80)$

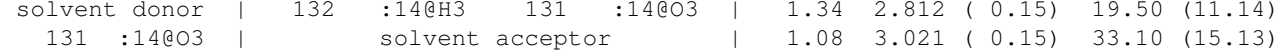

\begin{tabular}{|c|c|c|}
\hline \multicolumn{3}{|c|}{ lifetime maxocc } \\
\hline 2.1 & 1.9) & 29 \\
\hline 3.2 & 3.8) & 41 \\
\hline 1.5 & $0.9)$ & 10 \\
\hline 6.6 & 7.7) & 62 \\
\hline 13.2 & 23.31 & 202 \\
\hline 1.3 & $0.7)$ & 8 \\
\hline
\end{tabular}

Figure S48b - Occupancy rate of the HB between solvent molecules and the carboxylic acid groups of Q3 tetramer initially involved in the formation of intersheet HB. The formation of $\mathrm{HB}$ was followed along the $100 \mathrm{~ns}$ of the whole trajectory. The data reported come from one MD trajectory representative of all the MD trajectories performed in this work. 
HBOND SUMMARY:

Data was saved to series hbond, output to file HBond_info_intraF_325.txt,

data was sorted, intra-residue interactions are NOT included,

Distance cutoff is 3.25 angstroms, angle cutoff is 120.00 degrees

Hydrogen bond information dumped for occupancies $>0.00$

Dumping schematic of time series after each h-bond, key follows:

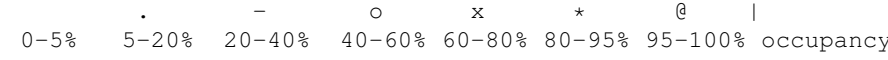

DONOR

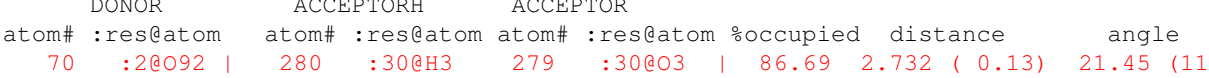

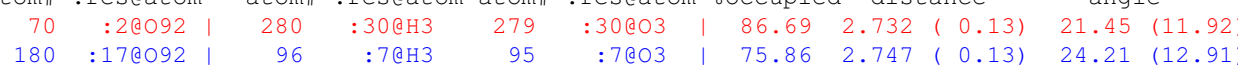

$\begin{array}{lllllllllll}180 & : 17092 & 96 & : 7 @ \mathrm{H3} & 95 & : 7003 & 75.86 & 2.747 & (0.13) & 24.21 & (12.91)\end{array}$

$\begin{array}{llllllllllll}542 & : 55 @ 02 & 132 & : 14 @ \mathrm{H} 3 & 131 & : 14 @ 03 & 42.72 & 2.859 & (0.16) & 19.46 & (11.55)\end{array}$

\begin{tabular}{ll|lllllllllll}
282 & $: 30002$ & | & 392 & $: 39 @ \mathrm{H} 3$ & 391 & $: 39 @ 03$ & $\mathbf{4 2 . 1 6}$ & 2.877 & $(0.17)$ & 21.20 & $(12.26)$
\end{tabular}

$\begin{array}{llllllllllll}514 & : 50 \mathrm{eO} & \mid & 428 & : 46 \mathrm{eH} 3 & 427 & : 46 \mathrm{eO} & 38.38 & 2.754 & (0.13) & 23.32 & (12.74)\end{array}$

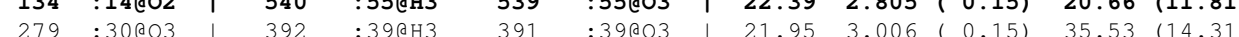

$\begin{array}{lllllllllll}279 & : 30 \mathrm{eO} & 392 & : 39 \mathrm{eH} 3 & 391 & : 39 \mathrm{eO} 3 & 21.95 & 3.006 & (0.15) & 35.53 & (14.31) \\ 539 & : 55 \mathrm{eO} & 132 & : 140 \mathrm{AH} 3 & 131 & : 14003 & 14.89 & 3.005 & (0.15) & 35.63 & (14.13)\end{array}$

$\begin{array}{llllllllll}394: 39 @ 02 & 280 & : 30 @ \mathrm{H} & 279 & : 30 @ 03 & 6.65 & 2.817 & (0.15) & 20.61 & (11.70)\end{array}$

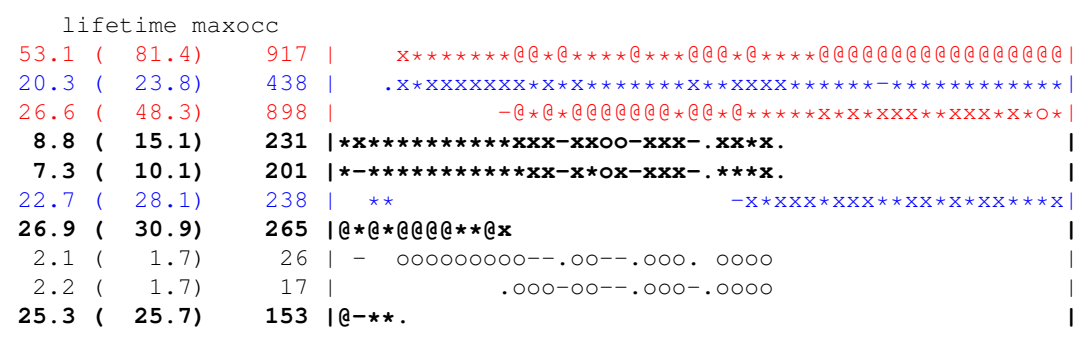

Figure S48c - Occupancy rate of the HB in the Q4 tetramer. The intersheet HB (in bold) are broken while inter-FPD HB are formed. The first intersheet $\mathrm{HB}$ to be broken are the one between atom $\mathrm{O} 2$ of residue 39 and atom $\mathrm{O} 3$ of residue 30 and the one between atom $\mathrm{O} 2$ of residue 14 and atom $\mathrm{O} 3$ of residue 55 which are the most accessible to solvent. Then, the two other intersheet HB, the one between atom O2 of residue 30 and atom O3 of residue 39 and the one between atom $\mathrm{O} 2$ of residue 55 and atom $\mathrm{O} 3$ of residue 14 are also broken. These intersheet HB are overtaken by two inter-FPD HB (colored in red), one between atom $\mathrm{O} 92$ of residue 33 and atom O3 of residue 55 and the other one between $\mathrm{O} 92$ of residue 2 and atom O3 of residue 30 . The data also reveal two transitory Hydrogen-bonding patterns involving atom O3 of residue 30 or residue 55 and atom O3 of residue 39 or residue 14 respectively. Atom $\mathrm{O} 92$ of residue 17 and atom $\mathrm{O} 3$ of residue 7 on the one side and atom $\mathrm{O} 92$ of residue 50 and atom O3 of residue 46 on the other side of Q4 structure form inter-FPD HB (colored in blue) as well. The formation of HB was followed along the $100 \mathrm{~ns}$ of the whole trajectory. The data reported come from one MD trajectory representative of all the MD trajectories performed in this work. 
HBOND SUMMARY:

saved to series hbond, output to file HBond_info_325_wat.txt,

data was sorted, intra-residue interactions are NOT included,

Distance cutoff is 3.25 angstroms, angle cutoff is 120.00 degrees

Hydrogen bond information dumped for occupancies $>0.00$

Dumping schematic of time series after each h-bond, key follows:

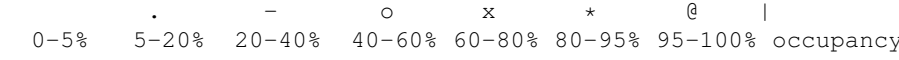

DONOR

atom\# :reseato

$394: 39002$

$\begin{aligned} 282 & : 30002 \\ 134 & : 14002\end{aligned}$

$\begin{array}{rr}134 & : 14002 \\ 542 & : 55002\end{array}$

solvent donor

solvent donor

$\begin{array}{ll}539 & : 55003 \\ 391 & : 39003 \\ 2 & : 3003\end{array}$

$\begin{aligned} 391 & : 39003 \\ 279 & : 30003\end{aligned}$

$279: 3003$

solvent donor

solvent donor

$$
\begin{aligned}
& \text { ACCEPTORH ACCEPTOR } \\
& \text { :rescatom atom\# : } \\
& \text { solvent acceptor } \\
& \text { solvent acceptor } \\
& \text { solvent acceptor }
\end{aligned}
$$$$
\begin{aligned}
& \text { ACCEPTORH ACCEPTOR } \\
& \text { tom\# : reseatom atom\# }: \text { reseatom }
\end{aligned}
$$$$
\text { 1 } 112.88 \text { distance }
$$$$
112.882 .885(0.17) \quad 27.09(14.020
$$$$
\text { ( }
$$$$
\begin{array}{ll} 
&
\end{array}
$$$$
\text { . }
$$$$
48.26 \quad 2.813(0.15) \quad 19.47(11.54)
$$$$
48.14=2.793(0.15)-19.47(11.54)
$$

$4.46 \quad 2.800(0.15) \quad 21.60(11.94)$ lifetime maxocc

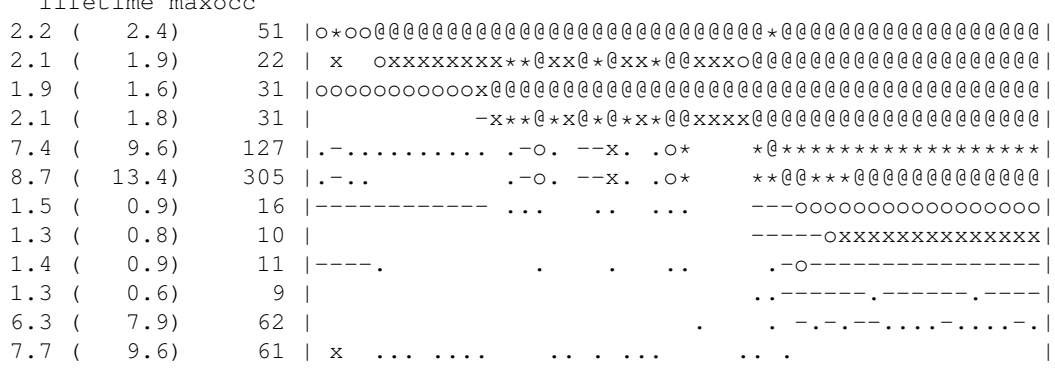

Figure S48d - Occupancy rate of the HB between solvent molecules and the carboxylic acid groups of tetramer Q4 initially involved in the formation of intersheet HB. The formation of HB was followed along the $100 \mathrm{~ns}$ of the whole trajectory. The data reported come from one MD trajectory representative of all the MD trajectories performed in this work. 


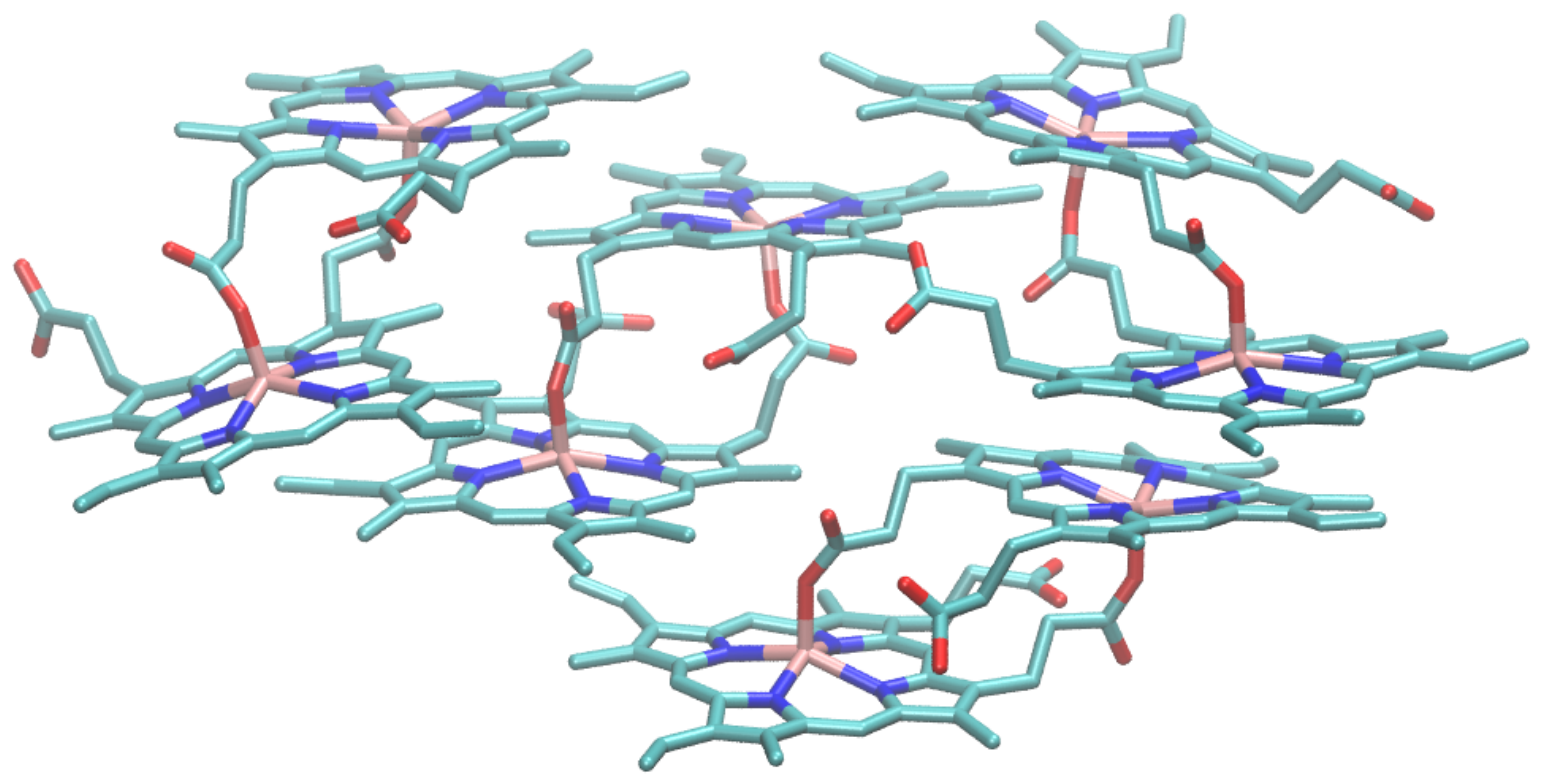

Figure S49 - Illustration of the evolution of the Q5 tetramer toward molecular substructures with reduced SASA. FPD enities are represented by sticks, carbon, nitrogen, oxygen, and iron atoms are colored in green, blue, red, and pink respectively. 